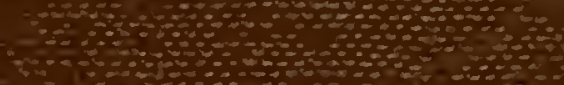


The Generic Names of

Moths of the World

Volume I Noctuoidea (part):

Noctudac, Agaristidac, and Nolidac

by Ian W B Nyc

The ain of this new series is to provide a complete list of all the generic nancs, their type-species, designations, and appropriate references, for cach of the families of moths treated. By ensuring that each generic name has its ty pe-species fixed in accordance w ith the Internutional Code of Zoologion Nomenclature, this series will rectify present confusion and form a basis for a more stable nomenclature. All generic names have been checked for homonymy, and if necessary replaced.

The first volume deals with the families Noctuidac, Agaristidac and Nolidac. It contains entrics for 5300 generic names, each placed to family or subfamily, with adelitional information giving the type-locality of the type-species and in the majority of cases the depository of the type-specimens.

This new series for the moths will be a companion to The Gencric Names of the Butterflics and their Type-species by F. Hemmng, published 111967 (reprinted 1972).

Dr I W B Nye graduated from Imperial College of Scicnee and Tee hnology, Unuversity of London, and then specialized in insect pests of cercal crope in this country. After joining the Commonwcalth Institute of Entomology he extended his studies to pests of cereal crops in East Africa, and of coconut palms in the Seychelles. For the last twelve years Dr Nye has becn on the staff of the British Museum (Natural History) making a special study of the Noctuid moths and their names. He represents the United Kingdom on the International · Commission on Zoological Nomenclature. 



\section{FRONTISPIECE}

FIG. I. Phalaena prasinana Linnaeus, lectotype $q$, [Europe].)

FIG. 2. Bena prasinana (Linnaeus), ô, Germany. See Bena Billberg, 1820.

FIG. 3. Pyralis fagana Fabricius, lectotype + , [Europe].)

FIG. 4. Pseudoips fagana (Fabricius), $q$, Switzerland. $\}$ See Pseudoips Hübner, I822.

FIG. 5. Zanclognatha lunalis (Scopoli), $\hat{\delta}$, Austria. See Zanclognatha Lederer, 1857.

FIG. 6. Herminia tarsicrinalis (Knoch), $\hat{\sigma}$, Austria. See Herminia, Latreille, 1802.

FIG. 7. Pachythrix hampsoni sp. n., holotype đ̃. Australia. \} See Pachythrix, Turner,

FIG. 8. Euplexia smaragdina Bethune-Baker, $\hat{0}$, New Guinea. $\} \quad$ I942.

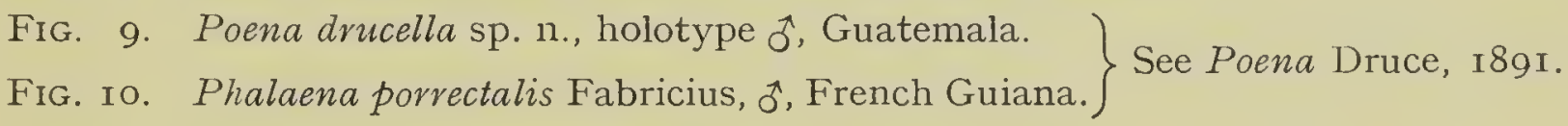

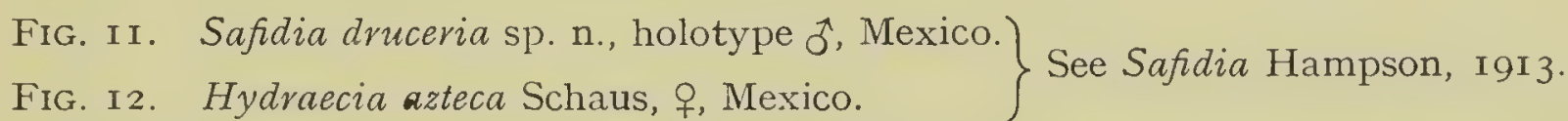

The black line under each figure represents the natural size of the moth, from the centre of its thorax to the apex of its fore wing. 



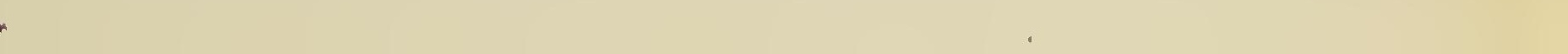


IRONTISLIECI:
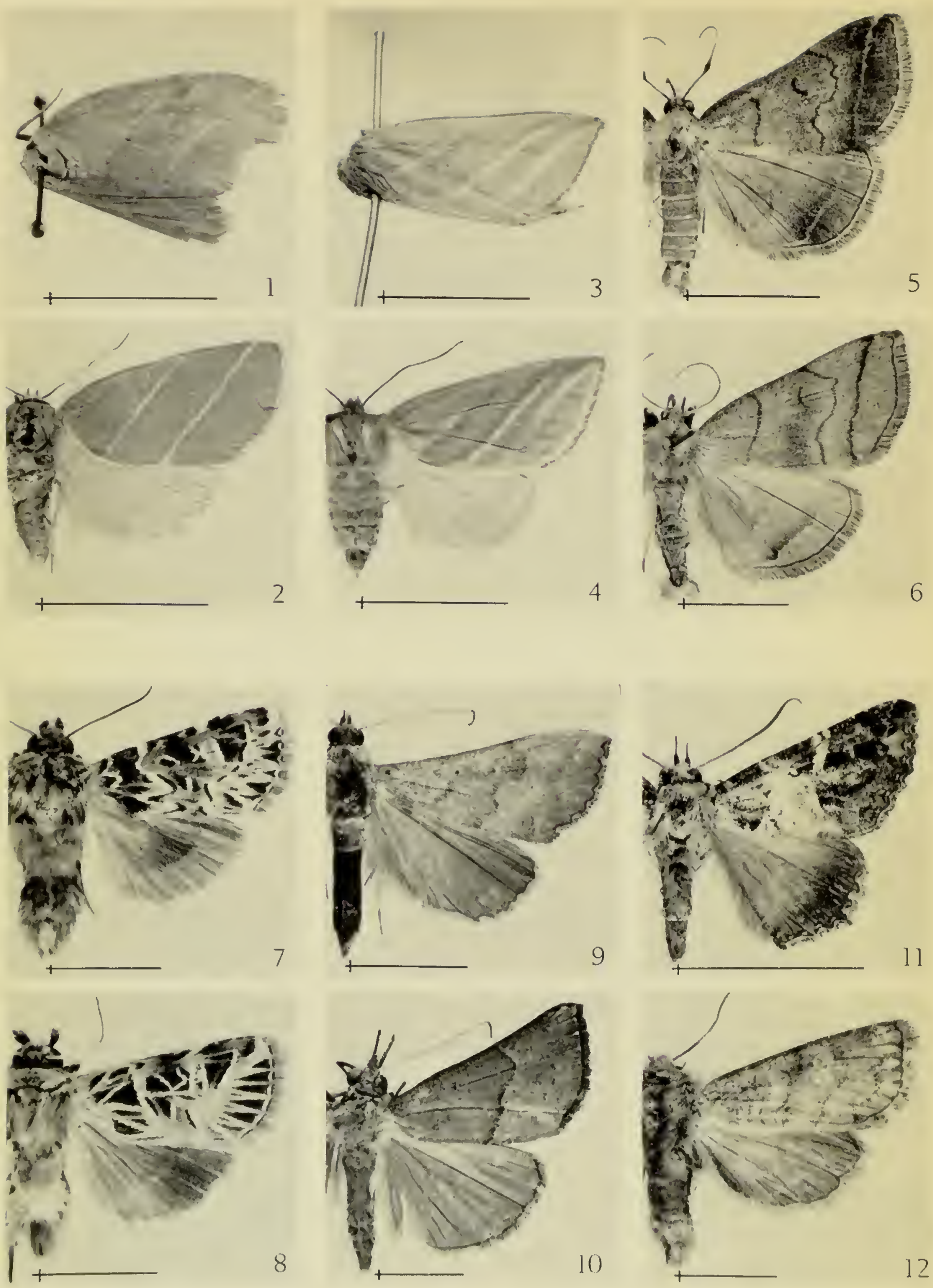


\title{
The Generic Names of Moths of the World
}

\author{
Volume I \\ Noctuoidea (part):
}

Noctuidae, Agaristidae, and Nolidae

By Ian W. B. Nye

Trustees of the
British Museum (Natural History) 
(C) Trustees of the British Museum (Natural History), 1975

Publication Number $77^{\circ}$

ISBN o $56500770 \mathrm{x}$

B.MNH/207/1m/7.75

Printed by Unwin Brothers Limited, The Gresham Press, Old Woking, Surrey, England 
Dedicated to the memory of the late Reginald J. Collins who, with the indefatigable assistance of Mrs Elsa B. Cummins, compiled the comprehensive card indexes to the names of the Lepidoptera, upon which this work is based. 



\section{Acknowledgements}

Many specialists have contributed either with information or by helping during my visits to their museums to verify the type-material in the collections under their care. In particular I would like to thank Mons. L. A. Berger, Tervuren; Dr E. Berio, Genoa; Dr I. F. B. Common, Canberra; Mr E. C. Dahms, Brisbane; Dr W. Dierl, Munich; Herr G. Ebert, Karlsruhe; Prof. J. G. Franclemont, Ithaca; Dr G. Friese, Eberswalde; Dr L. A. Gozmány, Budapest; Mr B. Gustafsson, Stockholm; Dr H. J. Hannemann, Berlin; Dr H. Inoue, Fujisawa; Dr F. Kasy and Herr R. Imb, Vienna; Dr P. Köhler, Buenos Aires; Dr N. P. Kristensen, Copenhagen; Mr I. Lansbury, Oxford; Dr E. G. Matthews, Adelaide; Mr A. Neboiss, Melbourne; Dr U. Roesler, Karlsruhe; Dr H. Schröder, Frankfurt-am-Main; Dr S. Sugi, Tokyo; Mrs I. L. Sukhareva, Leningrad; Mr E. Taylor, Oxford; Dr E. L. Todd, Washington; Dr L. Vári, Pretoria; Dr P. Viette, Paris; and Dr N. L. Wolff, Copenhagen. I greatly appreciated the comments given to me from time to time by the late Mons. C. Boursin, Paris.

In addition to those to whom this catalogue is dedicated, I wish to thank my colleagues Mr D. S. Fletcher, Mr A. H. Hayes, Mr A. Watson, Mr P. E. S. Whalley and Dr K. Sattler for their helpful discussion and constructive criticism on the many complex problems which continually developed. Mr W. H. T. Tams, my most able and generous mentor, was unstinting with his encyclopaedic knowledge and time whenever I needed to draw upon them. Our librarians, in particular Mr B. J. Clifton and Miss P. M. Gilbert were always most helpful and cheerfully traced some very cryptic references which had defeated me. Mr W. G. Tremewan and the staff of the Indexing Section are continually updating our records and have greatly contributed to the completeness of this work.

My special thanks are given to Mr P. Hurworth, Mrs B. A. Carter (née Spark), Mr M. A. K. Habershon and Mr R. M. Rowden who in succession over the years have so willingly assisted me in the many tedious stages in the preparation of this work, culminating in the excellent and accurate typescript produced by Mrs S. D. M. Knapp from the 6400 often complex manuscript entries.

Finally I wish to express my appreciation to my wife, Mary, for her assistance in so many ways throughout this entel prise, which was so much more extensive than I had anticipated. 



\section{Contents}

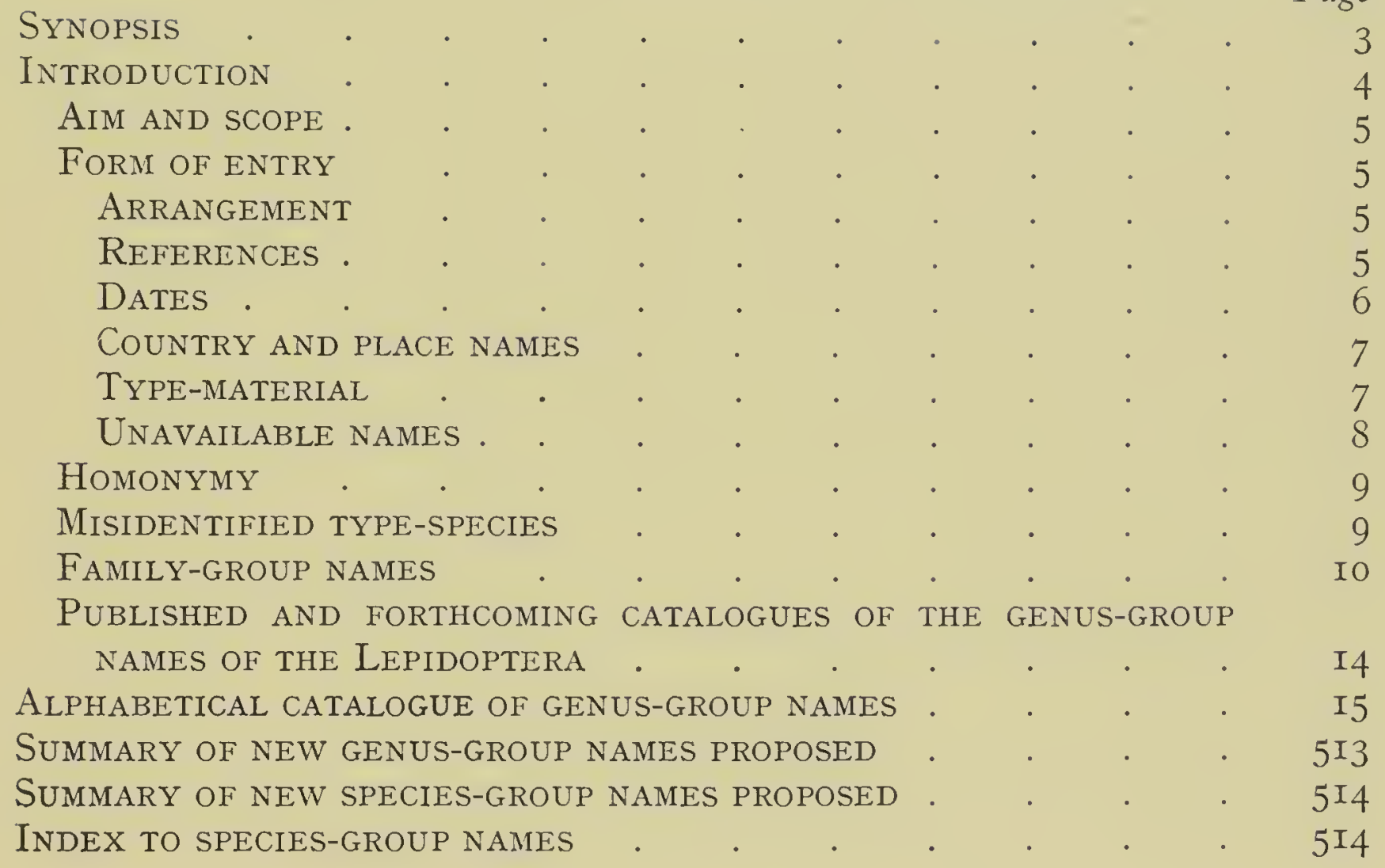





\section{Synopsis}

The 5300 genus-group names of the lepidopterous families Noctuidae, Agaristidae and Nolidae are listed alphabetically, with their type-species and relevant references. For each nomenclaturally available name the type-locality of the type-species is given, and for the majority the depository of the type or type-material. The family or subfamily placement of each name is indicated. New genus-group names are proposed for 55 junior homonyms for which no other replacement names are available. Two new species-group names are proposed for junior primary homonyms. Three type-species based on misidentifications are described as new. 


\section{Introduction}

CARD indexes are important individual tools for systematists, and over the decades those left by specialists have accumulated at this museum. During the period from I955 up to his death in I969, Mr R. J. Collins set himself the task of amalgamating those indexes relating to Lepidoptera into one systematic arrangement of $5^{\prime \prime} \times 3^{\prime \prime}$ cards of reasonably standard format, each bearing bibliographical data for one name. Every name was additionally cross-referenced by means of a second card filed in alphabetic order. Concurrently, other works, journals, catalogues and the Zoological Record were scanned, new cards added and old cards retyped. This operation has continued, and there now exists for the Lepidoptera a virtually complete file of over 280000 genus-group, species-group and infrasubspecific names with original references, forming the raw data on which to work.

In addition to the above Herculean task a supplementary Generic Index for each of the 26400 genus-group names of the Lepidoptera was started. Each card carries the genus-group name and basic bibliographic reference; a list of the species-group names originally included when it was proposed; the type-species and mode of type-fixation; the references to subsequent type-speciss designations; and the family or subfamily in which the name is placed.

Once 'The Generic Names of the Butterflies and their Type-species' by Hemming, I967, Bull. Br. Mus. nat. Hist. (Ent.) Suppl. 9: 509 pp. had been published, Reg Collins hoped that generic catalogues of the moths would soon follow as companion volumes. However, at the time of his pre-retirement death, the Generic Index, although it contained the vast majority of genus-group names, still lacked much of the detail and all of the checking required before any part of it could be published.

Among other things, D. S. Fletcher and I have searched more literature for typespecies designations and have checked the entire Generic Index against Neave, I939-I966, Nomenclator Zoologicus 1-6 to ensure that all names marked as 'Lep.' have been included and placed to family and in the majority of cases to subfamily level. Additionally, all genus-group names have been checked for homonymy, and the reference of the senior homonym entered on each of the relevant I6oo cards. 


\section{Aim and Scope}

The aim of this work has been to compile as complete a catalogue as possible of all the genus-group names in the Noctuidae, Agaristidae and Nolidae and to objectively define each of the nomenclaturally available names by linking it with its type-species determined according to The International Code of Zoological Nomenclature (Edn 2). To this has been added the family or subfamily placement of each name, the typelocality and in the majority of cases the depository of the type or type-material.

This catalogue contains all genus-group names of the appropriate families occurring in Neave's Nomenclator Zoologicus 1-6; together with additions gleaned from the Systematic Index described in the Introduction; and from the Zoological Record including volume $\mathbf{1 0 8}$ for I97I, published in I975. More recent relevant names that have come to my notice from journals and separates have been included up to the time of going to press.

No subjective synonymy of genus-group names has been attempted, except in the case of

(a) junior homonyms having no objective replacement name

(b) two or more genera having type-species which are subjectively synonymous; the genus-group names have then been cross-referenced by the phrase: 'See also Genus Author, date'.

In the case of a genus having a type-species which is a junior subjective synonym, then the senior synonym and reference is also included.

\section{Form of entry}

\section{ARRANGEMENT}

This catalogue is alphabetically arranged with junior homonyms, junior objective synonyms, and names not nomenclaturally available grouped together chronologically under their nomenclaturally available name. In every case these names are cross-referenced in the main alphabetic sequence.

\section{REFERENCES}

Titles of journals have been abbreviated as in the World List of Scientific Periodicals (Edn 4) or, if not included therein, according to the methods used in that edition. Nouns always start with a capital letter and adjectives with a small letter, unless it is customary in the country of origin of the journal for the word to begin with a capital, for example, Bull. mens. Soc. linn. Lyon but J. Proc. Linn. Soc. Similarly, Annls Mus.r. Hist. nat. belg. but Proc. R. ent. Soc. Lond. Abbreviations are followed by a full stop but contractions are not, for example, Coll. for College, but Colln for Collection; Stettin. for Stettiner, but Stettin for Stettin itself; univl. for universalis, but univl for universal. One other irritation, $J$. is for Journal, except in those references in which it is followed by a one letter abbreviation; it is then cited in the World List as $J l$, for example, $J l N$.Y. ent. Soc. 
Titles of books and other works have been abbreviated as though they were journals, using the 'Select List of Abbreviations Used' in volume 3 of the World List.

A full bibliography has not been included in this catalogue. It would amount to over I500 references. If necessary full titles of journals may be found from the World List or from the List of serial Publications in the British Museum (Natural History) Library. Similarly nearly all the other works cited are already in the Catalogue of Books, Manuscripts, Maps and Drawings in the British Museum (Natural History).

\section{DATES}

Following the Code, Recommendation 22A, dates of publication have been cited in square brackets if they have been determined from external evidence, and in parentheses if determined by evidence from the volume concerned. This has been followed in many cases by the better known title-page date to assist in finding the work in library catalogues, for example, [I825] I8I6, Verz. bekannter Schmett.

For the dates of publication, Sherborn's Index Animalium and the Catalogue of Books, Mamuscripts, Maps and Drawings in the British Museum (Natural History) have been accepted except where subsequent bibliographical research has shown that changes should be made. Hemming's classical work on the dates of Hübner has been followed. In the case of Seitz's Die Gross-Schmetterlinge der Erde, the English edition The Macrolepidoptera of the World was sometimes published ahead of the German; as an extreme example Plagideicta Warren was proposed in volume 11, page 339, and the part which contained it was dated 29 June I9I4 in the English, and as 20 July I937 in the German edition. I have cited in each case the edition having the earlier date printed at the top of the first page of each part. Although these dates indicated the intended rather than the actual date of publication, I have accepted them in preference to the date of receipt of the parts in London as listed by Griffin (1936, Trans. R. ent. Soc. Lond. 85: 243-280).

The dates of issue of the parts of Walker, F., I854-I866, List of the Specimens of lepidopterous Insects in the Collection of the British Museum, have not hitherto bcen published. In the table below, the date on the title page is compared with the date on which each printed part was submitted to and approved by the Trustees of the British Museum. It should be noted that parts I0, I2, I3, I6, 26, 3I, and 34 actually appeared in the year after that printed on the title page.

\begin{tabular}{|c|c|c|c|c|c|}
\hline Part & $\begin{array}{l}\text { Date on } \\
\text { title page }\end{array}$ & $\begin{array}{l}\text { Date on which } \\
\text { available for issue }\end{array}$ & Part & $\begin{array}{l}\text { Date on } \\
\text { title page }\end{array}$ & $\begin{array}{l}\text { Date on which } \\
\text { available for issue }\end{array}$ \\
\hline I & 1854 & I I Feb. I 854 & 9 & 1856 & 22 Nov. 1856 \\
\hline 2 & 1854 & I 3 May I 854 & Io & I 856 & ${ }_{14}$ Feb. 1857 \\
\hline 3 & 1855 & 9 Jun. I 855 & I I & I 857 & 9 Мау 1857 \\
\hline 4 & 1855 & I I Aug. I 855 & I 2 & I 857 & 9 Jan. 1858 \\
\hline 5 & 1855 & Io Nov. I 855 & I3 & I 857 & 9 Jan. 1858 \\
\hline 6 & 1855 & 8 Dec. I 855 & I4 & I 858 & 22 Мау 1858 \\
\hline 7 & I 856 & Io May 1856 & 15 & I 858 & 9 Oct. I 858 \\
\hline 8 & I 856 & 9 Aug. I 856 & I 6 & I 858 & 22 Jan. 1859 \\
\hline
\end{tabular}




\begin{tabular}{|c|c|c|c|c|c|}
\hline Part & $\begin{array}{l}\text { Date on } \\
\text { title page }\end{array}$ & $\begin{array}{l}\text { Date on which } \\
\text { available for issue }\end{array}$ & Part & $\begin{array}{l}\text { Date on } \\
\text { title page }\end{array}$ & $\begin{array}{l}\text { Date on which } \\
\text { available for issue }\end{array}$ \\
\hline I 7 & I 859 & I4 May I859 & 27 & I 863 & I 8 Apr. I 863 \\
\hline I 8 & I 859 & 8 Oct. I 859 & 28 & I 863 & I9 Dec. I 863 \\
\hline I9 & I 859 & Io Dec. I859 & 29 & I 864 & I9 Mar. I 864 \\
\hline 20 & I 860 & Io Mar. I 860 & 30 & I 864 & Io $\mathrm{Dec} .1864$ \\
\hline $2 \mathrm{I}$ & I 860 & Io Nov. I 860 & $3 I$ & 1864 & I I Feb. I 865 \\
\hline 22 & I 86 I & I 8 May I 86I & 32 & I 865 & 29 Apr. I 865 \\
\hline 23 & I 86I & I 4 Dec. I86I & 33 & 1865 & I4 Oct. I 865 \\
\hline 24 & I 862 & 8 Mar. I 862 & 34 & I865 & I3 Jan. I 866 \\
\hline 25 & I 862 & II Oct. I 862 & 35 & I 866 & 4 Aug. I 866 \\
\hline 26 & I 862 & I4 Feb. I863 & & & \\
\hline
\end{tabular}

\section{COUNTRY AND PLACE NAMES}

Country and place names are quoted from the original description, not from typespecimens. Names of countries in their current English form are given additionally in square brackets when they differ from or are lacking in the original description. The Times Atlas of the World (Edn 2) has been used as a standard except for some recently changed names such as Zaire for what was formerly Belgian Congo, and Bangladesh for East Pakistan. In general, geographical names are used for islands and island groups.

Place names are usually quoted unaltered from the original description unless they may be misleading out of context, for example, Wienergegend is cited as Vienna district. Added information is given in square brackets. Where the type-locality is not known or not stated in the original description, the country, or some of the countries, from which there are specimens in the BMNH collection is cited in square brackets after the depository, if known.

\section{TYPE-MATERIAL}

The use of the expression 'Type(s)' means that the author did not indicate whether his description was based on one or more than one specimen.

Other information on type-material has been taken from the original description, except for the depository which is only included if I have seen the type or been reliably informed of its presence. The depositories have been added as a useful bonus to over half the type-species where the information was fairly readily available from my own studies or from overseas colleagues. It has not been possible in the time available to make prolonged searches for types, and because no information is given it must not be assumed that the type is not in the British Museum (Natural History), or in any other museum. Similarly, in the case of syntypes when a depository is given, there may also be other syntypes in other collections.

The abbreviations used for depositories are listed here in alphabetical order of the town in which they are situated.

(SAM, Adelaide)

(MNHU, Berlin)
South Australian Museum.

Museum für Naturkunde der Humboldt-Universität. 
(MAK, Bonn)

(MCZ, Boston)

(QM, Brisbane)

(IRSNB, Brussels)

(TM, Budapest)

(Univ. Buenos Aires)

(ANIC, Canberra)

(UZM, Copenhagen)

(DEI, Eberswalde)

(SNG, Frankfurt)

(MCSN, Genoa)

(Colln E. Berio, Genoa)

(MZC, Havana)

(CU, Ithaca)

(Collu J. G. Franclemont, Ithaca)

(LN, Karlsruhe)

(RNH, Leiden)

(ZI, Leningrad)

(UZI, Lund)

(BMNH)

(Colln E. P. Wiltshire, London)

(LS, London)

(NMV, Melbourne)

(ZSBS, Munich)

(AMNH, New York)

(CNC, Ottawa)

(UM, Oxford)

(MNHN, Paris)

(C.I, Pittsburgh)

(TM, Pretoria)

(EIHU, Sapporo)

(Ehime Univ. Shikoku)

(NR, Stockholm)

(AM, Sydney)

(MRAC, Tervuren)

(Colln H. Inoue, Tokyo)

(Colln S. Sugi, Tokyo)

(IZUU, Uppsala)

(NM, Vienna)

(USNM, Washington)

(S.M, Wiesbaden)
Zoologisches Forschungsinstitut und Museum Alexander líoenig.

Museum of Comparative Zoology, Harvard University.

Queensland Museum.

Institut Royal des Sciences Naturelles de Belgique.

Természettudományi Múzeum.

University of Buenos Aires.

Australian National Insect Collection, C.S.I.R.O.

Universitetets Zoologiske MIuseum.

Deutsches Entomologisches Institut.

Forschungsinstitut Senckenberg der Senckenbergischen

Naturforschenden Gesellschaft, Franlifurt-am-Main.

Museo Civico di Storia Naturale.

Dr E. Berio, c/o Museo Civico di Storia Naturale.

Museo Zoologico Cubano.

Cornell University.

Professor J. G. Franclemont, Cornell University.

Landessammlungen für Naturkunde.

Rijksmuseum van Natuurlijke Historie.

Zoological Institute, Academy of Sciences of the U.S.S.R.

Universitetets Zoologislia Institution.

British Museum (Natural History), London.

Mr E. P. Wiltshire, c/o British Museum (Natural History).

Linnean Society.

National Museum of Victoria.

Zoologische Sammlung des Bayerischen Staates.

American Museum of Natural History.

Canadian National Collection, Entomology Research

Institute.

University Museum.

Musćum National d'Histoire Naturelle.

Carnegie Museum.

Transvaal Museum.

Entomological Institute, Holkaido University.

Ehime University, Shikoku 1., Japan.

Naturhistoriska Riksmuseet.

Australian Museum.

Musée Royal de l’Afrique Centrale.

Dr H. Inoue, Otsuma Woman's University.

Dr S. Sugi, I4-I2, Omori-IKita 4, Otaku, Tokyo.

Institute of Zoology, University of Uppsala.

Naturhistorisches Museum.

National Museum of Natural History, formerly United States

National Museum.

Stadtisches Museum.

\section{UNAVAILABLE NAMES}

Unavailable names, i.e. names which are not nomenclaturally available under the provisions of the Code, are preceded by a double dagger $\left(\begin{array}{l}+ \\ +\end{array}\right)$ and the reason why they must not be used is given.

Names were often recorded in Neave's Nomenclator Zoologicus as '(pro A-us Author, date)' without positive indication as to whether they were misspellings 
having no nomenclatural availability or whether they were emendations having nomenclatural availability. They were usually misspellings, but all have been checked. Other misspellings have been included if they have come to my notice, but no deliberate search has been made for them.

Some manuscript names have been included as ' $B$-US Author, no published reference found'. The majority consist of about 80 genus-group names placed in the BMNH collection of Hypeninae by Hampson for genera which he intended to describe as new. Some of these names, for example Eugoniella Kaye \& Lamont, I927, have been unintentionally made available by authors who did not know that they were at that time unpublished.

\section{Homonymy}

Names that have been proposed expressly to replace junior homonyms, and junior objective synonyms that have been used for the same purpose, are referred to in this catalogue as objective replacement names. Junior subjective synonyms that have been used to replace preoccupied senior synonyms are referred to as subjective replacement names.

Out of the 4800 nomenclaturally available generic names covered in this catalogue 280 were found to be junior homonyms, and of these, objective replacement names were available for I50. Each of the remaining I30 homonymous generic names was studied and wherever it was possible to find a genus whose type-species was congeneric with that of the homonym then the latter has been placed as a synonym of it. There remained, however, 55 for which there were neither objective nor subjective replacement names and new names have been proposed for these. A summary of the new names is given towards the end of the catalogue on page $5 \mathrm{r} 3$.

During the preparation of his Catalogue of the Lepidoptera Phalaenae in the British Museum from I 898 to I920, Hampson placed in the collection labels bearing the manuscript names of genera which he intended to describe as new. He also used these names on his determination labels on specimens returned to collectors or curators. As a result of this, some of the names were used in works by authors who did not realise that they were at that time unpublished. Such genus-group names, if associated with nomenclaturally available species-group names and published before I93I are thereby made nomenclaturally available and have to be attributed to the author who first published them. Collins (1962, Ann. Mag. nat. Hist. (I3) 5: I2I-I26) has published notes on 56 of Hampson's generic names which were made available by other authors who anticipated him. The 27 which belong to families relevant to this catalogue have been treated as junior homonyms.

\section{Misidentified Type-species}

Under the Code, Article 70, it is to be assumed that an author has correctly identified the nominal species that he

(I) referred to a new genus when he proposed it, or

(2) designated as the type-species of a new or established genus. 
In the present catalogue there are several genera having type-species considered to be misidentifications. Under the Code, Article 70, these cases should be referred to the Commission to designate as the type-species whichever species will in its judgement best serve stability and uniformity of nomenclature. In the catalogue I have suggested the species that appears to fulfil these conditions.

In three genera the nominal species which have been designated as type-species have been shown to be misidentifications of hitherto unnamed species. In these instances I have described them as new species. This will enable cases to be submitted to the Commission. The genera and new specific names are summarised towards the end of the catalogue on page 5I4.

\section{Family-group Names}

No serious study of the family-group names associated with the Noctuidae and related families has yet been published, and considerable confusion at present exists. It is exceptional to find any two authors who use the same combination of subfamily names within the Noctuidae. Having started a study with the intention of basing the family-gioup names for this catalogue on unassailable foundations of priority I rapidly found myself in a predicament. The universally used names of the two large worldwide subfamilies Hadeninae and Cuculliinae certainly do not have priority and would have to be changed to Orthosiinae and Xylininae respectively. To do so would merely add to the confusion as in all probability neither of these names has been used for decades. It will be necessary to invoke the recently amended Articles $23(\mathrm{a}-\mathrm{b}$ ) and 79, as published in 1972 (Bull. zool. Nom. 29: I85) but although many more than the required ten usages of the well known junior names could be quoted, it is not possible with the very scattered literature on the Noctuidae to know that the senior names have not been applied at all in the last 50 years.

In the hope of attaining acceptable stability for the family-group names, I have studied each and have tried to select the one having the greatest current usage. The Comparative Table of Family-Group Name Usages summarises the classification in three standard works of the first half of the century, and, a further six, more recent works representing various parts of the world.

No problem arises with the usage of Nolidae and Agaristidae. These names have been consistently applied for many years to groups considered to be either subfamilies of the Arctiidae and Noctuidae respectively, or families in the Noctuoidea.

The Noctuidae have in the past also been known as Phalaenidae, Caradrinidae and Agrotidac, but under a ruling of the Commission (1957, Opin. Decl. int. Commn zool. Nom. 15: Opinion 450) the family name Noctuidae and the nominate subfamily Noctuinae are now stabilized and almost universally used.

The Heliothinae though recognized as a subfamily have not been separated from the Noctuinae in this catalogue. The name is sometimes spelled as Heliothiinae or Heliothidinae, but under the amended Code, Article 29(d), as it was based on Heliothidi Boisduval, I828, the stem Helioth- should not be changed. In any case, it is customary in the Lepidoptera to avoid the clumsy -ididac termination, for 
example, Pyralis, family Pyralidae (Opinion 450) not Pyralididae; Pieris, family Pieridae (Opinion 500). The name Heliothinae has priority over Melicleptriinae, used in parts of Europe.

The Hadeninae and Cuculliinae have been mentioned above and so far as I am aware are universally used.

The Acronictinae and Amphipyrinae though recognized as subfamilies have not been separated in this catalogue. The Acronictinae are known as the Apatelinae in Japan and parts of Europe.

The Acontiinae are also known as the Erastriinae and Jaspidiinae. The former is incorrect as Erastria, although it has in the past been wrongly used in the Noctuidae, is correctly placed in the Geometridae. The name Jaspidiinae has limited usage in parts of Europe.

The Euteliinae and Stictopterinae both have universal usage, as also has Sarrothripinae except in Japan and in limited parts of Europe.

The Chloephorinae have been known under several names none of which has gained general acceptance. The problem was discussed with Mr D. S. Fletcher and we agreed that in the circumstances priority was the best arbiter. Chloephorinae Stainton, I859, was the earliest suprageneric usage that could be found and it has been used within the last 50 years by Gaede (I929, Die Tierwelt Deutschlands 14(2): 22) and by Fletcher (1972, in Kloet \& Hincks, A Check List of British Insects (Edn 2)).

The Pantheinae and Plusinae have had general usage for at least 35 years; and the Catocalinae for over 70 years.

The greatest problem concerns the name of the subfamily containing the largest number of genera and species, the Ophiderinae of this catalogue (the Noctuinae of Hampson). This subfamily was separated from the Catocalinae by Hampson on the absence of spines on the mid tibiae, an admittedly poor character as in some genera both conditions occur. McDunnough (I938) and Sugi (1958) have united the two as Catocalinae, while Forbes (I954) retained the Catocalinae in a restricted sense and combined the remainder as the Erebinae which has priority dating back to I8I5. In this catalogue I am retaining the traditional Hampsonian Catocalinae (having spines on mid tibiae), but recognize that they must be subdivided at least into the Catocalinae in the strict sense plus the Erebinae. Those genera without mid tibial spines are retained as Ophiderinae. An immense amount of research is required into this large and uncatalogued subfamily of over IIoo genera which must be reclassified particularly where they merge into the following two subfamilies.

The Hypeninae and Herminiinae have had general usage this century but as in this catalogue are frequently not separated.

The Hyblaeidae, a very small group, was formerly regarded as an aberrant subfamily of the Noctuidae but has now been placed by Brock (I97I, J. nat. Hist. 5: 35$)$ as a family in the Aegerioidea. 
Hampson

I 900-I 920

Cat. Lepid.

Phalaenae Br. Mus.

2-13, Suppls

Nolinae

AGARISTIDAE

NOCTUIDAE

Agrotinae

Hadeninae

Cucullianae

Acronyctinae

(Zenobianae)

Erastrianae

(Eustrotiinae)

Eutelianae

(Phlogophorinae)

Stictopterinae

(Odontodinae)

Sarrothripinae

Acontianae

(Vestermannianae)

Mominae

(Diphtherinae)

Phytometrinae

(Plusianae)

Catocalinae

Noctuinae

Hypeninae

(Polypogoninae)

Hyblaeinae
2

Warren/Gaede/Draudt I 909-I 937

in Seitz,

Gross-Schmett.

Erde 3, 7, 11, 15

Nolinae

AGARISTIDAE

NOCTUIDAE

(AGROTIDAE)

$\left\{\begin{array}{c}\text { Agrotinae } \\ \text { (Euxoinae) } \\ \text { Melicleptriinae }\end{array}\right.$

Hadeninae

Cucullianae

$\left\{\begin{array}{c}\text { Bryophilinae } \\ \text { (Netachrostinae) } \\ \text { Acronyctinae } \\ \text { (Acronictinae) } \\ \text { Amphipyrinae } \\ \text { (Zenobiinae) }\end{array}\right.$

Erastrianae

Eutelianae

Stictopterinae

Sarrothripinae

Acontianae

Acronyctinae (part)

Phytometrinae

Catocalinae

Noctuinae

Noctuinae (part)

Hyblaeinae
3

McDunnough

I 938

Mem. sth. Calif.

Acad. Sci. 1

(Check List Lepid.

Canada \& U.S.A.)

NOLIDAE

NOLIDAE

AGARISTIDAE

AGARISTIDAE

PHALAENIDAE

NOCTUIDAE

Phalaeninae

Heliothiinae

Hadeninae

Cuculliinae

\} Acronictinae

Amphipyrinae

Acontiinae

Euteliinae

Absent from fauna Absent from fauna

Sarrothripinae

Sarrothripinae

Absent from fauna

Absent from fauna

Pantheinae

Pantheinae

Plusiinae

Plusiinae

Catocalinae

$\left\{\begin{array}{l}\text { Catocalinae } \\ \text { Erebinae }\end{array}\right\}$

$\left\{\begin{array}{l}\text { Catocalinae (part) } \\ \text { Rivulinae }\end{array}\right\}$ Erebinae (part)

$\{$ Hypeninae

Herminiinae

Hypeninae

Herminiinae

Hyblaeinae

Absent from fauna
NOLIDAE

AGARISTIDA

NOCTUIDAE

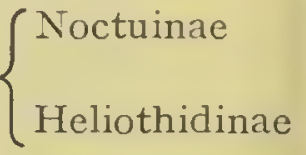

Hadeninae

Cuculliinae

$\left\{\begin{array}{l}\text { Cryphiinae } \\ \text { Apatelinae } \\ \text { Amphipyrinae }\end{array}\right.$

Acontiinae

Euteliinae

Stictopterinae

Nycteolinae

Nycteolinae (part)

Pantheinae

Plusiinae

Catocalinae

Catocalinae (part)

Hypeninae Herminiinae

H I BLAEIDAF

NOTES

Column I. The names in parentheses are those used by Hampson as subfamily names either before or after the relevant volume of his catalogue had been published.

Column 2. The names in parentheses are those used by Warren or by Gaede as subfamily names in the earlie volumes of the work.

Column 4. The subfamily name Hypenodinae was also used. Forbes applicd tlis name to a group of four smal genera placed between the Erebinae and Hypeninae, with the comment that he was doubtful whether the group should be rated as a subfamily or as a tribc. 
6

Forster \&

Wohlfahrt

I $95^{6}$, I 97 I

Schmett.

Mitteleur. 3, 4

NOLIDAE

Absent from fauna

NOCTUIDAE

Noctuinae

Melicleptriinae

Hadeninae

Cuculliinae

Bryophilinae

Apatelinae

Amphipyrinae

Jaspidiinae

Euteliinae

Absent from fauna

Nycteolinae

Beninae

Apatelinae (part)

Plusinae

Catocalinae

Ophiderinae

Hypeninae

HYBLAEIDAE
7

Dufay

I960

Vie Milieu

12 (Suppl.)

(Faune...

Pyrénées-Orientales)

NOLINAE

Absent from fauna

NOCTUIDAE

Noctuinae

Melicleptriinae

Hadeninae

Cuculliinae

\} Apatelinae

Amphipyrinae

Jaspidiinae

Euteliinae

Absent from fauna

Nycteolinae

Westermanniinae

Pantheinae

Plusinae

Catocalinae

$\left\{\begin{array}{l}\text { Catocalinae } \\ \text { Erebiinae }\end{array}\right.$
$\left\{\begin{array}{l}\text { Erebiinae (part) } \\ \text { Rivulinae }\end{array}\right.$

$\left\{\begin{array}{l}\text { Hypeninae } \\ \text { Herminiinae }\end{array}\right.$
Catocalinae

\}Ophiderinae

\} Hypeninae

HYBLAEIDAE

IO

Family and subfamily names

recognized

NOLIDAE NOLIDAE

AGARISTIDAE AGARISTIDAE

NOCTUIDAE NOCTUIDAE

Noctuinae

Noctuinae

Heliothidinae

Heliothinae

Hadeninae

Cuculliinae

Acronictinae

Amphipyrinae

Amphipyrinae

Erastriinae

Acontiinae

Euteliinae

Stictopterinae

Sarrothripinae

Chloephorinae

Pantheinae

Plusiinae

$\left\{\begin{array}{l}\text { Catocalinae } \\ \text { Erebinae }\end{array}\right.$

Ophiderinae

$\{$ Hypeninae

$\{$ Herminiinae

HYBLAEIDAE
HYBLAEIDAE

Column ıо. The Heliothinae, Amphipyrinae, Erebinae and Herminiinae are recognized as subfamilies, but the constituent genera have not yet been separated on a world basis in any work. It has therefore been impracticable to do so in this catalogue, and so the genera have been listed (with some reacrangements) within the larger subfamilies as used by Hampson. 


\section{Published and Forthcoming Catalogues of the Genus-group Names of the Lepidoptera}

The following catalogues, dealing with some of the 26500 genus-group names of the Lepidoptera, have already been published in the Bulletin of the British Museum (Natural History) Entomological series.

Hemming, F. 1967. The generic names of the butterflies and their type-species (Lepidoptera: Rhopalocera). Bull. Br. Mus. nat. Hist. (Ent.) Suppl. 9. 509 pp. [3097 genus-group names].

Whalley, P. E. S. I970. A synonymic catalogue of the genera of Phycitinae (Lepidoptera: Pyralidae) of the world. Bull. Br. Mus. nat. Hist. (Ent.) 25: 31-72 [673 genus-group names].

TREMEWAN, W. G. I973. A catalogue of the genus-group names of the Zygaenidae (Lepidoptera). Bull. Br. Mus. nat. Hist. (Ent.) 28: II I-I 5I [37I genus-group names].

SATtLER, K. I973. A catalogue of the family-group and genus-group names of the Gelechiidae, Holcopogonidae, Lecithoceridae and Symmocidae (Lepidoptera). Bull. Br. Mus, nat. Hist. (Ent.) 28: I 53-282 [3 I family-group and Iro3 genus-group names].

Sattler, K. \& Tremewan, W. G. 1974. A catalogue of the family-group and genus-group names of the Coleophoridae (Lepidoptera). Bull. Br. Mus. nat. Hist. (Ent.) 30:I83-2I 4 [28 family-group and 76 genus-group names].

The present volume is the first of a series of catalogues which will include the genus-group names of the following families and superfamilies:

Noctuoidea (part): Arctiidae and Ctenuchidae by A. Watson.

Noctuoidea (part): Lymantriidae, Notodontidac, Thyretidae and Dioptidae by I. W. B. Nye. Geometroidea by D. S. Fletcher.

Bombycoidea and Sphingoidea by I. W. B. Nye \& A. H. Hayes.

Cossoidea and Castnioidea by I. W. B. Nye. 


\section{Alphabetical Catalogue of Genus-group Names}

SYMBOLS USED

‡. A name not nomenclaturally available under the Code.

[ ]. Added information derived from external evidence.

[1825] I8I6. Date of publication derived from external evidence followed by the title-page date.

ABbreviations USED FOR FAMILY-GROUP NAMES

ACoN Acontiinae

ACRO Acronictinae + Amphipyrinae

AGAR Agaristidae

CATo Catocalinae + Erebinae

CHLO Chloephorinae

cucu Cuculliinae

EUTE Euteliinae

HADE Hadeninae
HYPE Hypeninae + Herminiinae

Noct Noctuinae + Heliothinae

Noli Nolidae

OPHI Ophiderinae

PANT Pantheinae

PLUS Plusiinae

SARR Sarrothripinae

sTIC Stictopterinae

ABABLEMMA nom. n. for Microblemma Hampson, I9io.

ACON

Type-species: Microblemma discipuncta Hampson, I9Io, Cat. Lepid. Phalaenae Br. Mus. 10: 33, fig. I2, by original designation (for Microblemma Hampson). Holotype $ᄋ$, PANAMA : La Chorrera (BMNH).

MICROBLEMMA Hampson, I9io, Cat. Lepid. Phalaenae Br. Mus. 10: 3, 33.

Type-species: Microblemma discipuncta Hampson, I9Io, by original designation.

A junior homonym of Microblemma Semenow, I889, Horae Soc. ent. ross. 24: 213,-Coleoptera. The objective replacement name is Abablemma nom. $\mathrm{n}$.

ABACENA Walker, [1866] I 865, List Specimens lepid. Insects Colln Br. Mus. 34: I270. ACON Type-species: Abacena discalis Walker, [1866] I865, ibidem 34: I270, by monotypy. Holotype , , [BraziL]: [R. Amazon], [Tefé] Ega (BMNH).

ABAGROTIS Smith, I89o, Bull. U.S. natn. Mus. 38: 9, 49.

NOCT

Type-species: Agrotis erratica Smith, I89o, Trans. Am. ent. Soc. 17: 4I, by original designation, on page 9. Syntypes, [U.S.A.]: Cal[if]., Sierra Nevada; Calif. (USNM, Washington).

ABLEPHARON Grote, I873, Bull. Buffalo Soc. nat. Sci. 1: III.

ACRO

Type-species: Leucania henrici Grote, I873, ibidem 1 : Io, pl. I, fig. I 5, by subsequent designation by Grote, 1874, ibidem 2: 2 I. Syntypes ô. ㅇ, [U.S.A.]: New York State (BMNH).

ABLEPTINA Prout, I927, Trans. ent. Soc. Lond. 75:227. HYPE

Type-species: Ableptina delospila Prout, I927, ibidem 75: 227, by original designation. Syntypes I $\mathcal{O}^{\prime}, 4$ ㅇ, [SÃo Tomé I., W. Africa] São Thomé I. (BMNH).

ABLITA Dyar, I9I4, Proc. U.S. natn. Mus. 47: I83.

ACRO

Type-species: Neolita adin Schaus, I9I I, Ann. Mag. nat. Hist. (8) 8: 107, by original designation. Type(s) 우, Costa RICA: Laguna (USNM, Washington).

ABOLLA Rogenhofer, 1874, in Felder \& Rogenhofer, Reise öst. Fregatte Novara (Zool.) 2 (Abt. 2): pl. III.

OPHI

Type-species: Abolla pellicosta Felder \& Rogenhofer, 1874, ibidem 2 (Abt. 2): pl. IIx, fig. 5 , by monotypy. Type(s) $\delta$, Brazil: Maranham (BMNH).

In the caption to pl. II , fig. 5, Abolla was clearly attributed to Rogenhofer whereas pellicosta was attributed to both authors. 
ABRIESA Swinhoe, I900, Ann. Mag. nat. Hist. (7) 6:3 32. OPHI Type-species: Abriesa derna Swinhoe, I900, ibidem (7) 6:312, by original designation. Holotype 웅. Australia: Qd, Dawson (BMNH).

ABROMIAS Billberg, I820, Enumeratio Insect. Mus. G. J. Billberg: 88.

ACRO

Type-species: Phalaena polyodon Clerck sensu Linnaeus, I76I, Fauna Suecica (Edn 2): 322 [a misidentification later described as Phalaena monoglypha Hufnagel, 1766, Berlin. Mag. 3: 308], by subsequent designation by Berio, 1966, Annali Mus. civ. Stor. nat. Giacomo Doria 76: 47. Syntypes (of P. monoglypha), Germany: Berlin.

Incorrect type-species designation by Tams, I939, Entomologist 72: I 36 of $P$. monoglypha Hufnagel, a name not originally included in Abromias and not linked by Tams with one of the originally included names.

Abromias when proposed contained 13 species. 'The first two 'hepatica Linn.' and 'polyodon Linn.' were originally proposed by Clerck and it has long been accepted that $P$. polyodon Clerck sensu Linnaeus, I76I, was at that time a new species (later described as P. monogly pha Hufnagel, I 766) and was a misidentification of Phalaena polyodon Clerck, I 759, Icones Insect. rariorm 1: pl. 2, fig. 2. This is a case of a misidentified type-species which under the Code, Article $70(a)$ should be referred to the Commission.

XYLOPHASIA Stephens, I 829, Illust. Br. Ent. (Haustellata) 2: 174 .

Type-species: Phalaena polyodon Clerck sensu Linnaeus, I76I [= Phalaena monoglypha Hufnagel, I766], by subsequent designation by INestwood, I840, Synopsis Genera $\mathrm{Br}$. Insects: 94 but cited as 'polyodon L'.

Invalid designation of type-species: $P$. polyodon was also designated by Boisduval, I 836, Hist. nat. Insectes (Lépid.) 1: 144. Boisduval in his lengthy Introduction, up to page I54, reviewed earlier classifications and designated up to three different type-species for each generic name. In his 'Exposé de notre méthode' from page i 55-690, no typespecies designations were made for the genera he himself used. Under the Code, Article 69 (a)(iii) the type-designation of an author is eligible for consideration if he states that it is the type ' . . . and if it is clear that he himself accepts it as the type-species'. Boisduval's type-designations although clearly stated do not fulfil the last requirement and so are invalid. Even though Boisduval's i 836 work was well known to lepidopterists, the typedesignations contained in it have not in the past been accepted by Hemming or by other authors. The acceptance of Boisduval's designation in this case would make no difference to the generic concept.

Xylophasia when proposed contained nine species including 'polyodon Linne'. There is no doubt, from Stephens' description, that the species included was that now known as monoglypha Hufnagel. The information given above for Abromias also applies here.

$X y l o p h a s i a$ is a junior objective synonym of Abromias Billberg, I8zo.

AGROSTOBIA Boie, x835. Isis, Leipzig 19: 325 .

Type-species: Phalaena polyodon Clerck sensu Linnaeus, I76I, [ = Phalaena monoglypha Hufnagel, I766], by PRESENT DESIGNATION.

Agrostobia when proposed contained five species including 'polyodon'. There is no doubt from the context that the species which Boie included was that now known as monoglypha Hufnagel. The information given above for Abromias also applies here.

Agrostobia is a junior objective synonym of Abromias Billberg, I820.

ABROSTOLA Ochsenheimer, I816, Schmett. Eur. 4: 88.

PLUS

Type-species: Phalaena triplasia Linnaeus, 1758 , Syst. Nat. (Edn 10) 1: 5I7, by subsequent designation by Duponchel, 1829, in Godart \& Duponchel, Hist. nat. Lépid. Papillons Fr. 7 (2): 72. Type(s), Type-Locality not stated (LS, London) [Europe]. $\ddagger U N C A$ Oken, I8I 5, Okens Lehrbuch Naturg. 3: 689.

Included in a work rejected for nomenclatural purposes by Int. Commn zool. Nom., I956, Opin. Decl. int. Commn zool. Nom. 14 Opinion 4I 7: 3 .

Originally included species: Phalaena triplasia Linnaeus, I758; Phalaena gamma Linnaeus, 1758; Phalaena mi Clerck, 1759. 
HABROSTOLA Sodoffsky, i837, Bull. Soc. imp. Nat. Moscou 1837 (6): 88.

An unjustified emendation of Abrostola Ochsenheimer, I8I6.

UNCA Lhomme, [1929], Cat. Lépid. Fr. Belg. 1: 32 I.

Type-species: Phalaena triplasia Linnaeus, I758, by original designation.

Although Unca was attributed to Oken by Lhomme, this was the next published usage after $\ddagger U n c a$ Oken, I8I5, and so became nomenclaturally available. On page II 5 of his catalogue Lhomme stated that he was following Hampson's classification, but Hampson, 1913, Cat. Lepid. Phalaenae Br. Mus. 13: 582 used Abrostola Ochsenheimer for triplasia. However, in his personal interleaved copy Hampson had changed this to 'Unca Oken'. It is probable that Lhomme was directly, or indirectly through Joannis, in communication with Hampson, and was informed of the unpublished change.

Unca Lhomme is a junior objective synonym of Abrostola Ochsenheimer, I816.

ABSEUDRAPA Berio, 1956, Memorie Soc. ent. ital. 35: 27.

OPHI

Type species: Tachosa metaphaearia Walker, 1869, in Chapman, Proc. nat. Hist. Soc.

Glasgow 1: 346 , by original designation. Type(s) $q$, Congo (BMNH).

ABURINA Möschler, I887, Abh. senckenb. naturforsch. Ges. 15: 90.

OPH I

Type-species: Aburina sobrina Möschler, I887, ibidem 15: 9I, fig. I3, by monotypy.

Syntypes 4 잉. [GHana] Gold Coast: Aburi (MNHU, Berlin).

See also Ugana Swinhoe, Igog.

ACACALIS Agassiz, I846. See Acacallis Hübner, [1823].

OPHI

ACACALLIS Hübner, [1823] I816, Verz. bekannter Schmett.: 265.

OPHI

Type-species: Phalaena procus Cramer, I777, Uitlandsche Kapellen 2:85, pl. I49, fig.

$\mathrm{G}$, by monotypy, but cited as $\$$ procax Cram.', an incorrect subsequent spelling. Type(s),

SURINAM.

$A C A C A$ LIS Agassiz, 1846, Nomencl. zool. Index univl.: I.

An unjustified emendation of Acacallis Hübner, [1823].

ACACHMENA Turner, 1908, Trans. Proc. R. Soc. S. Aust. 32: 62

CHLO

Type-species: Acachmena oenocrossa Turner, I908, ibidem 32: 62, by monotypy. Holotype + , Australia: Qd, Kuranda (ANIC, Canberra).

ACAENICA Hampson, I918, Novit. zool. 25: i82.

$\operatorname{ACON}$

Type-species: Acaenica diaperas Hampson, I9I8, ibidem 25 : I83, by original designation.

Holotype $\hat{\sigma}$, [Mozambioue] Port. E. Afr.: Mt Chiperone (BMNH).

ACANTHERMIA Hampson, 1926, Descr. new Genera Species Lepid. Phalaenae Subfamily

Noctuinae Br. Mus.: 248.

OPHI

Type-species: Acanthermia dyari Hampson, I 926, ibidem: 249, by original designation. Holotype $\sigma^{*}$. Mexico: Venadio, Sinaloa, bearing a label in Dyar's handwriting †'Rhosologia stigmatica Dyar', but no published description under this name has been found (BMNH).

ACANTHOCOLES Turner, Igo3, Trans. Proc. R. Soc. S. Aust. $27: 25$.

CATO

Type-species: Acanthocoles eucesta Turner, I903, ibidem 27: 26, by monotypy. Holotype 9 , Australia: N.S.W., Cobar (ANIC, Canberra).

ACANTHODELTA Hampson, I908, Ann. Mag. nat. Hist. (8) 1: 487.

CATO

Type-species: Acanthodelta distriga Hampson, I908, ibidem (8) 1:487, by monotypy. Holotype $\hat{\sigma}$, South Africa: Cape Province, Table Mt (BMNH).

ACANTHODICA Schaus, I894, Trans. Am. ent. Soc. 21: 24I.

CATO

Type-species: Acanthodica grandis Schaus, 1894, ibidem 21: 24 I, by subsequent designation by Hampson, I9I3, Cat. Lepid. Phalaenae Br. Mus. 12: 249. Type(s) 9 , Mexico: Jalapa (USNM, Washington). 
ACANTHOFRONTIA Hampson, rgio, Cat. Lepid. Phalaenae Br. Mus. 10: 3, 644.

ARCTIIDAE

Type-species: Acanthofrontia lithosiana Hampson, I9Io, ibidem 10: 644, fig. I77, by original designation.

Acanthofrontia was originally proposed in the Noctuidae.

ACANTHOLEUCANIA Rungs, I953, Bull. Soc. ent. Fr. 58: I 39.

HADE

Type-species: Noctua loreyi Duponchel, 1827, in Godart \& Duponchel, Hist. nat. Lépid. Papillons Fr. 7 (I): 8r, pl. I05, fig. 7, by original designation. Syntypes, France: Provence (MNHN, Paris).

Acantholencania was originally placed as a subgenus of Lencania Ochsenheimer, I8I6.

ACANTHOLIPES Lederer, I857, Noctuinen Eur.: 44, I98.

OPHI

Type-species: Noctua regularis Hübner, [I8I3], Samml. eur. Schmett. 4: pl. I28, fig. 588 , by monotypy. Type(s), Europe.

${ }_{+}^{+}$ACANTHOLIPIS; Hampson, I907, J. Bombay nat. Hist. Soc. 17: 662.

An incorrect subsequent spelling.

$\ddagger$ ACANTHOLIPIS, misspelling. See Acantholipes Lederer, I857.

OPHI

ACANTHONYX Hampson, I902. See Ctemusa Hampson, I9io.

CATO

ACANTHOPLUSIA Dufay, I970, Bull. mens. Soc. limn. Lyon 39: io4.

PLUS

Type-species: Phytometra tarassota Hampson, I9I3, Cat. Lepid. Phalaenae Br. Mus. 13: 473, pl. 237, fig. I3, by original designation. Syntypes, [India]: [W. Bengal], Darjiling (BMNH).

$P$. tarassota was proposed as the objective replacement name for Plusia confusa Moore, I882, in Hewitson \& Moore, Descr. new Indian lepid. Insects Colln late Mr W. S. Atkinson: I49, a junior primary homonyn of Plusia confusa Stephens, I850.

ACANTHOPRORA Hampson, I926, Descr. new Genera Species Lepid. Phalaenae Subfamily Noctuinae Br. Mus.: 85 . OPHI

Type-species: Acanthoprora melanoleuca Hampson, I926, ibidem: 86, by original designation. Holotype ô, Australia: WV. Aust., Sherlock R. (BMNH).

ACANTUERTA Hampson, 1907, Ann. Mag. nat. Hist. (7) 19: $24 \mathrm{I}$.

AGAR

Type-species: Tuerta thomensis Jordan, I904, Novit. zool. 11: 445, by original designation. Holotype 오 [SÃo Tomé I., West Africa], St. Thomé (BMNH).

ACATAPAUSTUS Bethune-Baker, 1904, Novit. zool. 11: 4I 5.

NOLI

Type-species: Acatapaustus basifusca Bethune-Baker, I904, ibidem 11: 4I 5, pl. 6, fig. 25 , by original designation. Holotype $\vec{\delta}$, NEw Gunnea: [Papua], Dinawa (BMNH).

ACERRA Grote, I874, Bull. Buffalo Soc. nat. Sci. 2: 162.

HADE

Type-species: Acerra normalis Grote, I874, ibidem 2: I62, by monotypy. Syntypes o. [U.S.A.]: California (BMNH).

ACHAEA Hübner, [1823] i8I6, Verz. bekannter Schmett.: 269.

CATO

Type-species: Phalaena melicerta Drury, [I773] I 770 , Illust. nat. Hist. 1 ( I 770 ) : 4 6, pl. 23. fig. I, but without scientific names until the Index was issued with 2 (I773), by subsequent designation by Moore, [1885] I 887, Lepid. Ceylon 3: I63, but cited as †'melicerte Drury', an incorrect subsequent spelling. Type(s), [INDIA]: Bombay.

$P$. melicerta was originally included in Achaea by Hübner, as 'melicerta Cramer', an incorrect authorship. It is a junior subjective synonym of Phalaena janata Linnaeus, I758, Syst. Nat. (Edn Io) 1: 527 . 
ACHAEOPS Hampson, 1926, Descr. new Genera Species Lepid. Phalaenae Subfamily Noctuinae Br. Mus.: I03.

OPHI

Type-species: Ophisma esperanza Schaus, I9I I, Ann. Mag. nat. Hist. (8) 7: 63, by original designation. Syntypes, Cosra Rica: Esperanza; Sixola (USNM, Washington).

$\lceil$ ACHALIA, misspelling. See Achatia Hübner, [1813].

HADE

ACHANTODES Guenée, I852, in Boisduval \& Guenée, Hist. nat. Insectes (Lépid.) 6: 386.

Type-species: Achantodes cerusicosta Guence, 1852, PYRALAE Type-species: Achantodes cerusicosta Guenée, I852, ibidem 6: 387, pl. I3, fig. 3, by monotypy.

Achantodes was originally placed in the Noctuidae.

ACHARYA Moore, 1882, in Hewitson \& Moore, Descr. new Indian lepid. Insects Colln late Mr W.S. Atkinson: 185 .

$\mathrm{OPHI}$

Type-species: Acharya crassicornis Moore, I882, ibiden : I 85, pl. 6, fig. 3, by monotypy. Type(s) ô. [BAngladesh]: [E. Bengal], Silhet.

$\ddagger \boldsymbol{A C H} \boldsymbol{A} \boldsymbol{T E} \boldsymbol{A}, \mathrm{misspelling.} \mathrm{See} \mathrm{Achatia} \mathrm{Hübner,} \mathrm{[1813].}$

HADE

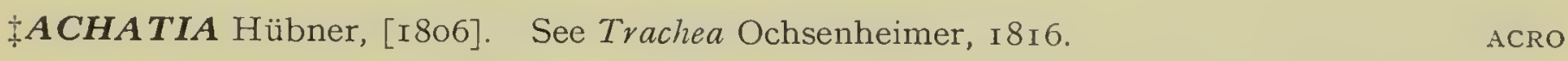

$\ddagger$ ACHATIA Hübner, I 808. See Cerma Hübner, I8 88. ACro

ACHATIA Hübner, [I813], Samml. exot. Schmett. 1: pl. [I94]. HADE

Type-species: Achatia distincta Hübner, [I8I3], ibidem 1: pl. [194], figs I-4, by monotypy. Syntypes $\widehat{0}$,, , TYPE-LOCALITY not stated [U.S.A.].

ASTRAPETIS Hübner, I821, Index exot. Lepid.: [3].

Type-species: Achatia distincta Hübner, [I8I3], by monotypy.

Astrapetis was also used as Astrapetis Hübner, [1821] I 816, Verz. bekannter Schmett.: 219, for nine species including distincta. I am following Hemming, 1937, Hübner 2: I 59 in giving priority to the Index exot. Lepid. usage.

Incorrect type-species designation: Phalaena genistae Villers, 1789 , (a name not originally included in the Index although it was included in the Verzeichniss) when cited by Moore, x884, Lepid. Ceylon 3: 38 .

Astrapetis is a junior objective synonym of Achatia Hübner, [1813].

$\ddagger$ ACHALIA ; Hübner, [1825], Cat. Lépid. composent Colln feu Mr Franck: 91.

An incorrect subsequent spelling.

$¥ A C H A T E A$; Curtis, I826, Br. Ent. 3: I I 7 .

An incorrect subsequent spelling.

$\ddagger$ ASTRAPETES; Agassiz, 1846, Nomencl. zool. (Lepid.): 9. Index univl.: 38 .

An incorrect subsequent spelling.

ACHATIS Billberg, I820. See Trachea Ochsenheimer, I816.

ACRO

ACHATODES Guenée, I852, in Boisduval \& Guenée, Hist. nat. Insectes (Lépid.) 5: I32. ACro Type-species: Achatodes sandix Guenée, I852, ibidem 5: I32, pl. 6, fig. 4, by monotypy. Syntypes I $\tilde{\sigma}, \mathrm{I}$ 우, [U.S.A.]: New York State (BMNH).

A. sandix is a junior subjective synonym of Gortyna zeae Harris, I84I, Rep. Insects Mass. injurious Vegn: 3 I9.

ACHERDOA Walker, I865, List Specimens lepid. Insects Colln Br. Mus. 32: 45I.

Type-species: Acherdoa ferraria Walker, I 865, ibidem 32:45I, by monotypy. Syntypes 2 o, [U.S.A.]: Florida (BMNH).

See also Varina Neumoegen, 1884 . 
ACHLYA Billberg, I820, Enumeratio Insect. Mus. G. J. Billberg: 87.

THYATIRIDAE

Type-species: Phalaena flavicornis Linnaeus, I758, Syst. Nat. (Edn ro) 1: 518, by subsequent designation by Tams, 1939, Entomologist 72: 70 .

Achlya was originally placed in the Noctuidae.

CY'MATOPHORA Treitschke, 1825, Schmett. Eur. 5 (I) : 77.

Type-species: Phalaena flavicornis Linnaeus, I758, by subsequent designation by Duponchel, 1829, in Godart \& Duponchel, Hist. nat. Lépid. Papillons Fr. 7 (2): 7 I, but cited as the type-species of $\ddagger$ Cymathophora, an incorrect subsequent spelling.

A junior homonym of Cymatophora Hübner, [1812], Samml. exot. Schmett. 1: pl. [205], Lepid., Geometridae. The objective replacement name is Achlya Billberg, I 820.

Cymatophora was originally placed in the Noctuidae.

$¥$ CYMATHOPHORA; Duponchel, I829, in Godart \& Duponchel, Hist. nat. Lépid. Papillons Fr. 7 (2): 7 I. An incorrect subsequent spelling.

ACHYTONIX McDunnough, 1937, Can. Ent. 69: 61.

ACRO

Type-species: Homohadena epipaschia Grote, I883, Trans. Kans. Acad. Sci. 8: 49, by original designation. Type(s), [U.S.A.]: New Mexico.

ACIDALIA Treitschke, I825. See Cymatida Sodoffsky, I 837.

GEOMETRIDAE

ACIDALIODES Hampson, I910, Cat. Lepid. Phalaenae Br. Mus. 10 : vii, I7.

$\mathrm{ACON}$

Type-species: Prolophota perstriata Hampson, I907, J. Bombay nat. Hist. Soc. 17: 677.

by original designation. Holotype $\hat{o}$, CEYLON: Hantone (BMNH).

ACIDON Hampson, I896, Fauna Br. India (Moths) 4: 462.

HYPE

Type-species: Acidon paradoxa Hampson, 1896, ibidem 4: 462, fig. 247, by original designation. Holotype ô. BHutan (BMNH).

This genus was originally placed in the Notodontidae.

†CIDONISTIS Hampson, no published reference found.

MYPE

ACIGONA Hübner, [1825] 1816, Verz. bekannter Schmett.: 342.

OPHI

Type-species: Phalaena manto Cramer, 1775, Uitlandsche Kapellen 1: 15, pl. Io, fig. F, by subsequent designation by Hampson, 1926, Descr. new Genera Species Lepid. Phalaenae Subfamily Noctuinae Br. Mus.: 52ł. Type(s), Surinam.

Acigona has recently been used, wrongly, in the Pyralidae, Crambinae, following the designation of the incorrect type-species Tinea cicatricella Hübner, [1824], by Błeszyński \& Collins, 1962, Acta zool. cracov. 7: 198.

$\ddagger$ $\$ C I G O N I A$ : Hübner, [1826] I 816, Verz. bekannter Schmett. Anzeiger: 57.

An incorrect subsequent spelling.

$\ddagger$ ACIGONIA, misspelling. See Acigona Hübner, [1825].

OPH I

ACMANA Schaus, 1916, Proc. U.S. natn. Mus. 50: 264, 384.

II Y PE

Type-species: Herminia moeonalis Walker, I859, List Specimens lepicl. Insects Colln Br. Mus. 19: 857, by original designation. Type(s) $q$, Brazil (UM, Oxford).

ACNISSA Turner, Igoz December, Trans. Proc. R. Soc. S. Aust. 26: i 80.

ACON

Type-species: Acnissa pyrrhias Turner, I902, ibidem 26: 180, by monotypy. Syntypes I 0,2 ㅇ. Australia: Qd, Townsville (ANIC, Canberra).

A. pyrrhias is a junior subjective synonym of Haplopsenstis erythrias Meyrick, I902 April

See also /Iaplopseustis Meyrick, I902 April.

$\ddagger$ ACOLASIA, misspelling See Acolasis Hübner, [1 $82 \mathrm{I}]$.

OPHI

ACOLASIS Hübner, [1821] 1816, Verz. bekannter Schmett.: 256.

OPHI

Type-species: Phalaena tanais Cramer, 1775, Uitlandsche Kapellen 1: 106, p1. 68, 
fig. D, by subsequent designation by Hampson, I926, Descr. new Genera Species Lepid.

Phalaenae Subfamily Noctuinae Br. Mus.: i Io. Type(s) 9 , Surinam.

$\ddagger$ ACOLASIA; Hübner, [1826] I8I6, Verz. bekannter Schmett. Anzeiger: 36 .

An incorrect subsequent spelling.

ACONTIA Ochsenheimer, I816, Schmett. Eur. 4: 91.

$\operatorname{ACON}$

Type-species: Noctua solaris [Denis \& Schiffermüller], I775, Ankündung syst. Werkes

Schmett. Wienergegend: 90, by subsequent designation by Duponchel, I829 [March 2Ist],

in Godart \& Duponchel, Hist. nat. Lépid. Papillons Fr. 7 (2): 72. Type(s), [Austria]:

Vienna district (Collection destroyed, Horn \& Kahle, 1936, Ent. Beih. Bevl.-Dahlem 3: 243).

$N$. solaris is a junior subjective synonym of Phalaena lucida Hufnagel, 1766, Berlin. Mag.

$3(3): 302$.

Incorrect type-species designation: Noctua luctuosa [Denis \& Schiffermüller], I775, in the Ophiderinae, cited by Curtis, I 829 September Ist, Br. Ent. 6: 276. This designation was published five months after that of Duponchel, dated from Biblphie Fr. 1829: 198.

HELIOTHERA Sodoffsky, I837, Bull. Soc. imp. Nat. Moscou 1837 (6): 89.

Proposed, unnecessarily, as an objective replacement name for Acontia Ochsenheimer, I 816, which Sodoffsky considered to be inappropriate.

PORROTHA Gistl, I 848 , Naturg. Thierveichs: viii.

Proposed, unnecessarily, as an objective replacement name for Acontia Ochsenheimer, I8I6, which Gistl considered to be a junior homonym of Acontias Cuvier, I8I7, - Reptilia.

$\$$ ACONTIA Hübner, [1823]. See Xanthodes Guenée, 1852.

CHLO

ACONTIOLA Staudinger, I90o, Dt. ent. Z. Iris 12: 382.

$\mathrm{ACON}$

Type-species: Acontiola sancta Staudinger, I9oo, ibidem 12: 382, pl. 5, fig. 4, by monotypy. Syntypes 3 ( $\sigma$, 아. , [LEBANoN]: Beirut district (MNHU, Berlin). [Jordan] Palestine: Jordan valley (MNHU, Berlin).

ACOPA Harvey, I875, Bull. Buffalo Soc. nat. Sci. 2: 279.

ACRO

Type-species: Acopa carina Harvey, I875, ibidem 2: 279, pl. 3, fig. 7, by monotypy. Syntypes $\widehat{0}$, ㅇ, [U.S.A.]: Texas (BMNH).

ACOSMETIA Stephens, I829 [June], Nom. Br. Insects: 43.

ACRO

Type-species: Noctua caliginosa Hübner, [I8I3], Samml. eur. Schmett. 4: pl. Ioo, fig. 474, by subsequent designation by Curtis, I831, Br. Ent. 8: 356 . Type(s), Europe.

HYDRILLA Boisduval, I840, Geneva Index Meth. Eur. Lepid.: I38.

Type-species: Noctua caliginosa Hübner, [1813] (but included by Boisduval as 'caliginosa Tr.', an incorrect authorship), by subsequent designation by Duponchel, I845, in d'Orbigny. Dict. univl Hist. nat. 6: 743 , but cited as 'caliginosa Treits'.

Hydrilla is a junior objective synonym of Acosmetia Stephens, I829.

ACRAPEX Hampson, I894, Fauna Br. India (Moths) 2: xiii, 286

ACRO

Type-species: Leucania prisca Walker, I866, List Specimens lepid. Insects Colln Br. Mus. 35: 1953, by original designation. Holotype q, CEylon (BMNH).

ACRARMOSTIS Meyrick, I889, Trans. ent. Soc. Lond. 1889: 479.

HYPE

Type-species: Acrarmostis dryopa Meyrick, I 889, ibidem 1889: 479, by monotypy. Holotype $\widehat{\sigma}$, NEw Guinea: [Papua], Mt Obree or adjoining ranges.

ACREMMA Berio, 1959, Annali Mus. civ. Stor. nat. Giacomo Doria 71 : 88.

$\operatorname{ACON}$

Type-species: Acremma albipoda Berio, I959, ibidem 71:88, fig. 2, by original designation. Holotype $\hat{\sigma}$, Madagascar: Nossi Bé (MNHN, Paris).

ACRIPIA Walker, r863, List Specimens lepid. Insects Colln Br. Mus. 27: 9. CHLO Type-species: Acripia subolivacea Walker, 1863, ibidem 27: 9, by monotypy. Holotype $\vec{\sigma}$. South AFrica (BMNH). 
ACRIPIOIDES Strand, I920, Arch. Naturgesch. 84(A) I2: I21.

CHLO

Type-species: Acripioides trimacula Strand, I920, ibidem 84 (A) I2: I2 I, by monotypy. Holotype $\tilde{\sigma}$. [TAIwaN] Formosa: Kosempo (DEI, Eberswalde).

ACROBYLA Rebel, I903, Dt. ent. Z. Iris 16: 68.

OPHI

Type-species: Acrobyla kneuckeri Rebel, I903, ibidem 16:69, pl. 2, fig. 9, by monotypy. Holotype ô, [EGypt/IsRaEL]: between Mt Sinai and Mt Serbal, Wadi Esch-Schêch (LN, IVarlsruhe).

ACRONICTA Ochsenheimer, I 816, Schmett. Eur. 4:62.

ACRO

Type-species: Phalaena leporina Linnaeus, 1758, Syst. Nat. (Edn Io) 1: 510, by subsequent designation by Curtis, I826, Br. Ent. 3: I36. Type(s), TyPE-Locality not stated (LS, London) [EUROPE].

$¥$ ¥PATELE Hübner, [I806], Tentamen determinationis digestionis . . . : [r] .

Included in a work rejected for nomenclatural purposes by Int. Commn zool. Nom., 1926, Smithson. misc. Collns 73 (4) Opinion 97: 19. Also idem, 1954, Opin. Decl. int. Commn zool. Nom. 6 Opinion 278: I 4 O.

Only included species: Phalaena aceris Linnaeus, I758, Syst. Nat. (Edn Io) 1: 5I4, congeneric with Phalaena leporina Linnaeus.

†PATELAE Ochsenheimer, I8 , 6, Schmett. Eur. 4:62.

Published as a junior synonym of Acronicta Ochsenheimer, i 816 , and not subsequently treated as an available name under the Code, Article II (d).

$+A P A T E L E$ Hübner, 1818, Zuträge Samml. exot. Schmett. 1:21, 33.

Not nomenclaturally available as a genus-group name. Hübner used Apatele, on both pages, in a suprageneric sense for one of the names of his tribes (Namen der Stämme) listed on page 33. Hübner listed his genus-group names on page 35 .

AP.ATELE Hübner, 1822, Syst.alphab. Verz.: 21, 28.

Type-species: Phalaena leporina Linnaeus, I758, by subsequent designation by Westwood, I840, Synopsis Genera Br. Insects: 95.

A patele is a junior objective synonym of Acronicta Ochsenheimer, I8I6.

ACRONYCTA Treitschke, I825, Schmett. Eur. 5 (I): 3.

An unjustified emendation of Acronicta Ochsenheimer, 1816.

$\ddagger$ APATELA; Stephens, I829, Nom. Br. Insects: 42 .

An incorrect subsequent spelling.

$\ddagger A C R O N Y C T I A$; Meigen, I831, Syst. Beschreibung Eur. Schmett. 3: 65 .

An incorrect subsequent spelling.

COMETA Sodoffsky, i 837, Bull. Soc. imp. Nat. Moscou 1837 (6): 85 .

Proposed, unnecessarily, as an objective replacement name for Acronycta Treitschlie, I 825, which Sodoffsky considered to be inappropriate.

ACRONICTOIDES Kiozhanchikov, I950, Fanna SSSR (Insecta, Lepid.) 12: 4 ro.

ACRO

Type-species: Graphiphora lichenodes Graeser, 1892, Berl. ent. Z. 37: 216, by original designation. Holotype $q,[$ U.S.S.R.]: Amurland, Chabarofka.

ACRONYCTA Treitschke, I825. See Acronicta Ochsenheimer, I8I6.

ACRO

$\ddagger$ ACRONYCTIA, misspelling. See Acronicta Ochsenheimer, I816.

ACRO

ACROPSEROTARACHE Berio, 1937, Annali Mus. civ. Stor. nat. Giacomo Doria 59: 391. ACON

Type-species: Acropserotarache elegantissima Berio, 1937, ibidem 59: 391, by original designation. Holotype $\hat{\sigma}$, [Somali Republic] Somalia Ital.: Ogaden, Uarder.

ACRORIA Walker, 1858, List Specimens lepid. Insects Colln Br. Mus. 15: 1730.

Type-species: Acroria villipes Walker, I 858, ibidem 15: I730, by monotypy. Type(s) o., [Brazil]: Rio Janeiro (UM, Oxford). 

586.

A. villipes is a junior subjective synonym of Hadena terens Walker, 1857 , ibidem 11:

ACRORIESIS Hampson, I916, in Poulton, Proc. zool. Soc. Lond. 1916: I Io.

$\mathrm{ACR}()$

Type-species: Acroriesis ignifusa Hampson, I9I6, ibidem 1916: I Io, pl. I, fig. 6, by original designation. Holotype 우, [ Somali Republic] Somaliland: Mandera (BMNH).

ACRORIODES Druce, 1908 April, Ann. Mag. nat. Hist. (8) 1: 303.

ACRO

Type-species: Acroriodes diplolopha Druce, 1908, ibidem (8) 1: 303, by monotypy. Type(s) ô. Peru: Oconeque, Carabaya $7000 \mathrm{ft}(\mathrm{BMNH})$.

Acroriodes was used by Druce without any indication that it was new even though his new species, described on the same page, were marked sp. n. The generic name was probably obtained from Hampson as an identification and was unintentionally made nomenclaturally available before Hampson's generic description was published.

ACRORIODES Hampson, 1908. [December], Cat. Lepid. Phalaenae Br. Muss. 7: 14, and Hampson, 1909, ibidem 8: 279.

Type-species: Acroriodes diplolopha Druce, I908, by subsequent designation by Hampson, 1909, ibidem 8: 279.

A junior homonym of Acroriodes Druce, 1908 April, - Lepid., Noctuidae. This generic name was used and made nomenclaturally available by Druce prior to its proposal and generic description by Hampson. The objective replacement name is Acroriodes Druce, I908 April.

ACRORIODES Hampson, I908 [December]. See Acroviodes Druce, I908 April. ACRo

ACROSPHALIA Rebel, I918, Verh. zool.-bot. Ges. Wien 68: (I 58).

ACRO

Type-species: Acrosphalia kulmburgi Rebel, I918, ibidem 68: (I58), figs 2, 3, by monotypy. Syntypes $\hat{0}$, ㅇ, [U.S.S.R.]: Ussuri district, Nikolsk (NM, Vienna).

A. kulmburgi is a junior subjective synonym of Usbeca cornuta Püngeler, I9I4.

See also Usbeca Püngeler, I9I4.

ACTEBIA Stephens, I829 [June], Nom. Br. Insects: 4 I.

NOCT

Type-species: Phalaena praecox Linnaeus, 1758, Syst. Nat. (Edn Io) 1: 517, by monotypy. Type(s), TyPE-LOCALITY not stated (LS, London) [EUROPE].

ACTOBIA Agassiz, I 846, Nomencl. zool. Index univl.: 6, 7.

An unjustified emendation of Actebia Stephens, I 829.

HAPALIA Hübner, [1821] I816, Vevz. bekannter Schmett.: 220.

Type-species: Phalaena praecox Linnaeus, $175^{8}$, by subsequent designation by Curtis, I835, Br. Ent. 12: 539.

A junior homonym of Hapalia Hübner, 1818, Zuträge Samml. exot. Schmett. 1: 19,Lepid., Pyralidae. The objective replacement name is Actebia Stephens.

ACTENIA Guenée, 1854, in Boisduval \& Guenée, Hist. nat. Insectes (Lépid.) 8: I34. Pyralidae Type-species: Pyralis honestalis Treitschke, 1829, Schmett. Eur. 7: 49, by original designation.

Actenia has been used as the original generic name for species which are now in the Noctuidae.

ACTINOTIA Hübner, [1821] I816, Verz. bekannter Schmett.: 244.

NOCT

Type-species: Phalaena perspicillaris Linnaeus, I76I, Fauna Suecica (Edn 2): 317, by subsequent designation by Grote, 1874, Bull. Buffalo Soc. nat. Sci. 2: 16. Type(s), SwEDEN (LS, London).

$P$. perspicillaris is a junior subjective synonym of Phalaena polyodon Clerck, I759, Icones Insect. varionum 1: pl. 2, fig. 2.

$\ddagger$ ACTINOTIS; Schulze \& Kükenthal, 1926, Nomencl. Anim. Generum Subgenerum 1: 49.

An incorrect subsequent spelling by Schulze \& Kükenthal who misquoted a correct use of Actinotia by Morrison, 1875, Proc. Acad. nat. Sci. Philad. 1875: 62. 
$\ddagger$ ACTINOTIS, misspelling. See Actinotia Hübner, [182I]

ACTOBIA Agassiz, I846. See Actebia Stephens, 1829.

NOCT

ACUTIPENNA Hampson, I894, Fauna Br. India (Moths) 2: I66, 287.

ACRO

Type-species: Acutipenna acuminata Hampson, r894, ibidem 2: 287, fig. I55, by original designation. Holotype ${ }^{*}$, [I.via] : [Madras], Nilgiris (BMNH).

ACYGNATHA Hampson, 1926, Descr. new Genera Species Lepid. Phalaenae Subfamily Noctuinae Br. Mus.: 482 .

OPH I

Type-species: Raparna atrapex Hampson, I895, Fauna Br. India (Moths) 3: 25, by original designation. Type(s) +, IndiA: Assam, Margarita.

ACYGONIA Roeplie, 1948, Tijdschr. Ent. 89: 225.

OPHI

Type-species: Acygonia difformis Roepke, 1948, ibidem 89: 226, pl. 13, fig. 5, by original designation. Holotype $\hat{\jmath}$, Sumatra: Brastagi.

A. difformis is a junior subjective synonym of Chilkasa falcata Swinhoe, I 885.

See also Chilkasa Swinhoc, I 885 .

ACYGONIODES Hampson, I926. See Amphigonia Guenée, I852.

OPHI

ACYLITA Hampson, igo8, Cat. Lepid. Phalaenae Br. Mus. 7: xi. Available, but without included species until Hampson, I910, ibidem 9: xi, 304.

ACRO

Type-spccies: Doryodes sanguifusa Jones, igo8, Trans. ent. Soc. Lond. 1908: i65, by subscquent designation by Hampson, IgIo, ibidem 9: 30 . Type(s) $\hat{0}$. Brazil: Parana, Castro (BMNH).

ACYRA Gistl, I848. See Mormo Ochsenheimer, I8I6.

ACRO

ADAPHAENURA Hampson, 1905, Cat. Lepid. Phalaenae Br. Muts. 5: xiv, 460.

CUCU

Type-species: Daphoenura minuscula Butler, 1882, Cistula ent. 3: 3, by original designation. Type(s) ㅇ, Madagascar: Betsileo, Ankafana (BMNH).

D. minuscula was originally proposcd as $\ddagger$ Daphaenura, an incorrect subsequent spelling.

ADELPHAGROTIS Smith, I89o, Bull. U.S. natn. Mus. 38: 9, 38.

NOCT

Type-species: Agrotis stellaris Grote, I88o, Can. ent. 12: I53, by original designation (on page 9). Syntypes 우, [U.S.A.]: Wash[ington] T[erritory] (BMNH); Nevada.

ADENA Agassiz, i846. See Hadena Schrank, i802.

HADE

ADEVA McDunnough, 1944, Mem. sth. Calif. Acad. Sci. 2: 2 I3.

PLUS

Type-specics: Autographa albavitta Ottolengui, I902, Jl N.Y. ent. Soc. 10: 75, pl. 8, fig. 8, by original designation. Holotype 오. [U.S.A.]: Middle California.

ADIOPA Schaus, 1940, Scient. Surv. P. Rico 12: 246.

OPHI

Type-species: Hadena disgrega Möschler, 1890, Abh. senckenb. naturforsch. Ges. 16: I28, by original designation. Syntypes $\hat{0},+$, , [Puerto Rico] Portorico I.

ADIPSOPHANES Grote, 1873, Bull. Buffalo Soc. nat. Sci. 1: i 81.

ACRO

Type-species: Adipsophanes miscellus Grote, 1873, ibidem 1: 18I, by monotypy. Syntypes $\hat{\delta}$,, , [U.S.A.]: N.Y., Albany; Pennsylvania; Massachusetts; (BMNH, without locality data).

A. miscellus is a junior subjective synonym of Catabena lineolata Walker, I865.

See also Catabena Walker, I865; and Turbula Walker, I869.

ADISURA Moore, 1881, Proc. zool. Soc. Lond. 1881: 367.

NOCT

Typc-species: Adisura atkinsoni Moore, I88I, ibidem 1881: 368, pl. 37, fig. 6, by original designation. Syntypes $\nrightarrow,+$, [IndiA]: [W. Bengal], Darjiling (MNHU, Berlin).

ADITA Grote, 1874, Bull. Buffalo Soc. nat. Sci. 2: 1 2, 63.

NOCT

Type-species: Phalaena chionanthi Smith, 1797, in Smith \& Abbot, Nat. Hist. rarer lepid. Insects Georgia 2: 195, pl. 98, by original designation. Typc(s), [U.S.A.]: Georgia. 
ADMETOVIS Grote, I873, Bull. Buffalo Soc. nat. Sci. 1: I33.

HADE Type-species: Admetovis oxymorus Grote, I873, ibidem 1: I33, pl. 4, fig. 5, by monotypy. Type(s) 오 [U.S.A.]: California, Sierra Nevada (BMNH).

ADONISEA Grote, I875, Bull. Buffalo Soc. nat. Sci. 2: 220. NOCT

Type-species: Heliothis pulchripennis Grote, I874, Proc. Boston Soc. nat. Hist. 16: 24I, by monotypy. Syntypes 0 , [U.S.A.]: California (MCZ, Boston; AMNH, New York).

ADRA Walker, I863, List Specimens lepid. Insects Colln Br. Mus. 27: 137. OPн I Type-species: Adra argentilinea Walker, 1863, ibidem 27: I38, by monotypy. Type(s) ð., BORNEO: Sarawak (BMNH).

ADRANA Walker, 1858, List Specimens lepid. Insects Colln Br. Mus. 15: I783

EUTE Type-species: Adrana pseudopsis Walker, I858, ibidem 15: I784, by monotypy. Type(s) ô. [BRazIL]: Rio Janeiro (UM, Oxford).

A. pseudopsis is a junior subjective synonym of Edema fuscescens Walker, I855, ibidem 5: IO3I.

ADRAPSA Walker, [I859] I 858, List Specimens lepid. Insects Colln Br. Mus. 16: I69. HYFE Type-species: Adrapsa ablualis Walker, [I859] I858, ibidem 16: I70, by monotypy. Holotype ô, CEYLon (BMNH).

ADRAPSOIDES Matsumura, 1925, J. Coll. Agric. Hokkaido Imp. Univ. 15: I53. HYPE Type-species: Adrapsa reticulatis Leech, I900, Trans. ent. Soc. Lond. 1900: 6I6, by original designation. Holotype ${ }^{\text {, }}$ CHINA: [Hupei], Chang-yang (BMNH).

ADRIS Moore, I88I, Trans. zool. Soc. Lond. 11: 69.

OPHI

Type-species: Ophideres tyrannus Guenée, I852, in Boisduval \& Guenée, Hist. nat. Insectes (Lépid.) 7: I Io, by original designation. Holotype 요 INDIA : Central (BMNH).

ADROCAMPA Schaus, I894, Trans. Am. ent. Soc. 21: 234.

OPHI

Type-species: Adrocampa pallescens Schaus, 1894, ibidem 21: 234, by monotypy. Type(s), Brazil: Parana, Castro.

Adrocampa when originally proposed contained a second species, but this was doubtfully included, and under the Code, Article 68(c) is not eligible for designation as type-species.

ADYROMA Möschler, I88o, Verh. zool.-bot. Ges. Wien 30 (Abh.) : $455 . \quad$ OPH I

Type-species: Adyroma reposita Möschler, I 88o, ibidem 30 (Abh.) : 456, by monotypy. Syntypes 2 s, I 9 , SuRINAm: interior (MNHU, Berlin).

AEDEMON Turner, 1944, Proc. R. Soc. Qd 55: 47.

NOLI

Type-species: Aedemon eurapta Turner, 1944, ibidem 55:47, by monotypy. Holotype ô. Australia: Queensland, Stanthorpe.

AEDIA Hübner, [1823] I 8I6, Verz. bekannter Schmett.: 260.

OPHI

Type-species: Noctua leucomelas Linnaeus sensu Hübner, [1803], Samml. eur. Schmett. 4: pl. 62, fig. 304 [a misidentification already named as Noctua funesta Esper, I786, Die Schmett. 4 (I): pl. 88, fig. 6; I787, ibidem: 72], by subsequent designation by Hampson, I902, Ann.S. Afv. Mus. 2: 355, but cited as †'leucamelas Hübner', an incorrect subsequent spelling. Type(s) ơ (of $N$. funesta), Germany (West) : Frankfurt am Main (SM, Wiesbaden).

$N$. leucomelas Linnaeus sensu Hübner has been long established as a misidentification of Phalaena leucomelas Linnaeus, I758, which is the type-species of Anophia Guenée, I84I. This is a case of a misidentified type-species which under the Code, Article 7o(a) should be referred to the Commission.

AEDOPHRON Lederer, I857, Noctuinen Eur.: 43, I80.

NOCT

Type-species: Heliothis rhodites Eversmann, I851, Bull. Soc. Nat. Moscou 24: 635, by monotypy. Syntypes, [U.S.S.R.]: [Krasnoarmeysk] Sarepta (ZI, Leningrad); Black Sea Coast.

C 
AEGARA Walker, [I 866] I865, List Specimens lepid. Insects Colln Br. Mus. 34: I I27. HYPE Type-species: Aegara interruptalis Walker, [1866] I 865, ibidem 34: I I 28, by monotypy. Holotype f, [BrazII] : [R. Amazon], [Tefé] Ega (BMNH).

A. interruptalis is a junior subjective synonym of Megatomis polycletusalis Walker, I 858 , ibidem 16: 145 .

AEGILIA Walker, [1858] I857, List Specimens lepid. Insects Colln Br. Mus. 13: I I22, I I38. STIC

Type-species: Aegilia describens Walker, [1858] I857, ibidem 13: I I39, by monotypy. Holotype ô, CEYLON (BMNH).

AEGLE Hübner, [1823] I816, Verz. bekannter Schmett: 257.

ACRO

Type-species: Tortrix kaekeritziana Hübner, [1799], Samml. eur. Schmett. 7: pl. 26, fig. I63, by subsequent designation by Hampson, igIo, Cat. Lepid. Phalaenae Br. Mus. 9: 380 , but cited as coeceritziana Hampson, an unjustified emendation. Type(s), Europe. Aegle had already been used as a generic name in the Mollusca by Oken, is I5, Okens Lehrbuch Naturg. 3: 326, but this work has been rejected for nomenclatural purposes by Int. Commn zool. Nom., 1956, Opin. Decl. int. Commn zool. Nom. 14 Opinion 417: 3. Between $18 I_{5}$ and 1823 there seems to be only one usage (and that as a nomen nudum) by Audouin et al., 1822, Dictionnaire classique Hist. nat. 1: 124, 'Aegle Moll. (Ocken) V. Eglée.' But in the same series (1824) 6 there is no reference to Eglée in the alphabetic sequence. Aegle Hübner is therefore nomenclaturally available and may continue in use in the Noctuidae.

See also Metoponia Duponchel, [1845].

AEGLEOIDES Berio, 1937, Annali Mus. civ. Stor. nat. Giacomo Doria 59: 385.

ACON

Type-species: Aegleoides paolii Berio, I937, ibidem 59: 386, by original designation. Holotype $\hat{\sigma}$. [Somali Republic] Somalia Ital.: Villaggio Duca degli Abruzzi (Colln E. Berio, Genoa).

AEGOCERA Latreille, I 809, Genera Crustaceorum Insect. 4: 2 I I.

AGAR

Type-species: Phalaena venulia Cramer, 1777, Uitlandsche Kapellen 2: 107, pl. 165, fig. D, by monotypy. Syntype(s), [Tndia/Bangladesi] : Côte de Bengale.

$P$. venulia is dated from the wrapper of the part and not from the title-page of the volume, I 779 .

$\ddagger$ OEGOCERA Boisduval, I844, in Guérin-Méneville, Iconographie Règne anim. G. Cuvier

(Insectes) : 498 .

An incorrect subsequent spelling.

AEGOCEROPSIS Karsch, i 895, Ent. Nachr. 21: 348.

AGAR

Type-species: Aegoceropsis norma Karsch, I895, ibidem 21:348, 352, pl. 2, fig. 6, by original designation. Holotype $\hat{\jmath}$, Africa: Mkaramo (MNHU, Berlin).

AENIGMA Strecker, 1876. See Hyblaea Fabricius, I793.

HYBLAEIDAE

AEOLOGRAMMA Strand, I9Io, Int. ent. Z. 4 (18): 98.

ACRO

Type-species: Poecilogramma picatum Butler, 1892, Proc. zool. Soc. Lond. 1892: I 26 , pl. 6, fig. 4, by monotypy (of Poecilogramma Butler, I892). Holotype ô, Borneo: North Borneo, Sandakan (BMNH).

Acologramma was proposed as the objective replacement name for Poecilogramma Butler, 1892.

POECILOGRAMMA Butler, i 892, Proc. zool. Soc. Lond. 1892: i 26.

Type-species: Poecilogramma picatum Butler, I892, by monotypy.

A junior homonym of Poecilogramma Karsch, 1887, Ent. Nachr. 13: 52, - Orthoptera. The objective replacement name is Aeologramma Strand, I9Io.

AESCHRADIA Hampson, igr2, Cat. Lepid. Phalaenae Br. Mus. 11: xii, 378. SARR

Type-species: Apamea mammida Druce, I898, Biologia cent.-am. (Zool.) Lepid. 
Heterocera 2: 477, pl. 93, fig. 20, by original designation. Syntypes 2 ex., GuAtemala: Volcan de Atitlan (BMNH).

AETHALINA Turner, I902, Proc. Linn. Soc. N.S.W. 27: I07.

Type-species: Aethatir

Type-species: Aethalina asaphes Turner, r902, ibidem 27: 108, by monotypy. Holotype
+. Australia: Qd, Cardwell (ANIC, Canberra).

AETHIA Hübner [1825]. See Trisateles Tams, I939.

HYPE

AETHODES Hampson, x918, Novit. zool. 25: 216.

AGAR

Type-species: Aethodes angustipennis Hampson, I9I8, ibidem 25: 217, by original designation. Holotype $\widehat{\jmath}$, Nigeria: northern, Minna (BMNH).

AETHRIA Hübner, [1821]. See Mamestra Ochsenheimer, r816.

HADE

AFOTELLA Barnes \& Benjamin, 1925, Bull. Brooklyn ent. Soc. 20 : 195.

ACRO

Type-species: Hadena cylindrica Grote, r88o, Can. Ent. 12: 214, by original designation. Type(s), [U.S.A.]: Nevada (BMNH).

AFRENELLA Berio, 1965, Memorie Soc. ent. ital. 44: 94.

ACRO

Type-species: Afrenella jansei Berio, I965, ibidem 44: 94, by original designation.

Holotype ô., [ZAIRE] Congo: [Katanga], Elisabethville (MRAC, Tervuren).

AFRICALPE Krüger, 1939, Annali Mus. libico Stor. nat. 1: 348.

OPHI

Type-species: Africalpe intrusa Krüger, I939, ibidem 1: 349, pl. I3, figs I 8, r9, by monotypy. Holotype $\tilde{\sigma}^{*}$ [LIByA]: WV. Sirtica; Uadi Merdum or Uadi Gobin (Mus. Libico Storia Nat., Tripoli).

$\ddagger$ AGAB.4, misspelling. See Agabra Walker, I862.

ACRO

AGABRA Valker, r862, J. Proc. Linn. Soc. (Zool.) 6: г 36.

ACRO

Type-species: Agabra trilineata Walker, I 862, ibidem 6: 137, by monotypy. Lectotype o. Børneo: Sarawak (UM, Oxford), designated (as type) by Swinhoe, rgoo, Cat. east. and Aust. Lepid. Heterocera 2: 37.

$\ddagger A G A B A$; Pagenstecher, r909, Geogr. Verbreitung Schmett.: 425 .

An incorrect subsequent spelling.

AGAMANA Walker, [1866] I865, List Specimens lepid. Insects Colln Br. Mus. 34: Ir 52. OPHI Type-species: Agamana cavatalis Walker, [1866] r865, ibidem 34: II 52, by monotypy. Holotype , Australia (BMNH).

AGANZAGARA Walker, [1866] 1865, List Specimens lepid. Insects Colln Br. Mus. 34: Ir 46. Hy PE Type-species: Aganzagara disparatalis Walker, [1866] I865, ibidem 34: Ir 46, by monotypy. Holotype , Honduras: Limas (BMNH).

AGARISTA Leach, r8r4, Zool. Miscellany 1: 37.

AGAR

Type-species: Agarista picta Leach, I8r $_{5}$, ibidem 1:38, pl. I5, by monotypy. Syntypes o., , [Australia] New Holland.

AGARISTODES Hampson, I908, Cat. Lepid. Phalaenae Br. Mus. 7: 7. Available, but without included species until Hampson, rgro, ibidem 9: xiv, $45^{6}$.

AGAR

Type-species: Agarista feisthamelii Herrich-Schäffer, 1853 , Samml. newer oder wenig bekannter aussereur. Schmett. 1 (I): wrapper, pl. 6, fig. 25; I856, r858, ibidem 1: 13, 7I, 78, by subsequent monotypy. Type(s), [Australia] Nov. Holl.

A. feisthamelii was attributed to Boisduval by Herrich-Schäffer. The species is dated from the wrapper of the plates and not from the title-page of the volume, $1850-1858$.

AGASSIZIA Behr, r870, Trans. Am. ent. Soc. 3: 23.

HYPE

Type-species: Agassizia urbicola Behr, 1870, ibidem 3: 23, by subsequent designation 
by Grote, 1874, Bull. Buffalo Soc. nat. Sci. 2: 45. Syntypes 3 ex., [U.S.A.]: California, 'different parts' and San Francisco.

Not a junior homonym of $\ddagger$ Agassizia; Agassiz \& Desor, 1847, Annls Sci. Nat. (Zool.) (3) 8: 20, which is an incorrect subsequent spelling of Agassisia Valenciennes, 1846, Echinoderma.

AGGUSTIANA Schaus, I916, Proc. U.S. natn. Mus. 50: 334.

OPH I

Type-species: Gustiana libitina Druce, I890, Biologia cent.-am. (Zool.) Lepid. Heterocera 1: 425, pl. 35, fig. x, by original designation. Syntypes $\hat{\jmath},+$, M Mexico: Guerrero, Omilteme (BMNH); Morelos, Cuernavaca; Jalapa.

AGINNA Walker, I865, List Specimens lepid. Insects Colln Br. Mus. 33: 1022.

HYPE

Type-species: Aginna circumscripta Walker, 1865, ibidem 33: 1023, by monotypy. Holotype $\hat{\jmath}$, [West Malaysia]: [Malaya], Penang (BMNH).

AGLAONICE Möschler, I890 [May], Abh. senckenb. naturforsch. Ges. 16:226. HYPE

Type-species: Aglaonice snelleni Möschler, I890, ibidem 16: 227, by monotypy. Syntypes, Puerto Rico: i ô (MNHU, Berlin). Surinam: $2 \hat{\jmath}$ (MNHU, Berlin).

Möschler intended that this new genus and species should be named $\ddagger$ Snellenia oppositalis. Möschler's manuscript was, however, published posthumously and in a footnote the editor explained that as Snellenia was preoccupied, the generic name was changed. In order to preserve Möschler's intention to dedicate a name to his friend and eminent lepidopterist P. C. T. Snellen, the species name was also changed.

A. snelleni is a junior subjective synonym of Bleptina hirtipalpis Walker, [1859].

See also Metina Druce, I 890 December.

AGLOSSESTRA Hampson, I905, Cat. Lepid. Phalaenae Br. Mus. 5: 3, 225.

HADE

Type-species: Aglossestra deserticola Hampson, I905, ibidem 5: 225, fig. 38, by original designation. Holotype ô, Egypt: Suez (BMNH).

AGLOSSOSTOLA Hampson, 1926, Descr. new Genera Species Lepid. Phalaenae Subfamily Noctuinae Br. Mus.: 606. OPHI

Type-species: Stellidia diana Schaus, I904, Trans. Am. ent. Soc. 30: i66, by original designation. Type(s), [BraziL]: Parana, Castro.

AGNOMONIA Hübner, [1831] I825, Zuträge Samml. exot. Schmett. 3: Io.

CATO

Type-species: Agnomonia sequistriaris Hübner, [1831] I825, ibidem 3: Io, figs 4I9, 420, by subsequent designation by Hampson, I913, Cat. Lepid. Phalaenae Br. Mris. 13: I5I, but cited as anilis Drury, 1773, which Hampson placed (on page I56) as the senior synonym of sequistriaris Hübner. Holotype $q$, [U.S.A.]: Georgia.

A. sequistriaris is a junior subjective synonym of Phalaena anilis Drury, I 773.

See also Argyrostrotis Hübner, [I82 I].

AGONISTA Rogenhofer, I875. See Lygniodes Guenée, 1852.

CATO

AGRAGA Walker, I858, List Specimens lepid. Insects Colln Br. Mus. 15: I772. ACRO

Type-species: Agraga fimbripes Walker, I 858, ibidem 15: 1773, by monotypy. Type(s) o., [BRAzIL]: Rio Janeiro (UM, Oxford).

AGRAPHA Hübner, [182I] i816, Verz. bekannter Schmett.: 250.

PLUS

Type-species: Agrapha ahenea Hübner, [1821] I8I6, ibidem: 250 (the objective replacement name for Phalaena glauca Stoll, 1780 , in Cramer, Uitlandsche Krapellen 4: 45, pl. 3 I , fig. G.), by subsequent designation by Grote, I 896, Entomologist's Rec. J. Var. 8: 303, but cited as ¥'glauca Cram.', an incorrect authorship for this species. Type(s), SURINAM.

P. glauca Stoll is a junior primary homonym of Phalaena glanca Cramer, 1777 , ibidem 2: I7, pl. 107, fig. E, - Lepid., Hypsidae. 
AGRIOPIS Boisduval, I840. See Griposia Tams, I939.

CUCU

AGRIOPODES Hampson, I908, Cat. Lepid. Phalaenae Br. Mus. 7: 16. Available, but without included species until Hampson, I909, ibidem 8: vii, 37. ACRO

Type-species: Moma fallax Herrich-Schäffer, 1854, Samml. neuer oder wenig bekannter ausseveur. Schmett. 1 (I): wrapper, pl. 42, fig. 2I I; I858, ibidem, 1:68, 80, by subsequent designation by Hampson, I909, ibidem 8: 37 . Type(s) [U.S.A.]: Tennessee.

$M$. fallax is dated from the wrapper of the plates and not from the title-page of the volume, I $850-1858$.

AGROCHOLA Hübner, [1821] I8I6, Vevz. bekannter Schmett.: 229.

CUCU

Type-species: Noctua pistacina [Denis \& Schiffermüller], 1775, Ankündung syst. Werkes Schmett. Wienergegend: 77 , by subsequent designation by Hampson, I906, Cat. Lepid. Phalaenae Br. Mus. 6: 470. Type(s), [Austria]: Vienna district (Collection destroyed, Horn \& Kahle, 1936, Ent. Beih. Berl.-Dahlem 3: 243).

$N$. pistacina is a junior subjective synonym of Noctua lychnidis [Denis \& Schiffermüller], I 775 , ibidem: 76 .

A MATHES Hübner sensu Hampson, 1906, Cat. Lepid. Phalaenae Br. Mus. 6: 470.

Used in the Cuculliinae based on the incorrect type-species Phalaena litura Linnaeus, I76I, at present placed in Agrochola Hübner, [I82I].

AGRONOMA Hübner, [1821] I816, Verz. bekannter Schmett.: 227.

Nocr

Type-species: Noctua valligera [Denis \& Schiffermüller], I775, Ankündung syst. Werkes Schmett. Wienergegend: 80, by subsequent designation by Grote, I895, Abh. naturw. Ver. Bremen 14: 64. Type(s), [Austria]: Vienna district (Collection destroyed, Horn \& Kahle, 1936, Ent. Beih. Berl.-Dahlem 3: 243).

$N$. valligera is a junior subjective synonym of Phalaena vestigialis Hufnagel, 1766 , Berlin. Mag. 2: 422 .

Throughout the Verzeichniss all the generic names were proposed in the plural and then used in the singular for the first binomen. This genus was proposed as $\ddagger$ Agronomae and then used again in the plural for the first binomen. This clearly inadvertent error was later corrected in Hübner, [1826] I8I6, Vevz. bekannter Schmett. Anzeiger: 36.

$\ddagger A G R O N O M A E$; Hübner, [I82 I] I8I6, Verz. bekannter Schmett.: 227.

An incorrect original spelling.

$\ddagger$ AGRONOMAE, misspelling. See Agronoma Hübner, [I82I].

NOCT

AGROPERINA Hampson, I908, Cat. Lepid. Phalaenae Br. Mus. 7: xii, 398.

ACRO

Type-species: Phalaena lateritia Hufnagel, I 766, Berlin. Mag. 3: 306, by original designation. Type(s), Germany: Berlin.

AGROPHILA Boisduval, I840. See Emmelia Hübner, [182 I].

$\triangle \mathrm{CON}$

AGROSTOBIA Boie, I835. See Abromias Billberg, 1820.

ACRO

AGROTANA Bethune-Baker, IgI I, Ann. Mag. nat. Hist. (8) 8: 509.

Type-species: Agrotana jacksoni Bethune-Baker, I9II, ibidem (8) 8:509, by original designation. Type(s) $\hat{o}$, [Kenya] B.E. Africa: Nairobi (BMNH).

AGROTIMORPHA Barnes \& Benjamin, 1929, Bull. Brooklyn ent. Soc. 24: I74.

NOCT

Type-species: Agrotis staudingeri Möschler, I862, Wien. ent. Monatschr. 6: I32, pl. I, fig. 4 , by original designation. Syntypes $3 \hat{\sigma},[$ CANADA] : Labrador.

AGROTIPHILA Grote, I875, Ann. Lyceum nat. Hist. 11: Io8.

NOCT

Type-species: Agrotiphila colorado Smith, I89I, Trans. Am. ent. Soc. 18: 133, by subsequent designation by the International Commission on Zoological Nomenclature, I972, Bull. zool. Nom. 29 Opinion 983: i I3. Syntypes ô, ㅇ. [U.S.A.]: Colorado (USNM, Washington). 
Agrotiphila was proposed as a monotypic genus by Grote, based on a specimen which he determined as Agrotis montana Morrison, 1875. Later, McDunnough, 1947, Can. Ent. 79: 38, showed that Grote's description was based on a specimen of colorado and not montana. Because Agrotiphila was based on a misidentified type-species, a case was submitted to the Commission by Hardwick \& McDunnough, 1969, Bull. zool. Nom. 26: 97. which resulted in the above type-species designation. Agrotiphila Grote has been placed on the Official List of Generic Names in Zoology: Name No. I96I.

$\ddagger$ AGROTIS Hübner, [1806]. See Agrotis Ochsenheimer, I816.

NOC'

$\ddagger$ AGROTIS Hübner, I808. See Elaphria Hübner, I818.

ACRO

AGROTIS Ochsenheimer, I8I6, Schmett. Eur. 4: 66.

NOCT

Type-species: Noctua segetum [Denis \& Schiffermüller], r775, Ankïndung Syst. Werkes Schmett. Wienergegend: 81, by subsequent designation by Curtis, I827, Br. Ent. 4: 165, but cited as ' $N$. segetis Fab.' Type(s), [AusTria]: Vienna district (Collection destroyed, Horn \& Kahle, 1936, Ent. Beih. Berl.-Dahlem 3: 243).

Noctua segetis Fabricius, I781, Species Insect. 2: 223, was an emendation of $N$. segetum. ¥AGROTIS Hübner, [1806], Tentamen determinationis digestionis . . . : [1].

Included in a work rejected for nomenclatural purposes by Int. Commn zool. Nom., 1926, Smithson. misc. Collns 73 (4) Opinion 97: 19. Also idem, 1954, Opin. Decl. int. Commn zool. Nom. 6 Opinion 278: I40.

Only included species: 'Agrotis segetis'.

GEORYX Hübner, [1821] I816, Verz. bekannter Schmett.: 227.

Type-species: Noctua segetum [Denis \& Schiffermüller], I775, by subsequent designation by Hampson, 1903, Cat. Lepid. Phalaenae Br. Mus. 4: I 53.

Georyx is a junior objective synonym of Agrotis Ochsenheimer, I816.

NOCTUA Boisduval, I828, Eur. Lepid. Index meth.: 63.

Type-species: Phalaena exclamationis Linnaeus, 1758, Syst. Nat. (Edn Io) 1: 515, by subsequent designation by Duponchel, I829, in Godart \& Duponchel, Hist. nat. I-épid. Papillons Fr. 7 (2): 7 I.

A junior homonym of Noctua Linnaeus, 1758. There is no objective replacement name, but $P$. exclamationis is congeneric with Noctua segetum [Denis \& Schiffermüller], 1775, the type-species of Agrotis Ochsenheimer, 1816. The latter is therefore available for use as the subjective replacement name.

PSAMMOPHILA Stephens, 1850, List Specimens Br. Anim. Colln Br. Mus. 5: 72.

Type-species: Noctua ripae Hübner, [1823], Samml, eur. Schmett. 4: pl. I 51, figs 702, ;03, by monotypy. Type(s), Europe.

A junior homonym of Psammophila Brown, 1827, Illust. Conchology Gt Br. and Ir.: pl. I2, fig. I, - Mollusca. There is no objective replacement name but $N$. ripae is congeneric with Noctua segetum [Denis \& Schiffermüller], 1775, the type-species of Agrotis Ochsenheimer, I8I6. The latter is therefore available for use as the subjective replacement name.

AGROTISIA Hampson, I908, Cat. Lepid. Phalaenae Br. Mus. 7: 18, 692.

ACRO

Type-species: Agrotisia subhyalina Hampson, 1908, ibidem 7:692, fig. I84, by original designation. Holotype ơ. PERU: Callao (BMNH).

HARRISONIA Schaus, I923, Zoologica, N.Y. 5: 36.

Type-species: Harrisonia williamsi Schaus, I923, ibidem 5:36, pl. I, fig. 7, by original designation. Icctotype ô. Galapagos Is: South Seymour (USNM, Washington), designated by Todcl, 1973, Proc. ent. Soc. Wash. 75: 36 .

A junior homonym of Harrisonia Ferris, 1922, Parasitology 14: 80, - Mallophaga. There is no objective replacement name, but $1 \%$. williamsi is congeneric with Agrotisia subhyalina Hampson, 1908, the type-species of Agrotisia Hampson, 1908. The latter is therefore available for 11 se as a subjective replacement name. 
$\ddagger$ AGRYPHIA, misspelling. See Argyphia Saalmüller, I $89 \mathrm{I}$.

OPHI

AGYRA Guenée, I852, in Boisduval \& Guenée, Hist. nat. Insectes (Lépid.) 7: 373. Type-species: Agyra marchandi Guenée, 1852, ibidem 7: 373, by monotypy.

o., Type-locality 'probablement américain' [Central \& South America].

See also Marthama Walker, I858.

AIRAMIA Barnes \& Benjamin, I926, Pan-Pacif. Ent. 3: 73.

$\operatorname{ACON}$

Type-species: Hoplotarache albiocula Barnes \& McDunnough, I9I8, Contr. nat. Hist. N. Am. 4: I I 4, pl. I 7, fig. I 2, by original designation. Holotype 오, [U.S.A.]: Calif., Inyo Co., Olancha.

AITETA Walker, I856, List Specimens lepid. Insects Colln Br. Mus. 9: 69, I 3 I.

CHLO Type-species: Aiteta musculina Walker, I 856, ibidem 9: I 3 I, by monotypy. Holotype

‥ Philippines (BMNH).

See also Chaladra Walker, I 865.

BRADA Walker, I858, List Specimens lepid. Insects Colln Br. Mus. 15: 1665.

Type-species: Brada truncata Walker, I 858, ibidem 15: I666, by monotypy. Holotype o., CEYLON (BMNH).

A junior homonym of Brada Stimpson, I854, Smithson. Contr. Knowl. 6 (5): 32,Vermes. There is no objective replacement name but $B$. truncata is congeneric with Aiteta musculina Walker, I856, the type-species of Aiteta Walker, I856. The latter is therefore available for use as a subjective replacement name.

AKONIODES Matsumura, 1929, Insecta matsum. 3 : I 77.

ACRO

Type-species: Akoniodes kuyanianus Matsumura, I929, ibidem 3: II7, by original designation. Holotype $\widetilde{\jmath}$, [TAIWAN] Formosa: Tappan, Kuyania (EIHU, Sapporo).

AKONUS Matsumura, I929, Insecta matsum. 3: ir6.

ACRO

Type-species: Akonus formosanus Matsumura, I929, ibidem 3: I16, by original designation. Holotype $\widehat{\partial},[$ TAIwAN] Formosa: Ako (EIHU, Sapporo).

ALA Staudinger, I882. See Trichanarta Hampson, I896.

NOCT

ALABAMA Grote, I895, Abh. nature. Ver. Bremen 14 I 22.

OPHI

Type-species: Aletia argillacea Hübner, I823, Zuträge Samml. exot. Schmett. 2: 32, figs 399,400 , by original designation. Syntypes $\hat{\sigma}$, [BRAzIL]: Bahia.

EUALABAMA Grote, I896, Proc. Am. Phil. Soc. 34: 4 I9.

Proposed, unnecessarily, as an objective replacement name for Alabama Grote, I895, in case the latter was ever regarded as a junior homonym of Alibama Möschler, I886, Abh. Senckenb. Ges. 14: 43, - Lepid., Noctuidae.

$\ddagger$ ALAEOGNATHA, misspelling. See Elaeognatha Hampson, I905.

SARR

ALAMIS Guenée, 1852, in Boisduval \& Guenée, Hist. nat. Insectes (Lépid.) 7: 3. Cato Type-species: Alamis umbrina Guenée, I 852 , ibidem 7:4, by subsequent designation by Hampson, I913, Cat. Lepid. Phalaenae Br. Mus. 13: 297. Syntypes 2 9 , East Indies (UM, Oxford).

ALAPADNA Turner, 1902, Proc. Linn. Soc. N.S.W. 27: 106.

OPHI

Type-species: Alapadna pauropis Turner, I002, ibidem 27: I06, by monotypy. Syntypes, I $\overrightarrow{0}$. I 9 , Australia: Qd, Brisbane (ANIC, Canberra).

ALARIA Duncan [\& Westwood], I84I. See Rhodophora Guenée, I852.

NOCT

ALBERTICODES Biezanko \& Ruffinelli, I 963, Rerta Soc. umg. Ent. 5: 45.

HYPE

Type-species: Tortricodes pterophoralis Guenée, 1854, in Boisduval \& Guenée, Hist. nat. Insectes (Lépid.) 8: 72, pl. 7, fig. 2, by subsequent designation (for Tortricodes Guenée, I 854) by Desmarest, (I857), in Chenu, Encycl. Hist. nat. Papillons nocturnes: 2I8. Holotype ふ, Brazil (BMNH). 
Alberticodes was proposed as the objective replacement name for Tortricodes Guenée, I 854. Alberticodes was also proposed in a Portuguese translation of the paper, published in 1963 in Agros Pelotas 11 (I).

TORTRICODES Guenée, I854, in Boisduval \& Guenée, Hist. nat. Insectes (Lépid.) 8: 7 I.

Type-species: Tortricodes pterophoralis Guenée, r 854 , by subsequent designation by Desmarest, (1857).

A junior homonym of Tortricodes Guenée, r845, Annls Soc. ent. Fr. (2) 3: 305, - Lepid., Tortricidae. The objective replacement name is Alberticodes Biezanko \& Ruffinelli, I963.

ALELIMMA Hampson, I895, Fauna Br. India (Moths) 3: 31, 47.

HYPE

Type-species: Alelimma pallidifusca Hampson, I 895, ibidem 3: 47, by original designation. Holotype , [ [India]: [Assam], Nagas, $6000 \mathrm{ft}$.

ALELIMMINOLA Strand, I9I 7, Arch. Naturgesch. 83 (A) Io: 156.

HYPE

Type-species: Alelimminola dubiosa Strand, I917, ibidem 83(A) ro: 156, by monotypy. Holotype ô. [TAIwan] Formosa: Suisharyo (DEI, Eberswalde).

ALEPTINA Dyar, I902, Can. Ent. 34: 105.

ACRO

Typc-species: Aleptina inca Dyar, 1902, ibidem 34: 105, by monotypy. Syntypes 2 o, 2 ㅇ, [U.S.A.]: Arizona; Texas, Comfort and Kerrville.

ALEPTINOIDES Barnes \& McDunnough, I912, Contr. nat. Hist. Lepid. N. Am. 1(5): 24. ACro

Type-species: Aleptinoides ochrea Barnes \& McDunnough, I9r2, ibidem 1 (5): 24 , pl. 2, fig. 17 , by original designation. Syntypes 2 9, [U.S.A.]: New Mexico, Deming.

ALESUA Dyar, 1918, Proc. U.S. natn. Mus. 54: 353.

HYPE

Type-species: Alesua etialis Dyar, 1918, ibidem 54: 353, by original designation. Holotype ô, Mexico (USNM, Washington).

$\ddagger$ ALETERIA Hampson, no published reference found.

HYPE

$\ddagger$ ALETHMIA, misspelling. See Atethmia Hübner, [1 $82 \mathrm{I}]$.

CUCU

ALETIA Hübner, [I82I] I8I6, Verz. bekannter Schmett.: 239.

HADE

Type-species: Noctua vitellina Hübner, [i 8o8], Samml. eur. Schmett. 4: pl. 81, fig. 379,

by subsequent designation by Moore, I881, Proc. zool. Soc. Lond. 1881: 333. Type(s), EUROPE.

Incorrect type-species designation: Aletia argillacea Hübner, I 823, a name not originally included in Aletia, and not linked with one of the originally included names when cited by Grote, I874, Bull. Buffalo Soc. nat. Sci. 2: 24.

$\ddagger$ HELIOPHILA Hübner, [1806], Tentamen determinationis digestionis . . . : [1].

Included in a work rejected for nomenclatural purposes by Int. Commn zool. Nom., 1926, Smithson. misc. Collns 73 (4) Opinion 97: 19. Also idem, 1954, Opin. Decl. int. Commn zool. Nom. 6 Opinion 278: I 40.

Only included species: Phalaena pallens Linnaeus, 1758 , which is congeneric with Noctua vitellina Hübner, [1808].

HELIOPHILA Hübner, 1822, Syst.-alphab. Verz.: 20, 32.

Type-species: Phalaena pallens Linnaeus, I 758, Syst. Nat. (Edn ro) 1:5 Io, by subsequent designation by Grote, 1874, Bull. Buffalo Soc. nat. Sci. 2: 21.

A junior homonym of Heliophila Klug, I807, Mag. Insektenk. (Illiger) 6: 227,Hymenoptera. There is no objective replacement name, but $P$. pallens is congeneric with $N$. vitellina Hübner, the type-species of Aletia Hübner, $[\mathrm{r} 82 \mathrm{r}]$, which should therefore be used as the subjective replacement name.

LEUCANIA Boisduval, I 828, Eur. Lepid. Index meth.: 82.

Type-species: Phalaena pallens Linnaeus, I 758, by subsequent designation by Duponchel, 1829, in Godart \& Duponchel, Hist. nat. Lépid. Papillons F'. 7 (2): 72.

A junior homonym of Leucania Ochsenheimer, 1816,-Lepid., Noctuidac. There is no 
objective replacement name, but $P$. pallens is congeneric with $N$. vitellina Hübner, the type-species of Aletia Hübner, [I82I], which should therefore be used as the subjective replacement name.

ALETOPUS Jordan, 1926, Novit. zool. 33: 376.

AGAR

Type-species: Aletopus imperialis Jordan, I926, ibidem 33:377, by original designation. Holotype \%, [TANZANIA]: Tanganyika, Usambara, Bungu (BMNH).

ALEUCANITIS Warren, I913, in Seitz, Gross-Schmett. Erde 3: 389.

OPHI

Type-species: Heliothis cailino Lefebvre, i 827, Mém. Soc. linn. Paris 6: 94, pl. 5, fig. I, by original designation. Syntypes $2 \hat{0}$, [ItALy]: Sicily, jardin de botanique Palerme.

$H$. cailino was originally described as ‘'Heliotis', an incorrect subsequent spelling.

The date of publication of the part of Seitz containing Aleucanitis was stated on page 389 as I9I 3 December Ist in both the English and in the German editions.

BOLINA Duponchel, [1845], Cat. méth. Lépid. Eur. (2): 180.

Type-species: Heliothis cailino Lefebvre, 1827, by monotypy.

A junior homonym of Bolina Rafinesque, I8I5, Analyse: I44,-Mollusca. A nomenclaturally available name and not a nomen nudum as stated by Neave, I939, Nomencl. zool. 1: 447. The objective replacement name is Aleucanitis Warren, I9I3. $\ddagger B O L I N A$ Walker, [1858] r 857, List Specimens lepid. Insects Colln Br. Mus. 13: I I 44 .

Listed by Neave, 1939, Nomencl. zool. 1: 447 as an avajlable genus. Walker was not proposing a new genus; the first of the species he included was $A$. cailino Lefebvre.

ALGONIA Möschler, I886, Abh. senckenb. naturforsch. Ges. 14 (3):39. SARR

Type-species: Algonia mollis Möschler, I886, ibidem 14 (3): 39, fig. 3, by monotypy. Holotype q, JamaICa (MNHU, Berlin).

ALIBAMA Möschler, I886, Abh. senckenb. naturforsch. Ges. 14 (3): 43.

ACRO

Type-species: Alibama pulchra Möschler, r 886, ibidem $14(3): 44$, fig. 27, by subsequent designation by Hampson, I909, Cat. Lepid. Phalaenae Br. Mus. 8: 276, but cited as 'Hadena terens Walker, I 857 ', which Hampson placed as the senior synonym of Alabama [misspelling] pulchra. Holotype $\widehat{\jmath}$. JamaICa (MNHU, Berlin).

ALIKA Strand, I920, Arch. Naturgesch. 84(A) I 2: I43.

ACRO

Type-species: Alika typica Strand, I920, ibidem 84(A) I2: I43, by original designation, under the Code, Article 68(b). Holotype $\widehat{\sigma}$. [TArwan] Formosa: Alikang (DEI, Eberswalde).

ALIKANGIANA Strand, I920, Arch. Naturgesch. 84(A) I2: I23.

ACON

Type-species: Alikangiana signicosta Strand, I920, ibidem 84(A) I 2: I23, by monotypy. Holotype $\hat{\sigma}$, [TAIWan] Formosa: Alikang (DEI, Eberswalde).

ALIMALA Walker, I862, J. Proc. Linn. Soc. (Zool.) 6: 132.

HADE Type-species: Alimala limacodoides Walker, I862, ibidem 6: I33, by monotypy. Lectotype ô. Borneo: Sarawak (UM, Oxford), designated (as type) by Swinhoe, rgoo, Cat. east. and Aust. Lepid. Heterocera 2: 38.

A. limacodoides is a junior subjective synonym of Elusa ceneusalis Walker, [1859].

See also Elusa Walker, [1859].

ALINZA Walker, [I866] I865, List Specimens lepid. Insects Colln Br. Mus. 34: I I48. HyPE Type-species: Alinza discessalis Walker, [1866] I865, ibidem 34: II 49, by monotypy.

Holotype ô. [BraziL]: [R. Amazon], [Tefé] Ega (BMNH).

$\ddagger$ ALINZIA; Warren, I889, Trans. ent. Soc. Lond. 1889: 233.

An incorrect subsequent spelling.

$\ddagger$ ALINZIA, misspelling. See Alinza Walker, [1866].

HYPE 
ALLAGRAPHA Franclemont, 1964, Proc. ent. Soc. Wash. 66: 73.

Type-species: Noctua aerea Hübner, [1803], Samml. eur. Schmett. 4: pl. 56, fig. 271, by original designation. Type(s), EuropE.

ALLIA Wralker, I867, J. Proc. Limn. Soc. (Zool.) 9: I88.

OPHI

Type-species: Allia ocellata VValker, I867, ibidem 9: I89, by monotypy. Type(s) $\widehat{\sigma}$, [Colombia]: Bogota.

ALLOMECIA Dumont, I928. See Pseudomecia Hampson, I9I8.

CUCU

ALLOPHYES Tams, I942, Entomologist 75: 209.

CUCU

Type-species: Phalaena oxyacanthae Linnaeus, I758, Syst. Nat. (Edn Io) 1: 5I6, by original designation. Type(s), TyPE-LOCALITY not stated (LS, London) [EUROPE].

+ MISELIA Hübner, [1806], Tentamen determinationis digestionis . . . : [I].

Included in a work rejected for nomenclatural purposes by Int. Commn zool. Nom., 1926, Smithson. misc. Collns 73(4) Opinion 97: I9. Also idem, I954, Opin. Decl. int. Commu zool. Nom. 6 Opinion 278: i 40.

Only included species: Phalaena oxyacanthae Linnaeus, $175^{8}$.

MISELIA Boisduval, I828, Eur. Lepid. Index meth.: 73

Type-species: Phalaena oxyacanthae Linnaeus, I758, by subsequent designation by Duponchel, I829, in Godart \& Duponchel, Hist. nat. Lépid. Papillons Fr. 7(2): 7I, but cited as ¥'oxiacanthae', an incorrect subsequent spelling.

A junior homonym of Miselia Ochsenheimer, I 816, - Lepid., Noctuidae. The objective replacement name is Allophyes Tams.

ALLORHODOECIA Brèthes, 1923, Revta Fac. Agron. Univ. nac. La Plata 15(I): 5. ACro Type-species: Allorhodoecia hampsoni Brèthes, I923, ibidem 15(I): 5, figs I-3, by original designation. Syntypes, Argentrxa: between San Anselmo and Coronel Suárez.

ALLOTRIA Hübner, [I823] isi6, Verz. bekannter Schmett.: 280.

CATO

Type-species: Ephesia elonympha Hübner, I I 8, Zuträge Samml. exot. Schmett. 1: I I, figs 29, 30, by monotypy. Type(s) , , [U.S.A.]: Georgia [formerly] in Florida.

${ }_{+}$BLEPHARA Hübner, I 808, Erste Zuträge Samml. exot. Schmett.: 4.

Included in a work rejected for nomenclatural purposes by Int. Commn zool. Nom. 1966, Bull.zool. Nom. 23 Opinion 789: 213.

Placed on the Official Index of Rejected and Invalid Generic Names in Zoology: Name No. I 829 .

Only included species: †Blephara elonympha Hübner, I808, later made nomenclaturally available as Ephesia elon'mpha. Hiibner, I8 8 .

ALOGONIA Schaus, InI6, Proc. U.S. natn. Mus. 50: 279.

HYPE

Type-species: Alogonia perissalis Schaus, I I6, ibidem $\mathbf{5 0}$ : 280, by original designation. Type(s) ơ, Surinam: Geldersland. Trinidad: Montserrat (USNM, Washington).

ALOPHOSOMA Turner, I929, Trans. R. Soc. S. Aust. 53: 302.

OPHI

Type-species: Alophosoma syngenes Turner, I929, ibidem 53: 302, by monotypy. Holotype $\hat{\jmath}$. Australia: Qd, Cairns, Kuranda (ANIC, Canberra).

ALOTSA Swinhoe, Igoo, Cat. east. and Aust. Lepid. Heterocera 2: 87.

EUTY

Type-species: Eutelia discitriga Walker, I 865, List Specimens lepid. Insects Colln Br. Mus. 33: 823, by subsequent designation by Hampson, I912, Cat. Lepid. Phalaenae Br. Mus. 11: I 5, but cited as +'discistriga', an incorrect subsequent spelling. Holotype $q$. [INDIA]: South Hindostan (BMNH).

E. discitriga is a junior subjective synonym of Eurhipia blandiatrix Cnence I852, in Boisluval \& Guenée, Hist. nat. Insectes (1.épid.) 6:307, pl. I 4, fig. 8.

ALPESA Tralker, I858, List Specimens lepid. Insects Colln Br. MIus. 15: I662.

ACRO

Type-species: Alpesa villicosta Walker, I 858, ibidem 15: I663, by monotyly. Type(s) $\hat{o}$, [BRAzIL]: Rio Janeiro (UM, Oxford). 
ALUACA Waller, [1866] i 865, List Specimens lepid. Insects Colln Br. Mus. 34: I I 52. OpH I Type-species: Aluaca eubolialis Walker, [1 866] 1865, ibidem 34: I I53, by subsequent designation by Hampson, 1926, Descr. new Genera Species Lepid. Phalaenae Subfamily, Noctuinae Br. Mus.: 332. Holotype q, [Dominican Republic] St. Domingo (BMNH).

$\ddagger$ ALUCULA, misspelling. See Aucula Walker, I 862 .

AGAR

ALURA Möschler, I884, Vevh. zool.-bot. Ges. Wien 33 (Abh.) : 303.

CATO

Type-species: Alura caupona Möschler, I884, ibidem 33 (Abh.) : 304, pl. I6, fig. I8, by monotypy. Holotype $\hat{0}$. [South AFrica]: Kaffernlandes.

A. caupona is a junior subjective synonym of Phoberia catocala Felder \& Rogenhofer, 1874, Reise öst. Fregatte Novara (Zool.) 2 (Abt. 2): pl. I 16, fig. 2.

ALYPIA Hübner, I818, Zuträge Samml. exot. Schmett. 1: 22.

AGAR

Type-species: Alypia octomaculalis Hübner, I8I8, ibidem 1: 22, figs II9, 120, by subsequent designation by Grote, 1873, Bull. Buffalo Soc. nat. Sci. 1: 30, but cited as Zygaena octomaculata Fabricius, which Grote placed as the senior synonym of octomaculalis Hübner. Type(s) ô, [U.S.A.]: Georgia [formerly] in Florida.

A. octomaculalis is a junior subjective synonym of Sesia octomaculata Fabricius, I775, Syst. Ent.: 830 (the type-locality, India, cited by Fabricius was probably incorrect).

See also Leucosemia Ménétriés, I 857.

AL YPIODES Grote, I883, Trans. Kans. Acad. Sci. 8: 46.

Type-species: Alypiodes flavilinguis Grote, I 883, ibidem 8: 4 , by monotypy. Type(s)

우, [U.S.A.]: New Mexico, near Las Vegas (USNM, Washington).

A. flavilinguis is a junior subjective synonym of Agarista bimaculata Herrich-Schäffer,

I 853, Samml. neuer oder wenig bekannter aussereur. Schmett. 1 (I): wrapper, pl. 4, fig. 26; I858, ibidem 1: 7 r.

$\ddagger$ AL YPIODES Dyar, 1902; Neave, 1939, Nomencl. zool. 1 : 130.

An incorrect citation of author by Neave. Dyar, 1902, Bull. U.S. natn. Mus. 52: 97, correctly attributed Alypiodes to Grote, I 883.

†LYPIOIDES; Neave, 1939, Nomencl. zool. 1: i 30.

An incorrect subsequent spelling.

$\ddagger$ ALYPIODES Dyar, Ig02. See Alypiodes Grote, 1883 .

AGAR

$\ddagger$ AL YPIOIDES, misspelling. See Alypiodes Grote, 1883 .

AGAR

AL YPOPHANES Turner, rgo8, Trans. Proc. R. Soc. S. Aust. 32: 62.

ACON

Type-species: Alypophanes iridocosma Turner, 1908, ibidem 32: 63, by monotypy. Syntypes, Australia: $2 \hat{\jmath}, 2$ 9 , Qd, Kuranda (ANIC, Canberra).

ALYSIA Guenée, I868. See Alysina Cockerell, I9I3.

HADE

ALYSINA Cockerell, 19I3, Entomologist 46: I 5.

HADE

Type-species: Alysia specifica Guenée, 1868, Entonologist's mon. Mag. 5: 3, by monotypy (of Alysia Guenée, I868). Syntypes ô, 우, New ZEALANd: Canterbury (BIINH).

A. specifica is a junior subjective synonym of Agrotis nullifera Wallier, I 857, I-ist Specimens lepid. Insects Colln Br. Mus. 11: 742.

Alysina was proposed as the objective replacement name for Alysia Guenée, i 868.

ALYSIA Guenée, I868, Entomologist's mon. Mag. 5: 3.

Type-species: Alysia specifica Guenée, I868, by monotypy.

A junior homonym of Alysia Latreille, r8o4, Nowv. Dict. Hist. nat. 24: 173, - Hymenoptera. The objective replacement name is Alysina Cockerell, i913.

AMABELA Möschler, 1880, Verh. zool.-bot. Ges. Wien 30 (Abh.) : 435.

OPHI

Type-species: Amabela delicata Möschler, r88o, ibidem 30 (Abh.) : 435, pl. 9, fig. 34, by monotypy. Holotype $\hat{\sigma}$, Surinam: Paramaribo. 
${ }_{+}^{+}$AMALOPTERA, misspelling. See Amaloptila Turner, IgO3. SARR

AMALOPTILA Turner, 1903, Trans. Proc. R. Soc. S. Aust. 27: 6. SARR Type-species: Amaloptila triorbis Turner, I903, ibidem 27:6, by monotypy. Holotype o. Australia: N.S.W., Newcastle (NMV, Melbourne).

A. triorbis is a junior subjective synonym of Elesma subglauca Walker, I 865.

See also Elesma Walker, I865.

$¥$ † MALOPTERA; Hampson, igr2, Cat. Lepid. Phalaenae Br. Mus. 11: 369.

An incorrect subsequent spelling.

AMARNA Walker, [1857] I 856, List Specimenslepid. Insects Colln Br. Mus. 10: 4I 3, 477 . OPHI Type-species: Amarna discursa Walker, [1 857] 1856, ibidem 10: 478, by monotypy. Holotype of. TŸPE-LOCALITY not stated (BMNH). [The only specimen of this species in BMNH is the holotype without pin-label data. Related species occur in central and southern America].

AMATHES Hübner, [1821] 1816, I'erz. bekannter Schmett.: $222 . \quad$ Noct

Type-species: Noctua baja [Denis \& Schiffermüller], 1775, Ankündung syst. Werkes Schmett. Wienergegend: 77 , by subsequent designation by Grote, 1895, Abh. naturw. Ver. Bremen 14: 62. Type(s), [Austria]: Vienna district (Collection destroyed, Horn \& Kahle, 1936, Ent. Beih. Berl.-Dahlem 3: 243).

Confusion has arisen as this generic name has been used by authors, based on the incorrect type-species, Phalaena (Noctua) lituva Linnaeus, I76r, -Cuculliinae.

AMATHES Hübner sensu Hampson, 1906. See Agrochola Hübner, [1821]. cUcU

† AMAURIDIA Iampson, no published reference found. HYPE

AMAZELA Boisduval, I874, Revne Mag. Zool. (3) 2:66. AGaR

Type-species: Amazela calisto Boisduval, r 874 , ibidem (3) 2: 66, by subsequent designation by Hampson, 1910, Cat. Lepid. Phalaenae Br. Nus. 9: 449. Syntypes, [Australia] New Holland (BMNH).

A. calisto was treated by Boisduval as though it was a new species although it is a junior subjective synonym of Apina callisto Walker, 1855, the type-species of Apina Walker, I 855 .

AMAZONIDES Fletcher, 196r, Ruwenzori Exped. I952 1: I79. NOCT

Type-species: Axylia putrefacta Guenée, 1852, in Boisduval \& Guenée, Hist. nat. Insectes (I.épid.) 5: 134, by original designation. Lectotype $\hat{\sigma}$, [ETHIopia] Abyssinia (MNHN, Paris), designated by Viette, 1951, Bull. mens. Soc. linn. Lyon 20: 160.

AMBLYGOES Butler, 1879, Illust. typical Specimens Lepid. Heterocera Colln Br. Mus. 3: 69. OPHI

Type-species: Apphadana evulsalis Walker, [1866] I 865, List Specimens lepid. Insects Colln Br. Mus. 34: I2I3, by monotypy (of Apphadana Waller, [1866]). Syntypes II $(0,9)$, Ceylon (BMNH).

A. evulsalis is a junior subjective synonym of Gesonia obeditalis Walker, [1859].

Amblygoes was proposed as the objective replacement name for Apphadana Walker.

See also Gesonia Walker, [1859]; Dragana Walker, [1859]; Hileia Walker, 1861; and Maresia Walker, 1866.

APPHADANA Walker, [1866] 1865, List Specimens lepid. Insects Colln Br. Muss. 34: I 2 I2.

Type-species: Apphadana evulsalis Walker, [1866] 1865, by monotypy.

A junior homonym of Apphadana Walker, I865, ibidem 33: 1094, - Iepid., Noctuidae. The objective replacement name is Amblygoes Butlcr, 1879 .

AMBLYGONIA Herrich-Schäffer, I855, Samml. neuer oder wenig bekannter antssereur. Schmett. 1 (1): wrapper, pl. 69, fig. 391; r 858 , ibidem 1: 69, 82. OPHI 
Type-species: Amblygonia atlantica Herrich-Schäffer, I 855, ibidem 1 (I): wrapper, pl. 69, fig. 391; i 858, ibidem 1: 69, 82, by monotypy. Type(s), Venezuela.

Amblygonia and atlantica are dated from the wrappers of the plates.

See also Juncaria Walker, I 858 .

AMBLYPRORA Bethune-Baker, I9I I, Ann. Mag. nat. Hist. (8) 8: 534.

OPHI

Type-species: Catephia acholi Bethune-Baker, I906, ibidem (7)18: 344 , by original designation. Holotype, UGANDA: northern, Patigo (BMNH).

AMBLYZANCLA Turner, I936, Proc. R. Soc. Qd 47: 34

OPHI

Type-species: Amblyzancla declivis Turner, I936, ibidem 47: 34, by monotypy.

Holotype, Australia: N. Qd, Cape York (QM, Brisbane).

$A$. declivis is a junior subjective synonym of Midea rectalis Walker, I863.

See also Arsacia Walker, [I866]; and Notocyma Snellen, I872.

AMEFRONTIA Hampson, I899, Bull. Liverpool Mus. 2: 37.

ACRO

Type-species: Amefrontia purpurea Hampson, I899, ibidem 2: 37, by original designation. Holotype $\hat{\sigma}$, Socotra I. [Indian Oc.]: Hadibu Plain (BMNH).

PALAFRONTIA Hampson, 19o8, Cat. Lepid. Phalaenae Br. Mus. $7: 6$.

Available, but without included species in volume 7. Hampson's key to genera in volume 7 is repeated with amendments in volume 8 . The couplet in volume $7: 6$ leading to Palafrontia is repeated in volume 8: 4 but leads to Amefrontia Hampson, which has an included species, A. purpurea Hampson, in 8: $4 \mathrm{I} 7$.

Type-species: Amefrontia purpurea Hampson, I 899, by subsequent monotypy.

Palafrontia is a junior objective synonym of Amefrontia Hampson, I 899.

AMELIA Wagner, I93I. See Amelina Draudt, I934.

ACRO

AMELINA Draudt, I934, in Seitz, Gross-Schmett. Erde 3 Suppl.: I 74.

ACRO

Type-species: Amelia gracilis Wagner, I93I, Int. ent. Z. 25: 369, by monotypy (of Amelia Wagner, I93I). Holotype $\hat{\delta}$. [TuRkey]: Akschehir.

A. gracilis is a junior subjective synonym of Victrix karsiana Staudinger, I 879.

Amelina was proposed as the objective replacement name for Amelia Wagner, I93I, but Draudt implied in the accompanying text that Amelina had already been proposed by Wagner as a replacement. However, $\ddagger$ Amelina Wagner is not recorded at all either in Neave's Nomencl. zool. 1-6, or in the Zool. Rec. I93I-I934.

The date of publication of the part of Seitz containing page I74, was stated on page I 69 as I 934 October $24^{\text {th }}$ in the German and the English editions.

See also Victvix Staudinger, I 879.

AMELIA Wagner, I93I, Int. ent. Z. 25: 368.

Type-species: Amelia gracilis Wagner, I931, by monotypy.

A junior homonym of Amelia Hübner, [I825] I8I6, Vevz. bekannter Schmett.: 390, Lepid., Tortricidae. The objective replacement name is Amelina Draudt, I934.

AMEPHANA Hampson, igo6, Cat. Lepid. Phalaenae Br. Mus. 6: viii, io6.

CUCU

Type-species: Cleophana anarrhini Duponchel, [1840] I836, in Godart \& Duponchel, Hist. nat. Lépid. Papillons Fr., Suppl. 3: 387, pl. 35, fig. 2, by original designation. Syntypes, France: Aix and Lyon.

C. anarrhini was attributed by Duponchel to Boisduval, I 840 , Genera Index meth. Eur.

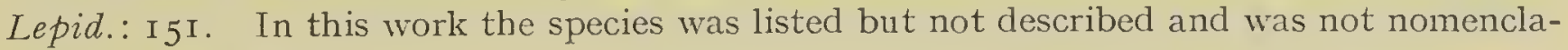
turally available.

AMETROPALPIS Mabille, i 884, C. r. Séanc. Soc. ent. Belg. 1884: clxxxix.

HYPE

Type-species: Ametropalpis nasuta Mabille, I 884, ibidem 1884: clxxxix, by monotypy. Type(s), Madagascar (not found, see Viette \& Fletcher, I968, Bull. Br. Mus. nat. Hist. (Ent.) $21: 4$ IO). 
AMIANA Dyar, 1904, Proc. ent. Soc. Wash. 6: 104.

ACRO

Type-species: Amiana niama Dyar, 1904, ibidem 6: 105, by original designation. Syntypes 8 ex., [U.S.A.]: Huachuca Mits and Nogales (USN.I, Washington).

AMILAGA Swinhue, Igor, Ann. Mag. nat. Hist. (7)8: r6.

HYPE

Type-species: Lusia geometroides Waller, [1 858] I857, List Specimens lepid. Insects Colln Br. Mus. 13: rrr3, by monotypy (of Lusia Walker, [1858]). Holotype ô. Ceylon (BMNH).

Amilaga was proposed as the objective replacement name for + Lucia, an error for Lusia Walker, [1 858 ].

LUSIA Walker, [1858] i 857, List Specimens lepid. Insects Colln Br. Mus. 13: Io79, I I 3.

Type-species: Lusia geometroides Walker, $[1858]$ i 857 , by monotypy.

A junior homonym of Lusia Milne-Edwards, 1836, in Lamarck, Hist. nat. Anim. sans I'ertèbres (Edn 2) 2: 72, - Bryozoa. The objective replacement name is Amilaga Swinhoe, I $90 \mathrm{I}$.

†LUCIA; Swinhoe, I901, Ann. Mag. nat. Hist. (7)8: 16.

An incorrect subsequent spelling.

AMMETOPA Hampson, i9o6, Cat. Lepid. Phalaenae Br. Mus. 6: ix, I 20.

CUCU

Type-species: Metopoceras codeti Oberthür sensu Hampson, I906, [a misidentification later described as Erythrophaia canroberti Oberthür, rgr8, Études Lépid. comparée 16: 182, fig. 4[30], by original designation. Syntypes (of E. canroberti) I $\hat{O}, \mathrm{I}$ ㅇ, Algeria: Constantine, El-Outaya (BIINH).

Hampson misidentified Metopoceras codeti Oberthür, I88I, Études Ent. 6: 88, pl. Ir, fig. 10. Hampson's specimens were of a species undescribed at that time. This is a case of a misidentified type-species which under the Code, Article $70(a)$, should be referred to the Commission.

${ }_{\ddagger}^{\ddagger}$ AMMOCANIA, misspelling. See Ammoconia Lederer, 1857 NOCT

AMMOCONIA Lederer, 1857, Noctuinen Eur.: 33, $97 . \quad$ NOCT

Type-species: Noctua caecimacula [Denis \& Schiffermüller], I775, Ankïndung syst. Werkes Schmett. Wienergegend: 8r, by subsequent designation by Hampson, 1903, Cat. Lepid. Phalaenae Br. Mus. 4: 602. Type(s), [Austria]: Vienna district (Collection destroyed, Horn \& Krahle, 1936, Ent. Beih. Berl.-Dahlem 3: 243).

† MMOCANIA; Tschetveriliov, I904, Revue russe Ent. 4: 78.

An incorrect subsequent spelling.

AMMOGROTIS Staudinger, I895, Dt. ent. Z. Iris 8: 358.

NOCT

Type-species: Ammogrotis suavis Staudinger, I 895 , ibidem 8: 358, pl. 6, fig. II, by monotypy. Syntypes $2 \vec{\jmath}$, [Mongolia]: Uliassutai (MNHU, Berlin).

AMMOPHANES Turner, 1932, Trans. R. Soc. S. Aust. 56 : 179.

OPIII

Type-species: Ammophanes deserticola Turner, I932, ibidem 56: I 79, by monotypy. Holotype ô, Australia: Qd, Charleville (ANIC, Canberra).

AMMOPOLIA Boursin, 1955, Bull. mens. Soc. linn. Lyon 24: 219.

NOCT

Type-species: Orthosia witzenmanni Standfuss, I 890, Nitt. schweiz. ent. Ges. 8: 233 , by original designation. Syntypes $\widehat{\sigma}$. Francl: Basses-Alpes, Digne.

AMOLITA Grote, 1874, Bull. Buffalo Soc. nat. Sci. 2: 158.

ACRO

Type-species: Amolita fessa Grote, I874, ibidem 2: I 58, by monotypy. Holotype 0 , [U.S.A.]: New York (BMNH).

AMPELASIA Schaus, I9I 3, Ann. Mag. nat. Hist. (8)11: 3.

OPHI

Type-species: Ampelasia azelinoides Schaus, r9r3, ibidem (8)11:3, by original designation. Type(s) $q$, Costa Rica: Juan Viñas. 
AMPHIA Guenée, I852, in Boisduval \& Guenée, Hist. nat. Insectes (Lépid.) 5: 224. ACro

Type-species: Amphia hepialoides Guenée, I852, ibidem 5: 224, pl. 6, fig. I2, by subsequent designation by Viette, 1965, Faune Madagascar 20 (I): 405. Lectotype o, [Ethiopia] Abyssinia (MNHN, Paris), designated by Viette, I95 I, Bull. mens. Soc. linn. Lyon 20: 160.

AMPHIDRINA Staudinger, I891, Dt. ent. Z. Iris 4: 293.

ACRO

Type-species: Amphidrina agrotina Staudinger, I89 I, ibidem 4: 293, pl. 3, fig. I I, by monotypy. Syntypes ô, ㅇ. [Turkey]: Amasia (MNHU, Berlin); südlichen Anti-Taurus, Eibes (MNHU, Berlin).

AMPHIGONIA Guenée, 1852, in Boisduval \& Guenée, Hist. nat. Insectes (Lépid.) 7: 337.

OPHI

Type-species: Amphigonia hepatizans Guenée, I852, ibidem 7:338, pl. 24, fig. I2, by subsequent designation by Moore, [1885] I887, Lepid. Ceylon 3: 215. Type(s), EAST INDIES (BMNH).

ACYGONIODES Hampson, 1926, Descr. new Genera Species Lepid. Phalaenae Subfamily Noctuinae Br. Mus.: 5 I 7 .

Type-species: Amphigonia hepatizans Guenée, I 852, by original designation.

Acygoniodes is a junior objective synonym of Amphigonia Guenée, I 852.

AMPHILITA Hampson, I908, Cat. Lepid. Phalaenae Br. Mus. 7: i 8. Available, but without included species until Hampson, I910, ibidem 9: xi, 303.

ACRO

Type-species: Parvapenna punctilinea Jones, I908, Trans, ent. Soc. Lond. 1908: I65. by subsequent designation by Hampson, igro ibidem 9: 303. Type(s) J゙, Brazil: São Paulo (BMNH).

AMPHIONGIA Hampson, 1926, Descr. new Genera Species Lepid. Phalaenae Subfamily Noctuinae Br. Mus.: 516.

OPHI

Type-species: Zethes chordophoides Lucas, I892, Proc. R. Soc. Qd 8: 89, by original designation. Syntypes $\hat{0}$, ㅇ․, Australia: Brisbane to Mackay (SAM, Adelaide).

AMPHIPOEA Billberg, I820, Enumeratio Insect. Mus. G. J. Billberg: 87.

ACRO

Type-species: Phalaena nictitans Linnaeus, I767, Syst. Nat. (Edn I2) 1: 847, by subsequent designation by Franclemont, 1950, Bull. Brooklyn ent. Soc. 45: I48. Type(s), EUROPE (LS, London).

$P$. nictitans is a junior subjective synonym of Phalaena oculea Linnaeus, I76I, Fanna Suecica (Edn 2): $32 \mathrm{I}$.

Incorrect type-species designation: Phalaena secalis Linnaeus, I758, a name not originally included in Amphipoea, and not linked with one of the originally included names when cited by Tams, 1939, Entomologist 72: 136.

Berio, I966, Annali Miss. civ. Stor. nat. Giacomo Doria 76:48, considered that Franclemont's designation was incorrect as there was no mention of the combination Amphipoea nictitans. I cannot agree that this invalidated the designation.

AMPHIPYRA Ochsenheimer, I816, Schmett. Eur. 4: 70.

ACRO

Type-species: Phalaena tragopoginis Clerck, I759, Icones Insect. rariorum 1: pl. [I], fig. 5 (but included by Ochsenheimer as ‡'tragopogonis’, an incorrect subsequent spelling), by subsequent designation by Duponchel, I829, in Godart \& Duponchel, Hist. nat. Lépid. Papillons Fr. 7(2): 7I, but cited as ł'tragopogonis'. Type(s), TYPE-LoCALITY not stated (NR, Stockholm) [EUROPE].

$\ddagger$ PYROPHYLA Hübner, [1806], Tentamen determinationis digestionis . . . : [I] .

Included in a work rejected for nomenclatural purposes by Int. Commn zool. Nom., 1926, Smithson. misc. Collns 73(4) Opinion 97: I9. Also idem, I954, Opin. Decl. int. Commn zool. Nom. 6 Opinion 278: I40.

Only included species: Phalaena pyramidea Linnaeus, 1758 , which is congeneric with $P$. tragopoginis Clerck, I759. 
Pyrophyla was not used again in zoology. A similar name Pyrophila Stephens, 1829, was subsequently proposed.

$\ddagger$ PYROPHILAE Ochsenheimer, 1816, Schmett. Eur. 4: 70 .

Published as a junior synonym of Amphipyra Ochsenheimer, I8I6, and not subsequently treated as an available name under the Code, Article II(d).

SCOTOPHILA Hübner, [182I] I8I6, Verz. bekannter Schmett.: 209.

Type-species: Phalaena tragopoginis Clerck, i759 (but included by Hübner as \$tragopogonis Linn.', an incorrect subsequent spelling and authorship), by subsequent designation by Blanchard, I840, in Castelnau, Hist. nat. Anim. articulés (Insectes) 3: 500

(but cited as 'tragopoginis Linn.', an incorrect authorship).

Scotophila is a junior objective synonym of Amphipyra Ochsenheimer, 1816.

PYROPHILA Stephens, I 829, Illust. Br. Ent. (Haustellata) 2: i64.

Type-species: Phalaena tragopoginis Clerck, I759, by subsequent designation by Westwood, I840, Synopsis Genera Br. Insects: 94.

Invalid designation of type-species: P. tragopoginis was also designated by Boisduval, 1836, Hist. nat. Insectes (Lépid.) 1: 144. Boiscluval in his lengthy Introduction, up to

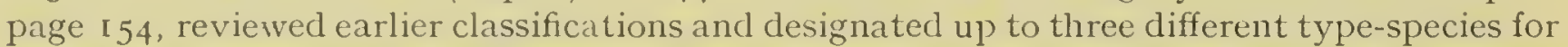
each generic name. In his 'Exposé de notre méthode' from page I 55-69o, no type-species designations were made for the genera he himself used. Under the Code, Article 69(a) (iii), the type-designation of an author is eligible for consideration if he states that it is the type '. . . and if it is clear that he himself accepts it as the type-species.' Boiscluval's type-designations although clearly stated do not fulfil the last requirement and so are invalid. Even though Boisduval's I 836 work was well known to lepidopterists, the type-designations contained in it have not in the past been accepted by Hemming or by other authors. The acceptance of Boiscluval's designation in this case would make no difference to the generic concept.

Pyrophila is a junior objective synonym of Amphipyra Ochsenheimer, I8I6.

PHILOPYRA Guenée, 1837, Amis Soc. ent. Fr. 6: 222.

Proposed, unnecessarily, as an objective replacement name for Pyrophila Stephens, I 829, which Guenée considered to be a junior homonym of Noctua pyrophila [Denis \& Schiffermüller], 1775 .

Philopyra is cited in Neave, 1940, Nomencl.zool. 3: 713 as being proposed by Guenée, I 838 , ibidem $7:$ i 6 , where it is used for the second time.

AMPHITROGIA Hampson, 1926, Descr. new Genera Species Lepid. Phalaenae Subfamily Noctuinae Br. Mus.: 589 .

OPHI

Type-species: Marmorinia amphidecta Butler, 1879, Illust. typical Specimens Lepid. Heterocera Colln Br. Mus. 3: xvi, 6n, pl. 57, fig. I2, by original designation. Type(s), [JAPAN]: Iokohama (BMNH).

AMPHITROTA Warren, 1909, in Seitz, Macrolepid. W'orld 3: 57.

NOCT

Type-species: Mamestra unicolor Walker, I856, List Specimens lepid. Insects Coln Br. Mus. 9: 233, by original designation. Syntypes 8 ․ [U.S.A.]: New York, Trenton Falls (BMNH). [CANAdA]: Hudson's Bay, R. Albany; Nova Scotia.

The date of publication of the part of Seitz containing page 57, was stated on page 53 as 1909 October 28th in the English edition and Igog November 19th in the German edition.

AMPHODIA Nöschler, I88o, Verh. zool.-bot. Ges. Wien 30 (Abh.): 386.

ACON

Type-species: Amphodia prolata Möschler, I880, ibidem 30 (Abh.): 387, pl. 9, fig. 45, by monotypy. Holotype $\hat{\sigma}$. SURINAm: interior (MNHU, Berlin).

AMPHORACERAS Bethune-Baker, 1904, Novit. zool. 11: 427.

CA'TO

Type-species: Amphoraceras rothschildi Bethune-Baker, 1904, ibidem 11: 428, p1. 5 , fig. I, by monotypy. Syntypes $\hat{0}$,, , New Guinea: [Papua], Dinawa (BMNH). 
AMRELLA Moore, I882, in Hewitson \& Moore, Descr. new Indian lepid. Insects Colln late MrW.S. Atkinson: 158 .

Type-species: Amrella angulipennis Moore, I882, ibidem: I 58, pl. 5, fig. 6, by monotypy. Type(s), India: [W. Bengal], Darjiling (MHNU, Berlin).

AMYNA Guenée, 1852, in Boisduval \& Guenée, Hist. nat. Insectes (Lépid.) 5: 406 ACON

Type-species: Amyna selenampha Guenée, I852, ibidem 5: 406, by subsequent designation by Hampson, I 894, Fauna Br. India (Moths) 2:250. Syntypes, [Bangladesh]: [E. Bengal], Silhet (BMNH); [Philippines]: [Manila] Manille.

A. selenampha is a junior subjective synonym of Noctua punctum Fabricius, I794, Ent. Syst. $3(2): 34$.

See also Hesperimorpha Saalmüller, I880.

AMYNODES Warren, I913, in Seitz, Gross-Schmett. Erde 11: 273.

ACON

Type-species: Erastria distigmata Hampson, I896, Fauna Br. India (Moths) 4: 513, by original designation. Holotype, BHutan (BMNH).

The date of publication of this part of Seitz was stated on page 273 as I9I3 October $5^{\text {th }}$ in both the English and German editions.

†AMYTUS, misspelling. See Sutyna Todd, I958.

NOC'T

ANABATHRA Möschler, 1887, Abh. senckenb. naturforsch. Ges. 15: 89.

CATO

Type-species: Anabathra una Möschler, I887, ibidem 15: 89, fig. 22, by monotypy.

Holotype $\widehat{\sigma}$, [Ghana] Gold Coast: Accra.

A. una is a junior subjective synonym of Tachosa acronyctoides Walker, I869.

See also Tachosa Walker, I869.

ANABLEMMA Schaus, I9I I, Ann. Mag. nat. Hist. (8)8: Iog.

ACON

Type-species: Anablemma lebana Schaus, I 9 I I, ibidem (8)8: Io9, by original designation.

Type(s) ô. Costa Rica: Sixola.

A. lebana is a junior subjective synonym of Lambana cucullatalis Walker, [1866].

See also Lambana Walker, [1866].

ANACHROSTIS Hampson, I893, Illust. Lepid. Heterocera Br. Mus. 9: 20, 98.

OPHI

Type-species: Anachrostis nigripuncta Hampson, I893, ibidem 9: 20, 98, pl. I62, fig. I9,

by monotypy. Type(s) 오, Ceylon: Pundaloya (BMNH).

ANACRONICTA Warren, I9o9, in Seitz, Macrolepid. World 3: I8.

PANT

Type-species: Aplectoides caliginea Butler, I88I, Trans. ent. Soc. Lond. 1881: I85, by subsequent designation by Hampson, I9I3, Cat. Lepid. Phalaenae Br. Mus. 13: 339. Type(s) JaPAN: Tokei (BMNH).

The date of publication of the part of Seitz containing page I8, was stated on page I3 as I 909 February 24th in the English edition, and Igog May 2Ist in the German edition.

ANACRONYCTA Bryk, I94I, Ent. Tidskr. 62: I 49.

An unjustified emendation of Anacronicta Warren, Igog.

ANACRONYCTA Bryk, I94I. See Anacronicta Warren, Igog.

PANT

$\ddagger$ ANACTA, misspelling. See Semiophora Stephens, I829.

HADE

ANADEVIDIA Kostrowicki, I961, Acta zool. cracov. 6: 384.

PLUS

Type-species: Noctua peponis Fabricius, I 775, Syst. Ent.: 608, by original designation.

Type(s) India : eastern (UZM, Copenhagen).

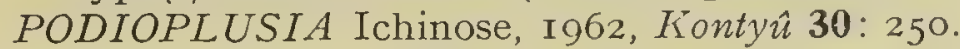

Type-species: Noctua peponis Fabricius, I775, by original designation.

Podioplusia Ichinose is a junior objective synonym of Anadevidia Kostrowicki, I96I.

ANAGOA Möschler, I886, Abh. senckenb. naturforsch. Ges. 14(3): 62.

HYPE

Type-species: Anagoa ophiusioides Möschler, I886, ibidem 14(3): 62, fig. 3I, by monotypy. Holotype $\widehat{\jmath}$. Jamaica (MNHU, Berlin).

$\mathrm{n}$ 
ANAGRAPHA McDunnough, I944, Mem. sth. Calif. Acad. Sci. 2: 202.

Type-species: Plusia falcifera Kirby, I837, in Richardson, Fauna Boreali-Am. 4: 308, by original designation. Type(s) [CANADA]: Nova Scotia.

ANALETIA Calora, 1966, Philipp. Agric. 50: 709.

HADE

Type-species: Leucania micacea Hampson, I891, Illust. typical Specimens Lepid. Heterocera Colln Br. Mus. 8: 1 1, 67, pl. I44, fig. 8, by original designation. Syntypes of. INDIA: S., Nilgiri Hills (BMNH).

ANAMECIA Boursin, 1958, Bull. mens. Soc. linn. Lyon. 27: 5.

ACRO

Type-species: Pseudohadena deceptrix Staudinger, I900, Dt. ent. Z. Iris 12:369, pl. 5, fig. I 5, by original designation. Syntypes I $\hat{0}$, I $q$, [Jordan] Palestine: Jordan valley (MNHU, Berlin).

ANANEPA Hampson, 1926, Descr. new Genera Species Lepid. Phalaenae Subfamily Noctuinae Br. Mus.: 205 .

OPH I

Type-species: Hypena doda Swinhoe, 1902, Ann. Mag. nat. Hist. (7) 9: 180, by original designation. Syntypes , [West Malaysia]: [Malay], Perak, Goping (BMNH).

ANAPLECTOIDES MicDunnough [1929] 1928, Bull. Dep. Mines, Can. 55: 65.

NOCT

Type-species: Eurois pressus Grote, 1874, Trans. Am. ent. Soc. 5: 90, by original designation. Syntypes of, $q$, [U.S.A.]: New York (BMNH). Canada: St Catharines.

ANARTA Ochsenheimer, I8I6, Schmett. Eur. 4: 90.

HADE

Type-species: Phalaena myrtilli Linnaeus, I761, Fanna Suecica (Edn 2): 311, by subsequent designation by Samouelle, I819, Entomologist's useful Compendium: 252. Type(s), Sweden (LS, London).

CHARELIA Sodoffsky, r837, Bull. Soc. imp. Nat. Moscou 1837 (6) : 88.

Proposed, unnecessarily, as an objective replacement name for Anarta Ochsenheimer, I 8 I6, which Sodoffsky considered to be inappropriate.

ANARTODES Culot, 1915, Noctuelles Géomètres Eur. 2: 125.

HADE

Type-species: Mamestra rangnovi Püngeler, 1909, Dt.ent. Z. Iris 21:288, by monotypy. Syntypes I ô, I ․, [SwEDEN]: Lappmark, Lulea (MNHU, Berlin).

Anartodes was attributed to Oberthür by Culot, but no earlier published reference has been found. Anartodes is dated from the wrappers of the part and not the title-page of the volume.

$M$. rangnovi is often cited as +'rangnowi', an incorrect subsequent spelling. Püngeler's latinisation of the personal name Rangnow is permitted under the Code, Article 32(a).

$M$. rangnovi is a junior subjective synonym of Anarta lamuta Hertz, 1903, Anmu. Mus. zool. Acad. imp. Sci. St Pétersb. 8: 82.

ANARTOMIMA Boursin, 1952, Z. Lepid. 2: 55.

HADE

Type-species: Anarta bohemani Staudinger, I861, Stettin. ent. Ztg 22: 370, 403, by original designation (of Pseudanarta Kozhanchikov). Syntypes $\hat{0},+$, [Scandinavia]: Lappland, Munioniska and other localities (MNHU, Berlin has $2 \hat{0}, 2$ q from Alten).

$A$. bohemani has often been spelled ¥'bohemanni', an incorrect subsequent spelling. Staudinger referred frequently to Professor Boheman in the text.

Anartomima was proposed as the objective replacement name for Pseudanarta Kozhanchikov, 1947.

PSEUDANARTA Kozhanchikov, I947, Ent. Obozr. 29: I 4, I8.

Type-species: Anarta bohemani Staudinger, by original designation, but cited as ‡'bohemanni Stgr.'

A junior homonym of Pseudanarta Grote, 1878,-Lepid., Noctuidae. The objective replacement name is Anartomima Boursin, 1952. 
ANARTOMORPHA Alphéraky, 1892, in Romanoff, Mém. Lépid. 6: 39.

NOCT

Type-species: Anartomorpha potanini Alphéraky, I892, ibidem 6: 39, pl. 2, fig. 8, by monotypy. Holotype ㅇ, Chrna: [Kansu], Lagi (ZI, Leningrad).

ANATAELIA Draudt, I933. See Paranataelia Draudt, I935.

ACRO

ANA TATHA Hampson, I926, Descr. new Genera Species Lepid. Phalaenae Subfamily Noctuinae Br. Mus.: 291.

OPHI

Type-species: Catada nigrisigna Hampson, I895, Fauna Br. India (Moths) 3: 62, by original designation. Type(s), Indra: [Punjab], Simla (BMNH).

ANATEINOMA Möschler, I890, Abh. senckenb. naturforsch. Ges. 16: I69.

ACON

Type-species: Anateinoma affabilis Möschler, I 890, ibidem 16 : I 70, fig. I4, by monotypy. Syntypes $3 \hat{0}, \mathbf{I}$ q, Puerto Rico (MNHU, Berlin).

ANATHETIS Janse, 1938, Moths S. Afr. 3: 280.

ACRO

Type-species: Caradrina atrirena Hampson, 1902, Ann.S. Afr. Mus. 2: 304, by original designation. Type(s) $\tilde{\sigma}$. [South AFrica]: Cape Colony, Worcester district (BMNH).

ANATHIX Franclemont, 1937, Can. Ent. 69: I27.

CUCU

Type-species: Xanthia puta Grote \& Robinson, I868, Trans. Am. ent. Soc. 1: 347, pl. 7 , fig. 50, by original designation. Type(s) $\hat{0}$, [U.S.A.]: Atlantic District, N.Y., Trenton Falls.

ANCARA Walker, I858, List Specimens lepid. Insects Colln Br. Mus. 15: I 7 I 4.

ACRO

Type-species: Ancara replicans Walker, I858, ibidem 15: I 7 I5, by subsequent designation by Hampson, I894, Fauna Br. India (Moths) 2: 223. Holotype ô, Borneo (BMNH).

ANCARISTA Jordan, I921, Novit. zool. 28: 73.

AGAR

Type-species: Ovios laminifera Saalmüller, I878, Ber. senckenb. naturf. Ges. 1877-78: $9 \mathrm{I}$, by original designation. Holotype, Madagascar.

ANCAROIDES Bethune-Baker, 1906, Novit. zool. 13: 196.

ACRO

Type-species: Ancaroides kebea Bethune-Baker, 1906, ibidem 13: 196, by subsequent designation by Collins, I962, Ann. Mag. nat. Hist. (13)5: 5. Syntypes, New Gurnea: [Papua], Kebea Range (BMNH); Ekeikei (BMNH); Aroa River.

ANCATA Căpușe, 1958. See Semiophora Stephens, I829.

HADE

ANCHIROE Saalmüller, I89I, Lepid. Madagascar: $35^{\circ}$.

$A C O N$

Type-species: Anchiroe flavofimbria Saalmüller, I891, ibidem: 350, pl. I3, fig. 231, by subsequent designation by Hampson, igio, Cat. Lepid. Phalaenae Br. Mus. 10: 497. Syntypes $\hat{\jmath}, q$, MADAGASCAR: S.-Betsileo-Lande.

Anchiroe was cited as $¥$ 'Anchiroa', an incorrect original spelling, in the caption to pl. Io, fig. I67. In the text, index and caption to fig. 23I, the correct spelling has been used. ANCHIRRHOE Bertkau, I892, Arch. Naturgesch. 58(2) (2): I88.

An unjustified emendation of Anchiroe Saalmüller, I891.

Anchirrhoe was proposed as ¥'Anchir $(r h) \circ \ddot{e}$ ', an incorrect original spelling which under the Code, Article 32(c)(i) must be corrected by the deletion of parentheses and diaeresis.

ANCHIRRHOE Bertkau, i892. See Anchivoe Saalmüller, I89i.

$\operatorname{ACON}$

†ANCHOCELIS, misspelling. See Anchoscelis Guenée, I839.

CUCU

ANCHOSCELIS Guenée, I839, Annls Soc. ent. Fr. 8: 483.

CUCU

Type-species: Noctua nitida [Denis \& Schiffermüller], I775, Ankündung syst. Werkes Schmett. Wienergegend: 86 (but included by Guenée as 'nitida Fab.', an incorrect authorship), by subsequent designation by Duponchel, I84I, in d'Orbigny, Dict. univl Hist. nat. 1: 
473, but cited as 'nitida Fabr.' Type(s), [Austria]: Vienna district (Collection destroyed, Horn \& Kahle, I936, Ent. Beih. Berl.-Dahlem 3: 243).

$\ddagger$ ANCHOCELIS; Stephens, 1850, List Specimens Br. Anim. Colln Br. Mus. 5: 85.

An incorrect subsequent spelling.

$¥ A N C I O L A$, misspelling. See Anicla Grote, I 874 .

ANCISTRIS Mabille, I898, Annls Soc. ent. Fr. 66: 225.

OPHI

Type-species: Ancistris saturnina Mabille, I 898 , ibidem $66: 225$, by monotypy. Type(s), Madagascar (not found by Viette \& Fletcher, I968, Bull. Br. Mus. nat. Hist. (Ent.) 21 : 4 IO).

ANDESIA Hampson, 1906, Cat. Lepid. Phalaenae Br. Mus. 6: 6, I42.

CUCU

Type-species: Andesia oenistis Hampson, I906, ibidem 6: 142, fig. 44, by original designation. Syntypes $2 \hat{0}, \mathrm{I}+$, Argenrina: Mendoza, Puenta del Inca (BMNH).

ANDICOLA Staudinger, r894, Dt. ent. Z. Iris 7: 86.

CUCU

Type-species: Andicola huallatani Staudinger, I894, ibidem 7: 86, pl. 2, fig. I 3, by monotypy. Syntypes I $\hat{O}, \mathrm{I} \rho,[$ France/ITALy]: Mont Blanc.

ANDOBANA Viette, 1965, Faune Madagascar 20:357.

ACRO

Type-species: Perigea duchesnei Viette, (1960), Bull. Soc. ent. Fr. 64:228, by original designation. Holotype $\overrightarrow{0}$, MADAGascar: S.E. of Diego-Suarez, Analamerana forest (MNHN, Paris).

ANDRAPHA Walker, I866, List Specimens lepid. Insects Colln Br. Mus. 35: I74I.

SARR

Type-species: Andrapha basalis Walker, I 866, ibidem 35: I742, by monotypy. Type(s) , BoRNEO: Sarawak (BMNH).

ANDREUSIA Hampson, I913. See Andrewsia Grote, I882.

CATO

ANDREWSIA Grote, 1882, New Check List N. Am. Moths: $4 \mathrm{I}$.

CATO

Type-species: Catocala belfragiana Harvey, 1875, Bull. Buffalo Soc. nat. Sci. 2: 281, by monotypy. Type(s) [U.S.A.]: Texas (BMNH).

ANDREUSIA Hampson, I913, Cat. Lepid. Phalaenae Br. Mus. 12: 206.

An unjustified emendation of Andrewsia Grote, I882.

ANDRHIPPURIS Karsch, 1895, Ent. Nachr. 21: 348.

AGAR

Type-species: Andrhippuris caudaequina Karsch, 1895, ibidem 21: 348, 353, pl. I, fig. I, by original designation. Syntypes $\delta$, $q$, WEST AfrICA: Lower Guinea, Mukenge; Quango (MNHU, Berlin).

ANDRIANAM Viette, 1954, Bull. Soc. zool. Fr. 78: 34r.

OPHI

Type-species: Andrianam poinimerina Viette, 1954, ibidem 78: 342, fig. 4, by original designation. Holotype ô, Madagascar: S.E., env. de Fort-Dauphin, forêt d'Isaka (MNHN, Paris).

ANDRODES Turner, I920, Trans. Proc. R. Soc. S. Aust. 44: I29, I34.

NOCT

Type-species: Agrotis tibiata Guenée, 1852, in Boisduval \& Guenée, Hist. nat. Insectes (Lépid.) 5: 273, by original designation. I,ectotype $\hat{\sigma}$, [AusTraLIA] Nouvelle-Hollande (MNHN, Paris), designated by Viette, I95I, Bull. mens. Soc. linn. Lyon 20: I60.

ANDROLOMA Grote, 1873, Bull. Buffalo Soc, nat. Sci. 1: 30.

Type-species: Alypia lorquinii Grote \& Robinson, I868, Trans. Am. ent. Soc. 1: 328,

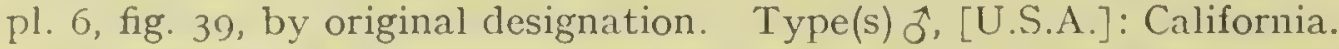

A. lorquinii is a junior subjective synonym of Alypia maccullochii Kirby, 1837 , in Richardson, Fauna Boreali-Am. 4: 3or, pl. 4, fig. 5.

ANDROLYMNIA Hampson, I908, Cat. Lepid. Phalaenae Br. Mus. 7: 15. Available, but without included species until Hampson, I9Io, ibidem 9: ix, I79.

ACRO 
Type-species: Ozarba emarginata Hampson, I891, Illust. Lepid. Heterocera Br. Mus. 8: I 3, 76, pl. I 45, fig. 22, by subsequent designation by Hampson, I91o, ibidem 9: 179 . Type(s) ठ̊. India: [Madras prov.], Nilgiri slopes 3000-4000 ft (BMNH).

ANDROPOLIA Grote, 1895, Abh. naturw. Ver. Bremen 14: 82.

Type-species: Apatele theodori Grote, I 878, Can. Ent. 10:237, by original designation.

Type(s) ô. [U.S.A.]: Colorado (BMNH).

$A$. theodori was originally proposed as $¥$ Apatela, an incorrect subsequent spelling.

ANEDA Sukhareva, 1973, Ent. Obozr. 52: 404, 410.

HADE

Type-species: Noctua rivularis Fabricius, $\mathrm{I} 775$, Syst. Ent.: 6I3, by original designation. Type(s), [Germany (EAst)]: Saxony (Type(s) not found by Zimsen, I964, Type Material of I. C. Fabricius: 26I).

Aneda was proposed as a subgenus of Hadena Schrank, r8o2.

ANEDHELLA Viette, I965, Bull. Soc. ent. Fr. 70: 88.

ACRO

Type-species: Hadenella thermodesa Viette, (1958) I957, Bull. Soc. ent. Fr. 62: 275, by original designation. Holotype $q$, Madagascar: Route d'Anosibe, $\mathrm{Km} 52$ (MNHN, Paris).

ANELIOPIS Bethune-Baker, I908, Novit. zool. 15: 208.

HYPE

Type-species: Aneliopis alampeta Bethune-Baker, 1908, ibidem 15: 208, by original designation. Syntypes ô, ㅇ, New Guinea: [PAPuA], Owgarra (BMNH); Dinawa.

ANEPA Swinhoe, I905. See Folka Swinhoe, I917.

OPHI

$\ddagger$ ANEPHISCHETOS, misspelling. See Anepischetos Smith, Igoo.

HYPE

ANEPHOLCIA Prout, 1924, in Prout \& Talbot, Bull. Hill Mus., Witley 1: 40 I.

PANT

Type-species: Anepholcia talboti Prout, 1924, ibidem 1:402, pl.13, fig. 9, by original designation. Syntypes i Io $\hat{\jmath}, 5$ q, Sumatra: S.W., Mt Korintji (BMNH); N. Korintji valley.

ANEPIA Hampson, I9r8, Novit. zool. 25: II6.

HADE

Type-species: Phalaena echii Borkhausen, I792, Naturg. eur. Schmett. 4: I66, by subsequent designation (for Epia Hübner, [I82I]) by Hampson, 1905, Cat. Lepid. Phalaenae Br. Mus. 5: 226. Syntypes ơ, ㅇ, Europe.

$P$. echii is a junior subjective synonym of Phalaena irregularis Hufnagel, I766, Berlin. Mag. 3 (4): 394.

Anepia was proposed as the objective replacement name for Epia Hübner, [I82I].

EPIA Hübner, [I82I] I8I6, Verz. bekannter Schmett.: 2 I4.

Type-species: Phalaena echii Borkhausen, I 792, by subsequent designation by Hampson, 1905, Cat. Lepid. Phalaenae Br. Mus. 5: 226, but cited as 'Noctua irregularis Hufnagel, I766,' which Hampson placed (on page 228) as the senior synonym of $P$. echii.

A junior homonym of Epia Hübner, [1820] r8I6, Verz. bekannter Schmett.: I88, - Lepid., Bombycidae. The objective replacement name is Anepia Hampson, I9I8.

ANEPILECTA Warren, I9I2, in Seitz, Gross-Schmett. Erde 11: 64.

NOCT

Type-species: Spintherops accipiter Felder \& Rogenhofer, 1874, Reise öst. Fregatte

Novara (Zool.) 2 (Abt. 2): pl. I I , fig. 29, by original designation. Type(s) ㅇ, [?INDIA]: [? Kashmir], Pangi (BMNH).

The date of publication of the part of Seitz containing page 64 was stated on page 57 as I9I2 October Ioth in both the German and in the English editions.

See also Perinaenia Butler, I878.

ANEPISCHETOS Smith, I90o, Proc. U.S. natn. Mus. 22: 482.

Type-species: Anepischetos bipartita Smith, I90o, ibidem 22: 482, by monotypy. Syntypes 4 ठే. [U.S.A.]: Florida, Key West (USNM, Washington). 
A. bipartita is a junior subjective synonym of Phalaena lividalis Hübner, I790.

See also Ophiuche Hübner, [1825].

$\ddagger$ ANEPHISCHETOS; McDunnough, 1938, Mem. sth. Calif. Acad Sci. 1: I28.

An incorrect subsequent spelling.

ANEREUTHINA Hübner, I 823, Zuträge Samml. exot. Schmett. 2: 23.

OPHI

Type-species: Anereuthina renosa Hübner, I823, ibidem 2: 23, figs 325, 326, by monotypy. Type(s) $\sigma^{2}$ Java.

HYPAETRA Guenée, 1852, in Boisduval \& Guenée, Hist. nat. Insectes (Lépid.) 7: 259.

Type-species: Anereuthina renosa Hübner, I 823, by subsequent designation by Desmarest, (1857), in Chenu, Encycl. Hist. nat. Papillons nocturnes: I 32.

Hypaetra is a junior objective synonym of Aneveuthina Hübner, I823.

$\ddagger$ HYPETRA; Herrich-Schäffer, I854, Samml. newer oder wenig bekannter ausseveur. Schmett.

1 (I): wrapper, pl. 27, fig. I33; I 858, ibidem 1:68, 79.

An incorrect subsequent spelling of Hypaetra Guenée, 1852. Neave, 1939, Nomencl. zool. 2: 732 listed $¥$ Hypetra as a nomenclaturally available name but there is no evidence to show that Herrich-Schäffer was proposing a new generic name. The new species which he figured as 'Hyp[a]etra megastigma' is within Guenée's generic concept.

ANEREUTHINULA Strand, I920, Arch. Naturgesch. 84(A) I 2: I 38.

ACRO

Type-species: Anereuthinula lyncestidis Strand, I920, ibidem 84(A)I2: I38, by monotypy. Holotype + , [TAIWAN] Formosa: Kosempo (DEI, Eberswalde).

ANEURETA Turati \& Krrüger, 1936, Memorie Soc. ent. ital. 15: 66.

CHLO

Type-species: Aneureta eureka Turati \& Krüger, 1936, ibidem 15:67, figs, by monotypy. Syntypes I $\hat{O}^{*}, \mathrm{I}$ ㅇ. [LIBYA]: Cirenaica, Uadi Cuf.

ANGITIA Walker, I858. See Blancharditia Biezanko \& Ruffinelli, I963.

ACON

ANHAUSTA Hampson, 1907, Ann. Mag. nat. Hist. (7) 19: 250.

NOCT

Type-species: Dasypolia exprimata Staudinger, I896, Dt. ent. Z. Iris 9: 190, 37 I, pl. 4, fig. I 5, by original designation. Syntypes 3 \&, [U.S.S.R.]: [Kirghizia], N.E. of Issyk Kul (MNHU, Berlin).

ANHIMELLA McDunnough, I943, Can. Ent. 75: 48.

HADE

Type-species: Celaena contrahens Walker, I860, in D'Urban, Can. Nat. E Geol. 5: 255, by original designation. Type(s) ô. Canada: Montreal.

ANIANA Walker, [1866] I865, List Specimens lepid. Insects Colm Br. Mus. 34: I I24. OPH Type-species: Aniana straminealis Walker, [1866] I 865, ibidem 34: I I 24, by monotypy. Holotype ㅇ, [BraziL]: [R. Amazon], [Tefé] Ega.

ANICLA Grote, 1874, Bull. Buffalo Soc. nat. Sci. 2: 159.

NOCT

Type-species: Anicla alabamae Grote, I 874, ibidem 2: I59, by monotypy. Syntypes o, 우 [U.S.A.]: Central Alabama (BMNH).

A. alabamae is a junior subjective synonym of Agrotis infecta Ochsenheimer, I8I6, Schmett. Eur. 4:67.

$\ddagger$ ANIDA; Grote, 1875, Stettin. ent. Ztg 36: 200.

An incorrect subsequent spelling.

$\ddagger$ ANCIOLA; Neave, 1939, Nomencl.zool. 1: 186.

An incorrect subsequent spelling.

$\ddagger A N I D A$, misspelling. See Anicla Grote, I874.

NOCT

ANIGRAEA Walker, i 862, J. Proc. Limn. Soc. (Zool.) 6: I 39.

EUTE

Type-species: Anigraea rubida Walker, i862, ibidem 6: I 39, by monotypy. Lectotype o. Bonneo: Sarawak (UM, Oxford), designated (as type) by Swinhoe, 190o, Cat. east. and Aust. Lepid. Heterocera 2: 93. 
ANIGRAEOPSIS Warren, I9I 4, Novit. zool. 21 : 279.

EUTE

Type-species: Anigraeopsis subalbiplaga Warren, I9I4, ibidem 21: 279, by original designation. Holotype $q$, New Guinea: [Papua], R. Angabunga, above $6000 \mathrm{ft}$ (BMNH).

ANISONEURA Guenée, I852, in Boisduval \& Guenée, Hist. nat. Insectes (Lépid.) 7 : I60. CATo

Type-species: Anisoneura salebrosa Guenée, I852, ibidem 7: I6I, by subsequent designation by Desmarest, (I857), in Chenu, Encycl. Hist. nat. Papillons nocturnes: I 30. Syntypes ô. [BAngladesh]: [E. Bengal], Silhet (BMNH).

$\ddagger A N I S O Z E U X I S$ Prout, no published reference found.

HYPE

ANITHA Walker, [1866] I865, List Specimens lepid. Insects Colln Bv. Mus. 34: I 206. HyPE Type-species: Anitha mundiferalis Walker, [1 866] I 865, ibidem 34: I 206, by monotypy. Type(s) ô, BORneo: Sarawak (BMNH).

ANNAPHILA Grote, 1873, Bull. Buffalo Soc. nat. Sci. 1: I 49.

ACRO

Type-species: Annaphila diva Grote, I873, ibidem 1: I50, pl. 4, fig. I4, by subsequent designation by Grote, 1874 , ibidem 2: 35 . Syntypes 5 ( $(\hat{\jmath}$, ㅇ), [U.S.A.]: California (BMNH).

ANOBA Walker, 1858, List Specimens lepid. Insects Colln Br. Mus. 15: i $844 . \quad$ opHI Type-species: Anoba trigonoides Walker, I858, ibidem 15: I845 (but cited as ‘'Onoba', an incorrect original spelling), by monotypy. Type(s) $\widehat{\sigma}$. [BRAzIL]: Rio Janeiro (UM, Oxford).

Anoba was the spelling used for the generic heading and in the index on page I864; $\ddagger$ Onoba used for the new species, was an inadvertent error.

$\ddagger O N O B A$; Walker, I858, List Specimens lepid. Insects Colln Br. Mus. 15: I845.

An incorrect original spelling.

ANODAPHA Moore, [I885]. See Polydesma Boisduval, I833.

OPH I

ANODONTA Rambur, I858. See Raphia Hübner, [I82I].

OPHI

ANODONTODES Hampson, I895, Trans. ent. Soc. Lond. 1895: 302.

ACRO

Type-species: Anodontodes rotunda Hampson, I895, ibidem 1895: 302, fig. [13], by original designation. Syntypes $\widehat{\jmath},+$, [ [Sıккıм] Sikhim (BMNH).

ANOMIS Hübner, [1821] I8I6, Verz. bekannter Schmett.: 249. Nomenclaturally available but including two species, both nomina nuda until they were described, one in [1822] and the other in 1823 .

OPHI

Type-species: Anomis exacta Hübner, [1822], Samml. exot. Schmett. 2: pl. [198], figs I-4, by subsequent monotypy. Syntypes $\hat{\sigma}, q$, TyPE-LOCALity not stated [MeXico. Peru]. ANOMUS Agassiz, I846, Nomencl. zool. Index univl.: 24.

An unjustified emendation of Anomis Hübner, [I 82 I].

ANOMOCALA Tams, I935, Insects Samoa 3(4): 2 I I.

CATO

Type-species: Anomocala hopkinsi Tams, I935, ibidem 3(4): 2 I I, pl. 6, fig. I6, pl. 7, fig. 5, by original designation. Holotype + , SAMOA: Upolu, Malololelei, $2000 \mathrm{ft}(\mathrm{BMNH})$.

ANOMOGYNA Staudinger, I871, in Staudinger \& Wocke, Cat. Lepid. eur. Faunengeb.: Iro.

NOCT

Type-species: Hadena laetabilis Zetterstedt, I839, Insecta Lapponica:940, by monotypy. Type(s) $\widehat{\sigma}$, [Scandinavia]: [Lappland] Lapponia borealis.

ANOMOPHLEBIA Turner, igo8, Trans. Proc. R. Soc. S. Aust. 32: 75.

HYPE

Type-species: Anomophlebia furtiva Turner, I908, ibidem 32: 75, by monotypy. Holotype ô, Australia: Qd, Kuranda (ANIC, Canberra).

ANOMUS Agassiz, 1846. See Anomis Hübner, [182 I]. 
ANOPHIA Guenée, I 84 I, Annls Soc. ent. Fr. 10: 248.

OPHI

Type-species: Phalaena leucomelas Linnaeus, I758, Syst. Nat. (Edn I0) 1: 51 8, by subsequent designation by Moore, [1885] i887, Lepid. Ceylon 3: i I7. Type(s), Europe (LS, London).

ANOPHIODES Hampson, i9r3, Cat. Lepid. Phalaenae Br. Mus. 13: 332.

CATO

Type-species: Catephiodes meeki Bethune-Baker, 1908, Novit. zool. 15: 20 I, by original designation (for Catephiodes Bethune-Baker, I908). Type(s) ठే. NEw Gurnea: [Papua], R. Aroa (BMNH).

Anophiodes was proposed as the objective replacement name for Catephiodes BethuneBaker, igo8.

CATEPHIODES Bethune-Baker, i908, Novit. zool. 15: 200.

Type-species: Catephiodes meeki Bethune-Baker, 1908, by original designation.

A junior homonym of Catephiodes Hampson, 1905, Cat. Lepid. Phalaenae Br. Mus. 5: vii, 36,-Lepid., Noctuidae. The objective replacement name is Anophiodes Hampson, I9I 3 .

ANORATHA Moore, i 867, Proc. zool. Soc. Lond. 1867: 82.

HYPE

Type-species: Anoratha costalis Moore, I 867, ibidem 1867: 82, pl. 7, fig.9, by monotypy. Syntypes $\hat{0}$, ‥ India: Bengal, Darjeeling (BMNH).

ANORENA Schaus, I9I 4, Proc. U.S. natn. Mus, 46: 5 I 3.

OPHI

Type-species: Anorena hyrtacides Schaus, I9I 4, ibidem 46:513, by original designation.

Type(s) ô. [Surinam]: R. Surinam, Geldersland (USNM, Washington).

ANORTHODES Smith, I891, Trans. Am. ent. Soc. 18: i I 4.

ACRO

Type-species: Anorthodes prima Smith, I89I, ibidem 18: I I 5, by monotypy. Syntypes,

[U.S.A.]: Fla., Archer; D.C., Washington; O., Columbus.

A. prima is a junior subjective synonym of Caradrina tarda Guenée, 1852 , in Boisduval

\& Guenée, Hist. nat. Insectes (Lépid.) 5: 243.

ANSA Walker, I858, List Specimens lepid. Insects Colln Br. Mus. 15: I73 I.

OPHI

Type-species: Ansa filipalpis Walker, I 858, ibidem 15: I 73r, by monotypy. Holotype

o. CEYLON (BMNH).

A. filipalpis is a junior subjective synonym of Briarda precedens Walker, I 857.

See also Briarda Walker, [I858]; and Erygansa Bethune-Baker, I 906.

ANTACHARA Walker, I858, List Specimens lepid. Insects Colln Br. Mus. 15: I 740. ACRO

Type-species: Antachara rotundata Walker, I858, ibidem 15: $174 \mathrm{I}$, by monotypy.

Holotype $\hat{\sigma}$. [Dominican Republic] St. Domingo (BMNH).

A. rotundata is a junior subjective synonym of Xylophasia diminuta Cuenée, 1852 , in Boisduval \& Guenée, Hist. nat. Insectes (Lépid.) 5: I 4 I.

ANTAEA Hübner, [1823] i 816, Verz. bekannter Schmett.: 226.

NOTODONTIDAE

Type-species: Phalaena juturna Cramer, I777, Uitlandsche liapellen 2: 48, pl. I29, fig. E, by subsequent designation by Schaus, igor, Trans. ent. Soc. Lond. 1901: 342.

Antaea was originally placed in the Noctuidae.

ANTAPISTIS Hübner, [1825] I8I6, Vevz. bekannter Schmett.: 3.t I.

OPIII

Type-species: Capnodes leucospila Walker, I865, List Specimens lepid. Insects Colln Br. Mus. 33: 1076, by subsequent monotypy. Holotype $\widehat{0}$. TYPE-Localrty not known (BMINH). [JAMAICA. BRAzIL.]

Antapistis was briefly clescribed and so made nomenclaturally available from 1825 but the only originally included name $\ddagger A$. mixtalis was and still remains without any description or indication. There has hitherto been no other associated specific name, so in order to place this genus, C. leucospila, which conforms with Hubner's generic description, has now been included.

See also Nazuda Walker, 1865. 
ANTAPLAGA Grote, I877, Can. Ent. 9: 70.

Type-species: Antaplaga dimidiata Grote, I877, ibidem 9: 71, by monotypy. Type(s) [U.S.A.]: Colorado (BMNH).

ANTARCHAEA Hübner, [I82I] I8I6, Verz. bekannter Schmett.: 254.

OPH I

Type-species: Noctua numisma Hübner, [1803], Samml.eur.Schmett. 4: pl. 55, fig. 270, by monotypy. Type(s), Europe.

ANTEOIS Warren, I900, Novit. zool. 7: I 46.

GEOMETRIDAE

Type-species: Phalaena muricata Hufnagel, I769, Berlin. Mag. 4: 606, by original designation.

HYRIA Stephens, I829, Nom. Br. Insects: 45 .

Type-species: Pyralis auroralis [Denis \& Schiffermüller], I775, Ankïndung syst. Werkes Schmett. Wienergegend: I24, by monotypy, but included as auroraria, an unjustified emendation by Borkhausen, I794.

A junior homonym of Hyria Lamarck, I8 19,-Mollusca. There is no objective replacement name but $P$. auroralis is a junior subjective synonym of $P$. muricata, the type-species of Anteois Warren. The latter is thus available for use as a subjective replacement name.

Hyria has been used as the original generic name for species which are now in the Noctuidae.

ANTHA Staudinger, I892, in Komanoff, Mém. Lépid. 6: 448.

ACRO

Type-species: Antha pretiosa Staudinger, I 892, ibidem 6: 448, pl. 7, fig. 6, by monotypy. Holotype ㅇ, [U.S.S.R.]: Amur district, Baranowka (MNHU, Berlin).

A. pretiosa is a junior subjective synonym of Leptina grata Butler, 188I, Trans. ent. Soc. Lond. 1881: I 72.

$\ddagger$ ANTHAECIA, misspelling. See Anthracia Hübner, [I823].

ACRO

ANTHEMOESSA Agassiz, I846. See Polydesma Boisduval, I833.

OPHI

ANTHEMOISIA Blanchard, I840. See Polydesma Boisduval, I833.

OPHI

ANTHOCITTA Hübner, [I823] I8 I6, Verz. bekannter Schmett.: 259.

OPHI

Type-species: Phalaena capensis Cramer, i777, Uitlandsche Liapellen 2: 109, pl. I67, fig. C, by subsequent designation by Hampson, I926, Descr. new Genera Species Lepid. Phalaenae Subfamily Noctuinae Br. Mus.: ito. Type(s), Trye-LOCALITy stated to be Cap de bonne Espérance, but this was probably an error as the species illustrated is not known to occur in Africa but is widespread in equatorial South America.

See also Hypogramma Guenée, I 852 .

ANTHODES Dognin, I9I4, Hétérocères nouv. An. Sud 8: I8.

ACRO

Type-species: Anthodes acynodonta Dognin, I9I 4, ibidem 8: 18, by monotypy. Holotype 9 , URUGUAY (USNM, Washington).

ANTHODES Hampson, I9I 8, Novit. zool. 25: I42.

Type-species: Anthodes acynodonta Dognin, I9I 4, by original designation.

A junior homonym of Anthodes Dognin, I9I 4. This generic name was used and made available by Dognin prior to its proposal and generic description by Hampson. The objective replacement name is Anthodes Dognin, I9If.

ANTHODES Hampson, I9I8. See Anthodes Dognin, I9I4.

ACRO

ANTHOECIA Boiscluval, i84o. See Melicleptria Hübner, [1823]. NOCT

ANTHOPHILA Ochsenheimer, I8I6. See Porplyyrinia Hübner, [I821]. ACON

†ANTHOPHILAE Hübner, [1806]. See Porphyrinia Hübner, [182 I]. ACoN 
ANTHRACIA Hübner, [1823] I816, Verz. behannter Schmett.: 275.

ACRO

Type-species: Noctua ephialtes Hübner, [1822], Samml. eur. Schmett. 4: pl. I42, fig. $6_{52}$, by subsequent designation by Berio, 1957, Memorie Soc. ent. ital. 36: 7. Type(s), EUROPE.

$\ddagger$ ANTHRACIA Guenée, I 852; Hampson, I913, Cat. Lepid. Phalaenae Br. Mus. 13: 207.

This genus was correctly attributed to Hübner, [1823], by Guenée, I852, Hist. nat.

Insectes (Lépid.) 7: I 8. Guenée was not proposing a new genus; he was adding two new species to Anthracia Hübner, [I823]. Hampson, I9I3, was incorrect in designating Anthracia coracias Guenée, 1852 as the type-species of $\ddagger$ Anthracia Guenée, 1852 .

$\ddagger$ ANTHAECIA; Butler, I883, Proc. zool. Soc. Lond. 1883: I62.

An incorrect subsequent spelling.

GRACILIPALPUS Calberla, i888, Corresp.-Bl. ent. Ver. Iris 1: 270.

Type-species: Noctua ephialtes Hübner, [1822], by monotypy.

Gracilipalpus is a junior objective synonym of Anthracia Hübner, [1823].

$\ddagger$ ANTHRACIA Guenée, I852. See Anthracia Hübner, [1823].

ACRO

ANTIBLEMMA Hübner, I823, Zuträge Samml. exot. Schmett. 2: 18, 27.

OPHI

Type-species: Antiblemma acclinalis Hübner, i 823, ibidem 2: 27 figs 355, 356, by subsequent designation by Grote, 1874, Bull. Buffalo Soc. nat. Sci. 2: 44 . Type(s) . , Surinam.

ANTICARSIA Hübner, I818, Zuträge Samml. exot. Schmett. 1: 26.

OPHI

Type-species: Anticarsia gemmatalis Hübner, 1818, ibidem 1: 26, figs I 53, I 54, by subsequent designation by Grote, 1874, Bull. Buffalo Soc. nat. Sci. 2: 44. Type(s) . SURINAM.

ANTIOPHLEBIA Felder, I874, in Felder \& Rogenhofer, Reise öst. Fregatte Novara (Zool.) 2 (Abt. 2): pl. 99.

OPHI

Type-species: Antiophlebia bracteata Felder, I874, ibidem 2 (Abt. 2): pl. 99, fig. I8, by monotypy. Type(s) $\vec{\sigma}$, [South Africa]: [Cape of Good Hope] Cap. b. sp. (BMNH).

$\ddagger A N T I T Y P A$, misspelling. Sce Antitype Hübner, [182I].

CUCU

ANTITYPE Hübner, [182I] 1816, Verz. bekannter Schmett.: 212.

CUCU

Type-species: Phalaena chi Linnaeus, I 758, Syst. Nat. (Edn Io) 1: 5 I 4, by subsequent designation by Hampson, igo6, Cat. Lepid. Phalaenae Br. Mus. 6: 357 . Type(s), TyPELOCALITY not stated (LS, London) [EUROPE].

$\ddagger$ ANTITYPA; Agassiz, I 846, Nomencl. zool. Index univl.: 27.

An incorrect subsequent spelling.

$\ddagger$ ANTOPHILA Hübner, [1806]. See Porphyrinia Hübner, [I821]. ACON

${ }_{\ddagger}$ ANTOPHYLA, misspelling. See Porphyrinia Hübner, [1821]. ACON

ANUA Walker, I858, List Specimens lepid. Insects Colln Br. Mus. 15: I788. Cato

Type-species: Anua amplior Walker, I 858, ibidem 15: I789, by monotypy. Holotype

ㅇ. [South Africa]: Port Natal (BMNH).

A. amplior is a junior subjective synonym of Nephelodes finifascia Walker, I 858 , ibidem 15: 1676 .

ANUGA Guenée, 1852, in Boisduval \& Guenée, Hist. nat. Insectes (Lépid.) 6: 307.

EUTE

Type-species: Anuga constricta Guence, I 852, ibidem 6: 308, by monotypy. Syntypes

$\delta$, $q$, INDIA : central (BMNH).

See also Caecila Walker, I858; and Spersara Walker, [1863].

ANUGANA Strand, 1924, Dt. ent. Z. Iris 38: I4 I.

OPH I

Type-species: Anugana limbatis Strand, 1924, ibidem 38: I 42 , by original designation. Holotype ô, Australia: S.W., Eradu (MINHU, Berlin). 
ANUMETA Walker, 1858, List Specimens lepid. Insects Colln Br. Mus. 15: I 769 OPH Type-species: Anumeta atrosignata Walker, I858, ibidem 15: I770, by monotypy. Holotype ,. [INDIA]: N. Hindostan (BMNH).

ANYCTEOLA Barnes \& Benjamin, 1929, Bull. Brooklyn ent. Soc. 24: I68.

ACRO

Type-species: Stilbia fotelloides Barnes \& McDunnough, I916, Contr. nat. Hist. N. Am. 3: II, pl. 3, fig. I, by original designation. Holotype Q, [U.S.A.]: Ariz., Baboquavaria Mts.

ANYDROPHILA John, I909, Rev. russe Ent. 9: I23.

CATO

Type-species: Euclidia mirifica Ershov, 1874, in Fedchenko, Reise in Turkestan 2 (5) 3: 54, pl. 4, fig. 56, by original designation. Syntypes ơ, q, [U.S.S.R.]: Turkestan, Kisilkum desert (ZI, Leningrad).

ANYTUS Grote, 1873. See Sutyna Todd, 1958.

NOCT

AON Neumoegen, I892, Ent. News 3: 258 .

EUTE

Type-species: Aon noctuiformis Neumoegen, I892, ibidem 3: 258, by monotypy. Syntypes 2 ô, [U.S.A.]: S.W. Texas, Nueces River.

APAEGOCERA Hampson, I905, Ann. Mag. nat. Hist. (7) 15: 449.

AGAR

Type-species: Apaegocera argyrogramma Hampson, 1905, ibidem (7)15: 449, by original designation. Holotype ô. [GHANA] : Ashanti, Obuassi (BMNH).

APAMEA Ochsenheimer, I816, Schmett. Eur. 4: 75.

ACRO

Type-species: Noctua basilinea [Denis \& Schiffermüller], I775, Ankündung syst. Werkes Schmett. Wienergegend: 78, by subsequent designation by Samouelle, I819, Entomologist's useful Compendium: 25I, but cited as 'basilinea Fabr.', an incorrect authorship. Type(s), [Austria]: Vienna district (Collection destroyed, Horn \& Kahle, I936, Ent. Beih. Berl.Dahlem 3: 243).

$N$. basilinea is a junior subjective synonym of Phalaena sordens Hufnagel, r766, Berlin. Mag. 3(3): 306.

HAMA Stephens, I 829 [June], Nom. Br. Insects: 4 I.

Type-species: Noctua basilinea [Denis \& Schiffermüller], I775, by subsequent designation by Westwood, I840, Synopsis Genera Br. Insects: 95 (but cited as 'basilinea F.', an incorrect authorship).

Incorrect type-species designation: Noctua testacea [Denis \& Schiffermüller], I775, cited by Boisduval, I 836, Hist. nat. Insectes (Lépid.) 1 : I 44. Boisduval, in his lengthy I 54 page Introduction, reviewed earlier classifications and designated up to three different typespecies for each generic name. In his 'Exposé de notre méthode' from page I 55-690, no type-species designations were made for the genera he himself used. Under the Code, Article 69(a)(iii), the type-designation of an author is eligible for consideration if he states that it is the type '. . . and if it is clear that he himself accepts it as the type-species.' Boisduval's type-designations although clearly stated do not fulfil the last requirement and so are invalid. Even though Boisduval's I 836 work was well known to lepidopterists, the type-designations contained in it have not in the past been accepted by Hemming or by other authors.

Hama is a junior objective synonym of Apamea Ochsenheimer, I816.

SYMA Stephens, 1850, List Br. Anim. 5: 28I.

Type-species: Noctua hirticornis Haworth, I812, Trans. ent. Soc. Lond. 1: 336, by monotypy. Type(s), Great Britain (Types destroyed, Stephens, i829, Illust. Br. Ent. (Haustellata) 2: I 77).

$N$. hirticornis is a junior subjective synonym of Phalaena crenata Hufnagel, I766, Berlin. Mag. 3(3): 402 .

Syma Stephens is a junior homonym of Syma Lesson, 1827, in Férussac, Bull. Sci. nat. et Géol. 11: 443,-Aves. There is no objective replacement name but $N$. hivticornis 
is congeneric with Noctua basilinea [Denis \& Schiffermüller], I775, the type-species of Apamea Ochsenheimer, I816. The latter is therefore available for use as a subjective replacement name.

DIMYA Mloore, I882, in Hewitson \& Moore, Descr. new Indian lepid. Insects Colln late Mr W. S. Atkinson: I 21 .

Type-species: Dimya simuata Moore, 1882 , ibidem: I22, pl. 4 , fig. I7, by monotypy. Syntypes $\widehat{\jmath}$. 9 , India: Jongei, I $3500 \mathrm{ft}$; Darjiling, I $0000 \mathrm{ft}$.

A junior homonym of Dimya Rouault, 1850, Mém. Soc. géol. Fr. (2)3(2): 470,-Mollusca. There is no objective replacement name but $D$. sinuata is congeneric with Noctua basilinea [Denis \& Schiffermüller], I775, the type-species of Apamea Ochsenheimer, 1816. The latter is therefore available for use as a subjective replacement name.

APANDA Noore, 1882, in Hewitson \& Moore, Descr. new Indian lepid. Insects Colln late Mr W. S. Atkinson: I86. HYPE

Type-species: Apanda denticulata Moore, I882, ibidem: I87, pl. 6, fig. 24, by monotypy. Syntypes, India: [IT. Bengal], Darjiling (BMNH).

†APATELA, misspelling. See Acronicta Ochsenheimer, I8I 6 .

ACRO

†APA TELAE Ochsenheinier, I816. See Acronicta Ochsenheimer, I8I6. ACRO

$\ddagger A P A T E L E$ Hübner, [1806]. See Acronicta Ochsenheimer, I8I6. ACRO

$\ddagger$ APATELE Hübner, I808. See Triaena Hübner, 1818. ACRO

$\ddagger$ †PATELE Hübner, I8I8. See Acronicta Ochsenheimer, I8I6. ACRO

APATELE Hübner, I822. See Acronicta Ochsenheimer, I8I6. ACRO

APAUSTIS Hübner, [1823] I816, Verz. bekannter Schmett.: [258]. ACRO

Type-species: Noctua heliophila Hübner, [1803], Samml. eur. Schmett.4: pl. 64, fig. 3 I 7 (a junior homonym), by monotypy. Type(s), Europi.

$N$. heliophila Hübner, [1803], is a junior primary homonym of Noctua heliophila Paykull I793, Skr. Naturh.-Selskabet 2 (2): ro2, pl. 2, fig. 5. There is no objective replacement name but $N$. heliophila Hübner is a junior subjective synonym of Noctua rupicola [Denis \& Schiffermüller], 1775, Ankïndung syst. Werkes Schmett. Wienergegend: 90. The latter is therefore available for use as the subjective replacement name.

APHARETRA Grote, I9oi, Proc. ent. Soc. Wash. 4: 368.

CUCU

Type-species: Apatela dentata Grote, I875, Can. Ent. 7:222, by original designation. Type(s) ơ. [CANADA]: Quebec (BMNH).

APHLYCTAENA Kaye \& Lamont, 1927. See Phlyctaina Möschler, I 890.

HYPE

APHOMIA Hübner, [1825] I8I6, Verz. bekannter Schmett.: 369.

PYRALIDAE

Type-species: Phalaena sociella Linnaeus, $175^{8}$, Syst. Nai. (Edn Io) $1: 534$, by subsequent designation by Hampson, 1917, Novit. zool. 24: 37 .

Included in this catalogue owing to the confused usage, in the Noctuidae, of its junior objective synonym Melia Curtis, 1828, see below.

MELIA Curtis, I828, Br. Ent. 5: 201.

Type-species: Phalaena sociella Linnaeus, 1758, Syst. Nat. (Edn ro) 1: 543, by original designation, but cited by Curtis as 'sociella Fab.', an incorrect authorship.

Melia Curtis had four originally included species; P. sociella, - Pyraliclae; Melia flammea Curtis, I 828, - Noctuidae, I Tadeninae; and two others. Melia has subsecuently been in use both in the Pyralidae and in the Noctuidae.

A junior homonym of Melia Bosc, 1813, in Risso, Nouv. Bull. sci. Soc. philomath. Paris 3: 233, - Crustacea. The objective replacement name is Aphomia Hübner, [1825].

For usage in the Noctuidae, see under Senta Stephens, 1834. 
APHORISMA Hampson, 1898, J. Bombay nat. Hist. Soc. 11: 461.

HYPE

Type-species: Aphorisma albistriata Hampson, I898, ibidem 11: 462 , fig., by original designation. Syntypes $\hat{\sigma},+$, India: Assam, Khasis (BMNH).

APHUSIA Walker, [1858] 1857, List Specimens lepid. Insects Colln Br. Mus. 12: 766, 769. chlo Type-species: Aphusia speiplena Walker, [1858] 1857, ibidem 12: 770, by monotypy. Holotype $\hat{\sigma}$. [INDIA]: Punjaub (BMNH).

APHYPENA Swinhoe, I9o I, Ann. Mag. nat. Hist. (7)8: 21 .

$\mathrm{OPH}$ l

Type-species: Hypena dissimulans Hampson, i898, J. Bombay nat. Hist. Soc. 11: 707 . by original designation. Holotype , India: $[$ Assam], Khasis (BMNH).

APINA Walker, 1855, List Specimens lepid. Insects Colln Br. Mus. 3: $587,756$.

AGAR

Type-species: Apina callisto Walker, 1855 , ibidem 3: 756, by subsequent designation by Kirby, 1892, Synonymic Cat. Lepid. Heterocera 1: 442. Syntypes 6 ex., Australia: [Tasmania] Van Dieman's Land (BMNH); [Qd], Moreton Bay.

APISTIS Hübner, 1823, Zuträge Samml. exot. Schmett. 2: 30.

OPH I

Type-species: Apistis fellearis Hübner, 1823, ibidem 2: 30, figs 379, 380, by subsequent designation by Hampson, 1926, Descr. new Genera Species Lepid. Phalaenae Subfamily Noctuinae Br. Mus. : 444. Type(s) ㅇ. [BrazIL]: Bahia.

Apistis was also used by Hübner, [1823] 1816, Verz. bekannter Schmett.: 271. Hemming, 1937, Hiibner 2: 155, gave priority to the Zuträge.

APISTUS Agassiz, 1846, Nomencl. zool. Index univl.: 29.

An unjustified emendation of Apistis Hübner, I823; and a junior homonym of Apistus Cuvier, 1829, in Cuvier \& Valenciennes, Hist. nat. Poissons 4: 39I, - Pisces.

APISTUS Agassiz, I846. See Apistis Hübner, 1823.

OPHI

APLADRAPSA Warren, 1913, in Seitz, Gross-Schmett. Erde 3: 423.

HYPE

Type-species: Adrapsa ochracea Leech, 1900, Trans. ent. Soc. Lond. 1900: 614, by original designation. Syntypes $4 \hat{0}, 3$ ㅇ, Crina: [Hupeh], Ichang (BMNH); [Szechwan], Moupin (BMNH).

The date of publication of the part of Seitz containing page 423 was stated on page $42 \mathrm{I}$ as 1913 December $5^{\text {th }}$ in both the English and the German editions.

APLECTA Guenée, I838. See Polia Ochsenheimer, 1816.

HADE

APLECTOIDES Butler, 1878, Ann. Mag. nat. Hist. (5)1: 193.

NOCT

Type-species: Aplecta condita Guenée, 1852, in Boisduval \& Guenée, Hist. nat. Insectes (Lépid.) 6: 78, pl. 8, fig. 5, by original designation. Holotype ㅇ․ [U.S.A.]: New York State (BMNH).

APLOCAMPA Schaus, 1894, Trans. Am. ent. Soc. 21: 234.

OPHI

Type-species: Aplocampa fumida Schaus, I 894, ibidem $21: 234$, by monotypy. Type(s), Brazil: Parana, Castro.

APLOTELIA Warren, I914, Novit. zool. 21: 409.

EUTE

Type-species: Ingura tripartita Semper, I9oo, Reisen Archipel Philippinen (2)6 Schmett. 2: 532, pl. 60 , fig. 8 , by original designation, but cited as 'tripartita Snell.', an incorrect authorship. Syntypes $3(\widehat{0}$, $)$, Philippines: Luzon.

APOCAL YMNIA Hampson, 1908, Cat. Lepid. Phalaenae Br. Mus. 7: 7. Available but without included species until Hampson, 1910, ibidem 9: ix, 184. ACRO

Type-species: Caradrina tenebrosa Hampson, 1902, J. Bombay nat. Hist. Soc. 14: 204, by subsequent monotypy. Holotype , India: Cuddapah, Horsleykhonda (BMNH).

APOPESTES Hübner, [1823] I816, Verz. bekannter Schmett.: 275.

OPHI

Type-species: Noctua spectrum Esper, 1787, Die Schmett. 4(1): pl. 100, figs 3, 4; 7788 , ibidem: 13 I, by monotypy. Syntypes $\hat{\sigma}, \hat{q}$, ITALY: southern. 
SPINTHEROPS Boisduval, i 840, Genera Index Meth. Eur. Lepid.: 98.

Type-species: Noctua spectrum Esper, I 788 , by PRESENT DESIGNATION.

Spintherops is a junior objective synonym of Apopestes Hübner, [1823].

${ }_{+}^{+}$SPHINTEROPS; Kiollar, [1849] I850, in Kollar \& Redtenbacher, Denkschr. Akad. Wiss.

Wien 1: 53 .

An incorrect subsequent spelling.

APOROPHYLA Guenée, I84I, Annls Soc. ent. Fr. 10: 246.

CUCU

Type-species: Xylina australis Boisduval, 1829, Eur. Lepid. Index Meth., Errata

Addenda: 6, by monotypy. Type(s), [FrancE] Galloprov.

$\ddagger$ APOROPH YLLA; Neave, 1939, Nomencl. zool. 1: 261.

An incorrect subsequent spelling.

†APOROPHYLLA, misspelling. See A porophyla Guenée, I $84 \mathrm{I}$.

CUCU

APOSPASTA Fletcher, I959, Entomologist 92: 45.

HADE

Type-species: Apospasta sabulosa Fletcher, I959, ibidem 92: 46, figs I, 4, 5, and pl. 2, fig. 8, by original designation. Holotype $\hat{0}$, ErHiopia: northern, Simien, Lori i $1,500 \mathrm{ft}$ (BMNH).

APOSTEMA Warren, 1913, in Seitz, Gross-Schmett. Erde 11: i I6.

CUCU

Type-species: Cosmia distigmata Hampson, I906, Cat. Lepid. Phalaenae Br. Mus. 6: 509, pl. 107, fig. 19, by original designation. Holotype + , [INDIA] : Punjab, Kulu, Sultanpur (BMNH).

The date of publication of the part of Seitz containing page I I 6 was stated on page I I 3 as I9I3 August $5^{\text {th }}$ in both the Finglish and the German editions.

APOTHRIGUNA Berio, I062, Annali Mus. civ. Stor. nat. Giacomo Doria 73: I77.

SARR

Type-species: Apothriguna legrandi Berio, 1962, ibidem 73: 177, fig. 8, by original designation. Holotype ô. Seychelles: Mahé [I.], B[eau] Vallon (MNHN, Paris).

APOTHRIPA Hampson, 1907, J. Bombay nat. Hist. Soc. 17:653

SARR

Type-species: Clettharra iphida Swinhoe, I90I, Ann. Mag. nat. Hist. (7)7: 490, by original designation. Type(s) $\widehat{A}$. [INDIA]: [Assam], Jaintia Hills (BMNH).

APOXESTIA Draudt, I924, in Seitz, Gross-Schmett. Erde 7: 85.

NOCT

Type-species: Agrotis ioglauca Zerny, I916, Annln naturh. Mus. Wien 30: 175, pl. 5, fig. 23, by monotypy. Holotype ${ }^{\prime}$, Bolivia: Cuesta von Cillutincara $3500 \mathrm{~m}$. (NM, Vienna).

The date of publication of the part of Seitz containing page 85 was stated on page 85 as 1924 July 23 rd in the German edition, and 1925 October 23 rd in the English edition.

APPANA Moore, 1881, Proc. zool. Soc. Lond. 1881: 355.

ACRO

Type-species: Phlogophora indica Moore, I867, ibidem 1867:57, by monotypy. Type(s) ㅇ, [INDia $]$ : Bengal.

APPHADANA Walker, I865, List Specimens lepid. Insects Colln Br. Mus. 33: I094. OPII Type-species: Apphadana liturata Walker, 1865, ibidem 33: 1094, by monotypy. Syntypes 2 , , [BraziL]: [R. Amazon], [Tefé] Ega (BMNH); Para (BMNH).

APPHADANA Walker, [1866] I865, List Specimens lepid. Insects Colln Br. Mus. 34: I 212. See Amblygoes Butler, I 879.

OPHI

APSAPHIDA Franclemont, i973, Proc, ent. Soc. Wash. 75: i72. CUCU

Type-species: Apsaphida eremna Franclemont, 1973, ibidem 75: 173, figs I-9, by original designation. Holotype ô. U.S.A.: Arizona, Santa Cruz Co., Santa Rica Mits, Madera Canyon (Colln Franclemont, Ithaca). 
APSARANYCTA Hampson, I 9I 4, Ann. Mag. nat. Hist. (8)13: i64.

ACRO

Type-species: Apsaranycta bryophilina Hampson, I9I 4, ibidem (8)13: 165, by original designation. Holotype 9 , India: Bombay, Anshi (BMNH).

$\ddagger$ APSARANYET A; Neave, I939, Nomencl. zool. 1: 264.

An incorrect subsequent spelling.

† APSARANYETA, misspelling. See Apsaranycta Hampson, I9I 4 .

ACRO

APSARASA Moore, I 867, Proc. zool. Soc. Lond. 1867: 665.

ACRO

Type-species: Noctua radians Westwood, I848, Cabinet Oriental Ent.: 58, pl. 28, fig. 4, by monotypy. Type(s), India: Assam (UM, Oxford).

$N$. radians was originally described in the subgenus $\ddagger$ Apatela, an incorrect subsequent spelling of Apatele Hübner, I 822 .

APUSTIS Schaus, I913, Ann. Mag. nat. Hist. (8)11: 18.

HYPE

Type-species: Apustis sabulosa Schaus, I9I3 ibidem (8)11: I8, by original designation.

Type(s) ô, Costa Rica: Sixola.

AQUIS Walker, 1858, List Specimens lepid. Insects Colln Br. Mus. 15: I652.

SARR

Type-species: Aquis viridisquama Walker, I858, ibidem 15: I652, by monotypy.

Holotype $\widehat{\jmath}$. Borneo: Sarawak (BMNH).

See also Dimirica Walker, [I863].

AQUITA Walker, I863, List Specimens lepid. Insects Colln Br. Mus. 27: 200.

NOLI

Type-species: Aquita horridella Walker, I863, ibidem $27: 200$, by monotypy. Syntypes

$4\left(0^{*}\right.$, O), Australia (BMNH).

$A$. horridella is a junior subjective synonym of Zia tactalis Walker, I863.

ZIA Walker, 1863, List Specimens lepid. Insects Colln Br. Mus. 27: Io9.

Type-species: Zia tactalis Walker, I863, ibidem 27: I Io, by monotypy. Holotype ô. [Australia]: Sydney (BMNH).

A junior homonym of Zia Koch, I84I, Dtl. Crustaceen Heft 34: Tab. 2 I-23, - Crustacea. There is no objective replacement name but $Z$. tactalis is conspecific with Aquita horridella Walker, I863, the type-species of Aquita Walker, 1863. The latter is therefore available for use as a subjective replacement name.

ARABRIGA Walker, I 869, Characters undescr. Lepid. Heterocera: 52.

OPHI

Type-species: Arabriga bimaculata Walker, I869, ibidem: 52, by monotypy. Type(s)

ㅇ, [HoNDURAs]: Limas.

ARACHNOGNATHA Hampson, I 894, Fauna Br. India (Moths) 2: 365, 373.

SARR

Type-species: Arachnognatha meterythra Hampson, I894, ibidem 2: 373, fig. 205, by original designation. Holotype ㅇ, [Burma]: E. Pegu (BMNH).

ARADRAPHA Walker, [1866] I865, List Specimens lepid. Insects Colln Br. Mus. 34: I I82. Noli Type-species: Aradrapha partitalis TValker, [I 866] I 865, ibidem 34: I I 82, by monotypy. Holotype $\hat{\sigma}$, [South Africa]: Natal (BMNH).

ARAEA Hampson, I908, Cat. Lepid. Phalaenae Br. Mus. 7: xv, 688.

ACRO

Type-species: Araea attenuata Hampson, 1908, ibidem 7:688, fig. I8I, by original designation. Holotype $\hat{\sigma}$, [INDIA]: Kashmir, Scinde Valley, $8000 \mathrm{ft}$ (BMNH).

MICHENERIA Orfila \& Rossi, 1956, Revta Soc. ent. Avgent. 19: 28.

Proposed, unnecessarily, as an objective replacement name for Avaea Hampson, I908, which Orfila \& Rossi considered to be a junior homonym of Area Ragonot, [I89I] I890, Annls Soc. ent. Fr. (6) 10: 483, - Lepid., Pyralidae.

ARAEOGNATHA Hampson, I 893, Illust. Lepid. Heterocera Br. Mus. 9: 3I, I 29.

OPHI

Type-species: Araeognatha umbrosa Hampson, I 893, ibidem 9: 31, 129, pl. 167, fig. 2, by monotypy. Type(s) ふ̊. CEyLon: Hewahetta (BMNH). 
ARAEOPTERA Hampson, igio. See Araeopteron Hampson, I893.

$\operatorname{ACON}$

ARAEOPTERELLA Dyar, 1914, Proc. U.S. natn. Mus. 47: I89.

$\operatorname{ACON}$

Type-species: Araeopterella miscidisce Dyar, I914, ibidem 47: I89, by original designation. Holotype q, Panama: R. Trinidad (USNM, Washington).

ARAEOPTERON Hampson, I893, Illust. typical Specimens Lepid. Heterocera Colln Br. Mus.

9: 33, I 36. $A C O N$

Type-species: Araeopteron pictale Hampson, I893, ibidem 9: 33, 137, pl. I68, fig. I9, by monotypy. Type(s) ô, CEYLON: Pundaloya (BMNH).

ARAEOPTERUII Hampson, 1895, Fanna Br. India (Moths) 3: 64.

An unjustified emendation of Araeopteron Hampson, I 893.

ARAEOPTERA Hampson, igro, Cat. Lepid. Phalaenae Br. Mus. 10: 22.

An unjustified emendation of Araeopteron Hampson, I 893.

ARAEOPTERUM Hampson, I895. See Avaeopteron Hampson, I 893.

$A C O N$

$\ddagger$ ARAEOSTOMA Hampson, no published reference found.

HYPE

ARAMUNA Moore, i 884, Lepid. Ceylon 3: 3.5.

OPHI

Type-species: Aramuna marginata Moore, I884, ibidem 3: 36, pl. I49, fig. 6, by monotypy. Type(s) ô. CEYLON (BMNH).

A. marginata Noore, I884, has as an objective replacement name Bocula xanthostola Hampson, 1926, Descr. new Genera Species Lepid. Phalaenae Subfamily Noctuinae Br. Mus.: 227. The latter is the valid name as the type-species is a junior secondary homonym of Borsippa marginata Moore, I882. Both species are currently placed in Bocula Guenée, I 852 .

ARASADA Moore, [1885] 1887, Lepid. Ceylon. 3: i88. $\operatorname{ACON}$

Type-species: Arasada pyraliformis Moore, [1885] I887, ibidem 3: I89, pl. I72, fig. I, by monotypy. Syntypes 0 , ㅇ, CEYLON (BMNH).

ARBASERA Walker, I865, List Specimens lepid. Insects Colln Br. Mus. 32: 638.

ACRO

Type-species: Arbasera candida Walker, 1865 , ibidem $32: 638$, by subsequent designation by Berio, I966, Annali Mus. civ. Stor. nat. Giacomo Doria 76: 48. Lectotype ô. Camiodia (UM, Oxford), designated (as type) by Swinhoe, igoo, Cat. east. and Aust. Lepid. Heterocera 2: 4 S.

See also Clinophlebia Hampson, 1893.

ARBINIA Möschler, I88o, V'erh. zool.-bot. Ges. Wien 30 (Abh.) : 466.

PYRALIDAE

Type-species: Arbinia todilla Möschler, I88o, ibidem 30 (Abh.) : 467, pl. 9, fig. 20, by monotypy.

Originally placed in the Noctuidae.

ARBORICORNUS Hampson, I894, Fauna Br. India (Moths) 2: 164, 258.

ACRO

Type-species: Arboricornus ruber Hampson, I 894, ibidem 2: 258, fig. I 46, by original designation. Holotype $\hat{\sigma},[$ [NDIA] : [Assam], Nagas, $6000 \mathrm{ft}$ (BMNH).

ARBOSTOLA Druce, I 9oo, Ann. Mag. nat. Hist. (7)5: 5 I9.

OPIII

Type-species: Arbostola viridis Druce, I 900, ibidem (7)5:519, by monotypy. Type(s) o. Colombia: Bonda (BMNH).

Arbostola probably originated as a misspelling of Abrostola Ochsenlieimer, i 8 i 6 , but based on viridis is in a different subfamily. Dyar, I92I, Insecutor Inscit. menst. 9: 45, pointed this out, accepted it as a good genus, and described another species in it.

$\ddagger A R C A N A$, misspelling. See Aroana Bethune-Baker, 1906 .

$\operatorname{ACON}$

ARCHANA Walker, I 865, List Specimens lepid. Insects Colln Br. Mus. 33: 1097.

OPY1

Type-species: Archana certa Walker, 186,5, ibidem 33: 1098, by monotypy. Holotype o., [BRAzIL] : [R. Amazon], Santarem (BMNH). 
A. certa is a junior subjective synonym of Orthogramma coppryi Guenée, I852, in Boisduval \& Guenée, Hist. nat. Insectes (Lépid.) 3: 348.

ORTHOGR AMMA Guenée, I852, in Boisduval \& Guenée, Hist. nat. Insectes (Lépid.) 7: 347.

Type-species: Orthogramma coppryi Guenée, I852, ibidem $7: 348$, by subsequent designation by Desmarest, (I 857), in Chenu, Encycl. Hist. nat. Papillons nocturnes: I 34. Holotype o., [FRENCH GuIANA]: Cayenne (BMNH).

A junior homonym of Orthogramma R.L., I8 17, Jenaische Allg. Lit.-Ztg 1: 285, - Lepid., Noctuidae. There is no objective replacement name, but $O$. coppryi is conspecific with Archana certa Walker, I865, the type-species of Archana Walker, I865. The latter is therefore available for use as a subjective replacement name.

ARCHANARA Walker, I 866, List Specimens lepid. Insects Colln Br. Mus. 35: I 737. ACRo

Type-species: Archanara nonogriella Walker, I866, ibidem 35: 1737 , by monotypy. Holotype $\sigma^{\star}$, [CHINA]: Shanghai (BMNH).

A. nonogriella is a junior subjective synonym of Nonagria polita Walker, $186_{5}$, ibidem 32: 629 .

$\ddagger A R C H A R A N A$; Viette, I947, Revue fr. Lépidopt. 11:60.

An incorrect subsequent spelling.

ARCHANARTA Barnes \& Benjamin, I929, Bull. Brooklyn ent. Soc. 24: I73. Noct

Type-species: Noctua quieta Hübner, [I8I3], Samml. eur. Schmett. 4: pl. I03, fig. 485 , by original designation. Type(s), Europe.

$\ddagger$ ARCHARANA, misspelling. See Avchanava Walker, I 866.

ACRO

ARCHEPHIA Hampson, I926, Descr. new Genera Species Lepid. Phalaenae Subfamily Noctuinae Br. Mus.: 88.

OPHI

Type-species: Archephia olivacea Hampson, I 926, ibidem: 88, by original designation. Holotype ô. [South Africa]: Natal, Durban (BMNH).

A. olivacea is a junior subjective synonym of Polydesma basilinea Hampson, I902, Ann. S. Afr. Mus. 2: 359 .

ARCHIEARIS Hübner, [I823] I 8 I6, Verz. bekannter Schmett.: 279, 280. GeOMETRIdaE Type-species: Phalaena parthenias Linnaeus, I76I, Fauna Suecica (Edn 2): 308, by subsequent designation by Hulst, I896, Trans. Am. ent. Soc. 23: 3 I 7 .

Archiearis was originally placed in the 'Noctuae'.

$\ddagger B R E P H A$ Hübner, [1806], Tentamen determinationis digestionis . . . : [2].

$\ddagger$ BREPHOS Hübner, [1806], ibidem: [2].

Included in a work rejected for nomenclatural purposes by Int. Commn zool. Nom., 1926, Smithson. misc. Collns 73(4) Opinion 97: 19. Also idem, 1954, Opin. Decl. int. Commn zool. Nom. 6 Opinion 278: I 40.

Only included species: Phalaena parthenias Linnaeus, I76I.

ARCHINOLA Hampson, I896, Proc. zool. Soc. Lond. 1896: 258.

NOLI

Type-species: Archinola pyralidia Hampson, I896, ibidem 1896: 259, pl. Io, fig. 23, by monotypy. Type(s) $\overrightarrow{0}$, [SoUthern Yemen]: Aden (BMNH).

ARCILASISA Walker, I 865, List Specimens lepid. Insects Colln Br. Mus. 32: 470. ACro

Type-species: Arcilasisa sobria Walker, I 865, ibidem 32: 470 , by monotypy. Lectotype ๙. [INDIA]: South Hindostan (UM, Oxford), designated (as type) by Swinhoe, I9oo, Cat. east. and Aust. Lepid. Heterocera 2: 47.

$\ddagger$ ARCTA, misspelling. See Arcte Kollar, I 844 .

OPHI

ARCTE Kollar, 1844, in Hügel, Kaschmir und des Reich der Siek 4: 477. OPHI

Type-species: Arcte polygrapha Kollar, I 844 , ibidem 4:477, by monotypy. Holotype

ㅇ, [INDia]: Himalaya, [Mussooree] Massuri.

$\ddagger$ ARCT A ; Pagenstecher, I909, Geogr. Verbreitung Schmett.: 424 .

An incorrect subsequent spelling.

$\mathrm{E}$ 
ARCTIA Schrank, i8o2, Famna Boica 2(2): I 52.

ARCTIIDAE

Type-species: Phalaena caja Linnaeus, I758, Syst. Nat. (Edn Io) 1 : 500, by subsequent designation by Westwood, I840, Synopsis Genera Br. Insects: 92.

Arctia has been used as the original generic name for species which are now in the Noctuidae.

ARCTINIA Möschler, I880, T'erh. zool.-bot. Ges. Wien 30 (Abh.) : 432.

OPHI

Type-species: Arctinia diffumata Möschler, I88o, ibidem 30 (Abh.) : 433, pl. 9, fig. 25, by subsequent designation by Hampson, 1926, Descr. new Genera Species Lepid. Phalaenae Subfamily Noctuinae Br. Mus.: I72. Syntypes 20 . Surinam: Paramaribo.

Arctinia Möschler is not preoccupied by $\ddagger$ Arctinia; Eichwald, I830, Zool. specialis 2: I95, an incorrect subsequent spelling of Arctia Schrank, I8o2.

ARCTIOPAIS Jordan, I896, in Rothschild \& Jordan, Novit. zool. 3: 46.

AGAR

Type-species: Hypsa ambusta Nabille, I88 I, Bull. Soc. ent. Belg. (3)1881 : lv, by original designation. Holotype . MADAGASCAR (BMNH).

ARCTOMYSCIS Hübner, [I820] I8I6, Vevz. bekanuter Schmett.: 202.

ACRO

Type-species: Phalciena aceris I,innaeus, I 758, Syst. Nat. (Edn Io) 1: $5 \mathrm{I} 4$, by subsequent designation by Butler, I879, Frans. ent. Soc. Lond. 1879: 3I5, but cited for $₫$ Artomyscis, an incorrect subsequent spelling. Type(s), TrPE-LOCALITy not stated (LS, London) [EUROPE].

$\ddagger$ ARTOM YSCIS; Butler, I879, Trans. ent. Soc. Lond. 1879: 31 5 .

An incorrect subsequent spelling.

ARCYOPHORA Guenée, I852, in Boisduval \& Guenée, Hist. nat. Insectes (Lépid.) 6: 378 . CHLO

Type-species: Arcyophora longivalvis Guenée, I 852, ibidem 6: 379, pl. I2, fig. 9, by monotypy. Syntypes ô,, , [South AFrica], Port Natal.

$\ddagger$ ARYOPHORA; Grünberg, I91о, Arch. Naturgesch. (1908) 74 (2) 2: 230.

An incorrect subsequent spelling.

$\ddagger$ ARCYOSPHORA; Bryk, I913, Int. ent. Z. 7: 213 .

An incorrect subsequent spelling.

$\ddagger A R C Y O S P H O R A$, misspelling. See Arcyophora Guencee, I852. CrrLo

$\ddagger A R C Y R A$, misspelling. See Momno Oclisenheimer, i8 I6. ACRo

ARENARBA Berio, I950, Annali Mus. civ. Stor. nat. Giacomo Doria 64: I $57 . \quad$ ACoN

Type-species: Ozarba arenacea Hampson, I893, Illust. typical Specimens Lepid. Heterocera Br. Mus. 9: 19, 97, pl. 162, fig. 3, by original designation. Type(s) \&, CEYLON: Pundaloya (BMNH).

ARENOSTOLA Hampson, I908, Cat. Lepid. Phalaenae Br. Mus. 7: I8. Available, but without included species until Hampson, I9I0, ibidem 9: xi, 28I.

ACRO

Type-species: Noctua phragmitidis Hübner, [1803], Samml. eur. Schmett. 4: pl. 47. fig. $33^{\circ}$ [recte 230], by subsequent designation by Hampson, I910, ibidem 9:28I. Type(s), EUROPE.

$\ddagger$ AREOLA, misspelling. See Aviola VValker, [1 858 ].

CHLO

ARETYPA Smith, 1903, Trans. Am. ent. Soc. 29: $222 . \quad$ OPHI

Type-species: Feltia pectinicornis Smith, I89o, Bull. U.S. natn. Mus. 38: I I4, by original designation. Syntypes, [U.S.A.]: Texas (USNM, Washington).

$F$. pectinicornis is a junior subjective synonym of Pseudorgyia russula Grote, I883. Papilio 3: 75 .

ARGADESA Moore, I88I. See Elygea Billberg, I820. 
ARGANIA Druce, I89r, Biologia cent.-am. (Zool.) Lepid. Heterocera 1: 480.

HYPE

Type-species: Argania pilosa Druce, I891, ibidem 1: 480, pl. 40, figs 6, 7, by original designation. Syntypes $\hat{\sigma}, q$, Guatemala: Cerro Zunil, $4000 \mathrm{ft}$; Volcan de Atitlan $2500-$ $3500 \mathrm{ft}$.

ARGIDIA Guenée, 18 52, in Boisduval \& Guenée, Hist. nat. Insectes (Lépid.)7: 345.

OPHI

Type-species: Phalaena tomyris Cramer, I779, Uitlandsche Kapellen 3: 123, p1. 262, figs F, G, by subsequent designation by Hampson, 1926, Descr. new Genera Species Lepid. Phalaenae Subfamily Noctuinae Br. Mus.: 459. Holotype, SuRinam.

ARGILLANA Bethune-Baker, Igo8, Novit. zool. 15: 237.

HYPE

Type-species: Argillana albistrigata Bethune-Baker, 1908, ibidem 15:237, by original designation. Type(s) $\hat{\delta}$, New Guinea: [Papua], Dinawa (BMNH).

ARGILLOPHORA Grote, 1873, Bull. Buffalo Soc. nat. Sci. 1: I24.

ACON

Type-species: Argillophora furcilla Grote, I873, ibidem, 1: I24, by monotypy. Syntypes $\widehat{0}$,, , [U.S.A.]: Central Alabama (BMNH).

ARGIVA Hübner, [1823] I8I6, Vevz. bekannter Schmett.: 272.

CATO

Type-species: Phalaena hieroglyphica Drury, I773, Illust. nat. Hist. 2: 3, Index, pl. 2, fig. I, by subsequent designation by Desmarest, I857, in Chenu, Encycl. Hist. nat. Papillons nocturnes: i 30. Type(s), [INDia]: Madras.

See also Coria Walker, I866.

ARGYPHIA Saalmüller, I89I, Lepid. Madagascar: 4II.

OPHI

Type-species: Argyphia modesta Saalmüller, I89I, ibidem: 4II, pl. II, fig. I88, by subsequent designation by Berio, 1966, Annali Mus. civ. Stor. nat. Giacomo Doria 76: 49. Holotype, Madagascar: N.W., Nossi-Bé I. (SNG, Frankfurt).

A. modesta is a junior subjective synonym of Orthosia arcifera Mabille, I88I, Bull. Soc. ent. Belg. 1881 : Ivi.

+ +AGRYPHI A; Neave, 1939, Nomencl. zool. 1: 96.

An incorrect subsequent spelling.

ARG YRANA Köhler, (1952) I95I, Acta zool. lilloana 12: I47.

CuCU

Type-species: Argyrana excellens Köhler, (1952) I95I, ibidem 12: I47, by original designation. Syntypes $\hat{0}$, $q$, ARgentina: Chubut [Prov.], Comodoro Rivadavia (ZSBS, Munich); Santa Cruz.

ARGYRARGENTA Berio, I939. Boll. Soc. ent. ital. 71: i86.

ACON

Type-species: Argyrargenta giacomellii Berio, 1939, ibidem 71: 187, by original designation. Holotype $\widehat{o}$, Argentina: La Rioja (Colln E. Berio, Genoa).

ARGYRHODA Hampson, I908, Cat. Lepid. Phalaenae Br. Mus. 7: 8. Available, but without included species until Hampson, I9I0, ibidem 9: x, 2 I6. ACRO

Type-species: Thalpochares laronia Druce, I89o, Proc. zool. Soc. Lond. 1890: 516, by subsequent monotypy. Holotype, Mexico: Guerrero, Tierra Colorada (BMNH).

ARGYRITIS Hübner, [I82I] I8I6, Verz. bekannter Schmett.: 247. cucu

Type-species: Noctua artemisiae [Denis \& Schiffermüller], I775, Ankündung syst. Werkes Schmett. Wienergegend: $3^{\mathrm{I} 2}$, by subsequent designation by Hampson, I906, Cat. Lepid. Phalaenae Br. Mus. 6: I4. Type(s), [Austria]: Vienna district (Collection destroyed, Horn \& Kahle, 1936, Ent. Beih. Berl.-Dahlem 3: 243).

$N$. artemisiae is a junior subjective synonym of Phalaena argentea Hufnagel, $\mathrm{r} 766$, Berlin.

Mag. 3: 286.

See also Argyrogalea Hampson, I906.

ARGYROGALEA Hampson, 1906, Cat. Lepid. Phalaenae Br. Mus. 6: viii, 81.

cucu

Type-species: Phalaena argentea Hufnagel, 1766, Berlin. Mag. 3: 286, by original designation. Type(s), [Germany]: Berlin.

See also Argyritis Hübner, [182 I]. 
ARGYROGRAMMA Hübner, 1823, Zuträge Samml. exot. Schmett. 2: 29.

PLUS

Type-species: Argyrogramma omega Hübner, 1823, ibidem 2: 29, figs 373, 374, by subsequent designation by McDunnough, I944, Mem. sth. Calif. Acad.Sci. 2: 206. Type(s) ô. [U.S.A.]: Savannah.

A. omega is a junior subjective synonym of Noctua vernuca Fabricius, 1794, Ent. Syst. $3(2): 8 \mathrm{r}$.

ARGYROLEPIDIA Hampson, I901, Cat. Lepid. Phalaenae Br. Mus. 3: xvii, 55o. Agar

Type-species: Phalaena pamphilia Stoll, r78I, in Cramer, Uitlandsche Iiapellen 4: I53, pl. 368, fig. G, by original designation. Type(s), [Moluccas]: Amboina I.

$P$. pamphilia is dated from the wrapper of the part and not from the title-page of the volume, 1782 .

ARGYROLOPHA Hampson, I914, Ann. Mag. nat. Hist. (8) 13: 205.

OPHI

Type-species: Argyrolopha costibarbata Hampson, 19r4, ibidem (8)13: 205, by original designation. Syntypes $\mathrm{I} \hat{\jmath}, 2$ ㅇ, Mauritrus: Curepipe (BMNH).

ARG YROMATA Hampson, Igo6, Cat. Lepid. Phalaenae Br. Mus. 6: viii, 82.

CUCU

Type-species: Phalaena splendida Stoll, $\mathrm{I}_{7} 82$, in Cramer, Uitlandsche Fiapellen 4:242, pl. 400, fig. F, by original designation. Type(s), [U.S.S.R.]: southern Russia.

ARGYROPASTA Hampson, I91o, Cat. Lepid. Phalaenae Br. Mus. 10: 3, 482.

$A C O N$

Type-species: Argyropasta thermopera Hampson, I9ro, ibidem 10: 483 , fig. I39, by original designation. Syntypes $x \hat{0}, 2$ ㅇ, BrazIL: Santa Catherina (BMNH).

ARG YROPHYES Grote, 1873, Bull. Buffalo Soc. nat. Sci. 1: 175 .

NOLI

Type-species: Argyrophyes cilicoides Grote, I873, ibidem 1: I75, by monotypy. Type(s) [U.S.A.]: New Jersey.

ARGYROSPILA Herrich-Schäffer, [1851] 1845, Syst. Bearb. Schmett. Eur. 2: 374. Acro

Type-species: Leucania maculata Eversmann, 1842, Uchen. Zap. imp. Iiazan Univ. 1842 (I): 96, by monotypy. Syntypes, [U.S.S.R.]: [Krasnoarmeysk] Sarepta; Ural Mts (ZI, Leningrad).

L. maculata was also proposed in Eversmann, 1842 May 23rd, Bull. Soc. Nat. Moscou 15: 549, pl. 5, fig. 4 .

L. maculata is a junior subjective synonym of Phalaena succinea Esper, [1796], Die Schmett. 4(2) Abschnitt 2: 37, pl. r9o, fig. 3.

ARGYROSTICTA Hübner, [1821] I816, Verz. bekannter Schmett.: 253. ACRO

Type-species: Phalaena amoenita Stoll, I780, in Cramer, Uitlandsche Kapellen 4: 47, pl. 3I2, fig. D, by subsequent designation by Hampson, 1908, Cat. Lepid. Phalaenae Br. Mus. 7: $5^{\mathrm{I}} 7$, but cited in error under Argyrostrotis Hübner, [182x]. Holotype, Surinam.

ARGYROSTROTIS Hübner, [1821] 1816, Verz. bekannter Schmett.: 253. CATo

Type-species: Phalaena anilis, Drury, 1773, Illust. nat. Hist. 2: 21, Index, pl. 12, fig. 3 , by subsequent designation by Hampson, r913, Cat. Lepid. Phalaenae Br. Mus. 13: I5I. Type(s), [U.S.A.]: Virginia.

Incorrect type-species designation: Phalaena amoenita Stoll, I 780 , a name not originally included in Argyrostrotis and not linked with one of the originally included names when cited by Hampson, 1908, Cat. Lepid. Phalaenae Br. Mus. 7: 517 . Hampson's use here of Argyrostrotis was an error for Argyrosticta Hübner, [1821], as pointed out by Hampson, rgr 3 , ibidem 13 : r 5 r.

See also Agnomonia Hübner, [1831].

ARGYROSTROTUS Agassiz, i 846, Nomencl. zool. Index univl.: 33.

An unjustified emendation of Argyrostrotis Hübner, [I82 I].

ARGYROSTROTUS Agassiz, 1846. See Argyrostrotis Hübner, [1821]. 
ARGYROTHRIPA Hampson, I894, Fauna Br. India (Moths) 2: xvii, 380.

Type-species: Symitha lilacina Moore, I888, in Hewitson \& Moore, Descr. new Indian lepid. Insects Colln late $M r W$. S. Atkinson: 289, by original designation. Type(s) o, $^{2}$ India: [W. Bengal], Darjiling (MNHU, Berlin).

S. lilacina is a junior subjective synonym of Ptisciana seminivea Walker, 1865.

See also Ptisciana Walker, 1865.

ARIATHISA Walker, I 865, List Specimens lepid. Insects Colln Br. Mus. 33: 747.

ACRO Type-species: Ariathisa atrosignata Walker, I865, ibidem 33: 747, by monotypy. Holotype ô. [South Africa]: Natal (BMNH).

A. atrosignata is a junior subjective synonym of Noctua excisa Herrich-Schäffer, 1854 , Samml. neuer oder wenig bekannter aussereur. Schmett. 1(I): wrapper, pl. 27, fig. I 29; I 858 , ibidem 1: 68.

See also Eulaphygma Butler, I890.

ARIDAGRICOLA Shchetkin/Stshetkin, 1965, Vysshie cheshuekrylye peskov Vakhshskoi Doliny: I73. ACRO

Type-species: Aridagricola rufulus Shchetkin/Stshetkin, 1965, ibidem: 176, by original designation. Holotype $\hat{\jmath}$, U.S.S.R.: Tadzhikistan, Vakhsh Valley, Kara-Dum (ZI, Leningrad).

ARIOLA Walker, [1858] 1857, List Specimens lepid. Insects Colln Br. Mus. 12: 766, 768. chlo Type-species: Ariola coelisigna Walker, [1 858] 1857, ibidem 12: 769 , by monotypy. Syntypes 3 ex., CEylon (BMNH). BoRneo: Sarawak (BMNH).

$\$ A R E O L A$; Walker, $186_{5}$, List Specimens lepid. Insects Colln Br. Mus. 33: 774.

An incorrect subsequent spelling.

ARIOLICA Walker, [1863], I864, J. Proc. Linn. Soc. (Zool.) $7: 53$.

CHLO

Type-species: Ariolica lineolata Walker, [1863] 1864, ibidem 7: 53, by monotypy. Lectotype 9 , Borneo: Sarawak (UM, Oxford), designated (as type) by Swinhoe, I90o, Cat. east. and Aust. Lepid. Heterocera 2: 589.

$\ddagger$ $\$$ RTOLICA; Pagenstecher, I909, Geogr. Verbreitung Schmett.: 422.

An incorrect subsequent spelling.

ARIPHRADES Druce, I891, Biologia cent.-am. (Zool.) Lepid. Heterocera 1:48I.

HYPE

Type-species: Ariphrades setula Druce, 1891, ibidem 1: 482, pl. 40, fig. 9, by original designation. Holotype $\widehat{\sigma}$, Panama: Chiriqui, Caldera (BMNH).

ARISTARIA Guenée, I 854, in Boisduval \& Guenée, Hist. nat. Insectes (Lépid.) 8: 70. HYPE Type-species: Aristaria cellulalis Guenée, 1854, ibidem 8:70, by monotypy. Holotype o., Brazil (BMNH).

ARMACTICA Walker, I865, List Specimens lepid. Insects Colln Br. Mus. 33: 8o8.

CHLO

Type-species: Armactica columbina Walker, I865, ibidem 33: 808, by monotypy. Holotype ô., [Australia]: [Qd], Moreton Bay (BMNH).

ARMADA Staudinger, I884, in Romanoff, Mém. Lépid. 1: 142.

OPHI

Type-species: Acontia dentata Staudinger, I884, ibidem 1: 142, pl. 9, fig. 3, by subsequent designation by Warren, 1913, in Seitz, Gross-Schmett. Erde 3: 393. Syntypes o. ․, [U.S.S.R.]: [Turkmenistan], Askhabad (MNHU, Berlin).

ARMANA Swinhoe, I89o, Trans, ent. Soc. Lond. 1890: 250.

OPHI

Type-species: Armana nigraericta Swinhoe, I890, ibidem 1890: 250, pl. 8, fig. I, by monotypy. Type(s) o, Burma: Bassein (BMNH).

AROANA Bethune-Baker, 1906, Novit. zool. 13: 280.

$\mathrm{ACON}$

Type-species: Aroana olivacea Bethune-Baker, 1906, ibidem 13: 28I, by original designation. Syntypes $\hat{\jmath}$, 우, New Guinea: [Papua], Aroa River (BMNH). 
$\ddagger A R C A N A$; Grünberg, I9ro, Avch. Naturgesch. (1907) 73(2) 2: 248.

An incorrect subsequent spelling.

ARPIA Schaus, I896, Jl N.Y.ent. Soc. 4: I48.

AGAR

Type-species: Arpia janeira Schaus, I896, ibidem 4: 148, by monotypy. Syntypes, o. ㅇ. [BRAzIL]: Rio Janeiro (USNMI, Washington).

ARRADE Walker, r863, List Specimens lepid. Insects Colln Br. Mus. 27: 82.

HYPE

Type-species: Arrade erebusalis Walker, 1863 , ibidem 27: 82, by monotypy. Syntypes 2 ㅇ, CEYLON (BMNH).

ARRHAPA Walker, I862, J. Proc. Linn. Soc. (Zool.) 6: I 74.

CHLO

Type-species: Arrhapa frontalis Walker, I862, ibidem 6: 174, by monotypy. Lectotype o. Borneo: Sarawak (UMI, Oxford), designated (as type) by Swinhoe, r892, Cat. east. and Aust. lepid. Heterocera 1: 240.

ARROTHIA Jordan, I896, in Rothschild \& Jordan, Novit. zool. 3: 46.

AGAR

Type-species: Arrothia bicolor Rothschild, I896, ibidem 3: 46, by original designation.

Holotype , MADAGASCAR: Morondava (BMNH).

ARSACIA Walker, [1 866] I 865, List Specimens lepid. Insects Colln Br. Mus. 34: I259. OPHI

Type-species: Arsacia saturatalis Walker, [1866] I865, ibidem 34: I260, by monotypy.

Holotype , [West Malaysia]: [Malaya], Penang (BMNH).

$A$. saturatalis is a junior subjective synonym of Midea rectalis Walker, I863.

See also Notocyma Snellen, I872; and Amblyzancla Turner, 1936.

MIDEA Walker, I863, List Specimens lepid. Insects Colln Br. Mus. 27: 2 I.

Type-species: Midea rectalis Walker, I863, ibidem 27: 21, by monotypy. Type(s) $q$, BORNEO: Sarawak (BMNH).

A junior homonym of Midea Bruzelius, 1854, Beskrifning Hydrachnider: 35, - Arachnida. There is no objective replacement name but $M$. rectalis is the senior subjective synonym of Arsacia saturatalis Walker, [1866], the type-species of Arsacia Walker, [1866]. The latter is therefore available for use as a subjective replacement name.

ARSACIODES Schaus, rgi2, Ann. Mag. nat. Hist. (8) 9: 207.

OPHI

Type-species: Arsaciodes rufa Schaus, 1912, ibidem (8)9: 207, by monotypy. Type(s) , Costa Rica: R. Banana, Sixola.

ARSILONCHE Lederer, I857, Noctuinen Eur.: 28, 70.

ACRO

Type-species: Phalaena albovenosa Goeze, I78I, Ent. Beyträge 3(3):25I, by monotypy, but included by Lederer as 'venosa Bkh.' i.e. Phalaena venosa Borkhausen, I792, Naturg. eur. Schmett. 4: 7I6, an unjustified emendation of $P$. albovenosa Goeze, I78I, and also a junior homonym of Phalaena venosa Fourcroy, 1785, Ent. Paris.: 266,-Lepidoptera, Notodontidae. Type(s), Type-Locality not stated [EuRope].

ARSINA Guenće, I862, in Maillard, Notes sur l'Isle de la Réunion 2: G.58.

HYPL

Type-species: Arsina silenalis Guenée, 1862, ibidem 2: G.59, by monotypy. Syntypes o. ㅇ, RÉunion.

ARSISACA Walker, [I866] I 865, List Specimens lepid. Insects Colln Br. Mus. 34: I26I. OPHI Type-species: Arsisaca bolinalis Walker, [1866] I 865, ibidem 34: 1262, by monotypy. Holotype ㅇ. JAMAICA (BMINH).

A. bolinalis is a junior subjective synonym of Bolina brunnearis Guenée, 1852 .

See also Bulia Walker, 1858.

ARTENA Walker, I858, List Specimens lepid. Insects Colln Br. Mus. 14: 1343, I388. CATo Type-species: Artena submira Walker, i 858, ibidem 14: I389, by monotypy. Holotype o. [INDIA]: Hindostan (BMNH). 
ARTHISMA Moore, I883, Proc. zool. Soc. Lond. 1883: 20.

OPH I

Type-species: Arthisma scissuralis Moore, 1883, ibidem 1883: 20, by monotypy. Type(s) ô. Singapore (BMNH).

$\ddagger$ ARTHRACHLORA, misspelling. See Arthrochlora Grote, I875.

CUCU

ARTHROCHLORA Grote, I875, Stettin. ent. Ztg 36: 197.

CUCU

Type-species: Feralia februalis Grote, I874, Bull. Buffalo Soc. nat. Sci. 2: 6o, by monotypy. Type(s) ㅇ․ [U.S.A.]: [Calif.], Sanzalito (BMNH).

$\ddagger$ ARTHRACHLORA; Hampson, igo6, Cat. Lepid. Phalaenae Br. Mus. 6: r99.

An incorrect subsequent spelling.

$\Varangle A R T I C O X I S$, misspelling. See Artiloxis Schaus, I9r3.

OPHI

ARTIGISA Walker, [1863] i 864, J. Proc. Linn. Soc. (Zool.) 7: 160.

OPHI

Type-species: Artigisa nigrosignata Walker, [I863] I 864, ibidem 7: I60, by monotypy. Lectotype $\hat{\jmath}$, Borneo: Sarawak (UM, Oxford), designated (as type) by Swinhoe, I9oo, Cat. east. and Aust. Lepid. Heterocera 2: r29.

ARTILOXIS Schaus, I9I3, Ann. Mag. nat. Hist. (8)11: 43.

OPH I

Type-species: Artiloxis vitiosa Schaus, r913, ibidem (8)11: 43, by original designation.

Syntypes $\widehat{0}$, ㅇ, Costa Rica: Sixola.

$\ddagger$ †RTICOXIS; Neave, I939, Nomencl. zool. 1: 309.

An incorrect subsequent spelling.

†ARTOLICA, misspelling. See Aviolica Walker, [1863].

CHLO

$\ddagger A R T O M Y S C I S$, misspelling. See Arctomyscis Hübner, [I820].

ACRO

ARUGISA Walker, I865, List Specimens lepid. Insects Colln Br. Mus. 33: I023.

HYPE

Type-species: Arugisa aliena Walker, I865, ibidem 33: I023, by monotypy. Holotype o. [BrazIL]: [R. Amazon], [Tefé] Ega (BMNH).

ARVADUCA Walker, r869, Characters undescr. Lepid. Heterocera: 27.

OPH I

Type-species: Arvaduca dotata Walker, 1869, ibidem: 28, by monotypy. Type(s)

․, [HONDURAS]: Limas (BMNH).

$\ddagger$ ARYOPHORA, misspelling. See Arcyophora Guenée, I 852 .

CHLO

ARYTRURA John, I912, Revue russe Ent. 12: 133.

OPHI

Type-species: Zethes musculus Ménétriés, I859, Bull. phys.-math. Acad. Sci. St. Pétersb. 17: 220, by original designation. Syntypes 2 ex., [U.S.S.R.]: Amur, Ussuri (ZI, Leningrad). JAPAN (ZI, Leningrad).

Arytruva was attributed to Zahn, an incorrect authorship, by Neave, 1939, Nomencl. zool. 1: 3II.

MEGAZETHES Warren, I9I3, in Seitz, Gross-Schmett. Erde 3: 405.

Type-species: Zethes musculus Ménétriés, 1859 , by original designation.

Megazethes is a junior objective synonym of Arytrura John, I9I2.

The date of publication of this part of Seitz was stated on page 405 as I9I3 December Ist in both the English and German editions.

ARYTRURIDES Hampson, I926, Descr. new Genera Species Lepid. Phalaenae Subfamily Noctuinae Br. Mus.: 5I 5 .

OPHI

Type-species: Deva inornata Walker, 1865, List Specimens lepid. Insects Colln Br. Mus. 33: 848 , by original designation. Holotype of. TYPE-LOCALITY not stated (BMNH) [INDIA].

ARZAMA Walker, 1865, List Specimens lepid. Insects Colln Br. Mus. 32: 644.

Type-species: Arzama densa Walker, 1865, ibidem 32:645, by monotypy. Holotype o. [U.S.A.]: Georgia (BMNH). 
ARZAMOPSIS Dyar, 1922, Insecutor Inscit. menstr. 10: 50.

ACRO

Type-species: Arzama diffusa Grote, i 878, Bull. U.S. geol. geogr. Surv. Territ. 4: I 79 , by original designation. Type(s) 으, [U.S.A.]: Maine (BMNH).

ASAPHES Turner, 1945. See Paventia Rossi, I958. ACON

$+A S C A L A P H A$ Hübner, [I806]. See Minucia Moore, [i 855]. CATo

† ASCALAPHA Hübner, I 808. See Parallelia Hübner, I8I8. CATo

ASCALAPHA Hübner, [1809], Samml. exot. Schmett. 1: pl. [196]. OPHI

Type-species: Phalaena odorata Linnaeus, I 758, Syst. Nat. (Edn Io) 1 : 505, by monotypy, but cited by Hübner as †'odora’, an incorrect subsequent spelling by Linnaeus, I 764 Mus. Lud. Ulr.: 374. Type(s), America (LS, London).

IDECHTHIS Hübner, I82 I, Index exot. Lepid. : [5].

Type-species: Phalaena odorata Linnaeus, I758, by monotypy, but cited by Hubneı

as +'odora', an incorrect subsequent spelling by Linnaeus, I 764 .

Idechthis is a junior objective synonym of Ascalapha Hübner, [r809].

OTOSEMA Hübner, [I823], Samml. exot. Schmett. 2: pl. [206].

Type-species: Phalaena odorata Linnaeus, 1758 , by monotypy, but cited by Hübner as †'odora', an incorrect subsequent spelling by Linnaeus, I 764 .

Otosema was also used for the same concept by Hübner, [1823] i8I6, Verz. bekannter Schmett.: 273 .

Otosema is a junior objective synonym of Ascalapha Hübner, [1809].

ASEMA Sodoffsky, I837. See Simyra Ochsenheimer, I8r6.

ACRO

ASEPTIS McDunnough, I937, Can. Ent. 69:59. ACRO

Type-species: Hadena genitrix Grote, I878, ibidem 10: 237, by original designation. Syntypes . , [U.S.A.]: Colorado (B.MNH); Nevada (BMNH); Nebraska.

$\pm H$. genetrix is an incorrect subsequent spelling.

ASICCIA Hampson, I926, Descr. new Genera Species Lepid. Phalaenae Subfamily Noctuinae Br. Mus: : I97.

OPHI

Type-species: Asiccia lithosiana Hampson, I926, ibidem: I97, by original designation. Syntypes 3 \%, [INDiA]: Assam, Khasis (BMNH).

+ASINCHRONA, misspelling. See Asinduma Walker, [1863].

SARR

ASINDUMA Walker, [1863] i 864, J. Proc. Linn. Soc. (Zool.) 7: i67.

SARR

Type-species: Asinduma exscripta Walker, [1863] I864, ibidem 7: I67, by monotypy. Lectotype 9 , Borneo: Sarawak (UM, Oxford), designated (as type) by Swinhoe, I9oo,

Cat. east. and Aust. Lepid. Heterocera 2: 78.

$¥$ ASINCHRONA; Fryer, I9I 2, Trans. Limn. Soc. Lond. (2. Zool.) 15: I I.

An incorrect subsequent spelling.

+ ASINDURA Hampson, no published reference found.

HYPI:

†ASINENTA Hampson, no published reference found.

HYPE

+ASINGANA Hampson, no published reference found.

IIYPF

ASIS YRA Grote, 1873, Bull. Buffalo Soc. nat. Sci. 1: I69.

ACRO

Type-species: Asisyra zelleri Grote, I873, ibidem 1: 169, by monotypy. Type(s) $\hat{0}$, NorTh AMERICA.

4. zelleri is a junior subjective synonym of Cargaza tristrigella Walker, 1866.

See also Gargaza Walker, 1866.

ASPHALIA Hübner, [1821] 1816, Vevz. bekannter Schmett.: 238.

THYATIRIDAE

Type-species: Noctua ruficollis [Denis \& Schiffermüller], I 775, A nkiundung syst. Werkes 
Schmett. Wienergegend: 87 , by subsequent designation by Harvey, 1874, Bull. Buffalo Soc. nat. Sci. $1: 277$.

Asphalia was originally placed in the Noctuidae.

ASPIDHAMPSONIA Berio, 1964, Annali Mus. civ. Stor. nat. Giacomo Doria 74: 232. HADE Type-species: Aspidifrontia glaucescens Hampson, 1905, Cat. Lepid. Phalaenae Br. Mus. 5: 45 I, pl. 9I, fig. 5, by original designation. Syntypes $4 \sigma^{\hat{\alpha}}$, [RHodesiA]: Mashonaland, Salisbury (BMNH).

ASPIDIFRONTIA Hampson, I902, Ann. S. Afr. Mus. 2: 269.

HADE

Type-species: Aspidifrontia semipallida Hampson, I902, ibidem 2: 269, by original designation. Type(s) [RHodesia]: Mashonaland, Umtali (BMNH).

ASPILA Guenée, r852. See Chloridea Duncan [\& Westwood], r841.

NOCT

ASPLENIA Hampson, I916, in Poulton, Proc. zool. Soc. Lond. 1916: 133. O Phi Type-species: Asplenia rubrescens Hampson, I9I6, ibidem 1916: I33, pl. 2, fig. I, by monotypy. Holotype $\widehat{\jmath}$. [Somali Republic]: Somaliland, Mandera (BMNH).

A. rubrescens is a junior subjective synonym of Melipotis melanodonta Hampson, I 896 . ASPLENIA Hampson, 1926, Descr. new Genera Species Lepid. Phalaenae Subfamily Noctuinae Br. Mus.: 77 .

Type-species: Melipotis melanodonta Hampson, 1896, in Walsingham \& Hampson, Proc. zool. Soc. Lond. 1896: 263, by original designation. Type(s), [Southern Yemen]: Aden (BMNH). '

A junior homonym of Asplenia Hampson, 1916, - Lepid., Noctuidae. This name was used and made available by Hampson in I 9 I 6 for a similar concept but having a different type-species. There is no objective replacement name but $M$. melanodonta is certainly congeneric and probably conspecific with Asplenia rubrescens Hampson, I9I6, the typespecies of Asplenia Hampson, I9I6. The latter is therefore available for use as a subjective replacement name.

ASPLENIA Hampson, 1926. See Asplenia Hampson, 1916.

OPH1

ASTA Walker, [1863] I864, J. Proc. Linn. Soc. (Zool.) 7: I70.

OPH1

Type-species: Asta quadrilinea Walker, [1863] I864, ibidem 7: I7I, by monotypy. Lectotype ô. Borneo: Sarawak (UM, Oxford), designated (as type) by Swinhoe, I9oo, Cat. east. and Aust. Lepid. Heterocera 2: I94.

$\ddagger$ ASTEROMESA Hampson, no published reference found.

HYPE

ASTEROPETES Hampson, igo I, Cat. Lepid. Phalaenae Br. Mus. 3: xviii, 605. AGar Type-species: Seudyra noctuina Butler, 1878, Entomologist's mon. Mag. 14: 206, by original designation. Syntypes, JAPAN: Hakodadi (BMNH).

S. noctuina was originally described as ‡'Sendyra', an incorrect subsequent spelling.

ASTEROSCOPUS Boisduval, I828, Eur. Lepid. Index meth.: 59.

CUCU

Type-species: Bombyx cassinia [Denis \& Schiffermüller], I775, Ankündung syst. Werkes Schmett. Wienergegend: $6 \mathrm{I}$, by subsequent designation by Duponchel, 1829 , in Godart \& Duponchel, Hist. nat. Lépid. Papillons Fr. 7 (2): 71. Type(s), [Austria]: Vienna district (Collection destroyed, Horn \& Kahle, 1936, Ent. Beih. Berl.-Dahlem 3: 243).

$B$. cassinia is a junior subjective synonym of Phalaena sphinx Hufnagel, I766, Berlin. $\operatorname{Mag} .3(4): 400$.

PETASIA Stephens, 1829, Illust. Br. Ent. (Haustellata) 2: 31.

Type-species: Bombyx cassinia [Denis \& Schiffermüller], I775, by monotypy, but cited as + 'Bo. cassinea Fabricius', an incorrect subsequent spelling and authorship.

Petasia is a junior objective synonym of Asteroscopus Boisduval, I828. 
ASTHA Walker, 1865, List Specimens lepid. Insects Colln Br. Mus. 33: $975 . \quad$ OPHI Type-species: Astha spectabilis Walker, I 865, ibidem 33: 975, by monotypy. Holotype 9, TYPE-LOCALITY not stated no specimens in BIINH, but from the description very close to Khadiva aurantia (Moore, I877) from S.E. Asia].

ASTHALA Noore, I882, in Hewitson \& Moore, Descr. new Indian lepid. Insects Colln late Mr IV. S. Atkinson: I 96.

Type-species: Bocana silenusalis Walker, [1859] I858, List Specimens lepid. Insects Collu Br. Mus. 16: I 79, by monotypy. Syntypes I $\hat{\sigma}$, I 9 , Borneo: Sarawak (BMNH).

ASTHANA Walker, 1862, Trans. ent. Soc. Lond. (3)1: 100.

OPHI

Type-species: Asthana erecta Walker, I 862 , ibidem 1: IOI, by subsequent designation by Hampson, 1926, Descr. new Genera Species Lepid. Phalaenae Subfamily Noctuinae Br. Mils.: i8 8 . Type(s) ô, Type-Locality 'unknown' (UMI, Oxford) [Brazil. Guiana]. See also Rethma Walker, I 866.

${ }_{\ddagger}$ ASTHATE WValker, I862; Schulze \& Kükenthal, 1926, Nomencl. Anim. Generum Subgenerum 1: 307 , cited the original description as Walker, I862, Trans. ent. Soc. Lond. (3) 1: 87. No usage by Walker of this generic name has been found. It is probably an error for Asthana Walker, r862.

†ASTHATE Wilker, i 862. See Asthana Walker, I862.

OPH I

ASTICTA Hiibner, [1823] i816, Verz. bekannter Schmett.: 266.

OPHI

Type-species: Noctua procax Hübner, [1813], Samml. eur. Schmett. 4: pl. Io9, fig. 5Io. by monotypy, but included by Hübner as 'proclivis Hübner', an unjustified emendation, Type(s), EuROPE.

See also Toxocampa Guenéc, i 8 \&1.

$\ddagger$ ASTIODES, misspelling. See.4stiotes Hübner, [1823]. CATO

ASTIOTES Hübner, [1823] 1816, Verz. bekannter Schmett.: 277. CATO

Type-species: Noctua dilecta Hübner, [18o8], Samml. eur. Schmett. 4: pl. 83, fig. 388, by subsequent designation by Hampson, 1913, Cat. Lepid. Phalaenae Br. Mus. 12: 42 . Type(s), Europe.

In his I'erzeichniss, Hübner [ I 823] listed the type-species as 'Astiotes dilecta Borkh. Noc. I 2. Hübn.Noc.[fig.]388'. Unfortunately Hiibner's [1808] fig. 388 was not of Phalaena dilecta Borkhausen, I792, but of a then undescribed species which ever since has been known as Noctua clilecta Hübner. Strictly, Noctua dilecta Hübner is not nomenclaturally available as it is a misidentification of Phalaena dilecta Borkhausen, I792, Naturg. eur. Schmett. 4:38, a junior subjective synonym of Noctua promissa [Denis \& Schiffermüller], I775. The next nomenclaturally available name for dilecta Hübner is dayvemi Oberthür, I 907 which was published as a var. of Catocala dilecta Hübner from Algeria. To change dilecta, after over I50 years of stability, to the name of an Algerian subspecies would cause unnecessary confusion, and so in order to ensure stability an application should be submitted to the Commission under Article 79, requesting that Noctua dilecta Hübner, [I 808] be validated.

†STIODES; Forster \& Wohlfahrt, I970, Schmett. Mitteleur. 4: 280, 319.

An incorrect subsequent spelling.

ASTONYCHA Turner, I920, Trans. Proc. R. Soc. S. Aust. 44: I29, I3I. NOCT

Type-species: Astonycha litarga Turner, I920, ibidem 44: I 3 I, by monotypy. Holotype $\hat{o}$. Australia: Qd, Rosewood (ANIC, Cauberra).

$\ddagger$ ASTRAPETES, nisspelling. See Achatia Hübner, [ [ $\left.1 \&_{1} 3\right]$.

HADI:

ASTRAPETIS, Hübner, I82I. Sce Achatia Hübner, [18I3].

HADE

ASYLAEA Möschler, 1883, Verh.zool.bot. Ges. Wien 32 (Ablu.): 357.

HYPE:

Type-species: Asylciea inflexa Möschler, 1883 , ibidem 32 (Abh.): $35^{8}$, pl. is, fig. 43 , by monotypy. Holntype ô. Surinam: Paramaribo (MNHU, Berlin). 
AS YMBA TA Gerstaecker, I871, Arch. Naturgesch. 37(I): 360.

OPHI

Type-species: Asymbata roseiventris Gerstaecker, I 87 I, ibidem 37(1) : 360, by monotypy. Type(s) 우 [KenYa]: L. Jipe.

A. roseiventris is a junior subjective synonym of Calesia zambesita Walker, I865, List Specimens lepid. Insects Colln Br. Mus. 33: 962.

Asymbata and roseiventris were both described again as new in Gerstaecker, I873, Baron C. C. von der Decken's Reisen in Ost-Afrika 3 (2): 377, pl. I 5, fig. 8.

ASYMBLETIA Herrich-Schäffer, I856, Sammlung neuer oder wenig bekannter aussereur. Schmett. 1(1): wrapper, pl. 79, figs 456,457 ; I 858 , ibidem 1: 69, 83 .

OPHI

Type-species: Asymbletia dispar Herrich-Schäffer, I856, ibidem 1(1): wrapper, pl. 79, figs 456,$457 ; 1858$, ibidem 1: 69, 83 , by monotypy. Type(s) Type-Locality not stated [Equatorial S. America].

AS YNEDA Richards, 1936, Can. Ent. 67: 265.

OPHI

Type-species: Syneda mendozina Hampson, 1926, Descr. new Genera Species Lepid. Phalaenae Subfamily Noctuinae Br. Mus.: 44, by original designation. Syntypes I $\delta^{\hat{\sigma}}$ I + , Argentina: Mendoza (BMNH).

ATACIRA Swinhoe, I90o, Cat. east. and Aust. Lepid. Heterocera 2: 87.

EUTE

Type-species: Eutelia approximata Walker, [1863] 1864, J. Proc. Linn. Soc. (Zool.)

7: 65 , by monotypy. Lectotype $\hat{0}$, BORNEO: Sarawak (UM, Oxford), designated (as type) by Swinhoe, r 900, ibidem 2:87.

ATENERIA Schaus, I9I4, Proc. U.S. natn. Mus. 46: 5I 4.

OPH I

Type-species: Ateneria crinipuncta Schaus, I9I 4, ibidem 46:5I 4, by original designation. Type(s) ô. [French GuIANA]: R. Maroni, St. Jean (USNM, Washington).

ATETHMIA Hübner, [I82I], I8I6, Verz. bekannter Schmett.: 238.

CUCU

Type-species: Phalaena xerampelina Esper, I793, Die Schmett. 4(2) Abschnitt 2: pl. I83, fig. 4; [I796], ibidem: I5, by subsequent designation by Grote I874, Bull. Buffalo Soc. nat. Sci. 2: 24, but cited as xerampelina Hübner, an incorrect authorship. Type(s) $\hat{0}$, HUNGARY.

P. xerampelina is a junior subjective synonym of Noctua ambusta [Denis \& Schiffermüller],

I775, Ankündung syst. Werkes Schmett. Wienergegend: 88.

See also Brachycosmia Butler, I89o.

†LETHMIA ; Hübner, [1826], I8I6, Verz. bekannter Schmett. Anzeiger: 36.

An incorrect subsequent spelling.

$\ddagger$ ATHETMIA; Hübner, [I826], I816, Verz. bekannter Schmett. Anzeiger: 37 .

An incorrect subsequent spelling.

ATHAUMASTA Hampson, I906, Cat. Lepid. Phalaenae Br. Mus. 6: 380.

CUCU

Type-species: Polia expressa Lederer, I855, Verh. zool.-bot. Ver. Wien 5: Io9, pl. I, fig. 8, by monotypy (of Thaumasta Staudinger, I87I). Syntypes $2 \hat{\delta}$, [U.S.S.R.]: Siberia, Altai Mits.

Athaumasta was proposed as the objective replacement name for Thaumasta Staudinger, I87I.

THAUMASTA Staudinger, I871, in Staudinger \& Wocke, Cat. Lepid. eur. Fannengeb.: 79.

Type-species: Polia expressa Lederer, I 855 , by monotypy.

A junior homonym of Thaumasta Gistl. I848, Naturg. Thierreichs: ix, - Crustacea. The objective replacement name is Athaumasta Hampson, I906.

ATHETIS Hübner, [1821] I816, Verz. bekannter Schmett. : 209.

ACRO

Type-species: Noctua dasychira Hübner, [I8I7], Samml. eur. Schmett. 4: pl. I38, fig. 634, by subsequent designation by Hampson, I909, Cat. Lepid. Phalaenae Br. Mus. 8: 299, but cited as 'furvula Hübner, [I808]', which Hampson placed (on page 3I8) as the senior synonym of dasychira Hübner. Type(s), Europe. 
$N$. dasychiva is a junior subjective synonym of Noctua furvula Hübner, [I808], ibidem 4: pl. 83, fig. 390 .

$\ddagger$ ATHETMIA, misspelling. See Atethmia Hübner, [r82r].

CUCU

ATHURMODES Dognin, I9I4, Hétérocères nouv. Am. Sud. 8: 73.

HYPE

Type-species: Athurmodes spreta Dognin, I9I 4 , ibidem 8: 73, by original designation.

Holotype of. Colombia: San Antonio r8oo m.

$\ddagger$ ATHYANIA, misspelling. See Athysania Hübner, [1823].

OPH I

${ }_{\ddagger}^{\dagger}$ ATHYMA, misspelling. See Athyrma Hübner, [1823].

OPHI

ATHYRMA Hübner, [1823] I816, Verz. bekannter Schmett.: 267.

OPHI

Type-species: Phalaena adjutrix Cramer, I780, Uitlandsche Fiapellen 3: I44, pl. 272, figs E, F, by monotypy. Type(s), Surinam.

Athyrma had three originally included species of which two, + A. ganglio Hübner, [1823], and $\ddagger$ A interpuncta Hübner, [1823], were nomina mula.

$P$. adjutrix is dated from the wrapper of the part and not from the title-page of the volume, 1782 .

¥THYMA; Pagenstecher, i 888, Jb. nassau. I'er. Naturk. 41: I 49.

An incorrect subsequent spelling.

A THYRMELLA Roepke, I941, Zoöl. Meded., Leiden 23: 26.

OPHI

Type-species: Athyrmella priangani Roepke, I94I, ibidem 23: 26, pl. I, figs 3, 4, by original designation. Holotype $\hat{0}$. JAYA: WV., Preanger.

The type-species was proposed as priangani, but the locality was cited as Preanger.

ATHYRMINA Hampson, 1926, Descr. new Genera Species Lepid. Phalaenae Subfamily Noctuinae Br. Mus.: I 79.

OPHI

Type-species: Talapa albigutta Swinhoe, I 895, Ann. Mag. nat. Hist. (6)15: i6, by original designation. Syntypes I ô, 3 우, [INDIA]: [Assam], Shillong (BMNH).

A THYSANIA Hübner [1823] I 8 I6, Verz. bekannter Schmett.: 263.

OPHI

Type-species: Phalaena chorinea Stoll, 1780 , in Cramer, Uitlandsche Kapellen 4: 42 , pl. 3ro, fig. F, by monotypy. Type(s), Surinam (not found in RNH, Leiden; see Todd, I959, Tech. Bull. U.S. Dep. Agric. 1201: 29).

$P$. chorinea is the spelling in the Dutch text and in the Index 1782 , page 248 . In the French text the spelling is ‘'choninea', an incorrect original spelling.

$P$. chorinea is dated from the wrapper of the part and not from the title-page of the rolume, 1782.

† ATHYANIA; Walker, [1858] I857, List Specimens lepid. Insects Colln Br. Mus. 12: 946.

An incorrect subsequent spelling.

A TIMAEA Hampson, I908, Cat. Lepid. Phalaenae Br. Mus. 7: I8. Available, but without included species until Hampson, I9 Io, ibidem 9: viii, I25.

$\triangle \mathrm{CRO}$

Type-species: Timaea pictura Saalmüller, I 891, Lepid. Madagascar: 309, pl. I3, fig. 240 , by subsequent designation by Hampson, I9 Io, ibidem 9: i25. Syntypes, Madagascar (Z.MH U, Berlin).

ATLANTAGROTIS Köhler, 1955, Rev. Soc. ent. Avgent. 18: 2.

NOCT

Type-species: Stenagrotis hesperoides Köhler, 1945, Acta zool.lilloana 3: 74, by original designation. Holotype $q$, Argentina: Chubut [Prov.], Comodoro Rivadavia (ZSBS, Munich).

ATOPOMORPHA Warren, I889, Trans, ent. Soc. Lond. 1889: 252.

HYPE

Type-species: Atopomorpha singularis Warren, I889, ibidem 1889: 253, by monotypy. Holotype $\vec{\sigma}$, [BrazıL]: Amazon basin, Para (BMNH). 
ATRACHEA Warren, I9I I, in Seitz, Gross-Schmett. Erde 3: I75.

Type-species: Spaelotis nitens Butler, I878, Ann. Mag. nat. Hist. (5)1: I64, by original designation. Type(s), JAPAN: Yokohama (BMNH).

The date of publication of the part of Seitz containing page I75, was stated on page I73 as I9II February 8th in both the English and the German editions.

ATREPHES Jones, I908 June, Trans. ent. Soc. Lond. 1908: I63.

Type-species: Atrephes phocea Jones, I908, ibidem 1908: 163, by monotypy. Type(s) o. Brazil: Parana Castro (BMNH).

ATREPHES Hampson, 1908 [December], Cat. Lepid. Phalaenae Br. Mus. 7: 284.

Type-species: Atrephes albiluna Hampson, I908, ibidem 7: 284, fig. 45, by original designation. Syntypes $2 \hat{\jmath}$, I 9, Brazil: Porto Alegre (BMNH).

A junior homonym of Atrephes Jones, I908,-Lepid., Noctuidae. This generic name probably originated from Hampson, and was used and unintentionally made nomenclaturally available by Jones prior to its proposal and generic description by Hampson for a similar concept but having a different type-species. There is no objective replacement name but $A$. albiluna is congeneric with Atrephes phocea Jones, I908, the type-species of Atrephes Jones, I 908 . The latter is therefore available for use as a subjective replacement name.

The date of publication of Hampson's Catalogue volume 7 is taken as 1908 December I 4 th from Collins, I962, Ann. Mag. nat. Hist. (13)5: I26.

AT REPHES Hampson, I908 [December]. See Atrephes Jones, I908 June.

ACRO

ATTATHA Moore, I878, Proc. zool. Soc. Lond. 1878: 847 .

CATO

Type-species: Hypercompa regalis Moore, I872, ibidem 1872: 575, pl. 33, fig. 7, by monotypy. Lectotype, InDia: northern (UM, Oxford), designated (as type) by Swinhoe, I900, Cat. east. and Aust. Lepid. Heterocera 2: I55.

ATTONDA Swinhoe, I919, Ann. Mag. nat. Hist. (9)4: I23.

OPH I

Type-species: Capnodes trifasciata Moore, 1877, Proc. zool. Soc. Lond. 1877: 612, by original designation. Type(s) 9 , Andaman Is (BMNH).

C. trifasciata is a junior subjective synonym of Felinia adspersa Felder \& Rogenhofer, I874, Reise öst. Fregatte Novara (Zool.) 2 (Abt. 2): pl. II7, fig. 23.

ATYPHA Hübner, [I82I] I8I6, Verz. bekannter Schmett.: 2 I3.

ACRO

Type-species: Phalaena pulmonaris Esper, I790, Die Schmett. 4 (2) Abschnitt I: pl. I 5I, fig. 5; [I798-I805], ibidem: 499, by subsequent designation by Hampson, I909, Cat. Lepid. Phalaenae Br. Mus. 8: 299. Type(s) ô. Germany.

AUCHA Walker, [1858] I857, List Specimens lepid. Insects Colln Br. Mus. 13: II37. ACRO Type-species: Aucha velans Walker, [1858] I857, ibidem 13: II38, by monotypy. Holotype ô. Ceylon (BMNH).

AUCHENISA Hampson, 1905, in Holland, Ann. Mag. nat. Hist. (7) 16: i8.

OPHI

Type-species: Auchenisa schausi Hampson, 1905, ibidem (7)16: 18, by monotypy. Holotype + , Sierra Leone (CM, Pittsburgh).

AUCHMIS Hübner, [I82I] I8I6, Verz. bekannter Schmett: 243.

ACRO

Type-species: Phalaena petroriza Borkhausen, I792, Naturg. eur. Schmett. 4: 343, by subsequent designation by Warren, I9I4, in Seitz, Gross-Schmett. Erde 3: 509, but cited as 'comma Schiff.', see below. Type(s), Europe.

$P$. petroriza is a junior subjective synonym of Noctua detersa Esper, I791, Die Schmett. $4(\mathrm{I}): 260$, [no fig.].

Auchmis Hübner contained, as the first of the originally included species, the following entry' '2422. Auchmis petroriza Borkh. Noc. I43. comma Schiff. Verz. Noc. R. 9. Hübn. Noc. 25I'. Warren cited the type-species as 'comma Schiff.' and included +petrorhiza Bkh.' (an incorrect subsequent spelling) in its synonymy. But 'comma [sensu] Schiff.' 
(although one of the originally included names of Auchmis) was cited by [Denis \& Schiffermüller], I775, Ankïndung syst. Werkes Schmett. Wienergegend: 76, as 'Noctua comma L.' and was actually a misidentification of the Linnaean species (in the Hadeninae) and so has no nomenclatural status.

Incorrect type-species designation: Phalaena polyodon Clerck, I759, a name not originally included in Auchmis, and not linked with one of the originally included names when cited by Hampson, I894, Fanna Br. India (Moths) 2: 283 .

RHIZOGRAMMA Lederer, I857, Noctuinen Eur.: 35, I09.

Type-species: Phalaena petroriza Borkhausen, I792, by monotypy, but cited by Lederer as +'petrorhiza Blkh.', an incorrect subsequent spelling.

Rhizogramma is a junior objective synonym of Auchmis Hübner, [I82 I].

AUCHMOPHANES Turner, igo8, Trans. Proc. R. Soc. S. Aust. 32: 64.

HYPE

Type-species: Auchmophanes ochrospila Turner, I908, ibidem 32: 65, by monotypy. Holotype , Australia: Qd, R. Mulgrave, near Cairns (NMT, Melbourne).

AUCULA Walker, 1862, Trans. ent. Soc. Lond. (3)1: 253.

AGA R

Type-species: Aucula josioides Walker, I862, ibidem (3) 1:253, by monotypy. Type(s) o., [BRAZIL]: Rio Janeiro.

$\ddagger$ †LUCULA ; Jörgensen, 1935, An. Mus, argent. Cienc. nat. 38: 125.

An incorrect subsequent spelling.

AUDEA Walker, [1858] I857, List Specimens lepid. Insects Colln Br. Mus. 13: I I22, I I34. Cato Type-species: Audea bipunctata Walker, [1858] i857, ibidem 13: II35, by monotypy. Syntypes ô, [South AFrica]: Port Natal (BMNH); Caffraria.

AUDELA Walker, I86I, in D'Urban, Can. Nat. E Geol. 6: 37.

PANT

Type-species: Audela acronyctoides Walker, I 861, ibidem 6:37, by monotypy. Type(s) o., [CANADA]: [Quebec], Montcalm.

AULOCHETA Prout, I927, Trans. ent. Soc. Lond. 75: 228.

MYPE

Type-species: Aulocheta violacea Prout, I927, ibidem 75:228, pl. 2I, fig. 2I, by original designation. Syntypes I $\hat{\jmath}, \mathrm{I}$ q, [S̃̃o Tomé I., West Africa] São Thomé I. (BMNH).

AULOTARACHE Hampson, igio, Cat. Lepid. Phalaenae Br. Mus. 10: xviii, 722. ACoN

Type-species: Tarache decoripennis Mabille, I900, Annls Soc.ent.Fr. 68:728, by original designation. Type(s) E. Africa (not found by Viette \& Fletcher, 1968, Bull. Br. Mus. nat. Hist. (Ent.) 21: $4 \mathrm{I} 6$; but Hampson, 1910, when he proposed the genus indicated that he had examined the type from the Mabille Colln).

AUSAVA Walker, I858, List Specimens lepid. Insects Colln Br. Mus. 15: I644. $\operatorname{ACON}$

Type-species: Ausava triplaga Walker, I858, ibidem 15: I644, by monotypy. Type(s) o., [BRAzIL]: Rio Janeiro (UM, Oxford).

AUSINZA Walker, 1864, J. Proc. Linn. Soc. (Zool.) 7: I92.

$\operatorname{ACON}$

Type-species: Ausinza aequa Walker, 1864, ibidem 7: 192, by monotypy. Lectotype 9 , BorNeo: Sarawak (UM, Oxford), designated (as type) by Swinhoe, I9oo, Cat. east. and Aust. Lepid. Heterocera 2: 70.

A. aequa is a junior subjective synonym of Corgatha zonalis Walker, [1859].

See also Corgatha Walker, [1859].

AUSTRAMATHES Hampson, I906, Cat. Lepid. Phalaenae Br. Mus. 6: xiv, 492.

CUCU

Type-species: Graphiphora purpurea Butler, 1879, Cistula ent. 2: 490, by original designation. Type(s), New ZEaland: Otago (BMNH).

AUSTRANDESIA Köhler, 1967, Acta zool. lilloana 21: 272.

NOCT

Type-species: Austrandesia argentina Köhler, 1967, ibidem 21: 272, figs 7 \& I04. Holotype $\hat{\delta}$, Argentina: Chubut [Prov.], Lago Argentino, Punta Bandera. 
AUSTRAZENIA Warren, 1913, in Seitz, Gross-Schmett. Evde 11: 196.

ACRO Type-species: Megalodes tusa Swinhoe, I902, Ann. Mag. nat. Hist. (7) 9: 168, by original designation. Syntypes $2 \AA, 4$, Australia: W. Aust., Sherlock River (BMNH).

The date of publication of the part of Seitz containing page I96, was stated on page 193 as I9I3 August $5^{\text {th }}$ in both the German and the English editions.

AUTANTHEMA Warren, I9I2, Novit. zool. 19: 45.

CHLO

Type-species: Autanthema diversicolor Warren, I9I2, ibidem 19: 45, by original designation. Syntypes I $\widetilde{J}, 3$ + , NEw GuINEA: [West Irian], Snow Mts, upper R. Setekwa.

AUTHADISTIS Hampson, I902, Ann.S. Afr. Mus. 2: $36 \mathrm{I}$.

OPHI

Type-species: Authadistis metaleuca Hampson, I902, ibidem 2: 361, by monotypy.

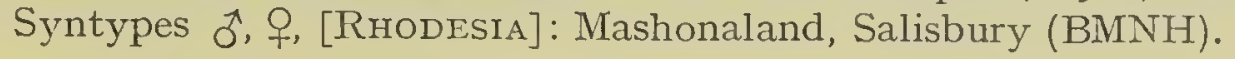

AUTOBA Walker, [1863] I864, J. Proc. Linn. Soc. (Zool.) 7: 57.

ACON

Type-species: Autoba versicolor Walker, [I863] I864, ibidem 7: 58, by monotypy. Lectotype of, Borneo: Sarawak (UM, Oxford), designated (as type) by Swinhoe, I9oo, Cat. east. and Aust. Lepid. Heterocera 2: 65.

AUTOGRAPHA Hübner, [I82I] I8I6, Verz. bekannter Schmett.: 251.

PLUS

Type-species: Phalaena gamma Linnaeus, I758, Syst. Nat. (Edn Io) 1: 5I3, by subsequent designation by Grote, I896, Entomologist's Rec. J. Var. 8: 303. Type(s), TypeLOCALITY not stated (LS, London) [EUROPE].

AUTOMALA Walker, [1863] I864, J. Proc. Linn. Soc. (Zool.) 7: 60.

NOLI

Type-species: Automala semidolosa Walker, [1863] I864, ibidem 7: 60, by monotypy. Type(s) ô. Borneo: Sarawak (UM, Oxford).

AUTOPHILA Hübner, [I823] I816, Verz. bekannter Schmett.: 274.

OPHI

Type-species: Noctua dilucida Hübner, [1808], Samml. eur. Schmett. 4: pl. 82, fig. 383, by subsequent designation by Warren, I9I3, in Seitz, Gross-Schmett. Erde 3: 37 I. Type(s), Europe.

AUTOPLUSIA MIcDunnough, I944, Mem. sth. Calif. Acad. Sci. 2: 203.

PLUS

Type-species: Plusia egena Guenée, I852, in Boisduval \& Guenée, Hist. nat. Insectes (Lépid.) 6:328, by original designation. Syntypes 5 ex., BrazIL: Nouvelle-Fribourg.

$\boldsymbol{A V A T H A}$ Walker, [I 858] I857, List Specimens lepid. Insects Colln Br. Mus. 13: Io79, I Io6. CAтo Type-species: Avatha includens Walker, [1858] I857, ibidem 13: I107, by monotypy. Syntypes 우 [India] Hindostan.

$A$. includens is a junior subjective synonym of Noctua discolor Fabricius, I794, Ent. Syst. $3(2): 50$.

AVENTIA Duponchel, I829. See Laspeyria Germar, I8II.

OPHI

AVENTINA Staudinger, I892, in Romanoff, Mém. Lépid. 6: 604.

ACON

Type-species: Aventiola costimacula Staudinger, I892, ibidem 6: 604, pl. I4, fig. 4, by subsequent designation by Berio, 1966, Annali Mus. civ. Stor. nat. Giacomo Doria 76 : 50. Syntypes $2 \hat{0}$, I 우 [U.S.S.R.]: Amur district, Baranowka (MNHU, Berlin); Sutschan (MNHU, Berlin).

A. costimacula is a junior subjective synonym of Egnasia argillacea Butler, 1879, Ann. Mag. nat. Hist. (5) 4: 450.

Aventina was originally proposed as a subgenus of Aventiola Staudinger, I892.

AVENTIOLA Staudinger, I892, in Romanoff, Mém. Lépid. 6: 602. HYPE

Type-species: Aventiola maculifera Staudinger, I892, ibidem 6:602, pl, I4, fig. 3, by monotypy. Holotype ô, [U.S.S.R.]: Amur district, Sidemi (MNHU, Berlin).

A. maculifera is a junior subjective synonym of Egnasia pusilla Butler, I879, Illust. typical Specimens Lepid. Heterocera Colln Br. Mus. 3: xv, 67, pl. 5\%, fig. 9. 
AVIROSTRUM Bethune-Baker, I908, Novit. zool. 15: 227.

HYPE

Type-species: Avirostrum pratti Bethune-Baker, 1908, ibidem 15: 227, by original designation. Syntypes ${ }^{\jmath}$, 우 New Guinea: [Papua], Dinawa; R. Aroa; 'other localities'.

AVITTA Walker, I858, List Specimens lepid. Insects Colln Br. Mus. 15: I674. OPHI

Type-species: Avitta subsignans Walker, I 858 , ibidem 15: I675, by monotypy. Type(s) $\widehat{o}$, [INDiA]: [Mysore], Canara (BMNH).

See also Oroba Walker, [1863].

AVITTONIA Hampson, 1926, Descr. new Genera Species Lepid. Phalaenae Subfamily Noctuinae Br. Mus.: 496.

$\mathrm{OPHI}$

Type-species: Avittonia albidentata Hampson, I926, ibidem: 496, by original designation. Holotype + , Singapore (BMNH).

AXENUS Grote, I873, Bull. Buffalo. Soc nat. Sci. 1: I 52.

ACRO

Type-species: Axenus arvalis Grote, I873, ibidem 1: I52, pl. 4, fig. 8, by monotypy. Syntypes $8(\hat{\sigma}$, o) $)$ [U.S.A.]: California (BMNH).

AXIA Hübner, [1821] I816, Verz. bekannter Schmett.: 249.

TIHYATIRIDAE

Type-species: Noctua margarita Hübner, [18I3], Samml. eur. Schmett. 4: pl. I Io, fig. 514, by monotypy.

Axia was originally placed in the Noctuidae.

CIMELIA Lederer, I853, Verh. zool.-bot. Ges. Wien 3 (Abh.): I8o, 228, 237.

Type-species: Noctua margarita Hübner, [I8I3], by monotypy.

Cimelia has been used as the original generic name for species which are now in the Noctuidae.

$\boldsymbol{A X I A}$ Hübner, [1825]. See Nycteola Hübner, I822.

SARR

AXIOCTETA Turner, 1902, Proc. Linn. Soc. N.S.IW. 27: 105.

OPHI

Type-species: Axiocteta oenoplex Turner, 1902, ibidem 27: 106, by monotypy. Holotype + , Australia: Qd, Brisbane (ANIC, Canberra).

AXIORATA Turner, 1902, Proc. Linn. Soc. N.S.W. 27: I 20.

ACRO

Type-species: Axiorata leucozona Turner, I902, ibidem 27: I 2 I, by original designation. Syntypes, AUstralia: $3 \hat{0}$, Qd, Townsville (ANIC, Canberra); Rockhampton; Brisbane.

AXYLIA Hübner, [1821] i 816, Verz. bekannter Schmett.: 242.

NOCT

Type-species: Phalaena putris Linnaeus, I76I, Fauna Suecica (Edı 2): 315, by subsequent designation by Guenée, 1852 , in Boisduval \& Guenée, Hist. nat. Insectes (Lépid.) 5: 134. Type(s), Sweden (LS, London).

AZAMORA Walker, I858, List Specimens lepid. Insects Colln Br. Mus. 15: i 757 . PyralddaE Type-species: Azamora tortriciformis Walker, i858, ibidem 15: I 757, by monotypy. Azamora was originally placed in the Noctuidae.

AZATHA Walker, I 858, List Specimens lepid. Insects Colln Br. Mus. 15: I 522, I $534 . \quad$ opHI Type-species: Azatha retardens Walker, I858, ibidem 15: I 534, by monotypy. Holotype ô. Honduras (BMNH).

AZAZIA Walker, i 858, List Specimens lepid. Insects Colln Br. Mus. 15: I 546, I 576.

OPHI Type-species: Ophiusa rubricans Boisduval, 1833, Nouv. Ann. Mus. Hist. nat. Paris 2: 254, by monotypy. Type(s), Madagascar.

O. rubricans was also proposed in 1833 in Faune ent. Madagascar (Lépid.): 106, pl. I6, fig. I.

AZENIA Grote, i882, Papitio 2: i 86.

Type-species: Azenia implora Grote, I882, ibidem 2: 186, by monotypy. Type(s), [U.S.A.]: Arizona. 
AZETA Guenée, 1852, in Boisduval \& Guenée, Hist. nat. Insectes (Lépid.) 7: 358 OPHI Type-species: Azeta uncas Guenée, I 852, ibidem 7:359, by subsequent designation by Desmarest, (1857), in Chenu, Encycl. Hist. nat. Papillons nocturnes: I35. Holotype o, Jamaica (UM, Oxford).

See also Milyas Walker, I858.

AZIRISTA Walker, I858, List Specimens lepid. Insects Colln Br. Mus. 15: I 547, I638. opH I Type-species: Azirista intracta Walker, I858, ibidem 15: 1639, by monotypy. Holotype ㅇ, [BRAzIL]: Santarem (BMNH).

A. intracta is a junior subjective synonym of Phalaena tarchon Cramer, I777, Uitlandsche Kapellen 2: 65, pl. I39, fig. C.

BADAUSA Walker, [1863] I864, J. Proc. Linn. Soc. (Zool.) 7: I 70.

HYPE

Type-species: Badausa hypenoides Walker, [1863] I864, ibidem 7: I70, by monotypy. Lectotype + , BoRneo: Sarawak (UM, Oxford), designated (as type) by Swinhoe, I90o, Cat. east. and Aust. Lepid. Heterocera 2: 212.

B. hypenoides is a junior subjective synonym of Hypena rhombalis Guenée, I854, in Boisduval \& Guenée, Hist. nat. Insectes (Lépid.) 8: 33.

BADIZA Walker, I864, J. Proc. Linn. Soc. (Zool.) 7: 195.

HYPE

Type-species: Badiza ereboides Walker, I864, ibidem 7: 196, by monotypy. Type(s) J゙, BorNEO: Sarawak (BMNH).

BAECULA Walker, I856, List Specimens lepid. Insects Colln Br. Mus. 9: I37, I47. OPH Type-species: Baecula gallopavo Walker, I 856 , ibidem 9: I48, by monotypy. Holotype ô. VENEZUela (BMNH).

Baecula when originally proposed contained a second species, but this was only doubtfully included, and under the Code, Article 68(c), is not eligible for designation as type-species. $\ddagger$ BOECULA; Walker, I858, List Specimens lepid. Insects Colln. Br. Mus. 15: 1669.

An incorrect subsequent spelling.

BAGADA Walker, I858, List Specimens lepid. Insects Colln Br. Mus. 15: I753.

ACRO

Type-species: Bagada pyrochroma Walker, I858, ibidem 15: I753, by monotypy. Holotype 9 , CEylon (BMNH).

B. pyrochroma is a junior subjective synonym of Perigea spicea Guenée, 1852, in Boisduval \& Guenée, Hist. nat. Insectes (Lépid.) 5: 226.

BAGISARA Walker, I 858, List Specimens lepid. Insects Colln Br. Mus. 15: I770. ACON Type-species: Bagisara incidens Walker, I858, ibidem 15: I77I, by monotypy. Holotype + , JAMAICA (BMNH).

B. incidens is a junior subjective synonym of Bombyx repanda Fabricus, I793, Ent. Syst. 3(I): 462. LECTOTYPE, [WEST INDIEs] Americae meridionalis Insulis (UZM, Copenhagen), here designated [photograph examined].

BAGISARA Walker, I 858, List Specimens lepid. Insects Colln Br. Mus. 15: I79I. See Caroia Walker, 1858 . NOTODONTIDAE

BAGISTANA Valker, I864, J. Proc. Linn. Soc. (Zool.) 7: I94.

SARR

Type-species: Bagistana rudis Walker, I864, ibidem 7: I94, by monotypy. Lectotype $\hat{0}$. Borneo: Sarawals (UM, Oxford), designated (as type) by Swinhoe, I9oo, Cat. east. and Aust. Lepid. Heterocera 2: 79.

$B$. rudis is a junior subjective synonym of Nanaguna brevinuscula Walker, I863.

See also Nanagına Walker, I863; Clettharra Walker, I863; and Thrypticodes Lucas, I89o.

BAILEYA Grote, I895, Abh. naturw. Ver. Bremen 14: I22.

SARR

Type-species: Leptina dormitans Guenée, 1852 , in Boisduval \& Guenée, Hist. nat. Insectes (Lépid.) 5: 15, by subsequent designation (for Leptina Guenée, I 852) by Grote, 1873, Bull. Buffalo Soc. nat. Sci. 1: 76. Holotype 3. [U.S.A.]: New York (BMNH).

Baileya was proposed as the objective replacement name for Leptina Guenée, 1852. 
$\ddagger B A Y L E Y A ;$ Lucas, I900, Arch. Naturgesch. 63 (2) (2): 507.

An incorrect subsequent spelling.

LEPTINA Guenée, 1852, in Boisduval \& Guenée, Hist. nat. Insectes (Lépid.) 5: I 4.

Type-species: Leptina dormitans Guenée, 1852 , by subsequent designation by Grote, 1873, Bull. Buffalo Soc. nat. Sci. 1: 76.

A junior homonym of Leptina Meigen, 1830, Syst. Beschr. zweifl. Insekt. 6:283,-Diptera. The objective replacement name is Baileya Grote, 1895 .

BALLATHA Walker, [1866] I865, List Specimens lepid. Insects Colln Br. Mus. 34: I2 I4. chLo

Type-species: Ballatha laeta Walker, [1866] I865, ibidem 34: I2 I5, by subsequent designation by Kirby, I892, Synonymic Cat. Lepid. Heterocera 1: 284. Syntypes $3 q$, [INDia]: North Hindostan (BMINH); Hindostan (BMNH).

HYPOSCOT A Hampson, I912, Cat. Lepid. Phalaenae Br. Mus. 11 : xiii, 460.

Type-species: Ballatha laeta Walker, [1866], by original designation.

Hyposcota is a junior objective synonym of Ballatha Walker, [I866].

BALLONICHA Möschler, 1886, Abh. senckenb. naturforsch. Ges. 14 (3): 60. HYPE

Type-species: Ballonicha recurvata Möschler, I886, ibidem 14 (3): 60, by monotypy. Holotype q, JamaICa (MNHU, Berlin).

BALSA Walker, 1860, in D'Urban, Can. Nat. E Geol. 5: 250.

ACRO

Type-species: Balsa obliquifera Walker, I860, ibidem 5: 25I, by monotypy. Type(s)

o. [CANADA]: Montreal.

B. obliquifera is a junior subjective synonym of Brachytaenia malana Fitch, I856.

See also Nolaphana Grote, I873.

BAMBUSIPHILA Sugi, 1958, Tinea, Tokyo 4: 184.

$\mathrm{ACRO}$

Type-species: Polydesma vulgaris Butler, 1886, Trans. ent. Soc. Lond. 1886: 135, by original designation. Syntypes $2 \hat{0}, 3$ f, JAPAN: Tokei (BMNH); Yokohama (BMNH). ChINA: Chekiang (BMNH).

BAMRA Moore, I882, in Hewitson \& Moore, Descr. new Indian lepid. Insects Colln late Mr W. S. Atkinson: I 59 .

OPHI

Type-species: Agriopis discalis Moore, I867, Proc. zool. Soc. Lond. 1867: 57, pl. 7 , fig. 2, by original designation. Syntypes $\hat{0}, q$, India: Bengal (BMNH).

A. discalis is a junior subjective synonym of Felinia albicola Walker, 1858 , List Specimens lepid. Insects Colln Br. Mus. 14: I 5 I5.

BANASSA Walker, I863, List Specimens lepid. Insects Colln Br. Mus. 27: 19. OPH

Type-species: Banassa rutilans Walker, 1863, ibidem 27: 20, by monotypy. Type(s)

, BORNEO: Sarawak.

B. nutilans is a junior subjective synonym of Nolasena ferrifervens Walker, [I 858 ].

See also Nolasena Walker, [1858].

BANDELIA Lindsey, 1923, Ent. News 34: I 23.

ACON

Type-species: Nothophila angulata Barnes \& Lindsey, 1922, Bull. Brooklyn ent. Soc. 17: 76, by original designation (for Nothophila Barnes \& Lindsey). Holotype ô, [U.S.A.]: Ariz., Cochise Co., Chiricahua Mts.

Bandelia was proposed as the objective replacement name for Nothophila Barnes \& Lindsey, 1922.

NOTHOPHILA Barnes \& Lindsey, 1922 June, Bull. Brooklyn ent. Soc. 17: 75.

Type-species: Nothophila angulata Barnes \& Lindsey, I922, by original designation.

A junior homonym of Nothophila Alexander, 1922 May, Ann. Mag. nat. Hist. (9)9:

512, - Diptera. The objective replacement name is Bandclia Lindsey, 1923.

BANIANA Walker, I858, List Specimens lepid. Insects Colln Br. Mus. 15: I843.

OPIII

Type-species: Baniana significans Walker, 1858, ibidem 15: I843, by monotypy. Holotype ô. [Dominican Republic] St. Domingo (BMNH). 
B. significans is a junior subjective synonym of Poaphila relapsa Walker, 1858 , ibidem 15 : 1836 .

BANIOPIS Hampson, I926, Descr. new Genera Species Lepid. Phalaenae Subfamily Noctuinae Br. Mus.: 208 .

OPHI

Type-species: Baniopis pulverea Hampson, I926, ibidem: 208, by original designation. Holotype ㅇ, [GHANA] Gold Coast: Bibianaha (BMNH).

BANKIA Guenée, I852. See Deltote R. L., I8I7.

ACON

BANTANA Walker, [1863] I864, J. Proc. Linn. Soc. (Zool.) 7: 52.

SARR

Type-species: Bantana albida Walker, [1863] I864, ibidem 7: 52, by monotypy. Lectotype 9, Borneo: Sarawak (UM, Oxford), designated (as type) by Swinhoe, I9oo, Cat. east. and Aust. Lepid. Heterocera 2: 82.

BAORISA Moore, I882, in Hewitson \& Moore, Descr. new Indian lepid. Insects Colln late Mr W.S. Atkinson: I33.

OPHI

Type-species: Baorisa hieroglyphica Moore, I882, ibidem: I33, pl. 4, fig. I4, by monotypy. Type(s) §., India: [W. Bengal], Darjiling.

$\ddagger \boldsymbol{B} \boldsymbol{A P A} \boldsymbol{T} \boldsymbol{A}$, misspelling. See Baputa Walker, [1865].

OPHI

BAPTARMA Smith, 1904, Psyche, Camb. 11: 59.

NOCT

Type-species: Baptarma felicita Smith, I904, ibidem 11: 59, by monotypy. Syntypes I ${ }^{A}, 2$ ㅇ, [U.S.A.]: Arizona, Yuma County.

BAPUTA Walker, [1865] i864, List Specimens lepid. Insects Colln Br. Mus. 31: 58.

OPHI

Type-species: Baputa dimidiata Walker, [1865] I864, ibidem 31: 58, by monotypy. Lectotype $q$, New Guinea (UM, Oxford), designated (as type) by Swinhoe, I892, Cat. east. and Aust. Lepid. Heterocera 1: 95.

$\ddagger B A P A T A$; Kirby, 1892, Synonymic Cat. Lepid. Heterocera 1: 387 .

An incorrect subsequent spelling.

BARASA Walker, 1862, J. Proc. Linn. Soc. (Zool.) 6: 192.

SARR

Type-species: Barasa acronyctoides Walker, I862, ibidem 6: 192, by monotypy. Lectotype 0 . Borneo: Sarawak (UM, Oxford), designated (as type) by Swinhoe, I9oo, Cat. east. and Aust. Lepid. Heterocera 2: 83.

BARASTROTIA Warren, I913, in Seitz, Gross-Schmett. Erde 11: 29I.

$\mathrm{ACON}$

Type-species: Barasa metalophota Hampson, I898, J. Bombay nat. Hist. Soc. 11: 452, by original designation. Type(s) ô, India: [Assam], Khasis (BMNH).

The date of publication of the part of Seitz containing page 29I was stated on page 289 as I9I3 October $5^{\text {th }}$ in both the English and the German editions.

BARATHA Walker, I865, List Specimens lepid. Insects Colln Br. Mus. 33: I02I.

CATO

Type-species: Baratha acuta Walker, I865, ibidem 33: 1022, by monotypy. Holotype 9 , TYPE-LOCALITY unknown (BMNH) [PANAMA].

B. acuta is a junior subjective synonym of Remigia disseverans Walker, 1858 , ibidem 14: I 495 .

BA $\boldsymbol{R} \boldsymbol{A T H R A}$ Hübner, [I82I]. See Mamestra Ochsenheimer, I8I6.

HADE

BARBESOLA Walker, I862, J. Proc. Linn. Soc. (Zool.) 6: i 87.

OPHI

Type-species: Barbesola defixa Walker, I862, ibidem 6: I88, by monotypy. Lectotype o. Borneo: Sarawak (UM, Oxford), designated (as type) by Swinhoe, I900, Cat. east. and Aust. Lepid. Heterocera 2: 40.

BARCITA Möschler, I886, Abh. senckenb. naturforsch. Ges. 14 (3): 54.

OPHI

Type-species: Barcita muscosa Möschler, I886, ibidem $14(3): 54$, fig. I I, by monotypy. Holotype ㅇ. Jamaica (MNHU, Berlin). 
B. muscosa is a junior subjective synonym of Hadena subviridescens Walker, 1858 , List Specimens lepid. Insects Colln Br. Mus. 15: 1724 .

BAREIA Walker, I858, List Specimens lepid. Insects Colln Br. Mus. 15: I840.

OPHI

Type-species: Bareia incidens Walker, I858, ibidem 15: 1840, by monotypy. Holotype o. [South Africa]: Port Natal (BMNH).

BARIANA Walker, 1865, List Specimens lepid. Insects Colln Br. Mus. 32: 603.

SARR

Type-species: Bariana submuscosa Valker, I865, ibidem 32: 604, by monotypy. Holotype q, JAva (BMNH).

BARROVIA Barnes \& McDunnough, I9I6, Can. Ent. 48: 29 I.

NOCT

Type-species: Psychophora fasciata Skinner, 1902, Ent. News 13: 14I, by original designation. Syntypes several $\hat{\jmath}, \mathbf{I}$, , [U.S.A.]: Alaska, Point Barrow.

BARYBELA Turner, I944, Trans. R. Soc. S. Aust. 68: 7 .

ACRO

Type-species: Barybela chionostigma Turner, 1944, ibidem 68: 7 , by monotypy. Holotype q, Australia: IW. Aust., Yanchep (ANIC, Canberra).

BARYDIA Guenée, I852, in Boiscluval \& Guenée, Hist. nat. Insectes (Lépid.) 6: 409. NOTODONTIDAE

Type-species: Barydia bufo Guenée, I852, ibidem 6:4IO, pl. I8, fig. I, by monotypy.

Barydia was originally placed in the Noctuidae.

BARYPHANES Turner, 1933, Trans. Proc. R. Soc. S. Aust. 57: I67.

OPHI

Type-species: Monoctenia niphosema Lower, I908, ibidem 32: II4, by monotypy. Holotype q, Australia: W. Aust., Perth.

BASILICA Hampson, I908, Cat. Lepid. Phalaenae Br. Mus. 7: 8. Available, but without included species until Hampson, I9ro, ibidem 9: x, 209.

ACRO

Type-species: Basilodes chrysosticta Hampson, I895, Trans. ent. Soc. Lond. 1895: 306, by subsequent monotypy. Type(s) q. Ixvia: Bombay (BMNH).

Hampson, I9Io, cited the original description of the type-species as B. chrysosticta Hampson, 1896, Fauna Br. India (Moths) 4:535, but at this reference he refers back to the I 895 description.

BASILODES Guenée, I 852, in Boisduval \& Guenée, Hist. nat. Insectes (Lépid.) 6: 358. ACro Type-species: Basilodes pepita Guenée, 1852 , ibidem 6: 358 , pl. I2, fig. I, by monotypy. Holotype ô. [U.S.A.]: Florida.

See also Deobriga Walker, I 869.

BASTILLA Swinhoe, I9I8, Ann. Mag. nat. Hist. (9)2: 78.

CATO

Type-species: Ophiusa redunca Swinhoe, I900, Cat. east. and Aust. Lepid. Heterocera

2: I 4 I, by original designation. Holotype ô. Australia: Brisbane (UM, Oxford).

BATHYRA Walker, I865, List Specimens lepid. Insects Colln Br. Mus. 32: $403 . \quad$ PANT Type-species: Diphtera sagata Walker, I 856 , ibidem 9: 38 , by monotypy. Holotype, TYPE-LOCALITY not stated (BMNH) [VENEZUELA].

BATHYSTOLMA Turner, 1932, Trans. R. Soc. S. Aust. 56: 183.

OPHI

Type-species: Bathystolma brunnea Turner, 1932, ibidem 56: I83, by monotypy. Syntypes, Australia: I $\hat{\delta}, \mathrm{I}$ q, Qd, Kuranda (ANIC, Canberra); Cooktown; Yeppoon.

BATHYTRICHA Turner, I920, Trans. Proc. R. Soc. S. Aust. 44: I4 I, I $50 . \quad$ ACro

Type-species: Leucania truncata Walker, I 856, List Specimens lepid. Insects Colln Br.

Mus. 9: il i, by monotypy. Syntypes 4 ex., [Australia]: Adelaide (BMNH).

BATINA Walker, I865, List Specimens lepid. Insects Colln Br. Mins. 33: 865.

OPHI

Type-species: Batina marginalis Walker, 1865 , ibidem 33: 865, by monotypy. Holotype q. [Dominican Republic] St. Domingo. 
BATRACHARTA Walker, i 862, J. Proc. Linn. Soc. (Zool.) 6: 196.

OPHI

Type-species: Batracharta obliqua Walker, I862, ibidem 6: 196, by monotypy. Lectotype of. Borneo: Sarawak (UM, Oxford), designated (as type) by Swinhoe, I9oo, Cat. east. and Aust. Lepid. Heterocera 2: 104.

BATYMA Schaus, I906, Proc. U.S. natn. Mus. 30 : I зо.

HYPE

Type-species: Batyma onesalis Schaus, I906, ibidem 30: I30, by subsequent designation by Schaus, I9i6, Proc. U.S. natn. Mus. 50: 338. Type(s), Brazil. Parana, Castro (USNM, Washington).

BA VILIA Möschler, I880, Verh. zool.-bot. Ges. Wien 30 (Abh.) : 471.

OPHI

Type-species: Bavilia flavocostata Möschler, I880, ibidem 30 (Abh.) : 472, pl. 9, fig. 30,

by monotypy. Holotype $q$, Surinam: interior (MNHU, Berlin).

BAXAGHA Walker, I865, List Specimens lepid. Insects Colln Br. Mus. 33: 1002.

OPHI

Type-species: Baxagha serpentina Valker, I865, ibidem 33: I002, by monotypy.

Type(s) ô. [South AFrica]: Natal.

$B$. serpentina is a junior subjective synonym of Tephrias plumipes Wallengren, I860.

See also Tephrias Wallengren, I860.

${ }_{+} \boldsymbol{B} \boldsymbol{A} \boldsymbol{Y} \boldsymbol{L} \boldsymbol{E} \boldsymbol{Y} \boldsymbol{A}$, misspelling. See Baileya Grote, 1895 .

SARR

BEANA Walker, I862, J. Proc. Linn. Soc. (Zool.) 6: I83.

SARR

Type-species: Beana polychroma Walker, I862, ibidem 6: I83, by monotypy. Lectotype ô, Borneo: Sarawak (UM, Oxford), designated (as type) by Swinhoe, I9oo, Cat. east. and Aust. Lepid. Heterocera 2: I 26.

$B$. polychroma is a junior subjective synonym of Felinia terminigera Walker, I858.

See also Pitacota Moore, I 884 .

BEARA Walker, I866, List Specimens lepid. Insects Colln Br. Mus. 35: I703.

CHLO

Type-species: Beara dichromella Walker, I866, ibidem 35: 1703, by subsequent designation by Hampson, I894, Fauna Br. India (Moths) 2: 427. Holotype $q$, Type-Locality not known (BMNH) [INDIA].

BEHRENSIA Grote, I875, Can. Ent. 7: 70.

CUCU

Type-species: Behrensia conchiformis Grote, 1875, ibidem 7: 7I, by monotypy. Type(s) ô., [U.S.A.]: California (BMNH).

BEIHANIA Wiltshire, I967, J. Bombay nat. Hist. Soc. 64: 232.

OPHI

Type-species: Metoponrhis anartoides Warnecke, I937, Mitt. münch. ent. Ges. 27: 47 ,

pl. 3, fig. I, by original designation. Holotype ô. [Southern Yemen]: [Mukalla] Makalla.

BELCIADES Kozhanchikov, I950, Fauna SSSR (Insecta, Lepid.) 12: 443.

OPHI

Type-species: Habrostola niveola Motschoulsky, I866, Bull. Soc. imp. Nat. Moscou 39

(I): I95, by original designation. Type(s), Japan.

BELCIANA Walker, I 862, J. Proc. Linn. Soc. (Zool.) 6: I82.

OPHI

Type-species: Dandaca biformis Walker, I858, List Specimens lepid. Insects Colln Br.

Mus. 15: I671, by monotypy. Holotype $\hat{\sigma}$, Borneo: Sarawak (BMNH).

NALCA Walker, I866, List Specimens lepid. Insects Colln Br. Mus. 35: I983.

Proposed, unnecessarily, as an objective replacement name for Belciana Walker, I 862, which is not preoccupied. Walker may have been intending to replace Belciana Walker, [1865] I864, ibidem 31: I56, - Lepid., Geometridae.

BELLURA Walker, I865, List Specimens lepid. Insects Colln Br. Mus. 32: 465.

ACRO

Type-species: Bellura gortynoides Walker, 1865, ibidem 32: 465 , by monotypy. Holotype $q$, U.S.A. (BMNH). 
BELOSTICTA Butler, I879, Ann. Mag. nat. Hist. (5)4: 357.

CUCU

Type-species: Belosticta extensa Butler, I879, ibidem (5)4:357, by original designation. Type(s), JAPAN (BMNH).

BEMATHA Walker, 1865, List Specimens lepid. Insects Colln Br. Mus. 33: 1095.

OPHI

Type-species: Bematha extensa Walker, r865, ibidem 33: I095, by monotypy. Holotype 3. [INDIA] : N. Hindostan (BMNH).

BEN A Billberg, I820, Enumeratio Insect. Mus. G. J. Billberg: 90.

CHLO

Type-species: Phalaena prasinana Linnaeus, I758, Syst. Nat. (Edn Io) 1: 530, by subsequent designation by Tams, I939, Entomologist 72: 70. Lectotype ㅇ, TYPE-LOCALITY not stated (LS, London) [EUROPE], designated by Lempke, I947, Entomologist 80: I 29.

Great confusion has existed between the interpretations of Bena Billberg, 1820, and the distinct genus Pseudoips Hübner, I822, the type-species of which was misidentified as Phalaena prasinana Linnaeus (see Pseudoips Hübner, I822). The main synonymy is as follows:

Bena Billberg, I820

prasinana Linnaeus, $175^{8}$

(The Scarce Silver Lines)

bicolorana Fuessly, I775

quercana [Denis \& Schiffermüller], I775.
Pseudoips Hübner, I 822

fagana Fabricius, I $78 \mathrm{I}$

(The Green Silver Lines) sylvana Fabricius, 1794

prasinana Linnaeus sensu Hübner.

Bena originally included both prasinana and fagana so Billberg was aware of both species; thus the designation by Tams is of the valid prasinana Linnaeus.

See Frontispiece, fig. I, Phalaena prasinana Linnaeus, lectotype \%; fig. 2, Bena prasinana (Linnaeus), o; fig. 3, Pyralis fagana Fabricius, lectotype O+; fig. 4, Pseudoips fagana (Fabricius), 우.

See also Hylophilina Warren, I9I3.

†PSEUDOIPS Hübner, [1806], Tentamen determinationis digestionis . . . : [2].

Included in a work rejected for nomenclatural purposes by Int. Commn zool. Nom., r926, Smithson. misc. Collns 73 (4) Opinion 97: 19. Also idem, 1954, Opin. Decl. int. Commn zool. Nom. 6 Opinion 278: 1 4o.

Only included species: Tortrix quercana [Denis \& Schiffermüller], I775, a junior subjective synonym of $P$. prasinana Linnaeus, 1758 .

BENDIS Hübner, [I823] I8I6, Verz. bekannter Schmett.: 269.

OPHI

Type-species: Noctua irregularis Hübner, [1808], Samml. eur. Schmett. 4: pl. 78, fig. 36r, by subsequent designation by Grote, 1874, Bull. Buffalo Soc. nat. Sci. 2: 45. Type(s), EUROPE.

BENDISODES Hampson, 1924, Ann. Mag. nat. Hist. (9) 13: 440.

OPHI

Type-species: Isogona aeolia Druce, I890, Biologia cent.-am. (Zool.) Lepid. Heterocera 1: 390, pl. 33, fig. I, by original designation. Syntypes, Guatemala: San Gerónimo (BMNH). Panama: Chiriqui (BMNH).

BENDISOPIS Hampson, 1926, Descr. new Genera Species Lepid. Phalaenae Subfamily Noctuinae Br. Mus.: 433. OPHI

Type-species: Ephyrodes remissa Waller, I858, List Specimens lepid. Insects Colln Br. Mus. 15: 1592, by original designation. Holotype +, Venezuela (BMNH).

BENJAMINIOLA Strand, I928, Arch. Naturgesch. 92 (A) 8: 50.

ACRO

Type-species: Arsilonche colorada Smith, I900, Proc. U.S. natn. Mus. 22: 4I 4, by original designation (for Buchholzia Barnes \& Benjamin). Syntypes 2 우 [U.S.A.]: Colorado, Glenwood Springs.

Benjaminiola was proposed as the objective replacement name for Buchholzia Barnes \& Benjamin, 1926. 
BUCHHOLZIA Barnes \& Benjamin, I926, Pan-Pacif. Ent. 3: 68.

Type-species: Arsilonche colorada Smith, I900, by original designation.

A junior homonym of Buchholzia Michaelsen, I886, Arch. mikrosk. Anat. 28 (3) : 293, Vermes. The objective replacement name is Benjaminiola Strand, I928.

EUBUCHHOLZIA Barnes \& Benjamin, I929, Bull. Brooklyn ent. Soc. 24: i 84.

Proposed, unnecessarily, as an objective replacement name for Buchholzia Barnes and Benjamin, 1926. The objective replacement name Benjaminiola Strand, I928, had already been proposed.

BEREGRA Walker, 1858, List Specimens lepid. Insects Colln Br. Mus. 14: I 29I, I3 I5. OPHI Type-species: Beregra replenens Walker, I858, ibidem 14: I3 I5, by monotypy.

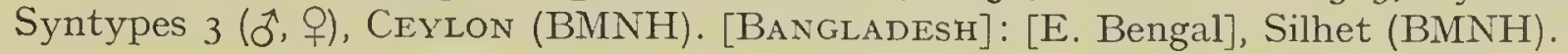

$B$. replenens is a junior subjective synonym of Evebus omma Hoeven, I 840 .

See also Cyclodes Guenée, I 852.

BERIOANA Fletcher \& Viette, I962, Bull. mens. Soc. linn. Lyon 31: 7.

ACRO

Type-species: Trachea limbulata Berio, I 955, Mem. Inst. sci. Madagascar (E)6: I I, fig. I3, by original designation. Holotype $\sigma^{\lambda}$, MADAGASCAR: central, massif de l'Ankaratra (MNHN, Paris).

BERIOHANSA nom. n. for Hansa Berio, I959.

CATO

Type-species: Remigia hansali Felder \& Rogenhofer, 1874, Reise öst. Fregatte Novara (Zool) 2 (Abt. 2): pl. I 7, fig. 5, by original designation (for Hansa Berio, I959). Type(s) ㅇ, [ETHIOPIA ]: Bogos [I $5^{\circ} 4 \mathrm{O}^{\prime}$ N. $38^{\circ} 45^{\prime}$ E.] (BMNH).

Dr E. Berio was informed of the homonymy and has permitted me to propose the replacement name.

HANSA Berio, 1959, Annali Mus. civ. Stor. nat. Giacomo Doria 71: 320.

Type-species: Remigia hansali Felder \& Rogenhofer, I 874, by original designation.

A junior homonym of Hansa Evans, 1955, Cat. Am. Hesperiidae Br. Mus. nat. Hist. 4 : 303, 370, - Lepid., Hesperiidae. The objective replacement name is Beriohansa nom. 11.

BERIOTISIA Köhler, I967, Acta zool. lilloana 21: 275.

NOCT

Type-species: Metalepsis fuegensis Hampson, I907, Ann. Mag. nat. Hist. (7) 19: 244, by original designation, but cited as ¥‘fueguensis’, an incorrect subsequent spelling. Holotype $\tilde{\delta}$, [Argentina/Chile] : Tierra del Fuego, Cheena Creek (BMNH).

BEROCYNTA Möschler, I886, Abh. senckenb. naturforsch. Ges. 14(3):61.

HYPE

Type-species: Berocynta simplex Möschler, I886, ibidem 14(3): 6I, by monotypy. Holotype , Jamaica (MNHU, Berlin).

BERRESA Walker, [1859] i 858, List Specimens lepid. Insects Colln Br. Mus. 16: 214. ACoN Type-species: Berresa natalis Walker, [1859] I 858, ibidem 16: 214, by monotypy. Syntypes 20 , CeYlon (BMNH).

BERRHAEA Walker, I858, List Specimens lepid. Insects Colln Br. Mus. 15: I 72 I. ACRO Type-species: Berrhaea aurigera Walker, I858, ibidem 15: I72I, by monotypy. Type(s) ò. [InDIA] Hindostan (UM, Oxford).

BERTULA Walker, [1859] I858, List Specimens lepid. Insects Colln Br. Mus. 16: I62. HyPE

Type-species: Bertula abjudicalis Walker, [1859] I 858, ibidem 16: I63, by original designation. Syntypes 30 , CEYLON.

BERTULANIA Strand, I9I4, Arch. Naturgesch. 80(A)2: I 42.

OPHI

Type-species: Bertulania corticea Strand, I9I4, ibidem $80(\mathrm{~A}) 2$ : I43, by original designation. Holotype $\widehat{\jmath}$, Caneroun: Dschang (DEI, Eberswalde).

BESSACTA Warren, I9I 2, Novit. zool. 19: 52.

OPHI

Type-species: Athyrma polyspila Walker, I865, List Specimens lepid. Insects Colln Br. Mus. 33: 966, by original designation. Syntypes 400 , [INDIA]: Hindostan (BMNH). [BAngLadesh]: [E. Bengal], Silhet (BMNH). 
BESSARA Walker, I862, J. Proc. Limn. Soc. (Zool.) 6: 180.

CHLO

Type-species: Bessara quadratipennis Vialker, I862, ibidem 6: I8 I, by monotypy. Lectotype ơ. Borveo: Sarawak (UM, Oxford), designated (as type) by Swinloe, I9oo, Cat. east. and Aust. Lepid. Heterocera 2: 98.

BESSULA Grote, I 88 I, Papilio 1: I 76.

NOCT

Type-species: Bessula luxa Grote, I88 I, ibidem 1: I 76, by monotypỹ. Syntypes 07, 우, [U.S.A.]: New Mexico (BMNH).

†ETONSA, misspelling. See Bucinna Walker, I 866.

OPHI

BETOUSA Walker, I865. See Bucinna Walker, I 866

OPH1

BETUSA Malker, I856, List Specimens lepid. Insects Colln Br. Wus. 9: 137, I5I. OPHI

Type-species: Betusa phasianus Walker, i 856 , ibidem 9: i jI, by monotypy. Holotype ô, [BRAzIL]: Parà (BMNH).

$B$. phasianus is a junior subjective synonym of Phalaena amynta Cramer, I 779.

See also Ceroctena Guenée, I 852.

BIAGICOLA Strand, I9I 4, Ent. Witt. 3: I.

OPII

Type-species: Biagicola signipennis Strand, I9I 4, ibidem 3: 2, fig. I, by original designation. Holotype ơ, [Australian] New Guinea: R. Mambare, Biagi (DEI, Eberswalde).

BIAREOLIFERA Strand, I915, Ent. Mitt. 4: I 65.

OPH I

Type-species: Biareolifera geometriformis Strand, I9I5, ibidem 4: I65, by original designation. Holotype ?. CAmERoun: Bonaberi (DEI, Eberswalde).

BIBACTA Moore, I882, in Hewitson \& Moore, Descr. nere Indian lepid. Insects Colln late Mr W. S. Atkinson: 197.

'Type-species: Bibacta truncata Moore, I 882, ibidem: 198, pl. 6, fig. 25, by monotypy. Syntypes ô. TyPE-LOCALITY not stated (BMNH) [INDIA].

BIFURCA Joannis, 1929, Annls Soc. ent. Fr. 98: 781.

LYMANTRIIDAE

Type-species: Bifurca longinasus Joannis, I 929, ibidem 98: 782 , by original designation. Holotype $\tilde{\sigma}$, [NoRTh VIETNAM] : Tonkin, Cha-pa (MNHN, Paris).

$B$. longinasus was originally placed in the Lymantriidae but was transferred to the Hypeninae by Collenette, 1935, Stylops 4:242. On venation and antennal characters it does, however, fit better into the Lymantriidae.

BIREGULA Saalmüller, I89I, Lepid. Madagascar : 49 I .

OPHI

Type-species: Biregula recens Salmüller, I89 I, ibidem: 491, fig. I50, by monotypy. Type(s), MADAGascar (SNG, Frankfurt)

BIRTHA Walker, I865, List Specimens lepid. Insects Colln Br. Mus. 33: 976.

OPHI

Type-species: Birtha insulata Walker, I865, ibidem 33: 976, by monotypy. Holotype o. CEYLON but probably an error; the species is known only from West Africa (BMNH).

BISTICA Dyar, I9I2, Proc. U.S. natn. Mlus. 42:67.

ACRO

Type-species: Bistica noela Dyar, I9I2, ibidem 42: 67, by original designation. Holotype f, MExico: Tehuacan (USNM, Washington).

B. noela Dyar is a junior subjective synonym of Mychonia nocla Druce, I 892, Biologia. cent.am. Zool. Lepid. Heterocera 2: 69, pl. 47, fig. 28, originally placed in the Geometridae.

BISULCIA Chapman, i890, See Craniophora Snellen, I 867.

ACRO

BITHIASA VValker, I865, List Specimens lepid. Insects Colln Br. MMs. 33: I I 6.

IIX'PL

Type-species: Bithiasa determinata Walker, 1865, ibidem 33: 11 I6, by monotypy. Holotype ô, CEYLON (BMNH). 
B. determinata is a junior subjective synonym of Bocana manifestalis Walker, [ 1859$]$.

See also Bocana Walker, [1859], ibidem 16: I70; and Lamuva Walker, [1859], ibidem 16: I 89 .

BITHIGA Walker, I865, List Specimens lepid. Insects Colln Br. Mus. 33: 832.

OPHI

Type-species: Bithiga rubrisparsa Walker, I865, ibidem 33: 832, by monotypy. Holotype ô, Venezuela (BMNH).

BIT YLA Walker, I865, List Specimens lepid. Insects Colln Br. Mus. 33: 869.

ACRO

Type-species: Bityla thoracica Walker, 1865 , ibidem 33: 869, by monotypy. Syntypes 20 . New Zealand (BMNH).

B. thoracica is a junior subjective synonym of Xylina defigurata Walker, 1865, ibidem 33: 756 .

$\ddagger B Y T I L A ;$ Warren, I913, in Seitz, Gross-Schmett. Erde 11: i I9.

An incorrect subsequent spelling in the German and in the English edition.

BIULA Walker, [1858]. See Bulia Walker, I 858.

OPHI

BLANCHARDITIA Biezanlso \& Ruffinelli, I963, Rev. Soc. urng. Ent. 5: 45. ACON

Type-species: Angitia directa Walker, 1858, List Specimens lepid. Insects Colln Br.

Mus. 15: I7 I9, by monotypy (of Angitia Walker, i 858). Type(s) ठో, [BRAzIL]: Rio Janeiro (UM, Oxford).

Blancharditia was proposed as the objective replacement name for Angitia Walker, I858. Blancharditia was also proposed in a Portuguese translation of the paper also published in 1963 in Agros Pelotas 11 (I).

ANGITIA Walker, I858 [October 9th], List Specimens lepid. Insects Colln Br. Mus. 15: I7I9.

Type-species: Angitia directa Walker, i 858 , by monotypy.

A junior homonym of Angitia Holmgren, I 858 [June 9th], K. svenska Vetens-Akad. Handl. 2(8): I06,-Hymenoptera. The objective replacement name is Blancharditia Biezanko \& Ruffinelli, I963.

BLANONA Walker, I865, List Specimens lepid. Insects Colln Br. Mus. 33: I I05. OPHI Type-species: Blanona selenisoides Walker, I865, ibidem 33: I105, by monotypy. Holotype ô. [BrazIL]: [R. Amazon], [Tefé] Ega (BMNH).

Blanona when originally proposed contained a second species, but this was doubtfully included and under the Code, Article 68(c), is not eligible for designation as type-species.

BLASTICORHINUS Butler, I893, Ann. Mag. nat. Hist. (6)12: $46 . \quad$ opHI

Type-species: Thermesia rivulosa Walker, I865, List Specimens lepid. Insects Colln Br. Mus. 33: 1060, by monotypy. Holotype $\sigma^{2}$ [INDIA]: Hindostan (BMNH).

BLEMMATIA Herrich-Schäffer, I 870, CorrespBl. zool.-min. Ver. Regensburg 24: 102, 107. HYPE

Type-species: Blemmatia gallinalis Felder \& Rogenhofer, I8 †, Reise öst. Fregatte Novara (Zool.) 2 (Abt. 2): pl. I 20, fig. 22, by PRESENT DESIGNATION. Syntypes $\hat{\sigma}$, ㅇ, Venezuela (BMNH).

This genus was made nomenclaturally available by Herrich-Schäffer without any included species. Felder \& Rogenhofer added two species, but each of them was doubtfully included in the genus. The Code does not cover such a case so both species have been taken to be eligible for designation as the type-species as both more or less fit the very brief generic description. Herrich-Schäffer, however, associated Blemmatia with 'a characteristic insect from Venezuela'. B. gallinalis has therefore been designated as type-species in preference to B. cavapa Felder \& Rogenhofer, I874, from Brazil.

BLENINA Walker, [I858] I857, List Specimens lepid. Insects Colln Br. Mus. 13: I I78, I214.

SARR

Type-species: Blenina accipiens Walker, [1 858] i 857, ibidem 13: I2 I5, by subsequent 
designation by Moore, [1885] 1887, Lepid. Ceylon 3: i29. Syntypes 7 (ðં, 9), Australia (BMNH). CEYLON.

$\ddagger$ BLEPHARA Hübner, I808. See Allotria Hübner, [1823].

CATO

¥BLEPHARA Ochsenheimer, 1816. See Catocala Schrank, I802.

CATO

Name not used again in Lepidoptera. A similar name Blepharidia Hübner, I822, has been subsequently proposed.

$\ddagger$ BLEPHARIDIA Hübner, I818. See Corisce Hübner, [1823].

CATO

BLEPHARIDIA Hübner, I 822. See Hemigeometra Haworth, 1809.

CATO

BLEPHARIDIA Püngeler, 1900. See Blepharosis Boursin, 1964.

CUCU

BLEPHARITA Hampson, 1907, Ann. Mag. nat. Hist. (7) 19: 25 I.

CUCU

Type-species: Hadena amica Treitschke, I825, Schmett. Eur. 5(1): 332, by original designation. Syntypes $\hat{\sigma}$,,, [U.S.S.R.] Russia (TM, Budapest).

BLEPHAROA Hampson, 1907, Ann. Mag. nat. Hist. (7) 19: $25 \mathrm{I}$.

NOCT

Type-species: Agrotis mamestrina Butler, i 882, Trans. ent. Soc. Lond. 1882: 1 30, by original designation. Syntypes $\hat{0},+$,, CHILI: Las Zorras (BMNH).

$\ddagger$ BLEPHARONIA Hübner, 1823. See Blepharonia [Hübner], [1825].

CATO

BLEPHARONIA [Hübner], [1825], Cat. Lépid. composent Colln feu Mr Franck: 94. CATo

Type-species: Noctua pellex Hübner, [1809], Samml. eur. Schmett. 4: pl. 92, fig. 435, by PRESENT DESIGNATION. Type(s), EUROPE.

$N$. pellex is a junior subjective synonym of Phalaena puerpera Giorna, 179I, Cal. Ent.: 104 .

$N$. pellex has been designated the type-species as in addition to being the first of the five originally included species, it is congeneric with the type-species of Catocala Schrank, I 802. Blepharonia thus becomes a synonym and will cause no further confusion in the complex usage of the similar generic names Blephara, Blepharidia and Blepharum, all proposed by Hübner.

See also Eunetis Hübner, 1823.

$\ddagger$ BLEPHARONIA Hübner, 1823, Zuträge Samml. exot. Schmett. 2: 26, 33 .

Not nomenclaturally available as a genus-group name. Hübner used Blepharonia, on both pages, in a suprageneric sense for one of the names of his tribes (Namen der Stämme), listed on page 33. Hübner listed lis genus-group names on page 35 .

BLEPHAROSIS Boursin, 1964, Lepid. Dt. Nepal-Exped. 2: 34.

CUCU

Type-species: Heliophobus lama Püngeler, 1900, Dt. ent. Z. Iris 12: 293, pl. 9, fig. 8, by original designation (for Blepharidia Püngeler). Syntypes $2 \hat{\sigma}, 2$ 우, [CHrna]: [Tsinghai], Kuku-Noor (MNHU, Berlin).

Blepharosis was proposed as the objective replacement name for Blepharidia Püngeler, Igoo.

BLEPHARIDIA Püngeler, 1900, Dt. ent. Z. Iris 13: I 22.

Type-species: Heliophobus lama Püngeler, I900, by original designation.

A junior homonym of Blepharidia Hübner, I822,-Lepid., Noctuidae. The objective replacement name is Blepharosis Boursin, 1964.

Blepharidia Püngeler has been placed on the Official Index of Rejected and Invalid Generic Names in Zoology: Name No. I83 I.

$\ddagger$ \$LEPHARUM Hübner, [1806]. See Catocala Schrank, I802.

CATO

$\ddagger$ BLEPHARUM Hübner, I808. See Corisce Hübner, [1823].

CATO

Name not used again in Lepidoptera. A similar name Blepharidia Hübner, I822, has been subsequently proposed. 
BLEPTINA Guenée, 1854, in Boisduval \& Guenée, Hist. nat. Insectes (Lépid) 8: $66 . \quad$ HYPE Type-species: Bleptina caradrinalis Guenée, 1854 , ibidem $8: 67$, by subsequent designation by Grote, I874, Bull. Buffalo Soc. nat. Sci. 2: 50. Syntypes I ô, I q, NorTH AMERICA.

BLEPTINODES Hampson, 1925, Ann. Mag. nat. Hist. (9) 15: 409.

HYPE

Type-species: Nodaria perumbrosa Hampson, 1898, J. Bombay nat. Hist. Soc. 11: 701 , by original designation. Holotype, India: [Assam], Khasis (BMNH).

BLEPTIPHORA Schaus, I916, Proc. U.S. natn. Mus. 50: 27 I.

HYPE

Type-species: Bleptiphora laurentia Schaus, I916, ibidem 50: 272, by original designation. Type(s) 우, [FRench Guiana]: R. Maroni, St. Laurent (USNM, Washington).

BLOSYRIS Hübner, [1822], Samml. exot. Schmett. 2: pl. [2II].

OPHI

Type-species: Blosyris opigena Drury sensu Hübner, [1822], ibidem 2: pl. [2II], figs I-4, [=Brujas malitiosa Guenée, 1852, in Boisduval \& Guenée, Hist. nat. Insectes (Lépid.) 7: I40], by monotypy. Type(s) of malitiosa, BRAzIL (BMNH).

Guenée loc. cit. proposed his new species malitiosa and in its synonymy placed opigena Drury sensu Hübner, [1822], remarking that Hübner had mistaken this species for Phalaena opigena Drury, I773, Illust. nat. Hist. 2: 39, Index [9I], pl. 22, fig. 4.

In Hübner, [1823], Vevz. bekannter Schmett.: 273 under Blosyris four species were listed including '2697. B. opigena Drur. II. [pl] 22.4. Hübn. Asc. und. opigena' thus showing that Hübner regarded the species he figured as being conspecific with that figured by Drury. I have compared the figures in the two works together with specimens in BMNH and agree with Guenée that there are two distinct species. This is a case of a misidentified typespecies which under the Code, Article 70, should be referred to the Commission.

BRUJAS Guenée, 1852, in Boisduval \& Guenée, Hist. nat. Insectes (Lépid.) 7: 139.

Type-species: Brujas malitiosa Guenée, 1852, by subsequent designation by Hampson, I926, Descr. new Genera Species Lepid. Phalaenae Subfamily Noctuinae Br. Mus.: I 20.

Brifjas is a junior objective synonym of Blosyris Hübner, [1822].

$\ddagger B R U N J I A$; Pagenstecher, I909, Geogr. Verbreitung Schmett.: 424 .

An incorrect subsequent spelling.

BOALDA Schaus, 1929, Proc. ent. Soc. Wash. 31: 49.

HYPE

Type-species: Boalda gyona Schaus, I929, ibidem 31: 49, pl. 4, fig. 27, by original designation. Type(s) ô, Brazil: Santa Catharina (USNM, Washington).

BOANA Walker, I862. See Naboa nom.n.

HYPE

BOCANA Walker, [1859] I858, List Specimens lepid. Insects Colln Br. Mus. 16: I70. HYPE Type-species: Bocana manifestalis Walker, [1859] I 858, ibidem 16: I7I, by original designation. Holotype , CEYLON (BMNH).

See also Lamura Walker, [1859], ibidem 16: I89; and Bithiasa Walker, I865.

BOCANA Walker, [1865]. See Coria Walker, I866.

CATO

BOCULA Guenée, I 852, in Boisduval \& Guenée, Hist. nat. Insectes (Lépid.) 7: 295. OPHI Type-species: Bocula caradrinoides Guenée, 1852, ibidem 7: 296, by monotypy. Holotype of. Java (BMNH).

$\ddagger$ BOECULA, misspelling. See Baecula Walker, 1856 .

OPHI

BOETHANTHA Walker, r865, List Specimens lepid. Insects Colln Br. Mus. 33: 982. OPHI Type-species: Boethantha bisignata Walker, 1865, ibidem 33: 983 , by monotypy. Lectotype ô. Timor [I.] (UM, Oxford), designated (as type) by Swinhoe, I900, Cat. east. and Aust. Lepid. Heterocera 2: I86.

BOLETOBIA Boisduval, I840. See Parascotia Hübner, [I825].

OPHI 
BOLICA Vialker, I862, Trans. ent. Soc. Lond. (3) 1: I I 7 . OPHI

Type-species: Bolica armata VValker, I862, ibidem (3) 1: I I 8, by monotypy. Type(s) o., Brazil (UM, Oxford).

BOLINA Duponchel, [I8+5]. See Aleucanitis IVarren, I9I3. OPHI †BOLINA Walker, [1858]. See Aleucanitis Warren, I9I 3 . OPH I BOLITOBIA Agassiz, I846. See Parascotia Hübner, [I825]. OPHI

BOMBOTELIA Hampson, i912, Cat. Lepid. Phalaenae Br. Mus. 11: vii, 6. EuTE Type-species: Penicillaria jocosatrix Guenéc, 1852, in Boisduval \& Guenée, Hist. nat. Insectes (Lépid.) 6: 304, by original designation. Lectotype q, JAVA (MNHN, Paris), designated by Viette, I95I, Bull. mens. Soc. linn. Lyon 20: I6I.

+BOMBYCIA Hübner, [1806]. Sec Tethea Ochsenheimer, I 8 I6.

THYATIRIDAE BOMBYCIA Hübner, I 822. See Tethea Ochsenhcimsr, I8I6. THYATIRIDAE

BOMBYCIA Hübner sensu Stephens, I 829. See Iteophaga Boursin, I965.

CUCU

†BOMBYCIAE Ochsenheimer, I 8 I6. See Tethea Ochsenheimer, i 8 i6.

THYATIRIDAE

BOMBYCIELLA Draudt, I950, Mitt. mïnch. ent. Ges. 40:63.

CUC U

Type-species: Bombyciella talpa Draudt, I950, ibidem 40:63, pl. 4, fig. 19, by original designation. Syntypes, China: [Shensi Prov.], Tai-pei-shan (MAK, Bonn).

BOMBYX I.innaeus, I758, Syst. Nat. (Edn Io) 1 : 495.

BOMBYCIDAE

Type-species: Phalaena mori Linnaeus, I 758, ibidem 1 : 499, by subsequent designation by Int. Commn zool. Nom., 1957, Opin. Decl. int. Commn zool. Nom. 15 Opinion 450: 254.

Bombyx was placed on the Official List of Generic Names in Zoology: Name No. Io56.

Bombyx was originally proposed as a subgenus of Phalaena Linnaeus, i758. Many species of Noctuidae have been originally described in Bombyx.

BOMOLOCHA Hübner, [1825] I816, Verz. bekannter Schmett.: 343.

HYPE

Type-species: Phalaena crassalis Fabricius, I 787, Mantissa Insect. 2: 217 , by subsequent designation by Grote, I874, Bull. Buffalo Soc. nat. Sci. 2: 5 I. Type(s), Austria (UZM, Copenliagen).

BONABERIANA Strand, I9 I 5, Ent. Mitt. 4: I6o.

OPHI

Type-species: Bonaberiana crassisquama Strand, I9I5, ibidem 4: I6I, fig. 3, by original designation. Type(s) $\tilde{\jmath}$. Cameroun: Bonaberi (DEI, Eberswalde).

BONONIA Walker, I862, Trans. ent. Soc. Lond. (3) 1 : I I .

ACRO

Type-species: Bononia niveilinea Walker, I862, ibidem (3) 1: I I , by monotypy. Type(s) ô, BrazIL (UM, Oxford).

B. niveilinea is a junior subjective synonym of Licha undilinealis Walker, 1859.

Sec also Licha Walker, I859; and Tendarba Walker, [I866].

†BORALIA, misspelling. See Borolia Moore, $188 \mathrm{I}$.

HADE

BORBOTANA Walker, I 858, List Specimens lepid. Insects Colln Br. Mus. 15: i65I. ACRO Type-species: Borbotana nivifascia Valker, I 858, ibidem 15: I65I, by monotypy. Holotype $\hat{0}$, Bornio: Sarawak (BMNH).

See also Choluata Wallier, [1863].

BORISIA Filipjev, I949. See Sirioba nom. 11.

CUCU

BOROLIA Moore, t88 I March, Proc.zool. Soc. Lond. 1881: 334.

HADE

Iype-species: Borolia fasciata Moore, I 88 I March, ibidem 1881: 334, pl. 37, fig. I2, by monotypy. Syntypes ơ, q., [INDIA]: [W. Bengal], Darjiling (BMNH). 
Incorrect type-species designation: $\ddagger$ Borolia furcifera Moore, I88I March (nomen mudum), by original designation of Moore. This name was not nomenclaturally available until Moore, I88I November, in Descr. new Indian lepid. Insects Colln late Mr W. S. Atkinson: 98. The other originally included nominal species must therefore be the type-species.

$\ddagger$ BORALIA; Wileman, igा 2, Entomologist 45: I47.

An incorrect subsequent spelling.

BORSANIA Köhler, (1952) I95I, Acta zool. lilloana 12: 14I.

CUCU

Type-species: Borsania mendozina Köhler, (I952) 195I, ibidem 12: I4I, by original designation. Syntypes $\hat{\sigma}, q$, Argentina: [Mendoza Prov.], Mendoza (ZSBS, Munich).

BORSIPPA Walker, I858, List Specimens lepid. Insects Colln Br. Mus. 15: 1756.

OPHI

Type-species: Borsippa quadrilineata Walker, 1858, ibidem 15: 1756, by monotypy. Syntypes $20 \hat{0}$, BoRneo: Sarawak (BMNH).

BORYZA Walker, 1858, List Specimens lepid. Insects Colln Br. Mus. 15: 1655.

OPHI

Type-species: Boryza commiscens Walker, I858, ibidem 15: I656, by monotypy. Holotype $\hat{\sigma}$. [BraziL]: Parà (BMNH).

BORYZOLA Hampson, 1926, Descr. new Genera Species Lepid. Phalaenae Subfamily Noctuinae Br. Mus.: 46 .

OPHI

Type-species: Polia lorina Druce, I89o, Proc. zool. Soc. Lond. 1890: 5I 5, by original designation. Type(s), Mexico: Presidio (BMNH).

$P$. lorina is a junior subjective synonym of Orodesma apicina Herrich-Schäffer, I868.

See also Orodesma Herrich-Schäffer, 1868.

BORYZOPS Richards, I936, Revta Ent., Rio de J. 6: 369.

OPH

Type-species: Iscadia purissima Dyar, I910, Proc. U.S. natn. Mus. 38: 252, by original designation. Holotype 9 , Mexico: Vera Cruz state, Misantla (USNM, Washington).

BOSTRODES Hampson, I910, Cat. Lepid. Phalaenae Br. Mus. 10: xi, 280.

$\operatorname{ACON}$

Type-species: Bostrodes proleuca Hampson r9 Io, ibidem 10: 280, fig. 82, by original designation. Syntypes $7 \hat{0}, 5$ ㅇ, Java (BMNH).

BOSTRYCHARIA Schaus, 1916, Proc. U.S. natn. Mus. 50: 395.

HYPE

Type-species: Phaeochlaena cuprea Schaus, I906, Proc. U.S. natn. Mus. 30: Iro, by original designation. Holotype, BRAzIL: São Paulo (USNM, Washington).

BOTIS Swainson, I821. See Botys Latreille, I802.

PYRALIDAE

BOTYS Latreille, [1802], in Sonnini's Buffon, Hist. nat. générale particulière Crustacés Insectes 3: 4I 4 . PYRALIDAE

Type-species: Phalaena punctalis Fabricius, 1775, Syst. Ent.: 644, by subsequent designation by Stephens, r834, Illustr. Br. Ent. (Haustellata) $4: 46$, but cited as 'erigatus Fab.'

Crambus evigatus Fabricius, I798, Ent. Syst (Suppl.): 469, one of the nominal species originally included in Botys, was an unjustified emendation of Phalaena erigalis Fabricius, I 794, Ent. Syst. 3(2): 237. The latter was an objective replacement name for Phalaena punctalis Fabricius.

Botys has been used as the original generic name for species which are now in the Noctuidae.

BOTIS Swainson, I82I, Zool. Illust. (1)2: pl. 77.

An unjustified emendation of Boty's Latreille, I802.

Botis has been used as the original generic name for species which are now in the Noctuidae.

BOUDA Dyar, 1918, Proc. U.S. natn. Mus. 54: 347.

ACRO

Type-species: Bouda pallipars Dyar, I9I8, ibidem 54:347, by original designation. Holotype $\widehat{\jmath}$, Mexico: Mexico City (USNM, Washington). 
BOURSINANIA Rungs, I955, Mem. Inst. scient. Madagascar (E) 6: $76 . \quad$ HADE Type-species: Leucania insulicola Guenée, I852, in Boisduval \& Guenée, Hist. nat. Insectes (Lépid.) 5: 82, by monotypy. Lectotype q, [Mauritrus] Ile de France (MNHN, Paris), designated by Viette, I95 I, Bull. mens. Soc. linn. Lyon 20: I 59.

Boursinania was originally described as a subgenus of Leucania Ochsenheimer.

BOURSINIA Brandt, I938, Ent. Rdsch. 55: 518.

ACRO

Type-species: Boursinia symmicta Brandt, I938, ibidem 55:5 I9, by original designation. Syntypes ô, ㅇ, Iran: Keredj (NR, Stockholm).

BOURSINIDIA Köhler, I953, Revta Soc.ent. Argent. 16: I7. NOCT

Type-species: Boursinidia petrowskyi Köhler, I953, ibidem 16: I 7, figs 2, 3, by original designation. Syntypes $\widehat{\sigma}$,, Argentrna: Nahuel Huapi; Neuquén, San Martén de los Andes (ZSBS, Munich).

$B$. petrowskyi is a junior subjective synonym of Orthosia darwini Staudinger, I 899 Ergebn. Hamb. Magalhaensischen Sammelreise 4 (7): 74, fig. 24.

BRABANTIA Dyar, I9I3, Insecutor Inscit. menstr. 1: 60.

SARR,

Type-species: Pseudacontia rhizoleuca Brabant, I912, Bull. Soc. ent. Fr. 1912:330, by monotypy. Syntypes, French Guiana: St. Laurent; St. Jean du Maroni.

$+B R A T A N I A$; Neave, 1939, Nomencl. zool. 1: 483.

An incorrect subsequent spelling of Brabantia Dyar, I9I3. Neave cited 'Bratania Dyar, I9I4 [sic], Insecutor Inscit. menst. 1: 60', but at this reference Brabantia Dyar is proposed. Neave omitted this latter name.

BRACHARTHRON Hampson, I89I, Illust. typical Specimens Lepid. Heterocera Colln Br. Mus. 8: 25, 99.

HYPE

Type-species: Bracharthron maculapex Hampson, I89 I, ibidem 8: 25, 99, pl. I48, figs 4, I I, by monotypy. Syntypes $\sigma$, , I, InDIA: Nilgiri dist., S. slopes (BMNH).

B. maculapex is a junior subjective synonym of Lysimelia neleusalis IValker, [I859].

See also Lysimelia IValker, [1859].

BRACHIONYCHA Hübner, [1819] I8I6, Vevz. bekannter Schmett.: I 44.

CUCU

Type-species: Bomby:x nubeculosa Esper, I785, Die Schmett. 3: 248, pl. 48 [not 68], fig. 6, by subsequent designation by Hampson, I906, Cat. Lepid. Phalaenae Br. Miss. 6: 202. Type(s) $\hat{\jmath}$, [GERMANY].

+ BRACHIONYX; Meigen, I832, Syst. Beschreibung Eur. Schmett. 3 (4): I 78.

An incorrect subsequent spelling.

BRACHYONYCIIA Agassiz, I846, Nomencl. zool. (Index univl.): 50, 5 I.

An unjustified emendation of Brachionycha Hübner, [18I9].

SELENOSCOPUS Heinemann, I 859, Schmett. Dtl. Schweiz 1: 445.

Type-species: Bombyx nubeculosa Esper, I785, by monotypy.

Selenoscopus is a junior objective synonym of Brachionycha Hübner, [I8I9].

\pm BRACHYONIX; Berio, I966, Annali Mus. civ. Stor. nat. Giacomo Doria 76: 5I.

An incorrect subsequent spelling of Meigen's misspelling.

$\ddagger$ ¿RACHIONYX, misspelling. See Brachionycha Hübner, [ 8 I9].

CUCU

BRACHYBLEMMA Hampson, 1926. See Habershonia nom. 1.

OPHI

BRACHYCOSMIA Butler, I89o, Trans. ent. Soc. Lond. 1890: 680.

CUCU

Type-species: Noctua ambusta [Denis \& Schiffermüller], I775, Ankiindung syst.

Werkes Schmett. Wienergegend: 88, by monotypy. Type(s), [Austria]: Vienna district

(Collection destroyed, Horn \& Kahle, I936, Ent. Beih. Berl.-Dahlem 3: 243).

See also Atethmia Hiibler, [182I].

BRACHYCOSMIA Hampson, I906. See Lemmeria Barnes \& Benjamin, I926. 
BRACHYCYTTARA Turner, I933, Trans. R. Soc. S. Aust. 57 : I65.

OPHI

Type-species: Brachycyttara crypsipyrrha Turner, I933, ibidem 57: 165, by monotypy. Holotype 0 , Australia: N.S.W., Bourke (AM, Sydney).

BRACHYGALEA Hampson, I906, Cat. Lepid. Phalaenae Br. Mus. 6: 2, 8.

CUCU

Type-species: Brachygalea leucorhabda Hampson, I906, ibidem 6: 8, fig. 3, by original designation. Syntypes $2 \hat{0}, 2$ 우, Algeria: Biskra (BMNH).

BRACHYHERCA Hampson, 1926, Descr. new Genera Species Lepid. Phalaenae Subfamily Noctuinae Br. Mus.: 527.

OPHI

Type-species: Brachyherca saphobasis Hampson, 1926, ibidem: 527, by original designation. Syntypes 30 , Cameroun: R. Ja, Bitje (BMNH).

BRACHYLOMIA Hampson, I906, Cat. Lepid. Phalaenae Br. Mus. 6: x, 220.

CUCU

Type-species: Cleoceris populi Strecker, I898, Lepid. Rhopaloceres \& Heteroceres, indigenous \& exotic. Suppl. 1: 8, by original designation. Holotype, [U.S.A.]: Colorado, Loveland.

$\ddagger B R A C H Y M A S T I X$ Hampson, no published reference found.

HYPE

BRACHYONA Hampson, I926, Descr. new Genera Species Lepid. Phalaenae Subfamily Noctuinae Br. Mus.: 84 .

OPHI

Type-species: Brachyona xylodesma Hampson, I926, ibidem: 85 , by original designation. Syntypes I $\sigma^{\lambda}, I$ ㅇ, Australia: W. Aust., R. Sherlock (BMNH).

BRACHYONIX, misspelling. See Brachionycha Hübner, [I8I9].

CUCU

BRACHYONYCHA Agassiz, I846. See Brachionycha Hübner, [1819].

CUCU

BRACHYPTERAGROTIS Viette, I959, Bull. Soc. ent. Fr. 64: 25.

NOCT

Type-species: Brachypteragrotis patricei Viette, I959, ibidem 64: 25, figs 2-5, by original designation. Holotype ${ }^{\star}$, INdian OcEan: Amsterdam I. (MNHN, Paris).

BRACHYTAENIA Stephens, I852, List Specimens Br. Anim. Colln Br. Mus. 10: 25.

Type-species: Tortrix semifasciana Haworth, I8I I, Lepid. Br. (3): 43I, by subsequent designation by Fernald, I908, Genera Tortricidae: 35 .

Brachytaenia has been used as the original generic name for species which are now in the Noctuidae.

BRACHYTEGMA Berio, I962, Annali Mus. civ. Stor. nat. Giacomo Doria 73: 202.

NOCT

Type-species: Brachytegma rotunda Berio, I962, ibidem 73: 202, fig. 6, by original designation. Holotype $\hat{\sigma},\left[Z_{A I R E}\right]$ : [Equateur], Duma (MRAC, Tervuren).

BRACHYXANTHIA Butler, I878, Ann. Mag. nat. Hist. (5)1 : I69.

ACRO

Type-species: Brachyxanthia peculiaris Butler, I878, ibidem (5) 1: I69, by original designation. Syntypes $\sigma^{7}$, ㅇ․, JAPAN: Yolkohama (BMNH); Hakodaté (BMNH).

$B$. peculiaris is a junior subjective synonym of Xanthia zelotypa Lederer, I853, Verh. zool.-bot. Ver. Wien 3: 373, pl. 3, fig. 4.

BRADA Walker, I858. See Aiteta Walker, I 856 .

CHLO

BRADUNIA Schaus, I9I6, Proc. U.S. natn. Mus. 50: 297.

OPHI

Type-species: Bradunia improba Schaus, I9I6, ibidem 50: 298, by original designation. Syntypes $\sigma^{\lambda}$, 우, FRENCH GuIANA: Cayenne (USNM, Washington).

BRANA Walker, [I858] I857, List Specimens lepid. Insects Colln Br. Mus. 13: I079, I099. opHI Type-species: Brana calopasa Walker, [I858] I857, ibidem 13: I Ioo, by monotypy. Syntypes 2 ex., CEYLON (BMNH). 
BRANDTINA Bytinski-Salz \& Brandt, I937, Entomologist's Rec.J.Var. 49(9): (I I). PANT Type-species: Brandtina albonigra Bytinski-Salz \& Brandt, I937, ibidem 49 (9): (I I), by monotypy. Holotype ô. [IRAN] Persia: Nissa $2300 \mathrm{~m}$.

$\ddagger$ ¿RATANIA, misspelling. See Brabantia Dyar, I9I3.

SARR

$\ddagger$ BREPHA Hübner, [1806]. See Archiearis Hübner, [1 823$]$.

GEOMETRIDAE

BREPHA Hübner, [1813]. See Brephos Hübner, [1813].

AGAR

BREPHA Billberg, I 820. See Brephos Hübner, [1813].

AGAR

$\ddagger$ BREPHIA Hübner, I8I8. See Brephos Hübner, [18I3].

AGAR

BREPHIA Hübner, I 822. See Brephos Hübner, [I813].

AGAR

$\ddagger$ BREPHOS Hübner, [1806]. See Avchiearis Hübner, [1823].

GEOMETRIDAE

BREPHOS Hübner, [1813], Samml. exot. Schmett. 1 : pl. [198]. AGAR

Type-species: Phalaena julia Cramer, I775, Uitlandsche Liapellen 1: I I, pl. 7, figs E, F,

by monotypy. Syntypes $\hat{\sigma}, q$, [South AFricA] : Cape of Good Hope.

+ BREPHA Hübner, [18r3], Samml. exct. Schmett. 1: pl. [198].

Used as the plural form of Brephos Hübner, [r8r3].

$\ddagger$ BREPHIA Hübner, I818, Zuträge Samml. exot. Schmett. 1:28, 33.

Brephia is not nomenclaturally available for use from i 818 as a genus-group name as

Hübner used it, on both pages, in a suprageneric sense for the name of a tribe (Namen

der Stamme) as listed on page 33. Hübner listed his genus-group names on page 35.

BREPHA Billberg, I820, Enumeratio Insect. Mus. G. J. Billberg: 86.

Also used by Curtis, I 826, Br. Ent. 3: 121.

An unjustified emendation of Brephos Hibner, [18I3].

BREPHIA II übner, i 822 , Syst.-alphab. Verz.: 3I, 32, 36.

An unjustified emendation of Brephos Hiibner, [18I3].

BREVIPECTEN Hampson, I 894, Fauna Br. India (Moths) 2: xvi, 36I.

OPHI

Type-species: Euclidia captata Butler, I 889, Illust. typical Specimens Lepid. Heteroceva Colln Br. Mus. 7: 1 5, 79, pl, I 33, fig. I, by original designation. Syntypes, [India]: [Punjab], Liangra (BMNH).

$\ddagger \boldsymbol{B R I A D A}$, misspelling. See Briarda Walker, [1858].

OPHI

BRIARDA Walker, [ I 858] i 857, List Specimens lepid. Insect. Colln Br. Mus. 13: I079, io97. Opн I Type-species: Briarda precedens Walker, [1858] I 857, ibidem 13: I098, by subsequent designation by Noore, [1885] i 887, Lepid. Ceylon 3: 98, but cited for $\ddagger$ Briada, an incorrect original spelling. Syntypes 4 9 , CEYLON (BMNH). [INDIA]: North Hindostan.

Briarda was the spelling used in the Key to Genera on page 1079, and for three new species on pages I098-I099; $\$$ Briada, used as the heading, was an inadvertent error. Walker himself continued to use Briarda, e.g. I 865, ibidem 33: 894 .

See also Ansa Walker, 1858 ; and Erygansa Bethune-Baker, I go6.

$\ddagger$ \$RIADA; Walker, [1858] I857, List Specimens lepid. Insects Colln Br. Mus. 13: 1097.

An incorrect original spelling.

BRITHA Walker, [1 866] i 865, List Specimens lepid. Insects Colln Br. Mus. 34: I I 46. HyPE Type-species: Britha biguttata Walker, [I 866] 1865, ibidem 34: I I 47, by monotypy. Holotype ô. [Australia]: [Qd], Moreton Bay.

See also Hyposemeia Hampson, I 893.

BRITHODES Bethune-Baker, I go8, Novit. zool. 15: 237.

HYPE

Type-species: Brithodes quadrilineata Bethune-13aker, I908, ibidem 15: 238, by monotypy. Syntypes $\overrightarrow{0}$,, , NEw Guinea: [Papua], Dinawa (BMNH). 
Incorrect type-species: $\ddagger$ Brithodes tetralinea Bethune-Baker, designated in the original description of Brithodes. No species of this name has been described by Bethune-Baker, and its use was an inadvertent error for $B$. quadrilineata.

$\ddagger \boldsymbol{B R I T H Y A}$, misspelling. See Brithys Hübner, [I82 I] .

HADE

BRITHYS Hübner, [182I] I816, Verz. bekannter Schmett.: 226.

HADE

Type-species: Noctua pancratii Cyrillo, 1787, Entomologiae Neapolitanae: pl. I2, fig. 4, by subsequent designation by Blanchard, 1840 , in Castelnau, Hist. nat. Anim. articulés (Insectes) 3: 505, but cited as ' $N$. pancratii Hübn.', an incorrect authorship, for $\ddagger$ Brithya, an incorrect subsequent spelling. Type(s), [ITALY]: [Naples].

COCYTIA Treitschke, I835, Schmett. Eur. 10 (2): 29.

Type-species: Noctua pancratii Cyrillo, 1787 , by subsequent designation by Guenée, I 852, for Glottula, Guenée, 1837.

A junior homonym of Cocytia Boisduval, 1828, Essai Monogr. Zygénides: 22, - Lepid., Cocytiidae. The objective replacement name is Brithys Hübner, [182I].

GLOTTULA Guenée, I837, Annls Soc. ent. Fr. 6: 359.

Type-species: Noctua pancratii Cyrillo, 1787 , by subsequent designation by Guenée, 1852, in Boisduval \& Guenée, Hist. nat. Insectes (Lépid.) 5: II 5.

Glottula was proposed as the objective replacement name for Cocytia Treitschke, 1835 , and is also a junior objective synonym of Brithys Hübner, [182I].

$\ddagger$ BRITHYA; Boisduval, r840, Genera Index meth. Eur. Lepid.: II 7 .

An incorrect subsequent spelling.

$\ddagger$ BRYTHIS; Stephens, 1850, List Specimens Br. Anim. Coln Br. Mus. 5: 282.

An incorrect subsequent spelling.

$\ddagger$ \$RYTIS; Ragusa, I885, Naturalista sicil. 4: 272.

An incorrect subsequent spelling.

BRITHYSANA Viette, [1963] 1962, Annls Soc. ent. Fr. 131: 238.

HADE

Type-species: Polia maura Saalmüller, I89 I, Lepid. Madagascar: 308, pl. I3, fig. 235, by original designation. Holotype $\precsim$, MADAGascar (MNHU, Berlin).

BRONTYPENA Holland, I90o, Novit. zool. 7 : 576.

OPHI

Type-species: Hypena eximia Pagenstecher, I886, Corresp.-Bl. ent. Ver. Iris 1: 43, pl. 2, fig. 8, by original designation. Type(s) $\hat{\jmath}$, [Moluccas]: Ceram I. (SM, Wiesbaden).

BROTIS Hübner, [1821] I816, Verz. bekannter Schmett.: 226.

NOCT

Type-species: Noctua fumosa [Denis \& Schiffermüller], I775, Ankïndıng syst. Werkes Schmett. Wienergegend: 8r, by subsequent designation by Hampson, r903, Cat. Lepid. Phalaenae Br. Mus. 4: I53, but cited as 'nigricans Linn.' which Hampson placed (on page 249 ) as the senior synonym of fumosa. Type(s), [Austria]: Vienna district (Collection destroyed, Horn \& Kahle, 1936, Ent. Beih. Berl.-Dahlem 3: 243).

$N$. fumosa is a junior subjective synonym of Phalaena nigricans Linnaeus, 176r, Fauna Suecica (Edn 2): 322 .

BROTOLOMIA Lederer, 1857. See Phlogophora Treitschke, I825.

ACRO

BRUJAS Guenée, I852. See Blosyris Hübner, [1822].

OPHI

$\ddagger \boldsymbol{B R} \boldsymbol{U} \boldsymbol{N} \boldsymbol{J I} \boldsymbol{A}$, misspelling. See Blosyris Hübner, [1822].

OPHI

BRYOCODIA Hampson, I9ro, Cat. Lepid. Phalaenae Br. Mus. 10: xiv, 476.

$\operatorname{ACON}$

Type-species: Jaspidia lepidula Grote, 1874, Annual Rep. Trustees Peabody Acad. Sci.

6: 23 , by original designation. Syntypes $\hat{\jmath}$, [U.S.A.]: Maine (BMNH); New York (BMNH); Penn. (BMNH).

BR YOGRAMMA Schaus, I9I I, Ann. Mag. nat. Hist. (8)8: 1o6.

ACRO

Type-species: Bryogramma sisera Schaus, I9 I I, ibidem (8)8; Io6, by original designation.

Type(s) ㅇ, Costa Rica: Volcano Poas (USNM, Washington).

G 
BRYOLEUCA Hampson, I908, Cat. Lepid. Phalaenae Br. Mus. 7: xv, 686.

ACRO

Type-species: Miana trilinea Bethune-Baker, I894, Trans. ent. Soc. Lond. 1894: 4I, pl. I, fig. Io [this fig. was omitted from the plate], by original designation. Type(s), EGyPT: Alexandria (BMNH).

BR YOL YMNIA Hampson, I9o8, Cat. Lepid. Phalaenae Br. Mus. 7: ro. Available but without included species until Hampson, I9ro, ibidem 9: viii, Ir9.

ACRO

Type-species: Dacira roma Druce, I894, Ann. Mag. nat. Hist. (6)13:36I, by subsequent designation by Hampson, igro, ibidem 9: r19. Syntypes, Mexico: Jalapa (BMNH). Guatemala: Guatemala City (BMNH).

BRYOMIMA Staudinger, I900, Dt. ent. Z. Iris. 12: 357 .

CUCU

Type-species: Bryomima carducha Staudinger, I90o, ibidem 12: 357, pl. 6, fig. 9, by monotypy. Syntypes $2 \hat{0}, 2$ ㅇ, [Turkey]: N. Mesopotamia, Mardin (MNHU, Berlin).

BRYOMIMA Turner, 1902. See Trissernis Meyrick, 1902.

$\operatorname{ACON}$

BRYOMOEA Hampson, I908. See Bryomoia Staudinger, I892. ACro

BRYOMOIA Staudinger, I892, in Romanoff, Mém. Lépid. 6: 397.

ACRO

Type-species: Bryophila melachlora Staudinger, I892, ibidem 6: 397, pl. 5, fig. IO, by monotypy. Holotype , [U.S.S.R.]: Amur district, Askold (MNHU, Berlin).

Bryomoia was proposed as a subgenus of Bryophila Treitschke, 1825 .

BRYOMOEA Hampson, I908, Cat. Lepid. Phalaenae Br. MIus. 7: 6I 5.

An unjustified emendation of Bryomoia Staudinger, 1892.

BRYONOLA Toulgoët, I955, Revue fr. Ent. 22: $20 \mathrm{I}$.

ACRO

Type-species: Nola parmelia Toulgoët, 1954, Mem. Inst. scient. Madagascar (E)5: I76, fig. 7, pl. 9, fig. 6, by original designation. Holotype 0 , MADAGaScar: massif de l'Ankaratra, Manjakatompo r850 m (MNHN, Paris).

BRYONYCTA Boursin, I955, Bull.mens. Soc. linn. Lyon 24: 220.

ACRO

Type-species: Bryophila pineti Staudinger, I859, Stettin ent. Ztg 20: 212, by original designation. Holotype ${ }^{\Im},[$ SPAIN] : Andalusia, Chiclana (MNHU, Berlin).

BRYOPHILA Treitschke, I 825, Schmett. Eur. 5(I): 57.

ACRO

Type-species: Noctua perla [Denis \& Schiffermüller], I775, Ankundïng syst. Werkes Schmett. Wienergegend: 70, by subsequent designation by Westwood, 1840, Synopsis Genera Br. Insects: 96. Type(s), [Austria]: Vienna district (Collection destroyed, Horn \& Kahle, I936, Ent. Beih. Berl.-Dahlem 3: 243).

$N$. perla is a junior subjective synonym of Phalaena domestica Hufnagel, I 766, Berlin. Mag. $3(4): 406$.

Incorrect type-species designation: Noctua glandifera [Denis \& Schiffermüller], I775, a name not originally included in Bryophila, and not linked with one of the originally included names when cited by Duponchel, I829, in Godart \& Duponchel, Hist. nat. Lépid. Papillons Fr. 7 (2): 7 r.

Bryophila was proposed as the objective replacement name for Poecilia Schrank, I80z. POECILIA Schrank, I802, Fauna Boica 2(2): I 57.

Type-species: Noctua perla [Denis \& Schiffermüller], I775, by subsequent designation (for Bryophila Trcitschke) by Westwood, 1840, Synopsis Genera Br. Insects: 96.

A junior homonym of Poecilia Schneider, I8or, in Bloch, Syst. Ichthyologiae: 452, - Pisces. The objective replacement name is Bryophila Treitschke, I825.

BRYOPHILINA Staudinger, i892, in Romanoff, Mém. Lépid. 6: 398.

ACRO

Type-species: Bryophilina blandula Staudinger, I892, ibidem 6: 398, pl. 5, fig. II, by monotypy. Holotype $\hat{\sigma}$, [U.S.S.R.]: Amur district (MNHU, Berlin). 
BRYOPHILOPSIS Hampson, I894, Fauna Br. India (Moths) 2: $36 \mathrm{r}$.

Type-species: Bryophilopsis griseat $a$ Hampson, I 894, ibidem $2: 36 \mathrm{r}$, fig. 194, by original designation. Type(s) ô, India: [Punjab], Simla (BMNH).

BRYOPHILOPSIS Dyar, I920. See Parabryophila Dyar, I92 I.

ACRO

BRYOPOLIA Boursin, I954, Bull. Soc. Fouad I. Ent. 38: 86.

CUCU

Type-species: Polia virescens Hampson, 1894, Fauna Br. India (Moths) 2: 234, by original designation. Type(s) ô. India: Kashmir (BMNH).

BRYOPSIS Boursin, 1969, Z. wien. ent. Ges. 54: 46.

ACRO

Type-species: Phalaena muralis Forster, 1771, Novae Species Insect.: 74, by original designation. Type(s), [Great Britain]: England.

Bryopsis was proposed as a subgenus of Cryphia Hübner, I8I8.

BRYOTHRIPA Hampson, I9I2, Cat. Lepid. Phalaenae Br. Mus. 11: 223, 310.

SARR

Type-species: Bryothripa miophaea Hampson, I9I2, ibidem 11: 3I0, fig. Iog, by original designation. Holotype $\widehat{\sigma}$, Nigeria: Lagos $(\mathrm{BMNH})$.

$\ddagger B R Y O T Y P A$, misspelling. See Bryotype Hampson, 1906.

CUCU

BRYOTYPE Hampson, I906, Cat. Lepid. Phalaenae Br. Mus. 6: xii, 382.

CUCU

Type-species: Euplexia mesomelana Hampson, I902, J. Bombay nat. Hist. Soc. 14: 200, by original designation. Holotype $\hat{0}$, India: [Himachal Pradesh], Simla $7000 \mathrm{ft}$ (BMNH).

$\ddagger B R Y O T Y P A$; Grünberg, 1910, Avch. Naturgesch. 73 (2) (2) (2): 249.

An incorrect subsequent spelling.

$\ddagger$ BRYTHIS, misspelling. See Brithys Hübner, [I $82 \mathrm{I}$ ].

HADE

$\ddagger$ BRYTIS, misspelling. See Brithys Hübner, [I $82 \mathrm{I}]$.

HADE

BUCHHOLZIA Barnes \& Benjamin, 1926. See Benjaminiola Strand, r928. ACRO

BUCIARA Walker, I869, Chavacters undescr. Lepid. Heterocera: 36.

NOCT

Type-species: Buciara bipartita Walker, I869, ibidem: 36, by monotypy. Type(s)

o., TyPe-locality not stated (BMNH) [Australia].

BUCINNA Walker, I866, List Specimens lepil. Insects Colln Br. Mus. 35: 1983.

OPHI

Type-species: Betousa divisalis Walker, [1866] I865, ibidem 34: I209, by monotypy (of

Betousa Walker, [I 866]). Holotype ô. [Brazil]: [R. Amazon] [Tefé] Ega (BMNH).

Bucinna was proposed as the objective replacement name for Betousa Walker, [1866].

BETOUSA Walker, [I 866] 1865, List Specimens lepid. Insects Colln Br. Mus. 34: 1208.

Type-species: Betousa divisalis Walker, [1866], ibidem 34: r209, by monotypy.

A junior homonym of Betousa Walker, r865, ibidem 33: I I I I, - Lepid., Thyrididae. The objective replacement name is Bucinna Walker, I866.

$\ddagger$ BETONSA; Waller, I866, List Specimens lepid. Insects Colln Br. Mus. 35: 1983.

An incorrect subsequent spelling.

BULIA Walker, 1858, List Specimens lepid. Insects Colln Br. Mus. 15: 1815.

Type-species: Biula propria Walker, [1858] 1857, ibidem 13: I 170, by monotypy (of

Binla Walker, [1858]). Syntypes 30 . [Dominican Republic] St. Domingo (BMNH).

$B$. propria is a junior subjective synonym of Bolina brunnearis Guenée, 1852 , in

Boisduval \& Guenée, Hist. nat. Insectes (Lépid.) 7: 68.

Bulia was proposed as the objective replacement name for Biula Walker, [1858].

See also Arsisaca Walker, [1866].

BIULA Walker, [1858] I857, List Specimens lepid. Insects Colln Br. Mus. 13: I I 42, I 69.

Type-species: Biula propria Walker, [1858] I 857 , by monotypy.

A junior homonym of Biula WValker, 1857, ibidem 11: 7r4,-Lepid., Notodontidae. The objective replacement name is Bulia Walker, $\mathrm{x} 85^{8}$. 
BULNA Tralker, I865, List Specimens lepid. Insects Colln Br. Mus. 33: 943.

OPHI

Type-species: Bulna glaucineta Walker, ibidem 33: 944, by monotypy. Syntypes $2 q$ (not ô as described), Jamaica (BMNH).

$B$. glaucineta is a junior subjective synonym of Phalaena cynara Cramer, I 775 .

See also Macrodes Guenée, 1854.

BUPHANA Möschler, I880, Verh. zool.-bot. Ges. Wien. 30 (Abl.): 462.

OPHI

Type-species: Buphana zopissa Möschler, I 88o, ibidem 30 (Abh.) : 462, pl. 9, fig. 35, by monotypy. Holotype ठ․ SURINAm: interior (MNHU, Berlin).

BURDETTIA Schaus, I9I6, Proc. U.S. natn. Mus. 50:364.

OPHI

Type-species: Hypena braziliensis Schaus, 1904, Trans. Am. ent. Soc. 30: I74, by original designation. Type(s), [BRAzIL]: Rio Janeiro.

BURDRIA Walker, I869, Chavacters undescr. Lepid. Heterocera: 50.

STIC

Type-species: Burdria edemoides Walker, I869, ibidem: 50, by monotypy. Syntypes

ㅇ. [INDIA] Hindostan (BMNH).

$B$. edemoides is a junior subjective synonym of Odontodes aleuca Guenée, I 852.

See also Odontodes Guenée, I852; and Nedroma Walker, I869.

BURGENA Walker, [1865] I864, List Specimens lepid. Insects Colln Br. Mus. 31: 55. AGAR Type-species: Burgena transducta Walker, [1865] I 864, ibidem 31: 56, by subsequent designation by Kirby, I892, Synonymic Cat. Lepid. Heterocera 1: 31. Holotype ô, [Moluccas]: [Halmahera I.] Gilolo (BMNH).

$B$. transducta is a junior subjective synonym of Damias varia Walker, 1854 , ibidem 1 : 15 .

BUSMADIS Walker, I866, List Specimens lepid. Insects Colln Br. Mus. 35: 1984. ACON Type-species: Phanaspa thermesialis Walker, [1866] 1865, ibidem 34: I2I I, by monotypy (of Phanaspa Walker, [i 866]). Holotype ㅇ. [South AFrica]: Natal (BMNH).

Busmadis was proposed as the objective replacement name for Phanaspa IVallier, [1866].

PHANASPA Walker, [1866] 1865, List Specimens lepid. Insects Colln Br. Muıs. 34: i I I.

Type-species: Phanaspa thermesialis Walker, [1866] I 865, by monotypy.

A junior homonym of Phanaspa Walker, [1866] I865, ibidem 34: I 92, - Lepid., Noctuidae. The objective replacement name is Busmadis Walker, I 866.

BUSSEOLA Thurau, 1904, Berl. ent. Z. 49: 55.

ACRO

Type-species: Busseola sorghicida Thurau, I904, ibidem 49: 56, by monotypy. Syntypes ô, 우, [TANZANiA] : [Tanganyika], Lindi district.

$B$. sorghicida is a junior subjective synonym of Sesamia fusca Fuller, igor, Natal Dep. Agric., First Rep. Gout Entomologist I899-I900: 45, pl. I I.

See also Calamistis Hampson, 1908.

BUTLERONEA Viette, 1968, Bull. Soc. ent. Fr. 73: 77.

ACRO

Type-species: Butleronea tsara Viette, 1968, ibidem 73: 78, figs I, 2, pl. 2, fig. 4, by original designation. Holotype $\widehat{\jmath}$. Madagascar: W., $64 \mathrm{~km}$ à l'Est de Tuléar, forêt d'Andranovory (MNHN, Paris).

BUZARA Walker, [1865] i864, List Specimens lepid. Insects Colln Br. Mus. 31: I 59. opH

Type-species: Buzara chrysomela Walker, [1865] I864, ibidem 31: I59, by inonotypy. Lectotype + , New Guinea (UM, Oxford), designated (as type) by Swinhoe, igoo, Cat. east. and Aust. Lepid. Heterocera 2: 590.

BYAS Billberg, I820. See Evebus Latreille, I810.

CATO

$\ddagger \boldsymbol{B Y T I L A}$, misspelling. See Bityla Walker, i 865 .

ACRO

BYTURNA Moore, I883, Proc. zool. Soc. Lond. 1883: 28.

OPHI

Type-species: Bocana digramma Walker, [ I 866] i 865, List Specimens lepid. Insects Colln

Br. Mus. 34: i 7 7o, by monotypy. Holotype ô, Cerlon (BMNH). 
B. digramma is a junior subjective synonym of Daona mansueta Walker, I864.

See also Daona Walker, I 864 .

CABRALIA Moore, I882, Proc. lit. phil. Soc. Lpool 36: 374.

OPHI

Type-species: Cabralia trifasciata Moore, I882, ibidem 36:374, pl. 6, fig. 20, by monotypy. Syntypes, BrazIL: São Paulo.

CACOFOTA Dyar, I9I 4, Proc. U.S. natn. Mus. 47: 376.

CUCU

Type-species: Cacofota inermis Dyar, I9I 4, ibidem 47:377, by original designation. Holotype ô, Mexico: Zacualpan (USNM, Washington).

CACYPARIS Walker, [1863] I862, List Specimens lepid. Insects Colln Br. Mus. 26: I 572. CHLO Type-species: Cacyparis insolitata Walker, [I863] I 862, ibidem 26: I 572, by monotypy. Holotype 9 . [INDIA]: Hindostan (BMNH).

†COCYPARIS; Moore, [I886] i 887, Lepid. Ceylon 3: 440.

An incorrect subsequent spelling.

$\ddagger C \boldsymbol{A D A} \boldsymbol{T} \boldsymbol{A}$ Warren, no published reference found.

HYPE

CADIORAPA nom. n. for Paracodia Hampson, I 910.

$\operatorname{ACON}$

Type-species: Tarache puella Schaus, I904, Trans. Am. ent. Soc. 30: I58, by original designation (for Paracodia Hampson). Type(s), Brazil: S.E., São Paulo.

PARACODIA Hampson, I9Io, Cat. Lepid. Phalaenae Br. Mus. 10: $35^{2}$.

Type-species: Tarache puella Schaus, I904, by original designation.

A junior homonym of Paracodia Druce, I909,-Lepid., Noctuidae. The objective replacement name is Cadiorapa nom. $\mathrm{n}$.

CADUCA Moore, [1885] i 887, Lepid. Ceylon 3: 96.

OPHI

Type-species: Alamis meleagris Felder \& Rogenhofer, I874, Reise öst. Fregatte Novara (Zool.) 2 (Abt. 2) : pl. I I , fig. 32, by original designation. Type(s) ô., CEylon: Rambodde. A. meleagris is a junior subjective synonym of Homoptera albopunctata Walker, [1858] I 857, List Specimens lepid. Insects Colln Br. Mus. 13: I068.

CAECILA Walker, I858, List Specimens lepid. Insects Colln Br. Mus. 15: I824.

EUTE

Type-species: Caecila complexa Walker, I 858 , ibidem 15: I825, by monotypy. Lectotype $\hat{0}$. Singapore (UM, Oxford), designated (as type) by Swinhoe, I9oo, Cat. east. and Aust. Lepid. Heterocera 2: 84 .

C. complexa is a junior subjective synonym of Anuga constricta Guenée, 1852.

See also Anuga Guenée, I852; and Spersara Walker, [1863].

CAEDESA Walker, 1862, J. Proc. Linn. Soc. (Zool.) 6: 193.

Type-species: Caedesa agropoides Walker, I 862, ibidem 6: 193, by monotypy. Type(s)

ㅇ, BORnEo: Sarawak (UM, Oxford).

$\ddagger$ CAENIPETA, misspelling. See Coenipeta Hübner, I8 8 8.

OPHI

$\ddagger$ CAENOPHILA, misspelling. See Coenophila Stephens, I85o.

NOCT

CAENURGIA Walker, I858, List Specimens lepid. Insects Colln Br. MIus. 14: I464, I49I.

CATO

Type-species: Caenurgia socors Walker, I 858, ibidem 14: I 492, by subsequent designation by Hampson, I9I3, Cat. Lepid. Phalaenae Br. Mus. 13: 65, but cited as convalescens Guenée, I 852, which Hampson placed (on page 67 ) as the senior synonym of socors Walker. Syntypes $4(\hat{\partial}$, o $)$, [U.S.A.]: East Florida (BMNH); New York.

C. socors is a junior subjective synonym of Drasteria convalescens Guenée, 1852 , in Boisduval \& Guenée, Hist. nat. Insectes (Lépid.) 7: 289, pl. 22, fig. 9.

$\ddagger$ GLOIA Hübner, I8I8, Zuträge Samml. exot. Schmett. 1: I6, 33.

Not nomenclaturally available as a genus-group name. Hübner used Gloia, on both pages, in a suprageneric sense for one of the names of his tribes (Namen der Stämme) 
listed on page 33. Gloia is included here as Berio, 1957, Memorie Soc. ent. ital. 36: I4, has accepted it as an available generic name from I818, and had designated as type-species, Xestia chloropha Hübner, I818, which is congeneric with Caenurgia socors Walker, 1858 , the type-species of Caenurgia Walker, 1858 .

CAENURGINA McDunnough, I937, Can. Ent. 69: 65.

CATO

Type-species: Drasterin caerulea Grote, r873, Bull. Buffalo Soc. nat. Sci. 1: I55, by original designation. Syntypes $5(\hat{0}$, o $)$, [U.S.A.]: California (BMNH).

CAFFRISTIS Hampson, 1906, Cat. Lepid. Phalaenae Br. Mus. 6: x, 227. CUCU

Type-species: Heliophobus ferrogrisea Hampson, Ig02, Ann. S. Afr. Mus. 2: 442, by original designation. 'Type(s) $\vec{\sigma}$. [LEsotho] Basutoland: Masite (BMNH).

CALAMIA Hübner, [I821] i 816, Verz. bekannter Schmett. : 240.

ACRO

Type-species: Phalciena virens Linnaeus, I 767 , Syst. Nat. (Edn I 2) 1: 847, by subsequent designation by Hampson, i9ıo, Cat. Lepid. Phalaenae Br. Mus. 9: 340. Type(s), GERMANY (LS, London).

$P$. virens is a junior subjective synonym of Phalaena tridens Hufnagel, I766, Berlin. Mag. $3(3): 300$.

LUCERIA Heinemann, I859 [December 3Ist], Schmett. Dtl. Schweiz 1: xvi, 442.

Type-species: Phalaena virens Linnaeus, I 767 , by monotypy.

A junior homonym of Luceria Walker, I 859 [1)cember Ioth], - Lepid., Noctuidae. The objective replacement name is Calamia Hübner, [I $82 \mathrm{I}]$.

Zuchold, [1860], Bibl. Hist. nat. 9: I60, listed Heinemann's work as being published between July and December 1859 . In the absence of other evidence and under the Code, Article $2 \mathrm{I}(\mathrm{b})$ the date of publication is to be taken as the last day of the year. Luevia Heinemann is, in any case, a junior objective synonym and so will never be required for use; it is therefore reasonable to continue to use Luceria Walker as an available name.

LEDERERIA Grote, I874, Bull. Buffalo Soc. nat. Sci. 2: 54.

Type-species: Phalaena rivens Linnaeus, I 767 , by subsequent designation by Grote, I896, Entomologist's Rec. J. I'ar. 8: I83.

A junior homonym of Ledereria Narschall, i 873, Nomencl. zool.: 299 (cited under Margarodes Guenée), - Lepid., Pyralidae. The objective replacement name is Calamia Hübner, [I82I].

Ledereria Grote was proposed as a new genus for Luperina Boisduval sensu Lederer, 1857 , Noctuinen Eur.: 34, I04.

CALAMISTIS Hampson, I908, Cat. Lepid. Phalaenae Br. Mus. 7: II. Available but with ou included species until Hampson, I9Io, ibidem 9: xi, 273.

ACRO

Type-species: Sesamia fusca Hampson, I902, Ann. S. Afr. Mus. 2: 296, (a junior primary homonym), bysubsequent designation by Hampson, Igro, ibidem 9:273. Syntypes o. 옹, SOUTH AFrica (BMNH).

The binomen Sesamia fusca originated from Hampson but was described and unintentionally made nomenclaturally available as Sesamia fusca Fuller, igor, Natal Dep. Agric., First Rep. Govt Entomologist I899-I900: 45, pl. I I, prior to its proposal and description by Hampson for the same species.

S. fusca Hampson, 1902, is a junior primary homonym and also a junior subjective synonym of $S$. fusca Fuller, Igor; the latter should therefore be used as the subjective replacement name.

See also Busseola Thurau, I904.

CALATHUSA Walker, 1858, List Specimens lepid. Insects Colln Br. Mus. 15: 1645. SARR Type-species: Calathusa basicunea Walker, I858, ibidem 15: 1645, by monotypy. Syntypes $3(\hat{0}$, ) $)$, [Australia]: Sydney (BMNH); [Qd], Moreton Bay.

See also Sina Walker, 1865; and Hisbanda Walker, [1866]. 
CALDUBA Walker, I858, List Specimens lepid. Insects Colln Br. Mus. 15: I8 I 5. C CHLO Type-species: Calduba obtenta Walker, I858, ibidem 15: I8 I 5 , by monotypy. Holotype o., Ceylon (BMNH).

C. obtenta is a junior subjective synonym of Maceda mansueta Valler, [1858], ibidem 13: I I $4 \mathrm{r}$.

See also Maceda Walker, [1858].

CALESIA Guenée, I852, in Boisduval \& Guenée, Hist. nat. Insectes (Lépid.) 7: 257 OPH Type-species: Calesia comosa Guenée, I852, ibidem 7: 258, pl. 2 I, fig. 7, by subsequent designation by Desmarest, (1857), in Chenu, Encycl. Hist. nat. Papillons nocturnes: I32. Holotype ô, [BAngladesh]: [E. Bengal], Silhet (BMNH).

C. comosa is a junior subjective synonym of Evebus dasypterus Kollar, 1844, in Hügel, Kaschmir und das Reich der Siek 4: 476.

CALESIDESMA Strand, I920, Arch. Naturgesch. 84(A) I 2: I 44.

OPHI

Type-species: Calesidesma fraternella Strand, I920, ibidem 84(A) I 2: I 44, by monotypy. Holotype ō. [TAIwaN] Formosa: Alikang (DEI, Eberswalde).

Calesidesma Strand n.g. was the spelling used as the heading but was immediately followed by $\ddagger$ Calisidesma as the generic name for the new species fraternella Strand. There is no doubt concerning the correct spelling as on page 145 Strand associates his new name with Calesia Guenée, I852.

$\ddagger_{+}$CALISIDESMA; Strand, I920, Arch. Naturgesch. 84(A) I 2: I 44. An incorrect original spelling.

CALESIODES Roepke, I94I, Zoöl. Meded., Leiden 23: 28.

OPHI

Type-species: Calesiodes punctigera Roepke, I94I, ibidem 23: 28, pl. I, fig. 6, by original designation. Holotype ô., Borneo: E., Kariorang (RNH, Leiden).

CALICULA Walker, I858, List Specimens lepid. Insects Colln Br. Mus. 15: I807.

OPHI

Type-species: Calicula exempta Walker, I858, ibidem 15: I808, by subsequent designation by Berio, I966, Annali Mus. civ. Stov. nat. Giacomo Doria 76: 5I. Holotype , [INDIA]; Hindostan (BMNH).

C. exempta is a junior subjective synonym of Erygia apicalis Guenée, 1852.

See also Erygia Guenée, I 852.

CALIGATUS Wing, [I850], Proc. zool. Soc. Lond. 1849: I04.

EUTE

Type-species: Caligatus angasii Wing, [1850], ibidem 1849: I04, pl. I4, figs 2, 3, by original designation. Type(s) $\vec{\delta}$. [South Africa]: Cape of Good Hope.

See also Pacidara Walker, I865.

$\ddagger$ CALISIDESMA, misspelling. See Calesidesma Strand, I920.

OPH I

CALLAENIA Hübner, [182I]. See Cucullia Schrank, I802.

CUCU

$\ddagger$ CALLAINIA, misspelling. See Cucullia Schrank, I802.

CUCU

CALLARG YRA Hampson, I908, Cat. Lepid. Phalaenae Br. Mus. 7: 5, 5I 5 .

ACRO

Type-species: Callargyra bayni Hampson, I908, ibidem 7: 5I5, fig. ro8, by original designation. Holotype $\widehat{\sigma}$. Argentina: Mendoza, Trotero Soza (BMNH).

CALLHYCCODA Berio, 1935, Annali Mus. civ. Stor. nat. Giacomo Doria 58: 63.

ACRO

Type-species: Callhyccoda viriditrina Berio, I935, ibidem 58: 64, fig. 6, by original designation. Holotype , [Somali Republic] Italian Somaliland: Belet Amin.

CALLICAMPA Agassiz, I846. See X̌lena Ochsenheimer, I816. Cucu

$\ddagger$ CALLIERGES, misspelling. See Calliergis Hübner, [I82 I].

CUCU 
CALLIERGIS Hübner, [1821] 1816, Verz. behannter Schmett.: $244 . \quad$ cucu Type-species: Bombyx ramosa Esper, 1786, Die Schmett. 3: 391, pl. 78, fig. 3, by subsequent designation by Hampson, 1906, Cat. Lepid. Phalaenae Br. Mus. 6: 93.

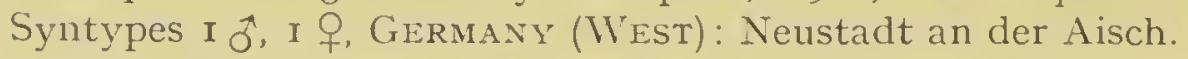

LITHOCAMPA Guenée, 1852, in Boisduval \& Guenée, Hist. nat. Insectes (Lépid.) 6: 108.

Type-species: Bombyx ramosa Esper, 1786 , by monotypy.

Lithocampa is a junior objective synonym of Calliergis Hübner, [182I].

†CALLIERGES; Hampson, I 9o6, Cat. Lepid. Phalaenae Br. Mus. 6: 93.

An incorrect subsequent spelling.

CALLIGRAPHIDIA Gaede, 1939, in Seitz, Gross-Schmett. Erde 15: $28 \mathrm{r}$.

OPHI

Type-species: Ophisma opulenta Möschler, 1887, Abh. senckent. naturforsch. Ges.

15: 86, fig. I5, by monotypy. Holotype ㅇ, [GHana] Gold Coast: Aburi (MNHU, Berlin).

The date of publication of the part of Seitz containing page 28I was stated on page 279 as 1939 June 2oth in both the German and English editions.

CALLIMORPHA Latreille, I 809, Genera Crustaceonum Insectornm 4: $220 . \quad$ ARCTIDAE Type-species: Phalaena dominula Linnaeus, I758, Syst. Nat. (Edn ro) 1: 509, by subsequent designation by Desmarest, (1857), in Chenu, Encycl. Hist. nat. Papillons nocturnes: 33 .

HYPERCOMPA Rambur, I866, Cat. syst. Lépid. Andalousie (2): 230.

Type-species: Phalaena dominula Linnaeus, $\mathrm{r} 75^{8}$, by monotypy.

Hypercompa has been used as the original generic name for species which are now in the Noctuidae.

CALLINGURA Butler, 1894, Entomologist 27: 284.

FUTE

Type-species: Ingura cristat rix Guenée, 1852 , in Boisduval \& Guenée, Hist. nat. Insectes (Lépid.) 6: 313, pl. I4, fig. Io, by monotypy. Holotype ô. TYPE-Locality stated to be 'Amérique Septentrionale? Coll. Cie des Indes' (BMNH). [The species illustrated is not known to occur in the New World but is present in the East Indies.]

$\ddagger$ CALLINODES, misspelling. See Calliodes Guenée, 1852.

CATO

CALLINOLA Butler, i889, Illust. typical Specimens Lepid. Heterocera Colln Br. Mus. 7: 33.

NOLI

Type-species: Roeselia antennata Butler, 1878, Trans. ent. Soc. Lond. 1878: 57, by original designation. Syntypes $\hat{\sigma},+$, $[$ BrazIL]: Amazon, lRio Solimões, Tamandare (BMNH).

CALLIOCLOA Draudt, 1950, Mitt. münch. ent. Ges, 40: I 29.

ACRO

Type-species: Calliocloa trapezoides Draudt, I950, ibidem 40: I29, pl. 8, fig. I7, by monotypy. Syntypes, CHrna: [Yunnan Prov.], Li-kiang (MAK, Bonn); [Szechwan Prov.], Batang.

CALLIODES Guenée, 1852, in Boisduval \& Guenée, Hist. nat. Insectes (Lépid.) 7: 192. Cato

Type-species: Calliodes appollina Guenée, r852, ibidem 7: 193, pl. 20, fig. 5, by subsequent designation by Hampson, 1902, Ann. S. Afr. Mus. 2: 319 , but cited as †'apollina', an incorrect subsequent spelling. Holotype $\widehat{\jmath}$. Senegal (BMNH).

C. appollina was attributed to Feisthamel by Gucnée, but no earlier published reference has been found.

$\ddagger$ CALI.INODES; Pagenstecher, I909, Geogr. Verbreitung Schmett.: 425.

An incorrect subsequent spelling.

CALLIPHASIA Agassiz, 1846. See Calophasia Stephens, 1829.

CUCU

CALLIPYRIS Meyrick, I891, Trans. R. Soc. S. A ust. 14: 195.

$\triangle \mathrm{CON}$

Type-species: Callipyris arosera Meyrick, I 891 , ibidem 14: 195, by monotypy. Syntypes $3(\hat{o}, 9)$, Australia: Qd, Brisbane; N.S.W., Sydney. 
CALLISTEGE Hübner, [1823] I8 I6, Verz. bekannter Schmett. : 28 I.

Type-species: Phalaena mi Clerck, 1759, Icones Insect. rariorum 1: pl. 9, fig. 5 (but included by Hübner as 'mi Linn.', an incorrect authorship), by subsequent designation by Berio, 1957, Memorie Soc. ent. ital. 36: i2, but cited as 'mi Linn.' Type(s), TypeLOCALITY not stated (NR, Stockholm) [probably SwEDEN].

EUCLIDIMERA Hampson, I9I3, Cat. Lepid. Phalaenae Br. Mus. 12: 4. Available but without included species until Hampson, I9I3, ibidem 13: vii, 45.

Type-species: Phalaena mi Clerck, I 759, by subsequent designation by Hampson, I9I3, ibidem 13: 45 .

Euclidimera is a junior objective synonym of Callistege Hübner, [1823].

CALLITAENIA Agassiz, I846. See Staurophora R.L., i 8 I 7.

CUCU

CALLIXENA Saalmüller, I 89 I, Lepid. Madagascar: 324.

ACRO

Type-species: Callixena versicolora Saalmüller, I89 I, ibidem:325, fig. I64, by monotypy. Syntypes $5(\widehat{0}$, $)$, Madagascar: W., Nossi-Bé I. (SNG, Frankfurt).

CALLOECIA Hampson, igo8, Cat. Lepid. Phalaenae Br. Mus. 7: 8. Available but without included species until Hampson, I910, ibidem 9: vii, 56.

CUCU

Type-species: Anthracia swinhoei Butler, I883, Proc. zool. Soc. Lond. 1883: i62, by subsequent monotypy, but cited by Hampson as 'C. svinhoei', an unjustified emendation. Type(s), India: [Madhya Pradesh], [Asirgarh] Assirghur (BMNH).

$A$. swinhoei was orginally proposed as $\ddagger$ Anthaecia, an incorrect subsequent spelling.

CALLOGONIA Hampson, I908. See Goonallica nom. n.

ACRO

CALLOPHISMA Hampson, I913, Cat. Lepid. Phalaenae Br. Mus. 12: 8, 263.

CATO

Type-species: Callophisma flavicornis Hampson, I913, ibidem 12:263, fig. 36, by original designation. Holotype ô, Nigeria: Akassa (BMNH).

CALLOPISTRIA Hübner, [1821] i 816, Verz. bekannter Schmett. : 216.

ACRO

Type-species: Phalaena juventina Stoll, I782, in Cramer, Uitlandsche Kapellen 4: 245, pl. 400, fig. N, by subsequent designation by Grote, I874, Bull. Buffalo Soc. nat. Sci. 2: I 7, but cited as Noctua pteridis Fabricius, I794, Ent. Syst. 3 (2): 90, one of the names originally included in Callopistria and also an unnecessary objective replacement name for Phalaena lagopus Esper, I 788, Die Schmett. 4 (I): 356, pl. I25, fig. 7, itself an unnecessary objective replacement name for $P$. juventina. Type(s). TYPE-LOcality stated by Stoll to be Surinam, but this was probably an error as the species illustrated is not known to occur in the New World but is widespread in Europe and parts of Asia.

Under the Code, Article 67 (e) the senior objective synonym is to be cited as the name of the type-species.

LAGOPUS R. L., i 8 I 7, Jenaische Allg. Lit.-Ztg 1: 285 .

Type-species: Phalaena juventina Stoll, I782, by monotypy, but cited as $N$. pteridis Fabricius, I794, an unnecessary objective replacement name.

A junior homonym of Lagopus Brisson, i760, Ornith. 1: 216,-Aves. The objective replacement name is Callopistria Huibner, [1 $82 \mathrm{I}]$.

ERIOPUS Treitschke, I825, Schmett. Eur. 5 (I): 365 .

Proposed as the objective replacement name for Lagopus R. L., I8I7.

$\ddagger$ CALOPISTRIA ; Stephens, 1850, List Specimens Br. Anim. Colln Br. Mus. 5: 286.

An incorrect subsequent spelling.

$\ddagger$ CALLORUGA, misspelling. See Calloruza Hampson, I9 I8.

$A C O N$

CALLORUZA Hampson, i 9 i 8, Novit. zool. 25: I69.

ACON

Type-species: Corgatha pulchra Bethune-Baker, I906, ibidem 13: 213, by original designation. Holotype $\hat{\sigma}$, New Guinea: [Papua], R. Aroa.

${ }_{+}$CALLORUGA; Neave, 1939, Nomencl. zool. 1: 543 .

An incorrect subsequent spelling. 
CALLOSTOLIS Dyar, I928, in Walter, Proc. ent. Soc. Wash. 30: I38.

ACON

Type-species: Callostolis polyrrhoda Walter, I928, ibidem 30: 138 , by original designation. Holotype ô. [U.S.A.]: Arizona, Tempe (USNM, Washington).

C. polyrrhoda is a junior subjective synonym of Phoenicophanta bicolor Barnes \& McDunnough, 1916, Contr. nat. Hist. Lepid. N. Am. 3: 15, pl. 3, fig. I5.

CALLOSTROTIA Hampson, I914, Ann. Mag. nat. Hist. (8) 13: 2 I I.

ACON

Type-species: Callostrotia flavizonata Hampson, I9I 4, ibidem (8) 13:2 I I, by original designation. Holotype ô, NigERIA: N., Zungeru (BMNH).

CALLYNA Guenée, 1852, in Boisduval \& Guenée, Hist. nat. Insectes (Lépid.) 5: I I $2 . \quad$ ACro

Type-species: Callyna siderea Guenée, I852, ibidem 5: II3, by monotypy. Syntypes o. ㅇ. [Bangladesh]: [E. Bengal], Silhet (BMNH).

${ }_{\ddagger}$ CALOBOCHYLA, misspelling. See Colobochyla Hübner, [1825].

OPH I

CALOCAMPA Stephens, I829. See Xylena Ochsenheimer, I816.

CUCU

$\ddagger$ CALOCASIA, misspelling. See Colocasia Ochsenheimer, I816.

PANT

CALOCEA Dyar, 1914, Proc. U.S. natn. Mus. 47: 375.

CUCU

Type-species: Calocea eucraspedica Dyar, I9I 4, ibidem 47:375, by original designation. Holotype 3 , Mexico: [Puebla], Tehuacan (USNM, Washington).

CALOGRAMMA Guenće, I 852, in Boisduval \& (iuenée, Hist. nat. Insectes (Lépid.) 5: 165.

$\mathrm{ACRO}$

Type-species: Polia picta Guérin-Méneville, 1838, in Duperrey, Voyage autour du Monde sur la Corvette La Coquille (Zool.) 2 (2(I)) : 285, proposed as the objective replacement name for Phalaena (Noctua) festiva Donovan, I805, Epitome nat. Hist. Insects of New Hollancl etc. : facing page \& pl. 36, fig. I, by monotypy. Syntypes (of $P$. festiva), [Australia] New Holland: New South IVales.

P. festiva Donovan is a junior primary homonym of Phalaena (Geometra) festiva Cramer, I775. Uitlandsche Kapellen 1: I I2, pl. 7I, fig. C, - Lepid., Geometridae.

CALOPHASIA Stephens, I829) [June], Nom. Br. Insects: 42.

CUCU

Type-species: Noctua linariae [Denis \& Schiffermüller], 1775, Ankïndung syst. Werkes Schmett. Wienergegend: 73, by monotypy. Type(s), [Austria]: Vienna district (Collection destroyed, Horn \& Kahle, I936, Ent. Beih. Berl.-Dahlem 3: 243).

$\Lambda$. linariae is a junior subjective synonym of Phalaena lnmula Hufnagel, $\mathbf{I} 766$, Berlin. Mag. $3(4): 394$.

Calophasia became available together with included species in I829 [June]. The generic description followed in i 829 September, Illust. Br. Ent. (Haustellata) 3: 93.

CALLIPHASIA Agassiz, r846, Nomencl. zool. Index univl.: 59, 61.

An unjustified emendation of Calophasia Stephens, I829.

CALOPHASIDIA Hampson, I908, Cat. Lepid. Phalaenae Br. Mus. 7: 5. Available, but without included species until Hampson, 1909, ibidem 8: ix, i8o.

ACRO

Type-species: Megalodes lucala Swinhoe, 1902, Ann. Mag. nat. Hist. (7) 9: 168, by subsequent designation by Hampson, 1909, ibidem 8: i8o. Holotype ô. Australia: W'. Aust., Sherlock River (BMNH).

${ }_{\ddagger}$ CALOPISTRIA, misspelling. See Callopistria Hübner, [182I].

ACRO

CALOPLUSIA Smith, i 884, Bull. Brooklyn ent. Soc. 7: 68. Available, but without included species until Smith, I891, List Lepid. boreal Am.: 52 .

PLUS

Trpe-species: Phalaena hochenwarthi Hochenwarth, I785, Schr. berl. Ges. naturf. Freunde 6: 33.5, pl. 7, fig. 2, loy subsequent designation by Warren, I9I3 April, in Seitz. 
Gross-Schmett. Erde 3: 344, but cited as ‡'hochenwarthii', an incorrect subsequent spelling. Type(s), [Germany].

$P$. hochenwarthi was also designated as type-species by Hampson, I9I3 [November], Cat. Lepid. Phalaenae Br. Mus. 13: 405 .

CALOPTERA Blanchard, 1845. See Egybolis Boisduval, I847. CAto

CALOTAENIA Stephens, I830. See Staurophova R.L., I8I7. Cucu

$\ddagger$ $\boldsymbol{C A L P A}$, misspelling. See Calyptra Ochsenheimer, I8I6. OPHI

CALPE Treitschke, I825. See Calyptra Ochsenheimer, I8I6. OPHI

CALPIFORMIS Hampson, I908, Cat. Lepid. Phalaenae Br. Mus. 7: xiii, 504. ACRo

Type-species: Conservula craushayi Hampson, 1905, Ann. S. Afr. Mus. 3: 427, by original designation. Type(s) $\hat{o}$, [LEsotho] Basutoland: Mahalishoek (BMNH).

CALYDIA Bar, 1875, Annls Soc. ent. Fr. (5)5: 291. OPHI

Type-species: Calydia bourgaulti Bar, I 875, ibidem (5)5: 292, pl. 5, fig. I, by subsequent designation by Dyar, r9i 4, Proc. U.S. natn. Mus. 47: 96. Type(s), French Guiana.

CALYMERA Moore, I882, in Hewitson \& Moore, Descr. new. Indian lepid. Insects Colln late Mr W. S. Atkinson: I04.

CHLO

Type-species: Calymera picta Moore, I882, ibidem: 104, pl. 4, fig. 7, by monotypy. Type(s) 9 , India: Darjiling (MNHU, Berlin).

CALYMMA Hübner, [1823] I816, Verz. bekannter Schmett.: 28ז.

ACON

Type-species: Noctua communimacula [Denis \& Schiffermüller], I775, Ankündung syst. Werkes Schmett. Wienergegend: 85, by subsequent designation by Hampson, I9Io, Cat. Lepid. Phalaenae Br. Mus. 10: 63. Type(s), [Austria]: Vienna district (Collection destroyed, Horn \& Kahle, 1936, Ent. Beih. Berl.-Dahlem 3: 243).

ORATOSCELIS Guenée, I84I, Annls Soc. ent. Fr. 10: $23 \mathrm{I}$.

Type-species: Noctua communimacula [Denis \& Schiffermüller], I775, by monotypy, but cited as 'communimacula Fab.', an incorrect authorship.

Oratoscelis is a junior objective synonym of Calymma Hübner, [1823].

HORATOSCELIS Agassiz, I846, Nomencl. zool. Index univl.: I86, 262.

An unjustified emendation of Oratoscelis Guenée, I84I.

$\ddagger$ CALYMNA; Walker, [I859] I858, List Specimens lepid. Insects Colln Br. Mus. 16: I3.

An incorrect subsequent spelling.

$\ddagger$ ORATOCELIS; Spuler, I907, in Hofmann, Schmett. Eur. 1: 29 I.

An incorrect subsequent spelling.

$\ddagger$ CALYMNA, misspelling. See Calymma Hübner, [1823]. ACON

CALYMNIA Hübner, [182I] I8I6, Verz. bekannter Schmett.: 235. ACRO

Type-species: Phalaena trapezina Linnaeus, I758, Syst. Nat. (Edn Io) 1: 510, by subsequent designation by Grote, 1874, Bull. Buffalo Soc. nat. Sci. 2: 24. Type(s), EuropE (LS, London).

CALYMNIODES Dognin, I907, Annls Soc. ent. Belg. 51: 237.

ACRO

Type-species: Calymniodes pyrostrota Dognin, 1907, ibidom 51: 237, by subsequent designation by Collins, I962, Ann. Mag. nat. Hist. (13)5: I21. Holotype $ᄋ$, PERU: Carabaya, Santo Domingo (USNM, Washington).

CAL YMNIODES Hampson, I908. See Hampsonodes nom. n.

ACRO

CALYMNIOPS Hampson, 1926, Descr. new Genera Species Lepid. Phalaenae Subfamily Noctuinae Br. Mus.: 205. OPHI

Type-species: Elydna trapezata Moore, 1887, Lepid. Ceylon 3: 547, p1. 214, fig. 9, by original designation. Type(s), CEYLon: Wattegama (BMNH). 
CALYPTIS Guenée, I 852, in Boisduval \& Guenée, Hist. nat. Insectes (Lépid.) 6: 323. Cato Type-species: Calyptis iter Guenée, I 852, ibidem 6:324, pl. I2, fig. 3, by monotypy. Type(s) q, TyPE-Locality 'Amérique Septentrionale?', but as Guenée suspected this was probably incorrect as the species illustrated is not known to occur in North America but is widespread in Central and equatorial South America.

CALYPTRA Ochsenheiner, i 816, Schmett. Eur. 4: 78.

OPHI

Type-species: Phalaena thalictri Borkhausen, I790, Naturg. eur. Schmett. 3: 425, by subsequent designation by Duponchel, I826, in Godart \& Duponchel, Hist. nat. Lépid. Papillons Fr. 6: [(3)]. Syntypes o, ㅇ, Europe.

P. thalictri was figured by Esper, I 789, Die Schmett. 3 (Suppl. I) Abschnitt 3: 7, pl. 8I, figs I-3, as P. capucina Linnaeus [Notodontidae], but Esper had misidentified what was then a new species, not named till a year later by Borkhausen.

Caly'ptra had already been used as a generic name by Klein, I 753, and also by Humphreys, I797, Mus. Calonnianum: 5, for two different concepts both in the Mollusca. However, neither of these usages is nomenclaturally available, the first being pre-1758, and the second being included in a work rejected for nomenclatural purposes by Int. Commn zool. Nom., I9I2, Smithson. Inst. Publ. 2060 Opinion 5I : I I6. I have found no other citation of Calyptra in the Mollusca until Gray, I847, Proc. zool. Soc. Lond. 15: I 57, I8I, where the usage of both Kilein and of Humphreys was recorded.

CALPE Treitschke, I 825, Schmett. Eum. 5(2): I68.

Proposed as an objective replacement name for Calyptra Ochsenheimer, I816. When Treitschke proposed Calpe he stated 'Nach dem Dictionnaive des sciences naturelles, T[ome] II [recte V1]. pag. 274, wurde Calvptra von Lamarck schon für eine Gattung der Mollusken verwendet'. The actnal entry in Levrault, I8I7, Dict. Sci. nat. 6: 274 reads 'Calyptrée (Moll.) Lam., genre de mollusques a coquilles en cône, etabli par Lamarck'. In fact the genus concerned had a multiple original spelling Calyptroea/Calyptraea Lamarck, I799, Mém. Soc. Hist. nat. Paris 1799: 78, 9r (table),-Mollusca. The name Calyptraea is now in general use in the Mollusca but neither of the spellings is homonymous with Calyptra Ochsenheimer.

${ }_{+}^{+}$CALPA; Pagenstecher, 1909, Geogr. T'erbreitung Schmett.: 424.

An incorrect subsequent spelling.

CAMADENIANA Strand, igr5, Ent. Mitt. 4: igr.

TORTRICIDAE

Type-species: Camadeniana capitalis Strand, I9 I5, ibidem 4: I93, by original designation. Holotype ô, Cameroun: Bonaberi (DEI, Eberswalde).

This genus was originally placed in the Thyrididae but was transferred to the Noctuidae by Gaede, I9I 7 , Mitt. zool. Mus. Berl. 8: 359 and more recently has been transferred to the Tortricidae, Olethreutinae by Whalley, I964, Ann. Mag. nat. Hist. (13) 7: I 8.

CAMPHYPENA Prout, 1927, Trans, ent. Soc. Lond. 75: 230.

II'PE

Type-species: Camphypena thomensis Prout, 1927, ibidem 75: 230, pl. 21, fig. 8, by original designation. Syntypes $4 \hat{o}$, [SÃo Tomé I., West Africa] São Thomé I. (BMNH).

CAMPOMETRA Guenée, I852, in Boisduval \& Guenée, Hist. nat. Insectes (Lépid.) $7: 25$.

CATO

Type-species: Campometra amella Guenée, I 852 , ibidem $7: 25$, pl. I 8, fig. 8, by monotypy. Type(s) ơ, America: northern.

CAMPTOCHILUS Hampson, I895. See Ectogonia Hampson, I 896.

OPHI

CAMPTOCROSSA Turner, I04, Trans. R. Soc. S. Aust. 68: I6.

IYPE

Type-species: Camptocrossa selenotypa Turner, 1944, ibidem 68: 16, by original designation. Holotype ${ }^{*}$, Australia: Qd, $\Lambda$ therton Plateau, L. Barrine (ANIC, Canberra). 
CAMPTOZADA Hampson, I912, Cat. Lepid. Phalaenae Br. Mus. 11: xv, 575. Chlo

Type-species: Clettharra mirabilis Swinhoe, I890, Trans. ent. Soc. Lond. 1890: 237, by original designation. Type(s), Burma: Rangoon (BMNH).

C. mivabilis was originally proposed as $\ddagger$ Cletthora, an incorrect subsequent spelling.

CAMP TYLOCHILA Stephens, I834, Illust. Br. Ent. (Haustellata) 4:21. HYPE

Type-species: Camptylochila undulalis Stephens, I834, ibidem 4:22, by subsequent designation by Westwood, I840, Synopsis Genera Br. Insects: I05. Syntypes 2 ex., TYPE-LOCALITy not stated but described in a 'work on British insects (BMNH). This species is occasionally recorded from Britain but is not established in the Old World; it is a New World species common in U.S.A. and Canada.

CAMPYLOCHILA Agassiz, I846, Nomencl. zool. Index univl.: 63.

An unjustified emendation of Camptylochila Stephens, I834.

CAMPYDELTA Berio, I964, Doriana 3 (I 44) : 2.

ACRO

Type-species: Delta campyla Hampson, 1909, Cat. Lepid. Phalaenae Br. Mus. 8: I94, pl. I27, fig. 25, by original designation. Syntypes 4 오․ Sierra Leone (BMNH).

CAMPYLOCHILA Agassiz, I846. See Camptylochila Stephens, I 834.

HYPE

CANA THA Walker, [I866] I 865, List Specimens lepid. Insects Colln Br. Mus. 34: I I 25. OPHI Type-species: Canatha confutalis Walker, [I866] I865, ibidem 34: I I25, by subsequent designation by Berio, I966, Annali Mus. civ. Stor. nat. Giacomo Doria 76: 5I. Holotype ㅇ. [BRazil]: [R. Amazon], [Tefé] Ega.

CANIDIA Grote, I890. See Schinia Hübner, I8I8.

NOCT

CANNA Walker, I865. See Nacna Fletcher, I961.

ACRO

CANODIA Guenée, 1852, in Boisduval \& Guenée, Hist. nat. Insectes (Lépid.) 6: 377.

NOTODONTIDAE

Type-species: Canodia carmelitoides Guenée, I852, ibidem 6: 378, pl. I2, fig. 8, by monotypy.

Canodia was originally placed in the Noctuidae.

CANTHYLIDIA Butler, I886, Trans. ent. Soc. Lond. 1886: 406.

Noct

Type-species: Canthylidia pallida Butler, I886, ibidem 1886: 406, by monotypy. Type(s), [Australia]: [Qd], Gayndah (BMNH).

C. pallida is a junior subjective synonym of Leucania invaria Walker, I 856 , List Specimens lepid. Insects Colln Br. Mus. 9: I I I.

CAPELICA Turner, I944, Trans. R. Soc. S. Aust. 68: I3.

OPHI

Type-species: Capelica oxylopha Turner, I944, ibidem 68: 13, by monotypy. Holotype o. Australia: W. Aust., Yanchep (ANIC, Canberra).

CAPHORNIA Köhler, 1958, Rev. Soc. ent. Argent. $20: 9$.

NOCT

Type-species: Agrotis xanthostola Mabille, I885, Bull. Soc. philomath. Paris (7) 9:6I, by original designation. Lectotype $\hat{\sigma},[\mathrm{CH} I \mathrm{LE}]$ : Terre de Feu [Hardy Penin.], Orange $\mathrm{B}[\mathrm{ay}]$ (MNHN, Paris), designated (as holotype) by Viette \& Fletcher, 1968, Bull. Br. Mus. nat. Hist. (Ent.) $21: 410$.

CAPILLAMENTUM Pinhey, 1956, Occ. Pap. natn. Mus. Sth. Rhod. 3(2 I B): 81.

HADE

Type-species: Capillamentum nigrofasciatum Pinhey, I956, ibidem 3 (2 I B): 82, fig. 4, pl. I, fig. 6 , by original designation. Holotype $\hat{\sigma},[$ KENYA]: Kitale district (BMNH).

CAPIS Grote, 1882, Can. Ent. 14:20.

OPHI

Type-species: Capis curvata Grote, I882, ibidem 14: 20, by monotypy. Type(s) [U.S.A.]: New York (BMNH). 
CAPITARIA Walker, I869, Characters undescr. Lepid. Heterocera: 42.

Type-species: Capitaria sublineata Walker, I869, ibidem: 42 , by nonotypy. Type(s) 7 , [HONDURAS]: Limas (BMNH).

CAPNISTIS Warren, I9I3, in Seitz, Gross-Schmett. Erde 3: 426.

HYPE

Type-species: Amblygoes albinotata Butler, 1879, Ann. Mag. nat. Hist. (5) 4: 449, by original designation. Type(s) JAPAN (BMNH).

The date of publication of the part of Seitz containing page 426 was stated on page $42 \mathrm{I}$ as 1913 December $5^{\text {th }}$ in both the English and German editions.

CAPNODES Guenée, I852, in Boisduval \& Guenée, Hist. nat. Insectes (Lépid.) 7: $374 . \quad$ opHI

Type-species: Capnodes irene Guenée, I852, ibidem 7:375, by subsequent designation by Grote, I874, Bull. Buffalo Soc. nat. Sci. 2: 45. Holotype ô., Brazil: Nouvelle-Fribourg (BMNH).

CAPOTENA Walker, 1857, List Specimens lepid. Insects Colln Br. Mus. 11: 7 I4.

CHLO

Type-species: Capotena apriformis Walker, I857, ibidem 11: 7I5, by monotypy. Lectotype ?, TYPE-LOCALITY stated to be Java, but this was probably an error which was corrected by Hampson, I894, Fauna Br. India (Moths) 2: 4I9, to Ceylon (BMNH). Lectotype designated (as type) by Hampson, ig 2, Cat. Lepid. Phalaenae Br. Mus. 11: 534 .

CARADJIA Zerny, 1928, Verh.zool.-bot. Ges. Wien 78(2):(86).

ACRO

Type-species: Caradjia sericea Zerny, I928, ibidem 78 (2):(87), by monotypy. Syntypes 3 , Mongolia: Chingan mont., Inu Shan (NM, Vienna).

CARADRINA Ochsenheimer, i 816, Schmett. Eur. 4: 80.

ACRO

Type-species: Phalaena morpheus Hufnagel, I 766, Berlin. Mag. 3(3): 302, by subsequent designation by Samouelle, I8I9, Entomologist's useful Compendium: 251. Type(s), [GERMANY]: Berlin.

CHARADRINA Agassiz, I846, Nomencl. zool. Index univl.: 65, 77.

An unjustified emendation of Caradrina Ochsenheimer, I8I6.

CARANDANA Moore, [1884] 1887, Lepid. Ceylon 3: 45.

ACRO

Type-species: Acontia fasciculosa Walker, 1858 , List Specimens lepid. Insects Colln Br. Mus. 15: i 760 , by monotypy. Holotype $q$, Ceylon (BMNH).

CARANILLA Moore, [1885] I887, Lepid. Ceylon 3: I69.

CATO

Type-species: Naxia onelia Guenée, 1852, in Boisduval \& Guenće, Hist. nat. Insectes (Lépid.) 7: 256, by original designation. Type(s), [Bangladesh]: [E. Bengal], Silhet (TMI, Oxford).

CARBONA Schaus, igo6, Proc. U.S. natn. Mus. 30: 102.

ACRO

Type-species: Carbona obscura Schaus, I906, ibidem 30: 102, by monotypy. Type(s), BrazIL: Petropolis (USNMI, IVashington).

$\ddagger$ CARCA, misspelling. See Carea Walker, 1856 .

CHLO

CARCHARODA Hampson, I9Io, Cat. lepid. Phalaenae Br. Mus. 9: 4, 2 I I.

ACRO

Type-species: Carcharoda flavirosea Hampson, I9Io, ibidem 9: 2 I I, fig. 76, by original designation. Holotype $\widehat{A}$, [Kenya] Brit. E. Afr.: Mombasa (BMNH).

CARDALENA Walker, I859, List Specimens lepid. Insects Colln Br. Mus. 19: 888. IYPE

Type-species: Neviasca tespisalis Walker, [1859] I 858, ibidem 16: 200, by monotypy (of Neviasca Walker, [1859], ibidem 16: I99). Lectotype ô. Borneo: Sarawak (UM, Oxford), designated (as type) by Swinhoc, I900, Cat. east. and Aust. lepid. Heterocera 2: 200.

Cardalena was proposed as the objective replacement name for Neviasca Valker, [I859], ibidem 16: 199 .

NEVIASCA Walker, [1859] I858, List Specimens lepid. Insects Colln Br. Mus. 16: I99. 
Type-species: Neviasca tespisalis Walker, [1859], by monotypy.

A junior homonym of Neviasca Walker, [1859], ibidem 16: 7,-Lepid., Noctuidae. The objective replacement name is Cardalena Walker, 1859.

GABRISA Walker, [1866] I865, List Specimens lepid. Insects Colln Br. Mus. 34: I I 74.

Proposed, unnecessarily, as an objective replacement name for Neviasca Walker, [1859], ibidem 16: 199. The objective replacement name Cardalena Walker, I 859, had already been proposed.

CARDEPIA Hampson, 1905, Cat. Lepid. Phalaenae Br. Mus. 5: x, 234.

$\mathrm{HADE}$

Type-species: Mamestra irrisoria Ershov, 1874 , in Fedchenko, Reise in Turkestan 2 (5) $3: 42, \mathrm{I} 28, \mathrm{pl} .4$, fig. 53, by original designation. Syntypes ${ }^{7}$, q, [U.S.S.R.]: Turkestan town.

$M$. irrisoria is a junior subjective synonym of Hadena sociabilis Graslin, I850, Ammls Soc. ent. Fr. (2) 8: 4I2, pl. 10, fig. I3.

CARDIESTRA Boursin, I963, Z. wien. ent. Ges. 48: 86.

HADE

Type-species: Mamestra eremistis Püngeler, 1904, Societas ent. 19: I30, by original designation. Syntypes ơ. [U.S.S.R.]: [Turkmenistan], Merw (MNHU, Berlin).

CARDIOSACE Hampson, I gIo, Cat. Lepid. Phalaenae Br. Mus. 10: xviii, 706.

ACON

Type-species: Tarache sphendonistis Hampson, I902, Ann. S. Afr. Mus. 2: 384, by original designation. Syntypes + , [South Africa]: Natal, Estcourt (BMNH). [Lesotho] Basutoland: Maseru.

CAREA Walker, [1857] I856, List Specimens lepid. Insects Colln Br. Mus. 10: 4I3, 474. chlo Type-species: Carea varipes Walker, [1857] 1856, ibidem 10: 475, by monotypy. Lectotype ô. [West Malaysia]: [Malaya], Malacca (UM, Oxford), designated (as type) by Swinhoe, I900, Cat. east. and Aust. Lepid. Heterocera 2: 100.

$\ddagger$ CARCA; Pagenstecher, 1909, Geogr. Verbreitung Schmett.: 424 .

An incorrect subsequent spelling.

CAREADES Bethune-Baker, 1906, Novit. zool. 13: 243.

CHLO

Type-species: Careades sanguinea Bethune-Baker, 1906, ibidem 13: 243, by original designation. Syntypes ô, + , NEw Guinea: [Papua], Ekeikei (BMNH).

C. sanguinea is a junior subjective synonym of Thyrsoscelis huntei Warren, 1903, Novit. zool. 10: 123.

CARELIS Bowden, 1956, Bull. ent. Res. 47: 4 I6.

ACRO

Type-species: Carelis albula Bowden, I956, ibidem 47: 4I6, figs I-5, by original designation. Holotype $\tilde{\sigma}$, [GHana] Gold Coast: Kumasi, Kwadaso (BMNH).

CARILLADE Schaus, I913, Ann. Mag. nat. Hist. (8) 11: 39.

OPHI

Type-species: Carillade harmonia Schaus, I913, ibidem (8)11:39, by original designation. Type(s) $\hat{o}$, Costa Rica: Carillo (USNM, Washington).

CARIONA Swinhoe, I918, Ann. Mag. nat. Hist. (9)2:83.

CATO

Type-species: Erebus albicinctus Kollar, 1844, in Hügel, Kaschmir und das Reich der Siek 4: 474, pl. 22, by original designation. Holotype, [INDIA]: Himalayas, [Mussoorie] Massuri.

CARISSA VValker, [1863] I864, J. Proc. Linn. Soc. (Zool.) 7: I68.

OPHI

Type-species: Carissa cossoides Walker, [1863] I864, ibidem 7: I68, by monotypy. Lectotype $\tilde{0}$. Borneo: Sarawak (UM, Oxford), designated (as type) by Swinhoe, I9oo, Cat. east. and Aust. Lepid. Heterocera 2: 104.

CARMARA Walker, [1863] i864, J. Proc. Linn. Soc. (Zool.) 7: 62. $\operatorname{ACON}$ Type-species: Carmara subcervina Walker, [1863] 1864, ibidem 7:63, by monotypy. Lectotype +. Borneo: Sarawak (UM, Oxford), designated (as type) by Swinhoe, Igoo, Cat. east. and Aust. Lepid. Heterocera 2: 70.

See also Zagira Walker, r866; and Gyrognatha Hampson, 1893. 
CARNEADES Grote, 1883. See Paragrotis Dyar, 1902.

CAROGA Schaus, I906, Proc. U.S. natn. Mus. 30 : i io.

Type-species: Caroga costalis Schaus, I906, ibidem 30: I Io, by monotypy. Type(s), BRAzIL: Rio Janeiro (USNM, Washington).

CAROIA Walker, I858, List Specimens lepid. Insects Colln Br. Mius. 15: i861.

Type-species: Bagisara bombycoides Walker, i 858, ibidem 15: I792, by monotypy (of Bagisara Walker, I 858, ibidem 15: I791).

Caroia was proposed as the objective replacement name for Bagisara Walker, I 858 , ibidem 15: I 79I.

Caroia was originally placed in the Noctuidae.

BAGISARA Walker, I 858 , List Specimens lepid. Insects Colln Br. Mus. 15: I79I.

Type-species: Bagisara bombycoides Walker, i 858 , by monotypy.

A junior homonym of Bagisara Walker, i 858 , ibidem 15: I770, - Lepid., Noctuidae. The objective replacement name is Caroia Walker, 1858 .

CARPHERIA Hampson, 1926, Descr. new Genera Species Lepid. Phalaenae Subfamily Noctuinae Br. Mus.: 60o.

OPHI

Type-species: Zethes aroa Bethune-Baker, 1906, Novit. zool. 13: 273, by original designation. Holotype, NEw Guinea: [Papua], R. Aroa (BMNH).

CARPHOLITHIA Butler, I 882, Trans. ent. Soc. Lond. 1882: 426.

OPHI

Type-species: Carpholithia cinerea Butler, I882, ibidem 1882: 426 , by PRESENT DESIGNATION. Syntypes, CHILI: Las Zorras (BMINH).

CARSINA Hampson, I924, in Tams, J. nat. Hist. Soc. Siam 6: 251.

OPHI

Type-species: Phurys obliqua Mloore, I 867, Proc. zool. Soc. Lond. 1867: 8o, by original designation. Type(s) ô, INdia: Bengal (BMNH).

CARTEIA Walker, [1863] i 864, J. Proc. Linn. Soc. (Zool.) 7: 82.

OPHI

Type-species: Carteia nebulilinea Walker, [1863] I864, ibidem 7: 83, by monotypy. Lectotype 9, Borneo: Sarawak (UM, Oxford), designated (as type) by Swinhoe, Igoo, Cat. east and Aust. Lepid. Heterocera 2: I50.

CARTERIS Dognin, I9I4, Hétérocères nouv. Am. Sud. 8: 65.

HYPE

Type-series: Carteris incana Dognin, I9I4, ibidem 8: 66, by original designation Holotype ô. PERU: Rio Colorado, $2500 \mathrm{~m}$.

CARTHARA Walker, I865, List Specimens lepid. Insects Colln Br. Mus. 33: 914. PyRALIDAE Type-species: Carthara albicosta Walker, 1865, ibidem 33: 915, by monotypy. Holotype $\hat{o},[$ [BraziL] : Amazon region (UM, Oxford).

CARYONOPERA Hampson, 1926, Descr. new Genera Species Lepid. Phalaenae Subfamily Noctuinae Br. Mus.: 230. OPHI

Type-species: Deinypena triangularis Bethune-Baker, I9I I, Amn. Mag. nat. Hist. (8) 8: 539, by original designation. Holotype ô., [NIGERIA]: Ioo miles N. of Lokoja (BMNH).

CASAMBA Walker, I866, List Specimens lepid. Insects Colln Br. Mus. 35: I919.

ACRO

Type-species: Casamba zonata Walker, I 866, ibidem 35: 1920, by monotypy. Holotype o., [BRAZIL]: Bahia.

CASANDRIA Walker, 1857, List Specimens lepid. Insects Colln Br. Mus. 11: 494, 6o6. Cato Type-species: Casandria emittens Walker, 1857, ibidem 11: 606, by monotypy. Holotype q. Jamaica (UM, Oxford).

Walker described a new species Casandria emittens on page 606 and another new species 
Agrotis emittens on page 737. Both species have extremely similar descriptions and were based on material from Jamaica 'in Mr Saunders' collection'. In this collection there is a specimen from Jamaica labelled 'emittens' in Walker's handwriting, and subsequently labelled 'Agrotis emittens Walker. Type Lep.: No. I833'. No separate type for Casandria emittens has been found. I have examined the specimen labelled emittens by Walker and it fits exactly the detailed original description of both species. It seems that Walker inadvertently described the same species twice and that the same specimen must be the type for both names.

Hampson, 1912, Cat. Lepid. Phalaenae Br. Mus. 11: 350, used Casandria as a genus in the Sarrothripinae, citing Casandria emittens Walker as the type-species. Hampson then described or redescribed with synonymy i 8 species in Casandria but emittens was not mentioned, apart from the type-species designation. At the end of his subfamily revision, on page 454, Hampson listed Casandria emittens in a list of unrecognized species. $\ddagger$ CASSANDRIA; Hampson, 1918, Novit. zool. 25: 199 .

An incorrect subsequent spelling.

CASPERIA Walker, I867, J. Proc. Linn. Soc. (Zool.) 9: I8I, I94.

HYPE

Type-species: Casperia erebipennis Walker, 1867, ibidem 9: 181, 194, by monotypy. Type(s) ô. [Colombia]: Bogota.

$\ddagger$ CASSANDRIA, misspelling. See Casandria Walker, I857.

CATO

CASSANIA Berio, 1972, Memorie Soc. ent. ital. 51 : I79.

NOCT

Type-species: Cassania hyperacantha Berio, I972, ibidem 51 : 179, fig. 27, by original designation. Holotype $\hat{\jmath}$, Tanzania: Ikonda (Colln E. Berio, Genoa).

CA TABAPTA Hulst, I884, Bull. Brooklyn ent. Soc. 7: 49.

CATO

Type-species: Ephesia antinympha Hübner, [1823] I816, Verz. bekannter Schmett: 278, by subsequent designation by Hampson, I913, Cat. Lepid. Phalaenae Br. Mus. 12: I I. Holotype, [U.S.A.]: New York.

E. antinympha was the name proposed for Phalaena paranympha Linnaeus sensu Drury, I 773, Illust. nat. Hist. exot. Insects 1 (I770): 49, pl. 23, fig. 6, but without scientific names until the Index was issued with 2 (I 773 ).

CA TABENA Walker, i865, List Specimens lepid. Insects Colln Br. Mus. 32: 631.

ACRO

Type-species: Catabena lineolata Walker, 1865, ibidem $32: 63$ I, by monotypy. Holotype $\hat{0}$, TYPE-LOcAlity not known (BMNH) [U.S.A.].

See also Turbula Walker, I869; and Adipsophanes Grote, I873.

$\ddagger$ CATACOLA, misspelling. See Catocala Schrank, I802.

CATO

CATADA Walker, [1859] I 858, List Specimens lepid. Insects Colln Br. Mus. 16: 209. HyPE Type-species: Catada glomeralis Walker, [1859] 1858, ibidem 16: 2 I0, by monotypy. Syntypes 2 으, CEYLON (BMNH).

C. glomeralis is a junior subjective synonym of Bocana vagalis Walker, [1859] I 858 , ibidem 16: I 72 .

Catada when originally proposed contained a second species, but this was doubtfully included and under the Code, Article 68(c) is not eligible for designation as type-species.

CATADELLA Strand, I919, Avch. Naturgesch. 83(A)(10): i6o.

HYPE

Type-species: Catada pyralistis Strand, I9I9, ibidem 83(A)(Io): I58, by monotypy. Holotype $\hat{\jmath}$. [TAIwan] Formosa: Suisharyo (DEI, Eberswalde).

Catadella was proposed as a subgenus of Catada Walker, [I 859] I 858.

$\ddagger$ CATADELPHA Hampson, no published reference found.

HYPE

$\ddagger$ CATADELTA Hampson, no published reference found.

HYPE 
CATADOIDES Bethune-Baker, I908, Novit. zool. 15: 2 I8.

Type-species: Catadoides punctata Bethune-Baker, 1908, ibidem 15: 219, by original designation. Type(s) ô, New Guinea: [Papua], Dinawa (BMNH).

CATALANA Viette, I954, Annls Mus. r. Congo Belge (4 ${ }^{\circ}$ 1: $55^{8}$.

OPH I

Type-species: Catalana vohilava Viette, I954, ibidem $\left(4^{\circ}\right)$ 1:559, figs I2, I3, by original designation. Holotype ô, Madagascar: S.E., Sandramora, Vohilava (MNHN, Paris).

CA TALOXIA Hampson, 1926, Descr. new Genera Species Lepid. Phalaenae Subfamily Noctuinae Br. Mus. : 295.

OPHI

Type-species: Cataloxia diagrapta Hampson, 1926, ibidem: 295, by original designation. Holotype , Borneo: [Sabah], Sandakan (BMNH).

CATAMECIA Staudinger, I897, Dt. ent. Z. Iris 10: 288.

ACRO

Type-species: Catamecia jordana Staudinger, I897, ibidem 10: 288, pl. 4, fig. 23, by monotypy. Syntypes $3 \hat{0}, 3$ q , [JoRdan] Palestine: Jordan valley (MNHU, Berlin).

C. jordana is a junior subjective synonym of Apamea minima Swinhoe, I889, Proc. zool. Soc. Lond. 1889: 410.

CATAMELAS Rogenhofer, I874, in Felder \& Rogenhofer, Reise öst. Fregatte Novara (Zool.) 2 (Abt. 2): pl. II 9.

$\mathrm{OPHI}$

Type-species: Catamelas caripina Felder \& Rogenhofer, I874, ibidem 2 (Abt. 2): pl. 1 I9, fig. 2I, by monotypy. Type(s) ô., [Brazil]: R. Amazon (BMNH).

CATASEMA Staudinger, I 888, Stettin. ent. Ztg 49: 20.

CUCU

Type-species: Episema vulpina Staudinger, I888, ibidem 49: I8, by monotypy. Syntypes I Ő, I 오. [U.S.S.R.]: [Uzbekistan], Margelan (MNHU, Berlin).

Catasema was proposed as a subgenus of Episema Ochsenheimer, I8I6.

CATASTIA Hübner, [1825] I816, Vevz. bekannter Schmett.: 372.

PYRALIDAE

Type-species: Noctua marginea [Denis \& Schiffermüller], I775, Ankündung syst. Werkes Schmett. Wienergegend: 69, 3I7, (but included by Hübner as 'marginalis Schiff.', an unjustified emendation), by subsequent designation by Ragonot, 1893, in Romanoff, Mém. Lépid. $7: 479$.

DIOSIA Duponchel, 1832, in Godart \& Duponchel, Hist. nat. Lépid. Papillons Fr. 8(2):12, 278.

Type-species: Noctua marginea [Denis \& Schiffermüller], I775, by original designation, but cited as 'Pyralis marginalis [Denis \& Schiffermüller]', I775, an unjustified emendation.

Diosia Duponchel, 1832, was cited by Neave, 1939, Nomencl. zool. 2: 102 as a Noctuid, followed by a separate entry for Diosia Duponchel, I836, ibidem 10: I0, I43, as a Pyralid. The second entry was not a homonym but was a redescription and family transfer from Noctuidae to Pyralidae.

CA TEPHIA Ochsenheimer, i816, Schmett. Eur. 4: $94 \quad$ орні

Type-species: Noctua alchymista [Denis \& Schiffermüller], I775, Ankündung syst. Werkes Schmett. Wienergegend: 89, by subsequent designation by Duponchel, 1829 , in Godart \& Duponchel, Hist. nat. Lépid. Papillons Fr. 7(2): 72. Type(s), [AustriA]: Vienna district (Collection destroyed, Horn \& Kahle, 1936, Ent. Beih. Berl.-Dahlem 3: 243) $\ddagger_{\ddagger}$ CATOPHIA ; Walker, r 858, List Specimens lepid. Insects Colln Br. Mus. 15: i 81 o.

An incorrect subsequent spelling.

MAGEUTICA Hampson, I926, Descr. new Genera Species Lepid. Phalaenae Subfamily Noctuinae Br. Mus.: 64 .

Type-species: Noctua alchymista [Denis \& Schiffermiiller], I775, by original designation. Mageutica is a junior objective synonym of Catephia Ochsenheimer, I8I6.

CATEPHIODES Hampson, I905, Cat. Lepid. Phalaenae Br. Mus. 5: vii, 36.

HADE

Type-species: Hadena zuelana Schaus, I898, Jl N.Y. ent. Soc. 6: I43, by original designation. Syntypes 8 ex., Venezuela: Aroa. 
CATEPHIODES Bethune-Baker, I908. See Anophiodes Hampson, I9r3.

CATO

CATEPHIONA Hampson, 1926, Descr. new Genera Species Lepid. Phalaenae Subfamily Noctuinae Br. Mus. : 62.

OPHI

Type-species: Catephia lichenea Hampson, I895, Trans. ent. Soc. Lond. 1895: 306, by original designation. Holotype 9 , Bhutan (BMNH).

CA TOBLEMMA Hampson, I910, Cat. Lepid. Phalaenae Br. Mus. 10: vii, 36.

$\mathrm{ACON}$

Type-species: Catoblemma sumbavensis Hampson, I910, ibidem 10: 38, pl. I49, fig. 26, by original designation. Holotype ㅇ. [Indonesia]: Sumbawa [I.] (BMNH).

CATOCALA Schrank, I802, Fauna Boica 2 (2): I 58.

CATO

Type-species: Phalaena nupta Linnaeus, I767, Syst. Nat. (Edn I2) 1: 841, by subsequent designation by Curtis, I828, Br. Ent. 5: 217 . Syntypes, Germany. [N.W. Africa] Barbariae (LS, London).

$\ddagger$ †LEPHARUM Hübner, [1806], Tentamen determinationis digestionis . . . : [2].

Included in a work rejected for nomenclatural purposes by Int. Commn zool. Nom., I926, Smithson. misc. Collns 73 (4) Opinion 97: 19. Also idem, 1954, Opin. Decl. int. Commn zool. Nom. 6 Opinion 278: I40.

Only included species: Phalaena sponsa Linnaeus, I767, which is congeneric with Phalaena nupta Linnaeus, I 767.

$\ddagger$ CATACOLA ; Oken, I8 I 5, Okens Lehrbuch Naturg. 3 (I): 69 I.

An incorrect subsequent spelling.

$\ddagger$ BLEPHARA Ochsenheimer, I816, Schmett. Eur. 4: 94 .

Published as a junior synonym of Catocala Schrank, 1802 , and not subsequently treated as an available name under the Code, Article II (d).

$\ddagger C A T O C A L L A ;$ Hübner, [I823] I 8I6, Verz. bekannter Schmett. : 276.

An incorrect subsequent spelling.

CATOCALIRRHUS Andrews, I877. See Euparthenos Grote, I876. CATo

$\ddagger$ CATOCALLA, misspelling. See Catocala Schrank, I802. CATo

$\ddagger$ СA TOPHIA, misspelling. See Catephia Ochsenheimer, i8i6. OPH

CAULARIS Walker, [1858]1857, List Specimens lepid. Insects Colln Br. Muls.12: 772, 800.AGAR Type-species: Caularis undulans Walker, [1858] I 857, ibidem 12: 80I, by monotypy. Holotype $\hat{\sigma}$, [Dominican Republic] St. Domingo (BMNH).

CAUNINDA Moore, [1885] I887, Lepid. Ceylon 3: 190.

CATO

Type-species: Phalaena archesia Cramer, I780, Uitlandsche Kapellen 3: I45, pl. 273, figs F, G, by original designation. Syntypes ${ }^{\lambda}$, 우, [INDiA]: Côte de Coromandel.

$P$. archesia is dated from the wrapper of the part and not from the title-page of the volume, I782; it is also a junior subjective synonym of Noctua undata Fabricius, I775, Syst. Ent.: 600 .

See also Mocis Hübner, [1823].

CAUTAESCHRA Hampson, I910, Cat. Lepid. Phalaenae Br. Mus. 10: vii, 39. ACON

Type-species: Micraeschus ustipennis Hampson, 1894, Fauna Br. India (Moths) 2: 353, by original designation. Type(s) $q$, India: [Assam], Nagas (BMNH).

CAUTATHA Hampson, r9ıo, Cat. Lepid. Phalaenae Br. Mus. 10: 5, 284. ACON

Type-species: Cautatha phoenicea Hampson, I9 10, ibidem 10: 285, fig. 86, by original designation. Syntypes $30^{\hat{N}},[\mathrm{GHANA}$ ] Gold Coast: Kumasi (BMNH).

$\ddagger$ CAUTHATA ; Gaede, 1935, in Seitz, Gross-Schmett. Erde 15: I33.

An incorrect subsequent spelling in both the German and English editions.

$\ddagger$ CAUTHATA, misspelling. See Cautatha Hampson, I9io.

ACON 
CEA Grote, I883. See Sparkia nom. n.

CECHARISMENA Möschler, I 890, Abh. senckenb. naturforsch. Ges. 16: I64.

Type-species: Cecharismena nectarea Möschler, I890, ibidem 16; I65, by PRESENT DESIGNATION. Holotype f, [Puerto Rico] Portorico I.

CEILODIASTROPHON Bethune-Baker, I908, Novit. zool. 15: 2 I3.

HYPE

Type-species: Ceilodiastrophon brunneum Bethune-Baker, I9o8, ibidem 15: 213, by original designation. Holotype $\hat{\sigma}$, NEw Guınea: [Papua], Dinawa (BMNH).

CELAENA Stephens, I 829 [June], Nom. Br. Insects: 4 I.

ACRO

Type-species: Apamea haworthii Curtis, 1829, Br. Ent. 6: 260, pl. 260 (but included by Stephens as +'haworthi', an incorrect subsequent spelling), by subsequent designation by Westwood, I840, Synopsis Genera Br. Insects:95. Syntypes, Great BritaIN: [England], Whittlesea Mere; Windermere.

‡CELAENO; Herrich-Schäffer, I868, Corresp.-Bl. zool.-min. Ver. Regensburg 22: I53.

An incorrect subsequent spelling.

‡ELOEN A ; Druce, I89o, Proc. zool. Soc. Lond. 1890: 5 I 2.

An incorrect subsequent spelling.

$\ddagger$ †ELAENO, misspelling. See Celaena Stephens, I 829 .

ACRO

CELAMA Walker, I865, List Specimens lepid. Insects Colln Br. Mus. 32: 500.

NOLI

Type-species: Celama liparisalis Walker, I 865, ibidem 32: 500, by monotypy. Lectotype $\hat{o}$, Borneo: Sarawak (UMI, Oxford), designated (as type) by Swinhoe, I892, Cat. east. and Aust. Lepid. Heterocera 1: I32.

CELAMOIDES Eecke, I920, Zö̈l. Meded., Leiden 5: I 20,

NOLI

Type-species: Celamoides pseudastigma Eccke, I920, ibidem 5 : I 20, figs 8,9 , by original designation under the Code, Article 68(a)(i). Syntypes I 0 , 8 ㅇ, JAva: WV., Preanger; Malabar, Tjinjiroean.

The genus was originally proposed as \$Celamoides but has been corrected under the Code, Article 32(c)(i).

CELAZIA Joannis, I930, Annls Soc. ent. Fr. 98: 751.

NOLI

Type-species: Celazia bipuncta Joannis, I930, ibidem 98: 75I, by original designation. Holotype $\hat{\jmath}$. [North Vietnam]: Tonkin, Cha-pa (MNHN, Paris).

CELEOPS YCHE Butler, I879, Illust. typical Specimens Lepid. Heterocera Colln Br. Mus. 3 : 68. ACON

Type-species: Celeopsyche nitens Butler, I879, ibidem 3: 68, pl. 57, fig. Io, by original designation. Type(s), [JAPAN]: Yokohama (BMNH).

CELIPTERA Guenée, I 852, in Boisduval \& Guenée, Hist. nat. Insectes (Lépid.) 7: $308 . \quad$ cato Type-species: Celiptera frustulum Guenée, I852, ibidem 7: 308, by monotypy. Syntypes 0 , 우, America: northern. BraziL.

See also Litomitus Grote, I864.

CELLACRINA TA Bethune-Baker, I908, Novit. zool. 15: 2 I7.

Type-species: Cellacrinata grisea Bethune-Baker, I908, ibidem 15: 2I7, by original designation. Type(s) $َ$, NEw Guinea: [Papua], Kebea Range (BMNH).

$\ddagger$ CELOENA, misspelling. See Celaena Stephens, I 829.

ACRO

†CELSIA Stephens, I829 [June]. See Staurophora R.L., I8 I7.

CUCU

CELSIA Stephens, I829 [August]. See Staurophora R.L., I8I7.

CUCU 
CENTRARTHA Hampson, 1908, Cat. Lepid. Phalaenae Br. Mus. 7: 5. Available, but without included species until Hampson, I909, ibidem 8: 284 when republished as +'Centrarthra', an incorrect subsequent spelling.

ACRO

Type-species: Centrartha furcivitta Hampson, I909, ibidem 8: 284, fig. 80, but described as ¥'Centrarthra', by subsequent monotypy. Holotype + , [Sourt AfrICA]: Cape Colony, Deelfontein (BMNH).

Centrartha was the spelling originally used both in the phylogenetic table facing page 2, and in the Key to genera on page 5. There is no clear evidence of an inadvertent error; the original spelling should therefore be retained.

$\ddagger$ CENTRARTHRA; Hampson, i 9o9, Cat. Lepid. Phalaenae Br. Mus. 8: x, 284.

An incorrect subsequent spelling.

$\ddagger$ CENTRARTHRA, misspelling. See Centrartha Hampson, I908.

ACRO

CENTROCHLORA Dyar, I9i2, Proc. U.S. natn. Mus. 42:6I.

ACRO

Type-species: Centrochlora esmeralda Dyar, I9I2, ibidem 42: 6I, by original designation. Holotype ${ }^{\hat{*}}$, Mexico: Mexico City (USNM, Washington).

CENTROGONE Hampson, I9o8, Cat. Lepid. Phalaenae Br. Mus. 7: 4. Available, but without included species until Hampson, I910, ibidem 9: 223.

ACRO

Type-species: Centrogone chlorochrysa Hampson, I9Io, ibidem 9: 223, fig. 85, by subsequent monotypy. Holotype $\hat{\sigma}$, [Soutr AFRICA]: Transvaal, Johannesburg (BMNH).

CENTROPODIA Hampson, I908. See Scythocentropus Speiser, I902. ACRO

CENTROPUS Christoph, I889. See Scythocentropus Speiser, I902. ACRo

CEPARCHA Meyrick, i889, Trans. ent. Soc. Lond. 1889: $476 . \quad$ SARR

Type-species: Ceparcha cymatistis Meyrick, I889, ibidem 1889: 476, by monotypy. Syntypes $4(\widehat{\jmath}$, +$)$, NEw Guinea: [Papua], Mt Obree or adjoining ranges (BNNH).

†CEPHALOSPARGESTA, misspelling. See Cephalospargeta Möschler, I890. ACRO

CEPHALOSPARGETA Möschler, 1890, Abh. senckenb. naturforsch. Ges. 16: I19. ACRo

Type-species: Cephalospargeta elongata Möschler, I890, ibidem 16 : I 20, by monotypy. Syntypes $2 \hat{0}$, I , PUerto Rico.

$\ddagger$ CEPHALOSPARGEST A; Strand, I9I2, in Aurivillius \& Wagner, Lepid. Cat. 5: 73.

An incorrect subsequent spelling.

CEPHENA Moore, 1882, in Hewitson \& Moore, Descr. new Indian lepid. Insects Colln late Mr W. S. Atkinson: ig6.

OPHI

Type-species: Cephena costata Moore, I882, ibidem: 196, pl. 6, fig. I 7, by monotypy. Syntypes, India: [Assam], Khasia Hills; [W. Bengal], Darjiling (BMNH).

CERALA Hampson, i9i2. See Kerala Moore, I88x.

CHLO

CERAMICA Guenée, I852, in Boisduval \& Guenée, Hist nat. Insectes (Lépid.) 5: 343. HadE Type-species: Ceramica exusta Guenée, I 852, ibidem 5: 344, pl. 5, fig. 9, by subsequent designation by Grote, 1874 , Bull. Buffalo Soc. nat. Sci. 2: 22. Lectotype $\sigma^{\prime}$. North AMerica (MNHN, Paris), designated by Viette, I95I, Bull. mens. Soc. limn. Lyon 20: I60.

CERAPODA Smith, i 894, Trans. Am. ent. Soc. 21: 68, 88.

CUCU

Type-species: Cerapoda stylata Smith, I894, ibidem 21:69, 88, pl.2, fig. 4, by monotypy. Syntypes I 0 . 3 ㅇ, [U.S.A.]: Colorado.

Cerapoda was the spelling adopted by Dyar, I902, Bull. U.S. natn. Mus. 52: I28, as first reviser, from a multiple original spelling. $¥$ Ceropoda was used for the description of the genus and species on pp. 68 \& 69. Cerapoda was used on page 88 in the legend to pl. 2. $\ddagger$ †EROPODA; Smith, 1894, Trans. Am. ent. Soc. 21: 68 .

An incorrect original spelling. 
CERAPTER YX Curtis, I833, Br. Ent. 10:45I.

HADE

Type-species: Phalaena graminis Linnaeus, I758, Syst. Nat. (Edn Io) 1 : 506, by original designation. Type(s), Type-LOCALITy not stated (LS, London) [Europe].

CERAPTILA Guenée, I854, in Boisduval \& Guenée, Hist. nat. Insectes (Lépid.) 8: 24. HYPE Type-species: Ceraptila reniferalis Guenée, I 854 , ibidem $8: 24$, by monotypy. Lectotype $\hat{\sigma}$. [South Africa]: Cap de Bonne-Espérance (MNHN, Paris), designated by Viette, I95I, Bull. mens. Soc.linn. Lyon 20: I62.

CERASTIA Stephens, I850. See Cerastis Ochsenheimer, I8I6.

NOCT

CERASTIS Ochsenheimer, I8r6, Schmett. Eur. 4: 84 . NOCT

Type-species: Noctua rubricosa [Denis \& Schiffermüller], 1775, Ankündung syst. Werkes Schmett. Wienergegend: 77 , by subsequent designation by Duponchel, I 829 , in Godart \& Duponchel, Hist. nat. Lépid. Papillons Fr. 7(2): 72. Type(s), [Austria]: Vienna district (Collection destroyed, Horn \& Kahle, I936, Ent. Beih. Berl.-Dahlem 3: 243). $\ddagger$ GLAEAE Ochsenheimer, 1816, Schmett. Eur. 4:84.

Published as a junior synonym of Cerastis Ochsenheimer, I8I6, and not subsequently treated as an available name uncler the Code, Article I I (d).

CERASTIA Stephens, I850, List Specimens Br. Anim. Colln Br. Mus. 5: 79.

An unjustified emendation of Cerastis Ochsenheimer, I8I6.

MATUTA Grote, I874, Can. Ent. 6: II6.

Type-species: Matuta catherina Grote, I874, ibidem 6: II6, by monotypy. Type(s) ô. CANada: [L. Ontario], St. Catherines.

$M$. catherina is a junior subjective synonym of Hadena tenebrifera Walker, 1865 , List Specimens lepid. Insects Colln Br. Mus. 33: 727.

A junior homunym of Matuta Weber, I795, Nomencl. Ent. secundum Ent. Syst. ill. Fabricii: 92,-Crustacea. There is no objective replacement name but $M$. catherina is congeneric with Noctua nubricosa [Denis \& Schiffermüller], the type-species of Cerastis Ochsenheimer, I8r6. The latter is therefore available for use as a subjective replacement name.

CERATHOSIA Smith, I887, Entomologica am. 3: 79.

ACON

Type-species: Cerathosia tricolor Smith, I887, ibidem 3: 79, by monotypy. Syntypes, [U.S.A.]: Texas.

$\ddagger$ CERA TOPACHA, misspelling. See Tethea Ochsenheimer, I8I6.

THYATIRIDAE

CERATOPACHA Stephens, I850. See Tethea Ochsenheimer, I8I6.

THYATIRIDAE

$\ddagger$ CERATOSCOPA Hampson, no published reference found.

HYPE

CERA TOSTROTIA Warren, 1913, in Seitz, Gross-Schmett. Erde 11: 291.

$\mathrm{ACON}$

Type-species: Tarache melanchlaena Swinhoe, I89I, Trans. ent. Soc. Lond. 1891: I 48, by original designation. Syntypes ${ }^{*}$, 오, India: [Mysore], North Kanara (BMNH).

The date of publication of the part of Seitz containing page $29 \mathrm{I}$ was stated on page 289 as $191_{3}$ October $5_{\text {th }}$ in both the Enghish and German editions.

CERBIA Walker, I858, List Specimens lepid. Insects Colln Br. Muss. 14: I 343, I365.

Type-species: Cerbia fugitiva Walker, I 858, ibidem 14: ${ }_{3} 355$, by monotypy. Syntypes 3 ex., [INDiA]: Punjaub (BMNH); N. Hindostan.

C. fugitiva is a junior subjective synonym of Thria robusta Walker, [I858].

See also Thria Walker, [1858].

CERIGO Stephens, 1829. See Thalpophila Hübner, [1820].

ACRO

†CERINA; Vuillet, I91 I, Insecta 1: I67.

An incorrect subsequent spelling of Cirina Walker, 1855, List Specimens lepid. Insects 
Colln Br. Mus. 6: 1382,-Lepid., Saturniidae. Not of Cerma Hüibner, 1818, - Lepid., Noctuidae, as stated by Neave, 1939, Nomencl. zool. 1: 645 .

CERMA Hübner, I8I8, Zuträge Samml. exot. Schmett. 1: I4.

ACRO

Type-species: Cerma cora Hübner, I8I8, ibidem 1: I4, figs 59, 60, by monotypy. Type(s), [U.S.A.]: Georgia [formerly] in Florida.

$\ddagger$ ACHATIA Hübner, I 808, Erste Zuträge Samml. exot. Schmett.: 4, 6.

Included in a work rejected for nomenclatural purposes by Int. Commn zool. Nom., 1966, Bull. zool. Nom. 23 Opinion 789: 213. Placed on the Official Index of Rejected and Invalid Generic Names in Zoology: Name No. I825.

$\ddagger$ Achatia contained two nomenclaturally unavailable names of which $\ddagger A$. cora Hübner, I 808 , ibidem: 4 , has been designated and treated as a type-species by Berio, 1957, Memorie Soc. ent. ital. 36: 6.

CEROCALA Boisduval, I828, Eur. Lepid. Index meth.: ioo.

CATO

Type-species: Noctua scapulosa Hübner, [1808], Samml. eur. Schmett.4: pl. 77, fig. 360, by monotypy. Type(s), Europe.

CEROCTENA Guenée, I852, in Boisduval \& Guenée, Hist. nat. Insectes (Lépid.) 5: 9. OPHI

Type-species: Phalaena amynta Cramer, I779, Uitlandsche Kapellen 3: 10o, pl. 25 I, fig. E, by PRESENT DESIGNATION. Syntypes $\hat{0}$, 우, Surinam.

See also Betusa Walker, 1856.

CEROMACRA Guenée, I 852, in Boisduval \& Guenée, Hist. nat. Insectes (Lépid.) 7: 327. OPHI

Type-species: Phalaena tymber Cramer, I777, Uitlandsche Kapellen 2: 109, pl. I67, fig. D, by monotypy. Type(s), Surinam.

$P$. tymber is dated from the wrapper of the part and not from the title-page of the volume, I 779 .

CEROPACHA Stephens, I829. See Tethea Ochsenheimer, I8I6.

THYATIRIDAE

$\ddagger$ CEROPODA, misspelling. See Cerapoda Smith, i 894 .

CUCU

CERTILA Walker, I865, List Specimens lepid. Insects Colln Br. Mus. 32: 448.

OPHI

Type-species: Certila flexuosa Walker, I865, ibidem 32: 449, by monotypy. Syntypes $2 \hat{0}$. North America (BMNH).

C. flexuosa is a junior subjective synonym of Raphia abrupta Grote, i863, Proc. ent. Soc. Philad. 2: 336, pl. 8, fig. 3 .

CER YNEA Walker, I859, List Specimens lepid. Insects Colln Br. Mus. 19: 878.

ACON

Type-species: Cerynea omphisalis Walker, I 859, ibidem 19:878, by original designation. Lectotype $\hat{\delta}$. Borneo: Sarawak (UM, Oxford), designated (as type) by Swinhoe, I90o, Cat. east. and Aust. Lepid. Heterocera 2: 67.

CETOLA Walker, I 855, List Specimens lepid. Insects Colln Br. Mus. 5: 980, Ior 5. ACRo Type-species: Cetola dentata Walker, I855, ibidem 5: 10I6, by monotypy. Holotype o. NEPAL (BMNH).

CHABORA Walker, I865, List Specimens lepid. Insects Colln Br. Mus. 33: I II4. OPHI

Type-species: Chabora undulifera Walker, I865, ibidem 33: III4, by monotypy. Holotype $\hat{0}$. [Dominican Republic] St. Domingo (BMNH).

C. undulifera is a junior subjective synonym of Noctua versicolor Fabricius, I794, Ent. Syst. 3 (2): 49 .

CHABORA Walker, [1866] I 865, List Specimens lepid. Insects Colln Br. Mus. 34: I 84 . See Duriga Schaus, 1940.

$\mathrm{OPHI}$

CHABUATA Walker, [1858] I 857, List Specimens lepid. Insects Colln Br. Mus. 13: I022, I034. HADE

Type-species: Chabuata ampla Walker, [1858] 1857, ibidem 13: I034, by monotypy. Type.(s) ô. Brazil (UM, Oxford). 
CHADACA Walker, I858, List Specimens lepid. Insects Colln Br. Mus. 15: I 545, I6 fo. OPHI Type-species: Chadaca atrosignata Walker, $185^{8}$, ibidem 15: I64 I, by monotypy. Holotype , Venezuela (BIINH).

$\ddagger$ CHADOCA ; Neave, 1939, Nomencl. zool. 1: 654 .

An incorrect subsequent spelling.

${ }_{+}^{+}$CHADOCA, misspelling. See Chadaca Walker, I 858 . OPHI

CHAETAGLAEA Franclemont, 1943, Ent. News 54:94. cucu

Type-species: Chaetaglaea cerata Franclemont, 1943, ibidem 54: 94, fig. 2, by original designation. Holotype $\sigma^{\widehat{T}}$, [U.S.A.]: Connecticut, Mystic (Colln J. G. Franclemont, Ithaca, U.S.A.).

CHAETOLOMA Felder, I874, in Felder \& Rogenhofer, Reise öst. Fregatte Novara (Zool.) 2 (Abt. 2): pl. 99.

OPHI

Type-species: Chaetoloma actinobola Felder, 1874 , ibidem 2 (Abt. 2): pl. 99, fig. 20, by monotypy. Type(s) $\hat{\sigma},[$ BraziL]: R. Amazonas (in Explanation to pls 75-107:3).

C. actinobola is a junior subjective synonym of Sosxetra grata Walker, I 862.

See also Sosxetra Walker, i 862.

CHAETOSTEPHANA Jordan, I9I 3, in Seitz, Gross-Schmett. Erde 15: 20.

AGAR

Type-species: Metagarista rendalli Rothschild, 1896, Novit. zool. 3: 97, pl. I 4, fig. 2 I, by original designation. Holotype $\hat{\jmath}$. [Malawi] Br. Cent. Afr.: S. of L. Nyasa, Zomba (BMNH).

The date of publication of the part of Seitz containing page 20 was stated on page 15 as I9I3 December Ioth in both the German and English editions.

CHALADRA Walker, i 865, List Specimens lepid. Insects Colln Br. MIus. 32: 639.

CHLO Type-species: Chaladra cucullioides Walker, I865, ibidem 32: 640, by monotypy. Type(s) ô. [Moluccas] : Ceram [1.] (UM, Oxford).

C. cucullioides is a junior subjective synonym of Aiteta musculina Walker, i 856.

See also Aiteta Wálker, I 856.

CHALCAMISTIS Dyar, 1916, Proc. U.S. natn. Mus. 51: 17.

ACRO

Type-species: Chalcamistis autoplusia 1)yar, 1916, ibidem 51: I7, by original designation. Holotype ${ }^{\star}$, Mexico: Zacualpan (USNMI, Washington).

CHALCIOPE Hübner, [1823] i \& I6, Verz. bekannter Schmett. : 268.

CATO

Type-species: Phalaena mygdon Cramer, 1777, Uitlandsche Kapellen 2: 94, pl. I56, fig. Gr, by subsequent designation by Moore, [1885] i 887, Lepid. Ceylon 3: i 80. Type(s), TYPE-LOCALITY stated as Surinam, but this was probably an error as the species illustrated is not known to occur in the New World but is widespread in the Oriental Region.

EUCLIDISEMA Hampson, I9I3, Cat. Lepid. Phalaenae Br. Mus. 12: 9. Available, but without included species until Hampson, I913, ibidem 13: vii, 40.

Type-species: Phalaena mygdon Cramer, 1777, by subsequent designation by Hampson, I913, ibidem 13: 40.

Euclidisema is a junior objective synonym of Chalciope Hübner, [1823].

CHALCOECIA Hampson, 1908, Cat. Lepid. Phalaenae Br. Mus. 7: 9. Available, but without included species until Hampson, Io Io, ibidem 9: ix, 182. ACRO

Type-species: Anthoecia emessa Druce, I889, Biologia cent.-am. (Zool.) Lepid.

Heterocera 1: 300 , pl. 28, fig. 4, by subsequent monotypy. Holotype, Guatrarala: Pantaleon $1700 \mathrm{ft}(\mathrm{BMNH})$.

$\ddagger$ CHALCOESIA; Schaus, I9I I, Amn. Mag. nat. Hist. (8)7: 47 .

An incorrect subsequent spelling.

‡CHALCOESIA, misspelling. See Chalcoecia Hampson, in ro.

ACRO 
CHALCOPASTA Hampson, 1908, Cat. Lepid. Phalaenae Br. Mus. 7: 4. Available, but without included species until Hampson, I910, ibidem 9: x, 2 I8.

Type-species: Basilodes territans Edwards, 1884, Papilio 4: 45, by subsequent designation by Hampson, I9I0, ibidem 9: 2 I8. Syntypes 3 Õ. I + , [U.S.A.]: Arizona.

CHALENATA Walker, I864, List Specimens lepid. Insects Colln Br. Mus. 30: IOOI. ACON Type-species: Chalenata micaceella Walker, I864, ibidem 30: IooI, by monotypy. Holotype ㅇ, [Brazil]: [R. Amazon], [Tefé] Ega (BMNH).

$\ddagger$ CHALEPOTA; Grünberg, I912, Arch. Naturgesch. 77,(4) (2): I3 I.

An incorrect subsequent spelling.

$\ddagger$ CHALEPOTA, misspelling. See Chalenata Walker, I86 4 .

$\mathrm{ACON}$

CHALESTRA Walker, I859, List Specimens lepid. Insects Colln Br. Mus. 19: 852. OpH Type-species: Chalestra podaresalis Walker, I859, ibidem 19: 853, by monotypy. Type(s) ô, [South Africa]: Cape.

$\ddagger$ CHALICHARES, misspelling. See Chelichares Hampson, I9Io. ACON

CHAMAECLEA Grote, I883, Can. Ent. 15: 76.

ACRO

Type-species: Chariclea pernana Grote, I88I, Papilio 1: I55, by original designation.

Type(s), [U.S.A.]: Arizona, Tucson.

$\ddagger$ CHAMOCLEA; Hill, 1924, Bull. Sth. Calif. Acad. Sci. 23: i 58.

An incorrect subsequent spelling.

CHAMAEPORA Warren, I909. See Viminia Chapman, I890. ACRo

$\ddagger$ CHAMINA, misspelling. See Chamyna Hübner, [I $82 \mathrm{I}]$. OPH

$\ddagger$ CHAMOCLEA, misspelling. See Chamaeclea Grote, I 883 . ACRO

CHAMYLA Staudinger, r9oo, Dt. ent. Z. Iris. 12: $3+3$. Nocr

Type-species: Chamyla idia Staudinger, I9oo, ibidem 12:343, pl. 7, fig. 9, by monotypy. Syntypes I ô, 3 오, [China]: E. Tien Shan Mts; Mts near Korla.

C. idia is a junior subjective synonym of Isochlora arctomys Alphéraky, I897, in Romanoff, Mém. Lépid. 9: 43, pl. 2, figs i i, i 2.

CHAMYNA Hübner, [1821], Samml. exot. Schmett. 2: p]s [204], [205].

OPHI

Type-species: Chamyna homichlodes Hübner, [182 I], ibidem 2 : pl. [204], by subsequent designation by Hampson, 1926, Descr. new Genera Species Lepid. Phalaenae Subfamily Noctuinae $\mathrm{Br}$. Mus.: 463 , but cited for $\$ C h a m i n a$, an incorrect subsequent spelling.

Syntypes 0 , ㅇ, TYPE-LOCALITY not stated [BRAZIL].

See also Varia Walker, [1867].

$\ddagger$ CHAMINA; Hübner, [1823] I8I6, Verz. bekannter Schmett.: $27 \mathrm{I}$.

An incorrect subsequent spelling.

CHAMYRIS Guenée, 1852, in Boisduval \& Guenée, Hist. nat. Insectes (Lépid.) 6: 225. ACON

Type-species: Acontia cerintha Treitschke, I 826, Schmett. Eur. 5 (3): 240, by monotypy. Holotype 0 . Type-Localitr stated to be Spain, but as pointed out by Guenée, when he described Chamyris, this locality was probably incorrect as cerintha was common in U.S.A. but was not found in Europe (TM, Budapest).

CHAMYRISILLA Draudt, I950, Mitt. münch. ent. Ges. 40: I38.

ACON

Type-species: Chamyrisilla ampolleta Draudt, I950, ibidem 40: 138, pl. 8, fig. 29, by monotypy. Syntypes, China: [Chekiang Prov.], W. Tien-mu-shan (MAK, Bonn); [Hunan Prov.], Hoeng-shan.

CHANDATA Moore, I882, in Hewitson \& Moore, Descr. new. Indian lepid. Insects Colln late $\operatorname{Mr}$ IV.S. Atkinson: I I3.

Type-species: Chandata partita Moore, I882, ibidem: I I4, pl. 4, fig. I6, by monotypy. Syntypes $\tilde{\sigma},+$, India: [W. Bengal], Darjiling. 
CHANDICA Moore, I888, in Hewitson \& Moore, Descr. new Indian lepid. Insects Colln late Mr W. S. Atkinson: 286.

Type-species: Chandica quadripennis Moore, I888, ibidem: 286, by monotypy. Type(s) ô. India: [W. Bengal], Darjiling (MNHU, Berlin).

CHAOGRAPTIS Meyrick, I902, Trans. ent. Soc. Lond. 35: 42.

ACON

Type-species: Chaograptis crystallodes Meyrick, I902, ibidem 35: 42, by monotypy. Syntypes 3 , Australia: Qd, Duaringa.

CHARA Staudinger, I892, in Romanoff, Mém. Lépid. 6: 559.

ACON

Type-species: Chara albosignata Staudinger, I 892, ibidem 6: 559, pl. I4, fig. 2, by subsequent designation by Warren, 1913, in Seitz, Gross-Schmett. Erde 3: 402. Holotype O. [U.S.S.R.]: Amur district, Askold (MNHU, Berlin).

CHARACOMA Walker, i863, List Specimens lepid. Insects Collu Br. Mus. 27: Io6. SARR

Type-species: Characoma albulalis Walker, I863, ibidem 27: 107, by monotypy. Type(s) , Borneo: Sarawak (BMNH).

CHARADRA Walker, I865, List Specimens lepid. Insects Colln Br. Mus. 32: 445.

PANT

Type-species: Charadra contigua Walker, I865, ibidem 32: 446, by monotypy. Holotype $\delta$. TYPE-LOCALITY 'Georgia?' (BMNH). Locality probably correct as this species is known from U.S.A.: Georgia.

C. contigua is a junior subjective synonym of Acrouycta circulifera Walker, 1857 , ibidem 11: 709 .

CHARADRINA Agassiz, I846. See Caradrina Ochsenheimer, I816.

ACRO

CHARAEAS Stephens, I829. See Tholeva Hübner, [1821].

HADE

CHARANYCA Billberg, I 820, Enumeratio Insect. MIıs. G.J. Billberg: 85.

ACRO

Type-species: Noctua quercus Fabricius, I775, Syst. Ent.: 594, by subsequent designation by Boursin, 1965 , Bull. mens. Soc. limn. Lyon 34: i86. Type(s), Europe (Type(s) not found by Zimsen, I964, Type Material of I. C. Fabricius: 522).

$N$. quercus is a junior subjective synonym of Phalaena trigrammica Hufnagel, I766, Berlin. Mag. 3 (4): 408.

Incorrect type-species designation: Phalaena claripalpis Scopoli, I763, a name not originally included in Charanyca and not linked with one of the originally included names when cited by Tams, 1939, Entomologist 72: 137.

See also Meristis Hübner, [1821].

CHARELIA Sodoffsky, I837. See Anarta Ochsenheimer, I8I6.

HADE

CHARICLEA Curtis, I 825. See Periphanes Hübner, [I82I].

NOCT

CHARICLEA Stephens, 1829. See Periphanes Hübner, [1821].

NOCT

CHARIDEA Guenée, I852. See Compsotata Prout, I907.

CUCU

†CHARIELLA, misspelling. See Periphanes Hübner, [1821].

NOCT

CHARIERGES Draudt, I950, Mitt. mïnch. ent. Ges. 40: 59.

CUCU

Type-species: Chavierges nigralba Draudt, I9.50, ibidem $40: 60, \mathrm{pl} .4$, fig. I 4 , by original designation. Syntypes ô, CHind: [Yunnan Prov.], Ji-kiang; A-tun-tse (MAK, Bonn).

CHARILINA Walker, I854. See Crameria Hübner, [1819].

AGAR

CHARIPTERA Guenée, I 838. See Lamprosticta Hübner, [I820].

CUCU

CHARITOSEMIA Kiriakoff, I955, Bull. Inst. r. Sci.nat. Belg. 31(69): r.

AGAR

Type-species: Pristoceraea geraldi Kirby, I 896, Amm. Mag. nat. IIist. (6) 18: 377, pl. 19, fig. 3, by original designation. Holotype ${ }^{2}$, UGanna: Narogare (BMNH). 
$P$. gevaldi was originally proposed as $₫$ Protoceraea, an incorrect subsequent spelling.

$P$. gevaldi is a junior subjective synonym of Pristoceraea albigutta Karsch, I895, Ent. Nachr. 21: 357, pl. 2, fig. 2.

CHARMODIA Möschler, I883, Verh. zool.-bot. Ges. Wien 32 (Abh.): 356.

HYPE

Type-species: Charmodia vectis Möschler, I883, ibidem 32(Abh.): 357, pl. I8, fig. 42, by monotypy. Holotype $\widehat{\delta}$. Surinam: interior (MNHU, Berlin).

CHAROBLEMMA Dyar, I9I4, Proc. U.S. natn. Mus. 47: 19o. ACON

Type-species: Charoblemma unilinea Dyar, I9I4, ibidem 47: 190, by original designation. Holotype $q$, Panama: La Chorrera (USNM, Washington).

¥CHASINIA Hampson, no published reference found.

HYPE

CHASMINA Walker, I856, List Specimens lepid. Insects Colln Br. Mus. 9: 69, I33, I46. Acro

Type-species: Chasmina cygnus Walker, I 856 , ibidem 9 : I 47 , by subsequent designation by Hampson, I910, Cat. Lepid. Phalaenae Br. Mus. 9: 35I, but cited as tibialis Fabricius, I775, which Hampson placed (on page 353) as the senior synonym of cygnus Walker. Syntypes 5 ex., India: North (BMNH). 578.

C. cygnus is a junior subjective synonym of Bombyx tibialis Fabricius, I775, Syst. Ent.:

CHASMINODES Hampson, I908, Cat. Lepid. Phalaenae Br. Mus. 7: 4. Available, but without included species until Hampson, I910, ibidem 9: xii, 349. ACRO

Type-species: Acontia albonitens Bremer, I86I, Bull. Acad. imp. Sci. St. Petersb. 3: 490, by subsequent designation by Hampson, I910, ibidem 9: 349. Syntypes, [U.S.S.R.]: Amur, Ussuri above the Ema.

CHECUPA Moore, 1867, Proc. zool. Soc. Lond. 1867: 60.

ACRO

Type-species: Checupa fortissima Moore, I 867, ibidem 1867: 60, pl. 6, fig. 5, by monotypy. Syntypes $\hat{\sigma}$,, India: [W.] Bengal, Darjeeling (BMNH).

CHEILLOPHOTA Bethune-Baker, I 908, Novit. zool. 15: 217.

HYPE

Type-species: Cheillophota costistrigata Bethune-Baker, I908, ibidem 15: 217, by original designation. Type(s) $\widehat{\jmath}$, New Guinea: [Papua], Kebea Range (BMNH).

CHEIROPHANES Boursin, 1955, Z. wien. ent. Ges. 40: I 70.

OPHI

Type-species: Amphipyra ligaminosa Eversmann, I85I, Bull. Soc. Nat. Moscou 24: 630, by original designation. Syntypes, [U.S.S.R.]: Georgia; Armenia (ZI, Leningrad).

CHELAPRORA Hampson, 1926, Descr. new Genera Species Lepid. Phalaenae Subfamily Noctuinae Br. Mus.: 84 . OPHI

Type-species: Chelaprora dichroa Hampson, 1926, ibidem: 84 , by original designation. Holotype $\hat{\sigma}$, Australia: W. Aust., R. Sherlock (BMNH).

CHELECALA Hampson, I913, Cat. Lepid. Phalaenae Br. Mus. 12: ix, 240.

Type-species: Gnamptonyx trefoliata Butler, I898, Proc. zool. Soc. Lond. 1898: 423, by original designation. Holotype 9 , [KenYa] Br. E. Africa: between Voi and Ndi (BMNH).

CHELICHARES Hampson, I910, Cat. Lepid. Phalaenae Br. Mus, 10: 6, 718. ACoN

Type-species: Chelichares nubifera Hampson, I9I0, ibidem 10: 7I8, fig. 205, by original designation. Holotype $\widehat{0}$. Argentina: Metan (BMNH).

$\ddagger$ CHALICHARES; Neave, 1939, Nomencl. zool. 1: 665 .

An incorrect subsequent spelling.

CHELIGALEA Hampson, I906, Cat. Lepid. Phalaenae Br. Mus. 6: vii, I3.

CUCU

Type-species: Cucullia scopariae Dorfmeister, I853, Verh. zool.-bot. Ver. Wien 3: 4I3, by original designation. Syntypes, [AUSTRIA]: Vienna district. 
CHELONOMORPHA Motschulsky, [1861] is6o, Etud. ent. 9: 30.

AGAR

Type-species: Chelonomorpha japana Motschulsky, [186I] 1860, ibidem 9: 30, by subsequent designation by Kirby, 1892, Synonymic Cat. Lepid. Heterocera 1: 30 , but cited as ‘japona', an incorrect subsequent spelling. Type(s), JAPAN.

${ }_{+}$CHILONOMORPHA; Pagenstecher, 1909, Geogr. Verbreitung Schmett. : 423.

An incorrect subsequent spelling.

CHERA Hübner, [1821] i 816, Verz. bekannter Schmett.: 2 I I.

HADE

Type-species: Polia serratilinea Ochsenheimer, I 816 , Schmett. Eur. 4: 74, by subsequent designation by Hampson, igoz, Ann.S. Afr. Mus. 2: 258. Type(s), Europe (TM, Budapest).

$P$. serratilinea was the name proposed for Noctua polyodon Linnaeus sensu Hübner, [1803], Samml. eur. Schmett. 4: pl. 78, fig. 365.

CHERSOTIS Boisduval, I840, Genera Index meth. Eur. Lepid.: 103.

NOCT

Type-species: Noctua rectangula [Denis \& Schiffermüller], 1775, Ankündung syst. Werkes Schmett. Wienergegend: 78 (but included by Boisduval as 'rectangula F.', an incorrect authorship), by subsequent designation by Duponchel, I843, in d'Orbigny, Dict. universel Hist. nat. 3: 47x, but cited as 'rectangula Fabr.'. Type(s), [Austria]: Vienna district (Collection destroyed, Horn \& Kahle, I936, Ent. Beih. Berl.-Dahlem 3: 243).

CHESIAS Treitschke, I 825, Schmett. Eur. 5(2): 437.

GEOMETRIDAE

Type-species: Phalaena spartiata Herbst, I 782, in Fuessly, Arch. Insektengesch. 2: pl. 5, by subsequent designation by Duponchel, I 29 , in Godart \& Duponchel, Hist. nat. Lépid. Papillons Fr. 7(2): I 12.

Chesias has been used as the original generic name for species which are now in the Noctuidae.

CHILKASA Swinhoc, i 885, Proc. zool. Soc. Lond. 1885: 852.

OPHI

Type-species: Chilkasa falcata Swinhoe, I 885 , ibidem 1885: 853, pl. 56, fig. 2, by monotypy. Type(s) ô, [INDIA]: Bombay (BMNH).

Chilkasa was originally placed in the Geometridae.

See also Acygonia Roepke, 1948.

CHILODES Herrich-Schäffer, [1849] 1845, Syst. Bearb. Schmett. Eur. 2: 226.

ACRO

Type-species: Noctua ulvae Hübner, [1817], Samml. eur. Schmett. 4: pl. I39, figs 635, 636, by subsequent designation by Hampson, i910, Cat. Lepid. Phalaenae Br. Mus. 9: 321 , but cited as maritima Tauscher, i 806 , which Hampson placed as the senior synonym of ulvae Hïbner. Type(s), Europe.

N. ulvae is a junior subjective synonym of Noctua maritima Tauscher, iso6, Mém. Soc. imp. Nat. Moscou 1: 2 I I.

† CHILONOMORPHA, misspelling. See Chelonomorpha Motschulsky, [186I].

AGAR

CHIONOMERA Butler, I881, Trans. ent. Soc. Lond. 1881: i8.

CHLO

Type-species: Tyana superba Moore, 1867, Proc. zool. Soc. Lond. 1867: 668, pl. 33. fig. 15, by original designation. Type(s), InDra: [W.] Bengal, Darjeeling (BMNH).

CHIONOXANTHA Hampson, igl 4, Ann. Mag. nat. Hist. (8)13: 202.

$\operatorname{ACON}$

Type-species: Erastria staudingeri Standfuss, I892, in Romanoff, Mém. Lépid. 6: 667. pl. 15, fig. 7, by original designation (for Xantholeuca Hampson, igro). Holotype, [TURKEY]: [Kurdistan], Mardin.

Chionoxantha was proposed as the objective replacement name for Xantholeuca Hampson, I910.

XANTHOLEUCA Hampson, iq о, Cat. Lepid. Phalaenae Br. Mus. 10: xiii, 378.

Type-species: Errestria staudingeri Standfuss, I802, by original designation.

A junior homonym of Xantholeuca Stephens, 183 I, Illust. Br. Ent. Haustellata 3: 329 , 333, - Lepid., Noctuidae. The objective replacement name is Chionorantha Hampson, I 914. 
CHIRCONIA Schaus, I9I6, Proc. U.S. natn. Mus. 50:319.

OPHI

Type-species: Plusiodonta anartoides Walker, I865, List Specimens Lepid. Insects

Colln Br. Mus. 33: 843, by original designation. Holotype $q$, Venezuela (BMNH).

CHIRIPHA Walker, I856, List Specimens lepid. Insects Colln Br. Mus. 9: I65, 200. ACro Type-species: Chiripha involuta WValker, I 856, ibidem 9: 20I, by monotypy. Lectotype $\sigma^{7}$, [INDIA]: Hindostan (UM, Oxford), designated (as type) by Swinhoe, Igoo, Cat. east. and Aust. Lepid. Heterocera 2: 27.

CHITASIDA Hampson, I926, Descr. new Genera Species Lepid. Phalaenae Subfamily Noctuinae Br. Mus.: 73 .

OPHI

Type-species: Catephia diplogramma Hampson, I905, Ann. S. Afr. Mus. 3: 432, by original designation. Syntypes $\hat{\sigma}$, + , [South Africa]: Transvaal, Potchefstroom district (BMNH); Lydenberg district (BMNH).

CHLAMIFERA Hübner, I822. See Nola Leach, [I8I5].

NOLI

†HLAMIPHORA Hübner, [I806]. See Nola Leach, [I8I5].

NOLI

CHLOANTHA Boisduval, Rambur \& Graslin, [1836] I832, Colln iconographique hist. Chenilles Eur.: Noctuélides text \& pl. 22, figs 5, 6.

NOCT

Type-species: Noctua hyperici [Denis \& Schiffermiiller], I775, Ankündung syst. Werkes Schmett. Wienergegend: 76, by monotypy. Type(s), [Austria]: Vienna district (Collection destroyed, Horn \& Kahle, I936, Ent. Beih. Berl.-Dahlem 3: 243).

The date of publication of Chloantha is accepted from Cowan, I970, J. Soc. Biblphy nat. Hist. $5: 323$.

$\ddagger C L O A N T H A$; Guenée, I839, Annls Soc. ent. Fr. 8: 509 .

An incorrect subsequent spelling. Although Guenée clearly attributed $\ddagger$ Cloantha to Boisduval and mentioned the original publication in the text, Staudinger, I90I, in Staudinger \& Rebel, Cat. Lepid. palaearct. Fanmengeb. 1: 183, used Chloantha Guenée, an incorrect authorship. Others have used $\ddagger$ Cloantha Guenée as a nomenclaturally available name.

CHLOANTHA Agassiz, I 846, Nomencl. zool. Index univl.: 82, 90.

Proposed as a new name for $\ddagger$ Cloantha; Guenée, I839.

A junior homonym of Chloantha Boisduval et al., [1 836], - Lepid., Noctuidae, which is also the objective replacement name.

CHLOANTHA Agassiz, I846. See Chloantha Boisduval et al., [1836]. Noct

$\ddagger$ CHLOEOPHORA, misspelling. See Pseudoips Hübner, I 822. CHLO

$\ddagger$ CHLOEPHILA Guilding, I 830, Phil. Mag. (N. S.) 7: 206.

PYRALIDAE

A nomen nudum; neither the generic name nor the included specific name was described and neither of the names has been used again for this concept.

CHLOEPHILA Costantini, I920, Atti Soc. Nat. Mat. (5)5: 4.

CHLO

Type-species: Hylophila fiorii Costantini, I9I I, ibidem (4)13: 8I, figs I, 2, by monotypy. Syntypes $\hat{\sigma}$, 웅. Italy: Modena plain; Lombardia.

Chloephila was proposed as \#'Chloëphila' which under the Code, Article 32, was an incorrect original spelling and must be changed.

H. fiorii is a junior subjective synonym of Pyralis fagana Fabricius, I78I, Species Insect.

2: 276 .

See also Pseudoips Hübner, I 822.

CHLOEPHORA Stephens, I827. See Pseudoips Hübner, I822.

CHLO

CHLOETHRIPA Hampson, I9I2, Cat. Lepid. Phalaenae Br. Mus. 11: x, 259.

SARR

Type-species: Sarrothripa chlorana Hampson, I896, Fauna Br. India (Moths) 4: 528, by original designation. Type(s) Ĵ. [SıккIM] Sikhim (BMNH). 
CHLORHODA Hampson, I9Io. See Prasinopyra Hampson, I9I4.

$\operatorname{ACON}$

CHLORIDEA Duncan [\& Westwood], I84I, in Jardine, Naturalist's Library (Edn I) 33 (Ent. 7): 198

Type-species: Phalaena rhexiae Smith, I797, in Smith \& Abbot, Nat. Hist.rarerlepid. Insects Georgia: 199, pl. I0o, by monotypy. Syntypes, [U.S.A.]: Georgia.

Chloridea was attributed to Westwood, by Sherborn, I924, Index Anim. (I80I-1850): I 240, and by Neave, 1939, Nomencl.zool.1:705. Although Westwood was not acknowledged by Duncan in the original description, justification can be found in Stainton, I885, Entomologist's mon. Mag. 21: I8I-I86, who quoted from a letter by Westwood. The difficulty can be overcome by attributing Westwood with anonymous junior authorship.

$P$. rhexiae is a junior subjective synonym of Noctua virescens Fabricius, [I777]. ASPILA Guenée, 1852, in Boisduval \& Guenée, Hist. nat. Insectes (Lépid.) 6: I 74 .

Type-species: Noctna vivescens Fabricius, [I777], Genera Insect.: 282, by subsequent designation by Hampson, 1903, Cat. Lepid. Phalaenae Br. Mus. 4: 34. Type(s), [West InDies]: [Virgin Is], [St. Croix] St. Crux (Type(s) not found by Zimsen, I964, Type Material of I. C. Fabricius: 532 ).

A junior homonym of Aspila Stephens, I834, Illust. Br. Ent. Haustellata 4: 104, - Lepid., Olethreutidae. There is no objective replacement name but $N$. virescens is the senior synonym of Phalaena rhexiae Smith, I797, the type-species of Chloridea Duncan [\& Westuood], I84I. The latter is thus available for use as a subjective replacement name.

CHLORIOLA Hampson, 1912, Cat. Lepid. Phalaenae Br. Mus. 11 : xiv, 515.

CHLO

Type-species: Nolasena gratissima Walker, [1863] 1864, J. Proc. Linn. Soc. (Zool.)

7: 75, by original designation. Lectotype , BonNEo: Sarawak (UM, Oxford), designated (as type) by Swinhoe, igoo, Cat. east. and Aust. Lepid. Heterocera 2: 590.

CHLOROCLEPTRIA Hampson, I903, Cat. Lepid. Phalaenae Br. Mus. 4: x, 50.

NOCT

Type-species: Schinia simplex Sinith, I891, Trans. Am. ent. Soc. 18: I29, by original designation. Syntypes, [U.S.A.]: Colorado (USNM, Washington).

CHLOROCODIA Hampson, igro, Cat. Lepid. Phalaenae Br. Mus. 10: 7, 350.

$\operatorname{ACON}$

Type-species: Chlorocodia olivescens Hampson, I9ro, ibidem 10:35I, fig. Io6, by original designation. Holotype $\widehat{\sigma}$, BrazIL: Rio Janeiro (BMNH).

CHLOROGNESIA Warren, I9I3, in Seitz, Gross-Schmett. Erde 11: I 50.

ACRO

Type-species: Ancara glaucochlora Hampson, I894, Fauna Br. India (Noths) 2: 226, by original designation. Holotype $\hat{0}$. [SIKkı $]$ Sikhim (BMNH).

The date of publication of the part of Seitz containing page r5o, was stated on page I 45 as I9I 3 January 2oth in both the German and English editions.

CHLOROGRAPTA Hampson, 1926, Descr. new Genera Species Lepid. Phalaenae Subfamily Noctuinae Br. MIus.: 596.

OPHI

Type-species: Pradiota variegata Walker, I866, List Specimens lepid. Insects Colln Br. Mus. 35: I 573, by original designation. Holotype . Sierra Leone (BMNH).

CHLOROPLAGA Hampson, I912, Cat. Lepid. Phalaenae Br. Mus. 11: xvi, $596 . \quad$ Chlo

Type-species: Acontia nygmia Swinhoe, I893, Ann. Mas. nat. Hist. (6) 12: 217 , by original designation. Syntypes $2 \hat{o}$, I $q$, Singapore (BMNH).

CHLOROTHRIX Dyar, I92I, Insecutor Inscit. menst. 9: 64.

ACRO

Type-species: Chlorothrix zothecaea Dyar, I92I, ibidem 9: 64, by monotypy. Holotype 9, Argentina: La Rioja (USNM, Washington).

CHLOROZADA Hampson, I9I 2, Cat. Lepid. Phalaenae Br. Mus. 11 : xiv, 517.

CHLO

Type-species: Erizada verna Hampson, 1902, Ann. S. Afr. Mus. 2: $3^{\text {I8 }}$, by original designation. Type(s) ô. [RHodesia]: Mashonaland, Salisbury (BMNH). 
CHLUMETIA Walker, [1866] I865, List Specimens lepid. Insects Colln Br. Mus. 34: I270. EUTE Type-species: Chlumetia guttiventris Walker, [1866] 1865, ibidem 34: I27I, by monotypy. Syntypes $2 \hat{0}$, CeYLON (BMNH).

Chlımetia was cited by Neave, 1939, Nomencl. zool. 1:710 as an objective replacement name for Nachaba Walker, I863. This is strictly incorrect as Chlumetia Walker was proposed without any mention of Nachaba. These genera are, however, subjective synonyms.

NACHABA Walker, I863, List Specimens lepid. Insects Colln Br. Mus. 27: I I3.

Type-species: Nachaba transversa Walker, I863, ibidem 27: II4, by monotypy. Holotype $\delta$, Type-Locality not known (BMNH) [CEyLon. India].

A junior homonym of Nachaba Walker, I859, ibidem 19: 834, - Lepid., Pyralidae. There is no objective replacement name but $N$. transversa is the senior synonym of Chlumetia guttiventris Walker, [1866], the type-species of Chlumetia Walker, [1866]. The latter is thus available for use as a subjective replacement name.

CHOBATA Walker, [1858] I 857, List Specimens lepid. Insects Colln Br. Mus. 12: 81 2, 837.

ACON

Type-species: Chobata discalis Walker, [1858] 1857, ibidem 12: 838, by monotypy. Holotype $\widehat{o}$. [Dominican Republic] St. Domingo (BMNH).

CHODDA Walker, [1863] 1864, J. Proc. Limn. Soc. (Zool.) 7: 64.

OPHI

Type-species: Chodda sordidula Walker, [1863] 1864, ibidem 7: 64, by monotypy. Lectotype ․, Borneo: Sarawak (UM, Oxford), designated (as type) by Swinhoe, I90o, Cat. east. and Aust. Lepid. Heterocera 2: I 5 I.

CHOEPHORA Grote \& Robinson, I868, Trans. Am. ent. Soc. 2: 199.

NOCT

Type-species: Choephora fungorum Grote \& Robinson, I 868, ibidem 2: 200, pl. 3, fig. 74, by monotypy. Syntypes $\hat{0}$, 우, [U.S.A.]: Atlantic district.

CHOEROPAIS Jordan, I9I3, in Seitz, Gross-Schmett. Evde 15: I3.

AGAR

Type-species: Pseudospiris jucunda Jordan, 1904, Novit. zool. 11: 444, by monotypy. Holotype $\hat{0}$. ANGola: North Bailundu (BMNH).

The date of publication of the part of Seitz containing page I 3 was stated on page 7 as I9I3 November 25th in both the German and English editions.

CHOLIMMA Walker, [I863] I864, J. Proc. Linn. Soc. (Zool.) 7: 6o.

OPHI

Type-species: Cholimma leucanioides Walker, [1863] I864, ibidem 7: 61, by subsequent designation by Hampson, 1926, Descr. new Genera Species Lepid. Phalaenae Subfamily Noctninae Br. Mus.: 252. Lectotype 오. BorNeO: Sarawak (UM, Oxford), designated (as type) by Swinhoe, 1900, Cat. east. and Aust. Lepid. Heterocera 2: 66.

$\ddagger$ CHOLOBOCHYLA, misspelling. See Colobochyla Hübner, [I825].

OPHI

CHOLUATA Walker, [1863] I864, J. Proc. Linn. Soc. (Zool.) 7: 57.

ACRO

Type-species: Choluata eburneifera Walker, [I863] I864, ibidem 7: 57, by monotypy. Lectotype $\hat{0}$. Borneo: Sarawak (UM, Oxford), designated (as type) by Swinhoe, I9oo, Cat. east. and Aust. Lepid. Heterocera 2: 39.

C. eburneifera is a junior subjective synonym of Borbotana nivifascia Walker, 1858 .

See also Borbotana Walker, I 858 .

CHOPARDIANA Viette, [1963] I962, Annls Soc. ent. Fr. 131: 257.

CUCU

Type-species: Perigea zebrina Viette, [1958] 1957, Bull. Soc. ent. Fr. 62: 273, by original designation. Holotype ô. MADAGASCAR: Route de Lakato, forêt d'Ankasoka (MNHN, Paris).

CHORA Walker, I862, J. Proc. Linn. Soc. (Zool.) 6: I88.

CHLO

Type-species: Chora repandens Walker, I862, ibidem 6: I88, by subsequent designation by Hampson, I9I2, Cat. Lepid. Phalaenae Br. Mus. 11: 542. Lectotype 우, Borneo: 
Sarawak (UM, Oxford), designated (as type) by Swinhoe, 1900, Cat. east. and Aust. Lepid. Heterocera 2: Ioo.

CHORIZAGROTIS Smith, I890, Bull. U.S. natn. Mus. 38: Io, 98.

NOCT

Type-species: Agrotis auxiliaris Grote, 1873, Bull. Buffalo Soc. nat. Sci. 1: 96, by original designation (on page Io). Type(s) ô, [U.S.A.]: Colorado Territory (BMNH).

CHORIZOMENA Turner, I939, Proc. R. Soc. Qd 50: I 4 S.

OPH I

Type-species: Chorizomena nivosa Turner, I939, ibidem 50: I48, by monotypy. Type(s) + , Australia: N. Qd, Cooktown, R. Endeavour (NMV, Melbourne).

CHORSIA Walker, [1863] i 864, J. Proc. Linn. Soc. (Zool.) 7: 59.

OPHI

Type-species: Chorsia maculosa Walker, [I863] I864, ibidem 7: 60, by monotypy. Lectotype ô, Borneo: Sarawak (UM, Oxford), designated (as type) by Swinhoe, I900, Cat. east. and Aust. Lepid. Heterocera 2: 587.

CHORTODES Tutt, I897, Entomologist 30: 284.

ACRO

Type-species: Acosmetia morrisii Dale, I837, in Morris, Naturalist (Mannd, eic.) 2 : 88, 505, by monotypy. Syntypes, [Great Britain]: [England], Dorsetshire, Charmouth.

${ }_{\ddagger}$ CHRYSAECIA, misspelling. See Chrysoecia Hampson, I910.

ACRO

CHRYSANYMPHA Grote, I896, Proc. Am. phil. Soc. (I 895) 34: 4 I7.

PLUS

Type-species: Leptina formosa Grote, I865, Proc. ent. Soc. Philad. 4: 323, by original designation. Type(s) ふે. [U.S.A.]: Mass., Lawrence.

CHRYSASPIDIA Hiibner, [1821]. See Plusia Ochsenheimer, 1816.

PLUS

CHRYSOCRASPEDA Swinhoe, i 893, Ann. Mag. nat. Hist. (6) 12: 157.

GEOMETRIDAE

Type-species: Chrysocraspeda cerasina Swinhoe, I 893, ibidem (6) 12 : I 57, by monotypy.

Chrysocraspeda has been used as the original generic name for species which are now in the Noctuidae.

${ }_{\ddagger}$ CHR YSODEICIS, misspelling. See Chrysodeixis Hübner, [I8zI].

PLUS

${ }_{\ddagger}$ CHRYSODEIXIA, misspelling. See Chrysodeixis Hübner, I82I.

PLUS

CHRYSODEIXIS Hübner, [I82I] I 816, Verz. bekannter Schmett.: $25^{2}$.

PLUS

Type-species: Phalaena chalcites Esper, I789, Die Schmett. 4 (2) Abschnitt I: pl. I41, fig. 3; [1798-1805], ibidem: 447 (but included by Huibner as ¥'chalcitis', an incorrect subsequent spelling), by subsequent designation by Dyar, I902, $J l N . Y$. ent. Soc. 10: $\delta$ I, for ‡Chrysodeiria Hübner, an incorrect subsequent spelling. Type(s) ô. ITALy: central.

$\ddagger$ CHR YSODEICIS; Hübner, [I 826] i 816, Verz. bekannter Schmett. Anzeiger: 37.

An incorrect subsequent spelling.

CHRYSODIXIS Agassiz, i846, Nomencl. zool. Index univl.: 84.

An unjustified emendation of Chry'sodeixis Hiibner, [I $82 \mathrm{r}]$.

†CHR YSODEIXIA; Dyar, I902, Jl N.Y. ent. Soc. 10:81.

An incorrect subsequent spelling.

CHRYSODIXIS Agassiz, I846. See Chrysodeixis Hübner, [1821].

PLUS

CHR YSOECIA Hampson, I908, Cat. Lepid. Phalaenae Br. Mus. 7: 9. Available, but without included species until Hampson, I9Io, ibidem 9: ix, I82.

ACRO

Type-species: Anthoecia scira Druce, I889, Biologia cent.-am. (Zool.) Lepid. Heterocera 1: 30 I, pl. 28, fig. 5, by subsequent monotypy. Holotype q. Mexico: Guerrero, Rincon (BMNH).

$\ddagger$ CHRYSAECIA; Neave, 1939, Nomencl. zool. 1: 724 .

An incorrect subsequent spelling. 
CHRYSOGRAPTA Hampson, 1926, Descr. new Genera Species Lepid. Phalaenae Subfamily Noctuinae Br. Mus.: 597

OPHI

Type-species: Egnasia igneola Swinhoe, 1890, Trans. ent. Soc. Lond. 1890: 254, by original designation. Syntypes, Burma: Rangoon (BMNH).

CHRYSONICARA Draudt, I937, in Seitz, Macrolepid. World 3 Suppl.: 262.

ACRO

Type-species: Chrysoptera aureus Bang-Haas, 1927, Horae Macrolepid. Reg. palaearct.

1: 9 I, pl. Io, fig. 42, by monotypy. Syntypes $3 \hat{0}$, China: S.W., Tsekou (MNHU, Berlin).

The date of publication of the part of Seitz containing page 262 was stated on page 257 as 1937 January I $^{\text {th }}$ in the English edition, and 1937 January I9th in the German edition.

CHRYSOPERA Hampson, I894, Fauna Br. India (Moths) 2: xx, 493.

OPHI

Type-species: Achaea combinans Walker, 1858, List Specimens lepid. Insects Colln Br. Mus. 14: I 399, by original designation. Holotype ô, CEYLoN (BMNH).

†CHRYSOPTERA Zincken, I8I7; Sherborn, 1924, Index Anim. (I801-1850): 1266.

LYCAENIDAE

An incorrect subsequent spelling of Chysoptera Zincken, I817, Allg. Lit.-Ztg, Halle [Jena] 3: 75, - Lepid., Lycaenidae.

CHRYSOPTERA Berthold, 1827. See Lamprotes R.L., I8I7.

PLUS

CHRYSORITHRUM Butler, 1878, Ann. Mag. nat. Hist. (5)1: 292.

$\mathrm{OPHI}$

Type-species: Catocala amata Bremer \& Grey, 1853, in Motschulsky, Etud. ent. 1: 66, by original designation. Type(s), [CHINA]: Pekin district.

CHRYSOZONATA Hampson, I9I4, Ann. Mag. nat. Hist. (8)13: 175.

ACON

Type-species: Chrysocraspeda latiflavaria Swinhoe, I904, Trans. ent. Soc. Lond. 1904: 562 , by original designation, but cited by Hampson as ¥'Crysocraspeda flavaria', an incorrect subsequent spelling of both the generic and specific names. Type(s) $q$, [MALAwI] Nyassaland: Likoma (BMNH).

CHUBUTIANA Köhler, (1952) I95I, Acta zool. lilloana 12: I 40.

CUCU

Type-species: Chubutiana nigripes Köhler, (1952) I95I, ibidem 12: $\mathrm{I}_{4} \mathrm{O}$, by original designation. Syntypes $\hat{\sigma},+$, Argentina: Chubut [Prov.], Comodoro Rivadavia (ZSBS, Munich).

CHUDUCA Walker, [1863] x864, J. Proc. Linn. Soc. (Zool.) 7: x64.

STIC

Type-species: Chuduca pyraloides Walker, [1863] 1864, ibidem 7: I65, by monotypy. Lectotype + , Borneo: Sarawak (UM, Oxford), designated (as type) by Swinhoe, I9oo, Cat. east. and Aust. Lepid. Heterocera 2: 93.

CHURIA Moore, I88I, Proc. zool. Soc. Lond. 1881: 359.

CHLO

Type-species: Churia nigrisigna Moore, I88I, ibidem 1881: 360, pl. 37, fig. $\mathrm{x}$, by original designation. Syntypes ô, + , [INDIA]: Calcutta (MNHU, Berlin).

CHUSARIS Walker, [I859] I 858, List Specimens. lepid Insects Colln Br. Mus. 16: 2I 5. HyPE Type-species: Chusaris retatalis Walker, [1859] 1858, ibidem 16: 2I 5, by monotypy. Holotype , CEYLON (BMNH).

CHUTAPHA Moore, $\mathrm{x} 882$, in Hewitson \& Moore, Descr. new Indian lepid. Insects Colln late Mr W. S. Atkinson: I3I.

ACRO

Type-species: Chutapha costalis Moore, I882, ibidem: I3I, by monotypy. Syntypes o, +, INDIA: Darjiling (BMNH).

CHYTOBRYA Draudt, 1950, Mitt. münch. ent. Ges. 40: I 5 .

ACRO

Type-species: Chytobrya bryophiloides Draudt, 1950, ibidem 40: 16, pl. 3, fig. 7, by original designation. Syntypes, China: [Yunnan Prov.], A-tun-tse; [Szechwan Prov.], Mien-shan (MAK, Bonn). 
CHYTOLITA Grote, I873 January, Trans. Am. ent. Soc. 4: 309.

HYPE

Type-species: Herminia morbidalis Guenée, I854, Hist. nat. Insectes (Lépid.) 8: 56, pl. 6, fig. 3 with caption $\ddagger H$. morbillosalis (an incorrect original spelling), by monotypy. Syntypes I ô, I \%, AMERICA: northern.

Chytolita was again described by Grote, I 873 May, Bull. Buffalo Soc. nat. Sci. 1: 39.

CHYTONIDIA Schaus, I914, Proc. U.S. natn. Mus. 46: 489.

ACRO

Type-species: Chytonidia chloristis Schaus, I9I4, ibidem 46: 489, by original designation. Type(s) , [French Guiana]: R. Maroni, St. Jean (USNM, Washington).

CHYTONIX Grote, 1874, Bull. Buffalo Soc. nat. Sci. 2: 14, 66. ACRO

Type-species: Apamea iaspis Guenée, I852, Hist. nat. Insectes (Lépid.) 5: 209, by original designation. Holotype ô. [U.S.A.]: Etat de New Yorck (BMNH).

$\ddagger$ CHYTONYX; Neave, 1939, Nomencl. zool 1: 733 .

An incorrect subsequent spelling by Neave who misquoted a correct use of Chytonix by Smith, I 894, Trans. Am. ent. Soc. $21: 42$.

†CHYTONYX, misspelling. See Chytonix Grote, 1874 .

$\Lambda \mathrm{CRO}$

†CHYTORHIZA, misspelling. See Chytoryza Grote, I876.

ACON

CHYTORYZA Grote, I876, Can. Ent. 8: 190.

ACON

Type-species: Chytoryza tecta Grote, I876, ibidem 8: I90, by monotypy. Syntypes o. ‥ [U.S.A.]: Texas (BMNH).

C. tecta is a junior subjective synonym of Perigea octo Guenée, 1852 .

See also Ilattia Walker, [1859]; and Stridova Walker, I869.

$\ddagger$ CHYTORIIIZA; Lucas, I902, Arch. Naturgesch. 65(2): 646 .

An incorrect subsequent spelling.

CIASA Waller, [I863] I 864, J. Proc. Limn. Soc. (Zool.) 7: I65.

STIC

Type-species: Ciasa pustulifera Walker, [1863] 1864, ibidem 7: 165, by monotypy. Lectotype $q$, Borneo: Sarawak (UMI, Oxford), designated (as type) by Swinhoe, I9oo, Cat. east. and Aust. Lepid. Heterocera 2: 92.

CIDARIPLURA Butler, I879, Ann. Mag. nat. Hist. (5) 4: 449.

HYPE

Type-species: Cidariplura gladiata Butler, I879, ibidem (5)4: 449, by original designation. Type(s), Japan (BMNH).

CILLA Grote, I880, N. Am. Ent. 1: 99.

OPHI

Type-species: Cilla distema Grote, 1880, ibidem 1: 99, by monotypy. Syntypes o. [U.S.A.]: Florida, Enterprise (BMNH); 'Texas, Bosque Co. (BMNH).

CIMELIA Lederer, I853. See Axia Hübner, [I813].

THYATIRIDAE

CINGALESA Hampson, I894, Fauna Br. India (Moths) 2: xv, 336.

ACON

Type-species: Microphysa strigicosta Hampson, 1893, Illust. typical Specimens Lepid. Heterocera Colln Br. Mus. 9: 20, Ioo, pl. I62, fig. I4, by original designation. Type(s) $q$, CEYLon: Pundaloya (BMNH).

CIRIS Grote, I863. See Xerociris Cockerell, 1904.

AGAR

CIRPHIS Walker, 1865, List Specimens lepid. Insects Colln Br. Mus. 32: 622.

HADE

Type-species: Cirphis costalis Walker, I865, ibidem 32: 623, by monotypy. Syntypes 2 o., [Australia]: Tasmania (BMNH).

†CIRRAEDIA, misspelling. See Cirroedia Guenée, I839.

CUCU

†CIRREDIA, nisspelling. See Cirroedia Guenće, I 839 .

cucu

$\ddagger$ CIRRHBOLINA, misspelling. See Cirrhobolina Grote, I875.

OPHI 
CIRRHIA Hübner, [1821] 1816, Verz. bekannter Schmett.: 234.

CUCU

Type-species: Phalaena fulvago Clerck 'sensu Linnaeus, I761, Fauna Suecica (Edn 2): 3I 2 [= Phalaena icteritia Hufnagel, 1766 , Berlin. Mag. 3: 296], by subsequent designation by Grote, I902, Allg. Z. Ent. 7: 398, who stated 'Der Typus ist die Art, welche Hübner Verz. als "fulvago Linn. Syst. Phal. I9o" bestimmt hat.' Syntypes of icteritia, Germany: Berlin, given in Berlin. Mag. 2: I 75 .

Phalaena fulvago Clerck, I759, Icones Insect. rariorum 1: pl. [6], fig. $\mathrm{I}_{5}$ and Phalaena fulvago Clerck sensu Linnaeus, I76r, are two congeneric species. Werneburg, I864, Beitr. Schmett. 1: 21 7, resolved the problem of synonymy and equated $P$. fulvago Clerck sensu Linnaeus with $P$. icteritia. This is a case of a misidentified type-species which, under the Code, Article 70 (a), should be referred to the Commission.

${ }_{+} X A N T H I A$ Hübner, [1806], Tentamen determinationis digestionis . . . : [I].

Included in a work rejected for nomenclatural purposes by Int. Commn zool. Nom., 1926, Smithson. misc. Collns 73 (4) Opinion 97: 19. Also idem, 1954, Opin. Decl. int. Commn zool. Nom. 6 Opinion 278: I 40.

Only included species: Phalaena fulvago Clerck sensu Linnaeus, i 76r.

CIRRHOBOLINA Grote, I875, Trans. Am. ent. Soc. 5 : i 7.

$\mathrm{OPHI}$

Type-species: Syneda deducta Morrison, 1875, Proc. Boston Soc. nat. Hist. 17: 220, by subsequent designation by Kirby, 1877, Zool. Rec. 12: 442 . Type(s), [U.S.A.]: Texas, Waco.

†CIRRHBOLINA; Dyar, 1902, Bull. U.S. natn. Mus. 52: 222.

An incorrect subsequent spelling.

CIRRHOIDIA Agassiz, I846. See Cirroedia Guenée, I839.

CUCU

$\ddagger$ CIRRHÖ̈DIA, misspelling. See Cirroedia Guenée, I839.

CUCU

†IRRHOPHANES, misspelling. See Cirrhophanus Grote, I872. ACRO

CIRRHOPHANUS Grote, I872, Can. Ent. 4: i 87.

$\mathrm{ACRO}$

Type-species: Cirrhophanus triangulifer Grote, I872, ibidem 4: I87, by monotypy. Holotype, [U.S.A.]: Missouri.

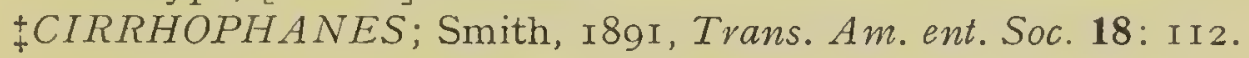

An incorrect subsequent spelling.

CIRRODES Hampson, Igio, Cat. Lepid. Phalaenae Br. Mus. 9: 6, 2 Io.

ACRO

Type-species: Cirrodes phoenicea Hampson, Igro, ibidem 9: 210, fig. 75, by original designation. Syntypes $\mathrm{I} \delta, \mathrm{x}$ ㅇ, [ZAmbIA] N. Rhodesia: Luangwa Distr. (BMNH).

CIRRODIANA Betlune-Baker, I9I I, Ann. Mag. nat. Hist. (8)8: 5 I5.

Type-species: Cirrodiana bella Bethune-Baker, I9I I, ibidem (8)8: $5^{1} 5$, by original designation. Holotype $\hat{\sigma}, \mathrm{W}$. Africa [Angola]: Malange (BMNH).

CIRRODISTIS Dyar, I9I2, Proc. U.S. natn. Mus. 42: $7 \mathrm{I}$.

ACRO

Type-species: Cirrodistis benedicta Dyar, I91 2, ibidem 42: 7I, by original designation. Holotype , Mexico: Zacualpan (USNM, Washington).

CIRROEDIA Guenée, 1839, Annls Soc. ent. Fr. 8: 489.

CUCU

Type-species: Noctua xerampelina Esper sensu Hübner, [1809], Samml. eur. Schmett. 4: pl. 9o, fig. 42 I [a misidentification described later in the same year as Noctua centrago Haworth, I809, Lepid. Br.: 236], by monotypy. Syntypes (of $N$. centrago), Great BRITAIN.

Haworth referred to 'xevampelina Hübner' in his description of $N$. centrago. Guenée originally included only one species which he cited as 'xerampelina Hüb., centrago Curt., Steph.'; but this usage was a misidentification of Phalaena xerampelina Esper, [I796], Die Schmett. 4 (Abschnitt 2): I5, pl. I83, fig. 4, and so is not nomenclaturally available. This is a case of a misidentified type-species which under the Code, Article 7o (a), should be referred to the Commission. 
+CIRRAEDIA; Doubleday, I 842, Entomologist 1: 378 .

Also Walker, 1858, List Specimens lepid. Insects Colln Br. Mus. 15: I7II.

An incorrect subsequent spelling.

$\ddagger$ CIRRHOËDIA; Agassiz, I 846, Nomencl. zool. Index univl.: 87 .

An incorrect subsequent spelling.

CIRRHOIDIA Agassiz, I846, Nomencl. zool. Index univl.: 87.

An unjustified emendation of Cirroedia Guenée, 1839 , but cited as $\ddagger$ Cirrhoëdia Guenée.

†CIRREDIA; Doubleday, [1849] I850, Synonymic List Br. Lepid. (Edn 2): 9.

An incorrect subsequent spelling; as $\ddagger$ Cirraedia in the Ist edition.

CISAUCULA Todd, ig66, Proc. U.S. natn. Mus. 120: I4.

AGAR

Type-species: Copidryas peruviana Druce, I9I0, Ann. Mag. nat. Hist. (8) 6: I68, by original designation. Lectotype $\delta$, PERU: S.E., Santo Domingo (BMNH), designated by Todd, I966, ibidem 120: I5.

CISSUSA Walker, I856, List Specimens lepid. Insects Colln Br. Mus. 9: I 37, I 53. OPH I

Type-species: Phalaena spadix Cramer, I780, Uitlandsche Kapellen 3: 149, pl. 275 , fig. F, by monotypy. Type(s), [U.S.A.]: Virginia.

$P$. spadix is dated from the wrapper of the part and not from the title-page of the volume, 1782 .

CITRIA Hübner, [1821]. See Xanthia Ochsenheimer, I8I6.

CUCU

CLADENIA Möschler, I88o, Verh.zool.-bot. Ges. Wien 30 (Abh.) : 474.

OPHI

Type-species: Cladenia mocha Möschler, r 880, ibidem 30 (Abh.): 474, pl. 9, fig. 32, by monotypy. Holotype $q$, Surinam: interior.

CLADOCEROTIS Hampson, I903, Cat. Lepid. Phalaenae Br. Mus. 4: xi, I38.

NOCT

Type-species: Heliophobus optabilis Boisduval, [1837] I834, Icones hist. Lepid. 2: pl. 74, figs 2, 3, by original designation. Syntypes 0 , ㅇ, EUROPE.

CLANYMA Guenée, I854. See Palthis Hübner, [1825].

HYPE

CLAPRA Möschler, I88o, Verh. zool.-bot. Ges. Wien 30 (Abh.) : 457.

OPHI

Type-species: Clapra asthenoides Möschler, I88o, ibidem 30 (Abh.) : 457, pl. 9, fig. 37,

by subsequent designation by Berio, I966, Annali Mus. civ. Stor. nat. Giacomo Doria 76: 52 .

Syntypes I $\tilde{\sigma}, \mathrm{I}$ ㅇ, Surinam: Paramaribo, and the interior (MNHU, Berlin).

CLAPRONIA Hampson, 1926, Descr. new Genera Species Lepid. Phalaenae Subfamily Noctuinae Br. Mus.: 3I6.

OPH I

Type-species: Clapronia albicilia Hampson, I926, ibidem: 3I 7 , by original designation. Holotype ô., British Gutana: Demerara (BMNH).

CLARGIA Schaus, I916, Proc. U.S. natn. Mus. 50: 3 I7.

OPHI

Type-species: Clargia bonema Schaus, I916, ibidem 50:317, by original designation. Type(s) $\precsim$, French Guiana: Cayenne (USNM, Washington).

CLATERNA Walker, I858, List Specimens lepid. Insects Colln Br. Mus. 15: I537, I 543. OPH

Type-species: Claterna exagens Valker, I858, ibidem 15: I 543, by monotypy. Holotype ō. [Sulawesi] Celebes (BMNH).

C. exagens is a junior subjective synonym of Phalaena cydonia Cramer, [1775] 1779 (see below).

TRIGONIA Guenée, I 854, in Boisduval \& Guenée, Hist. nat. Insectes (Lépid.) 8: I I.

Type-species: Phalaena cydonia Cramer, [I775] I779, Uitlandsche Kapellen 1: 34, pl. 22, fig. F, by monotypy, but cited as ‘'T. cydonialis Cr.', an incorrect subsequent spelling. Syntypes, TyPE-LOcality stated to be Surinam and [Guyana]: Berbice, but these were probably errors as the species illustrated is not known to occur in the New World but is widespread from India through to New Guinea. 
A junior homonym of Trigonia Bruguière, [I789] I792, Encycl. Méth. (Hist. nat. Vers.) 1 : xiv,-Mollusca. There is no objective replacement name but $P$. cydonia is conspecific with Claterna exagens Walker, 1858 , the type-species of Claterna Walker, 1858 . The latter is thus available for use as a subjective replacement name.

$\ddagger$ CLAVICORNIS Warren, no published reference found.

$\operatorname{ACON}$

CLAVIPALPA Joicey \& Talbot, I9I 5, in Joicey, Noakes \& Talbot, Trans. ent. Soc. Lond. 1915: 377.

ACRO

Type-species: Clavipalpa monogramma Joicey \& Talbot, I9I5, ibidem 1915: 378, pl. 6I, fig. 8, by original designation. Syntypes I ${ }^{2}, 2$ ㅇ, New Guinea: [West Irian], Angi Lakes (BMNH).

CORDYLEPALPA Joicey \& Talbot, I9I 7, Ann. Mag. nat. Hist. (8)20: 5 I .

Proposed, unnecessarily, as an objective replacement name for Clavipalpa Joicey \& Talbot, I9I5, which the authors considered to be a junior homonym, presumably of Clavipalpus Laporte, I833, - Coleoptera. No true homonym has been found.

CLAVIPALPULA Staudinger, I 892, in Romanoff, Mém. Lépid. 6: 499.

HADE

Type-species: Taeniocampa aurariae Oberthür, I880, Études Ent. 5: 76, pl. 3, fig. 6, by monotypy. Syntypes I $\cong$, I , [U.S.S.R.]: near Vladivostok, Askold I. (BMNH).

CLEDEOBIA Stephens, I829. See Synaphe Hübner, [1825].

PYRALIDAE

CLEOCERIS Boisduval, [1836] I834, Icones hist. Lépid. nouv. ou peu connus 2: pl. 71, fig. 2.

CUCU

Type-species: Phalaena scoriacea Esper, I789, Die Schmett. 3 (Suppl. I) Abschnitt 3: 22, pl. 83, figs 4, 5, by PRESENT DESIGNATION. Syntypes $\hat{\jmath},+$, Germany.

Both the nominal species originally included in Cleoceris are type-species of other genera. The first, P. scoriacea Esper, is the type-species of Derthisa Walker, I857, and so with the present designation, Derthisa becomes a junior objective synonym of Cleoceris. $P$. scoriacea is, however, congeneric with $P$. glaucina Esper, the type-species of Episema Ochsenheimer, I8I6; so Cleoceris and Derthisa are both junior subjective synonyms of Episema. The second of the nominal species originally included in Cleoceris was Noctua hybris Hübner, the type-species of Raphia Hübner, [1821], in the Ophiderinae.

Cleoceris was used by Duponchel, [1837] 1836, in Godart \& Duponchel, Hist. nat. Lépid. Papillons Fr. (Suppl.) 3: I57, for the nominal species scoriacea Esper, [1789], and saliceti Borkhausen, I792. Cleoceris was also used by Boisduval, I840, Genera Index meth. Eur. Lepid.: 93, for viminalis Fabricius, [1777], and oo Linnaeus, 1758. Both these usages have been cited over the years by authors as the original bibliographic reference of the proposal of Cleoceris. I have accepted the date of publication of Cleoceris Boisduval, [1836], from Cowan, 1970, J. Soc. Biblphy nat. Hist. 5: 300.

Incorrect type-species designation: Noctua viminalis Fabricius, a name not originally included in Cleoceris, and not linked with one of the originally included names when cited by Grote, 1874, Bull. Buffalo Soc. nat. Sci. 2: 25; and subsequently by other authors.

DERTHISA Walker, I857, List Specimens lepid. Insects Colln Br. Mus. 11: 495, 524.

Type-species: Phalaena scoriacea Esper, [I789], by subsequent designation by Hampson, 1906, Cat. Lepid. Phalaenae Br. Mus. 6: 229.

Derthisa is a junior objective synonym of Cleoceris Boisduval, [1836].

CLEONYMIA Berio, I966, Annali Mus. civ. Stor. nat. Giacomo Doria 76: 53.

CUCU

Type-species: Cleophana baetica Rambur, [1837], Faune ent. Andalousie 2: pl. I8, fig. 4, by original designation. Type(s) of, [Spain]: Andalusia.

CLEOPHANA Boisduval, Rambur \& Graslin, I832, Colln iconogr. hist. Chenilles: Noctuélides pl. 4 \& 4 pp. text; pl. $22 \& 2$ pp. text.

CUCU

Type-species: Phalaena casta Borkhausen, I793, in Scriba, Beitr. Insekten-Gesch. 3: 212, pl. I7, fig. 7, by subsequent designation by Hampson, I906, Cat. Lepid. Phalaenae Br. Mus. 6: I23. Syntypes, [France]: Languedoc (SM, Wiesbaden). 
P. casta was included in the genus by Boisduval et al. under Phalaena opalina Esper, [I796], in the text to pl. 4 .

Incorrect type-species designation: Noctua antirrhinii Hübner, [1803], a name not originally included in Cleophana and not linked with one of the originally included names when cited by Guenée, I852, in Boisduval \& Guenée, Hist. nat. Insectes (Lépid.) 6: I6I.

Incorrect type-species designation: Cleophana baetica Rambur, [1837], a name not originally included in Cleophana and not linked with one of the originally included names when cited by Hampson, Igo6, Cat. Lepid. Phalaenae Br. Mus. 6: 97.

Cleophana as used by Boisduval, I840, Genera Index meth. Eur. Lepid.: I 5 I, has been cited as a genus differing from that of 1832 , by Hampson, I906, Cat. Lepid. Phalaenae Br. Mus. 6: 97, I23. This is not justifiable as all three of the originally included nominal species were also included in the 18 fo concept, which was not proposed as a new genus but as an amplification of the 1832 concept. Boisduval, 1840 , gave the derivation of the name as 'Cleophanae Tr.' but no such published usage by Treitschke has been found.

RHABDOPHANA Sodoffsky, i837, Bull. Soc. imp. Nat. Moscou 1837 (6) : 88.

Proposed, unnecessarily, as an objective replacement name for Cleophana Boisduval, I 832, which Sodoffsky considered to be inappropriate.

CLEPTOMITA Grote, I873, Trans, Am. ent. Soc. 4: 301.

HYPE

Type-species: Cleptomita atrilineella Grote, I873, ibidem 4: 30I, by monotypy. Holotype ô, [U.S.A.]: Texas.

CLETHROPHORA Hampson, 1894, Fauna Br. India (Moths) 2: xviii, 4 I6.

CHLO

Type-species: Gonitis distincta Leech, I889, Proc. zool. Soc. Lond. 1889: 506, pl. 52, fig. 7 , by original designation. Syntypes 3 ex., JAPAN: Nagahama (BMNH). KorEA: Gensan.

CLETHRORASA Hampson, I908, Cat. Lepid. Phalaenae Br. Mus. 7: I5. Available, but without included species until Hampson, I9 Io, ibidem 9: xii, 343.

ACRO

Type-species: Leocyma pilcheri Hampson, I 896, Fauna Br. India (Moths) 4: 512, by subsequent monotypy. Type(s) ô. [Sikкim] Sikhim (BMNH).

$\ddagger$ CLETTHARA, misspelling. See Clettharra Walker, I863.

SARR

CLETTHARINA Hampson, I894, Fauna Br. India (Moths) 2: 365, 379.

SARR

Type-species: Clettharina nitens Hampson, I 894, ibidem 2: 379, fig. 210, by original designation. Type(s) ô, [BURMA $]$ : E. Pegu (BMNH).

CLETTHARRA Walker, I863, List Specimens lepid. Insects Colln Br. Mus. 27: Ior. SARR Type-species: Clettharra valida Walker, I863, ibidem 27: IOI, by monotypy. Type(s) ô., Borneo: Sarawak (BMNH).

C. valida is a junior subjective synonym of Nanaguna breviuscula Walker, 1863.

See also Nanaguna Walker, I863, ibidem 27: 85; Bagistana Valker, I864; and Thrypticodes Lucas, I89o.

†CLETTHARA; Moore, [1885] i887, Lepid. Ceylon 3: 105.

An incorrect subsequent spelling.

$\ddagger$ CLETTHORA; Swinhoe, I 890, Trans. ent. Soc. Lond. 1890: 237.

An incorrect subsequent spelling.

†CLETTHORA, misspelling. See Clettharra Walker, I863. SARR

CLIDIA Boisduval, [1837]. See Oxicesta Hübner, [1819]. cucu

CLINA Walker, [1865] 1864, List Specimens lepid. Insects Colln Br. Mus. 31:257. STic

Type-species: Clina lapidaria Walker, [1865] I864, ibidem 31: 257, by monotypy. Holotype . [IN1)IA]: S. Hindostan (BMNH). 
CLINOPHLEBIA Hampson, I893, Illust. typical Specimens Lepid. Heterocera Colln Br. Mus.

9: $17,92$.

Type-species: Clinophlebia sericea Hampson, I893, ibidem 9: I7, 92, pl. I6I, fig. 7. by monotypy. Type(s) ô, CEYlon: Colombo (BMNH).

C. sericea is a junior subjective synonym of Arbasera candida Walker, 1865.

See also Arbasera Walker, I865.

CLITIS Walker, [I858] I857, List Specimens lepid. Insects Colln Br. Mus. 12: 94I, 96I. AGAR Type-species: Phalaena proserpina Stoll, I782, in Cramer, Uitlandsche Kapellen 4: 239, pl. 399, fig. I, by monotypy. Type(s), Surinam.

See also Darceta Herrich-Schäffer, [1856].

$\ddagger$ CLOANTHA, misspelling. See Chloantha Boisduval, Rambur \& Graslin, [1836] I832. NOCT

CLONIATARPHES Schaus, I9I6, Proc. U.S. natn. Mus. 50:287. HYPE

Type-species: Cloniatarphes carunalis Schaus, I9I6, ibidem 50:288, by original designation. Type(s) $\hat{0}$, BrazIL: Petropolis (USNM, Washington).

CLOSTEROMORPHA Felder, I874, in Felder \& Rogenhofer, Reise öst. Fregatte Novara (Zool.) 2 (Abt. 2): pl. 83.

ACRO

Type-species: Closteromorpha reniplaga Felder, I874, ibidem 2 (Abt. 2): pl. 83, fig. I6, by monotypy. Type(s), BraziL (BMNH).

CLYTIE Hübner, [1823] I8I6, Verz. bekannter Schmett.: 267.

CATO

Type-species: Noctua illunaris Hübner, [1813], Samml. eır. Schmett. 4: pl. I22, fig. 565; and pl. 124, fig. 574, by subsequent designation by Hampson, 1913, Cat. Lepid. Phalaenae Br. Mus. 13: 289. Syntypes, Europe.

PSEUDOPHIA Guenée, I 852, in Boisduval \& Guenée, Hist. nat. Insectes (Lépid.) 7: 234.

Type-species: Noctua illunaris Hübner, [I 8 I3], by subsequent designation by Desmarest, (1857), in Chenu, Encycl. Hist. nat. Papillons nocturnes: I32.

Pseudophia is a junior objective synonym of Clytie Hübner, [1823].

CLYTOMORPHA Turner, I932, Trans. R. Soc. S. Aust. 56: I82.

OPHI

Type-species: Clytomorpha psilozona Turner, I932, ibidem 56: I82, by monotypy. Holotype $₫$, Australia: N. Qd, Kuranda (ANIC, Canberra).

CLYTOPHYLLA Turner, 1929, Trans. R. Soc. S. Aust. 53: 299.

CHLO

Type-species: Clytophylla artia Turner, I929, ibidem 53:300, by monotypy. Syntypes, Australia: I $\tilde{\sigma}$, I $q, Q d$, Bunya Mts (ANIC, Canberra); I ex., Qd, Toowoomba.

CLYTOSCOPA Turner, I93I, Proc. Linn. Soc. N.S.W. 56: 340.

ACON

Type-species: Clytoscopa iorrhoda Turner, I93I, ibidem 56: 340, by original designation. Holotype, Australia: Qd, Bunya Mts (QM, Brisbane).

CNEPHATA Billberg, I820. See Simyra Ochsenheimer, I8I6.

ACRO

CNEPHOZETA Billberg, I820. See Gortyna Ochsenheimer, I8I6.

ACRO

CNODIFRONTIA Hampson, igo2, Ann. S. Afr. Mus. 2: 375 .

ACRO

Type-species: Polydesma dissimilis Distant, I898, Ann. Mag. nat. Hist. (7) 1: 228, by original designation. Type(s), South Africa: Transvaal, Waterberg (BMNH).

COARICA Moore, I882, in Hewitson \& Moore, Descr. new Indian lepid. Insects Colln late Mr W. S. Atkinson: I53.

OPHI

Type-species: Coarica fasciata Moore, I882, ibidem: I53, pl. 5, fig. I, by monotypy. Type(s) ô, India: [W. Bengal], Darjiling.

COBALIODES Dyar, I902. See Cobalos Smith, I899.

ACRO 
COBALOS Smith, 1899, Jl $N^{\top}$.Y. ent. Soc. $7: 223$.

Type-species: Cobalos angelicus Smith, 1899, ibidem 7:223, by subsequent designation by Hampson, 1908, Cat. Lepid. Phalaenae Br. Mus. 7: 505. Syntypes I ô, 2 q, [U.S.A.]: California, Los Angeles.

COBALIODES Dyar, I902, Bull. U.S. natn. Mus. 52: I68.

Proposed, unnecessarily, as an objective replacement name for Cobalos Smith, I899, which Dyar considered to be a junior homonym of Cobalus Hübner, [I819] I8I6, Verz. bekannter Schmett.: I I 5, - Lepid., Rhopalocera.

COBUBA THA Walker, 1863, List Specimens lepid. Insects Colln Br. Mus. 27: I 26.

ACON

Type-species: Cobubatha metaspilaris Walker, I863, ibidem 27: I26, by monotypy. Holotype , [Dominican Republic] St. Domingo (BMINH).

COCCIDIPHAGA Spuler, 1907 March I6th, in Hofmann, Schmett. Eur. 1: 291.

ACON

Type-species: Erastria scitula Rambur, I833, Annls Soc.ent. Fr. 2: 26, pl. 2, fig. I6, by monotypy. Type(s), [Fra.NCE]: Corsica.

ZONESTHIOUSA Thierry-Xieg, I 907 September I 5th, Naturaliste (2)29: 2 I2.

Type-species: Erastria scitula Rambur, I833, by monotypy.

Zonesthiousa is a junior objective synonym of Coccidiphaga Spuler, I907.

$\ddagger$ COCYPARIS, misspelling. See Cacyparis Walker, [1863].

CHLO

COCYTIA Boisduval, r828, Essai Monogr. Zygénides: 22.

COCYTIIDAE

Type-species: Cocytia durvillii Boisduval, I 828 , ibidem: 22, pl. I, fig. I, by monotypy. Syntypes 3 ex., New Guivea.

RHOPTROPHALAENA Hampson, 1918, Novit.zool. 25: 366.

Proposed, unnecessarily, as an objective replacement name for Cocytia Boisduval, I828, which Hampson considered to be a homonym of Cocytius Hübner, [1819] I816, Verz. bekannter Schmett.: I 4 O, - Lepid., Sphingidae.

$\ddagger$ ROPTROPHALAENA; Neave, I940, Nomencl. zool. 4:83.

An incorrect subsequent spelling.

COCYTIA Treitschke, I835. See Brithys Hübner, [I82I].

HADE

COCYTODES Guenée, I 852, in Boisduval \& Guenée, Hist. nat. Insectes (Lépid.) 7: 41. OPH Type-species: Cocytodes coerula Guenée, I852, ibidem 7: 4I, pl. I3, fig. Io, by subsequent designation by Hampson, 1913, Cat. Lepid. Phalaenae Br. Mus. 12: 258, but cited as + caevulea, an incorrect subsequent spelling. Syntypes 0 . EAst INDIES (BMNH.)

CODONODES Hampson, I907, J. Bombay nat. Hist. Soc. 17:667.

OPH I

Type-species: Codonodes rectigramma Hampson, I907, ibidem 17: 667, fig., by monotypy. Syntypes ơ. q, India: Canara, Karwar (BMNH); Sikhim.

COELITES Turati, I926. See Coeloturatia Strand, I928.

$\triangle \mathrm{CON}$

COELOPHORIS Mabille, I9oo, Annls Soc. ent. Fr. 68: 733.

OPHI

Type-species: Coelophoris trilineata Mabille, I9oo, ibidem 68: 733, by monotypy.

Lectotype on. Madagascar: Baie d'Antongil (MNHN, Paris), designated (as holotype) by

Viette, I954, Mém. Inst. scient. Madagascar (E) $5: 384$.

†COLEOPHORIS; Viette, 1965, Bull. Soc. ent. Fr. 70: 152.

An incorrect subsequent spelling.

COELOTURATIA Strand, I928, Arch. Naturgesch. 92 (A)8: 74.

$\triangle \mathrm{CON}$

Type-species: Coelites patanei Turati, 1926, Atti Soc. ital. Sci.nat. 65: 47, fig. 9, by monotypy (of Coelites Turati). Syntypes 20 , [LiBYA]: Cirenaica.

Coeloturatia was proposed as the objective replacement name for Coelites Turati, r926. COELITES Turati, 1926, Atti Soc. ital. Sci.nat. 65: 45, fig. 8.

Type-species: Coelites patanei Turati, 1926, by monotypy. 
A junior homonym of Coelites Westwood, $185^{\circ}$, in Doubleday \& Westwood, Genera diumal Lepid. (2): pl. 66,-Lepid., Satyridae. The objective replacement name is Coeloturatia Strand, I928.

COENAGRIA Staudinger, I892, in Romanoff, Mém. Lépid. 6: 473

ACRO

Type-species: Tapinostola nana Staudinger, I 892, ibidem 6: 473, by monotypy. Syntypes 2 ㅇ. [U.S.S.R.]: Amur district, Raddefka; Ussuri.

Coenagria was proposed as a subgenus of Tapinostola Lederer, 1857.

COENIPETA Hübner, 1818, Zuträge Samml. exot. Schmett. 1: 22.

OPHI

Type-species: Coenipeta colliquens Hübner, I8I8, ibidem 1:22, figs II7, II8, by subsequent designation by Hampson, 1926, Descr. new Genera Species Lepid. Phalaenae Subfamily Noctuinae Br. Muss.: I Io. Type(s) $\hat{D}_{\text {, BrazIL. }}$

Incorrect type-species designation: Helia bibitrix Hübner, I823, a name not originally included in Coenipeta and not linked with one of the originally included names when cited by Desmarest, (1857), in Chenu, Encycl. Hist. nat. Papillons nocturnes: I 27.

COENOPETA Agassiz, I846, Nomencl. zool. Index univl.: 93.

An unjustified emendation of Coenipeta Hübner, I8I8.

$\ddagger$ CAENIPETA; Guenée, I 852, in Boisduval \& Guenée, Hist. nat. Insectes (Lépid.) 7: 29.

An incorrect subsequent spelling.

COENOBELA Hampson, 1926, Descr. new Genera Species Lepid. Phalaenae Subfamily Noctuinae Br. Mus.: 426.

OPHI

Type-species: Poaphila paucula Walker, I858, List Specimens lepid. Insects Colln Br. Mus, 15: 1838, by original designation. Holotype 우, Honduras (BMNH).

COENOBIA Stephens, i850, List Specimens Br. Anim. Colln Br. Mus. 5: I 34 . ACro

Type-species: Phytometra rufa Haworth, I809, Lepid. Br.: 260, by monotypy. Type(s), Great Britain.

COENOPETA Agassiz, I846. See Coenipeta Hübner, I8I8.

OPHI

COENOPHILA Stephens, 1850, List Specimens Br. Anim. Colln Br. Mus. 5: 74.

NOCT

Type-species: Graphiphora subrosea Stephens, I829, Illust. Br. Ent. (Haustellata) 2: I28, pl. I9, fig. I, by monotypy. Syntypes 4 ex., [Great Britain]: England, Whittlesea Mere.

$\ddagger$ CAENOPHILA; Walker, [1857] I 856, List Specimens lepid. Insects Colln Br. Mus. 10: 384 . An incorrect subsequent spelling.

COENOTOCA Turner, I903, Trans Proc. R. Soc. S. Aust. 27: 2.

AGAR

Type-species: Coenotoca monophyes Turner, I903, ibidem 27: 2, by monotypy.

Holotype 0 , Australia: N.S.W., Ballina (Richmond River) (ANIC, Canberra).

C. monophyes is a junior subjective synonym of Melanchroia subaspersa Walker, [1865] I 864, List Specimens lepid. Insects Colln Br. Mus. 31: 2 Iо.

COERIANA Walker, I 858, List Specimens lepid. Insects Colln Br. Mus. 15: 1852.

OPHI

Type-species: Coeriana clandestina Walker, I 858, ibidem 15: I852, by monotypy. Type(s) q, [BraziL]: Rio Janeiro (UM, Oxford).

COESA Walker, 1866, List Specimens lepid. Insects Colln Br. Mus. 35: I728.

NoLI

Type-species: Coesa viduella iValker, 1 866, ibidem 35: 1729, by monotypy. Holotype

$\hat{\jmath}$, [Australia]: Tasmania (BMNH).

C. viduella is a junior subjective synonym of Uraba lugens Walker, I863.

See also Uraba Walker, I863; and Toxoloma Felder, I874.

COLA Dyar, I9I 4, Proc. U.S. natn. Mus. 47: 219.

OPHI

Type-species: Cola nabis Dyar, I9I4, ibidem 47: 219, by original designation. Syntypes 2 ex., 'selected from a series of thirteen', Panama: Porto Bello; Canal Zone, Corozal; Cabina; La Chorrera. (USNM, Washington.) 
COLBUSA Walker, I865, List Specimens lepid. Insects Colln Br. Mus. 33: 978.

Type-species: Colbusa euclidica Walker, 1865, ibidem 33: 978, by monotypy. Syntypes $3(\hat{o}, \underline{q}),[$ GHANA] : Ashanti $(\mathrm{BMNH})$.

‡COLEOPHORIS, misspelling. See Coelophoris Mabille, Igoo.

OPHI

COLLOMENA Möschler, I890, Abh. senckenb. naturforsch. Ges. 16: I 45 .

SARR

Type-species: Collomena elota MIöschler, I89o, ibidem 16: I45, fig. I7, by monotypy. Syntypes $\hat{\jmath}, \underline{q}$, Puerto Rico.

COLOBOCHILA Agassiz, 1846. See Colobochyla Hübner, [1825].

OPHI

COLOBOCHYLA Hübner, [1825] I816, Verz. bekannter Schmett. : 344.

OPHI

Type-species: Pyralis salicalis [Denis \& Schiffermüller], 1775, Ankündung syst. Werkes Schmett. Wienergegend: 122, by monotypy. Type(s), [Austria]: Vienna district (Collection destroyed, Horn \& Kahle, 1936, Ent. Beih. Berl.-Dahlem 3: 243).

$\ddagger$ SALIA Hübner, [1806], Tentamen determinationis digestionis . . .:[2].

Included in a work rejected for nomenclatural purposes by Int. Commn zool. Nom., 1926, Smithson. misc. Collns 73 (4) Opinion 97: 19. Also idem, 1954, Opin. Decl. int. Commn zool. Nom. 6 Opinion 278: 140.

Only included species: Pyralis salicalis [Denis \& Schiffermüller], I775.

${ }_{\ddagger}$ CHOLOBOCHYLA; Hübner, [1826] I 816, Verz. bckannter Schmett. Anzeiger: 57.

An incorrect subsequent spelling.

MADOPA Stephens, i 829 [August], Syst. Cat. Br. Insects 2: i 59.

Type-species: Pyralis salicalis [Denis \& Schiffermüller], 1775, by monotypy.

Madopa is a junior objective synonym of Colobochyla Hübner, [I825].

COLOBOCHILA Agassiz, I 846, Nomencl. zool. Index univl.: 94.

An unjustified emendation of Colobochyla Hübner, [1 825].

${ }_{+}^{+C A L O B O C H Y L A}$; Walker, [1 859] I 858, List Specimens Lepid. Insects Colln Br. Mus. 16: I8. An incorrect subsequent spelling.

${ }_{\ddagger}$ COLOCAMPA, misspelling. See Xylena Ochsenheimer, I8I6.

CUCU

COLOCASIA Ochsenheimer, I 816, Schmett. Eur. 4: 63.

PANT

Type-species: Phalaena coryli Linnaeus, I758, Syst. Nat. (Edn ro) 1: 503, by subsequent designation by Samouelle, I8I9, Entomologist's useful Compendium: 250. Type(s), TY'PE-LOCALITY not stated (IS, London) [EUROPE].

See also Phineca Walker, 1856.

LEPTOSTOLA Billberg, I820, Enumeratio Insect. Mus. G. J. Billberg: 87.

Type-species: Phalaena coryli Linnaeus, $175^{8}$, by monotypy.

Leptostola is a junior objective synonym of Colocasia Ochsenheimer, 1816.

DEMAS Stephens, I829, Illust. Br. Ent. (Haustellata) 2: 59.

Type-species: Phalaena coryli Linnacus, I758, by inonotypy.

Demas is a junior objective synonym of Colocasia Ochsenheimer, I8I6.

$\ddagger$ CALOCASIA; Hampson, I9 13, Cat. Lepid. Phalaenac Br. Mus. 13: 360 .

An incorrect subsequent spelling.

COLOCASIODES Matsumura, I931, 6000 illust. Insects Japan-Empire: 782.

PANT

Type-species: Colocasiodes jezoensis Matsumura, I93I, ibidem: 782, fig., by monotypy. Type(s) ô. JapAN : Hokkaido (EIHU, Sapporo).

C. jezoensis is a junior subjective synonym of Diloba mus Oberthiir, r884, Etudes Ent. 10: 17, pl. 2, fig. 4 .

COLODES Schaus, 1914, Proc. IJ.S. natn. Mus. $46: 493$.

ACRO

Type-species: Colodes selecta Schaus, I914, ibidem 46:493, by original designation. Type(s) $\sigma^{\lambda}$, [French Gutana]: R. Maroni, St. Jean (USNM, Washington). 
COLPOCHEILOPTERYX Wallengren, 1865, K. svenska VetenskAkad. Handl. (N.F.) 5(4): 60 .

Type-species: Eurhipia operatrix Wallengren, I860, Wien. ent. Monatschr. 4: I70, by monotypy. Type(s), [Sourh AFrICA] Caffraria (NR, Stockholm).

COLPOSIA Hübner, [1823]. See Laspeyria Germar, i8 II.

OPH I

COMACHARA Franclemont, 1939, Ent. News 50: 216.

ARCTIIDAE

Type-species: Comachara cadburyi Franclemont, I939, ibidem 50: 216, fig., by original designation.

Originally placed in the Noctuidae; transferred to the Arctiidae, Lithosiinae by McDunnough, I943, Can. Ent. 75: 62.

COMETA Sodoffsky, I837. See Acronicta Ochsenheimer, i8i6.

ACRO

COMETASTER Hampson, I9I3, Cat. Lepid. Phalaenae Br. Mus. 12: x, 362.

CATO

Type-species: Spirama pyrula Hopffer, I857, in Peters, Mber. K. preuss. Akad. Wiss. 1857: 422, by original designation. Type(s), Mozambique.

COMOCRUS Jordan, I 896, in Rothschild \& Jordan, Novit. zool. 3: 40.

AGAR

Type-species: Agarista contorta Walker, [1865] 1864, List Specimens lepid. Insects Colln Br. Mus. 31: 45, by original designation, but cited as ¥'cortutus', an incorrect subsequent spelling. Syntypes $2 \hat{\sigma}$, Australia (BMNH).

A. contorta is a junior subjective synonym of Agarista behri Angas, 1847, South Aust. illust.: pl. 37, figs $21,22$.

COMODORIA Köhler, (I952) I95I, Acta zool. lilloana 12: I4I.

CUCU

Type-species: Comodoria splendida Köhler, (1952) I95I, ibidem 12: I42, by original designation. Holotype $\hat{\sigma}$. Argentina: Chubut [Prov.], Comodoro Rivadavia (ZSBS, Munich).

COMOPHORUS Alphéraky, I887. See Lycophonus Staudinger, I9oi.

NOCT

COMPLUTIA Walker, I869, Characters undescr. Lepid. Heterocera: $5_{8 .}$

OPHI

Type-species: Complutia transversa Walker, I869, ibidem: 59, by monotypy. Type(s) ô. [Honduras]: Limas.

COMPSENIA Dognin, I9I4, Hétérocères nouv. Am. Sud 8: 49.

HYPE

Type-species: Compsenia furtiva Dognin, I9I4, ibidem 8: 49, by monotypy. Holotype $\sigma^{3}$, Colombia: Cali, San Antonio.

COMPSENIA Schaus, I9i6, Proc. U.S. natn. Mus. 50:260, 274.

Type-species: Compsenia catagrapha Schaus, I9I6, ibidem $50: 275$, by original designation. Type(s) . . [French GuiAna]: R. Maroni, St. Jean (USNM, Washington).

A junior homonym of Compsenia Dognin, I9I4. This generic name was used and made available by Dognin prior to its proposal and generic description by Schaus, for a similar concept. There is no objective replacement name but C. catagrapha is congeneric with C. furtiva Dognin, I9I4, the type-species of Compsenia Dognin, I9I4. The latter is therefore available for use as a subjective replacement name.

COMPSENIA Schaus, I9I6. See Compsenia Dognin, I9I4.

HYPE

COMPSOPTERA Blanchard, I845, Hist. Insectes $2: 394,397$.

GEOMETRIDAE

Type-species: Geometra jourdanaria Serres, I 826, in Villiers, Mém. Soc. linn. Paris $5: 48$ o, pl. I I, fig. 3 , by original designation.

LIGIA Duponchel, I829, in Godart \& Duponchel, Hist. nat. Lépid. Papillons Fr. 7(2) : I07. 462.

Type-species: Geometra jourdanaria Serres, I826, by original designation. 
A junior homonym of Ligia Weber, I795,-Crustacea. The objective replacement name is Compsoptera Blanchard, I 845 .

Ligia has been used as the original generic name for species which are now in the Noctuidae.

COMPSOTATA Prout, I907, Entomologist 40: 109.

CUCU

Type-species: Charidea elegantissima Guenée, I852, in Boisduval \& Guenée, Hist. nat. Insectes (Lépid.) 6: 61, pl. 8, fig. I2, by subsequent designation (for Charidea Guenée, 1852) by Hampson, 1906, Cat. Lepid. Phalaenae Br. Mus. 6: I 40 . Syntypes 4 $(\hat{o},+)$, [South AFrica]: Cape of Good Hope.

Compsotata was proposed as the objective replacement name for Charidea Guenée, I 852. CHARIDEA Guenée, I 852, in Boisduval \& Guenée, Hist. nat. Insectes (Lépid.) 6: 60.

Type-species: Charidea elegantissima Guenée, I 852, by subsequent designation by Hampson, I906, Cat. Lepid. Phalaenae Br. Mus. 6: I40.

A junior homonym of Charidea Dalman, I816, $K$. svenska VetenskAkad. Handl. 1816: 225, - Lepid., Zygaenidae. The objective replacement name is Compsotata Prout, I907.

TIMAEA Saalmüller, I891, Lepid. Madagascar: 309.

Proposed as the objective replacement name for Charidea Guenée, I852; but Timaea Saalmüller, I89I, is itself a junior homonym of Timaea Walker, I863, List Specimens lepid. Insects Colln Br. Mus. 28: 520, - Lepid., Tineidae.

CONACONTIA Smith, I90o, Trans. Am. ent. Soc. $27: 49,55$.

ACON

Type-species: Conacontia flavicosta Smith, I900, ibidem 27: 58, by subsequent designation by Hampson, I9Io, Cat. Lepid. Phalaenae Br. Mus. 10: 702. Syntypes 50 , 2 ㅇ. [U.S.A.]: New Mexico, Hot Springs; Colorado; Montana, Black Hills.

CONCANA Walker, [1 858] 1857, List Specimens lepid. Insects Colln Br. Mus. 12: 880, 939.

OPHI

Type-species: Concana mundissima Walker, [1 858] i 857, ibidem 12 : 940 , by monotypy. Syntypes 20 , [Dominican Republic] St. Domingo (BMNH).

See also Thelidora Möschler, i 880 .

CONDATE Walker, I862, Trans. ent. Soc. Lond. (3)1: 99.

OPHI

Type-species: Condate hypenoides Wallker, I862, ibidem (3)1: 99, by monotypy. Type(s) ㅇ․ CHina.

CONDICA Walker, I856, List Specimens lepid. Insects Colln Br. Mus. 9: 214, 239. ACro

Type-species: Condica palpalis Walker, I 856 , ibidem 9: 240 , by monotypy. Holotype o., TYPE-Locality not stated (BMNH) [WESt Indies].

C. palpalis is a junior subjective synonym of Phalaena cupentia Cramer, 1779, Uitlandsche Kapellen 3: 103, pl. 252, fig. E.

CONICOCHYTA Hampson, I9I8, Novit. zool. 25: I 37.

ACRO

Type-species: Chytonix olivacea Wileman, I9I4, Entomologist 47: I65, by original

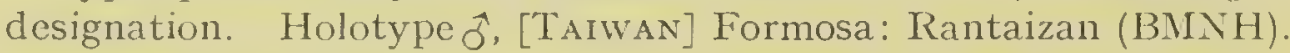

CONICOFRONTIA Hampson, I902, Ann. S. Afr. Mus. 2: 296.

ACRO

Type-species: Conicofrontia sesamoides Hampson, 1902, ibidem 2: 296, by monotypy. Holotype $q$, South Africa: Cape Colony, Transkei (BMNH).

CONICOPHORIA Matsumura, I929, Insecta matsum. 3: i I8.

ACRO

Type-species: Conicophoria formosana Matsumura, I929, ibidem 3: i 8, by original designation. Holotype 9 , [Tamwan] Formosa: [Taipei] Taihoku (EIHU, Sapporo).

CONISANIA Hampson, ig05, Cut. Lepid. Phalaenae Rr. Mus. 5: xiv, 472.

HADE

Type-species: Apamea leineri Freyer, I836, Nenere Beitr. Schmett. 2: 145, p1. I84, fig. 3, by original designation. Holotype, Type-Locality not stated. [Hungary, Austria]. 
CONISTRA Hübner, [I82I] I 8I6, Verz. bekannter Schmett.: 229.

CUCU

Type-species: Noctua veronicae Hübner, [I8I3], Samml. eur. Schmett. 4: pl. II6, fig. 54I, by subsequent designation by Hampson, I906, Cat. Lepid. Phalaenae Br. Mus. 6 : 443. Type(s), Europe.

CONOCHARES Smith, I905, Jl N.Y. ent. Soc. 13: 207.

ACON

Type-species: Conochares acutus Smith, I905, ibidem 13: 207, by subsequent designation by Hampson, I910, Cat. Lepid. Phalaenae Br. Mus. 10: 682. Syntypes $2 \hat{0}$, I ․, [U.S.A.]: [Arizona], Santa Catalina Mts.

†ONOCHARIS; McDunnough, I943, Can. Ent. 75: 59 .

An incorrect subsequent spelling.

$\ddagger$ CONOCHARIS, misspelling. See Conochaves Smith, I905.

$\operatorname{ACON}$

CONOCHUZA Berio, 1962, Annali Mus. civ. Stor. nat. Giacomo Doria 73: I 73.

ACRO

Type-species: Conochuza lineola Berio, I962, ibidem 73: I75, fig. 3, by original designation. Holotype ?, Aldabra I. (MNHN, Paris).

CONOCRANA Turner, 1942, Proc. R. Soc. Qd 53: 72.

ACRO

Type-species: Conocrana ochthera Turner, 1942, ibidem 53: 73, by monotypy. Holotype , , [Australia]: Qd, Cunnamulla (ANIC, Canberra).

C. ochthera is a junior subjective synonym of Macroprova chionobola Turner, I94I.

See also Macroprova Turner, I94I.

CONOSEMA Hampson, 1926, Descr. new Genera Species Lepid. Phalaenae Subfamily Noctuinae Br. Mus.: 236.

OPH I

Type-species: Zethes pratti Bethune-Baker, 1908, Novit. zool. 15: 202, by original designation. Syntypes $\hat{0},+$, New Guinea: [Papua], Dinawa (BMNH).

CONSERVULA Grote, I874, Bull. Buffalo Soc. nat. Sci. 2: I7.

ACRO

Type-species: Phlogophora anodonta Guenée, 1852, in Boisduval \& Guenée, Hist. nat. Insectes (Lépid.) 6: 63 , pl. 7, fig. 8, by original designation. Holotype ô. [U.S.A.]: New York State (BMNH).

CONSTANTIODES Hampson, I9i 6, in Poulton, Proc. zool. Soc. Lond. 1916: Io7.

ACRO

Type-species: Constantiodes pyralina Hampson, I9I6, ibidem 1916: I07, pl. I, fig. 35, by original designation. Holotype $\delta$. [Somali Republic] Somaliland: Mandera $(\mathrm{BMNH})$.

CONTORTIVENA Bethune-Baker, I906, Novit. zool. 13: 283.

OPH I

Type-species: Contortivena umbrosa Bethune-Baker, I906, ibidem 13: 283, by original designation. Syntypes $\hat{\jmath}$,, , NEw Guinea: [Papua], Babooni (BMNH).

C. umbrosa is a junior subjective synonym of Zethes distorta Warren, I903, Novit. zool. 10: I 26.

COPA BLEPHARON Harvey, I 878, Can. Ent. 10: 56.

NOCT

Type-species: Ablepharon absidum Harvey, I875, Bull. Buffalo Soc. nat. Sci. 2: 275, by monotypy. Type(s), [U.S.A.]: Oregon.

COPANARTA Grote, I895, Abh. naturw. Ver. Bremen 14: III.

CUCU

Type-species: Hadena aurea Grote, I879, Bull. U.S. geol. geogr. Surv. Territ. 5: 205, by original designation. Type(s) $\hat{\sigma}$, [U.S.A.]: Southern Texas (BMNH).

COPHANTA Walker, I 864, List Specimens lepid. Insects Colln Br. Mus. 30: 964.

ACON

Type-species: Cophanta funestalis Walker, I864, ibidem 30: 964, by monotypy. Type(s) $9,[$ Borneo]: Sarawak (BMNH).

See also Toxophleps Hampson, I893. 
COPIBRYOPHILA Sinith, I90o, Proc. U.S. natn. Mus. $22: 4 \mathrm{I} 6$. ACRO

Type-species: Copibryophila angelica Smith, I900, ibidem 22: +16 , by monotypy. Syntypes 7 ex., [U.S.A.]: California, Los Angeles (USNM, Washington).

COPICUCULLIA Smith, I894, Trans. Am. ent. Soc. $21: 84$.

CUCU

Type-species: Cleophana eulepis Grote, 1876, Bull. Buffalo Soc. nat. Sci. 3: 86, by subsequent designation by Hampson, I906, Cat. Lepid. Phalaenae Br. Mus. 6: 9. Type(s) ㅇ, [U.S.A.]: Oregon.

COPIDRYAS Grote, I876, Can. Fnt. 8: 99.

AGAR

Type-species: Euscirrhopterus gloveri Grote \& Robinson, I868, Trans. Am. ent. Soc. 2: 185, by monotypy. Type(s) 9, [U.S.A.]: Texas.

COPIFRONTIA Hampson, I905, Ann. S. Afr. Mus. 3: 433.

ACRO

Type-species: Copifrontia xantherythra Hampson, 1905, ibidem 3: 433, by originai designation. Holotype $\hat{o}$, [RHodesIA]: Mashonaland (BMNH).

COPIHADENA Morrison, I875, Can. Ent. 7: 91.

CUCU

Type-species: Homohadena atricollaris Harvey, 1875 , Bull. Buffalo Soc. nat. Sci. 2: 273, by monotypy. Type(s), [U.S.A.]: Texas (BMNH).

COPIMAMESTRA Grote, I883. See Mamestra Ochsenheimer, I8I6.

HADE

COPIPANOLIS Grote, I874, Annual Rep. Trustees Peabody Acad. Sci. 6: 25.

CUCU

Type-species: Copipanolis cubilis Grote, 1874 , ibidem 6:26, by monotypy. Syntypes o. [U.S.A.]: [Mich.,], Lansing (BMNH); Massachusetts (BMNH).

COPIPHANA Hampson, I906, Cat. Lepid. Phalaenae Br. Mus. 6: viii, 95.

CUCU

Type-species: Cleophana olivina Herrich-Schäffer, (1852), Syst. Bearb. Schmett. Eur. 2: pl. II3, fig. 578; 6(Nachtrag): 57, by original designation. Type(s) $q$ Asia Minor.

C. olivina was figured and given only a specific name in (185I) vol. 2 . In (1852) vol. 6 olivina was associated with its generic name and became nomenclaturally available. The dates of publication are taken from the original wrappers.

COPITARSIA Hampson, I906, Cat. Lepid. Phalaenae Br. Mus. 6: ix, I83. Cucu

Type-species: Polia turbata Herrich-Schäffer, (1855), Syst. Bearb. Schmett. Eur. 2: pl. I24, figs 639,$640 ; 6$ (Index to 2): 60, by original designation. Syntypes, TYPELOCALITY see below [COLOMBIA. VENEZUELA].

$P$. turbata was figured and given only a specific name in (1852) vol. 2. In (1855) vol. 6 turbata was associated with its generic name and became nomenclaturally available. In (1856) vol. 6 (Nachtrag): I77 the type-locality was given as Dalmatia and Asia Minor. This is probably an incorrect locality and subsequent authors e.g. Staudinger, Hampson and Draudt have all regarded it as a South American species. The dates of publication are taken from original wrappers and listed by Hemming, 1937, Hïbner 1: 585,589 .

COPIT YPE Hampson, I906, Cat. Lepid. Phalaenae Br. Mus. 6: x, 206.

CUCU

Type-species: Dasypolia pagodae Alphéraky, I892, Horae Soc. ent. ross. 26: 45I, by original designation. Type(s) $\hat{o}$. Cirnna: [Tibet], Amdo district, Myn-dyn-scha (ZI, Leningrad).

COPIVALERIA Grote, I883, Proc. Am. phil. Soc. 21: I 46, I68. cucu

Type-species: Valeria grotei Morrison, 1874, Bull. Buffalo Soc. nat. Sci. 1: 274, by original designation. Syntypes, [U.S.A.]: Massachusetts, Cambridge (BMNH).

COPTOCNEMIA Zeller, I872, Verh. zool.-bot. Ges. Wien 22 (Abh.): $475 . \quad$ орні

Type-species: Coptocnemia floccalis Zeller, 1872, ibidem 22 (Abh.): 476 , pl. 2 , fig. Io, by monotypy. Holotype 3 , [U.S.A.]: Texas.

C. floccalis is a junior subjective synonym of Phytometra scopulepes Haworth, I8og.

See also Hemeroplanis Hübner, I818; and Scopelopus Stephens, I829. 
CORCOBARA Moore, I882, in Hewitson \& Moore, Descr. new Indian lepid. Insects Colln late Mr W. S. Atkinson: 186. OPHI

Type-species: Corcobara angulipennis Moore, I882, ibidem: I86, pl. 6, fig. 16, by monotypy. Syntypes, CEY'Lon (BMNH). India: [W. Bengal], Darjiling.

CORDYLEPALPA Joicey \& Talbot, I9I7. See Clavipalpa Joicey \& Talbot, I9I7.

ACRO

COREMAGNATHA Hampson, I924, Ann. Mag. nat. Hist. (9) 13: 445.

HYPE

Type-species: Bocana orionalis Walker, [1859] 1858, List Specimens lepid. Insects Colln Br. Mus. 16: 186, by original designation. Type(s) 우 Brazil (UM, Oxford).

CORETHROBELA Turner, 1908, Trans. Proc. R. Soc. S. Aust. 32: 73.

HYPE

Type-species: Corethrobela melanophaes Turner, 1908, ibidem 32: 74, by monotypy. Holotype $\hat{O}$, Australia: Qd, Kuranda (ANIC, Canberra).

CORGATHA Walker, [1859] I 858, List Specimens lepid. Insects Colln Br. Mus. 16: 21 5. AcoN Type-species: Corgatha zonalis Walker, [1859] I858, ibidem 16: 216 , by monotypy. Holotype ô, CEYLON (BMNH).

See also Ausinza Walker, I864.

CORGATHALIA Berio, I966, Annali Mus. civ. Stor. nat. Giacomo Doria 76: I 20.

$\operatorname{ACON}$

Type-species: Corgathalia viettei Berio, I966, ibidem 76: I2I, fig. I2, by original designation. Holotype $\hat{\sigma}$. Madagascar: E., env. de Perinet, Forêt de l'Analamazoatra (MNHN, Paris).

CORIA Walker, I866, List Specimens lepid. Insects Colln Br. Mus. 35: 1982.

CATO

Type-species: Bocana lunaris Walker, [1865] I864, ibidem 31: 57, by monotypy (of Bocana Walker, [1865]). Lectotype 9 , [Sulatvesi] Celebes (UM, Oxford), designated (as type) by Swinhoe, 1900, Cat. east. and Aust. Lepid. Heterocera 2: i 16.

$B$. Innaris is a junior subjective synonym of Phalaena hievoglyphica Drury, 1773.

Coria was proposed as the objective replacement name for Bocana Walker, [1865].

See also Argiva Hübner, [1823].

BOCANA Walker, [1865] 1864, List Specimens lepid. Insects Colln Br. MTus. 31: 57.

Type-species: Bocana lunaris Walker, [1865] 1864, ibidem 31: 57, by monotypy.

A junior homonym of Bocana Walker, [I859] I858, ibidem 16: I70,-Lepid., Noctuidae. The objective replacement name is Coria Walker, I866.

CORISCE Hübner, [I823] I8I6, Verz. bekannter Schmett.: 279.

CATO

Type-species: Ephesia amica Hübner, I8I8, Zutväge Samml. exot. Schmett. 1: I4, figs $57,5^{8}$, by monotypy. Type(s) $q$, [U.S.A.]: Florida [formerly] in Georgia.

$\ddagger$ †ORISEE; Walker, [1858] ז857, List Specimens lepid. Insects Colln Br. Mus. 13: I I79.

An incorrect subsequent spelling.

$\ddagger$ BLEPHARUM Hübner, I808, Erste Zuträge Samml. exot. Schmett.: 4 .

Included in a work rejected for nomenclatural purposes by Int. Commn zool. Nom., 1966, Bull. zool. Nom. 23 Opinion 789:213. Placed on the Official Index of Rejected and Invalid Generic Names in Zoology: Name No. 1830.

Only included species $\$$ Blepharmm amica, Hübner, I808, ibidem: 4, later published and made nomenclaturally available as Ephesia amica Hübner, I8I8.

$\ddagger$ BLEPHARIDIA Hübner, r818, Zuträge Samml. exot. Schmett. 1: Ir, r4, 33.

Not nomenclaturally available as a genus-group name. Hübner used Blepharidia on all three pages, in a suprageneric sense for one of the names of his tribes (Namen der Stämme) listed on page 33. Hübner listed his genus-group names on page 35. Blepharidia is included here as Berio, 1957, Memorie Soc. ent. ital. 36: 9, has accepted it as an available generic name from 1818 and has designated as type-species, Ephesia amica Hübner, I8I8. 
CORNA Walker, 1865, List Specimens lepid. Insects Colln Br. Mus. 33: 1005.

OPHI

Type-species: Corna inconspicua Walker, I865, ibidem 33: I006, by monotypy. Holotype ô, [Brazil]: [R. Amazon], [Tefé] Ega (BMNH).

CORNUTIPLUSIA Kostrowicki, I961, Acta zool. cracov. 6: 432.

PLUS

Type-species: Phalaena circumflexa Linnaeus, 1767, Syst. Nat. (Edn 12) 1: 8.4, by original designation. Type(s), EUROPE (LS, London).

CORONIS Hübner, [I823]. See Feventa Walker, [I858].

OPHI

CORONTA Walker, [1858] i 857, List Specimens lepid. Insects Colln Br. Mus. 13: I I42, I 7 o.

Type-species: Coronta surrepens tWalter, [It Type(s) $\delta^{\star}$. Haiti (UM, Oxford).

C. surrepens is a junior subjective synonym of Bolina strigifera Valker, [1858] I857, ibidem 13: I I53.

CORRHA Walker, [1858] 1857, List Specimens lepid. Insects Colln Br. Mus. 13: 1079, Iogo.

Type-species: Corrha difficilis Walker, [1858] 1857, ibidem 13: Iogo, by monotypy. Syntypes I 0 , I + , [Australia]: Tasmania (BMNH).

CORSA Walker, [I858] I 857, List Specimens lepid. Insects Colln Br. Mus. 13: Io79, I Ior. oph I Type-species: Corsa lignicolora Walker, [1858] I857, ibidem 13: I Io I, by monotypy. Holotype + , CEYLON (BMNH).

CORTICATA Walker, [I863] I 864, J. Proc. Limn. Soc. (Zool.) 7: 52.

SARR

Type-species: Corticata scoparioides Walker, [1863] 1864, ibidem 7: 53, by monotypy. Lectotype , Borneo: Sarawak (UM, Oxford), designated (as type) by Swinhoe, I90o, Cat. east. and Aust. Lepid. Heterocera 2: 78.

CORTYTA Walker, [1858] 1857, List Specimens lepid. Insects Colln Br. Mus. 13: 1045, Io76.

Type-species: Cortyta canescens Walker, [1858] I857, ibidem 13: I077, by monotypy. Holotype $q$. [South Africa] Caffraria (BMNH).

CORUBIA Schaus, I906, Proc. U.S. natn. Mus. 30: Iog.

OPHI

Type-species: Corubia testacea Schaus, I906, ibidem 30: 109, by monotypy. Type(s), Brazil: Parana, Castro (USNMI, Washington).

CORULA Walker, I856, List Specimens lepid. Insects Colln Br. Mus. 9: I I. Geometridae Type-species: Corula geometroides Walker, I 856 , ibidem 9: II, by monotypy.

Conula has been used as the original generic name for species which are now in the Nolidae.

CORUNCALA Walker, I865, List Specimens lepid. Insects Colln Br. Mus. 33: $866 \quad$ орн1

Type-species: Coruncala latipennis Walker, I 865, ibidem 33: 866, by monotypy. Type(s) ô. [BraziL]: Amazon Region.

CORYCIA Hübner, [1823] I8I6, Vevz. bekannter Schmett.: 265.

OPH I

Type-species: Phalaena cajeta Cramer, I775, Uitlandsche Kapellen 1: 48, pl. 30, figs A-C, by subsequent designation by Hampson, 1926, Descr. new Genera Species Lepid. Phalaenae Subfamily Noctuinae Br. Mus.: 335. Syntypes of, ․, [India]: Coromandel. [Java]: [Djakarta] Batavia.

CORYMBIA Walker, I865, List Specimens lepid. Insects Colln Br. Mus. 33: 765.

NOTODONTIDAE

Type-species: Corymbia smerinthoides Walker, 1865, ibidem 33:765, by subsequent designation by Berio, 1966, Annali Mus. civ. Stor. nat. Giacomo Doria 76: 54.

Corymbia was originally placed in the Noctuidae. 
CORYNITIS Geyer, I832, in Hübner, Zuträge Samml. exot. Schmett. 4: 26.

Type-species: Corynitis penicillalis Geyer, I832, ibidem 4: 26, figs 707, 708, by

PRESENT DESIGNATION. Type(s) ô, [BRAzIL]: Rio Janeiro.

Corynitis was proposed for $C$. penicillalis, with one other species mentioned for comparison.

CORYTHURUS Hampson, I893, Illust. typical Specimens Lepid. Heterocera Colln Br. Mus. 9: 27. II 2 .

ACRO

Type-species: Corythurus nocturnus Hampson, I893, ibidem 9: 27, II 3, pl. 65 , figs 4 , Io, by monotypy. Syntypes $0 *$, $q$, CEYLon: Colombo (BMNH); Udagama (BMNH).

COSCAGA Schaus, I9o6, Proc. U.S. natn. Mus. 30: I 3 I.

HYPE

Type-species: Coscaga angulata Schaus, I906, ibidem 30: I3 I, by monotypy.

Type(s), Mexico: Jalapa (USNM, Washington).

$\ddagger$ COSMIA Hübner, [I806]. See Cosmia Ochsenheimer, I8I6.

ACRO

COSMIA Ochsenheimer, i8i6, Schmett. Eur. 4: 84.

ACRO

Type-species: Phalaena diffinis Linnaeus, 1767 , Syst. Nat. (Edn I2) 1: 848, by subsequent designation by Duponchel, 1829, in Godart \& Duponchel, Hist. nat. Lépid. Papillons Fr. 7(2): 72. Type(s), [Portugal] Lusitania (LS, London).

$\ddagger$ COSMIA Hübner, [1806], Tentamen determinationis digestionis . . . : [I].

Included in a work rejected for nomenclatural purposes by Int. Commn zool. Nom., 1926, Smithson. misc. Collns 73 (4) Opinion 97: I9. Also idem, 1954, Opin. Decl. int. Commn zool. Nom. 6 Opinion 278: I 40.

Only included species: Phalaena affinis Linnaeus, I767, Syst. Nat. (Edn I2) 1: 848, which is congeneric with Phalaena diffinis Linnaeus, 1767.

EUSTEGNIA Hübner, [1821] I8I6, Vevz. bekannter Schmett.: 236.

Type-species: Phalaena diffinis Linnaeus, I 767 , by subsequent designation by Hampson, I9Io, Cat Lepid. Phalaenae Br. Mus. 9: i87.

Eustegnia is a junior objective synonym of Cosmia Ochsenheimer, I8I6.

COSMODES Guenée, I852, in Boisduval \& Guenée, Hist. nat. Insectes (Lépid.) 6: 289. ACRo

Type-species: Phalaena elegans Donovan, I805, Epitome nat. Hist. Insects of New Holland etc.: pl. 36, fig. 5, text overleaf, by monotypy. Type(s), [Australia] New Holland: New South Wales.

COSMOPHILA Boisduval, I833, Nouv. Ann. Mus. Hist. nat. Paris 2: 242.

OPHI

Type-species: Cosmophila xanthindyma Boisduval, I833, ibidem 2: 242, by monotypy. Syntypes, Mauritius. [Réunion] Bourbon.

Also published in I833 as Faune ent. Madagascar (Lépid.): 94, pl. I3, fig. 7 .

C. xanthindyma is a junior subjective synonym of Noctua flava Fabricius, I775, Syst. Ent. : 601.

COSSEDIA Walker, [I 866] i 865, List Specimens lepid. Insects Colln Br. Mus. 34: I277. chLO Type-species: Cossedia erateinalis Walker, [1866] I 865, ibidem 34: I277, by monotypy. Lectotype ô, Borneo: Sarawak (UM, Oxford), designated (as type) by Swinhoe, Igoo, Cat. east. and Aust. Lepid. Heterocera 2: 590.

COSSUS Fabricius, I793, Ent. Syst. 3(I): Characteres Generum. Available but without included species until I794, ibidem 3(2): [3].

COSSIDAE

Type-species: Cossus ligniperda Fabricius, I794, ibidem 3(2): [3], by subsequent designation by Latreille, I8Io, Considérations générales Ordre nat. Anim.: 44I.

Cossus has been used as the original generic name for species which are now in the Noctuidae.

COTANDA Moore, I 88I, Proc. zool. Soc. Lond. 1881: 374.

ACRO

Type-species: Eriopus placodoides Guenée, 1852, in Boisduval \& Guenée, Hist. nat. Insectes (Lépid.) 6: 296, by monotypy. Holotype ô. JAVA.

$\mathrm{K}$ 
COTARSINA Köhler (1952) I951, Acta zool. lilloana 12: i66.

CUCU

Type-species: Cotarsina clavata Köhler, (I952) I95I, ibidem 12: I66, by original designation. Syntypes $\delta$, ㅇ, Argentina: Chubut [Prov.], Comodoro Rivadavia (ZSBS, Munich).

COTUZA Walker, I858, List Specimens lepid. Insects Colln Br. Mis. 15: I 545, I 55I. OPHI

Type-species: Cotuza drepanoides Walker, I 858 , ibidem 15: I 552, by monotypy. Syntypes $4 \hat{\jmath},[$ India]: Hindostan (BMNH). Hong Kong (BMNH). [West Malaysia]: Penang (BMINH).

COXINA Guenée, I852, in Boisduval \& Guenée, Hist. nat. Insectes (Lépid.) 6: 297.

OPH I

Type-species: Coxina ensipalpis Guenée, I $8_{52}$, ibidem 6: 298, pl. I4, fig. 5, by subsequent designation by Hampson, 1926, Descr. new Genera Species Lepid. Phalaenae Subfamily Noctuinae Br. Mus.: I 4. Holotype j, Mexico.

CRAMBIFORMA Hampson, 1926, Descr. new Genera Species Lepid. Phalaenae Subfamily Noctuinae Br. Mus.: 6I 4 . OPHI

Type-species: Crambiforma leucostrepta Hampson, I926, ibidem: 6I4, by original designation. Holotype $\hat{0}$. [EтHropia] Abyssinia: [Harar] Harrar (BMINH).

CRAMBODES Guenée, I 852, in Boisduval \& Guenée, Hist. nat. Insectes (Lépid.) 6 : I 52.

Type-species: Crambodes talidiformis Guenée, I 852 , ibidem 6: I 52, pl. 7, fig. I 2 , by monotypy. Syntypes $3 \hat{\jmath}$, America: northern (BMNH). BrazIL.

CRAMBOPHILIA Dyar, I9I4, Proc. U.S. natn. Mus. 47:220. HYPE

Type-species: Crambophilia majorcula Dyar, I9I4, ibidem 47: 220, by original designation. Syntypes 3 ex., Panama: Porto Bello; Alhajuelo; Cabima. (USNM, Washington).

CRAMBOPSIS Walker, I865, List Specimens lepid. Insects Colln Br. Mus. 32: 634. HADE Type-species: Crambopsis excludens Walker, I865, ibidem 32: 634, by monotypy. Type(s) T, CeYlon.

CRAMBUS Fabricius, I 798, Ent. Sy'st. (Suppl.) : 464. PYRALIDAE

Type-species: Phalaena pascuella Linnaeus, I758, Syst. Nat. (Edn io) $1: 535$, by subsequent designation by Curtis, I826, Br. Ent. 3 : 109.

Crambus has been used as the original generic name for species which are now in the Noctuidae.

CRAMERIA Hübner, [I8I9] I8I6, Verz. bekannter. Schmett. : I68.

AGAR

Type-species: Phalaena amabilis Drury, I773, Illust. nat. Hist. 2: 23, pl. I3, fig. 3, and Index, by subsequent designation by Grote, I865, Proc. ent. Soc. Philad. 5: 236. Holotype, AFRICA: about $6^{\circ} \mathrm{N}$.

CHARILINA Walker, I 854, List Specimens lepid. Insects Colln Br. Mus. 2: 560.

Type-species: Phalaena amabilis Drury, I 773, by monotypy.

Charilina when originally proposed contained a second species, but this was doubtfully included, and under the Code, Article 68 (c), is not eligible for designation as type-species.

Charilina is a junior objective synonym of Crameria Hübner, [I8I9].

CRANIONYCTA Lattin, I949, Z. wien. ent. Ges. 34: Io8.

ACRO

Type-species: Cranionycta oda Lattin, I949, ibidem 34: Io8, by original designation. Holotype ô, [U.S.S.R.]: Sutschanski-Rudnik, Ussuri.

CRANIOPHORA Snellen, I 867, Vlinders Nederland 1867: 262.

ACRO

Type-species: Noctua ligustri [Denis \& Schiffermüller], I775, Ankïndung syst. Werkes Schmett. Wienergegend: 70, by monotypy. Type(s), [Austris]: Vienna district (Collection destroyed, Horn \& Kahle, i936, Ent. Beih. Berl.-Dahlem 3: 243). 
BISULCIA Chapman, I890, Entomologist's Rec. J. Vur. 1: 28.

Type-species: Noctua ligustri [Denis \& Schiffermüller], I775, by monotypy.

Bisulcia is a junior objective synonym of Craniophora Snellen, I867.

CRAPTIGNAPA nom. n. for Pangraptica Hampson, I926.

OPHI

Type-species: Egnasia delicata Bethune-Baker, I906, Novit. zool. 13: 279, by original designation (for Pangraptica Hampson). Holotype $\hat{\jmath}$. New Guinea: [Papua], Ekeikei (BMNH).

PANGRAPTICA Hampson, 1926, Descr. new Genera Species Lepid. Phalaenae Subfamily Noctuinae Br. Mus.: 557.

Type-species: Egnasia delicata Bethune-Baker, 1906, by original designation.

A junior homonym of Pangraptica Wileman \& South, I92I, - Lepid., Noctuidae. The objective replacement name is Craptignapa nom. n.

CRASIA Aurivillius, I891, Nordens Fjärilar: I62.

CUCU

Type-species: Hadena iris Zetterstedt, I839, Insecta Lapponica: 94I, by monotypy. Type(s) + , [Scandinavia]: [Lappland] Lapponia borealis.

See also Hillia Grote, I 883.

CRASPEDOGONIA Hampson, I893, Illust. typical Specimens Lepid. Heterocera Colln Br. Mus. 9: 29, II7.

CHLO

Type-species: Craspedogonia nubes Hampson, I893, ibidem 9: 29, II7, pl. I66, fig. 3, by monotypy. Type(s) ㅇ, Ceylon: Kelani Valley (BMNH).

CRASSIVESICA Hardwick, 1970, Mem. ent. Soc. Can. 67: I 56.

NOCT

Type-species: Agrotis bochus Morrison, 1874, Proc. Boston Soc. nat. Hist. 17: 163, by original designation (but cited as $\ddagger A$. brocha Morrison, an incorrect subsequent spelling). Type(s) ô, [U.S.A.]: Nebraska (not found in Colln Tepper, Michigan State Univ., so presumed lost, Hardwick, 1970, ibidem 67: 1 57).

A. bochus was misspelled as 'brochus' by Morrison, 1875, Proc. Acad. nat. Sci. Philad. 1875: 56. Smith, I890, Bull. U.S. natn. Mus. 38: I48 used 'Cameades brocha Morr.' and also cited ' $A$. bochus Morr.' Under the Code, Article 33 (a) the binomen Carneades brocha must therefore be attributed to Smith, I890, as an unjustified emendation.

CRATERESTRA Hampson, 1905, Cat. Lepid. Phalaenae Br. Mus. 5: vii, i7. Hade

Type-species: Stibaera lucina Druce, I889, Biologia cent.-am. (Zool.) Lepid., Heterocera 1: 296, pl. 27, fig. 27, by original designation. Holotype, Mexico: Tabasco, Teapa (BMNH).

CREMNODES Felder, I874. See Mocrendes nom. n.

OPHI

†CREMNOPALPUS, misspelling. See Cremopalpus Strand, I909.

SARR

CREMNOPHORA Hampson, Igor, Cat. Lepid. Phalaenae Br. Mus. 3: 453.

AGA R

Type-species: Apina angasii Walker, I855, List Specimens lepid. Insects Colln Br. Mus. 3: 757 , by original designation, but cited as ' $A$. angasi', an unjustified emendation, by Hampson. Syntypes 2 ex., Australia: S. (BMNH).

A. angasii Walker is a junior subjective synonym of Agarista angasii Angas, I847, South Aust. illust.: pl. 37, fig. 24.

Cremnophora was originally placed in the Arctiidae.

CREMOPALPUS Strand, 1909, Arch. Naturgesch. 75 (I) 3: 381.

SARR

Type-species: Cremopalpus inquirendus Strand, 1909, ibidem 75(I) 3: 383, by original designation. Holotype $\hat{0}$, [Zambia/Rhodesia]: Zambesi bank (MNHU, Berlin). $\ddagger$ CREMNOPALPUS; Hampson, ig12, Cat. Lepid. Phalaenae Br. Mus. 11: 454 .

An incorrect subsequent spelling.

†CRENOTERMES Hampson, no published reference found.

HYPE 
$\ddagger$ CRENULA, misspelling. See Crinala Jordan, I 896 .

CRENULARIA Bethune-Baker, I906, Novit. zool. 13: 280.

Type-species: Crenularia concolor Bethune-Baker, 1906, ibidem 13: 280, by original designation. Syntypes $\hat{\jmath}$, ㅇ, New Guinea: [Papua], Ekeikei (BMNH).

CRETONIA Walker, I866, List Specimens lepid. Insects Colln Br. Mus. 35: I 735.

ACON

Type-species: Cretonia platyphaeella Walker, I866, ibidem 35: I736, by monotypy. Holotype ô. Sierra Leone (BMNH).

CRIMONA Smith, 1902, Jl N.Y. ent. Soc. 10: 49.

ACRO

Type-species: Crimona pallimedia Smith, I902, ibidem 10: 49, by monotypy. Holotype 오, [U.S.A.]: California, Walters Station.

CRINALA Jordan, I 896, in Rothschild \& Jordan, Novit. zool. 3: 35.

AGAR

Type-species: Crinala mimetica Rothschild, 1896 , ibidem 3: 36 , by original designation. Holotype $\hat{\sigma}$. [PHilippines]: N. Luzon (BMNH).

$\ddagger$ CRENULA; Pagenstecher, I909, Geogr. Verbreitung Schmett.: 423.

An incorrect subsequent spelling.

CRINISINUS Bryk, 1949, Ark. Zool. 41(A)I: I35.

HYPE

Type-species: Crinisinus turbo Bryk, I949, ibidem 41(A)I: I36, pl. 5, fig. I4, by original designation. Holotype $\widehat{\jmath}$, Korea (NORTH): Shuotsu (NR, Stockholm).

C. turbo is a junior subjective synonym of Herminia trilinealis Bremer, I864, Mém. Acad. imp.Sci.St. Pétersb. (7) 8(r): 64, pl. 5, fig. 23, but proposed as †'Herminea', an incorrect subsequent spelling.

CRINO Hübner, [1821]. See Tarsolepis Butler, I872.

NOTODONTIDAE

CRINO Hübner sensu Hampson, I906. See Mniotype Franclemont, r94 I. CUCU

CRINOCULA Jordan, I896, in Rothschild \& Jordan, Novit. zool. 3: 36.

AGAR

Type-species: Crinocula kinabaluensis Rothschild, I896, ibidem 3: 36 , by original designation. Syntypes I ô, I ㅇ, Borneo: [Sabah], Kina Balu (BMNH).

CRIOA Walker, [1858] I857, List Specimens lepid. Insects Colln Br. Mus. 13: Io78, I Iro. opH Type-species: Crioa acronyctoides Walker, [1 858] i 857, ibidem 13: Ir Ir, by monotypy. Holotype ㅇ, [Australia]: [Qd], Moreton Bay (BMNH).

See also Piana Walker, I 869.

CRIONICA Hampson, 1926, Descr. new Genera Species Lepid. Phalaenae Subfamily Noctuinae Br. Mus.: 82.

OPH I

Type-species: Pteronycta cervicornis Fawcett, I9I8, Proc. zool. Soc. Lond. 1917: 240 , pl. I, fig. $2 \mathrm{I}$, by original designation. Holotype + (not $\hat{\sigma}$ as stated), [KeNYA]: [Teita Hills], Kedai (BMNH).

CRIOPHASIA Hampson, rgo6, Cat. Lepid. Phalaenae Br. Mus. 6: ix, I 22.

CUCU

Type-species: Calophasia albolineata Blachier, 1905, Bull. Soc. ent. Fr. 1905: 53, by original designation, but cited as ' $C$. albolineata Bang-Haas', an incorrect authorship. Holotype ơ, Tunisia: Gafsa.

In the original description Blachier attributed the name albolineata to Bang-Haas who authorized him to describe the species.

CRISHNA Kirby, I897. See Eupatula Ragonot, I 895.

CATO

CRISTATOPALPUS Bethune-Baker, 1908, Novit. zool. 15: 209.

IIY PE

Type-species: Cristatopalpus olivens Bethune-Baker, I908, ibidem 15: 210, by original designation. Syntypes $\hat{\sigma}$, New Guinea: [Papua], Mount Kebea (BMNH); Dinawa. 
CRITHOTE Walker, I864, J. Proc. Linn. Soc. (Zool.) 7 : 182.

OPHI

Type-species: Crithote horridipes Walker, 1864, ibidem 7: 183, by monotypy. Lectotype $\hat{0}$, BoRneo: Sarawak (UM, Oxford), designated (as type) by Swinhoe, I9oo, Cat. east. and Aust. Lepid. Heterocera 2: 163.

CROCHIPHORA Hübner, [183I] I825, Zuträge Samml. exot. Schmett. 3: 26, 35.

CATO

Type-species: Crochiphora flavistriaria Hübner, [I83I] I825, ibidem 3: 35, figs 503, 504, by subsequent designation by Hampson, I913, Cat. Lepid. Phalaenae Br. Mus. 13: ro5. Type(s), U.S.A.

CROCIGRAPHA Grote, I875, Can. Ent. 7: 57.

HADE

Type-species: Perigrapha normani Grote, I874, ibidem 6: II5, by monotypy. Syntypes of, q, CANADA: St Catharines (BMNH).

CROMOBERGIA Bourquin, 1937, Revta Soc. ent. argent. 9: 67.

OPHI

Type-species: Speocropia smilacis Bourquin, I937, ibidem 9:67, pl. 3, 4, by monotypy, but cited as 'S. smilacis Hayw.', an incorrect authorship. Syntypes 0 . 9 , ArgentinA: Concordia.

S. smilacis was first proposed in a lecture delivered by K. J. Hayward to the Segunda Reunion de Ciencias Naturales at Mendoza in 1937 April. This lecture was published in the Proceedings in Physis B. Aires 17, dated 1939 May. During the delay the use of $S$. smilacis accompanied by the description given by Bourquin in 1937 December made it nomenclaturally available.

S. smilacis is a junior subjective synonym of Cucullia teichii Berg, I885.

CROMOBERGIA Köhler, 1943, Revta Soc. ent. argent. 12: 30.

Type-species: Cucullia teichii Berg, 1885, An. Soc. cient. argent. 19: 27x, by original designation. Syntypes, Argentina: Buenos Aires province (Univ. Buenos Aires).

A junior homonym of Cromobergia Bourquin, I937,-Lepid., Noctuidae. The name Cromobergia originated from Köhler but was used and unintentionally made nomenclaturally available by Bourquin. Köhler (1943: 30) stated that Cromobergia Bourquin was not nomenclaturally available as it did not comply with the Code which requires that genus-group names published after 1930 must be accompanied by the definite fixation of a type-species. In this case the genus was monotypic and under Article 68 (c) the typespecies was therefore fixed. There is no objective replacement name but $C$. teichii is the senior synonym of Speocropia smilacis Bourquin, 1937, the type-species of Cromobergia Bourquin, x937. The latter is therefore available for use as a subjective replacement name.

CROMOBERGIA Köhler, I943. See Cromobergia Bourquin, I937.

OPHI

CROPIA Walker, [1858] I857, List Specimens lepid. Insects Colln Br. Mus. 13: Io78, III2. ACRO

Type-species: Cropia hadenoides Walker, [1858] I857, ibidem 13: I I I2, by monotypy. Holotype $\widehat{0}$, [Colombia]: Bogotà (BMNH).

EUDIPNA Walker sensu Schaus, 1906, Proc. U.S. natn. Mus. 30: 97.

Hampson, 1908, Cat. Lepid. Phalaenae Br. Mus. 7: 260, treated this usage as a junior homonym of Eudipna Walker, I856, - Noctuidae, Ophid., but Schaus attributed Eudipna to Walker and was not proposing a new genus, even though he used it for a concept differing somewhat from that of Walker. The only species included by Schaus at this date was Eudipna templada Schaus, x906, which is congeneric with Cropia hadenoides Walker, [1858].

CROSIA Dupont, r91o, Bull. Soc. ent. Fr. 1910: 369. ACRO

Type-species: Crosia hachem Dupont, I9I0, ibidem 1910: 369, fig. I, by monotypy. Holotype, Algeria: Oran prov., Mascara (MNHN, Paris).

CRURIA Jordan, I896, in Rothschild \& Jordan, Novit. zool. 3: 39.

AGAR

Type-species: Agarista donovani Boisduval, I832, Voyage de Découvertes de l'Astrolabe 1: I76, 67I, by original designation. Type(s), [Australia] Nouvelle Hollande (BMNH). 
The type-species was proposed by Boisduval as $A$. donowani but was indexed on page $67 \mathrm{I}$ as donovani. Subsequently the spelling donovani has been used except by Jordan when he proposed Cruvia. The species was named after E. Donovan and so ‡donowani should be treated as an incorrect original spelling.

CRURIOPSIS Jordan, Igr2, in Seitz, Gross-Schmett. Erde 11: 2 I.

AGAR

Type-species: Eusemia funebris Moore, I872, Proc. zool. Soc. Lond. 1872: 569, by monotypy. Type(s) J., India: [W. Bengal], Darjiling (BMNH).

The date of publication of the part of Seitz containing page 2 I was stated on page I 7 as I912 February 22nd in both the German and the English editions.

CRUSISETA Schultze, Igo8, Philipp. J. Sci. (A)3: 33 .

OPHI

Type-species: Crusiseta basipuncta Schultze, I908, ibidem 3: 33, pl. I, fig. 9, by original designation. Holotype đ̋, Philippines: Manila.

CRYASSA Walker, I858, List Specimens lepid. Insects Colln Br. Mus. 15: I745. EUTE

Type-species: Cryassa bifacies Walker, I858, ibidem 15: 1745, by monotypy. Holotype ô, CEYLON (BMNH).

C. bifacies was cited by Hampson, I912, Cat. Lepid. Phalaenae Br. Mus. 11: 81 as ¥'bifascies Wlk.', an incorrect subsequent spelling.

CRYMODES Guenée, I84I, Annls Soc. ent. Fr. 10: 238.

ACRO

Type-species: Hadena groenlandica Duponchel, [I838] I836, in Godart \& Duponchel, Hist. nat. Lépid. Papillons Fr. Suppl. 3: 228, pl. 2I, fig. 3, by subsequent designation by Guenée, I852, in Boisduval \& Guenée, Hist. nat. Insectes (Lépid.) 5: 185. Syntypes ô. 9 , GREENLAND.

H. groenlandica is a junior subjective synonym of Hadena exulis Lefebvre, 1836, Annls Soc. ent. Fr. 1836: 392, pl. Io. fig. 2.

CRYMONA Walker, I862, Trans, ent. Soc. Lond. (3)1: I I 7.

HYPE

Type-species: Crymona receptalis Walker, I862, ibidem (3)1: II7, by monotypy. Type(s) o, Brazil (UM, Oxford).

C. receptalis is a junior subjective synonym of Hypena hastatalis Walker, [I859] I858, List Specimens lepid. Insects Colln Br. Mus. 16:81.

CR YPHIA Hübner, I8 8, Zuträge Samml. exot. Schmett. 1: I4.

ACRO

Type-species: Noctua receptricula Hübner, [1803], Samml. eur. Sclumett. 4: pl. 6, fig. 27, by subsequent designation by Hampson, 1908, Cat. Lepid. Phalaenae Br. Mus. 7 : 6i8. Type(s), Europe.

CRYPHIMAEA Turner, I933, Trans. R. Soc. S. Aust. 57: I62. SARR

Type-species: Cryphimaea poliophasma Turner, I933, ibidem 57: I63, by monotypy. Holotype $q$, Austral1a: Qd, Yeppoon (ANIC, Canberra).

CRYPHIOIDES Berio, I964, Boll. Soc. ent. ital. 94: I24.

$\operatorname{ACON}$

Type-species: Cryphioides ocellata Berio, I964, ibidem 94: I25, by original designation. Holotype $q$, MAdagascar: Plateau de l'Imerina (MNHN, Paris).

Berio himself stated in the original description of his species that the type-species is possibly the same as Bryophila ocellata Saalmüller, I89I, Lepid. Madagascar: 25I, fig. 25I.

CRYPSEDRA Warren, igro, in Seitz, Macrolepid. World 3: i 33.

CUCU

Type-species: Miselia gemmea Treitschke, I825, Schmett. Eur. 5(I): 393, by original designation. Syntypes, Austria: Turnitz; Salzburg.

The date of publication of the part of Seitz containing page I 33 was stated on page I 33 as I9Io May $5^{\text {th }}$ in the English edition and as I9I I January I 3 th in the German edition.

¡Miselia gemmea Ochsenheimer, I816, Schmett. Eur. 4: 73 when first used was a nomen nudum. 
CR YPSIPRORA Meyrick, 1902, Trans. ent. Soc. Lond. 35: 29.

OPHI

Type-species: Crypsiprora ophiodesma Meyrick, I902, ibidem 35: 30, by monotypy. Holotype + , Australia: W. Aust., Geraldton (BMNH).

†CRYSIPRORA; Turner, I94I, Mem. Qd Mus. 12: 50 .

An incorrect subsequent spelling.

CRYPSOTIDIA Rothschild, I9or, Novit. zool. 8: 432.

CATO

Type-species: Crypsotidia wollastoni Rothschild, I90I, ibidem 8: 432 , by original designation. Holotype ô, Sudan: Shendi (BMNH).

$\ddagger$ CRYPTOTIDIA ; Hayward, I926, Entomologist's Rec.J. Var. 38 (Suppl.) Lepid. Aswan: (20).

CRYPTASTRIA Hampson, 1926, Descr. new Genera Species Lepid. Phalaenae Subfamily Noctuinae Br. Mus.: 595.

OPHI

Type-species: Zethes fuscomarginata Bethune-Baker, 1906, Novit. zool. 13: 272, by original designation. Holotype 0 , New Guinea: [Papua], Dinawa (BMNH).

CRYPTOCALA Benjamin, I921, Bull. sth. Calif. Acad. Sci. 20: гз3.

NOCT

Type-species: Agrotis gilvipennis Grote, 1874, Annual Rep. Trustees Peabody Acad. Sci. 6: 24 , by original designation. Syntypes $5(\hat{0}$, f), [CANADA]: [Quebec], Anticosti I. $(\mathrm{BMNH})$.

CRYPTOCHROSTIS Hübner, [I823] I8I6, Verz. bekannter Schmett.: 297.

OPHI

Type-species: Cryptochrostis suppulchraria Hübner, [1823] 1816, ibidem: 297, the objective replacement name for Phalaena hyphinoe Stoll, I78I, in Cramer, Uitlandsche Kapellen 4: r 30 , pl. 357, figs G, H (a primary homonym), by monotypy. Type(s) (of $P$. hyphinoe Stoll), Surinam.

P. hyphinoe Stoll, I78I, is a junior homonym of Phalaena hyphinoe Cramer, I777, ibidem 2: 9 I, - Lepid., Cossidae.

$P$. hyphinoe Stoll is dated from the wrapper of the part and not from the title-page of the volume, 1782 .

CRYPTOCHRYSA Hampson, 1926, Descr. new Genera Species Lepid. Phalaenae Subfamily Noctuinae Br. Mus. : rog.

OPH I

Type-species: Hypogramma auripennis Schaus, igr2, Ann. Mag. nat. Hist. (8)10: 5³, by original designation. Syntypes ô, Costa RICA: Sitio; Sixola (USNM, Washington).

CRYPTOMERIA Saalmüller, i88o, Ber. senckenb. naturforsch. Ges. 1879-1880: 290. OPHI

Type-species: Cryptomeria mabillei Saalmüller, I880, ibidem 1879-1880: 29r, by monotypy. Type(s), Madagascar (SNG, Frankfurt).

CRYPTOTHRIPA Hampson, I912, Cat. Lepid. Phalaenae Br. Mus. 11: xi, 309.

SARR

Type-species: Selepa occulta Swinhoe, r 885, Proc. zool. Soc. Lond. 1885: 461, pl. 27 , fig. I I, by original designation. Syntypes $\widehat{\sigma}, q,[$ INDIA]: [Bombay], Poona (BMNH).

$\ddagger$ CRYPTOTIDIA, misspelling. See Crypsotidia Rothschild, I9oI.

CATO

$\ddagger$ CRYSIPRORA, misspelling. See Crypsiprora Meyrick, I902.

OPHI

CTEIPOLIA Staudinger, I896, Dt. ent. Z. Iris 9: I9I.

CUCU

Type-species: Cteipolia sacelli Staudinger, r896, ibidem 9: I9r, by monotypy. Syntypes 5 q. [U.S.S.R.]: [Kirghizia], N.E. of Issyk Kul (MNHU, Berlin).

CTENOPLUSIA Dufay, 1970, Faune Madagascar 31: 91.

PLUS

Type-species: Plusia limbirena Guenée, 1852, in Boisduval \& Guenée, Hist. nat. Insectes (Lépid.) 6: 350, by original designation. Lectotype 9 , MADAGASCAR (MNHN, Paris), designated by Paulian \& Viette, [1956] 1955, Mém. Inst. scient. Madagascar (E)6: 232. 
CTENUSA Hampson, I9I O, Proc. zool. Soc. Lond. 1910: 422.

CATO

Type-species: Chalciope carnicolor Hampson, 1902, Ann. S. Afr. Mus. 2: 329, by original designation. Type(s) $\hat{o}$, [South West Africa]: Damaraland (BMNH).

C. carnicolor is a junior subjective synonym of Calliodes pallida Hampson, I902, ibidem 2: $32 \mathrm{I}$.

ACANTHON YX Hampson, 1902, Ann. S. Afr. Mus. 2: 323.

Type-species: Agrotis marginalis Walker, 1858 , List Specimens lepid. Insects Colln Br. Mus. 15: I700 (a primary homonym), by original designation. Holotype $\sigma^{\wedge}$ (not $q$ as stated), South Africa (BMNH).

Agrotis marginalis Walker, I 858, is a junior primary homonym of Agrotis marginalis Walker, [1857] I856, ibidem 10:339, - Noctuidae, Noctuinae. The objective replacement name is Agrotis marginifera Walker, 1865 , ibidem 32: 706, but this is a junior subjective synonym of Agrotis varians Wallengren, 1863, Wien. ent. Monatschr. 7: 147. Ctenusa varians (Wallengren) should be used as the valid name.

Acanthonyx Hampson is junior homonym of Acanthony'x Latreille, [1828] 1825, Ency'cl. méth. Hist. nat. 10(2): 698,-Crustacea. There is no objective replacement name but $A$. marginalis Walker, I 858 , is congeneric with Chalciope carnicolor Hampson, I902, the type-species of Ctenusa Hampson, I9Io. The latter is therefore available for use as a subjective replacement name.

CTENYPENA Prout, 1927, Trans. ent. Soc. Lond. 75: 229.

HYPE

Type-species: Ctenypena tenuis Prout, 1927, ibidem 75: 229, pl. 21, fig. 23, by original designation. Holotype $\tilde{\sigma}$. [São Tomé I., West Africa] São Thomé I. (BMNH).

CTYPANSA Walker, I 858, List Specimens lepid. Insects Colln Br. Mus. 15: I548, i6 го. орні Type-species: Ctypansa inconstans Walker, I 858 , ibidem 15: I6I I, by monotypy. Holotype ô., [BraziL]: [R. Amazon], [Tefé] Ega (BMINH).

${ }_{+}$CT YPA USA; Neave, 1939, Nomencl. zool. 1: 899.

An incorrect subsequent spelling.

†TYPAUSA, misspelling. See Ctypansa Walker. $185^{8}$.

OPHI

CUBENA Walker, I 856, List Specimens lepid. Insects Colln Br. Mus. 9: I 37, I 50. Plus

Type-species: Phalaena polydamia Stoll, I782, in Cramer, Uitlandsche Kapellen 4: $242, \mathrm{pl} .400$, fig. D, by monotypy. Type(s), Surinam.

$P$. polydamia is a junior subjective synonym of Phalaena c-aureum Knoch, I781.

See also Lamprotes R.L. I 817.

CUCULLIA Schrank, 1802, Fauna Boica 2(2): 157.

CUCU

Type-species: Phalaena umbratica Linnaeus, I758, Syst. Nat. (Edn Io) 1: 5I5, by subsequent designation by Curtis, I $824, B r$. Ent. 1: 45 . Type(s), Type-Locality not stated (LS, London) [EUROPE].

${ }_{+} T R I B O N O P H O R A$ Hübner, [1806], Tentamen determinationis digestionis . . . : [I] .

Included in a work rejected for nomenclatural purposes by Int. Commn zool. Nom., 1926, Smithson. misc. Collns 73(4) Opinion 97: 19. Also idem, 1954, Opin. Decl. int. Commn zool. Nom. 6 Opinion 278: I 4 o.

Only included species: Phalaena umbratica Linnaeus, 1758.

${ }_{+}^{+}$TRIBONOPHORAE Ochsenheimer, I8I6, Schmett. Fur. 4:87.

First published as a junior synonym of Cucullia Schrank, I802, and not sibsequently treated as an available name under the Code, Article I I(d).

TRIBUNOPHORA Hübner, I822, Syst.-alphab. Verz.: 20, 37.

Type-species: Phalaena umbratica linnaeus, I 758 , by subsequent designation by Nye, 1964, Bull. zool. Nom. 21: 75 .

Tribunophora is a junior objective synonym of Cucullia Schrank, I 802.

CALLAENIA Hübner, [1821] 1816, Verz. bekannter Schmett.: 246. 
Type-species: Phalaena umbratica Linnaeus, $175^{8}$, by subsequent designation by

Hampson, 1906, Cat. Lepid. Phalaenae Br. Mus. 6: 14.

Callaenia is a junior objective synonym of Cucullia Schrank, I8o2.

$\ddagger$ CALLAINIA; Hübner, [1826] I816, Verz. bekannter Schmett. Anzeiger: 37 .

An incorrect subsequent spelling.

LOPHIA Sodoffsky, r837, Bull. Soc. imp. Nat. Moscou 1837 (6): 88.

Proposed, unnecessarily, as an objective replacement name for Cucullia Schrank, I802, which Sodoffsky considered to be inappropriate.

CUCULLUNA Köhler, (1952) I951, Acta zool. lilloana 12: I 39.

CUCU

Type-species: Cuculluna cristagalli Köhler, (I952) I95I, ibidem 12: 139 , by original designation. Syntypes $\hat{\sigma}, \mathcal{q}$, Argentina: [La Rioja Prov.], La Rioja (ZSBS, Munich).

CULASTA Moore, I881, Proc. zool. Soc. Lond. 1881: 376.

OPHI

Type-species: Culasta indecisa Moore, I88I, ibidem 1881; 377, by monotypy. Syntypes, [India]: Madras (BMNH); Bombay (BMNH); Benares.

CULICULA Walker, [1863] I864, J. Proc. Linn. Soc. (Zool.) 7: i 78.

H YPE

Type-species: Culicula bimarginata Walker, [1863] I864, ibidem 7: 178, by monotypy. Lectotype $\tilde{\sigma}$, Borneo: Sarawak (UM, Oxford), designated (as type) by Swinhoe, 1900, Cat. east. and Aust. Lepid. Heterocera 2: 197.

C. bimarginata is a junior subjective synonym of Bocana schaldusalis Walker, [1859] 1858, List Specimens lepid. Insects Colln Br. Mus. 16: I80.

See also Nabartha Moore, [1885].

CULTRIPALPA Guenée, in Boisduval \& Guenée, I852, Hist. nat. Insectes (Lépid.) 7: 332. OPHI Type-species: Cultripalpa partita Guenée, I852, ibidem 7: 332, by monotypy. Holotype $\hat{o}$, India : central (BMNH).

CUNEISIGNA Hampson, I9I3, Cat. Lepid. Phalaenae Br. Muss. 12: 7. Available, but without included species until Hampson, I9I3, ibidem 13: vii, 3I.

CATO

Type-species: Trigonodes obstans Walker, I858, List Specimens lepid. Insects Colln Br. Mus. 14: I 449, by subsequent designation by Hampson, I9I3, ibidem 13: 31. Holotype , South Africa (BMNH).

CUPHANOA Hübner, [I82I] I8I6, Verz. bekannter Schmett.: 230.

HADE

Type-species: Noctua stabilis [Denis \& Schiffermüller], I775, Ankündung syst. Werkes Schmett. Wienergegend: 76 , by subsequent designation by Hampson, 1905, Cat. Lepid. Phalaenae Br. Mus. 5: 409. Type(s), [Austria]: Vienna district (Collection destroyed, Horn \& Kahle, 1936, Ent. Beih. Berl.-Dahlem 3: 243).

TAENIOCAMPA Guenée, 1839, Annls Soc. ent. Fr. 8: 477.

Type-species: Noctua stabilis [Denis \& Schiffermüller], I775, by subsequent designation

by Guenée, I 852, in Boisduval \& Guenée, Hist. nat. Insectes (Lépid.) 5: 354.

Taeniocampa is a junior objective synonym of Cuphanoa Hübner, [I82I].

CYPHONOA Agassiz, I846, Nomencl. zool. Index univl.: 108, II3.

An unjustified emendation of Cuphanoa Hübner, [1 821 ].

CURGIA Walker, [1863]. See Leucocosmia Butler, I886.

ACRO

CURUBASA Moore, i 881, Proc. zool. Soc. Lond. 1881: 366.

NOCT

Type-species: Alaria lanceolata Walker, I865, List Specimens lepid. Insects Colln Br. Mus. 33: 767, by original designation. Holotype $\hat{\delta}$, [INDIA]: S. Hindostan (BNMH).

A. lanceolata is a junior subjective synonym of Leocyma galatheae Wallengren, 1856 , Anteckningar $i$ Zool. Lund: $5^{8 .}$

CURVATULA Staudinger, 1892, in Romanoff, Mém. Lépid. 6: 601. $\operatorname{ACON}$ Type-species: Curvatula pallicostata Staudinger, I 892, ibidem 6: 601, pl. I2, fig. 6, by 
monotypy. Syntypes I $\hat{0}$, I + , [U.S.S.R.]: Amur district, Chingan (MNHU, Berlin); Sutschan (MNHU, Berlin).

C. pallicostata is a junior subjective synonym of Selenis mira Butler, I1879, Illust. typical Specimens Lepid. Heterocera Colln Br. Mus. 3: x, 29, pl. 47, fig. 6.

CUSPIDIA Chapman, I89o. See Triaena Hübner, I818.

ACRO

CUTINA Walker, I866, List Specimens lepid. Insects Colln Br. Mus. 35: I734.

CATO

Type-species: Cutina albopunctella Walker, I866, ibidem 35: I735, by monotypy. Holotype ô. U.S.[A.] (B.MNH).

CYATHISSA Grote, I88I, Bull. U.S. geol. geogr. Surv. Territ. 6: 576.

ACRO

Type-species: Bryophila percara Morrison, 1875, Proc. Boston Soc. nat. Hist. 17: 2I3, by monotypy. Type(s), [U.S.A.]: Texas, Waco.

CYCLODES Guenée, I852, in Boisduval \& Guenée, Hist. nat. Insectes (Lépid.) 7: $26 . \quad$ opHI Type-species: Erebus omma Hoeven, 1840, Tijdschr. Natuurl. Gesch. Physiol. 7: 28I, pl. 7, fig. 7, by monotypy. Type(s), JAvA.

See also Beregra Walker, 1858.

CYCLOPERA Hampson, 1908, Cat. Lepid. Phalaenae Br. Mus. 7: 6. Available, bint without included species until Hampson, I9Io, ibidem 9: xiii, 389.

ACRO

Type-species: Megalodes similis Hampson, 1902, Ann. S. Afr. Mus. 2: 382, by subsequent designation by Hampson, I9Io, ibidem 9: 389. Type(s) ô. [RHodesia]: Mashonaland, Salisbury (BMNH).

CYCLOPIS Hübner, [182 I May], Samml. exot. Schmett. 2: pl. [213].

OPHI

Type-species: Cyclopis caecutiens Hübner, [I82I], ibidem 2: pl. [2I3], by monotypy. Type(s) + , Type-Locality not stated [Equatorial South America].

Cyclopis was also used by Hübner, I82 I December, Index exot. Lepid.: [2], for the same concept.

CYCLOPRORA Turner, i9zo Trans. (Proc.) R. Soc. S. Aust. 44: I4O, I44.

ACRO

Type-species: Prometopus nodyna Turner, I904, ibidem 28: 215, by monotypy. Holotype ô, Australia: Qd, Brisbane (ANIC, Canberra).

CYCLOPRORA Turner, I93I. See Hedymiges Turner, 1933.

ACRO

CYCLOPROSOPUS Graede, I939, in Seitz, Gross-Schmett. Erde 15: 280.

OPHI

Type-species: Alamis strigifera Pagenstecher, I907, in Voeltzkow, Reise Ostafrika 2: i I , pl. 6, fig. I 4 , by monotypy. Type(s), Madagascar.

The date of publication of the part of Seitz containing page 280 was stated on page 279 as 1939 June 2oth in both the German and English editions.

CYCLOPTER YX Guencee, 1854, in Boisduval \& Guenée, Hist. nat. Insectes (Lépid.) 8: 46.

Type-species: Cyclopteryx observalis Guenée, I854, ibidem 8: 47, by subsequent designation by Schaus, i916, Proc. U.S. natn. Mus. 50: 366. Holotype ㅇ, [French GuiAna]: Cayenne (BMNH).

CYDOSIA Duncan [\& Westwood], 1841, in Jardine, Naturalist's Library 33 (Ent. 7): I93.

$\triangle \mathrm{CON}$

Type-species: Phalaena nobilitella Cramer, 1779, Uitlandsche Kapellen 3: 1 28, pl. 264. fig. G, by monotypy. Holotype, Curaçao [I.]

Cydosia was attributed to Dincan, by Sherborn, 1924, Index Anim. (1801-1850): 1743, and was attributed to Westwool, by Neave, 1939, Nomencl. zool. 1:924. Justification for the latter can be found in Stainton, I885, Entomologist's mon. Mag. 21: I8I-186, who quoted from a letter by Westwood. The difficulty can best be overcome by attributing Westwood with anonymous junior authorship. 
CYLIGRAMMA Boisduval, I833, Nouv. Annls Mus. Hist. nat. Paris 2: 257.

Type-species: Cyligramma joa Boisduval, I833, ibidem 2: 258, by subsequent designation by Desmarest, (1857), in Chenu, Encycl. Hist. nat. Papillons nocturnes: I 30. Type(s), Madagascar (BMNH).

Cyligramma and C. joa were also published in 1833 in Faune ent. Madagascar, Bourbon et Maurice (Lépid.): 109-1 Io, pl. 16, fig. 2.

CYLOGRAMMA Agassiz, I846, Nomencl. zool. Index univl.: I I I, I I 2.

An unjustified emendation of Cyligramma Boisduval, I833.

CYLOGRAMMA Agassiz, I846. See Cyligramma Boisduval, I833.

CATO

$\$$ CYMATHOPHORA, misspelling. See Achlya Billberg, 1820 .

THYATIRIDAE

CYMATIDA Sodoffsky, I837, Bull. Soc. imp. Nat. Moscou 1837 (6) : 91.

GEOMETRIDAE

Type-species: Geometra strigaria Hübner, [I799] I796, Samml. eur. Schmett. 5 : pl. 18, fig. 95, by subsequent designation (for Acidalia Treitschke) by Duponchel, 1829, in Godart \& Duponchel, Hist. nat. Lépid. Papillons Fr. 7(2) : 108.

ACIDALIA Treitschke, I825, Schmett. Eur. 5(2): 438 .

Type-species: Geometra strigaria Hübner, by subsequent designation by Duponchel, I 829 .

A junior homonym of Acidalia Hübner, [I8I9] I8I6, Vevz. bekannter Schmett.: 3I,Lepid., Nymphalidae. The objective replacement name is Cymatida Sodoffsky, 1837 .

Acidalia has been used as the original generic name for species which are now in the Noctuidae.

CYMATOPHORA Treitschke, I 825. See Achlya Billberg, I 820.

THYATIRIDAE

CYMATOPHOROPSIS Hampson, I894, Fauna Bv. India (Moths) 2: xvii, $397 . \quad$ OPH Type-species: Gluphisia sinuata Moore, 1879, Proc. zool. Soc. Lond. 1879: 405, by original designation. Type(s) , , [INDIA]: N.E. Bengal.

CYMOBLEMMA Hampson, I926, Descr. new Genera Species Lepid. Phalaenae Subfamily Noctuinae Br. Mus.: 409 .

OPHI

Type-species: Acantholipes crenelata Hampson, 1907, J. Bombay nat. Hist. Soc. 17: 662 (but proposed as ¥'Acantholipis', an incorrect subsequent spelling), by original designation. Holotype $\sigma^{t}$, India: Travancore, Pirmad (BMNH).

CYMODEGMA Tams, I935, Insects Samoa 3(4): 2 I 2.

CATO

Type-species: Cymodegma buxtoni Tams, 1935, ibidem 3(4): 212, pl. 6, fig. I7, pl. 7, fig. 6, by original designation. Holotype 9 , SamoA: Upolu, Malololelei (BMNH).

CYMONIA Dyar, I920, Insecutor Inscit. menst. 8: I9I.

$A C O N$

Type-species: Cymonia harminella Dyar, I920, ibidem 8: I9I, by monotypy. Holotype , Mexico: Sinaloa, Venadio (USNM, Washington).

CYMOSAFIA Hampson, I913, Cat. Lepid. Phalaenae Br. Mus. 12: 9. Available, but without included species until Hampson, I913, ibidem 13: ix, I58.

CATO

Type-species: Focilla laba Druce, 189o, Biologia cent.-am. (Zool.) Lepid. Heterocera 1: 392, pl. 33, fig. 3, by subsequent designation by Hampson, I9I3, ibidem 13: I58. Syntypes 2 ex., Guatemala: Cerro Zunil (BMNH); Volcan de Atitlan (BMNH).

CYNISCA Fawcett, r918. See Siccyna nom. n.

OPHI

CYPHANTA Walker, I865, List Specimens lepid. Insects Colln Br. Mus. 33: 855.

ACRO

Type-species: Cyphanta xanthochlora Walker, I865, ibidem 33: 856 , by monotypy. Holotype ô., [IndiA]: Hindostan (BMNH). 
CYPHOTOPS YCHE Hampson, I 895, Trans, ent. Soc. Lond. 1895: 297.

Type-species: Cyphotopsyche ustipennis Hampson, I895, ibidem 1895: 297, by original designation. Syntypes $\tilde{\jmath}$, BHutan (BMNH). CEYLon (BMNH).

C. ustipennis is a junior subjective synonym of Sarbena lignifera Walker, I862.

See also Sarbena Walker, I862.

CYREBIA Guenée, 1852, in Boisduval \& Guenée, Hist. nat. Insectes (Lépid.) 6: 195. Noct Type-species: Cyrebia luperinoides Guenée, I852, ibidem 6: 196, pl. 4, fig. I2, by subsequent designation by Hampson, 1903, Cat. Lepid. Phalaenae Br. Mus. 4: 467. Syntypes 40 . [U.S.S.R.]: S. Russia.

CYRIMA Schaus, 1904, Trans. Am. ent. Soc. 30: I 52.

ACRO

Type-species: Cyrima muscosa Schaus, I904, ibidem 30: 152, by monotypy. Type(s), MExico: Coatepec (USNM, Washington).

CYRTANDRA Roepke, I9łI, Zoöl. Meded., Leiden 23: 2n. OPHi

Type-species: Cyrtandra borneensis Roepke, 1941, ibidem 23: 29, pl. I, fig. 7, by original designation. Holotype ô, BorNEo: E., Kariorang (RNH, Leiden).

CYTOCANIS Hampson, I908, Cat. Lepid. Phalaenae Br. Mus. 7: 8. Available, but without included species until Hampson, Igro, ibidem 9: viii, I Io. ACRO

Type-species: Miana denticulosa Walker, I 865, List Specimens lepid. Insects Colln Br. Mus. 32: 676, by subsequent monotypy. Holotype $q$, [INDIA]: S. Hindostan (BMNH).

CYTOTHYMIA Hampson, 1908, Cat. Lepid. Phalaenae Br. Mus. 7: 5. Available, but without included species until Hampson, r910, ibidem 9: xiii, 370.

ACRO

Type-species: Metoptria absita Felder \& Rogenhofer, I874, Reise öst. Fregatte Novara (Zool.) 2 (Abt. 2): pl. Io8, fig. 36, by subsequent monotypy, but included by Hampson as ‡ 'obsita Feld.', an incorrect subsequent spelling. Type(s) $\hat{o}$, [South Africa]: Promont. b. spei [Cape of Good Hope] (BMNH).

CYTTARALOPHA Bethune-Baker, 1908, Novit. zool. 15: 223.

HYPE

Type-species: Cyttaralopha loxographa Bethune-Baker, I908, ibidem 15: 224, by original designation. Type(s) ô, New Guinea: [Papua], R. Aroa (BMNH).

DABARITA Walker, [I857] I 856, List Specimens lepid. Insects Colln Br. Mus. 10: 4I2, 478.

CHLO

Type-species: Dabarita subtilis Walker, [1 857 ] I856, ibidem 10: 479 , by monotypy. Syntypes $3(0$, O), INDIA: northern (BMNH).

D. subtilis is a junior subjective synonym of Bombyx angulata Fabricius, r 793, Ent. Syst. 3 (I) : $48 \mathrm{r}$.

DACIRA Walker, [1858] I 857, List Specimens lepid. Insects Colln Br. Mus. 12: 772, 801. Acro Type-species: Dacira combusta Walker, [1858] I857, ibidem 12: 802, by monotypy. Holotype ô. [BRazIL]: [Amazon], Villa Nova (BMNH).

DADDALA Walker, I865, List Specimens lepid. Insects Colln Br. Mus. 33: 974.

OPHI

Type-species: Daddala quadrisignata Walker, 1865, ibidem 33: 974, by monotypy. Type(s) q. TYPE-LOCALITY not stated (BMNH) [INDIA].

DADICA Muore, I881, Proc. zool. Soc. Lond. 1881 : 349.

ACRO

Type-species: Dadica lineosa Moore, r88 r, ibidem 1881:349, by monotypy. Type(s) ऽ. [INDIA]: Punjab Hills (BMNH).

DAEDALINA Möschler, I880, I'erh. zool.-bot. Ges. Wien 30(Abh.): 385 .

$\triangle \mathrm{CON}$

Type-species: Daedalina clevia Möschler, I880, ibidem 30 (Abh.): 386, pl. 9, fig. 44,

by monotypy. Holotype $\hat{\sigma}$. Surinam: Paramaribo (MNHU, Berlin). 
DAGASSA Walker, I 858, List Specimens lepid. Insects Colln Br. Mus. 15: I 546, I592. OPH I Type-species: Dagassa eupithecioides Valker, I 858 , ibidem 15: I 593, by monotypy. Syntypes 2 으. [Dominican Republic] St. Domingo (BMNH).

DAHLIA Pagenstecher, I900, Zoologica, Stuttg. 12 (29): I 20.

OPHI

Type-species: Dahlia hesperioides Pagenstecher, I90o, ibidem 12 (29): I 20, pl. I, fig. I 3, by monotypy. Type(s) $\hat{0}$, New Guinea: Bismarck Archipelago.

DALLOLMOIA Berio, I972, Memorie Soc. ent. ital. 51: i 76.

NOCT

Type-species: Dallolmoia triangula Berio, I972, ibidem 51: I76, fig. I8, by original designation. Holotype $\hat{o}$, Tanzania: Ikonda (Colln E. Berio, Genoa).

DANDACA Walker, I856, List Specimens lepid. Insects Colln Br. Mus. 9: I37, I 49.

Type-species: Dandaca columba Walker, 856 , ibidem 9 . I q. INDIA: northern (BMNH).

DANTONA Walker, I 859, List Specimens lepid. Insects Colln Br. Mus. 19: IO2I.

ACRO

Type-species: Dantona busalis Walker, I 859, ibidem 19: I02 I, by monotypy. Type(s) o., [BRazIL]: Rio Janeiro (UMI, Oxford).

D. busalis is a junior subjective synonym of Nonagria stillata Guenée, 1852, in Boisduval \& Guenée, Hist. nat. Insectes (Lépid.) 5: 107.

DAONA Walker, I864, J. Proc. Linn. Soc. (Zool.) 7: I9o.

OPHI

Type-species: Daona mansueta Walker, I864, ibiclem 7: I90, by monotypy. Lectotype ㅇ, Borneo: Sarawak (UM, Oxford), designated (as type) by Swinhoe, Igoo, Cat. east. and Aust. Lepid. Heterocera 2: 57.

See also Byturna Moore, I 883.

GAUZANIA Walker, [1866] i 865, List Specimens lepid. Insects Colln Br. Mus. 34: I 208.

Type-species: Gauzania mundalis Walker [I866] I865, ibidem 34: I208, by monotypy. Holotype 9 , [Australia]: [W. Aust.], R. Swan (BMNH).

G. mundalis is a junior subjective synonym of Catada detersalis Walker, [1866] I865, ibidem 34: I I 75 .

A junior homonym of Gauzania Walker, [1856] 1857, J. Proc. Linn. Soc. (Zool.) 1: 130, Diptera. There is no objective replacement name but $G$. mundalis is congeneric with Daona mansueta Walker, I864, the type-species of Daona Walker, I864. The latter is therefore available for use as a subjective replacement name.

DAPHA Walker, I863 [April I 8th], List Specimens lepid. Insects Coln Br. Mus. 27: I 24. CHLO Type-species: Dapha valeusalis Walker, I863, ibidem 27: I25, by monotypy. Holotype 9 , Ceylon (BMNH).

$\boldsymbol{D} \boldsymbol{A P H A}$ Walker, [1863 May I 3 th]. See Hapda noñ. n. HYPE

$\ddagger$ DAPHAENURA, misspelling. See Daphoenuva Butler, I878. CUCU

$\ddagger$ DAPHENURA, misspelling. See Daphoenura Butler, I878. CUCU

$\ddagger$ DAPHNAEURA, misspelling. See Daphoenura Butler, I $878 \quad$ CUcU

DAPHOENURA Butler, I878, Ann. Mag. nat. Hist. (5) 2: 457. CUCU

Type-species: Daphoenura fasciata Butler, I878, ibidem (5) 2: 457 , by original designation. Lectotype 9 , MAdagascar: Ellongo (BMNH), designated by Viette, [1963] 1962, Annls Soc. ent. Fr. 131:281.

$\ddagger D A P H N A E U R A$; Mabille, I 880, Bull. Soc. ent. Fr. (5) 9 : clxxiv.

An incorrect subsequent spelling.

$\ddagger D A P H A E N U R A$; Butler, I 882, Cistula ent. $3: 3$.

An incorrect subsequent spelling, also used by Kirby, I892, Synonymic Cat. I.epid. Heterocera 1: 204. 
$\ddagger D A P H E N U R A$; Strand, in Aurivillius \& Wagner, Lepid. Cat. 5: 73.

An incorrect subsequent spelling.

DARCETA Herrich-Schäffer, I856, Samml. nener oder wenig bekannter aussereur. Schmett. 1:

I3, pl. 92, figs 524-526.

AGAR

Type-species: Darceta hesperina Herrich-Schäffer, 1856, ibidem 1: I3, pl. 92, figs 524-526, by monotypy. Syntypes, BrazIL: interior (BMNH).

The type-species was proposed as hesperina on page I 3 and hesperica on the wrapper of the plates. Both names were published in I 856 but the exact dates of publication of the text and of the plates are not known. The spelling hesperina as used in the text on page 13 and later (in I858) on pages 7 I and 84 has usually been used. Herrich-Schäffer attributed both Darceta and hesperina to Boisduval. The two syntypes have been labelled in Boisduval's handwriting as hesperina.

$D$. hesperina is a junior subjective synonym of Phalaena proserpina Stoll, I782.

See also Clitis Walker, [1858].

DARCETINA Felder, 1874, in Felder \& Rogenhofer, Reise öst. Fregatte Novara Zool. 2 (Abt. 2): pl. 99.

AGAR

Type-species: Darcetina cinerosa Felder, I874, ibidem 2 (Abt. 2): pl. 99, fig. I9, by monotypy. Type(s) $q$, [Brazil $]:$ R. Amazonas.

D. cinerosa is a junior subjective synonym of Bepara sublata Walker, [I865] I864, List Specimens lepid. Insects Colln Br. MIus. 31: I 43.

DARGIDA Walker, I856, List Specimens lepid. Insects Colln Br. Mus. 9: I65, 20 I.

HADE

Type-species: Dargida grammivora Walker, I856, ibidem 9: 202, by monotypy. Syntypes 30 , VenEzLELA (BMNH).

DASEOCHAETA Warren, [1907], in Seitz, Gross-Schmett. Erde 3: I I.

ACRO

Type-species: Agriopis viridis Leech, 1889, Proc. zool. Soc. Lond. 1889: 502, pl. 5I, fig. 6 , by monotypy. Syntypes $4(\hat{0}$, o) JaPan: [Honshu], Yokohama (BMNH).

The date of publication of the part of Seitz containing page I I is not stated in the text. Copies of these parts of both the German and English editions were received at BMNH in 1907 and this is the date accepted by Hampson, I909, Cat. Lepid. Phalaenae Br. Mus. 8: 22; and by Neave, 1939, Nomencl. zool. 2: I5.

DASEUPLEXIA Hampson, igo6, Cat. Lepid. Phalaenae Br. Mus. 6: xi, 303.

CUCU

Type-species: Luperina lagenifera Moore, I882, in Hewitson \& Moore, Descr. new Indian lepid. Insects Colln late Mr W.S. Atkinson: I I4, by original designation. Type(s) o., , Inda: [W. Bengal], Darjiling (BMNH).

DAS YBLEMMA Dyar, 1923, Insecutor Inscit. menstr. 11: I8.

ACON

Type-species: Dasyblemma straminea Dyar, I923, ibidem 11: 19, by monotypy. Holotype ô, U.S.A.: California, Palm Springs (USNM, Washington).

DASYCAMPA Guenée, I837, Annls Soc. ent. Fr. 6: 224.

CUCU

Type-species: Noctua rubiginea [Denis \& Schiffermüller], I775, Ankündung syst. Herkes Schnett. Wienergegend: 86, by monotypy. Type(s), [AustriA]: Vienna district (Collection destroyed, Horn \& Kahle, 1936, Ent. Beih. Berl.-Dahlem 3: 243).

$\ddagger$ OMALOSOMA Walker, [1857] i 856, List Specimens lepid. Insects Colln Br. Mus. 10: 455 .

Published as a junior symonym of Dasycampa Guenée, i 837, and not subsequently treated as an available name under the Code, Article I I (d).

DASYERGES Draudt, I950, Mitt. münch. ent. Ges. 40: 59.

CUCU

Type-species: Dasyerges poliastis 1)raudt, I950, ibidem 40: 59, pl. 4, fig. I2, by monotypy. Syntypes $\hat{0}, \mathcal{q}$, Cruna: [Yunnan Prov.], A-tun-tse and Li-kiang (MAK, Bonn).

D. poliastis was described as 'poliasits' but figured as poliastis. Under the Code, Article $32(\mathrm{~b})$, I have accepted poliastis as the correct original spelling. 
DASYGASTER Guenée, I852, in Boisduval \& Guenée, Hist. nat. Insectes (Lépid.) 5 : 20 I.

HADE

Type-species: Dasygaster hollandiae Guenée, I852, ibidem 5: 20I, p1. 6, fig. I I, by subsequent designation by Hampson, 1905, Cat. Lepid. Phalaenae Br. Mus. 5: 473. Lectotype ô., [Australia] Nouvelle-Hollande (MNHN, Paris), designated by Viette, I95 I, Bull. mens. Soc. linn. Lyon 20: 160.

DAS YPODIA Guenée, 1852, in Boisduval \& Guenée, Hist. nat. Insectes (Lépid.) 7: I74. OPHI

Type-species: Dasypodia selenophora Guenée, 1852, ibidem 7: I75, by subsequent designation by Meyrick, I9I2, Trans. Proc. N.Z. Inst. 44: I05. Syntypes ${ }_{0}$, [Australia]: Tasmania. INDIA: central (BMNH).

DASYPOLIA Guenée, I852, in Boisduval \& Guenée, Hist. nat. Insectes (Lépid.) 6: 44. cucu

Type-species: Noctua templi Thunberg, 1792, Diss. Ent. sistens Insecta Suecica (4): 56, pl. 4, by monotypy. Type(s), Sweden.

DASYSPOUDAEA Smith, I882, Trans. Am. ent. Soc. 10: 2 I 3.

NOCT

Type-species: Heliothis lucens Morrison, I875, Proc. Acad. nat. Sci. Philad. 27: 69, by original designation. Syntypes 2 ex., [U.S.A.]: Mass., Nebraska.

DASYSTERNUM Staudinger, I896, Dt. ent. Z. Iris 8: 327.

CUCU

Type-species: Dasysternum tibetanum Staudinger, I896, ibidem 8: 327, pl. 6, fig. 9, by monotypy. Syntypes $4 \hat{\jmath}$, [CHInA]: Tibet, between Lob Noor and Kuku Noor (MNHU, Berlin).

DASYTHORAX Staudinger, I889, Stettin. ent. Ztg $\mathbf{5 0}: 38$.

CuCU

Type-species: Dasythorax polianus Staudinger, I889, ibidem 50: 38, by monotypy. Syntypes $3 \hat{\jmath}$. [U.S.S.R.]: [Kirghizia], Mts S. of Issyk-Kul (MNHU, Berlin).

DATA Walker, I862, J. Proc. Linn. Soc. (Zool.) 6: I9I.

ACRO

Type-species: Data thalpophiloides Walker, 1862, ibidem 6: 192, by monotypy. Lectotype + , Borneo: Sarawak (UM, Oxford), designated (as type) by Swinhoe, I9oo, Cat. east. and Aust. Lepid. Heterocera 2: 26.

DATUNGIA Boursin, I940, Mém. Mus. natn. Hist. nat. Paris 13: $324 . \quad$ ACro

Type-species: Datungia argillosa Boursin, I940, ibidem 13: 325, pl. 9, fig. Io, by original designation. Holotype $\widehat{\sigma}$, [China]: Kan-Su, R. Datung (LN, Karlsruhe).

DAULA Saalmüller, I89 I, Lepid. Madagascar: 302.

ACRO

Type-species: Daula abscissa Saalmüller, I891, ibidem: 303, fig. I80, by monotypy. Holotype $\widehat{\jmath}$, Madagascar: Nossi-Bé I. (SNG, Frankfurt).

DAVEA Berio, 1959, Annali Mus. civ. Stor. nat. Giacomo Doria 71: 309.

CATO

Type-species: Audea antennalis Berio, I954, Boll. Soc. ent. ital. 84: 56, by original designation. Holotype $\hat{\sigma}$. South West Africa: Tsumeb (Colln E. Berio, Genoa).

DAXATA Walker, r865. See Tadaxa nom. n.

OPHI

DECARYNODES Viette, 1961, Bull. Soc. ent. Fr. 66: 52.

ACRO

Type-species: Decarynodes ankasoka Viette, I96I, ibidem 66: 52, by original designation. Holotype 07 , Madagascar: Lakato, Ankasoka (MNHN, Paris).

DECELEA Walker, [1858] I857, List Specimens lepid. Insects Colln Br. Mus. 13: Io79, I I I 5. ACro Type-species: Decelea indigna Walker, [1858] I857, ibidem 13: III5, by subsequent designation by Hampson, r9o8, Cat. Lepid. Phalaenae Br. Mus. 7: 260. Syntypes $2 \hat{f}$, [Dominican Republic] St. Domingo (BMNH).

DECLANA Walker, 1858 , List Specimens lepid. Insects Colln Br. Mus. 15: I649.

GEOMETRIDAE

Type-species: Declana floccosa Walker, I858, ibidem 15: I649, by monotypy.

Declana was originally placed in the Noctuidae. 
DECTICRYPTIS Hampson, i910, Cat. Lepid. Phalaenae Br. Mus. 10 : x, i94.

Type-species: Niaccaba deleta Moore, [1885] I887, Lepid. Ceylon 3: 304, pl. I79, fig. I3, by original designation. Type(s), CEYLON (BMNH).

†DICTICRYPTIS; Neave, I939, Nomencl. zool. 2: 77.

An incorrect subsequent spelling.

DECTOCRASPEDON Schaus, I916, Proc. U.S. natn. Mus. 50: 262, 328.

HYPE

Type-species: Dectocraspedon braziliensis Schaus, I916, ibidem 50:328, by original designation. Type(s) ô. Brazil: Rio Janeiro, Espiritu Santo (USNM, Washington).

†EINHYPENA, misspelling. Sec Deinypena Holland, I 894 .

OPHI

DEINOPA Walker, I856, List Specimens lepid. Insects Colln Br. Mus. 9: 69, I35. OPнI

Type-species: Deinopa notabilis Walker, I856, ibidem 9: 135, by monotypy. Holotype

ㅇ, [BRAZIL]: Santarem (BMINH).

DEINOPALPUS Holland, I894, Psyche, Camb. 7 : 47.

OPHI

Type-species: Deinopalpus africana Holland, I894, ibidem 7: 47, fig., by original designation. Type(s) $\hat{0}$, West Africa (CMI, Pittsburgh).

D. africana is a junior subjective synonym of Gonitis leona Schaus, 1893, in Schaus \& Clements, On a Colln of Sierra Leone Lepid.: 36, pl. 3, fig. Io.

DEINYPENA Holland, I894, Psyche, Camb. 7: I23.

OPHI

Type-species: Deinypena lacista Holland, I894, ibidem 7: 124, pl. 2, figs 3, 4, by original designation. Syntypes $\widehat{\jmath},+$, West Africa: Benita; Kangwé. (CM, Pittsburgh). $\ddagger$ DEINHYPENA; Hampson, I9Io, Proc. zool. Soc. Lond. 1910: 448.

An incorrect subsequent spelling.

DEL GAMMA Moore, [1885] i 887, Lepid. Cevlon 3: I68.

OPHI

Type-species: Naxia calorifica Walker, 1858, List Specimens lepid. Insects Colln Br.

Mus. 14: I 406, by original designation. Syntypes, [BAngladesh]: [E. Bengal], Silhet (BMNH). [TNdiA]: N. Hindostan (BMNH). CEYlon.

DELOCOMA Swinhoe, 1905, Ann. Mag. nat. Hist. (7) 16: I 53.

OPH I

Type-species: Delocoma marmorea Swinhoe, 1905, ibidem (7) 16: I53, by monotypy.

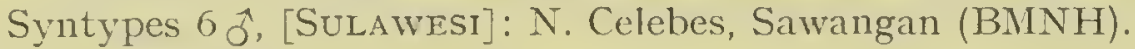

DELTA Saalmüller, I891. See Lepidodella Viette, I967.

ACRO

DELTAPTERUM Hampson, I894, Fanna Br. India (Moths) 2: 138.

NOLI

Type-species: Deltapterum peguense Hampson, I894, ibidem 2: 138, fig. 93, by original designation. Holotype $\hat{\jmath}$, Burma: E. Pegu (BMNH).

$\ddagger$ DEL TINELLA Hampson, no published reference found.

HYPE

DELTOTE R. L., I8I7, Jenaische Allg. Lit.-Ztg 1: 288.

ACON

Type-species: Phalaena argentula Hübner, [1787], Beitr. Gesch. Schmett. 1 (2): 9, pl. 2, fig. F, by PRESENT DESIGNATION. Type(s), [Germany]: Augsburg district.

$P$. argentula was attributed to Esper, [I79I], by Ochsenheimer, Treitschke, Guenée and others. At times it has been referred to under its senior subjective synonyms Pyralis bankiana Fabricius, I775, and Tortrix olivana [Denis \& Schiffermuiller], I775. As a result of Opin. Decl. int. Commn zool. Nom. (1958) 19 Opinion 5I6, the Fabrician name has priority and thus the valid name of the type-species is Deltote bankiana (Fabricius, 1775), Syst. Ent: 645 .

BANIIIA Guencee, i 852, in Boisduval \& Guenće, Hist. nat. Insectes (Lépid.) 6: 23 I.

Type-species: Phalaena argentula Hübner, [ $\left.{ }^{7} 787\right]$, by original designation, but cited as 'B. argentula Esper', an incorrect authorship.

A junior homonym of Bankia Gray, I842, Synopsis Contents Br. Mus. (Edn 44): 76, Mollusca. The objective replacement name is Deltote R.L., I8I7. 
DEMAS Stephens, I829. See Colocasia Ochsenheimer, I8I6.

DENDROTHRIPA Hampson, I 896, Fauna Br. India (Moths) 4: 527.

SARR

Type-species: Dendrothripa rufipuncta Hampson, I896, ibidem 4: 527, fig. 275, by original designation. Type(s) $\vec{b}$. India: [Punjab], Kangra (BMNH).

D. rufipuncta is a junior subjective synonym of Garella rotundipennis Walker, [1863].

See also Gavella WValker, [I863].

DEOBRIGA Walker, I869, Characters undescr. Lepid. Heterocera.: ${ }_{4} \mathrm{I}$.

Type-species: Deobriga chrysopasa Walker, I 869, ibidem: 4I, by monotypy. Type(s)

0. TYPE-LOCAlity not stated (BMNH) [U.S.A.].

D. chrysopasa is a junior subjective synonym of Basilodes pepita Guenée, I 852 .

See also Basilodes Guenée, 1852.

DEPALPATA Rothschild, I9I9, Ann. Mag. nat. Hist. (9) 3: 488.

AGAR

Type-species: Depalpata mirabilis Rothschild, I919, ibidem (9) 3: 488, by monotypy.

Type(s) ô. New Guinea: [Papua], Hydrographer Mts (BMNH).

DERCETIS Grote, I878. See Redectis nom. n.

OPHI

DEREMMA Walker, I865, List Specimens lepid. Insects Colln Br. Mus. 33: 863.

OPHI

Type-species: Deremma simulatrix Walker, I865, ibidem 33: 864, by monotypy. Holotype $\hat{\sigma}$. Sierra Leone (BMNH).

DERMALEIPA Saalmüller, i891, Lepid. Madagascar: 460.

CATO

Type-species: Ophiodes parallelipipeda Guenée, I852, in Boisduval \& Guenée, Hist. nat. Insectes (Lépid.) 7: 230, by monotypy. Type(s), Senegal.

DERRIMA Walker, [1858] i 857, List Specimens lepid. Insects Colln Br. Mus. 12: 766, 770. Noct Type-species: Derrima stellata Walker, [1858] I857, ibidem 12: 770 , by monotypy. Holotype q, U.S.A. (BMNH).

See also Philomma Grote, $186_{4}$.

DERTHISA Walker, I857. See Cleoceris Boisduval, [1836].

CUCU

DESANA Swinhoe, I9oo, Cat. east. and Aust. Lepid. Heterocera 2: 26.

HADE

Type-species: Plusia peninsulata Walker, I865, List Specimens lepid. Insects Colln Br. Mus. 33: 840, by monotypy. Lectotype ô, [Moluccas]: Mysol (UM, Oxford), designated (as type) by Swinhoe, I9oo, ibidem 2: 26.

DESERTULLIA Köhler, (1952) I95I, Acta zool. lilloana 12: 157.

CUCU

Type-species: Desertullia argyrofulva Köhler, (I952) I95I, ibidem 12: I58, by original designation. Syntypes $\hat{\jmath}, q$, Argentina: Chubut [Prov.], Comodoro Rivadavia (ZSBS, Munich); [Mendoza Prov.], Mendoza (ZSBS, Munich).

$\$$ DESMOPHORA Stephens, I 829 [June]. See Desmophora Stephens, I829 [August]. ACON

DESMOPHORA Stephens, I 829 [August], Syst. Cat. Br. Insects 2: Iog.

ACON

Type-species: Phalaena catena Sowerby, I 805, Br. Miscellany 1 (4): 29, pl. I4, by monotypy, but cited as Desmophora elegans Stephens, I829, an unnecessary replacement name, with catena in synonymy. Type(s), [GreAt Britain]: [London], [formerly in] Surr[e]y, Brixton.

$P$. catena is a junior subjective synonym of Bombyx nitidula Fabricius, 1787, Mantissa Insect. 2: 126.

†DESMOPHORA Stephens, I829 [June], Nom. Br. Insects: 43.

Both the generic and the only included name +elegans Stephens were nomina nuda.

EUPHASIA Stephens, I830, Illust. Br. Ent. (Haustellata) 3: II 5.

Type-species: Phalaena catena Sowerby, 1805 , by monotypy.

Euphasia is a junior objective synonym of Desmophora Stephens, I829.

L 
TIMA Walker, [1858] i 857, List Specimens lepid. Insects Colln Br. Mus. 12: 839.

Type-species: Phalaena margaritata Drury, I782, Illust. nat. Hist. 3: 28, pl. 21, fig. 6, Index (but included by Walker as +'margarita', an incorrect subsequent spelling), by monotypy. TyPe-Locality stated to be New York, but this was probably an error as this species is not known to occur in the New World, but is found in southern Africa.

A junior homonym of Tima Eschscholtz, I829, Syst. Acalephen: I03,-Coelenterata. There is no objective replacement name but $P$. margaritata Drury is congeneric with Phalaena catena Sowerby, the type-species of Desmophora Stephens, I829. The latter is therefore available for use as a subjective replacement name.

DESPUMOSIA nom. n. for Pseudosmia Köhler, (1952).

CUCU

Type-species: Pseudosmia rubescens Köhler, (1952) I95I, Acta zool. lilloana 12: I6I, by original designation (for Pseudosmia Köhler). Syntypes $\hat{0}, \underline{q}$, Argentina: Chubut [Prov.], Comodoro Rivadavia (ZSBS, Munich).

PSEUDOSMIA Köhler, (1952) I95 I, Acta zool. lilloana 12: г6r.

Type-species: Psendosmia rubescens Köhler, (1952), by original designation.

A junior homonym of Pseudosmia Radoszkovsky, 1872, Horae Soc. ent. ross. 8: xviii, - Hymenoptera. The objective replacement name is Despumosia nom. n.

DETOUNDA Walker, i864, J. Proc. Linn. Soc. (Zool). 7: I93.

SARR

Type-species: Detounda spurcata Walker, 1864, ibidem 7: 194, by monotypy. Lectotype , Borneo: Sarawak (UMI, Oxford), designated (as type) by Swinhoe, I90o, Cat. east. and Aust. Lepid. Heterocera 2: 77.

DEVA Walker, [1858] i 857, List Specimens lepid. Insects Colln Br. Mus. 12: 94r, 962. OPH Type-species: Deva conducens Walker, [1858] 1857, ibidem 12:963, by subsequent designation by Warren, 1913, in Seitz, Gross-Schmett. Erde 3: 384, but cited as Plusia coelonota Kollar, I844, which Warren placed as the senior synonym of $D$. conducens. Syntypes 2 ô. CEYLON (BMNH).

D. conducens is a junior subjective synonym of Plusia coelonota Kollar, I844, in Hügel, Kaschmir und das Reich der Siek 4: 482.

DEVENA Walker, [1858] I857, List Specimens lepid. Insects Colln Br. Mus. 12: 941, 965. OPH Type-species: Devena atomifera Walker, [1858] I857, ibidem 12: 966, by monotypy. Holotype 9 , TyPE-LOCALITY not stated (BMNH) [MALAWI].

DEXIADENA Filipjev, 1927, Annu. Mus. zool. Acad.imp. Sci. Leningrad 28: $246 . \quad$ Acro Type-species: Hadena arcta Lederer, 1853, Verh. zool.-bot. Ver. Wien 3: 370, pl. 2, fig. 5, by original designation. Holotype ㅇ, [U.S.S.R.]: Siberia (MNHU, Berlin).

DIACHRYSIA Hübner, [I82 I] I8I6, Vevz. bekannter Schmett.: $252 . \quad$ PLUS

Type-species: Diachry'sia orichalcea Fabricius sensu Hübner, [1821] I816, ibidem: 252, [= Phalaena chryson Esper, I789, Die Schmett.4(2) Abschnitt I, pl. I4I, fig. 2 (not fig. I as stated in text); [I798-1804], ibidem: 446], by subsequent designation by Dyar, I902, $J l$ N.Y. ent. Soc. 10: 8 I (but cited as 'orichalcea Fab.'). Syntypes + (of chryson), Italy': central.

D. orichalcea Fabricius sensu Hübner has for long been accepted as a misidentification of Noctua orichalcea Fabricius, I775, Sy'st. Ent.: 607. Dyar, I902, stated 'Diachrysia contains cight species in Hübner's work, but they appear congeneric and orichalcea Fab., the second species mentioned, may be regarded as the type.' Hübner, [I 821 ], cited the second species as 'D. orichalcea Fabr. Mant. Noc. I75. Chryson Esp. Noc. 62.2. Hüibn. Noc. 278.' This is a case of a misidentified type-species which under the Code, Article 70 (a) should be referred to the Commission.

$\ddagger$ †LUSIA Hübner, [1806], Tentamen determinationis digestionis . . : : [2].

Included in a work rejected for nomenclatural purposes by Int. Commn zool. Nom., 1926. Smithson, misc. Collns 73 (4) Opinion 97: 19. Also idem, 1954, Opin. Decl. int. Commn zool. Nom. 6 Opinion 278: I40. 
Only included species: Phalaena chrysitis Linnaeus, 1758 , which is congeneric with Phalaena chryson Esper.

$\ddagger D Y A C H R Y S I A$; Geyer, I832, in Hübner. Zuträge Samml. exot. Schmett. 4: 22.

An incorrect subsequent spelling.

DIACOPE Hübner, [1820]. See Staurophora R.L., I8I 7 .

CUCU

DIADOCHIA Püngeler, I9I4, Dt. ent. Z. Iris 28: $4^{2}$.

ACRO

Type-species: Diadochia saca Püngeler, I9I 4, ibidem 28: 43, pl. 2, fig. I 2, by original designation. Syntypes 2 ô, I + , [U.S.S.R.]: Syr-Daria, Baigacum (MNHU, Berlin); Transcaspia (MNHU, Berlin).

DIADOCIS Saalmüller, I89I, Lepid. Madagascar: 294.

$\mathrm{HADE}$

Type-species: Diadocis longimacula Saalmüller, I89I, ibidem: 295, fig. 204, by monotypy. Holotype ô, Madagascar: Nossi-Bé I. (SNG, Frankfurt).

DIAGRAPtA Hampson, 1926, Descr. new Genera Species Lepid. Phalaenae Subfamily Noctuinae Br. Mus.: $54^{0}$.

OPHI

Type-species: Tyrissa laminata Butler, 1879, Trans. ent. Soc. Lond. 1879: 65, by original designation. Holotype, [BRazIL]: Amazons, Manaos (BMNH).

DIALITHIS Hübner, [I82I] I 8I6, Verz. bekannter Schmett.: 255.

OPHI

Type-species: Dialithis gemmifera Hübner, [1821] I8I6, ibidem: 255, by monotypy. Type(s) 9 , Type-Locality stated (in 1823, Zuträge Samml. exot. Schmett. 2: 30) to be Neuholland [Australia], but this was probably an error as the species illustrated is not known to occur in the Oriental Region but is present in equatorial South America.

DIALITHOPTERA Hampson, I900, Cat. Lepid. Phalaenae Br. Mus. 2: 4, 50. Noli

Type-species: Pisara gemmata Hampson, I896, Fauna Br. India (Moths) 4: 506, by original designation. Holotype $\widehat{\jmath}$, [SıkкıM] Sikhim (BMNH).

DIALLAGMA Smith, 1900, Proc. U.S. natn. Mus. 22: 483.

HYPE

Type-species: Diallagma lutea Smith, I90o, ibidem 22: 483 , by monotypy. Syntypes o. +, [U.S.A.]: Florida, Charlotte Harbor and Archer. (USNM, Washington).

D. lutea is a junior subjective synonym of Acrobasis latiorella Walker, I863, List Specimens lepid. Insects Colln Br. Mus. 27: 29.

DIALOXA Hampson, 1926, Descr. new Genera Species Lepid. Phalaenae Subfamily Noctuinae Br. Mus.: 299.

OPHI

Type-species: Ledaea arduine Druce, I89I, Biologia cent.-am. (Zool.) Lepid. Heterocera 1: 485 , pl. 40, figs I7, I8, by original designation. Syntypes 0 , 9 , Guatemala: Volcan de Atitlan (BMNH).

DIAMUNA Walker, [1858] I 857, List Specimens lepid. Insects Colln Br. Mus. 12:94I, 960. AGAR Type-species: Phalaena severa Stoll, I782, in Cramer, Uitlandsche Kapellen 4: 235 , pl. 398, fig. L, by monotypy. Type(s), Surinam.

†DIANTHAECIA, misspelling. See Hadena Schrank, I802。 HADE

$\ddagger$ DIANTHECIA, misspelling. See Hadena Schrank, I802. HADE

DIANTHOECIA Boisduval, I834. See Hadena Schrank, I802. HAdE

DIANTHOECIA Agassiz, I846. See Hadena Schrank, I802. HADE

DIAPERA Hampson, 1926, Descr. new Genera Species Lepid. Phalaenae Subfamily Noctuinae Br. Mus.: 377 .

OPHI

Type-species: Parora snowi Smith, I908, Jl N.Y. ent. Soc. 16: 96, by original desig-

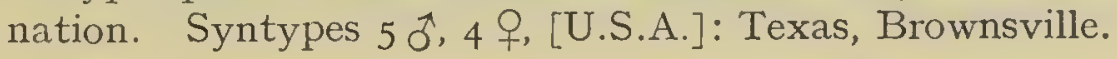


DIAPHONE Hübner, [1 820] I 8 I6, Verz. bekannter Schmett.: I 88.

HADE

Type-species: Bombyx elegans Fabricius, I 787, Mantissa Insect. 2: I i , by subsequent designation by Kirby; I892, Synonymic Cat. Lepid. Heterocera 1: 909. Type(s), [Soutu AFRICA]: [Cape of Good Hope] Cap. Bon. Spei.

$B$. elegans is a junior subjective synonym of Phalaena eumela Stoll, I 78 I, in Cramer, Uitlandsche Kapellen 4: I I I, pl. 347, fig. G.

Sce also Taeniopyga Wallengren, i 858.

DIAPOLIA Hampson, r926, Descr. new Genera Species Lepid. Phalaenae Subfamily Noctuinae Br. Mus.: 597 . OPHI

Type-species: Zethes magna Leech, I900, Trans, ent. Soc. Lond. 1900: 608, by original designation. Syntypes I J, I f, CHINA: [Szechwan], Moupin (BMNH); [Hupeh], Ichang (BMNH).

DIARSIA Hübner, [I82 I] I 8I6, Verz. bekannter Schmett.: 222.

NocT

Type-species: Noctua dahlii Hübner, [ISI3], Samml. eur. Schmett. 4: pl. 99, figs 465 , 466, by subsequent designation by Hampson, r9o3, Cat. Lepid. Phalaenae Br. Mus. 4: 363, 423, but cited as dahli Hampson, I903, an unjustified emendation. Syntypes, Europe.

DIASCIA Hampson, 1926, Descr. new Genera Species Lepid. Phalaenae Subfamily Noctuinae Br. Mus.: 273 .

$\mathrm{OPHI}$

'Type-species: Hingula transvitta Moore, I887, Lepid. Ceylon 3: 55I, pl. 2I5, fig. I, by original designation. Type(s), CEYLON (BMNH).

DIASTEMA Guenée, I 852, in Boisduval \& Guenée, Hist nat. Insectes (Lépid.) 6: 3I7. ACON

Type-species: Diastema tigris Guenée, I852, ibidem 6:3 I 7, pl. I 5, fig. 2, by subsequent designation by Hampson, igro, Cat. Lepid. Phalaenae Br. Mus. 10: 645. Syntypes, Colombia.

See also Nipista Walker, [1858].

DIASTREPTONEURA Warren, I 889, Trans. ent. Soc. Lond. 1889: 279.

$A C O N$

Type-species: Diastreptoneura distorta Warren, r 889, ibidem 1889: 280, by monotypy. Syntypes 2 oै. [BraziL]: Amazon basin, R. Jurua (BMNH).

DIATARAXIA Hübner, [I82 I] I8I6, Vevz. bekannter Schmett.: 2 I 9.

HADE

Type-species: Noctua splendens Hübner, [1808], Samml. cur. Schmett. 4: pl. 85, fig. 400 , by subsequent designation by Hampson, I905, Cat. Lepid. Phalaenae Br. Mus. 5 : 6o. Type(s), Europe.

DIA TENES Guenée, I 852, in Boisduval \& Guenée, Hist. nat. Insectes (Lépid.) 6: 442. OPHI Type-species: Diatenes gerula Guenée, I 852, ibidem 6: 443, pl. I 8, fig. 5, by subsequent designation by Viette, I95I, Bull. mens. Soc.linn. Lyon 20: I6I. Holotype ô. Australia (MNHN, Paris).

†DIAUTHOECIA, misspelling. See Hadena Schrank, r 802.

HADE

DICEROGASTRA Fletcher, I96I, Ruwenzovi. Exped. I952 1: I90.

HADE

Type-species: Miselia proleuca Hampson, I9I3, Ann. Mag. nat. Hist. (8) 12: 591, by original designation. Holotype o, [KEnya] Br. E. Africa: Makuru (BMNH).

†DICESTRA, misspelling. See Discestra Hampson, I905.

HADE

DICHAGRAMMA Grote, I 864, Proc. ent. Soc. Philad. 2: 439.

CUCU

Type-species: Dichagramma walkerii Grote, I864, ibidem 2: 439, pl. 9, fig. 5, by subsequent designation by Hampson, igo6, Cat. Lepid. Phalaenae Br. Mus. 6: 443, but cited as valceri Hampson, rgo6, an unjustified emendation. Type(s) 9. Canada: Middle States.

D. walkerii is a junior subjective synonym of Scopelosoma sidus Guenée, I 852 , in Boisduval \& Guenće, Hist. nat. Insectes (Lépid.) 5: 386. 
$\ddagger$ DICHAG YRIA, misspelling. See Dichagyris Lederer, 1857 .

NOCT

DICHAG YRIS Lederer, 1857, Noctuinen Eur.: 45, 206.

NOCT

Type-species: Agrotis melanura Carrara, I846, La Dalmazia descritta (13): 99, by monotypy, but included as 'melanura H.-Sch.', an incorrect authorship. Holotype, [Yugoslavia]: Dalmatia, Ragusa.

Carrara attributed $A$. melanura to Kollar, and Herrich-Schäffer did likewise. Lederer attributed this species to Herrich-Schäffer who had figured it.

$\ddagger$ DICHAGYRIA; Walker, r866, List Specimens lepid. Insects Colln Br. Mus. 33: 87 I.

An incorrect subsequent spelling.

DICHONIA Hübner, [1821] 1816, Verz. bekannter Schmett.: 217.

CUCU

Type-species: Noctua aeruginea Hübner, [1803], Samml. eur. Schmett. 4: pl. 8o, fig. 374, by subsequent designation by Butler, i89o, Trans. ent. Soc. Lond. 1890:67r. Type(s), EUROPE.

HORMA Walker, 1857, List Specimens lepid. Insects Colln Br. Muss. 11: 494, 603.

Type-species: Noctua aeruginea Hübner, [1803], by subsequent designation by Berio, r966, Annali Mus. civ. Stor. nat. Giacomo Doria 76: 60.

Horma is a junior objective synonym of Dichonia Hübner, [1821].

DICHONIOPSIS Warren, 1913, in Seitz, Gross-Schmett. Erde 11: 108.

CUCU

Type-species: Polia obliquisigna Hampson, 1902, J. Bombay nat. Hist. Soc. 14: 202, by original designation. Holotype $ᄋ$, India: [Himachal Pradesh], Simla (BMNH).

The date of publication of the part of Seitz containing page ro8 was stated on page 105 as rgr3 January 2oth in both the English and the German editions.

DICHROMIA Guenée, 1854, in Boisduval \& Guenée, Hist. nat. Insectes (Lépid.) 8: r8. HyPE Type-species: Phalaena orosia Cramer, r78o, Uitlandsche Kapellen 3: 149, pl. 275, fig. D (but included as 'D. orosialis Cr.', an unjustified emendation by Guenée, 1854), by subsequent designation by Moore, [1885] 1887, Lepid. Ceylon 3: 220. Syntypes, [INDIA] : Coromandel Coast.

P. orosia is a junior subjective synonym of Noctua sagitta Fabricius, r775, Syst. Ent.: 597 .

DICOPIS Grote, 1874, Annual Rep. Trustees Peabody Acad. Sci. 6: 23.

CUCU

Type-species: Dicopis muralis Grote, 1874, ibidem 6:23, by monotypy. Syntypes $\overrightarrow{0}$,

[U.S.A.]: Pennsylvania (BMNH); New York (BMNH).

D. muralis is a junior subjective synonym of Psaphida resumens Walker, I865.

See also Psaphida Walker, 1865 .

$\ddagger$ DICTICRYPTIS, misspelling. See Decticryptis Hampson, I9ro.

$\mathrm{ACON}$

DICYCLA Guenée, I852, in Boisduval \& Guenée, Hist. nat. Insectes (Lépid.) 6: 7 .

ACRO

Type-species: Phalaena oo Linnaeus, r758, Syst. Nat. (Edn ro) 1: 507, by monotypy. Type(s), TYPE-LOCALITY not stated (LS, London) [EUROPE].

Dicycla when originally proposed contained a second species, but this was doubtfully included and under the Code, Article 68(c), is not eligible for designation as type-species.

EUGRAMMA Stephens, r85o, List Specimens Br. Anim. Colln Br. Mus. 5: r2r.

Type-species: Phalaena oo Linnaeus, $\mathrm{r} 75^{8}$, by monotypy.

A junior homonym of Eugramma Billberg, 1820, Enumeratio Insect. Mus. G. J. Billberg: 78, - Lepid., Nymphalidae. The objective replacement name is Dicycla Guenée, 1852.

DIDA Druce, r891, Biologia cent.-am. (Zool.) Lepid. Heterocera 1: 490.

HYPE

Type-species: Dida cidaria Druce, I891, ibidem 1: 490, pl. 4I, fig. 8, by original designation. Holotype $\widehat{o}^{\hat{n}}$ Mexico: Las Vigas.

DIDIGUA Walker, 1862, J. Proc. Linn. Soc. (Zool.) 6: 181.

CHLO

Type-species: Didigua purpureoscripta Walker, 1862, ibidem 6: 181, by monotypy. 
Lectotype ․, Bonneo: Sarawak (UM, Oxford), designated (as type) by Swinhoe, Igoo, Cat. east. and Aust. Lepid. Heterocera 2: 99.

D. purpureoscripta is a junior subjective synonym of Nonagria seticornis Mallker, I862, ibidem 6: I 80 .

DIDUGUA Druce, I89 I, Biologia cent.-am. (Zool.) Lepid. Heterocera 1: 483. Totodontidae Type-species: Didugua argentilinea Druce, I $89 \mathrm{I}$, ibidem 1: 483, pl.40, fig. I3, by original designation.

Didugua was originally placed in the Noctuidae.

DIERNA Walker, [1859] I858, List Specimens lepid. Insects Colln Br. Mus. 16: 204. opH Type-species: Dierna acanthusalis Walker, [1859] I 858, ibidem 16: 205, by monotypy. Holotype ô. [INDIA]: N. Hindostan (BMNH).

D. acanthusalis is a junior subjective synonym of Noctua patibulum Fabricius, I794, Ent. Syst. 3 (2): 89.

See also Nahara Walker, $186_{5}$.

DIETHUSA Walker, [1859] I 858, List Specimens lepid. Insects Colln Br. Mus. 16: 205. ACro Type-species: Diethusa emiliusalis Walker, [1 859] I 858, ibidem 16: 206, by monotypy. Holotype ô., Borneo: Sarawak (BMNH).

See also Pachydasys Butler, I892.

DIGBA Walker, i862, J. Proc. Linn. Soc. (Zool.) 6: 197.

CHLO

Type-species: Digba uninotata Walker, 1862, ibidem 6: 198, by monotypy. Lectotype o. Borneo: Sarawak (UM, Oxford), designated (as type) by Swinhoe, 190o, Cat. east. and Aust. Lepid. Heterocera 2: I34.

D. uninotata is a junior subjective synonym of Earias flavida Felder, I86r, Sber. Akad. Wiss. Wien 43 ( 1 ) : 34 .

DILOBA Boisduval, I840, Genera Index meth. Eur. Lepid.: 88.

NOTODONTIDAE

Type-species: Phalaena caeruleocephala Linnaeus, 1758 , Syst. nat. (Edn 10)1: 504, by monotypy. Type(s), TrPe-Locality not stated (LS, London) [EuRope].

Diloba Boisduval, I840, was considered by the International Commission on Zoological Nomenclature, 1957, Opin. Decl. int. Conimn zool. Nom. 17 Opinion 494: 267, and placed on the Official List of Generic Names in Zoology: Name No. I233. There was, however, an earlier usage i.e. Diloba Boisduval, [1837] 1834, Icones hist. Lépid. nouv. ou peu conmus 2: I89. This original reference was overlooked by the Commission in Opinion 494 but fortunately Diloba Boisduval [1837] was based on the same species.

$P$. caenuleocephala has been placed in the Notodontidae, Thyatiridac, and in the Noctuidae subfamily Plusinae. Boursin, 1953, Bull. mens. Soc. limn. Lyon 22: I25, placed it in the Pantheiinac. Kiriakoff, 1970, NachrBl. bayer. Ent. 19: Ior, considered that, on a basis of tympanal characters, $D$. caeruleocephala does not belong to the Noctuidae but should be placed on its own in the family Dilobidae. This family-group name had already been proposed as Dilobinae Aurivillius, 1889, Nordens Fjärilar: 79, 95, and placed on the Official List of Family-group Names in Zoology: Name No. 198. Fletcher, 1972, in Kloet \& Hinchs, Check List Br. Insects (Edn 2) 2: 70, has retained Diloba in the Notodontidae.

$\ddagger$ †ETEROMORPHA Hübner, [1806], Tentamen determinationis digestionis . . . [1].

Included in a work rejected for nomenclatural purposes by Int. Commn zool. Nom., I926, Smithson. misc. Collns 73 (4) Opinion 97: 19. Also idem, 1954, Opin. Decl. int. Commn zool. Nom. 6 Opinion 278: I 40. Placed on the Official Index of Rejected and Invalid Generic Names in Zoology: Name No. 1061.

Phalaena caeruleocephala I,innaeus, 1758 , was the only included species.

HETEROMORPHA Hübner, I822, Syst.alphab. V'erz.: I 5 , i 8.

Type-species: Phalaena caeruleocephala Linnaeus, $175^{8}$, by subsequent designation by Kirby, 1892, Synonymic Cat. Lepid. Heteroceva 1: 585 .

Heteromorpha was suppressed for the purposes of the I.aw of Priority but not for those 
of the Law of Homonymy in Opin. Decl. int. Commn zool. Nom. (1957) 17 Opinion 494: 267. Placed on the Official Index of Rejected and Invalid Generic Names in Zoology: Name No. 1062 .

DILOPHOTHRIPA Hampson, I898, J. Bombay nat. Hist. Soc. 11: $45^{2}$.

SARR

Type-species: Dilophothripa chrysorrhaea Hampson, I898, ibidem 11: 453, fig., by original designation. Type(s) ô, InDia: Assam, Khasis (BMNH).

DILOPHOTHRIPOIDES Strand, I9I 7, Arch. Naturgesch. 82 (A) 3: I 32.

SARR

Type-species: Dilophothripoides noliformis Strand, I9I7, ibidem 82 (A) 3: I32, by monotypy. Holotype $q$, [Tarwan] Formosa: Sokutsu (DEI, Eberswalde).

†IMEROTA Hampson, no published reference found.

HYPE

DIMIRICA Walker, [1863] i864, J. Proc. Limn. Soc. (Zool.) 7: 56.

SARR

Type-species: Dimirica nubifera Walker, [1863] I864, ibidem 7: 56, by monotypy. Type(s) Õ. BORNEo: Sarawak (BMNH).

D. nubifera is a junior subjective synonym of Aquis vividisquama Walker, I 858 .

See also Aquis Walker, I 858 .

DIMONA Walker, I858, List Specimens lepid. Insects Colln Br. Mus. 15: I650.

NOLI

Type-species: Dimona porrigens Walker, I858, ibidem 15: I650, by monotypy. Holotype đ̋, [Australia]: Sydney (BMNH).

DIMORPHINOCTUA Viette, 1952, Results Norw. scient. Exped. Tristan da Cunha 1937-I938 $3(23): 6$.

Type-species: Agrotis pilifera Walker, [1857] 1856, List Specimens lepid. Insects Colln $B r$. Mus. 10: 343, by original designation. Syntypes 3 ex., Tristan DA Cunha I. [S. Atlantic Oc.] (BMNH).

DIMYA Moore, I882. See Apamea Ochsenheimer, I8I6.

ACRO

DINOPRORA Turner, I920, Trans. Proc. R. Soc. S. Aust. 44: I4I, I 53.

ACRO

Type-species: Prometopus endesma Lower, 1902, Proc. Linn. Soc. N.S.W. 26: 648, by original designation. Syntypes $2 \pi$, Australia: S.A., Parkside (SAM, Adelaide).

DINUMMA Walker, 1858, List Specimens lepid. Insects Colln Br. Mus. 15: I805. OPHI Type-species: Dinumma placens Walker, I858, ibidem 15: I806, by subsequent designation by Moore, [r885] i887, Lepid. Ceylon 3: ror. Holotype ô, Ceylon (BMNH).

DIODINES Schaus, I9I6, Proc. U.S. natn. Mus. 50: 264, 373.

OPHI

Type-species: Diodines trilinea Schaus, I9I6, ibidem 50:373, by original designation. Type(s) $\lesssim$, EcUADor: Loja (USNM, Washington).

DIOMEA Walker, [I 858] I857, List Specimens lepid. Insects Colln Br. Mus. 13: ro79, I I 09. OPHI Type-species: Diomea rotundata Walker, [1858] I857, ibidem 13: I I Io, by monotypy. Holotype ㅇ, CEYLON (BMNH).

†DIOMYX, misspelling. See Dyomyx Guenée, I 852 .

OPHI

DIOPA Walker, [1858] r857, List Specimens lepid. Insects Colln Br. Mus. 13: II 42, I I 7I. OPHI Type-species: Diopa furcula Walker, [1858] 1857, ibidem 13: II 72 , by monotypy. Holotype q, [BRAzIL]: [R. Amazon], [Tefé] Ega (BMNH).

DIOPTIS Hübner, I8I 8, Zuträge Samml. exot. Schmett. $1: 9$.

DIOPTIDAE

Type-species: Dioptis cyma Hübner, I818, ibidem $1: 9$, figs I7, I 8 , by subsequent designation by Kirby, I892, Synonymic Cat. Lepid. Heterocera $1: 4^{\mathrm{I}} 3$.

Dioptis has been used as the original generic name for species which are now in the Agaristidae. 
DIOSIA Duponchel, I832. See Catastia Hübner, [1825].

PYRALIDAE

DIPAROPSIS Hampson, 1902, Ann. S. Afr. Mus. 2: 294.

ACRO

Type-species: Diparopsis castanea Hampson, 1902, ibidem Holotype ․ [Mozambique]: Delagoa Bay (BMNH).

DIPAUSTICA Meyrick, 19I2, Trans. N. Z. Inst. 44: 98.

HADE

Type-species: Leucania epiastra Meyrick, I9I I, ibidem 43: 58, by monotypy. Syntypes I $\hat{O}$, I + , NEW Zealand: Makara.

$\doteqdot$ DIPHTERA, misspelling. See Moma Hübner, [1 820$]$.

ACRO

DIPHTERA Stephens, I 850. See Diphthera Hübner, [1809]. OPHI

$\ddagger$ DIPHTHERA Hübner, [1806」. See Moma Hübner, [1820]. ACro

${ }_{\ddagger}$ DIPHTHERA Hübner, r808. See Polygrammate Hübner, I8I8. ACRo

DIPHTHERA Hübner, [1809], Samml. exot. Schmett. 1: pl. [193]. OPHI

Type-species: Diphthera elegans Hübner, [I809], ibidem 1: pl. [193], figs I-4, by monotypy. Syntypes $\sigma^{\wedge}$,, Type-locality not stated. [Central \& South America].

Diphthera had two earlier (but nomenclaturally unavailable) usages by Hübner for moths of somew hat similar appearance to that of the third. By 820 Hübner had separated all three into different genera and used Diphtherae in a suprageneric sense. Owing to the doubtful nomenclatural status of the first two usages there has been confusion in the application of Diphthera. The works containing the first two usages have now been rejected for nomenclatural purposes by the Commission leaving the third usage available and valicl.

D. elegans is a junior subjective synonym of Bombyx festiva Fabricius, I775, Syst. Ent.: 579 .

See also Noropsis Guenée, 1852.

EUGLYPHIA Hübner, [1820] I816, Verz. bekannter Schmett.: 203.

Type-species: Diphthera elegans Hübner, [1809], by monotypy.

D. elegans was used by Hübner with Bombyx festiva Fabricius, 1775, and Phalaena hieroglyphica Cramer, 1777 , both placed in synonymy. Hübner used elegans although it was the junior name as he considered that Bombyx festiva Fabricius, I775, was a junior homonym of Noctua festiva [Denis \& Schiffermüller], I775 (see Verz. bekannter Schmett.: 222), and that Phalaena hieroglyphica Cramer, I777, was a junior homonym of Phalaena hieroglyphica Drury, I773 (see I'erz. bekannter Schmett.: 272). Under the Code although the two hieroglyphica species are primary homonyms the two festiva species are not.

Euglyphia is a junior objective synonym of Diphthera Hübner, [1809].

DIPHTERA Stephens, r85o, List Specimens Br. Anim. Colln Br. Mus. 5 : I I 4.

An unjustified emendation of Diphthera Hübner, [1 809].

DIPHTHERA Hübner sensu Hampson, r913. See Panthea Hübner, [1820].

PANT

DIPHTHEROCOME Warren, [1907], in Seitz, Gross-Schmett. Erde 3: r r. ACro

Type-species: Diphthera pallida Moore, I867, Proc. zool. Soc. Lond. 1867: 46, pl. 6, fig. 6 (but clescribed as \$Diphtera, an incorrect subsequent spelling), by subsequent designation by Hampson, 1909, Cat. Lepid. Phalaenae 13r. Mus. 8:22. Type(s), [India]: Bengal (BMNH).

The date of publication of the part of Seitz containing page I I was not stated in the text. Copies of the English and German editions were received at BMNH in r907 April and this is the date accepted by Neave, 1939, Nomencl. zool. 2: ro4. The next part of Seitz, commencing on page $\mathrm{r} 3$, is dated 1909 in both editions.

†DIPHTHEROIDES, misspelling. See Diptheroides Bethune-Baker, 1906 .

OPHI 
DIPINACIA Dognin, I907, Annls Soc. ent. Belg. 51: 239.

Type-species: Dipinacia schiniodes Dognin, 1907, ibidem 51: 239, by monotypy. Holotype + , Argentina: Tucuman.

DIPINACIA Hampson, I9o8, Cat. Lepid. Phalaenae Br. Mus. 7: 6, and Hampson, igio, ibidem 9: 370.

Type-species: Dipinacia schiniodes Dognin, 1907, Annls Soc. ent. Belg. 51: 239, by subsequent designation by Hampson, I910, ibidem $9: 370$.

A junior homonym of Dipinacia Dognin, 1907. This generic name was first made available, by Dognin, prior to its proposal and generic description (with the same typespecies) by Hampson. The objective replacement name is Dipinacia Dognin, I907.

DIPINACIA Hampson, 1908. See Dipinacia Dognin, I907. ACro

$\ddagger$ DIPLODINA, misspelling. See Diplodiva Schaus, I9I6. HYPE

DIPLODIRA Schaus, I9I6, Proc. U.S. natn. Mus. 50: 260, $280 . \quad$ HyPE

Type-species: Diplodira jamaicalis Schaus, I9I6, ibidem $50: 280$, by original designation. Type(s) ô. JamaICA (USNM, Washington).

$\ddagger D I P L O D I N A$; Neave, 1939, Nomencl. zool. 2: 108 .

An incorrect subsequent spelling.

DIPLOLOPHA Dognin, I II , Mém. Soc. ent. Belg. 18: I54.

SARR

Type-species: Diplolopha cycloptera Dognin, I9I I, ibidem 18: I5t, by monotypy. Syntypes 2 . Colombia: Cali, San Antonio. Argentina. (USNM, Washington).

DIPLOLOPHA Hampson, I91 4, Ann. Mag. nat. Hist. (8) 13: 219.

Type-species: Diplolopha cycloptera Dognin, I9I I, by original designation.

A junior homonym of Diplolopha Dognin, I9I. This generic name probably originated from Hampson, but it was used and unintentionally made nomenclaturally available by Dognin prior to its proposal and generic description by Hampson. The objective replacement name is Diplolopha Dognin, I9II.

DIPLOLOPHA Hampson, I9I4. See Diplolopha Dognin, I9II.

SARR

DIPLONEPHRA Turner, 1920, Trans. Proc. R. Soc. S. Aust. 44: I4 I, I5I. Acro

Type-species: Luperina ditata Lucas, i 892, Proc. Linn. Soc. N.S.W. (2) 7: 254, by monotypy. Syntypes $\hat{\jmath}, q$, Australia: [Qd], Duaringa; Brisbane (SAM, Adelaide).

DiPLOTHECTA Turner, i920, Trans. Proc. R. Soc. S. Aust. 44: i 58, I67. Acon

Type-species: Diplothecta digonia Turner, 1920, ibidem 44: 167, by monotypy. Holotype $\widehat{\jmath}$, Australia: Qd, Cairns (NMV, Melbourne).

DIPTERYGIA Agassiz, I846. See Dypterygia Stephens, I829. ACro

DIPTERYGINA Sugi, I954, Tinea, Tokyo 1:25. ACRo

Type-species: Dipterygia japonica Leech, I889, Proc. zool. Soc. Lond. 1889: 489, pl. 50, fig. 9, by original designation. Holotype ô. JAPAN (BMNH).

$\ddagger$ DIPTHERA, misspelling. See Moma Hübner, [1820]. ACro

DIPTHEROIDES Bethune-Baker, I906, Novit. zool. 13: 203.

Type-species: Diptheroides kenricki Bethune-Baker, 1906, ibidem 13: 203, by original designation. Syntypes 0 , 오, New Guinea: [Papua], Dinawa (BMNH).

$\ddagger$ DIPHTHEROIDES; Neave, 1939, Nomencl. zool. 2 : I04.

¥DIRCETIS, misspelling. See Redectis nom. n.

OPHI

DISCESTRA Hampson, I905, Cat. Lepid. Phalaenae Br. Mus. 5: vii, I4. HADE

Type-species: Mamestra chartaria Grote, 1873, Bull. Buffalo Soc. nat. Sci. 1: I38, pl. 4, fig. I2, by original designation. Syntypes, [U.S.A.]: California (BMNH). 
SALACIA Boie, I839, in Freyer, Z. Ent. (Germar) 1: 39 I.

Type-species: Mamestra sodae Boisduval, i 829, Eur. Lepid. Index meth. Errata Addenda: 5, by PRESENT DESIGNATION. Syntypes, [FRANCE]: [Hérault], [Montpellier] Monspelii.

A junior homonym of Salacia Lamouroux, i816, Hist. Polypiers Coralligénés flexibles: 2 I2, - Coelenterata. There is no objective replacement name but $M$. sodae is congeneric with Mamestra chartaria Grote, I873, the type-species of Discestra Hampson, 1905. The latter is therefore available for use as the subjective replacement name.

$M$. sodae has usually been attributed to Rambur, I829 May, Annls Sci. Observ. 2: 260, but Higgins, 1965, Entomologist 98: 242 has reasoned that Boisduval had priority.

†ICESTRA; McDunnough, I937, Can. Ent. 69: 4 I.

An incorrect subsequent spelling.

DISCHALIS Hampson, I918, Novit. zool. 25: I88.

ACON

Type-species: Dischalis leucomera Hampson, 1918, ibidem 25: I88, by original

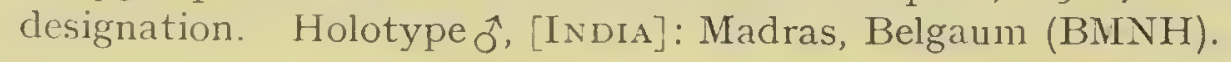

DISCOSEMA Hampson, 1926, Descr. new Genera Species Lepid. Phalaenae Subfamily Noctuinae Br. Mus.: 425 .

OPHI

Type-species: Renodes birenosa Felder \& Rogenhofer, I874, Reise öst. Fregatte Novara (Zool.) 2 (Abt. 2): pl. I 19, fig. 19; Inhalts-Verz.: 17, by original designation. Type(s) ô, [Brazil]: Amazon (BMNH).

DISEPHOLCIA Prout, 1924, in Prout \& Talbot, Bull. Hill. Mus., Witley 1: 404. PANT

Type-species: Trisuloides caerulea Butler, I889, Illust. typical Specimens Lepid. Heterocera Colln Br. Muts. 7: 5, 35, pl. I28, fig. 3, by original designation. Syntypes I ot, I.$+[$ INDIA]: [Punjab], Dharmsala (BMNH).

DISSOLOPHUS Butler, I891, Ann. Mag. nat. Hist. (6) 8: 71, 73.

ACRO

Type-species: Eriopus chloriza Guenée, 1852 , in Boisduval \& Guenée, Hist. nat. Insectes (Lépid.) 6: 296, pl. I 4, fig. 3, by original designation. Holotype ơ, Java (BMNH).

DISTAGMA Staudinger, I898, Ergebn. Hamb. Magalhaensischen Sammelveise 4 (7): 80.

GEOMETRIDAE

Type-species: Distagma desolata Staudinger, i 898, ibidem: 80, by monotypy.

D. desolata is a junior subjective synonym of Honorana aenea Butler, I882, Trans. ent. Soc. Lond. 1882: $36_{5}$.

Distagma was not originally placed in a family, but was compared with a Noctuid genus. It has since been placed in the Geometridae, Ennominae.

DISTICTA Hampson, 1902, Ann. S. Afr. Muss. 2: 424 .

$\mathrm{OPHI}$

Type-species: Achantodes atava Felder \& Rogenhofer, 1874, Reise öst. Fregatte Noiara (Zool.) 2 (Abt. 2): pl. II9, fig. 4; Inhalts-Verz.: [I], by monotypy. Type(s) ô, Sovith Africa: [Cape Prov.], Knysna (BMNH).

DITROGOPTERA Hampson, I898, Trans. ent. Soc. Lond. 1898: 256.

ACON

Type-species: Ditrogoptera trilineata Hampson, I 898, ibidem 1898: 256, pl. 17, fig. 20, by monotypy. Type(s) $q$, Lesser Antilles: St. Vincent I. (BMNH).

DNOPHEROPIS Turner, 1902, Proc. Limn. Soc. N.S.W. 27: 103.

OPHI

Type-species: Dnopheropis scotaea Turner, 1902, ibidem 27: 104, by monotypy. Holotype ô. Austraima: Qd, Brisbane (ANIC, Canberra).

DOCELA Walker, [1866] I 865, List Specimens lepid. Insects Colln Br. Mus. 34: 1258. OPHI

Type-species: Docela vetustalis Walker, [1866] I 865, ibidem 34: 1258, by monotypy. Holotyle ?, Congo (BMNH).

D. vetustalis is a junior subjective synonym of Hydretia circumdata Walher, 1858 , ibidem 15: 1763 . 
DOCHMIOGRAMMA Hampson, 1926, Descr. new Genera Species Lepid. Phalaenae Subfamily Noctuinae Br. Mus.: 499.

Type-species: Thiona filamentosa Felder \& Rogenhofer, 1874, Reise öst. Fregatte Novara (Zool.) 2 (Abt. 2) : pl. II9, fig. I8; Inhalts-Verz.: I9, by original designation. Type(s) 우, [BrazIL]: R. Amazon (BMNH).

T. filamentosa is a junior subjective synonym of Gigia obliqua Walker, 1865.

See also Gigia Walker, I865.

DOERRIESA Staudinger, I90o, Dt. ent. Z. Iris 12: 373.

ACRO

Type-species: Ragonotia striata Staudinger, I90o, ibidem 12:372, pl. 6, fig. I5, by monotypy (of Ragonotia Staudinger). Syntypes o, ․, [U.S.S.R.]: S. E. Amur, Askold; Wladiwostock; Suifun; Sutschan.

Doerriesa was proposed as the objective replacement name for Ragonotia Staudinger, I900.

RAGONOTIA Staudinger, I90o, Dt. ent. Z. Iris 12: 372.

Type-species: Ragonotia striata Staudinger, I900, by monotypy.

A junior homonym of Ragonotia Grote, I888, Can. Ent. 20: 75,--Lepid., Pyralidae. At the end of the paragraph containing the original description of his new genus Staudinger stated that if Ragonotia was preoccupied then the name Doerriesa should be used. This set an unbeatable record for the minimum time required for the proposal of an objective replacement name.

DOGNINADES Schaus, I916, Proc. U.S. natn. Mus. 50: 26I, 308.

HYPE

Type-species: Hypena jactatalis Walker, [1859] I 858, List Specimens lepid. Insects Colln Br. Mus. 16: 49, by original designation. Holotype of, [BRAzIL]: Parà (BMNH).

$\ddagger$ DOLICHOSCYTA Hampson, no published reference found.

HYPE

DOLICHOSOMASTIS Hampson, 1924, Ann. Mag. nat. Hist. (9)13: 436.

$\mathrm{OPHI}$

Type-species: Capnodes dorsilinea Dognin, I912, Hétérocères nouv. Am. Sud 6: 35, by original designation. Syntypes $3 \hat{\jmath}$, PerU: S.E., La Oroya; Rio Inambari; Carabaya.

The type-species is a junior secondary homonym of Thermesia dorsitinea Dyar, I9Io, Zoologica, N.Y. 1: I 30, fig. 4I. The objective replacement name is Dolichosomastis leucogrammica Hampson, 1924, Ann. Mag. nat. Hist. (9) 13: 436.

DONACESA Walker, 1858, List Specimens lepid. Insects Colln Br. Mus. 15: I859. OPH

Type-species: Donacesa miricornis Walker, I 858 , ibidem 15: I 859 , by monotypy. Type(s) $\widehat{\jmath},[$ BRAzIL]: Rio Janeiro (UM, Oxford).

DONACHLORA Sodoffsky, I837. See Lencania Ochsenheimer, I8 I6.

HADE

DONACOCHLORA Agassiz, I846. See Lencania Ochsenheimer, I8I6.

HADE

DONDA Moore, I882, in Hewitson \& Moore, Descr. new Indian Lepid. Insects Colln late Mr W. S. Atkinson: r6r.

Type-species: Dandaca eurychlora Walker, I858, List Specimens lepid. Insects Colln Br. Mus. 15: I670, by original designation. Type(s) $q$, [India]: Hindostan, Canara $(\mathrm{BMNH})$.

DONUCA Walker, 1865, List Specimens lepid. Insects Colln Br. Mus. 33: 925.

CATO

Type-species: Donuca spectabilis VValker, 1865, ibidem 33: 926, by subsequent designation by Hampson, I913, Cat. Lepid. Phalaenae Br. Mus. 12: 364. Syntypes I j, I q, [Australia]: [TV. Austr.], Swan River (BMNH).

DONUCTENUSA Berio, I940, Memorie Soc. ent. ital. 19: I 27.

CATO

Type-species: Donuctenusa fiorii Berio, I940, ibidem 19: I28, by original designation. Holotype ô, Somalia: Uarder. 
DORANAGA Moore, I88`, Lepid. Ceylon 3: 553.

Type-species: Doranaga apicalis Moore, I 887, ibidem 3: 553, pl. 214, fig. 7, by original designation. Syntypes, CEYLON (BMNH).

D. apicalis is a junior subjective synonym of Aviolica signata Waiker, [1863] r864, J. Proc. Linn. Soc. (Zool.) $7: 54$.

See also Nertobriga Walker, [I863].

DORDURA Moore, I882, in Hewitson \& Moore, Descr. new Indian lepid. Insects Colln late Mr W.S. Atkinson: I 70 .

OPHI

Type-species: Dordura apicalis Moore, I882, ibidem: I7o, pl. 5, fig. 20, by original designation. Type(s), [INDIA]: Calcutta.

DORIKA Moore, I88 I, Proc. zool. Soc. Lond. 1881: 363 .

NOCT

Type-species: Dorika sanguinolenta Moore, I88I, ibidem 1881: 363, by original designation. Type(s) , [INDIA $]$ : Bombay (BMNH).

DORSIPPA Walker, [1863] i864, J. Proc. Linn. Soc. (Zool.) 7:81.

OPHI

Type-species: Dorsippa notabilis Walker, [1863] I864, ibidem 7: 82, by monotypy. Lectotype 3 . Borneo: Sarawak (UM, Oxford), designated (as type) by Swinhoe, I90o, Cat. east. and Aust. Lepid. Heterocera 2: 58 .

D. notabilis is a junior subjective synonym of Oylasa lagusalis Walker, I 858 .

See also Oglasa Walker, [1859].

DORYODES Guenée, I857, in Boisduval \& Guenée, Hist. nat. Insectes (Lépid.) 10: 233. Cato Type-species: Ligia acutaria Herrich-Schäffer, [1852] I843-I856, Syst. Bearb. Schmett. Eur. 6: 74, pl. 73, fig. 447, by subsequent designation by Grote, 1874, Bull. Buffalo Soc. nat. Sci. 2: 20. 'Type(s), Type-Locality stated to be 'Sudrussland?' but as HerrichSchäffer suspected, this was incorrect. The species illustrated is not known to occur in the Old World but is found along the eastern coast of Canada and U.S.A.

L. acutaria is a junior subjective synonym of Agriphila bistrialis Geyer, 1832, in Hübner, Zuträge Saniml. exot. Schmett. 4: 38, figs 775, 776 .

HERMOEA Walker, [1 859] i 858, List Specimens lepid. Insects Colln Br. Mus. 16: I 2, 73.

Type-species: Doryodes acutalis Walker, [1859] I858, ibidem 16: 73, by monotypy.

D. acutalis is an unjustified emendation of Ligia acutaria Herrich-Schäffer, [1 852 ].

The species described by Waller as $D$. acutalis was misidentified and was not Ligia acutaria, but was at that time undescribed. This was noticed by two authors who independently named and placed the species in new genera, i.e. Phyprosopus callitrichoides Grote, I872 September, and Sudariophora nasutaria Zeller, 1872 [December].

Hermoea was made nomenclaturally available by Walker in a key on page 12 , but on page 73 was placed in the synonymy of Doryodes Guence, I 857 .

DOSA Walker, [1865] 1864, List Specimens lepid. Insects Colln Br. Mus. 31: I44.

OPHI

Type-species: Dosa obsea Walker, [1865] I 864, ibidem 31: I 4 , by monotypy. Syntypes 3 (ō, 우), [BRAzIL]: [R. Amazon], [Tefé] Ega (BMNH).

DRAGANA Walker, [I859] I858, List Specimens lepid. Insects Colln Br. Mus. 16: 200. орн I Type-species: Dragana pansalis Walker, [1859] I858, ibidem 16: 200, by monotypy. Holotype $\hat{j}$, CEYLON (BMNH).

D. pansalis is a junior subjective synonym of Gesonia obeditalis Walker, [1859].

See also Gesonia Walker, [I859]; Hileia Walker, I86I; Maresia Walker, 1866; and Amblygoes Butler, r879.

DRASTERIA Hübner, 1818, Zuträge Samml. exot. Schmett. 1: 8.

OPHI

Type-species: Drasteria graphica Hübner, I8 18, ibidem 1: 8, figs I I, I2, by subsequent designation by Hampson, 1926, Descr. new Genera Species Lepid. Phalaenae Subfamily Noctuinae Br. Mus.: 38 . Type(s) ô, [U.S.A.]: Georgia [formerly] in Florida. 
Incorrect type-species designation: Phalaena evechtea Cramer, i 780 , a name not originally included in Drasteria, and not linked with one of the originally included names when cited by Desmarest, I 857, Encyl. Hist. nat. Papillons Nocturnes: I33.

SYNEDA Guenée, I 852, in Boisduval \& Guenée, Hist nat. Insectes (Lépid.) 7 : 7 I.

Type-species: Drasteria graphica Hübner, I818, by subsequent designation by Grote, I 874, Bull. Buffalo Soc. nat. Sci. 2: 4 o.

Syneda is a junior objective synonym of Drasteria Hübner, I8I8.

$\ddagger_{\ddagger}$ DRASTORIA; Walker, I858, List Specimens lepid. Insects Colln Br. Mus. 14: I 447.

An incorrect subsequent spelling.

DRASTERIODES Hampson, 1926, Descr. new Genera Species Lepid. Phalaenae Subfamily Noctuinae Br. Mus.: 4 I. OPHI

Type-species: Photedes limata Christoph, I884, in Romanoff, Mém. Lépid. 1: I35, pl. 8, fig. 7, by original designation. Syntypes $\hat{0}$, ㅇ, [U.S.S.R.]: [Turkmenistan], Acha]Tekke district (ZI, Leningrad).

$P$. limata was originally proposed as $\$$ Phothedes, an incorrect spelling of Photedes Lederer, I 857 .

†DRASTORIA, misspelling. See Drasteria Hübner, I8I8.

OPHI

DRAUDTIA Barnes \& Benjamin, 1926, Pan-Pacif. Ent. 3: 66.

ACRO

Type-species: Namangana revellata Barnes \& Benjamin, 1924, Contr. nat. Hist. Lepid. N. Am. 5 (3): I 56, by original designation. Holotype J. [U.S.A.]: [Ariz.], Cochise Co., Paradise.

DRAUDTIANA Turati, I934, Atti Soc. ital. Sci.nat. 73: I67.

ACRO

Type-species: Draudtiana castanea Turati, I934, ibidem 73: 168, fig., by monotypy. Holotype ô. [Libya]: Cirenaica, U. Bakur.

DREPANOBLEMMA Hampson, 1926, Descr. new Genera Species Lepid. Phalaenae Subfamily Noctuinae Br. Mus.: 38 I.

$\mathrm{OPH}$ I

Type-species: Capnodes incurvata Schaus, I901, Ann. Mag. nat. Hist. (7) 8: 93, by original designation. Type(s), Brazil: Casa Branca.

DREPANOFODA Hampson, 1926, Descr. new Genera Species Lepid. Phalaenae Subfamily Noctuinae Br. Mus.: 203.

OPH I

Type-species: Fodina juncta Hampson, I894, Fauna Br. India (Moths) 2: 530, by original designation. Type(s) ô. [SIKkIM] Sikhim (BMNH).

DREPANOPALPIA Hampson, I898, Trans. ent. Soc. Lond. 1898: 255.

HYPE

Type-species: Drepanopalpia polycyma Hampson, I898, ibidem 1898: 256, pl. I7, fig. I 4, by monotypy. Syntypes ô, $q$, [West Indies]: [Windward Is], St. Lucia (BMNH).

$D$. polycyma is a junior subjective synonym of Mastigophora latipennis Herrich-Schäffer, I 870, Corresp.-Bl. zool.-min. Ver. Regensburg 34: I05.

DREPANOPERAS Hampson, 1926, Descr. new Geneva Species Lepid. Phalaenae Subfamily Noctuinae Br. Mus.: 5I I.

OPHI

Type-species: Plaxia falcigera Walker, I858, List Specimens lepid. Insects Colln Br. Mus. 15: i629, by original designation. Type(s) ㅇ, Brazil (UM, Oxford).

DREPANOPHILETIS Hampson, 1926, Descr. new Genera Species Lepid. Phalaenae Subfamily Noctuinae Br. Mus.: 34I.

OPHI

Type-species: Rhescipha siderosticta Holland, I894, Psyche, Camb. 7: 32, pl. I, fig. 9, by original designation. Syntypes 2 ㅇ, West AfriCA (CM, Pittsburgh).

DREPANOPSES Hampson, 1926, Descr. new Genera Species Lepid. Phalaenae Subfamily Noctuinae Br. Muss.: 506.

OPHI

Type-species: Drepanopses rufipicta Hampson, 1926, ibidem: 507, by original designation. Syntypes i $\hat{O}$, I $q$, Nigeria : Sapele (BMNH). 
DREPANORHINA Hampson, 1926, Descr. new Genera Species Lepid. Phalaenae Subfamily Noctuinae Br. Mus.: 285.

Type-species: Pseudaglossa shelfordi Swinhoe, 1904, Trans. ent. Soc. Lond. 1904: 142 , by original designation. Syntypes $5 \hat{\sigma}$, BORNEO: [Sarawak], Kuching (BMNH); [Kalimantan], Pulo Laut (BMNH).

$\ddagger$ DROBEDA, misspelling. See Drobeta Walker, I 858 .

$\operatorname{ACON}$

DROBETA Walker, I 858, List Specimens lepid. Insects Colln Br. Mus. 15: 1755 .

$\operatorname{ACON}$

Type-species: Drobeta exscendens Walker, I858, ibidem 15: 1755, by monotypy. Holotype +, Venezuela (BMNH).

$\ddagger D R O B E D A$; Kaye, I901, Trans. ent. Soc. Lond. 1901 : 123.

An incorrect subsequent spelling.

DRUCUMA Schaus, I9i 6, Proc. U.S. natn. Mus. 50:262, 322.

HYPE

Type-species: Aristaria apicata Druce, I89I, Biologia cent.-am. (Zool.) Lepid. Heterocera 1: 459, pl. 37, fig. 23, by original designation. Syntypes o, + , Mexico: Mazatlan; Tabasco, Teapa. Guatemala: San Gerónimo. Costa Rica: Caché (BMNH).

DRYOBOTA Lederer, I857, Noctuinen Eur.: 33, 100.

CUCU

Type-species: Noctua occlusa Hübner, [1824], Samml. eur. Schmett. 4: pl. I56, fig. 732, by subsequent designation by Hampson, I906, Cat. Lepid. Phalaenae Br. Mus. 6: 302, but cited as furva Esper which Hampson placed as the senior synonym of occlusa Hübner. Type(s), Europe.

N. occlusa is a junior subjective synonym of Phalaena labecula Esper, 1788, Die Schmett. 4(2) Abschnitt I: pl. 129, fig. 5; 1796, ibidem: 393.

DRYOBOTODES Warren, I910, in Seitz, Macrolepid. World 3: 133.

CUCU

Type-species: Noctua protea [Denis \& Schiffermüller], I775 [December 8th], Ankündung syst. Werkes Schmett. Wienergegend: 84, by original designation, but cited as 'protea Bkh.', an incorrect authorship. Type(s), [Austr1A]: Vienna district (Collection destroyed, Horn \& Kahle, 1936, Ent. Beih. Berl.-Dahlem 3: 243).

$N$. protea is a junior subjective synonym of Noctua eremita Fabricius, 1775 [April 17 th], Syst. Ent.: 616.

The date of publication of the part of Seitz containing page I33 was stated on page I33 as I9Io May $5^{\text {th }}$ in the English edition and as I9II January I $3^{\text {th }}$ in the German edition.

DRYOTYPE Hampson, 1906, Cat. Lepid. Phalaenae Br. Mus. 6: x, $228 . \quad$ cucu

Type-species: Dryobota opina Grote, 1878, Bull. U.S. geol. geogr. Surv. Territ. 4: I 78, by original designation. Syntypes 0 , ㅇ, [U.S.A.]: California (BMNH).

DUGARIA Walker, [1858] 1857, List Specimens lepid. Insects Colln Br. Mus. 13: 1045, I075.

CATO

Type-species: Dugaria cilipes Walker, [1858] 1857, ibidem 13: 1076, by monotypy. Syntypes ô, + , Congo (BMNH). [South Africa]: Port Natal.

D. cilipes is a junior subjective synonym of Alamis mendax Walker, [1858] I857, ibidem 13: $10+7$.

DUHEMIA Rungs, 1943, Bull. Soc. Sci. nat. Maroc 22: r 66.

$\operatorname{ACON}$

Type-species: Duhemia metachrostina Rungs, 1943, ibidem 22: 167, pl. 1, fig. 19, by original designation. Holotype ô, Morocco: El-Aïoun-du-Dra.

D. metachrostina is a junior subjective synonym of Jugurthia iariegata Lucas, 1932, Bull. Soc. ent. Fr. 37: 166.

DUMATHA Walker, 1864, J. Proc. Linn. Soc. (Zool.) 7: 195.

SARR

Type-species: Dumatha herbida Walker, 1864, ibidem 7: 195, by monotypy. Lectotype + , Borneo: Sarawak (UM, Oxford), designated (as type) by Swinlıoe, rgoo. Cat. east. and Aust. Lepid. Heterocera 2: 78. 
DUMIGANIA Turner, I933, Trans. R. Soc. S. Aust. 57: r63.

CHLO

Type-species: Dumigania iochlora Turner, 1933, ibidem 57: I63, by monotypy. Holotype \%, Australia: Qd, Mt Tambourine (ANIC, Canberra).

DUNIRA Moore, [1885] I887, Lepid. Ceylon 3: 206.

OPHI

Type-species: Thermesia scitula Walker, 1865, List Specimens lepid. Insects Colln Br. Mus. 33: IO6I, by original designation. Holotype ${ }^{\star}$, CEYLON (BMNH).

DURDARA Moore, 1882, in Hewitson \& Moore, Descr. new Indian lepid. Insects Colln late MrW.S. Atkinson: I76. THYRIDIDAE

Type-species: Phalaena myrtaea Drury, 1773, Illust. nat. Hist. 2: Index \& 4, pl. 2, fig. 3 , by original designation.

Durdara was originally placed in the Noctuidae.

DURIGA Schaus, I940, Scient. Surv. P. Rico 12: 255 .

OPHI

Type-species: Megatomis nealcesalis Walker, I859, List Specimens lepid. Insects Colln Br. Mus. 19: 883, by original designation. Holotype $\tilde{\sigma}$. [Dominican RePublic]: St. Domingo (BMNH).

CHABORA Walker, [1866] 1865, List Specimens lepid. Insects Colln Br. Mus. 34: Ir84.

Type-species: Chabora tauralis Walker, [1866] 1865, ibidem 34: II85, by monotypy. Holotype ô. [Dominican Republic]: St. Domingo (BMNH).

A junior homonym of Chabora Walker, 1865, ibidem 33: II 44,-Lepid., Noctuidae. There is no objective replacement name but $C$. tauralis is conspecific with Megatomis nealcesalis Walker, 1859, the type-species of Duriga Schaus, 1940. The latter is thus available for use as a subjective replacement name.

DUSPONERA Schaus, 1916, Proc. U.S. natn. Mus. 50 : 26I, 299.

HYPE

Type-species: Dusponera fannia Schaus, x 916 , ibidem 50:299, by original designation.

Type(s) o, French GuIANA: R. Maroni, St. Jean (USNM, Washington).

$\ddagger$ DYACHRYSIA, misspelling. See Diachrysia Hübner, [1821]

PLUS

DYMBA Dyar, I914, Proc. U.S. natn. Mus. 47: 185.

$\operatorname{ACON}$

Type-species: Dymba coryphata Dyar, I9I4, ibidem 47: I86, by original designation.

Holotype $\widehat{\sigma}$. Panama: R. Trinidad (USNM, Washington).

$\ddagger D Y O M I X$, misspelling. See Dyomyx Guenée, I852.

OPHI

DYOMYX Guenée, I852, in Boisduval \& Guenée, Hist. nat. Insectes (Lépid.) 6: 281. OPHI

Type-species: Dyomyx cimolia Guenée, 1852, ibidem 6:282, by subsequent designation

by Dyar, I9I4, Proc. U.S. natn. Mus. 47: II3. Holotype ô., Brazil (BMNH).

$\ddagger D Y O M I X$; Bar, 1876, Annls Soc. ent. Fr. (5) 6: 436 .

An incorrect subsequent spelling.

†IOMYX; Hampson, 1926, Descr. new Genera Species Lepid. Phalaenae Subfamily Noctuinae

$B r . M u s .:$ I 3 I.

An incorrect subsequent spelling.

DYOPS Guenée, 1852, in Boisduval \& Guenée, Hist. nat. Insectes (Lépid.) 6: 283.

OPHI

Type-species: Phalaena ocellata Cramer, 1780 [a junior primary homonym], Uitlandsche Kapellen 3: I5I, pl. 276, fig. E [but not fig. D], by subsequent designation by Hampson, 1926, Descr. new Genera Species Lepid. Phalaenae Subfamily Noctuinae Br. Mus.: I28. Lectotype, SuRINAM (not found in RNH, Leiden nor in BMNH), designated as fig. E (but not fig. D), by Wiltshire, 1962, J. Lepid. Soc. 16: 48. Neotype ô, French Guiana (BMNH), designated by Wiltshire, I962, ibidem 16: 48 .

$P$. ocellata Cramer is a junior homonym of Phalaena ocellata Linnaeus, I758, - Lepid., Geometridae. The subjective replacement name is Boecula chromatophila Walker, I858, List Specimens lepid. Insects Colln Br. Mus. 15: 1669. 
DYPTERYGIA Stephens, I829, Illust. Br. Ent. (Haustellata) 2: I67.

ACRO

Type-species: Phalaena scabriuscula Linnaeus, 1758, Syst. Nat. (Edn to ) 1: 516, by monotypy, but included as pinastri Linnaeus, see below. Type(s), Europe (LS, London).

Phalaena pinastri Linnaeus, I761, Famna Suecica (Edn 2): 315, was proposed as the objective replacement name for $P$. scabriuscula presumably because pine woodland was cited as the habitat in the Famna Succica. Under the Code Article I8(a), a nomenclaturally available species-group name cannot afterwards be rejected even by its own author, because of inappropriateness.

DIPTERYGIA Agassiz, I8 66, Nomencl. zool. Index univl.: I27, I32.

An unjustified emendation of Dypterygia Stephens, I829.

DYRZELA Walker, 1858, List Specimens lepid. Insects Colln Br. Mus. 15: 1758.

ACRO

Type-species: Dyrzela plagiata IValker, i 858 , ibidem 15: I 758 , by subsequent designation by Hampson, I910, Cat. Lepid. Phalaenae Br. MIus. 9: I58. Syntypes 20 , [INDiA]: Hindostan (BMNH).

DYSAPURA Turner, 1936, Proc. R. Soc. Qd 47: 39.

SARR

Type-species: Dysapura xanthosticha Turner, 1936, ibidem 47: 39, by monotypy. Type(s) ô, Australia: N. Qd, Cape York (QMI, Brisbane).

DYSCHORISTA Lederer, I857. See Parastichtis Hübner, [I82I].

CUCU

DYSEDIA Rogenhofer, I874, in Felder \& Rogenhofer, Reise öst. Fregatte Novara (Zool.) 2 (Abt. 2): pl. II2; Inhalts-Yerz.: 8.

OPHI

Type-species: Dysedia zibellina Felder \& Rogenhofer, I87t, ibidem 2 (Abt. 2): pl. II 2, fig. 8; Inhalts-Verz.: 8, by monotypy. Type(s) 3 . Borneo: Sarawak.

D. zibellina is a junior subjective synonym of Premusia intrahens Walker, $185^{8}$.

See also Premusia Walker, $185^{8 .}$

DYSGLYPTOGONA Warren, I889, Trans. ent. Soc. Lond. 1889: 248.

OPH I

Type-species: Dysglyptogona dissimilis Warren, I 889, ibidem 1889:249, by monotypy. Syntypes I $\hat{\jmath}, I$ \&, [BRAzIL]: Amazon basin, R. Jurua (BMNH); Juruapuca.

DYSGNATHIA Warren, I913, in Seitz, Gross-Schmett. Erde 11: 246.

$\mathrm{ACON}$

Type-species: Corgatha nigropunctata Bethune-Baker, I906, Novit. zool. 13: 2I 4, by original designation. Holotype ô, NEw Gurnea: [Papua], Aroa River (BMNH).

The date of publication of the part of Seitz containing page 246 was stated on page $24 \mathrm{I}$ as 1913 September $15^{\text {th }}$ in both the English and German editions.

DYSGONIA Hübner, [1823] i8 86, Verz. bekannter Schmett.: 269.

CATO

Type-species: Phalaena algira Linnaeus, I767, Syst. Nat. (Edn I2) 1:836, by subsequent designation by Moore, [1885] i 887, Lepid. Ceylon 3: I75. Type(s), Algeria (LS, London).

DYSMILICHIA Speiser, I902, Berl. ent. Zeit. 47: I 40.

ACRO

Type-species: Perigea gemella Leech, I889, Proc. zool. Soc. Lond. 1889: 492, pl. 53, fig. I 2, by monotypy (of Phalacra Staudinger, I 892). Syntypes 8 (ふ઼, q), KorEa: Gensan (BMNH). Japan: Yokohama (BMNH).

Dysmilichia was proposed as the objective replacement name for Milichia Snellen, [1 899].

PHALACRA Staudinger, 1892, in Romanoff, Mém. Lépid. 6: 568.

Type-species: Perigea gemella Leech, I889, by monotypy.

A junior homonym of Phalacra Walker, 1866, List Specimens lepid. Insects Colln Br. Mus. 35: I638,-Lepid., Drepanidae. The objective replacement name is Dysmilichia Speiser, 1902 .

MILICH*I A Snellen, [1899], I898, Tijdschr. Ent. 41: i94.

Proposed as the objective replacement name for Phalacra Staudinger, 1892.

A junior homonym of Milichia Meigen, 1830, Syst. Beschreibung bekannten eur. 
zweiflïgeligen Insekten 6: I3I, - Diptera. The objective replacement name is Dysmitichia Speiser, I902.

$\ddagger$ PHALAKRA; Speiser, I902, Berl. ent. Zeit. 47: ז40.

An incorrect subsequent spelling.

DYSOCNEMIS Grote, I89o, Revised Check List N. Am. Noct. 1: 34.

NOCT

Type-species: Melicleptria belladonna Edwards, I88I, Papilio 1: 20, by monotypy. Syntypes 30,2 , [U.S.A.]: Southern Utah.

DYSPYRALIS Warren, I89I, Ann. Mag. nat. Hist. (6) 8: 64.

HYPE

Type-species: Dyspyralis illocata Varren, 189I, ibidem (6) 8: 64, by original desig-

nation. Holotype ô, TYPE-LOCALITy not known [U.S.A.].

Dyspyralis was originally placed in the Pyralidae.

DYSTHYMIA Newman, I 868, Entomologist 4: I74.

OPHI

Type-species: Noctua luctuosa [Denis \& Schiffermüller], I775, Ankündung syst. Werkes Schmett. Wienergegend: 90, by monotypy. Type(s), [Austria]: Vienna district (Collection destroyed, Horn \& Kahle, I936, Ent. Beih. Berl.-Dahlem 3: 243).

See also Tyta Billberg, I820.

EARIAS Hübner, [1825] I816, Verz. bekannter Schmett.: 395.

CHLO

Type-species: Phalaena clorana [sic] Linnaeus, I76I, Fauna Suecica (Edn 2): 343, by subsequent designation by Westwood, I84o, Synopsis Genera Br. Insects: Io 7 (but cited for $\ddagger$ Earis, an incorrect subsequent spelling). Type(s), SwEDEN.

$\ddagger$ EARIS; Stephens, I 834, Illust. Br. Ent. (Haustellata) 4:67.

An incorrect subsequent spelling.

$\ddagger E A R I S$, misspelling. See Earias Hübner, [1825].

CHLO

EBERTIDIA Boursin, I968, Entomops, Nice 2: I23. HADE

Type-species: Epunda mamestrina Butler, I889, Illust. typical Specimens Lepid. Heterocera Colln Br. Mus. 7: II, 59, pl. I28, fig. 5, by original designation. Type(s), [INDiA]: [Punjab], Dharmsala (BMNH).

ECBOLEMIA Hampson, igo8, Cat. Lepid. Phalaenae Br. Mus. 7 : xii, $44^{8}$.

ACRO

Type-species: Margelana misella Püngeler, 1907, Dt. ent. Z. Iris 19:219, pl. 8, fig. I, by original designation. Syntypes I $\hat{O}$, I ㅇ, [U.S.S.R.]: [Kazakhstan], Ili district. [CHINA]: [Sinkiang-Uigur], Kuldja (MNHU, Berlin).

ECCLETA Turner, 1902, Proc. Linn. Soc. N.S.W. 27: 86.

ACRO

Type-species: Eccleta xuthophanes Turner, I902, ibidem 27: 87, by monotypy Syntypes, Australia: I 0 , Qd, Stradbroke (ANIC, Canberra); 2 ex., Qd, Townsville.

ECCOPTEROMA Staudinger, I892, in Romanoff, Mém. Lépid. 6: 506.

CHLO

Type-species: Calymnia falcata Graeser, I889, Berl. ent. Z. 33: 257, by monotypy. Holotype 오, [U.S.S.R.]: Amur, probably Raddefka.

C. falcata is a junior subjective synonym of Macrochthonia fervens Butler, I88I.

Eccopteroma when proposed contained a second species, but this was doubtfully included, and under the Code, Article 68(c) is not eligible for designation as type-species.

See also Macrochthonia Butler, I88I.

ECCRITA Lederer, I857, Noctuinen Eur.: 45, 207.

CATO

Type-species: Phalaena ludicra Hübner, I790, Beitr. Gesch. Schmett. 2(4): 95, pl. 3, fig. R, by monotypy. Syntypes + , Germany: Sachsen. Austria.

ECHANA Walker, [1859] I858, List Specimens lepid. Insects Colln Br. Mus. 16: I95. HyPE

Type-species: Echana abavalis Walker. [1859] I858, ibidem 16: I95, by original designation. Syntypes 3 of, CEylon (BMNH). Borneo: Sarawak (BMNH). 
ECHANELLA Bethune-Baker, 1908, Novit. zool. 15: 216.

HY'PE

Type-species: Echanella purpurea Bethune-Baker, r9o8, ibidem 15: 216, by original designation. Syntypes ô. New Guinea: [Papua], Dinawa (BMNH); R. Aroa.

ECHINOCAMPA Franclemont, I949, Proc. ent. Soc. Wash. 51: 279.

OPHI

Type-species: Echinocampa cocophaga Franclemont, 1949, ibidem 51: 280, figs I, 4, 7, by original designation. Holotype $\hat{\jmath}$, CuBA: Havana, Marianao (USNM, Washington).

ECLIPSEA Hampson, I926, Descr. new Genera Species Lepid. Phalaenae Subfamily Noctuinae Br. Mits.: 420 .

$\mathrm{OPHI}$

Type-species: Avitta luna Hampson, I891, Illust. typical Specimens Lepid. Heterocera Colln Br. Mus. 8: 25, IOo, pl. I48, fig. 2I, by original designation. Type(s) Jे, INDIA: [Madras prov.], Nilgiri S. slopes (BMNH).

ECNOMIA Turner, 1936, Proc. R. Soc. Qd 47: 33.

$\operatorname{ACON}$

Type-species: Ecnomia hesychima Turner, 1936, ibidem 47: 34, by monotypy. Holotype $\hat{0}$, Australia: N. Qd, Kuranda (ANIC, Canberra).

ECPATIA Turner, 1902, Proc. Linn. Soc. N.S.IV. 27: 85.

OPHI

Type-species: Anophia dulcistriga Walker, $185^{8}$, List Specimens lepid. Insects Colln Br. Mus. 15: I8I I, by original designation. Holotype ô, [South AFricA]: Caffraria (BMNH).

ECREGMA Walker, [1859]. See Glympis Walker, I859.

OPHI

ECTHETIS Hübner, [1823] I816, Verz. bekannter Schmett.: 257.

$A C O N$

Type-species: Noctua pura Hübner, [1813], Samml. eur. Schmett. 4: pl. I25, fig. I79 [recte 579], by monotypy. Type(s), Europe.

ECTHYMIA Berio, 1940, Memorie Soc. ent. ital. 19: I 26.

ACRO

Type-species: Ecthymia lemonia Berio, 1940, ibidem 19: 127, by original designation.

Holotype + , EThiopia: Borana, Zona di Neghelli.

ECTOCHELA Hampson, I902, Ann. S. Afr. Mus. 2: 279.

cucu

Type-species: Acronycta canina Felder, 1874, in Felder \& Rogenhofer, Reise öst.

Fregatte Novara (Zool.) 2 (Abt. 2) : pl. Ioo, fig. Io; Erklärung: [I], by monotypy. Type(s) $\hat{\sigma}$, [South Africa]: [Cape of Good Hope] Cap. b. sp. (BMNH).

ECTOGONIA Hampson, I896, Fauna Br. India (Moths) 4: 543.

OPHI

Type-species: Camptochilus viola Hampson, I895, ibidem 3: 68, fig. 35, by original designation (for Camptochilus Hampson, i895). Type(s) ơ. Sikкim (BMNH).

Ectogonia was proposed as the objective replacement name for Camptochilus Hampson, I 895 .

CAMPTOCHILUS Hampson, I895, Fanna Br. India (Moths) 3: 33, 68.

Type-species: Camptochilus viola Hampson, I895, by original designation.

A junior homonym of Camptochilus Hampson, [1893] I892, ibidem 1: 351, -Lepid, Thyrididae. The objective replacement name is Ectogonia Hampson, I896.

ECTOGONIELLA Strand, 1920, Arch. Naturgesch. 84(A) I2: I 55. HYPE

Type-species: Ectogoniella pangraptalis Strand, 1920, ibidem $84(\mathrm{~A}) \mathrm{r}_{2}$ : 155, by monotypy. Holotype $q$, [TaIWan] Formosa: Kosempo (DEI, Eberswalde).

ECTOGONITIS Hampson, I896. See Goniocraspidum Hampson, I894.

OPHI

ECTOLOPHA Hampson, I902, Ann. S. Afr. Mus. 2: 382.

ACRO

Type-species: Ectolopha viridescens Hampson, I902, ibidem 2: 383, by monotypy. Syntypes ㅇ, [Rhodesia]: Mashonaland, Salisbury (BMNH). [Kenya] Br. E. Afr.: Kikuyu. 
ECTOPATRIA Hampson, 1903, Cat. Lepid. Phalaenae Br. Mus. 4: xx, 652.

Type-species: Agrotis subrufescens Walker, I865, List Specimens lepid. Insects Colln Br. Mus. 32: 705, by original designation. Holotype $\tilde{\jmath}$, Australia (BMNH).

ECTROGATHA Hampson, I9 Io, Cat. Lepid. Phalaenae Br. Mus. 10: xi, 285. $\operatorname{ACON}$

Type-species: Erosia himerata Walker, [1863] I862, List Specimens lepid. Insects Colln Br. Mus. 26: I757, by original designation. Holotype $\tilde{\sigma}$, BrazIL: [R. Amazon], [Tefé] Ega (BMNH).

$\ddagger E C T R O G N A T H A$; Neave, I939, Nomencl. zool. 2: 195 .

An incorrect subsequent spelling.

$\ddagger$ ECTROGNATHA, misspelling. See Ectrogatha Hampson, I9io.

$\mathrm{ACON}$

ECT YPA Billberg, I820. See Euclidia Ochsenheimer, I8I6.

CATO

EDESSENA Walker, [1859] I858, List Specimens lepid. Insects Colln Br. Mus. 16: I62. HyPE

Type-species: Edessena gentiusalis Walker, [I859] I 858, ibidem 16: I62, by monotypy. Syntypes $2 \hat{\sigma}$, CHina: northern (BMNH).

EDMONDSIA Butler, I882, Trans. ent. Soc. Lond. 1882: го6.

OPHI

Type-species: Edmondsia sypnoides Butler, I882, ibidem 1882: I07, fig., by monotypy. Syntypes 0 , 우, Chili (BMNH).

EDWARDSIA Neumoegen, I880. See Eupseudomorpha Dyar, I893.

AGAR

EDYMA Walker, I858, List Specimens lepid. Insects Colln Br. Mus. 15: I 548, I639. OPH Type-species: Edyma significans Walker, 1858, ibidem 15: I640, by monotypy. Type(s) ô. Brazil (UM, Oxford).

EGABRA Walker, I858, List Specimens lepid. Insects Colln Br. Mus. 15: I668. Geometridae Type-species: Egabra certissima Walker, I 858, ibidem 15: I669, by monotypy.

E. certissima is a junior subjective synonym of Azelina hoedularia Guenée, I857.

Egabra was originally proposed in the Noctuidae.

EGCHIRETES Dyar, I9I4, Proc. U.S. natn. Mus. 47: 202.

SARR

Type-species: Egchiretes nomimus Dyar, I9I4, ibidem 47: 202, by original designation. Holotype , Panama: Alhajuelo (USNM, Washington).

EGELESTA Walker, I858, List Specimens lepid. Insects Colln Br. Mus. 15: I747.

SARR

Type-species: Egelesta rudivitta Walker, I858, ibidem 15: 1748 , by monotypy.

Type(s) ㅇ, CEILON (BMNH).

E. rudivitta is a junior subjective synonym of Gadirtha decrescens Walker, [1858] I857, ibidem 13: I IO4.

See also Plotheia Walker, [I858]; Galleriomorpha Nietner, I86I; and Othora Walker, I865.

EGGYNA Walker, I866, List Specimens lepid. Insects Colln Br. Mus. 35: I894.

ACON

Type-species: Eggyna mimica Walker, I866, ibidem 35: I895, by subsequent designation by Hampson, igı Io, Cat. Lepid. Phalaenae Br. Mus. 10: Io. Holotype ô., [Brazil]: [Amazon], Villa Nova (BMNH).

EGIRA Duponchel, [1845] I844, Cat. méth. Lépid. Eur. (2): I62.

HADE

Type-species: Phalaena conspicillaris Linnaeus, I758, Syst. Nat. (Edn Io) 1: 5I5, by subsequent designation by Desmarest, (1857), in Chenu, Encycl. Hist. nat. Papillons nocturnes: 84. Type(s), Europe (LS, London).

XYLOMYGES Guenée, I852, in Boisduval \& Guenée, Hist. nat. Insectes (Lépid.) 5: I47.

Type-species: Phalaena conspicillaris Linnaeus, $\mathbf{1 7 5 8}$, by subsequent designation by Grote, I874, Bull. Buffalo Soc. nat. Sci. 2: 27.

Xylomyges is a junior objective synonym of Egiva Duponchel. Confusion has been caused by the use of Xylomyges based on an incorrect type-species, Phalaena eridania Stoll, $\mathrm{I} 78 \mathrm{I}$, in the Acronictinae. 
$¥ X Y L O M I G E S$; Grote, I8 7 , Bull. Buffalo Soc. nat. Sci. 2: 27.

An incorrect subsequent spelling; also used by Hering, I932, Tierwelt Mitteleur. Ergänz. 1: 459 .

EGNASIA Walker, [I 859] I 858, List Specimens lepid. Insects Colm Br. Mius. 16: 2 I6. OPH

Type-species: Egnasia ephyrodalis Walker, [I 859] I 858, ibidem 16:217, by subsequent designation by Hampson, I 895, Fauna Br. India (Moths) 3: I5. Syntypes 4 ㅇ. [INDIA]: North Hindostan (BMNH). [Bangladesh]: Silhet (BMNH). Ceylon.

EGNASIDES Hampson, 1926, Descr. new Genera Species Lepid. Phalaenae Subfamily Noctuinae Br. Mus.: $54^{\mathrm{I}}$.

OPHI

Type-species: Egnasia rudmuna Swinhoe, 1905, Ann. Mag. nat. Hist. (7)16:623, by original designation. Syntypes $3 \hat{0}$, Borneo: [Sabah], Kina Balu (BMNH).

EGONE Walker, I863, List Specimens lepid. Insects Colln Br. Mus. 27: 4.

OPHI

Type-species: Egone bipunctalis Walker, 1863, ibidem 27:4, by monotypy. Holotype , [Australia]: Sydney (BMNH).

$\ddagger$ EGRYRION, misspelling. See Egryrlon Smith, I900.

OPHI

EGRYRLON Sinith, I900, Jl N.Y. ent. Soc. 8: 176.

OPHI

Type-species: Egryrlon filaria Smith, I900, ibidem 8: I77, by monotypy. Holotype

․, [U.S.A.]: Florida, Biscayne Bay.

†EGRYRION; Neave, I939, Nomencl. zool. 2: i99.

An incorrect subsequent spelling. Neave then cited 'Egryrlon (pro -rion Smith 190o), Dyar, I902, Bull. U.S. nat. Mus. No. 52, 238. - Lep.' thereby meaning that Dyar had either misspelled or had emended Egryrion Smith to Egryrlon Dyar. In fact Dyar had done no such thing and had merely correctly cited Egryrlon Smith.

$\ddagger E G Y B O L I A$, misspelling. See Egybolis Boisduval, I847.

CATO

EGYBOLIS Boiscluval, I 847, in I)elegorgue, I'oy'age dans l'A frique australe 2: 595 . CAто

Type-species: Phalaena vaillantina Stoll, I790, in Cramer, Uitlandsche Kapellen Aanhangsel: I42, pl. 31, fig. 3, by subsequent designation by Kirby, I892, Synonymic Cat. Lepid. Heterocera 1: 393. Type(s), [Soutu AFRICA]: Land der Caffers.

$P$. vaillantina is dated from the wrapper of the part and not from the title-page of the volume, I 79r.

$\ddagger E G Y B O L I A$; Walker, I 855, List Specimens lepid. Insects Colln Br. Mus. 3: $587,755$.

An incorrect subsequent spelling.

CALOPTERA Blanchard, I 845, Hist. Insect. 2: 362, 376.

Type-species: Caloptera formosa Blanchard, I845, ibidem 2: 376, pl. I7, fig. 6, by monotypy. Type(s), [South Arrica]: Port Natal.

A junior homonym of Caloptera Gistl, I834, Insect.-Doubletten Samml. R. J. Walworth: Io,-Coleoptera. A nomenclaturally available generic name although cited as a nomen mudum by Neave. There is no objective replacement name but $C$. formosa is a junior subjective synonym of Phalaena iaillantina Stoll, I790, the type-species of Egybolis Boiscluval, I847. The latter is therefore available for use as a subjective replacement naine.

EICOMORPHA Standinger, I888, Stettin. ent. Ztg 49: 24.

NOC $\mathrm{r}$

Type-species: Eicomorpha antiqua Staudinger, I 888, ibidem 49: 24, by monotypy. Syntypes 7 ô. 3 f, [U.S.S.R.]: [Uzbekistan], Namangan; Transalai; Samarkand.

ELAEMIMA Berio, 1970, Boll. Soc. ent. ital. 102: 24

HADF:

Type-species: Elaemima brunnea Berio, I970, ibidem 102: 24, fig. 4, by original designation. Holotype ô. [ZAIRE]: [Equateur], Itoko à Combe (MRAC, Tervuren).

ELAEODES Hampson, 1913. See Nyodes Laporte, 1970. 
ELAEODOPSIS Prout, 1927, Trans. ent. Soc. Lond. 75: 207.

ACRO

Type-species: Elaeodopsis loxoscia Prout, 1927, ibidem 75: 208, pl. 21, fig. 6, by original designation. Syntypes i +2 0 , [SÃo Tomé I., W. Africa] São Thomé I. (BMNH).

ELAEOGNATHA Hampson, I 905, Ann. Mag. nat. Hist. (7) 16: 577.

SARR

Type-species: Elaeognatha argyritis Hampson, 1905, ibidem (7) 16: 577 , by original designation. Holotype 9, Panama : Cana Mines (BMNH).

$\ddagger$ ALAEOGNATHA; Druce, i9io, Ann. Mag. nat. Hist. (8)6: i 79 .

An incorrect subsequent spelling.

ELAPHRIA Hübner, I818, Zuträge Samml. exot. Schmett. 1: I6.

ACRO

Type-species: Elaphria grata Hübner, I818, ibidem 1: I6, figs 71, 72, by subsequent designation by Barnes \& Benjamin, I929, Bull. Brooklyn ent. Soc. 24: i 81. Type(s) ô, [U.S.A.]: Georgia [formerly] in Florida.

Incorrect type-species designation: Phalaena morpheus Hufnagel, I766, a name not originally included in Elaphria, and not linked with one of the originally included names when cited by Hampson, 1909, Cat. Lepid. Phalaenae Br. Mus. 8: 299, 357.

$\ddagger_{\ddagger}$ AGROTIS Hübner, I808, Erste Zuträge Samml. exot. Schmett.: 4 .

Included in a work rejected for nomenclatural purposes by Int. Commn zool. Nom. I966, Bull. zool. Nom. 23 Opinion 789: 2 I 3.

Placed on the Official Index of Rejected and Invalid Generic Names in Zoology: Name No. 1826 .

Only included species: $\ddagger$ Agrotis grata Hübner, 1808 , later made nomenclaturally available as Elaphria grata Hübner, i 818.

ELAPHRISTIS Meyrick, i89i, Trans. (Proc.) R. Soc. S. Aust. 14: 198.

HYPE

Type-species: Elaphristis anthracia Meyrick, I89I, ibidem 14: 198, by monotypy. Syntypes 2 đ., Australia: Qd, Brisbane (BMNH).

ELATINA Duponchel, [1845]. See Panthea Hübner, [I820].

PANT

ELEALE Walker, I862. See Tamseale nom. n.

EUTE

ELECUSSA Schaus, I912, Ann. Mag. nat. Hist. (8) 10: 531 .

Type-species: Elecussa displosa Schaus, i912, ibidem (8)10: 53 I, by monotypy. Type(s) , Costa Rica: Juan Viñas.

ELEEMOSIA Prout, I90I, Entomologist's Rec. J. Var. 13: i 83.

ACRO

Type-species: Noctua abjecta Hübner, [I8I3], Samml. eur. Schmett. 4: pl. I I6, fig. 539, by original designation. Type(s), Europe.

N. abjecta is a junior subjective synonym of Noctua oblonga Haworth, i 809, Lepid. Br.: I 88 .

ELEGARDA Walker, I 865, List Specimens lepid. Insects Colln Br. Mus. 33: 7 I2.

NOCT

Type-species: Agrotis dorsicinis Walker, I858, ibidem 15: I70I, by PRESENT DESIGNATION. Holotype $\widehat{\sigma}$, [Australia]: [Qd], Moreton Bay (BMNH).

Incorrect type-species designation: Perigea albinasus Walker, I 856, a name not originally included in Elegarda, cited by Hampson, I903, Cat. Lepid. Phalaenae Br. Mus. 4: I53, who then on page 165 placed albinasus, together with all three of the originally included nominal species (dorsicinis Walker, summa Walker and orthosioides Walker), all as junior synonyms of Agrotis porphyricollis Guenée, I 852. From this designation it is not possible to know which of the three originally included names should be the type-species.

A. dorsicinis is a junior subjective synonym of Agrotis porphyricollis Guenée, 1852, in Boisduval \& Guenée, Hist. nat. Insectes (Lépid.) 5: 259.

ELEGOCAMPA Franclemont, 1949, Proc. ent. Soc. Wash. 51:281. OPHI Type-species: Herminodes catharina Schaus, 1933, Ann. Mag. nat. Hist. (10)12: 
382, by original designation. Holotype ㅇ, BrazIL: Sta Catharina, Blumenau (USNM, Washington).

ELESMA Walker, 1865, List Specimens lepid. Insects Colln Br. Mus. 32: 608.

SARR

Type-species: Elesma subglauca VValker, I865, ibidem 32: 608, by monotypy.

Holotype $q$, Australia (BMNH).

See also Amaloptila Turner, I903.

ELESMOIDES Prout, I927, Trans. ent. Soc. Lond. 75: 21 4.

SARR

Type-species: Elesmoides thomae Prout, I927, ibidem 75: 214, pl, 21, fig. 22, by original designation. Syntypes I $\hat{0}, 7$, [S̃̃o Tomé I., WV. Africa] São Thomé I. (BMNH).

ELIGMA Hübner, [1819] I8I6, Verz. bekannter Schmett.: I65.

CHLO

Type-species: Phalaena narcissus Cramer, I775, Uitlandsche Kapellen 1: II6, pl. 73, figs E, F, by monotypy. Syntypes 2 우, China.

$P$. narcissus is dated from the wrapper of the part and not from the title-page of the volume, I 779 .

HELIGMA Agassiz, 18 4 6, Nomencl. zool. Index univl.: I 36, I75.

An unjustified emendation of Eligma Hübner, [1819].

PANGLIMA Noore, I858, in Horsfield \& Moore, Cat. lepid. Insects Mus. Hon. East-India Company 2: 207 .

Type-species: Phalaena narcissus, Cramer, 1775, by monotypy.

Panglima is a junior objective synonym of Eligma Hübirer, [ISI9].

ELIOCROEA Walker, I865, List Specimens lepid. Insects Colm Br. Mus. 33: 935. SARR

Type-species: Eliocroea chrysochlora Walker, 1865, ibidem 33: 935, by monotypy. Holotype f, [Moluccas]: Ceram [I.] (BMNH).

ELIXOIA Wallier, i 865, List Specimens lepid. Insects Colln Br. Mus. 33: i Ioo. OpH

Type-species: Elixoia subocellata Wallier, I865, ibidem 33: IIoo, by monotypy. Holotype ô, [BraziL]: Rio Janeiro.

ELOCUSSA Walker, 1865, List Specimens lepill. Insects Colln Br. Mus. 33: Ioor. ophi

Type-species: Elocussa gortynoides Walker, I 865, ibidem 33: Ioo I, by monotypy. Holotype ô, [Brazil]: [R. Amazon], [Tefé] Ega (BMNH).

ELOUSA Walker, [1858] i 857, List Specimens lepid. Insects Colln Br. Mus. 13: I o78, i I 7. OPHI Type-species: Elousa albicans Walker, [1858] I857, ibidem 13: III8, by monotypy. Syntypes 3 9, [Dominicax Republic] St. Domingo (BMNH).

See also Masebia Walker, 1858.

ELPIA Walker, I865, List Specimens lepid. Insects Colln Br. Mus. 33: 983.

OPHI

Type-species: Elpia achacoides Walker, 1865, ibidem 33: 984, by monotypy. Lectotype ô, [Sulawesi] Celebes: Makian (UNI, Oxford), designated (as type) by Swinhoe, I9oo, Cat. east. and Aust. Lepid. Heterocera 2: io6.

ELUSA Walker, [1859] I 858, List Specimens lepid. Insects Colln Br. Mus. 16: 202. HADE Type-species: Elusa ceneusalis Valker, [I859] I 858, ibidem 16: 202, by monotypy. Syntypes 3 ô, BORnEo: Sarawak (BMNH).

Sce also Alimala Walker, I 862.

ELVESIA Hampson, I906. See Elwesia Himpsou, I 894.

CUCU

ELWESIA Hampson, I89., Fauna Br. India (Moths) 2: 164, I 7.

CUCU

Type-species: Elwesia diplostigma Hampson, I894, ibidem 2: I72, fig. II I, by original designation. Holotype ․ [SIkkim] Sikhim (BMNH).

ELVESIA Hampson, I906, Cat. Lepid. Phalaenae Br. MIus. 6: 434.

An unjustified emendation of Elwesia Hampson, 1894. 
ELYDNA Walker, 1858, List Specimens lepid. Insects Colln Br. Mus. 15: I7I2.

ACRO

Type-species: Elydna transversa Wallier, I858, ibidem 15: I7I3, by monotypy. Holotype $q$ (not $\delta$ as stated), [India] Hindostan (BMNH with added labəl Burma, Moulmein).

ELYDNODES Hampson, I913, Cat. Lepid. Phalaenae Br. Mus. 13: xi, 399.

PANT

Type-species: Carea variegata Leech, Igoo, Trans. ent. Soc. Lond. 1900: 524, by original designation. Holotype o, CHINA: western, Chia-ting-fu (BMNH).

EL YGEA Billberg, I820, Enumeratio Insectorum Mus. G. J. Billberg: 85.

OPHI

Type-species: Phalaena materna Linnaeus, I767, Syst. Nat. (Edn I2) 1: 840, by subsequent designation by Berio, I966, Annali Mus. civ. Stor. nat. Giacomo Doria 76: 57. Type(s), India (LS, London).

ARGADESA Moore, I88I, Trans. zool. Soc. Lond. 11: 74 .

Type-species: Phalaena materna Linnaeus, I767, by monotypy.

Argadesa is a junior objective synonym of Elygea Billberg, ISzo.

ELYPTRON Saalmüller, I89I, Lepid. Madagascar: 300.

ACRO

Type-species: Elyptron cinctum Saalmüller, I891, ibidem: 301, fig. 254, by monotypy. Holotype ô. Madagascar (MNHU, Berlin).

EL YRA Walker, [1859] I858, List Specimens lepid. Insects Colln Br. Mus. 16: 203.

HYPE Type-species: Elyra phlegeusalis Waller, [1859] 1858, ibidem 16: 203, by monotypy. Lectotype ô. BorNeo: Sarawak (UM, Oxford), designated (as type) by Swinhoe, I900, Cat. east. and Aust. Lepid. Heterocera 2: 200.

Elyra when originally proposed contained a second species, but this was doubtfully included and under the Code, Article'68 (c) is not eligible for designation as type-species.

See also Eordaea Walker, [I859].

EMARGINEA Guenée, I852, in Boisduval \& Guenée, Hist. nat. Insectes (Lépid.) 6: 288. ACro Type-species: Emarginea gammophora Guenée, I852, ibidem 6:289, pl. I4, fig. I by monotypy. Lectotype $\delta$, [URUGUAY]: Montevideo (MNHN, Paris), designated by Viette, I95 I, Bull. mens. Soc. linn. Lyon 20: I6I.

EMARIANNIA Benjamin, I933, Pan-Pacif. Ent. 9: 2.

CUCU

Type-species: Emariannia cucullidea Benjamin, 1933, ibidem 9: 3, by original designation. Holotype $\sigma^{\lambda}$, [U.S.A.]: Texas, Alpine (USNM, Washington).

EMBOL OECIA Hampson, I908, Cat. Lepid. Phalaenae Br. Mius. 7: Io. Available, but without included species until Hampson, r9ro, ibidem 9: vii, 59.

ACRO

Type-species: Ochria sauzalitae Grote, I875, Bull. Buffalo Soc. nat. Sci. 2: 216, by subsequent monotypy. Syntypes, [U.S.A.]: California (BMNH).

EMEA Walker, I865. See Erebostrota Warren, I889.

OPHI

†EMESIA, misspelling. See Episteme Hübner, [1820].

AGAR

EMMELIA Hübner, [182I] I8I6, Verz. bekannter Schmett.: 254.

$\mathrm{ACON}$

Type-species: Phalaena sulphuralis Linnaeus, I767, Syst. Nat. (Edn I2) 1:881, by monotypy, but included as 'E. sulphurea Schiff.', an unjustified emendation. Type(s), GERMANY (LS, London).

[Denis \& Schiffermüller], I775, Ankündung. syst. Werkes Schmett. Wienergegend: 93, transferred $P$. sulphuralis Linnaeus from the 'Pyralides' to the 'Noctuae' and emended the ending of the name.

P. sulphuralis is a junior subjective synonym of Phalaena trabealis Scopoli, 1763, Ent. Carniolica: 240 . 
†EROTI"LA Hübner, [1806], Tentamen determinationis digestionis . . . : [2].

Included in a work rejected for nomenclatural purposes by Int. Commn zool. Nom.. 1926, Sinithson. misc. Collns 73(4) Opinion 97: 19. Also idem, 1954, Opin. Decl. int. Commn zool. Nom. 6 Opinion 278: I 40.

Only included species: Noctua sulphurea [Denis \& Schiffermüller], I775.

EROT Y'LA Hübner, I 822, Syst.-alphab. Verz.: 22, 36.

Type-species: Phalaena sulphuralis Linnaeus, I 767 (but included by Hübner as 'sulphurea S.'), by subsequent designation by Grote, i 874, Bull. Buffalo Soc. nat. Sci. 2: 37, but cited as 'Noctua sulphurea'.

Erotyla is a junior objective synonym of Emmelia Hübner, [182I].

AGROPHILA Boisduval, i8 fo, Genera Index meth. Eur. Lepid.: I 75.

Type-species: Phalaena sulphuralis Linnaeus, 1767 , by subsequent designation by Blanchard, I840, in Castelnau, Hist. nat. Anim. articulés (Insectes) 3:522 (but cited as 'sulphurea Hübn.', an incorrect authorship of Noctua sulphurea [Denis \& Schiffermüller], I775, a name originally included in Agrophila and also an unjustified emendation of sulphuralis Linnaeus).

Agrophila is a junior objective synonym of Emmelia Hübner, [1821].

$\ddagger$ EMMONODIA, misspelling. See Enmonodia Walker, I 858 .

CATO

EMPELATHRA Walker, i858, List Specimens lepid. Insects Colln Br. Mus. 15: $15+7,1633$.

OPH I

Type-species: Empelathra amplificans Walker, i 858, ibidem 15: I633, by monotypy. Holotype ô. BraziL (BMNH).

†EMPISEME, misspelling. Sec Episema Ochsenheimer, i 8 r6.

CUCU

EMPUSA Hübner, [1821]. See Emprusada Hampson, I906.

CUCU

EMPUSADA Hampson, I906, Cat. Lepid. Phalaenae Br. Mus. 6: viii, 85. cucu

Type-species: Noctua lactea Fabricius, I787, Mantissa Insect. 2: 182, by monotypy (of Empusa Hübner, 182I). Type(s), [U.S.S.R.] Russia: southern (Type(s) not found by Zimsen, 1964, Type Material of 1. C. Fabricius: 550).

Empusada was proposed as an objective replacement name for Empusa Hübner, I82 I.

EMPUSA Hübner, [1821] г 816, Verz. bekannter Schmett. : 247.

Type-species: Noctua lactea Fabricius, 1787 , by monotypy.

A junior homonym of Empusa Illiger, I798, in Kugelann, Verz. Käfer Preussens: 499, Orthoptera. The objective replacement name is Empusada Hampson, 1906.

ENARGIA Hübner, [1821] i816, Verz. bekannter Schmett.: 235.

ACRO

Type-species: Phalaena paleacea Esper, 1788, Die Schmett. 4(1): pl. 122, figs 3, 4; I791, ibidem: 323 , by monotypy, but cited by Hübner as $\ddagger E$. palleacea, an incorrect subsequent spelling. Syntypes $\hat{o}, \underline{q}$. [Germany].

EUPERIA Guence, 1839, Annls Soc. ent. Fr. 8(3): 486.

Type-species: Noctua fulvago Clerck sensı Hübner, [1803], Samml. eur. Schmett. 4: pl. 4I, figs I98, I99 [= Phalaena paleacea Esper, I788], by subsequent designation by Guenée, 1852, in Boisduval \& Guenée, Hist. nat. Insectes (Lépid.) 6: 6.

$N$. fulvago Clerck sensu Hübner, [1803] has long been accepted as a misidentification of Phalaena fulvago Clerck, I759, Icones Insect. rarionum 1: pl. 6, fig. ${ }^{5} 5$. This is a case of a misidentified type-species which under the Code, Article 70 (a) should be referred to the Commission.

Euperia is a junior objective synonym of Enargia Hübner, [1821].

ENCALYPTA Möschler, I890, Abh. senckenb. naturforsch. Ges. 16: I 47.

Type-species: Encalypta schildei Möschler, 1890, ibidem 16:1 18 , fig. 23, by monotypy. Syntypes 3 ․ Puerto Rico. 
E. schildei is a junior subjective synonym of Iscadia aperta Walker, I857.

See also Iscadia Walker, 1857.

$\ddagger E U C A L Y P T A$; Hampson, I912, Cat. Lepid. Phalaenae Br. Mus. 11: 363 .

An incorrect subsequent spelling.

ENCRUPHION Schaus, I914, Proc. U.S. natn. Mus. 46: 506.

OPHI

Type-species: Encruphion porrima Schaus, I914, ibidem 46: 506, by original designation. Type(s) ô, British Guiana: Omai (USNM, Washington).

ENEA Valker, [1863] r864, J. Proc. Linn. Soc. (Zool.) 7: 63.

$\operatorname{ACON}$

Type-species: Enea signicosta Walker, [1863] 1864, ibidem 7: 63, by monotypy. Lectotype of. BORNEO: Sarawak (UM, Oxford), designated (as type) by Swinhoe, I90o, Cat. east. and Aust. Lepid. Heterocera 2: 197.

ENEDENA Dognin, I9I4, Hétérocères nouv. Am. Sud. 8: 68.

HYPE

Type-species: Enedena punctilinea Dognin, I914, ibidem 8: 69, by original designation. Holotype o, Ecuador: Environs de Loja.

ENGELHARDTIA Barnes \& Benjamin, I923, Bull. Brooklyn ent. Soc. 18: I 2.

HADE

Type-species: Lathosea ursina Smith, 1898, Can. Ent. 30:324, by original designation. Syntypes II ठ, I ㅇ, [U.S.A.]: Colo., Glenwood Springs and Garfield county.

ENGUSANACANTHA Berio, 194I, Annali Mus. civ. Stor. nat. Giacomo Doria 61: I83. Noct

Type-species: Engusanacantha bilineata Berio, 1941, ibidem 61: 183, by original designation. Holotype ô, [ETHIopia]: Eritrea, Elaberet (Holotype destroyed in MCSN, Genoa during World War II. Paratype o in Colln E. Berio, Genoa).

ENISPA Walker, [1866] I865, List Specimens lepid. Insects Colln Br. Mus. 34: I 275. ACON

Type-species: Enispa eosarialis WValker, [1866] I865, ibidem 34: I275, by monotypy. Lectotype ${ }^{\top}$ (not $q$ as stated by Walker), Borneo: Sarawak (UNI, Oxford), designated (as type) by Swinhoe, 1900, Cat. east. and Aust. Lepid. Heterocera 2: 70.

ENISPADES Bethune-Baker, I91 I, Ann. Mag. nat. Hist. (8)8: 5 18.

ACON

Type-species: Enispades angola Bethune-Baker, I9II, ibidem (8)8: 519, by original designation. Type(s) $\vec{o},[$ Angola $]$ : N'Dalla Tando (BMNH).

ENISPODES Warren, 1913, in Seitz, Gross-Schmett. Erde 11: $21 \mathrm{r}$.

ACON

Type-species: Enispa purpurea Hampson, I9Iо, Cat. Lepid. Phalaenae Br. Mus. 10: 40, fig. I 8, by original designation. Holotype o, Singapore (BMNH).

The date of publication of the part of Seitz containing page 2 I I was stated on page 209 as I9r3 August 2oth in both the English and the German editions.

ENMONODIA Walker, r858, List Specimens lepid. Insects Colln Br. Mus, 14: $1332 . \quad$ Cato

Type-species: Enmonodia hypopyroides Walker, I858, ibidem 14: 1333, by mono-

typy. Holotype ô. TyPe-Locality unknown (BMNH). [INDia].

E. hypopyroides is a junior subjective synonym of Noctua vespertilio Fabricius, ${ }^{7} 787$.

See also Hypopyra Guenée, 1852.

$\ddagger E M M O N O D I A$; Butler, i 893, Entomologist 26: 353 .

An incorrect subsequent spelling.

ENMONODIOPS Hampson, 1926, Descr. nere Genera Species Lepid. Phalaenae Subfamily

Noctuinae Br. Mus.: 530.

OPHI

Type-species: Enmonodiops ochrodiscata Hampson, 1926, ibidem: 53 I, by original designation. Holotype $\hat{o}$. [CAMeroun] Cameroon: R. Ja, Bitje (BMNH).

ENSIPIA Walker, [I859] I858, List Specimens lepid. Insects Colln Br. Mrus. 16: 207. opHI Type-species: Ensipia lamusalis Walker, [1859] I858, ibidem 16: 208, by monotypy. Holotype ô. [BrazIL]: R. Amazon, [Tefé] Ega (BMNH). 
${ }_{+}^{+}$ENTELIA, misspelling. See Eutelia Hübner, [I823].

ENTERIONA Sodoffisy;, I837. See Nonagria Ochsenheimer, rSr6.

ACRO

ENTERPIA Guenée, I850, Amls Soc.ent. Fr. (2)8: 235.

ACRO

'Type-species: Cleophana laudeti Boisduval, I8 70 , Genera Inder meth. Eur. Lepid.: 152, by monotypy. Type(s), [SwitzerLAND]: [Valais] V'alesia.

†EUTERPIA; Guenée, IS52, in Boisduval \& Guenée, Hist. nat. Insectes (Lépid.) $6:$ I 7 I.

An incorrect subsequent spelling.

ENTOMOGRAMMA Guence, I852, in Boiscluval \& Guenée, Hist. nat. Insectes (Lépid.) 7: 203 . cato

Type-species: Entomogramma fautrix Guenée, 1852 , ibidem 7: 204, by subsequent designation by Moore, [1885] I887, Lepid. Ceylon 3: I53. Syntypes, [BA.valADESH]: [E. Bengal], Silhet (BMNH).

ENYDRA Walker, 1862, Trans. ent. Soc. Lond. (3) 1: 77.

OPHI

Type-species: Enydra cinctigutta Walker, I862, ibidem (3) 1: 77, by monotypy. Syntypes $\hat{0}$, ?, [South AFrica?: Port Natal.

EOGENA Guenće, I852, in Boisduval \& Guenćc, Hist. nat. Insectes (Lépid.) 5: 340. ACro

Type-species: Noctua contaminei Eversmann, 1847, Bull. Soc. Nat. Moscou 20(3): 77, pl. 5, fig. 6, by monotypy. Type(s), [U.S.S.R.]: [Krasnoarmeysk] Sarepta (ZI, Leningrad).

EOPAECTES Hulstacrt, 1924, Am. Mag. nat. Hist. (9)13: ro4.

OPH I

Type-species: Eopaectes alboniacula Hulstaert, I924, ibidem (9)13: 105, by original designation. Holotype $\hat{\sigma}$. [Moluccas]: Jamdena, Alusi.

EORDAEA VIalker, [1859] I858, List Specimens lepid. Insects Colln Br. Mus. 16: 206. HYPE Type-species: Eordaea peleusalis Walker, [1859] 1858, ibidem 16: 207, by monotypy. Lectotype ơ, Borneo: Sarawak (UM, Oxford), designated (as type) by Swinhoe, I9oo, Cat. east. and Aust. Lepid. Heterocera 2: 200.

E. pelensalis is a junior subjective synonym of Elyma phlegeusalis Walker, [I859] I858, ibidem 16:203.

See also Elyra Wallicr, [1859].

EOSPHOROPTERYX Dyar, I902, Jl N.Y.ent. Soc. 10: 8o.

PLUS

Type-species: Plusia thyatyroides Guenée, 1852 , in Boisduval \& Guenéc, Hist. nat. Insectes (Lépid.) 6: 337, pl. Ir, fig. 8, by original designation, but cited as \$'thyatiroides Guen.', an incorrect subsequent spelling. Fo!ntype ô, [U.S.A.]: New York State (BMNH).

EPA Bethune-Baker, I906, Novit. zool. 13: 192.

ACRO

Type-species: Epa pratti Bethune-Baker, Igo6, ibidem 13: I93, by original designation. Type(s) ô, New Guinea: [Papua], Jinawa (BMNH).

EPHARMOTTOMENA John, I 909, Revue russe Ent. 9: I I8.

OPIII

Type-species: Leucanitis nana Staudinger, r88., in Romanoff, Mém. Lépid. 1: I.47, pl. 9, fig. 6, by original designation. Syntypes, [U.S.S.R.]: [Turkmenistan], Askhabad (MNHU, Berlin).

EPHEMIAS Hübner, [1821]. See Scoliopteryx Germar, I8II.

OPHI

EPHESIA Hübner, I8 I 8, Zuträge Samml. exot. Schmett. 1: I r.

CATO

Type-species: Phalaena paranympha Linnac1s, I 767, Syst. Nat. (Edn 12) 1: 8.42. by subscquent designation by Hampson, 19 3, Cat. Lepid. Phalaenas Bir. MIus. 12: 143, but cited as fulminea Scopoli, I $76_{3}$, which Hampson placed, on page 202, as the senior synonym of $P$. paranympha. Type(s), Gernany (LS, London). 
P. paranympha is a junior subjective synonym of Phalaena fulminea Scopoli, I763, Ent. Carniolica: 206.

EPHYRODES Guenée, I852, in Boisduval \& Guenée, Hist. nat. Insectes (Lépid.) 7: 365. opH I Type-species: Ephyrodes cacata Guenée, 1852, ibidem 7: 366, pl. 24, fig. 7, by subsequent designation by Hampson, 1926, Descr. new Genera Species Lepid. Phalaenae Subfamily Noctuinae Br. Mus.: 455. Syntypes Ŏ, Colombia (BMNH). Cuba.

EPIA Hübner, [1821]. See Anepia Hampson, I9I 8.

HADE

EPICARSIA Hampson, 1926, Descr. new Genera Species Lepid. Phalaenae Subfamily Noctuinae Br. Miss.: $45 \mathrm{I}$.

OPHI

Type-species: Thermesia bigutta Walker, I858, List Specimens lepid. Insects Colln Br. Mus. 15: I 857 , by original designation. Type(s) 오, [BrazIL]: Rio Janeiro (UM, Oxford).

EPICAUSIS Butler, I880, Ann. Mag. nat. Hist. (5)5: $34 \mathrm{I}$.

CUCU

Type-species: Epicausis lanigera Butler, I880, ibidem (5)5:342, by original designation. Lectotype q, Madagascar (BMNH), designated by riette, [1963] ig62, Annls Soc. ent. Fr. $131: 284$.

E. lanigera Butler, r88o April, is a junior subjective synonym of Daphoenura smithii Mabille, I880 March, Bull. Soc. ent. Fr. (5)9: clxxiv. D. smithii was proposed as $\$$ Daphnaeura, an incorrect subsequent spelling.

Epicausis was originally placed in the Arctiidae.

EPICERYNEA Hampson, I9I4, Ann. Mag. nat. Hist. (8)13: I73.

$\mathrm{ACON}$

Type-species: Epicerynea goniosema Hampson, I9I4, ibidem (8)13: I 74, by originaI designation. Holotype ô, [Ghana] Gold Coast: Kumasi (BMNH).

EPICONCANA Hampson, 1926, Descr. new Genera Species Lepid. Phalaenae Subfamily Noctuinae Br. Mus. I27.

OPHI

Type-species: Concana leucomera Dyar, I9I2, Proc. U.S. natn. Mus. 42: 80, by original designation. Syntypes $\tilde{\sigma}$, 우, Mexico: Misantla; Orizaba. (USNM, Washington).

EPICORIA Walker, I 865, List Specimens lepid. Insects Colln Br. Mus. 33: 852. votodontidaE Type-species: Epicoria canosparsa Walker, I865, ibidem 33: 853, by subsequent designation by Berio, 1966, Annali Mus. civ. Stor. nat. Giacomo Doria 76: 57.

Epicoria was originally placed in the Noctuidae.

EPICYRTICA Turner, I908, Trans. Proc. R. Soc. S. Aust. 32: 6I.

OPHI

Type-species: Epicyrtica lathridia Turner, I908, ibidem 32: 6I, by monotypy. Holotype $\widehat{\sigma}$, Australia: near Melbourne, Leopold.

$\ddagger$ EPIDELTA Hampson. See Epidelta nom.n.

HYPE

EPIDELTA nom. n. for Philometra Grote, I872.

HYPE

Type-species: Herminia longilabris Grote, I872, Trans. Am. ent. Soc. 4: 99, by subsequent designation (for Philometra Grote) by Grote, I873, ibidem 4: 308. Syntypes $\widehat{0}$,, , [U.S.A.]: Pennsylvania; Virginia.

H. longilabris is a junior subjective synonym of Herminia metonalis WValker, [1859] I858, List Specimens lepid. Insects Colln Br. Mus. 16: 236.

PHILOMETRA Grote, I872, Trans. Am. ent. Soc. 4: 98.

Type-species: Herminia longilabris Grote, 1872 , by subsequent designation by Grote, I873, ibidem 4: 308 .

Philometra Grote was originally placed as a subgenus of Herminia Latreille, r8oz.

A junior homonym of Philometra Costa, 1846, Annali Accad. Aspir. Nat. Napoli 3: 8o, - Vermes, Nematoda. The objective replacement name is Epidelta nom. n. 
$\ddagger E P I D E L T A$ Hampson, no published reference found.

Epidelta was used by Hampson in his manuscript and collection as a new genus for Herminia metonalis Walker, [I859].

EPIDEMAS Sinith, r894, Trans. Am. ent. Soc. 21: 40.

CUCU

Type-species: Epidemas cinerea Smith, I894, ibidem 21: 4I, pl. 4, figs I0, I2, by monotypy. Syntypes ro $(\hat{0},+$ ) $)$, [U.S.A.]: Colo., Glenwood Springs.

EPIDROMIA Guenée, 1852, in Boisduval \& Guenée, Hist. nat. Insectes (Lépid.) 7: 325. OPH Type-species: Epidromia pannosa Guenée, I852, ibidem 7: 326, by subsequent designation by Berio, I966, Annali Mus. civ. Stor. nat. Giacomo Doria 76: 57. Syntypes ô. BRAZIL (BMNH).

E. pannosa is a junior subjective synonym of Bendis poaphiloides Guenée, 1852 , ibidem 7: 215 .

EPIGIAURA Prout, I927, Trans. ent. Soc. Lond. 75: 2 I 3.

SARR

Type-species: Epigiaura trachylepis Prout, 1927, ibidem 75: 213, pl. 21, fig. 10, by original designation. Syntypes I ô, I $q$, [S̃̃o Tomé I., West Africa] São Thomé I. (BMNH).

EPIGLAEA Grote, 1878, Bull. U.S. geol. geogr. Surv. Territ. 4: I8I.

CUCU

Type-species: Orthosia apiata Grote, I874, Anmul Rep. Trustees Peabody Acad. Sci.

6: 30, by subsequent designation by Grote, 1895, Abh. naturw. Ver. Bremen 14: 95.

Type(s), [U.S.A.]: Ill., Chicago (BMNH).

See also Harpaglaea Hampson, I 906.

EPIGRYPERA Hampson, rgio, Cat. Lepid. Phalaenae Br. Mus. 10: 9, 282.

ACON

Type-species: Epigrypera eriogona Hampson, I9I0, ibidem 10: 282, fig. 84, by original designation. Holotype ô, BoRneo: Sarawak (BMNH).

EPIGRYPODES Turner, 1936, Proc. R. Soc. Qd 47: 42.

OPHI

Type-species: Epigrypodes amplipennis Turner, 1936, ibidem 47 : 42, by monotypy. Holotype 0 . Australia: N. Qd, Cape York.

EPILECTA Hübner, [I82I] I8I6, Verz. bekannter Schmett.: 22I.

NOCT

Type-species: Noctua linogrisea [Denis \& Schiffermüller], 1775, Ankïndung syst. Werkes Schmett. Wienergegend: 79, by monotypy. Type(s), [AusTRIA]: Vienna district (Collection destroyed, Horn \& Kahle, 1936, Ent. Beih. Berl.-Dahlem 3: 243).

HIRIA Duponchel, [1845], Cat. méth. Lépid. Europe (2): I45.

NOCT

Type-species: Noctua linogrisea [Denis \& Schiffermüller], I 775 (but included by Duponchel as 'linogrisea F.', an incorrect authorship), by subsequent designation by Hampson, 1903. Cat. Lepid. Phalaenae Br. Mus. 4: 620.

Hiria is a junior objective synonym of Epilecta Hübner, [182I].

$\ddagger$ EPILOMIA Hampson, no published reference found.

HYPE

EPIMECIA Guenée, I 839, Annls Soc. ent. Fr. 8: 5 I 4.

ACRO

Type-species: Cleophana ustulata Boisduval, Rambur \& Graslin, [1836] 1832, Colln iconographique hist. Chenilles Eur.: Noctuélides, text \& pl. 22, figs I-4, by monotypy. Syntypes, [France]: Montpellier district; Provence.

The date of publication of the type-species has been accepted from Cowan, I970, J. Soc. Biblphy nat. Hist. 5: 323 .

C. ustulata is a jumior subjective synonym of Cymatophora ustula Freyer, 1835 . Nevere Beitr. Schmett. 2: 90, p]. I 48 , fig. I.

EPIMECIODES Hampson, 1926, Descr. new Genera Species Lepid. Phalaenae Subfamily Noctuinae Br. Mus.: 6I 5 . OPHI Type-species: Epimecia abunda Felder \& Rogenhofer, 1874, Reise öst. Fregatte Novara 
(Zool.) 2 (Abt. 2): pl. Io8, fig. 46; Inhalts-Verz.: 8, by original designation. Type(s) , [South Africa]: [Cape of Good Hope] Promont. b. spei (BMNH).

EPINEURONIA Rebel, I90I. See Neuronia Hübner, [I82I].

HADE

EPINYCTIS Grote, I882, April, Can. Ent. 14: 75.

CUCU

Type-species: Epinyctis notatella Grote, I882, ibidem 14:75, by monotypy. Type(s), [U.S.A.]: Montana.

E. notatella is a junior subjective synonym of Cucullia luna Morrison, I875, Proc. Boston Soc. nat. Hist. 18: I 22.

See also Nycterophaeta Smith, I882 February.

EPIOECIA Petrowsky, I958, Ent. Z., Frankf. a. M. 68: I 58.

HADE

Type-species: Epioecia kohleriana Petrowsky, I958, ibidem 68: I59, figs I-3, by original designation. Holotype, ARgenrina: Neuquen province, Cerro Malo (ZSBS, Munich).

EPIPHANIS Schaus, 1913. See Ipanephis nom. n.

HYPE

EPIPSAMMIA Staudinger, I879, Stettin. ent. Ztg 40:320.

ACRO

Type-species: Epipsammia deserticola Staudinger, I879, ibidem 40: 320, by monotypy. Syntypes, [U.S.S.R.]: S.E. European Russia, Narün sandy region (MNHU, Berlin).

EPIPSILIA Hübner, [182I] I816, Verz. bekannter Schmett.: 210.

NOCT

Type-species: Noctua latens Hübner, [1809], Samml. eur. Schmett. 4: pl. 89, fig. 419, by subsequent designation by Hampson, I903, Cat. Lepid. Phalaenae Br. Mus. 4: 467, but cited for $\ddagger$ Episilia Hübner, an incorrect subsequent spelling. Type(s), Europe.

$\ddagger$ EPISILIA; Hampson, I903, Cat. Lepid. Phalaenae Br. Mus. 4: 467 .

An incorrect subsequent spelling.

EPIPSILIAMORPHA Barnes \& Benjamin, 1929, Bull. Brooklyn ent. Soc. 24: i 73.

NOCT

Type-species: Agrotis alaskae Grote, 1876, Bull. Buffalo Soc. nat. Sci. 3: 84, pl. 4, fig. I, by original designation. Holotype $\hat{\delta}$, [U.S.A.]: Alaska (BMNH).

EPISCOTIA Berio, 1962, Annali Mus. civ. Stor. nat. Giacomo Doria 73: 201.

NOCT

Type-species: Episcotia bicostata Berio, I962, ibidem 73:201, by original designation.

Holotype ô, [ZaIRE]: [Kivu], [Bakavu] Costermansville (MRAC, Tervuren).

EPISEMA Ochsenheimer, I8I6, Schmett. Eur. 4: 65.

cucu

Type-species: Bombyx glaucina Esper, I789, Die Schmett. 3 (Suppl. I) Abschnitt 3: II, pl. 8I, figs 4,5 , by subsequent designation under the plenary powers of Int. Commn zool. Nom., 1957, Opin. Decl. int. Commn zool. Nom. 17 Opinion 494: 267. Syntypes ô, O, Germany: Frankfurt am Main and other parts.

Placed on the Official List of Generic Names in Zoology: Name No. I234.

Under the Plenary Powers all designations of type-species for Episema made prior to Opinion 494, in 1957 , were set aside, including -

(I) Phalaena caeruleocephala Linnaeus, I758, - Pantheinae, cited by Duponchel, I829, in Godart \& Duponchel, Hist. nat. Lépid. Papillons Fr. 7(2): 7I. The genus Diloba Boisduval, I 840 , is now used for this concept.

(2) Noctua cincta Fabricius, I787, an unjustified emendation of Noctua i-cinctum [Denis \& Schiffermüller], I775, - Hadeninae, cited by Duponchel, I845, in d'Orbigny, Dict. univl Hist. nat. 5: 367 . The genus Perigrapha Lederer, I857, is now used for this concept.

(3) Bombyx trimacula [Denis \& Schiffermüller], I775, - Cuculliinae cited by Guenée, I 852, in Boisduval \& Guenée, Hist. nat. Insectes (Lépid.) 5: I74. B. trimacula is a junior subjective synonym of Noctua tersa [Denis \& Schiffermüller], I775, and is congeneric with $B$. glaucina, the type-species of Episema Ochsenheimer. 
†EMPISEME; Freyer, 1848, Nenere Beitr. Schmett. 6: 56 .

An incorrect subsequent spelling.

$\ddagger E P I S I L I A$, misspelling. See Epipsitia Hübner, [1821].

NOCT

EPISPARINA Berio, I964, Doriana 4(I5I): 2.

OPHI

Type-species: Episparis hieroglyphica Holland, I894, Psyche, Camb. 7: I 20, pl. 4, fig. 9, by original designation, but cited as łhyeroglyphica, an incorrect subsequent spelling. Syntypes ô, 7 , [Gabon]: Kangwé. [Equatorial Gunnea]: [Rio Muni], Benita. (CM, Pittsburgh).

EPISPARIS Walker, [1857] 1856, List Specimens lepid. Insects Colln Br. Mus. 10: + I3, 475. OPHI Type-species: Episparis penetrata Valker, [1 857] 1856, ibidem 10: 476, by monotypy. Syntypes I $\hat{0}$, I q, AFrICA (BMNH).

EPISPARONIA Berio, 1964, Doriana 4(151): 2.

OPHI

Type-species: Episparis angulatilinea Bethune-Balier, 1906, Novit. zool. 13: 268, by original designation. Holotype $\hat{o}$. New Gunna: [Papua], Dinawa (BMNH).

EPISTEME Hübner, [1820] I816, V'orz. bekannter. Schmett.: I 80.

AGAR

Type-species: Phalaena lectrix Linnaeus, 1764, Mus. Ludovicae Ulricae: 389 , by monotypy. Type(s), ChrNa (IZUU, Uppsala).

EUSEMIA Dalman, 1825, Prodromus. Monogr. Castniae: 26.

Type-species: Phalaenae lectrix Linnaeus, I 764 , by monotypy.

Eusemia has been incorrectly attributed to Westwood, I84I, in Jardine, Naturalist's Libr. (Edn I) 33: 86, 88.

Eusemia is a junior objective synonym of Episteme Hübner, [1820].

†EMESIA; Waterhouse, [1885], Aid Ident. Insects 2: I 7, pl. I 55, figs 3, 4.

An incorrect subsequent spelling.

EPISTONA Möschler, I 884, T'erh.zool.-bot. Ges. Wien 33 (Abh.) : 302.

CATO

Type-species: Epistona limula MIöschler, I884, ibidem 33 (Abh.): 303, pl. I6, fig. I7, by monotypy. Syntypes $\hat{\sigma}$, 옹, [South AFriCa] : Kaffernlandes.

EPIS TREMA Schaus, I9I3, Ann. Mag. nat. Hist. (8)11: 40.

ACON

Type-species: Epistrema ora Schaus, I9I3, ibidem (S) 11:40, by original designation. Type(s) ô, Costa Rica: Carillo.

EPIT A USA Walker, [1857] I 856, List Specimens lepid. Insects Colln Br. Mus. 10: 4I 3, 476. opH I Type-species: Epitausa laetabilis Walker, [1857] I856, ibidem 10: 477, by monotypy. Holotype $\widehat{\sigma}$, [Dominican Republic] St. Domingo (BMNH).

EPITOMIPTERA Kaye, 1923, Proc. zool. Soc. Lond. 1922: 994.

HYPE

Type-species: Epitomiptera purpurascens Kaye, 1923, ibidem 1922: 994, pl. I, figs 4 \& 23 , by PRESENT DESIGNATION. Type(s), TRINIDAD (BMNH).

EPITRIPTA Turner, 1902, Proc. Limn. Soc. N.S.W. 27: I23. HYPE

Type-species: Epitripta acosmopis Turner, 1902, ibinlem 27: 1 23, by monotypy. Type(s) ㅇ. Australia.

EPIZEUC'TIS Meyrick, 1889, Trans. ent. Soc. Lond. 1889: 463.

NOLI

Type-species: Nola innocuc Butler, i 88o, Proc. zool. Soc. Lond. 1880: 671, by monotypy. Holotype, [Taiwan] Formosa: Takow (BMNH).

EPIZEUXIS Hübncr, I 81 8, Zuträge Samml. exot. Schmett. 1: 9.

II Y PE

Type-species: Noctua calvaria [Denis \& Schiffermüller], I775, Ankündung syst. Werkes Schmett. Wienergegend: 7 I, 3r6, by subsequent designation by Grote, 1874, Bull. Buffalo Soc. nat. Sci. 2: 47 but cited as Pyralis calvarialis [Denis \& Schiffermüller], an unjustified emendation (proposed on page $3 \mathrm{I}(1)$ and the name originally included in Epizentis. 
Ty'pe(s), [Austria]: Vienna district (Collection destroyed, Horn \& Kahle, I936, Ent. Beih. Berl.-Dahlem 3: 243).

HELIA Duponchel, [1845], I844, Cat. méth. Lépid. Eur.: 2 I4.

Type-species: Noctua calvaria [Denis \& Schiffermüller], I775, by monotypy.

Helia was attributed to Guenée by Duponchel who by using it unintentionally made it nomenclaturally available prior to its proposal and generic description by Guenée, 1854, for the same concept.

A junior homonym of Helia Hübner, I818,-Lepid., Noctuidae, Ophiderinae. The objective replacement name is Epizenxis Hübner, I8I8.

HELIA Guenée, I854, in Boisduval \& Guenée, Hist. nat. Insectes (Lépid.) 8: 75.

Type-species: Noctrua calvaria [Denis \& Schiffermüller], I775, by original designation on page 76 , but cited and originally included in the genus as Pyralis calvarialis [Denis \& Schiffermüller], an unjustified emendation.

A junior homonym of Helia Hübner, 1818,-Lepid., Noctuidae, Ophiderinae. The objective replacement name is Epizenxis Hübner, I8I8.

EPOPSIMA Turner, 1920, Trans. Proc. R. Soc. S. Aust. 44: I58, I7 I. ACON

Type-species: Erastria fasciolata Butler, I886, Trans. ent. Soc. Lond. 1886: 403, by monotypy. Type(s), Australia: [Qd], Peak Downs (BMNH).

EPORECTIS Meyrick, 1902, Trans. ent. Soc. Lond. 35: 30.

OPHI

Type-species: Eporectis phenax Meyrick, I902, ibidem 35: 30, by monotypy. Holotype ô, Australia: Qd, Rosewood (BMNH).

EPUNDA Duponchel, [1845]. See Eumichtis Hübner, [I82 I].

CUCU

EQUATOSYPNA Berio, 1959, Annali Mus. civ. Stor. nat. Giacomo Doria 71: $304 . \quad$ cato

Type-species: Sypna equatorialis Holland, 1894, Psyche, Camb. 7: 69, by original designation. Type(s) o, West Africa.

ERAGISA Walker, I858, List Specimens lepid. Insects Colln Br. Mus. 15: I656. NotodontidaE Type-species: Eragisa lanifera Walker, 1858, ibidem 15: 1657, by monotypy.

Eragisa was originally placed in the Noctuidae.

ERANA Walker, I857. See Feredayia Kirkaldy, I910.

HADE

$\ddagger$ ERASTRIA Hübner, [I806]. See Timandra Duponchel, I829.

GEOMETRIDAE

${ }_{\ddagger}$ ERASTRIA Hübner, I808. See Hypagyrtis Hübner, I818.

GEOMETRIDAE

ERASTRIA Hübner, [1813], Samml. exot. Schmett. 1: pl. [203].

GEOMETRIDAE

Type-species: Erastria dissimilaria Hübner, [1813], ibidem 1 : pl. [203], by monotypy.

E. dissimilaria is a junior subjective synonym of Phalaena coloraria Fabricius, I798, Suppl. Ent. Syst.: 452 .

This Geometrid generic name is included here because Erastria has been incorrectly used in the Noctuidae, both as a generic name and as a family-group name, the Erastriinae, based on the usage by Ochsenheimer, I8I 6 .

ERASTRIA Ochsenheimer, I8I6. See Eustrotia Hübner, [I82I].

ACON

ERASTRIFACIES Dyar, 1925, Insecutor Inscit. menstr. 13: 4 .

HYPE

Type-species: Erastrifacies schedocala Dyar, I925, ibidem 13: 4, by monotypy. Holotype 9 , Mexico: Colima (USNM, Washington).

ERASTRIOPIS Hampson, I926, Descr. new Genera Species Lepid. Phalaenae Subfamily Noctuinae Br. Mus.: I 96.

OPHI

Type-species: Eudorea lativitta Moore, 1883, Proc. zool. Soc. Lond. 1883: 29, by original designation. Syntypes, [India]: [W. Bengal], Darjiling (BMNH). 
ERASTROIDES Hampson, I893, Illust. typical Specimens Lepid. Heterocera Colln Br. Mus. 9: $20,98$.

Type-species: Erastroides oliviaria Hampson, I893, ibidem 9: 20, 99, pl. 162, fig. 23, by monotypy. Type(s) +, CEYLON: Dickoya (B.INH).

ERCHEIA Walker, [1858] 1857, List Specimens lepid. Insects Colln Br. Mus. 13: 1078, I 107. CATo Type-species: Ercheia diversipennis Walker, [1 858] I 857, ibidem 13: I 108, by monotypy. Holotype , Ceylon (BMNH).

EREBOPHASMA Boursin, 1963, Forsch. Ber. Lands Nordrhein-Westfalen 1170: 73. NOCT

Type-species: Erebophasma haematina Boursin, 1963 , ibidem 1170: 75, by original designation. Holotype $\hat{\jmath}$, CHINA: Si-kang, Batang (MAK, Bonn).

EREBOSTROTA W'arren, I889, Trans. ent. Soc. Lond. 1889: 250.

OPHI

Typc-species: Erebostrota albocincta Warren, I 889, ibidem 1889:251, by monotypy. Holotype $\hat{o},[$ BraziL]: Amazon basin, R. Manhes, Laranjal (BMNH).

E. albocincta is a junior subjective synonym of Phalaena stenelea Stoll, 1780 , in Cramer, Uitlandsche Kapellen 4: 38, pl. 308, fig. B.

EMEA Walker, 1865, List Specimens lepid. Insects Colln Br. Mus. 33: 1099.

Type-species: Emea palpalis Walker, 1865, ibidem 33: I100, by monotypy. Type(s) o. [Colombia]: Bogota.

A junior homonym of Emea Leidy, I 850, Proc. Acad. nat. Sci. Philad.5(6): 125, - Vermes. There is no objective replacement name but $E$. palpalis is a senior subjective synonym of Evebostrota albocincta Warren, 1889, the type-species of Evebostrota Warren, 1889. The latter is therefore available for use as a subjective replacement name.

EREBOTHRIX Warren, I889, Trans, ent. Soc. Lond. 1889:241.

OPHI

Type-species: Erebothrix semiusta Warren, 1889, ibidem 1889: 242, by monotypy. Holotype ${ }^{\hat{D}},[$ BrazIL] : Amazon basin, R. Jurua, Lago Cerrada (BMNH).

EREBUS Latreille, I810, Considérations générales sur l'Ordre naturel des Animanx: 365. Cato

Type-species: Phalaena crepuscularis Linnacus, 1758, Syst. Nat. (Edn 10) 1: 509, by original designation on page 44I. Type(s), TYPE-LOCALITY stated to be America but this was probably an error as this species is from the EAST INDIES and is not known to occur in the New World (IZUU, Uppsala).

The type-species designations of Latreille, r8Io, were accepted by Int. Commn zool. Nom., 1939, Opin. Decl. int. Commn zool. Nom. 2 Opinion 136: 15, for those genera in which Latreille in his 'Table des genres avec l'indication de l'espèce qui leur sert de type' cited only one of the species included in the genus by the original author. This was so in the case of Erebus.

B Y'AS Billberg, I 820 , Emumeratio Insect. Mus. G. J. Billberg: 85 .

Type-species: Phalaena crepuscularis Linnaeus, 1758 , by subsequent designation by Berio, I966, Annali Mus. civ. Stor. nat. Giacomo Doria 76: 51 .

Byas is a junior objective synonym of Evebus Latreille, I 8 Io.

NYCTIPAO Hübner, [1823] 1816, Verz. bekannter Schmett.: 272.

Type-species: Phalaena crepuscularis Linnaeus, 1758 , by subsequent designation by Desmarest, (1857), in Chenu, Encyl. Hist. nat. Papillons nocturnes: r 30.

Ayctipao is a junior objective synonym of Evebus Latreille, I8Io.

†NYCTIPAON; Hübner, [1826] 1816, Verz. bekannter Schmett. Alizeiger: 38.

An incorrect subsequent spelling.

EREMAULA Turner, 1942, Proc. R. Soc. Qd 53: 74.

ACRO

Type-species: Eremaula ptilopleura Turner, 1942, ibidem 53: 74, by monotypy. Holotype o, [Australia]: Qd, Cunnamulla (ANIC, Canberra).

E. ptilopleura is a junior subjective synonym of Crambodes minor Butler, I886, Trans. cnt. Soc. Lond. 1886: 397. 
EREMNOPHANES Turner, 1944, Trans. R. Soc. S. Aust. 68: I 2.

Type-species: Eremnophanes apicinota Turner, I944, ibidem 68: I2, by monotypy. Syntypes 2 , Australia: Qd, Cunnamulla (ANIC, Canberra).

EREMOBATES Turner, 1939. See Tabomeeres nom. n.

$\mathrm{ACON}$

EREMOBIA Stephens, I 829 [June], Nom. Br. Insects : 42.

ACRO

Type-species: Noctua ochroleuca [Denis \& Schiffermüller], I775, Ankündung syst. Werkes Schmett. Wienergegend: 87, by monotypy. Type(s), [Austria]: Vienna district (Collection destroyed, Horn \& Kahle, I936, Ent. Beih. Berl.-Dahlem 3: 243).

Evemobia was also proposed by Stephens, I 829 September, Illust. Br. Ent. Haustellata 3: 94

EREMOBINA McDunnough, 1937, Can. Ent. 69: 60.

ACRO

Type-species: Hadena claudens Walker, I857, List Specimens Lepid. Insects Colln Br. Mus. 11: 753, by original designation. Holotype ô, [CANADA]: Newfoundland (BMNH).

EREMOCHLAENA Boursin, I953, Z. wien. ent. Ges. 38: 240.

CUCU

Type-species: Episema orana Lucas, I849, Explor. scient. Algérie Anim. articulés 3: 384 , pl. 3, fig. 7, by original designation. Syntypes, Algeria: western.

EREMOCHROA Meyrick, I897, Trans. ent. Soc. Lond. 1897: 368.

ACRO

Type-species: Eremochroa psammias Meyrick, I 897, ibidem 1897:368, by original designation. Syntypes $5(\hat{0}$, $q)$, Australia: W. Austr., Carnarvon (BMNH).

EREMODRINA Boursin, I937, Ent. Rdsch. 54: 422.

ACRO

Type-species: Caradrina vicina Staudinger, 1870, Berl. ent. Z. 14: i 8, by original designation. Syntypes 30 , I ․, [U.S.S.R.]: [Krasnoarmeysk] Sarepta (MNHU, Berlin).

Evemodrina was proposed as a subgenus of Elaphria Hübner, I8I8, but has since been placed as a subgenus of Caradrina Ochsenheimer, I8I6.

EREMONOMA Warren, I913, in Seitz, Gross-Schmett Erde 3: 394.

OPHI

Type-species: Palpangula straminea Bang-Haas, I906, Dt. ent. Z. Iris 19: I35, pl. 5, fig. II, by original designation. Syntypes 0 , $q$, Tunisia: Gafsa (MNHU, Berlin); Foum-Tatahouine.

The date of publication of the part of Seitz containing page 394 , was stated on page 389 as i913 December ist in both the English and German editions.

EREMOPHYSA Boursin, 1958, Bull. mens. Soc. linn. Lyon 27: 4.

ACRO

Type-species: Sidemia apotheina Brandt, I938, Ent. Rdsch. 55: 522, figs 82-84, by original designation. Syntypes ô. IRAN: Keredj (NR, Stockholm).

EREMOPOLA Warren, I9II, in Seitz, Gross-Schmett. Erde 3: I8I.

CUCU

Type-species: Phoebophilus lenis Staudinger, I892, Dt. ent. Z. Iris 4: 279, by original designation. Holotype $\hat{\sigma}$, [ISRAEL]: Jerusalem (MNHU, Berlin).

The date of publication of the part of Seitz containing page I8I was stated on page I8I as I9I I February 8th in both the English and German editions.

See also Libyana Turati, I924.

ERICEIA Walker, [1858] I 857, List Specimens lepid. Insects Colln Br. Mus. 13: 1078, I089. opH

Type-species: Ericeia sobria Walker, [I 858] I857, ibidem 13: Io89, by monotypy. Holotype ô, [Australia]: [Qd], Moreton Bay (BMNH).

ERICHILA Billberg, I820. See Hypena Schrank, I802.

HYPE

ERIOCERA Guenée, I 852, in Boisduval \& Guenée, Hist. nat. Insectes (Lépid.) 6: 40 I. OPHI

Type-species: Eriocera mitrula Guenée, I852, ibidem 6: 402, pl. I I, fig. 7, by monotypy. Lectotype ô. TyPE-LOCAlity 'Sans indication de patrie; mais je la crois américaine' (MNHN, Paris), designated by Viette, I95 I, Bull. mens. Soc. linn. Lyon 20: I6I. [No other specimens known to me]. 
ERIOPUS Treitschke, I825. See Callopistria Hübner, [1821].

ERIOPYGA Guenée, I 852, in Boisduval \& Guenée, Hist. nat. Insectes (Lépid.) 5: 203. HADE Type-species: Eriopyga punctulum Guenée, I 852, ibidem 5: 203, by monotypy. Syntypes $30^{*}$. BrazIL: Nouvelle-Fribourg (BMNH).

ERIOPYGODES Hampson, 1905, Cat. Lepid. Phalaenae Br. Mus. 5: xii, 353. HAdE Type-species: Noctua imbecilla Fabricius, I 794, Ent. Syst. 3(2): i I 3, by original desigtion. Type(s), [Germany]: [Kiel] IKilia (Type(s) not found by Zimsen, I964, Type Material of I. C. Fabricius: 570 ).

ERIOSCELE Möschler, I880, Verh. zool.-bot. Ges. Wien 30 (Abh.) : 38 .

Type-species: Erioscele rureoides Möschler, I880, ibidem monotypy. Holotype ô. Surram: interior (MNHU, Berlin).

ACRO

30 (Abh.): 385, by

ERIZADA Walker, I865, List Specimens lepid. Insects Colln Br. Mus. 32: 506.

CHLO

Type-species: Erizada lichenaria Walker, I865, ibidem 32: 507, by monotypy. Holotype ô. Java (BMNH).

E. lichenaria is a junior subjective synonym of Gadivtha semifervens Walker, [1863] I864, J. Proc. Linn. Soc. (Zool.) 7: 163.

See also Rhizana Walker, 1865, List ... 33: 719; and Tinosoma Hampson, I894.

ERNA Strand, I9I 5, Ent. Mitt. 4: I66.

HYPE

Type-species: Erna cara Strand, I9I 5, ibidem 4: I67, fig. 4, by original designation.

Type(s) ô. Cameroun: Bonaberi (DEI, Eberswalde).

EROCHA Walker, I854, List Specimens lepid. Insects Colln Br. Mus. 2: 3 I 8.

AGAR

Type-species: Dioptis discreta Walker, I854, ibidem 2:319, by monotypy. Holotype ô. [BRazIL]: R. Amazon, [Tefé] Ega (BMNH).

D. discreta is a junior subjective synonym of Phalaena mummia Cramer, I779, Uitlandsche Kapellen 3: 6I, pl. 228, fig. C.

Erocha was proposed as a subgenus of Dioptis Hübner, I8 8 8, - Lepid., Dioptidae.

EROMENE Hübner, [I82I] i 8I6, Verz. bekannter Schmett.: 256.

ACON

Type-species: Noctua ostrina Hübner, [I8o8], Samml. eur. Schmett 4: pl. 85, fig. 399, by subsequent designation by Hampson, 1910, Cat. Lepid. Phalaenae Br. Mus. 10: 63. Type(s), Europe.

EROMIDIA Schaus, I914, Proc. U.S. natn. Mus. 46: 520.

OPHI

Type-species: Eromidia clotho Schaus, I9I4, ibidem 46:52I, by original designation.

Type(s) $\hat{o}$, [FREnch Guiana]: R. Maroni, St. Laurent (USNM, Washington).

EROSIA Guenée, I 857, in Boisduval \& Guenée, Hist. nat. Insectes (Lépid.) $10: 34$. URANIIDAE

Type-species: Erosia incendiata Guenée, I857, ibidem $10: 35$, pl. 8, fig. 4, by PRESENT DESIGNATION.

Erosia has been used as the original generic name for species which are now in the Noctuidae.

$\ddagger$ EROTYLA Hübner, [1806]. See Emmelia Hübner, [1821]. ACON

EROTYLA Hübner, I822. See Emmelia Hübner, [1821]. ACON

$\ddagger \boldsymbol{E R O T Y L A E}$ Ochsenheimer, I8I6. See Eustrotia Hübner, [I82r]. ACON

$\ddagger$ ERPYZON Hübner, [1806]. See Pechipogo Hübner, [18 25]. HYPE

$\ddagger$ ERPYZON Hübner, 1808. See Megachyta Grote, I873. HYPE

Erpyzon has not been used again in zoology. A similar name Herpyzon Hübner, I822, was subsequently proposed. 
ERSCHOVIELLA Obraztsov, 1953, Eos, Madr. 29: I68.

SARR

Type-species: Sarrothripa musculana Ershov, 1874, in Fedchenko, Reise in Turkestan (2) 5 (3): 3I, pl. 2, fig. 27, by original designation, but proposed as †Sarothripa, an incorrect subsequent spelling. Type(s) 우 [U.S.S.R.]: Turkestan, Sarafschan valley near Iori.

ERYGANSA Bethune-Baker, 1906, Novit. zool. 13: 246.

OPHI

Type-species: Erygansa kebea Bethune-Baker, 1906, ibidem 13: 246, by original designation. Type(s) $\hat{\sigma}$. New Guinea: [Papua], Kebea Range (BMNH).

E. kebea is a junior subjective synonym of Briarda precedens Walker, [1858] I857.

See also Briarda Walker, [1858] I857; and Ansa Walker, I858.

ERYGIA Guenée, I852, in Boisduval \& Guenée, Hist. nat. Insectes (Lépid.) 7: 49.

Type-species: Erygia apicalis Guenée, 1852, ibidem 7:50, by monotypy. Holotype o. East Indies (BMNH).

See also Calicula Walker, 1858 .

ERYMELLA Walker, [r867] r868, J. Linn. Soc. (Zool.) 9: r89.

OPHI

Type-species: Erymella hieroglyphica Walker, [1867] 1868, ibidem 9: 190, by monotypy. Type(s), [Colombia]: Bogota (BMNH).

ERYSTHIA Walker, I862, Trans. ent. Soc. Lond. (3) 1: 93.

EUTE

Type-species: Erysthia obliquata Walker, I862, ibidem (3)1: 94, by monotypy. Type(s) , TyPE-LOCALITy not known (Type(s) not found in UM, Oxford or in BMNH). [No specimens in UM, Oxford or in BMNH.]

ERYTHROCHRUS Herrich-Schäffer, 1855, Samml. neuer oder wenig bekannter aussereur. Schmett. 1(I): wrapper, pl. 70. figs 397, 398; 1858, ibidem 1: 75, 82. HYBLAEIDAE

Type-species: Erythrochrus bicolor Herrich-Schäffer, r855, ibidem 1(r): wrapper, pl. 70, figs 397,398 ; I 858, ibidem 1: 75, 82, by monotypy. Type(s), [LEEWARd Is]: Martinique I. Erythrochrus and bicolor are both dated from the wrapper of the plates.

See also Torone Walker, 1863.

ER YTHROECIA Hampson, 1908, Cat. Lepid. Phalaenae Br. Mus. 7: ro. Available, but without included species until Hampson, I910, ibidem 9: vii, 57.

NOCT

Type-species: Heliothis suavis Edwards, I884, Papilio 4: 45, by subsequent designation by Hampson, rgro, ibidem 9: 57. Syntypes, [U.S.A.]: New Mexico, Las Vegas, near Hot Springs.

ERYTHROPHAEA Rebel, I901. See Erythrophaia Staudinger, I892.

NOCT

ERYTHROPHAIA Staudinger, I892, Dt. ent. Z. Iris 4: 316.

NOCT

Type-species: Erythrophaia eudoxia Staudinger, I892, ibidem 4:316, pl. 4, fig. 9, by monotypy. Syntypes $\hat{\sigma}^{*}$, + , [CHINA]: Kaschgar district, Kysil Jart (MNHU, Berlin).

ERYTHROPHAEA Rebel, I9or, in Staudinger and Rebel, Cat. Lepid. palaearct. Faunengeb.

1: 224 .

An unjustified emendation of Erythrophaia Staudinger, I892.

ERYTHROPLUSIA Ichinose, I962, Konty $\hat{\imath} 30: 249$.

PLUS

Type-species: Plusia rutilifrons Walker, I858, List Specimens lepid. Insects Colln Br. Mus. 15: $\mathrm{7}_{785}$, by original designation. Holotype ${ }^{\circ}$. CHina: northern (BMNH).

Erythroplutsia Ichinose was originally proposed as a subgenus of Autographa Hübner, [182 $\mathrm{I}]$.

ERYTHROTIS Bryk, I949, Ark. Zool. 41(A) I : 78.

HADE

Type-species: Erythrotis cedermarki Bryk, 1949, ibidem 41(A)x: 78, by original designation. Holotype $q$, Korea: Shuotsu (NR, Stockholm). 
ESCANDIA Dyar, I9I 4, Proc. U.S. natn. Mus. 47: 297.

ACON

Type-species: Escandia fimbrialis Dyar, I914, ibidem 47:297, by originaldesignation. Holotype ㅇ, Panama: Canal Zone, Corozal (USNM, Washington).

ESCARIA Grote, I882, Papilio 2: I86.

ACRO

Type-species: Escaria clauda Grote, I882, ibidem 2: 186, by monotypy. Syntypes $4(\widehat{\jmath}$, P), [U.S.A.]: Arizona.

ESCUA Walker, I 858, List Specimens lepid. Insects Colln Br. Mus. 15: I85I.

OPHI

Type-species: Escua extollens Walker, I 858, ibidem 15: I 85 I, by monotypy. Type(s)

․, [BRazIL]: Rio Janeiro (UM, Oxford).

$\ddagger E X U A$; Schaus, I9I I, Ann. Mag. nat. Hist. (8) 8: 227.

An incorrect subsequent spelling of Escua Walker, I858, not of Eఒxoa Hübner, [I82I], as cited by Neave, I939, Nomencl. zool. 2: 393.

ESSONISTIS Meyrick, I902, Trans. ent. Soc. Lond. 35: 36.

$A C O N$

Type-species: Essonistis micraeola Meyrick, 1902, ibidem 35: 36, by monotypy. Syntypes 2 \&, Australia: Qd, Brisbane.

ESTAGROTIS nom. n. for Setagrotis Warren, I9I2.

NOCT

Type-species: Gortyna cuprea Moore, I867, Proc. zool. Soc. Lond. 1867: 50, pl. 6, fig. 8, by original designation (for Setagrotis Warren). Syntypes ô, India: [W.] Bengal, Darjeeling (MNHU, Berlin).

SET AGROTIS Warren, 1912, in Seitz, Gross-Schmett. Erde 11: 66.

Type-species: Gortyna cuprea Moore, 1867, by original designation.

The date of publication of the part of Seitz containing page 66 was stated on page 65 as I9I 2 September $25^{\text {th }}$ in both the English and the German editions.

A junior homonym of Setagrotis Smith, i89o, Bull. U.S. natn. Mus. 38: 59,-Lepid., Noctuidae. The objective replacement name is Estagrotis nom. n.

ESTEPARIA Fernández, I93I, Eos, Madr. 7: 2 I I.

ACRO

Type-species: Esteparia agenjoi Fernández, I93I, ibidem 7: 2I I, pl. 2, fig. I, by original designation, but cited as +'E. ambrosiana nov.sp.', a nomen nudum and error which was corrected by an errata-slip in vol. 7 part 3. Type(s) ठૈ. Spain: Burgos, Estépar.

ESTHLODORA Tumer, igo2, Proc. Limn. Soc. N.S.W. 27: 1зо.

HYPE

Type-species: Esthlodora versicolor Turner, 1902, ibidem 27: I30, by monotypy. Syntypes, Australia: I ठే, Qd, Brisbane (ANIC, Canberra); 2 ㅇ, Brisbane.

ESTIMAJA Kozhanchikov, 1937. See Estimata Kozhanchikov, 1928.

NOCT

ESTIMATA Kozhanchiliov, 1928, Revue russe Ent. 22: 94.

NOCT

Type-species: Agrotis herrichschaefferi Alphéraky, 1895, Dt. ent. Z. Iris 8: I91, by original designation, but cited by Kozhanchikov as ‘'E. herrich-schäfferi Stgr.', an incorrect subsequent spelling and authorship. Syntypes ô., [U.S.S.R.]: [Mongolia], Ourga (ZI, Leningrad).

A. herrichschaefferi was originally proposed as $\ddagger A$. Herrich-Schaefferi, an incorrect original spelling under the Code, Articles 28 and 32 (c)(i).

ESTIMAJA Kozhanchikov, I937, Fanna SSSR (Insecta, Lepid.) 13(3) : 220, 6I4.

An unjustified emendation of Estimata Kozhanchikov, 1928.

ETANNA Walker, I 862, J. Proc. Linn. Soc. (Zool.) 6: I I8.

SARR

Type-species: Etanna basalis Walker, 1862, ibidem 6: II9, by original designation. Lectotype 9. Borneo: Sarawak (UM, Oxford), designated (as type) by Siwinhoe, I90o, Cat. east. and Aust. Lepid. Heterocera 2: 78.

${ }_{\ddagger}$ ETHIOMINIA Hampson, no published reference found.

HYPE 
ETHIONODES Hampson, I918, Novit. zool. 25: I46.

ACRO

Type-species: Xanthograpta brunneaplaga Bethune-Baker, I9I I, Ann. Mag. nat. Hist. (8) 8: 523, by original designation. Holotype $\hat{\sigma},[$ Angola] Port W. Africa: Gunnal (BMNH).

ETHIOPICA Hampson, I908, Cat. Lepid. Phalaenae Br. Mus. 7: I I. Available, but without included species until Hampson, I909, ibidem 8: xii, 4I8.

ACRO

Type-species: Charidea vinosa Hampson, 1902, Ann. S. Afr. Mus. 2: 292, by subsequent designation by Hampson, I909, ibidem 8: 4 I8. Type(s) ô., [Rhodesia]: Mashonaland, Salisbury (BMNH).

ETHIOTERPIA Hampson, I9Io, Cat. Lepid. Phalaenae Br. Mus. 9: I, II2.

ACR O

Type-species: Ethioterpia neavi Hampson, I9Io, ibidem 9: II2, fig. 3I, by original designation. Holotype ô, [Zambra] N. Rhodesia: Tanganyika Plateau, Lope Valley $(\mathrm{BMNH})$.

EUAETHIOPS Hampson, 1926, Descr. new Genera Species Lepid. Phalaenae Subfanily Noctuinae Br. Mus.: 487. OPHI

Type-species: Selenis limbata Holland, I894, Psyche, Camb. 7: I12, pl. 5, fig. 7, by original designation. Type(s) + , West AfRICA.

EUAGROTIS McDunnough [1929] 1928, Bull. Dep. Mines, Can. 55: $43 . \quad$ Noct

Type-species: Noctua lubricans Guenée, 1852 , in Boisduval \& Guenée, Hist. nat. Insectes (Lépid.) 5: 323, pl. 5, fig. 7, by original designation. Syntypes ô. ․, [U.S.A.]: Florida (BMNH).

EUALABAMA Grote, I896. See Alabama Grote, I895.

OPHI

EUAMIANA Barnes \& Benjamin, I927, Insecutor Inscit. menstr. 14: I82.

ACRO

Type-species: Perigea contrasta Barnes \& McDunnough, I91о, Jl N.Y. ent. Soc. 18: I 54, by original designation. Holotype ${ }^{+},[$U.S.A.]: Ariz., Redington.

EUAONTIA Barnes \& McDunnough, I9Io, Jl N.Y. ent. Soc. 18: I59.

ACON

Type-species: Euaontia semirufa Barnes \& McDunnough, I910, ibidem I8: I 59, by original designation. Holotype $\sigma^{*}$, [U.S.A.]: Nevada, Esmeralda Co.

EUBLARGINEA Berio, I966, Annali Mus. civ. Stor. nat. Giacomo Doria 76: I21.

ACON

Type-species: Eublarginea argentifera Berio, I966, ibidem 76: I22, fig. I3, by original designation. Holotype $\widehat{\sigma}$, MAdagascar: E., env. de Perinet, Forêt d'Analamazoatra (MNHN, Paris).

EUBLEMMA Hübner, [I82I] I8I6, Verz. bekannter Schmett.: 256.

$\operatorname{ACON}$

Type-species: Noctua amoena Hübner, [I803] Samml eur. Schmett. 4: pl. 6r, fig. 300, by subsequent designation by Moore, I884, Lepid. Ceylon 3: 54. Type(s), Europe.

N. amoena is a junior subjective synonym of Phalaena respersa Hübner, I790, Beitr. Gesch. Schmett. 2(r): I4, pl. 2, fig. H.

EUBLEMMARA Bethune-Baker, I9I I, Ann. Mag. nat. Hist. (8)8: $52 \mathrm{I}$.

HYPE

Type-species: Eublemmara tandoana Bethune-Baker, I9I I, ibidem (8)8: 522, by original designation. Syntypes $\hat{\jmath}$, Angola: N'Dalla Tando (BMNH).

EUBLEMMISTIS Hampson, I902, Ann. S. Afr. Mus. 2: 406.

$\operatorname{ACON}$

Type-species: Eublemmistis chlorozonea Hampson, 1902, ibidem 2: 406, by monotypy. Holotype , South Africa: Natal, Durban (BMNH).

EUBLEMMOIDES Bethune-Baker, I906, Novit. zool. 13: 212.

ACON

Type-species: Eublemmoides dinawa Bethune-Baker, I906, ibidem 13: 212, by original designation. Type(s) ô, New Guinea: [Papua], Dinawa (BMNH). 
EUBOLINA Harvey, I875, Bull. Buffalo Soc. nat. Sci. 2: 280.

Type-species: Eubolina impartialis Harvey, I875, ibidem 2: 28I, by monotypy. Type(s), [U.S.A.]: Texas (BMNH).

EUBR YOPTERELLA Roepke, I938, Bull. Mus, r. Hist. nat. Belg. 14( ( 3$): 56 . \quad$ OPH I

Type-species: Eubryopterella vaneeckei Roeple, I938, ibidem 14(13): 56, by monotypy. Holotype ô, [SulawesI]: N. Celebes (IRSNB, Brussels).

EUBUCHHOLZIA Barnes \& Benjamin, 1929. See Benjaminiola Strand, I928. ACro

EUCALIMIA Hübner, [I82I] I8I6, Verz. bekannter Schmett.: $2+5$.

Type-species: Noctua gnaphalii Hübner, [1813], Samml. eur. Schmett. 4: pl. I26, figs 582,583 , by subsequent designation by Hampson, 1906, Cat. Lepid. Phalaenae Br. Mus. 6: 14. Type(s), Europe.

$\ddagger$ †UCALYPTA, misspelling. See Encalypta Möschler, I89o.

SARR

EUCALYPTRA Morrison, 1875, Ann. Lyceum nat. Hist. 11: 103.

OPH I

Type-species: Eucalyptra bipuncta Morrison, 1875, ibidem 11: 104, by monotypy. Type(s), [U.S.A.]: Massachusetts.

EUCAMPIMA Wileman \& South, I92I, Entomologist 54:202. OPH I

Type-species: Eucampima griseisigna Wileman \& South, I92 I, ibidem 54: 202, by monotypy. Syntypes $2 \hat{0}$. Philippines: Luzon I., Benguet, Palali (BMNH).

EUCAMPIMA Hampson, 1926, Descr. new Genera Species Lepid. Phalaenae Subfamily Noctuinae Br. Mus.: $54^{2}$.

Type-species: Zethes coenotype Hampson, I910, Proc. zool. Soc. Lond. 1910: 447, pl. 38 , fig. 8 , by original designation. Holotype $\hat{\sigma}$. [ZA.MBIA] Rhodesia: N.E., Chinsali district (BMNH).

A junior homonym of Eucampima Wileman \& South, I92I. This generic name probably originated from Hampson, but was used and unintentionally made nomenclaturally available by Wileman \& South prior to its proposil and generic description by Hampson for a similar concept but having a different type-species. There is no objective replacement name but $Z$. coenotype is probably congeneric with $E$. griseisigna and no replacement name should be proposed until the complex of species and related genera have been studied.

EUCAMPIMA Hampson, I926. See Eucampina Wileman \& South, I92I. OPHI

EUCAPNODES Holland, I894, Psyche, Camb. 7: I Io. OPH I

Type-species: Capnodes sexmaculata Walker, I865, List Specimens lepid. Insects Colln Br. Mus. 33: 10 75 , by monotypy. Holotype ô. Sierra Leone (BMNH).

C. sexmaculata is a junior subjective synonym of Macella euritiusalis Walker, i 858.

See also Macella Walker, [1859].

EUCARTA Lederer, I857, Noctuinen Eur.: 4I, I68.

ACRO

Type-species: Noctua amethystina Hübner, [1803], Samml. eur. Schmett. 4: pl. I4, fig. 69, by subsequent designation (for Placodes Boisduval) by Blanchard, 1840 , in Castelnau, Hist. nat. Anim. articulés (Insectes) 3: 508. Type(s), Europe.

Eucarta was proposed as the objective replacement name for Placodes Boisduval, i840. PLACODES Boisduval, 1840, Genera Index meth. Eur. Lepid.: I29.

Type-species: Noctua amethystina Hübner, [1803], by subsequent designation by Blanchard, 18 40 , in Castelnau, Hist. nat. Anim. articulés (Insectes) 3: 508 .

A junior homonym of Placodes Erichson, I 834, in Klug, Jb. Insectenkunde 1: 86, 103, Coleoptera. The objective replacement name is Eucarta Leclerer, 1857.

TELESILLA Herrich-Schäffer, i 856, Syst. Bearb. Schmett. Eur. 6 1ndex univl.: 47.

Telesilla was proposed as the objective replacement name for Placodes Boisduval, 1840 . Unfortunately it was a junior homonym of Telesilla Reichenbach, I853, J. Orn. Lpz. 1 Extraheft Beil. (Aufz. Colibris): 5,-Ares. As an addecl complication the name was 
spelled as Telesiella on page I3. From this multiple original spelling Cabanis \& Heine, I86o, Mus. Orn. Heinearm 3: 27 selected Telesilla and listed Telesiella in synonymy. Simon, I92 I, Hist. nat. Trochilidae (Synopsis et Cat.): 272 used Telesiella and listed Telesilla as an emendation by Cabanis \& Heine. Neither of these multiple original spellings are now in use for humming birds as they are junior subjective synonyms. I can find no reference to these spellings between 1853 and I860 so accept the selection of Cabanis \& Heine as first revisers under the Code, Article 32 (b).

EUCATEPHIA Hampson, 1926, Descr. new Genera Species Lepid. Phalaenae Subfamily Noctuinae Br. Mus.: 62 .

OPHI

Type-species: Catephia dinawa Bethune-Baker, I906, Novit. zool. 13: 252, by original designation. Holotype 3 , New Guinea: [Papua], Dinawa (BMNH).

EUCHALCIA Hübner, [1821] I8I6, Verz. bekannter Schmett.: 250.

PLUS

Type-species: Noctua illustris Fabricius, I787, Mantissa Insect. 2: 164, by subsequent designation by Dyar, I902, Jl N.Y. ent. Soc. 10:81. Type(s), Germany: southern (UZM, Copenhagen).

$N$. illustris is a junior subjective synonym of Noctua variabilis Piller \& Mitterpacher, I783, Iter per Poseganam Sclavoniae Provinciam: 70, pl. 6, fig. 3 .

EUCHORISTEA Warren, I9Io, in Seitz, Macrolepid. World 3: 93.

HADE

Type-species: Apamea limbata Butler, i 879, Ann. Mag. nat. Hist. (5)4: 360, by original designation. Type(s), JAPAN (BMNH).

The date of publication of the part of Seitz containing page 93, was stated on page 93 as I9Io February Ist in the English edition and I9Io April 25th in the German edition.

EUCHROMALIA Schaus, I916, Proc. U.S. natn. Mus. 50:260, $277 . \quad$ орн I

Type-species: Tarache violetta Schaus, 1904, Trans. Am. ent. Soc. 30: 159, by original designation. Type(s), BrazIl: S.E., São Paulo.

EUCIRROEDIA Grote, I875, Can. Ent. 7 : 205.

CUCU

Type-species: Cirroedia pampina Guenée, 1852 , in Boisduval \& Guenée, Hist. nat. Insectes (Lépid) 5: 402, pl. 7, fig. 2, by monotypy. Syntypes 30․ I q, AMERıCA: northern (BMNH). [U.S.A.]: New York; Baltimore.

EUCLADODES Fletcher, I961, Ruwenzori Exped. I952 1: 187.

HADE

Type-species: Cladocerotis oeneus Fawcett, 1918, Proc. zool. Soc. Lond. 1917: 236, pl. I, fig. I2, by original designation. Syntypes $2 \hat{0}$, I 우, [Kenya] Br. E. Africa: Meru $(\mathrm{BMNH})$.

${ }_{\ddagger}$ EUCLIDESIS Hampson, no published reference found.

HYPE

$\ddagger$ EUCLIDIA Hübner, [1806]. See Euclidia Ochsenheimer, I8I6.

CATO

$\ddagger$ EUCLIDIA Hübner, 1808. See Schinia Hübner, I818.

NOCT

EUCLIDIA Ochsenheimer, I816, Schmett. Eur. 4: 96.

CATO

Type-species: Phalaena glyphica Linnaeus, i758, Syst. Nat. (Edn 10) 1: 510, by subsequent designation by Duponchel, 1829, in Godart \& Duponchel, Hist. nat. Lépid. Papillons Fr. 7(2): 72. Type(s), TyPe-Locality not stated (LS, London) [Europe].

†UCLIDIA Hübner, [1806], Tentamen determinationis digestionis . . . : [2].

Included in a work rejected for nomenclatural purposes by Int. Commn zool. Nom., 1926, Smithson. misc. Collns 73(4) Opinion 97: I9. Also idem, I954, Opin. Decl. int. Commn zool. Nom. 6 Opinion 278: I 40.

Only included species: Phalaena glyphica Linnaeus, 1758.

ECT YPA Billberg, I820, Enumeratio Insect. Mus. G. J. Billberg: 86.

Type-species: Phalaena glyphica Linnaeus, I 758 , by subsequent designation by Tams, 1939, Entomologist 72: 138 .

Ectypa is a junior objective symonym of Euclidia Ochsenheimer, I8I6. 
EUCLIDIMERA Hampson, I9I3. See Callistege Hübner, [1823].

EUCLIDINA McDunnough, I937, Can. Ent. 69: 66.

CATO

Type-species: Drasteria cuspidea Hübner, I8I8, Zuträge Samml. exot. Schmett. 1: I6, figs 69, 70 , by original designation. Type(s) ㅇ. [U.S.A.]: Georgia [formerly] in Florida.

EUCLIDISEMA Hampson, I9I3. See Chalciope Hübner, [I823]. CATo

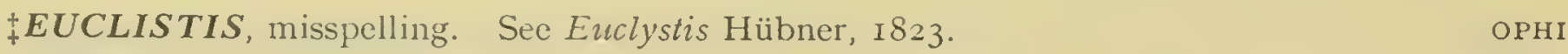

EUCLYSTIS Hübner, I823, Zuträge Samml. exot. Schmett. 2: $20 . \quad$ орHI

Type-species: Euclystis centurialis Hübner, I823, ibidem 2: 20, figs 295, 296, by subsequent designation by Hampson, 1926, Descr. new Genera Species Lepid. Phalaenae Subfamily Noctuinae Br. Mus.: 524. Type(s) Õ. Americi. [Costa Rica. Venezuela]. $\ddagger$ †UCLISTIS; Hübner, [I826] I8I6, Verz. bekannter Schmett. Anzeiger: 57 .

An incorrect subsequent spelling.

FOCILLA Guenée, I 852, in Boiscluval \& Guenée, Hist. nat. Insectes (Lépid.) 7: 333.

Type-species: Euclystis centurialis Hübner, I823, by subsequent designation by Desmarest, (1857), in Chenu, Encycl. Hist. nat. Papillons nocturnes: I34.

Focilla is a junior objective synonym of Euclystis Hübner, I 823.

$\ddagger J O C I L L A$; Herrich-Schäffer, I858, Samml. neıer oder wenig bekannter aussereur. Schmett. 1: 68 .

An incorrect subsequent spelling. Neave, 1939, Nomencl. zool. 2: 810, cited Jocilla as an available name.

EUCOCYTIA Rothschild \& Jordan, 1905, Novit. zool. 12: 474.

COCYTIIDAE

Type-species: Eucocytia meeki Rothschild \& Jordan, I905, ibidem 12: 474, by original designation. Syntypes I $\partial$, I + , NEw Guinea: [Papua], R. Angabunga (BMNH); Owgarra.

EUCOPTOCNEMIS Grote, 1874, Bull. Buffalo Soc. nat. Sci. 2: I 3.

NOCT

Type-species: Heliophobus fimbriaris Guenée, I852, in Boisduval \& Guenée, Hist. nat. Insectes (Lépid.) 5: I72, by original designation. Holotype $\hat{\sigma}$, AmERICA: northern (BMNH).

EUCORA Hübner, [1823] 1816, Verz. bekannter Schmett.: 278.

CATO

Type-species: Phalaena neonympha Esper, [1796], Die Schmett. 4 (2) Abschnitt 2: 75 , pl. I98, figs I, 2, by subsequent designation by Hampson, I9I3, Cat. Lepid. Phalaenae Br. Mus. 12: 58. Syntypes $\widehat{\sigma}$, ․․, [U.S.S.R.]: [Krasnoarmeysk] Sarepta.

EUCOSMOCARA Swinhoc, Igor, Ann. Mag. nat. Hist. (7) 8: 22.

HYP E

Type-species: Eucosmocara plumifera Swinhoe, I9or, ibidem (7) 8: 22, by monotypy. Syntypes $\hat{o}$, ㅇ, Borneo: Sarawak (BMINH).

EUCROPIA Hampson, I9o8, Cat. Lepid. Phalaenae Br. Muss. 7: x, 286.

ACRO

Type-species: Hadena pallirena Felder \& Rogenhofer, 1874, Reise öst. Fregatte Novara (Zool.) 2 (Abt. 2) : pl. I Io, fig., 9; Inhalts-Terz.: I I, by original designation. Syntypes ${ }^{*}$, Venezuela (BMNH). Brazil.

EUCYCLOMMA Butler, I893, Entomologist 26: 292, 293.

CATO

Type-species: Bombyx castalia Fabricius, I775, Syst. Ent.: 559, by subsequent designation by Hampson, 1913. Cat. Lepid. Phalaenae Br. Mus. 12: 364. Syntypes, [Australia] Nova Hollandia (BMNH).

In addition to $B$. castalia, Butler on page 292 referred to Calliodes Guenée, 1852, and correctly stated that it 'was fonnded for the reception of two species, an $\Lambda$ frican and an Australian.' Butler then differentiated the Australian species C. orbigera Guenée from the African C. appollina Guenée and continued 'I would, therefore, propose the name Eucyclomma for the Australian forms.' The originally inchuded species in the genus inust 
therefore be Calliodes orbigera Guenée, 1852 , as well as Bombyx castalia Fabricius, I793, included by Butler on page 293.

EUDAPHAENURA Viette, [1963] 1962, Annls Soc. ent. Fr. 131: 286.

CUCU

Type-species: Epicausis splendens Viette, I954, Lambillionea 54: I0, fig. I, by original designation. Holotype 9 , Madagascar: Fianarantsoa, Sahambava (MNHN, Paris).

Eudaphaenura is the correct original spelling, used repeatedly by Viette, although based on the related genus Daphoenura Butler, 1878, also used by Viette in the same work on page 279 .

EUDERAEA Hübner, [182 I] I816, Verz. bekannter Schmett.: 245.

CUCU

Type-species: Noctua asteris [Denis \& Schiffermüller], 1775, Ankündung syst. Werkes Schmett. Wienergegend: 3I2, by subsequent designation by Stephens, 1835, Illust. Br. Ent. (Haustellata) 4: 390. Type(s) [Austria]: Vienna district (Collection destroyed, Horn \& Kahle, 1936, Ent. Beih. Berl.-Dahlem 3: 243).

EUDESMEOLA Hampson, 1926, Descr. new Genera Species Lepid. Phalaenae Subfamily Noctuinae Br. Mus.: 32.

OPHI

Type-species: Diatenes lawsoni Felder \& Rogenhofer, I874, Reise öst. Fregatte Novara (Zool.) 2 (Abt. 2): pl. Ir r, fig. 30; Inhalts-Verz.: 8, by original designation. Type(s) 우, Australia.

EUDIPHTHERA Nordström, I94I. See Griposia Tams, I939.

CUCU

EUDIPNA Walker, I856, List Specimens lepid. Insects Colln Br. Mus. 9: I37, I49. орні Type-species: Phalaena phasis Cramer, 1777, Uitlandsche Kapellen 2: 106, pl. I65. fig. B, by subsequent designation by Hampson, 1926, Descr. new Genera Species Lepid. Phalaenae Subfamily Noctuinae Br. Mus.: i io. Type(s), Surinam.

$P$. phasis is dated from the wrapper of the part and not the title-page of the volume, I779.

EUDIPNA Walker sensu Schaus, I906. See Cropia Walker, I857.

ACRO

EUDOCIMA Billberg, I820, Enumeratio Insect. Mus. G.J. Billberg: 85.

OPHI

Type-species: Phalaena salaminia Cramer, 1777, Uitlandsche Kapellen 2: I I7, pl. I74, fig. A, by monotypy. Type(s), Surinam.

$P$. salaminia is dated from the wrapper of the part, and not the title page of the volume, I779.

LEPTOPHARA Billberg, I820, Enumeratio Insect. Mus. G.J. Billberg: 85. Available but hitherto without any included species.

Type-species: Phalaena salaminia Cramer, I777, by subsequent monotypy.

Leptophava was partially described and therefore nomenclaturally available, but had no originally included species. No differential diagnosis between Leptophara and Eudocima was given so in order to place Leptophara I here include $P$. salaminia Cramer.

Leptophara is a junior objective synonym of Eudocima Billberg, I820.

MAENAS Hübner, [1823] i816, Verz. bekannter Schmett.: 264.

Type-species: Phalaena salaminia Cramer, 1777, by monotypy, but cited as †'salaminea Cram.', an incorrect subsequent spelling.

A junior homonym of Maenas Walbaum, 1792, in Artedi, P. Artedi renovati... Ichthyologiae (Edn 2) 3: 585,- Pisces. The objective replacement name is Eudocima Billberg, I 820 .

†MOENAS; Walker, [1858] I857, List Specimens lepid. Insects Colln Br. Mus. 13: I 2 I7.

An incorrect subsequent spelling.

EUMAENAS Hampson, 1924, in Tams, J. nat. Hist. Soc. Siam 6: 252.

Proposed, unnecessarily, as the objective replacement name for Maenas Hübner, [I823]. 
EUDOREA Curtis, I $827, \mathrm{Br}$. Ent. 4 : I 70.

Type-species: Tinea pyralella [Denis \& Schiffermüller], I775, Ankündung syst. Werkes Schmett. Wienergegend: I35, by original designation, but cited as pyralella Hübner, an incorrect authorship.

Eudorea has been used as the original generic name for species which are now in the Noctuidae.

EUDRAGANA Butler, I888, Proc. zool. Soc. Lond. 1888: 545.

OPHI

Type-species: Eudragana limbata Butler, I888, ibidem 1888: 545, by monotypy. Type(s) 을 Christmas I. [Indian Ocean] (BMNH).

E. limbata is a junior subjective synony'm of $H_{y}$ pocala subsatura Guenée, 1852 , in Boisduval \& Guenée, Hist. nat. Insectes (Lépid.) 7: 75.

EUDRAPA Walker, [1858] i 857, List Specimens lepid. Insects Colln Br. Mus. 13: io79, i Ioo.

OPHI

Type-species: Eudrapa mollis Walker, [1 858] I857, ibidem 13: I Ior, by monotypy. Syntypes 2 웅 [GHANA]: Ashanti (BMNH).

EUDRYAS Boisduval, I836, Hist. nat. Insectes (Lépid.) 1 (Explication des planches) : 4. AGAR Type-species: Euthisanotia unio Hübner, [I831] I825, Zuträge Samml. exot. Schmett. 3: I 2, figs 431, 432, by monotypy. Type(s) 9 , [U.S.A.]: Savannah.

EUDYOPS Hampson, 1926, Descr. new Genera Species Lepid. Phalaenae Subfamily Noctuinae Br. Mus.: I26.

OPHI

Type-species: Dyops xantholepis Dyar, I912, Proc. U.S. natn. Mus. 42: 77, by original designation. Syntypes $2 \hat{\jmath}, 2$ ㅇ, Mexico: Misantla (USNM, Washington).

EUEDWARDSIA Grote, I882, Papilio 2: I22.

ACRO

Type-species: Xanthothrix neumoegeni Edwards, I88I, Papilio 1: Io I, by original designation. Syntypes, [U.S.A.]: Cal., Sta Barbara Co.; S. California.

EUEDWARDSIA Kirby, I892. See Eupseudomorpha Dyar, I893.

AGAR

EUERETAGROTIS Smith, I890, Bull. U.S. natn. Mus. 38: 9, 47.

NOC T

Type-species: Noctua sigmoides Guenée, 1852, in Boisduval \& Guenée, Hist. nat. Insectes (Lépid.) 5: 325, by original designation (on page 9). Holotype ô, [U.S.A.]: New York State $(\mathrm{BMNH})$.

EUGATHA Hampson, 19ा1, Ann. Mag. nat. Hist. (8) 8:444. ACON

Type-species: Eugatha thermochroa Hampson, I9I1, ibidem (8)8: 444, by original designation. Holotype ô, NEw GuiNea: [West Irian], Fak-fak (BMNH).

EUGLYPHIA Hübner, [I820]. See Diphthera Hübner, [1809]. OPнI

EUGLYPHINA Hampson, 1926. See Metapliusia Dyar, 1925. OPH I

EUGLYPHIS Hübner, [1825]. See Hulypegis nom. n. OPH

EUGNATHIA Warren, 1912, in Seitz, Gross-Schmett. Evde 3: $271 . \quad$ acon

Type-species: Selenis longipalpis Walker, 1865, List Specimens lepid. Insects Colln Br. Mus. 33: io68, by original designation. Holotype 우, [INDia]: Hindostan (BMNH with added label Burma, Moulmein).

The date of publication of the part of Seitz containing page 27 I was stated on page 209 as 1912 January 10 th in both the German and the English editions.

EUGNORISMA Boursin, I946, Revue fr. Lépidopt. 10: 188.

NOCT

Type-species: Graphiphora insignata Jederer, i 853, Verh. zool.bot. Ver. Wien 3: 360, 1]. 4, fig. 2, by original designation, but cited as $\ddagger$ Graphophora, an incorrect subsequent spelling. Ty'pe(s), [U.S.S.R.]: Siberia. 
EUGONIELLA Kaye \& Lamont, 1927, Mem. Dept. Agric. Trinidad E Tobago 3: 78. Hy PE Type-species: Megatomis sapota Felder \& Rogenhofer, 1874 , Reise öst. Fregatte Novara (Zool.) 2 (Abt. 2) : pl. I20, fig. I2; Inhalts-Verz.: I3, by monotypy. Type(s) GUIANA (BMNH).

EUGORNA Holland, I894, Psyche, Camb. 7: I 22.

OPHI

Type-species: Eugorna vidua Holland, I894, ibidem 7: I 23, pl. 4, fig. I, by original designation. Syntypes $\widehat{0},+$, West Africa (CM, Pittsburgh).

EUGRAMMA Stephens, 1850. See Dicycla Guenée, 1852.

ACRO

EUGRAMMODES Hampson, 1926, Descr. new Genera Species Lepid. Phalaenae Subfamily Noctuinae Br. Mus.: 300.

OPHI

Type-species: Noctua esquina Dognin, i 897, Annls Soc. ent. Belg. 41: 29, by original designation. Holotype $\hat{\sigma},[$ ECUADOR]: Loja.

${ }_{+} \boldsymbol{E U G R A P H A}$, misspelling. See Eugraphe Hübner, [1 $\left.82 \mathrm{I}\right]$.

NOCT

EUGRAPHE Hübner, [1821] I 816, Verz. bekannter Schmett.: 224.

NOCT

Type-species: Noctua sigma [Denis \& Schiffermüller], I775, Ankündnng syst. Werkes Schmett. Wienergegend: 78 (but included by Hübner as sigma Hübner, an incorrect authorship), by subsequent designation by Hampson, 1903, Cat. Lepid. Phalaenae Br. Mus. 4: 364 , but cited as ¥'signa Fabr', which Hampson placed (on page 407) as the senior synonym of sigma. Type(s), [Austria]: Vienna district (Collection destroyed, Horn \& Kahle, 1936, Ent. Beih. Berl.-Dahlem 3: 243).

$¥$ Noctua signum Fabricius, I787, Mantissa Insect. 2: I54, is an incorrect subsequent spelling, repeated in 1794, Ent. Syst. 3(2):65. Hampson confused the latter reference with Syst. Ent., dated it 1775 , and accepted $\ddagger$ signa Fabricius as the valid name.

${ }_{\ddagger} E U G R A P H A$; Hübner, [1826] i 816, Verz. bekannter Schmett. Anzeiger: 37 .

An incorrect subsequent spelling.

EUGRAPHIA Guenée, I852. See Eusceptis Hübner, I 823.

$\operatorname{ACON}$

EUGRAPTA Wileman \& South, I9I 7, Entomologist 50: 146.

OPHI

Type-species: Eugrapta igniflua Wileman \& South, I9I 7 , ibidem 50: 146 , by monotypy. Holotype ‥ JaPAN (BMNH).

EUGRAPTA Hampson, 1926, Descr. new Genera Species Lepid. Phalaenae Subfamily

Noctuinae Br. Mus.: 544 .

Type-species: Egnasia venusta Hampson, I898, J. Bombay nat. Hist. Soc. 11: 460, by original designation. Holotype ‥ InDIA: [Assam], Khasis (BMNH).

A junior homonym of Eugrapta Wileman \& South, I917. This generic name probably originated from Hampson but was used and unintentionally made available by Wileman \& South prior to its proposal and generic description by Hampson. There is no objective replacement name but E. venusta is congeneric with Eugrapta ignifua Wileman \& South, the type-species of Eugrapta. Wileman \& South. The latter is therefore available for use as the subjective replacement name.

EUGRAPTA Hampson, I926. See Eugrapta Wileman \& South, I9I7. OPHI

EUGRAPTOBLEMMA Warren, I9I3, in Seitz, Gross-Schmett. Erde 11: $205 . \quad$ ACON

Type-species: Mimoruza pictalis Hampson, I 898, J. Bombay nat. Hist. Soc. 11: 450, by original designation. Holotype $\widehat{0}$. India: [Assam], Khasis, (BMNH).

The date of publication of the part of Seitz containing page 205 was stated on page 20 I as I9I3 August 2oth in both the German and English editions.

EUHARVEYA Grote, 1894, Can. Ent. 26: 81.

CUCU

Type-species: Lithophane carbonaria Harvey, I876, ibidem 8: 55 , by monotypy. Holotype ㅇ. [U.S.A.]: California. 
EUHERRICHIA Grote, I882, Papilio 2: r22.

Type-species: Eriopus monetifera Guenée, 1852, in Boisduval \& Guenée, Hist. nat. Insectes (Lépid.) 6: 295, pl. I4, fig. 4, by original designation (for Herrichia Grote, I882). Type(s) ô. TrPe-Locality ?America (UM, Oxford). [Canada. U.S.A:]

Euherrichia was proposed as the objective replacement name for Herrichia Grote, I882. HERRICHIA Grote, 1882, New Check List N. Am. Moths: 38, 64.

Type-species: Eriopus monetifera Guenée, 1852, by original designation on page 64 .

A junior homonym of Herrichia Staudinger, 1871, Berl. ent. Z. 14: 292, - Lepid., Oecophoridae. The objective replacement name is Euherrichia Grote, 1882.

EUHETEROSPILA Strand, 1912, Revue zool. afr. 2: 89.

OPHI

Type-species: Euheterospila antennalis Strand, 1912, ibidem 2: 89, by original designation. Holotype $\sigma^{*},\left[Z_{A} r R E\right]$ Belgian Congo: R. Congo, Poste de Bois No. 7 (MRAC, Tervuren).

EUHYPENA Grote, 1873 January, Trans. Am. ent. Soc. 4: 310.

HYPE

Type-species: Hypena toreuta Grote, 1872, ibidem 4: 24 (the objective replace ment name for Hypena internalis Robinson, 1870, see below), by subsequent designation by Grote, 1874, Bull. Buffalo Soc. nat. Sci. 2: 51, but cited as internalis. Type(s) [U.S.A.]: Pennsylvania.

H. toreuta was proposed by Grote as the objective replacement name for Hypena internalis Robinson, 1870, Ann. Lycerm nat. Hist. N.Y. 9: 311, a junior primary homonym of Hypena internalis Guenće, 1854, in Boisduval \& Guenće, Hist. nat. Insectes (Lépid.) 8: 41, Noctuidae, Hypeninae.

Euhypena was also proposed in Grote, 1873 May, Bull. Buffalo Soc. nat. Sci. 1: 38 with the same included species.

EUIPPODES Hampson, 1926, Descr. new Genera Species Lepid. Phalaenae Subfamily Noctuinae Br. Muss.: 486 .

OPHI

Type-species: Euippodes euprepes Hampson, 1926, ibidem 486, by original designation. Types $2 \hat{0}$, I 우 [GHana] Gold Coast: Kumasi (BMNH).

EULAPHYGMA Butler, 189o, Trans, ent. Soc. Lond. 1890: 668.

ACRO

Type-species: Spodoptera abyssinia Guenée, 1852, in Boisduval \& Guenée, Hist. nat. Insectcs (Lépid.) 5: 154, pl. 6, fig. 6, by monotypy. Lectotype ô. [EтHropia] Abyssinia (MNHN, Paris), designated by Viette, 1951, Bull. mens. Soc.linn. Lyon 20: i60.

S. abyssinia is a junior subjective synonym of Noctua excisa Herrich-Schäffer, 1854 , Samml. neuer oder wenig bekannter aussereur. Schmett. 1 (I): wrapper, pl. 27, fig. 129; 1858, ibidem 1: 68, 79 .

See also Ariathisa Walker, 1865.

EULEPA W'alker, [1863] 1864, J. Proc. Linn. Soc. (Zool.) 7: 54. $\triangle \mathrm{CRO}$

Type-species: Eulepa niveigutta Walker, [1863] 1864, ibidem 7: 54, by monotypy. Lectotype ô. Borneo: Sarawak (UM, Oxford), designated (as type) by Swinhoe, 1900, Cat. east. and Aust. lepid. Heterocera 2: 52.

EULEPIDOTIS Hübner, I823, Zuträge Samml. exot. Schmett. 2: 22.

OPHI

Type-species: Eulepidotis alabastraria Hübner, 1823, ibidem 2: 22, figs 311, 312, by subsequent designation by Grote, 1870, Bull. Buffalo Soc. nat. Sci. 2: 24. Type(s) $q$, [U.S.A.]: Savannah.

†EULEPIDOTUS; Herrich-Schäffer, 1856, Samml. newer oder wenig bekannter aussereur.

Schmett. 1: 28, $4 \mathrm{~T}$.

An incorrect subsequent spelling.

${ }_{+}^{+}$EULEPIDOTUS, misspelling. See Eulepidotis Hübner, I 823 .

OPHI 
EULEUCYPTERA Grote, r865, Proc. ent. Soc. Philad. 4: 329.

NOCT

Type-species: Euleucyptera cumatilis Grote, 1865, ibidem 4: 330, p1. 2, fig. 6, by monotypy. Holotype ㅇ, [U.S.A.]: Colorado Territory.

EULINTNERIA Grote, 188 I, Bull. U.S. geol. geogr. Surv. Territ. 6: 564 .

HYPE

Type-species: Tortricodes bifidalis Grote, I872, Trans. Am. ent. Soc. 4: 105, by original designation. Type(s) ठ̂. [U.S.A.]: Atlantic district, New York to Texas.

$T$. bifidalis is a junior subjective synonym of Gaberasa ambigualis Walker, [1866].

See also Gaberasa Walker, [1866].

$\ddagger$ EULISSA, misspelling. See Zalissa Walker, 1865 .

AGAR

EULITHOSIA Edwards, I884, Papilio 4: 43.

ACRO

Type-species: Eulithosia composita Edwards, I884, itidem 4: 44, by subsequent designation by Kirby, I892, Synonymic Cat. Lepid. Heterocera 1:371. Holotype + , [U.S.A]: Arizona.

Eulithosia was originally placed in the 'Lithosiidae.'

EULOCASTRA Butler, I 886, Trans. ent. Soc. Lond. 1886: 4r9.

ACON

Type-species: Eulocastra fasciata Butler, I886, ibidem 1886: 4r9, by monotypy. Type(s) ô., [Australia]: [N.S.W.], Sydney (BMNH).

EULONCHE Grote, I873, Bull. Buffalo Soc. nat. Sci. 1: 81.

ACRO

Type-species: Phalaena oblinita Smith, 1797, in Smith \& Abbot, Nat. Hist. raver lepid. Insects Georgia 2: 187, pl. 94, by original designation, but cited as oblinita Guenée, an incorrect authorship. Syntypes, [U.S.A.]: Georgia; Virginia.

EUL YMNIA Hampson, 1908, Cat. Lepid. Phalaenae Br. Mus. 7: r6. Available, but without included species until Hampson, I9ro, ibidem 9: viii, II I.

ACRO

Type-species: Conservula pulcherrima Hampson, I902, Ann. S. Afr. Mus. 2: 295, by subsequent monotypy. Type(s) ô, [RHodesia]: Mashonaland, Salisbury (BMNH).

EUMAENAS Hampson, 1924. See Eudocima Billberg, I820.

OPHI

EUMESTLETA Butler, I892, Entomologist 25: i 89.

$\mathrm{ACON}$

Type-species: Tarache patula Morrison, 1875, Proc. Acad. nat. Sci. Philad. 27: 69, by monotypy. Holotype, [U.S.A.]: Texas.

T. patula is a junior subjective synonym of Phalaena obliqualis Fabricius, r794, Ent. Syst. 3 (2): 224, but this binomen is a primary junior homonym of Phalaena obliqualis Gmelin, I790, in Linnaeus, Syst. Nat. (Edn I3) 1 (5): 2528. There is no objective replacement name but Barnes \& Benjamin, 1927, Pan-Pacif. Ent. 4:39, have examined the probable holotype of Micra recta Guenée, I852, in Boisduval \& Guenée, Hist. nat. Insectes (Lépid.) 6: 245 , and placed it as a junior subjective synonym of obliqualis Fabricius. M. recta is thus available for use as a subjective replacement name.

EUMICHTHIS Agassiz, 1846. See Eumichtis Hübner, [1821].

CUCU

EUMICHTIS Hübner, [182I] I8I6, Verz. bekannter Schmett.: 2II.

CUCU

Type-species: Noctua lichenea Hübner, [18r3], Samml. eur. Schmett. 4: pl. I22, figs 562,563 , by monotypy. Syntypes of, ㅇ, Europe.

EPUNDA Duponchel, [1845], Cat. méth. Lépid. Eur. (2): $\mathrm{r}_{4} \mathrm{r}$.

Type-species: Noctua lichenea Hübner, [1813], by subsequent designation by Guenée, 1852, in Boisduval \& Guenée, Hist. nat. Insectes (Lépid.) 6: 48.

Epunda is a junior objective synonym of Eumichtis Hübner, [I82I].

EUMICHTHIS Agassiz, I846, Nomencl. zool. Index univl.: $x_{4} 8$.

An unjustified emendation of Eumichtis Hübner, [r82 I]. 
EUMICHTOCHROA Hampson, 1926, Descr. new Genera Species Lepid. Phalaenae Subfamily

Voctuinae Br. Mus.: Ioo.

OPH I

Type-species: Trisula dudgeoni Hampson, is96, Fauna Br. India. (Moths) 4: 530, by original designation. Type(s) 우, BHUtan (BMNH).

EUMICREMMA Berio, I954, Mem. Inst. sci. Madagascar (E) 5: 139.

ACON

Type-species: Micra minima Guenée, I852, in Boisduval \& Guenée, Hist. nat. Insectes (Lépid.) 6:245, by original designation. Holotype ô. [West INDiEs]: [Virgin Is], Île SaintThomas (not found in IINHN, Paris; nor in BMNH).

Eumicremma was proposed for M. minima on the basis of I $\overrightarrow{0}$, I $q$ from Madagascar. Berio stated that the male had been compared for him with Guenée's type in BMNH. I have been unable to find this type, so it is probable that the male from Madagascar had been compared only with the series of specimens in BMNH, as listed by Hampson, I9IO, Cat. Lepid. Phalaenae Br. MIus. 10: I52, and not with the type itself. The specimens of this series range in distribution from U.S.A. to South Africa and probably represent more than one species.

EUMILICHIA Warren, I937. See Protheodes Swinhoe, I 885.

$\triangle \mathrm{CRO}$

EUMINUCIA Hampson, I913, Cat. Lepid. Phalaenae Br. Mus. 12: 7, 421.

CATO

Type-species: Euminucia conflua Hampson, I9I3, ibidem 12:421, fig. 98, by original designation. Holotype ô., [GHANA] Gold Coast: Bibianaha (BMNH).

EUMUELLERIA Dyar, igi 8, Proc. U.S. natn. Mus. 54: 343.

HADE

Type-species: Eumuelleria cliopis Dyar, I918, ibidem 54:343, by original designation. Holotype 3 . Mexico (USNM, Washington).

‡EUMULLERIA Dyar, 1918, Proc. U.S. natn. Mus. 54: 343.

An incorrect original spelling under the Code, Article 32 (c) (i), which states that in a German word the umlaut sign is to be deleted and the letter ' $e$ ' is to be inserted after that vowel. In the example given in the Code 'mülleri becomes muelleri and is not a homonym of mullevi.' In the case of Eumiilleria Dyar which is dedicated to 'Mr Roberto Müller of Mexico City' I have taken Müller to be a German personal name even though the gentleman concerned was probably not of that nationality. If the name was treated as not being German then it would become a homonym of Eumulleria Anthony, 1907, Annls Soc. $r$. zool. malacol. Belg. 41: 407, - Mollusca.

†UMÜLLERIA Dyar, I9I8. See Eumuelleria Dyar, I918.

HADE

EUNEOPHLEBIA Berio, 1940, Boll. Soc. cnt. ital. 72: I23.

NOCT

Type-species: Euneophlebia pruinosa Berio, I940, ibidem 72: I23, by original designation. Holotype ô, [Eтhiopia] Eritrea: Dorfù (Colln E. Berio, Genoa).

EUNETIS Hübner, I823, Zuträge Samml. exot. Schmett. 2: 26.

CA TO

Type-species: Phalaena amasia Esper, [1796], Die Schmett. 4 (2) Abschnitt 2: 55, pl. I94, figs I, 2, by subsequent designation by Hampson, I9I3, Cat. Lepid. Phalaenae Br. Mus. 12: 58, but cited as puevpera Giorna, I 79I, which Hampson placed (on page I I 5) as the senior synonym of amasia Esper. Syntypes $\hat{o}$,, , [TURkey]: Taurien. Italy: southern.

$P$. amasia is a junior subjective synonym of Phalaena puerpera Giorna, I79I, Cal. Ent.: I04.

See also Blepharonia [Hübner], [1825].

EUNIMBATANA Fletcher \& Viette, I955, Bull. Inst. fr. Afr. noire 17 (A): I77.

OPH I

Type-species: Eunimbatana lobata Fletcher \& Viette, I955, ibiclem 17 (A): I78, pl. I, fig. I, by original designation. Holotype $\hat{\sigma}$, [Guinea] French Guinea: Mont Nimba, Ziela (MNHN, Paris).

EUONYCHODES Warren, 1914, Ann. S. Afr. Mus. 10: 480.

CATO

Type-species: Euonychodes albivenata Warren, I9T4, ibidem 10: 480, pl. 40, fig. I4, by original designation. Holotype $q$, Soutir ArRicA. 
EUPALINDIA Schaus, 1904, Trans. Am. ent. Soc. 30: 165.

OPHI

Type-species: Eupalindia magnifica Schaus, I904, ibidem 30: I65, by subsequent designation by Hampson, I926, Descr. new Genera Species Lepid. Phalaenae Subfamily Noctuinae Br. Mus.: 369 . Type(s), BrazIL: São Paulo.

EUPALINDIA Hampson, 1926, Descr. new Genera Species Lepid. Phalaenae Subfamily Noctuinae Br. Mus.: 369 .

OPHI

Type-species: Eupalindia magnifica Schaus, 1904, by original designation.

A junior homonym of Eupalindia Schaus, I904. This generic name was used and made available by Schaus prior to its proposal and generic description by Hampson for exactly the same concept.

${ }_{\ddagger} \boldsymbol{E} \boldsymbol{U} \boldsymbol{P} \boldsymbol{A} \boldsymbol{L} \boldsymbol{T H} \boldsymbol{I} \boldsymbol{S}$ Hampson, no published reference found.

HYPE

EUPANYCHIS Grote, I890, Revised Check List N. Am. Noctuidae 1:34. Noct

Type-species: Heliothis spinosae Guenée, 1852 , in Boisduval \& Guenée, Hist. nat.

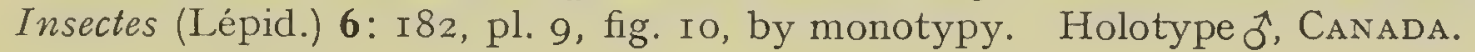

EUPARTHENOS Grote, I876, Ann. Lyceum nat. Hist. 11: зог.

CATO

Type-species: Parthenos nubilis Hübner, [1823], Samml. exot. Schmett. 2: pl. [215],

by monotypy (of Parthenos Hübner, [1823]). Type(s), Type-Locality not stated. [N. AMERICA].

Euparthenos was proposed as the objective replacement name for Parthenos Hübner, [1823].

PARTHENOS Hübner, [1823], Samml. exot. Schmett. 2: pl. [215].

Type-species: Parthenos nubilis Hübner, [1823], by monotypy.

A junior homonym of Parthenos Hübner, [I 819] I 8 16, Verz. bekannter Schmett.: 38, -Lepid., Nymphalidae. The objective replacement name is Euparthenos Grote, I876.

CATOCALIRRHUS Andrews, I877, Can. Ent. 9: 20.

Proposed, unnecessarily, as an objective replacement name for Parthenos Hübner, [1823]. The objective replacement name Euparthenos Grote, I876, had already been proposed.

EUPATULA Ragonot, I894, Bull. Soc. ent. Fr. 1894: ccvii.

СATO

Type-species: Phalaena macrops Linnaeus, I 768, Syst. Nat. (Edn I 2) 3: 225, by subsequent designation by Hampson, I9I3, Cat. Lepid. Phalaenae Br. Mus, 12: 273. Type(s), [INDIA]: India Orientali (LS, London).

Eupatula was proposed as the objective replacement name for Patula Guenée, I852.

PATULA Guenée, I852, in Boisduval \& Guenée, Hist. nat. Insectes (Lépid.) 7: 176.

Type-species: Phalaena macrops Linnaeus, 1768 , by subsequent designation by Hampson, I9I3, Cat. Lepid. Phalaenae Br. Mus. 12: 273.

A junior homonym of Patula Held, I837, Isis, Jena 1837: 916, - Mollusca. The objective replacement name is Eupatula Ragonot, I894.

CRISHNA Kirby, I897, Handb. Order Lepid. 5: r53.

Proposed, unnecessarily, as an objective replacement name for Patula Guenée, I852.

The objective replacement name Eupatula Ragonot, I 895 had already been proposed.

EUPERIA Guenée, I839. See Enargia Hübner, [I82I].

ACRO

$\ddagger$ EUPHAIS Hübner, I818. See Hypsoropha Hübner, I818.

OPH I

EUPHAIS Hübner, I822. See Scoliopteryx Germar, I8I I.

OPHI

EUPHASIA Stephens, I830. See Desmophora Stephens, I829. ACON

$\ddagger$ EUPHIUCHE Hampson, no published reference found. HYPE

EUPHIUSA Hampson, I913, Cat. Lepid. Phalaenae Br. Mus. 12: xii, 550. Cato

Type-species: Chalciope harmonica Hampson, I902, Ann. S. Afr. Mus. 2: 335, by original designation. Holotype $\widetilde{\sigma}$, [BotswaNA]: N'Gamiland (BMNH). 
EUPITHECIA Curtis, I 825, Br. Ent. $2: 64$.

Type-species: Phalaena absinthiata Clerck, I759, Icon. Insect. rariorum 1 : pl. 8, fig. 9, by original designation, but included as absinthiata Linnaeus, an incorrect authorship.

Eupithecia has been used as the original generic name for species which are now in the Noctuidae.

EUPLEXIA Stephens, I829, [June], Nom. Br. Insects: $4 \mathrm{I}$.

ACRO

Type-species: Phalaena lucipara Linnaeus, I76I, Fauna Suecica (Edn 2): 318, by monotypy. Type(s), Sweden (LS, London).

EUPLEXIDIA Hampson, I 896, Fauna Br. India (Moths) 4: $46 \mathrm{I}$.

ACRO

Type-species: Euplexidia noctuiformis Hampson, I896, ibidem 4: 46I, fig. 246, by original designation. Type(s) ô, INDiA [Assam], Khasis (BMNH).

Hampson attributed both Euplexidia and noctuiformis to 'Swinh. MS'., and placed them in the Notodontidae.

EUPLEXIDIA Swinhoe, I897, Ann. Mag. nat. Hist. (6) 19: 165.

Type-species: Euplexidia noctuiformis Hampson, I 896, by original designation.

A junior homonym of Euplexidia Hampson, I 896 . This generic name was used and unintentionally made nomenclaturally available by Hampson prior to its proposal and generic description by Swinhoe for the same concept. The objective replacement name is Euplexidia Hampson, 1896.

EUPLEXIDIA Swinhoe, IS97. See Euplexidia Hampson, I 896.

ACRO

EUPOLIA Smith, I894. See Trichopolia Grote, IS83.

HADE

EUPRORA Hampson, 1926. See Roperua nom. n.

ACRO

EUPSEPHOPAECTES Grote, I873, Bull. Buffalo Suc. nat. Sci. 1: I 37.

HADE

Type-species: Eupsephopaectes procinctus Grote, I873, ibidem 1: 138, pl. 4, fig. 6, by monotypy. Type(s) $q$, [U.S.A.]: California (BMNH).

EUPSEUDOMORPHA Dyar, 1893. Can. Ent. 25: 28.

AGAR

Type-species: Edwardsia brillians Neumoegen, I880, ilidem 12: 68, by monotypy

(of Edwardsia Neumoegen, IS80). Type(s), [U.S.A.]: S. W. Texas (USNM, Washington).

Eupseudomorpha was proposed as the objective replacement name for Edwardsia

Neumoegen, 1880 .

EDWARDSI.1 Neumoegen, i 880, Can. Ent. 12:67.

Type-species: Edwardsia brillians Neumoegen, isSo, by monotypy.

A junior homonym of Edwardsia Costa, IS34, Cenni zool.: 62, - Crustacea. The objective replacement name is Eupseudomorpha Dyar, I893.

EUEDWARDSIA Kirby, IS92, Synonymic Cat. Lepid. Heterocera 1: 36.

Proposed as the objective replacement name for Edwardsia Neumoegen, I880.

A junior homonym of Euedwardsia Grote, 1882, Papilio 2: i22,-Lepid., Noctuidae.

The objective replacement name is Eupsendomorpha Dyar, I893.

EUPSILIA Hübner, [182I] I816, Verz. bekannter Schmett.: 231.

CUCU

Type-species: Phalaena satellitia Linnaeus, I767, Syst. Nat. (Edn I2) 1: 855, by monotypy. Type(s), Germany (LS, London).

$P$. satellitia is a junior subjective synonym of Phalaena transversa Hufnagel, 1766, Berlin. Mag. 3: 4 18.

SCOPELOSOMA Curtis, x 836, Br. Ent. 14:635.

Type-species: Phalaena satellitia Linnaeus, 1767 , by original designation.

Scopelosoma is a junior objective synonym of Eupsilia Hübner, [I $\mathbf{S} 2 \mathbf{I}$ ].

MECOPTERA Guenée, 1837, Annls Soc. ent. Fr. 6: 225.

Type-species: Phalaena satellitia Linnaeus, 1767 , by monotypy.

Mecopteva when originally proposed contained a second species, but this was doubtfully 
included and, under the Code, Article 68(c), is not eligible for designation as type-species.

Mecopteva is a junior objective synonym of Eupsitia Hübner, [182I].

EUPSOROPSIS Berio, 1969, Boll. Soc ent. ital.99-101 [sic]: 45.

ACON

Type-species: Eupsoropsis robertsi Berio, I969, ibidem 99-101: 45, figs I, 2, by original designation. Holotype $\hat{\jmath}$. Nigeria: N., Samaru (BMINH).

EURABILA Butler, 1889, Illust. typical Specimens Lepid. Heterocera Colln Br. Mus. 7: 39.

ACRO

Type-species: Eurabila lignea Butler, I889, ibidem 7: 40, pl. I 28, fig. 6, by monotypy. Syntypes ô, ㅇ. [INDIA]: Dharmsala (BMNH).

EURHIPIA Boisduval, I828. See Eutelia Hübner, [1823].

EUTE

EURHOIS Agassiz, I846. See Eurois Hübner, [1821].

NOCT

EUROGRAMMA Hampson, 1926, Descr. new Genera Species Lepid. Phalaenae Subfamily Noctuinae Br. Mus.: 498.

OPH I

Type-species: Talapa obliquilineata Leech, Igoo, Trans. ent. Soc. 'Lond. 1900: 646, by original designation. Syntypes I $\hat{\delta}, 2$ ㅇ, CHINA: western, Omei-shan (BMNH).

EUROIS Hübner, [1821] I8I6, Verz. bekannter Schmett.: 2 I8. NocT

Type-species: Phalaena occulta Linnaeus, I758, Syst. Nat. (Edn Io) 1: 5I 4, by subsequent designation by Grote, 1874, Bull. Buffalo Soc. nat. Sci. 2: 12, but cited as E. occulta Hübner, an incorrect authorship. Type(s), Europe (LS, London).

EURHOIS Agassiz, I846, Nomencl. zool. Index univl.: I 50.

An unjustified emendation of Eurois Hübner, [1821].

EUROMOEA Hampson, I908. See Euromoia Staudinger, I892.

ACRO

EUROMOIA Staudinger, I892, in Romanoff, Mém. Lépid. 6: 632.

ACRO

Type-species: Euromoia mixta Staudinger, I 892, ibidem 6: 632, pl. I2, fig. 10, by monotypy. Holotype ơ. [U.S.S.R.]: Amur district, Sutschan area (MNHU, Berlin).

EUROMOEA Hampson, I908, Cat. Lepid. Phalaenae Br. Mus. 7: 425.

An unjustified emendation of Euromoia Staudinger, r 892.

EUROS Edwards, I881, Papilio 1: I9.

CUCU

Type-species: Euros proprius Edwards, I881, ibidem 1: 19, by monotypy. Holotype o. [U.S.A.]: Calif., Siskiyou Co., Strawberry Valley.

EUROT YPE Hampson, I906. See Platypolia Grote, I895.

CUCU

EURYNOLA Hampson, 1905, Ann. Mag. nat. Hist. (7) 15: 432.

NOLI

Type-species: Corula mesoleuca Lower, 1903, Trans. R. Soc. S. Aust. 27: 39, by original designation. Holotype $\hat{0}$, Australia: Queensland, Cooktown (SAM, Adelaide).

EUR YPS YCHE Butler, I886, Trans. ent. Soc. Lond. 1886: 392.

HADE

Type-species: Eurypsyche similis Butler, 1886, ibidem 1886: 392, by monotypy. Type(s) ô. [Australia]: [Qd], Gayndah (BMNH).

E. similis is a junior subjective synonym of Lewcania lewinii Butler, r886, ibidem 1886: 390.

EURYSCHEMA Turner, 1925, Trans. R. Soc. S. Aust. 49: 38.

OPHI

Type-species: Euryschema tricycla Turner, 1925, ibidem 49: 38, by monotypy. Holotype $q$, Australia: Qd, Toowoomba (ANIC, Canberra).

EURYTHMUS Butler, I886, Trans. ent. Soc. Lond. 1886: 409.

OPHI

Type-species: Eurythmus bryophiloides Butler, I886, ibidem 1886: 4IO, by monotypy. Type(s), [Australia]: [Qd], Peak Downs (BMNH).

$\mathrm{O}$ 
EUSCEPTIS Hübner, I823, Zuträge Samml. exot. Schmett. 2: 21.

Type-species: Eusceptis irretita Hübner, I823, ibidem 2: 2I, figs 305, 306, by monotypy. Type(s) ?Q, BraziL.

EUGRAPHIA Guenée, I 852, in Boisduval \& Guenée, Hist. nat. Insectes (Lépid.) 6: 208.

Type-species: Eusceptis irretita Hübner, I 823, by monotypy.

Eugraphia is a junior objective synonym of Eusceptis Hübner I 823 .

EUSCHESIS Hübner, [1821] 1816, Verz. bekannter Schmett.: 221.

NOCT

Type-species: Noctua janthina [Denis \& Schiffermüller], I775, Ankündung syst. Werkes Schmett. Wienergegend: 78, by subsequent designation by Hampson, 1903, Cat. Lepid. Phalaenae Br. Mus. 4: 622. Type(s), [Austria]: Vienna district (Collection destroyed, Horn \& Kahle, 1936, Ent. Beih. Berl.-Dahlem 3: 243).

†EUSCHIRRHOPTERUS, misspelling. See Euscirrhopterus Grote, I 866.

AGAR

${ }_{\ddagger}^{+}$EUSCHIRROPTERUS, misspelling. See Euscirrhopterus Grote, I866.

AGAR

EUSCIRRHOPTERUS Grote, I 866 July, Proc. ent. Soc. Philad. 6: i76.

AGAR

Type-species: Euscirrhopterus poeyi Grote, I 866 July, ibidem 6: I78, by monotypy. Syntypes 0 . ㅇ, CuBA.

The date of publication of Euscirrhopterus has been discussed by Todd, 1966, Proc. U.S. natn. Mus. 120:4.

See also Hetevandra Herrich-Schäffer, I866 [September]; and Laquea Jordan, I 896.

${ }_{+}^{+E U S C H I R R H O P T E R U S ; ~ K i r b y, ~ I ~ 892, ~ S y n o n y m i c ~ C a t . ~ L e p i d . ~ H e t e r o c e r a ~ 1: ~} 40$.

An incorrect subsequent spelling.

$\ddagger$ EUSCHIRROPTERUS; Hampson, igor, Cat. Lepid. Phalaenae Br. Mus. 3: 619.

An incorrect subsequent spelling.

EUSCOTIA Butler, I889, Illust. typical Specimens Lepid. Heterocera Colln Br. Mus. 7: 47.

CUCU

Type-species: Rhizogramma inextricata Moore, I88 I, Proc. zool. Soc. Lond. 1881: 342, by monotypy. Syntypes $\hat{0}$, ㅇ, [India]: [Himachal Pradesh], Dalhousie (BMNH); Punjab, Solun (BMNH).

Euscotia when originally proposed contained a second binomen, but this was doubtfully included as a probable synonym of inextricata and, under the Code, Article 68 (c), is not eligible for designation as type-species.

EUSEMIA Dalman, 1825. See Episteme Hübner, [1820].

AGAR

EUSIMARA Walker, I865, List Specimens lepid. Insects Colln Br. Mus. 33: 1098.

OPHI

Type-species: Eusimara subfervida Walker, 1865, ibidem 33: I099, by monotypy. Holotype , [Colombia]: Bogota (not found in $\mathrm{BMNH}$ ).

EUSTEGNIA Hübner, [182I]. See Cosmia Ochsenheimer, I8I6.

ACRO

EUSTROTIA Hübner, [I82I] I8I6, Verz. behannter Schmett.: 253.

$\operatorname{ACON}$

Type-species: Phalaena uncula Clerck, I759, Icones Insect. rariorum 1: pl. 3, fig. 7 (but included by Hübner as 'E. unca Schiff.'), by monotypy. Type(s), Type-Locality not stated [EUROPE].

Noctua unca [Denis \& Schiffermüller], I775, Ankündung syst. Werkes Schmett. Wienergegend: $9 \mathrm{I}$, is an unjustified emendation of Phalaena uncana Linnaeus, I76I, Fauna Succica (Edn 2): 342 , which is an unjustified emendation of P. uncula Clerck, I 759.

ERASTRIA Ochsenheimer, I8I6, Schmett. Eur. 4: 92.

Type-species: Phalaena uncula Clerck, I759, by subsequent designation by Samouelle, I8I9, Entomologist's useful Compendium: 252, but cited as unca, a name originally included in the genus, and also an unjustified emendation.

A junior homonym of Erastria Hübner, [I8I3], Samml. exot. Schmett. 1: pl. [203], Lepid., Geometridae. The objective replacement name is Eustrotia Hübner. 
Erastria Ochsenheimer has been placed on the Official Index of Rejected and Invalid Generic Names in Zoology: Name No. I 840.

$\ddagger$ EROTYLAE Ochsenheimer, I8I6, Schmett. Eur. 4: 92.

Published as a junior synonym of Evastria Ochsenheimer, I8I6, and not subsequently treated as an available name under the Code, Article i I (d).

$\ddagger$ HEMERAPTENA; Sodoffsky, i837, Bull. Soc. imp. Nat. Moscou 1837 (6): 89, Errata 99.

An incorrect original spelling, changed to Hemeroptena in the errata published in the same work and at the same time.

HEMEROPTENA Sodoffsky, i 837, Bull. Soc. imp. Nat. Moscou 1837 (6) : 89; Errata 99.

Proposed as the objective replacement name for Evastria Ochsenheimer, I8I6, which Sodoffsky considered to be inappropriate. The name Hemeroptena Sodoffsky is however unnecessary as there is an earlier objective replacement name, Eustrotia Hübner, [I82 I].

HYDRELIA Guenée, I84I, Annls Soc. ent. Fr. 10: 227.

Type-species: Phalaena uncula Clerck, I759 (but included by Guenée as 'unca Esp.', an incorrect authorship), by subsequent designation by Guenée, I 852 , in Boisduval \& Guenée, Hist. nat. Insectes (Lépid.) 6: 235, but cited as 'unca Lin.', an incorrect authorship.

A junior homonym of Hydrelia Hübner, [I825] I8I6, Vevz. bekannter Schmett.: 322, Lepid., Geometridae. The objective replacement name is Eustrotia Hübner, [I82I].

HYELA Stephens, I85o, List Specimens Br. Anim. Colln. Br. Mus. 5: I 49.

Type-species: Phalaena uncula Clerck, I759 (but included by Stephens as $P$. uncana Linnaeus), by subsequent designation by Moore, I884, Lepid. Ceylon 3: 56, but cited as unca.

Hyela is a junior objective synonym of Eustrotia Hübner, [I82I].

†EUTROSTIA; Chrétien, I93I, Amat. Papillons 5: 26r.

An incorrect subsequent spelling.

EUSTROTIOPIS Hampson, I926, Descr. new Genera Species Lepid. Phalaenae Subfamily Noctuinae Br. Mus.: 207.

OPHI

Type-species: Eustrotiopis chlorota Hampson, I926, ibidem: 207, by original designation. Holotype ô, [KenYa] Brit. E. Afr.: Nairobi (BMNH).

EUTACTIS Hübner, I8 18, Zuträge Samml. exot. Schmett. 1: 28.

AGAR

Type-species: Eutactis tristifica Hübner, I 8I 8, ibidem 1: 28, figs I65, I66, by subsequent designation by Hampson, igor, Cat. Lepid. Phalaenae Br. Mus. 3: 555. Type(s) ô. [Australia] Neuholland.

$\ddagger$ EUT AETIS; Strand, I912, in Aurivillius \& Wagner, Lepid. Cat. 5: 73.

An incorrect subsequent spelling.

†UTAETIS, misspelling. See Eutactis Hübner, I8I8.

AGAR

EUTAMSIA Fletcher, I96I, Ruwenzori Exped. I952 1: I98.

ACRO

Type-species: Hadena indistans Guenée, 1852 , in Boisduval \& Guenée, Hist nat. Insectes (Lépid.) 6:87, by original designation. Type(s), India: central (BMNH).

EUTELEPHIA Hampson, I926, Descr. new Genera Species Lepid. Phalaenae Subfamily Noctuinae Br. Mus.: 49.

ACRO

Type-species: Eutelia aureopicta Kenrick, I9I7, Trans. ent. Soc. Lond. 1917: 9I, pl. 4 , by original designation. Type(s), Madagascar (BMNH).

EUTELES Gistl, I848. See Laspeyria Germar, I8I .

OPHI

EUTEL1A Hübner, [I823] I8I6, Verz. bekannter Schmett.: 259.

EUTE

Type-species: Noctua adulatrix Hübner, [I8I3], Samml. eur. Schmett. 4: pl. I I I, fig. 5I7, by monotypy. Type(s), Europe.

†EUTESIA; Hübner, [I 826] r8I6, Verz. bekannter Schmett. Anzeiger: 38.

An incorrect subsequent spelling. 
EURHIPIA Boisduval, I828, Eur. Lepid. Index meth.: 73.

Type-species: Noctua adulatrix Hübner, [I8I3], by monotypy.

Eurhipia is a junior objective synonym of Eutelia Hübner, [1823].

$\ddagger$ ENTELIA; Lower, Igor, Proc. Linn. Soc. N.S.W. 26: 659 .

An incorrect subsequent spelling.

EUTELIELLA Roepke, 1938, Bull. Mus. r. Hist. nat. Belg. 14 (13): 16, 72.

EUTE

Type-species: Euteliella eriopoides Roepke, I938, ibidem 14 (13): I6, by monotypy.

Holotype ơ. [Sulawesi]: N. Celebes (IRSNB, Brussels).

EUTERMINA Hampson, 1926, Descr. new Genera Species Lepid. Phalaenae Subfamily Noctuinae Br. Mus.: 487 .

OPHI

Type-species: Bareia tenebrosa Holland, I894, Psyche, Camb. 7: 90, pl. 3, fig. I7, by original designation. Holotype 9 , WEST AFricA.

$\ddagger$ EUTERPIA, misspelling. See Enterpia Guenée, I850.

ACRO

EUTERPIODES Hampson, I908, Cat. Lepid. Phalaenae Br. Mus. 7: 4. Available, but without included species until Hampson, I910, ibidem 9: xiii, 394. ACRO

Type-species: Megalodes pienaari Distant, I 898, Ann. Mag. nat. Hist. (7) 1: 223, by subsequent monotypy. Syntypes, South AFrica: Transvaal, Pienaars River (BMNH); Pretoria (BMNH).

$\ddagger$ EUTESIA, misspelling. See Eutelia Hübner, [I 823].

EUTE

EUTHALES Hübner, [I820] I816, Verz. bekannter Schmett.: 205.

ACro

Type-species: Noctua algae Fabricius, I775, Syst. Ent.: 6I 4, by subsequent designation by Hampson, igo8, Cat. Lepid. Phalaenae Br. Mus. 7: 618. Type(s), Germany (Type(s) not found by Zimsen, 1964, Type Material of I. C. Fabricius: 524).

See also Jaspidia Hübner, 1822.

EUTHEIAPLUSIA Dufay, 1970, Faune Madagascar 31: Io.

PLUS

Type-species: Phytometra pratti Kenrick, r917, Trans. ent. Soc. Lond. 1917: 94, pl. 3, by original designation. Lectotype + , Madagascar (BMNH), designated (as holotype) by Dufay, I970, ibidem 31: I 2.

EUTHEMONIA Gistl, I848. See Xanthia Ochsenheimer, I8I6.

CUCU

EUTHERMESIA Butler, I896, Entomologist 29: 280.

$\mathrm{OPHI}$

Type-species: Thermesia inexacta Walker, I865, List Specimens lepid. Insects Colln Br. Mus. 33: I038, by monotypy. Syntypes 2 ö, U.S.[A.] (BMNH).

T. inexacta is a junior subjective synonym of Thermesia absumens Walker, I862, Trans. ent. Soc. Lond. (3) 1 : 106.

See also Metallata Möschler, I89o.

EUTHISANOTIA Hübner, [I83I]. See Xanthopastis Hübner, [1821]. HADE

EUTHYTOMA Turner, 1920. See Psendozarba Warren, 1913. Acon

EUTOLYPE Grote, 1874, Proc. Acad. nat. Sci. Philad. 1874: 198. cucu

Type-species: Eutolype rolandi Grote, 1874, ibidem 1874: 198, by monotypy. Syntypes of, [U.S.A.]: Massachusetts; Missouri; (BMNH, without locality data).

EUTOREUMA Grote, 1872, Trans, Am. ent. Soc. 4: 21.

OPHI

Type-species: Eutoreuma tenuis Grote, 1872 , ibidem 4:22, by monotypy. Syntypes §. ㅇ [U.S.A.]: central Alabama (BMNH).

EUTORNOPTERA Hampson, 1926, Descr. new Genera Species Lepid. Phalaenae Subfamily. Noctuinae Br. Mus.: 44 r. OPIII

Type-species: Eutornoptera endosticta Hampson, 1926, ibidem: 44I, by original designation. Holotype $\hat{\sigma}$, Cameroun: R. Ja, Bitje (BMNH). 
EUTRICHOPIDIA Hampson, I9OI, Cat. Lepid. Phalaenae Br. Mus. 3: xvii, 554. AGAR

Type-species: Phalaena latinus Donovan, $180_{5}$, Epitome nat. Hist. Insects of New Holland etc.: pl. 32, fig. 4, text overleaf, by original designation. Syntypes [Australia] New Holland: New South Wales and Botany Bay.

EUTRICOPIS Morrison, I875, Ann. Lyceum nat. Hist. 11: 102.

NocT

Type-species: Eutricopis nexilis Morrison, 1875, ibidem 11: 102, by monotypy. Type(s), [U.S.A.]: Colorado.

EUTRINITA Hampson, I924, Ann. Mag. nat. Hist. (9) 13: $45 \mathrm{I}$.

Type-species: Eutrinita ferruginea Hampson, 2024 ibidem (9) 13: 452 , by original designation. Holotype $\hat{o}$, TRINidAD: Caparo (BMNH).

EUTROGIA Hampson, 1926, Descr. new Genera Species Lepid. Phalaenae Subfamily Noctuinae Br. Mus.: 575 .

OPHI

Type-species: Egnasia castanea Moore, 1882, in Hewitson \& Moore, Descr. new Indian lepid. Insects Colln late MrW.S. Atkinson: 184, by original designation. Syntypes, India: [Assam], Khasia Hills (BMNH); [W. Bengal], Darjiling (MNHU, Berlin).

$\ddagger$ EUTROSTIA, misspelling. See Eustrotia Hübner, [182 I] 。 ACON

EUXENISTIS Warren, I910, in Seitz, Macrolepid. World. 3: I40.

CUCU

Type-species: Hadena amicina Staudinger, I896, Dt. ent. Z. Iris. 8: 322 , by original designation. Syntypes $3 \hat{0}$, [CHINA]: Tibet (MNHU, Berlin).

The date of publication of the part of Seitz containing page I4O, was stated on page I33 as I9ro May 5th in the English edition and as I9I I January I3th in the German edition.

EUXESTIS Lederer, I 869, Horae Soc. ent. ross. 6: 88.

CHLO

Type-species: Euxestis dentula Lederer, r869, ibidem 6: 89, pl. 5, fig. 8, by monotypy. Type(s) ô. [IRAN] Persia: [Gorgan] Astrabad.

PSEUDOCALPE Hampson, I894, Fauna Br. India (Moths) 2: xxi, 566.

Type-species: Bankia angulifera Moore, i 881, Proc. zool. Soc. Lond. 1881: 373, by original designation. Type(s), [INDIA]: N. W. Himalaya (BMNH).

A junior homonym of Pseudocalpe Möschler, I890,-Lepid., Noctuidae. There is no objective replacement name but $B$. angulifera is a junior subjective synonym of Euxestis dentula Lederer, I869, the type-species of Euxestis Lederer, I869. The latter is therefore available for use as a subjective replacement name.

EUXOA Hübner, [I82 I] I8I6, Verz. bekannter Schmett. : 209.

NOCT

Type-species: Noctua decora [Denis \& Schiffermüller], I775, Ankündung syst. Werkes Schmett. Wienergegend: 313, by subsequent designation by Hampson, 1903, Cat. Lepid. Phalaenae Br. Mus. 4: I53. Type(s), [Austria]: Vienna district (Collection destroyed, Horn \& Kahle, 1936, Ent. Beih. Berl.-Dahlem 3: 243).

$N$. decora has been cited by some authors as Euxoa nivens Hübner, [1821] r816, ibidem: 209, proposed, unnecessarily, as an objective replacement name for Noctua decora which Hübner considered to be a junior homonym of Phalaena (Noctua) decora Linnaeus, I764, Mus. Lud. Ulv.: 382, - Lepid., Agaristidae.

MIMETES Hübner, [1821] I816, Verz. bekannter Schmett.: 2 Io.

Type-species: Noctua nebulosa Hübner, [r8o8], Samml. eur. Schmett. 4: pl. 86, fig. 402 (but included by Hübner, [I82I], as $¥ M$. mubilosa, an incorrect subsequent spelling), by subsequent designation by Hampson, 1903, Cat. Lepid. Phalaenae Br. Mus. 4: i 53, (but cited as Noctua decora [Denis \& Schiffermüller, I775], which was placed on page 240 as the senior synonym of $N$. nebulosa). Type(s), Europe.

A junior homonym of Mimetes Eschscholtz, r818, Mém. Acad. imp. sci. St. Petersb. 6: 467 ,-Coleoptera. There is no objective replacement name but $N$. nebulosa is a junior subjective synonym of Noctua decora [Denis \& Schiffermüller], I775, the type-species of 
Euxoa Hübner, [182 r]. The latter is therefore available for use as a subjective replacement name.

†.MIMETIS; Hampson, 1903, Cat. Lepid. Phalaenae Br. Mus 4: I53.

An incorrect subsequent spelling.

EUXOAMORPHA Franclemont, 1950, Proc. ent. Soc. Wash. 52: 40.

NocT

Type-species: Euxoamorpha eschata Franclemont, 1950, ibidem 52: 40, fig. I, by original designation. Holotype $\hat{\sigma}$, ChILE: [Tierra del Fuego], Punta Arenas (USNM, Washington).

EUXOOTERA Fletcher, 1961, Ruwenzori Exped. I952 1: I85.

NOCT

Type-species: Euxootera callima Fletcher, I96I, ibidem 1: I85, figs II \& I55-157,

by original designation. Holotype $\hat{\jmath}$, UGanda: Ruwenzori, Nyamaleju (BMNH).

EUXOULLIA Berio, 1973, Annali Mus. civ. Stor. nat. Giacomo Doria 79 : 135.

Cucu

Type-species: Euxoullia tanzaniae Berio, I973, ibidem 79: I35, fig. 18, by original designation. Holotype $\widehat{0}$, Taxzania: [Tanganyika], Ikonda (Colln E. Berio, Genoa).

EUZANCLA Turner, 1932, Trans. R. Soc. S. Aust. 56: 184.

HYPE

Type-species: Euzancla rhopalophora Turner, 1932, ibidem 56: I84, by monotypy. Holotype 3. Australia: Qd, Coolangatta (ANIC, Canberra).

EVANINA Boursin, 1963, Bull. mens. Soc. linn. Lyon 32: 297.

CUCU

Type-species: Evisa wiltshirei Boursin, 1957, ibidem 26: 247, by original designation. Holotype ․․, AfGhanistan: Djebel Seratsch, Kohdaman.

EVIA Walker, i863, List Specimens lepid. Insects Colln Br. Mus. 27: 89.

STIC

Type-species: Evia ferrinalis Walker, I863, ibidem 27:89, by monotypy. Type(s) 우, BORNEO: Sarawak (BMNH).

EVIRIDEMAS Barnes \& Benjamin, 1929, Bull. Brooklyn ent. Soc. 24: т 66.

ACRO

Type-species: Viridemas minuta Barnes \& McDunnough, I910, Jl N.Y. ent. Soc. 18: I56, by original designation. Holotype ô. [U.S.A.]: Tex., Kerrville.

EVISA Reisser, 1930, Z. öst. EntVer. 15: 2.

ACRO

Type-species: Evisa schawerdae Reisser, I930, ibidem 15: 4, pl. I, by original designation. Syntypes $2 \hat{\jmath}$, [France] : Corsica, Evisa, Col de Sevi (NM, Vienna).

EVONIMA Walker, I865, List Specimens lepid. Insects Colln Br. Mus. 32: $505 . \quad$ Noli

Type-species: Evonima aperta IValker, I 865, ibidem 32: 506, by monotypy. Syntypes 2 q, JAVA (BMNH).

EXAERETA Hübner, [1820] I8 I6, Verz. bekannter Schmett.: 200.

NOTODONTIDAE

Type-species: Noctua ulmi [Denis \& Schiffermüller], 1775, Ankïndung syst. Werkes

Schmett. Wienergegend: 66 , by monotypy.

Exaereta was originally placed in the Noctuidac.

$\ddagger E X E R E T A$; Hübner, [1826] I816, Verz. bekannter Schmett. Anzeiger: 38 .

An incorrect subsequent spelling.

EXARNIS Hübner, [I82I] I8I6, Verz. behannter Schmett.: 225.

NOCT

Type-species: Noctua ruris Hübner, [I809], Samml. eur. Schmett. 4: pl. 89, fig. 4I6, by subsequent designation by Hampson, 1903, Cat. Lepid. Phalaenac Br. Mivs. 4: I53, but cited as 'obelisca Schiff.' which Hampson placed, on page 182, as the senior synonym of ruris (sce below). Type(s), Europe.

$N$. ruris is a junior subjective synonym of Noctua temera Hübner, [1808], Samml. eur. Schmett. 4: pl. 84, fig. 393. Both are nowadlays accepted as referring to a species distinct from Noctua obelisca [Denis \& Schiffermüller], I775. 
EXATHETIS Janse, 1938, Moths S. Afr. 3: 283.

ACRO

Type-species: Athetis strigata Hampson, I9I I, Ann. Mag. nat. Hist. (8) 8: 437, by original designation. Holotype ㅇ, [KENYA $]$ Br. E. Africa (BMNH).

$\ddagger$ EXERETA, misspelling. See Exaeveta Hübner, [I820].

NOTODONTIDAE

$\ddagger$ EXOPHILA, misspelling. See Exophyla Guenée, I $84 \mathrm{I}$.

OPHI

EXOPHYLA Guenée, I84I, Ammls Soc. ent. Fr. 10: 248.

OPHI

Type-species: Noctua rectangularis Hübner, [I 828], Samml. eur. Schmett. 4: pl. I59, figs 743, 744, by subsequent designation by Warren, 19I3, in Seitz, Gross-Schmett. Evde 3: 375 , but cited for $\ddagger$ Exophila, an incorrect subsequent spelling. Type(s), Europe.

$\ddagger E X O P H I L A$; Guenée, in Boisduval \& Guenée, Hist. nat. Insectes (Lépid.) 6: 4 I 9.

An incorrect subsequent spelling.

EXSULA Jordan, I896, in Rothschild \& Jordan, Novit. zool. 3: 35, 4I.

AGAR

Type-species: Eusemia dentatrix Westwood, I848, Cabinet oriental Ent.: 68, pl. 33, fig. 5, by original designation. Lectotype, InDIA: Assam (UM, Oxford), designated (as type) by Swinhoe, I892, Cat. east. and Aust. Lepid. Heterocera 1: I6I.

$\ddagger \boldsymbol{E X U A}$, misspelling. See Escua Walker, I858.

OPHI

EXYRA Grote, 1875, Check List Noctuidae Am. N. of Mex.: 26.

ACON

Type-species: Xanthoptera semicrocea Guenée, I852, in Boisduval \& Guenée, Hist. nat. Insectes (Lépid.) 6:24I, by original designation. Type-specimen, none; in the original description Guenée states 'Je ne connais cette Xanthoptère que par un dessin d'Abbot, d'après lequel elle différerait de la $[X$.$] semiflava ...' Abbot's drawing is not known$ to have been published, but from Guenée's description the identity of this species is not in doubt. TYPE-LOCALITY not stated [U.S.A.].

FACIDIA Walker, I865, List Specimens lepid. Insects Colln Br. Mus. 33: 95 I.

OPHI

Type-species: Facidia nigrofusca Walker, I865, ibidem 33: 952, by monotypy. Holotype $\hat{\sigma}$, [South Africa]: Natal (BMNH).

$F$. nigrofusca is a junior subjective synonym of Tavia vacillans Walker, I858, ibidem 15: 1820 .

FACIDINA Hampson, 1926, Descr. new Genera Species Lepid. Phalaenae Subfamily Noctuinae Br. Mus.: 94.

OPHI

Type-species: Ischyja polystigma Lower, I903, Trans. R. Soc. S. Aust. 27: 36, by original designation. Holotype, Australia: Qd, Mackay (SAM, Adelaide).

FAGITANA Walker, I865, List Specimens lepid. Insects Colln Br. Mus. 32: 645.

ACRO

Type-species: Fagitana lucidata Walker, I865, ibidem 32: 645, by monotypy.

Holotype + , TYPE-LOCALITY not known (BMNH). [U.S.A.].

$F$. lucidata is a junior subjective synonym of Leucania littera Guenée, I 852, in Boisduval

\& Guenée, Hist. nat. Insectes (Lépid.) 5: $7 \mathrm{I}$.

See also Psendolimacodes Grote, I874.

FALA Grote, I875, Proc. Acad. nat. Sci. Philad. 1875: 425.

ACRO

Type-species: Fala ptycophora Grote, 1 875, ibidem 1875: 426, by monotypy. Type(s) $\widehat{0}$. [U.S.A.]: California (BMNH).

FALANA Moore, I882, in Hewitson \& Moore, Descr. new Indian lepid. Insects Colln late Mr W. S. Atkinson: I 53 .

OPHI

Type-species: Falana sordida Moore, I882, ibidem: I54, by monotypy. Syntypes, India: Assam, Cherra Punji (BMNH).

FALCAPYRIS Berio, I959, Annali Mus. civ. Stor. nat. Giacomo Doria 71: 93.

$\operatorname{ACON}$

Type-species: Cerynea falcigera Berio, I959, ibidem 71: 93, fig. I3, by original desig- 
nation. Holotype 3., MADAgAScAR: Perinet, forêt d'Analamazoatrá.

Falcapyris was proposed as a subgenus of Cerynea Walker, I 859.

FALCIMALA Hampson, I 895, Fanna Br. India (Moths) 3: xiv, 5 I.

HYPE

Type-species: Pseudaglossa atrata Butler, 1889, Illust. typical Specimens Lepid.

Heterocera Colln Br. Mus. 7: 17, 88, pl. I34, fig. 6, by original designation. Syntypes

[INDIA]: [Punjab], Dharmsala (BMNH).

FARARA Bethune-Baker, 1908, Novit. zool. 15: 203.

$A C O N$

Type-species: Farara pulchra Bethune-Baker, 1908, ibidem 15: 203, by original designation. Type(s) ô, NEw Guinea: [Papua], Dinawa (BMNH).

FARONTA Smith, 1908, Ann. N.Y. Acad. Sci. 18: 106.

HADE

Type-species: Faronta aleada Smith, 1908, ibidem 18: 107, by monotypy. Syntypes I o. 3 \%. [U.S.A.]: Tex., Brazos.

FAUTAUA Collenette, 1929, Trans. ent. Soc. Lond. 76: 484.

HY PE

Type-species: Fautaua diagonalis Collenette, 1929, ibidem 76:484, pl, 21, fig. 7, by original designation. Holotype $\widehat{\sigma}$. Society Is: Tahiti (B.INH).

FELINIA Guenée, I852, in Boisduval \& Guenée, Hist. nat. Insectes (Lépid.) 7: 322.

OPHI

Type-species: Felinia spissa Guenée, I852, ibidem 7: 322, by monotypy. Type(s),

[Banglanesh]: [E. Bengal], Silhet (UM, Oxford).

FELINIOPSIS Roepke, I938, Bull. Mus. r. Hist. nat. Belg. 14(I3): I4, 72.

ACRO

Type-species: Feliniopsis incerta Roepke, 1938, ibidem 14 (13): I5, by monotypy. Holotype ô. [Sulawesi] : న. Celebes.

FELTIA Walker, I856, List Specimens lepid. Insects Colln Br. Mus. 9: 165, 202.

NOCT

Type-species: Feltia ducens Walker, 1856 , ibidem 9: 203, by monotypy. Holotype

o. Canada: [Ontario, Orillia] Orilla (BMNH).

FENARIA Grote, 1882, Papilio 2: 132.

AGAR

Type-species: Fenciria sevorsa Grote, 1882 , ibidem 2: 132, by monotypy. Holotype, [U.S.A.]: Arizona.

FERALIA Grote, 1874, Bull. Buffalo Soc, nat. Sci. 2: 6, 58.

CUCU

Type-species: Diphtera jocosa Guenée, I 852, in Boisduval \& Guenée, Hist. nat. Insectes (Lépid). 5: 37, by original designation. Holotype +, Norti America (BMNH).

FEREDAYIA Kirkaldy, i910, Can. Ent. 42:8.

HADE

Type-species: Erana graminosa Walker, 1857, List Specimens lepid. Insects Colln Br. Mus. 11: 605, by monotypy (of Evana Walker). Holotype 3 . New Zealand (BMNH).

Feredayia was proposed as the objective replacement name for Erana Walker, I 857.

ERANA Walker, 1857, List Specimens lepid. Insects Colln Br. Mus. 11: 495, 605.

Type-species: Erana graminosa Walker, 1857 , by monotypy.

A junior homonym of Erana Gray, 18.40 , List Genera Birds: $4^{8},-1$ ves. The objective replacement name is Feredayia Kirkaldy, 1910.

FERENTA Walker, [1 858] I 857, List Specimens lepid. Insects Colln Br. MIus. 12:941, 96 I. opuI Type-species: Phalaena stolliana Stoll, I780, in Cramer, Uitlandsche Kapellen 4: 4I, pl. 3io, figs A, B, by monotypy. Type(s), Surinam.

$P$. stolliana is dated from the wrapper of the part and not from the title-page of the volume, 1782 .

CORONIS Hübner, [1823] i8 16, Verz. bekannter. Schmett.: 265

Type-species: Phalaena stolliana Stoll, I 780 , by monotypy, but cited by Hübner as C. stollii, an unjustified emenclation.

A junior homonym of Coronis Desmarest, i 823, Dictionnaire Sci. nat. 28: 345, - Crustacea. The objective replacement name is Ferenta Walker, [1858]. 
FERGANA Staudinger, I892, Dt. ent. Z. Iris 5: 166.

ACRO

Type-species: Fergana oreophila Staudinger, I892, ibidem 5: 166, pl. 2, fig. 9, by monotypy. Syntypes ô. [U.S.S.R.]: [Fergana dist.], Alai Mts; Samarkand Prov. (MNHU, Berlin).

Fergana was originally placed in the Geometridae.

FISHIA Grote, I877, Can. Ent. 9:21.

NOCT

Type-species: Fishia enthea Grote, 1877, ibidem 9: 21, by monotypy. Type(s), [U.S.A.]: Maine, Oldtown (BMNH).

FLAMMONA Walker, [1863] i 864, J. Proc. Linn. Soc. (Zool.) 7: 55.

$\mathrm{ACON}$

Type-species: Flammona quadrifasciata Walker, [1863] I 864, ibidem 7: 55, by monotypy. Lectotype, Borneo: Sarawak (UM, Oxford), described (as type) by Swinhoe, I90o, Cat. east. and Aust. Lepid. Heterocera 2: 66.

FLA VALA Berio, 1966. See Thioptera Franclemont, I950.

ACON

FLETA Jordan, I896, in Rothschild \& Jordan, Novit. zool. 3: 34.

AGAR

Type-species: Agarista belangeri Guérin-Méneville, I834, in Bélanger, Toyage aux Indes-Orientales (Zool.): 506, Insectes pl. 5, fig. 3, by original designation. Type(s), JAVA.

FLETCHEREA Viette, 1961, Bull. Soc. ent. Fr. 66: 45.

CUCU

Type-species: Fletcherea pauliani Viette, I96r, ibidem 66: 46 , by original designation. Holotype ô., Madagascar: Betsileo, forêt d'Ambatofitorahana (MNHN, Paris).

FLOCCIFERA Hampson, I 894, Fauna Br. India (Moths) 2: xiii, 28 I.

ACRO

Type-species: Aletia erigida Swinhoe, 1890, Trans. ent. Soc. Lond. 1890: 221, by original designation. Type(s), Burma: Thyetmyo (BMNH).

FOCILLA Guenée, I852. See Euclystis Hübner, I823.

OPHI

FOCILLIDIA Hampson, I9I3, Cat. Lepid. Phalaenae Br. Mus. 12: 7. Arailable but without included species until Hampson, I9I3, ibidem 13: I 48.

CATO

Type-species: Focillidia texana Hampson, I9I3, ibidem 13: I48, by subsequent designation by Hampson, I9I3, ibidem 13: I+8. Holotype 9 , U.S.A. (BMNH).

FOCILLSTIS Hampson, I926, Descr. new Genera Species Lepid. Phalaenae Subfamily Noctuinae Br. Mus.: 599.

OPHI

Type-species: Zethes salsoma Swinhoe, I902, Ann. Mag. nat. Hist. (7) 10: 499, by original designation. Syntypes $\hat{o}$,, , Borneo: [Sabah], Kina Balu (BMNH).

FOCILLODES Bethune-Baker, I 906, Novit. zool. 13: 28 I.

OPHI

Type-species: Focillodes dinawa Bethune-Baker, 1906, ibidem 13: 282, by original designation. Syntypes $\hat{0}$, 오, New Guinea: [Papua], Dinawa (BMNH); Aroa River.

FOCILL OPIS Hampson, I926, Descr. new Genera Species Lepid. Phalaenae Subfamily . Voctuinae Br. Mus.: 5 I0.

OPHI

Type-species: Focillopis eclipsia Hampson, I926, ibidem: 5 Io, by original designation. Syntypes I $\delta, 2$ ㅇ. CAMERoun: R. Ja, Bitje (BMNH).

FODINA Guenée, I 852, in Boisduval \& Guenée, Hist. nat. Insectes (Lépid) 7: 274 . OpH

Type-species: Fodina oriolus Guenée, I852, ibidem 7: 274, pl. 22, fig. 5, by subsequent designation by Moore, [1885], i887, Lepid. Ceylon 3: I8 r. Type(s), [Bangladesh]: [E. Bengal], Silhet (BMNH).

FOLKA Swinhoe, 1917, Ann. Mag. nat. Hist. (8)20: I63.

OPHI

Type-species: Hypena oxydata Hampson, I898, J. Bombay nat. Hist. Soc. 11: 707. by original designation (for Anepa Swinhoe, i905). Holotype ô. India: [Assam], Khasis (BMNH). 
Folka was proposed as the objective replacement name for Anepa Swinhoe, I905.

ANEPA Swinhoe, 1905, Ann. Mag. nat. Hist. (7) 15: 502.

Type-species: Hypena oxydata Hampson, by original designation.

A junior homonym of Anepa Swinhoe, 1903, Trans. ent. Soc. Lond. 1903: 478, - Lepid.,

Lymantriidae. The objective replacement name is Folka Swinhoe, rgr 7.

FORMOSAMYNA Strand, 1920, Arch. Naturgesch. 84 (A) I2: Ir9.

$\operatorname{ACON}$

Type-species: Amyna frontalis Strand, r920, ibidem 84 (A)I2: II9, by monotypy.

Syntypes 20 , [TaIvan] Formosa: Kosempo (DEI, Eberswalde).

Formosamyna was described as a subgenus of Amyna Guenée, I 852.

FORSEBIA Richards, 1936, Can. Ent. 67: 264.

OPH I

Type-species: Melipotis perlaeta Edwards, I882, Papilio 2: 14, by original designation.

Holotype, [U.S.A.]: Arizona, Prescott.

FOTA Grote, I882, Can. Ent. 14: I74.

ACRO

Type-species: Fota armata Grote, I882, ibidem 14: 175, by monotypy. Type(s) 웅. [U.S.A.]: Arizona.

FOTELLA Grote, I882, Can. Ent. 14: I8I.

ACRO

Type-species: Fotella notalis Grote, I882, ibidem 14: r8r, by monotypy. Type(s)

Q. [U.S.A.]: Arizona.

FOTOPSIS Dyar, I918, Proc. U.S. natn. Mus. 54: 347.

ACRO

Type-species: Fotopsis sparganiotis Dyar, I91 8, ibidem $54: 347$, by original designation.

Holotype ô, Mexico: Sierra de Gucrrero (USNM, Washington).

${ }_{\ddagger}$ POTOPSIS; Neave, I940, Nomencl. zool. 3: 882.

An incorrect subsequent spelling.

$\ddagger$ FOVAREOLA Hampson, no published reference found.

HYPE

FOVEADES Bethune-Baker, 1908, Novit. zool. 15: 239.

HYPE

Type-species: Foveades aroensis Bethune-Baker, I9o8, ibidem 15: 239, by original designation. Type(s) $\hat{O}$, NEw Gurnea: [Papua $]$, R. Aroa (BMNH).

FRACARA Walker, I856, List Specimens lepid. Insects Colln Br. Mus. 9: r 37, I 46.

ACRO

Type-species: Phalaena viridata Stoll, I780, in Cramer, Uitlandsche Kapellen 4: 44, pl. 3ir, fig. D, by monotypy. Type(s), Surinam.

$P$. vividata is dated from the wrapper of the part and not from the title-page of the volume, 1782 .

FREDINA Brandt, 1939, Ent. Rdsch. 56: 242.

ACRO

Type-species: Fredina esmeralda Brandt, 1939, ibidem 56: 242, pl. r, fig. 6, by monotypy. Syntypes $\hat{0}$, ㅇ. Iran: Baluchistan, Bender Tchahbahar.

FREILLA Druce, I 89o, Biologia cent.-am. (Zool.) Lepid. Heterocera 1: 4 ro.

OPHI

Type-species: Freilla variabilis Druce, I890, ibidem 1: 4 IO, pl. 33, fig. I9, by subsequent designation by Hampson, 1926, Descr. new Genera Species Lepid. Phalaenae Subfamily Noctuinae 73r. Mus.: 453. Syntypes, Mexico: Tabasco, Teapa (BMNH). Guatemala (BMNH). Panama (BMNH).

FRIESIA Barnes \& McDunnough, I9I2, Can. Ent. 44: 18, 57.

OPHI

Type-species: Friesia anormalis Barnes \& McDunnough, I912, ibidem 44: I 8, fig. I, by monotypy. Type(s), North America.

F. anomalis is a junior subjective synonym of Prosoparia perfuscaria Grote. I883, Can. Ent. 15: гзо.

See also Prosoparia Grote, 1883. 
FRU V A Grote, 1877, Can. Ent. 9: 69.

$\operatorname{ACON}$

Type-species: Spragueia fasciatella Grote, I875, ibidem 7:225, by original designation. Syntypes 2 ex., [U.S.A.]: Texas (BMNH).

FULVARBA Berio, 1950, Annali Mus. civ. Stor. nat. Giacomo Doria 64: 56.

ACON

Type-species: Ozarba fulvescens Hampson, I910, Cat. Lepid. Phalaenae Br. Mus. 10 : 427, pl. I6I, fig. 26, by original designation. Holotype $\sigma^{\star}$. [South AFricA]: Transvaal (BMNH).

GAALA Walker, [1866] i 865, List Specimens lepid. Insects Colln Br. Mus. 34: I I28. HyPE Type-species: Gaala dispunctalis Walker, [1 866] I 865, ibidem 34: I I 29, by monotypy. Type(s) ô, BraziL.

G. dispunctalis is a junior subjective synonym of Platydia abditalis Walker, [1859] I858, ibidem 16: I I.

See also Gustiana Walker, I86r.

GABALA Walker, [1866] i 865, List Specimens lepid. Insects Colln Br. Mus. 34: I 220. CHLO Type-species: Gabala polyspilalis Walker, [1866] I865, ibidem 34: I221, by monotypy. Holotype ô. [INDIA]: Hindostan (BMNH).

GABARA Walker, I866, List Specimens lepid. Insects Colln Br. Mus. 35: I 740.

OPH I

Type-species: Gabara subnivosella Walker, I866, ibidem 35: I740, by monotypy. Holotype o., U.S.[A.] (BMNH).

GABERASA Walker, [1866] I865, List Specimens lepid. Insects Colln Br. Mus. 34: I I97. HYPE Type-species: Gaberasa ambigualis Walker, [1 866] I 865, ibidem 34: I I98, by monotypy. Holotype ô, North America (BMNH).

See also Eulintneria Grote, I88I.

GABRISA Walker, I865. See Cardalena Walker, I 859.

HYPE

GABYNA Möschler, I88o, Verh. zool.-bot. Ges. Wien 30 (Abh.): $445 . \quad$ орн I

Type-species: Gabyna coerulina Möschler, I 88o, ibidem 30 (Abh.) 445, pl. 9. fig. 47, by subsequent designation by Berio, 1966, Annali Mus. civ. Stor. nat. Giacomo Doria 76: 58. Holotype $\widetilde{đ}$, Surinam: interior (MNHU, Berlin).

Incorrect type-species designation: Amphigonia placida Butler, I879, a name not originally included in Gabyna, and not linked with one of the originally included names when cited by Hampson, 1926, Descr. new Genera Species Lepid. Phalaenae Subfamily Noctuinae Br. Mus.: 470.

G. coerulina is a junior subjective synonym of Amphigonia placida Butler, I879, Trans. ent. Soc. Lond. 1879: 57 .

GADERA Walker, [I 858] i 857, List Specimens lepid. Insects Colln Br. Mus. 12: 94I, 964. OPH I Type-species: Gadera incitans Walker, [1858] 1857, ibidem 12: 964, by subsequent designation by Hampson, 1926, Descr. new Genera Species Lepid. Phalaenae Subfamily Noctuinae Br. Mus.: 36r. Holotype ô, Type-Locality not known (BMNH). [Mexico. Costa Rica].

GADIRTHA Walker, [1858] I 857, List Specimens lepid. Insects Colln Br. Mus. 13: I079, I I02.

SARR

Type-species: Gadirtha impingens Walker, [1858] I857, ibidem 13: II03, by subsequent designation by Moore, [I885] I887, Lepid. Ceylon 3: Ior. Syntypes [India]: I o. Hindostan (BMNH with added label Burma, Moulmein). Hong Kong: i ㅇ.

G. impingens is a junior subjective synonym of Gadivtha inexacta Walker, [1858] I857, ibidem 13: I I02.

GAEDEODES Fletcher \& Viette, 1955, Bull. Inst. fr. Afr. noive 17(A): I81. HYPE

Type-species: Gaedeodes testacea Fletcher \& Viette, I955, ibidem 17 (A): 182, p1. I, 
fig. 9, by original designation. Holotype of. French Guinea: Mont Nimba, Zicla (MNHN, Paris).

GAEDONEA Berio, r966, Annali Mus. civ. Stor. nat. Giacomo Doria 76: i 28.

OPHI

Type-species: Gaedonea rosealutea Berio, I966, ibidem 76: 128 , fig. 17 , by original designation. Holotype $\widehat{\jmath}$. Madagascar: N.E., Maroantsetra, Forêt d'Ambodivoangy (MNHN, Paris).

$\ddagger G A L A C R A S P I A$ Hampson, no published reference found.

HYPE

GALACTOMOIA Fawcett, i 9i 6, Proc. zool. Soc. Lond. 1916: 7i 6.

OPHI

Type-species: Galactomoia berenice Fawcett, I9I6, ibidem 1916: 7I7, pl. I, fig. I 5, by original designation. Syntypes I $\hat{\delta}$, I $q$, [Kenya]: Masongaleni (BMNH). [Ghana]: Kumasi (BMNH).

GALANDA Walker, [1859] i 858, List Specimens lepid. Insects Colln Br. Mus. 16: 250. opH Type-species: Galanda hebrusalis Walker, [1 859$]$ I 858 , ibidem 16: $25 \mathrm{I}$, by monotypy. Type(s) ô. Brazil (UM, Oxford).

GALAPHA Walker, I858, List Specimens lepid. Insects Colln Br. Mus. 15: I537, I 544. OPH Type-species: Galapha antecedens Walker, I858, ibidem 15: 1544, by monotypy. Holotype đే, [BRAzIL]: [R. Amazon], [Tefé] Ega (BMNH).

GALAPHA Walker, I858, List Specimens lepid. Insects Colln Br. Mus. 15: i850. See Tephrias Wallengren, i 860 .

OPHI

GALEANA Köhler, (1952) r95I, Acta zool. lilloana 12: I 50.

CUCU

Type-species: Galeana basilinea Köhler, (1952) I95I, ibidem 12: I50, by original designation. Syntypes $\hat{\delta}, \mathcal{q}$. Argentrina: Chubut [Prov.], Comodoro Rivadavia (ZSBS, Munich); [Mendoza Prov.], Mendoza (ZSBS, Munich).

GALGULA Guenée, I 852, in Boisduval \& Gucnée, Hist. nat. Insectes (Lépid.) 6: $239 . \quad$ acro Type-species: Galgula hepara Guencee, i 852, ibidem 6:239, pl. 1o, fig. I r, by subsequent designation by Grote, 1874, Bull. Buffalo Soc. nat. Sci. 2: 36. Syntypes 2 q. North AMERICA.

G. hepara is a junior subjective synonym of Galgula partita Guenée, I 852, ibidem 6: 239.

GALLERIDIA Hampson, I 896, Fauna Br. India (Moths) 4: 499.

OPHI

Type-species: Galleridia atrisigna Hampson, 1896, ibidem 4: 499, fig. 266, by original designation. Type(s) ठ̊. CEYLON (BMNH).

GALLERIOMORPHA Nietner, I86I, Observations Enemies Coffee Tree Ceylon: 3, 23. SARR Type-species: Galleriomorpha lichenoides Nietner, I86I, ibidem: 3, 23, by monotypy. Type(s), Cerlon.

The binomen of the type-species was attributed by Nietner to 'Feld. in litt.' Nictner must therefore have obtained the names from Felder presumably associated with specimens determined by Felder.

G. lichenoides is a junior subjective synonym of Gadirtha decrescens Walker, [1858].

See also Plotheia Walker, [1858]; Egelesta Walker, I858; Othora Walker, I865.

GALLERIOMORPHA Felder \& Felder, 1862, Wien. ent. Monatschr. 6: 37.

Type-species: Galleriomorpha lichenoides Nietner, 1861, by monotypy.

A junior homonym of Galleviomorpha Nietner, r $86 \mathrm{r}$. The binomen of the type-species was used, described and made nomenclaturally available by Nietner. Felder \& Felder attributed $G$. lichenoides to themselves but cited Nietner as the reference. The objective replacement name is Galleriomorpha Nietner, I86r.

GALLERIOMORPHA Felder \& Felder, I 862. See Galleviomorpha Nietner, 1861. 
GAMMACE Walker, 1862, Trans. ent. Soc. Lond. (3)1: 103.

OPH I Type-species: Gammace magniplaga Walker, 1862, ibidem (3)1: 103, by monotypy. Type(s) ô, BrazIL (UM, Oxford).

GANGRA Walker, x862, Trans. ent. Soc. Lond. (3)1: 104.

OPHI Type-species: Gangra atripustula Walker, 1862, ibidem (3)1: 105, by monotypy. Type(s) 우, Type-locality unknown (UM, Oxford) [Central America].

GAPHARA Walker, 1862, Trans. ent. Soc. Lond. (3)1: 96.

Type-species: Gaphara sobria Walker, I862, ibidem (3)1: 96, by monotypy. Type(s) o., TYPE-LOCALITY unknown (UM, Oxford). [BRAZIL].

G. sobria is a junior subjective synonym of Celaena inquieta Walker, 1858 , List Specimens lepid. Insects Colln Br. Mus. 15: 1687.

GARELLA Walker, [1863] 1864, J. Proc. Limn. Soc. (Zool.) 7: 5 I.

Type-species: Garella rotundipennis Walker, [1863] I864, ibidem 7: 52, by monotypy. Type(s) +, BORnEo: Sarawak (UM, Oxford).

See also Dendrothripa Hampson, I 896.

GARGAZA Walker, I866, List Specimens lepid. Insects Colln Br. Mus. 35: 1733.

ACRO Type-species: Gargaza tristrigella Walker, I866, ibidem 35: I734, by monotypy. Holotype $\hat{\sigma}$, TYPE-LOCALITY unknown (BMNH) [CANADA. U.S.A.].

See also A sisyra Grote, 1873 .

GARIGA Walker, [I863] I 864, J. Proc. Linn. Soc. (Zool) 7: 59.

CHLO

Type-species: Gariga argentilinea Walker, [1S63] i 864, ibidem 7: 59, by monotypy. Lectotype $\hat{\sigma}$, Borneo: Sarawak (UM, Oxford), designated (as type) by Swinhoe, I9oo, Cat. east. and Aust. Lepid. Heterocera 2: 587.

GAUJONIA Dognin, I891, Naturaliste (2)5: 125.

PANT

Type-species: Gaujonia arbosi Dognin, I891, ibidlem (2)5: I 26, by monotypy. Holotype + , [ECUADOR]: Loja (USNM, Washington).

GAURENA Walker, I865, List Specimens lepid. Insects Colln Br. Mus. 32: 6ig. thyatiridae Type-species: Gaurena florens Walker, I 865, ibidem 32:620, by subsequent designation by Hampson, I893, Fauna Br. India (Moths) 1: 18 I.

Gauvena was originally placed in the Noctuidae.

GAURENOPSIS Houlbert, I92I, in Oberthür, Études Lépid. comparée 18(2): $232 . \quad$ cucu

Type-species: Gaurenopsis velutina Houlbert, I92I, ibidem 18(2): 232, pl. 489, fig. 4028, by original designation. Syntypes 6 o. [CHINA]: [Szechwan Prov.], Ta-tsien-lou, (BMNH); Thibet (BMNH).

G. velutina is a junior subjective synonym of Polia conspicua Leech, I9oo, Trans. ent. Soc. Lond. 1900: 94 .

GAUZANIA Walker, [1866]. See Daona Walker, I864.

OPHI

GELASTOCERA Butler, I877, Ann. Mag. nat. Hist. (4)20: 476 .

CHLO

Type-species: Gelastocera exusta Butler, I877, ibidem (4)20:476, by original designation. Type(s), JaPAN: Hakodaté (BMNH).

Gelastocera was originally placed in the Notodontidae.

GELENIPSA Dyar, 1914, Proc. U.S. natn. Mus. 47: 192.

ACON

Type-species: Gelenipsa psychodidarum Dyar, 1914, ibidem 47: 192, by original designation. Syntypes 5 ô, I , Panama: R. Trinidad; Porto Bello; Cabima; La Chorrera. (USNM, Washington).

GENIASCOTA Hampson, 1926, Descr. new Genera Species Lepid. Phalaenae Subfamily Noctuinae Br. Mus.: 2 II.

OPHI 
Type-species: Geniascota trichoptycha Hampson, 1926, ibidem: 2II, by original designation. Syntypes $2 \hat{0}$, Nigeria: Lagos, Ebute Meta (BMNH).

GEOMETRIMIMA Holland, I894, Psyche, Camb. 7: I 42.

OPHI

Type-species: Geometrimima callista Holland, I894, ibidem 7: I 42, pl. 4, fig. 2, by original designation. Holotype + , West AFrICA (CM, Pittsburgh).

GEORYX Hübner, [1821]. See Agrotis Ochsenheimer, I8I6.

NOCT

GERARCTIA Hampson, 1905, Ann. Mag. nat. Hist. (7)15: 448.

ACON

Type-species: Gerarctia poliotis Hampson, 1905, ibidem (7)15: 449, by original. designation. Holotype q, CANARY Is: Teneriffe (BMNH).

GERBATHA Walker, 1865, List Specimens lepid. Insects Colln Br. Mus. 33: 9r 3. SARR

Type-species: Gerbatha laticincta Walker, 1865, ibidem 33: 913, by monotypy. Holotype ô, CEYLON (BMNH).

G. laticincta is a junior subjective synonym of Bryophila semipars Walker, $185^{8}$, ibidem 15: 1647 .

GERBATHODES Warren, I9I I, in Seitz, Gross-Schmett. Erde 3: 175.

ACRO

Type-species: Gerbatha angusta Butler, I879, Illust. typical Specimens Lepid. Heterocera Colln Br. Mus. 3: ix, 24, pl. 47, fig. 2, by original designation. Type(s), JAPAN: Yokohama (BMNH).

The date of publication of the part of Seitz containing page I 75, was stated on page I73 as I9I I February 8th in both the German and English editions.

GERESPA Walker, [1858] i 857, List Specimens lepid. Insects Colln Br. Mus. 13: I I 42, I I 68. OPH I

Type-species: Gerespa prolata Walker, [I858] 1857, ibidem 13: I I69, by monotypy. Holotype + , JamaICA (BMNH).

GERIA Walker, 1858, List Specimens lepid. Insects Colln Br. Mus. 14: г343, I $366 . \quad$ Cato Type-species: Geria restituta Walker, I 858 , ibidem 14: I366, by monotypy. Syntypes 20 . [Dominican Republic] St Domingo. Venezuela.

G. restituta is a junior subjective synonym of Ophisma ablunaris Guenée, $\mathbf{1} 852$, in Boisduval \& Guenée, Hist. nat. Insectes (Lépid) 7: 237.

GERISA Walker, I858, List Specimens lepid. Insects Colln Br. Mus. 15: I 547, i6 2.

OPHI

Type-species: Gerisa discerpta Walker, I 858, ibidem 15: г6 2, by monotypy. Holotype o. Honduras.

G. discerpta is a junior subjective synonym of Capnodes rufinans Guenée, 1852, in Boiscluval \& Guenée, Hist. nat. Insectes (Lépid.) 7: 377, pl. 24, fig. 9.

GERODA Walker, I 859, List Specimens lepid. Insects Colln Br. Mus. 19: 832.

ACRO

Type-species: Geroda xeneusalis Walker, i 859 , ibidem 19:833, by monotypy. Holotype $\hat{\jmath},[$ BRAzIL] : Rio Janeiro (UM, Oxford).

Geroda when originally proposed contained a second species, but this was doubtfully included and, under the Code, Article 68 (c), is not eligible for designation as type-species.

GERRA Walker, [1865] i 864, List Specimens lepid. Insects Colln Br. Mus. 31: I4t. AGAR

Type-species: Gerra radicalis Walker, [1865] i864, ibidem 31: I4I, by subsequent designation by Kirby, 1892, Synonymic Cat. Lepid. Heterocera 1: 406. Type(s), TypeLOCALITY not stated (BMNH). [MEXICO].

GERRODES Hampson, 1908, Cat. Lepid. Phalaenae Br. Mus. 7: 7. Available, but without included species until Hampson, igIo, ibidem 9: xiii, 4 I8.

AGAR

Type-species: Diamuna longipes Druce, ז889, Biologia cent.-am. (Zool.) Lepid. Heterocera 1: 334, pl. 30, fig. I 8, by subsequent monotypy. Holotype ô. Mexico: Jalapa (BMNH). 
GESONIA Walker, [1859] I858, List Specimens lepid. Insects Colln Br. Mus. 16: 75. OPH Type-species: Gesonia obeditalis Walker, [1859] 1858, ibidem 16: 75, by monotypy. Holotype ô. CEYLON (BMNH).

See also Dragana Walker, [1859]; Hileia Walker, I86I; Maresia Walker, I866; and Amblygoes Butler, I879.

GESPANNA Swinhoe, I9oo, Cat. east. and Aust. Lepid. Heterocera 2: 162.

OPHI

Type-species: Ginaea pectoralis Walker, 1864, J. Proc. Linn. Soc. (Zool.) 7: 190, by monotypy. Lectotype q, Borneo: Sarawak (UM, Oxford), designated (as type) by Swinhoe, 1900, ibidem 2: 163.

GIACLA Walker, I855. See Metopta Swinhoe, I900.

CATO

GIAURA Walker, 1863, List Specimens lepid. Insects Colln Br. Mus. 28: 434. SARR Type-species: Giaura repletana Walker, I863, ibidem 28: 434, by monotypy. Holotype + , Type-locality unknown (BMNH) [Ivory Coast. Sierra Leone. TANZANIA].

GIGAGLOSSA Berio, I966, Annali Mus. civ. Stor. nat. Giacomo Doria 76: I32.

OPHI

Type-species: Gigaglossa macrocula Berio, 1966, ibidem 76: I34, fig. 24, by original designation. Holotype $\hat{\jmath}$, Madagascar: Massif d'Ankaratra, Manjakatompo, Forêt d'Ambahona (MNHN, Paris).

GIGANTOCERAS Holland, I893, Psyche, Camb. 6: 549.

SARR

Type-species: Gigantoceras solstitialis Holland, I893, ibidem 6:549, pl. 2 I, fig. 9, by original designation. Type(s) $\vec{\delta}$. West AFriCA (CM, Pittsburgh).

GIGIA Walker, 1865, List Specimens lepid. Insects Colln Br. Mus. 33: $94 \mathrm{I}$.

OPHI

Type-species: Gigia obliqua Walker, I865, ibidem 33: 942, by monotypy. Holotype o., Type-locality unknown (BMNH) [Eguatorial South America].

See also Dochmiogramma Hampson, 1926.

GIGIDES Hampson, I926, Descr. new Genera Species Lepid. Phalaenae Subfamily Noctuinae Br. Mus.: 455 .

OPHI

Type-species: Gigides megalops Hampson, 1926, ibidem: $45^{6}$, by original designation. Holotype ô. Peru: El Porvenir (BMNH).

GINAEA Walker, I858, List Specimens lepid. Insects Colln Br. Mus. 15: I 546, I637. OPH Type-species: Ginaea removens Walker, 1858 , ibidem 15: 1638, by monotypy. Holotype o, [INDIA]: N. Hindostan (BMNH).

G. removens is a junior subjective synonym of Phalaena umminia Cramer, 1780 .

See also Platyja Hübner, [I823].

GIRIA Fawcett, I9I6, Proc. zool. Soc. Lond. 1916: 7 17.

OPHI

Type-species: Giria bubastis Fawcett, I916, ibidem 1916: 718, pl. I, fig. I4, by original designation. Holotype 우, [KenYa]: Mombasa (BMNH).

$G$. bubastis is a junior subjective synonym of Ophiusa pectinicornis Bethune-Baker, I9o9, Ann. Mag. nat. Hist. (8)3: 432.

GIRPA Walker, I858, List Specimens lepid. Insects Colln Br. Mus. 15: 1849.

OPHI

Type-species: Girpa aliena Walker, I858, ibidem 15: 1849, by monotypy. Holotype o. [Australia]: [Qd], Moreton Bay (BMNH).

GIRTESMA Schaus, I913, Ann. Mag. nat. Hist. (8)11: 13.

HYPE

Type-species: Girtesma messala Schaus, I913, ibidem (8)11: I3, by original designation. Type(s) ô, Costa Rica: Juan Viñas.

GISCALA Walker, I858, List Specimens lepid. Insects Colln Br. Mus. 15: I67x.

OPHI

Type-species: Giscala quadricolor Walker, I858, ibidem 15: I672, by monotypy. Syntypes 30 , Venezuela (BMNH). Brazil: [R. Amazon], [Tefé] Ega (BMNH). 
GISIRA Walker, [1859] I858, List Specimens lepid. Insects Colln Br. Mus. 16: 7I. HYPE Type-species: Gisira acclamalis Walker, [1859] I858, ibidem 16: 7 1, by monotypy. Holotype ô, VeNezuela (BMNH).

GIUBICOLANTA Berio, 1937, Annali Mus. civ. Stor. nat. Giacomo Doria 58: i 76. ACON

Type-species: Giubicolanta orientalis Berio, 1937, ibidem 58: 177 , by original designation. Holotype, [Somali Republic]: Giuba, Belet Amin.

GIZAMA Walker, [1859] I 858, List Specimens lepid. Insects Colln Br. Mus. 16: 192. HYPE Type-species: Gizama midasalis Walker, [1859] 1858, ibidem 16: 193, by monotypy. Holotype ô. VenezUela (B.INH).

Gizama when originally proposed contained a second species, but this was doubtfully included and, under the Code, Article 68 (c), is not eligible for designation as type-species.

$\ddagger G \boldsymbol{L A} \boldsymbol{E} \boldsymbol{A}$, misspelling. See Gloia Hübner, I8 22.

CUCU

GLAEA Stephens, I829. See Gloia Hübner, I822.

CUCU

$\ddagger_{+} \boldsymbol{L} \boldsymbol{A} \boldsymbol{E A E}$ Ochsenheimer, i 816. See Cerastis Ochsenheimer, i 8 I6.

NOCT

$\ddagger$ GLAEE Hübner, [I806]. See Gloia Hübner, 1822.

CUCU

Glaee has not been 11sed again in zoology. A similar name Gloia Hübner, IS22, was subsequently proposed.

GLAPHYRA Guenée, 18+1. See Rhypagla nom. n.

$\operatorname{ACON}$

GLAUCICODIA Hampson, igio, Cat. Lepid. Phalaenae Br. Mus. 10: 4, 35I.

ACON

Type-species: Glaucicodia leuconephra Hampson, I910, ibidem 10: 352, fig. I07, by original designation. Holotype $q$, CUBA: Baracoa (BMNH).

$\ddagger G L E N O P T E R A$, misspelling. Sec Glenopteris Hübner, [182I].

HYPE

GLENOPTERIS Hübner, [1821] I8I6, Verz. bekannter Schmett.: 255.

HYPE

Type-species: Glenopteris oculifera Hübner, [1821] 1816, ibidem: 255 (the objective replacement name for Phalaena oculata Stoll, 1780 , see below), by monotypy. Type(s) (of $P$. oculata), Surinam.

G. oculifera was proposed by Hübner as the objective replacement name for Phalaena (Noctua) oculata Stoll, I780, in Cramer, Uitlandsche Kapellen 4: 7I, pl. 324, fig. F (dated from the wrapper of the part); a junior primary homonym of Phalaena (Geometra) oculata Linnacus, I766, Syst. Nat. (Edn I 2) 1:872 and Errata at the end of vol. I.

Glenopteris was also used by Hübner, [182I], Samml. exot. Schmett. 2: pl. [200], for the same concept. I am following Hemming, 1937, Hübner 2: 201 in giving priority to the Terzeichniss.

${ }_{+}^{\ddagger}$ GLENOPTERA; Schaus, I9I I, Ann. Mag. nat. Hist. (8)8: 230.

An incorrect subsequent spelling.

GLOANNA nom. n. for Langona Barnes \& Lindsey, I92I.

ACRO

Type-species: Langona grisescens Barnes \& Lindsey, I921, Psyche, Camb. 28: I 58, by original designation (for Langona Barnes \& Lindsey). Holotype ô. [U.S.A.]: Ariz., Palmerlee.

LANGONA Barnes \& Lindsey, I921, Psyche, Camb. 28: I 58.

Type-species: Langona grisescens Barnes \& Lindsey, I92I, by original designation.

A junior homonym of Langona Simon, I90 , Annls Soc.ent. Fr. 70: 70, - Arachnida. The objective replacement name is Gloanna nom. n.

GLOBOSUSA Swinhoe, I9I 8, Ann. Mag. nat. Hist. (9)2: 91.

HYPE

Type-species: Globosusa curiosa Swinhne, I9I 8, ibidem (9) 2: 91, by original designation. Type(s) ô. [Sulawesi]: [Sangihe Is], Sangir I. (BMNH). 
† GLOCE, misspelling. See Monogona Guenée, 1852 .

OPHI

GLOEA Agassiz, I846. See Gloia Hübner, I822.

CUCU

† GLOEE Hübner, I808. See Monogona Guenée, 1852 .

OPHI

Gloee has not been used again in zoology. A similar name Gloia Hübner, I822, was subsequently proposed.

$\ddagger$ GLOIA Hübner, I818. See Caenurgia Walker, I858.

CATO

GLOIA Hübner, I822, Syst.-alphab. Verz.: 21, 37.

CUCU

Type-species: Phalaena vaccinii Linnaeus, I76I, Fauna Suecica (Edn 2): 320, by subsequent designation by Curtis, I829, Br. Ent. 6: 268, but cited for Glaea Stephens, I829, an unjustified emendation. Type(s), SwEDEN (LS, London).

$\ddagger$ GLAEE Hübner, [1806], Tentamen determinationis digestionis . . . [I].

Included in a work rejected for nomenclatural purposes by Int. Commn zool. Nom., 1926, Smithson. misc. Collns 73 (4) Opinion 97: 19. Also idem, 1954, Opin Decl. int. Commn zool. Nom. 6 Opinion 278: I40.

Only included species: Phalaena vaccinii Linnaeus, I $76 \mathrm{I}$.

GLAEA Stephens, I829, Illust. Br. Ent. (Haustellata) 2: I 59.

An unjustified emendation of $\ddagger$ Glaee Hübner, [I806], and Gloia Hübner, I822.

$\ddagger G L A E A$; Curtis, I829, Br. Ent. 6: 268.

An incorrect subsequent spelling of Gloia Hübner, I822. Placed on the Official Index of Rejected and Invalid Generic Names in Zoology: Name No. 1847.

GLOEA Agassiz, I846, Nomencl. zool. Index univl.: 162, I63.

An unjustified emendation of Glaea Stephens, r829.

GLORIANA Kirby, I897, Handb. Order Lepid. 5: I68.

OPHI

Type-species: Phyllodes ornata Moore, 1882, in Hewitson \& Moore, Descr. new Indian

lepid. Insects Colln late $M r W$. S. Atkinson: 166, by monotypy. Syntypes + , India:

[W. Bengal], Darjiling (BMNH).

GLOTTULA Guenée, I837. See Brithys Hübner, [I82I].

HADE

GLUPHISIA Boisduval, I828, Eur. Lepid. Index meth.: 56.

NOTODONTIDAE

Type-species: Bombyx crenata Esper, 1785, Die Schmett. $3: 245$, pl. 47, figs 3, 4, by monotypy.

Gluphisia has been used as the original generic name for species which are now in the Noctuidae.

GLYMPIS Walker, I859, List Specimens lepid. Insects Colln Br. Mus. 19: 852.

OPH I

Type-species: Glympis eraconalis Walker, I859, ibidem 19: 852, by monotypy.

Holotype ô. [BrazIL]: Villa Nova (BMNH).

G. eraconalis is a junior subjective synonym of Ecregma damoetesalis Walker, [I859].

ECREGMA Walker, [1859] I858, List Specimens lepid. Insects Colln Br. Mus. 16: 25 I.

Type-species: Ecregma damoetesalis Walker, [1859] I858, ibidem 16: 252, by monotypy. Holotype o, Venezuela (BMNH).

A junior homonym of Ecregma Walker, [1858] I857, ibidem 12: 966, - Lepid., Notodontidae. There is no objective replacement name but $E$. damoetesalis is conspecific with Glympis evaconalis Walker, I859, the type-species of Glympis Walker, I859. The latter is thus available for use as a subjective replacement name.

GNAMPTOCERA Butler, r891, Ann. Mag. nat. Hist. (6) 8: 7r, 73.

ACRO

Type-species: Callopistria minuta Butler, I889, Illust. typical Specimens Lepid. Heterocera Colln Br. Mus. 7: 70, pl. ×30, fig. 4, by original designation. Type(s), [INDIA]: [Punjab], Dharmsala (BMNH). 
GNAMPTOGYIA Hampson, I 894, Fauna Br. India (Moths) 2: 432, 478.

OPHI

Type-species: Gnamptogyia multilineata Hampson, I894, ibidem 2: 479, fig. 266, by original designation. Type(s) ơ, India: [Punjab], Simla (BMNH).

GNAMPTONYX Hampson, I894, Fauna Br. India (Moths) 2: xix, 480.

CATO

Type-species: Homoptera vilis Walker, 1865 , List Specimens lepid. Insects Colln Br. Mus. 33: 889, by original designation. Holotype 오, [INDIA]: Punjab (BMNH).

GNATHOGONIA Hampson, 1926, Descr. new Genera Species Lepid. Phalaenae Subfamily Noctuinae Br. Mus.: 234 .

OPHI

Type-species: Bocula plecopteridia Hampson, I907, J. Bombay nat. Hist. Soc. 17: 66I, by original designation. Holotype $\hat{\sigma}$. India: Bombay (BMNH).

$\ddagger$ GNATHOMECES Hampson, no published reference found.

HYPE

†OANA, misspelling. See Toana Walker, I 865 .

ACON

GOEDNES Schaus, I9I6, Proc. U.S. natn. Mus. 50: 331.

HY PE

Type-species: Goednes abnormalis Schaus, I9I6, ibidem 50:33 I, by original designation. Type(s) ô, Surinam: Geldersland (USNM, Washington).

GOENYCTA Hampson, 1908, Cat. Lepid. Phalaenae Br. Mus. 7: 9. Available, but without included species until Hampson, Igog, ibidem 8: vii, 49.

ACRO

Type-species: Erastria niveiguttata Hampson, I902, J. Bombay nat. Hist. Soc. 14: 205, by subsequent monotypy. Type(s) 옹 India: Sikkim (BMNH).

GOMORA Walker, I869, Characters undescr. Lepid. Heterocera: 53.

OPHI

Type-species: Gomora argentipes IValker, I869, ibidem: 54, by monotypy. Type(s) o., [HoNDURAS]: Limas (BMNH).

GONAGYRA Hampson, I926, Descr. new Genera Species Lepid. Phalaenae Subfamily Noctuinae Br. Mus.: $46 \mathrm{I}$.

OPHI

Type-species: Gonagyra metabrachys Hampson, I926, ibidem: 462, by original designation. Syntypes 207, PERU: Yahuarmayo (BMNH).

GONDYSIA Berio, I955, Boll. Soc. ent. ital. 85: I 46.

CATO

Type-species: Gondysia pertorrida Berio, I 955, ibidem 85: I 47, by original designation. Holotype $\hat{\sigma}$. MadaGascar (BMNH).

GONELYDNA Hampson, igio, Cat. Lepid. Phalaenae Br. Mus, 9: 2, 529.

ACRO

Type-species: Gonelydna acutangula Hampson, I9IO, ibidem 9: 529, fig. 247, by original designation. Holotype $\sigma^{\star}$. [GHANA] Gold Coast: Ashanti, Obuassi (BMNH).

GONIAPTER YX Perty, [1833], Delectus Anim. articulatorum Brasil. (3): i62.

OPHI

Type-species: Goniapteryx tullia Perty, [1833], ibidem (3): I62, pl. 32, fig. 9, by monotypy. Type(s), Brazil: equatorial.

GONIOPTERYX Agassiz, i 846, Nomencl. zool. Index univl.: 165.

An unjustified emendation of Goniapteryx Perty, [1883).

GONIOCALPE Hampson, I912, Cat. Lepid. Phalaenae Br. Mus. 11: xvi, 597.

CHLO

Type-species: Erizada sericealis Hampson, Igo2, Ann. S. Afr. Mus. 2: 31 8, by original designation. Type(s) ㅇ․ [South AFricA]: Natal, Durban (BMNH).

GONIOCARSIA Hampson, I926, Descr. new Genera Species Lepid. Phalaenae Subfamily Noctuinae Br. Mus.: 425 .

OPII

Type-species: Thermesia electrica Schaus, 1894, Trans. Am. ent. Soc. 21: 244, by original designation. Type(s), MExico: Jalapa. 
GONIOCRASPEDON Hampson, I893, Illust. typical Specimens Lepid. Heterocera Colln Br. Mus. 9: 3I, I25.

OPHI

Type-species: Nagadeba mistura Swinhoe, I891, Trans. ent. Soc. Lond. 1891: I5I, by original designation. Syntypes $\hat{\alpha}$, $q$, India: [Bombay], Poona (BMNH).

GONIOCRASPEDUM Hampson, I 895, Fauna Br. India (Moths) 3: xvi, IoI.

An unjustified emendation of Goniocraspedon Hampson, I 893.

GONIOCRASPEDUM Hampson, I 895. See Goniocraspedon Hampson, I893.

OPHI

GONIOCRASPIDUM Hampson, I894, Fauna Br. India (Moths) 2: 407, 4I6.

OPHI

Type-species: Goniocraspidum ennomoide Hampson, I894, ibidem 2: 4I 6, fig. 232, by original designation. Syntypes $\hat{\delta}$, [PAkistan]: Murree (BMNH). India: Dharmsala (BMNH).

ECTOGONITIS Hampson, I896, Fauna Br. India (Moths) 4: 530.

Proposed, unnecessarily, as an objective replacement name for Goniocraspidum Hampson, I 894, which Hampson considered to be a homonym of Goniocraspedon Hampson, I893, Lepid., Noctuidae.

GONIOHELIA Hampson, I926, Descr. new Genera Species Lepid. Phalaenae Subfamily Noctuinae Br. Mus.: 3 I 8.

OPHI

Type-species: Selenis gallinago Felder \& Rogenhofer, I874, Reise öst. Fregatte Novara (Zool.) 2 (Abt. 2): pl. I I , fig. 9; Inhalts-Verz.: I7, by original designation. Type(s) 우, [BraziL]: R. Amazon (BMNH).

GONIOPHILA Hampson, I926, Descr. new Genera Species Lepid. Phalaenae Subfamily Noctuinae Br. Mus.: 342.

OPHI

Type-species: Cosmophila excavata Swinhoe, I905, Ann. Mag. nat. Hist. (7)15: I 58, by original designation. Type(s) 우, Borneo: [Sarawak), Sadong (BMNH).

GONIOPHYLLA Turner, I945, Mem. Qd Mus. 12: I 55.

ACON

Type-species: Goniophylla fragilis Turner, 1945, ibidem 12: 155, by monotypy. Holotype $q$, Australia: Qd, Cape York.

GONIOPTERYX Agassiz, I846. See Goniapteryx Perty, [I833].

OPHI

GONIOSCIA Hampson, I926, Descr. new Genera Species Lepid. Phalaenae Subfamily Noctuinae $B r$. Mus. : 443.

OPHI

Type-species: Gonioscia meroleuca Hampson, I926, ibidem: 444, by original designation. Holotype 우 [GHana] Gold Coast: Bibianaha (BMNH).

† GONIOS TIXIS Hampson, no published reference found.

HYPE

GONIOTERMASIA Hampson, I9I8, Novit. zool. 25: I36.

ACRO

Type-species: Perciana bistrigata Bethune-Baker, 1906, ibidem 13: 206, by original designation. Holotype đ., New Guinea: [Papua], Dinawa (BMNH).

GONIOXESTIS Hampson, I9I2, Cat. Lepid. Phalaenae Br. Mus. 11 : xvi, 637.

CHLO

Type-species: Arcyophora zanderi Felder \& Rogenhofer, 1874, Reise öst. Fregatte Novara (Zool.) 2 (Abt. 2): pl. I I I, fig. I 5; Inhalts-Verz: 3, by original designation. Type(s) 우, [Ethlopia] Abyssinia (BMNH).

¡GONIOXESTRIS; Neave, 1939, Nomencl. zool. 2: 502.

An incorrect subsequent spelling.

†GONIOXESTRIS, misspelling. See Gonioxestis Hampson, I9I2.

CHLO

GONIPPA Möschler, I883, Verh. zool.-bot. Ges. Wien 32 (Abh): 354 .

OPHI

Type-species: Gonippa perusia Möschler, I883, ibidem 32 (Abh): 354, pl. I8, fig. 39, by monotypy. Syntypes $2 \pi$, Surinam: Paramaribo and the interior (MNHU, Berlin). 
GONITIS Guenée, I 852, in Boisduval \& Guenée, Hist. nat. Insectes (Lépid.) 6: 403. OPH I

Type-species: Gonitis editrix Guenée, I 852 , ibidem 6: 404, pl. I I, fig. 5, by subsequent designation by Hampson, 1926, Descr. new Genera Species Lepid. Phalaenae Subfamily Noctrinae Br. Mus.: 345. Type(s), Haiti [Republic] (BMNH).

$\ddagger$ GONOTIS; Moore, I882, in Hewitson \& Moore, Desc. new Indian lepid. Insects Colln late Mr. W. S. Atkinson: I 53 .

An incorrect subsequent spelling.

GONODES Druce, I 908 April, Ann. Mag. nat. Hist. (8)1 : 298.

Type-species: Gonodes albifissa Druce, I 908, ibidem (8)1:298, by monotypy. Type(s), Peru: S.E., Santo Domingo (BMNH).

Gonodes was used by Druce without any indication that it was new even though his new species, described on the same page, were marked sp. n. The generic name was probably obtained from Hampson as an identification and was used by Druce before Hampson's generic description was published.

GONODES Hampson, 1908 [December], Cat. Lepid. Phalaenae Br. Mus. 7: I5. Available but without included species until Hampson, I909, ibidem 8: 450 .

Type-species: Ipimorpha liquida Möschler, I 886, Abh. Senckenb. naturforsch. Ges. 14(3): 48, fig. I7 (but proposed in ${ }_{+}^{+}$Iphimorpha, an incorrect subsequent spelling), by subsequent designation by Hampson, I909, ibidem 8: 450. Syntypes $\hat{0}$, 우, Jamarca (MNHU, Berlin).

A junior homonym of Gonodes Druce, 1908 April. This generic name probably originated from Hampson, but it was used and unintentionally made nomenclaturally available by Druce, prior to its proposal and generic description by Hampson, for a similar concept but having a different type-species. There is no objective replacement name but $I$. liquida is congeneric with Gonodes albifissa Druce, 1908, the type-species of Gonodes Druce, I908 April. The latter is thus available for use as a subjective replacement name.

GONODES Hampson, I908. See Gonodes Druce, I908.

ACRO

GONODONTA Hübner, i818, Zuträge Samml. exot. Schmett. 1: i I.

OPHI

Type-species: Gonodonta uncina Hübner, I8 I8, ibidem 1: I I, figs 35, 36, by subsequent designation by Grote, I902, Allg. Z. Ent. 7: 472. Type(s) ô, Brazil.

G. uncina is a junior subjective synonym of Phalaena sicheas Cramer, I777, Uitlandsche Kapellen 2: 86, pl. I 50, fig. E.

$\ddagger$ PTILODON Hübner, I808, Erste Zuträge Samml. exot. Schmett.: 4.

Included in a work rejected for nomenclatural purposes by Int. Commn zool. Nom., I966, Bull. zool. Nom. 23 Opinion 789: 2 I6.

Placed on the Official Index of Rejected and Invalid Generic Names in Zoology: Name No. I881.

The only included name + Ptilodon uncium Hübner, I808, was subsequently made nomenclaturally available as Gonodonta uncina Hübner, I8 8.

$\ddagger$ MEROPIS Hübner, I818, Zuträge Samml. exot. Schmett. 1: I I, 34.

Not nomenclaturally available as a genus-group name. Hübner used Meropis, on both pages, in a suprageneric sense for one of the names of his tribes (Namen der Stämme) listed on pages 33-34. Hübner listed his genus-group names on page 35 .

GONODONTODES Hampson, I913, Cat. Lepid. Phalaenae Br. Mus. 12: 6. Available bint without included species until Hampson, I9I3, ibidem 13: ix, I46.

CATO

Type-species: Gonodontodes dispar Hampson, I9I3, ibidcm 13: ix, I47, fig. 42 (but included by Hampson as 'dispar Herr.-Schäff.', an incorrect authorship) by subsequent designation by Hampson, I913, ibidem 13: i 46. Holotype ô, CuBA: Santiago (BMNH).

G. dispar was first described by Herrich-Schäffer, I868, Corresp.-Bl. zool.-min. Ver. Regensburg 22: I 8 I but was without a generic name and so not nomenclaturally available. Gundlach, I88I, Contribucion Ent. Cubana 1: 329, repeated the description but still did not place dispar in a genus. Hampson attributed dispar to Herrich-Schäffer. 
GONOGLASA Hampson, I924, in Tams, J. nat. Hist. Soc. Siam 6: 250.

OPHI

Type-species: Gonoglasa camptogramma Hampson, I924, ibidem 6: 250, pl. I7, fig. I8, by original designation. Holotype $q$, BorNeo: [Sabah], Sandakan (BMNH).

GONOPHAEA Hampson, I910, Cat. Lepid. Phalaenae Br. Mus. 10: xiii, 362. ACON

Type-species: Tarache villica Schaus, 1904, Trans. Am. ent. Soc. 30: 159, by original designation. Type(s), Brazil: São Paulo.

GONOPTERA Berthold, I827. See Scoliopteryx Germar, r8I I.

OPHI

GONOPTERONIA Bethune-Baker, I9o6, Novit. zool. 13: 239.

OPH

Type-species: Gonopteronia albopunctata Bethune-Baker, 1906, ibidem 13: 239 , by original designation. Syntypes $\hat{\sigma}$, New Guinea: [Papua], Ekeikei (BMNH).

GONOSPILEIA Hübner, [1823] I816, Verz. bekannter Schmett.: 28I.

CATO

Type-species: Noctua munita Hübner, [18r3], Samml. eur. Schmett. 4: pl. r29, figs 593, by subsequent designation by Hampson, I913. Cat. Lepid. Phalaenae Br. Mus. 13: 50. Type(s), Europe.

GONOSTYGIA Hampson, I908, Cat. Lepid. Phalaenae Br. Mus. 7: 4. Available, but without included species until Hampson, I9Io, ibidem 9: viii, I24.

ACRO

Type-species: Anarta agonax Druce, r89o, Proc. zool. Soc. Lond. 1890: 5I5, by subsequent monotypy. Type(s), Mexico: Jalapa (BMNH).

†GONOTIS, misspelling. See Gonitis Guenée, I852.

OPHI

GONURIS Möschler, I880, Verh. zool.-bot. Ges. Wien 30 (Abh) : 397.

OPHI

Type-species: Gonuris flaminia Möschler, I 88o, ibidem 30 (Abh.): 398, pl. 9, fig. 43 , by monotypy. Holotype + , Surinam: Paramaribo (MNHU, Berlin).

GOONALLICA nom. n. for Callogonia Hampson, Igo8.

ACRO

Type-species: Abrostola virgo Treitschke, I 835, Schmett. Eur. 10(2): I 30, by original designation (for Callogonia Hampson). Holotype + , [Yugoslavia]: Syrmia [district, $45^{\circ} \mathrm{N}$. I $9^{\circ} \mathrm{E}$.] (TM, Budapest).

CALLOGONIA Hampson, I9o8, Cat. Lepid. Phalaenae Br. Mus. 7 : xiv, 589.

Type-species: Abrostola virgo Treitschke, I835, by original designation.

A junior homonym of Callogonia Dall, I889, Bull. Mus. comp. Zool. Havv. 18: 440,Mollusca. The objective replacement name is Goonallica nom. n.

GORGONE Hübner, I82I, Index exot. Lepid.: [5].

OPHI

Type-species: Ascalapha orphna Hübner, [18167, Samml. exot. Schmett. 1: pl. [195],

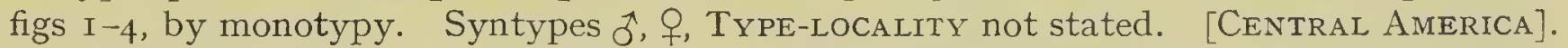

A. orphna is a junior subjective synonym of Phalaena augusta Stoll, I782, in Cramer,

Uitlandsche Kapellen 4: 227, pl. 397, fig. F.

†GORGONIA; Hübner, [1823] I816, Verz. bekannter Schmett.: 270.

An incorrect subsequent spelling.

$\ddagger$ GORGONIA, misspelling. See Gorgone Hübner, I82I.

OPHI

GORGORA Dyar, I914, Proc. U.S. natn. Mus. 47: 377.

ACON

Type-species: Gorgora morga Dyar, I9I 4, ibidem 47: 378, by original designation. Holotype 9 Mexrco: Mexico City (USNM, Washington).

$\ddagger$ GORNA, misspelling. See Gorıa Walker, I 865 .

OPHI

GOROSINA Schaus, I9I3, Ann. Mag. nat. Hist. (8)11:2I.

HYPE

Type-species: Gorosina ampla Schaus, I9I3, ibidem (8)11:2I, by original designation. Type(s) o, Costa Rrca: Tuis. 
GORTYNA Ochsenheimer, I816, Schmett. Eur. 4: 82.

ACRO

Type-species: Noctua flavago [Denis \& Schiffermüller], I775, Ankïndung syst. Werkes Schmett. Wienergegend: 86, by subsequent designation by Samouelle, 1819, Entomologist's useful Compendium: 252, but cited as 'flavago Hüb.', an incorrect authorship. Type(s), [Austria]: Vienna district (Collection destroyed, Horn \& Kahle, 1936, Ent. Beih. Berl.Dahlem 3: 243).

CNEPHOZETA Billberg, I820, Enumeratio Insect. Mus. G. J. Billberg: 88.

Proposed, unnecessarily, as an objective replacement name for Gortyna Ochsenheimer, I8r6.

OCHRIA Hübner, [1821] I816, Verz. bekannter. Schmett.: 233.

Type-species: Noctua flavago [Denis \& Schiffermüller], I775, by subsequent designation by Grote, 1874, Bull. Buffalo Soc. nat. Sci. 2: 19, but cited as 'flavago Lin.', an incorrect authorship.

Ochria is a junior objective synonym of Gortyna Ochsenheimer, i8i6.

XANTHOECIA Hampson, 1908, Cat. Lepid. Phalaenae Br. Mus. 7: 9. Available, but without included species until Hampson, I910, ibidem 9: vii, 32.

Type-species: Noctua flavago [Denis \& Schiffermüller], 1775, by subsequent designation by Hampson, 1910, ibidem 9: 32 .

A junior objective synonym of Gortyna Ochsenheimer, 1816.

GORTYNODES Bethune-Baker, I9I I, Ann. Mag. nat. Hist. (8)8: $5^{\text {I } 2 .}$

ACRO

Type-species: Gortynodes holophaea Bethune-Baker, I9II, ibidem (8)8: 5I3, by original designation. Type(s) $\tilde{\sigma},[$ ANGola $]$ : Gunnal.

GORUA Walker, 1865, List Specimens lepid. Insects Colln Br. Mus. 33: 942.

OPHI

Type-species: Gorua partita Walker, 1865, ibidem 33: 943, by monotypy. Holotype o. Sierira leone (BMNH).

$\ddagger$ GORNA; Holland, 1894, Psyche, Camb. 7: 121.

An incorrect subsequent spelling.

GRACILIPALPUS Calberla, 1888. See Anthracia Hübner, [1823]. ACro

GRACILLINA Hampson, 1924, Entomologist 57: 182.

OPHI

Type-species: Gracillina prosthenia Hampson, 1924, ibidem 57: 182, by original designation. Holotype $\widehat{0}$. Singapore (BMNH).

GRACILODES Guenće, I 852, in Boisduval \& Guenée, Hist. nat. Insectes (Lépid.) 7: 369. opHI Type-species: Gracilodes caffra Guenée, 1852, ibidem 7:370, by subsequent designation by Desmarest, (1857), in Chenu, Encycl. Hist. nat. Papillons nocturnes: 135. Holotype $q$, [South AFrica]: Cafrerie (BMNH).

GRACILOPSIS Hampson, 1926, Descr. new Genera Species Lepid. Phalaenae Subfamily' Noctuinae Br. Mus.: 586.

OPHI

Type-species: Gracilopsis guianensis Hampson, 1926, ibidem: 586 , by original designation. Syntypes I ô, I + , [Guyana] British Guiana: Demerara (BMNH).

GRAEPERIA Grote, I895. See Heliodora Neumoegen, I89I. ACON

GRAMMESIA Stephens, I829, Illust. Br. Ent. (Haustellata) 2: I5I.

$\triangle \mathrm{CRO}$

Type-species: Noctua bilinea Hübner, [1803], Samml. eur. Schmett. 4: pl. 45, fig, 2 I7. by subsequent designation by Westwood, 1840, Synopsis Genera Br. Insects: 94 . Type(s), EUROPE.

GRAMMODES Guenée, 1852, in Boisduval \& Guenée, Hist. nat. Insectes (Lépid.) 7: 275. Cato Type-species: Noctua geometrica Fabricius, 1775, Syst. Ent.: 599, by original designation but cited as geometrica Rossi, an incorrect authorship. Type(s), India: eastern (Type(s) lost, Zimsen, 1964, Type Material of I. C. Fabricius: 523). 
GRAMMOPHORA Guenée, I852. See Polygrammate Hübner, I8I8.

ACRO

GRAMMOSCELIS Hampson, I906, Cat. Lepid. Phalaenae Br. Mus. 6: 3, 468.

$\mathrm{CUCU}$

Type-species: Grammoscelis leuconeura Hampson, I906, ibidem 6: 468, fig. I60, by original designation. Holotype $\hat{\sigma}$, [South Africa]: Cape Colony, Deelfontein (BMNH).

GRAPHANIA Hampson, I905, Cat. Lepid. Phalaenae Br. Mus. 5: xiv, 468.

HADE

Type-species: Heliophobus disjungens Walker, 1858 , List Specimens lepid. Insects Colln Br. Mus. 15: I68I, by original designation. Holotype $\hat{\sigma}$. New Zealand (BMNH). MAORIA Warren, 1912, in Seitz, Gross-Schmett. Erde 11: 76.

Type-species: Evana plena Walker, 1865, List Specimens lepid. Insects Colln Br. Mus. 33: 744, by original designation. Holotype $\AA$. New ZEaland: Auckland (BMNH).

A junior homonym of Maoria Laporte, I868, Trans. Proc. R. Soc. Vict. 8: I63,Coleoptera. There is no objective replacement name but E. plena is congeneric with Heliophobus disjungens Walker, 1858, the type-species of Graphania Hampson, 1905. The latter is thus available for use as a subjective replacement name.

The date of publication of the part of Seitz containing page 76 was stated on page 73 as I9I 2 September $25^{\text {th }}$ in both the English and German editions.

GRAPHIGONA Walker, [1858] I857, List Specimens lepid. Insects Colln Br. Mus. 13: I2I7, I 230.

OPHI

Type-species: Ophideres regina Guenée, 1852 , in Boisduval \& Guenée, Hist. nat. Insectes (Lépid.) 7: I 18, by subsequent designation by Berio, I966, Annali Mus. civ. Stor. nat. Giacomo Doria 76: 59. Holotype ô. Colombia (BMNH).

†GRAPHIPHORA Hübner, [1806]. See Semiophora Stephens, I829.

HADE

GRAPHIPHORA Ochsenheimer, I816, Schmett. Eur. 4: 68.

NOCT

Type-species: Noctua augur Fabricius, 1775, Syst. Ent.: 604, by subsequent designation by Samouelle, I8I9, Entomologist's useful Compendium: 25I. Type(s), Germany (Type(s) not found by Zimsen, 1964, Type Material of I. C. Fabricius: 524).

GRAPHOPHORA Agassiz, I846, Nomencl. zool. Index univl: I67.

An unjustified emendation of Graphiphora Ochsenheimer, I8I6.

GRAPHOPHORA Agassiz, i846. See Graphiphora Ochsenheimer, I8I6.

NOCT

GRAPTOCULLIA Köhler, (1952) i95I, Acta zool. lilloana 12: I43.

CUCU

Type-species: Graptocullia sagittata Köhler, (1952) I95I, ibidem 12: I44, by original designation. Syntypes $\hat{\sigma}$, 우, Argentina: [Chubut Prov.], Comodoro Rivadavia (ZSBS, Munich); Neuquén [Prov.], Rio Agrio (ZSBS, Munich).

GRAPTOLITHA Hübner, [1821] 1816, Verz. bekannter Schmett.: 243.

CuCu

Type-species: Noctua conformis [Denis \& Schiffermüller], I775, Ankündung syst. Werkes Schmett. Wienergegend: 76, by subsequent designation by Grote, 1874, Annual Rep. Trustees Peabody Acad. Sci. 6: 34. Type(s), [Austria]: Vienna district (Collection destroyed, Horn \& Kahle, I936, Ent. Beih. Berl.-Dahlem 3: 243).

$N$. conformis is a junior subjective synonym of Phalaena furcifera Hufnagel, I766, Berlin. Mag. 3 (4): 402.

GRAVODOS Rothschild, I920, J. fed. Malay St. Mus. 8: Iзо.

HYPE

Type-species: Gravodos robinsoni Rothschild, I920, ibidem 8: I30, by monotypy. Holotype ô. Sumatra: W., Sandaran Agong, Korinchi (BMNH).

GRIPOSIA Tams, 1939, Entomologist 72: 72.

CUCU

Type-species: Phalaena aprilina Linnaeus, 1758, Syst. Nat. (Edn Io) 1: 5I4, by monotypy (of Agriopis Boisduval, i 840). Type(s), Europe (LS, London).

Griposia was proposed as the objective replacement name for Agriopis Boisduval, I 840 . 
AGRIOPIS Boisduval, I840, Genera Index meth. Eur. Lepid.: I 23.

Type-species: Phalaena aprilina Linnaeus, I 758, by monotypy.

A junior homonym of Agriopis Hübner, [1825] I8I6, Verz. bekannter Schmett.: 320, Lepid., Geometridae. The objective replacement name is Griposia Tams, 1939.

EUDIPHTHERA Nordström, I94I, Svenska Fjärilar 2: 152, 343.

Proposed, unnecessarily, as an objective replacement name for Agriopis Boisduval, 1840.

GRISANA Kölıler, (1952) I95I, Acta zool. lilloana 12: I 50.

CUCU

Type-species: Grisana praetiosa Köhler (1952) 195I, ibidem 12: 15I, by original

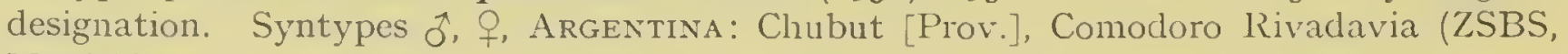
Munich).

GRIVEAUDIA Viette, 1958, Mem. Inst. scient. Madagascar (E)9: I 73.

OPHI

Type-species: Griveaudia vieui Viette, I958, ibidem (E)9: 174, pl. I, figs I, 2, by original designation. Holotype ô, MADAGASCAR: E., forest $6 \mathrm{~km}$ N.W. of Fanovana (MNHN, Paris).

GROTELLA Harvey, I 875, Bull. Buffalo Soc. nat. Sci. 2: 278.

NOCT

Type-species: Grotella septempunctata Harvey, I875, ibidem 2: 278, pl. 3, fig. I, by monotypy. Type(s) ô, [U.S.A.]: Texas (BMNH).

GROTELLAFORMA Barnes \& Benjamin, 1922, Contr. nat. Hist. Lepid. N. Am. 5: 9, 24. NOCT

Type-species: Grotella calora Barnes, 1907, Can. Ent. 39: 68, 93, by original designation. Lectotype of, [U.S.A.]: Arizona, Redington, designated by Barnes \& Benjamin, 1922, ibidem 5: 26 .

G. calora is a junior subjective synonym of Cisthene lactea Stretch, 1885, Ent. Am. 1: IO3.

GRUMIA Alphéraliy, I892, Horae Soc. ent. ross. 26: 453.

NOCT

Type-species: Grumiaflora Alphéraky, I 892, ibidem 26:453, by monotypy. Syntypes o., [China]: Amdo region, Sinin-schan Mits (ZI, Leningrad).

GRYPHADENA Kusnezov, I908, Ezheg.zool. Muz. 13: 66.

ACRO

Type-species: Pseudohadena minuta Püngeler, 190o, Dt. ent. Z. Iris 12: 293, pl. 9, fig. Io, by original designation. Syntypes 4 ㅇ, [U.S.S.R.]: [Kazakhstan], Ili district; [Turkmenistan], Merw (MNHU, Berlin).

GRYPOSOBA Hampson, I912, Cat. Lepid. Phalaenae Br. Mus. 11 : xiii, 423.

SARR

Type-species: Cymatophoropsis catagrapha Tumer, 1903, Trans. R. Soc. S. Aust. 27: 7, by original designation. Holotype $\sigma^{2}$, Australia: N. Qd, Cairns.

GRYPOTES Dyar, I9I7. See Zatilpa Dyar, 1920.

ACRO

GURIAUNA Walker, I86I, List Specimens lepid. Insects Colln Br. Mus. 23: 948. Available but witlout included species until W'alker, I862, ibidem 24: I Io I.

$\operatorname{ACON}$

Type-species: Guriauna semipardata Walker, I862, ibdem 24: I ror, by subsequent monotypy. Lectotype $q$, Borneo: Sarawak (UM, Oxford), designated (as type) by Swinhoe, 1900, Cat. east. and Aust. Lepid. Heterocera 2: 2 I 5.

GUSTIANA Walker, I86I, List Specimens lepid. Insects Colln Br. Mus. 23: 947. Available but without included species until Walker, i 862, ibidem 24: 1099.

II Y'PE

Type-species: Gustiana subflexata Walker, I862, ibidem 24: 1100, by subsequent monotypy. Type(s) ô. Brazil (UM, Oxford).

G. subflexata is a junior subjective synonym of Platydia abditalis Waller, [1859] I858, ibidem 16: I I.

See also Gaala Walker, [1866].

GYMNOPA Stephens, I829. See Panemeria Hübner, [1823]. 
GYNAEPHILA Staudinger, I892, in Romanoff, Mém. Lépid. 6: 629.

HYPE

Type-species: Gynaephila maculifera Staudinger, I892, ibidem 6:629, pl. I4, fig. I3,

by monotypy. Syntypes 8 ․ [U.S.S.R.]: Amur district, Vladivostock; Askold; Sutschan.

China: N. of Peking (MNHU, Berlin).

GYOPHORA Warren, I913, in Seitz, Gross-Schmett. Erde 11: 217.

ACON

Type-species: Thalpochares quadrilineata Moore, i 88I, Proc. zool. Soc. Lond. 1881:

370, pl. 38, fig. I4, by original designation. Type(s), [India]: Calcutta (MNHU, Berlin).

The date of publication of the part of Seitz containing page 2I 7 was stated on page 2I 7

as I9I3 August 2oth in both the English and German editions.

$\ddagger$ GYROPHORA; Neave, I939, Nomencl. zool. 2: 538 .

An incorrect subsequent spelling.

GYPSITEA Tams, 1939, Entomologist 72: 135.

NOCT

Type-species: Noctua leucographa [Denis \& Schiffermüller], I775, Ankündung syst.

Werkes Schmett. Wienergegend: 83, by monotypy (of Sora Heinemann, i 859). Type(s),

[Austria]: Vienna district (Collection destroyed, Horn \& Kahle, I936, Ent. Beih. Berl.Dahlem 3: 243).

Gypsitea was proposed as the objective replacement name for Sora Heinemann, I 859.

SORA Heinemann, I 859 [December], Schmett. Dtl. Schweiz 1: xvii, 459.

Type-species: Noctua leucographa [Denis \& Schiffermüller], I 775, by monotypy.

A junior homonym of Sora Walker, 1859, April, Ann. Mag. nat. Hist. (3)3: 259, Coleoptera. The objective replacement name is Gypsitea Tams, I939.

Zuchold, [I860], Bibl. Hist. nat. 9: I60 lists Heinemann's work as being published between July and December I 859 .

GYROGNATHA Hampson, I893, Illust. typical Specimens Lepid. Heterocera Colln Br. Mus.

9: 32, I34.

ACON

Type-species: Gyrognatha atriceps Hampson, I893, ibidem 9: 32, 134, pl. I68, fig. Io,

by monotypy. Type(s) $\widehat{\delta}$, Ceylon: Pundaloya (BMNH).

G. atriceps is a junior subjective synonym of Carmara subcervina Walker, [I 863].

See also Carmara Walker, [1863]; and Zagira Walker, I 866.

GYROHYPSOMA Staudinger, I 888, Stettin. ent. Ztg 49: 47.

ACON

Type-species: Gyrohypsoma sterrha Staudinger, I888, ibidem 49: 47, by monotypy. Syntypes 2 ô, [U.S.S.R.]: [Uzbekistan], Margelan (MNHU, Berlin). [China]: [Sinkiang], Kuldja district (MNHU, Berlin).

† GYROPHORA, misspelling. See Gyophora Warren, т913.

$\operatorname{ACON}$

GYROPRORA Turner, igr I, Ann. Qd Mus. 10: 64.

CUCU

Type-species: Gyroprora ochrias Turner, I9I I, ibidem 10:64, by monotypy. Holotype o. Australia: [Vict.], Melbourne (NMV, Melbourne).

GYROS Edwards, I88I, Papilio 1: I I 7.

PYRALIDAE

Type-species: Oribates muirii Edwards, I 881, ibidem $1: 22$, by subsequent designation by Grote, 1882, Can. Ent. 14: I96. Syntypes 2 ô, 3 오, [U.S.A.]: Cal., Tuolumne Co.

Gyros was proposed as the objective replacement name for Oribates Edwards, I88I.

ORIBATES Edwards, I 88 I, Papilio 1: 22.

Type-species: Oribates muirii Edwards, I88I, by subsequent designation (for Gyros

Edwards, I881) by Grote, i882, Can. Ent. 14: 196.

Oribates was originally placed in the Noctuidae.

A junior homonym either of Oribates Dugès, I834, Annls Sci.nat.(Zool.) (2)1: 21, or of Oribates Koch, I 835, Dt. Crust. (2): tab. I3,-- both Arachnida, and both either unjustified emendations or incorrect subsequent spellings of Oribata Latreille, r802, Sonnini's Buffon (Insect.) 3: 65. The name Oribates has been used so frequently in the nomenclature of the 
mites that although there is some doubt as to when it became nomenclaturally available, there is no doubt that it should be stabilised for use, if required, in that group.

‡GYROTHRIPA, misspelling. See Gyrtothripa Hampson, I 912. SARR

GYRTONA Walker, 1863, List Specimens lepid. Insects Colln Br. Mus. 27 : 89. STIC Type-species: Gyrtona proximalis IValker, I863, ibidem 27:90, by original designation. Type(s) q. BORneo: Sarawak (BMNH).

GYRTONIDES Hampson, 1912, Cat. Lepid. Phalaenae Br. Mus. 11: 223, 453. SARR

Type-species: Gyrtonides albifascia Hampson, I9I 2, ibidem 11:453, fig. I65, by original designation. Holotype $\hat{\sigma}$, BraziL: Amazons, [Tefé] Ega (BNINH).

GYRTOTHRIPA Hampson, 1912, Cat. Lepid. Phalaenae Br. Mus. 11: xiii, 448.

SARR

Type-species: Gyrtona pusilla Moore, I888, in Hewitson \& Moore, Descr. new Indian lepid. Insects Colln late $M r W$. S. Atkinson: 289, by original designation. Syntypes, INDIA : Calcutta (BMNH).

† YROTHRIPA; Gaede, I935, in Seitz, Gross-Schmett. Erde 15: i 90.

An incorrect subsequent spelling both in the German and in the English edition.

HABERSHONIA nom. n. for Brachyblemma Hampson, I926.

OPHI

Type-species: Phalaena areos Cramer, I777, Uitlandsche Kapellen 2: 50, pl. I30, fig. D, by original designation (for Brachyblemma Hampson, 1926). Type(s), IVEST INDIES: [Virgin Is], St. Thomas I.

$P$. areos is dated from the wrapper of the part and not from the title-page of the volume, I 779 .

This name is proposed in honour of Mr Mark A. K. Habershon in appreciation of his unstinted assistance during the compilation of this catalogue.

BRACHYBLEMMA Hampson, 1926, Descr. new Genera Species Lepid. Phalaenae Subfamily Noctuinae Br. Mus.: II9.

Type-species: Phalaena areos Cramer, I777, by original designation.

A junior homonym of Brachyblemma Wagler, I833, Isis, Jena 1833: 904,-Reptilia. The objective replacement name is Habershonia nom. n.

HABROPHYES Turner, I920, Trans. Proc. R. Soc. S. Aust. 44: I 58, I 7 I. ACON

Type-species: Tarache xuthosoma Turner, 1909, Proc. Limn. Soc. N.S.W. 34: 349, by monotypy. Holotype $\hat{\sigma}$, Australia: N. Qd, Kuranda, Stannary Hills (ANIC, Canberra).

HABROSTOLA Sodoffsky, I837. See Abrostola Ochsenheimer, 1816.

PLUS

HABROSTOLODES Hampson, 1926, Descr. new Genera Species Lepid. Phalaenae Subfamily Noctuinae Br. Mus.: 22 . OPHI

Type-species: Abrostola congressa Wa!ker, I858, List Specimens lepid. Insects Colln Br. Mus. 15: i 781, by original designation. Holotype $q$, Venezuela (BMNH).

HABROSYNE Hübner, [I82I] I816, I'erz. bekannter Schmett.: 236.

THYATIRIDAE Type-species: Phalaena derasa Linnacus, 1 767, Syst. Nat. (Edn 1 2) 1:85 I, by monotypy. Habrosyne was originally placed in the 'Noctuae.'

HABR YNTIS Lederer, I857, Noctuinen Eur.: 35, II 4. ACRO Type-species: Phalaena scita Hübner, I 790, Beitr. Gesch. Schmett. 2 (2): 35, pl. I, fig. A, by monotypy. Type(s) $q$, Italy.

HADA Billberg, I 820, Enumeratio Insect. Mus. G.J. Billberg: 86.

HADE Type-species: Noctua dentina [Denis \& Schiffermüller], I775, Ankündung syst. IVerkes Schmett. Wienergegend: 82 , by subsequent designation by Boursin, 1965, Bull. mens. Soc. limn. Lyon 34: I83, but cited as 'nana Hfn.' which Boursin referred back to his earlier work (Boursin, 1964, ibidem 33: 217) where he placed nana as the senior synonym of 
dentina. Type(s), [Austria]: Vienna district (Collection destroyed, Horn \& Kahle, I936, Ent. Beih. Berl.-Dahlem 3: 243).

$N$. dentina is a junior subjective synonym of Phalaena nana Hufnagel, i 766, Berlin. Mag. $3(4): 398$.

Incorrect type-species designation: Phalaena nana Hufnagel, a name not originally included in Hada, and not linked with one of the originally included names when cited by Tams, 1939, Entomologist 72: 72.

HADENA Schrank, I802, Fauna Boica 2 (2): I 58.

HADE

Type-species: Noctua capsincola [Denis \& Schiffermüller], I775, Ankündung syst. Werkes Schmett. Wienergegend: 84 , by subsequent designation by Curtis, I830, Br. Ent. 7: 308, but cited as 'capsincola Esp.', an incorrect authorship. Type(s), [Austria]: Vienna district (Collection destroyed, Horn \& Kahle, I936, Ent. Beih. Berl.-Dahlem 3: 243).

N. capsincola is a junior subjective synonym of Phalaena bicruris Hufnagel, 1766, Berlin. Mag. 3(3): 302 .

Hadena when proposed by Schrank included not only the species in sections M \& N in his Fanna Boica 2 (I) : $347-354$ but also the species in sections O \& P of [Denis \& Schiffermüller], I775, ibidem: 8I-84. The type-species, $N$. capsincola, was among the latter.

Incorrect type-species: Phalaena typica Linnaeus, I 758, although the first of the species originally included in Hadena, was not a new species proposed in Hadena and so cannot, under the Code, Article 68 (b), be regarded as the type-species.

Incorrect type-species designation: Phalaena genistae Villers, I 789, a name not originally included in Hadena, and not linked with one of the originally included names when cited by Duponchel, 1829, in Godart \& Duponchel, Hist. nat. Lépid. Papillons Fr. 7 (2): 7 I.

ADENA Agassiz, I846, Nomencl. zool. Index univl.: 8, I70.

An unjustified emendation of Hadena Schrank, I802.

DIANTHOECIA Boisduval, I 834, Revue ent. (Silbermann) 2: 246.

Type-species: Noctua capsincola [Denis \& Schiffermüller], I775, (but included by Boisduval as 'capsincola Hübn.', an incorrect authorship), by subsequent designation by Duponchel, 1844, in d'Orbigny, Dict. univl. Hist. nat. 4: 727, but cited as 'capsincola Hübn.'

Dianthoecia is a junior objective synonym of Hadena Schrank, I802.

$\ddagger D I A N T H A E C I A$; Guenée, I838, Annls Soc. ent. Fr. 7(2): 204.

An incorrect subsequent spelling.

$\$ D I A N T H E C I A$; Taylor, I855, New Zealand and its Inhabitants: xiii.

An incorrect subsequent spelling, also used by Moore, I88I, Proc. zool. Soc. Lond. 1881: 354 , and in other works.

DIANTHOECIA Agassiz, I846, Nomencl. zool. Index univl.: I 21.

Proposed, unnecessarily, as an emendation of $\ddagger$ Dianthaecia; Guenée, I838.

$\ddagger$ DI A UTHOECI A; Möschler, I864, Wien. ent. Monatschr. 8: I97.

An incorrect subsequent spelling.

HADENELLA Grote, I883, Papilio 3: I23.

ACRO

Type-species: Hadenella pergentilis Grote, I883, ibidem 3: I 23, by monotypy. Type(s),

[U.S.A.]: Washington Territory.

HADENNIA Moore, [I885] I 887, Lepid. Ceylon. 3: 236.

HYPE

Type-species: Bocana hypenalis Walker, [I 859] I 858, List Specimens lepid. Insects Colln

Br. Mus. 16: I74, by original designation. Holotype , CEYLON (BMNH).

$B$. hypenalis is a junior subjective synonym of Bocana jutalis Waller, [I859] I858, ibidem 16: I72.

$\ddagger$ HADENNINA; Bertkau, I89I, Arch. Naturgesch. 57 (2) 2: I89.

An incorrect subsequent spelling.

$\ddagger$ HADENNINA, misspelling. See Hadennia Moore, [I885].

HYPE 
HADERONIA Staudinger, I896, Dt. ent. Z. Iris 8: 320.

HADE

Type-species: Hadena subarschanica Staudinger, 1896, ibidem 8: 320, pl. 6, fig. I 2, by monotypy. Syntypes $4 \hat{0}, \mathrm{I}$ ㅇ, [CHINA]: Tibet, between Lob Noor and Kuku Noor (MNHU, Berlin).

Haderonia was originally placed as a subgenus of Hadena Schrank, I802.

HADJINA Staudinger, I S92, Dt. ent. Z. Iris 4: 286.

ACRO

Type-species: Hadjina lutosa Staudinger, IS92, ibidem 4: 286, pl. 4, fig. I, by monotypy. Syntypes $\hat{0}, \underline{\text { ㅇ. }}$ [Turker]: Hadjin (MNHU, Berlin); MIespotamien, Mardin (MNHL, Berlin).

$\ddagger$ HADROECIA, misspelling. See Hydraecia Guenée, I8 4 I.

HADE

HADULA Staudinger, I889, Stettin. ent. Ztg $\mathbf{5 0}: 43$. HADE

Type-species: Hadula insolita Staudinger, I889, ibidem 50: 43, by monotypy. Syntypes ô, 우 [U.S.S.R.]: [Kirghizia], S. of Issyk Ǩul (MNHU, Berlin).

HADULIPOLIA Boursin, 1964, I'eröff. zool. StSamml. Wünch. 2: 22.

HADE

Type-species: Mamestra odiosa Staullinger, $1896, D t$. ent. Z. Iris 8: 315, by original designation. Syntypes 3 o, I 우, [CHiNa]: Tibet (MNHU, Berlin).

HAEMABASIS Hampson, i913, Cat. Lepid. Phalaenae Br. Mus. 12: ix, 266.

CATO

Type-species: Buzara calodesma Rothschild \& Jordan, I905, Novit. zool. 12: 474, by original designation. Syntypes $\delta$, ㅇ, NEw GuineA: [Papua], R. Angabunga (BMNH).

HAEMAPHLEBIA Hampson, y 9 Io, Cat. Lepid. Phalaenae Br. Mus. 10: 3, $203 . \quad$ ACoN

Type-species: Haemaphlebia atripalpis Hampson, I9Io, ibidem 10: 203, fig. 45, by original designation. Holotype $\hat{o}$, [GHana] Gold Coast: Kumasi (BMNH).

HAEMASSIA Hampson, I908. See Haemerosia Boisduval, 1840. ACro

HAEMATOSTICTA Hampson, I896, Famna Br. India (Moths) 4:5 $42 . \quad$ ACON

Type-species: Haematosticta sanguiguttata Hampson, 1896, ibidem 4: 542, fig. 281, by original designation. Type(s) ô, CEYLON (BMNH).

HAEMEROSIA Boisduval, i 840, Genera Index meth. Eur. Lepid.: i63. ACRo

Type-species: Pyralis renalis Hübner, [1813], Samml. eur. Schmett. 6: pl. 24, fig. I57, by subsequent designation by Duponchel, 1845, in d'Orbigny, Dict. univl Hist. nat. 6: 455 , but cited as renifera, an unjustified emendation by Boisduval, I 840 , ibidem: I 64 . Type(s), Europe.

HAEMASSIA Hampson, 1908, Cat. Lepid. Phalaenae Br. Mus. 7: Io. Available, but without included species until Hampson, I909, ibidem 8: xii, 438.

Type-species: Pyralis renalis Hübner, [18I3], by subsequent monotypy.

Haemassia is a junior objective synonym of Haemerosia Boisduval, r 840 .

HALASTUS Butler, I892, Ann. Mag. nat. Hist. (6) 9: 376.

OPHI

Type-species: Halastus intricatus Butler, 1892, ibidem (6) 9: 376, by monotypy. Syntypes [Nigeria]: Old Calabar (BMNH); R. Niger (BMNH). [Angola]: Ambriz (BMNH). Sierra Leone (BMNH).

Butler proposed his new genus and species for Ophideres princeps Boisduval sensu Guenée, I 852; Walker, I857; and Moore, I88 r.

H. intricatus is a junior subjective synonym of Ophideres divitiosa Wallier, r869, Proc. nat. Hist. Snc. Glasgow 1(2): 356, pl. 7, fig. I I.

${ }_{+}^{+}$HALASTL'S Snellen, I 895, Tijdschr. Ent. 38: 205.

Neave, 1939, Nomencl. zool. 2: 549 cited the above as an original reference, but Snellen attributed Halastus to Butler and was not proposing a new name.

$\ddagger$ HALASTUS Snellen, I 895. See Halastus Butler, I 892.

OPHY 
HALIAS Treitschlke, I829. See Pseudoips Hübner, I 822.

CHLO

HALIOPHYLE Warren, I9I2, in Seitz, Gross-Schmett. Erde 11: 76.

Type-species: Leucania compsias Meyrick, I899, in Sharp, Fauna Hawaiiensis 1(2): I39, pl. 4, fig. 2 , by original designation. Syntypes $5(\pi, 9)$, Hawalran Is: IKilauea (BMNH); Olaa (BMNH).

The date of publication of the part of Seitz containing page 76 was stated on page 73 as I9I 2 September $25^{\text {th }}$ in both the English and German editions.

HAL OCHROA Hampson, I926, Descr. new Genera Species Lepid. Phalaenae Subfamily Noctuinae Br. Mus.: 48 .

OPHI

Type-species: Diphthera aequatoria Mabille, I879, Guide du Naturaliste. Revue biblphiq. Sci. 1: 26, by original designation. Lectotype +, Congo (BMNH), designated (as holotype) by Viette \& Fletcher, I968, Bull. Bv. Mus. nat. Hist. (Ent.) 21: 4 I 4.

HAMA Stephens, I829. See Apamea Ochsenheimer, I8I6.

ACRO

HAMAXIA Walker, I863. See Olulis Walker, I 863.

OPHI

HAMODES Guenée, I 852, in Boisduval \& Guenée, Hist. nat. Insectes (Lépid.) 7: 202. OPH

Type-species: Ophiusa propitia Boisduval, I832, in d'Urville, Voyage de Découvertes de l'Astrolabe 1: 244, by subsequent designation by Hampson 1894, Fauna Br. India (Moths) 2: 547, but cited as 'propitia Guen.', an incorrect authorship. Type(s), [New GurNeA]: [Bismarck Archipelago], Nouvelle-Irlande.

Boisduval attributed $O$. propitia to Guérin-Méneville who did not, however, publish his description of propitia until I 838 .

HAMPSONIA Kozhanchikov, 1950. See Hampsonidia Inoue, I958.

ACRO

HAMPSONIDIA Inoue, 1958, in Inoue \& Sugi, Check List Lepid. Jap. 5: 436.

ACRO

Type-species: Apatela jankowskii Oberthür, I880, Études Ent. 5: 69, pl. 7, fig. I, by original designation (for Hampsonia Kozhanchikov, I950). Holotype $\hat{0}$, [U.S.S.R.]: near Vladivostok, Askold I. (BMNH).

Hampsonidia was proposed as the objective replacement name for Hampsonia Kozhanchikov, 1950.

HAMPSONIA Kozhanchikov, I950, Fanna SSSR (Insecta, Lepid.) 12: 535.

Type-species: A patela jankowskii Oberthür, I880, by original designation.

A junior homonym of Hampsonia Swinhoe, I894, Ann. Mag. nat. Hist. (6) 14: 443, Lepid., Zygaenidae. The objective replacement name is Hampsonidia Inoue, 1958.

HAMPSONODES nom. n. for Calymniodes Hampson, I908.

ACRO

Type-species: Hadena basicarnea Walker, I857, List Specimens lepid. Insects Colln $B r$. Mus. 11: 587 , by original designation (for Calymniodes Hampson). Holotype $\tilde{\sigma}$, VenEZUELA (BMNH).

CALYMNIODES Hampson, 1908, Cat. Lepid. Phalaenae Br. Mus. 7: I8. Available, but without included species until Hampson, I9Io, ibidem 9: I28.

Type-species: Hadena basicarnea Walker, I 857 , by original designation.

A junior homonym of Calymniodes Dognin, 1907,-Lepid., Noctuidae. This generic name was used and made available by Dognin prior to its proposal and generic description by Hampson for a related but distinct genus. The objective replacement name is Hampsonodes nom. n.

HANSA Berio, I959. See Beriohansa nom. n.

CATO

HAPALIA Hübner, [I82I]. See Actebia Stephens, I829.

NOCT

HAPALOTIS Hübner, [I82I] I8I6, Verz. bekannter Schmett.: 254.

ACRO

Type-species: Phalaena venustula Hübner, I790, Beitv. Gesch. Schmett. 2(3): 78, pl. 4, fig. Z, by subsequent designation by Butler, I891, Entomologist 24: I 3 . Type(s), TYPE-LOCALITY not stated [EUROPE]. 
HAPDA nom. n. for Dapha Walker, [1863 May i 3 th]. HYPE

Type-species: Dapha exhibens Walker, [I863] 1864, J. Proc. Limn. Soc. (Zool.) 7: 55, by monotypy (of Dapha Walker). Lectotype +. Borneo: Sarawak (UM, Oxford), designated (as type) by Swinhoe, I900, Cat. east. and Aust. Lepid. Heterocera 2: 216.

D.APHA Walker, [1863 May I3tli] 1864, J. Proc. Linn. Soc. (Zool.) 7: 54.

Type-species: Dapha exhibens Walker, [1863], by monotypy.

A junior homonym of Dapha Walker, 1863 [April I8th], List Specimens lepid. Insects Colln Br. Mus. 27: I24, - Noctuidae, Chloephorinae. The objective replacement name is Hapda nom. n.

HAPIGIA Guenée, I 852, in Boisduval \& Guenée, Hist. nat. Insectes (Lépid.) 6: 375. NOTODON TIDAE

Type-species: Hapigia nodicornis Guenée, I852, ibidem 6: 376, pl. I2, fig. 7, by monotypy.

Hapigia was originally placed in the Noctuidae.

HAPLOCESTRA Aurivillius, ig Io, Annali Mus. civ. Stor. nat. Genova 44: $498 . \quad$ Hade Type-species: Haplocestra similis Aurivillius, I9I0, ibidem 44: 499. Syntypes 2 J. Cape T'erde Is: Razo I.

Haplocestra is dated from the bottom of page 497 as I9Io April Ioth; Neave, 1939, Nomencl. zool. 2: 566 , cited I 909 .

HAPLOOLOPHUS Butler, I891, Ann. Mag. nat. Hist. (6) 8: 71, 73.

ACRO

Type-species: Eriopus mollissima Guenée, 1852 , in Boisduval \& Guenée, Hist. nat. Insectes (Lépid.) 6: 294, by original designation. Holotype ô. [U.S.A.]: Florida (BMNH).

HAPLOPSEUSTIS Meyrick, I 902 April, Trans. ent. Soc. Lond. 35: 34. ACON Type-species: Haplopseustis erythrias Meyrick, I902, ibidem 35: 34, by monotypy. Syntypes 20 . Australia: N. Aust., Port Darwin (BMNH); Qd, Brisbane.

See also Acnissa Turner, 1902 December.

HAPLOSTOLA Möschler, I890, Abh. senckenb. naturforsch. Ges. 16: I62.

ACON

Type-species: Haplostola aphelioides Möschler, I890, ibidem 16: I63, by monotypy. Holotype ô. [Puerto Rico] Portorico I. (MNHU, Berlin).

HARITA Moore, I882, in Hewitson \& Moore, Descr. new Indian lepid. Insects Colln late Mr W. S. Atkinson: 187 . HYPE

Type-species: Harita rectilinea Moore, 1882, ibidem: 187, pl. 6, fig. 23, by monotypy. Syntypes, Inda: [Assam], Khasia Hills (BMNH).

HARITALOPHA Hampson, I895, Trans. ent. Soc. Lond. 1895: 309.

OPHI

Type-species: Haritalopha biparticolor Hampson, I 895, ibidem 1895: 309, by original designation. Type(s) ô. BHutan (BMNH).

HARMATELIA Moore, I882. See Imaharela nom. n.

OFHI

HARMODIA Hübner, [1820]. See Miselia Ochsenheimer, I8I6.

HADE

HARPAGLAEA Hampson, I906, Cat. Lepid. Phalaenae Br. Mus. 6: xiii, 429.

cucu

Type-species: Glaea pastillicans Morrison, 1874, Proc. Boston Soc. nat. Hist. 17: I5I, by original designation. Holotype, [U.S.A.]: New Hampshire.

G. pastillicans is a junior subjective synonym of Orthosia apiata Grote, 1874, Anmeal Rep. Trustees Peabody Acad. Sci. 6: 30.

See also Epiglaea Grote, 1878.

HARPAGOPHANA Hampson, I906, Cat. Lepid. Phalaenae Br. Mus. 6: viii, I 5.

CUCU

Type-species: Metopoceras hilaris Staudinger, I895, Dt. ent. Z. Iris 7:275, by original designation. Holotype $\hat{\sigma},[$ Sp'sin]: Murcia prov., Algezares (MNHU, Berlin). 
HARRISIMEMNA Grote, I873, Trans. Am. ent. Soc. 4: 293.

ACRO

Type-species: Notodonta sexguttata Harris, I869, Occ. Pap. Boston Soc. nat. Hist.

1: 175, figs 24, 25, by monotypy. Syntypes $\hat{\delta}$, + , [U.S.A.]: [Mass.], Cambridge.

$N$. sexguttata is a junior subjective synonym of Grammophora trisignata Walker, 1856, List Specimens lepid. Insects Colln Br. Mus. 9: 29.

HARRISONIA Schaus, I923. See Agrotisia Hampson, I908.

ACRO

HAR VEYA Grote, I 873 , Bull. Buffalo Soc. nat. Sci. 1: 125.

OPH I

Type-species: Harveya auripennis Grote, I873, ibidem 1: I 26, by monotypy. Syntypes o. [U.S.A.]: Florida; Kentucky.

$H$. auripennis is a junior subjective synonym of Siavana repanda Walker, [1858].

See also Siavana Walker, [1858].

HAYESIA nom. n. for Stictothripa Hampson, I 912.

SARR

Type-species: Thalpochares grisella Schaus, I904, Trans. Am. ent. Soc. 30: I62, by original designation (for Stictothripa Hampson). Type(s) Venezuela: Aroa.

This new name is proposed in honour of Mr Alan $\mathrm{H}$. Hayes in appreciation of his enthusiastic help and textual criticism.

STICTOTHRIPA Hampson, r9i2, Cat. Lepid. Phalaenae Br. Mus. 11: $45^{\circ}$.

Type-species: Thalpochares grisella Schaus, 1904, by original designation.

A junior homonym of Stictothripa Schaus, I9II,-Lepid., Noctuidae. This generic name probably originated from Hampson, but was used and unintentionally made nomenclaturally available by Schaus, prior to its proposal and generic description by Hampson for a similar concept but having a different type-species. The objective replacement name is Hayesia nom. $\mathrm{n}$.

HEBDOMOCHONDRA Staudinger, I879, Stettin. ent. Ztg 40:321.

NOCT

Type-species: Hebdomochondra syrticola Staudinger, I879, ibidem 40: 32I, by monotypy. Syntypes $\widehat{\jmath}$, q [U.S.S.R.]: S.E. european Russia, Narün sandy region (MNHU, Berlin).

$\ddagger$ HEPDOMOCHONDRA; Staudinger, I9or, in Staudinger \& Rebel, Cat. Lepid. palaearct. Faunengeb. 1:223.

An incorrect subsequent spelling.

HECATERA Guenée, I852, in Boisduval \& Guenée, Hist. nat. Insectes (Lépid.) 6: 27. HADE

Type-species: Nociua dysodea [Denis \& Schiffermüller], 1775, Ankïndung syst. Werkes Schmett. Wienergegend: 72, by original designation. Type(s), [Austria]: Vienna district (Collection destroyed, Horn \& Kahle, 1936, Ent. Beih. Berl.-Dahlem 3: 243).

HECATESIA Boisduval, I828, Essai Monogr. Zygénides: Ir. AGAR

Type-species: Hecatesia fenestrata Boisduval, 1828, ibidem: II, pl. I, fig. 2, by monotypy. Type(s), [Australia] Nouvelle-Hollande.

HED YMIGES Turner, 1933, Trans. R. Soc. S. Aust. 57: I60.

Type-species: Cycloprora aridoxa Turner, I931, Proc. Linn. Soc. N.S.W. 56: 338, by monotypy (of Cycloprova Turner, r93I). Holotype $\widehat{\jmath}$. Australia: Qd, Toowoomba.

Hedymiges was proposed as the objective replacement name for Cycloprova Turner, I93I.

CYCLOPRORA Turner, I931, Proc. Linn. Soc. N.S.W. 56: 338.

Type-species: Cycloprova aridoxa Turner, I93I, by monotypy.

A junior homonym of Cycloprova Turner, 1920, - Lepid., Noctuidae. The objective replacement name is Hedymiges Turner, 1933.

HELIA Hübner, I818, Zuträge Samml. exot. Schmett. 1: 27.

OPHI

Type-species: Helia calligramma Hübner, I8I8, ibidem 1: 27, figs I57, I58, by subsequent designation by Berio, 1957, Memorie Soc. ent. ital. 36: I5. Type(s) ô, SURINAM. 
HELIA Duponchel, [1845]. See Epizeuxis Hübner, I8I8.

HYPE

HELIA Guenée, I854. See Epizeuxis Hübner, I8I8.

HYPE

HELIACA Herrich-Schäffer, [I85I]. See Heliodes Guenée, I $84 \mathrm{I}$ ACRo

HELICOVERPA Hardwick, 1965, Mem. ent. Soc. Can. 40: 9. NOCT

Type-species: Noctua armigera Hïbner, [I808], Samml. eur. Schmett. 4: pl. 79, fig. 370 , by original designation. Type(s), Europe.

HELIGMA Agassiz, I846. See Eligma Hübner, [1819].

CHLO

HELIOCHEILUS Grote, 1865, Proc. ent. Soc. Philad. 4: 328.

NOCT

Type-species: Heliocheilus paradoxus Grote, I865, ibidem 4: 329, pl. 2, figs 3-5, by monotypy. Syntypes $2 \hat{\sigma}$, I ㅇ, [U.S.A.]: Colorado Territory.

HELIOCONTIA Hampson, igio, Cat. Lepid. Phalaenae Br. MIus. 10: xvii, 662.

ACON

Type-species: Emmelia apicella Grote, I872, Trans. Am. ent. Soc. 4: 21, by original designation. Syntypes $\hat{\jmath}$, 우, [U.S.A.]: central Alabama.

HELIODES Guenée, I 4 I, Annls Soc. ent. Fr. 10: 6 I.

ACRO

Type-species: Noctua arbuti Fabricius, I 775, Syst. Ent. : 619, by subsequent designation by Guenée, 1852, in Boiscluval \& Gucnée, Hist. nat. Insectes (Lépid.) 6: I97. Type(s), [Great Britain]: [England] Anglia (Type(s) not found by Zimsen, I964, Type Material of I.C. Fabricius: 525).

N. arbuti is a junior subjective synonym of Phalaena tenebrata Scopoli, I763, Ent. Carniolica: 230 .

See also Panemeria Hïbner, [1823].

HELIACA Herrich-Schäffer, [185I] 1845, Syst. Bearb. Schmett. Euv. 2: 370.

Type-species: Noctua arbuti Fabricius, 1775 (but included by Herrich-Schäffer as 'arbuti L.', an incorrect authorship), by subsequent designation by Hampson, i9ıo, Cat. Lepid. Phalaenae Br. Mus. 9: 487, but cited as tenebrata Scopoli, 1763 , which Hampson placed, on page 488 , as the senior synonym of arbuti Fabricius, 1775 .

A junior homonym of Heliaca Hübner, 1822, Syst.-alphab. Verz.: 52, - Lepid., Pyralidae. The objective replacement name is Heliodes Guence.

Incorrect type-species designation: Phalaena tenebrata Scopoli, I763, a name not originally included in Heliaca and not linked with one of the originally included names when cited by Grote, I895, Abh. naturw. Ver. Bremen 14: I I3, as well as by Bode, I907, Mitt. Roemer-Mus., Hildesheim 22: 52.

HELIODORA Neumoegen, I891, Can. Ent. 23: 125. ACON

Type-species: Heliodora magnifica Neumoegen, I 891, ibidem 23: I 25, by monotypy. Holotype , [U.S.A.]: Texas, Houston.

H. magnifica is a junior subjective synonym of Acontia costalis WValker, [I858] I857, List Specimens lepid. Insects Colln Br. Mus. 12: 787.

GRAEPERIA Grote, i 895, Abh. naturw. Ver. Bremen 14: io8.

Proposed, unnecessarily, as an objective replacement name for Heliodora Neumoegen, I 89 I, which Grote considered to be a junior homonym of Heliodore Stål, i 867,-- Hemiptera. Under the Code, Article 56(a), a one-letter difference is sufficient to prevent homonymy in genus-group names.

HELIOLONCHE Grote, 1873, Bull. Buffalo Soc. nat. Sci. 1: i I 5. NOCT

Type-species: Heliolonche modicella Grote, I873, ibidem 1: I16, pl. 3, fig. I2, by monotypy. Syntypes ő, ㅇ, [U.S.A.]: California (BMNH).

HELIOMANES Sodoffsky, I837. See Porphyrinia Hübner, [i82 I].

ACON

†HELIOPEPLA Hampson, no published reference found.

HYPE 
HELIOPHANA Grote, I875, Bull. Buffalo Soc. nat. Sci. 2: 220.

NOCT

Type-species: Heliothis mitis Grote, I873, Bull. Buffalo Soc. nat. Sci. 1: i I6, pl. 3, fig. 7, by monotypy. Holotype \%. [U.S.A.]: central Alabama (BMNH).

Heliophana Grote is not preoccupied by $\ddagger$ Heliophana Simon, I864, Hist. nat. Araignées (Edn I): 332, an incorrect subsequent spelling of Heliophanus Koch, I833, in Panzer, Faun. Insect. Germanicae initia Heft 119: pl. I, - Arachnida.

$\ddagger$ HELIOPHILA Hübner, [I806]. See Aletia Hübner, [1821]. HADE

HELIOPHILA Hübner, I822. See Aletia Hübner, [I82I]. HadE

$\ddagger$ HELIOPHILAE Ochsenheimer, I8I6. See Leucania Ochsenheimer, I8I6. HadE

HELIOPHISMA Hampson, I913, Cat. Lepid. Phalaenae Br. Mus. 12: xi, 46r. CAto

Type-species: Ophisma croceipennis Walker, 1858, List Specimens lepid. Insects Colln Br. Mus. 14: I377, by original designation. Syntypes 8 ex., South Africa: Port Natal (BMNH). Sierra Leone. Congo.

O. croceipennis is a junior subjective synonym of Ophiusa klugii Boisduval, I833, Nouv. Annls Mus. Hist. nat. Paris 2: $25 \mathrm{I}$.

HELIOPHOBUS Boisduval, I828, Eur. Lepid. Index Meth.: 69.

HADE

Type-species: Phalaena saponariae Borkhausen, I792, Naturg. eur. Schmett. 4: 370, by subsequent designation by Duponchel, I829, in Godart \& Duponchel, Hist. nat. Lépid. Papillons Fr. 7(2): 71. Syntypes ô, P, Europe.

$P$. saponariae is a junior subjective synonym of Phalaena reticulata Goeze, I $78 \mathrm{r}$, Ent. Beyträge 3(3): 254 .

NEURIA Guenée, I84I, Annls Soc. ent. Fr. 10:24I.

Type-species: Phalaena saponariae Borkhausen, I792, by subsequent designation by

Guenée, I 852, in Boisduval \& Guenée, Hist. nat. Insectes (Lépid.) 5: I66.

$P$. saponariae was included by Guenée as 'saponariae Esper' an incorrect authorship.

Neuria Guenée is a junior objective synonym of Heliophobus Boisduval, I 828 .

NEVRIA Agassiz, I846, Nomencl. zool. Index univl.: 249.

An unjustified emendation of Neuria Guenée, I84 r.

HELIOSCOTA Grote, I895, Abh. naturw. Ver. Bremen 14: 77.

ACIRO

Type-species: Hadena miselioides Guenée, I852, in Boisduval \& Guenée, Hist. nat.

Insectes (Lépid.) 6: 89, by original designation. Syntypes, [U.S.A.]: Carolina; Florida;

New York state (BMNH).

HELIOSEA Grote, I 875, Bull. Buffalo Soc. nat. Sci. 2: 220.

NOCT

Type-species: Heliosea pictipennis Grote, I875, ibidem 2: 220, by monotypy. Type(s)

[U.S.A.]: California (BMNH).

$\ddagger$ †HELIOTHENTES Ochsenheimer, I8I6. See Heliothis Ochsenheimer, I8I6. Nocr

HELIOTHERA Sodoffsky, I837. See Acontia Ochsenheimer, I8I6. ACON

$\ddagger$ †ELIOTHIS Hübner, [I806]. See Heliothis Ochsenheimer, I8I6. NOCT

$\ddagger$ †ELIOTHIS Hübner, I808. See Melipotis Hübner, I8I8. OPн I

HELIOTHIS Ochsenheimer, I8I6, Schmett. Eur. 4:91. NOCT

Type-species: Phalaena dipsacea Linnaeus, 1767, Syst. Nat. (Edn I2) 1: 856, by subsequent designation by Samouelle, I8I9, Entomologist's useful Compendium: 252. Type(s), Sweden.

$P$. dipsacea is a junior subjective synonym of Phalaena viriplaca Hufnagel, I766, Berlin. Mag. 3(4): 406 .

Q 
‡HELIOTHIS Hübner, [1806], Tentamen determinationis digestionis . . . : [2].

Included in a work rejected for nomenclatural purposes by Int. Commn zool. Nom., 1926, Smithson. misc. Collns 73(4) Opinion 97: 19. Also idem, 1954, Opin. Decl. int. Commn zool. Nom. 6 Opinion 278: 140. Only included species: Phalaena dipsacea Linnaeus, 1767 .

${ }_{\ddagger}$ HELIOTHENTES Ochsenheimer, I8I6, Schmett. Eur. 4: 91.

Published as a junior synonym of Heliothis Ochsenheimer, I8I6, and not subsequently treated as an available name under the Code, Article II (d).

$\ddagger$ HELIOTIS; Lefebvre, I 827, Mém. Soc. linn. Paris 6: 94.

An incorrect subsequent spelling.

HELIOTHISA Meigen, 1832, Syst. Beschreibung Eur. Schmett. 3: 224.

An unjustified emendation of Heliothis Ochsenheimer. Meigen did not mention Heliothis in the generic diagnosis, but in some specific synonymies e.g. on the first lines of pages 230 and 233, the genus Heliothis was cited.

HELIOTIS Sodoffsky, 1837, Bull. Soc. imp. Nat. Moscou 1837 (6) : 88.

An unjustified emendation of Heliothis Ochsenheimer, I8I6. Neave. I939, Nomencl. zool. 2: 595, listed two earlier usages of Heliotis, neither of which was nomenclaturally available. The first, $\ddagger$ Heliotis Lefebvre, 1827 (see above), was an incorrect subsequent spelling. The second, + Heliotis Laporte, 1836 , Revue Ent. (Silbermann) 4: r8, was listed as the minor name of a multiple original spelling of Hellotis Laporte, - Coleoptera, but I cannot find this usage in the original reference.

$\ddagger$ †ELIOTHRIS; Olliff, I890, Agric. Gaz. N.S.W. 1(1): I25. Also [Anonymous], r893, ibidem 4 (3): 2 I 3.

An incorrect subsequent spelling.

HELIOTHISA Meigen, r832. See Heliothis Ochsenheimer, I816.

NOCT

HELIOTHODES Hampson, 1908, Cat. Lepid. Phalaenae Br. Mus. 7: 8. Available, but without included species until Hampson, I910, ibidem 9: xiv, 464.

NOCT

Type-species: Heliothis diminutivus Grote, 1873, Bull. Buffalo Soc. nat. Sci. 1: 148, by subsequent designation by Hampson, I9Io, ibidem 9: 464 . Syntypes ro $(\widehat{o}, q)$, [U.S.A.]: California (BMNH).

†HELIOTHRIS, misspelling. See Heliothis Ochsenheimer, I8I6.

NOCT

†HELIOTIS, misspelling. See Heliothis Ochsenheimer, r816.

NOCT

HELIOTIS Sodoffsky, I837. See Heliothis Ochsenheimer, I816.

NOCT

HELOTROPHA Lederer, 1857, Noctuinen Eur.: 35, II 8.

ACRO

Type-species: Noctua leucostigma Hübner, [1808], Samml. eur. Schmett. 4: pl. 80, fig. 375, by monotypy. Type(s), Europe.

HEMACHRA Sodoffsky, r837. See Ophiusa Ochsenheimer, I8I6.

CATO

$\ddagger$ HEMERAPTENA, misspelling. See Eustrotia Hübner, [I82 I] .

$A C O N$

HEMEROBLEMMA Hïbner, I8 8, Zuträge Samml. exot. Schmett. 1: 26.

OPHI

Type-species: Hemeroblemma amethystina Hübner, I8I8, ibidem 1: 26, figs 147, 148, by subsequent designation by Desmarest, 1857, in Chenu, Encycl. Hist. nat. Papillons nocturnes: 130 , but cited as \$ametystina, an incorrect subsequent spelling. Type(s) $\widetilde{\sigma}$, SURINAM.

H. amethy'stina is a junior subjective synonym of Phalaena dolon Cramer, 1777 , Uitlandsche Kapellen 2: 7, pl. Iо I, figs D-F.

HEMEROPLANIS Hübner, 1818, Zuträge Samml. exot. Schmett. 1: 23.

OPIII

Type-species: Hemeroplanis pyralis Hübner, 1818 , ibidem 1:23, figs $127, \mathrm{r} 28$, by monotypy. Type(s) $\tilde{\sigma}$, [U.S.A.]: Georgia [formerly] in Florida. 
H. pyralis is a junior subjective synonym of Phytometra scopulepes Haworth, I809, Lepid. Br.: 260.

See also Scopelopus Stephens, I829; and Coptocnemia Zeller, I872.

PLEONECTYPTERA Grote, I872, Trans. Am. ent. Soc. 4: 23.

Type-species: Hemeroplanis pyralis Hübner, I8I8, by subsequent designation by Grote, I874, Bull. Buffalo Soc. nat. Sci. 2: 44.

Pleonectyptera is a junior objective synonym of Hemeroplanis Hübner, I8r8.

HEMEROPTENA Sodoffsky, I837. See Eustrotia Hübner, [I82I].

$\operatorname{ACON}$

HEMIBR YOMIMA Barnes \& Benjamin, I927, Pan-Pacif. Ent. 3: II I.

Type-species: Hadena chryselectra Grote, I880, Can. Ent. 12: 244 by original designation. Type(s) $\hat{o}$, [U.S.A.]: Colorado.

HEMICEPHALIS Möschler, I 890, Abh. senckenb. naturforsch. Ges. 16: I74.

OPH I

Type-species: Phalaena characteria Stoll, I790, in Cramer, Uitlandsche Kapellen Aanhangsel: 154 , pl. 34, fig. 5, by monotypy. Type(s), Surinam.

$P$. characteria is dated from the wrapper of the part and not from the title-page of the volume, I 79 I.

HEMICERAS Guenée, I852, in Boisduval \& Guenée, Hist. nat. Insectes (Lépid.) 6: 379. NOTODONTIDAE

Type-species: Hemiceras cadmia Guenée, I 852 , ibidem 6:383, pl. I3, fig. 2, by subsequent designation by Grote, 1874, Bull Buffalo Soc. nat. Sci. 2: 29.

Hemiceras was originally placed in the Noctuidae.

HEMICERATOIDES Strand, I9I I, Fauna exot. 1 : 42.

OPHI

Type-species: Hemiceras hieroglyphica Saalmüller, I89I, Lepid. Madagascar: 405, fig. 208, by monotypy. Holotype $\hat{0}$, Madagascar: N.W., Nossi-Bé I.

HEMICHLORIDIA Hampson, I926, Descr. new Genera Species Lepid. Phalaenae Subfamily Noctuinae Br. Mus.: I43.

OPHI

Type-species: Westermannia euprepia Hampson, I902, J. Bombay nat. Hist. Soc. 14: 209, by original designation. Syntypes ô. India: Cuddapah, Horsleykhonda (BMNH). CEYLON (BMNH).

HEMICTENOPHORA Sugi, I970, Tinea 8: 222.

ACRO

Type-species: Hemictenophora euplexiodes Sugi, I970, ibidem 8: 223, figs I4, 27, by original designation. Holotype ô, Ryukyu Is: Ishigaki I., Air Port (Colln S. Sugi, Tokyo).

HEMIEUXOA McDunnough, [1929] I928, Bull. Dep. Mines, Can. 55: 44.

NOCT

Type-species: Agrotis rudens Harvey, I 875, Bull. Buffalo Soc. nat. Sci. 2: 271, by original designation. Syntypes $\hat{\jmath}$, 우, [U.S.A.]: Texas (BMNH).

HEMIEXARNIS Boursin, I948, Revue fr. Lépidopt. 11: 256.

NOCT

Type-species: Euxoa moechilla Püngeler, I906, Dt. ent. Z. Iris 19: 8I, pl. 6, fig. I3, by original designation. Holotype $\widehat{\delta},[\mathrm{CHINA}]$ : E. Turkestan [Sinkiang-Uigur], Aksu (MNHU, Berlin).

HEMIGEOMETRA Haworth, I 809, Lepid. Br.: 267.

CATO

Type-species: Phalaena fraxini Linnaeus, I758, Syst. Nat. (Edn Io) 1: 5I2, by subsequent designation by Berio, 1966, Annali Mus. civ. Stor. nat. Giacomo Doria 76: 6o. Type(s), TyPE-Locality not stated (LS, London) [Europe].

BLEPHARIDIA Hübner, I822, Syst.-alphab. Verz.: 21, 26.

Type-species: Phalaena fraxini Linnaeus, I758, by subsequent designation by Berio, 1957, Memorie Soc. ent. ital. 36: 9.

Blepharidia Hübner is a junior objective synonym of Hemigeometra Haworth, I809. 
HEMIGRAPHIPHORA McDunnough, [1929] I928, Bull. Dep. Mines, Can. $55: 69 . \quad$ Noct Type-species: Noctua plebeia Smith, i 898, Jl N.Y. ent. Soc. 6: 105, by original designation. Syntypes $0,+$, [CANADA]: Vancouver; Br. Columbia. [U.S.A.]: California.

HEMIGROTELLA Barnes \& McDunnough, I9I8, Contr. nat. Hist. Lepid. N. Am. 4: 91.

Type-species: Hemigrotella argenteostriata Barnes \& McDunnough, I9r8, ibidem 4: 91, pl. I7, fig. I5, by original designation. Syntypes 8 J., 3 ․․ [U.S.A.]: Calif., Riverside Co., Palm Springs.

HEMINOCLOA Barnes \& Benjamin, 1924, Contr. nat. Hist. Lepid. N. Am. 5: 165. ACRo Type-species: Basilodes mirabilis Neumoegen, I884, Papilio 4: 94, by original designation. Holotype $\hat{\sigma}$, [U.S.A.]: S.W. Arizona.

HEMIOSLARIA Barnes \& Benjamin, 1924, Contr. nat. Hist. Lepid. N. Am. 5: 167. ACRo Type-species: Hemioslaria pima Barnes \& Benjamin, I924, ibidem 5: 167, by original designation. Holotype $\hat{\sigma},[$ U.S.A.]: Arizona, Pima Co., Baboquivari Mts.

HEMIPACHNOBIA McDunnough, [1929] 1928, Bull. Dep. Mines, Can. 55: $47 . \quad$ Noct

Type-species: Agrotis monochromatea Morrison, 1874, Proc. Boston Soc. nat. Hist. 17 : I65, by original designation. Type(s) ô. [U.S.A.]: Massachusetts.

HEMIPACHYCERA Butler, r891, Ann. Mag. nat. Hist. (6) 8:71, 75.

ACRO

Type-species: Callopistria rivularis Walker, [1858] I857, List Specimens lepid. Insects Colln $\mathrm{Br}$. Mus. 12: 867, by original designation. Holotype , INDiA: northern (BMNH).

HEMIPSECTRA Hampson, I891, Illust. typical Specimens Lepid. Heterocera Colln Br. Mus. 8: 17,84 . OPHI

Type-species: Hemipsectra plumipars Hampson, I891, ibidem 8: r7, 84, pl. I 47 , fig. 23, by monotypy. Type(s) ठે. INDiA: Nilgiri district, plateau (BMNH).

HEMISPRAGUEIA Barnes \& Benjamin, 1923, Contr. nat. Hist. Lepid. N. Am. 5: 85. ACON

Type-species: Cerathosia idella Barnes, 1905, Can. Ent. 37 : 193, by original designation. Syntypes ô. ㅇ. [U.S.A.]: Arizona, Pima Co.

HEMISTILBIA Barnes \& Benjamin, I929, Bull. Brooklyn ent. Soc. 24: i68.

ACRO

Type-species: Stilbia apposita Barnes \& McDunnough, 1918, Contr. nat. Hist. Lepid. N. Am. 4: I06, pl. I6, fig. 8, by original designation. Syntypes 2 ㅇ. [U.S.A.]: Arizona, Prescott.

HEOEUGORNA Hampson, 1924, in Tams, J. nat. Hist. Soc. Siam 6: 254.

OPHI

Type-species: Eugorna alboarcuata Bethune-Baker, 1906, Novit. zool. 13: 269, by original designation. Holotype ô. New Guinea: [Papua], R. Aroa (BMNH).

HEORTA Walker, I858, List Specimens lepid. Insects Colln Br. Mus. 15: r664.

NOTODONTIIDAE

Type-species: Heorta roseoalba Walker, I 858, ibidem 15: 1665, by monotypy.

Heorta was originally placed in the Noctuidae.

HEPATICA Staudinger, I892, in Romanoff, Mém. Lépid. 6: 634.

$\mathrm{OPHI}$

Type-species: Hepatica anceps Staudinger, r 892 , ibidem 6: 634, pl. I2, fig. I I, by monotypy. Holotype $\tilde{\sigma}$, [U.S.S.R.]: Amur district, Sutschan area (MNHU, Berlin).

${ }_{+}^{+}$HEPDOMOCHONDRA, misspelling. See Hebdomochondra Staudinger, I 874 .

NOCT

HEPSIDERA Swinhoe, 1902, Ann. Mag. nat. Hist. (7) 9: 176.

HYPE

Type-species: Hepsidera lignea Swinhoe, 1902, ibidem (7) 9: I 76, by monotypy. Type(s)

o., [Malaysia]: Perak, Goping (BMNH). 
HEPTAGROTIS McDunnough, [1929] I928, Bull. Dep. Mines, Can. 55: 52.

NOCT

Type-species: Agrotis phyllophora Grote, I874, Bull. Buffalo Soc. nat. Sci. 2: 61, by original designation. Syntypes 2 J. [U.S.A.]: New York (BMNH). CAnAda (BMNH).

HEPTAPO TAMIA Alphéraky, I882, Horae Soc. ent. ross. 17: 75.

ACRO

Type-species: Heptapotamia eustratii Alphéraky, I882, ibidem 17: 75, pl. 3, fig. 57,

by monotypy. Holotype 0 . [CHrNA]: Kouldja $\left[44^{\circ} \mathrm{N} .8 \mathrm{I}^{\circ} \mathrm{E}.\right]$, Arganaty (ZI, Leningrad).

$\ddagger$ HE RACLEA, misspelling. See Hevaclia Hübner, [1820].

AGAR

HERACLIA Hübner, [1820] i816, Verz. bekannter Schmett.: i 80.

AGAR

Type-species: Phalaena euphemia Stoll, I78I, in Cramer, Uitlandsche Kapellen 4: 105, pl. 345, fig. A, by subsequent designation by Hampson, I920, Cat. Lepid. Phalaenae Br. Mus. Suppl. 2: 549, but cited as geryon Fabricius, I 78 I, and referred back to Hampson, I90I, ibidem 3: 565 , where geryon was placed as the senior synonym of euphemia Stoll. Type(s), Moluccas: Amboina I.

$P$. euphemia is a junior subjective synonym of Noctua geryon Fabricius, I78I, Species Insect. 2: 2 I6.

See also Xanthospilopteryx Wallengren, I 858.

$\ddagger$ HERACLEA ; Butler, I877, Illust. typical Specimens Lepid. Heterocera Colln Br. Mus. 1: 54 .

An incrrrect subsequent spelling.

HERAEMA Staudinger, I892, in Romanoff, Mém. Lépid. 6: 501. ACRO

Type-species: Pachnobia mandschurica Graeser, I889, Berl. ent. Z. 33: 256, by monotypy. Syntypes 40 , [U.S.S.R.]: Amurland, Raddefka and Blagoweschtschensk.

HERCHUNDA Swinhoe, I90o, Cat. east. and Aust. Lepid. Heterocera 2: 592.

OPHI

Type-species: Premusia hirtissima Walker, [1863] I864, J. Proc. Linn. Soc. (Zool). 7: 173 , by monotypy. Lectotype $\hat{\sigma}$, Borneo: Sarawak (UM, Oxford) designated (as type) by Swinhoe, I900, ibidem 2: 592 .

$P$. hirtissima was originally proposed as $\ddagger$ Remusia, an incorrect subsequent spelling.

$\ddagger$ HERMINEA, misspelling. See Herminia Latreille, I 802 .

HYPE

HERMINIA Latreille, I802, in Sonnini's Buffon, Hist. nat. générale particulière Crustacés Insectes 3: 4I3.

HYPE

Type-species: Phalaena ventilabris Fabricius, I787, Mantissa Insect. 2: 216, by subsequent designation by Duponchel, I83I, in Grodart \& Duponchel, Hist. nat. Lépid. Papillons Fr. 8(2): 8, but cited as tarsiplumalis Hübner, I796 which on page 3I was placed as the senior synonym of ‡'ventilabve Latr.,' an incorrect subsequent spelling and authorship. Type(s), [Austria]: [Karnten prov.] Carinthia (Type(s) not found by Zimsen, 1964, Type Material of I. C. Fabricius: 552).

The citation by Duponchel is acceptable as a type-species designation as it is contained in the continuation of a layout in which Duponchel stated, in the same work 7 (2): I02, that the species so cited were the types of genera.

$P$. ventilabris is a junior subjective synonym of Phalaena tarsicrinalis Knoch, I782, Beitr. Insekt. 2: 75, pl. 4, figs I-I 2.

See Frontispiece, fig. 6, Herminia tarsicrinalis (Knoch), $0^{\star}$.

Incorrect type-species designation: Crambus barbatus Fabricius, I798, and Phalaena tentacularis Linnaeus, I758. Neither of these names were originally included in Herminia, nor were they linked with one of the originally included names when cited by Latreille, I 8Io, Considérations générales sur l'Ordre naturel des Animaux: 44I. The Int. Commn zool. Nom., 1939, Opin. Decl. int. Commn zool. Nom. 2 (Opinion I36): I5 have ruled that the designation can be accepted in the case of the 'Table des genres avec l'indication de l'espèce qui leur sert de type' forming part of Latreille's 18 Io work if one only of the species 
originally included in the genus was cited even though other species not originally included were also cited.

$\ddagger$ HERMINEA; Sodoffsky, r837, Bull. Soc. imp. Nat. Moscou 1837 (6): [91].

An incorrect subsequent spelling.

POGONITIS Sodoffsky, ז837, Bull. Soc. imp. Nat. Moscou 1837(6): [91], [97].

Proposed, unnecessarily, as an objective replacement name for Herminia Latreille, 1802 , which Sodoffsky considered to be inappropriate.

HERMINIELLA Kaye \& Lamont, 1927. See Heterogramma Guenée, I854. HyPE

HERMINIOCALA Hampson, I913, Cat. Lepid. Phalaenae Br. Mus. 12: ix, 234. Cato

Type-species: Ophiodes daona Druce, I894, Ann. Mag. nat. Hist. (6) 13: 362, by original designation. Type(s), Mexico: Orizaba (BMNH).

HERMINODES Guenée, 1852, in Boisduval \& Guenée, Hist. nat. Insectes (Lépid.) 6: 43 I.

OPHI

Type-species: Herminodes nigripalpis Guenée, I 852, ibidem 6: 43I, pl. I 8, fig. 4, by subsequent designation by Hampson, 1926, Descr. new Genera Species Lepid. Phalaenae Subfamily Noctuinae Br. Mus.: 2.49. Holotype $q$, [French Guiana]: Cayenne (BMNH).

HERMOEA Walker, [1859]. See Doryodes Guenée, I 857. Cato

HERMONASSA Walker, I865, List Specimens lepid. Insects Colln Br. Mus. 32: 631. NocT

Type-species: Hermonassa consignata Walker, 1865, ibidem 32: 632, by monotypy. Syntypes $\sigma^{7}$, ㅇ․ [INDIA]: Darjeeling (BMINH).

HERMONASSOIDES Strand, I9I 5, Ent. Mitt. 4: I 57.

NoCT

Type-species: Agrotis problematica Strand, I915, ibidem 4: 157, fig. 2, by monotypy. Holotype of. [CAmerour] Cameroon: Bonaberi.

Hermonassoides was originally proposed as a subgenus of Agrotis Ochsenheimer, i8i6.

HERPEPERAS Hampson, 1926, Descr. ncw Genera Spccies Lepid. Phalaenae Subfamily Noctuinae Br. Mus.: 512 .

OPHI

Type-species: Amphigonia rudis Walker, 1865, List Specimens lepid. Insects Colln Br. Mus. 33: I032, by original designation. Holotype , Sierra LEone (BMNH).

HERPOPERASA Hampson, 1926, Descr. ncw Genera Species Lepid. Phalaenae Subfamily Noctuinae Br. MIus.: 499 .

$\mathrm{OPHI}$

Type-species: Ephyrodes apicata Dognin, I9I2, Hétérocères nouv. Am. Sud. 6: 33, by original designation. Holotype $\sigma$, Bolivia: Yungas de la Paz.

HERPYZON Hübner, I822. See Hypena Schrank, I 80z.

HYPE

HERRICHIA Grote, I882. See Euherrichia Grote, I 882.

HESPAGARISTA Walker, I 854, List Specimens lepid. Insects Colln Br. Mus. 1: I3. AGar Type-species: Hespagarista interlecta Valker, I854, ibidem 1: I4, by monotypy. Syntypes 20 , South Africa: Port Natal (BMNH).

$H$. interlecta is a junior subjective synonym of Agarista cchione Boisduval, i 847 , in Delegorgue, Voyage dans l'A frique australe 2: 595.

HESPERIMORPHA Saalmüller, I880, Ber. senckenb. naturf. Ges, 1879-80: 283.

ACON

Type-species: Hesperimorpha paradoxa Saalmüller, I880, ibidcm 1879-80: 283, by monotypy. Type(s), Madagascar (SNG, Frankfurt).

H. paradoxa is a junior subjective synonym of Noctua punctum Fabricius, I794, Ent. Syst. 3(2): 34 .

See also Amyna Guenée, I 852 . 
HESPEROCHROA Hampson, I926, Descr. new Genera Species Lepid. Phalaenae Subfamily Noctuinae Br. Mus.: 95.

OPHI

Type-species: Eudrapa multiscripta Holland, I894, Psyche, Camb. 7: 50, pl. 2, fig. I, by original designation. Holotype $\widehat{\jmath}$. West Africa: Benita (CM, Pittsburgh).

HESPEROTHRIPA Hampson, I9I2, Cat. Lepid. Phalaenae Br. Mus. 11: 22I, 227. SARR Type-species: Hesperothripa dicyma Hampson, I9I2, ibidem 11:227, fig. 74, by original designation. Holotype $q$, Srerra Leone (BMNH).

HETERANASSA Smith, (1899), Proc. U.S. natn. Mus. 22: 105.

OPHI

Type-species: Homoptera mima Harvey, I876, Can. Ent. 8: I55, by PRESENT DESIGNATION. Holotype, [U.S.A.]: Texas (BMNH).

HETERANDRA Herrich-Schäffer, I 866 [September], Corvesp.-Bl zool.-min. Vev. Regensburg 20: I 34 . AGAR

Type-species: Heterandra disparilis Herrich-Schäffer, I866 [September], ibidem 20: I34, by monotypy. Syntypes $\hat{0},+$, CUBA.

$H$. disparilis is a junior subjective synonym of Euscirrhopterus poeyi Grote, I866 July.

The date of publication of Heterandra has been discussed by Todd, I966, Proc. U.S. natn. Mus. 120: 6 .

See also Euscirrhopterus Grote, I866 July; and Laquea Jordan, I896.

HETEROCAMPA Doubleday, I84I, Entomologist $1: 55$.

NOTODONTIDAE

Type-species: Heterocampa astarte Doubleday, I84I, ibidem $1: 57$, by monotypy.

Heterocampa has been used as the original generic name for species which are now in the Noctuidae.

HETEROCHROMA Guenée, I852, in Boisduval \& Guenée, Hist. nat. Insectes (Lépid.) 6: 69. ACRO

Type-species: Heterochroma eriopioides Guenée, I 852, ibidem 6: 69, pl. 8, fig. 8, by subsequent designation by Hampson, 1908, Cat. Lepid. Phalaenae Br. Mus. 7: 27I. Syntypes ô, + , Brazil: Nouvelle-Fribourg (BMNH).

HETERODELTA Berio, I964, Doriana 3 (I 44): 5.

ACRO

Type-species: Heterocampa nea Druce, I898, Biologia cent.-am. (Zool.) Lepid. Heterocera $2: 45^{8}$, pl. 9o, fig. 22, by original designation. Holotype $\widehat{\sigma}$, Panama: Chiriqui.

HETEROGRAMMA Guenée, I 854, in Boisduval \& Guenée, Hist. nat. Insectes (Lépid.) 8: 91. HYPE

Type-species: Heterogramma circumflexalis Guenée, I 854, ibidem 8: 92, pl. 6, fig. 4, by subsequent designation by Grote, 1874, Bull. Buffalo Soc. nat. Sci. 2: 52 . Syntypes 2 ô, I 9, BRAzIL (BMNH).

HERMINIELLA Kaye \& Lamont, 1927, Mem. Dept. Agric. Trinidad \& Tobago 3: 76.

Type-species: Bleptina ceusalis Walker, I859, List Specimens lepid. Insects Colln Br. Mus. 19: 868, by PRESENT DESIGNATION. Type(s) ㅇ, [BRAzIL]: Rio Janeiro (UM, Oxford).

A junior homonym of Herminiella Blattný, 1925, Sb. ent. Odd. nár. Mus. Praze 3(26): 2II, - Coleoptera. There is no objective replacement name but $B$. ceusalis is congeneric with $H$. circumflexalis Guenée, the type-species of Heterogramma Guenée, I854. The latter is therefore available for use as a subjective replacement name.

HETEROGRAPHA Standinger, I877, Stettin. ent. Ztg 38: I85.

ACRO

Type-species: Luperina mira Staudinger, I877, ibidem 38: I85, by monotypy. Holotype ô, [U.S.S.R.]: Turkestan, Krasnowodsk.

L. miva is a junior subjective synonym of Mamestra zelleri Christoph, I876, Horae Soc. ent. ross. $12: 250$, pl. 6, fig. 24 .

Heterographa was proposed as a subgenus of Luperina Boisduval, I828. 
HETEROMALA Hampson, I895, Fanna Br. India (Moths) 3: 31, 39.

OPH I

Type-species: Heteromala thyrophora Hampson, I895, ibidem 3: 40, fig. I 8, by original designation. Syntypes ot, [Sikkim] Sikhim. India: [Assam], Khasis (BMNH, but without locality).

HETEROMMA Warren, IgII. See Heterommiola Strand, IgI 2.

ACRO

HETEROMMIOLA Strand, 1912, Ent. Rdsch. 29: i6.

ACRO

Type-species: Hadena alpigena Boisduval, [1837] 1834, Icones hist. Lépid. 2: pl. 84, fig. 5, by original designation (for Heteromma Warren, I9II). Type(s), Europe.

Heterommiola was proposed as the objective replacement name for Heteromma Warren, I9I I.

HETEROMIIA Warren, I9I I, in Seitz, Gross-Schmett. Erde 3: I8o.

Type-species: Hadena alpigena Boisduval, [1837], by original designation.

A junior homonym of Heteromma Menge, 1856, Progr. Petrischule Dantzig: 8, - Arachnida. The objective replacement name is Heterommiola Strand, I9I2.

The date of publication of the part of Seitz containing page r 80 was stated on page I 73 as I91 I February 8th in both the English and German editions.

${ }_{\ddagger}$ HETEROMORPHA Hübner, [I806]. See Diloba Boisduval, I84o.

NOTODONTIDAE

HETEROMORPHA Hübner, I822. See Diloba Boisduval, I84o.

NOTODONTIDAE

HETEROMORPHA Failla-Tedaldi, I89o. See Orrhodiella Spuler, I907.

CUCU

HETERONOTA Meyrick, I902, Trans. ent. Soc. Lond. 35: 47.

SARR

Type-species: Heteronota ochthias Meyrick, I902, ibidem 35: 48, by monotypy.

Holotype q, New Guinea (BMNH).

H. ochthias is a junior subjective synonym of Selepa vitea Swinhoe, I 885.

See also Lophothripa Hampson, I 896.

HETEROPALPIA Berio, 1939, Memorie Soc. ent. ital. 17: 55.

CATO

Type-species: Heteropalpia cortytoides Berio, I939, ibidem 17: 56, pl. 2, fig. Io, by original designation. Holotype $\hat{\sigma}$, [ETHIOpia] Eritrea: Dorfù (Colln E. Berio, Genoa).

$\ddagger$ HETEROPHYES, no published reference found.

HYPE

HETEROPHYSA Boursin, 1953, Z. Lepid. 3: 47.

ACRO

Type-species: Noctua dumetorum Geyer, in Hübner, [1834], Samml. eur. Schmett. 4: pl. I 76 , fig. 839, by original designation. Type(s), Europe.

HETEROPYGAS Guenée, I 852, in Boisduval \& Guenée, Hist. nat. Insectes (Lépid.) 7: 285.

OPHI

Type-species: Heteropygas oppilata Guenće, 1852 , ibidem 7: 285 , pl. 22, fig. 7, by monotypy. Syntypes of, [URUGUay]: Montevideo. [U.S.A.]: Savannah.

HETERORMISTA Swinhoe, I90I, Amn. Mag. nat. Hist. (7) 8: i $9 . \quad$ орні

Type-species: Heterormista modesta Swinhoe, Igo I, ibidem (7) 8: 19, by monotypy. Syntypes ô, 9 , Australia: Qd, Dawson.

HETERORTA Warren, 1913, in Seitz, Gross-Schmett. Erde 11: 21 I.

Type-species: Thalpochares plutonis Lucas, 1895, Trans. nat. Hist. Soc. Qd 1: 109, by original designation. Syntypes $\hat{\sigma}$,, , Australia: Rockhampton (SAM, Adelaide).

The date of publication of the part of Seitz containing page 2 I I was stated on page 209 as 19 r 3 August 2oth in both the English and German editions.

HETEROSCOTIA Bryk, i949, Ark. Zool. 41 (A) I: 138.

OPHI

Type-species: Heteroscotia stygia Bryk, 1949, ibidem 41 (A) I: 138, pl. 5, fig. 9, by original designation. Holotype $\tilde{\sigma}$, Korea: Shuotsu (NR, Stockholm). 
HETEROSPILA Guenée, I 852, in Boisduval \& Guenée, Hist. nat. Insectes (Lépid.) 7 : 35 I. OPH I Type-species: Heterospila fulgurea Guenée, I852, ibidem 7: 352, by monotypy. Holotype ô. Java (BMNH).

HEXAMITOPTERA Pagenstecher, i 885, Jb. nassau. Ver. Naturk. 38: I80.

CATO

Type-species: Poeciloptera lawinda Pagenstecher, 1885 , ibidem 38: 35, pl. 2, fig. 5 , by monotypy (of Poeciloptera Pagenstecher, I885). Syntypes several $\hat{\sigma}$, I 9 , Sumatra: Nias I. (SM, Wiesbaden).

Hexamitoptera was proposed as the objective replacement name for Poeciloptera Pagenstecher, i 885 .

POECILOPTERA Pagenstecher, I 885, J6. nassau. Ver. Natuvk. 38: 35.

Type-species: Poeciloptera lawinda Pagenstecher, by monotypy.

Poeciloptera was originally described as a subgenus of Hypopyra Guenée, I 852.

A junior homonym of Poeciloptera Loew, i 8+6, Stettin. ent. Ztg 1846: 95, - Diptera. The objective replacement name is Hexamitoptera Pagenstecher, I 885 .

HEXORTHODES McDunnough, I943, Can. Ent. 75: 55.

HADE

Type-species: Trichopolia serrata Smith, I90o, Proc. U.S. natn. Mus. 22: 479, by original designation. Holotype oే, [U.S.A.]: Texas (USNM, Washington).

HIASPIS Walker, [1866] I 865, List Specimens lepid. Insects Colln Br. Mus. 34: I I88. OPH

Type-species: Hiaspis closteroides Walker, [1866] I 865, ibidem 34: I I89, by monotypy. Type(s) $\widehat{\jmath}$. BORneo (UM, Oxford).

HICCODA Moore, I882, in Hewitson \& Moore, Descr. new Indian lepid. Insects Colln late $\operatorname{Mr} W$. S. Atkinson: I 34 . ACON

Type-species: Hiccoda dosaroides Moore, I 882, ibidem: I35, by monotypy. Syntypes, India: Bombay (BMNH); Calcutta. Ceylon (BMNH).

HILARUS Agassiz, I846. See Ilarus Boisduval, I 828.

HADE

HILEIA Walker, I86I, List Specimens lepid. Insects Colln Br. Mus. 23: 948. Available, but without included species until Walker, i 862, ibidem 24: I Ioo.

OPHI

Type-species: Hileia crambisata Walker, I862, ibidem 24: I Ioo, by subsequent monotypy. Holotype $\hat{\sigma}$, [INDIA] : N. Hindostan (BMNH).

H. crambisata is a junior subjective synonym of Gesonia obeditalis Walker, [1859].

See also Gesonia IValker, [1 859]; Dragana Walker, [I859]; Maresia Walker, I866; and Amblygoes Butler, I 879 .

HILLIA Grote, i 883, Proc. Am. phil. Soc. 21: I46, I68.

CUCU

Type-species: Hadena senescens Grote, I 878, Can. Ent. 10: 235, by original designation.

Syntypes đ. ․ [U.S.A.]: N.Y., Lewis Co. (BMNH).

$H$. senescens is a junior subjective synonym of Hadena iris Zetterstedt, I839.

See also Crasia Aurivillius, i 89 I.

HIMELLA Grote, I 874, Proc. Acad. nat. Sci. Philad. 1874: 200.

HADE

Type-species: Himella fidelis Grote, I874, ibidem 1874: 201 , by original designation.

Type(s) ô. [U.S.A.]: Albany (BMNH).

H. fidelis is a junior subjective synonym of Taeniocampa intractata Morrison, I874, Proc. Boston Soc. nat. Hist. 17: i6o.

HIMEROIS Turner, I902, Proc. Linn. Soc. N.S.W. 27: I 2 I.

$\operatorname{ACON}$

Type-species: Himerois thiochroa Turner, I902, ibidem 27: I2I, by monotypy. Holotype $\widehat{o}$, Australia: Qd, Townsville (ANIC, Canberra).

HINGULA Moore, I882, in Hewitson \& Moore, Descr. new Indian lepid. Insects Colln late Mr W. S. Atkinson: i 80.

OPHI

Type-species: Hingula albolunata Moore, I882, ibidem: I $8 \mathrm{I}$, by subsequent designation 
by Warren, 1913, in Seitz, Gross-Schmett. Erde 3: 40I. Syntypes, India: [Madras], Nilgiris (BMNH); [Assam], Cherra Punji (BMNH).

$H$. albolunata is a junior subjective synonym of Rhesala imparata Walker, $185^{8}$.

See also Rhesala Walker, 1858 .

HIPOEPA Walker, [1859] 1858, List Specimens lepid. Insects Colln Br. Mus. 16: 188. HyPE

Type-species: Hipoepa lapsalis Walker, [1859] I858, ibidem 16: 188, by monotypy.

Holotype ô. CEYLON (BMNH).

$H$. lapsalis is a junior subjective synonym of Bocana biasalis Walker, [1859] I858, ibidem 16: 181.

HIPTELIA Guenée, 1852. See Xestia Hübner, r818. Noct

HIRIA Duponchel, [1845]. See Epilecta Hübner, [1821]. NOCT

HIRSUTIPES Bethune-Baker, 1906, Novit. zool. 13: 263 . OPHI

Type-species: Hypaetra trifasciata Swinhoe, 1905, Ann. Mag. nat. Hist. (7) 15: I60, by original designation. Holotype $\hat{\delta}$, NEw GuineA: [W. Irian], Kapaur (BMNH).

H. trifasciata is a junior subjective synonym of Remigia crinipes Felder \& Rogenhofer, I874, Reise öst. Fregatte Novara Zool. 2 (Lepid.) 2: pl. II4, fig. 4.

HIRSUTOPALPIS Bethune-Baker, 1904, Novit. zool. 11: 375.

OPHI

Type-species: Hirsutopalpis fasciata Bethune-Baker, 1904, ibidem 11:375, by original designation. Syntypes ô, New Guinea: [Papua], Ekeikei (BMNH); Dinawa.

HISBANDA Wallier, [1866] I865, List Specimens lepid. Insects Colln Br. Mus. 34: 1267. SARR Type-species: Hisbanda acronyctoides Walker, [1886] 1865, ibidem 34: I268, by monotypy. Holotype ô. [Australia]: Sydney (BMNH).

H. acronyctoides is a junior subjective synonym of Calathusa basicunea IValleer, 1858 , ibidem 15: 1645 .

See also Calathusa IValker, 1858 ; and Sina Walker, 1865.

HOENEIDIA Boursin, I954, Bonn. zool. Beitr. 5: 256.

NOCT

Type-species: Hoeneidia cidarioides Boursin, I954, ibidem 5: 257, pl. 4, figs I5, I8, by original designation. Holotype ô. CHInA: N. Y'unnan, A-tun-tse (MAK, Bonn).

$H$. cidarioides was originally described as Höneidia, an incorrect original spelling which uncler the Code, Article $32(\mathrm{c})(\mathrm{i})$, must be corrected.

${ }_{\ddagger}^{+H O ̈ N E I D I A}$ Boursin, 1954, Bonn. zool. Beitr. 5: 256.

An incorrect original spelling under the Code, Article 32(c)(i).

HOLLANDIA Butler, I 892, Ann. mag. nat. Hist. (6) 10: 295.

Type-species: Hollandia sigillata Butler, I892, ibidem (6) $\mathbf{1 0}: 296$, by original designation. Type(s), [GaBov] Gaboon.

HOLOCRYPTIS Lucas, I892, Proc. Linn. Soc. N.S.W. (2) 7: 256.

$\operatorname{ACON}$

Type-species: Holocryptis phasianura Lucas, r892, ibidem (2) 7: 257, by monotypy. Syntypes ô, $q$, Australia: [Qd], Brisbane to Mackay (SAM, Adelaide).

$\ddagger$ HOLOMELAENA Hampson, no published reference found.

HYPE

HOLOXANTHINA Hampson, 1926, Descr. new Genera Species Lepid. Phalaenae Subfamily Noctuinae Br. Mris.: 522.

OPHI

Type-species: Entomogramma lutosa Karsch, 1895, Ent. Nachr. 21: 376, pl. 4, fig. I3, by original designation. Ilolotype $\hat{\sigma}$, 'Togo: Station Bismarcliburg (MNHU, Berlin).

HOMAEA Guenće, i 852, in Boiscluval \& Guenće, Hist. nat. Insectes (Lépid.) 7: 206. Cato

Type-species: Homaea clathrum (inence, 1852, ibidem 7: 207, by monotypy. Holotype o. East Indies. 
HOMOANARTA Barnes \& Benjamin, 1923, Contr. nat. Hist. Lepid. N. Am. 5: $76 . \quad$ cucu Type-species: Papaipema peralta Barnes, 1907, Can. Ent. 39: 14, by original designation. Syntypes, [U.S.A.]: Ariz., Cochise Co.

${ }_{\ddagger}^{\ddagger} \boldsymbol{H O M O C A T A D A}$ Hampson, no published reference found.

HYPE

HOMOCERYNEA Barnes \& McDunnough, I9I3, Contr. nat. Hist. Lepid. N. Am. 2: Ir8. ACON Type-species: Homocerynea cleoriformis Barnes \& McDunnough, I9I3, ibidem 2: I I 9, pl. 4, figs IO, I I, by original designation. Syntypes I $\delta$, 3 ㅇ, [U.S.A.]: Ariz., Palmerlee.

HOMODES Guenée, I 852, in Boisduval \& Guenée, Hist. nat. Insectes (Lépid.) 6: 280. OPH Type-species: Homodes crocea Guenée, I 852 , ibidem 6: 280 , pl. I I, fig. 2, by subsequent designation by Hampson, I894, Fauna Br. India (Moths) 2: 364. Holotype of. Java $(\mathrm{BMNH})$.

HOMODINA Hampson, I926, Descr. new Genera Species Lepid. Phalaenae Subfamily Noctuinae Br. Mus.: I 42 . OPHI

Type-species: Homodina argentifera Hampson, I926, ibidem: I43, by original designation. Syntypes I $\sigma^{\wedge}$, I $q$, Nigeria: Old Calabar (BMNH).

HOMOGLAEA Morrison, i 876, Proc. Boston Soc. nat. Hist. 18: 240.

Type-species: Homoglaea hircina Morrison, I876, ibidem 18: 240, by monotypy. Type(s), [U.S.A.]: Illinois, Galena.

HOMOGR MMA Guenée, I 854. See Salia Hübner, [1818]. HYPE

HOMOHADENA Grote, r873, Bull. Buffalo Soc. nat. Sci. 1: 18o. cucu

Type-species: Hadena badistriga Grote, i 872, Trans. Am. ent. Soc. 4: 20, by monotypy. Syntypes $\overrightarrow{0}, q$, Canada. [U.S.A.]: New York State.

HOMOLAGOA Barnes \& McDunnough, I9I2, Can. Ent. 44: 9 I.

ACRO

Type-species: Homolagoa grotelliformis Barnes \& McDunnough, I9I 2, ibidem 44: 92, by original designation. Syntypes I $\hat{\delta}, 2$ ㅇ, [U.S.A.]: Ariz., Redington and Palmerlee.

$\ddagger$ HOMOLOCHA Hampson, no published reference found. HYPE

HOMONACNA Fletcher, 1961, Ruwenzori Exped. I952 1: I98. CUCU

Type-species: Homonacna alpnista Fletcher, I961, ibidem 1: I98, figs 26, 199, 200 $\& 202$, by original designation. Holotype $\sigma^{\star}$, UGanda: Ruwenzori, Nyinabitaba (BMNH).

HOMONCOCNEMIS Hampson, I906, Cat. Lepid. Phalaenae Br. Mus. 6: ix, 18o. cucu

Type-species: Homohadena fortis Grote, I88o, Can. Ent. 12: 257, by original designation. Type(s), [U.S.A.]: Nevada.

HOMOPHLEBIA Warren, I916, Novit. zool. 23: 2 I 8. CHLO

Type-species: Cletthora bilinea Swinhoe, I 890, Trans. ent. Soc. Lond. 1890: 237, by original designation. Type(s), Burma: Rangoon (BMNH).

HOMOPHOBERIA Morrison, I875, Proc. Boston Soc. nat. Hist. 18: 125. opHI

Type-species: Homophoberia cristata Morrison, I875, ibidem 18: I25, by monotypy. Holotype $\hat{\sigma}$. [U.S.A.]: N.J., Hoboken.

HOMOPTERA Guenée, I852. See Omoptera Guérin-Méneville, [1832].

CATO

HOMOPYRALIS Grote, 1874, Proc. Acad. nat. Sci. Philad. 1874: 2 I3.

OPHI

Type-species: Homopyralis tactus Grote, I874, ibidem 1874: 213, by subsequent designation by Grote, 1875, Bull. Buffalo Soc. nat. Sci. 2: 223. Syntypes ô, q. [U.S.A.]: Va. (BMNH); Texas; Mass.; N.Y.; (BMNH, without locality data).

$H$. tactus is a junior subjective synonym of Homoptera quadrisignata Walker, [1858] I857, List Specimens lepid. Insects Colln Br. Mus. 13: 1073. 
HOMORTHODES McDunnough, I943, Can. Ent.75:53. HADE

Type-species: Himella furfurata Grote, 1874, Proc. Acad. nat. Sci. Philad. 1874: 201, by original designation. Type(s) ô, [U.S.A.]: Albany (BMNH).

HONDRYCHES nom. n. for Rhynchodes Guenée, I 852.

OPHI

Type-species: Rhynchodes phalaeniformis Guenée, I852, in Boisduval \& Guenée, Hist. nat. Insectes (Lépid.) 6: 394, by monotypy. Syntypes $2 \hat{0}$. [South Africa]: Pays des Namaquois.

RHYNCHODES Guenée, 1852, in Boisduval \& Guenée, Hist. nat. Insectes (Lépid.) 6 : 393.

Type-species: Rhynchodes phalaeniformis Guencee, I 852, by monotypy.

A junior homonym of Rhynchodes White, IS46, Zool. Voyage H.M.S. Evebus \& Terror

(Insects): 16, - Coleoptera. The objective replacement name is Hondryches nom. n.

$\ddagger$ RH YNCODES; Hampson, I9 Io, Proc. zool. Soc. Lond. 1910: 433.

An incorrect subsequent spelling.

$\ddagger$ HÖNEIDIA Boursin, 1954. See Hoeneidia Boursin, I954.

NOCT

HOPETOUNIA Swinhoe, I902, Ann. Mag. nat. Hist. (7) 9: 173.

OPHI

Type-specics: Hopetounia carda Swinhoe, I902, ibidem (ᄀ) 9: 173, by monotypy. Syntypes 8 o., Australia: W. Aust., R. Sherlock (BMI.NH).

HOPLARISTA Hampson, I9Io, Proc. zool. Soc. Lond. 1910: 399.

AGAR

Type-species: Hoplarista haemaplaga Hampson, 1910, ibidem 1910: 399, by original designation. Syntypes I $\mathcal{O}^{*}, \mathrm{I}$ ?, [ZAmBIA] Rhodesia: L. Bangweolo (BMNH).

HOPLODRINA Boursin, 1937, Ent. Rdsch. 54: 367.

ACRO

Type-species: Phalaena alsines Brahm, I79I, Insecten-kalender Sammler Oekonomen

2: I I 4, by original designation. Syntypes, [GERMAny].

HOPLOLYTHRA Hampson, igro, Cat. Lepid. Phalaenae Br. Mus. 9: x, 214.

ACRO

Type-species: Lythrodes discistriga Smith, I903, Trans. Am. ent. Soc. 29: 207, by original designation. Syntypes $2 \overrightarrow{0}$. [U.S.A.]: California, Walter's Station; S. Arizona.

HOPLOTARACHE Hampson, I9Io, Cat. Lepid. Phalaenae Br. Mus. 10: xviii, 7I2. ACON

Type-species: Tarache mionides Hampson, 1905, Ann. S. Afr. Mus. 3: 435, by original designation. Holotype $\widehat{\delta}$. [Soutu AFrica]: Cape Colony, Deelfontein (BMNH).

HOPLOTARSIA Aurivillius, I922, in Aurivillius, Prout \& Meyrick, Nat. Hist. Juan Fernandez and Easter I. 3: 259.

cucu

Type-species: Hoplotarsia magna Aurivillius, I922, ibidem 3: 259, pl. Io, fig. 4, by monotypy. Holotype , Juan Fernandez I.: Masatierra.

HOPORINA Blanchard, I840. See Jodia Hübner, I8I8. cUCU

HOPOTHIA Dyar, 1914, Proc. U.S. natn. Mus. 47: $219 . \quad$ HYPE

Type-species: Hopothia histigma Dyar, I9I4, ibidem 47:220, by original designation. Syntypes $2 \hat{\delta}$. PANama: Taboga I.; La Chorrera (USNM, Washington).

HORATOSCELIS Agassiz, I8+6. See Calymma Hübner, [1823]. ACON

HORMA Walker, 1857. Sec Dichonia Hübner, [1821]. CUCU

HORMISA Walker, [1859] I 858, List Specimens lepid. Insects Colln Br. Mus. 16: 74. HYPE Type-species: Hormisa absorptalis Walker, [1859] r 858, ibidem 16:74, by monotypy . Holotype ô. U.S.[A.] (BMNH).

See also Litognatha Grote, I 873.

HORMOSCHISTA Möschler, I890, Abh. senckenb. naturforsch. Ges, 16: 221.

Type-species: Hormoschista pagenstecheri Nöschler, I890, ibidem 16: $22 \mathrm{I}$, fig. 3 , by monotypy. Syntypes $2 \hat{0}$. I 9 , [Puerto Rico] Portorico I. (MNHU, Berlin).

II. pasenstecheri is a junior subjective synonym of Hydrelia latipalpis Walker, 1858 , List Specimens lepid. Insects Colln Br. Mus. 15: 1763. 
HORTONIUS Dyar, I926, Insecutor Inscit. menstr. 14: 95.

ACRO

Type-species: Hortonius euenemus Dyar, 1926, ibidem 14: 95, by monotypy. Holotype $\widehat{0}$, [U.S.A.]: New Mexico, Tucumcari (USNM, Washington).

$\ddagger$ HOSPORINA, misspelling. See Jodia Hübner, I818.

CUCU

$\ddagger$ HÜBNERIUS Saalmüller, I891. See Huebnevius Saalmüller, I891.

$\mathrm{OPHI}$

HUEBNERIUS Saalmüller, I89I, Lepid. Madagascar: 446.

OPHI

Type-species: Phyllodes dux Saalmüller, I88I, Stettin. ent. Ztg 42: 44I, by monotypy. Syntypes i $\hat{0}$, I $q$, Madagascar: Nossi-Bé.

$\ddagger H U U B N E R I U S$ Saalmüller, i 89r, Lepid. Madagascar: 446 .

An incorrect original spelling under the Code, Article 32 (c)(i).

HUGONIA Alphéraky, I908. See Scythocentropus Speiser, I902.

ACRO

HULODES Guenée, I852, in Boisduval \& Guenée, Hist. nat. Insectes (Lépid.) 7: 207. OPHI

Type-species: Phalaena caranea Cramer, I780, Uitlandsche Kapellen 3: I40, I74, pl. 269, figs E, F, by subsequent designation by Desmarest, (1857), in Chenu, Encycl. Hist. nat. Papillons nocturnes: I3I. Type(s), JAVA: [Djakarta] Batavia.

The type-species was proposed by Cramer as $P$. carenea but was indexed on page I 74 as caranea. Guenée, I 852, ibidem 7: 208 cited both these multiple original spellings and adopted caranea which has subsequently been used.

HYLODES Hampson, I894, Fauna Br. India (Moths) 2: xix, 462.

An unjustified emendation of Hulodes Guenée, I 852.

A junior homonym of Hylodes Fitzinger, 1826, Nene Classification der Reptilien: 38, 64, -Amphibia.

HULYPEGIS nom. n. for Euglyphis Hübner, [1825].

OPHI

Type-species: Euglyphis procopialis Hübner, [1825] I8I6, Verz. bekannter Schmett.: 34I (the objective replacement name for Phalaena procopia Stoll, I790, see below), by monotypy (of Euglyphis Hübner). Type(s) (of Phalaena procopia Stoll, I79o), Surinam.

E. procopialis was proposed by Hübner as the objective replacement name for Phalaena (Noctua) procopia Stoll, I79o, in Cramer, Uitlandsche Kapellen Aanhangsel: 57, pl. I2, fig. 9, a junior primary homonym of Phalaena (Pyralis) procopia Stoll, I 78 I, in Cramer, ibidem 4: I 52, - Lepid., Pyralidae.

EUGLYPHIS Hübner, [1825], I8I6, Verz. bekannter Schmett.: 34I.

Type-species: Euglyphis procopialis Hübner, [1825] I816, by monotypy.

A junior homonym of Euglyphis Hübner, [1820] I816, ibidem: I90-Lepid., Lasiocampidae. The objective replacement name is Hulypegis nom. n.

HURWORTHIA nom. n. for Niphosticta Turner, I936.

OPH I

Type-species: Niphosticta apicipuncta Turner, I936, Proc. R. Soc. Qd 47: 43, by monotypy (of Niphosticta Turner). Holotype $q$ (not $\hat{\sigma}$ as stated), Australia: Qd, Yeppoon (ANIC, Canberra).

This name is proposed in honour of Mr Peter Hurworth, in appreciation of his assistance during the early stages of the compilation of this catalogue.

NIPHOSTICTA Turner, I936, Proc. R. Soc. Qd 47: 43.

Type-species: Niphosticta apicipuncta Turner, 1936, by monotypy.

A junior homonym of Niphosticta Hampson, 1926, Descr. new Genera Species Lepid. Phalaenae Subfamily Noctuinae Br. Mus.: 4I4,--Lepid., Noctuidae. The objective replacement name is Hurworthia nom. n.

HYADA Moore, I882, in Hewitson \& Moore, Descr. new Indian lepid. Insects Colln late Mr W. S. Atkinson: I29.

CUCU

Type-species: Hyada grisea Moore, I882, ibidem: I30, pl. 4, fig. 26, by monotypy. Type(s), Siккім: Alutong. 
HYALOBOLE Warren, I9II, Novit. zool. 18: I 4I.

Type-species: Hyalobole orthosioides Warren, I9II, ibidem 18: I42, by original designation. Syntypes $2 \hat{\sigma},[$ India $]$ : [W. Bengal], Darjiling.

HYAMIA Walker, [1859] I 858, List Specimens lepid. Insects Coln Br. MIus. 16: 72.

OPH I

Type-species: Hyamia palpitatalis Walker, [1859] I 858, ibidem 16: 72, by monotypy. Holotype ?, [BraziL]: [R. Amazon], [Tefé] Ega (BMNH).

JUNCARIA Walker, I863, List Specimens lepid. Insects Colln Br. MIus. 27: 187.

Type-species: Juncaria nonagrioides Walker, I863, ibidem 27: 187, by monotypy. Type(s) o., [BRazIL]: R. Amazon (BMNH).

A junior homonym of Juncaria Walker, 1858 , - Lepid., Noctuidae. There is no objective replacement name but $J$. nonagrioides is congeneric with Hyamia palpitatalis Walker, [1 859], the type-species of Hyamia Walker, [1859]. The latter is thus available for use as a subjective replacement name.

$\boldsymbol{H} \boldsymbol{Y} \boldsymbol{B L} \boldsymbol{A} \boldsymbol{E} \boldsymbol{A}$ Fabricius, I 793, Ent. Syst. 3 (1): [vi]. Available, but without included species until Fabricius, 1794, ibidem 3 (2): 127.

HYBLAEIDAE

Type-species: Noctua saga Fabricius, I787, Mantissa Insect. 2: 137, by subsequent designation by Hampson, I894, Fauna Br. India (Moths) 2:37 I, but cited as puera Cramer, I 777, which Hampson placed as the senior synonym of saga Fabricius.

N. saga is a junior subjective synonym of Phalaena pueva Cramer, 1777, Uitlandsche Kapellen 2: Io, pl. I03, figs D, E.

At the end of his generic redescription, Moore (I884, Lepid. Ceylon 3: 8I) where he usually but not always designated the type-species, on this occasion cited $H$. puera but did not state 'Ty'pe'; it is therefore not acceptable as a type-species designation.

Hyblaea was formerly the type-genus of a subfamily in the Noctuidae; it is currently accepted as the type-genus of a family in the Pyraloidea.

See also Nabara Walker, i 866.

NYCHOPHILA Billberg, I 820, Enmmeratio Insect. Mus. G.J. Billberg: 86.

Type-species: Noctua saga Fabricius, I 787 , by monotypy.

Nychophila is a junior objective synonym of Hyblaea Fabricius, I793.

AENIGMA Strecker, I876, Lepidoptera, Rhopaloceres \& Heteroceres, indigenous \& exotic: I 22.

Type-species: Aenigma mirificum Strecker, I876, ibidem: I22. Holotype ㅇ, [U.S.A.]: Texas.

A junior homonym of Aenigma Newman, i 836, Ent. Mag. 3: 499,-Coleoptera. There is no objective replacement name but $A$. mirificum is a junior subjective synonym of Noctua saga Fabricius, I787, the type-species of Hyblaea Fabricius, 1793. The latter is thus available for use as a subjective replacement name.

HYBOMA Hübner, [1820] I8I6, Verz. bekannter Schmett.: 200.

ACRO

Type-species: Noctua strigosa [Denis \& Schiffermüller], I775, Ankündung syst. Werkes Schmett. Wienergegend: 88, by subsequent designation by Moore, I884, Lepid. Ceylon 3: 5. Type(s), [Austria]: Vienna district (Collection destroyed, Horn \& Kahle, 1936, Ent. Beih. Berl.-Dahlem 3: 243).

HYDRAECIA Guenée, I84I, Annls Soc. ent. Fr. 10: 237.

ACRO

Type-species: Phalaena micacea Esper, 1789, Die Schmett. 4 (2) Abschnitt I: pl. I 45 , fig. 6; [I798-1804], ibidem: 466, by subsequent designation by Guenée, I852, in Boisduval \&E Guenée, Hist. nat. Insectes (Lépid.) 5: i 28, but cited for Hydroecia, an unjustified emendation. Holotype $\sigma^{\star},[$ AUstria]: Tyrol.

HYDROECIA Agassiz, i 846, Nomencl. zool. Index univl.: i87, i 88.

An unjustified emendation of Hydraecia Guenée, $184 \mathrm{r}$.

$\ddagger$ HADROECIA Schaus, r 894, Trans. Am. ent. Soc. 21: 231.

An incorrect subsequent spelling. Schaus described 'Hadroecia azteca sp. nov.' without any indication that he was proposing a new genus. In the same work four new genera are clearly indicated and described. 
HYDRELIA Guenée, 184I. See Eustrotia Hübner, [I82 I].

HYDRILLA Boisduval, I 840. See Acosmetia Stephens, I 829.

ACRO

HYDRILLODES Guenée, I 854, in Boisduval \& Guenée, Hist. nat. Insectes (Lépid.) 8: 65. HYPE

Type-species: Hydrillodes lentalis Guenée, I 854 , ibidem 8:66, pl. 5, fig. 3, by subsequent designation by Moore, [1885] I 887, Lepid. Ceylon 3: 237. Syntypes I Õ, I +, India: central (BMNH).

HYDRILLULA Tams, I938, Entomologist 71: I 23.

ACRO

Type-species: Noctua pallustris Hübner, [1808], Samml, eur. Schmett. 4: pl. 79, fig. 367, by original designation. Type(s), Europe.

Tams proposed this name for the part of Hydrilla Boisduval, I840, as restricted by Guenée, 1852, Hist. nat. Insectes (Lépid.) 5: 236, and at that time known as 'Hydrilla Guenée'. Tams also proposed the emendation of the spelling of the type-species to $H$. palustris. Under the Code, Article 32(a)(ii), the original spelling should be retained.

$\ddagger$ HYDRIODES, misspelling. See Hyriodes Hampson, I9Iо.

$\operatorname{ACON}$

HYDROECIA Agassiz, 1846. See Hydraecia Guenée, I841.

ACRO

HYDROECIODES Hampson, I905, Cat. Lepid. Phalaenae Br. Mus. 5: x, 253.

HADE

Type-species: Xanthia alala Druce, I890, Proc. zool. Soc. Lond. 1890: 5I 4, by original designation. Syntypes I $\hat{\sigma}, \mathrm{I}$ q, Mexico: Mexico City (BMNH).

HYELA Stephens, I850. See Eustrotia Hübner, [182 I].

$\operatorname{ACON}$

HYELOPSIS Hampson, I 894, Fauna Br. India (Moths) 2: xiv, 304.

ACON

Type-species: Acontia vialis Moore, 1882, in Hewitson \& Moore, Descr. new Indian lepid. Insects Colln late $M r W$. S. Atkinson: 135, by original designation. Syntypes ô, ㅇ. India: [Punjab], Dharmsala (BMNH); [W. Bengal], Darjiling.

HYGROSTOLA Warren, I9I3, in Seitz, Gross-Schmett. Erde 11: I90.

ACRO

Type-species: Nonagria robusta Hampson, I894, Famna Br. India (Moths) 2: 285, by original designation. Holotype $\hat{o}$, [Sikkim] Sikhim (BMNH).

The date of publication of the part of Seitz containing page I 90 was stated on page $\mathrm{r} 85$ as 1913 April 24 th in both the English and German editions.

HYGROSTOLIDES Strand, I920, Arch. Naturgesch. 84 (A) I 2: I I3.

ACRO

Type-species: Hygrostolides robustior Strand, I920, ibidem 84 (A) I 2: II3, by monotypy. Syntypes $3 \hat{0}, 3$ ㅇ․ [Tarwan] Formosa: Suisharyo (DEI, Eberswalde).

$\ddagger$ †YLINA, misspelling. See Xylena Ochsenheimer, I816. cucu

HYLODES Hampson, 1894. See Hulodes Guenée, I852. OPHI

HYLOPHILA Hübner, [1825]. See Pseudoips Hübner, I822. CHLO

HYLOPHILINA Warren, I9I3, in Seitz, Gross-Schmett. Erde 3: $297 . \quad$ chLO

Type-species: Phalaena bicolorana Fuessly, 1775, Vevz. Schweiz. Insecten: 4I, by original designation. Syntypes, Switzerland.

$P$. bicolorana was attributed by Fuessly to Geoffroy, 1762, Hist. abrégée Insectes 2: 172, No. 124, but this work has been rejected for nomenclatural purposes by the Int. Commn zool. Nom., 1954, Opin. Decl. int. Commn zool. Nom. 4 Opinion 228: 2 I I.

$P$. bicolorana is a junior subjective synonym of Phalaena prasinana Linnaeus, I758, Syst. Nat. (Edn Io) 1: 53 .

The date of publication of the part of Seitz containing page 297 was stated on page 293 as 1913 January 5 th in both the German and English editions.

See also Bena Billberg, I 820. 
HYLOPHILODES Hampson, 1912, Cat. Lepid. Phalaenae Br. Mus. 11 : xiv, 510.

CHLO

Type-species: Halias orientalis Hampson, I894, Fanna Br. India (Moths) 2: I32, fig. 87 , by original designation. Type(s) 을 India: [Assam] Naga Hills (BMNH).

${ }_{\ddagger}^{+}$HYLOPHILOIDES; Nagano, I918, Insect Wld 22: 191.

An incorrect subsequent spelling.

${ }_{\ddagger}$ HYLOPHILOIDES, misspelling. See Hylophilodes Hampson, I91 2.

CHLO

HYMENOCRYPHIA Boursin, 1968, Entomops, Nice 11: 87.

ACRO

Type-species: Bryophila modesta Moore, 1881, Proc. zool. Soc. Lond. 1881: 332, by original designation. Type(s), [INDIA]: N.W. Himalaya (BMNH).

Hymenocryphia Boursin was proposed as a subgenus of Cryphia Hübner, I8I 8.

HYMENODRINA Boursin, 1937, Ent. Rdsch. 54:420.

Type-species: Caradrina terrea Freyer, [1840] 1842, Newere Beitr. Schmett. 4: 26, pl. 303, figs 3, 4, by original designation. Type(s), [U.S.S.R.]: [Krasnoarmeysk] Sarepta.

Hymenodrina was proposed as a subgenus of Elaphria Hiibner, I818, but has since been placed as a junior subjective synonym of Platyperigea Smith, I894, a subgenus of Caradrina Ochsenheimer, 18 r 6.

HYPAENISTIS Warren, 1916, Nouit. zool. 23: 219.

HADE

Type-species: Hypaenistis purpurea Warren, 1916, ibidem 23: 219, by original designation. Type(s), Solomox Is: Choiseul I.

HYPAETRA Guenée, 1852 . See Aneveuthina Hübner, 1823.

OPHI

HYPAGYRTIS Hübner, 1818, Zuträge Samml. exot. Schmett. 1 : 20.

Type-species: Hypagyrtis pustularia Hübner, 1818, ibidem 1: 20, figs 103, 104, by monotypy.

H. pustularia is a junior subjective synonym of Phalaena unipunctata Haworth, I809, Lepid. Br.: 345 .

Hypagyrtis is included in this catalogue as it is linked with one of the early uses of Evastria.

†ERASTRIA Hübner, I808, Erste Zuträge Samml. exot. Schmett.: 5.

Included in a work rejected for nomenclatural purposes by Int. Commn zool. Nom., 1966, Bull. zool. Nom. 23 Opinion $789: 214$.

Placed on the Official Index of Rejected and Invalid Generic Names in Zoology: Name No. 1839 .

Only included species: ‡Evastria pustularia Hübner, 1808 , ibidem: 5, later made nomenclaturally available as Hypagyrtis pustulavia Hübner, 1818.

HYPANGITIA Hampson, I9I8, Novit. zool. 25: 178.

$\operatorname{ACON}$

Type-species: Hypangitia peratopis Hampson, 1918, ibidem 25: 178, by original designation. Holotype ô, PARAgUay: Sapucay (BMNH).

HYPANUA Hampson, 191 3, Cat. Lepid. Phalaenae Br. Mus. 12: 459.

CATO

Type-species: Ophiusa xylina Distant, 1898, Ann. Mag. nat. Hist. (7) 1: 229, by original designation. Syntypes, South AFriCA: Transvaal, Pretoria (BMNH).

HYPENA Schrank, 1802, Fauna Boica 2 (2): I63.

HYPE

Type-species: Phalaena proboscidalis Linnaeus, I758, Syst. Nat. (Edn 10) 1: 533 , by subsequent designation by Curtis, 1829, Br. Ent. 6: 288. Type(s), Europe (LS, london).

ERICHILA Billberg, 1820, Enumeratio Insect. Mus. G.J. Bitlberg: 89.

Type-species: Phalaena proboscidalis Linnaeus, I758, by subsequent designation by Tams, 1939, Entomologist 72: 138.

Erichila is a junior objective synonym of Hypena Schrank, 1802. 
HERPYZON Hübner, I822, Syst.-alphab. Verz.: 52, 56.

Type-species: Phalaena proboscidalis Linnaeus, I758 (but included by Hübner as ¥"procoscidalis L.', an incorrect subsequent spelling), by subsequent designation by Berio, 1957, Memorie Soc. ent. ital. 36: I3, but cited as 'proboscidalis Fab.,' an incorrect authorship.

Herpyzon is a junior objective synonym of Hypena Schrank, I8o2.

HYPENAGONIA Hampson, I893, Illust. typical Specimens Lepid. Heterocera Colln Br. Mus. 9: 30,122 .

HYPE

Type-species: Acidalia vexataria Walker, I86I, List Specimens lepid. Insects Colln Br. Mus. 23: 767, by subsequent designation by Hampson, 1895, Fauna Br. India (Moths) 3: Ioo. Lectotype q, Borneo: Sarawak (UM, Oxford), designated (as type) by Swinhoe, I90o, Cat. east. and Aust. Lepid. Heterocera 2: 2 I 6.

HYPENAGONIODES Strand, I920, Arch. Naturgesch. 84 (A) I 2: I77.

HYPE

Type-species: Hypenagoniodes vexatariola Strand, I920, ibidem 84 (A) I2: I77, by monotypy. Holotype o․ [Tarwan] Formosa: Suisharyo (DEI, Eberswalde).

$\ddagger$ †Y PMAGONIODES; Neave, 1939, Nomencl. zool. 2: 734 .

An incorrect subsequent spelling.

HYPENARANA Bethune-Baker, I908, Novit. zool. 15: 240.

HYPE

Type-species: Hypenarana rosacea Bethune-Baker, 1908, ibidem 15: 240, by original designation. Syntypes 0 , + , New Guinea: [Papua], Biagi, R. Mambaré (BMNH).

HYPENARIA Guenée, I852, in Boisduval \& Guenée, Hist. nat. Insectes (Lépid.) 7: 381. OpHI

Type-species: Phalaena ortilia Stoll, I78I, in Cramer, Uitlandsche Kapellen 4: 105, pl. 344, fig. F, by subsequent designation by Hampson, I926, Descr. new Genera Species Lepid. Phalaenae Subfamily Noctuinae Br. Mus.: 444. Type(s), Surinam.

$\ddagger$ †YPERNARIA; Walker, I858, List Specimens lepid. Insects Colln Br. Mus. 15: I6I3.

An incorrect subsequent spelling.

HYPENDALIA Berio, I962, Annali Mus. civ. Stor. nat. Giacomo Doria 73: 178.

HYPE

Type-species: Hypendalia microscopica Berio, I962, ibidem 73: I79, figs 5 \& 9, by original designation. Holotype $\widehat{0}$. Aldabra I. (MNHN, Paris).

HYPENODES Doubleday, I850, Zoologist 8 Appendix: cv.

HYPE

Type-species: Hypenodes humidalis Doubleday, I850, ibidem 8 Appendix: cv, by monotypy. Syntypes, Ireland.

$H$. humidalis is a junior subjective synonym of Hypena turfosalis Wocke, I850, $Z$. Ent. Breslau 4 (I6) Lepid.: pl. 5, fig. I7. These two names were both published at about the same time; $H$. humidalis in October and $H$. turfosalis in the last quarterly issue of I850. Ever since they were synonymized in 1854 , turfosalis has been accepted as the senior name.

See also Tholomiges Lederer, I857.

HYPENODES Guenée, 1854. See Schrankia Hübner, [1825].

HYPE

HYPENOPSIS Dyar, I913, Proc. U.S. natn. Mus. 44: 296.

HYPE

Type-species: Hypenodes macula Druce, I891, Biologia cent.-am. (Zool.) Lepid. Heterocera 1: 44I, pl. 36, fig. I, by original designation. Syntypes 2 ex., Panama: Chiriqui.

HYPENULA Grote, i876, Can. Ent. 8: 27.

HYPE

Type-species: Hypenula opacalis Grote, 1876 , ibidem 8: 27 , by monotypy. Syntypes

$3(\hat{0}$, q) , [U.S.A.]: Texas (BMNH).

H. opacalis is a junior subjective synonym of Hypena cacuminalis Walker, [1859] I858, List Specimens lepid. Insects Colln Br. Mus. 16: 37.

$\ddagger$ HYPER AENIA, misspelling. See Hypernaenia Hampson, I894.

NOCT 
HYPERAESCHRA Butler, I88o, Ann. Mag. nat. Hist. (5) $6: 65$. NOTODONTIDAE Type-species: Hyperaeschra pallida Butler, r88o, ibidem (5) $6: 65$, by monotypy.

Hyperaeschra has been used as the original generic name for species which are now in the Noctuidae.

HYPERAUCHA Meyrick, r897, Trans. ent. Soc. Lond. 1897: 383.

HYPE

Type-species: Hyperaucha octias Meyrick, r897, ibidem 1897: 384, by monotypy. Syntypes $\left.4(\bar{\sigma} \text {, }+)^{\prime}\right)$ New Zealand: Wellington. Australia: Victoria (BMNH); N.S.W., Sydney (BMNH); R. Richmond (BMNH).

$H$. octias is a junior subjective synonym of Lithilaria ossicolor Rosenstock, 1885.

See also Lithilaria Rosenstock, r885.

HYPERBANIANA Hampson, 1926, Descr. new Genera Species Lepid. Phalaenae Subfamily Noctuinae Br. Mus.: I 98. OPHI

Type-species: Hyperbaniana costinotata Hampson, 1926, ibidem: 199, by originai designation. Holotype $\delta$, [Sulawesi] Celebes: Sangir I. (BMNH).

HYPERCALYMNIA Hampson, 1909, Cat. Lepid. Phalaenae Br. Mus. 8: 6. Available, but without included species until Hampson, r9ro, ibidem 9: r85. ACRO

Type-species: Hypercalymnia metaxantha Hampson, 1910, ibidem 9: 185, fig. 69, by subsequent monotypy. Holotype ô. [ZAire] Congo: Katanga Distr. (BMNH).

HYPERCODIA Hampson, r910, Cat. Lepid. Phalaenae Br. Mus. 10: xv, $488 . \quad$ ACON

Type-species: Motina disparalis Walker, 1863, List Specimens lepid. Insects Colln Br. Mus. 27: r3, by original designation, but cited as $\$$ disparilis, an incorrect subsequent spelling. Type(s) ơ, Borneo: Sarawak (BMNH).

HYPERCOMPA Rambur, I866. See Callimorpha Latreille, I 809.

ARCTIIDAE

$\ddagger$ HYPERDASIS, misspelling. See Hyperdasys Butler, I891. ACRO

HYPERDASYS Butler, I891, Ann. Mag. nat. Hist. (6) 8:71, 74. ACro

Type-species: Eriopus exotica Guenée, I 852, in Boisduval \& Guenée, Hist. nat. Insectes

(Lépid.) 6:294, by original designation. Type(s), Java (BMNH).

$\ddagger$ HYPERDASIS; Swinhoe, rgor, Ann. Mag. nat. Hist. (7) 8: r29.

An incorrect subsequent spelling.

HYPEREPIA Barnes \& Lindsey, 1922, Bull. Brooklyn ent. Soc. 17: 56.

Type-species: Hyperepia pi Barnes \& Lindscy, r922, ibidem 17: 56, by original designation. Holotype $\hat{O}$. [U.S.A.]: N.M., Otero Co., High Rolls.

HYPERFRONTIA Berio, 1962, Annali Mus. civ. Stor. nat. Giacomo Doria 73: 198. Noct

Type-species: Hyperfrontia direae Berio, 1962, ibidem 73: 198, fig. 2, by original designation. Holotype $\hat{\sigma}$, [Eтhiopia]: Dire-Daoua (Colln E. Berio, Genoa).

HYPERIODES Warren, 1910. See Mythimna Ochsenheimer, 1816. HADE

HYPERLOPHA Hampson, I895, Fauna Br. India (Moths) 3: xiii, 19. OPH

Type-species: Ephyrodes cristifera Walker, 1865, List Specimens lepid. Insects Colm Br. Mus. 33: ro71, by original designation. Holotype of, CEYLON (BMNH).

HYPERLOPHOIDES Strand, 1917, Arch. Naturgesch. 83 (A) 10: 146.

OPHI

Type-species: Zethes compactilis Swinhoe, r890, Trans. ent. Soc. Lond. 1890: 25I, pl. 7, fig. 16, by monotypy. Type(s), Burma: Thyetmyo (BMNH).

HYPERMILICHIA Dognin, I914, Hétérocères nouv. Am. Sud 8: 30.

Type-species: Hypermilichia colon Dognin, r9r4, ibidem 8: 30, by monotypy.

Holotype of, Argentina: Los Vasquez (USNM, Washington).

HYPERMILICHIA Hampson, I918, Novit. zool. 25: 146.

Type-species: Hypermilichia colon Dognin, r9I4, by original designation. 
A junior homonym of Hypermilichia Dognin, I9 4 . This generic name was used and made available by Dognin prior to its proposal and generic description by Hampson. The objective replacement name is Hypermilichia Dognin, I9I4.

HYPERMILICHIA Hampson, I9I8. See Hypermilichia Dognin, I9r4.

ACRO

HYPERNAENIA Hampson, I894, Fauna Br. India (Moths) 2: xi, x94.

NOCT

Type-species: Ochropleura denticulata Warren, I888, Proc. zool. Soc. Lond. 1888: 307, by original designation. Syntypes, $3 \hat{0}$, India: [Punjab], Abbottabad, Thundiani (BMNH).

†HYPERAENIA; Neave, 1939, Nomencl. zool. 2: 729.

An incorrect subsequent spelling.

$\ddagger$ HYPERNARIA, misspelling. See Hypenaria Guenée, I 852 .

OPHI

HYPERSOPHTHA Berio, 1954, Mem. Inst. scient. Madagascar (E) 5: 142.

ACON

Type-species: Hypersophtha falcata Berio, I954, ibidem (E) 5: 142 , pl. 7, fig. 8, by original designation. Holotype ơ, MADAGASCAR: Massif de l'Ankaratra, forêt d'Ambahona (MNHN, Paris).

HYPERSTROTIA Hampson, I9Io, Cat. Lepid. Phalaenae Br. Mus. 10: xvi, 556. ACON Type-species: Thalpochares aetheria Grote, I879, N. Am. Ent. 1: 47, by original designation. Syntypes 3 ㅇ․ [U.S.A.]: Fl[orid]a, Tampa (BMNH); Enterprise (BMNH).

$T$. aetheria is a junior subjective synonym of Cryphia nana Hübner, 1818, Zuträge Samml. exot. Schmett. 1: I4, figs 53, 54 .

$\ddagger J A S P I D I A$ Hübner, 1808, Erste Zuträge Samml. exot. Schmett.: 4, 5 .

Included in a work rejected for nomenclatural purposes by Int. Commn zool. Nom., x 966, Bull. zool. Nom. 23 Opinion 789:215. Placed on the Official Index of Rejected and Invalid Generic Names in Zoology: Name No. I867.

Out of the two originally included names $\ddagger$ Jaspidia nana Hübner, I808, ibidem: 4, was cited as the type-species by Tams, 1939, Entomologist 72: 138 . $\ddagger J$. nana Hübner, I808, was later made nomenclaturally available as Cryphia nana Hübner, 1818, and has been placed by McDunnough, 1938, Check List. Lepid. Can. \& U.S.A. 1: 108, as the senior subjective synonym of Thalpochares aetheria Grote, I879.

$\ddagger J A S P I D I A$ Hübner, I818, Zuträge Samml. exot. Schmett. 1: 14, 18, 33 .

Not nomenclaturally available as a genus-group name. Hübner used Jaspidia, on all three pages, in a supra generic sense for one of the names of his tribes (Namen der Stämme) listed on page 33. Hübner listed his genus-group names on page 35 .

HYPERS YPNOIDES Berio, 1954, Annali Mus. civ. Stor. nat. Giacomo Doria 66: 34I. Cato

Type-species: Hypersypnoides congoensis Berio, I954, ibidem 66:342, figs I-3, by original designation. Holotype $\hat{\sigma}$, South West AFrICA: Tsumeb (MRAC, Tervuren).

HYPERTROCTA Hampson, I893, Illust. typical Specimens Lepid. Heterocera Colln Br. Mus.

9: 32, I3I. HYPE

Type-species: Hypertrocta marmorata Hampson, I893, ibidem 9: 32, I3 I, pl. I67, fig. 22, by monotypy. Type(s) ô, CEYlon: Nawala-pittia (BMNH).

H. marmorata is a junior subjective synonym of Moscha posticalis Walker, [r866].

MOSCHA Walker, [1866] I865, List Specimens lepid. Insects Colln Br. Mus. 34: I 199.

Type-species: Moscha posticalis Walker, [1866] I865, ibidem 34: I200, by monotypy. Lectotype $\widehat{\sigma}$, [India]: Hindostan (UM, Oxford), designated (as type) by Swinhoe, I9oo, Cat. east. and Aust. Lepid. Heterocera 2: 2 I 7.

A junior homonym of Moscha Brookes, I828, Cat. anat. zool. Mus. J. Brookes (2): 102, - Aves. There is no objective replacement name but $M$. posticalis is conspecific with Hypertrocta marmorata Hampson, I 893, the type-species of Hypertrocta Hampson, I893. The latter is therefore available for use as a subjective replacement name.

Not preoccupied by $\ddagger$ Moscha; (Leach MS) Stephens, 1824, in Shaw, Gen. Zool. 12 (2): 78 , - Aves; first published in synonymy. 
$\ddagger_{\ddagger} H Y P E T R A$, misspelling. See Aneveuthina Hübner, I823.

HYPEUTHINA Lederer, I 855 Verh. zool.-bot. Ver. I'ien 5: ig9.

ACRO

Type-species: Hypeuthina fulgurita Lederer, I 855, ibidem 5: I99, pl. 4, fig. I, by monotypy. Syntypes 30,2 아. [LEBAnon]: Beirut.

HYPHILARE Hübner, [1821] i 816, Vevz. bekannter Schmett.: 239.

HADE

Type-species: Noctua albipuncta [Denis \& Schiffermüller], I 775, Ankïndung syst. Werkes Schmett. Wienergegend: 84, by subsequent designation by Hampson, 1905, Cat. Lepid. Phalaenae Br. Mus. 5: 436. Type(s), [Austria]: Vienna district (Collection destroyed, Horn \& Kahle, I936, Ent. Beih. Bevl.Dahlem 3: 243).

HYPHYPENA Warren, I889, Trans. ent. Soc. Lond. 1889: 23I.

OECOPHORIDAE

Type-species: Hyphypena hipunctalis Warren, I 889, ibid:m 1889: 232, by monotypy.

Hyphypena was originaily placed in the Noctuidae.

$\ddagger$ HYPMAGONIODES, misspelling. See Hypenagoniodes Strand, I9zo.

HYPE

HYPNOT YPE Hampson, I906, Cat. Lepid. Phalaenae Br. Mus. 6: xiii, 4II.

CUCU

Type-species: Diphthera placens Walker, I857, List Specimens lepid. Insects Colln Br. Mus. 11: 708, by original designation. Holotype q, [Colonbia]: Bogota (BMNH).

HYPOBARATHRA Hampson, 1905, Cat. Lepid. Phalaenae Br. Mus. 5: x, $221 . \quad$ HAdE Type-species: Xylina icterias Eversmann, 1843, Bull. Soc. Nat. Moscou 16 (35): 548, by original designation. Syntypes, [U.S.S.R.]: Urals to R. Sacmara (ZI, Leningrad).

HYPOBLETA Turner, I908, Trans. R. Soc. S. Aust. 32: 77.

ACON

Type-species: Hypobleta cymaea Turner, 1908, ibidem 32: 78, by monotypy. Holotype o. Australia: Qd, Kuranda (ANIC, Canberra).

HYPOCALA Guenée, I852, in Boisduval \& Guenée, Hist. nat. Insectes (Lépid.) 7: $73 . \quad$ орн

Type-species: Hyblaea deflorata Fabricius, I 794, Ent. Syst. 3(2): I 27, by subsequent designation by Hampson, I894, Fauna Br. India (Moths) 2: 452. Type(s), [IndiA]: India orientali (UZM, Copenhagen).

HYPOCALAMIA Hampson, 1908, Cat. Lepid. Phalaenae Br. Mus. 7: 19. Available, but without included species until Hampson, I910, ibidem 9: xiv, 495. ACRO

Type-species: Bombycia meterythra Hampson, I894, Fauna Br. India (Moths) 2: 207, by subsequent monotypy. Type(s) $\delta$, [Pakistan]: Murree (BMNH).

H YPOCALPE Butler, I 883, Proc. zool. Soc. Lond. 1883: i $57 . \quad$ орн I

Type-species: Calpe fasciata Moore, $\mathrm{r} 882$, in Hewitson \& Moore, Descr. new Indian lepid. Insects Colln late Mr W. S. Atkinson: i 5 I, by monotypy. Syntypes, India: [WV. Bengal], Darjiling (BMNH).

HYPOCALPOPIS Hampson, I926. See Makapta Schaus, I904.

OP1I I

HYPOCAREA Hampson, I9I8, Novit. zool. 25: $20 \mathrm{I}$.

CHLO

Type-species: Remigia conspicua Leech, igoo, Trans. ent. Soc. Lond. 1900: 566, by original designation. Holotype $\sigma^{\imath}$, JAPAN : [Hokkaido], Hakodate (BMNH).

HYPOCOENA Hampson, igo8, Cat. Lepid. Phalaenae Br. Mus. 7: 19. Available, but without included species until Hampson, r9то, ibidem 9: xi, zо .

ACRO

Type-species: Leucania rufostrigata Packard, 1867, Proc. Boston Soc. nat. Hist. 11: 36 , but included by Hampson as $\ddagger L$. rufostriga, an incorrect subsequent spelling, by subsequent monotypy. Syntypes $\hat{\sigma}$, [CANADA]: Labrador, Str. of Belle I., Caribou I.

HYPODEVA Holland, i 894, Psyche, Camb. 7:27.

CHLO

Type-species: Hypodeva barbata Holland, I 894 , ibidem 7:27, pl. I, fig. 2, by original designation. Syntypes $\hat{\sigma}$, West Africa (CM, Pittsburgh). 
HYPOECHANA Druce, I89I, Biologia cent.-am. (Zool.) Lepid. Heterocera 1: $48 \mathrm{I}$.

HYPE

Type-species: Hypoechana fuliginosa Druce, I89I, ibidem 1: 48I, pl. 40, fig. 8, by original designation. Syntypes $\hat{\sigma}$, Mexıco: Vera Cruz, Atoyac (BMNH); Coatepec; Tabasco, Teapa. Guatemala: Volcan de Atitlan. Panama: Chiriqui.

HYPOEPA Leech, I 889, Entomologist 22: 63.

HYPE

Type-species: Hypoepa bambusalis Leech, I889, ibidem 22: 63, pl. 2, fig. 2, by monotypy. Holotype 0 , China: [Fukien], Foochau (BMNH).

HYPOGLAUCITIS Staudinger, I895, Dt. ent. Z. Iris 7: 284.

CATO

Type-species: Hypoglaucitis moses Staudinger, I 895, ibidem 7:284, pl. 9, fig. I7, by monotypy. Syntypes $4 \hat{\sigma}$, [Egypt]: Cairo. [IsRael] Palestine: Jaffa.

$H$. moses is a junior subjective synonym of Pandesma benenotata Warren, I888, Proc. zool. Soc. Lond. 1888: 3г 3.

HYPOGRAMMA Guenée, I852, in Boisduval \& Guenée, Hist. nat. Insectes (Lépid.) 7: 34 . opHI

Type-species: Phalaena sulima Stoll, I790, in Cramer, Uitlandsche Kapellen Aanhangsel: 175, pl. 40, fig. 5, by subsequent designation by Grote, r874, Bull. Buffalo Soc. nat. Sci. 2: 40. Syntypes, Surinam. The Cape of Good Hope was also cited by Stoll, but the species illustrated does not occur in South Africa.

P. sulima is a junior subjective synonym of Phalaena capensis Cramer, I777, ibidem 2: I09, pl. I67, fig. C.

See also Anthocitta Hübner, [1823].

HYPOGRAMMODES Hampson, I913 [January], Cat. Lepid. Phalaenae Br. Mus. 12: 8. Available but without included species until Hampson, І9г3 [November], ibidem 13: x, 253 .

CATO

Type-species: Coenipeta abscondens Walker, [1858] I 857, List Specimens lepid. Insects Colln Br. Mus. 13: I094, by subsequent designation by Hampson, I91 3, ibidem 13: 253. Type(s) $\tilde{\sigma}$, [BRAzIL]: Valley of the Amazon.

C. abscondens is a junior subjective synonym of Hypogramma balma Guenée, 1852 , in Boisduval \& Guenée, Hist. nat. Insectes (Lépid). 7: 36.

HYPOLISPA Turner, I926, Trans. R. Soc. S. Aust. 50: I 21.

SARR

Type-species: Hypolispa leucopolia Turner, 1926, ibidem 50: I2I, by monotypy. Holotype ․, Australia: Qd, Yeppoon (ANIC, Canberra).

HYPOMECIA Staudinger, I897, Dt. ent. Z. Iris. 10: 29I.

CUCU

Type-species: Epimecia quadrivirgula Mabille, I888, Bull. Soc. ent. Fr. 1888: li, by monotypy. Lectotype $\widehat{\sigma}$. Tunisia: Gabès (MNHN, Paris), designated (as holotype) by Viette \& Fletcher, I968, Bull. Br. Mus. nat. Hist. (Ent.) 21: 4I I.

HYPONEUMA Schaus, I906, Proc. U.S. natn. Mus. 30 : х 32.

HYPE

Type-species: Hyponeuma leucanioides Schaus, I906, ibidem 30: 133, by monotypy. Type(s), Brazil: São Paulo (USNM, Washington).

H. leucanioides is a junior subjective synonym of Herminodes taltula Schaus, 1904, Trans. Am. ent. Soc. 30: I67.

HYPOPERIGEA Hampson, I908, Cat. Lepid. Phalaenae Br. Mus. 7: 7. Available, but without included species until Hampson, I909, ibidem 8: xii, 422. ACRO

Type-species: Perigea tonsa Guenée, 1852 , in Boisduval \& Guenée, Hist. nat. Insectes (Lépid.) 5: 232, by subsequent designation by Hampson, I909, ibidem 8: 422. Syntypes o. ㅇ. [Australia] Nouvelle-Hollande (BMNH).

HYPOPLEURONA Hampson, 1926, Descr. new Species Lepid. Phalaenae Subfamily Noctuinae Br. Mus.: 5 oI. OPHI

Type-species: Parathermes acutissima Bethune-Baker, I9r I, Ann. Mag. nat. Hist.

(8) 8: 536 , by original designation. Holotype $\delta$, [ANGola] Port. W. Africa (BMNH). 
HYPOPLEXIA Hampson, I908, Cat. Lepid. Phalaenae Br. Mus. 7 : xii, 426.

ACRO

Type-species: Hadena algoa Felder \& Rogenhofer, I874, Reise öst. Fregatte Novara (Zool.) 2 (Abt. 2): pl. Io9, fig. 2I ; Inhalts-Verz.: I I, by original designation. Type(s) $\sigma^{*}$, South Africa: [Cape prov.], Knysna (BMNH).

HYPOPRORA Hampson, 1926, Descr. new Genera Species Lepid. Phalaenae Subfamily Noctuinae Br. Mus.: 82.

OPH I

Type-species: Prorocopis tyra Swinhoe, igo2, Ann. May. nat. Hist. (7) 9: 84, by original designation. Syntypes $\hat{\delta}$, 우, Australia: W. Aust., Sherlock River (BMNH).

HYPOPTERIDIA IVarren, I9I 2, Novit. zool. 19: II.

HADE

Type-species: Aletia reversa Moore, [1884] i 887, Lepid. Ceylon 3: 6, pl. I 44, fig. 5, by original designation. Syntypes $\hat{\jmath}, \underline{\text { q }}$, CEYLON (BMNH).

HYPOPYRA Guenée, I852, in Boisduval \& Guenée, Hist. nat. Insectes (Lépid.) 7: I98. CATC

Type-species: Noctua vespertilio Fabricius, I 787, Mantissa Insect. 2: I 36, by subsequent designation by Desmarest, (I857), in Chenu, Encycl. Hist. nat. Papillons nocturnes: I3I. Type(s), [India]: [Madras], Tranquebar (Type(s) not found by Zimsen, 1964, Type Material of I. C. Fabricius: 548 ).

See also Enmonodia Walker, 1858.

HYPOS ADA Hampson, ig Io August, Proc. zool. Soc. Lond. 1910: 4 I 2.

$\operatorname{ACON}$

Type-species: Phibalapteryx hydrocampata Guenée, 1857 , in Boisduval \& Guenée, Hist. nat. Insectes (Lépid.) 10: 438, by monotypy. Holotype 오. INDiA: northern (BMNH). HYPOSADA Hampson, I9Io [December], Cat. Lepid. Phalaenae Br. Mus. 10: xi, 273.

Type-species: Lycauges postvittata Moore, I887, Lepid. Ceylon 3: 456, pl. 20I, fig. I 2, by original designation. Type(s) $q$, CEYLon: Dickoya (BMNH).

A junior homonym of Hyposada Hampson, igro August. This generic name was used and unintentionally made available by Hampson in August, prior to its proposal and generic description by Hampson in December for the same concept, but having a different type-species. There is no objective replacement name but $L$. postvittata is congeneric with P. hydrocampata Guenée, I857, the type-species of Hyposada Hampson, I9Io August. The latter is therefore available for use as a subjective replacement name.

HYPOSADA Hampson, ig io [December]. See Hyposada Hampson, i9 Io August. ACoN HYPOSCOTA Hampson, igr2. See Ballatha Walker, [1866]. Chlo

HYPOSEMANSIS Hampson, i 895, Fauna Br. India (Moths) 3: xiii, $2 . \quad$ орн I

Type-species: Marmorinia singha Guenée, I852, in Boisduval \& Guenée, Hist. nat.

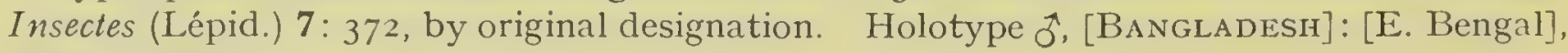
Silhet (BMNH).

HYPOSEMEIA Hampson, I893, Illust. typical Specimens Lepid. Heterocera Colln Br. Mus. 9: $30, \mathrm{I} 2 \mathrm{~T}$. HYPE

Type-species: Herminia incertalis Walker, [1866] 1865, List Specimens lepid. Insects Colln Br. Mus. 34: 15 8, by original designation. Holotype of. Java (BMNH).

$H$. incertalis is a junior subjective synonym of Britha biguttata Walker, [1866], ibidem 34: I I 47 .

See also Britha Walker, [1866].

HYPOSPILA Guenée, I 852, in Boisduval \& Guenée, Hist. nat. Insectes (Lépid.) 7: 358 . opH I Type-species: Hypospila bolinoides Guenée, I852, ibidem 7: 358, by monotypy. Syntypes I $\hat{O}$, I 9 , JAVA (BMNH). India: central.

See also Orrea Walker, i 866.

HYPOSTILBIA Hampson, igo8, Cat. Lepid. Phalaenae Br. Mus. 7: I7. Available, but without included species until Hampson, I909, ibidem 8: x, 293. ACRO

Type-species: Senta megastigma Püngeler, 1907, Dt. ent. Z. Iris 19:221, pl. 7, fig. I8, 
by subsequent designation by Hampson, I909, ibidem 8: 293. Syntypes 5 ô, I + , [CHINA]: [Sinkiang-Uigur], Lob-Noor (MNHU, Berlin).

S. megastigma is a junior subjective synonym of Caradrina distracta Eversmann, I848, Bull. Soc. imp. Nat. Moscou 21 (3): 217.

HYPOSTROTIA Hampson, I926, Descr. new Genera Species Lepid. Phalaenae Subfamily Noctuinae Br. Mus.: 293.

OPHI

Type-species: Capnodes cinerea Butler, i 878, Ann. Mag. nat. Hist. (5) 1: 294, by original designation. Syntypes $\hat{\jmath}$, ㅇ, JAPAN : Yokohama (BMNH).

HYPOS YPNOIDES Berio, 1954, Annali Mus. civ. Stor. nat. Giacomo Doria 66: 343. CATo

Type-species: Hypersypnoides flandriana Berio, I954, ibidem 66: 343 , by original designation. Holotype o., [ZAIRE]: Equateur, Flandria (MRAC, Tervuren).

Hyposypnoides Berio was proposed as a subgenus of Hypersypnoides Berio, I954.

HYPOT ACHA Hampson, i913, Cat. Lepid. Phalaenae Br. Mus. 12: ix, $243 . \quad$ Cato

Type-species: Audea retracta Hampson, 1902, Ann. S. Afr. Mus. 2: 329, by original designation. Syntypes, [South Africa]: Natal, Estcourt (BMNH); Cape Colony, Transkei (BMNH).

†HYPOTHENIA Schaus, no published reference found. HYPE

HYPOTHRIPA Hampson, I894, Fauna Br. India (Moths) 2: xvii, 383. SARR

Type-species: Selepa curiosa Swinhoe, I89o, Trans. ent. Soc. Lond. 1890: 235, by original designation. Type(s), Burma: Rangoon (BMNH).

HYPOTRISULA Hampson, I926, Descr. new Genera Species Lepid. Phalaenae Subfamily Noctuinae Br. Mus.: I $23 . \quad$ OPHI

Type-species: Steiria boarmioides Walker, 1865, List Specimens lepid. Insects Colln Br. Mus. 33: 923, by original designation. Holotype ․, [India]: N. Hindostan (BMNH).

HYPOTRIX Guenée, I852, in Boisduval \& Guenée, Hist. nat. Insectes (Lépid.) 5: 369. HADE Type-species: Hypotrix purpurigera Guenée, I 852, ibidem 5: 369, by subsequent designation by Hampson, I905, Cat. Lepid. Phalaenae Br. Mus. 5: 291. Syntypes I Õ, I $q$ BrazIL: Nouvelle- Fribourg (BMNH).

HYPO TYPE Hampson, I906, Cat. Lepid. Phalaenae Br. Mus. 6: xii, 384.

CUCU

Type-species: Polia scotomista Hampson, I902, Ann. S. Afr. Mus. 2: 278, by original designation. Syntypes $\widehat{\jmath}$, [South Africa]: Cape Colony, Capetown (BMNH); Natal, coastbelt.

HYPOXESTIA Hampson, 1903, Cat. Lepid. Phalaenae Br. Mus. 4: xix, 600.

NOCT

Type-species: Mesogona dilatata Butler, i 879, Ann. Mag. nat. Hist. (5) 4: 364, by original designation. Type(s), Japan (BMNH).

HYPPA Duponchel [1845] I844, Cat. méth. Lépid. Eur. (2): I6I.

ACRO

Type-species: Phalaena rectilinea Esper, I788, Die Schmett. 4 (2) Abschnitt I: pl. I 27 fig. I ; I 796, ibidem: 379, by monotypy. Type(s) $\hat{o}$, [Germany]: Fränkische district.

HYPSA Hübner, [1819] i 816, Verz. bekannter Schmett.: i 72. Hypsidae Type-species: Phalaena silvandra Stoll, [1781] I 782, in Cramer, Uitlandsche Kapellen 4 : I 55, pl. 369, fig. D, by subsequent designation by Kirby, i892, Synonymic Cat. Lepid. Heterocera $1: 388$.

Hypsa has been used as the original generic name for species which are now in the Agaristidae and Noctuidae.

HYPSIFORMA Oberthür, I923, Études Lépid. comparée 21 (2) : I 49.

OPHI

Type-species: Hypsiforma lambertoni Oberthür, I923, ibidem 21 (2): I49, pl. 566, figs 4887,4888 , by monotypy. Syntypes $\hat{\sigma}$,, , MADAgascar: southern (BMNH). 
HYPSOPHILA Staudinger, I 888, Stettin. ent. Ztg 49: 40.

CUCU

Type-species: Heliothis jugorum Ershov, 1874, in Fedchenko, Reise in Turkestan 2 (5) 3:48, pl. 3, fig. 46 , by monotypy. Holotype ơ. [U.S.S.R.]: Turkestan, Stschurowsky alps, near Djiptik.

HYPSOROPHA Hübner, I8 18, Zuträge Samml. exot. Schmett. 1: iо.

OPHI

Type-species: Noctua monilis Fabricius, [I777], Genera Insect.: 283, by subsequent designation by Grote, I874, Bull. Buffalo Soc. nat. Sci. 2: 29. Type(s), Type-Locality stated to be Anglia [England], but this was probably an error as the species described is not known to occur in the Old World but is widespread in southern U.S.A. (Type(s) not found by Zinusen, 1964, Type Material of I. C. Fabricius: 532).

$\ddagger$ EUPHAIS Hübner, I818, Zütrage Samml. exot. Schmett. 1: I0, 21, 33.

Not nomenclaturally available as a genus-group name. Hübner used Euphais, on all three pages, in a suprageneric sense for one of the names of his tribes (Namen der Stämme) listed on page 33. Hübner listed his genus-group names on page 35. Euphais is included here as Berio, 1957, Memorie Soc. ent. ital. 36: I t has accepted it as an available generic name from 1818 and has designated as type-species, Noctua monilis Fabricius, [1777], the type-species of Hypsoropha Hübner, i8is.

HYPTIOXESTA Rebel, I90I, in Staudinger \& Rebel, Cat. Lepid. palaearct. Fannengeb. 1: $23 I$.

ACON

Type-species: Erastria penthima Ershov, i 870, Trudy nussk. ent. Obshch. 4: 196, by monotypy. Holotype ô, [U.S.S.R.]: Irkutzkensi prov., Balagansk (ZI, Leningrad).

HYRCANYPENA Wagner, I937, Z. öst. EntVer. 22: 62.

HYPE

Type-species: Hyrcanypena schwingenschussi Wagner, I937, ibidem 22:63, pl. I, fig. 5, by monotypy. Syntypes ô, [Iran] Persia: [Elburz Mts], I 2 km E. of Demawend.

HYRIA Stephens, I829. See Anteois Warren, I90o.

GEOMETRIDAE

HYRIODES Hampson, I9Io, Cat. Lepid. Phalaenae Br. Mus. 10: 4, I6.

ACON

Type-species: Hyriodes leucocraspis Hampson, I9I0, ibidem 10: I6, fig. 5, by original designation. Holotype ?, Borneo: [Sabah], Sandakan (BMNH).

${ }_{\ddagger}^{\ddagger} H$ YDRIODES; Neave, 1939, Nomencl. zool. 2: 7 I 2 .

An incorrect subsequent spelling.

HYSSIA Guenée, I 852, in Boisduval \& Guenće, Hist. nat. Insectes (Lépid.) 5: $345 . \quad$ HADE

Type-species: Orthosia cavernosa Eversmann, I 842, Bull. Soc. Nat. Mcscou 15: 547 ,

pl. 5, fig. 3, by monotypy. Syntypes, [U.S.S.R.]: Urals and [Kazan] Casan (ZI, Leningrad).

IAMBIA Walker, I863, List Specimens lepid. Insects Colln Br. Mus. 27: 109.

ACRO

Type-species: Iambia inferalis Walker, I 863, ibidem 27: I09, by monotypy. Holotype

?. [South Africa]: Port Natal (BMNH).

$\ddagger J A M B I A$; Gaede, I934, in Seitz, Gross-Schmett. Errde 15: 73 .

An incorrect subsequent spelling.

IAMBIODES Hampson, I908, Cat. Lepid. Phalaenae Br. Mus. 7: xv, 659. ACro

Type-species: Tarache anormalis Hampson, I 906, J. Bombay nat. Hist. Soc. 17: 475,

by monotypy. Holotype , India: [Andhra Pradesh], Gooty (BMNH).

$\ddagger J A M B I O D E S$; Gaede, 1934, in Seitz, Gross-Schmett. Erde 15: 74.

An incorrect subsequent spelling.

IANIUS Richards, 1939, Entomologica am. 19: 22.

CATO

Type-species: Melipotis mosca Dyar, I9Io, Proc. U.S. natn. Mus. 38: 253, by original designation. Syntypes I $\hat{o}, 4$ ㅇ, Mexico: Mexico City (USNM, Waslington).

ICASMA Turner, 1902, Proc. Limn. Soc. N.S.W. 27: 90.

SARR

Type-species: Icasma minutum Turner, 1902, ibidem 27:91, by monotypy. Holotype $o$ (not $q$ as stated), Australia: Qd, Brisbane (ANIC, Canberra). 
ICHNEUTICA Meyrick, I887, Trans. N. Z. Inst. 19: $x_{3}$.

Type-species: Ichneutica ceraunias Meyrick, I887, ibidem 19: I3, by monotypy. Holotype ô. New Zealand: Mt Arthur (BMNH).

ICHTHYOPSELAPHA Aurivillius, I9ro, in Sjöstedt, Kilimandjaro Meru Exped. 9: 35. opHI Type-species: Ichthyopselapha insignis Aurivillius, I910, ibidem 9: 36, pl. 2, fig. 5 , by monotypy. Holotype + , [TANzANIA]: Kilimandjaro, Kibonoto (NR, Stockholm).

IDALIMA Turner, I903, Trans. R. Soc. S. Aust. $27: 2$.

Type-species: Agarista affinis Boisduval, I832, Voyage de Déconvertes de l'Astrolabe 1: $\mathrm{x} 77$, by original designation. Type(s), [Australia]: [N.S.W.], Port Jackson district.

IDECHTHIS Hübner, I82 I. See Ascalapha Hübner, [I809].

OPHI

$\ddagger$ IDIA Hübner, [I806]. See Synaphe Hübner, [I825].

PYRALIDAE

${ }_{\ddagger}$ IDIA Hübner, I 808. See Smicropus Warren, I 895 .

GEOMETRIDAE

IDIA Hübner, [г8I3], Samml. exot. Schmett. 1: pl. [2 I2].

HYPE

Type-species: Idia aemula Hübner, [I8I3], ibidem 1: pl. [2 I 2], figs I-4, by monotypy. Syntypes $\hat{\delta}$, + , Type-LOCALITY not stated [CANADA. U.S.A.].

A second species Idia retusalis Hübner, [I8I8], was published on pl. [2I I]. According to Hemming, 1937, Hübner 1:405 the date of publication of pl. [2 I I] was five years later than that of pl. [2.12] and so Idia when it became nomenclaturally available, was a monotypic genus. Pelecia Hübner, I $82 \mathrm{I}$, was later proposed for $I$. vetusalis.

See also Camptylochila Stephens, I834.

IDICARA Walker, [1863] I864, J. Proc. Limn. Soc. (Zool.) 7: I72.

OPHI

Type-species: Idicara olivacea Walker, [1863] I864, ibidem 7: I72, by monotypy. Lectotype ô. Borneo: Sarawak (UM, Oxford), designated (as type) by Swinhoe, rgoo, Cat. east. and Aust. Lepid. Heterocera 2: 29.

IDIOCYTTARA Turner, I944, Proc. R. Soc. Qd 55: 35.

NOLI

Type-species: Sorocostia tornotis Meyrick, I888, Proc. Linn. Soc. N.S.W. (2) 2: 923 , by original designation. Syntypes 20 . Australia: Queensland, Duaringa (BMNH).

IHERINGIA Jones, I9I4. See Lophomyra Schaus, I9II.

ACRO

IKONDIANA Berio, I972, Memorie Soc. ent. ital. 51 : I 79.

NOCT

Type-species: Ikondiana ikondae Berio, 1972, ibidem 51: I79, fig. 26, by original designation. Holotype ô, TANzANia: Ikonda (Colln E. Berio, Genoa).

ILARUS Boisduval, I 828, Eur. Lepid. Index meth.: 76.

HADE

Type-species: Phalaena piniperda Panzer, I786, in Kob, Die wahre Ursache der Baumtrockniss der Nadelwälder durch die Naturgeschichte der Forlphaläne (2): 5I, pl. I, figs I-I2, by subsequent designation by Duponchel, I829, in Godart \& Duponchel, Hist. nat. Lépid. Papillons Fr. 7 (2): 7 x. Syntypes ơ, 우, Germany.

$P$. piniperda is a junior subjective synonym of Noctıa flammea [Denis \& Schiffermüller], I 775 .

See also Panolis Hübner, [182 1 ].

HILARUS Agassiz, I846, Nomencl. zool. Index univl.: I 82, I94.

An unjustified emendation of Ilams Boisduval, I828.

ILA TTIA Walker, [1859] I858, List Specimens lepid. Insects Colln Br. Mus. 16: 208. ACON

Type-species: Ilattia cephusalis Walker, [1859] I858, ibidem 16: 209, by monotypy. Syntypes I $\hat{0}$, I ․, [CHINA]: Shanghai (BMNH.)

I. cephusalis is a junior subjective synonym of Perigea octo Guenée, 1852 , in Boisduval \&

Guenée, Hist. nat. Insectes (Lépid.) 5: 233.

See also Stridova Walker, I869, and Chytoryza Grote, I 876. 
ILSEA Schaus, 1906, Proc. U.S. natn. MIus. $30: 131$.

OPHI

Type-species: Ilsea bormia Schaus, 1906, ibidem 30: 131, by monotypy. Type(s), BrazIL: Petropolis (USNM, Washington).

$\ddagger$ ILURGIS, misspelling. See Ilyrgis Walker, I 859 .

OPHI

ILUZA Walker, I865, List Specimens lepid. Insects Colln Br. Mus. 33: 1006.

OPHI

Type-species: Iluza decisa IValker, I865, ibidem 33: I007, by original designation.

Holotype $\hat{\sigma}$, [INDIA]: N. Hindostan (BMNH).

$\ddagger I L U Z 1 A$; Moore, 1877, Proc. zool. Soc. Lond. 1877: 6ro.

An incorrect subsequent spelling.

${ }_{+}^{+} I L U Z I A$, misspelling. See Iluza Walker, I 865 .

OPHI

IL YRGIS Walker, 1859, List Specimens lepid. Insects Colln Br. Mus. 19: 879.

OPHI

Type-species: Ilyrgis echephurealis Walker, I859, ibidem 19: 880, by monotypy. Holotype ô. CEYLON (BMNH).

¥ILURGIS; Mabille, I900, Annls Soc. ent. Fr. 68: 735 .

An incorrect subsequent spelling.

IL YRGODES Hampson, I926, Descr. new Genera Species Lepid. Phalaenae Subfamily Noctuinae Br. Mus.: 6ro.

OPHI

Type-species: Ilyrgodes intacta Hampson, 1926, ibidem: 610, by original designation. Holotype J. New Gunnea: [Papua], Louisiade Is, St. Aignan I. (BMNH).

IMITATOR Alphéraky, I 883, Horae Soc. ent. ross. 17: 170.

OPHI

Type-species: Imitator dentistrigatae Alphéraky, I883, ibidem 17: I 7 I, pl. 9, figs 95, 96,

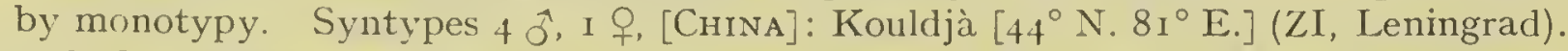

I. dentistrigatae is a junior subjective synonym of Ligia ciliaria Ménétriés, I849, Mém. Acad. imp. Sci. St. Petersb. (6) Sci. nat. 6 (4):296, pl. 6, fig. I I.

IMLEANGA Lucas, I90I, Proc. R. Soc. Qd 16:83.

OPHI

Type-species: Imleanga fluviatilis Lucas, 1901, ibidem 16:83, by monotypy. Syntypes 2 ô. [Australia]: Qd, Brisbane.

I. fluriatilis is a junior subjective synonym of Toxocampa lunifera Druce, I888, Proc. zool. Soc. Lond. 1888: 575 .

IMMETALIA Jordan, 1896, in Rothschild \& Jordan, Novit. zool. 3: 3 I.

AGAR

Type-species: Eusemia saturata Walker, [1865] I864, List Specimens lepid. Insects Colln Br. Mus. 31: 54, by original designation. Lectotype (sex not stated) Moluccas: [Buru I] Bourn (UNI, Oxford), designated (as type) by Swinhoe, I892, Cat. east. and Aust. I.epid. Heterocera 1: 162.

The older spelling of the type-locality was Bouru, but the final 'u' was incorrectly printed.

INCITA Grote, 1895, Abh. naturwe. Ver. Bremen 14: II I.

NOCT

Type-species: Annaphila aurantiaca Edwards, 1881, Papilio 1: 23, by original designation. Holotype ô. [U.S.A.]: Cal., Geysers.

INGURA Guenée, 1852, in Boisduval \& Guenée, Hist. nat. Insectes (Lépid.) 6: 309. EUTF

Type-species: Ingura lunodes Guenée, 1852 , ibidem 6: 3ro, by subsequent designation by Desmarest, (1857), in Chenu, Encycl. Hist. nat. Papillons nocturnes: 122. Syntypes 3 ö, [French Guiana]: Cayenne (BMNH). Brazil: Rio Janeiro. Honduras.

INGURIDIA Butler, 1879, Ann. Mag. nat. Hist. (5) 4:354.

PLUS

Type-species: Inguridia abrostolina Butler, 1879 , ibidem (5) 4:354, by original designation. Type(s), Japan (BMNH).

Inguridia was originally placed in the Notodontidae. 
INSOLENTIPALPUS Bethune-Baker, 1908, Novit. zool. 15: 2 I2.

Type-species: Insolentipalpus ochreopunctata Bethune-Baker, I9o8, ibidem 15: 2I2, by original designation. Type(s) $\tilde{\sigma}$, New Guinea: [Papua], Owgarra (BMNH).

INTERDELTA Berio, I964, Doriana 3 (I 44$): 4$.

Type-species: Interdelta mediafricana Berio, I964, ibidem 3 (I44): 4 , by original designation. Holotype $\hat{\sigma},\left[Z_{\text {AIRE }}\right]$ Congo: Kafakumba (MRAC, Tervuren).

IODOPEPLA Franclemont, 1964, Proc. ent. Soc. Wash. 66 : $7 \mathrm{I}$.

ACRO

Type-species: Ceramica u-album Guenée, 1852, in Boisduval \& Guenée, Hist. nat.

Insectes (Lépid.) 5: 345, by original designation. Holotype ㅇ. [U.S.A.]: Florida (BMNH).

IONTHA Doubleday, I 842, Entomologist 1: 297.

OPHI

Type-species: Iontha umbrina Doubleday, I 842 , ibidem $1: 298$, by monotypy. Type(s),

[Bangladesh]: [E. Bengal], Sylhet (BMNH).

$\ddagger$ JONTHE; Agassiz, [I 846], Nomencl. zool. Nomina syst. Generum Lepid.: 38 .

An incorrect subsequent spelling.

IPANA Walker, i858, List Specimens lepid. Insects Colln Br. Mus. 15: I66I. Geometridae Type-species: Ipana leptomera Walker, I 858, ibidem 15: I662, by monotypy.

Ipana was originally placed in the Noctuidae.

IPANA Jordan, I896. See Ipanica Hampson, I9o8.

AGAR

IPANEPHIS nom. n. for Epiphanis Schaus, I9I3.

HYPE

Type-species: Epiphanis esperanzalis Schaus, I9I3, Ann. Mag. nat. Hist. (8)11: 22, by original designation (for Epiphanis Schaus). Type(s) ${ }^{\lambda}$. Costa Rica: Esperanza.

EPIPHANIS Schaus, 1913, Ann. Mag. Nat. Hist. (8) 11: 22.

Type-species: Epiphanis esperanzalis Schaus, I913, by original designation.

A junior homonym of Epiphanis Eschscholtz, I829, in Thon, Ent. Archiv 2 (1): 35, Coleoptera. The objective replacement name is Ipanephis nom. $\mathrm{n}$.

IPANICA Hampson, I9o8, Cat. Lepid. Phalaenae Br. Mus. 7: 6. Available, but not proposed as the objective replacement name for Ipana Jordan, I896, until Hampson, I9Io, ibidem 9: $45^{\circ}$.

AGAR

Type-species: Aegocera cornigera Butler, 1886, Trans. ent. Soc. Lond. 1886: 381, by original designation (for Ipana Jordan, I896). Syntypes $\sigma^{*},+$, [Australia]: [Qd], Gayndah (BMNH); Peak Downs.

IPANA Jordan, I896, in Rothschild \& Jordan, Novit. zool. 3: 54.

Type-species: Aegoceva cornigera Butler, I 886, by original designation.

A junior homonym of Ipana Walker, 1858, List Specimens lepid. Insects Colln Br. Mus. 15: I66I, - Lepid., Geometridae. The objective replacement name is Ipanica Hampson, I908.

$\ddagger I P A R S A$; Pagenstecher, I909, Geogr. Verbreitung Schmett.: 423.

An incorrect subsequent spelling.

$\ddagger$ IPARSA, misspelling. See Ipanica Hainpson, I908.

AGAR

IPERMARCA Berio, I966, Annali Mus, civ. Stor. nat. Giacomo Doria 76: 135.

OPHI

Type-species: Ipermarca monovittata Berio, I966, ibidem 76: I36, fig. 26, by original designation. Holotype $\hat{\delta}$, Madagascar: Massif d'Ankaratra, Manjakatompo, Forêt d'Ambahona (MNHN, Paris).

$\ddagger$ †PHIMORPHA, misspelling. See Ipimorpha Hübner, [182I].

ACRO

IPIMORPHA Hübner, [182 I] I816, Verz. bekannter Schmett.: 238.

ACRO

Type-species: Noctua subtusa [Denis \& Schiffermüller], 1775, Ankündung syst. Werkes Schmett. Wienergegend: 88, by subsequent designation by Grote, 1874, Bull. Buffalo 
Soc. nat. Sci. 2: 24. Type(s), [Austria]: Vienna district (Collection destroyed, Horn \& Kahle, 1936, Ent. Beih. Berl.-Dahlem 3: 243).

¥ENOBIA Oken, 1815, Okens Lehrbuch Naturg. 3: 681.

Included in a work rejected for nomenclatural purposes by Int. Commn zool. Nom. 1956, Opin. Decl. int. Commn zool. Nom. 14 Opinion 41 7: 3.

Originally included species: Phalaena oo Linnaeus, I758; Phalaena delphinii Linnaeus, I 758; Phalaena retusa Linnaeus, I76I (see below).

PLASTENIS Boisduval, i 8 fo, Genera Index meth. Eur. Lepid.: 93.

Type-species: Noctua subtusa [Denis \& Schiffermüller], I775, by subsequent designation by Hampson, 1910, Cat. Lepid. Phalaenae Br. MIus. 9: I +7.

Plastenis is a junior objective synonym of I pimorpha Hübner, [I 82I].

ZENOBIA Agassiz, 1846, Nomencl. zool. Nomina syst. Generum Lepid.: 69.

Type-species: Phalaena retusa Linnaeus, I 76I, Fauna Suecica (Edn 2) : 32 I, by subsequent designation by Prout, 1901, Entomologist's Rec. J. I"ar. 13: I 8 , but cited for $\ddagger Z e n o b i a$ Oken. Type(s), Sweden.

As Agassiz did not include any species, but referred Zenobia to Oken, i8 5 (see above), then the type-species must be selected from one of the three species originally included by Oken.

A junior homonym of Zenobia Gray, I82 I, Lond. Medical Repository 15: 239, - Mollusca. There is no objective replacement name but $P$. retusa is congeneric with Noctua subtusa [Denis \& Schiffernuiller], I775, the type-species of Ipimorpha Hübner, [1821]. The latter is therefore available for use as a subjective replacement name.

$\ddagger I P H I M O R P H A$ Möschler, I886, Abh. senckenb. naturforsch. Ges. $14(3): 47$.

An incorrect subsequent spelling.

IPIRISTIS Draudt, I950, Mitt. mïnch. ent. Ges. 40: 124.

ACRO

Type-species: Ipiristis boursini Draudt, I950, ibidem 40 : I 25 pl. 8, fig. I 2, by monotypy. Syntypes 20 . CHINA: [Yunnan Prov.], Li-kiang (MAK, Bonn).

IPNEA Walker, [1859] I 858, List Specimens lepid. Insects Colln Br. Mus. 16: I90.

OPHI Type-species: Ipnea erebusalis Walker, [1859] I 858, ibidem 16: I90, by monotypy. Holotype , [Brazil]: Parà (UM, Oxford).

IPNISTA Schaus, 19i6, Proc. U.S. natn. Mus. 50: 264, 372.

OPHI

Type-species: Ipnea marina Druce, I89 I, Biologia cent.-am. (Zool.) Lepid. Heterocera 1: 482 , pl. 40 , fig. Io, by original designation. Syntypes $\hat{0}, ㅇ$, Guatemala: Quiche Mts (BMNH); Panajachel (BMNH); Volcan de Atitlan (BMNH). Ecuador: Loja.

TEINANOMIS Hampson, 1926, Descr. new Genera Species Lepid. Phalaenae Subfamily

Noctuinae Br. Mus.: 34I.

Type-species: Ipnea marina Druce, I $89 \mathrm{I}$, by original designation.

Teinanomis is a junior objective synonym of Ipnista Schaus, IgI6.

ISADELPHINA Hampson, 1926, Descr. new Genera Species Lepid. Phalaenae Subfamily Noctuinae Br. Mus.: 338. $\mathrm{OPHI}$

Type-species: Cosmophila retracta Hampson, igio, Proc. zool. Soc. Lond. 1910: 432, pl. 37 , fig. 20, by original designation. Syntypes $3 \hat{\sigma}$. [ZAIRE] Congo: Katanga, Kambove (BMNH).

IS ANA Walker, [1859] I858, List Specimens lepid. Insects Colln Br. Mus. 16: 21 3. HyPE Type-species: Isana larusalis Walker, [1859] I 858, ibidem 16: 2 I 3, by monotypy. Jectotype ơ, Borneo: Sarawak (UM, Oxford), designated (as type) by Swinhoe, I90o, Cat. east. and Aust. Lepid. Heterocera 2: $20 \mathrm{I}$.

ISATOOLNA nom. n. for Lasionota IVarren, I9I2.

OPHI

Type-species: Acantholipes hypenoides Moore, 188 I, Proc. zool. Soc. Lond. 1881: 372, by original designation (for Lasionota Warren). Syntypes o, q. [INDIA]: [W. Bengal], Darjiling. 


\section{LASIONOTA Warren, I912, Novit. zool. 19: 55.}

Type-species: Acantholipes hypenoides Moore, r88I, by original designation.

A junior homonym of Lasionota Mannerheim, r837, Bull. Soc. imp. Nat. Moscou 1837 (8): I02,-Coleoptera. An earlier usage of the name, probably for a different concept i.e. $\ddagger$ Lasionota Dejean, I833, Cat. Coléoptères Colln M. le Comte Dejean (Edn 2): 83, was undescribed and included a single undescribed species. The objective replacement name is Isatoolna nom. $\mathrm{n}$.

ISCADIA Walker, 1857, List Specimens lepid. Insects Colln Br. Mus. 11: 724.

SARR

Type-species: Iscadia aperta Walker, 1857, ibidem 11: 725, by monotypy. Holotype ${ }^{7}$. [Dominican Republic] St. Domingo (BMNH).

See also Encalypta Möschler, I890.

†SCHYIA, misspelling. See Ischyja Hübner, [I823].

OPHI

ISCHYJA Hübner, [I823] I 8I6, Verz. bekannter Schmett.: 265.

OPHI

Type-species: Phalaena manlia Cramer, I776, Uitlandsche Kapellen 1: I44, p1. 92, fig. A, by subsequent designation by Hampson, I 894, Fauna Br. India (Moths) 2: 537. Type(s), [INDiA]: Co:omandel Coast (BMNH).

$P$. manlia is dated from the wrapper of the part and not from the title-page of the volume, I 779 .

$\ddagger I S C H Y I A$; Agassiz, [I 846], Nomencl. zool. Nomina Syst. Generum Lepid.: 38.

An incorrect subsequent spelling.

POTAMOPHORA Guenée, I 852, in Boisduval \& Guenée, Hist. nat. Insectes (Lépid.) 7 : 122.

Type-species: Phalaena manlia Cramer, 1776, by monotypy.

Potamophora is a junior objective synonym of Ischyja Hübner, [1823].

ISOCHLORA Staudinger, I882, Stettin. ent. Ztg 43: 39.

NOCT

Type-species: Isochlora viridis Staudinger, I882, ibidem 43: 39, by monotypy. Syntypes $0^{\wedge}$, ㅇ, China: [approx. $46^{\circ}$ N. $86^{\circ}$ E.], Saisan (MNHU, Berlin).

ISOGONA Guenée, I852, in Boisduval \& Guenée, Hist. nat. Insectes (Lépid.) 7: $322 . \quad$ opHr Type-species: Isogona natatrix Guenée, I852, ibidem 7:323, by subsequent designation by Grote, I874, Bull. Buffalo Soc. nat. Sci. 2: 44. Holotype ô, North America (BMNH). See also Pincia Walker, I869.

ISOLASIA Warren, 1912, Novit. zool. 19: I3

CuCU Type-species: Isolasia biramata Warren, I912, ibidem 19: $\mathrm{I}_{3}$, by original designation. Syntypes $3 \hat{0}$, I ․ Sikisim.

ISOPOLIA Warren, I9I3, in Seitz, Gross-Schmett. Erde 11: IIo.

CUCU

Type-species: Pachypolia himalayensis Hampson, r9o6, Cat. Lepid. Phalaenae Br.

Mus. 6: 3I 2, fig. Iо I, by original designation. Holotype $\tilde{\delta}$. [SikкIm] Sikhim (BMNH).

$P$. himalayensis is a junior subjective synonym of Apamea strigidisca Moore, r88r, Proc. zool. Soc. Lond. 1881: 346, pl. 38, fig. 9 .

The date of publication of the part of Seitz containing page I Io, was stated on page Io5 as I9I3 January 2oth in both the German and the English editions.

ISOURA Hampson, I894, Fauna Br. India (Moths) 2: xx, 506.

OPHI

Type-species: Triphaena fuscicollis Butler, I889, Illust. typical Specimens Lepid. Heterocera Colln Br. Mus. 7: 10, 56, pl. I27, fig. 4, by original designation. Syntypes I ô, I 中, [INDIA]: [Punjab], Dharmsala (BMNH).

ISTARBA Walker, [1866] I865, List Specimens lepid. Insects Colln Br. Mus. 34: I I93. OPHI Type-species: Istarba varialis Walker, [I866] I865, ibidem 34: II94, by monotypy. Holotype 우, [Australia]: [Qd], Moreton Bay (BMNH).

I. varialis is a junior subjective synonym of Cidaria scitisignata Walker, 1862, ibidem 25: I 423 .

See also Sandava Walker, [1863]. 
ITEOPHAGA Boursin, I965 February, Ent. Ber., Berl. 1964 (3): 133.

CUCU

Type-species: Noctua viminalis Fabricius, [1777], Genera Insect.: 284, by original designation. Type(s), Germany (Type(s) not found by Zimsen, I964, Type Material of I. C. Fabricius: 532).

Iteophaga was proposed by Boursin as 'nom. nov. pro Cleoceris Dup.' [1837]. Later the same year Boursin, 1965 June, Bull. mens. Soc.linn. Lyon 34: 183, referred to Iteophaga as 'nom. nov. pro Cleoceris B. (1840).' Both Cleoceris as used by Duponchel, [1837] 1836, in Godart \& Duponchel, Hist. nat. Lépid. Papillons Fr. (Suppl.) 3: 157, and Cleoceris as used by Boisduval, 1840, Genera Index meth. Eur. Lepid.: 93, were, at that time, normal usages of Cleoceris Boisduval, [1836], even though the species included are now generically regrouped. Boursin implied that Cleoceris as used by Duponchel was a junior homonym for which he was proposing an objective replacement name. Although his intention was clear, Boursin, instead of proposing a replacement name for Cleoceris, should have described a new genus for viminalis. Whereas under the Code, Article r3(a), 'a statement that purports to give characters differentiating the taxon; or . . . a definite bibliographic reference to such a statement' is not required for a replacement name, such a statement is necessary for a new genus published after 1930. No generic description or indication accompanied the proposal of Iteophaga Boursin, 1965 February, or its later usage in June.

The problem of whether or not to accept Iteophaga Boursin as nomenclaturally available is somewhat academic as $N$. viminalis is congeneric with Cleoceris populi Strecker, the type-species of Brachylomia Hampson. Iteophaga Boursin, 1965, is therefore a junior subjective synonym of Brachylomia Hampson, I906.

$B O M B Y C I A$ Hübner sensu Stephens, I829, Illust. Br. Ent. (Haustellata) 3: 57.

Stephens attributed this genus to Hübner and was not proposing a new genus even though he used it for a concept now considered different from that of Hübner. Bombycia Hübner, I 822, had no generic description and because of this some authors have accepted Stephens' usage based on Stephens' generic description and the only species included by him, Noctua viminalis Fabricius.

ITMAHARELA nom. n. for Harmatelia Moore, I 882.

OPH I

Type-species: Harmatelia basalis Moore, 1882, in Hewitson \& Moore, Descr. new Indian lepid. Insects Colln late $M r W$. S. Atkinson: 183, pl. 6, fig. I3, by original designation (for Harmatelia Moore). Syntypes, India: [Assam], Cherra (BMNH); [W. Bengal], Darjiling.

HARMATELIA Moore, I882, in Hewitson \& Moore, Descr. new Indian lepid. Insects Colln late $\operatorname{Mr}$ W. S. Atkinson: 182.

Type-species: Harmatelia basalis Moore, 1882, by original designation.

A junior homonym of Harmatelia Walker, 1858, Ann. Mag. nat. Hist. (3)2: 281, Coleoptera. The objective replacement name is Itmaharela nom. $\mathrm{n}$.

ITOMIA Hübner, 1823, Zuträge Samml. exot. Schmett. 2: 22.

OPH I

Type-species: Itomia lignaris Hübner, 1823 , ibidem 2: 22, figs 317,318 , by monotypy. Syntypes , Surinam.

Neave, 1939, Nomencl. zool. 2: 801 cited Itomia Hübner, I823, as an error for Itonia Hübner, [1823]. Hemming, 1937, Hübner 2: 214 stated 'The name Itomia has priority over Itonia, which is, however, the spelling intended by Hübner.'

†ITON1A; Hübner, [1823] 1816, Verz. bekannter Schmett.: 271.

An incorrect subsequent spelling. Hübner cited without description 'Itonia [sic] lignaris Hübn. Zutr. 317, 318' as the sole species.

$\ddagger$ ITONIA, misspelling. See Itomia Hübner, I 823 .

OPH I

${ }_{\ddagger}^{\dagger}$ JALISSA, misspelling. See Zalissa Walker, 1865.

AGAR

$\ddagger J A M B I A$, misspelling. See Iambia Walker 1863 .

ACRO 
$\ddagger$ JAMBIODES, misspelling. See Iambiodes Hampson, I908.

ACRO

JANSEODES Viette, I967, Faune Madagascar 20: 743.

ACRO

Type-species: Euperia melanospila Guenée, I852, in Boisduval \& Guenée, Hist. nat. Insectes (Lépid.) 6: 4, pl. 8, fig. 4, by original designation. Lectotype 9 , Brazil (BMNH), designated by Viette, 1967 , ibidem $20: 745$.

JANTHINEA Guenée, 1852, in Boisduval \& Guenée, Hist. nat. Insectes (Lépid.) 6: I 88. ACRo

Type-species: Heliothis friwaldszkii Duponchel, r835, Annls Soc. ent. Fr. 4: 633, pl. $\mathrm{r}$, figs $\mathrm{B}_{2}, \mathrm{~B}_{3}$, by monotypy, but included as $\ddagger$ friwaldjzkyi, an incorrect subsequent spelling. Type(s) $\hat{o}$, [Bulgaria]: Turquie, Balkans Mts.

$H$. friwaldszkii was also proposed as a new species but spelled as frivaldszkyi by Treitschke in 1838 ; and unjustifiably emended to frivaldszci by Hampson, I910.

Janthinea should possibly be placed in the Heliothinae.

JARASANA Moore, I882, in Hewitson \& Moore, Descr. new Indian lepid. Insects Colln late Mr W. S. Atkinson: I32. OPHI

Type-species: Jarasana lativitta Moore, I882, ibidem: I32, by monotypy. Type(s) ㅇ, India: [Varanasi] Benares.

$\ddagger$ JASPIDEA, misspelling. See Staurophova R.L., I8I7.

CUCU

$+J \boldsymbol{H}$ SPIDIA Hübner, [1806]. See Jaspidia Hübner, I822. ACro

$\ddagger$ JASPIDIA Hübner, I808. See Hyperstrotia Hampson, I910. ACON

†JASPIDIA Ochsenheimer, I8I6. See Jaspidia Hübner, I822. ACRO

$\ddagger$ † J SPIDIA Hübner, I8I8. See Hyperstrotia Hampson, I9ro. ACON

JASPIDIA Hübner, I 822, Syst.-alphab. Verz.: 23, $36 . \quad$ ACro

Type-species: Noctua spoliatricula [Denis \& Schiffermüller], I775, Ankündung syst.

Werkes Schmett. Wienergegend: 89 , by subsequent designation by Grote, I874, Bull. Buffalo Soc. nat. Sci. 2: 8. Type(s) [Austria]: Vienna district (Collection destroyed, Horn \& Kahle, 1936, Ent. Beih. Berl.-Dahlem 3: 243).

$N$. spoliatricula is a junior subjective synonym of Noctua algae Fabricius, 1775, Syst. Ent.: 614.

See also Euthales Hübner, [1820].

$\ddagger J A S P I D I A$ Hübner, [1806], Tentamen determinationis digestionis . . . [1] .

Included in a work rejected for nomenclatural purposes by Int. Commn zool. Nom., 1926, Smithson. misc. Collns 73 (4) Opinion 97: 19. Also idem, 1954, Opin. Decl. int. Commn zool. Nom. 6 Opinion 278: I40.

Only included species: Noctua spoliatricula [Denis \& Schiffermüller], I775.

$\ddagger$ JASPIDIA Ochsenheimer, 1816, Schmett. Eur. 4: 63 .

Published as a junior synonym of Poecilia Schrank, I8o2.

Ochsenheimer was placing in synonymy $\ddagger$ Jaspidia Hübner, [1806], although the year and reference was not given.

JASPIDIA Boisduval, I840. See Staurophora R.L., I8I7.

CUCU

JAXARTIA Püngeler, I9I4, Dt. ent. Z. Iris 28: 43.

CUCU

Type-species: Jaxartia elinguis Püngeler, I9I4, ibidem 28: 44, pl. 2, figs 6, 7, by monotypy. Syntypes 4 ô, 6 , [U.S.S.R.]: Syr-Daria, Baigacum (MNHU, Berlin).

JOCHEAERA Hübner, [1820] I816, Verz. bekannter Schmett.: 201.

ACRO

Type-species: Phalaena alni Linnaeus, I 767, Syst. Nat. (Edn I2) 1: 845, by monotypy. Type(s), TYPE-LOCALITY not stated [EUROPE]. 
JOCHROA Felder, I874, in Felder \& Rogenhofer, Reise öst. Fregatte Novara (Zool.) 2 (Abt. 2): pl. 99; Erklärung: 5 . OPHI

Type-species: Jochroa chlorogastra Felder, I874, ibidem 2 (Abt. 2): pl. 99, fig. I7; Erklärung: 5, by monotypy. Type(s) ô, CHILE (BMNH).

$\ddagger$ JOCILLA, misspelling. See Euclystis Hübner, I 823 .

OPHI

JODIA Hübner, I8I8, Zuträge Samml. exot. Schmett. 1: I 5.

CUCU

Type-species: Noctua croceago [Denis \& Schiffermüller], I775, Ankündung syst. Werkes Schmett. Wienergegend: 86, by subsequent designation by Grote, i 874, Bull. Buffalo Soc. nat. Sci. 2: i 25. Type(s), [Austria]: Vienna district (Collection destroyed, Horn \& Kahle, 1936, Ent. Beih. Berl.-Dahlem 3: 243).

XANTHIA Boisduval, I 828, Eur. Lepid. Index meth.: 84.

Type-species: Noctua croceago [Denis \& Schiffermüller], I 775, by subsequent designation by Duponchel, I829, in Godart \& Duponchel, Hist. nat. Lépid. Papillons Fr. 7(2) : 72.

A junior homonym of Xanthia Ochsenheimer, i816, Schmett. Eur. 4: 82,-Lepid., Noctuidae. The objective replacement name is Jodia Hübner, I8I8.

LAMPETIA Curtis, [I830] i 829, Guide Br. Insects: I 53.

Type-species: Noctra croceago [Denis \& Schiffermüller], I775, by monotypy, but cited as 'croceago F.', an incorrect authorship.

A junior homonym of Lampetia Meigen, I80o, Nour. Classification Mouches: 34,Diptera. The objective replacement name is Jodia Hübner, I818.

XANTHOLEUCA Stephens, i 83I, Illust. Br. Ent. (Haustellata) 3: 329, 333.

Type-species: Noctua croceago [Denis \& Schiffermüller], I775, by original designation.

Xantholcuca is a junior objective synonym of Jodia Hübner, I8I 8.

HOPORINA Blanchard, I8 40, in Brullé, Hist. nat. Anim. articulés 3: 5 I 3.

Type-species: Noctua croceago [Denis \& Schiffermüller], I775, by monotypy.

Hoporina is a junior objective synonym of Jodia Hübner, I8I 8.

$\ddagger$ HOSPORINA; Duponchel, I845, Cat. méth. Lépid. Eur.: I I I, Errata.

An incorrect subsequent spelling.

OPORINA Agassiz, i 846 , Nomencl. zool. Index univ1.: I 86, 262.

An unjustified emendation of Hoporina Blanchard, I840.

$\ddagger$ JONTHE, misspelling. See Iontha Doubleday, 1842 .

OPHI

$\ddagger$ JUGURTHIA Oberthür, igo9. See Montcia Orfila \& Rossi, I956. ACro

JUGURTHIA Culot, I912. See Moureia Orfila \& Rossi, I956. ACRO

JUNCARIA Walker, I858, List Specimens lepid. Insects Colln Br. Mus. 15: I547, I636. OPHI Type-species: Juncaria dorsivitta Walker, 1858 , ibidem 15: I637, by monotypy. Holotype $\hat{o}$, Venezuela (BMINH).

$J$. dorsivitta is a junior subjective synonym of Amblygonia atlantica Herrich-Schäffer, I 855 .

See also Amblygonia Herrich-Schäffer, I 855.

JUNCARIA Walker, I863. See Hyamia Walker, [1859].

OPHI

KAKOPODA Smith, I900, Jl N.Y. ent. Soc. 8: I 75.

OPHI

Type-species: Kakopoda cincta Smith, i900, ibidem 8: 176, by monotypy. Holotype o. [U.S.A.]: Florida, Biscayne Bay.

KALLITRICHIA Ottolengui, I 898, Can. Ent. 30: I07.

ACRO

Type-species: Kallitrichia pendula Ottolengui, i 898, ibidem 30: Io8, pl. 5, fig. 6, by subsequent designation by Hampson, 1910, Cat. Lepid. Phalaenae Br. Mus. 9: 224, but cited as coronides which on page 225 was placed as the senior synonym of pendula. Holotype ô, [U.S.A.]: Arizona. 
$K$. pendula is a junior subjective synonym of Plusia coronides Druce, I889, Biologia cent.-am. (Zool.) Lepid. Heterocera 1: 328, pl. 30, fig. Io.

TRICHOCALA Dyar, I902, Bull. U.S. natn. Mus. 52: 196.

Proposed, unnecessarily, as an objective replacement name for Kallitrichia Ottolengui, I 898, which Dyar considered to be a junior homonym of Callitriche Poli, I79I, Test. Sicil. 1, Introd.: 32,-Mollusca. Dyar attributed Trichocala to Ottolengui, an incorrect authorship under the Code, Article 50.

KALMINA Swinhoe, I891, Trans. ent. Soc. Lond. 1891: 480.

OPHI

Type-species: Kalmina ochracea Swinhoe, I89I, ibidem 1891: 48I, pl. 19, fig. 3, by monotypy. Holotype ô, [INDia]: [Assam], Khasia Hills (BMNH).

$K$. ochracea is a junior subjective synonym of Ophisma lutea Walker, [1863] I864, J. Proc. Linn. Soc. (Zool.) 7: 180.

KARANA Moore, I882, in Hewitson \& Moore, Descr. new Indian lepid. Insects Colln late Mr W. S. Atkinson: 106.

ACRO

Type-species: Karana decorata Moore, I882, ibidem: 107, by original designation. Syntypes, India: [W. Bengal], Darjiling (BMNH).

KENRICKODES Viette, I96I, Bull. Soc. ent. Fr. 66: 50.

ACRO

Type-species: Perigea rubidata Kenrick, I9I7, Trans. ent. Soc. Lond. 1917: 88, pl. I, by original designation. Type(s), Madagascar (BMNH).

KERALA Moore, i 88 I, Proc. zool. Soc. Lond. 1881: 329.

CHLO

Type-species: Kerala punctilineata Moore, I881, ibidem 1881: 330, by monotypy. Syntypes $\hat{\sigma},+$,, [INDIA]: [W. Bengal], Darjiling (MNHU, Berlin).

CERALA Hampson, I9I2, Cat. Lepid. Phalaenae Br. Mus. 11: xiv, 489.

An unjustified emendation of Kevala Moore, I88I.

KHADIRA Moore, I881, Trans. zool. Soc. Lond. 11: 69.

OPHI

Type-species: Ophideres aurantia Moore, 1877, Proc. zool. Soc. Lond. 1877: 607, by monotypy. Type(s), S. Andaman Is (BMNH).

KITANOLA Matsumura, I925, J. Coll. Agric. Hokkaido Imp. Univ. 15: i 6.

NOLI

Type-species: Kitanola sachalinensis Matsumura, I925, ibidem 15: II6, pl. to, fig. 7 , by original designation. Holotype + , [U.S.S.R.]: [Sakhalin I.], South-Saghalien, Ichinosawa (EIHU, Sapporo).

KNAPPIA nom. n. for Lena Herz, I903.

ACON

Type-species: Lena poppiusi Herz, I903, Öfvers. finska Vetensk.-Soc. Förh. 45 (I5): 9, fig., by monotypy (of Lena Herz). Holotype ô. [U.S.S.R.]: [Siberia], Shigansk.

This name is proposed in honour of Mrs Sheila D. M. Knapp in appreciation of the excellent typescript she produced from the manuscript cards of this catalogue.

LENA Herz, 1903, Ofvers. finska Vetensk.-Soc. Förh. 45(I 5): 9.

Type-species: Lena poppiusi Herz, I903, by monotypy.

A junior homonym of Lena Casey, I886, Bull. Calif. Acad. Sci. 2: 21 I,-Coleoptera. The objective replacement name is Knappia nom. n.

KOHLERA Schaus, 1906, Proc. U.S. natn. Mus. 30: 99.

ACRO

Type-species: Kohlera nebula Schaus, I906, ibidem 30:99, by subsequent designation by Hampson, ı9ıо, Cat. Lepid. Phalaenae Br. Mus. 9: 96. Type(s), Brazil: Parana, Castro (USNM, Washington).

$\ddagger$ KOHLERIA; Schaus, I906, Proc. U.S. natn. Mus. 30: хоo.

An incorrect original spelling. The genus was proposed as Kohleva and the first of the two included species was described as Kohlera nebula. On the following page the second species was described as 'Kohleria bruma.'

$\ddagger$ KOHLERIA, misspelling. See Kohleva Schaus, 1906.

ACRO 
KOPTOPLAX Hampson, I891, Illust. typical Specimens Lepid. Heterocera Colln Br. Mus. 8: $33, \mathbf{1} 26$. OPHI

Type-species: Koptoplax lindsayi Hampson, I891, ibidem 8: 33, I27, pl. 154, fig. I7, by monotypy. Type(s) ô, INDIA: Nilgiri dist., S. slopes (BMNH).

KORAIA Herz, 1905, Annu. Mus. zool. Acad. imp. Sci. St. Pétersb. 9: $3^{1} 3$.

CATO

Type-species: Koraia pirata Herz, 1905, ibidem 9: 314, pl. I, fig. I8, by monotypy. Syntypes 2 , Korea (ZI, Leningrad).

Kovaia was originally proposed as $\ddagger$ Koraïa, an incorrect original spelling. Under the Code, Article 32 (c) (i), the diaeresis must be deleted.

KRUGIA Möschler, I890, Abh. senckenb. naturforsch. Ges. 16: 163.

OPHI

Type-species: Krugia operta Möschler, I890, ibidem 16: 164, by monotypy. Syntypes 2 ô. [Puerto Rico] Portorico I.

KUNUPIA Hampson, no published reference found.

HYPE

KURUSCHIA Boursin, 1940, Mitt. münch. ent. Ges. 30: 498.

HADE

Type-species: Kuruschia dianthoecioides Boursin, I940, ibidem 30:499, by original designation. Holotype ỗ, [U.S.S.R.]: Daghestan, Kurusch Dagh (LN, Karlsruhe).

KYNERIA Schaus, 1940, Scient. Surv. P. Rico 12: 27 I.

HYPE

Type-species: Kyneria utuadae Schaus, 1940, ibidem 12: 272, by original designation. Holotype ô, Puerto Rico: Utuado (USNM, Washington).

LABANDA Walker, 1859, List Specimens lepid. Insects Colln Br. Mus. 17: 284.

SARR Type-species: Labanda herbealis WValker, I 859 , ibidem 17: 284, by monotypy. Holotype §. Ceilon (BMNH).

LACANOBIA Billberg, I820, Enmmeratio Insect. Mus. G. J. Billberg: 87.

HADE

Type-species: Phalaena w-latinum Hufnagel, I 766, Berlin. Mag. 3 (3): 292 (but included by Billberg as 'w-latinum Esp.', an incorrect authorship), by subsequent designation by Tams, 1939, Entomologist 72: 139. Type(s), [Germany]: Berlin.

LACERA Guenée, 1852, in Boisduval \& Guenée, Hist. nat. Insectes (Lépid) 7: $336 . \quad$ ophi

Type-species: Phalaena alope Cramer, 1780, Uitlandsche Kapellen 3: 168, pl. 286, figs E, F, by subsequent designation by Hampson, I894, Fanna Br. India (Moths) 2: 491 . Syntypes $\overrightarrow{0}$, $q$. TYPE-LOCALITY stated to be Surinam, but this was probably an error as the species illustrated is not known to occur in the New World but is widespread in the Ethiopian and Oriental Regions.

LACIBISA Walker, [1863] 1864, J. Proc. Linn. Soc. (Zool.) 7: 82.

OPHI

Type-species: Lacibisa bifaria Walker, [1863] 1864, ibidem 7: 82, by monotypy. Lectotype $\hat{0}$. Borneo: Sarawak (UM, Oxford), designated (as type) by Sivinhoe, r9oo, Cat. east. and Aust. Lepid. Heterocera 2: 149.

LACINIPOLIA McDunnough, 1937, Can. Ent. 69: 43.

IIADE

Type-species: Mamestra illaudabilis Grote, 1875 , ibidem 7:27, by original designation. Syntypes ô. ‥ [U.S.A.]: California (BMNH); [CANADA]: Vancouver I.

The type-species name was originally proposed for a misidentification $M$. laudabilis Guenée sensu Grote, I874, ibidem 6: I57, but was described under the heading ' $M$. illaudibilis Grote.' Towards the bottom of the page $M$. illaudabilis was used. From this multiple original spelling illaudabilis has always been adopted.

LAGOPTERA Guenće, r 852, in Boisduval \& Guenće, IIist. nat. Insectes (Lépid.) 7: 223. Cato

Type-species: Ophideres elegans Hoeven, i 840, Tijdschr. Natumol. Gesch. Physiol. 7 : 280, pl. 6, fig. 6, by subsequent designation by Hampson, I894, Fauna Br. India (Moths) 2: 
505, but cited as 'juno Dalm.' which Hampson placed as the senior synonym of elegans. Types ô, ․, JAVA.

O. elegans is a junior subjective synonym of Noctua juno Dalman, I823, Analecta Ent.: 52.

LAGOPUS R.L., I8I7. See Callopistria Hübner, [182 I].

ACRO

$\ddagger \boldsymbol{L A} \boldsymbol{A} \boldsymbol{U} \boldsymbol{E} \boldsymbol{A}$, misspelling. See Laquea Jordan, I 896 .

AGAR

LAMBANA Walker, [1866] I865, List Specimens lepid. Insects Colln Br. Mus. 34: I I82. AcoN Type-species : Lambana cucullatalis Walker, [1866] I 865, ibidem 34: I I 83, by monotypy.

Holotype ơ. [BRAzIL]: [R. Amazon], [Tefé] Ega (BMNH).

See also Anablemma Schaus, IgI I.

LAMPADEPHORA Turner, 1932, Trans. R. Soc. S. Aust. 56: I82.

OPHI

Type-species: Lampadephora panimera Turner, I932, ibidem 56: I 83, by monotypy. Holotype ơ, Australia: N. Qd, Cape York (QM, Brisbane).

LAMPETIA Curtis, [1830]. See Jodia Hübner, I8I8.

CUCU

LAMPETIA Boie, I837. See Mesapamea Heinicke, I959.

ACRO

LAMPRA Hübner, [1821] I816, Verz. bekannter Schmett.: 221.

NOCT

Type-species: Phalaena fimbriata Schreber, I759, Novae Species Insect.: I3, fig. 9, but included by Hübner as fimbria Linnaeus (see below), by monotypy. Type(s), Germany.

$P$. fimbriata, although in current use, has in the past been well-known as $\ddagger$ fimbria Linnaeus, I767, Syst. Nat. (Edn I2) 1(2):842, an incorrect subsequent spelling.

LAMPROLOPHA Hampson, I9I4, Ann. Mag. nat. Hist. (8) 13: I72.

$\operatorname{ACON}$

Type-species: Lamprolopha melanephra Hampson, I9I4, ibidem (8) 13: I73, by

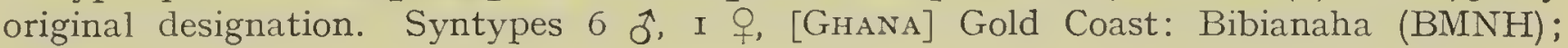
Kumasi.

LAMPROSIA Hübner, [I82I] I806, Samml. exot. Schmett. 2: pl. [2I4].

CATO

Type-species: Noctua amatrix Hübner, [I8I3], Samml. eur. Schmett. 4: pl. I03, fig. 487, by monotypy. Type(s), TYPE-LOCALITY by implication of title, Europe, but this was probably an error as the species illustrated is not known to occur in the Old World but is widespread in North America.

LAMPROSTICTA Hübner, [I820] I816, Verz. bekannter Schmett.: 207.

CUCU

Type-species: Noctua culta [Denis \& Schiffermuiller], I775, Ankïndung syst. Werkes Schmett. Wienergegend: 70, by monotypy. Type(s), [AusTrIA]: Vienna district (Collection destroyed, Horn \& Kahle, I936, Ent. Beih. Berl.-Dahlem 3: 243).

CHARIPTERA Guenée, I838, Anuls Soc. ent. Fr. 7: 124.

Type-species: Noctua culta [Denis \& Schiffermüller], I775, by subsequent designation by Guenée, 1852, in Boisduval \& Guenée, Hist. nat. Insectes (Lépid.) 6: 56.

Chariptera is a junior objective synonym of Lamprosticta Hübner, [1820].

LAMPROTES R.L., I 8 I, Jenaische Allg. Lit.-Ztg 1: 287.

PLUS

Type-species: Phalaena c-aureum Knoch, I781, Beitr. Insektengesch. 1: 7, pl. I, fig. 2 (but included by R.L., as Noctua concha Fabricius, 1787, Mantissa Insect. 2: I6I, an unnecessary replacement name), by PRESENT DESIGNATION. Type(s), TYPELOCALITY not stated. [GERMANY].

Lamprotes originally included two species, Noctua moneta Fabricius and P.c-aureum (as $N$. concha). Each is the type-species of a junior generic name i.e. Polychrysia Hübner, [I82I], and Chrysoptera Berthold, I827, respectively. P. c-aureum has been designated as the type-species of Lamprotes so as not to disturb the more widespread use, on both sides of the Atlantic, of Polychrysia.

See also Cubena Walker, 1856. 
CHRYSOPTERA Berthold, 1827, in Latreille, Naturliche Familien des Thierreichs: $4^{8} 3$.

Type-species: Phalaena c-aureum Knoch, I78I (but included by Berthold as Noctua concha Fabricius, 1787 , an unnecessary replacement name), by monotypy.

Chrysoptera is a junior objective synonym of Lamprotes R.L., I 817.

Chrysopteva originated from the vernacular name Chrysoptère used by Latreille, I825, Familles naturelles du Règne anim.: 476 .

LAMPROTHRIPA Hampson, 1912, Cat. Lepid. Phalaenae Br. Mus. 11 : xi, 288. SARR

Type-species: Ptisciana scotia Hampson, r902, J. Bombay nat. Hist. Soc. 14: 2 I , by original designation. Holotype $q$, [SIKKIM] Sikhim (BMNH).

LAMURA Walker, [1859] I 858, List Specimens lepid. Insects Colln Br. Mus. 16: I89. Hy Type-species: Lamura oberratalis Walker, [1859] I858, ibidem 16: 189, by monotypy. Holotype ô. CEYLON (BMNH).

L. oberratalis is a junior subjective synonym of Bocana manifestalis IValker, [1859] I 858, ibidem 16: 171 .

See also Bocana Walker, [1859] 1858, ibidem 16: I70; and Bithiasa Walker, I865.

LANGONA Barnes \& Lindsey, I92I. See Gloanna nom. n.

LANINIA Orfila \& Schajovskoi, 1957, Revta Soc. ent. avgent. 20:34.

Type-species: Laninia fletcheri Orfila \& Schajovskoi, 1957, ibidem 20:35, by original designation. Holotype $\hat{\sigma}$. Argentina: Neuquén, San Martin de los Andes.

LAPHYGMA Guenée, I 852, in Boisduval \& Guenée, Hist. nat. Insectes (Lépid.) 5: I 56. ACro Type-species: Noctua exigua Hübner, [1808], Samml. eur. Schmett. 4: pl. 78, fig. 362, by original designation. Type(s), Europe.

LAQUEA Jordan, 1896, in Rothschild \& Jordan, Novit. zool. 3: 4 I.

AGAR

Type-species: Euthisanotia argentata Druce, 1894, Ann. Mag. nat. Hist. (6) 13: 352, by original designation. Syntypes, Mexico: Orizaba (BMNH). Guatemala: Guatemala City.

E. argentata is a junior subjective synonym of Euscirrhopterus poeyi Grote, I 866 July.

See also Euscirrhopterus Grote, I 866 July; and Heterandra Herrich-Schäffer, I 866 [September].

$\ddagger L A G U E A$; Strand, I912, Lepid. Cat. 5: 74.

An incorrect subsequent spelling.

LARASSA Walker, [1866] I 865, List Specimens lepid. Insects Colln Br. Mus. 34: I 200. HYPE Type-species: Larassa condecoralis Walker, [1866] I 865, ibidem 34: I201, by monotypy. Holotype $\hat{\delta}$, [Australia]: [Qd], Moreton Bay (BMNH).

L. condecoralis is a junior subjective synonym of Madoce leucocosmalis Walker, 1863. See also Madoce Walker, I863.

LASCORIA Walker, I 859, List Specimens lepid. Insects Colln Br. Mus. 19: 838.

HYPE

Type-species: Lascoria phormisalis Walker, I859, ibidem 19: 839, by monotypy. Syntypes $\hat{0}$. [BraziL]: Rio Janeiro (UM, Oxford).

${ }_{+}^{+}$LASCORIDIA Hampson, no published reference found.

HYPE

LASIANOBIA Hampson, 1905, Cat. Lepid. Phalaenae Br. Mus. 5: xi, 274.

HADE

Type-species: Mamestra lauta Püngeler, 1900, Dt. ent. Z. Iris 13: I19, by original designation. Syntypes I ô, I 우, [CHina]: [Tsinghai], Kuku-Noor (MNHU, Berlin).

LASIESTRA Hampson, 1905, Cat. Lepid. Phalaenae Br. Mus. 5: vii, 47.

HADE

Type-species: Dianthoecia phoca Möschler, I864, Wien. ent. Monatschr. 8: 197, pl. 5. fig. I 5, by original designation. Holotype o, [CANADA]: Labrador.

$D$. phoca was described in the genus $\ddagger$ Diauthoecia, an incorrect subsequent spelling. The legend to fig. ${ }_{5}$ was spelled correctly. 
$\ddagger$ LASIOCRASPIS Hampson, no published reference found.

HYPE

LASIOLOPHA Turner, I920, Trans. R. Soc. S. Aust. 44: I85, I88.

CHLO

Type-species: Ariola saturata Walker, I 865, List Specimens lepid. Insects Colln Br. Mus.

33: 774, by monotypy. Type(s) 우, CEYLON.

A. saturata was originally proposed as $₫ A$ reola, an incorrect subsequent spelling.

LASIONOTA Warren, I9I2. See Isatoolna nom. n.

$\mathrm{OPH}$

LASIONOTELLA Warren, I9I6, Novit. zool. 23: 219.

CHLO

Type-species: Lasionotella exesa Warren, I9I6, ibidem 23: 219 , by original designation. Holotype ô. Borneo: [Sabah] North Borneo (BMNH).

LASIONYCTA Aurivillius, i 892, Ent. Tidskr. 13: 285.

HADE

Type-species: Phlogophora skraelingia Herrich-Schäffer, I852, Syst. Bearb. Schmett. Eur. 2: pl. I2I, fig. 624; 6 (Nachtrag): 57, by original designation. Holotype $\widehat{o}$, [Scandinavia]: Lappland.

$P$. skraelingia was figured and given only a specific name in 1852 , vol. 2 . In 1852 vol. 6, skraelingia was associated with its generic name and became nomenclaturally, available. The dates of publication are taken from original wrappers.

LASIOPODERES Bethune-Baker, I906, Novit. zool. 13: 263.

OPHI

Type-species: Lasiopoderes pratti Bethune-Baker, 1906, ibidem 13: 263, by original designation. Syntypes ô, New Gurnea: [Papua], Babooni (BMNH); Aora River.

LASIOSCELES Bethune-Baker, I906, Novit. zool. 13: 204.

ACRO

Type-species: Lasiosceles pratti Bethune-Baker, I906, ibidem 13: 205, by original designation. Syntypes $\delta$, ㅇ, New Guinea: [Papua], Ekeikei (BMNH); Kebea Range (BMNH).

†LASIOTHRIX Turner, no published reference found.

ACRO

LASIPLEXIA Hampson, I908, Cat. Lepid. Phalaenae Br. Mus. 7: xiii, 483.

ACRO

Type-species: Apamea cuprina Moore, I88 I, Proc. zool. Soc. Lond. 1881: 345, pl. 38, fig. 2, by original designation. Type(s), Sıккıм (MNHU, Berlin).

LASIRIDIA Draudt, I950, Mitt. münch. ent. Ges. 40: 39.

HADE

Type-species: Lasiridia iomelas Draudt, I950, ibidem 40: 39, pl. 2, fig. I9, by monotypy. Syntypes I 2 ðં. China: [Szechwan Prov.], Batang (MAK, Bonn).

LASPEYRESIA R.L., I8I7. See Laspeyria Germar, I8I I.

OPHI

LASPEYRESIA Herrich-Schäffer, I839. See Laspeyvia Germar, I8 I I.

OPHI

LASPEYRIA Germar, I8Io, Diss. sistens Bombycum Species: I 3.

OPHI

Type-species: Bombyx flexula [Denis \& Schiffermüller], I775, Ankündung syst.

Werkes Schmett. Wienergegend: 64, by monotypy. Type(s), [Austria]: Vienna district (Collection destroyed, Horn \& Kahle, 1936, Ent. Beih. Berl.-Dahlem 3: 243).

LASPEYRESIA R.L., I8I 7, Jenaische Allg. Lit.-Ztg 1: 288.

An unjustified emendation of Laspeyria Germar, I8I I.

COLPOSIA Hübner, [1823] I8I6, Verz. bekannter Schmett.: 287.

Type-species: Bombyx flexula [Denis \& Schiffermüller], I775, by monotypy, but included by Hübner as $C$. flexularia, an unjustified emendation.

Colposia is a junior objective synonym of Laspeyria Germar, I8I I.

A VENTIA Duponchel, I829, in Godart \& Duponchel, Hist. nat. Lépid. Papillons Fr. 7(2): I90.

Type-species: Bombyx flexula [Denis \& Schiffermüller], I775, by monotypy, but included by Duponchel as Geometra flexularia Hübner, an unjustified emendation.

Aventia is a junior objective synonym of Laspeyria Germar, I8II. 
LASPEYRESIA Herrich-Schäffer, I839, in Panzer, Dtl. Insecten Heft 165: I7 and end wrapper.

Type-species: Bombyx flexula [Denis \& Schiffermüller], 1775, by monotypy, but included by Herrich-Schäffer as flexulavia, an unjustified emendation.

Laspeyresia Herrich-Schäffer is a junior objective synonym of Laspeyria Germar, I8I I. EUTELES Gistl, I848, Naturg. Thierreichs höhere Schulen: ix.

Proposed, unnecessarily, as the objective replacement name for Laspeyresia HerrichSchäffer, I 839 .

Euteles Gistl is not preoccupied; $\ddagger$ Euteles Dejean, I835, Cat. Coléoptères Colln M. le Comte Dejean (Edn 2): 348 , and the only included species $\ddagger$ lurida Dejean are both nomina nuda.

LATEBRARIA Guenée, I 852, in Boisduval \& Guenée, Hist. nat. Insectes (Lépid.) 7: I 59. OPH I

Type-species: Latebraria amphipyroides Guenée, I 852, ibidem 7: I 59, pl. I7, fig. 2, by subsequent designation by Berio, 1966, Annali Mus. civ. Stor. nat. Giacomo Doria 76: 61. Syntypes, Brazil. Colombia.

LATHOSEA Grote, I881, Bull. U.S. geol. geogr. Surv. Tervit. 6: 270.

CUCU

Type-species: Lathosea pulla Grote, I88I, ibidem 6: 270, by monotypy. Holotype $\hat{o}$, [U.S.A.]: Oregon (BMNH).

LATHRAEOLIS Turner, I936, Proc. R. Soc. Qd 47: 38.

GEOMETRIDAE

Type-species: Lathraeolis spodochroa Turner, 1936, ibidem 47:38, by monotypy.

Lathraeolis has hitherto been placed in the Noctuidae, Sarrothripinae, but Dr I. F. B. Common, C.S.I.R.O., Canberra, has informed me that it should be transferred to the Geometridae, Ennominae.

LATIROSTRUM Hampson, I895, Fanna Br. India (Moths) 3: 33, 68.

OPHI

Type-species: Latirostrum bisacutum Hampson, I 895, ibidem 3: 69, fig. 36, by original designation. Syntypes ô. India: [Uttah Pradesh], Nussooree. [Sikkim] Sikhim $(\mathrm{BMNH})$.

LAUGASA Walker, I859, List Specimens lepid. Insects Colln Br. Mus. 19: 850.

Type-species: Laugasa perillalis Walker, I 859 , ibidem 19: $85^{\circ}$, by monotypy. Type(s) o., [BRAzIL]: Rio Janeiro.

LAZANDA Walker, I865, List Specimens lepid. Insects Colln Br. Mus. 32: 604.

SARR

Type-species: Lazanda fasciata Walker, I 865 , ibidem 32: 605, by monotypy. Holotype ㅇ. [INDIA]: Hindostan (BMNH).

LEBENA Walker, I866, List Specimens lepid. Insects Colln Br. Mus. 35: I901.

NOLI

Type-species: Lebena trinotata Walker, I866, ibidem 35: I902, by monotypy. Syntypes I $\hat{o}$, I $q$, NorTh Averica (BMNH).

L. trinotata is a junior subjective synonym of Brachytaenia triquetrana Fitch, I856, First \& second Rep. noxious, beneficial \& other Insects, State New-York: 244.

LECASIA Fawcett, I9I6, Proc. zool. Soc. Lond. 1916: 724.

OPH I

Type-species: Lecasia othello Fawcett, I9I6, ibidem 1916: 724, pl. I, fig. 4, by original designation. Syntypes I $\hat{\sigma}$, I $9,[$ Kenya]: Kedai (BMNH). [Soutr Africa]: Durban (BMNH).

LECERFIA Dumont, I920, Bull. Soc. cnt. Fr. 1920: I02.

NocT

Type-species: Lecerfia chitinipyga I) umont, I920, ibidem 1920 : I02, fig. I, by monotypy. Lectotype ô. AlGERIA: Grand Erg Occidental, W. of El-Goléa (MNHN, Paris), designated by Seymour, 1972, Bull. Br. Mus. nat. Hist. (Ent.) $27: 93$.

L. chitinipyga is a junior subjective synonym of Timora albida Hampson, i905, Ann. Mag. nat. Hist. (7) 15: 450 . 
LEDAEA Druce, I89I, Biologia cent.-am. (Zool.) Lepid. Heterocera 1: 484 opH I Type-species: Legna semilineata Walker, I865, List Specimens lepid. Insects Colln Br. Mus. 33: IIO2, by monotypy (of Legna Walker, 1865). Holotype đ̃. [U.S.A.]: Georgia (BMNH).

L. semilineata is a junior subjective synonym of Hyamia perditalis Walker, [1859] I 858 , ibidem 16: 234 .

Ledaea was proposed as the objective replacement name for Legna Walker, I865.

LEGNA Walker, 1865, List Specimens lepid. Insects Colln Br. Mus. 33: I102.

Type-species: Legna semilineata Walker, 1865 , by monotypy.

A junior homonym of Legna Walker, I858, Ann. Mag. nat. Hist. (3) 2: 281, - Coleoptera. The objective replacement name is Ledaea Druce, I $89 \mathrm{I}$.

LEDERERIA Grote, I874. See Calamia Hübner, [182I]. ACRO

LEGNA Walker, I865. See Ledaea Druce, I89I. OPHi

LEIANOPHERA Möschler, I 890, Abh. senckenb. naturforsch. Ges. 16: I 36 . SARR

Type-species: Leianophera transfossa Möschler, I890, ibidem 16: I36, fig. I6, by monotypy. Syntypes $4 \hat{\jmath}, 2$ + , PUerto Rico.

LEIDA Walker, I865, List Specimens lepid. Insects Colln Br. Mus. 33: I035. OPH

Type-species: Leida pallida Walker, I865, ibidem 33: 1036, by monotypy. Holotype $\hat{0}$, Venezuela (BMNH).

LEIOMETOPON Staudinger, I888, Stettin. ent. Ztg 49:37. ACRO

Type-species: Leiometopon simyrides Staudinger, I888, ibidem 49: 37, by monotypy. Holotype ठే, [CHina]: [Sin Kiang], Kuldja (MNHU, Berlin).

LEIORHYNX Hampson, I902, Ann. S. Afr. Mus. 2: 436.

OPHI

Type-species: Leiorhynx argentifascia Hampson, I902, ibidem 2: 436, by monotypy. Holotype đ, [Botswana]: N'Gamiland (BMNH).

LEIOSELIA Schaus, I9I I, Ann. Mag. nat. Hist. (8) 8: 94.

HADE

Type-species: Leioselia egregia Schaus, I9 I , ibidem (8) 8:95, by original designation. Type(s) \%, Costa Rica: Sixola (USNM, Washington).

LEIOSOMA Felder \& Rogenhofer, I874. See Leonides Druce, I896.

AGAR

LEIOSTOLA Hampson, I926, Descr. new Geneva Species Lepid. Phalaenae Subfamily Noctuinae $B r$. Mus.: 262.

OPHI

Type-species: Poaphila mollis Butler, I 879, Illust. typical Specimens Lepid. Heteroceva Colln Br. Mus. 3: x, 28, pl. 47, fig. Io, by original designation. Type(s), [Japan]: Yokohama (BMNH).

LEISTERA Swinhoe, I909, Ann. Mag. nat. Hist. (8) 3: 96.

OPHI

Type-species: Catephia pulchristrigata Bethune-Baker, I906, Novit. zool. 13: 253, by original designation. Holotype $\widehat{\partial}$, New Guinea: [Papua], Kebea Range (BMNH).

LEMMERIA Barnes \& Benjamin, I926, Pan-Pacif. Ent. 3: $69 . \quad$ cucu

Type-species: Anchoscelis digitalis Grote, I882, Bull. U.S. geol. geogr. Surv. Territ. 6 : 584, by original designation (for Brachycosmia Hampson, 1906). Type(s), U.S.A.: Maine, Orono. A. digitalis was proposed as $\$$ Anchocelis, an incorrect spelling.

Lemmeria was proposed as the objective replacement name for Brachycosmia Hampson, I 906.

BRACH Y COSMIA Hampson, I906, Cat. Lepid. Phalaenae Br. Mus. 6: xiv, 496.

Type-species: Anchoscelis digitalis Grote, I882, by original designation.

A junior homonym of Brachycosmia Butler, I890, Trans. ent. Soc. Lond. 1890: 680, Lepid., Noctuidae. The objective replacement name is Lemmeria Barnes \& Benjamin. 1926. 
+LEMUR Hübner, [1806]. See Mormo Ochsenheimer, I8I6.

ACRO

${ }_{+}^{+}$LEMUR Hübner, I 8oS. See Zale Hübner, I8I8.

CATO

†LEMUR Hübner, I818. See Zale Hübner, I818.

CATO

LEMUR Hübner, I822. See Mormo Ochsenheimer, I816. ACRO

†LEMURES Ochsenheimer, I8I6. See Mormo Ochsenheimer, I8I6. ACRO

†LEMURIS, misspelling. See Mormo Ochsenheimer, I8I6. ACRO

LENA Herz, igo3. See Knappia nom. n. ACON

†LENOSTOLA Hampson, no published reference found. HYPE

LEOCYMA Guenée, I 852, in Boisduval \& Guenée, Hist. nat. Insectes (Lépid.) 6: 2 I $2 . \quad$ CHLo

Type-species: Leocyma appollinis Guence, I852, ibidem 6: 2I2, by subsequent designation by Hampson, I9I2, Cat. Lepid. Phalaenae Br. Mus. 11: 66I. Holotype ơ, TYPE-LOCALITY 'je la crois africaine' (BMNH). [SOUTHERN AFriCA].

Incorrect type-species designation: Bombyx tibialis Fabricius, I 775, a name not originally included in Leocyma, and not linked with one of the originally included names when cited by Hampson, I894, Fauna Br. India (Moths) 2: 288. The same comments apply when B. tibialis was again cited by Hampson, ig02, Ann. S. Afr. Mus. 2: 298.

See also Thiganusa Walker, I865.

LEONIDES Druce, I 896, Biologia cent.-am. (Zool.) Lepid. Heterocera 2: 328. AGAR

Type-species: Leiosoma serpentinum Felder, 1874 , in Felder \& Rogenhofer, Reise öst. Fregatte Novara (Zool.) 2 (Abt. 2): pl. 99, fig. 23; Erklärung: 6, by monotypy (of Leiosoma Felder \& Rogenhofer, I874). Type(s) ô. [Colombia]: Bogota.

L. serpentinum is a junior subjective synonym of Euthisanotia leucotelus Walker, I865. List Specimens lepid. Insects Colln Br. Mus. 32: 635.

Leonides was proposed as the objective replacement name for Leiosoma Felder \& Rogenhofer, I874.

LEIOSOMA Felder \& Rogenhofer, I 874, Reise öst. Fregatte Novara (Zool.) 2 (Abt. 2): pl. 99 fig. 23; Erklärung: 6.

Type-species: Leiosoma serpentinum Felder, 1874, by monotypy.

A junior homonym of Leiosoma Stephens, I 829, Vom. Br. Insects: I 4 , Coleoptera. The objective replacement name is Leonides Druce, I896.

LEONILOMA Hampson, I926, Descr. new Genera Species Lepid. Phalaenae Subfamily Noctuinae Br. Mus.: I 44 .

OPH I

Type-species: Leoniloma convergens Hampson, I926, ibidem: I44, by original designation. Holotype $\hat{o}$, SiERra Leone: Bathanu (BMNH).

LEPHANA Walker, I866, List Specimens lepid. Insects Colln Br. Mus. 35: I702.

OPH I

Type-species: Lephana tetraphorella Walker, 1866, ibidem 35: 1702, by monotypy. Type(s) ․, [BraziL): Amazon Region (BMNH).

LEPIDODELTA Victte, I967, Fanne Madagascar 20: 502.

ACRO

Type-species: Delta stolifera Saalmüller, I89I, Lepid. Madagascar: 26ł, fig. 101, by monotypy (of Delta Saalmüller, I89I). Holotype q, Madagascar: Nossi-Bé I. (SNG, Frankfurt).

Lepidodelta was proposed as the objective replacement name for Delta Saalmïller, I891. DELTA Saalmüller, I89 I, Lepid. Madagascar: 263.

Type-species: Delta stolifera Salmüller, I 891 , by monotypy.

A junior homonym of Delta Saussure, I 885 , Eludes liamille Vespides 3: I30, Iymenoptera. The objective replacement name is Lepidodelta Viette, I967.

Victte, I967, Faune Madagascar 20:503, stated that as Delta Saussure was proposed as a 
name for a Division, it was not strictly nomenclaturally available. Viette nevertheless proposed his replacement name because a case had been submitted to the Commission asking them to declare as available the doubtful names of Saussure. In fact the Case No. Z.N.(S) 1689 published in 1967, Bull. zool. Nom. 24: 27-33, did not include Delta Saussure. The author of the case J. Van der Vecht (Rijksuniversiteit, Leiden) in an earlier letter to D. S. Fletcher (BMNH) in 1965 had stated that as Delta Saussure had been proposed in a subgeneric sense and was undoubtedly nomenclaturally available, there would be no need to include it in an application to the Commission. The 1972 amendment to Article II(f) of the Code confirmed this view.

LEPIDODES Guenée, 1852, in Boisduval \& Guenée, Hist. nat. Insectes (Lépid.) 7: 27 . OPH

Type-species: Lepidodes limbulata Guenée, 1852 , ibidem $7: 28$, pl. 18, fig. 9, by monotypy. Syntypes $\mathfrak{0}$, 우, Colombia (BMNH).

LEPIDOMYS Guenée, I852, in Boisduval \& Guenée, Hist. nat. Insectes (Lépid.) 6: $20 \mathrm{r}$.

Type-species: Lepidomys irrenosa Guenée, I852, ibidem 6: 202, pl. Io, fig. I, by monotypy.

Lepidomys was originally placed in the Noctuidae.

LEPIDOPALPIA Hampson, 1926, Descr. new Genera Species Lepid. Phalaenae Subfamily' Noctuinae Br. Mus.: rog.

OPHI

Type-species: Arctia bicolor Mabille, I 879, Bull. Soc. philomath. Paris (7) 3: 1 37, by original designation. Lectotype $q$, Madagascar (BMNH), designated (as holotype) by Viette \& Fletcher, 1968, Bull. Br. Mus. nat. Hist. (Ent.) 21: 4 Io.

LEPIDOPYRGA Warren, I914, Novit. zool. 21: 405.

$\operatorname{ACON}$

Type-species: Stenoloba viridimicta Hampson, I910, Cat. Lepicl. Phalaenae Br. Mus. 10: 369 , pl. I 59, fig. $3 \mathrm{r}$, by original designation. Holotype $\hat{\sigma}$, [India]: Assam, Khasis (BMNH).

LEPIDOTRAMA Cockerell, x9o3, Ent. News 14: i82.

OPHI

Type-species: Trama arrosa Harvey, 1875, Bull. Buffalo Soc. nat. Sci. 3: 13, by monotypy (of Trama Harvey, 1875). Syntypes $\lesssim$, . ., [U.S.A.]: Texas (BMNH).

$T$. arrosa is a junior subjective synonym of Poaphila detrahens Walker, $185_{5}^{8}$, List Specimens lepid. Insects Colln Br. Mus. 15: r834.

Lepidotrama was proposed as the objective replacement name for Trama Harvey, i 8 ; 5 .

TRAMA Harvey, I875, Bull. Buffalo Soc. nat. Sci. 3: r 3 .

Type-species: Trama arrosa Harvey, 1875 , by monotypy.

A junior homonym of Trama Heyden, 1837, Abh. Senckenb. Mus. 2 (3) : 293,-Hemiptera. The objective replacement name is Lepidotrama Cockerell, I903.

LEPIPOLYS Guenée, I852, in Boisduval \& Guenée, Hist. nat. Insectes (Lépid.) 6: I73. cucu

Type-species: Lepipolys perscripta Guenée, I 852, ibidem 6: 174, pl. 7, fig. ro, by monotypy. Syntypes 2 J. [U.S.A.]: Florida (BMNH).

LEPITOREUMA Grote, I873, Bull. Buffalo Soc. nat. Sci. 1 : 8 o.

ACRO

Type-species: Acronycta ovata Grote, I 873, ibidem 1:80, pl. 2, fig. I4, by original designation. Type(s) $\hat{\sigma}$. [U.S.A.]: Pennsylvania.

Lepitoreuma was proposed as a subgenus of Acronicta Ochsenheimer, I8I6 (but cited by Grote as Acronycta, an unjustified emendation by Treitschke, 1825).

LEPT AMMA Gaede, I939, in Seitz, Gross-Schmett. Erde 15: 279.

OPHI

Type-species: Leptamma flavalis Gaede, I939, ibidem 15: 279 [no figure], by monotypy. Type(s) q. [CAmeroun] Kamerun (MNHU, Berlin).

The date of publication of the part of Seitz containing page 279 was stated on page 279 as 1939 June 2oth in both the German and the English editions. 
LEPTERIA Schaus, I913, Ann. Mag.nat. Hist. (8) 11: 44.

Type-species: Rhyncholita viridicosta Schaus, I9I2, ibidem (8) 9:202, by original designation. Type(s) ô, Costa Rica: Turrialba.

LEPTINA Guenée, I852. See Baileya Grote, I895.

SARR

LEPTOCTENISTA Warren, I891, Ann. Mag. nat. Hist. (6) 7: 436.

OPH I

Type-species: Leptoctenista dubia Warren, I891, ibidem (6) $7: 437$, by original designation. Holotype ô, [BRazIL]: Rio Janeiro (BMNH).

LEPTOLOGIA Prout, I901, Entomologist's Rec.J. Var. 13: i 83.

CUCU

Type-species: Phalaena lota Clerck, I 759, Icones Insect. variorum 1: pl. 8, fig. I, by original designation. Type(s), TyPe-Locality not stated, but probably Sweden.

LEPTOPHARA Billberg, I820. See Eudocima Billberg, I820.

OPHI

LEPTOSIA Guenée, I84I. See Metachrostis Hübner, [1820].

$\operatorname{ACON}$

LEPTOSTOLA Billberg, i820. See Colocasia Ochsenheimer, i816.

PANT

LEPTOTROGA Hampson, 1926, Descr. new Genera Species Lepid. Phalaenae Subfamily Noctuinae Br. Mus.: 596.

OPH I

Type-species: Acharya costalis Moore, i 883, Proc. zool. Soc. Lond. 1883: 28, pl. 6, fig. Io, by original designation. Type(s), ANDaman Is (BMNH).

LESMONE Hübner, I818, Zuträge Samml. exot. Schmett. 1: 28.

OPH I

Type-species: Lesmone pelidnalis Hübner, I8I8, ibidem 1: 28, figs I69, I70, by monotypy. Type(s) 9 , Surinam.

L. pelidnalis is a junior subjective synonym of Phalaena porcia Stoll, I790, in Cramer,

Uitlandsche Kapellen Aanhangsel: 162, pl. 36, fig. 9.

LETABA Dyar, I912, Proc. U.S. natn. Mus. 42: 7 I.

AGAR

Type-species: Letaba noa Dyar, I9I2, ibidem 42: 72, by original designation. Holotype 우, Mexico: Zacualpan (USNMI, IVashington).

LETIS Hübner, [182 I May] 18o6, Samml. exot. Schmett. 2: pl. [212].

OPHI

Type-species: Letis specularis Hübner, [1821], ibidem 2: pl. [212], figs 1, 2, by monotypy. Type(s), Type-Locality not stated. [BrazIL].

Letis was also used by Hübner, I821 December, Index exot. Lepid.: [6].

LEUCADIA Sodoffsky, i 837. See Lencania Ochsenheimer, i816.

HADE

LEUCANIA Ochsenheimer, i 816, Schmett. Eur. 4: 81.

HADE

Type-species: Phalaena comma Linnaeus, I76I, Fauna Suecica (Edn 2): 316, by subsequent designation by Samouelle, I819, Entomologist's useful Compendium: 25I. Type(s), Sweden (LS, London).

†HELIOPHILAE Ochsenheimer, i816, Schmett. Eur. 4: 81.

Published as a junior synonym of Lencania Ochsenheimer, I 816 , and not subsequently treated as an available name under the Code, Article i I (d).

LEUCADIA Sodoffsky, I837, Bull. Soc. imp. Nat. Moscou 1837 (6): 87.

An unjustified emendation of Lencania Ochsenheimer, I8 6.

DONACHLORA Sodoffsky, i837, Bull. Soc. imp. Nat. Moscou 1837 (6): 87.

Proposed, unnecessarily, as an objective replacement name for Leucania Oclisenheimer, I 8 I6, which Sodoffsky considered to be inappropriate.

DONACOCHLORA Agassiz, i 846, Nomencl. zool. Index univl.: i 29.

An unjustified emendation of Donachlora Sodoffsky, 1837.

PUDORINA Gistl, 1848, Naturg. Thierreichs: xi.

Proposed, unnecessarily, as an objective replacement name for Leucania Ochsenheimer, I 8 I 6 .

LEUCANIA Boisluval, I828. See Aletia Hiibner. [1 821 ].

HADE 
LEUCANIMORPHA Walker, i 870, Entomologist 5: I33.

HYPE

Type-species: Leucanimorpha disjuncta Walker, i870, ibidem 5: I33, by monotypy. Type(s) ㅇ, [Somalia]: Red Sea, Dahleek I.

LEUCANITIS Guenée, I 852, in Boisduval \& Guenée, Hist. nat. Insectes (Lépid.) 7: 58. Cato Type-species: Microphisa rada Boisduval, I848, Bull. Soc. ent. Fr. (2) 6: xxx, by monotypy. Type(s), [U.S.S.R.]: Caucasus foothills (BMNH).

$M$. rada has been cited by Hampson and some other authors as $\ddagger$ Euclidia roda HerrichSchäffer, (1 85 I) i 845 , an incorrect subsequent spelling.

LEUCATOMIS Dognin, I9I4, Hétérocères nouv. Am. Sud 8: 56.

HYPE

Type-species: Leucatomis incondita Dognin, I9 4 , ibidem 8: 56 , by original designation. Holotype ô. French Guiana: Saint-Laurent du Maroni.

LEUCOCHLAENA Hampson, igo6, Cat. Lepid. Phalaenae Br. Mus. 6: ix, I 32.

Type-species: Noctua hispida Hübner, [1832], Samml. eur. Schmett. 4: pl. 166, figs 784786 , by original designation. Syntypes $\hat{\sigma}, \hat{+}$, Europe.

The original description (i.e. figures) of $N$. hispida was published by Geyer after Hübner's death. Hemming, 1937, Hübner 1: 220 stated that according to a manuscript by Geyer, the type-locality of $N$. hispida was Süd-Frankreich.

LEUCOCNEMIS Hampson, I908, Cat. Lepid. Phalaenae Br. Mus.7: 5. Available, but without included species until Hampson, I909, ibidem 8: ix, 22 I. ACRO

Type-species: Oxycnemis perfundis Smith, 1894, Trans. Am. ent. Soc. 21: 87, pl. 6, fig. 6, by subsequent designation by Hampson, I909, ibidem 8: 22 I. Syntypes, [U.S.A.]: Texas, Nueces River and S. Texas.

LEUCOCOSMIA Butler, i886, Trans. ent. Soc. Lond. 1886: 394.

ACRO

Type-species: Leucocosmia ceres Butler, I886, ibidem 1886: 394, by monotypy. Type(s) ô. [Frji Is]: Viti Islands (BMNH).

L. ceres is a junior subjective synonym of Curgia nonagrica Walker, [1863] I 864, J. Proc. Linn. Soc. (Zool.) 7: 166.

CURGIA Walker, [1863] i864, J. Proc. Linn. Soc. (Zool.) 7 : i66.

Type-species: Curgia nonagrica Walker, [1863] I864, ibidem 7: I66, by monotypy. Lectotype $\hat{\jmath}$, Borneo: Sarawak (UM, Oxford), designated (as type) by Swinhoe, I9oo, Cat. east. and Aust. Lepid. Heterocera 2: 4I.

A junior homonym of Curgia Walker, I860, Trans. ent. Soc. Lond. (2) 5 (2): I79, Neuroptera. There is no objective replacement name but $C$. nonagrica is the senior subjective synonym of Leucocosmia ceres Butler, I886, the type-species of Leucocosmia Butler, I886. The latter is therefore available for use as a subjective replacement name.

LEUCOGONIA Hampson, I908, Cat. Lepid. Phalaenae Br. Mus. 7: 7. Available, but without included species until Hampson, I910, ibidem 9: xiv, 446.

ACRO

Type-species: Zalissa ekeikei Bethune-Baker, 1906, Novit. zool. 13: 2 I , by subsequent designation by Hampson, I910, ibidem 9: 446, but cited as L. eceicei, an unjustified emendation by Hampson, igio. Holotype $\widehat{\sigma}$, New Guinea: [Papua], Ekeikei (BMNH).

LEUCOGRAMMA Hampson, 1926, Descr. new Genera Species Lepid. Phalaenae Subfamily Noctuinae Br. Mus. : 38 I. OPHI

Type-species: Phiprosopus hypenoides Schaus, 1906, Proc. U.S. natn. Mus. 30: 108, by original designation. Holotype, Brazıl: São Paulo (USNM, Washington).

LEUCOMELAS Hampson, Igr3 [January 3oth], Cat. Lepid. Phalaenae Br. Mus. 12: 6. Nomenclaturally available, but without included species until Warren, I9I3 April 5th, in Seitz, Gross-Schmett. Evde 3: 342. CATO

Type-species: Agnomonia juvenilis Bremer, I861, Bull. Acad.imp. Sci. St.-Pétersb. 3: 495, by subsequent designation by Warren, I9I3 April 5th, ibidem 3: $34_{2}$. Syntypes, [U.S.S.R.]: Amur, Bureja Mts; and between the Ussuri mouth and the lake. 
Lencomelas was made available by Hampson, I9r3 [January zoth], in a Key to Genera, but it was not until the next volume (Hampson, I9I3 [November I9th], ibidem 13: 44) that Hampson gave the full generic description and included a single species (Agnomonia jurenilis Bremer), designated as type-species. In between the publication of Hampson's volume I 2 and I3, Warren used Leucomelas for the same species and designated it as typespecies.

The exact dates of publication of Hampson's catalogues are taken from Collins, 1962, Ann. Mag. nat. Hist. (13) 5: 126. The date of publication of the part of Seitz containing page 342 was stated on page $34^{\mathrm{I}}$ as being $19 \mathrm{I} 3$ April $5^{\text {th }}$ in both the German and the English editions.

$\ddagger$ LEUCONEPHRA Hampson, no published reference found.

HYPE

LEUCONYCTA Hampson, 1908, Cat. Lepid. Phalaenae Br. Mus. 7: 1o. Available, but without included species until Hampson, I909, ibidem 8: vii, 35 . ACRO

Type-species: Microcoelia diphteroides Guenée, I 852, in Boisduval \& Guenée, Hist. nat. Insectes (Lépicl.) 5: 34, pl. 3, fig. 7 , by subsequent designation by Hampson, 1909, ibidem 8: 35. Holotype ô. [U.S.A.]: New York State (BMNH).

LEUCOPHANERA Joannis, I9I I, Bull. Soc. ent. Fr. 1911: I I 7.

CHLO

Type-species: Leucophanera argyrozona Joannis, I9I I, ibidem 1911 : I I 7 , by monotypy. Holotype 9 , [Gurnea] Haute-Guinée: Oudoula (MNHN, Paris).

LEUCOPHANERA Hampson, I9I2, Cat. Lepid. Phalaenae Br. Muıs. 11: xiv, $4^{81 .}$

Type-species: Leucophanera argyrozona Joannis, by original designation.

A junior homonym of Leucophanera Joannis, IgII. This generic name probably originated from Hampson, but was used and unintentionally made nomenclaturally available by Joannis prior to its proposal and generic description by Hampson for the same concept.

LEUCOPHANERA Hampson, I912. See Leucophanera Joannis, I9II.

CHLO

LEUCOSEMIA Ménétriés, I857, Enumeratio Corporum Anim. Mus. imp. Acad. Sci. Petropolitanae (Lepid.) 2 (Suppl. to Cat. 1): 87. AGAR

Type-species: Phalaena albomaculata Stoll, $178 \mathrm{I}$, in Cramer, Uitlandsche Kapellen 4: Io6, pl. 345, fig. C, by monotypy. Type(s), Guinea: coast.

$P$. albomaculata is a junior subjective synonym of Sesia octomaculata Fabricius, I775, Syst. Ent.: 830 (type-locality, India, cited by Fabricius was probably incorrect).

See also Alypia Hübner, I818.

LEUCOSIGMA Druce, Igo8 April, Ann. Mag. nat. Hist. (8) 1: 302.

ACRO

Type-species: Leucosigma uncifera Druce, I908, ibidem (8) 1: 302, by monotypy. Type(s) ô. PERU: La Oroya, Carabaya (BMNH).

LEUCOSIGMA Hampson, I9o8 [December], Cat. Lepid. Phalaenae Br. Mus. 7: 4. Available, but without included species until Hampson, I9Io, ibidem 9: 127.

Type-species: Leucosigma uncifera Druce, 1908, by subsequent designation by Hampson, 1910, ibidem 9: 127 .

A junior homonym of Leucosigma Druce, I908 April. This generic name probably originated from Hampson, but was used and unintentionally made nomenclaturally available by Druce prior to its proposal and generic description by Hampson for the same concept.

LEUCOSIGMA Hampson, I908. See Lencosigma Druce, 1908.

ACRO

LEUCOTELA Hampson, 1926, Descr. new Genera Species Lepid. Phalaenae Subfamily Noctuinae Br. Mus.: 33I.

OPHI

Type-species: Marimatha nigripalpis Walker, [1866] 1865, List Specimens lepid. Insects Colln Br. Mus. 34: 1204, by original clesignation. Holotype ô. Honduras (BMNH). 
LEUCOTELIA Hampson, 1926, Descr. new Geneva Species Lepid. Phalaenae Subfamily Noctuinae Br. Mus.: 234.

OPH I

Type-species: Eutelia ochreoplagata Fienrick, r917, Trans. ent. Soc. Lond. 1917: 91, pl. 2, by original designation. Type(s), Madagascar (BMNH).

LEUCOTRACHEA Janse, 1937, Moths S. Afr. 3: 58.

ACRO

Type-species: Bryophila melanoleuca Hampson, 1902, Ann. S. Afr. Mus. 2: 290, by original designation, but cited as Trachea melanodonta Hampson (see below). Type(s) ${ }_{\text {, }}$ South AFrica: Natal, Durban (BMNH).

B. melanoleuca Hampson, 1902, has as an objective replacement name Trachea melanodonta Hampson, rgo8, Cat. Lepid. Phalaenae Br. Mus. 7: r22. The latter is the valid name as the type-species is a junior secondary homonym of Euplexia melanoleuca Hampson, 1902, ibidem 2: 284. Both species are currently placed in Leucotrachea.

LEUCOVIS Hampson, 1908, Cat. Lepid. Phalaenae Br. Mus. 7: 8. Available, but without included species until Hampson, r910, ibidem 9: xiii, 428.

AGAR

Type-species: Pristoceraea alba Rothschild, r897, Novit. zool. 4: r83, by subsequent monotypy. Syntypes $2 \hat{0}, \mathrm{r}$ ㅇ, [Malawi] Nyassaland: Fort Johnstone (BMNH).

PERIMAGIA Strand, r909, Dt. ent. Z., 1909: 536.

Type-species: Pristoceraea alba Rothschild, I 897 , by monotypy.

Perimagia is a junior objective synonym of Leucovis Hampson, rgo8.

LEUMICAMIA Viette, 1965, Faune Madagascar 20: 416.

ACRO

Type-species: Dianthoecia graminicolens Butler, I878, Ann. Mag. nat. Hist. (5) 2 : 295, by original designation. Lectotype + , MADAGASCAR: Fianarantsoa (BMNH), designated by Viette, 1965, ibidem 20:417.

$\ddagger$ LIBA TRIX Berthold, I827. See Scoliopteryx Germar, I8I I.

OPHI

LIBISOSA Walker, [1859] I858, List Specimens lepid. Insects Colln Br. Mus. 16: I87. HyPE

Type-species: Libisosa butesalis Walker, [1859] 1858, ibidem 16: 187, by monotypy. Lectotype ô, Borneo: Sarawak (UM, Oxford), designated as (type) by Swinhoe, rgoo, Cat. east. and Aust. Lepid. Heterocera 2: 198.

LIBUNCA Walker, I869, Characters undescr. Lepid. Heterocera: 35.

SARR

Type-species: Libunca argentea Walker, 1869, ibidem: 35, by monotypy. Type(s)

\%. [HONDURAS]: Limas (BMNH).

+ ${ }_{\ddagger} I B U N E A$; Hampson, rgr2, Cat. Lepid. Phalaenae Br. Mus. 11: 334.

An incorrect subsequent spelling.

$\ddagger$ LIBUNEA, misspelling. See Libunca Walker, I 869 .

SARR

LIBYANA Turati, 1924, Atti Soc. ital. Sci. nat. 63: 93.

CUCU

Type-species: Libyana marmarides Turati, r924, ibidem 63: 93, pl. 4, figs ro, Ir, by monotypy. Syntypes I $\hat{0}, \mathrm{r}$, $[$ [LiBya]: Bengasi.

L. marmarides is a junior subjective synonym of Phoebophilus lenis Staudinger, I892, Dt. ent. Z. Iris 4: 279.

See also Evemopola Warren, I9II.

LIB YPHAENIS Hampson, r918, Novit. zool. 25: 141.

ACRO

Type-species: Libyphaenis virescens Hampson, 1918 , ibidem 25: $14 \mathrm{r}$, by original designation. Holotype ô, Nigeria: S., Yaba (BMNH).

LIB Y STICA Hampson, 1926, Descr. new Genera Species Lepid. Phalaenae Subfamily Noctuinae Br. Mus.: 529.

OPHI

Type-species: Episparis simplex Holland, I894, Psyche, Camb. 7: I21, pl. 4, fig. II, by original designation. Syntypes $\hat{\jmath}, 0+$, WEST AFrICA. 
LICHA Walker, 1859, List Specimens lepid. Insects Colln Br. Mus. 19:836. ACRO Type-species: Licha undilinealis Walker, I859, ibidem 19:836, by monotypy. Type(s) ô. [BRAzIL]: Rio Janeiro (UM, Oxford).

See also Bononia Walker, I862; and Tendarba Walker, [I866].

LICHNOPTERA Herrich-Schäffer, I856, Samml. neuer oder wenig behannter aussereur. Schmett. 1: I 4, I6, 72,84.

PANT

Type-species: Lichnoptera gulo Herrich-Schäffer, I856, ibidem 1: 16, 84, pl. 93, fig. 529, by subsequent designation by Kirby, I892, Synonymic Cat. Lepid. Heterocera 1: 213. Type(s), Venezuela (from the wrapper of the plates).

$\ddagger$ LICOPHOTIA, misspelling. See Lycophotia Hübner, [I82 I].

Nocr

LIGIA Duponchel, I829. See Compsoptera Blanchard, I845.

GEOMETRIDAE

LIGIDIA Walker, I862, J. Proc. Linn. Soc. (Zool.) 6: II 7.

ACON

Type-species: Ligidia decisissima Walker, 1862, ibidem 6: II7, by monotypy.

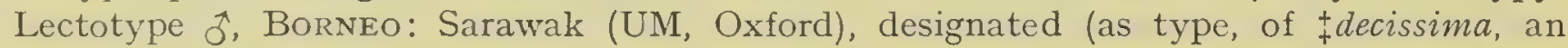
incorrect subsequent spelling) by Swinhoe, I90o, Cat. east. and Aust. Lepid. Heterocera 2: I04.

LIGNICIDA Swinhoe, 1905, Ann. Mag. nat. Hist. (7) 16: 625.

OPHI

Type-species: Lignicida echana Swinhoe, I905, ibidem (7) 16:625, by original designation. Holotype ô., [Philippine Is]: Palawan (BMNH).

LINEOPALPA Guenée, I 852, in Boisduval \& Guenée, Hist. nat. Insectes (Lépid.) 6: $290 . \quad$ OPH I Type-species: Lineopalpa horsfieldi Guenée, 1852, ibidem 6: 29I, pl. I4, fig. 2, by monotypy. Holotype , Java.

LIPATEPHIA Hampson, 1926, Descr. new Genera Species Lepid. Phalaenae Subfamily Noctuinae Br. Mus.: 79.

OPHI

Type-species: Anophia illegitima Wallengren, 1875, Öfvers. K. Vetensk Akad. Förh. Stockh. 1875 (I): Io8, by original designation. Holotype $\hat{\sigma}$, [South Africa]: Transvaal (UZI, Lund).

LIRA Billberg, I820. See Nola Leach, [1815].

NOLI

LISTA Walker, I859, List Specimens lepid. Insects Colln Br. Mus. 19: 877. PYRALIDAE Type-species: Lista genisusalis Walker, I859, ibidem 19: 877, by monotypy. Originally placed amongst Noctuidae.

$\ddagger$ LISTENIA, misspelling. See Listonia Möschler, I 886 .

ACRO

LISTONIA Möschler, I886, Abh. senckenb. naturforsch. Ges. 14(3): 37.

ACRO

Type-species: Listonia jamaicensis Möschler, I886, ibidem 14(3): 38 , fig. I3, by monotypy. Holotype ô. Jamaica (MNHU, Berlin).

Listonia was the spelling used once on page 37 for this new genus; the spelling $\ddagger$ Listenia was used in the legend of fig. I3. The first reviser was probably Hampson, I9o8, Cat. Lepid. Phalaenae Br. Mus. 7: 26o, who used the spelling Listonia when the genus was listed as a junior subjective synonym of Cropia Walker, [1858].

L. jamaicensis is a junior subjective synonym of Decelea infusa Walker, [I858] I857, List Specimens lepid. Insects Colln Br. Mus. 13: I I 6.

$\ddagger$ †ISTENIA; Möschler, I886, Abh. senckenb. naturforsch.Ges. 14(3): 37, fig. I3.

An incorrect original spelling.

LITA Harvey, I875. See Litocala Harvey, 1875.

OPIII

LITHACODIA Hübner, 1818, Zuträge Samml. exot. Schmett. 1: I8. ACON

Type-species: Lithacodia bellicula Hübner, I 8I 8, ibidem 1: I 8, figs 85, 86, by monotypy. Type(s) ô, [U.S.A.]: Georgia [formerly] in Florida. 
LITHILARIA Rosenstock, i 885, Ann. Mag. nat. Hist. (5) 16: 425.

HYPE

Type-species: Lithilaria ossicolor Rosenstock, I 885 , ibidem (5) 16:425, by monotypy.

Holotype $\sigma^{7}$. Australia: S. Aust. (BMNH).

See also Hyperaucha Meyrick, I897.

LITHOCAMPA Guenée, I852. See Calliergis Hübner, [I82I].

CUCU

LITHOLOMIA Grote, I875, Can. Ent. 7: 206.

CUCU

Type-species: Scopelosoma napaea Morrison, 1874, Proc. Boston Soc. nat. Hist. 17 : I52, by monotypy. Type(s), [U.S.A.]: Massachusetts.

$\ddagger$ LITHOMIA, misspelling. See Lithomoia Hübner, [I82I].

CUCU

LITHOMOEA Hampson, I909. See Lithomoia Hübner, [182I]. cucu

LITHOMOIA Hübner, [I82I] I8I6, Vevz. bekannter Schmett.: 244. cUCU

Type-species: Noctua solidaginis Hübner, [1803], Samml. eur. Schmett. 4: pl. 53,

fig. 256, by subsequent designation by Curtis, I838, Br. Ent. 15: 683. Type(s), Europe. †ITHOMI A; Curtis, I838, Br. Ent. 15:683.

An incorrect subsequent spelling.

LITHOMOEA Hampson, I909, Cat. Lepid. Phalaenae Br. Mus. 8: 208.

An unjustified emendation of Lithomoia Hübner, [I82I].

LITHOPHANE Hübner, [182 I] I8I6, Verz. bekannter Schmett.: 242.

CUCU

Type-species: Noctua petrificata [Denis \& Schiffermüller], I775, Ankündung syst. Werkes Schmett. Wienergegend: 75, by subsequent designation by Grote, i 874, Anmual Rep. Trustees Peabody Acad. Sci. 6: 3I. Type(s), [Austria]: Vienna district (Collection destroyed, Horn \& Kahle, 1936, Ent. Beih. Berl.-Dahlem 3: 243).

$N$. petrificata is a junior subjective synonym of Phalaena socia Hufnagel, I766, Berlin. Mag. 3 (4): 4r 8.

LITHOPHASIA Staudinger, I892, Dt. ent. Z. Iris. 4: 305.

CUCU

Type-species: Lithophasia venosula Staudinger, I892, ibidem 4:305, pl. 4, fig. 3, by monotypy. Syntypes ơ. ㅇ, [LEBANon]: Beirut (MNHU, Berlin).

LITHOPOMA Schaus, г9i6. See Mopothila nom. n.

OPHI

LITHOSIOPSIS Hampson, I895, Fauna Br. India (Moths) 3: 3 I, 34.

OPHI

Type-species: Lithosiopsis torsivena Hampson, I 895, ibidem 3: 34, fig. I5, by original designation. Type(s) $\widehat{\jmath}$, BHutan (BMNH).

LITOCALA Harvey, I875, Bull. Buffalo Soc. nat. Sci. 3: 3.

OPHI

Type-species: Lita sexsignata Harvey, I875, ibidem 2: 280, by monotypy (of Lita

Harvey, I875). Syntypes, [U.S.A.]: Nevada (BMNH).

Litocala was proposed as the objective replacement name for Lita Harvey, I875.

LITA Harvey, r875, Bull. Buffalo Soc. nat. Sci. 2: 280.

Type-species: Lita sexsignata Harvey, I875, by monotypy.

A junior homonym of Lita Treitschke, I833, Schmett. Eur. 9(2): 76, - Lepid., Tineidae. The objective replacement name is Litocala Harvey, I875.

LITOGNATHA Grote, I 873 July, Bull. Buffalo Soc. nat. Sci. 1: 85.

HYPE

Type-species: Litognatha nubilifascia Grote, 1873, ibidem 1: 85, p1. 2, figs 2, 3, by original designation. Syntypes, [U.S.A.]: Philadelphia; New York State, Buffalo and Olean and Albany. (I $\hat{\delta}, \mathrm{BMNH}$ no label data).

L. nubilifascia is a junior subjective synonym of Hormisa absorptalis Walker, [1859].

Litognatha was first used by Grote, I873 January, Trans. Am. ent. Soc. 4: 309 as a nomen nudum, and contained two species both nomina nuda.

See also Hormisa Walker, [1859]. 
LITOMITUS Grote, 1864, Proc. ent. Soc. Philad. 3: 84.

Type-species: Litomitus elongatus Grote, I864, ibidem 3: 85, pl. 2, fig. 6, by monotypy. Syntypes ô, ㅇ, [U.S.A.]: eastern and middle States.

L. elongatus is a junior subjective synonym of Celiptera frustulum Gưenée, 1852.

See also Celiptera Guenée, 1852.

LITOPROSOPUS Grote, I 869, Trans. Am. ent. Soc. 2: 309.

OPHI

Type-species: Noctua hatuey Poey, I832, Centurie Lépid. Ille Cuba (I): Num. [6], fig, by subsequent designation by Kirby, 187I, Zool. Rec. 7: 4 II, but cited as thatney, an incorrect subsequent spelling. Type(s) $\delta$, CuBA: S. E. of Matanzas, Lagunillas.

LITOSCELIS Turner, I944, Trans. R. Soc. S. Aust. 68: 8.

OPHI

Type-species: Euprora tanyphylla Turner, 1929, Trans. R. Soc. S. Aust. 53: 304, by monotypy. Syntypes 2 , Australia: N. Qd, near Cairns, Kuranda (ANIC, Canberra).

LITOSEA Grote, I875, Can. Ent. 7: 49.

CATO

Type-species: Litosea adversa Grote, 1875 , ibidem 7: 49, by monotypy. Syntypes, [U.S.A.]: California (BMNH.)

L. adversa is a junior subjective synonym of Anaitis togataria Walker, 1862, List Specimens lepid. Insects Colln Br. Mus. 25: I 445.

LIVIANA Walker, I865, List Specimens lepid. Insects Colln Br. Mus. 33: I034.

OPHI

Type-species: Liviana pallescens Walker, I865, ibidem 33: I035, by monotypy. Holotype $\hat{\sigma}$. [BRAzIL]: Para (BMNH).

LOBOCHEILOS Hampson, I891, Illust. typical Specimens Lepid. Heterocera Colln Br. Mus. $8: 24,98$. ACON

Type-species: Lobocheilos illattioides Hampson, I89I, ibidem 8: 24, 98, pl. I48, fig. Io, by monotypy. Type(s) $\overrightarrow{0}$. India: Nilgiri dist., plateau (BMNH).

L. illattioides is a junior subjective synonym of Apphadana plana Swinhoe, is9o, Trans. ent. Soc. Lond. 1890: $26 \mathrm{I}$.

Not preoccupied by $\ddagger$ Lobocheilos Bleeker, I853, Natuur. Tijdschr. Ned.-Indië 5: 520, an incorrect subsequent spelling of Lobocheilus Hasselt, I823, - Pisces.

LOBOCRASPIS Hampson, I 895, Trans. ent. Soc. Lond. 1895: 293.

CHLO

Type-species: Lobocraspis griseifusa Hampson, 1895, ibidem 1895: 293, fig. [9], by original designation. Syntypes $\hat{\sigma}, q$, Burma: Donaut Range, Tenasserim (BMNH); Yaw.

LOBOPHYLLODES Hampson, I913, Cat. Lepid. Phalaenae Br. Mus. 12: $400 . \quad$ cato

Type-species: Huebnerius miniatus Grünberg, 1907, Dt. ent. Z. 1907: 436, pl. 4, fig. 7 , by original designation. Holotype $q$, [CAmeroun] Kamerun: Nssanakang on the upper Mun Aya.

H. miniatus was proposed in the genus $\ddagger$ Hïbnerius, an incorrect original spelling.

LOBOPLUSIA Roepke, I94I, Zoöl. Meded., Leiden 23: 27.

PLUS

Type-species: Loboplusia vanderweelei Roepke, 194 I, ibidem 23: 27, pl. 2, fig. 8, by original designation. Holotype $\widehat{\jmath}$, JAVA: W., Tjinieruan.

LOBOTORNA Hampson, 1924, Ann. Mag. nat. Hist. (9) 13: 444.

Type-species: Lobotorna albapex Hampson, 1924, ibidem (9) 13: 445, by original designation. Holotype $\tilde{\sigma}$, Panama: Canu Mines (BMNH).

LOCHIA Walker, I865, List Specimens lepid. Insects Colln Br. Mus. 33: 748.

$\operatorname{ACON}$

Type-species: Lochia apicalis Walker, I865, ibidem 33: 748, by monotypy. Syntypes

3 P. Australia: [Qd], Moreton Bay (BMNH).

LOIS Dyar, 1924, Insecutor Inscit. menstr. 12: 16.

OPHI

Type-species: Lois monoflex Dyar, 1924, ibidem 12: 16, by monotypy. Holotype $\hat{\delta}$. Mexico: Colima (USNM, Washington). 
LOMANALTES Grote, I 873 April, Bull. Buffalo Soc. nat. Sci. 1: I 3.

HYPE

Type-species: Lomanaltes laetulus Grote, I873, ibidem 1: I4, p1. I, figs I2, I3, by monotypy. Syntypes, [U.S.A.]: Philadelphia (BMNH); New York, Albany (BMNH).

L. laetulus is a junior subjective synonym of Hypena eductalis Walker, [1859] I858, List Specimens lepid. Insects Colln Br. Mus. 16: 36.

Lomanaltes was first used by Grote, I 873 January, Trans. Am. ent. Soc. 4: 3 Io, but was a nomen nudum and contained one species, also a nomen nudum.

LOMILYSIS Franclemont, 1937, Can. Ent. 69: І 30.

CuCu

Type-species: Cleoceris discolor Smith, 1904, Psyche, Camb. 11: 55, by original designation. Holotype ㅇ, [U.S.A.]: New Mexico, Taos.

LONGICELLA Jordan, I896, in Rothschild \& Jordan, Novit. zool. 3: 5 I.

AGAR

Type-species: Eusemia mollis Walker, I856, List Specimens lepid. Insects Colln Br. Mus. 7: I774, by original designation. Syntypes I $\hat{\jmath}$, I $q$, EAST INDIES (BMINH). [West Malaysia]: [Malaya], Malacca (BMNH).

LONGIVESICA Hardwick, 1970, Mem. ent. Soc. Can. 67: 44.

NOCT

Type-species: Agrotis divergens Walker, [1857] I 856, List Specimens lepid. Insects Colln Br. Mus. 10:327, by original designation. Lectotype ô. [CANADA]: Nova Scotia (BMNH), designated by Hardwick, 1970, ibidem 67: 60 .

Longivesica was proposed as a subgenus of Euxoa Hübner, [182I].

LOPHARTHRUM Hampson, I895, Fauna Br. India (Moths) 3: xiii, I8.

OPHI

Type-species: Amphigonia comprimens Walker, 1858, List Specimens lepid. Insects Colln Br. Mus. 15: I540, by original designation. Holotype ơ. [Bangladesh]: [E. Bengal], Silhet (BMNH).

LOPHIA Sodoffsky, I837. See Cucullia Schrank, I802.

cucu

LOPHIOPHORA Bryk, I9I5, Arch. Naturgesch. 81 (A) $4: 7$.

OPHI

Type-species: Lophiophora fulminans Bryk, I9I 5, ibidem 81 (A) 4:8, pl. I, fig. 3, by monotypy. Holotype $\hat{\sigma}$. [CAMERoun]: Manenguba district (MNHU, Berlin).

See also Pyroblemma Hampson, 1926.

LOPHOCALAMA Hampson, 1908, Cat. Lepid. Phalaenae Br. Mus. 7: I7. Available, but without included species until Hampson, I910, ibidem 9: 266. ACRO

Type-species: Lophocalama neuritis Hampson, I9Io, ibidem 9: 266, fig. II , by subsequent monotypy. Holotype ô. [Australia]: New S. Wales, Sydney (BMNH).

LOPHOCERAMICA Dyar, I908, Proc. ent. Soc. Wash. 10: 32.

HADE

Type-species: Tricholita artega Barnes, I907, Can. Ent. 39: 64, by original designation. Syntypes of, ㅇ, [U.S.A.]: Ariz., Santa Catalina.

LOPHOCERYNEA Hampson, I9r8, Novit. zool. 25: i68.

$\operatorname{ACON}$

Type-species: Phanaspa punctata Bethune-Baker, 1906, ibidem 13: 216, by original designation. Holotype $\hat{\sigma}$, New Guines: [Papua], R. Aroa (BMNH).

LOPHOCOLEUS Butler, I886, Trans. ent. Soc. Lond. 1886: 4 I6.

HYPE

Type-species: Lophocoleus mirabilis Butler, I886, ibidem 1886: $4^{\mathrm{I} 6}$, by monotypy. Type(s) $\hat{\delta}$, [FIJI Is]: Viti Islands (BMNH).

Lophocoleus when originally proposed contained a second species, but this was doubtfully included and, under the Code, Article 68 (c), is not eligible for selection as type-species.

LOPHOCRAMA Hampson, I912, Cat. Lepid. Phalaenae Br. Mus. 11: xiv, 521.

CHLO

Type-species: Carea auritincta Hampson, 1905, Ann. Mag. nat. Hist. (7) 16: 597, by original designation. Holotype $\widehat{\sigma}$. Nigeria: Old Calabar (BMNH). 
LOPHOCRASPEDON Hampson, I893, Illust. typical Specimens Lepid. Heterocera Colln Br. Mus. 9:32, I 35 .

Type-species: Lophocraspedon cristatum Hampson, I 893, ibidem 9: 32, I35, pl. I68, fig. 4, by monotypy. Type(s), CEylon: Nawala-pittia (BMNH).

LOPHOCRYPTIS Hampson, I9I4, Ann. Mag. nat. Hist. (8) 13: I7 I.

ACON

Type-species: Lophocryptis argyrophora Hampson, I9I 4, ibidem (8) 13: I 72 , by original designation. Holotype , , [GHana] Gold Coast: Bibianaha (BMNH).

LOPHOCYTTARRA Hampson, I9I 4, Ann. Mag. nat. Hist. (8) 13: I97.

$\triangle \mathrm{CON}$

Type-species: Lophocyttarra phoenicoxantha Hampson, I9I4, ibidem (8) 13: I97, by original designation. Holotype $\hat{\sigma}$ [South AfricA]: Natal, Durban (BMNH).

LOPHODAXA Hampson, I926, Descr. new Genera Species Lepid. Phalaenae Subfamily Noctuinae Br. Mus.: 292.

OPHI

Type-species: Lophodaxa labandina Hampson, I926, ibidem: 292, by original designation. Holotype $\hat{\sigma},[$ GHANA $]$ Gold Coast: Kumasi (BMNH).

LOPHODELTA Hampson, I924, Ann. Mag. nat. Hist. (9) 13: 448.

HYPE

Type-species: Lophodelta argyrolepia Hampson, I924, ibidem (9) 13:449, by original designation. Holotype $\tilde{o}$, PERU: Chaquimayo (BMNH).

LOPHODITTA Möschler, I89o, Abh. senckenb. naturforsch. Ges. 16: 230.

HY PE

Type-species: Lophoditta perspicillaris Möschler, I S9o, ibidem 16: 23 I, by subsequent designation by Schaus, i9i6, Proc. U.S. natn. Mus. 50: 28I. Syntypes 2 J., [PUERTO Rrco] Portorico I. (MNHU, Berlin).

L. perspicillaris is a junior subjective synonym of Physula tuberculata Herrich-Schäffer, I870, Corresp.-Bl.zool.-min. Ver. Regensburg 24: Io6.

LOPHOGRAPTA Warren, I9 3 , in Seitz, Gross-Schmett. Erde 3: 4 I .

$\mathrm{ACON}$

Type-species: Egnasia pulcherrima Butler, I879, Illust. typical Specimens Lepid. Heterocera Colln Br. Mus. 3: xv, 67, pl. 57, fig. 8, by original designation. Type(s), JAPAN: Yokohania (BMNH).

The date of publication of the part of Seitz containing page 4 I I, was stated on page 405 as 1913 December Ist in both the German and the English editions.

LOPHOMILIA Warren, I9I3, in Seitz, Gross-Schmett. Erde 3: 4I I.

ACON

Type-species: Egnasia polybapta Butler, I879, Illust. typical Specimens Lepid. Heterocera Colln Br. Mus. 3: xv, 66, pl. 57, fig. 7, by original designation. Type(s), JAPAN: Yokohama (BMNH).

The date of publication of the part of Seitz containing page 4 I I, was stated on page 405 as I9I3 December Ist in both the German and the English editions.

LOPHOMYRA Schaus, I9I I, Ann. Mag. nat. Hist. (8) 8: 99.

ACRO

Type-species: Lophomyra tacita Schaus, I9I I, ibidem (8) 8: 99, by monotypy. Syntypes, Costa RicA: Sixola; Juan Vinas (USNM, Washington).

IHERINGIA Jones, I9I 4, Trans. ent. Soc. Lond. 1914: 439.

Type-species: Iheringia santista Jones, I9I4, ibidem 1914: 440, by original designation. Type(s) ô, Brazir: Santos, Alto da Serra (BMNH).

A junior homonym of Theringia Keyserling, I891, Spinnen Am. 3: 25, - Arachmida. There is no objective replacement name but $I$. santista is congeneric with Lophomyra tacita Schaus, I9I I, the type-species of Lophomyra Schaus, I9I I. The latter is therefore available for use as a subjective replacement name.

LOPHONOTIDIA Hampson, igor, Cat. Lepid. Phalaenae Br. Miıs. 3: 518, 6 I7. AGAR

Type-species: Lophonotidia nocturna Hampson, I901, ibidem 3: 6I7, fig. 268, by monotypy. Holotype $\tilde{\sigma}$, [Malawi] Brit. Central Afr.: Nyasa Land (BMNH). 
LOPHONOTINA Hampson, no published reference found.

HYPE

LOPHONYCTA Sugi, I970, Konty $\hat{\imath}$ 38: I34.

ACRO

Type-species: Moma confusa Leech, I889, Proc. zool. Soc. Lond. 1889: 480, pl. 50, fig. 5, by original designation. Holotype $0 \hat{\sigma}$, JAPAN: ?Yokohama (BMNH).

LOPHOPANILLA Hampson, 1926, Descr. new Genera Species Lepid. Phalaenae Subfamily Noctuinae Br. Mus.: 286.

$\mathrm{OPHI}$

Type-species: Panilla obscurissima Holland, I894, Psyche, Camb. 7: 48, pl. 2, fig. I8, by original designation. Type(s) $\delta$, West AfrICA (CM, Pittsburg).

LOPHOPHORA Möschler, I89o, Abh. senckenb. naturforsch. Ges. 16: 227.

HYPE

Type-species: Lophophora clanymoides Möschler, I89o, ibidem 16: 228, fig. 4, by monotypy. Syntypes $\hat{\sigma}, q$, CuBA (MNHU, Berlin).

$\ddagger$ PALINTHIS Hampson, no published reference found.

Proposed, unnecessarily, in Hampson's notes and collection, as the objective replacement name for Lophophora Möschler, I890, which is not preoccupied although Hampson considered it to be a junior homonym of Lophophorus Temminck, I8I3, - Aves.

LOPHOPLUSIA Zinmerman, I958, Insects Hawaii 7: 359.

PLUS

Type-species: Plusia pterylota Meyrick, 1904, in Sharp, Fauna Hawaiiensis 3: 348, by original designation. Holotype $\widehat{\jmath}$. Hawailan Is: Oahu, S.E. Koolau range (BMNH).

LOPHOPTERA Guenée, I852, in Boisduval \& Guenée, Hist. nat. Insectes (Lépid.) 7: 54 . STIC

Type-species: Lophoptera squammigera Guenée, I852, ibidem 7: 55, pl. I4, fig. I3, by subsequent designation by Hampson, 1912, Cat. Lepid. Phalaenae Br. Mus. 11: I75. Holotype ơ. [Australia] Nouvelle-Hollande (MNHN, Paris).

LOPHORACHE Hampson, I9Io, Cat. Lepid. Phalaenae Br. Mus. 10: 7, 648. ACoN

Type-species: Lophorache fulvirufa Hampson, I9I0, ibidem 10: 648, fig. I8I, by original designation. Holotype $\delta$, [Kenya] Brit. E. Afr.: Taveta (BMNH).

LOPHORUZA Hampson, I9Io, Cat. Lepid. Phalaenae Br. Mus. 10: x, 225.

ACON

Type-species: Lophoruza vacillatrix Hampson, I9I0, ibidem 10: 23I, fig. 62, by

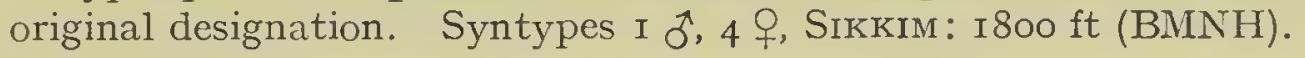

LOPHOSEMA Schaus, I9Io, Ann. Mag. nat. Hist. (8) 6: 204.

SARR

Type-species: Lophosema purpurascens Schaus, I9I0, ibidem (8) 6:204, by monotypy.

Type(s), Costa Rica: Juan Vinas (USNM, Washington).

LOPHOSEMA Hampson, I912, Cat. Lepid. Phalaenae Br. Mus. 11: 332.

Type-species: Lophosema purpurascens Schaus, I9Io, by original designation.

A junior homonym of Lophosema Schaus, i тіо. This generic name probably originated from Hampson, but was used and made nomenclaturally available by Schaus prior to its proposal and generic description by Hampson. The objective replacement name is Lophosema Schaus, igio.

LOPHOSEMA Hampson, I9i2. See Lophosema Schaus, I9io.

SARR

LOPHOTAGONIA Bethune-Baker, I9I I, Ann. Mag. nat. Hist. (8) 8:527. SARR

Type-species: Lophotagonia bostrycodes Bethune-Baker, I9II, ibidem (8) 8:528, by original designation. Type(s) $\tilde{\sigma}$, [ANGola] Portuguese W. Africa: Gunnal (BMNH).

†OPHOTOGONIA; Hampson, I9I2, Cat. Lepid. Phalaenae Br. Mus. 11: 670.

An incorrect subsequent spelling.

LOPHOTARSIA Hampson, I902, Ann. S. Afr. Mus. 2: 299.

ACRO

Type-species: Lophotarsia ochroprocta Hampson, I902, ibidem 2: 299, by monotypy. Holotype $\hat{0}$, South Africa: Natal, Estcourt (BMNH). 
LOPHOTAVIA Hampson, 1926, Descr. New Genera Species Lepid. Phalaenae Subfamily Noctunae Br. Mus.: 26.

Type-species: Homoptera globulipes Walker, I 865, List Specimens. lepid. Insects Colln Br. Mus. 33: 885, by original designation. Holotype , Sierra Leone (BMNH).

LOPHOTERGES Hampson, 1906, Cat. Lepid. Phalaenae Br. Mus. 6: viii, 9 I.

CUCU

Type-species: Lithocampa fatua Püngeler, 1904, Dt. ent. Z. Iris 16:288, pl. 6, fig. 4, by original designation. Syntypes $2 \hat{0}, \mathrm{I}, q_{0}[\mathrm{CHINA}]$ : [Tsinghai], Kuku-Noor (MNHU, Berlin).

LOPHOTHRIPA Hampson, i896, Fauna Br. India (Moths) 4: xxvi, 528.

SARR

Type-species: Selepa vitea Swinhoe, 1885, Proc. zool. Soc. Lond. 1885: 460, p]. 27, fig. I 7, by original designation. Syntypes, [INDIA]: Bombay (BMNH).

See also Heteronota Meyrick, I902.

LOPHOTIDIA Hampson, I913, Cat. Lepid. Phalaenae Br. Mus. 12: 4, 242.

Type-species: Lophotidia trisema Hampson, 1913, ibidem 12: 243, fig. 28, by original designation. Holotype $\hat{o},[$ KENYA $]$ Brit. E. Afr.: Kirbehs (BMNH).

†LOPHOTOGONIA, misspelling. See Lophotagonia Bethune-Baker, I 9 I I.

SARR

LOPHOTOMA Turner, 1902, Proc. Linn. Soc. N.S.W. 27 : 128.

OPHI

Type-species: Lophotoma diagrapha Turner, 1902, ibidem 27: 129, by subsequent designation by Berio, 1966, Annali Mus. civ. Stor. nat. Giacomo Doria 76: 61. Holotype o. Australia: Qd, Brisbane (ANIC, Canberra).

LOPHOTYNA Hampson, igo8, Cat. Lepid. Phalaenae Br. Mus. 7 : i2. Available, but without included species until Hampson, I910, ibidem 9: vii, I9.

ACRO

Type-species: Mamestra albirena Moore, I867, Proc. zool. Soc. Lond. 1867: 53, by subsequent monotypy. Syntypes $\hat{0}$. India: [W.] Bengal, Calcutta (BMNH); Darjeeling (BMNH).

LOPHOZANCLA Turner, 1932, Trans. R. Soc. S. Aust. 56: 180.

OPHI

Type-species: Lophozancla prolixa Turner, 1932, ibidem 56: i 80, by monotypy. Syntypes 20 , Australia: [N. Territ.], Darwin (ANIC, Canberra); Stapleton (ANIC, Canberra).

LOPHUDA Hampson, 1926, Descr. new Genera Species Lepid. Phalaenae Subfamily Noctuinae Br. Mus.: 382.

OPHI

Type-species: Margiza purpuraria Dognin, I9I4, Hétérocères nouv. Am. Sud 8: 72, by original designation. Holotype $\delta$, Colombia: Cali, San Antonio.

LOXAGROTIS McDunnough [1929] I928, Bull. Dep. Mines, Can. 55: 27.

NOCT

Type-species: Agrotis proclivis Smith, I 887, Proc. U.S. natn. Mus. 10: 453, by original designation. Syntypes I ô, I ㅇ, [U.S.A.]: Arizona.

LOXIODA Warren, 1913, in Seitz, Macrolepid. World 3: 386.

OPHI

Type-species: Phurys similis Moore, 1882, in Hewitson \& Moore, Descr. new Indian lepid. Insects Colln late $M r W$. S. Atkinson: I74, pl. 6, fig. 5, by original designation. Type(s) 오. India: [W. Bengal], Darjiling.

The date of publication of the part of Seitz containing page 386 was stated on page $38 \mathrm{I}$ as I913 November 2oth in the English edition, and I9I3 December ist in the German edition.

LUBERTA Schaus, Iऽ, 16, Proc. U.S. natn. Mus. 50:262, 320.

Type-species: Cuberta nymbisalis Schaus, I916, ibidem 50: 320, by original designation. Typn's) ?.. Bolivia (USNM, Washington). 
LUCASIDIA Boursin, I936, Bull. Soc. Sci. nat. Maroc 16: I47. ACRO

Type-species: Lucasidia phenax Boursin, I936, ibidem 16: I48, pl. I, figs I-4, by original designation. Holotype ô, Morocco: Merkala (LN, Karlsruhe).

LUCERIA Walker, I859 [December Ioth], List Specimens lepid. Insects Colln Br. Mus. 19: 853.

$\mathrm{H} \backslash \mathrm{PE}$

Type-species: Luceria novatusalis Walker, 1859 , ibidem 19: 854 , by original designation. Holotype of, CEYLON (BMNH).

The preface to Walker's List . . 19 was dated 1859 November I 2 th and so must have been published after that date. Manuscript annotations in the copies in BMNH give I 859 December Ioth as the date of publication.

LUCERIA Heinemann, I859 [December 3Ist]. See Calamia Hübner, [I82I]. ACRO

LUCERIOLA Strand, I920, Arch. Naturgesch. 84 (A) I2: I79.

HYPE

Type-species: Chusaris compripalpis Strand, I920, ibidem 84 (A) I 2: I79, by monotypy.

Holotype $q$, [TArwan] Formosa: Suisharyo (DEI, Eberswalde).

Luceriola was originally proposed as a subgenus of Chusaris Walker, [1859].

$\ddagger \boldsymbol{L U C I A}$, misspelling. See Amilaga Swinhoe, Igor.

HYPE

LUGANA Moore, 1882, in Hewitson \& Moore, Descr. new Indian lepid. Insects Colln late Mr W. S. Atkinson: I 45 .

HADE

Type-species: Lugana antennata Moore, I882, ibidem: $1_{4} 6$, by original designation. Syntypes, India: [W. Bengal], Darjiling (BMNH).

LUPERINA Boisduval, I828, Eur. Lepid. Index meth.: 77.

ACRO

Type-species: Noctua testacea [Denis \& Schiffermüller], I775, Ankündung syst. Werkes Schmett. Wienergegend: 8I, by subsequent designation by Duponchel, I829, in Godart \& Duponchel, Hist.nat. Lépid. Papillons Fr. 7 (2): 71. Type(s), [Austria]: Vienna district (Collection destroyed, Horn \& Kahle, 1936, Ent. Beih. Berl.-Dahlem 3: 243).

$\ddagger$ LUPERNIA; Doubleday, I850, Synonymic List. Br. Lepid.: 7, 27.

An incorrect subsequent spelling.

LYPERINA Spuler, (1905) I908, in Hofmann, Schmett. Eur. 1: 187.

An unjustified emendation of Luperina Boisduval, 1828.

PALLUPERINA Hampson, I920, Novit. zool. 26: 256.

Proposed, unnecessarily, as an objective replacement name for Luperina Boisduval, I828, which Hampson considered to be nomenclaturally unavailable as in his opinion it was not originally described.

$\ddagger$ LUPERNIA, misspelling. See Luperina Boisduval, I828.

ACRO

LUSIA Walker, [1858]. See Amilaga Swinhoe, I901.

HYPE

LUSSA Grote, 1883, Can. Ent. 15: I27.

SARR

Type-species: Lussa nigroguttata Grote, I883, ibidem 15: 127, by monotypy. Type(s) $\widehat{o}$. [U.S.A.]: Indian River.

L. nigroguttata is a junior subjective synonym of Motya abseuzalis Walker, I859.

See also Motya Walker, I859; and Pleurasympieza Möschler, I89o.

LUTOGONIA Schaus, 1913, Ann. Mag. nat. Hist. (8) 11: 3 o.

OPHI

Type-species: Lutogonia simplex Schaus, I913, ibidem (8) 11: 30, by original designation. Type(s) +, Costa RicA: Juan Vinas (USNM, Washington).

LYCANADES Franclemont, 1937, Can. Ent. 69: I28.

CUCU

Type-species: Xanthia pulchella Smith, Igoo, ibidem 32:223, pl. 5, fig. I, by original designation. Syntypes I $\widehat{O}, 2$, , [CANADA]: British Columbia, and Vancouver, Livingston 
LYCAUGESIA Dognin, I910 April, Hétérocères nouv. Am. Sud 1 : I 4.

$\operatorname{ACON}$

Type-species: Lycaugesia fuscifascia Dognin, I910, ibidem 1: I4, by monotypy. Holotype $\widehat{o}$, French Guiana: Saint-Laurent du Maroni.

LYCAUGESIA Hampson, I910 [December]. See Quandara nom. n.

$\operatorname{ACON}$

LYCIMNA Walker, I860, List Specimens lepid. Insects Colln Br. MIus. 20:214. OPHI

Type-species: Lycimna polymesata Walker, 1860, ibidem 20: 215, by monotypy. Holotype o. [Bangladesh]: [E. Bengal], Silhet (BMNH).

LYCOPHORUS Staudinger, I901, in Staudinger \& Rebel, Cat. Lepid. palaearct. Faunengeb.

1: 154. NOCT

Type-species: Comophorus villosus Alphéraky, I887, Stettin. ent. Ztg 48: I69, by monotypy (of Comophorus Alphéraky, I887). Syntypes ô. ‥ [U.S.S.R.]: Turkestan (ZI, Leningrad).

Lycophorus was proposed as the objective replacement name for Comophorus Alphéraky, 1887 .

COMOPHORUS Alphéraky, I887, Stettin. ent. Ztg 48: 168.

Type-species: Comophorus villosus Alphéraky, I887, by monotypy.

A junior homonym of Comophorus Agassiz, I848, Nomencl. zool. Index univl.: 95, 96, -

Pisces. The objective replacement name is Lycophoms Staudinger, I90 I.

LYCOPHOTIA Hübner, [1821] i816, Verz. bekannter Schmett.: 215.

NOCT

Type-species: Noctua porphyrea [Denis \& Schiffermüller], 1775, Ankïndung syst.

Werkes Schmett. Wienergegend: 82, by subsequent designation by Hampson, 1903, Cat.

Lepid. Phalaenae Br. Muss. 4: 5 I 4, but cited as ${ }_{+}$porphyria, an incorrect subsequent spelling.

Type(s), [Austria]: Vienna district (Collection destroyed, Horn \& Kahle, I936, Ent.

Beih. Berl.-Dahlem 3: 243).

SCOTOPHILA Stephens, 1829 [June], Nom. Br. Insects: 41.

Type-species: Noctua porphyrea [Denis \& Schiffermüller], 1775, by monotypy.

A junior homonym of Scotophila Hübner, [I821], - Lepid., Noctuidae. The objective replacement name is Lycophotia Hübner, [1821].

†LICOPHOTIA; Kozhanchikov, I937, Fauna SSSR (Insecta) Lepid. 13(3): ix, 227.

An incorrect subsequent spelling.

LYCOSELENE Möschler, I 887, Abh. senckenb. naturforsch. Ges. 15: 87.

SARR

Type-species: Lycoselene lunata Möschler, 1887, ibidem 15: 88, fig. 19, by monotypy. Holotype $q$, [GHANA] Gold Coast: Aburi.

LYGEPHILA Billberg, 1820, Enumeratio Insect. Mus. G. J. Billberg: 85.

OPHI

Type-species: Phalaena lusoria Linnaeus, 1758, Syst. Nat. (Edn 10) 1: 506, by subsequent designation by Tams, 1939, Entomologist 72: I40. Type(s), Germany (LS, London).

LYGNIODES Guenée, I 852, Hist. nat. Insectes (Lépid.) 7 : 123.

CATO

Type-species: Erebus endoleucus Guérin-Méneville, 1844, Iconographie Règne anim. G. Cuvier 3: 521, by subsequent designation by Hampson, 1913, Cat. Lepid. Phalacnae Rr. Mus. 12: 267. Type(s), Java.

AGONISTA Rogenhofer, 1875, in Felder \& Rogenhofer, Reise öst. Fregatte Novara (Zool.) 2

(Abt. 2): pl. 113 .

Proposed, unnecessarily, as an objective replacement name for Lygniodes Guenée, I 852, which Rogenlofer considered to be a junior homonym of Lignyodes Schocnherr, is.33, Genera Species Curculionidum 1 (1): 19, - Coleoptera.

Some authors including Neave, 1939, Nomencl. zool. 1:92, have attributed Agonista to

Felder. In the original description opposite pl. I 3 it is given as 'Agonista n.g. Roghf.' 
LYGRANTHOECIA Grote \& Robinson, I873, in Grote, Bull. Buffalo Soc. nat. Sci. 1: I I 5.

Type-species: Crambus marginatus Haworth, r809, Lepid. Br.: 374 (a junior primary homonym, see below), by subsequent designation by Grote, r874, ibidem 2: 33. Type(s), TYPE-LOCALITy stated to be Great Britain: [England] Anglia, but this was probably an error (or an accidental introduction) as this species is not known to occur in the Old World but is widespread in U.S.A. and CANADA.

The type-species is preoccupied by Crambus marginatus Fabricius, I798, Suppl. Ent. Syst.: 472, - Lepid., Pyralidae. There is no objective replacement name but $C$. marginatus Haworth is conspecific with Anthoecia rivulosa Guenée, 1852, in Boisduval \& Guenée, Hist. nat. Insectes (Lépid.) 6: 184, pl. 9, fig. I2. This latter is therefore available for use as a subjective replacement name.

LYNCESTIS Walker, I857, List Specimens lepid. Insects Colln Br. Mus. 11:6I I, 632. орн I Type-species: Phalaena amphix Cramer, 1777, Uitlandsche Kapellen 2: 59, pl. I34, fig. C, by monotypy. Type(s), [India]: Côte de Coromandel.

$P$. amphix is dated from the wrapper of the part and not from the title-page of the volume, I 779 .

LYPERINA Spuler, (I905). See Luperina Boisduval, I828.

ACRO

LYSIMELIA Walker, [1859] I858, List Specimens lepid. Insects Colln Br. Mus. 16: 20I. HyPE Type-species: Lysimelia neleusalis Walker, [1859] I858, ibidem 16: 201, by monotypy. Syntypes 2 d, Borneo: Sarawak (BMNH).

See also Bracharthron Hampson, I89I.

LYSSIA Guenée, I852, in Boisduval \& Guenée, Hist. nat. Insectes (Lépid.) 7: 296.

OPHI

Type-species: Lyssia orthosioides Guenée, 1852, ibidem 7:296, pl. 23, fig. I, by monotypy. Type(s) , America: northern.

L. orthosioides is a junior subjective synonym of Phoberia atomaris Hübner, 1818.

See also Phoberia Hübner, I8I8.

LY TAEA Stephens, I 829, Illust. Br. Ent. (Haustellata) 2: I07.

NOCT

Type-species: Noctua umbrosa Hübner, [1813], Samml. eur. Schmett. 4: pl. 97, figs 456, 457, by monotypy. Syntypes ठ․ ㅇ. Europe.

$N$. umbrosa is a junior subjective synonym of Noctua sexstrigata Haworth, r8og, Lepid. $B r .: 228$.

LYTHRODES Smith, 1903, Trans. Am. ent. Soc. 29: 206.

ACRO

Type-species: Lythrodes radiatus Smith, 1903, ibidem 29: 206, by subsequent designation by Hampson, rgro, Cat. Lepid. Phalaenae Br. Mus. 9: 214. Syntypes 2 ․ [U.S.A.]: Arizona, Yuma County.

MABILLEANA Fletcher \& Viette, 1962, Bull. mens. Soc. linn. Lyon 31: 5.

NOCT

Type-species: Agrotis pudens Mabille, I900, Annls Soc. ent. Fr. 68: 728, by original designation. Lectotype 우, [Comoro Is] E. Africa: Comores (BMNH), designated (as holotype) by Viette, [1963] 1962, Annls Soc. ent. Fr. 131: 134.

MABULIA Herrich-Schäffer, IS70, Corresp.-Bl. zool.-min. Ver. Regensburg 24: IO2. HYPE Nomenclaturally available, but without any included species. Proposed in a work on the Lepidoptera of Cuba.

MACALDENIA Moore, [1885] I887, Lepid. Ceylon 3: I62.

CATO

Type-species: Hulodes palumba Guenée, 1852, in Boisduval \& Guenée, Hist. nat. Insectes (Lépid.) 7: 21 I, by monotypy (of Pasipeda Moore, I882). Syntypes 3 J. Java (BMNH).

Macaldenia was proposed as the objective replacement name for Pasipeda Moore, I882. 
PASIPEDA Moore, I882, in Hewitson \& Moore, Descr. new Indian lepid. Insects Colln late Mr W. S. Atkinson: I 7 I.

Type-species: Hulodes palumba Guenée, r 852 , by monotypy.

A junior homonym of Pasipeda Walker, I858,-Lepid., Noctuidae. The objective replacement name is Macaldenia Moore, [1885].

MACAPTA Hampson, igio, Cat. Lepid. Phalaenae Br. Mus. 9:96. ACro

Type-species: Makapta carnescens Schaus, 1906, Proc. U.S. natn. Mus. 30: 99, by monotypy (of Makapta Schaus, 1906). Type(s), Brazil: São Paulo (USNMI, Washington).

Macapta was proposed by Hampson as an emendation of Makapta Schaus, I906, but as the latter is a junior homonym (see below), Hampson's emendation must be used as the objective replacement name.

MAKAPTA Schaus, 1906, Proc. U.S. natn. Mus. 30: 99.

Type-species: Makapta carnescens Schaus, rgo6, ibidem 30: 99, by monotypy.

A junior homonym of Makapta Schaus, I904,-Lepid., Noctuidae. This generic name was used and unintentionally made nomenclaturally available by Schaus, himself, in I904, prior to its proposal and description in 1906 for a genus having a different type-species.

† MACARIENTA Hampson, no published reference found.

HYPE

MACDUNNOUGHIA Kostrowicki, I961, Acta zool. cracov. 6: 402.

PLUS

Type-species: Plusia confusa Stephens, I850, List Specimens Br. Anim. Colln Br. Mus.

5: 29r, by original designation. Type(s) $\overrightarrow{0}$, EUROPE (BMNH).

$P$. confusa was proposed for the misidentification Noctua circumflexa Linnaeus sensu Hübner, [1803], Samml. eur. Schmett. 4: pl. 58, fig. 285.

†ARAPLUSIA Mukerji \& Krishnamorthy, I955, Proc. t2nd Indian Sci. Congr. Ass. (3): 295.

Type-species: Plusia confusa Stephens, I 850 , by original designation.

+Paraplusia is not nomenclaturally available as it does not satisfy the provisions of the Code, Article 13 (a), having been published after 1930 without a statement of characters differentiating the taxon.

SCLEROPLUSIA Ichinose, 1962, Kontyû 30: 249.

Type-species: Plusia confusa Stephens, I 850 , by original designation.

Scleroplusia Ichinose was originally proposed as a subgenus of Autographa Hübner,

[182 I]. It is, however, a junior objective synonym of Macdunnoughia Kostrowicki, I96I.

MACEDA Walker, [ I858] i 857, List Specimens lepid. Insects Colln Br. Mus. 13: I I 22, I I 40. CHLO Type-species: Maceda mansueta Walker, [1858] r857, ibidem 13: I I 4 I, by monotypy. Holotype ô. Borneo: Sarawak (BMNH).

See also Calduba Walker, 1858 .

MACELLA Walker, [i859] i 858, List Specimens lepid. Insects Colln Br. Mus. 16: i61. OPHI Type-species: Macella euritiusalis Walker, [1859] I 858, ibidem 16: I6I, by monotypy. Sytytyes 2 ô. Sierra Leone (BMNH).

See also Eucapnodes Holland, I 894.

MACELLOPIS Hampson, 1926, Descr. new Genera Species Lepid. Phalaenae Subfamily Noctuinae Br. Mus.: 4 I9.

OPHI

Type-species: Macellopis ustata Hampson, 1926, ibidem: $4 \mathrm{Ig}$, by original designation. Syntyles 5 ô. 3 ㅇ. Nigeria: Old Calabar (BMNH).

MACHAEROPALPUS Tams, 1935, Insect.s Samoa 3 (4): 229.

HYPE

Type-species: Machaeropalpus fasciatus Tams, 1935, ibidem $3(4): 230,1) l .6$, fig. I9, pl. 7, fig. 9, by original designation. Holotype q, SAmoa: Upolı, Malololelei (BMNH).

MACRHYPENA Grote, i 873 January, Trans. Am. ent. Soc. 4: 310.

HYPE

Type-species: Hypena deceptalis Walker, [1 859] I 858, List Specimens lepid. Insects Colln 
Br. Muss. 16: 30, by subsequent designation by Grote, I873, Bull. Buffalo Soc. nat. Sci. 1: 38. Syntypes 3 q. [U.S.A.]: New York (BMNH).

The later reference, Macrhypena Grote, 1873 May, ibidem 1:38, was cited by Neave, 1940 Nomencl. zool. 3: 8, as the original description.

MACRISTIS Schaus, 1916, Proc. U.S. natn. Mus. 50: 260, 276.

Type-species: Macristis geminipunctalis Schaus, I9I6, ibidem 50:277, by original designation. Type(s) $\hat{\jmath}$, CuBA: Santiago (USNM, Washington).

MACROBARASA Hampson, I9I2, Cat. Lepid. Phalaenae Br. Mus. 11: xiii, 442.

Type-species: Blenina xantholopha Hampson, 1896, Fauna Br. India (Moths) 4: 526 , by original designation. Type(s) $\hat{\sigma}$, India: [Assam], Khasis (BMNH).

$\ddagger$ IICROBARASA; Neave, 1940, Nomencl. zool. 3: 148 .

An incorrect subsequent spelling.

MACROCARSIA Hampson, I926, Descr. new Genera Species Lepid. Phalaenae Subfamily Noctuinae Br. Mus. : 296. OPHI

Type-species: Alamis holzi Pagenstecher, I 884, Jb. nassau Ver. Naturh. 37: 229, pl. 6, fig. 4, by original designation. Holotype 오. Moluccas: Amboina (SM, Wiesbaden).

†MACROCHILA, misspelling. See Macrochilo Hübner, [I825]. HYPE

†MACROCHILE, misspelling. See Macrochilo Hübner, [1825]. HYPE

MACROCHILO Hübner, [I825] I8I6, Verz. bekannter Schnett.: 345. HYPE

Type-species: Pyralis cribralis Hübner, I796, Samml. eur. Schmett. 6: pl. I, fig. 2, by subsequent designation by Westwood, I840, Synopsis Genera Br. Insects: I05 (but cited for $\$$ Macrochila, an incorrect subsequent spelling). Type(s), Europe.

$P$. cribralis is a junior subjective synonym of Phalaena cribrumalis Hübner, x793, Samml. auserlesener Vögel Schmett.: 7, pl. I5. P. cribralis was almost certainly an emendation of cribrumalis, but Hübner did not cite both names together in his Verzeichniss, or elsewhere. $P$. cribralis should strictly be treated as a subsequent incorrect spelling, but as it has in the past been so frequently used as a specific name in its own right, it is here also treated as such, and hence as a junior synonym.

$\ddagger$ MACROCHILA; Westwood, I840, Synopsis Genera Br. Insects: 105.

An incorrect subsequent spelling of Macrochilo Hübner, [1825]. Not to be confused with the available name Macrochila Stephens, I829, Nom. Br. Insects: 49, - Lepid., Oecophoridae.

† MACROCHILE; Walker, [ I 859] I 858, List Specimens lepid. Insects Colln Br. Mus. 16: 22, 96. An incorrect subsequent spelling.

MACROCHTHONIA Butler, r88x, Trans. ent. Soc. Lond. 1881: 599.

CHLO

Type-species: Macrochthonia fervens Butler, I88I, ibidem 1881 : 599, by monotypy. Type(s), Japan: Tokei (BMNH).

See also Eccopteroma Staudinger, I 892.

MACRODES Guenée, I854, in Boisduval \& Guenée, Hist. nat. Insectes (Lépid.) 8: I2. opHI

Type-species: Phalaena cynara Cramer, I775, Uitlandsche Kapellen 1: 23, pl. I5, figs C, D (but included as $M$. cynaralis Guenée, an unjustified emendation), by subsequent designation by Berio, I966, Amnali Mus. civ. Stor. nat. Giacomo Doria 76: 62, but cited as 'cynaralis Cr.,' an incorrect authorship. Type(s), Surinam.

An earlier usage of the name i.e. $\ddagger$ Macrodes Dejean, I833, Cat. Coléoptères Colln $M$. le Comte Dejean (Edn 2): 94, - Coleoptera, was undescribed and included a single indescribed species. Although the name has subsequently been listed in catalogues it has not been made nomenclaturally available in the Coleoptera.

See also Bulna Walker, r865. 
MARCODES Berio, 1966, Annali Mus. civ. Stor. nat. Giacomo Doria 76: 62.

Proposed, unnecessarily, as the objective replacement name for Macrodes Guenée, I 854 , which was not preoccupied by $\$$ Macrodes Dejean, 1833 .

MACRONOCTUA Grote, 1874, Anmual Rep. Trustees Peabody Acad. Sci. 6: 27. Acro Type-species: Macronoctua onusta Grote, I874, ibidem 6:27, by monotypy. Syntypes o. . . [U.S.A.]: Illinois (B.MNH).

MACROPRORA Turner, I94 I, Mem. Od Mus. 12: 48.

ACRO

Type-species: Macroprora chionobola Turner, I94I, ibidem 12: 48 , by monotypy. Holotype, Australia: Qd, Injune (QM, Brisbane).

See also Conocrana Turner, 1942.

MADATHISANOTIA Viette, 1970, Bull. Soc. ent. Fr. 75: 95.

ACRO

Type-species: Eudryas madagascariensis Rothschild, I924, Ann. Mag. nat. Hist.

(9) 14: 313, by original designation. Holotype , MAdagascar: Diego Suarez (BMNH).

MADECATHYMIA Viette, 1968, Annls Soc. ent. Fr. (N.S.) 4: 570.

ACRO

Type-species: Madecathymia cadoreli Viette, I968, ibidem 4: 57I, pl. A, fig. I, by original designation. Holotype ô, Madagascar: E., route d'Anosibe, forêt de Sandrangato (MNHN, Paris).

MADEGALATHA Viette, 1965, Bull. Soc. ent. Fr. 70:87.

ACRO

Type-species: Thalatha malagassica Hampson, 1909, Cat. Lepid. Phalaenae Br. Mus. 8: 42, fig. I i, by original designation. Holotype ô, Madagascar: Betsileo (BMNH).

MADEUPLEXIA Viette, (1960) 1959, Bull. Soc. ent. Fr. 64: 225.

ACRO

Type-species: Madeuplexia pretiosa Viette, (1960) 1959, ibidem 64: 226, by original designation. Holotype $\hat{\jmath}$, MadAGaSCAR: centre, Pays Betsileo, Ambatofitorahana forest (MNHN, Paris).

MADOCE Walker, I863, List Specimens lepid. Insects Colln Br. MIus. 27: I I7.

HYPE

Type-species: Madoce leucocosmalis Walker, I863, ibidem 27: I17, by PRESENT

DESIGNATION. Holutype q, [Australia]: [Qd], Moreton Bay (BMNH).

Madoce was originally proposed for two new species, but the type of the second, $M$. lineatula is lost, and the species unrecognised.

See also Larassa Walker, [1866].

MADOPA Stephens, 1829. See Colobochyla Hübner, [1825].

OPHI

MAENAS Hübner, [1823]. See Eudocima Billberg, I 820.

OPHI

MAGELLANA Staudinger, I899, Ergebn. Hamb. Magalhaensischen Sammelreise 4 (7): 77.

Type-species: Anarta trisema Mabille, 1885, Bull. Soc. philomath. Paris (7) 9: 64, by monotypy. Lectotype sं, [ARgentina'Chile]: [Tierra del Fuego] Terre de Feu (MNHN, Paris), designated (as holotype) by Viette \& Fletcher, 1968, Bull. Br. Mus. nat. Hist. (Ent.) $21: 4$ Io.

When Magellana was proposed, Staudinger listed '? Magellana Mabillei nov. spec.' under Anarta trisema and stated that if his specimen was not conspecific with $A$. trisema then it should be named $M$. mabillci. The latter was thus doubtfully included as a synonym and, under the Code, Article 68 (c), is not eligible for selection as type-species.

MAGEOCHAETA Victte, I965, Bull. Soc. ent. Fr. 70:86. ACRO

Type-species: Daseochaeta malgassica Kenrick, 1917. Trans. ent. Soc. Lond. 1917: 87, pl. 4, by original designation. Lectotype ?. MADAGASCAR (BMNH), designated (as holotype) by Viette, 1965, Fanne Madagascar 20(1): 485 .

MAGEUTICA Hampson, 1926. See Cutephia Ochsenheimer, r816.

OPHI 
MAGHADENA Viette, [1963] I962, Annls Soc. ent. Fr. 131: I 52.

HADE

Type-species: Xylomania betsileo Viette, (1960) 1959, Bull. Soc. ent. Fr. 64: 223, by original designation. Holotype $\widehat{\jmath}$. Madagascar: centre, Pays Betsileo, Ambatofitorahana forest (MNHN, Paris).

MAGUDA Walker, [1866] i865, List Specimens lepid. Insects Colln Br. Mus. 34: i I86. OpHI Type-species: Maguda immundalis Walker, [1866] i 865, ibidem 34: I 86, by monotypy. Lectotype ․ Borneo: Sarawak (UM, Oxford), designated (as type) by Swinhoe, I9oo, Cat. east. and Aust. Lepid. Heterocera 2: 203.

MAGULABA Walker, [1866] 1865, List Specimens lepid. Insects Colln Br. Mus. 34: I I 26. орні Type-species: Magulaba moestalis Walker, [I866] I865, ibidem 34: I I 27, by monotypy. Holotype , Sierra LeONe (BMNH).

See also Rimulia Heyden, i89i.

MAGUSA Walker, I 857, List Specimens lepid. Insects Colln Br. Mus. 11: 762.

ACRO

Type-species: Magusa strigifera Walker, i 857, ibidem 11:763, by monotypy. Holotype o. [Dominican Republic] St. Domingo (BMNH).

$M$. strigifera is a junior subjective synonym of Xylina orbifera Walker, 1857 , ibidem 11 : $76 \mathrm{r}$.

MAGUZA Walker, [1866] I865, List Specimens lepid. Insects Colln Br. Mus. 34: I223. HADE Type-species: Maguza albiguttalis Walker, [1866] I865, ibidem 34: I224, by monotypy. Type(s) ô. Brazil (UM, Oxford).

$M$. albiguttalis is a junior subjective synonym of Pastona rudis VValker, I858.

See also Pastona Walker, 1858.

MAIKONA Matsumura, I928, Insecta matsum. 2: I26.

AGAR

Type-species: Maikona jezoensis Matsumura, 1928, ibidem 2: 126, fig., by original designation. Syntypes 5 +, JAPAN: Holkkaido, Sapporo (EIHU, Sapporo).

$\ddagger \boldsymbol{M A} \boldsymbol{K} \boldsymbol{A I \boldsymbol { A }}$ Strand, no published reference found.

SARR

There is a specimen labelled $\ddagger$ Makaia schoutedeni Strand/Type, in MRAC, Tervuren. Both the generic and the specific names are probably unpublished.

MAKAPTA Schaus, I904, Trans. Am. ent. Soc. 30: I66.

OPHI

Type-species: Makapta argentescens Schaus, I904, ibidem 30: 166, by monotypy. Type(s), [BraziL]: Parana, Castro.

HYPOCALPOPIS Hampson, 1926, Descr. new Genera Species Lepid. Phalaenae Subfamily

Noctuinae Br. Mus.: 378 .

Type-species: Makapta argentescens Schaus, 1904, by original designation.

Hypocalpopis is a junior objective synonym of Makapta Schaus, I904.

MAKAPTA Schaus, I9o6. See Macapta Hampson, I9 Iо.

ACRO

MALAGONIA Hampson, 1926, Descr. new Genera Species Lepid. Phalaenae Subfamily Noctuinae Br. Mus.: 473 .

OPHI

Type-species: Talapa acypera Hampson, 1902, J. Bombay nat. Hist. Soc. 14: 217, by original designation. Holotype $\sigma^{\star}$ India: [Assam], Khasis (BMNH).

$\ddagger$ MALAMERA, misspelling. See Melamera Hampson, i9 го.

ACON

MALATROGIA Hampson, 1926, Descr. new Genera Species Lepid. Phalaenae Subfamily Noctuinae Br. Mus.: 594 . OPHI

Type-species: Zethes castanitis Hampson, I907, J. Bombay nat. Hist. Soc. 17: 666, by original designation. Holotype ô, India: Assam, Silchar (BMNH).

MALIA TTHA Walker, I863, List Specimens lepid. Insects Colln Br. Mus. 27: 86. ACON Type-species: Maliattha separata Walker, I863, ibidem $27: 86$, by monotypy. Type(s) o. BORNEO: Sarawak (BMNH). 
MALTANA Walker, I866, List Specimens lepid. Insects Colln Br. Mis. 35: 1974. OPH

Type-species: Maltana thermisioides Walker, I866, ibidem 35: I975, by monotypy. Holotype q. VENEZUELA (BMINH).

MAMERTHES Druce, I89I, Biologia cent.-am. (Zool.) Lepid. Heterocera 1: 449. HyPE Type-species: Mamerthes nigrilinea Iruce, I89 I, ibidem 1: 450, pl. 37, figs I, 2, by original designation. Syntypes I $\hat{\delta}, 5$ 9 , Guatenala: Volcan de Atitlan (BMNH). Panama: Tolcan de Chiriqui (BMNH); Chiriqui.

MAMESTRA Ochsenheimer, I 816, Schmett. Eur. 4: 76.

HADE

Type-species: Phalaena brassicae Linnaeus, I758, Syst. Nat. (Edn Io) 1: 5 I6, by subsequent designation by Duponchel, I829, in Godart \& Duponchel, Hist. nat. Lépid. Papillons Fr. 7 (2): 7r. Type(s), TyPe-Locality not stated (LS, London) [EURope]. AETHRIA Hübner, [1821] I816, V'erz. bekannter Schmett.: 2 I8.

Type-species: Noctua serena [Denis \& Schiffermüller], I775, Ankïndung Syst. Werkes Schmett. Wienergegend: 84, by subsequent designation by Hampson, 1905, Cat. Lepid. Phalaenae Br. Mus. 5: 60. Type(s), [Austria': Vienna district (Collection destroyed, Horn \& Kahle, 1936, Ent. Beih. Berl.-Dahlem 3: 2+3).

$N$. serena is a junior subjective synonym of Phalaena bi[c]olorata Hufnagel, I 766, Berlin. Mag. 3 (4): 4Io. The original spelling by Hufnagel was biolorata, but ever since, for over 200 years, it lias been known in all works and nomenclators (including that of Sherborn) as bicolorata. An application under the Code, Article 79, should be submitted to the Commission requesting that $P$. bicolorata be made nomenclaturally available from $\mathrm{I} 766$.

Aethria Hübner, [I82I], is a junior homonym of Aethria Hübner, [I8I9] I8I6, Verz. bekannter Schmett.: I20,-Lepid., Ctenuchidae. There is no objective replacement name but $N$. serena is congeneric with Phalaena brassicae Linnaeus, I758, the type-species of Mamestra Ochsenheimer, 1816. The latter is therefore available for use as a subjective replacement name.

BARATHRA Hiibner, [1821] I816, Verz. bekannter Schmett.: 218.

Type-species: Phalaena brassicae Linnaeus, I 758, by subsequent designation by Hampson, I905. Cat. Lepid. Phalaenae Br. Mus. 5: I I.

The first designation of the type-species of Barathra is in some doubt. Smith, I889, Proc. U.S. natn. Mus. 12: 457, implied that P. brassicae was the type-species; Grote, I895, Abh. naturw. Ver. Bremen 14: 7 I refuted this and implied that it should be Noctua albicolon Hübner, [I8I3]. Neither was an unequivocal type-species designation and so has not been accepted.

Barathra is a junior objective synonym of Mamestra Ochsenheimer, I8I6.

MAMISTRA Sodoffsky, I837, Bull. Soc. imp. Nat. Moscou 1837 (6) : 87.

An unjustified emendation of Mamestra Ochsenheimer, i8 6 .

COPIMAMESTRA Grote, I883, Ann. Mag. nat. Hist. (5) 11: 54.

Type-species: Phalaena brassicae Linnaeus, I7 758 , by subsequent designation by Grote, I895 March Ist, Entomologist's Rec. J. Var. 6: 79.

Copimamestra is a junior objective synonym of Mamestra Ochsenheimer, r816.

MAMISTRA Sodoffsky, i837. See Mamestra Ochsenheimer, I8I6.

HADE

MAMMIFRONTIA Barnes \& Lindsey, 1922, Bull. Brooklyn ent. Soc. 17: 75.

ACRO

Type-species: Mammifrontia leucania Barnes \& Lindsey, I922, ibidem 17: 75, by original designation. Holotype , [U.S.A.]: Utah, Cedar City.

MANBUTA Walker, I865, List Specimens lepid. Insects Colln Br. Mrus. 33: I I 5.

Type-species: Manbuta devia Walker, i 865, ibidem 33: I I 5, by monotypy. o., [BRazil]: [R. Amazon], [Tefé] Ega (BMNH).

OPHI

Holotype

MANDELA Walker, [1867] ז868, J. Limn. Soc. (Zool.) 9: 192.

OPHI

Type-species: Mandela crocea Walker, [1867] 1868, ibidem 9: 192, by monotypy. Type(s) ô. [Colomia ]: Bogota. 
MANGA Bowden, 1956, Bull. ent. Res. 47: 420.

Type-species: Manga basilinea Bowden, 1956, ibidem 47: 42I, figs IO-I3, by original designation. Holotype $\hat{\sigma}$. [Grana] Gold Coast: N. Territ., Tamale, Nyankpala (BMNH).

MANIA Treitschke, I825. See Mormo Ochsenheimer, i816.

ACRO

MANOBIA Staudinger, I896. See Niaboma nom. n.

HADE

MANRUTA Smith, 1903, Trans. Am. ent. Soc. 29: 205.

NOCT

Type-species: Manruta elingua Smith, 1903, ibidem 29: 205, by monotypy. Syntypes 2 o., [U.S.A.]: Arizona, Phoenix.

MAORIA Warren, I9I2. See Graphania Hampson, I905.

HADE

MARAPANA Moore, [1885] i 887, Lepid. Ceylon 3: 227.

OPHI

Type-species: Hypena raralis Walker, [I 859] I 858, List Specimens lepid. Insects Colln

$B r$. Mus. 16: 65, by original designation. Syntypes 2 of, CEYLoN (BMNH).

$H$. varalis is a junior subjective synonym of Sanys pulverata Guenée, I 852, in Boisduval \& Guenée, Hist. nat. Insectes (Lépid.) 7:351.

MARASCHIA Osthelder, 1933, Mitt. münch. ent. Ges. 23: 76.

ACRO

Type-species: Maraschia grisescens Osthelder, 1933, ibidem 23: 76, by monotypy. Syntypes $2 \hat{0}, \mathrm{I}$ 우, TuRkEY: Nordsyrien, Marasch.

MARASMALUS Grote, I8 $; 2$, Trans. Am. ent. Soc. 4: 89.

EUTE

Type-species: Marasmalus ventilator Grote, 1872, ibidem 4: 89, by monotypy.

Syntypes $\hat{0}$, [U.S.A.]: Mass., Andover; New York (BMNH).

$M$. ventilator is a junior subjective synonym of Marathyssa basalis Walker, i 865.

See also Marathyssa Walker, I 865.

MARATHYSSA Walker, i 865, List Specimens lepid. Insects Colln Br. Mus. 33: 1033. Eute Type-species: Marathyssa basalis Walker, I865, ibidem 33: I034, by monotypy. Syntypes 20 , TYPE-LOCAlity not known (BMNH). [U.S.A.].

See also Marasmalus Grote, i 872.

MARCA Saalmüller, I89I, Lepid. Madagascar: 486.

OPHI Type-species: Marca proclinata Saalmüller, I89I, ibidem: 486, fig. I38, by monotypy. Type(s), Madagascar: Nossi-Bé I. (SNG, Frankfurt).

MARCILLADA Walker, 1865, List Specimens lepid. Insects Colln Br. Mus. 33: 980. OPH Type-species: Marcillada rubricosa Walker, i 865, ibidem 33: 981, by monotypy. Lectotype ô. Cambodia (UM, Oxford), designated (as type) by Swinhoe, igoo, Cat. east. and Aust. Lepid. Heterocera 2: i 86.

See also Nasaya Moore, 1882.

MARCIPA Walker, I855, List Specimens lepid. Insects Colln Br. Mus. 4: 779, 807.

Type-species: Marcipa inscripta Walker, I 855 , ibidem $4: 808$, by monotypy. Holotype ô. Sierra Leone (BMNH).

MARCIPOPSIS Berio, I966, Annali Mus. civ. Stor. nat. Giacomo Doria 76: i 29.

OPHI

Type-species: Marcipopsis uniformis Berio, I966, ibidem 76: I 30, fig. I9, by original designation. Holotype $\hat{\delta}$, Madagascar: E., env. de Perinet, Forêt d'Analamazoatra (MNHN, Paris).

MARCODES Berio, I966. See Macrodes Guenée, 1854.

OPHI

MARDARA Waller, [1859] I858, List Specimens lepid. Insects Colln Br. Mus. 16: 157. Hy PE Type-species Mardara aracinthusalis Walker, [1 859] I 858, ibidem 16: I 58, by monotypy. Holotype 9 , TyPe-Locality not known (BMNH). [U.S.A. Canada].

$M$. aracinthusalis is a junior subjective synonym of Pyralis angulalis Hübner, $\mathrm{x} 796$.

See also Palthis Hübner, [1825]. 
MARESIA Walker, I866, List Specimens lepid. Insects Colln Br. Mus. 35: I637.

Type-species: Maresia binotata Walker, I866, ibidem 35: 1637, by monotypy. Type(s) o., [Moluccas]: Sula.

M. binotata is a junior subjective synonym of Gesonia obeditalis Walker, [I859].

See also Gesonia Walker, [1859]; Dragana Walker, [1859]; Hileia Walker, I86I; and Amblygoes Butler, I879.

MAREURA Walker, i865, List Specimens lepid. Insects Colln Br. Mus. 33: I Io7.

OPHI

Type-species: Mareura aurilinea Walker, I865, ibidem 33: II08, by monotypy. Holotype ô. [BrazIL]: [R. Amazon], [Tefé] Ega (BMNH).

MARGANA Walker, [1866] 1865, List Specimens lepid. Insects Colln Br. Mus. 34: I 520. OPHI Type-species: Margana seclusalis Walker, [1866] I 865, ibidem 34: I520, by monotypy. Holotype of. JaVA (BMNH).

MARGELANA Staudinger, I888, Stettin. ent. Ztg 49: 20.

ACRO

Type-species: Margelana versicolor Staudinger, I888, ibidem 49: 20, by monotypy. Syntypes $\sigma^{\star}$, 우 [U.S.S.R.]: [Uzbekistan], Margelan (MNHU, Berlin).

MARGITES Druce, I89I. See Margitesia Strand, I935.

HY'PE

MARGITESIA Strand, 1935, Folia zool. hydrobiol. 7: 304.

HYPE

Type-species: Margites bugaba Druce, I89I, Biologia cent.-am. (Zool.) Lepid. Heterocera 1: 480, pl. 40, fig. 5, by original designation (for Margites Druce, I89I). Syntypes 20 . PANama: Bugaba (BMNH).

Margitesia was proposed as the objective replacement name for Margites Druce, I89r.

MARGITES Druce, I89I, Biologia cent.-am. (Zool.) Lepid. Heterocera 1: 480.

Type-species: Margites bugaba Druce, I89 I, by original designation.

A junior homonym of Margites Gahan, I891, Ann. Mag. nat. Hist. (6) 7:26, - Coleoptera.

The objective replacement name is Margitesia Strand, 1935.

MARGIZA Schaus, I9I6, Proc. U.S. natn. Mus. 50: 260, 265.

HYPE

Type-species: Margiza terranea Schaus, I9I6, ibidem 50:265, by original designation. Type(s) , , [French Guiana]: R. Maroni, St. Laurent (USNM, Washington).

MARIMA THA Walker, [1866] I865, List Specimens lepid. Insects Colln Br. Mus. 34: I204. AcoN

Type-species: Marimatha dinumeratalis Walker, [1866] I865, ibidem 34: I204, by subsequent designation by Hampson, I894, Fauna Br. India (Moths) 2: 324. Holotype o, HONDLRAS (BMNH).

$M$. dinumeratalis is a junior subjective synonym of Xanthoptera botyoides Guenée, I852, in Boisduval \& Guenée, Hist. nat. Insectes (Lépid.) 6: 240.

MARMORINIA Guenée, 1852, in Boisduval \& Guenée, Hist. nat. Insectes (Lépid.) 7: 370.

OPHI

Type-species: Marmorinia epionoides Guenée, I 852, ibidem 7:371, by subsequent designation by Desmarest, (I857), in Chenu, Encycl. Hist. nat. Papillons nocturnes: I 35 . Holotype O, U.S.A.: Georgia (MNHN, Paris).

M. epionoides is a junior subjective synonym of Pangrapta decoralis Hübner, I818.

See also Pangrapta Hübner, i8r8.

MAROJALA Viette, I966, Bull. Soc. ent. Fr. 71: 150.

OPHI

Type-species: Actenia signata Butler, I880, Ann. Mag. nat. Hist. (5) 5: 393, by original designation (for Melanophthalma Hampson). Type(s) o. Madagascar (BMNH).

Marojala was proposed as the objective replacement name for Mclanophthalma Hampson, 1926.

MELANOPIITHALMA Hampson, 1926, Deser. new Genera Species Lepid. Phalaenae Subfamily Noctuinae Br. Mus.: 33 I.

Type-species: Actenia signata Butler, I880, by original designation. 
A junior homonym of Melanophthalma Motschulsky, I866, Bull. Soc. imp. Nat. Moscou 39 (2): 269, - Coleoptera. The objective replacement name is Marojala Viette, Ig66.

MARONIA Schaus, Igr6, Proc. U.S. natn. Mus. 50: 260, 273.

HYPE

Type-species: Maronia celadon Schaus, I916, ibidem 50:273, by original designation. Type(s) 우 [French Guiana]: R. Maroni, St. Jean (USNM, Washington).

MARONIS Saalmüller, I89I, Lepid. Madagascar: 409.

OPHI

Type-species: Maronis rivosa Saalmüller, r891, ibidem: 410, fig. I70, by monotypy. Syntypes I ô, I , MAdagascar: Nossi-Bé I. (SNG, Frankfurt).

MARSIPIOPHORA John, I909, Revue russe Ent. 9: II 7.

OPHI

Type-species: Calophasia christophi Ershov, 1874, in Fedchenko, Reise in Turkestan 2 (5) 3:46, pl. 3, fig. 44, by original designation. Holotype ․, [U.S.S.R.]: Turkesta n, Kisil-kum desert.

MARTHAMA Walker, I858, List Specimens lepid. Insects Colln Br. Mus. 15: I 547, I630. орHI Type-species: Marthama squamivaria Walker, 1858, ibidem 15: r63 I, by monotypy. Holotype $\hat{0}$, Brazil (BMNH).

$M$. squamivaria is a junior subjective synonym of Agyra marchandi Guenée, 1852.

See also Agyra Guenée, 1852.

MARZIGETTA Dyar, I9I8, Proc. U.S. natn. Mus. 54: 353.

HYPE

Type-species: Marzigetta obliqua Dyar, r9r 8, ibidem $\mathbf{5 4}: 353$, by original designation. Holotype ․ Mexico (USNM, Washington).

MASALIA Moore, I881, Proc. zool. Soc. Lond. 1881: 364.

NOCT

Type-species: Masalia radiata Moore, I88I, ibidem 1881:364, by original designation. Lectotype ô. India: [Uttar Pradesh] N.W., [Maimpuri] Manpuri (BMNH), designated by Seymour, 1972, Bull. Br. Mus. nat. Hist. (Ent.) 27: 52.

MASCA Walker, [1859] I858, List Specimens lepid. Insects Colln Br. Mus. 16: 8.

OPHI Type-species: Masca abactalis Walker, [1859] 1858, ibidem 16: 9, by monotypy. Holotype $\hat{0}$. Singapore (UM, Oxford).

See also Phagytra Walker, [I866].

MASEBIA Walker, 1858, List Specimens lepid. Insects Colln Br. Mus. 15: I771.

OPHI Type-species: Masebia famelica Walker, I858, ibidem 15: I772, by monotypy. Holotype $\hat{o}$, [Dominican Republic] St. Domingo (BMNH).

$M$. famelica is a junior subjective synonym of Elousa albicans Walker, [1858] I857, ibidem 13: Ir 8.

See also Elousa Walker, [1858].

MASOGA Walker, I863, List Specimens lepid. Insects Colln Br. Mus. 27: i6.

OPHI

Type-species: Masoga panagralis Walker, I863, ibidem 27: I6, by monotypy. Type(s) ô. [BRAzIL]: Rio Janeiro.

MASSAGA Walker, I 854, List Specimens lepid. Insects Colln Br. Mus. 2: 358.

AGAR

Type-species: Phalaena hesparia Cramer, I775, Uitlandsche Kapellen 1: 87, p1. 56. fig. C, by monotypy. Type(s), TYPE-Locality stated as 'Demerary', but this was probably an error as the species illustrated is not known to occur in the New World but is widespread in WEST AFrICA.

+ MESSAGA; Butler, I868, Proc. zool. Soc. Lond. 1868: 223.

An incorrect subsequent spelling.

MASSAGIDIA Hampson, Igor, Cat. Lepid. Phalaenae Br. Mus. 3: xix, 644.

Type-species: Phalaena hesparia Cramer, I775, by original designation.

Hampson, I901, ibidem 3: 642 designated, incorrectly, Massaga maritona Butler, I868, as the type-species of Massaga Walker, 1854. Hampson then proposed Massagidia 
for $P$. hesparia. The only justification that I can find for this action is that on page 643 , Hampson placed 'Massaga hesparia, Wlk. ii. $35^{S}$ ( 1854 ), nec Cram.' as a synonym of Massaga virescens Butler, 1874 , thus implying that Walker misidentified P. hesparia Cramer. This is not so. Walker's redescription of hesparia Cramer agrees with Cramer's original figure. Walker, however, did add 'Var. $\beta$ ' which Hampson in his manuscript notes has placed as M. virescens Butler.

MASSAGIDIA Hampson, I901. See Massaga Walker, I854.

AGAR

MASSALA Walker, 1865, List Specimens lepid. Insects Colln Br. Mus. 33: $977 . \quad$ орні

Type-species: Massala dimidiata Walker, 1865, ibidem 33: 977, by monotypy. Holotype of. [WEST INDIES]: Jamaica (BMNH).

M. dimidiata is a junior subjective synonym of Azeta quassa Walker, i 858 , ibidem 15: I $58 \mathrm{I}$.

MASSAVA Walker, I 865, List Specimens lepid. Insects Colln Br. Mus. 33: I Iog.

OPHI

Type-species: Massava scissa Walker, i 865, ibidem 33: i i o, by monotypy. Holotype $\hat{o}$, Venezuela (BMNH).

MASTIGIA Schaus, I916, Proc. U.S. natn. Mus. 50: 321.

HYPE

Type-species: Mastygophora epitusalis Walker, [1 859] I 858, List Specimens lepid. Insects Colln Br. Mus, 16: I 50, by original designation. Syntypes $+3{ }^{*}$. Venezuela (BMNH).

$\ddagger_{\dagger}$ MASTIGNATHA Hampson, no published reference found.

HYPE

† MASTIGOPHORA, misspelling. See Mastigophorus Poey, I 832 .

HYPE

MASTIGOPHORUS Poey, i 832, Centurie Lépid. Ile Cuba (1): Num.[8].

HYPE

Type-species: Mastigophorus parra Poey, I 832, ibidem (1): Num. [8], fig., by monotypy.

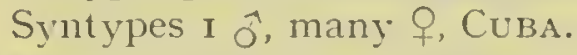

¥MAST YGOPHORA; Walker, [1850] I858, List Specimens lepid. Insects Colln Br. Mus. 16: 149 .

An incorrect subsequent spelling.

†MASTIGOPHORA; Felder \& Rogenhofer, 1875, Reise öst. Fregatte Novara (Zool.) 2 (Abt. 2): pl. I20, fig. II ; Inhalts-Verz.: I 3 .

An incorrect subsequent spelling, but cited by Neave, 1940, Nomencl. zool. 3: 54, as though an arailable name.

$\ddagger$ Mastigophora; Haase, 1888, Corresp.-Bl. ent. Ver. Iris Dresden 1: 327, an incorrect subsequent spelling, was also cited by Neave, but as an error.

MASTIPHANES Grote, I 882, New Check List N. Am. Moths: 23.

ACRO

Type-species: Acronycta xylinoides Guenée, 1852 , in Boisduval \& Guencee, Hist. nat. Insectes (Lépid.) 5: 56 (but included by Grote as xyliniformis Guenéc, an unnecessary replacement name), by subsequent designation by Grote, I895 May, Abh. naturn. Ver. Bremen 14: 55, but cited as xyliniformis Guenée. Lectotype +. [U.S.A.]: Géorgie (MNHN, Paris), designated by Viette, 195I, Bull. mens. Soc. linn. Lyon 20: i 59 (not by Hampson, 1909, Cat. Lepid. Phalaenae Br. Mus. 8: I57, as stated by Viette, because Hampson merely cited 'Type xylinoides in Mus. Paris').

Acronycta xyliniformis Guenéc, I 852, ibidem 7: 4 oo is an unnecessary replacement name: for A. xylinoides. Both are junior subjective synonyms of Acronycta longa Guence, i 852 , ibillem 5: 54 .

MASTIXIS Schaus, I913, Ann. Mag. nat. Hist. (8) 11:8.

IIY PE

Type-species: Mastixis chloe Schaus, 1913 , ibidem (8) 11: 8, by original designation. Type(s) ô, Costa Rica: Juan Vinas.

†MAST YGOPHORA, misspelling. Sec Mastigophorus Poey, I 832 .

HYPE 
MATAEOMERA Butler, I886, Trans, ent. Soc. Lond. 1886: 4I 8.

ACON

Type-species: Mataeomera dubia Butler, 1886, ibidem 1886: 4I8, by monotypy. Syntypes, [Australia]: [Qd], Peak Downs (BMNH); Gayndah (BMNH).

MATELLA Moore, [1885]. See Talmela nom. n.

OPH I

MATHURA Moore, I882, in Hewitson \& Moore, Descr. new Indian lepid. Insects Colln late Mr W. S. Atkinson: I88. HYPE

Type-species: Mathura albisigna Moore, I882, ibidem: 188, by monotypy. Syntypes o. India: [Assam] Cherra Punji (BMNH).

MATIGRAMMA Grote, I 872, Trans. Am. ent. Soc. 4: 22.

CATO

Type-species: Matigramma pulverilinea Grote, I872, ibidem 4: 22, by monotypy. Syntypes $\hat{0}$, ㅇ․ [U.S.A.]: Central Alabama (BMNH).

MATILOXIS Schaus, I9i6, Proc. U.S. natn. Mus. 50: 264, 380.

OPH I

Type-species: Rhaesena rubromarginata Schaus, 1906, Proc. U.S. natn. Mus. 30: 107, by original designation. Holotype, Mexico: Jalapa (USNM, Washington).

MATOPO Distant, I898, Ann. Mag. nat. Hist. (7) 1: 227.

ACRO

Type-species: Matopo typica Distant, I898, ibidem (7) 1:227, by original designation, under the Code, Article 68 (b). Type(s), [South Africa]: Transvaal, Pretoria (BMNH).

MATUTA Grote, I874. See Cerastis Ochsenheimer, I8I6.

NOCT

MAURILIA Möschler, I884, Verh. zool.-bot. Ges. Wien 33 (Abh.) : 298.

CHLO

Type-species: Maurilia lubina Möschler, I884, ibidem 33 (Abh.): 298, pl. I6, fig. I3, by monotypy. Syntypes $2 \hat{0}$. [South Africa]: Kaffernlandes.

$M$. lubina is a junior subjective synonym of Xanthodes arcuata Walker, [1858] I857, List Specimens lepid. Insects Colln Br. Mus. 12: 779 .

MAURILIADIA Berio, 1966, Annali Mus. civ. Stor. nat. Giacomo Doria 76: I 25.

CHLO

Type-species: Mauriliadia obscura Berio, I966, ibidem 76: I26, fig. I5, by original designation. Holotype $\hat{\sigma}$. [CAmeroun] Kamerun: Misellele (Colln E. Berio, Genoa).

MAXERA Walker, I865, List Specimens lepid. Insects Colln Br. Mus. 33: 8o9.

OPH I

Type-species: Maxera subocellata Walker, I865, ibidem 33: 809, by monotypy. Holotype $\hat{0}$. West Africa (BMNH).

MAXIA Heyden, I89I, in Saalmüller, Lepid. Madagascar: 488.

HYPE

Type-species: Maxia decora Saalmüller, I89I, ibidem: 489, fig. 207, by monotypy. Syntypes 2 ex., Madagascar: N.W., Nossi-Bé I. (SNG, Frankfurt).

MAXILUA Walker, [1863] i 864, J. Proc. Linn. Soc. (Zool.) 7: i68.

ACRO

Type-species: Maxilua frontalis Walker, [1863] 1864, ibidem 7: I69, by monotypy. Lectotype 9. Borneo: Sarawak (UM, Oxford), designated (as type) by Swinhoe, Igoo, Cat. east. and Aust. Lepid. Heterocera 2: 73.

$M$. frontalis is a junior subjective synonym of Dyrzela incrassata Walker, I858, List Specimens lepid. Insects Colln Br. Mus. 15: I759.

MAXULA Walker, I865, List Specimens lepid. Insects Colln Br. Mus. 33: Iog6.

Type-species: Maxula idonea Walker, I865, ibidem 33: 1096, by monotypy. Holotype o. [INDIA]: Hindostan (BMNH).

$M$. idonea is a junior subjective synonym of Hypopyra unistrigata Guenée, 1852 , in Boisduval \& Guenée, Hist. nat. Insectes (Lépid.) 7: 20I, pl. 2I, fig. I.

MAZACYLA Walker, 1865, List Specimens lepid. Insects Colln Br. Mus. 33: 945.

OPHI

Type-species: Mazacyla fusifera Walker, 1865 , ibidem 33:945, by monotypy. Holotype o., [BRazIL]: Rio Janeiro (BMNH).

$\mathrm{U}$ 
MAZUCA Walker, 1866, List Specimens lepid. Insects Colln Br. Mus. 35: 1777.

ACRO

Type-species: Mazuca strigicincta Walker, I866, ibidem 35: 1777, by monotypy. Type(s) ㅇ, [AFrICA]: S.E., Zambezi River.

${ }_{+}^{+}$MECISTOGNATHA Hampson, no published reference found.

HYPE

MECISTOPTERA Hampson, I893, Illust. typical Specimens Lepid. Heterocera Colln Br. Mus. 9:32, 134. HYPE

Type-species: Mecistoptera griseifusa Hampson, I893, ibidem 9: 32, I34, pl. I68, fig. I4, by monotypy. Syntypes $\hat{\sigma}, q$, Ceylox: Kelani Valley (BMNH).

MECODINA Guenée, I852, in Boisduval \& Guenée, Hist. nat. Insectes (Lépid.) 7: 372 . OPHI Type-species: Mecodina lanceola Guenée, 1852, ibidem 7:373, pl. 24, fig. 5, by monotypy. Holotype ô. [Bangladesh]: [E. Bengal], Silhet (BMNH).

MECODINOPS Hampson, 1926, Descr. new Genera Species Lepid. Phalaenae Subfamily Noctuinae Br. Mus.: 488 .

OPHI

Type-species: Thermesia anceps Mabille, I879, Bull. Soc. philomath. Paris (7) 3: 142, by original designation. Lectotype q, MADAGASCAR (BMNH), designated (as holotype) by Viette \& Fletcher, 1968, Bull. Br. Mus. nat. Hist. (Ent.) $21: 416$.

MECODOPSIS Hampson, 1926, Descr. new Genera Species Lepid. Phalaenae Subfamily Noctuinae Br. Mus.: 234. OPHI

Type-species: Mecodopsis conisema Hampson, I926, ibidem: 235, by original designation. Holotype +, [GHaNa] Gold Coast: Bibianaha (BMNH).

MECOPTERA Guenće, I837. See Eupsilia Hübner, [1821 ].

CUCU

MEC YRA Walker, I865, List Specimens lepid. Insects Colln Br. Mus. 33: 979.

OPHI

Type-species: Mecyra invaria Walker, 1865 , ibidem 33: 980, by monotypy. Holotype o. Java (BMNH).

$M$. invaria is a junior subjective synonym of Calesia gastropachoides Guenée, I852, in Boisduval \& Guenée, Hist. nat. Insectes (Lépid.) 7 : 258.

MEDAVA Walker, I863, List Specimens lepid. Insects Colln Br. Mus. 27: I I3.

SARR

Type-species: Medava diminuens Walker, 1863, ibidem 27: II3, by monotypy. Holotype $\hat{o}$, [BraziL]: [R. Amazon], [Tefé] Ega (BMNH).

$\ddagger$ MEDOPTA, misspelling. See Metopta Swinhoe, 1900 .

CATO

MEGACEPHALOMANA Strand, 1943, Folia zool. hydrobiol. 12: 2 I I.

OPHI

Type-species: Megacephalon rivulosum Saalmüller, I88o, Ber. senckenb. naturf. Ges. 1879-80: 286, by monotypy (of Megacephalon Saalmüller, 1880). Syntypes $\hat{\sigma}, \quad$, Madagascar (SNG, Frankfurt).

Megacephalomana was proposed as the objective replacement name for Megacephalon Saalmüller, I 880 .

MEGACEPHALON Saalmüller, 1880, Ber. senckenb. naturf. Ges. 1879-80: 286.

Type-species: Megacephalon rivulosum Saalmüller, I880, by monotypy.

A junior homonym of Megacephalon Temminck, in Gray, 1844, List Birds Br. Mus.

3: 21, - Aves. The objective replacement name is Megacephalomana Strand, $19+3$.

MEGACEPHALON Saalmiiller, I880. See Megacephalomana Strand, I9ł3. OPHI

MEGACHYTA Grote, r873, Trans. Am. ent. Soc. 4: $306 . \quad$ HyPE

Type-species: Epizeuxis lituralis Hübner, 1818, Zuträge Samml. exot. Schmett. 1: 9, figs 19,20 , by monotypy. 'Type(s) ô. [U.S.A.]: Florida [formerly] in Georgia.

$\ddagger$ ERPYZON Hübner, I808, Erste Zuträge Samml. exot. Schmett.: 4, 5 .

Included in a work rejected for nomenclatural purposes by Int. Commn zool. Nom., 
I966, Bull. zool. Nom. 23 Opinion 789:2I4. Placed on the Official Index of Rejected and Invalid Generic Names in Zoology: Name No. I84 I.

$\ddagger$ Erpyzon lituralis Hübner, I808, ibidem: 4, was subsequently made nomenclaturally available as Epizeuxis lituralis Hübner, I818, and cited as type-species by Berio, I957, Memorie Soc. ent. ital. 36: I3.

MEGACRONYCTA Grote, I873, Bull. Buffalo Soc. nat. Sci. 1: 79.

ACRO

Type-species: Phalaena hastulifera Smith, I797, in Smith \& Abbot, Nat. Hist. lepid. Insects Georgia 2: ז83, pl. 92, by original designation. Syntypes, [U.S.A.]: Georgia and Virginia.

MEGALOCTENA Warren, I9I3, in Seitz, Gross-Schmett. Erde 3: 4I2.

HYPE

Type-species: Nodaria mandarina Leech, I900, Trans. ent. Soc. Lond. 1900: 644, by original designation. Syntypes I I $\widehat{0}, 5$ ㅇ, China: [Szechwan], Moupin (BMNH).

The date of publication of the part of Seitz containing page $4 \mathrm{I} 2$ was stated on page 405 as I9I3 December ist in both the German and English editions.

MEGALODES Guenée, I852, in Boisduval \& Guenée, Hist. nat. Insectes (Lépid.) 6: 26 I. ACro

Type-species: Cosmia eximia Freyer, 1845, Neueve Beitr. Schmett. 5: 104, pl. 442, fig. 3, by monotypy. Type(s), Turkey: European.

MEGALONYCTA Viette, 1965, Bull. Soc. ent. Fr. 70: 86.

ACRO

Type-species: Acronycta mediovitta Rothschild, 1924, Ann. Mag. nat. Hist. (9) 14: 3I2, by original designation. Lectotype $\hat{\jmath}$, MadaGASCAR: Diego Suarez (BMNH), designated by Viette, I965, Faune Madagascar 20(I): 482 .

MEGALOPTERA Bethune-Baker, I908, Novit. zool. 15: 2 Io.

HYPE

Type-species: Megaloptera lophota Bethune-Baker, I908, ibidem 15: 210, by original designation. Syntypes $\delta$, New Guinea: [Papua], Kebea Range (BMNH); R. Aroa.

M. lophota is a junior subjective synonym of Piratisca minax Meyrick, I902.

See also Piratisca Meyrick, I902; and Systaticospora Bethune-Baker, I908.

Megaloptera is not preoccupied by $¥$ Megaloptera; Ashmead, I899, Trans. Am. ent. Soc. 26: 92, an incorrect subsequent spelling of Megalopta Smith, 1853, Cat. Hym. Insects Br. Mus. 1:83,-Hymenoptera.

MEGALOPTEROIDES Strand, I9I0, Ent. Rdsch. 27: I62.

Proposed, unnecessarily, as an objective replacement name for Megaloptera BethuneBaker, I908, which Strand considered to be a homonym of the sub-order Megaloptera in the order Neuroptera. The name Megalopteva was not, however, nomenclaturally available as a genus-group name in the Neuroptera.

MEGALOPTEROIDES Strand, I9io. See Megaloptera Bethune-Baker, Igo8. HyPE

MEGANEPHRIA Hübner, [I820] I816, Verz. bekannter Schmett.: 206.

CUCU

Type-species: Phalaena bimaculosa Linnaeus, I767, Syst. Nat. (Edn I2) 1: 856, by subsequent designation by Butler, 1890, Trans. ent. Soc. Lond. 1890: 670. Type(s), GERMANY (LS, London).

MEGANOLA Dyar, I898, $J l N . Y$. ent. Soc. 6: 43.

NOLI

Type-species: Meganola conspicua Dyar, I898, ibidem 6:43, by monotypy. Syntypes 3 ㅇ. [U.S.A.]: Texas; Colorado; Arizona, Fort Grant. (USNM, Washington).

${ }_{\ddagger}$ MEGARENIA Hampson, no published reference found.

HYPE

MEGASEMA Hübner, [I82I] I 8I6, Verz. bekannter Schmett.: 222.

NOCT

Type-species: Phalaena triangulum Hufnagel, I 766, Berlin. Mag. 3 (3):306, by subsequent designation by Moore, I881, Proc. zool. Soc. Lond. 1881: 352. Type(s), [Germany]: Berlin. 
MEGASTOPOLIA Hampson, igr8, Novit. zool. 25: ig6.

OPH I

Type-species: Polia ameria Druce, I 890, Proc. zool. Soc. Lond. 1890: 5I5, by original designation (for Poliothripa Hampson, I9I2). Holotype $\hat{\sigma}$. Guatemala: Volcan de Atitlan (BMNH).

Megastopolia was proposed as the objective replacement name for Poliothripa Hampson, I9I 2.

POLIOTHRIPA Hampson, igi2, Cat. Lepid. Phalaenae Br. Mus. 11: xii, 333

Type-species: Polia ameria Druce, I 890, by original designation.

A junior homonym of Poliothripa Hampson, i902,-Lepid., Nolidae. The objective replacement name is Megastopolia Hampson, I9I8.

MEGATHRIPA Hampson, i 91 2, Cat. Lepid. Phalaenae Br. Mus. 11: xi, 319.

SARR

Type-species: Sarrothripa rufimedia Hampson, 1905, Ann. S. Afr. Mus. 3: 429, by original designation. Type(s) $q$, RHodesia: Bulawayo (BMNH).

MEGATOMIS Hübner, I821. See Salia Hübner, [1818]. HYPE

MEGAZETHES Warren, I9I3. See Arytruva John, I9I2. OPH

MEGHYPENA Grote, I 873, Bull. Buffalo Soc. nat. Sci. 1: 86.

HYPE

Type-species: Meghypena velifera Grote, I 873 , ibidem 1:87, pl. 2, fig. 7, by subsequent designation by Grote, 1874, ibidem 2: 52. Type(s) ․․ [U.S.A.]: N.Y., Sharon Springs.

$M$. velifera is a junior subjective synonym of Hypena edictalis Walker, [1859] 1858, List Specimens lepid. Insects Colln Br. Mus. 16: 28.

MEGISTOCLISMA Hampson, I9I3, Cat. Lepid. Phalaenae Br. Mus, 12: 9. Available, but without included species until Hampson, I9I3, ibidem 13: vii, 55 .

Type-species: Nyctipao ribbei Pagenstecher, I886, Jb. nassau. Ver. Naturk. 39: I 4 o, pl. Io, fig. 2, by subsequent monotypy. Type(s) 9 , Moluccas: Aru I.

${ }_{\ddagger}$ MEGOSONA, misspelling. See Mesogona Boisduval, i 840 .

NOCT

MEIZOGLOSSA Hulstaert, 1924, Ann. Mag. nat. Hist. (9) 13: 100.

ACON

Type-species: Meizoglossa bipunctata Hulstaert, I924, ibidem (9) 13: 100, by original designation. Holotype $\hat{o}$, [Moluccas]: Tenimber, Jamdena, Olilit.

MELAGRAMMA Hampson, I908, Cat. Lepid. Phalaenae Br. Mus. 7: xv, 69i.

ACRO

Type-species: Bryophila expetita Walker, I858, List Specimens lepid. Insects Colln. Br. Mus. 15: I647, by original designation. Type(s), [BrazIL]: Rio Janeiro (UM, Oxford).

MELALEUCANTHA Draudt, 1950, Mitt. münch. ent. Ges. 40: I 37.

$\operatorname{ACON}$

Type-species: Melaleucantha albibasis Draudt, 1950, ibidem 40: 137, pl. 8, fig. 27 , by monotypy. Syntypes I $\hat{0}$, I $q$, China: [Szechwan Prov.], Mien-shan (MAK, Bonn).

MELALEUCIA Hampson, I9oo, Cat. Lepid. Phalaenae Br. Mus. 2: 4, 78.

NOLI

Type-species: Nola obliquifasciata Hampson, I896, Fauna Br. India (Moths) 4: 503.

by original designation. Holotype $q$, CEYLON (BMNH).

MELAMERA Hampson, igio, Cat. Lepid. Phalaenae Br. Mus. 10: 8, 327.

ACO.N

Type-species: Melamera velutina Hampson, I910, ibidem 10:327, fig. 94, by original

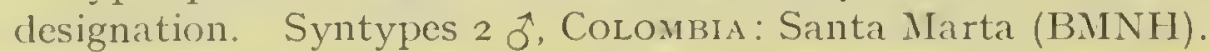

†MALAMERA; Grünberg, I912, Avch. Naturgesch. 77 (4) 2: 139.

An incorrect subsequent spelling.

MELANCHRA Hübner, [1820] i 8 I6, Verz. bekannter Schmett.: 207.

HADI:

Type-species: Phaldena persicariae Linnaeus, I761, Fauna Suecica (Edn 2): 319, by monotypy. Type(s), SwEDEN (IS, I,ondon). 
MELANCHROIA Hübner, [1819] I816, Verz. behannter Schmett.: I73.

GEOMETRIDAE

Type-species: Phalaena chephise Stoll, I782, in Cramer, Uitlandsche Kapellen 4: I 82, pl. 38I, fig. E (but included by Hübner as ¥'cephise Cram.', an incorrect subsequent spelling), by subsequent designation by Hulst, I 896, Trans. Am. ent. Soc. 23: 386, but cited as †'cephise Cr.'

Melanchroia Hübner has been used incorrectly in the Noctuidae based on Phalaena leontia Stoll, I790, the first of the originally included species. The correct usage is in the Geometridae, Ennominae.

MELANCHROIOPSIS Dyar, I9I 8, Proc. U.S. natn. Mus. 54:34I.

AGAR

Type-species: Melanchroiopsis acroleuca Dyar, I9I8, ibidem 54: 34 I, by original designation. Holotype $\hat{\jmath}$, Mexico: Sierra de Guerrero (USNM, Washington).

MELANEPHIA Le Cerf, 1922, Voyage Baron M. Rothschild Ethiopie Afr. orient. anglaise 1: 426.

OPHI

Type-species: Melanephia cinereovariegata Le Cerf, 1922, ibidem 1: 426, fig. 9, by monotypy. Holotype $\hat{0}$, [Kenya] Afr. Orient. Anglaise: Simba.

Melanephia was attributed to Warren by Le Cerf.

MELANEPHIA Hampson, 1926, Descr. new Genera Species Lepid. Phalaenae Subfamily Noctuinae Br. Mus.: 74 .

Type-species: Anophia nigrescens Wallengren, I856, Anteckningar $i$ Zool. 1: 64, by original designation. Type(s), [South AfrICA]: Kafferlandet (NR, Stockholm).

A junior homonym of Melanephia Le Cerf, I922. This generic name probably originated from Hampson, but was attributed to Warren by Le Cerf. It was used and unintentionally made nomenclaturally available by Le Cerf prior to its proposal and generic description by Hampson for a similar concept but having a different type-species. There is no objective replacement name but $A$. nigrescens is congeneric with Melanephia cinereovariegata Le Cerf, I922, the type-species of Melanephia Le Cerf, i922. The latter is therefore available for use as a subjective replacement name.

MELANEPHIA Hampson, 1926. See Melanephia Le Cerf, I922. OPH

MELANOGRAPHIA Hampson, i9oo, Cat. Lepid. Phalaenae Br. Mus. 2: $48 . \quad$ Noli

Type-species: Nola flexilineata Hampson, i 898, J. Bombay nat. Hist. Soc. 11: 440, by original designation. Holotype $\hat{\sigma}$. India: Assam, Khasis (BMNH).

MELANOMMA Grote, i875, Trans. Am. ent. Soc. 5: i I7.

OPHI

Type-species: Melanomma auricinctaria Grote, 1875, ibidem 5: I 7 , by monotypy. Type(s) $\hat{o}$, [U.S.A.]: Pennsylvania.

MELANOPHTHALMA Hampson, 1926. See Marojala Viette, 1966.

OPHI

$\ddagger$ MELANOPIS Hampson, no published reference found.

HYPE

$\ddagger$ MELANOTICA Hampson, no published reference found.

HYPE

MELAPERA Hampson, I908, Ann. Mag. nat. Hist. (8) 1: 488.

OPHI

Type-species: Melapera roastis Hampson, I908, ibidem (8) 1: 488, by monotypy. Holotype 9 , Madagascar: Forêt d'Ambre (BMNH).

MELAPIA Sugi, i 968, Kontyî́ 36: I64.

CATO

Type-species: Pelamia japonica Ogata, r96I, Publs ent. Lab. Univ. Osaka Prefect. 6: 79, fig. A, pl. I8, fig. E, by original designation. Holotype ô. JAPAN: Shikoku I., Tosa, Cape Muroto (Ehime Univ., Shikoliu).

MELAPORPHYRIA Grote, i 874, Bull. Buffalo Soc. nat. Sci. 2: 33, 75.

NOCT

Type-species: Melaporphyria immortua Grote, 1874 , ibidem 2: 75, by original designation. Syntypes, [U.S.A.]: Albany and Cambridge. 
MELENETA Smith, 1908, Ann. N.Y. Acad. Sci. 18: 92.

Type-species: Meleneta antennata Smith, I908, ibidem 18: 93, by monotypy. Syntypes 2 Oे, [U.S.A.]: Arizona, Huachua Mts and Cochise County, Palmerly.

MELIA Curtis, I 828. See Aphomia Hübner, [1825].

MELIABA Walker, [1859] 1858, List Specimens lepid. Insects Colln Br. Mus. 16: 2 I 2. OPHI Type-species: Meliaba pelopsalis Walker, [1859] I 858, ibidem 16: 212, by monotypy. Holotype $\widehat{\sigma}$. [South Africa]: Port Natal (BMNH).

MELIANA Curtis, 1836. See Senta Stephens, I834.

HADE

MELICLEPTRIA Hübner, [i 823] i 8I6, Verz. bekannter Schmett.: 262. NOCT

Type-species: Phalaena cardui Hübner, I790, Beitr. Gesch. Schmett. 2 (4): 84, pl. I, fig. B (a junior primary homonym), by subsequent designation by Grote, I873, Bull. Buffalo Soc. nat. Sci. 1: i 6 , but cited as cardui Esper, an incorrect authorship. Type(s) o., [Austria]: Vienna district.

Phalaena (Noctua) cardui Hübner, I790, is a junior primary homonym of Phalaena (Tortrix) cardui Ström, I783, Nye Saml. K. dansk. Vid. Selsk. Skr. 2: 87. There is no objective replacement name but a subspecific name Heliothis cardui purpurata Staudinger, I90I, in Staudinger \& Rebel, Cat. Lepid. palaearct. Faunengeb. (I): 22I, is available and could be used as a subjective replacement name, i.e. Melicleptria purpurata (Staudinger).

The senior homonym Phalaena cardui Ström has remained unused for over I 50 years, probably ever since it was published. The original description does not really fit any known species, and no Ström types are extant. In my opinion in this case the Int. Commn zool. Nom. should be asked to suppress the senior homonym under the Code, Article 79, for the purposes both of the Law of Priority and of the Law of Homonymy.

ANTHOECIA Boiscluval, i 840, Genera Index meth. Eur. Lepid.: i 62.

Type-species: Phalaena cardui Hübner, 1790 (but included by Boisduval as cardui Esper, an incorrect authorship), by subsequent designation by Guenée, 1852, in Boisduval \& Guenée, Hist. nat. Insectes (Lépid.) 6: I 87, but cited as cardui Esper.

Anthoecia is a junior objective synonym of Melicleptria Hübner, [1823].

TRYPANA Guenée, i 84I, Annls Soc. ent. Fr. 10: 58.

Type-species: Phalaena cardui Hübner, I 790, by subsequent designation by Desmarest, I 848, in d'Orbigny, Dict. univl Hist. nat. 12: 7 I I.

Trypana is a junior objective synonym of Melicleptria Hübner, [1823].

MELIONICA Berio, I970, Boll. Soc. ent. ital. 102: 25.

HADE

Type-species: Melionica citrinea Berio, I970, ibidem 102: 25, fig. 5, by original designation. Holotype $\hat{\delta},\left[Z_{A I R E}\right]$ : [Katanga], [Lubumbashi] Elizabethville (MRAC, Tervuren).

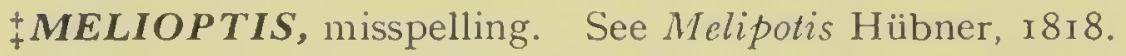

OPHI

MELIPOTIS Hübner, r8ı, Zuträge Samml. exot. Schmett. 1: i 7.

OPH I

Type-species: Melipotis jucunda Hübner, I 8I 8, ibidem 1: I7, figs 8I, 82, by monotypy.

Type(s) $\hat{o}$. [U.S.A.]: Georgia (formerly] in Florida.

† MELIOPTIS; Swinhoe, 1900, Ann. Mag. nat. Hist. (7) 6:3 I I.

An incorrect subsequent spelling.

†HELIOTHIS Hübner, I808, Evste Zuträge Samml. exot. Schmett.: 5 .

Inclucled in a work rejected for nomenclatural purposes by Int. Commn zool. Nom., I966, Bull. zool. Nom. 23 Opinion 789: 2 I5. Placed on the Official Index of Rejected and Invalid Generic Names in Zoology: Name No. 1857.

Only included species: †Heliothis jucunda Hübner, 1808 , subsequently made nomenclaturally available as Melipotis jucunda Hübner, I818. 
MELLINIA Hübner, [I821] I816, Vevz. bekannter Schmett.: 235.

CUCU

Type-species: Noctua palleago Hübner, [1803], Samml. eur. Schmett. 4: pl. 40, fig. 192, by subsequent designation by Hampson, I906, Cat. Lepid. Phalaenae Br. Mus. 6: 497. Type(s), Europe.

$N$. palleago is a junior subjective synonym of Noctua gilvago [Denis \& Schiffermüller], I775, Ankïndung syst. Werkes Schmett. Wienergegend: 87.

MENADA Kozhanchikov, I937, Fauna SSSR (Insecta) Lepid. 13 (3): 593, 65 I. NOCT

Type-species: Agrotis nomas Erschov, 1874 , in Fedchenko, Reise in Turkestan 2 (5) 3: 38 , pl. 3, fig. 36, by original designation. Syntypes $\hat{0}$, ㅇ, [U.S.S.R.]: Turkestan, Cocandensi near Karakasuk (ZI, Leningrad). [IRAN]: Isfairam.

MENDOZANIA Köhler, (1952) I95I, Acta zool. lilloana 12: I 45.

CUCU

Type-species: Mendozania sanmartini Köhler, (I952) I95I, ibidem 12: I45, by original designation. Syntypes + , Argentina: [Mendoza Prov.], Mendoza (ZSBS, Munich).

MENECINA Walker, I858, List Specimens lepid. Insects Colln Br. Mus. 15: I548, I 585. OPHI Type-species: Menecina bifacies Walker, 1858 , ibidem 15: 1585 , by monotypy. Holotype $\hat{\sigma},[$ Brazil $]:[R$. Amazon], [Tefé] Ega (BMNH).

MENEPTERA Hampson, igro, Cat. Lepid. Phalaenae Br. Mus. 10 : xvii, 640. $\mathrm{ACON}$

Type-species: Tarache diopis Hampson, 1905, Ann. S. Afr. Mus. 3: 435, by original designation. Type(s) $\hat{\sigma}$, [Sourh Africa]: Transvaal, Piet Retief (BMNH).

MENOPSIMUS Dyar, 1907, Jl N.Y. ent. Soc. 15: 1 I o.

HYPE

Type-species: Menopsimus caducus Dyar, I907, ibidem 15: I10, by monotypy. Syntypes 9 ex., [U.S.A.]: New Hampshire, Hampton; D.C., Washington (USNM, Washington).

MENTAXYA Geyer, I837, in Hübner, Zuträge Samml. exot. Schmett. 5: 10. Noct Type-species: Mentaxya muscosa Geyer, I837, ibidem 5: 10, figs 827, 828, by subsequent designation by Viette, 1958, Lambillionea 58: $4 \mathrm{I}$. Type(s) Õ. South AFrica. METAXYA Berio, 1955, Revue Zool. Bot. afr. 51: 212.

An unjustified emendation of Mentaxya Geyer, I 837.

$\ddagger$ MENTAXYIA; Berio, 1955, Revue Zool. Bot. afr. 51: 212.

An incorrect subsequent spelling.

†MENTAXYIA, misspelling. See Mentaxya Geyer, 1837 .

NOCT

MEPANTADREA Hampson, 1926, Descr. new Genera Species Lepid. Phalaenae Subfamily Noctuinae Br. Mus.: 558 .

$\mathrm{OPHI}$

Type-species: Zethes simia Saalmüller, I891, Lepid. Madagascar: 474, pl. Io, fig. I55, by original designation. Type(s), Madagascar (SNG, Frankfurt).

MERANDA Walker, [1866] I865, List Specimens lepid. Insects Colln Br. Mus. 34: I207. opH I Type-species: Meranda latalis Walker, [1866] I865, ibidem 34: I207, by monotypy. Holotype J. [Australia]: [W. Aust.], Swan River (BMNH).

MERIDYRIAS Hampson, I926, Descr. new Genera Species Lepid. Phalaenae Subfamily Noctuinae Br. Mus.: $20 . \quad$ OPHI

Type-species: Yrias progenies Guenée, 1852 , in Boisduval \& Guenée, Hist. nat. Insectes (Lépid.) 7:23, pl. I 5, fig. 10, by original designation. Syntypes, [WEST INDIES]: Île Saint-Thomas and Guadeloupe.

MERISTIDES Strand, I909, Dt. ent. Z. Iris 22: I I 5 .

ACRO

Type-species: Meristis umbripennis Strand, I909, ibidem 22: II4, by monotypy. Holotype , [Tanzania] Dt.-Ostafrica: [L. Nyasa], Ruanda. 
MERISTIS Hïbner, [I82I] I \&I6, Verz. bekannter Schmett.: 239.

ACRO

Type-species: Noctua trilinea [Denis \& Schiffermüller], I775, Ankündung syst. Werkes Schmett. Wienergegend: 84 , by subsequent designation by Grote, 1902, Allg. Z. Ent. 7 : 398 , but cited as Phalaena trigrammica Hufnagel, I 766 , which Grote placed as the senior synonym of $N^{\top}$. trilinea. Type(s), [Austria]: Tienna district (Collection destroyed, Horn \& Kahle, 1936, Ent. Beih. Berl.-Dahlem 3: 243).

$N$. trilinea is a junior subjective synonym of Phalaena trigrammica Hufnagel, i 766 , Berlin. Mag. 3 (4): 408.

See also Charanyca Billberg, I 820 .

MEROLEUCA Hampson, I908. See Moureia Orfila \& Rossi, I956.

ACRO

MEROLONCHE Grote, I882, Illust. Essay Noctuidae N. Am.: 50.

ACRO

Type-species: Apatela spinea Grote, 1875, Bull. Buffalo Soc. nat. Sci. 3: 78, pl. 4. fig. 7, by original designation. Holotype ․ [U.S.A.]: California (BMNH).

$\ddagger$ MEROPIS Hübner, I8I8. See Gonodonta Hübner, I8I8.

OPII

MEROPIS Hübner, 1822, Syst.-alphab. Verz.: 20, 22.

CATO

Type-species: Noctua auricularis Hübner, [1803], Samml. eur. Schmett. 4: pl. 66, fig. 32 I, by subsequent designation by Berio, I957, Memorie Soc. ent. ital. 36: I8. Type(s), Europe.

$N$. auricularis is a junior subjective synonym of Phalaena tirhaca Cramer, i 777.

See also Ophiusa Ochsenhemer, i 8 I6.

MEROPLEON Dyar, 1924, Insecutor Inscit. menstr. 12:2 I.

ACRO

Type-species: Meropleon cosmion Dyar, I924, ibidem 12:2I, by monotypy. Syntypes 4 ô, 2 ․, [U.S.A.]: Louisiana, Elm Park.

MERVIA Darichera, 1961, Ent. Obozr. 40:828.

CUCU

Type-species: Mervia kuznetzovi Daricheva, ibidem 40: 829, figs I-6, by original designation. Holotype $\hat{\sigma}$. U.S.S.R.: Turkmenia, $40 \mathrm{~km} \mathrm{~N}$. of Mary, Akibay (ZI, Leningrad).

MESAEGLE Dumont, 1922, Bull. Soc. ent. Fr. 1922: 242.

OPHI

Type-species: Mesaegle gouzzakouli Dumont, 1922, ibidem 1922: 243, by monotypy. Syntypes $\hat{\jmath}$, 우, [Algreria]: Hamâda d'El Golea, towards Ouargla (MNHN, Paris).

MESAPAMEA Heinicke, 1959, Dt. ent. Z. (N.F.) 6: 103.

ACRO

Type-species: Apamea moderata Eversmann, i843, Bull. Soc. Nat. Moscou 16 (35): 547, by original designation. Type(s), [U.S.S.R.]: Urals (ZI, Leningrad).

LAMPETIA Boie, I837, Naturhist. Tidsskr. 1: 536.

Type-species: Noctua didyma Esper, 1788, Die Schmett. 4 (2) Abschnitt I: pl. 126, fig. 7; 1 706 , ibidem: 378 , by PRESENT DESIGNATION. Syntypes ô. Geirmans:

A junior homonym of Lampetia Meigen, I800, Nouv. Classification Mouches: 34, Diptera. There is no objective replacement name but $N$. didyma is a junior subjective synonym of Phalaena secalis Linnaeus, 1758 , which is congeneric with Apamea moderata Eversmann, the type-species of Mesapamea Heinicke, 1959. The latter is thus available for use as a subjective replacement name.

MESASTERIA Hampson, 1926, Descr. new Genera Species Lepid. Phalaenae Subfamily Noctuinae Br. Mus.: 415 .

OPIII

Type-species: Mesasteria sanguilinea Hampson, 1926, ibidem: 415 , by original designation. Holotype q, Borneo: [Sabah], Sandakan (BMNH).

MESEMBRAGROTIS Barnes \& Benjamin, 1927, Pan-Pacific Ent. 4: 4. Noct

Type-species: Mesembragrotis ruckesi Barnes \& Benjamin, 1927, ibidem 4: 4, by original designation. Holotype $\hat{\sigma}$, [U.S.A.]: Texas, near $\mathrm{Al}_{\mathrm{l}}$ pine. 
MESEMBREOSA Hulstaert, 1924, Ann. Mag. nat. Hist. (9) 13: 103.

Type-species: Mesembreosa albatra Hulstaert, 1924, ibidem (9) 13: 103, by original designation. Holotype $\hat{\sigma},[$ Moluccas]: Tenimber, Jamdena, Olilit.

MESEMBREUXOA Hampson, I903, Cat. Lepid. Phalaenae Br. Mus. 4: 8, I37. Noct Type-species: Mesembreuxoa chilensis Hampson, I903, ibidem 4: I37, fig. 49, by original designation. Syntypes $4 \hat{\jmath}, 2$ q, Chile: Coquimbo (BMNH).

MESOCOPSIS Warren, I913, in Seitz, Gross-Schmett. Erde 11: 2 I6. ACON

Type-species: Eupithecia posticata Walker, I866, List Specimens lepid. Insects Colln Br. Mus. 35: I678, by original designation. Type(s) 9 , Borneo (UM, Oxford).

The date of publication of the part of Seitz containing page 2 I 6 was stated on page 209 as I9I 3 August 2oth in both the German and the English editions.

MESOCRAPEX Matsumura, I929, Insecta matsım. 3: i i8.

ACRO

Type-species: Mesocrapex punkikonis Matsumura, I929, ibidem 3: I I8, by original designation. Syntypes $2 \hat{0}, 2$ q, [Tarwan] Formosa: Punkiko (EIHU, Sapporo).

MESOEUXOA Corti, I932, in Seitz, Gross-Schmett. Erde 3 Suppl.: $38 . \quad$ Noct Type-species: Phalaena lidia Stoll, I782, in Cramer, Uitlandsche Kapellen 4: 222, pl. 396, fig. D, by subsequent designation by Kozhanchikov, I937, Fauna SSSR (Insecta) Lepid. $13(3): 52 \mathrm{I}$. Type(s), Type-Locality stated to be [Guyana]: Berbice, but this was probably an error as the species illustrated by Stoll is not known to occur in the New World but is found in Central Europe.

The date of publication of the part of Seitz containing page 38 was stated on page 33 as 1932 January 26th in both the German and the English editions.

Mesoeuxoa Corti is not strictly nomenclaturally available under Article I3 (6) as it was published after 1930 and had no definite fixation of a type-species: it is currently accepted as a junior subjective synonym of Chorizagrotis Smith, I89o.

MESOGENEA Hampson, 1902, Ann. S. Afr. Mus. 2: 363.

ACRO

Type-species: Mesogenea varians Hampson, I902, ibidem 2: 364 , by subsequent designation by Hampson, 1926, Descr. new Genera Species Lepid. Phalaenae Subfamily Noctuinae Br. Mus.: 337. Type(s), [Rhodesia]: Mashonaland, Salisbury (BMNH).

Not preoccupied by $\ddagger$ Mesogenea Gray, I863, Cat. Specimens Drawings Mammals Birds Reptiles Fishes Nepal Tibet (Edn 2): 52, - Aves; published in synonymy.

MESOGONA Boisduval, I840, Genera Index meth. Eur. Lepid.: I44.

NOCT

Type-species: Noctua acetosellae [Denis \& Schiffermüller], I775, Ankündung syst. Werkes Schmett. Wienergegend: 84 , by subsequent designation by Blanchard, I 840 , in Castelnau, Hist. nat. Anim. articulés (Insectes) 3: 5I2, but cited as 'acetosellae Hübn.,' an incorrect authorship. Type(s), [Austria]: Vienna district (Collection destroyed, Horn \& Kahle, I936, Ent. Beih. Berl.-Dahlem 3: 243).

$\ddagger$ MEGOSONA; Blanchard, I 840, in Castelnau, Hist. nat. Anim. articulés (Insectes) 3: 5 I 2. An incorrect subsequent spelling.

MESOLIGIA Boursin, I965, Bull. mens. Soc. linn. Lyon 34: I85.

ACRO

Type-species: Noctua furuncula [Denis \& Schiffermüller], I775, Ankiindung syst. Werkes Schmett. Wienergegend: 89, by original designation. Type(s), [Austria]: Vienna district (Collection destroyed, Horn \& Kahle, I936, Ent. Beih. Berl.-Dahlem 3: 243).

In proposing Mesoligia Boursin stated 'supprimer le genre Miana Steph., I829 (type literosa Haw.) qui est un synonyme d'Oligia Hb. et le remplacer par Mesoligia Brsn., I965, nom. nov., type furuncula Schiff.' This has been cited by Zool. Rec. I965, 102 (т3): 344 , as 'Mesoligia n.n. to replace Miana Steph.' Boursin was not proposing a replacement name for Miana Stephens, type-species Phalaena strigilis Linnaeus, but he was proposing a new genus for Miana sensu a uctorum, based on Noctua literosa Haworth which is congeneric with Noctua furuncula. 
MESOLOMIA Smith, I 893, Bull. U.S. natn. Mus. 44: i 7 I.

ACRO

Type-species: Phlogophora iris Guenée, I852, in Boisduval \& Guence, Hist. nat. Insectes (Lépid.) 6: 64, by monotypy. Holotype ․, [U.S.A.]: New York state (BMNH).

MESOPHRACTIAS Warren, I9I 3 in Seitz, Gross-Schmett. Erde 11: 212.

ACON

Type-species: Eublemma alstoni Hampson, I907, J. Bombay nat. Hist. Soc. 17: 647, by original designation. Holotype $\widehat{\jmath}$, CEYLon: Maskeliya (BMNH).

The date of publication of the part of Seitz containing page 212 , was stated on page 209 as I9I3 August 2oth in both the English and German editions.

MESOPLECTRA Butler, I879, Illust. Iypical Specimens Lepid. Heterocera Colln Br. MIus. 3: 65 .

IIY'PE

Type-species: Mesoplectra lilacina Butler, I 879, ibidem 3:65, pl. 57, fig. 3, by original designation. Type(s), [JAPAN]: Yokohama (BMNH).

MESOPLUS Boursin, 1949, Z. wien. ent. Ges. 34: I 58.

ACRO

Type-species: Agrotis contrita Christoph, I 884, in Romanoff, Mém. Lépid. 1: I I 8, pl. 7, fig. 8, by original designation. Holotype q, [U.S.S.R.]: [Turkmenistan], AchalTekke, Nuchur (ZI, I,eningrad).

MESORUZA Warren, I9I3, in Seitz, Gross-Schmett. Erde 11: $24^{8}$.

$\operatorname{ACON}$

Type-species: Mesoruza kuehni Warren, I9 I 3, ibidem 11:248, by original designation, but proposed by Warren as $+M$. kïhmi, an incorrect original spelling. Syntypes I $\vec{\sigma}$, I q, [Moluccas]: Kei Is (BMNH).

The date of publication of the part of Seitz containing page 248 , was stated on page 24 I as 1913 September I $5^{\text {th }}$ in both the German and the English editions.

MESOSCIERA Hampson, I926, Descr. new Genera Species Lepid. Phalaenae Subfamily Noctuinae Br. Mus.: 532 .

OPHI

Type-species: Mesosciera typica Hampson, 1926, ibidem: 534, by original designation. Holotype f. [GHana] Gold Coast: Kumasi (BMNH).

†MESOSTROTA, misspelling. Sec Mesotrosta Lederer, T857. ACRO

MESOTROSTA Lederer, I857, Nociuinen Eur.: 44, I9o.

ACRO

Type-species: Scopula signalis Treitschke, I829, Schmett. Eur. 7: 70, by monotypy. Syntypes ô. ㅇ, Europe (TM, Buclapest).

+MESOSTROT A; Möschler, i 890, Abh. senckenb. naturforsch. Ges. 16: I63.

An incorrect subsequent spelling.

† MESSAGA, misspelling. See Massaga Walker, I 854 .

AGAR

MESTLETA Walker, I 865, List Specimens lepid. Insects Colln Br. Mus. 33: 829.

$\operatorname{ACON}$

Type-species: Mestleta abrupta Waller, 1865, ibidem 33: 830, by monotypy.

Lectotype ơ, [INDIA]: Hindostan (UM, Oxford), designated (as type) by Swinhoe, I9oo, Cat. east. and Aust. Lepid. Heterocera 2: 64

METACAUSTA Hampson, igio, Cat. Lepid. Phalaenae Br. Mus, 10: x, 207. Acon

Type-species: Tathodelta ustata Hampson, i 898, J. Bombay nat. Hist. Soc. 11: 448 , by original designation. Holotype f, INIIA: [Assam], Khasis (BMNH).

METACHROSTIS Hübner, [1820] I 816, Verz. bekannter Schmett.: 204.

$\operatorname{ACON}$

Type-species: Noctua velox Hübner, [I8I3], Samml. eur. Schmett. 4: pl, io8, fig. 507, \& pl. IIO, fig. 5I5, by subsequent designation by Hampson, I894, Fanna. Br. India (Moths) 2: 325 . Syntypes, Europe.

LI:PTOSIA Guenće, 1841, Annls Soc. cnt. Fr. 10:225.

Type-species: Noctua relox Hübner, [1813], by subsequent designation by Guenée, I 852, in Boiscluval \& Guenée, llist. nat. Insectes (Lépid.) 6: 237.

I junion homonym of Leptosia Hiibner, 1818, Zatrïge Samml. exot. Schmett. 1: I3, Lepirl., Pieridac. The objective replacement name is Metachrostis Hübner, [1820]. 
METACINIA Schaus, I9I3, Ann. Mag.nat. Hist. (8) 11: i 8.

Type-species: Metacinia limonalis Schaus, I9I3, ibidem (8) 11: 19, by original designation. Syntypes $\hat{\sigma},+$, Costa Rica: Limon; Sixola; Juan Vinas. French Guiana.

METACULASTA Butler, I 898, Proc. zool. Soc. Lond. 1898: 444.

CHLO

Type-species: Metaculasta dives Butler, I 898, ibidem 1898: 444, by original designation. Type(s) 우, [KenYA]: Voi (BMNH).

METACULLIA Köhler, (1952) I95I, Acta zool. lilloana 12: I 59.

CUCU

Type-species: Metacullia bergi Köhler, (1952) I95I, ibidem 12: I59, by original designation. Syntypes $\hat{\sigma},+$, Argentina: Rio Negro [Prov.], Cipolletti (ZSBS, Munich)

$\ddagger$ METADELTA Hampson, no published reference found.

HYPE

METAEGLE Hampson, I908, Cat. Lepid. Phalaenae Br. Mus. 7: 4. Available, but without included species until Hampson, r 91 ro, ibidem 9: xiii, 379.

ACRO

Type-species: Metoponia pallida Staudinger, I 892, Dt. ent. Z. Iris 4: 323, by subsequent monotypy. Syntypes, [Turkey]: Mardin (MNHU, Berlin).

METAEMENE Hampson, i 9ro, Cat. Lepid. Phalaenae Br. Mus. 10: 801.

$\operatorname{ACON}$

Type-species: Lyclene atrigutta Walker, i862, J. Proc. Linn. Soc. (Zool.) 6: I 16, by original designation. Type(s) , Borneo: Sarawak (BMNH).

METAGARISTA Walker, I854, List Specimens lepid. Insects Colln Br. Mus. 1: 6o. A AGAR

Type-species: Metagarista triphaenoides Walker, I854, ibidem 1: 6I, by monotypy. Syntypes ô, [GHANA]: Ashanti (BMNH).

METAHADENA Morrison, I876, Proc. Acad. nat. Sci. Philad. 27: 431.

CUCU

Type-species: Metahadena atrifasciata Morrison, I 876, ibidem 27: 43 I, by monotypy. Syntypes, [U.S.A.]: Maine (BMNH); Orono.

$M$. atrifasciata is a junior subjective synonym of Phornacisa piffardi Walker, I 862.

See also Phornacisa Walker, I 862.

METALECTRA Hübner, I823, Zutväge Samml. exot. Schmett. 2: 29.

OPH I

Type-species: Metalectra praecisalis Hübner, 1823, ibidem 2: 29, figs 367, 368, by subsequent designation by Hampson, 1926, Descr. new Genera Species Lepid. Phalaenae Subfamily Noctuinae Br. Mus.: 280. Type(s) ठ઼, Surinam.

See also Stimmia Guenée, I 852.

METALEPSIS Grote, I875, Check List Noctuidae Am. N. of Mex.: 25.

NOCT

Type-species: Pachnobia cornuta Grote, I874, Bull. Buffalo Soc. nat. Sci. 2: 68, by original designation. Syntypes 2 J. [U.S.A.]: California (BMNH).

METALEPTINA Holland, I893, Psyche, Camb. 6: $55^{\circ}$.

CHLO

Type-species: Metaleptina nigribasis Holland, I893, ibidem 6: 55I, pl. 21, fig. I I, by original designation. Type(s) $\hat{\sigma}$, WVest Africa (CM, Pittsburgh).

METALLATA Möschler, I890, Abh. senckenb. naturforsch. Ges. 16: 2 I9.

OPHI

Type-species: Metallata variabilis Möschler, I89o, ibidem 16: 220, by monotypy. Syntypes $\hat{o}$, 오, [Puerto Rico] Portorico I. (MNHU, Berlin). [Venezuela]: Puerto Cabello. [Panama]: Chiriqui.

$M$. variabilis is a junior subjective synonym of Thermesia absumens Walker, I862, Trans. ent. Soc. Lond. (3) 1: 106.

See also Euthermesia Butler, I 896.

METALOPHA Staudinger, I892, Dt. ent. Z. Iris 4: 3 I 3 .

CUCU

Type-species: Metalopha plusina Staudinger, I891, ibidem 4: 313, pl. 4, fig. 8, by subsequent designation by Hampson, i906, Cat. Lepid. Phalaenae Br. Mus. 6: 105, but cited as $M$. liturata which Hampson placed as the senior synonym of $M$. plusina. Syntypes 
I 3., 2 \%, [IRAN] Persia; N., Schahrud (MNHU, Berlin). Turkey: N. of Antioch, Aintab (MHNU, Berlin); Mesopotamia, Mardin (MNHU, Berlin).

$M$. plusina is a junior subjective synonym of Megalodes liturata Christoph, i 887 , in Romanoff, Mém. Lépid. 3: 89, pl. 4, fig. io.

METANOLA Son, 1933, Ann. Transv. Mus. 15: 203.

NOLI

Type-species: Metanola myriostigma Son, 1933, ibidem 15: 203, pl. 1, fig. 4, pl. 5, fig. 5, pl. 8, fig. 7 , by original designation. Holotype $3 \overrightarrow{0}$, South Africa: Transvaal, Marieps Mountain (TMI, Pretoria).

METAPHOENIA Hampson, 1926, Descr. new Genera Species Lepid. Phalaenae Subfamily Noctuinae Br. Mrus.: 478 . $\mathrm{OPHI}$

Type-species: Thermesia plagifera Walker, I86., J. Proc. Linn. Soc. (Zool.) 7: I87, by original designation. Lectotype +, Borneo: Sarawak (UM, Oxford), designated (as type) by Swinhoe, 1900, Cat. east. and Aust. Lepid. Heterocera 2: 208.

METAPIOPLASTA Wallengren, I865, K. svenska I'etenskAkad. Handl. (N.F.) 5 (4): 70.

$A C O N$

Type-species: Acontia simo Wallengren, i860, Wien. ent. Monatschr. 4: I72, by

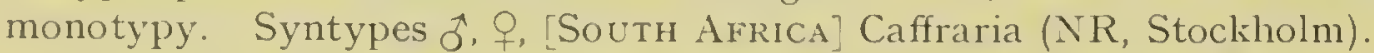

A. simo is a junior subjective synonym of Acontia insocia Walker, [I858] I857, List Specimens lepid. Insects Colln Br. Mus. 12: 788.

METAPLUSIA Dyar, I925, Insecutor Inscit. menstr. 13: 6.

PLUS

Type-species: Plusia argyra Druce, 1889, Biologia cent.-am. (Zool.) Lepid. Heterocera 1: 330, pl. 30, fig. I5, by original designation. Syntypes, MExico: Presidio (BMNH); Jalisco, Bolaños (BMNH).

EUGLYPHINA Hampson, 1926, Descr. new Genera Species Lepid. Phalaenae Subfamily

Noctuinae Br. Mus.: 145.

Type-species: Plusia argyra Druce, i 889, by original designation.

Euglyphina is a junior objective synonym of Metaphusia Dyar, 1925.

METAPONPNEUMATA Möschler, I890, Abh. senckenb. naturforsch. Ges. 16: I $59 . \quad$ opH I Type-species: Metaponpneumata rogenhoferi Möschler, I890, ibidem 16: I59, by monotypy. Syntypes 20,4 , Puerto Rico (MNHU, Berlin).

METAPPANA Viette, I965, Faune Madagascar 20: 433.

ACKO

Type-species: Atimaea crescentica Hampson, 1910, Cat. Lepid. Phalaenae Br. Mus. 9: 1 26, fig. 38, by original designation. Holotype ô. MAdagascar: Betsileo (BMNH).

METAPRIONOTA Hampson, 1926, Descr. new Genera Species Lepid. Phalaenae Subfamily Noctuinae Br. Mus.: I.

OPHI

Type-species: Ophisma sculpta Felder \& Rogenhofer, I874, Reise öst. Fregatte Novara (Zool.) 2 (Abt. 2): pl. I 7, fig. 26; Inhalts-Verz.: I 4, by original designation. Type(s) $\hat{o}$, French GuiAna (BNNH).

METAPROSPHERA Hampson, 1926, Descr. new Genera Species Lepid. Phalaenae Subfamily Noctuinae Br. Mus.: 316.

OPHI

Type-species: Renodes modesta Butler, 1879, Trans. ent. Soc. Lond. 1879: 67, by original designation. Syntypes, [BrazIL]: Amazons, Rio Purus, Sepatiny (BMNH): Rio Jutahi, Bôaventura (BMNH).

†METAPTILA, misspelling. See Metaptya Hampson, 1896 .

CIILO

METAPT YA Hampson, 1896, Fauna Br. India (Moths) 4:522.

CHLO

Type-species: Metaptya sericea Hampson, 1896, ibidem 4: 523, fig. 273, by original designation. Type(s) $\hat{\sigma}$, CEYLON (BMNH).

† METAPTILA; Lower, 1900, Proc.Linn. Soc. N.S.W. 25: 3 I. 
An incorrect subsequent spelling; but cited by Neave, I940, Nomencl. zool. 3: 132, as an available generic name. Lower, I900, under Metaptila(?) ptilomela n. sp. stated 'A new genus will no doubt be required to receive it.' He was not therefore proposing a new generic name.

METASADA Hampson, i9io, Cat. Lepid. Phalaenae Br. Mus. 10: xi, 257.

ACON Type-species: Zagira polycesta Turner, 1902, Proc. Linn. Soc. N.S.W. 27: I 20, by original designation. Holotype $\hat{\delta}$, Australia: Qd, Brisbane (not found in ANIC, Canberra).

METASARCA Dyar, I925, Insecutor Inscit. menstr. 13: 5.

OPHI

Type-species: Metasarca euphancra Dyar, I925, ibidem 13: 5, by monotypy. Holotype 1. Mexico: Cuernavaca (USNM, Washington).

METASARCA Hampson, 1926, Descr. new Genera Species Lepid. Phalaenae Subfamily Noctuinae Br. Mus.: I.

Type-species: Metasarca euphanera Hampson, 1926, ibidem: 2, by original designation. Holotype ơ, Mexico: Cuernavaca (BMNH).

A junior homonym of Metasarca Dyar, I925,-Lepid., Noctuidae. This name was used and made available by Dyar prior to its proposal and generic description by Hampson. There is no objective replacement name. Dyar's type-species was described as $M$. euphancra, and Hampson's type-species was described as $M$. euphanera. They were undoubtedly intended to be the same as Dyar acknowledged Hampson for the name, but each was proposed and described in good faith as a new species, each having a holotype. Both names are therefore nomenclaturally available, but both holotypes were collected from the same locality by W. Schaus and it is almost certain that when compared they will be found to be conspecific.

METASARCA Hampson, I926. See Metasarca Dyar, I925.

OPH I

METATACHA Hampson, I913, Cat. Lepid. Phalaenae Br. Mus. 12: ix, 238.

CATO

Type-species: Ercheia excavata Bethune-Baker, 1909, Ann. Mag. nat. Hist. (8) 3: 43I, by original designation. Holotype $ᄋ$, UGanda: N'tebi (BMNH).

METAXAGLAEA Franclemont, I937, Can. Ent. 69: I 29.

CUCU

Type-species: Orthosia viatica Grote, I874, Annual Rep. Trustees Peabody Acad. Sci. 6: 29, by original designation. Syntypes, [U.S.A.]: Alabama (BMNH); N.Y., Albany (BMNH).

METAXANTHIA Hampson, igo8. See Metaxanthiella Collins, I962.

ACRO

METAXANTHIELLA Collins, I962, Ann. Mag. nat. Hist. (13) 5: I25.

ACRO

Type-species: Britha cosmopis Lower, 1897, Proc. Linn. Soc. N.S.W. 22: 17, by subsequent monotypy (of Metaxanthia Hampson, i908). Syntypes 2 9, Australia: Qd, Cape York.

Metaxanthiella was proposed as the objective replacement name for Meta.ranthia Hampson, 1908.

METAX ANTHIA Hampson, i9o8, Cat. Lepid. Phalaenae Br. Mus. 7: 9, but without included species until Hampson, I9ro, ibidem 9: xiv, $44^{8}$.

Type-species: Britha cosmopis Lower, I897, by subsequent monotypy.

A junior homonym of Metaxanthia Druce, I 899, Ann. Mag. nat. Hist. (7) 3: 465 ,- Lepid., Arctiidae. The objective replacement name is Metaxanthiella Collins, I962.

$\ddagger$ MET $\boldsymbol{A X Y A}$, misspelling. See Metaxyja Hübner, [1 821]. NOCT

METAXYA Berio, 1955. See Mentaxya Geyer, I837. Noct

${ }_{+}^{\dagger}$ METAXYIA, misspelling. See Metaxyja Hübner, [I82 I]. NocT 
METAXYJA Hübner, [1821] 1816, Verz. bekannter Schmett.: 223.

NOCT

Type-species: Phalaena vitta Esper, 1789, Die Schmett. 4 (2) Abschnitt I: pl. I 43, fig. 6; [1798-1804], ibidem: 457, by subsequent designation by Hampson, 1903, Cat. Lepid. Phalaenae Br. Mus. 4: I53, but cited for \$Metaxyia, an incorrect subsequent spelling. Syntypes $\hat{o}$,, , GERMANY.

†.METAXYA; Walker, [1857] i 856, List Specimens lepid. Insects Colln Br. Mus. 10: 373, 377. An incorrect subsequent spelling.

† MET AXYIA; Hampson, I903, Cat. Lepid. Phalaenae Br. Mus. 4: 153.

An incorrect subsequent spelling.

METAXYLLIA Dyar, I922, Insecutor Inscit. menstr. 10: I I.

$\operatorname{ACON}$

Type-species: Metaxyllia metallicella Dyar, I922, ibidem 10: II, by monotypy. Syntypes 20 , MeXico: Cuernavaca (USNM, Washington).

METECIA Snellen, I879, Boln Acad. nac. Cienc., Córdoba 3: 94.

NOCT

Type-species: Metecia cornifrons Snellen, I879, ibidem 3: 96, pl. I, figs a-d, by monotypy. Holotype ô, Argentina: Cordova.

METERANA Butler, 1877, Proc. zool. Soc. Lond. 1877: 385.

HADE

Type-species: Dianthoecia pictula Taylor, I855, New Zealand and its Inhabitants: xiii, pl. I, fig. 3, by original designation. Type(s), NeW Zealand.

The type-species was proposed as $\ddagger$ Dianthecia, an incorrect subsequent spelling. The specific name has usually been attributed to White; there was, however, no mention of this author by Taylor.

METHORASA Moore, I88 I, Proc. zool. Soc. Lond. 1881: 374.

ACRO

Type-species: Noctua latreillei Duponchel, 1827 , in Godart \& Duponchel, Hist. nat. Lépid. Papillons Fr. 7 (1): 327, pl. 1 20, fig. 2, by original designation. Syntypes đ. France: Provence; Bretagne.

METINA Druce, I89o December, Biologia cent.-am. (Zool.) Lepid. Heterocera 1: 437. HyPE Type-species: Bleptina hirtipalpis Walker, [1 859] i 858, List Specimens lepid. Insects Colln Br. Mus. 16: 242, by original designation. Holotype 0 . Venezuela (BMNH).

See also Aglaonice Möschler, I 890 [May].

METLAOUIA Dumont, 1928, Encycl. ent. (B) 3 Lepid.: 20.

CUCU

Type-species: Simyra oberthueri Culot, [1910], Noctuelles Géomètres Eur. 1: 26, pl. 3, fig. I, by original designation. Type(s), Algeria: Aflou (BMNH).

$S$. oberthueri was, in the original description, attributed to Deckert but no earlier reference has been found.

METOPIORA Meyrick, I902, Trans. ent. Soc. Lond. 35: 25.

HADE

Type-species: Chariclea sanguinata Lucas, i 892, Proc. Linn. Soc. N.S.W. (2) 7: 254 , by monotypy. Syntypes ô. + , Australia: Brisbane (SAM, Adelaide).

METOPIS TIS Warren, 1913, in Seitz, Gross-Schmett. Erde 3: 395.

OPH I

Type-species: Phothedes erschoffi Christoph, 1884, in Romanoff, Mém. Lépid. 1: I33, pl. 8, fig. 8, by original designation. Holotype ó, [U.S.S.R.]: [Turkmenistan], Askhabad (ZI, Leningrad).

The date of publication of the part of Seitz containing page 395, was stated on page 389 as I9I 3 July ist in both the English and German editions.

$\ddagger_{\ddagger}$ METOPOCERA, misspelling. See Metopoceras Guenée, I 850 .

CUCU

METOPOCERAS Guenée, I 850, Bull. Soc. ent. Fr. (2) 8 : xviii.

CUCU

Type-species: Polia felicina lonzel, 18+4, Annls Soc. ent. Fr. (2)2: 199, pl. 6, fig. 2, by monotypy. Type(s), [France] : Marseille district. 
† METOPOCERA; Guenée, 1850, Annls Soc. ent. Fr. (2) 8: 233.

An incorrect subsequent spelling.

METOPODICHA Draudt, 1936, Ent. Rdsch. 53: 49 I.

CUCU

Type-species: Metopodicha ernesti Draudt, 1936, ibidem 53: 492, pl. 5, fig. 5c, by monotypy. Type(s) ô, [Turkey]: Achyr-Dagh, Maraș (Collection destroyed in World War II).

METOPONIA Duponchel, [1845] i 844, Cat. méth. Lépid. Eur. (2): i 87.

ACRO

Type-species: Anthophila flavida Ochsenheimer, I8I6, Schmett. Eur. 4: 93, by monotypy. Type(s) of $N$. flava Hübner, Europe.

A. flavida was proposed by Ochsenheimer as the objective replacement name for Noctua flava Hübner, [r809], Samml. eur. Schmett. 4: pl. 96, fig. 453, a junior primary homonym of Noctua flava Fabricius, I775, Syst. Ent.: 601, - Noctuidae, Ophiderinae.

A. flavida is a junior subjective synonym of Tortrix kaekeritziana Hübner, [I799], Samml. eur. Schmett. 7 : pl. 26, fig. I63.

Druce, I889, Biologia cent.-am. (Zool.) Lepid., Heterocera 1: 303, stated 'This generic name, as noted by Walker, is preoccupied in Diptera [by Metoponia], Macquart, 1847'. This error was due to the citation of the reference as 'Metoponia (Duponchel), Guenée, Sp. gén. des Lép. vi. p. 206 (I852)'; this was not the original reference and Metoponia Duponchel is not preoccupied.

See also Aegle Hübner, [1823].

METOPONRHIS Staudinger, I 888, Stettin. ent. Ztg 49: 54.

OPHI

Type-species: Photedes albirena Christoph, 1887, in Romanoff, Mém. Lépid. 3: 87, by monotypy. Syntypes $\hat{\delta}$, + , [U.S.S.R.]: [Turkmenistan], Achal-Tekke district (ZI, Leningrad). $P$. albivena was proposed as $\ddagger$ Phothedes, an incorrect spelling.

METOPOPLACIS Hampson, I909, Ann. Mag. nat. Hist. (8) 4:365.

NOCT

Type-species: Metopoplacis olivata Hampson, I909, ibidem (8) 4: 366, by original designation. Holotype 9, Brazil: Mato Grosso, Nivac (BMNH).

METOPOPLUS Alphéraky, I893, in Christoph, Dt. ent. Z. Iris 6: 92. ACRO

Type-species: Clidia excelsa Christoph, 1885, in Romanoff, Mém. Lépid. 2: pl. I3, fig. 4, by subsequent designation by Hampson, i9io, Cat. Lepid. Phalaenae Br. Mus. 9: 229. Holotype $\sigma^{-}$(not $q$ as stated], [IRAN] Persia: N., Shahkuh (ZI, Leningrad).

METOPOSCOPA Strand, I9I2, Arch. Naturgesch. 78 (A) I: 77.

OPHI

Type-species: Metoposcopa sagittalis Strand, I912, ibidem 78 (A) I: 78, by original designation. Syntypes I $\sigma^{\jmath}, \mathrm{I}$ ㅇ, [Tanzania] Dt. Ost-Afrika: Mkatta (MNHU, Berlin).

METOPTA Swinhoe, I9oo, Cat. east. and Aust. Lepid. Heterocera 2: 170.

CATO

Type-species: Spirama rectifasciata Ménétriés, 1863, Enumeratio Corporum Anim. Mus. imp. Acad. Sci. Petropolitanae (Lepid.): pl. 17, fig. 6, by original designation. Type(s), TYPE-LOCALITY not stated (ZI, Leningrad) [JAPAN].

GIACLA Walker, 1855, List Specimens lepid. Insects Colln Br. Mus. 6: I315. Available, but hitherto without included species.

Type-species: Spirama rectifasciata Ménétriés, r863, by subsequent monotypy.

Giacla was described very briefly among the Saturniidae and in the same paragraph transferred to the 'Noctuites', where it has remained in limbo ever since. No locality was given but from the generic description it is almost certain that Giacla was proposed to represent the concept now known as Metopta Swinhoe, I90o. In order to fix Giacla I have now placed in it S. rectifasciata as I can find no other species to fit the description. Unfortunately Giacla thus becomes the senior objective synonym of Metopta which has been in use for this concept since its proposal in I9oo. In the interest of stability under the Code, Article $23(\mathrm{a}-\mathrm{b})$, the Commission should be requested to use its plenary powers 
to suppress Giacla Walker, I855, for the purposes of the Law of Priority but not for those of the Law of Homonymy.

$\ddagger$ MEDOPTA; Warren, I9I 3, in Seitz, Gross-Schmett. Erde 3: 323.

An incorrect subsequent spelling.

METOPTRIA Guenée, [1 845]. See Synthymia Hübner, [I 823 ].

ACRO

METRIA Hübner, [1823] i8I6, Vevz. bekannter Schmett. : 26I.

OPH I

Type-species: Phalaena euristea Stoll, I780, in Cramer, Uitlandsche Kapellen 4: 7I, pl. 324, fig. A, by subsequent designation by Berio, I957, Mémorie Soc. ent. ital. 36 : I6. Type(s), Surinam.

MEYRICKELLA Berg, I898, Comun. Mus. nac. Buenos Aires 1: 42. OPH I

Type-species: Crambus ruptellus Walker, I863, List Specimens lepid. Insects Colln Br. Mus. 27: I73, by monotypy (of Prionophora Meyrick, 1879). Syntypes 4 ( $\vec{\sigma}, \quad$ ), Australia: [Qd], Moreton Bay (BMNH).

Meyrickella was proposed as the objective replacement name for Prionophora Meyrick, I 879.

PRIONOPHORA Meyrick, 1879, Proc. Linn. Soc. N.S.W. 3: I79.

Type-species: Crambus ruptellus Walker, I863, by monotypy.

A junior homonym of Prionophora Westwood, I848, Cabinet oriental Ent.: 85, - Coleoptera. The objective replacement name is Meyrickella Berg, I 898.

An earlier usage of the name i.e. \$Prionophora Dejean, I833, Cat. Coléoptères Colln $M$. le Comte Dejean (Edn 2): 78, was undescribed and included a single undescribed species.

$\ddagger$ PRONOPHORA; Turner, i 902, Proc. Limn. Soc. N.S.W. 27: 107.

An incorrect subsequent spelling.

MIANA Stephens, I829. See Oligia Hübner, [1821].

ACRO

MIAROMIMA Meyrick, i 889, Trans. ent. Soc. Lond. 1889: 47 I.

CHLO

Type-species: Miaromima dinotis Meyrick, I889, ibidem 1889: 47 I, by monotypy. Syntypes $4(\hat{\sigma}, \underline{f})$, New Guinea: [Papua], Nit Obree or adjoining ranges (BMNH).

MICARDIA Butler, I878, Ann. Mag. nat. Hist. (5) 1:81.

$\operatorname{ACON}$

Type-species: Micardia argentata Butler, I 878, ibidem (5) 1:81, by original designation. Type(s), JAPAN : Yokohama (BMNH).

MICHENERIA Orfila \& Rossi, I 956. See Araea Hampson, I908.

ACRO

MICHERA Walker, I865, List Specimens lepid. Insects Colln Br. Mus. 33: 8 Io.

OPHI

Type-species: Michera submurina Walker, I865, ibidem 33: 8Io, by monotypy. Holotype $\hat{\sigma},[$ Australia]: [IV. Aust.], Swan River (BMNH).

MICRA Guenée, I 84 I. See Trothisa Guenée, I 84 I .

ACON

MICRACONTIA Hampson, i 895, Trans. ent. Soc. Lond. 1895: $30 \mathrm{I}$.

$\operatorname{ACON}$

Type-species: Micracontia batisella Hampson, I895, ibidem 1895: 302, fig. [12], by original designation. Syntypes ô, CEYLON: Hambantota (BMNH).

MICRAESCHUS Butler, I878, Ann. Mag. nat. Hist. (5) 1: 402.

ACON

Type-species: Hyria elataria Walker, I86I, List Specimens lepid. Insects Colln Br. MIus. 22: 665, by original designation. Holotype $\sigma^{\wedge}$, CEYLON: Colombo (BMNH).

MICRAGROTIS Hampson, I903, Cat. Lepid. Phalaenae Br. Mus. 4: xii, I48.

NOCT

Type-species: Micragrotis axylides Hampson, I903, ibidem 4: I 50, fig. 56, l)y original designation. Syntypes $2 \hat{o}$, [INDIA]: Punjab, Mean Meer (BMNH).

MICRAMMA Schaus, 19 i6, Proc. IT.S. natn. MTus. 50: 261, 303.

IYPE:

Type-species: Micramma croceicosta Schaus, I9I6, ibidem 50:304, lyy original designation. 'Type(s) ô, liREncn Guana: R. Maroni, St. Jean (USNM, Washington). 
MICRANTHA Hampson, I9 10, Cat. Lepid. Phalaenae Br. Mus. 10 : xiv, 483.

Type-species: Erastria mirabilis Schaus, I904, Trans. Am. ent. Soc. 30: I6I, by original designation. Type(s), Brazıl: São Paulo.

MICRANTHOPS Hampson, 1926, Descr. new Genera Species Lepid. Phalaenae Subfamily Noctuinae Br. Mus.: 203.

OPHI

Type-species: Capnodes alceste Druce, I890, Biologia cent.-am. (Zool.) Lepid. Heterocera 1: 4I 7, pl. 33, fig. 24, by original designation. Type(s), Panama: Chiriqui.

MICRAPATETIS Meyrick, I897, Trans. ent. Soc. Lond. 1897: 369.

ACRO

Type-species: Micrapatetis orthozona Meyrick, i 897, ibidem 1897: 369, by monotypy.

Syntypes $7(\hat{0},+$ ) $)$, Australia: Qd, Duaringa (BMNH).

$\ddagger$ MICRAPATHETIS; Strand, I9I 2, in Aurivillius \& Wagner, Lepid. Cat. 5: 74.

An incorrect subsequent spelling.

†MICRAPATHETIS, misspelling. See Micrapatetis Meyrick, I897.

ACRO

MICRATHETIS Hampson, I908, Cat. Lepid. Phalaenae Br. Mus. 7: I8. Available, but without included species until Hampson, I909, ibidem 8: xii, 442.

ACRO

Type-species: Laphygma triplex Walker, 1857, List Specimens lepid. Insects Colln Br. Mus. 11:721, by subsequent designation by Hampson, I909, ibidem 8: 442. Holotype o. Honduras (BMNH).

Micrathetis was the spelling adopted by Hampson, I909, as first reviser from a multiple original spelling. Micrathetis was used in the phylogenetic table facing page 2, and Microthetis was used in the key to genera on page 18.

$\ddagger$ MICROTHETIS; Hampson, igo8, Cat. Lepid. Phalaenae Br. Mus. 7 : i 8.

An incorrect original spelling.

MICRAXYLIA Berio, I962, Annali Mus, civ. Stor. nat. Giacomo Doria 73: ig6.

NOCT

Type-species: Micragrotis delicatula Berio, I939, Memorie Soc. ent. ital. 17: 50, pl. I, fig. I4, by original designation. Holotype ô. [ETHiopia]: Eritrea, Tessenei (Colln E. Berio, Genoa).

MICREREMITES Warren, I89I, Ann. Mag. nat. Hist. (6) 8: 66.

HYPE

Type-species: Micreremites fatua Warren, I 89I, ibidem (6) 8: 66, by original designation. Holotype $\tilde{o}$. TyPE-LOCALITy stated as 'probably from Calcutta' (BMNH) [INDiA].

MICRIANTHA Hampson, I908, Cat. Lepid. Phalaenae Br. Mus. 7: 4. Available, but without included species until Hampson, I9Io, ibidem 9: xiv, 486. ACRO

Type-species: Heliothis decorata Frivaldszky, i 845, Evk. Királ. Magy. Term. Társ. 1: I 86, pl. 3, fig. 2, by subsequent monotypy. Type(s), [Turkey]: [Bursa] Brussa.

MICRISCADIA Hampson, I9I 2, Cat. Lepid. Phalaenae Br. Mus, 11: 222, $377 . \quad$ SARR

Type-species: Micriscadia metachryseis Hampson, I9I 2, ibidem 11: 377, fig. I 43, by original designation. Holotype $q$, [GuYanA] British Guiana: Roclsstone (BMNH).

†MICROBARASA, misspelling. See Macrobarasa Hampson, I9I 2.

SARR

MICROBLEMMA Hampson, igio. See Abablemma nom. n.

$\mathrm{ACON}$

MICROCOELIA Guenée, I 852, in Boisduval \& Guenée, Hist. nat. Insectes (Lépid.) 5: 33. ACro Type-species: Microcoelia fragilis Guenée, I852, ibidem 5: 34, by subsequent designation by Grote, I874, Bull. Buffalo Soc. nat. Sci. 2: 9. Holotype ô. [U.S.A.]: New York State $(\mathrm{BMNH})$.

MICROEDMA Warren, I9I3, in Seitz, Gross-Schmett. Erde 11: 2 I 7.

ACON

Type-species: Microedma extorris Warren, I913, ibidem 11: 217, by original designation. Syntypes, New Guinea: [Papua], [Louisiade Arch.], St. Aignan (BMNH).

$\mathrm{X}$ 
The date of publication of the part of Seitz containing page 2I7, was stated at the top of the page as I9I3 August 2oth, in both the German and the English editions.

MICROHELIA Hainpson, I908, Cat. Lepid. Phalaenae Br. Mus. 7: 8. Available, but without included species until Hampson, I9I0, ibidem 9: xiv, 463.

NOCT

Type-species: Heliodes angelica Smith, 1900, Proc. U.S. natn. Mus. 22: 484 , by subsequent designation by Hampson, I9 Io, ibidem 9: 463. Holotype, [U.S.A.]: California, Los Angeles County (USNM, Washington).

MICROLITA Hampson, I908, Cat. Lepid. Phalaenae Br. Mus. 7: 9. Available, but without included species until Hampson, I9IO, ibidem 9: xi, 302.

ACRO

Type-species: Doryodes leucorhabda Jones, I908, Trans. ent. Soc. Lond. 1908: I65, by subsequent monotypy. Type(s) , Brazil: Parana, Castro.

MICROMANIA Christoph, I893, Dt. ent. Z. Iris 6: 93.

ACRO

Type-species: Micromania turcomanica Christoph, I893, ibidem 6: 93, by monotypy. Syntypes ô. \&, [U.S.S.R.]: [Turkmenistan], Askhabad, Aidere (ZI, Leningrad).

Christoph attributed Micromania to Alphéraky.

MICROMONODES Hampson, I908, Cat. Lepid. Phalaenae Br. Mus. 7: 4. Available, but without included species until Hampson, I909, ibidem 8: xiv, 565.

ACON

Type-species: Thalpochares guarama Schaus, I904, Trans. Am. ent. Soc. 30: I62, by subsequent designation by Hampson, i 909 , ibidem 8: 565 . Type(s), [BrazIL]: São Paulo (USNM, Washington).

MICRONOLA Amsel, I935, Verëff. dt. Kolon. u. Übersee-Mus. Bremen 1: 2 I 5.

NOLI

Type-species: Micronola wadicola Amsel, I935, ibidem 1: 2 I6, pl. II, figs 23, 24, \& pl. I 2, figs I 5, I6, by monotypy. Holotype ô. [JoRDAN]: Jericho.

MICROPHAEA Hanpson, ig Io, Cat. Lepid. Phalaenae Br. Mus, 10: 6, 329.

$\mathrm{ACON}$

Type-species: Microphaea nyctichroa Hampson, I9Io, ibidem 10: 329, fig. 96, by original designation. Syntypes 2 \%, PANama: La Chorrera (BMNH).

MICROPHISA Boisduval, I84o, Genera Index meth. Eur. Lepid.: I 70.

ACON

Type-species: Noctua jucunda Hübner, [1813], Samml. eur. Schmett. 4: pl. I03, fig. 486, \& pl. I05, fig. 492, by subsequent designation by Desmarest, (1857), in Chenu, Encycl. Hist. nat. Papillons nocturnes: 104, but cited for Microphysa Boisduval, an incorrect authorship of an unjustified emendation. Syntypes, Europe.

MICROPHYSA Guenée, I8+I, Annls Soc. ent. Fr. 10: 233.

MICROPHYSA Agassiz, 1846, Nomencl. zool. Index univl.: 233.

Both unjustified emendations of Microphisa Boisduval, i 8 4o.

Both junior homonyms of Microphysa Westwood, i834, Annls Soc. ent. Fr. 3: 642,Hemiptera. The objective replacement name is Microphisa Boisduval, I840.

MIXOCHARIS Leclerer, I853, Verh. zool.-bot. Ver. Wien 3: 376.

Proposed, unnecessarily, as an objective replacement name for Microphisa Boisduval, I 840 , which is not preoccupied by Microphysa Westwood, I 834 .

MICROPHYSA Guenće, I84I. See Microphisa Boisduval, I840. ACoN

MICROPHYSA Agassiz, 1846. See Microphisa Boiscluval, I $840 . \quad$ ACon

MICROPIA Hampson, igog. See Pricomia nom.n. ACRo

MICROPLEXIA Hampson, 1908, Cat. Lepid. Phalaenae Br. Mus. 7: xi, 354. Acro

Type-species: Erastria elegans Saalmüller, 1891, Lepid. Madagascar: 347, pl. I3, fig. $2 \nmid 2$, by original designation. Syntypes $\hat{\jmath}$. $q$, MAdagascar: Betsilen-Land (SNG, Frankfurt). 
MICRORAPHE Berio, 1937, Annali Muts. civ. Stor. nat. Giacomo Doria 59: 390.

$\operatorname{ACON}$

Type-species: Microraphe fiorii Berio, I 937, ibidem 59: 390, by original designation. Holotype, [Somali Republic] Somalia Ital.: Ogaden, Uarder (Colln E. Berio, Genoa.)

$\ddagger$ MICROSADA, misspelling. See Microzada Hampson, I9I2.

CHLO

MICROSELENE Hampson, 1926, Descr. new Genera Species Lepid. Phalaenae Subfamily Noctrinae Br. Mus.: 582.

OPHI

Type-species: Microselene mesostipa Hampson, I926, ibidem: 582, by original designation. Holotype ô. Sikкim (BMNH).

MICROSEMYRA Butler, I883, Proc. zool. Soc. Lond. 1883: I55.

ACRO

Type-species: Microsemyra pallida Butler, I883, ibidem 1883: I55, by monotypy. Syntypes $\hat{\sigma}$, 우, [India]: [Madhya Pradesh], Mhow (BMNH).

$\ddagger$ MICROTHETIS, misspelling. See Micrathetis Hampson, I 908.

ACRO

MICROTHRIPA Hampson, I9I2, Cat. Lepid. Phalaenae Br. Mus. 11: x, 226.

SARR

Type-species: Dendrothripa baeota Turner, 1902, Proc. Linn. Soc. N.S.W. 27: 93, by original designation. Syntypes, Australia: I $\sigma$, I 9, Qd, Townsville (ANIC, Canberra).

MICROXESTIS Hampson, I9I 2, Cat. Lepid. Phalaenae Br. Mus. 11: xvii, 642.

CHLO

Type-species: Euxestis wutzdorffi Püngeler, I907, Dt. ent. Z. Iris 19: 223, pl. 7, fig. 5, by original designation, but cited by Hampson as $M$. vutzdorff, an unjustified emendation. Holotype ô. [JoRdan]: Palestine, Jordan valley (MNHU, Berlin).

MICROZADA Hampson, I91 2, Cat. Lepid. Phalaenae Br. Muss, 11: 456, 580.

CHLO

Type-species: Microzada anaemica Hampson, I9I2, ibidem 11: 580, fig. 226, by original designation. Syntypes $2 \hat{0}$, [GHana] Gold Coast: Kumasi (BMNH).

$\ddagger$ MICROSADA; Prout, I927, Trans. ent. Soc. Lond. 75: 2 I 5 .

An incorrect subsequent spelling.

MICTOCHROA Druce, I909, Ann. Mag. nat. Hist. (8) 3: 460.

ACON

Type-species: Mictochroa albirena Druce, I909, ibidem (8) 3: 460, by subsequent designation by Collins, I962, ibidem (I3) 5: I 23. Type(s) 우, Colombia: Sierra del Libane (BMNH).

Druce used this generic name without any indication that it was new, even though his new species described on the same page were marked sp. n. The generic name was probably obtained from Hampson as an identification and was unintentionally used and therefore made nomenclaturally available by Druce before Hampson's generic description was published.

MICTOCHROA Hampson, igio, Cat. Lepid. Phalaenae Br. Mus. 10: 355.

Type-species: Miana zonella Druce, I889, Biologia cent.-am. (Zool.) Lepid. Heterocera 1: 274, pl. 26, fig. 23, by original designation. Syntypes, Costa RicA: Volcan de Irazu (BMNH).

A junior homonym of Mictochroa Druce, I909, - Lepid, Noctuidae. This generic name was used and made nomenclaturally available by Druce prior to its proposal and generic description by Hampson. There is no objective replacement name but $M$. zonella is congeneric with Mictochroa albirena Druce, I909, the type-species of Mictochroa Druce, I 909. The latter is therefore available for use as a subjective replacement name.

MICTOCHROA Hampson, i9io. See Mictochroa Druce, I909. ACON

MIDEA Walker, I863. See Arsacia Walker, [I866]. OPHI

MILA Aurivillius, i892, Ent. Tidskr. 13: I87.

ACRO

Type-species: Hypsa concinnula Mabille, i878, Bull. Soc. zool. Fr. 3: 85, by original designation. Type(s), Congo (not found in BMNH or MNHN, Paris, see Viette \& Fletcher, I968, Bull. Br. Mits. nat. Hist. (Ent.) $21: 4^{\mathrm{I}} 3$ ). 
MILICHIA Snellen, [1899]. See Dysmilichia Speiser, I902.

MIL YAS Walker, I 858, List Specimens lepid. Insects Colln Br. Mus. 15: I 522, I $535 . \quad$ орH I Type-species: Milyas mixtura Walker, I 858 , ibidem 15: I536, by monotypy. Holotype of. TrPe-Locality stated to be Africa, but this was probably an error as this species is not known to occur in the Ethiopian Region but is found in Jamarca (BMNH). $M$. mixtura is a junior subjective synonym of Azeta uncas Guenée, I 852 .

See also Azeta Guenée, 1852.

MIMANUGA Warren, I9I3, in Seitz, Gross-Schmett. Erde 3: 288.

Type-species: Piada japonica Leech, I889, Proc. zool. Soc. Lond. 1889: 538, pl. 52, fig. 6, by original designation. Syntypes I 0,1 ㅇ, JAPAN: [Hokkaido], Yesso; [Kyushu], Sendai. (BMNH).

The type-species was originally described as Piada multiplicans var. japonica Leech. Under the amended Code, Article 45, a varietal name is to be accepted as a nomenclaturally available subspecific name. Warren changed its status to that of a species, without stating that he was so doing.

The date of publication of the part of Seitz containing page 288 was stated on page 285 as I9I 3 January 5 th in both the German and the English editions.

MIMASURA Hampson, I9Io [August], Proc.zool. Soc. Lond. 1910: 412.

$A C O N$

Type-species: Mimasura quadripancta Hampson, I9IO, ibidem 1910: 412, by subsequent designation by Collins, 1962, Ann. Mag. nat. Hist. (13) 5: I23. Syntypes I o. I 우, [ZAMBia] N.E. Rhodesia: Luangwa valley (BMNH).

MIMASURA Hampson, I9Io [December], Cat. Lepid. Phalaenae Bv. Mus. 10: xvii, 634.

Type-species: Xanthoptera tripuncta Hampson, 1902, Ann. S. Afr. Mus. 2: 398, by original designation.

A junior homonym of Mimasura Hampson, I9Io [August]. This generic name was used and made available by Hampson, r910 [August], prior to its proposal and generic description by Hampson, I9Io [December]. The date of publication of the former is printed in the separates of Hampson's Proc. zool. Soc. Lond. paper; the date of publication of the latter is given by Collins, 1962, Ann. Mag. nat. Hist. (13) 5: I26. There is no objective replacement name but $X$. tripuncta is congeneric with Mimasura quadripuncta Hampson, igio, the type-species of Mimasura Hampson, I9lo [August]. The latter is therefore available for use as a subjective replacement name.

MIMASURA Hampson, I9Io [December]. See Mimasura Hampson, I9Io [August]. ACON

†MIMERASTIA, misspelling. See Mimerastria Butler, I88I. Noli

MIMERASTRIA Butler, I88I, Ann. Mag. nat. Hist. (5) 7:236. Noli

Type-species: Erastria mandschuriana Oberthür, I880, Etudes Ent. 5: 83, pl. 2, fig. 9.

by monotypy. Holotype đ̃. [U.S.S.R.]: [near Vladivostok], Askold I.

†MIMERASTIA ; Pagenstecher, 1909, Geogr. Verbveitung Schmett.: 422.

An incorrect subsequent spelling.

MIMETES Hübner, [1 821 ]. See Euxoa Hübner, [1821].

NOCT

$\ddagger$ MIMETIS, misspelling. See Euxoa Hübner, [I 821$]$.

NOCT

MIMEUGOA Hampson, 1895, Trans. ent. Soc. Lond. 1895: 294.

O1'11

Type-species: Mimeugoa edentifascia Hampson, I 895, ibidem 1895: 295, by original designation. Syntypes +. [Sikkim] Sikhim (BMNH). Tndia: Simla and Murree.

$M$. edentifascia is a junior subjective synonym of Anachrostis bifasciata Hampson. 1894, Fauna Br. India (Moths) 2: 100.

MIMEUSEMIA Butler, 1875, Ann. Mag. nat. Hist. (4) 15: 397.

AGAR

Type-species: Mimeusemia persimilis Butler, 1875 , ibidem (4) 15: 397, by original designation. Type(s), [JAPAN]: Hakodadi (BMNH). 
MIMLEUCANIA Hampson, 1908, Cat. Lepid. Phalaenae Br. Mus. 7: 19. Available, but without included species until Hampson, I909, ibidem 8: $x, 285 . \quad$ ACRO Type-species: Mimleucania perstriata Hampson, I909, ibidem 8: 286, fig. 82, by subsequent designation by Hampson, r909, ibidem 8: 285. Syntypes 3 ot, I ‥ [South AfricA]: Orange River Colony, Bloemfontein (BMNH).

MIMOBARATHRA Barnes \& McDunnough, I9I5, Can. Ent. 47: 2 I. HADE Type-species: Mamestra antonito Barnes, I907, ibidem 39: I4, by original designation. Syntypes $\sigma^{*}$, [U.S.A.]: Ariz., Huachuca Mts.

The specimen labelled as type of (not described nor mentioned in the original description) of $M$. antonito Barnes, I907, was later described as a distinct species Leucocnemis barbara Barnes \& McDunnough, I9I5, ibidem 47: 2 I.

MIMOPHISMA Hampson, I926, Descr. new Genera Species Lepid. Phalaenae Subfamily Noctuinae Br. Mus.: I04. OPHI

Type-species: Ophisma delunaris Guenée, 1852, in Boisduval \& Guenée, Hist. nat. Insectes (Lépid.) 7:238, by original designation. Holotype $\widehat{0}$, TYPE-LocaliTY not known [S. America].

MIMORUZA Hampson, i 895, Trans. ent. Soc. Lond. 1895: 301. ACON

Type-species: Mimoruza nigriceps Hampson, I 895, ibidem 1895: 301, fig. [II], by original designation. Type(s)

MINDORA Möschler, I88o, Verh.zool.-bot. Ges. Wien 30 (Abh.) : 482.

CHLO

Type-species: Mindora tortriciformis Möschler, I880, ibidem 30 (Abh.): 483, pl. 9, fig. 33, by monotypy. Holotype ô. Surinam: Paramaribo (MNHU, Berlin).

MINICA Walker, [1858] I 857, List Specimens lepid. Insects Colln Br. Mus. 13: I I22, I I39. STIC Type-species: Minica confluens Walker, [I858] i 857, ibidem 13: I I40, by monotypy. Holotype 9 , TYPE-LOCALITY not known (BMNH) [AFRICA].

MINIODES Guenée, I 852, in Boisduval \& Guenée, Hist. nat. Insectes (Lépid.) 7: i I9. CATo Type-species: Miniodes discolor Guenée, I852, ibidem 7: I I9, pl. I6, fig. 4, by monotypy. Lectotype $\hat{\sigma}$. Africa: western, Côte de Guinée (MNHN, Paris), designated by Viette, I95I, Bull. mens. Soc. linn. Lyon 20: I62.

MINIOPHYLLODES Joannis, I9 I2, Bull. Soc. ent. Fr. 1912: 33 I.

Type-species: Miniophyllodes aurora Joannis, I9I2, ibidem 1912: 33I, by monotypy. Holotype + (not ơ as stated), Madagascar: Diego Suarez, Mt Ambre (MNHN, Paris).

MINNAGARA Walker, I866, List Specimens lepid. Insects Colln Br. Mus. 35: I902. Noli

Type-species: Minnagara fasciata Walker, I866, ibidem 35: I903, by monotypy. Lectotype ․, [Moluccas]: Sula [Is] (UM, Oxford), designated (as type) by Swinhoe, I 892, Cat. east. and Aust. Lepid. Heterocera 1: 99.

MINOFALA Smith, I905, Can. Ent. 37:65. ACRO

Type-species: Minofala instans Smith, 1905, ibidem 37: 65, by original designation. Holotype ô, [U.S.A.]: Texas, Galveston.

MINUCIA Moore, [I 885] I 887, Lepid. Ceylon 3: I59.

Type-species: Noctua lunaris [Denis \& Schiffermüller], I 775, Ankündung Syst. Werkes Schmett. Wienergegend: 94, by original designation. Type(s), [AustriA]: Vienna district (Collection destroyed, Horn \& Kahle, 1936, Ent. Beih. Berl.-Dahlem 3: 243). $\ddagger$ ASC ALAPHA Hübner, [1806], Tentamen determinationis digestionis . . . [2].

Included in a work rejected for nomenclatural purposes by Int. Commn zool. Nom., 1926, Smithson. misc. Collns 73 (4) Opinion 97: 19. Also idem, 1954, Opin. Decl. int. Commn zool. Nom. 6 Opinion 278: I 40.

Only included species: Noctua lunaris [Denis \& Schiffermüller), I 775. 
OPHIODES Guenée, I 84I, Annls Soc. ent. Fr. 10: 77.

Type-species: Noctua lunaris [Denis \& Schiffermüller], I 775, by subsequent designation by Guenée, 1852, in Boisduval \& Guenée, Hist. nat. Insectes (Lépid.) 7: $23 \mathrm{I}$.

A junior homonym of Ophiodes Wagler, I830, Nat.Syst. Amph.: I 59, - Reptilia. The objective replacement name is Minucia Moore, [1885].

NANTESIA Kirby, I897, Hand-Book Order Lepid. 5: I 73.

Proposed, unnecessarily, as the objective replacement name for $\ddagger$ Ascalapha Hübner, [1806], and for Ophiodes Guenée, I8+1.

MIODERA Smith, 1908, Ann. N.Y. Acad. Sci. 18: Ior.

HADE

Type-species: Miodera stigmata Smith, I908, ibidem 18: I02, by monotypy. Syntypes Io ô. [U.S.A.]: Cal., Witch Creek.

MIONIDES Hampson, I902, Ann. S. Afr. Mus. 2: $28 \mathrm{I}$.

Type-species: Mionides lichenea Hampson, I902, ibiden 2:28I, by monotypy. Type(s) o. South Africa: Natal, Estcourt (B.INH).

MIRACAVIRA Franclemont, 1937, Can. Ent. 69: I30.

CUCU

Type-species: Momaphana sylvia Dyar, 1913, Proc. U.S. natn. Mus. 44: 292, by original designation. Syntypes $7 \overrightarrow{0}, 5$ + , [U.S.A.]: S. Arizona. Mexico: Tehuacan; Guadalajara; Zacualpan and Cuernavaca. (USNM, Washington).

MIRACOPA Draudt, I950, Nitt. mïnch. ent. Ges. 40: I I9.

ACRO

Type-species: Miracopa prodigiosa Draudt, I950, ibidem 40: II9, pl. 8, fig. 5, by monotypy. Syntypes ơ, China: [Szechwan Prov.], Mien-shan (MAK, Bonn).

MIROPALPA Berio, I955, Mem. Inst. sci. Madagascar (E) 6: I 23.

ACRO

Type-species: Callopistria pauliani Berio, I955, ibidem (E) 6: I23, by original designation. Holotype $\vec{\delta}$, MADAGASCAR: forêt du domaine de l'Est, région de Périnet (MNHN, Paris).

Miropalpa was proposed as a subgenus of Callopistria Hübner, [I82 I].

MISA Karsch, I 895, Ent. Nachr. 21:349.

AGAR

Type-species: Misa memnonia Karsch, I895, ibidem 21: 349, 356, pl. 2, fig. 9, by original designation. Holotype \&, Togo: Station Misahöhe (MNHU, Berlin).

${ }_{\ddagger}^{\ddagger}$ MISELIA Hübner, [ISo6]. See Allophy'es Tams, I942.

$\mathrm{CUCl}^{\top}$

MISELIA Ochsenheimer, I 816, Schmett. Eur. 4: 72.

HADE

Type-species: Noctua compta [Denis \& Schiffermüller], 1775, Ankïndung syst. Werkes Schmett. Wienergegend: 70, by subsequent designation by Samouelle, I819, Entonologist's useful Compendium: 25I. Type(s), [Austria]: Vienna district (Collection destroyed, Horn \& Kahle, 1936, Ent. Beih. Berl-Dahlem 3: 243).

HARMODIA Hübner, [1820] I816, l'erz. bekannter Schmett.: 207.

Type-species: Noctua compta [Denis \& Schiffermüller], I 775, by subsequent designation by Hampson, 1905, Cat. Lepid. Phalaenae Br. Mus. 5: 6o.

Harmodia is a junior objective synonym of Miselia Ochsenheimer, I8I6.

MISELIA Boisduval, I 828. See Allophyes Tams, 1942.

CUCl:

MITHILA Moore, 1882, in Hewitson \& Moore, Descr. new Indian lepid. Insects Colln late Mr II. S. Atkinson: 156 .

OPHII

'Type-species: Mithila lichenosa Moore, I882, ibidem: I57, by monotypy. Syntypes, INDIA: Darjiling (BMNH).

MITHIMNA Sodoffsky, i 837. See Hythimna Ochsenheimer, 1816.

HADI 
MITOTHEMMA Butler, I883, Trans, ent. Soc. Lond. 1883: 62.

OPHI

Type-species: Mitothemma angulipennis Butler, I 883, ibidem 1883: 62, by PRESENT

DESIGNATION. Type(s), CHILE: Las Zorras (BMNH).

Mitothemma was originally placed in the Pyralidae, but was transferred to the Noctuidae by Hampson, i 895, Proc. zool. Soc. Lond. 1895: 974.

MITROPHRYS Karsch, 1895, Ent. Nachr. 21: 349.

AGAR

Type-species: Phalaena menete Cramer, I775, Uitlandsche Kapellen 1: 110, pl. 70, fig. D, by original designation. Type(s), West Indies.

$P$. menete is dated from the wrapper of the part and not from the title-page of the volume, I 779 .

MIXOCHARIS Lederer, I853. See Microphisa Boisduval, I 840.

$\triangle \mathrm{CON}$

MIXOMELIA Hampson, I898, J. Bombay nat. Hist. Soc. 11: 708.

HYPE

Type-species: Mixomelia decipiens Hampson, I898, ibidem 11: 708, fig., by original designation. Type(s) of. India: Assam, Khasis (BMNH).

MIXTILA Swinhoe, I900. See Pinacia Hübner, [I $83 \mathrm{I}]$.

HYPE

MNESIPYRGA Meyrick, I913, Trans, ent. Soc. Lond. 46: I 70.

$\operatorname{ACON}$

Type-species: Mnesipyrga trichostrota Meyrick, I9I3, ibidem 46: I 7I, by monotypy. Holotype + , PERU: Chanchamayo (BMNH).

Mnesipyrga was originally placed in the Tortricidae.

MNIO THRIPA Hampson, igi2, Cat. Lepid. Phalaenae Br. Mus. 11: x, 260.

SARR

Type-species: Giaura lichenigera Hampson, I905, Ann. Mag. nat. Hist. (7) 16: 543, by original designation. Syntypes 3 \% SINGAPORE (BMNH).

MNIOTYPE Franclemont, I94I, Ent. Neres 52: 201.

CUCU

Type-species: Hadena ducta Grote, i878, Bull. U.S. geol. geogr. Surv. Territ. 4: 176, by original designation. Type(s) ㅇ․ [U.S.A.]: Me., Orono (BMNH).

CRINO Hübner sensu Hampson, I906, Cat. Lepid. Phalaenae Br. Mus. 6: 32 I, 332.

Hampson used Hadena sommeri Lefebvre, 1836, - Noctuidae, Cuculliinae, as the typespecies of Crino, instead of Crino sommeri Hübner, [I82 I], - Notodontidae.

Hadena sommeri Lefebvre is congeneric with Hadena ducta Grote.

MOCIS Hübner, [1823] I8I6, Verz. bekannter Schmett.: 267.

CATO

Type-species: Phalaena virbia Cramer, I780, Uitlandsche Kapellen 3: I46, pl. 273 , fig. H, by subsequent designation by Hampson, I913, Cat. Lepid. Phalaenae Br. Mus. 13: 76, but cited as 'undata Fabr.', which Hampson placed, on page $9 \mathbf{I}$, as the senior synonym of virbia Cramer. Type(s), [INDIA]: Coromandel Coast.

$P$. virbia is a junior subjective synonym of Noctua undata Fabricius, I775, Sy'st. Ent.: 600.

Incorrect type-species designation: Mocis aurinia Geyer, I832, a name not originally included in Mocis and not linked with one of the originally included names when cited by Desmarest, 1857 , in Chenu, Encycl. Hist. nat. Papillons nocturnes: i 33.

See also Cauninda Moore, [1885].

MOCRENDES nom. n. for Cremnodes Felder, I 874.

OPHI

Type-species: Cremnodes lemur Felder, 1874 , in Felder \& Rogenhofer, Reise öst. Fregatte Novara (Zool.) 2 (Abt. 2): pl. II3, fig. 9, by monotypy (of Cremodes Felder). Type(s) ô, Molucca Is (BMNH).

CREMINODES Felder, I874, in Felder \& Rogenhofer, Reise öst. Fregatte Novara (Zool.) 2 (Abt. 2) : pl. II3.

Type-species: Cremnodes lemur Felder, 1874, by monotypy.

A junior homonym of Cremnodes Foerster, I850, Arch. Naturgesch. 16 (I): 70, 72, Hymenoptera. The objective replacement name is Mocrendes nom. n. 
MODUNGA Walker, I863, List Specimens lepid. Insects Colln Br. Mus. 27: 8f.

Type-species: Modunga palpigera Walker, 1863, ibidem 27:84, by monotypy.

Type(s)

ठ., BORnEO: Sarawak (BMNH).

$\ddagger$ MOENAS, misspelling. See Eudocima Billberg, I 820 .

OPH I

MOEPA Walker, I865, List Specimens lepid. Insects Colln Br. Mus. 33: 9 I 5.

C.ATO

Type-species: Moepa albidens Walker, I865, ibidem 33: 916, by monotypy. Holotype \%, [INDIA]: S. Hindostan (BMNH).

MOEPA Walker, r865, List. . 33: 981. See Orrea Walker, r 866.

OPHI

MOLOPA Swinhoe, 1902, Ann. Wag. nat. Hist. (7) 9: f20.

OPHI

Type-species: Molopa planalis Swinhoe, I902, ibidem (7) 9: 42I, by monotypy. Lectotype $\delta$, Australia: IV. Aust., Roebourne, designated by Common, 1963, J. ent. Soc. Qd 2: 6 .

MOLVENA Walker, [1866] I865, List Specimens lepid. Insects Colln Br. Mus. 34: I266. ACro Type-species: Molvena guttalis Walker, [1866] I865, ibidem 34: I267, by monotypy. Holotype $\overrightarrow{0}$, [AustraLia ]: [Qd], Moreton Bay (BMNH).

MOL YNDA Walker, I858, List Specimens lepid. Insects Colln Br. Mus. 15: I653. OPH Type-species: Molynda humeralis Walker, I858, ibidem 15: I653, by monotypy. Type(s) ô, [BrAzıL]: Rio Janeiro (UMI, Oxford).

MOMA Hübner, [1820] I816, V'erz. bekannter Schmett.: 203.

ACRO

Type-species: Noctua aprilina Linnaeus sensu Hübner, [1803], Samml eur. Schmett. 6: fig. 22 [a misidentification for Phalaena alpium Osbeck, I778, Götheborg. Samhalle Handl. (Wet. Afd.) 1: 52, pl. I, fig. 2], by subsequent designation by Grote, I874, Bull. Buffalo Soc. nat. Sci. 2: 7, but cited as 'Noctua orion Esper (aprilina Hübn.).' Type(s) of P. alpium, SWEDEN.

The second of the three species originally included in Moma was cited by Hübner as ' $H$. aprilina Linn., Syst., Phal. I38. Hübn., Noc. 22.' In Hübner, [1803], Samml. eur. Schmett. 4: figure 22 named aprilina was a misidentification. This mistake, repeated in Verz. bekannter Schmett.: 203, was corrected by Grote, 1874 (see above). Staudinger \& Rebel, I9o I, Cat. Lepid. palaearct. Faunengeb. (I): I30, placed Noctua orion Esper, I79I, and Noctua aprilina Linnaeus sensu Hübner, [ 1803 ], as junior synonyms of Phalaena alpium Osbeck, I778. I have examined the illustrations published in the original descriptions of these three taxa and agree that they are all conspecific. The true Phalaena aprilina Linnaeus, $175^{8}$ is the type-species of Griposia Tams, 1939.

This is a case of a misidentified type-species which under the Code, Article 7o (a), should be referred to the Commission.

$\ddagger$ DIPHTHERA Hübner, [1806], Tentamen determinationis digestionis . . . [1] .

Included in a work rejected for nomenclatural purposes by Int. Commn zool. Nom., 1926, Smithson. misc. Collns 73 (4) Opinion 97: 19. Also idem, 1954, Opin. Decl. int. Commn zool. Nom. 6 Opinion 278: r 40.

Only included species: Noctua aprilina Linnaeus sensu Hübner, [1803].

${ }_{+}$DIPHTERA; Ochsenheimer, i816, Schmett. Eur. 4: 63 .

An incorrect subsequent spelling of $\ddagger$ Diphthera Hübner, [1806], and Diphthera Hübner, 1809 .

Ochsenheimer attributed this genus to Hübner and was not proposing a new genus. Some authors, including Boisduval and Guenée, have used Ochsenheimer's incorrect spelling as an available name, but under the Code. Article 19, an incorrect spelling has no standing in nomenclature.

Ochsenheimer, Treitschke, Boiscluval and Guenée all followed Hübner's [1806] Tentamen concept based on the misidentification, Noctua aprilina Linnaeus sensu Hübner, [1803]. 
Hübner in 1809 broadened his concept (see Diphthera Hübner, [I809]) by including Diphthera elegans Hübner, [1809].

Sodoffsky, I837, Bull. Soc. imp. Nat. Moscou 1837 (6): 85, noted that Treitschke's spelling of the generic name was incorrect.

†DIPTHERA; [Hübner], [1825], Cat. Lépid. composent Colln feu Mr Franck: 90.

An incorrect subsequent spelling.

$\ddagger$ MOMAPHANA, misspelling. See Momophana Grote, 1875 .

CUCU

$\ddagger$ \OMOCATADA Hampson, no published reference found.

HYPE

MOMOPHANA Grote, I875, Stettin. ent. Ztg 36: 195.

CUCU

Type-species: Feralia comstocki Grote, 1874, Bull. Buffalo Soc. nat. Sci. 2: 59, by monotypy. Holotype o. [U.S.A.]: N.Y., I thaca (BMNH).

$\ddagger$ MOMAPHANA; Hampson, I906, Cat. Lepid. Phalaenae Br. Mus. 6: 199.

An incorrect subsequent spelling.

MONIMA Hübner, [1821] I816, Verz. bekannter Schmett.: 229.

HADE

Type-species: Noctua miniosa [Denis \& Schiffermüller], I 775, Ankündung syst. Werkes

Schmett. Wienergegend: 88, by subsequent designation by Hampson, 1905, Cat. Lepid. Phalaenae Br. Mus. 5: 409. Type(s), [Ausrria]: Vienna district (Collection destroyed, Horn \& Kahle, 1936, Ent. Beih. Berl.-Dahlem 3: 243).

MONOCHROIDES Kaye \& Lamont, 1927, Mem. Dept. Agric. Trinidad \& Tobago 3: 78 . HYPE

Type-species: Bleptina olivescens Warren, I889, Trans. ent. Soc. Lond. 1889: 240, by monotypy. Syntypes 2 ㅇ, BraziL: Basin of the Amazons, Trompetas (BMNH); R. Solimoes, Guará (BMNH).

MONOCTENIA Guenée, 1857, in Boisduval \& Guenée, Hist. nat. Insectes (Lépid.) 9 : I83. GEOMETRIDAE

Type-species: Monoctenia falernaria Guence, I 857, ibidem 9: I84, pl. 7, fig. 3, by monotypy.

Monoctenia has been used as the original generic name for species which are now in the Noctuidae.

MONOCYMIA Hampson, Igro, Cat. Lepid. Phalaenae Br. Mus.. 10 : xvii, 644.

$\operatorname{ACON}$

Type-species: Acontia harmina Schaus, 1904, Trans. Am. ent. Soc. 30: I63, by original designation. Type(s), [BrazIL]: Parana, Castro.

MONODES Guenée, I 852, in Boisduval \& Guenée, Hist. nat. Insectes (Lépid.) 5: 240. ACro Type-species: Monodes nucicolora Guenée, I 852, ibidem 5: 24I, pl. 4, fig. 9, by monotypy. Holotype , [U.S.A.] : Florida (BMNH).

MONOGONA Guenée, I852, in Boisduval \& Guenée, Hist. nat. Insectes (Lépid.) 6: 402. opHI

Type-species: Hypsoropha hormos Hübner, I818, Zuträge Samml. exot. Schmett.

1: I 4, figs 27, 28, by monotypy. Type(s) ㅇ, [U.S.A.]: Georgia [formerly] in Florida.

$\ddagger$ GLOEE Hübner, I8o8, Erste Zuträge Samml. exot. Schmett.: 4,5 .

Included in a work rejected for nomenclatural purposes by Int. Commn zool. Nom., I 966, Bull. zool. Nom. 23 Opinion 789:2 I4. Placed on the Official Index of Rejected and Invalid Generic Names in Zoology: Name No. I846.

Gloee monilis Fabricius sensu Hübner, I808, was designated as type-species by Berio, I957. Memorie Soc. ent. ital. 36: I4. The misidentification G. monilis Fabricius sensu Hübner, I 8o8, was replaced by Hypsoropha hormos Hübner, I8I8.

TIAUSPA Walker, [1858] I857, List Specimens lepid. Insects Colln Br. Mus. 13: 984, 995.

Type-species: Hypsoropha hormos Hübner, i8 8 , by monotypy.

Tiauspa is a junior objective synonym of Monogona Guenée, 1852 .

$\ddagger$ GLOCE; Neave, 1939, Nomencl. zool. 2: 477 .

An incorrect subsequent spelling. 
MONOPTYA Hampson, igo8, Cat. Lepid. Phalaenae Br. Mus. 7 : xii, 432. ACRO

Type-species: Euplexia leucobasis Hampson, 1904, Ann. Mag. nat. Hist. (7) 14: 167, by original designation. Holotype ㅇ, Bahamas: Abaco (BMNH).

MONOSCA Walker, I869, Characters undescr. Lepid. Heterocera: 29.

HADE

Type-species: Monosca subnotata Walker, I869, ibidem: 29, by monotypy. Type(s)

9 , TYPE-LOCALITY not stated (BMINH). [CANADA. U.S.A.].

M. subnotata is a junior subjective synonym of Nephelodes minians Guence, 1852.

See also Nephelodes Guenée, 1852.

MONOSTOLA Alphéraky, i892, in Romanoff, Mém. Lépid. 6: 37.

HADE

Type-species: Monostola asiatica Alphéraky, 1892, ibidem 6:37, pl. 2, fig. 7, by monotypy. Syntypes, CHINA: 8 ô. Prov. [Kansu] Gan-Sou, R. Honton (ZI, Leningrad); i + , N. Himalaya.

MOPOTHILA nom. n. for Lithopoma Schaus, I9I6.

Type-species: Ipnea ardalus Druce, I891, Biologia cent.-am. (Zool.) Lepid. Heterocera 1: 482, pl. 40, fig. II, by original designation (of Lithopoma Schaus). Holotype ${ }_{4}$ Panama: Volcan de Chiriqui (BMNH).

LITHOPOMA Schaus, 1916, Proc. U.S. natn. Mus. 50: 379.

Type-species: I pnea ardalus ])ruce, 1891 , by original designation.

A junior homonym of Lithopoma Gray, 1850, Figuves molluscous A nim. 4: 88, - Mollusca. The objective replacement name is Mopothila nom. $\mathrm{n}$.

MORMECIA Tams, 1935, Insects Samoa 3 (4): 231.

HYPE

Type-species: Mormecia lachnogyia Tams, 1935, ibidlem 3 (4): 23I, pl. 6, fig. 20, and pl. 7 , fig. 8, by original designation. Holotype ô., SAMOA (BMNH).

MORMO Ochsenheimer, I816, Schnett. Eur. 4: 70.

ACRO

Type-species: Phalaena maura Linnaeus, 1758, Syst. Nat. (Edn 10) 1: 512, by subsequent designation by Samo'selle, 1819, Entomologist's useful Compendium: 251, but cited as ' $N$. maura Fabr.', an incorrect authorship. Type(s), Mauritania (IS, London).

$\ddagger$ tLEMUR Hübner, [1806], Tentamen determinationis digestionis . . . [2].

Included in a work rejected for nomenclatural purposes by Int. Commn zool. Nom., 1926, Smithson. misc. Collns. 73 (4) Opinion 97: 19. Also idem, 1954, Opin. Decl. int. Comm. zool. Nom. 6: Opinion 278: 140.

Only included species: Phalaena maura Linnaeus, 1758.

+LEMURES Ochsenheimer, I8I6, Schmett. Eur. 4: 70.

Published as a junior synonym of Mormo Ochsenheimer, I8I6, and not subsequently treated as an available name under the Cocle, A ticle I 1 (d).

LE.MUR Hübner, 1822, Sy'st.-alphab. I'erz.: 23, 30.

Type-species: Phalaena maura Linnaeus, 1758 , by subsequent designation by Barnes \& Benjamin, 1923, Contr. nat. Hist. Lepid. N. Am. 5: $5^{8}$.

A junior homonym of Lemur Linnacus, 1758, Syst. Nat. (Edn ro) 1: 29, - Mammalia. The objective replacement name is Momo Ochsenheimer, I8r6.

Incorrect type-species designation: Phulaena typica J,innaeus, $175^{8}$, a name not originally: included in Lemur, and not linked with one of the originally included names when cited (for †Lemuris) by Westwood, 18ło. Synopsis Genera 13r. Insects: 94.

MANIA Treitschke, 1825, Schmett. Eur. 5 (I): 294.

Proposed, unnecessarily, as an objective replacement name for Mormo Ochsenheimer, I816, which Treitschle considered to be a junior homonym of Mormon Illiger, i8i1, Prodromus sist. Manmalium Aizum: 283 , - Aves.

A junior homonym of Mania Hübner, 1821 , lndex exot. Lepid.: [3], - Lepid., Uraniidae. +L.FLURIS; Curtis, 1831, Guide Arrangement lir. Insect: 1.18.

An incorrect subsequent spelling. 
ACYRA Gistl, I 848, Naturg. Thierreichs: x.

Proposed, unnecessarily, as the objective replacement name for Mormo Ochsenheimer, I8I6, which Gistl considered to be a junior homonym of Mormon Illiger, i8 I I, - Aves.

$\ddagger A R C Y R A$; Neave, 1939, Nomencl. zool. 1: 286.

An incorrect subsequent spelling.

MORMONIA Hübner, [1823] I8I6, Verz. bekannter Schmett.: 276.

CATO

Type-species: Phalaena epione Drury, I773, Illust. nat. Hist. 1 (1770): 47, pl. 23, fig. 2, but without names until the Index, issued with 2 (I773), by monotypy. Type(s), [U.S.A.]: New York.

†MORMOSIA; Walker, [1858] I857, List Specimens lepid. Insects Colln Br. Mus. 13: I I79.

An incorrect subsequent spelling.

MORMOSCOPA Meyrick, i897, Trans. ent. Soc. Lond. 1897: 37 I.

HYPE

Type-species: Mormoscopa crossodora Meyrick, I 897, ibidem 1897 : 37 I, by monotypy. Syntypes I 3 , I 9 , Australia: New South Wales, Mount Kosciusko (BMNH).

$M$. crossodora is a junior subjective synonym of Bleptina sordescens Rosenstock, I 885 , Ann. mag. nat. Hist. (5) $16: 424$, pl. I I, fig. I I.

$\ddagger$ \ORMOSIA, misspelling. See Mormonia Hübner, [1823].

CATO

MORRISONIA Grote, I874, Bull. Buffalo Soc. nat. Sci. 2: 53.

HADE

Type-species: Cloantha evicta Grote, 1873, ibidem 1: 84, pl. 2, fig. 18, by original designation. Type(s) ô, [U.S.A.]: New York State (BMNH).

MOSARA Walker, i 855, List Specimens lepid. Insects Colln Br. Mus. 5: 980, I032.

Type-species: Mosara apicalis Walker, I 855, ibidem 5: 1032, by monotypy. Holotype ठ., N. India (BMNH).

MOSCHA Walker, [1866]. See Hypertrocta Hampson, 1893.

HYPE

MOSOPIA Walker, [1866] I 865, List Specimens lepid. Insects Colln Br. MIus. 34: I I87. HyPE Type-species: Mosopia megaspila Walker, [I866] I865, ibidem 34: I I88, by monotypy. Holotype $\hat{0}$. [West Malaysia]: [Malaya], Penang (BMNH).

MOTAMA Moore, I882, in Hewitson \& Moore, Descr. new Indian lepid. Insects Colln late Mr W. S. Atkinson: i го.

ACON

Type-species: Motama cidarioides Moore, I882, ibidem: I Io, pl. 4, fig. 9, by subsequent designation by Hampson, I9 1o, Cat. Lepid. Phalaenae Br. Mus. 10: 497. Type(s), India: [W. Bengal], Darjiling (MNHU, Berlin).

$\ddagger$ MOTANA; Neave, 1940, Nomencl.zool. 3: 222.

An incorrect subsequent spelling.

$\ddagger$ MOTANA, misspelling. See Motama Moore, i 882 .

$\operatorname{ACON}$

MOTINA Walker, 1863, List Specimens lepid. Insects Colln Br. Mus. 27: I2. OPHI

Type-species: Motina aequalis Walker, 1863, ibidem 27: I2, by monotypy. Type(s)

, BORnEO: Sarawak.

Motina when originally proposed contained a second species, but this was doubtfully included and, under the Code, Article 68(c), is not eligible for selection as type-species.

MOTYA Walker, I 859, List Specimens lepid. Insects Colln Br. Mus. 19: I021.

Type-species: Motya abseuzalis Walker, I859, ibidem 19: 1022, by monotypy. Type(s)

o. [BRAZIL]: Rio Janeiro (UM, Oxford).

See also Lussa Grote, I883; and Pleurasy'mpieza Möschler, I890.

MOURALIA Walker, I858, List Specimens lepid. Insects Colln Br. Mus. 15: 1803.

PLUS

Type-species: Mouralia annulifera Walker, I858, ibidem 15: I803, by monotypy. Holotype $\hat{o}$, [Dominican Republic] St. Domingo (BMNH). 
MOUREIA Orfila \& Rossi, 1956, Rev. Soc. ent. Argent. 19: 29.

ACRO

Type-species: Poecilia microglossa Rambur, 1858 , Cat. syst. Lépid. Andalousie: pl. 7 , figs I, 2, and pl. 22, figs I, 2, by original designation (for Meroleuca Hampson, I908). Syntypes, [Sparn]: Andalusia.

Moureia was proposed as the objective replacement name for Meroleuca Hampson, I 908.

See also Oedibry'a Hampson, I9I8.

MEROLEUCA Hampson, I9o8, Cat. Lepid. Phalaenae Br. Mus. 7 : xv, 687.

Type-species: Poecilia microglossa Rambur, I 858 , by original designation.

A junior homonym of Meroleuca Packard, I904, Jl N.Y. ent. Soc. 12: 250,-Lepid., Saturniidae. The objective replacement name is Moureia Orfila \& Rossi, I956.

$\ddagger J U G U R T H I A$ Oberthür, 1909, in Culot; Neave, 1939, Nomencl. zool. 2: 8 1 3.

Culot attributed this generic name to Oberthür, but no earlier published reference has been found. Neave dated Jugurthia from the title-page of the volume and not from the wrappers of the parts which were published later.

JUGURTHIA Culot, I9I2, Noctuelles Géomètres Eur. 1: I 24.

Type-species: Poecilia microglossa Rambur, i 858 , by PRESENT DESIGNATION.

A junior homonym of Jugurthia Dalla Torre, I 894, Cat. Hymenopteron 9: 5, - Hymenotera. The objective replacement name is Moureia Orfila \& Rossi, I956.

Jugurthia Culot is dated from the wrappers of the parts and not from the title-page.

MUDARIA Moore, I893. Indian Mus. Notes 3: 68.

ACRO

Type-species: Mudaria cornifrons Noore, I 893 , ibidem 3: 69, fig., by monotypy. Syntypes ô, q. INDiA: Calcutta (BMNH).

MULELOCHA Walker, I 865, List Specimens lepid. Insects Colln Br. Mus. 33: I Iо3. орні

Type-species: Mulelocha frontalis Walker, I865, ibidem 33: iा 3, by monotypy. Holotype o., [BRAzIL]: Para (BMNH).

$M$. frontalis is a junior subjective synonym of Homoptera extranea Walker, I 865 , ibidem 33: $88 \mathrm{I}$.

MURGISA Walker, I864, J. Proc. Limn. Soc. (Zool.) 7: 193.

HYPE

Type-species: Murgisa orgyoides Walker, I864, ibidem 7: 193, by monotypy. Lectotype ô. Borneo: Sarawak (U1I, Oxford), designated by Swinhoe, 19oo, Cat. east. and Aust. Lepid. Heterocera 2: 200.

MURSA Walker, I 859, List Specimens lepid. Insects Colln Br. Mus. 19: 845.

Type-species: Mursa calisalis Walker, I 859, ibidem 19: 846, by monotypy. Type(s) ô. [BRazil]: Rio Janeiro (UM, Oxford).

M. calisalis is a junior subjective synonym of Physula phtisialis Guenée, 1854 , in Boisduval

\& Guenée, Hist. nat. Insectes (Lépid.) 8: 87.

See also Paramimetica Warren, I889, and Sisputa Möschler, I89o.

MUSOTHYMA Meyrick, I 897, Trans. ent. Soc. Lond. 1897: 375.

ACRO

Type-species: Musothyma cyanastis Meyrick, I 897, ibidem 1897: 375, by monotypy. Syntypes I ô, I + , Australia: Qd, Duaringa (BMNH).

MUSURGINA Jordan, I92I, Novit. zool. 28: 69, 71.

AGAR

Type-species: Musurgina laeta Jordan, I92I, ibidem 28: 71, figs $1-6$, by original designation. Holotype $\hat{o}$, Managascar: Diego Suarez (BMNH).

MYALILA Strand, 1909, Arch. Naturgesch. 75 (I) 3:381.

ACRO

Type-species: Myalila typica Strand, I 909, ibidem 75 (I) 3:38I, by original designation. Holotype, [Zambia/Rhodesia]: Zambesi I., Myalila (MNHU, Berlin).

MYANA Swinhoe, i884, Proc. zool. Soc. Lond. 1884: 522.

OPIII

Type-species: Myana sopora Swinhoe, I884, ibidem 1884: 522, pl. 48, fig. 4, by PRESENT DESIGNATION. Type(s), [Pakistan]: Sind, Kurrachee (BMNH). 
MYCTEROPHORA Hulst, i 896, Trans. Am. ent. Soc. 23: 298.

OPHI

Type-species: Mycterophora monticola Hulst, I896, ibidem 23: 299, by original designation. Type(s) $\hat{\jmath}$. [U.S.A.]: California, Sierra Nevada.

$M y c t e r o p h o r a$ was originally placed in the Geometridae.

$\ddagger$ MYCTEROPHORA Dyar, I904; Neave, I940, Nomencl. zool. 3: 235.

Listed by Neave as a nomenclaturally available name (and a junior homonym) of Mycterophora Hulst, I896. In my opinion the name is not available as Dyar, I904. Proc. U.S. natn. Mus. 27: 877, did not propose a new genus but stated, "The genus [Mycterophora Hulst], described as a Geometrid, is probably referable to the Noctuidae.'

$\ddagger$ MYCTEROPHORA Dyar, I904. See Mycterophora Hulst, I $896 . \quad$ OPHI

MYCTEROPLUS Herrich-Schäffer, [1850] i 845, Syst. Bearb. Schmett. Eur. 2: I91, 242. ACro

Type-species: Xanthia puniceago Boisduval, I840, Genera Index meth. Eur. Lepid.: I 46 , by monotypy. Syntypes, [U.S.S.R.] S. Russia.

The date of publication of Mycteroplus is accepted from Hemming, I937, Hübner 1: 585 .

STEPHANIA Guenée, I852, in Boisduval \& Guenée, Hist. nat. Insectes (Lépid.) 6: I72.

Type-species: Xanthia puniceago Boisduval, I 840, by monotypy.

Stephania is a junior objective synonym of Mycteroplus Herrich-Schäffer, [I 850 ].

MYDRODOXA Butler, i88o, Ann. Mag. nat. Hist. (5) 5: 340.

ACRO

Type-species: Mydrodoxa splendens Butler, I880, ibidem (5) 5: 34I, by original designation. Lectotype $q$, MADAGascar: Fianarantsoa (BMNH), designated (as type) by Hampson, i9ro, Cat. Lepid. Phalaenae Br. Mus. 9: 493.

$M y d r o d o x a$ was originally placed in the Arctiidae.

MYRTALE Druce, i891, Biologia cent.-am. (Zool.) Lepid. Heterocera 1: 443.

ACRO

Type-species: Myrtale imitata Druce, I89I, ibidem 1: 443, pl. 36, figs 6, 6a, 7, by original designation. Syntypes $\underset{\delta}{0}$,, Panama: Chiriqui. Brazil: S.E., Rio Janeiro.

${ }_{\ddagger}$ MYSTOMEMIA Hampson, no published reference found.

HYPE

MYSTROCEPHALA Herrich-Schäffer, I855, Sammlung neuer oder wenig bekannter aussereur.

Schmett. 1(I): wrapper, pl. 75, fig. 435; I 856 \& I858, ibidem, 1: 13, I7, 83. AGAR

Type-species: Mystrocephala quadrimacula Herrich-Schäffer, I855, ibidem 1(I): wrapper, pl. 75, fig. 435; I856 \& I858, ibidem 1: 13, 71, 83, by monotypy. Type(s) $\hat{o}$, [Australia] Nov. Holl.

MYTHIMNA Ochsenheimer, I8ı6, Schmett. Eur. 4: 78.

HADE

Type-species: Phalaena turca Linnaeus, I76I, Fauna Suecica (Edn 2): 322, by subsequent designation by Samouelle, I8I9, Entomologist's useful Compendium: 25I. Type(s), Sweden (LS, London).

PHILOSTOLA Billberg, I820, Enumeratio Insect. Mus. G.J. Billberg: 87.

Type-species: Phalaena turca Linnaeus, I76I, by subsequent designation by Tams, I939, Entomologist 72: I 40 .

Philostola is a junior objective synonym of Mythimna Ochsenheimer, i8r6.

MITHIMNA Sodoffsky, i837, Bull. Soc. imp. Nat. Moscou 1837 (6) : 87.

An unjustified emendation of Mythimna Ochsenheimer, i816.

HYPERIODES Warren, I9Io, in Seitz, Macrolepid. World 3: 94.

Type-species: Phalaena turca Linnaeus, I761, by original designation.

Hyperiodes is a junior objective synonym of Mythimna Ochsenheimer, I8I6.

The date of publication of the part of Seitz containing page 94 was stated on page 93 as I9Io February ist in the English edition, and as I9ro April 25th in the German edition.

МYTHYMIMA Berio, I970, Boll. Soc. ent. ital. 102: 22.

HADE

Type-species: Mythymima arcuata Berio, I970, ibidem 102: 22, fig. I, by original designation. Holotype $\hat{\jmath},[$ ZAIRE] Congo: [Katanga], Lulua, Kapanga (MRAC, Tervuren). 
NAARDA Walker, 1866, List Specimens lepid. Insects Colln Br. Mus. 35: I694.

Type-species: Naarda bisignata Walker, I866, ibidem 35: I695, by monotypy. Lectotype $\hat{0}$, [Moluccas]: Sula (UM, Oxford), designated (as type, from Sarawak) by Swinhoe, 1900, Cat. east. and Aust. Lepid. Heterocera 2: 2 I 5.

N. bisignata is a junior subjective synonym of Hypena ineffectalis Walker, [1859] I 858, ibidem 16:85.

$\ddagger N A A R O I D E S$ Prout, no published reference found.

HYPE

NABARA Walker, I866, List Specimens lepid. Insects Colln Br. Mus. 35: I705. Hyblaeidae Type-species: Nabara limacodella Walker, I866, ibidem 35: I706, by monotypy. Holotype , JaVA.

N. limacodella is a junior subjective synonym of Phalaena pueva Cramer, 1777, Uitlandsche Kapellen 2: 10, pl. I03, figs D, E.

See also Hyblaea Fabricius, I793.

NABARTHA Moore, [1885] I887, Lepid. Ceylon 3: 234.

HYPE

Type-species: Bocana schaldusalis Walker, [1859] I858, List Specimens lepid. Insects Colln Br. MU . 16: 180 , by original designation. Syntypes $3\left(0^{*}\right.$, O $\left.^{+}\right)$, Borneo: Sarawak (BMNH).

See also Culicula Walker, [1863].

NABOA nom. n. for Boana Walker, I862.

HYPE

Type-species: Boana semialba Walker, I862, Trans. ent. Soc. Lond. (3)1: i io, by monotypy (of Boana Walker, I862). Type(s) of (not $q$ as stated in description), BrazIL (UM, Oxford).

B. semialba is a junior subjective synonym of Hypena cataleuca Herrich-Schäffer, 1856 , Samml. nezuer oder wenig bekannter aussereur. Schmett. 1 (I): wrapper, pl. 79, fig. 452; I858, ibidem 1: 69.

BOANA Walker, i862, Trans. ent. Soc. Lond. (3) 1: i Io.

Type-species: Boana semialba Walker, i 862, by monotypy.

A junior homonym of Boana Gray, I825, Amm. Phil. 26: 214,-Amphibia. The objective replacement name is Naboa nom. $\mathrm{n}$.

NACERASA Walker, I866, List Specimens lepid. Insects Colln Br. Mus. 35: 1570.

ACON

Type-species: Nacerasa nana Walker, I866, ibidem 35: I 57 I, by monotypy. Holotype

․, [Australia]: [Qd], Moreton Bay (BMNH).

N. nana is a junior subjective synonym of Sophta concavata Walker, [1863] I862, ibidem 26: 1759 .

See also Sophta Walker, [1863].

NACHABA Walker, I863. See Chlumetia Walker, [1866]. EutE

NACNA Fletcher, 1961, Ruwenzori Exped. I952 1: 198. Aско

Type-species: Canna pulchripicta Walker, I865, List Specimens Lepid. Insects Colln Br. Mus. 33: 790, by monotypy (of Canna Walker, I865). Syntypes ô, ㅇ․, [INDia]: Darjecling (BMNH).

Nacna was proposed as the objective replacement name for Canna Walker, i865.

CANNA Walker, I865, List Specimens lepid. Insects Colln Br. MIts. 33: 790.

Type-species: Canna pulchripicta Walker, I865, by monotypy.

A junior homonym of Canna Gray, I82I, London Medical Repository 15: 307, - Mammalia. The objective replacement name is Nacna Fletcher, r96r.

NACOPA Barnes \& Benjamin, 1924, Contr. nat. Hist. Lepid. N. Am. 5: i5 5 .

CUCU

Type-species: Acopa bistrigata Barnes \& McDunnough, in 18 , ibidem 4: 107, pl. I7, fig. 18, by original designation. Syntypes 2 of. [U.S.A.]: Ariz., Cochise Co., Paradise; Ariz., Huachuca Mts. 
NAENIA Stephens, 1827, in Anonymous, Retrospective Rev. (2) 1: 243.

NOCT

Type-species: Phalaena typicoides Donovan sensu Stephens, 1827, ibidem (2) 1: 243 [a misidentification for Phalaena typica Linnaeus, 1758, Syst. Nat. (Edn 10) 1: 518], by monotypy. Type(s), TyPE-Locality (of P.typica) not stated (LS, London) [EUROPE].

$P$. typicoides Donovan sensu Stephens, 1827, was a misidentification of Phalaena typicoides Donovan, I811, Nat. Hist. Br. Insects 15: I, pl. 505. This is a case of a misidentified type-species which under the Code, Article 70 (a), should be referred to the Commission.

In Stephens, 1829, Illust. Br. Ent. (Haustellata) 2: 165, the error was corrected.

PHALAENA Linnaeus, I758, Syst. Nat. (Edn 10) 1: 495.

Type-species: Phalaena typica Linnaeus, $175^{8}$, by indication, under the Code, Articles 67 (b) and 68 (b).

Phalaena was suppressed for the purposes of the Law of Priority but not for those of the Law of Homonymy, by Int. Commn zool. Nom., 1957, Opin. Decl. int. Commn zool. Nom. 15 Opinion 450: 253. Placed on the Official Index of Rejected and Invalid Generic names in Zoology: Name No. 850.

Phalaena was used by Linnaeus to include all the Heterocera except for the Sphingidae. When supressed in Opinion 450, Phalaena was replaced by the following Linnaean subgenera, upgraded to genera:

Alucita L., I758, - Alucitidae.

Attacus L., I 767 , - Saturniidae.

Bombyx L., I 758 - Bombycidae.

Geometra L., I758, - Geometridae.
Noctua L., I 758 ,- Noctuidae.

Pyralis L., I758, - Pyralidae.

Tinea L., I 758, - Tineidae.

Tortrix L., I 758, - Tortricidae.

$\ddagger$ †HALENA Radermacher, I779; Neave, 1940, Nomencl. zool. 3: 693.

Neave cited this name as 'pro Phalaena L., I 758'. The reference given as Radermacher, I779, Verh. Batav. Genoot. 1: 9 I was incorrect. In vol. I of this journal (which did not appear until I 825) there is a paper by Radermacher on pages 85-93 but this was not on entomology and the name Phalena did not occur. \$Phalena Radermacher was not listed in Sherborn's Index Animalium (1758-1800), nor (180I-1850).

$\ddagger$ PHALENA; Rambur, I 829, Annls Sci. Observ. 2: 266.

An incorrect subsequent spelling.

${ }_{\ddagger}$ NAENIA Walker, [1858] 1857, List Specimens Lepid. Insects Colln Br. Mus. 13: IoI9.

Cited by Neave, 1940, Nomencl. zool. 3: 259, as an available name. This was not so; Walker was using Naenia Stephens and not proposing a new genus.

$\ddagger N A E N I A$ Walker, [1858]. See Naenia Stephens, I827.

NOCT

NAESIA Walker, I858, List Specimens lepid. Insects Colln Br. Mus. 15: I74I. HADE

Type-species: Naesia moesta Walker, 1858, ibidem 15: I742, by monotypy. Type(s) $\widehat{\jmath}$. Venezuela (UM, Oxford).

NAGADEBA Walker, [1866] r865, List Specimens lepid. Insects Colln Br. Mus. 34: I 52 I. OPHI Type-species: Nagadeba indecoralis Walker, [1866] I 865, ibidem 34: I 52 I, by monotypy. Holotype +, Java (BMNH).

NAGARA Walker, [1866] r865, List Specimens lepid. Insects Colln Br. Mus. 34: 1378. STIC Type-species: Nagara phryganealis Walker, [1866] I 865, ibidem 34: I378, by monotypy. Syntypes $3(\hat{o}$, $)$, Jamaica $(\mathrm{BMNH})$. [Brazil $]$ : Para (BMNH). America: W. Coast (BMNH).

$N$. phryganealis is a junior subjective synonym of Phalaena clara Stoll, I 781 , in Cramer, Uitlandsche Kapellen 4: 133 , pl. 358, fig. I.

Nagara when originally proposed contained a second species, but this was doubtfully included and, under the Code, Article 68 (c), is not eligible for selection as type-species.

NAGASENA Moore, 1882, in Hewitson \& Moore, Descr. new Indian lepid. Insects Colln late Mr W.S. Atkinson: $15 \mathrm{I}$. 
Type-species: Nagasena albescens Moore, I 882, ibidem: I52, by monotypy. Syntypes, INDIA: I)arjiling (BMNH).

$N$. albescens is a junior subjective synonym of Lrlona sublineata Walker, I862.

See also Urbona Walker, i 862.

NAGIA Walker, I858, List Specimens lepid. Insects Colln Br. Mus. 15: I739.

ОРн I

Type-species: Nagia gravipes Walker, I 858, ibidem 15: 1740 , by monotypy. Holotype ơ. Sierra Leone (BMNH).

NAHARA Walker, 1865, List Specimens lepid. Insects Colln Br. Mus. 33: Ioot.

OPHI

Type-species: Nahara clavifera Walker, I865, ibidem 33: I004, by monotypy. Holotype ô, [INDIA]: Hindostan (BMNH).

$N$. clavifera is a junior subjective synonym of Noctua patibulum Fabricius, I794, Ent. Syst. $3(2): 89$.

See also Diema Walker, [I859].

NAHARRA Walker, 1865, List Specimens lepid. Insects Colln Br. Mus. 33: 946.

OPH I

Type-species: Naharra contracta Walker, i 865 , ibidem 33:946, by monotypy. Lectotype ô, [Moluccas]: Aru [Is] (UMI, Oxford), designated (as type) by Swinhoe, I90o, Cat. east. and Aust. Lepid. Heterocera 2: 122.

NALCA Walker, I866. See Belciana Walker, i 862.

OPHI

NAMANGANA Staudinger, I 888, Stettin. ent. Ztg 49: 28.

ACRO

Type-species: Namangana cretacea Staudinger, I888, ibidem 49: 28 , by monotypy. Syntypes ô. ㅇ, [U.S.S.R.]: [Uzbekistan], Namangan (MNHU, Berlin).

NAMANGANA Staudinger, i 888, Stettin. ent. Ztg 49:52. See Sartha Staudinger, I89i. opH I

NANAGUNA Walker, I863, List Specimens lepid. Insects Colln Br. Mus. 27: 85. SARR

Type-species: Nanaguna breviuscula Walker, 1863 , ibidem 27: 85 , by monotypy. Type(s) ㅇ, Bokneo: Sarawak (BMNH).

Nanaguna when originally proposed contained a second species, but this was doubtfully included and, under the Code, Article 68 (c), is not eligible for selection as type-species.

See also Clettharra Walker, 1863; Bagistana Walker, I864; and Thrypticodes Lucas, I89o.

NANAMONODES Hampson, I9I 4, Am. Mag. nat. Hist. (8) 13: I 59.

Type-species: Nanamonodes albilinea Hampson, I914, ibidem (8) 13: I6́, by original designation. Holotype ô, V'ENezUela (BMNH).

NANARHYNCHA Hampson, I918, N'orit. zool. 25: 195.

SARR

Type-species: Nanarhyncha nolophaea Hampson, 1918, ibidem 25: I95, by original designation. Holotype + , SiERra LeONe (BMNH).

NANTESIA Kirby, I897. See Minucia Moore, [1885].

CATO

NANTHILDA Blanchard, I84o, in Castelnau, Hist. nat. Anim. articulés (Insectes) 3: 549. OpH I

Type-species: Nanthilala ernestinana Blanchard, I $8+0$, ibidem 3: 549, by monotypy. Lectotype ô. [U.S.A.]: Georgia, Savannah (MNHN, Paris), clesignated (as holotype) by Viette, I953, Bull. Mus. natn. Hist. nat. Paris (2) 24:556.

NARANGA Moore, i 88I, Proc. zool. Soc. Lond. 1881: $359 . \quad$ ACoN Type-species: Xanthodes diffusa Walker, 1865, List Specimens lepid. Insects Colln Br. Mus. 33: 779, by original designation. Holotype o, Cerlon (BMNH).

NARANGODES Hampson igro, Cat. Lepid. Phalaenae Br. Mus. 10: xvii, 633. ACON Type-species: Narangodes haemorranta Hampsoi, 19ro, ilidem 10: 633, fig. I68 [inaccurate], by original designation. Syntypes 2 ㅇ, [JAPAN]: [Ryukyu] Loo-choo Is $(B M N H)$. 
$\ddagger$ NARASODES, misspelling. See Narosodes Moore, I887.

ARCTIIDAE

NARCAEA Druce, r89I, Biologia cent.-am. (Zool.) Lepid. Heterocera 1: 449.

HYPE

Type-species: Narcaea villosa Druce, r891, ibidem 1:449, pl. 36, figs 21,24 , by original designation. Syntypes $\delta$, + , Panama: Chiriqui.

NAROSODES Moore, I887, Lepid. Ceylon $3: 535$.

ARCTIIDAE

Type-species: Tospitis punctana Walker, I863, List Specimens lepid. Insects Colln Br. Mus. 28 : 43I, by monotypy.

$\ddagger N A R A S O D E S$; Rothschild, I9I 3, Novit. zool. 20 : 224.

An incorrect subsequent spelling, repeated by Hampson, 1926, Descr. new Genera Species Lepid. Phalaenae Subfamily Noctuinae Br. Mus.: I92.

$\ddagger N$ arasodes has been used as the original generic name for species which are now in the Noctuidae.

NARTHECOPHORA Smith, I900, Proc. U.S. natn. Mus. 22: 485.

ACRO

Type-species: Narthecophora pulverea Smith, I900, ibidem 22: 486 , by monotypy. Syntypes 2 ô, 2 ㅇ, [U.S.A.]: Arizona, Tucson (USNM, Washington).

NARULLA Walker, I86I, List Specimens lepid. Insects Colln Br. Mus. 23: 949. Available but without included species until 1862, ibidem 24: 1083.

OPHI

Type-species: Narulla infixaria Walker, I862, ibidem 24: I083. by subsequent monotypy. Holotype đ., VenezUELa (BMNH).

NASAYA Moore, I882, in Hewitson \& Moore, Descr. new Indian lepid. Insects Colln late Mr W. S. Atkinson: I 73 .

OPHI

Type-species: Nasaya hepatica Moore, I882, ibidem: I73, by monotypy. Syntypes, INDIA: Darjiling (BMNH).

$N$. hepatica is a junior subjective synonym of Marcillada rubricosa Walker, 1865.

See also Marcillada Walker, 1865.

NAXIA Guenée, I852. See Xiana nom. n.

CATO

NAZUDA Walker, I865, List Specimens lepid. Insects Colln Br. Mus. 33: I I 7. OPHI

Type-species: Nazuda digestalis Walker, I865, ibidem 33: II 8, by monotypy.

Type(s) $\partial$, [Dominican Republic] St. Domingo (BMNH).

$N$. digestalis is a junior subjective synonym of Capnodes leucospila Walker, I865.

See also Antapistis Hübner, [1 825].

NEACHROSTIA Hampson, I907, J. Bombay nat. Hist. Soc. 17: 645.

OPHI

Type-species: Metachrostis brunneiplaga Swinhoe, 1905, Ann. Mag. nat. Hist. (7) 15: 500 , by original designation. Syntypes ô, ㅇ, [INDIA $]$ : [Assam], Khasia Hills (BMNH).

NEATHYRMA Hampson, 1926, Descr. new Genera Species Lepid. Phalaenae Subfamily Noctuinae Br. Mus.: I 76 .

OPHI

Type-species: Baniana iridescens Dognin, I9I4, Hétérocères nouv. Am. Sud 8: 42, by original designation. Syntypes 20 , Colombia: Cali, San Antonio (USNM, Washington).

NEAXESTIS Hampson, I902, Ann. S. Afr. Mus. 2: 316.

CHLO

Type-species: Neaxestis acutangula Hampson, I902, ibidem 2: 3I6, by subsequent designation by Hampson, 1912, Cat. Lepid. Phalaenae Br. Mus. 11: 638. Syntypes ô, , South Africa: Cape Colony, Annshaw (BMNH).

$N$. acutangula was the spelling adopted by Hampson, I9I2, as first reviser, from a multiple original spelling in which both acutangula and \$actuangula, an incorrect original spelling, were used on page 3 I 6.

NEBRISSA Walker, 1862, J. Proc. Linn. Soc. (Zool.) 6: I94.

ACRO

Type-species: Nebrissa bimacula Walker, 1862, ibidem 6: I94, by monotypy. Lectotype ot. Borneo: Sarawak (UM, Oxford), designated (as type) by Swinhoe, I9oo, Cat. east. and Aust. Iepid. Heterocera 2: $4 \mathrm{I}$. 
NECHESIA Walker, I862, List Specimens lepid. Insects Colln Br. Mus. 24: 1104.

HYPE

Type-species: Nechesia albotentata Walker, ibidem 24: I104, by monotypy. Lectotype 9, Borxeo: Sarawak (UM, Oxford), designated (as type) by Swinhoe, I9oo, Cat. east. and Aust. Lepid. Heterocera 2: 206.

Nechesia when originally proposed contained a second species, but this was doubtfully included and, under the Code, Article 68 (c), is not eligible for selection as type-species.

NECLA Waller, r863, List Specimens lepid. Insects Colln Br. Mus. 27: $99 . \quad$ NoLI

Type-species: Necla canioralis Walker, 1863, ibidem 27: 100, by monotypy. Type(s)

ㅇ, BorNEo: Sarawak (BMNH).

Necla when originally proposed contained a second species, but this was doubtfully included and, under the Code, Article 68 (c), is not eligible for selection as type-species.

NEDRA Clarke, 1940, Bull. sth. Calif. Acad. Sci. 39: 39.

ACRO

Type-species: Cloantha ramosula Guenée, 1852, in Boisduval \& Guenée, Hist. nat. Insectes (Lépid.) 6: I1 4, pl. 9, fig. 1, by original designation. Lectotype ô. [U.S.A.]: Géorgie, Savannah (MNHN, Paris), designated by Viette, 1951, Bull. mens. Soc. linn. Lyon 20: 161 .

NEDROMA Walker, I869, in Chapman, Proc. nat. Hist. Soc. Glasgow 1: 353.

STIC

Type-species: Nedroma ferruginea Walker, I869, ibidem 1: 353, by monotypy. Type(s) of. TrPe-LOC.1LITY stated to be Congo, but this was probably an error as the species is not known to occur in Africa, but is widespread in the Oriental Region. From the BMNH registration data on the type it probably came from India (BMNH).

$N$. ferruginea is a junior subjective synonym of Odontodes aleuca Guenée, I 852.

See also Odontodes Guenće, 1852; and Burdria Walker, 1869.

NEGETA Walker, 1862, List Specimens lepid. Insects Colln Br. Mus. 24: 1105.

CHLO

Type-species: Negeta contrariata Walker, 1862, ibidem 24: 1105, by monotypy.

Lectotype ô, BorNeo: Sarawak (UM, Oxford), designated (as type) by Swinhoe, 1900, Cat. east. and Aust. Lepid. Heterocera 2: 73.

NEGRITOTHRIPA Inoue, 1970, Bull. Japan ent. Acad. 5: 37.

SARR

Type-species: Asinduma hampsoni Wileman, 1911, Trans. ent. Soc. Lond. 1911:233, by original designation. Holotype ô, JAPAN: Yamato, Yoshino (BMNH).

NELEUCANIA Smith, I902, Proc. U.S. natn. Mus. 25: 203.

HADE

Type-species: Neleucania niveicosta Smith, 1902, ibidem 25: 205, Dy subsequent designation by Hampson, 1905, Cat. Lepid. Phalaenae Br. Mus. 5: 576 . Syntypes 5 ex., [U.S.A.]: Colorado, Glenwood Springs (USNM, Washington).

NEOBOROLIA Matsumura, 1926, Insecta matsum. 1: 59.

HADE

Type-species: Neoborolia nohirae Matsumura, I926, ibidem 1: 59, pl. I, fig. 40, by original designation. Holotype , JAPAN: Honshu, Kyoto (EIHU, Sapporo).

$N$. nohirae is a junior subjective synonym of Lencania insecuta Walker, I865, List Specimens lepid. Insects Colln Br. Mus. 32: 625.

NEOCALYMNIA Druce, 1908 April, Ann. Mag. nat. Hist. (8) 1: 302.

ACRO

Type-species: Neocalymnia obconica Druce, I908, ibidem (8) 1: 302, by monotypy. Type(s) ô, PERU: S.E., Santo Domingo (BMINH).

Neocalymnia was used by Druce without any indication that it was new even though his new species, described on the same page, were marked sp. n. The generic name was probably obtained from Hampson as an identification and was used and unintentionally made nomenclaturally available by Druce before Hampson's generic description was published.

NEOCALYMNIA Hampson, 1908 [December], Cat. Lepid. Phalaenae Br. Mus. 7: 4. Available but without included species until Hampson, 1910, ibidem 9: I 78 . 
Type-species: Neocalymnia obconica Druce, 1908, by subsequent designation by Hampson, I910, ibidem 9: I78.

A junior homonym of Neocalymnia Druce, I908 April, which is also the objective replacement name.

NEOCALYMNIA Hampson, I908 [December]. See Neocalymnia Druce, I908. ACRo

NEOCERYNEA Hampson, I918, Novit. zool. 25: i68.

$\mathrm{ACON}$

Type-species: Capnodes sabulosa Schaus, I901, Ann. Mag. nat. Hist. (7) 8: 98, by original designation. Type(s), [Mexico]: Orizaba.

NEOCHROSTIS Hampson, 1902, Ann. S. Afr. Mus. 2: 406.

$\operatorname{ACON}$

Type-species: Neochrostis diplogramma Hampson, I902, ibidem 2:407, by monotypy. Holotype $\widehat{\delta}$. South Africa: Transvaal (BMNH).

NEOCLEPTRIA Hampson, I903, Cat. Lepid. Phalaenae Br. Mus. 4: 7, $32 . \quad$ Noct

Type-species: Heliothis punctifera Walker, 1857, List Specimens lepid. Insects Colln. $B r$. Mus. 11: 69I, by original designation. Syntypes 2 ex., Type-Locality not known (BMNH) [Australia].

NEOCLETA Turner, I944, Trans. R. Soc. S. Aust. 68: II.

SARR

Type-species: Neocleta empyra Turner, I944, ibidem 68: I I, by monotypy. Holotype o. Australia: W. Aust., Merredin (ANIC, Canberra).

NEOCODIA Schaus, I9I I, Ann. Mag. nat. Hist. (8) 8: I I7.

ACON

Type-species: Neocodia asna Schaus, I9I I, ibidem (8) 8: I I7, by original designation. Syntypes +, Costa Rrca: Guapiles and Sixola.

NEOCOMIA Rougemont, Igor, Bull. Soc. Sci. nat. Neuchâtel 29: 372.

ACRO

Type-species: Amphipyra satinea Rougemont, I90I, ibidem 29: 37I, by monotypy. Syntypes 2 ex., [SwitzerLand]: Jura, Neuchâtel.

Neocomia was proposed provisionally in case $A$. satinea later required a separate genus

NEOCUCULLIA Janse, 1939, J. ent. Soc. sth. Afr. 1 : Io8.

CUCU

Type-species: Neocucullia albisignata Janse, I939, ibidem 1: III, fig. 3, pl. 3, figs I, 5, by original designation. Holotype ơ. South Africa: Durban.

NEOERASTRIA McDunnough, I937, Can. Ent. 69: 64.

$\operatorname{ACON}$

Type-species: Phytometra apicosa Haworth, I809, Lepid. Br.: 261, by original designation. Type(s), Great Britarn: England (BMNH).

NEOGABARA Wileman \& West, I929, Novit. zool. 35: 25.

OPHI

Type-species: Neogabara plagiola Wileman \& West, 1929, ibidem 35: 25, by original designation. Holotype 오. PHilippines: Mindanao I., Lanao Subprov., Kolambugan.

NEOGALEA Hampson, I9o6, Cat. Lepid. Phalaenae Br. Mus. 6: 2, 7.

ACRO

Type-species: Neogalea braziliensis Hampson, I906, ibidem 6: 8, fig. 2, by original designation. Holotype ㅇ, BRAzIL: Rio Janeiro (BMNH).

$N$. braziliensis is a junior subjective synonym of Xylina esula Druce, I889, Biologia cent.-am. (Zool.) Lepid. Heterocera 1: 297, pl. 28, fig. I.

NEOGROTELLA Barnes \& Benjamin, 1922, Contr. nat. Hist. Lepid. N. Am. 5: 9, 20. Noct

Type-species: Grotella spaldingi Barnes \& McDunnough, 1913, ibidem 2: 105, pl. 4, figs $\mathrm{I}, 2$, by original designation, but cited as $\ddagger$ spaulding $i$, an incorrect subsequent spelling (see page 52, Corrigenda). Lectotype o. [U.S.A.]: Utah, Vineyard, designation by Barnes \& Benjamin, 1922, ibidem 5: 22, but cited as $\ddagger$ spauldingi.

NEOHERMINIA Druce, I89I, Biologia cent.-am. (Zool.) Lepid. Heterocera 1: 47I. HYPE Type-species: Herminia pyramusalis Walker, [1859] 1858, List Specimens lepid. 
Insects Colln Br. Mus. 16: ro6, by original designation. Syntypes 4 ठ̊. U.S.A. (BMNH). See also Phalaenophana Grote, 1873 .

NEOLAPHYGMA Hampson, I908, Cat. Lepid. Phalaenae Br. Mus. 7: 3. Available, but without included species until Hampson, r909, ibidem 8: 266.

ACRO

Type-species: Neolaphygma leucoplaga Hampson, 1909, ibidem 8: 267, fig. 69, by

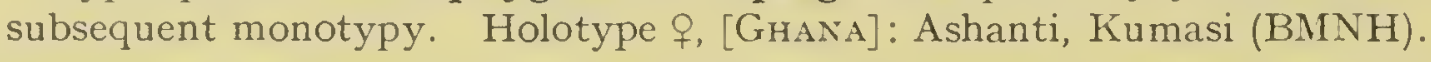

NEOLITA Hampson, 1908, Cat. Lepid. Phalaenae Br. Mus. 7: 3. Available, but without included species until Hampson, r9ro, ibidem 9: 307.

ACRO

Type-species: Neolita perstriata Hampson, I910, ibidem 9: 307, fig. 129, by subsequent monotypy. Syntypes 2 ô, Brazil: Rio Janeiro (BMNH).

NEOMANOBIA Dyar, r914, Proc. U.S. natn. Mus. 47: 373.

HADE

Type-species: Neomanobia thyodes Dyar, 1914, ibidem 47:373, by original designation. Holotype d, Mexico: Zacualpan (USNM, Washington).

NEOMILICHIA Hampson, 1908, Cat. Lepid. Phalaenae Br. Mus. 7: ro. Available, but without included species until Hampson, 1909, ibidem 8: xiii, 518. ACRO

Type-species: Phalaena hylea Stoll, r780, in Cramer, Uitlandsche Kapellen 4: 48, pl. 312, fig. E, by subsequent designation by Hampson, r9o9, ibidem 8: 518. Type(s), SURINAM.

$P$. hylea is dated from the wrapper of the part and not from the title-page of the volume, I 782.

NEOMONODES Hampson, 1908, Cat. Lepid. Phalaenae Br. Mus. 7: 3. Available, but without included species until Hampson, r 909, ibidem 8: xiv, 564.

$\operatorname{ACON}$

Type-species: Eustrotia bertha Schaus, I 898, Jl N.Y. ent. Soc. 6: II 8 , by subsequent monotypy. Syntypes ơ, q, BrazıL: S.E., São Paulo (USNM, Washington).

NEONEGETA Hampson, rgr2, Cat. Lepid. Phalaenae Br. Mus. 11: xvi, 617. chlo

Type-species: Westermannia trigonica Hampson, 1905, Ann. Mag. nat. Hist. (7) 16: 590 , by original designation. Holotype ơ. Nigeria: Old Calabar (BMNH).

NEONIGA Joannis, 1930, Annls Soc. ent. Fr. 98: 749. NOLI

Type-species: Neoniga costimacula Joannis, r930, ibidem 98: 750, by original designation. Holotype ô, [North Vietnam]: Tonkin, Cha-pa (MNHN, Paris).

NEONOLA Hampson, 1900, Cat. Lepid. Phalaenae Br. Mus. $2: 4$.

NOLI

Type-species: Neonola mesosticta Hampson, r9oo, ibidem 2: 4, fig. 2, by original designation. Holotype ô, [Borneo]: [S.E.], [Laut I.] Pulo Laut (BMNH).

NEOPALTHIS Druce, I 891, Biologia cent.-an. (Zool.) Lepid. Heterocera 1: 475. HYPE

Type-species: Neopalthis madates Druce, I891, ibidem 1: 475, pl. 39, figs r6, 17, by original designation. Syntypes $\hat{o}, \hat{q}$, Panama: Volcan de Chiriqui (BMNH). Mexico: Coatepec.

NEOPANGRAPTA Hampson, 1926, Descr. new Genera Species Lepid. Phalaenae Subfamily Voctuinae Br. Mus.: 598.

opH

Type-species: Neopangrapta stenothyris Hampson, 1926, ibidem: 598, by original designation. Holotype + , British Honderas (BMNH).

NEOPHAENIS Hampson, 1908, Cat. Lepid. Phalaenae Br. Mus. 7: xv, 68I.

ACRO

Type-species: Polyphaenis respondens Walker, i 858, List Specimens lepid. Insects Colln Br. Wus. 15: 1720 (but cited as ${ }_{\ddagger}$ Polyphlaenis, an incorrect subsequent spelling), by original designation. Syntypes $\mathrm{I} \hat{\sigma}, \mathbf{I}$ q, Brazil (BMNH). Honduras.

${ }_{+}$NEOPHOENIS; Neave, 1040, Nomencl. zool. 3: 304.

An incorrect subsequent spelling. Neave attributed a misspelling to Hampson, rgr $\mathrm{r}$, Ann. Jag. nat. Hist. (8) 8: 435, when no error lad been made. 
NEOPHAEUS Dyar, I9I8, Proc. U.S. natn. Mus. 54: 350.

ACRO

Type-species: Neophaeus chalcospilans Dyar, I9I8, ibidem 54: 350, by original designation. Holotype 9 , MExico (USNM, Washington).

$\$$ †EOPHOENIS, misspelling. See Neophaenis Hampson, I 908 .

ACRO

NEOPISTRIA Hampson, I908, Cat. Lepid. Phalaenae Br. Mus. 7: xiii, 530.

ACRO

Type-species: Perciana viridinotata Hampson, I894, Fanna Br. India (Moths) 2: 296, by original designation. Type(s) ô. [Sikkim] Sikhim (BMNH).

NEOPLUSIA Okano, 1963, Insecta matsum. 25: 91.

PLUS

Type-species: Neoplusia furihatai Okano, r963, ibidem 25: 91, fig. I, by original designation. Holotype $\tilde{\delta}$, JApan: N. Honshu, Aomori Pref., Fujisaki (EIHU, Sapporo).

$N$. furihatai is a junior subjective synonym of Plusia acuta Walker, [1858] I857, List Specimens lepid. Insects Colln Br. Mus. 12: 922.

NEOPTISTA Schaus, I9I6, Proc. U.S. natn. Mus. 50: 26I, 302.

ACRO

Type-species: Neoptista villalis Schaus, 1916, ibidem 50:302, by original designation. Type(s) $\delta$, Mexico: Jalapa, Orizaba (USNM, Washington).

NEOPTODES Schaus, I9I4, Proc. U.S. natn. Mus, 46: 5 I 5.

OPHI

Type-species: Neoptodes caicus Schaus, I9I4, ibidem 46: 5I 5 , by original designation. Type(s) $\hat{\sigma}$, [French Guiana]: R. Maroni, St. Jean (USNM, Washington).

NEOSEMA Rebel, 1907, Lepid. Südarabien und Insel Sokotra: 55.

NOCT

Type-species: Neosema sesamioides Rebel, I907, ibidem: 55, pl. I, fig. I 2, by monotypy. Holotype $\tilde{\sigma}$. [Southern Yemen]: [Socotra] Sokótra I. (NM, Vienna).

Separates of this paper by Rebel were dated 1907 on the original wrapper and were issued in that year, prior to its repaginated publication in r93 I in Denkschr. Akad. Wiss. Wien 71 (2): $3^{\mathrm{I}-\mathrm{I}} 3 \mathrm{O}$.

NEOSTICHTIS Janse, I937, Moths S. Afr. 3: 55 .

ACRO

Type-species: Parastichtis nigricostata Hampson, 1908, Cat. Lepid. Phalaenae Br. Mus. 7: 79, pl. I09, fig. 22, by original designation. Holotype + , Sierra LeONE (BMNH).

NEOS TICTOPTERA Druce, I900, Ann. Mag. nat. Hist. (7) 5: 519.

SARR

Type-species: Neostictoptera nigropuncta Druce, I9oo, ibidem (7) 5:519, by original designation. Type(s) ô, Colombia: Minca (BMNH).

NEOSTROTIA Hampson, 1908, Cat. Lepid. Phalaenae Br. Mus. 7: 3. Available, but without included species until Hampson, 1909, ibidem 8: xiv, 562.

ACON

Type-species: Eustrotia malonia Schaus, I898, Jl N.Y. ent. Soc. 6: I 44, by subsequent designation by Hampson, I909, ibidem 8: 562. Type(s) Brazil: S.E., São Paulo (USNM, Washington).

NEOT ARACHE Barnes \& Benjamin, 1922, Contr. nat. Hist. Lepid. N.Am. 5: $44 . \quad$ ACON

Type-species: Neotarache deserticola Barnes \& Benjamin, 1922, ibidem 5: 44, by original designation. Lectotype $\hat{\sigma},[$ U.S.A.]: S. Nevada, designated by Barnes \& Benjamin, I922, ibidem 5: 45.

Neotarache was the spelling used twice on page 44 and the name was derived from the genus Tarache Hübner which followed it on page 45; the spelling ${ }_{+}^{\dagger} N$ Neotarche used as the heading was an inadvertent error.

$\ddagger$ NEOT ARCHE Barnes \& Benjamin, 1922, Contr. nat. Hist. Lepid. N. Am. 5: 44.

An incorrect original spelling.

†NEOTARCHE Barnes \& Benjamin, 1922. See Neotarache Barnes \& Benjamin, I922. ACON

NEOTHRIPA Hampson, I894, Fauna Br. India (Moths) 2: 366, 382.

$\operatorname{ACON}$

Type-species: Neothripa punctistigma Hampson, I894, ibidem 2: 382, by original designation. Type(s), India: [Punjab], Simla (BMNH). 
NEPERIGEA McDunnough, I937, Can. Ent. 69:62.

ACRO

Type-species: Perigea continens Edwards, (I885) i884, Papilio 4: I22, by original designation. Holotype ô, [U.S.A.]: Arizona.

NEPHELEMORPHA Hampson, 1926, Descr. new Genera Species Lepid. Phalaenae Subfamily Noctuinae $B r$. Mus. : 99 .

OPHI

Type-species: Nephelemorpha semaphora Hampson, 1926, ibidem: 99, by original designation. Syntypes I $\hat{0}, \mathrm{I}$ ㅇ, Nigeria: Ilesha (BMNH).

NEPHELINA Kirby, I 897, Hand-Book Order Lepid. 5: I 30.

CATO

Type-species: Phalaena edusa Drury, I773, Illust. nat. Hist. 2: Index \& 42, pl. 24, fig. 4, by monotypy. Holotype, [U.S.A.]: New York.

$P$. edusa is a junior subjective synonym of Phalaena lunata Drury, [I 773].

See also Omoptera Guérin-Méneville, [1832].

NEPHELISTIS Hampson, I905, Cat. Lepid. Phalaenae Br. Mus. 5: x, 250.

HADE

Type-species: Nephelistis congenitalis Hampson, 1905, ibidem 5: 250, fig. 46, by original designation. Holotype $\hat{\sigma}$, Guatemala: Las Mercedes (BMNH).

NEPHELODES Guenée, I852, in Boisduval \& Guenée, Hist. nat. Insectes (Lépid.) 5: I29.

$\mathrm{HADE}$

Type-species: Nephelodes minians Guenée, I852, ibidem 5: I30, by subsequent designation by Grote, I874, Bull. Buffalo Soc. nat. Sci. 2: I8. Syntypes ô, 우, North AMERICA.

See also Monosca Walker, i869.

NEPTUNIA Barnes \& McDunnough, I9I.I. See L'niptena nom. n.

$\triangle \mathrm{CON}$

NERASTRIA McDunnough, 1937, Can. Ent. 69:65.

ACON

Type-species: Eustrotia dividua Grote, $1879, N$. Am. Ent. 1: 46 , by original designation. Syntypes ô, ․ [U.S.A.]: Texas (BMNH).

NEREISANA Strand, I 9 II, Annls Soc. ent. Belg. 55: 38.

$\triangle \mathrm{CON}$

Type-species: Chesias oranaria Lucas, I849, Explor. scient. Algérie Anim. articulés 3: 393, pl. 4, fig. t, by monotypy (of Psendosterrha Rebel, igor). Syntypes, Algeria: western.

Neveisana was proposed as the objective replacement name for Pseudostervha Rebel, I90I.

PSELDOSTERRH.t Rebel, igor, in Staudinger \& Rebel, Cat. Lepid. palaearct. Faunengeb.

$1: 373$.

Type-species: Chesius oranaria Lucas, I 849 , by monotypy.

A junior homonym of Pseudosterrha Warren, i 888, Proc. zool. Soc. Lond. 1888: 324, Lepid., Geometridae. The objective replacement name is Neveisana Strand, I9I I.

NOTHOSTERRHA Warren, i9i 2, in Seitz, Macrolepid. World 3: 283.

Proposed, unnecessarily, as an objective replacement name for Pseudosterrha Rebel, I 90 I .

The date of publication of the part of Seitz containing page 283 was stated on page 277 as I9I 2 January $15^{\text {th }}$ in the English edition and as I9I 3 January $15^{\text {th }}$ in the German edition.

NERTOBRIGA Walker, [1863] 1864, J. Proc. Linn. Soc. (Zool.) 7: 75.

CHLO

Type-species: Nertobriga reversa Walker, [1863] I864, ibidem 7: 75, by monotypy. Lectotype f, Borneo: Sarawak (UM, Oxford), designated (as type) by Swinhoe, I900, Cat. east. and Aust. Lepid. Heterocera 2: 74.

$N$. reversa is a junior subjective synonym of Ariolica signata Walker, [1863] 1864, ibidem 7: 54 .

See also Doranaga Moore, s 887. 
NESAMIPTIS Meyrick, I 899, in Sharp, Fauna Hawaiiensis 1 (2): I 56.

HYPE

Type-species: Nesamiptis plagiota Meyrick, I 899, ibidem 1 (2): I56, pl. 4, fig. I 5, by original designation. Syntypes $9(\hat{\jmath}$, $)$, HAwailan Is: Waianae Mts, Oahu (BMNH); Mts Waimea, Kauai (BMNH).

NESTIODES Turner, I939, Pap. Proc. R. Soc. Tasm. 1938: 7I.

NOLI

Type-species: Nestiodes eremnopa Turner, I939, ibidem 1938: 7I, by monotypy.

Holotype +, Australia: New South Wales, Ebor (ANIC, Canberra).

$N$. evemnopa is a junior subjective synónym of Sorocostia semograpta Meyrick, I886, Proc. Linn. Soc. N.S.W. (2) 1 : 720.

NETROCEROCERA Spuler, I906. See Netrocerocora Bartel, I902.

NOCT

NETROCEROCORA Bartel, 1902, Dt. ent. Z. Iris 15: 207.

NOCT

Type-species: Netrocerocora quadriplaga Bartel, 1902, ibidem 15: 208, by monotypy. Syntypes $\hat{o}, q$, , [U.S.S.R.]: S. Urals.

N. quadriplaga is a junior subjective synonym of Rusina coraxa Püngeler, I898, Societas ent. $13(8): 58$.

NETROCEROCERA Spuler, 1906, in Hofmann, Schmett. Eur. 1: 236.

An unjustified emendation of Netrocerocora Bartel, I902.

NEUMICHTIS Hampson, I906, Cat. Lepid. Phalaenae Br. Mus. 6: xi, 298.

CUCU

Type-species: Hadena trijuncta Walker, I857, List Specimens lepid. Insects Colln

Br. Mus. 11: 597, by original designation. Holotype ․ [Australia]: Tasmania (BMNH).

NEUMOEGENIA Grote, I882, Papilio 2: I32, I84.

ACRO

Type-species: Neumoegenia poetica Grote, I882, ibidem 2: 132, 184, by monotypy. Type(s), [U.S.A.]: Arizona.

NEUQUENIA Köhler, (1952). See Neuquenioa Rosas Costa, I954.

CUCU

NEUQUENIOA Rosas Costa, i 954, Neotropica 1 : 8.

CUCU

Type-species: Neuquenia nitida Köhler, (1952) I95I, Acta zool. lilloana 12: I44, by original designation (for Neuquenia Köhler). Syntypes $\hat{\delta}$, 우, Argentina: Rio Negro [Prov.], Cipolletti (ZSBS, Munich); Neuquen [Prov.], Rio Agrio (ZSBS, Munich).

Neuquenioa was proposed as the objective replacement name for Neuquenia Köhler, (1952).

NEUQUENIA Köhler, (1952) I95I, Acta zool. lilloana 12: I 44.

Type-species: Neuquenia nitida Köhler, (1952), by original designation.

A junior homonym of Neuquenia Mello-Leitão, 1940, Revta Mus. La Plata N.S. Zool.

2: I2, - Arachnida. The objective replacement name is Nerquenioa Rosas Costa, I954.

NEURANETHES Bethune-Baker, I9I I, Amn. Mag. nat. Hist. (8) 8: 508.

HADE

Type-species: Neuranethes angola Bethune-Baker, I9I I, ibidem (8) 8: 509, by original designation. Type(s) ơ, ANgolA: N'Dalla Tando (BMNH).

NEURIA Guenée, I84I. See Heliophobus Boisduval, I828.

HADE

NEUROIS Hampson, I903, Cat. Lepid. Phalaenae Br. Mus. 4: xix, 61 o.

NOCT

Type-species: Diphtera nigroviridis Walker, I865, List Specimens lepid. Insects Colln Br. Mus. 32: 6I5, by original designation. Syntypes 0 , $q$, [INDIA]: [W. Bengal], Darjeeỉing (BMNH).

NEURONIA Hübner, [I82 I] I8I6, V'erz. bekannter Schmett.: 2 I 5.

HADE

Type-species: Bombyx popularis Fabricius, 1775, Syst. Ent.: 577, by subsequent designation by Grote, I896, Entomologist's Rec. J. Var. 8: 304. Type(s), Germany (Type(s) not found by Zimsen, 1964, Type Material of I. C. Fabricius: 52 I).

$B$. popularis is a junior subjective synonym of Phalaena decimalis Poda, I761, Insecta Mus. Graecensis: 92. 
NEITRONLA Agassiz, I 846, Nomencl. zool. Index univl.: 249.

An unjustified emendation of Neuronia Hübner, [182I].

EPINEURONIA Rebel, Igor, in Staudinger \& Rebel, Cat. Lepid. palaearct. Faunengeb. 1: I 55 .

Proposed, unnecessarily, as the objective replacement name for Neuronia Hübner, [182I], which Rebel considered to be a junior homonym of ${ }_{+}$Neuronia Leach, I8I5, in Brewster, Edinburgh Encycl. 9: I69. In the index on page I69 is given:

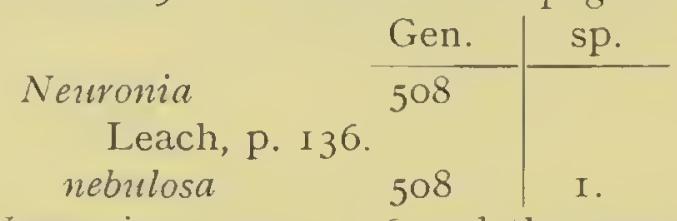

There is, however, no Nenronia on page I 36 and the genus 508, actually on page I39, is Nemoura Latreille which does contain Sp. I $N$. nebulosa Latreille, - Plecoptera. The genus Nemoura is omitted from the index. Evidently Nemoura has been indexed as Neuronia, and Rebel in checking through Leach's index for homonymous names accepted that Neuronia was preoccupied and proposed Epineuronia as its replacement name.

NEVIASCA Valker, [1859] i 858, List Specimens lepid. Insects Colln Br. MIus. 16: 7. OPH Type-species: Neviasca varialis Walker, [1859] I858, ibidem 16: 7 , by monotypy. Syntypes $3(\hat{\jmath}$, ㅇ). [INDIA] : North Hindostan (BMNH).

N. varialis is a junior subjective synonym of Phalaena liturata Fabricius, 1787 , Mantissa Insect. 2: 197. The latter is, however, a junior primary homonym of Phalaena liturata Clerck, I759, Icones Insect. rarionum 1: pl. 6, fig. 6,-Lepid., Geometridae. There is no objective replacement name for $P$. liturata Fabricius, therefore $N$. varialis Walker must be used as the subjective replacement name.

NEVIASCA Walker, [1859] I 858, List . . 16: I99. See Cardalena Walker, I 859.

HYPE

NEVRIA Agassiz, I846. See Heliophobus Boisduval, 1828.

HADE

NEVRONIA Agassiz, 18 46 . See Neuronia Hübner, [182 r].

HADE

NIABOMA nom. n. for Manobia Staudinger, I 896.

$\mathrm{HADE}$

Type-species: Manobia xena Staudinger, I 896, Dt. ent. Z. Ivis 8: 3I 7, pl. 6, fig. 8, by monotypy (of Manobia Staudinger). Syntypes ô, ㅇ, [CHIN.1]: Tibet, between Lob Noor and Kuku Noor (MNHU, Berlin).

MANOBIA Staudinger, I896, Dt. ent. Z. Iris 8: 317.

Type-species: Manobia xena Staudinger, I 896 , by monotypy.

A junior homonym of Manobia Jacoby, I885, Annali Mus. civ. Stor. nat. Genova 22: 73 - Coleoptera. The objective replacement name is Niaboma nom. n.

NIACCABA Walker, [1866] I 865, List Specimens lepid. Insects Colln Br. Mus. 34: I272. ACON

Type-species: Niaccaba sumptualis Walker, [1 866] I 865, ibidem 34: I272, by monotypy. Holotype ơ. CEYLoN (BMNH).

NIACCABANA Strand, 1920, Arch. Naturgesch. 84 (A) I 2: 180.

1IYPE

Type-species: Niaccabana siculipalpis Strand, I920, ibidem 84 (A) I2: I8I, by monotypy. Holotype $q$, [TAIWAN] Formosa: Suisharyo (DEI, Eberswalde).

$\ddagger$ NICARA, misspelling. See Nikara Moore, I882.

ACRO

NICARA Hampson, I 9 Io. See Nikara Moore, i 882.

ACRO

NICETAS Druce, I891, Biologia cent.-am. (Zool.) Lepid. Heterocera 1: $45^{\circ}$ HYPE

Type-species: Nicetas panamensis Druce, 1801, ibidem 1: 450, pl. 37, figs 3, 4, by original designation. Syntypes 3 . , , Costa Rica: Volcan de Irazu (BMNH). Panama: Chiriqui; Volcan de Chiriqui (BMNH). 
NICEVILLEA Hampson, i 895, Trans. ent. Soc. Lond. 1895: 307.

OPH I

Type-species: Nicevillea epiplemoides Hampson, I895, ibidem 1895: 307, fig. [I4], by original designation. Syntypes + , [Burma]: Tenasserim, Donaut Range (BMNH).

NIGETIA Walker, [1866] ז 865, List Specimens lepid. Insects Colln Br. Mus. 34: I 505. Noli Type-species: Nigetia formosalis Walker, [1 866] I 865, ibidem 34: I 506, by monotypy. Holotype $₫$, TYPE-LOCALITY not known (BMNH) [U.S.A.].

NIGRAMMA Walker, I863, List Specimens lepid. Insects Colln Br. Mus. 27: 77 STIC Type-species: Nigramma quadratifera Walker, I863, ibidem 27: 77, by monotypy. Holotype + , TYPE-LOCALITY not known (BMNH) [CEYLON].

NIGUZA Walker, I858, List Specimens lepid. Insects Colln Br. Mus. 15: i 855.

CATO

Type-species: Niguza spiramioides Walker, 1858, ibidem 15: 1855, by monotypy.

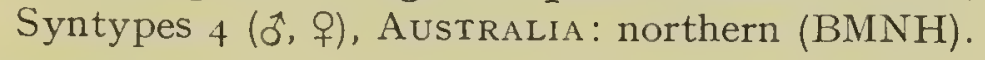

NIKARA Moore, I882, in Hewitson \& Moore, Descr. new Indian lepid. Insects Colln late Mr W. S. Atkinson: I 26. ACRO

Type-species: Nikara castanea Moore, I882, ibidem: I26, pl. 4, fig. 24 , by monotypy. Type(s) +, India: [W. Bengal], Darjiling (MNHU, Berlin).

$\ddagger$ NICARA; Hampson, 1908, Cat. Lepid. Phalaenae Br. Mus. 7: i8.

An incorrect subsequent spelling; there was no evidence of an intentional change of spelling.

NICARA Hampson, igro, Cat. Lepid. Phalaenae Br. Mus. 9: ix, is 3.

An unjustified emendation of Nikara Moore, 1882 ; there was evidence of an intentional change of spelling.

$\ddagger N I M Y R A$, misspelling. See Simyra Ochsenheimer, I8 6 .

ACRO

$\ddagger$ NIPHOCONA Hampson, no published reference found.

HYPE

NIPHOSTICTA Hampson, 1926, Descr. new Genera Species Lepid. Phalaenae Subfamily Noctuinae Br. Mus.: 4I 4 . OPHI

Type-species: Niphosticta stigmagrapta Hampson, I926, ibidem: 4I4, by original designation. Holotype ․, Nigeria: Lagos, Ebute Meta (BMNH).

NIPHOSTICTA Turner, 1936. See Hurworthia nom. n.

OPHI

NIPISTA Walker, [1858] I 857, List Specimens lepid. Insects Colln Br. Mus. 12: 772, 799. ACON Type-species: Nipista lineata Walker, [1858] i857, ibidem 12: 80o, by monotypy. Syntypes 2 ㅇ, VenezUELA (BMNH).

$N$. lineata is a junior subjective synonym of Diastema tigris Guenée, I852.

See also Diastema Guenée, 1852 .

NITOCRIS Guenée, i 868 . See Rictonis nom. n.

ACRO

NOCLOA Smith, i9o6, Jl N.Y. ent. Soc. 14: I 3.

ACRO

Type-species: Nocloa plagiata Smith, I906, ibidem 14: 13, by subsequent designation by Hampson, igro, Cat. Lepid. Phalaenae Br. Mus. 9: 253. Syntypes, [U.S.A.]: Arizona and Ariz., Colchise Co., Palmerly.

NOCTASOTA Clench, 1954, Revue Zool. Bot. afr. 50: 297.

EUTE

Type-species: Noctasota curiosa Clench, 1954, ibidem 50: 300, by original designation. Holotype J. Cameroun: Efulen (CM, Pittsburgh).

$N$. curiosa is a junior subjective synonym of Eutelia distorta Hampson, 1912, Cat. Lepid. Phalaenae Br. Mus. 11: т6, fig. 9.

Noctasota was originally placed in the Hypsidae. 
NOCTUA Linnaeus, 1758, Syst. Nat. (Edn ro) 1: 508.

Type-species: Phalaena pronuba Linnaeus, 1758, ibidem 1: 512 , by subsequent designation by Int. Commn zool. Nom., i 957, Opin. Decl. int. Commn zool.. Nom. 15 Opinion 450: 254. Type(s), TYPE-LOCALITY not stated (LS, London) [EUROPE?

Placed on the Official List of Generic Names in Zoology: Name No. I057, with P. promuba designated as type-species under the plenary powers of the Commission.

NOCTUELLA Rafinesque, I 8 I5, Analyse de la Natuere: 129.

Proposed as an objective replacement name for Noctua Fabricius, an incorrect authorship.

$¥$ NYCTEMIA Rafinesque, i8 15, Analyse de la Nature: i 29.

Rafinesque associated this generic name with Noctua but gave neither description, indication nor included species.

TRIPHAENA Ochsenheimer, i 816, Schmett. Eur. 4: 69.

Type-species: Phalaena pronuba Linnaeus, I $75^{8}$, by subsequent designation by Duponchel, I829, in Godart \& Duponchel, Hist. nat. Lépid. Papillons Fr. 7 (2): 7 I.

Triphaena is a junior objective synonym of Noctua Linnaeus, i 758 .

TRYPHAENA Meigen, I83I, Syst. Beschreib. Eur. Schmett. $3(2): 78$.

An unjustified emendation of Triphaena Ochsenheimer, i 8 I6.

XANTHOPTERA Sodoffsky, i837, Bull. Soc. imp. Nat. Moscou 1837 (6): 86.

Proposed, unnecessarily, as an objective replacement name for Triphaena Ochsenheimer, I 8 I6, which Sodoffsky considered to be inappropriate.

NOCTUA Boiscluval, I828. See Agrotis Ochsenheimer, i 8I6.

NOCT

NOCTUBOURGOGNEA Iiöhler, 1954, levta Soc. ent. argent. 17: 40.

NOCT

Type-species: Agrotis frigida Mabille, 1885, Bull. Soc. philomath. Paris (7) 9: 61, by original designation. Lectotype $\widehat{\sigma},[\mathrm{CHLL}]$ : Terre de Feu [Hardy Penin.], Orange B[ay] (MNHN, Paris), designated (as holotype) by Viette \& Fletcher, 1968, Bull. Br. Mus. nat. Hist. (Ent.) $21: 4 \mathrm{I} 0$.

NOCTUELLA Rafinesque, I815. See Noctua Linnaeus, I 758.

NOCT

NOCTUITES Heer, [1849] i85o, Neue Denkschr. Allg. schweiz. Ges. Naturw. 11: i 85 . Fossil

Type-species: Noctuites haidingeri Heer, [1849] I 850, ibidem 11: I 85, pl. I4, fig. 9, by PRESENT IDESIGNATION. Holotype, [Yugoslavia]: Croatia, Radoboj, from the Tertiary Formation.

The species designated as type-species is the first of two originally included by Heer, as fossil noctuids.

NOCTULIZERIA Köhler, 1945, Acta zool. lilloana 3: iog.

NOCT

Type-species: Noctulizeria elegantula Köhler, 1945, ibidem 3: 109, by original designation. Syntypes $\hat{\jmath}, \mathcal{\imath}$, Argentina: Chubut [Prov.], Comodoro Rivadavia (ZSBS, Munich).

N. elegantula is a junior subjective synonym of Synclerostola pampeana Berg, 1877.

See also Synclerostola Berg, i 877.

NODARIA Guenée, I854, in Boisduval \& Cruenée, Hist. nat. Insectes (Lépid.) 8: 63. HYPE Type-species: Nodaria hispanalis Guenée, I 854 , ibidem 8: 6.4, by subsequent designation by Desmarest, (1857), in Chenu, Encycl. Hist. nat. Papillons nocturnes: 218. Holotype ô., [Spain]: Midi de l'Espagne (BMNH).

$N$. hispanalis is a junior subjective synonym of Herminia nodosalis Herrich-Schäffer, [185I] i 845 , Syst. Beard. Schmett. Eur. 2: 385, pl. I 18, fig. 605.

NOLA Leach, [1815] 1830, in Brewster, Edinburgh Encycl. 9: 135.

NOLI

Type-species: Noctua palliola [Denis \& Schiffermüller], 1775, Ankïnlung syst. Werkes Schmett. Wienergegend: 69 (but included by Leach as $\ddagger$ palliolatis, an incorrect subsequent spelling), by monotypy. Type(s), [Austria]: Vienna district (Collection destroyed, Horn d Kahle, 1936, Ent. Beih. Berl.-Dahlem 3: 243). 
N. palliola is a junior subjective synonym of Phalaena cucullatella Linnaeus, I758, Syst. Nat. (Edn Io) 1: 537 .

See also Roeselia Huibner, [1825].

${ }_{\ddagger}^{\dagger}$ CHL A MIPHOR A Hübner, [1806], Tentamen determinationis digestionis . . . : [2].

Included in a work rejected for nomenclatural purposes by Int. Commn zool. Nom., r926, Smithson. misc. Collns 73 (4) Opinion 97: 19. Also idem, 1954, Opin. Decl. int.

Commn zool. Nom. 6 Opinion 278: 140.

Only included species: Noctua palliola [Denis \& Schiffermüller].

LIRA Billberg, I820, Enumeratio Insect. Mus. G. J. Billberg: 90.

Proposed, unnecessarily as an objective replacement name for Nola Leach, [I8I5].

CHLAMIFERA Hübner, x822, Syst.-alphab. Verz.: 52, 55.

Type-species: Noctua palliola [Denis \& Schiffermüller], I775 (but included by Hübner as $C$. palliolalis, an unjustified emendation), by PRESENT DESIGNATION.

Chlamifera is a junior objective synonym of Nola Leach, [I8I5].

$\ddagger$ NOLITES Bruand, I846, Mém. Soc. Emul. Doubs 2 (3) 5-6: I I3.

Proposed as a name for a tribe, but cited in Sherborn, I928, Index Anim. (I7): 4407, and in Neave, I940, Nomencl. zool. 3: 343, as a generic name.

NOLAPHANA Grote, I873, Bull. Buffalo Soc. nat. Sci. 1: I69.

ACRO

Type-species: Brachytaenia malana Fitch, I856, First \& Second Rep. noxious, beneficial \& other Insects, State New-York: 24I, pl. 3, fig. 5, by monotypy. Syntypes o, , U. U.S.A. : New York State.

See also Balsa Walker, I860.

NOLASENA Waller, [1858] I857, List Specimens lepid. Insects Colln Br. Mus. 12: 978, 981. OpHI Type-species: Nolasena ferrifervens Walker, [1858] I857, ibidem 12: 982 , by monotypy. Holotype $\widehat{\sigma}$, CEYLON (BMNH).

See also Banassa Walker, 1863 .

$\ddagger$ NOLASSENA; Neave, I940, Nomencl. zool. 3: 343 .

An incorrect subsequent spelling. Neave attributed a misspelling to Walker, I865, List Specimens lepid. Insects Colln Br. Mus. 33: 858 (recte 860), when no error had been made by Walker.

NOLASENIOLA Strand, 1920, Arch. Naturgesch. 84 (A) I2: I4I.

OPHI

Type-species: Acantholipes plecopteroides Strand, I920, ibidem 84 (A) I2: I4I, by monotypy. Holotype $\vec{\sigma}$, [TAIWAN] Formosa: Alikang (DEI, Eberswalde).

Nolaseniola was proposed as a subgenus of Acantholipes Lederer, I857.

NOLASODES Hampson, I9Io, Cat. Lepid. Phalaenae Br. Mus. 9: xv, 524.

ACRO

Type-species: Nolasodes atrisignata Hampson, I9Io, ibidem 9: 524, pl. 148, fig. 24 ,

by original designation. Holotype ô, French Guiana: St. Laurent Maroni (BMNH).

$\ddagger$ NOSALODES; Grünberg, I9I2, Arch. Naturgesch. 77 (4) 2: I4 I.

An incorrect subsequent spelling.

$\ddagger$ NOLASSENA, misspelling. See Nolasena Walker, [1858].

OPHI

NOLATHRIPA Inoue, I970, Bull. Japan ent. Acad. 5: 38.

SARR

Type-species: Nola lactaria Graeser, I892, Berl. ent. Z. 37: 2II, by original designation. Holotype + , [U.S.S.R.]: Amurland, Ussuri, Koslofska.

NOLATYPA Hampson, I920, Novit. zool. 26:26I.

SARR

Type-species: Nolatypa phoenicolepia Hampson, 1920, ibidem 26: 261, by original designation. Holotype $\tilde{\sigma}$, [Tanganyika] Germ. E. Afr.: Lulanguru (BMNH).

NOLIDIA Son, 1933, Ann. Transv. Mus. 15: 228.

NOLI

Type-species: Nolidia unipuncta Son, 1933, ibidem 15: 229, pl. 3, fig. 4, pl. 7, fig. 5, pl. 8, fig. 20, by original designation. Holotype $\hat{\sigma}$. South AFrica: Natal, Rietvlei (TM, Pretoria). 
$\ddagger$ NOLITES Bruand, I 846 . See Nola Leach, [1815].

NONAGRIA Ochsenheimer, isi6, Schmett. Eur. 4: 82.

ACRO

Type-species: Noctua typhae Thunberg, i 784 , Diss. Ent. sistens Insecta Suecica (I): 3 , by subsequent designation by Duponchel, I 829, in Godart \& Duponchel, Hist. nat. Lepid. Papillons Fr. 7 (2): 72. Type(s), Swenes.

$\ddagger N($ ) NAGRIA Hübner, [I82I] I8I6, Vevz. bekannter Schmett.: 24 I.

Cited by Neave, 1940, Nomencl. zool. 3: 344 , as a nomenclaturally available name, but this is not so as Hübner was merely using Ochsenheimer's genus. The first of the names included by Hübner was. . typhae.

ENTERIONA Sodoffsky, i 837, Bull. Soc. imp. Nat. Moscou 1837(6): 87.

Proposed, unnecessarily, as an objective replacement name for Nonagria Ochsenheimer, I 8 I6, which Sodoffsky considered to be inappropriate.

NONAGRIA Hübner, [I $82 \mathrm{I}]$. See Nonagria Ochsenheimer, I 816.

ACRO

NOROPSIS Guenée, I852, in Boisduval \& Guenée, Hist. nat. Insectes (Lépid.) 5: I I7. OPH

Type-species: Noropsis fastuosa Guenée, I 852, ibidem 5: i 7 , by monotypy. Type(s) of $P$. hieroglyphica Cramer, [Netherlands West Indies]: Curaçao I. (BMNH).

$\Lambda$. fastuosa was proposed as the objective replacement name for Phalaena hieroglyphica Cramer, 1777, Uitlandsche Kapellen 2: 8I, I 5 I, pl. I 47, fig. D, which was spelled thyroglyphica on page $8 \mathrm{I}$ but was corrected as a printer's error, on page ${ }_{5} \mathrm{I}$ to hieroglyphica. It thus became a junior primary homonym of Phalaena hieroglyphica Drury, I773, Illust. nat. Hist. 2: Index, 3, - Lepid., Noctuidae, Catocalinae.

N. fastıosa is a junior subjective synonym of Bombyx festiva Fabricius, 1775, Syst. Ent.: 579.

See also Diphthera Hübner, [1809].

NORRACA Moore, i 88I, Proc. zool. Soc. Lond. 1881:340.

NOTODONTIDAE

Type-species: Norraca longipennis Moore, I 88 I, ibidem 1881: 340, by monotypy.

Norraca was originally placed in the Noctuidae.

†NOSALODES, misspelling. See Nolasodes Hampson, i 9 io.

ACRO

NOSHIMEA Matsumura, 1931, Insecta matsum. 5: 2 I0.

ACON

Type-species: Noshimea fulgularis Matsumura, 1931, ibidem 5: 210, fig., by original designation. Holotype , Japan: Honshu, Aomori (EIHU, Sapporo).

N. fulgularis is a junior subjective synonym of Sinocharis korbae Püngeler, I9I 2.

See also Sinocharis Püngeler, I9I2.

NOTHOPHILA Barnes \& Lindsey, 1922. See Bandelia Lindsey, 1923.

ACON

NOTHOSTERRHA Warren, I9I2. See Neveisana Strand, I9II.

ACON

NOTOCYMA Snellen, i 872, Tijdschr. Ent. 15: 67.

OPHI

Type-species: Notocyma pruinosa Snellen, I 872, ibidem 15:67, pl. 5, figs I 1, 12, by monotypy. Holotype $q$, [Congo] Neder-Guinea: R. Congo.

$N$. pruinosa is a junior subjective synonym of Midea rectalis Walker, I863.

See also Arsacia Walker, [1866]; and Amblyzancla Turner, I936.

NOTODONTA Ochsenheimer, I 810 , Schmett. Eur. $3: 45$.

NOTODONTIDAE

Type-species: Phalaena dromedarius Linnaeus, I 767, Syst. Nat. (Edn I2) 1(2):827, by subsequent designation by Westwood, 1840, Synopsis Genera Br. Insects: 90.

Notodonta has been used as the original generic name for species which are now in the Noctuidae.

NUDIFRONS Staudinger, 1 897, Dt. ent. Z. Iris 10: pl. 4, fig. 24.

CUCU

Type-species: Nudifrons delicata Staudinger, 1897, ibidem 10: pl. 4, fig. 24. Also 
page $29 \mathrm{I}$ published $\mathrm{I} 898$, by monotypy. Holotype $\hat{O}$, [ISRAEL] Palestine: Jerusalem district (MNHU, Berlin).

NUMENIASTES Fletcher, 1963, Explor. Parc natn. Albert (2) 15: 89.

ACRO

Type-species: Numeniastes selenis Fletcher, 1963, ibidem (2) 15: 90, figs II \& 42-44, by original designation. Holotype $\widehat{\sigma}$. [ErHiopia] Abyssinia: Harar (BMNH).

NYCHIOPTERA Franclemont, 1966, Proc. ent. Soc. Wash. 68: 295.

OPHI

Type-species: Pleonectyptera noctuidalis Dyar, 1907, Jl N.Y. ent. Soc. 15: I07, by original designation. Lectotype $\hat{\sigma}$, U.S.A.: Arizona, Oracle (USNM, Washington), designated by Franclemont, 1966, ibidem 68: 300.

NYCHOPHILA Billberg, I 820. See Hyblaea Fabricius, I 793.

HYBLAEIDAE

†NCTEMIA Rafinesque, I815. See Noctua Linnaeus, I 758.

NOCT

NYCTENNOMOS Hampson, 1926, Descr. new Genera Species Lepid. Phalaenae Subfamily

Noctuinae Br. Mus.: 442.

OPHI

Type-species: Nyctennomos peratosema Hampson, 1926, ibidem: 442, by original designation. Holotype 9 , MADAGASCAR: Betsileo (BMNH).

$\ddagger$ †YCTEOLA Hübner, [1806]. See Sarrothripus Curtis, I 824.

SARR

NYCTEOLA Hübner, I 822, Syst.-alphab. Verz.: 60, 66.

SARR

Type-species: Tortrix undulana Hübner, [I799], Samml. eur. Schmett. 7: pl. 2, fig. 7 (a junior primary homonym), by subsequent designation by Prout, I9or, Entomologist's Rec.J. Var. 13: 183. Type(s), Europe.

T. undulana Hübner, [I799] (a junior primary homonym of Tortrix undulana [Denis \& Schiffermüller], I775, - Tortricidae) is a junior subjective synonym of Phalaena revayana Scopoli, I772, Annus Hist.-nat. 5: i 6 . There being no objective replacement name, the latter is thus available for use as a subjective replacement name.

See also Subrita Walker, I 866.

AXIA Hübner, [1825] I816, Verz. bekannter Schmett.: 395.

Type-species: Phalaena revayana Scopoli, I772, Annus Hist.-nat. 5: i 6, by subsequent designation by Hampson, I9I2, Cat. Lepid. Phalaenae Br. Mus. 11: 26I. Type(s), [Germany (EAST)]: [Karl Marx Stadt] Schemnitz.

A junior homonym of Axia Hübner, [182I] I 816, Vevz. bekannter Schmett.: 249, - Lepid., Thyatiridae. There is no objective replacement name but $P$. revayana is the senior subjective synonym of Tortrix undulana Hübner, [I799], the type-species of Nycteola Hübner, 1822 . The latter is therefore available for use as a subjective replacement name.

NYCTEOLA Herrich-Schäffer, [1851]. See Zebeeba Kirby, I892. AcoN

NYCTEROPHAETA Smith, I 882 February, in Hulst, Bull. Brooklyn ent. Soc. 4: 45 . cucu Type-species: Nycterophaeta magdalena Hulst, I 882, ibidem 4: 45, by monotypy. Type(s). [U.S.A.]: [S. Dakota], Black Hills.

N. magdalena is a junior subjective synonym of Cucullia luna Morrison, 1875 , Proc. Boston Soc. nat. Hist. 18: I 22.

See also Epinyctis Grote, I 882 April.

NYCTIPAO Hübner, [1823]. See Erebus Latreille, I810.

CATO

$\ddagger$ NYCTIPAON, misspelling. See Erebus Latreille, I8Io.

CATO

NYCTIPOLIA Schaus, i9i6, Proc. U.S. natn. Mus. 50: 261, 29 I.

HYPE

Type-species: Nyctipolia incondita Schaus, 19I6, ibidem 50:291, by original designation. Type(s) $\delta$, [French GUiANa]: R. Maroni, St. Laurent (USNM, Washington). 
NYCTOBRYA Boursin, 1957, Z. wien. ent. Ges. 42: I 43.

Type-species: Bryophila simonyi Rogenhofer, i889, T'erh. zool.-bot. Ges. Wien 39: 36, by original designation. Syntypes I $\hat{\sigma}, \mathrm{I}$ ㅇ, [CAnary Is]: Tenerife [I.], Monte Verde ( $\mathrm{NM}$, Vienna).

Nyctobrya was described as a subgenus of Cryphia Hübner, I8I8.

NYCTODRYAS Dyar, 1925, Insecutor Inscit. menstr. 13: 4.

ACRO

Type-species: Nyctodryas parthenomorpha Dyar, 1925, ibidem 13: 4, by monotypy. Holotype 3 , Mexico: Colima (USNM, Washington).

NYCT YCIA Hampson, I 9o6, Cat. Lepid. Phalaence Br. Mus. 6: xi, 299.

CUCU

Type-species: Bombycia persimilis Hampson, I 894, Fauna Br. India (Moths) 2: 206, fig. 125 , by original designation. Type(s) ô, India: [Punjab], Dharmsala (BMNH).

NYMBIS Guenée, 1852, in Boisduval \& Guenée, Hist. nat. Insectes (Lépid). 7: $320 . \quad$ Cato

Type-species: Nymbis iniqua Guenée, I 852, ibidem 7:32I, by subsequent designation by Hampson, 1913, Cat. Lepid. Phalaenae Br. Mus. 13: ir 5. Holotype q, Brazil; this locality was queried by Guenée, but is probably correct (BMNH).

NYODES Laporte, 1970, Biologia gabon. 6: I8I.

HADE

Type-species: Xanthia brevicornis Walker, [1857] I 856, List Specimens lepid. Insects Colln Br. Mus. 10: 466, by original designation (for Elaeodes Hampson, I913). Holotype o. CONGO (BMNH).

Nyodes was proposed as the objective replacement name for Elaeodes Hampson, I9I3. ELAEODES Hampson, igr3, Cat. Lepid. Phalaenae Br. Mus. 13: xi, 356.

Type-species: Xanthia brevicomis Walker, [1857], by original designation.

A junior homonym of Elaeodes Gemminger \& Harold, 1870, Cat. Coleopterorum 7: 1868, Coleoptera. The objective replacement name is Nyodes Laporte, 1970.

NYSSOCNEMIS Lederer, I 857, Noctuinen Eur.: 35, I I 7.

Type-species: Hadena eversmanni Lederer, i 853, Verh.zool.-bot. Ver. Wien 3: 369 (the objective replacement name for Noctua obesa Eversmann, I846, see below), by monotypy. Type(s) of $N$. obesa, [U.S.S.R.]: eastern Siberia.

$H$. eversmanni was proposed as the objective replacement name for Noctua obesa Eversmann, i 846, Bull. Soc. imp. Nat. Moscou 19 (3): 86, pl. 2, fig. 2, a junior primary homonym of Noctua obesa Boisduval I829, Eur. Lepid. Index Meth., Errata et Addenda: 3, - Lepid. Noctuidae.

NYSTALEA Guenée, I 852, in Boisduval \& Guenée, Hist. nat. Insectes (Lépid.) 6: I 22. NOTODONTIDAE

Type-species: Nystalea conchyfera Guenée, I 852 , ibidem 6: I22, pl. 9, fig. 2, by subsequent designation by Kirby, I 892, Synonymic Cat. Lepid. Heterocera 1: 6I 8.

Nystalea was originally placed in the Noctuidae.

OBANA Walker, i862 [March 8th], List Specimens lepid. Insects Colln Br. Mus. 24: I Io3. ACoN

Type-species: Obana vagipennata Walker, 1862, ibidem 24: I I03, by monotypy. Jectotype +, Borneo: Sarawak (UM, Oxford), clesignated (as type) by Swinhoe, rgoo, Cat. east. and Aust. Lepid. Heterocera 2: 67.

OBANA Walker, I 862 November, J. Proc. Linn. Soc. (Zool.) 6: igo. See Rhoptrotrichia Butler, I 89 I. ACRO

OBARZA Berio, 1950, Annali Mus. civ. Stor. nat. Giacomo Doria 64: I57. $\triangle \mathrm{CON}$

Type-species: Ozarba zernyi Berio, 1940, Memorie Soc. ent. ital. 19: 189, fig. 33, by original designation. Holotype $\delta$, [BRazıl]: Santa Catharina, Jaraguà (Colln E. Berio, Genoa). 
OBDORA NTalker, I869, Characters undescr. Lepid. Heterocera: 95.

Type-species: Obdora nigrilinea Walker, I869, ibidem: 95, by monotypy. Type(s) o., [INDIA]: [Varanasi] Benares (BMNH).

OBRIMA Walker, I856, List Specimens lepid. Insects Colln Br. Mus. 9: 69, I34.

OPHI

Type-species: Obrima pyraloides Walker, I 856, ibidem 9: I35, by monotypy. Holotype o. Honduras (BMNH).

OBROATIS Walker, I858, List Specimens lepid. Insects Colln Br. Mus. 15: I546, I635. OPHI Type-species: Obroatis negata Walker, I858, ibidem 15: I635, by monotypy. Holotype $\widehat{\jmath}$, Venezuela (BMNH).

OBUCOLA Walker, I858, List Specimens lepid. Insects Colln Br. Mus. 15: 1857.

OPHI Type-species: Obucola expandens Walker, I858, ibidem 15: I857, by monotypy. Type(s) $\hat{\jmath}$, [BrazIL]: Rio Janeiro (UM, Oxford).

O. expandens is a junior subjective synonym of Peosina staccata Guenée, I852, in Boisduval \& Guenée, Hist. nat. Insectes (Lépid.) 7 : I33.

OCALARIA Schaus, I906, Proc. U.S. natn. Mus. 30: I32

OPHI

Type-species: Ocalaria guarana Schaus, I906, ibidem 30: 132, by monotypy. Type(s), Brazil: São Paulo (USNM, Washington).

OCHRIA Hübner, [182I]. See Gortyna Ochsenheimer, I8I6.

ACRO

OCHROCALAMA Hampson, I908, Cat. Lepid. Phalaenae Br. Mus. 7: 6. Available, but without included species until Hampson, I9Io, ibidem 9: x, 226.

ACRO

Type-species: Megalodes xanthia Hampson, 1905, Ann. S. Afr. Mus. 3: 434, by subsequent monotypy. Lectotype $\hat{\sigma}$, [South AFrica]: Natal, Delvin (BMNH), designated (as type) by Hampson, I910, ibidem 9: 227.

OCHROPLEURA Hübner, [I82I] I8 I6, Verz. bekannter Schmett. : 223.

NOCT

Type-species: Phalaena plecta Linnaeus, I76I, Fauna Suecica (Edn 2): 32I, by subsequent designation by Grote, I895, Entomologist's Rec. J. Var. 6: 30. Type(s), SwEDEN (LS, London).

OCHROTHRIPA Hampson, I9I2, Cat. Lepid. Phalaenae Br. Mus. 11: xi, 3 I 7.

SARR

Type-species: Crioa leptochroma Turner, I902, Proc. Linn. Soc. N.S.W. 27: 96, by original designation. Syntypes I $\widehat{\jmath}, \mathrm{I}$ \&, Australia: Qd, Gympie (ANIC, Canberra).

OCHROTRIGONA Hampson, I926, Descr. new Genera Species Lepid. Phalaenae Subfamily Noctuinae Br. Mus.: 560 .

OPHI

Type-species: Bleptina triangulifera Hampson, I895, Fauna Br. India (Moths) 3: 46, by original designation. Type(s), [Sikкim] Sikhim (BMNH).

OCHTHOPHORA Turner, I902, Proc. Linn. Soc. N.S.W. 27: 89.

SARR

Type-species: Ochthophora sericina Turner, I902, ibidem 27: 90, by monotypy. Holotype $\widehat{\jmath}$. Australia: Qd, Brisbane (ANIC, Canberra).

ODICE Hübner, [1823] I8I6, Verz. bekannter Schmett.: [258].

$\mathrm{ACON}$

Type-species: Noctua inamoena Hübner, [1803], Samml. eur. Schmett. 4: pl. 6I, figs 30I, 302, by subsequent designation by Moore, I 884, Lepid. Ceylon 3: 57, but cited as †inamaena, an incorrect subsequent spelling. Syntypes, EUROPE.

$N$. inamoena is a junior subjective synonym of Phalaena arcuinna Hübner, I79o, Beitr. Gesch. Schmett. 2 (4): 93, pl. 2, fig. O.

ODONTELIA Hampson, 1905, Cat. Lepid. Phalaenae Br. Mus. 5: x, 2 I9.

HADE

Type-species: Thargelia margiana Püngeler, I9o1, Dt. ent. Z. Iris 14: I 82, pl. 2, fig. I 4 , by original designation. Syntypes $2 \pi$, , [U.S.S.R.]: [Turkmenistan], Merw district (MNHU, Berlin). 
ODONTESTIS Hampson, I912, Cat. Lepid. Phalaenae Br. Mus. 11: xvi, 632.

Type-species: Plusiocalpe prosticta Holland, I894, Psyche, Camb. 7: 29, pl. I, fig. 3, by original designation. Syntypes $\hat{\delta}, \underline{q}$. IVEST AFriCA (CM, Pittsburgh).

ODONTESTRA Hampson, 1905, Cat. Lepid. Phalaenae Br. Mus. 5: x, 205.

HADE

Type-species: Mamestra vittigera Hampson, I902, Ann. S. Afr. Mus. 2: 266, by original designation. Lectotype $\hat{\sigma}$. South AFricA: Natal, Victoria district (BMINH), designated (as type) by Hampson, 1905, ibidem 5: 206.

ODONTINA Guenée, I862. See Tinnodoa nom.n.

$\mathrm{OPHI}$

ODONTODES Guenée, I852, in Boisduval \& Guenée, Hist. nat. Insectes (Lépid.) 7: 50. STIC Type-species: Odontodes aleuca Guenée, I 852, ibidem 7 : 5 I, pl. I 3, fig. I I, by monotypy. Type(s), EAst Indies (BMINH).

See also Burdria W'alker, 1869, and Nedroma Walker, i 869.

ODONTORETHA Hampson, i9r6, in Poulton, Proc. zool. Soc. Lond. 1916: $105 . \quad$ ACRo Type-species: Odontoretha featheri Hampson, I9I 6, ibidem 1916: I06, pl. I, fig. 7 , by original designation. Holotype $\hat{\sigma}$. [Somalia] Somaliland: Mandera (BMNH).

ODONTOSEMA Warren, I9r3. See Tesomonoda nom. n.

$\operatorname{ACON}$

OEDEBASIS Hampson, Igo2, Ann. S. Afr. Mus. 2: $35^{2}$.

OPHI

Type-species: Oedebasis ovipennis Hampson, 1902, ibidem 2: 352, by monotypy. Syntypes $\hat{\sigma}$. 우, [Mozambigue]: Delagoa Bay (BMNH).

OEDERASTRIA Hampson, ig02, Ann. S. Afr. Mus. 2: 375.

ACON

Type-species: Oederastria ectorhoda Hampson, 1902, ibidem 2: 375, by monotypy. Holotype $\tilde{\sigma}$. [RHodesia]: Mashonaland, Salisbury (BMNH).

OEDEREMIA Hampson, igo8, Cat. Lepid. Phalaenae Br. Mus. 7: xii, 405.

ACRO

Type-species: Oederemia lithoplasta Hampson, 1908, ibidem 7: 406, by original designation. Syntypes $\hat{\jmath}$, 우, [U.S.S.R.]: IV. Turkestan, Tashkent (MNHU, Berlin).

Hampson cited the type-species as $\ddagger$ Bryophila lithoplasta Püngeler, ined. No earlier published reference has been found so Hampson's description made the name nomenclaturally available.

OEDIBLEMMA Hampson, i918, Norit. zool. 25: i 59.

ACON

Type-species: Oediblenma trogoptera Hampson, I918, ibidem 25: I60, by original designation. Holotype , [South Africa]: Transvaal, Pretoria, R. Pienaars (BMNH).

OEDIBRYA Hampson, I918, Novit. zool. 25: I 38.

ACRO

Type-species: Catamecia cinnamomina Rothschild, I9I 4, ibidem 21:336, by origina? designation. Syntypes $5\left(\sigma^{*}\right.$, ㅇ), Algeria: Alger Prov., Guelt-es-Stel (BMNH).

C. cinnamomina is a junior subjective synonym of Poecilia microglossa Rambur, 1858 , Cat. syst. Lépid. Andalousie: pl. 7, figs T, 2 and pl. 22, figs I, 2.

See also Moureia Orfila \& Rossi, 1956.

OEDICODIA Hampson, igro, Cat. Lepid. Phalaenae Br. Mus. 10:4,387. ACon

Type-species: Oedicodia violascens Hampson, I9I0, ibidem 10: 388, fig. 122, by original designation. Holotype , [Kenya] Brit. E. Afr.: Taveta (BMNH).

OEDICRASPIS Hampson, I9I2, Cat. Lepid. Phalaenae Br. Mus. 11: 224. 320. SARr

Type-species: Oedicraspis subfervida Hampson, I9I2, ibidcm 11: 320, fig. I I 5, by original designation. Syntypes 3 ㅇ. [KENYA] Brit. E. Afr.: Taveta (BMNH).

OEDIPLEXIA Hampson, 1908, Cat. Lepid. Phalaenae Br. Muss. 7: 9, 2 I 8.

ACRO

Type-species: Oediplexia mesophaea Hampson, Ino8, ibidcm 7: 218, fig. 32, by original clesignation. Holotype $\hat{\sigma}$, [TANzania] Brit. E. Afr.: [Tanganyilka], Kilima 'njaro $(\mathrm{BMNH})$. 
$\ddagger$ OEDIPOEPA Hampson, no published reference found.

HYPE

$\ddagger$ OEGOCERA Boisduval, I 844. See A egocera Latreille, I809.

AGAR

OENOPTERA Hampson, I910, Cat. Lepid. Phalaenae Br. Mus. 10: 3, 259.

ACON

Type-species: Oenoptera purpurea Hampson, I9ro, ibidem 10: 259, fig. 73, by original designation. Holotype $\widehat{0}$, CEYLON: Kegalle (BMNH).

OGDOCONTA Butler, I891, Ann. Mag. nat. Hist. (6) 7: 462 .

ACRO

Type-species: Placodes cinereola Guenée, I852, in Boisduval \& Guenée, Hist. nat.

Insectes (Lépid.) 6:3 16 , pl. I 5, fig. I, by original designation. Syntypes I ô, I q. [U.S.A.]:

New York State (BMNH). AMERICA: Northern.

OGLASA Walker, [1859] I858, List Specimens lepid. Insects Colln Br. Mus. 16: I94. OPH Type-species: Oglasa lagusalis Walker, [1859] I 858, ibidem 16: I94, by monotypy. Type(s) ô, Borneo: Sarawak (BMNH).

See also Dorsippa Walker, [1863].

OGLASODES Hampson, 1926, Descr. new Genera Species Lepid. Phalaenae Subfamily Noctuinae Br. Mus.: 209.

OPHI

Type-species: Oglasodes atrisignata Hampson, 1926, ibidem: 209, by original designation. Holotype +, [GHAna] Gold Coast: Bibianaha (BMNH).

OGOAS Druce, r89o, Biologia cent.-am. (Zool.) Lepid. Heterocera 1: 439.

HYPE

Type-species: Ogoas albipuncta Druce, I 890 , ibidem 1:439 pl. 35, fig. 24, by original designation. Syntypes $2 \hat{0}$, Guatemala: Sabo in Vera Paz (BMNH).

$\ddagger$ OGORIA, misspelling. See Ogovia Holland, I 892 .

OPHI

OGOVIA Holland, I 892, Entomologist 25 Suppl.: 95.

OPHI

Type-species: Ogovia tavetensis Holland, r892, ibidem 25 Suppl.: 95, by monotypy.

Type(s) $\sigma^{\star}$, AFrica : eastern (USNM, Washington).

†OGORIA; Grünberg, I9I I, Arch. Naturgesch. 76 (4) 2: I 8.

An incorrect subsequent spelling.

OGYGIA Hübner, [I82I]. See Yigoga nom. n.

NOCT

OIDEMASTIS Schaus, I916, Proc. U.S. natn. Mus. 50: 262, 323.

HYPE

Type-species: Oidemastis caliginosa Schaus, I916, ibidem 50:323, by original designation. Syntypes ô, ㅇ, French Guiana: R. Maroni, St. Jean (USNM, Washington).

OLIGIA Hübner, [1821] 1816, Vevz. bekannter Schmett.: 2 I3.

ACRO

Type-species: Phalaena strigilis Linnaeus, I758, Syst. Nat. (Edn I0) 1: ${ }_{\text {I }}^{\text {6, by subse- }}$ quent designation by Grote, 1895, Abh. naturw. Ver. Bremen 14: 79. Type(s), Europe (LS, London).

MIANA Stephens, I829 [June], Nom. Br. Insects: $4 \mathrm{I}$.

Type-species: Phalaena strigilis Linnaeus, I758, by subsequent designation by Westwood, I840, Synopsis Genera Br. Insects: 95.

Incorrect type-species designation: $P$. strigilis, cited by Boisduval, 1836 , Hist. nat. Insectes (Lépid.) 1: I44. Boisduval in his lengthy I 54 page Introduction, reviewed earlier classifications and designated up to three different type-species for each generic name. In his 'Exposé de notre méthode' from page I 55-69o, no type-species designations were made for the genera he himself used. Under the Code, Article 69(a)(iii), the type-designation of an author is eligible for consideration if he states that it is the type '... and if it is clear that he himself accepts it as the type-species.' Boisduval's type-designations although clearly stated do not fulfil the last requirement and so are invalid. Even though Boisduval's I 836 work was well known to lepidopterists, the type-designations contained in it have not in the past been accepted by Hemming or other authors.

Miana is a junior objective synonym of Oligia Hübner, [I 82 I] . 
OLULIS Walker, 1863, List Specimens lepid. Insects Colln Br. Mus. 27 : 126.

OPHI

Type-species: Olulis puncticinctalis WValker, I863, ibidem 27: I27, by monotypy. Type(s) \%. BORnEO: Sarawak (BMNH).

See also Phachthia Walker, I864.

HAMAXIA Walker, 1863, List Specimens lepid. Insects Colln Br. Mus. 27: I 28.

Type-species: Hamaxia lignulina Walker, 1863 , ibidem 27 : I 28, by monotypy. Holotype o. CEYLON (BMNH).

A junior homonym of Hamaxia Walker, [1860] I861, J. Proc. Linn. Soc. (Zool.) 5: I 53, Diptera. There is no objective replacement name but $H$. lignulina is a junior subjective synonym of Olulis puncticinctalis Walker, I863, the type-species of Olulis Walker, I863. The latter is thus available for use as subjective replacement name.

OLULODES Bethune-Baker, I908, Novit. zool. 15: 222.

OPHI

Type-species: Olulodes pulchra Bethune-Baker, 1908, ibidem 15: 222, by original designation. Type(s) q, New Guinea: [Papua], R. Aroa (BMNH).

OLYBAMA Walker, [1859] 1858, List Specimens lepid. Insects Colln Br. Mus. 16: 21 I. HYPE Type-species: Olybama thelephusalis Walker, [1859] 1858, ibidem 16: 21 I, by monotypy. Holotype , South AFrica (BMNH).

O. thelephusalis is a junior subjective synonym of Hydrillodes uliginosalis Guenée, I 854 , in Boisduval \& Guenée, Hist. nat. Insectes (Lépid.) 8: 66, pl. 6, fig. 6.

OLYSSA Walker, I858, List Specimens lepid. Insects Colln Br. Mus. 15: 1854.

OPH

Type-species: Olyssa calamitosa Walker, 1858, ibidem 15: I854, by monotypy. Holotype + , Australia: northern (BMNH).

†OMALOSOMA Walker, [1857]. See Dasycampa Guenée, I 837.

CUCU

OMBREA Walker, 1865, List Specimens lepid. Insects Colln Br. Mus. 33: I I Io.

OPH I

Type-species: Ombrea aenochromoides Walker, I 865, ibidem 33: I I I, by monotypy. Type(s) ô. Sumatra.

OMIA Hübner, [1821] i816, Verz. bekannter Schmett.: 244.

CUCU

Type-species: Noctua cymbalariae Hübner, [1809], Samml. eur. Schmett. 4: pl. 92, fig. 432, by subsequent designation by Guenée, 1852, in Boisduval \& Guenée, Hist. nat. Insectes (Lépid.) 6: i 56 . Type(s), Europe.

†OMIA Guenée, i 850, Annls Soc. ent. Fr. 8: 235.

Staudinger, I90I, in Staudinger \& Rebel, Cat. Lepid. palaearct. Faunengeb. 1: 220, as well as Neave, 1940, Nomencl. zool. 3: 410, treated Omia Guenée as nomenclaturally available. Guenée, however, was not proposing a new generic name but correctly attributed it to Hübner, and included in his grouping $N$. cymbalariae, the first of Hübner's originally included species.

† OMIA Guenée, I850. See Omia Hübner, [1821].

CUCU

OMMATOCHILA Butler, I894, Entomologist 27: 315 .

$\triangle \mathrm{CON}$

Type-species: Thalpochares mundula Zeller, I872, Verh.zool.-bot. Ges. Wien 22: 460, pl. 2, fig. 4 , by original designation. Holotype ơ, [U.S.A.]: Texas.

OMMATOPHORA Guenée, I 852, in Boisduval \& Guenće, Hist. nat. Insectes (Lépid.) 7: rgo. OPHI

Type-species: Phalaena luminosa Cramer, I780, Uitlandsche Kapellen 3: I 47, pl. 274, fig. D, by subsequent designation by Hampson, 1894, Fauna Br. India (Moths) 2: 55I. Type(s), Java: Samarang.

Not preoccupied by $\ddagger$ Ommatophora; Turner, 1848, Proc. zool. Soc. Lond. 16: 88, Mammalia, an incorrect subsequent spelling of Ommatophoca Gray, I844, - Mammalia. 
OMMATOSTOLA Grote, I873, Bull. Buffalo Soc. nat. Sci. 1: I I2.

ACRO

Type-species: Ommatostola lintneri Grote, I873, ibidem 1: II2, by monotypy. Type(s) ơ, [U.S.A.]: New York (BMNH).

OMMATOSTOLIDEA Benjamin, I933, Pan-Pacif. Ent. 9: i.

ACRO

Type-species: Ommatostolidea julitae Benjamin, I933, ibidem 9: 2, by original designation. Holotype , [U.S.A.]: Ariz., Douglas (USNM, Washington).

OMOPTERA Guérin-Méneville, [1832], Icon. Règne anim. Cuvier 2: Insectes pl. 89. cato

Type-species: Erebus putrescens Guérin-Méneville, [1832], ibidem 2: Insectes pl. 89, fig. 3, by monotypy, but cited as 'putrescens Boisd.', an incorrect authorship. Type(s), AMERICA: northern (from text ibidem 3: Insectes page 522).

Omoptera is dated from Cowan, 1971, J. Soc. Biblphy nat. Hist. 6: 18-29.

E. putrescens is a junior subjective synonym of Noctua lunata Drury, [I773] I770, Illust. nat. Hist. 1 (I 770 ): 40, pl. 20, fig. 3, but without scientific names until the Index was issued with 2 (I 773 ).

See also Nephelina Kirby, 1897.

†OMOPTERUS Guérin-Méneville, I 844, Icon. Règne anim. Cuvier 3: Insectes page 522.

An incorrect subsequent spelling of Omoptera. The plates (vol. 2) were published before the text (vol. 3), see Biblphie Fr., 1844, 33: 464 .

HOMOPTERA Guenée, I852, in Boisduval \& Guenée, Hist. nat. Insectes (Lépid.) 7: 8.

An unjustified emendation of Omoptera Guérin-Méneville, [1832]. Homoptera was attributed by Guenée to 'Bdv. Icon. du règne anim.?' There was no demonstrably intentional change of spelling by Guenée so Homoptera should strictly be treated as an incorrect subsequent spelling of Omoptera. However, for about sixty years following Guenée, Homoptera was in general use and has been used in the binomen of many new species. A case should be submitted to the Commission requesting the use of the Plenary Powers so that Homoptera Guenée, I 852 , may continue to be treated as an unjustified emendation. For the last sixty years Homoptera has been placed as a junior subjective synonym of Zale Hübner, I8 8 .

†OMOPTERUS Guérin-Méneville, I844. See Omopteva Guérin-Méneville, [1832].

CATO

OMORPHINA Alphéraky, I892, Horae Soc. ent. ross. 26: 452.

PLUS

Type-species: Omorphina aurantiaca Alphéraky, I892, ibidem 26: 452, by monotypy. Syntypes $\hat{\sigma},[\mathrm{CHINA}]$ : Amdo region, Sinin Mts (ZI, Leningrad).

OMPHALAGRIA Hampson, igro, Cat. Lepid. Phalaenae Br. Mus. 9: 7, 272.

ACRO

Type-species: Omphalagria hemiochra Hampson, I9Io, ibidem 9: 273, fig. I I 5, by original designation. Holotype . [Guinea] Fr. Guinea: Oudoula Siguiri (MNHN, Paris).

OMPHALESTRA Fletcher, I96I, Ruwenzori Exped. I952 1: I9I.

HADE

Type-species: Scotogramma submedianata Hampson, 1905, Cat. Lepid. Phalaenae Br. Mus. 5: 27, pl. 79, fig. 4, by original designation. Holotype + , [KENYA] Br. E. Africa: Machakos (BMNH).

OMPHALETIS Hampson, I908, Cat. Lepid. Phalaenae Br. Mus. 7: 5. Available, but without included species until Hampson, I909, ibidem 8: xi, 374.

ACRO

Type-species: Celaena florescens Walker, [1857] I856, List Specimens lepid. Insects Colln Br. Mus. 10: 268, by subsequent designation by Hampson, I909, ibidem 8: 374 . Lectotype $ᄋ$, [Australia]: New South Wales (UM, Oxford), designated (as type) by Swinhoe, I900, Cat. east. and Austr. Lepid. Heterocera 2: $3 \mathrm{I}$.

OMPHALOCEPS Hampson, I9o I, Cat. Lepid. Phalaenae Br. Mus. 3: xviii, 628. AGAR

Type-species: Eusemia triangularis Mabille, 1893, Annls Soc. ent. Belg. 37: 57, by original designation. Lectotype $\vec{\sigma}$. CAMERoun: lower (MNHU, Berlin), designated by Viette \& Fletcher, 1968, Bull. Br. Mus. nat. Hist. (Ent.) 21:4I 7. 
OMPHALOPHANA Hampson, i906, Cat. Lepid. Phalaenae Br. Muts. 6: viii, Iog.

Type-species: Noctua antirrhinii Hübner, [1803], Samml. eur. Schmett. 4: pl. 52, fig. 253 , by original designation. Type(s), Europe.

OMPHALOSCELIS Hampson, 1906, Cat. Lepid. Phalaenae Br. Mus. 6: xiii, $469 . \quad$ cucu

Type-species: Noctua lunosa Haworth, I809, Lepid. Br.: 230, by original designation. Syntypes 2 ex., Great Britain: [England], [Norfolk County] Com. Norf.

ONCOCNEMIS Lederer, I853, Verh. zool.-bot. Ver. Wien 3 (Abh.): 368.

CUCU

Type-species: Amphipyra confusa Freyer, [1840] I842, Nenere Beiträge Schmett. 4: 26, pl. 303, fig. I, by subsequent designation by Grote, 1874, Bull. Buffalo Soc. nat. Sci. 2: I3, but cited as 'confusa Ev.', an incorrect authorship. Type(s), [U.S.S.R.]: [Krasnoarmeysk] Sarepta.

Oncocnemis was also used by Lederer, I 857, Noctuinen Eur.: 32, 94, having the same included species.

ONCOTIBIALIS Janse, 1939, Moths S. Afr. 3: 201.

ACRO

Type-species: Oncotibialis flava Janse, 1939, ibidem 3: 202, fig. 55; pl. 37, fig. 21 ; pl. 40, fig. 7 , by original designation. Syntypes $\hat{\sigma},+$, , [RHodesia] S. Rhodesia: Wankie (TM, Pretoria).

¥ONEIDINA Hampson, no published reference found.

HYPE

ONEVATHA Walker, [i 859] I 858, List Specimens lepid. Insects Colln Br. Mus. 16: 225. Hy PE Type-species: Onevatha alsusalis Walker, [1859] I 858, ibidem 16: 226, by monotypy. Lectotype 9. Borneo: Sarawak (UM, Oxford), designated (as type) by Swinhoe, I90o, Cat. east. and Austr. Lepid. Heterocera 2: 199.

$\ddagger$ ONOBA, misspelling. See Anoba Walker, I 858 .

OPH I

ONYCHAGROTIS Hampson, 1903, Cat. Lepid. Phalaenae Br. Mus. 4: xvii, $465 . \quad$ Noct

Type-species: Agrotis rileyana Morrison, 1874, Proc. Boston Soc. nat. Hist. 17: I66, by original designation. Type(s), [U.S.A.]: Mo., St. Louis.

ONYCHESTRA Hainpson, 1905, Cat. Lepid. Phalaenae Br. Mus, 5: x, 223.

HADE

Type-species: Mamestra siccanorum Staudinger, i 870, Berl.ent. Z. 14: I I 4, by original designation. Syntypes $6(\hat{0}$, $)$ ), [U.S.S.R.]: [Krasnoarmeysk] Sarepta (MNHU, Berlin).

OORTIANA Köhler, 1947, Acta zool. lilloana 4: 90.

HADE

Type-species: Oortiana olivacea Köhler, 1947, ibidem 4: 90, by original designation. Holotype ô., Argentina: [Chubut Prov.], Comodoro Rivadavia (ZSBS, Munich).

OPHIDERES Boisduval, I832, Voyage de Découvertes de l'Astrolabe Ent. 1: 245.

OPHI

Type-species: Ophideres princeps Boisduval, 1832, ibidem 1: 245, by monotypy.

Type(s), New Guinea: [West Irian], Dorey.

OPHIODERES Agassiz, i 846, Nomencl. zool. Index univl.: 260.

An unjustified emendation of Ophideres Boisduval, 1832 .

OPHIODERES Agassiz, I 846. See Ophideres Boisduval, I832.

OPH I

OPHIODES Guenće, I841. See Minucia Moore, [1885].

CATO

OPHIOGENES R.L., I817. See Ophiusa Ochsenheimer, 1816.

CATO

OPHIOSEMA Romieux, I943, Mitt. schweiz. ent. Ges. 19: io8.

SARK

Type-species: Ophiosema jansei Romieux, I943, ibidem 19: 109, pl. 9, fig. 9, by original designation. Holotype $\hat{\delta}$, [ZArke] Congo: Upper Katanga, Kasolo, Tshinkolobwe.

OPHISMA Guenće, i 852, in Boisduval \& Guenće, Hist. nat. Insectes (Lépid.) 7: $236 . \quad$ Cato Type-species: Ophisma gravata Guence, I 852 , ibidem 7:237, by subsequent designation by Moore, [1885] i887, Lepid. Ceylon 3: i61. Syntypes ô. q, East Indies (BMNH). 
OPHIUCHE Hübner, [1825] I816, Verz. bekannter Schmett.: 344.

HYPE

Type-species: Phalaena lividalis Hübner, I790, Beitr. Gesch. Schmett. 2 (4): 86, pl. (4) I, fig. E, by monotypy. Type(s), Europe.

See also Anepischetos Smith, I9oo.

$\ddagger$ OPHIUCHIDIA Hampson, no published reference found.

HYPE

OPHIUSA Ochsenheimer, I8I6, Schmett. Eur. 4: 93.

CATO

Type-species: Phalaena tirhaca Cramer, I777, Uitlandsche Kapellen 2: I I6, pl. I72, fig. E (but included by Ochsenheimer as †'tirrhaea Fabr.'), by subsequent designation by Duponchel, 1829, in Godart \& Du ponchel, Hist. nat. Lépid. Papillons Fr. 7 (2): 72 , but cited as $\ddagger$ tirrhaea. Type(s), [South Africa]: Cape of Good Hope.

P. tirhaca was misspelled as $¥$ Noctua tirrhaea Cramer by Fabricius, I 78I, Species Insect. 2:

2I3. This incorrect subsequent spelling was used by Ochsenheimer and other authors.

See also Meropis Hübner, I 822.

OPHIOGENES R.L., i8I 7, Jenaische Allg. Lit.-Ztg 1: 288.

Type-species: Phalaena tirhaca Cramer, I777 (but included by R.L., as †tirrhaea, an incorrect subsequent spelling), by PRESENT DESIGNATION.

Ophiogenes R.L. is a junior objective synonym of Ophiusa Ochsenheimer, I8I6.

†OPHIUSSA; Hübner, [I823] I8I6, Verz. bekannter Schmett.: 266.

An incorrect subsequent spelling.

HEMACHRA Sodoffsky, i837, Bull. Soc. imp. Nat. Moscou 1837 (6): 89.

Proposed, unnecessarily, as an objective replacement name for Ophiusa Ochsenheimer, I8I6, which Sodoffsky considered to be inappropriate.

†OPHIUSSA, misspelling. See Ophiusa Ochsenheimer, I8I6.

CATO

OPHTHALMIS Hübner, [I819] I816, Verz. bekannter Schnett.: I66.

AGAR

Type-species: Phalaena lincea Cramer, I779, Uitlandsche Kapellen 3: 61, pl. 228, fig. B, by subsequent designation by Kirby, I892, Synonymic Cat. Lepid. Heterocera 1: 31. Type(s), Surinam.

OPHYX Guenée, I852, in Boisduval \& Guenée, Hist. nat. Insectes (Lépid.) 7: 233. OPH

Type-species: Ophyx ochroptera Guenée, I852, ibidem 7: 234, pl. 22, fig. I, by subsequent designation by Viette, I95 I, Bull. mens. Soc. linn. Lyon 20: i62. Holotype q. Australia (MNHN, Paris).

OPIGENA Boisduval, I840, Genera Index meth. Eur. Lepid.: Iо3.

NOCT

Type-species: Noctua polygona [Denis \& Schiffermüller], I775, Ankïndung syst. Werkes Schmett. Wienergegend: 78, by monotypy. Type(s), [Austria]: Vienna district (Collection destroyed, Horn \& Kahle, I936, Ent. Beih. Berl.-Dahlem 3: 243).

OPORINA Agassiz, I846. See Jodia Hübner, I8I8.

CUCU

OPOROPHYLLA Hampson, I913, Cat. Lepid. Phalaenae Br. Mus. 12: xi, 386.

CATO

Type-species: Phyllodes ustulata Westwood, I848, Cabinet Oriental Ent.: 57, pl. 28, fig. I, by original designation. Type(s), [Bangladesh] India: [E. Bengal], Sylhet (UM, Oxford).

OPOTURA Walker, [1859] I858, List Specimens lepid. Insects Colln Br. Mus. 16: I9I. HyPE Type-species: Opotura erinusalis Walker, [1859] I858, ibidem 16: I92, by monotypy. Holotype ơ, VENEZUELA (BMNH).

O. erimusalis is a junior subjective synonym of Bleptina confusalis Guenée, I854, in Boisduval \& Guenée, Hist. nat. Insectes (Lépid.) 8: 67, pl. 9, fig. 2.

OPSIGALEA Hampson, I906, Cat. Lepid. Phalaenae Br. Mus. 6: viii, 84.

CUCU

Type-species: Nystalea ocellata Walker, I865, List Specimens lepid. Insects Colln Br. Mus. 33: 760 , by original designation. Holotype $\hat{o}$. MExico: Oajaca (BMNH). 
OPSYRA Hampson, I908, Cat. Lepid. Phalaenae Br. Mus. 7: I5. Available, but without included species until Hampson, I9ro, ibidem 9: xiv, 445.

Type-species: Callyna chalcoela Hampson, 1902, J. Bombay nat. Hist. Soc. 14: 208, by subsequent monotypy. Syntypes, [Sikkim] Sikhim (BMNH). China.

ORAESIA Guenée, I 852, in Boisduval \& Guenée, Hist. nat. Insectes (Lépid.) 6: $362 . \quad$ OpH

Type-species: Noctua emarginata Fabricius, 1794, Ent. Syst. 3(2): 82, by subsequent designation by Warren, I9r3 November 2oth, in Seitz, Macrolepid. World 3: 383. Type(s), [INDIA]: [Madras Prov.], Tranquebar (UZM, Copenhagen).

Incorrect type-species: Oraesia rectistria Guenée, I852, was cited in parenthesis by Moore, I884, Lepid. Ceylon 3: 76, at the end of his generic redescription where he usually cited the type-species. On this occasion the word 'Type' was omitted and so no designation was made. O. rectistria is, however, congeneric with $N$. emarginata.

$\ddagger$ ORATOCELIS, misspelling. See Calymma Hübner, [1823].

$\operatorname{ACON}$

ORATOSCELIS Guenée, I84I. See Calymma Hübner, [I823].

$A C O N$

ORBIFRONS Staudinger, I877, Stettin. ent. Ztg 38: I87.

NOCT

Type-species: Orbifrons singularis Staudinger, 1877, ibidem 38: 187, by monotypy. Syntypes I ô, 2 ․ [U.S.S.R.]: Turkestan, Krasnowodsk (MNHU, Berlin).

ORBONA Hübner, [182I] I816, Verz. bekannter Schmett.: 232.

CUCU

Type-species: Cerastis serotina Ochsenheimer, I8I6, Schmett. Eur. 4: 85, by subsequent designation by Hampson, I906, Cat. Lepid. Phalaenae Br. Mus. 6: 443 (but cited as fragariae Esper, an incorrect authorship, placed by Hampson (on page 448) as the senior synonym of serotina Ochsenheimer). Type(s), Europe (TM, Budapest).

C. serotina was the name proposed for the misidentification Noctua orbona Hufnagel sensu Hübner, [1803], Samml. eur. Schmett. 4: pl. 22, fig. I04.

C. serotina is a junior subjective synonym of Noctua fragariae Vieweg, I 79o, Tabellarisches Verz. Churmark Brandenburg einheimischen Schmett. 2: 56, pl. 2, figs 4, 5 .

ORECTIS Lederer, I857, Noctuinen Eur.: 46, 216.

HYPE

Type-species: Helia proboscidata Herrich-Schäffer, [185I] I845, Syst. Bearb. Schmett. Eur. 2: 430, pl. I20, fig. 6I8, by monotypy. Type(s), Hungary.

The type-species was attributed to Kindermann by Herrich-Schäffer on page 430, but in the index to the whole work in volume 6 , Herrich-Schäffer cited himself as the author. No other reference to Kindermann as author of this species lias been found.

ORIA Hübner, [182 I] I8I6, Verz. bekannter Schmett.: 240.

ACRO

Type-species: Noctua musculosa Hübner, [1808], Samml. cur. Schmett. 4: pl. 78, fig. 363 , by monotypy. Type(s), Europe.

SYNIA Duponchel, [I845], Cat. méth. Lépid. Eur. (2): I20.

Type-species: Noctua musculosa Hübner, [r8o8], by monotypy.

Synia is a junior objective synonym of Oria Hübner, [I82I].

TAPINOSTOLA Lederer, I857, Noctuinen Eur.: 36, 123.

Type-species: Noctua musculosa Hübner, [ 1808$]$, by subsequent designation by Hampson, I9Io, Cat. Lepid. Phalaenae Br. Mus. 9: 333.

Tapinostola is a junior objective synonym of Oria Hübner, [1821].

ORIA Guenée, I852. See Porrima Grote, I877.

NOCT

ORIBATES Edwards, I88I. See Gyros Edwards, I88I.

PYRALIDAE

ORIXA Walker, I869. See Xoria nom. n.

HY PE

OROBA Walker, [1863] i864, J. Proc. Linn. Soc. (Zool.) 7: 8 I.

OPH I

Type-species: Oroba surrigens Walker, [1863] I864, ibidem 7: 81, by monotypy. 
Lectotype ô, Borneo: Sarawak (UM, Oxford), designated (as type) by Swinhoe, I9oo, Cat. east. and Aust. Lepid. Heterocera 2: 194.

$O$. surrigens is a junior subjective synonym of Avitta subsignans Walker, I 858.

See also Avitta Walker, I 858 .

ORODESMA Herrich-Schäffer, I868, CorrespBl. zool.-min. Ver. Regensburg 22: I 79.

OPH1

Type-species: Orodesma apicina Herrich-Schäffer, I 868, ibidem 22: I 79, by monotypy, but cited by Herrich-Schäffer as 'apicina Gn.', an incorrect authorship. Type(s) + , CubA (MZC, Havana).

$O$. apicina was attributed to Guenée, but no earlier reference has been found.

See also Boryzola Hampson, 1926.

$\ddagger$ ORODESMI A; Wolcott, I923, J. Dept. Agric. Porto Rico 7 (I): I74.

An incorrect subsequent spelling.

$\ddagger$ ORODESMIA, misspelling. See Orodesma Herrich-Schäffer, I 868.

OPHI

OROMENA Moore, I882, in Hewitson \& Moore, Descr. new Indian lepid. Insects Colln late Mr W. S. Atkinson: i6o.

OPHI

Type-species: Briada relinquenda Walker, 1858 , List Specimens lepid. Insects Colln Br. Mus. 15: I802, by original designation, but cited by Moore as $₫ B$. veliquenda, an incorrect subsequent spelling. Holotype 우, [INDIA]: North Hindostan (BMNH).

OROPLEXIA Hampson, I9o8, Cat. Lepid. Phalaenae Br. Mus. 7 : xiii, 5 Io.

ACRO

Type-species: Mamestra decorata Moore, I882, in Hewitson \& Moore, Descr. new Indian lepid. Insects Colln late $M v W$. S. Atkinson: I I I, pl. 4, fig. 8, by original designation. Type(s), India: [W. Bengal], Darjiling (MNHU, Berlin).

OROSA Walker, [I866] I865, List Specimens lepid. Insects Colln Br. Mus. 34: I222. SARR

Type-species: Orosa tortricoides Walker, [1866] I865, ibidem 34: 1223, by monotypy. Holotype , Ceylon (BMNH).

OROSAGROTIS Hampson, I903, Cat. Lepid. Phalaenae Br. Mus. 4: xi, 135, 658.

NOCT

Type-species: Agrotis montana Morrison, 1875, Ann. Lyceum nat. Hist. 11: 94, by original designation. Lectotype, [U.S.A.]: Colorado Mts (MCZ, Boston), designated (as type) by Smith, 1903, Trans. Am. ent. Soc. 29: 204.

Orosagrotis Hampson was considered by the Int. Commn zool. Nom., I972, Bull. zool. Nom. 29 Opinion 983: II3, and placed on the Official List of Generic Names in Zoology: Name No. 1962, with type-species Agrotis montana Morrison, by original designation. Hampson, on page 135, actually designated Agrotiphila rigida Smith, I891, Trans. Am. ent. Soc. 18: I34, and then dealt with rigida on page I36. In his Addenda on page 658, Hampson stated that Agrotis montana should be inserted on page I 36 as the senior synonym of rigida; he omitted, however, except by implication, to change his type-designation on page I 35 .

$\ddagger$ PROSAGROTIS; Warren, i9r I, in Seitz, Gross-Schmett. Erde 3: 25 I.

An incorrect subsequent spelling.

OROSCOPA Druce, I891, Biologia cent.-am. (Zool.) Lepid. Heterocera 1: 486.

OPHI

Type-species: Oroscopa concha Druce, I89I, ibidem 1:486, pl. 40, fig. 22, by original designation. Holotype + , Panama: Chiriqui.

OROTERMES Dognin, I919, Hétérocères nouv. Am. Sud 17: 5.

OPHI

Type-species: Orotermes monstrosa Dognin, I9I9, ibidem 17: 5, by monotypy. Holotype $\delta$, French Guiana: Maroni (USNM, Washington).

ORREA Walker, I866, List Specimens lepid. Insects Colln Br. Mus. 35: 1983.

OPHI Type-species: Moepa concisa Walker, I865, ibidem 33: 982, by monotypy (of Moepa Walker, I865, ibidem 33: 981). Type(s) o,. [Moluccas]: Ceram [I.] (BMNH).

$M$. concisa is a junior subjective synonym of Hypospila bolinoides Guenée, I 852. 
Orrea was proposed as the objective replacement name for .1/oepa Walker, I865, ibidem 33: $98 \mathrm{r}$.

See also Hypospila Guenée, 1852.

.MOEPA Walker, 1865, List Specimens lepid. Insects Colln Br. Mus. 33: 98r.

Type-species: Moepa concisa Walker, I865, by monotypy.

A junior homonym of Moepa Walker, r865, ibidem 33: 916, - Lepid., Noctuidae. The objective replacement name is Orrea Walker, I 866.

ORRHODIA Hübner, [I821] I8I6, Verz. bekannter Schmett.: 231.

CUCU

Type-species: Bombyx vaupunctatum Esper, 1786, Die Schmett. 3: 385, pl. 76, fig. 4, by subsequent designation by Hampson, 1906, Cat. Lepid. Phalaenae Br. Mus. 6: 443. Syntypes, [Germany].

B. vaupunctatum is a junior subjective synonym of Phalaena mibiginosa Scopoli, 1763 , Ent. Camiolica: 213.

ORRHODIELLA Spuler, 1907, in Hofmann, Schmett. Eur. 1: 255 footnote.

CUCU

Type-species: Heteromorpha ragusae Failla-Tedaldi, I890, Naturalista sicil. 10 : 30, pl. I, figs 6,7 , by monotypy (of Heteromorpha Failla-Tedaldi, r89o). Syntypes I $\hat{0}$, 2 ․ [ITALy]: Sicily, Madonie.

Orrhodiella was proposed as the objective replacement name for Heteromorpha FaillaTedaldi, i 890.

HETEROMORPHA Failla-Tedaldi, I890, Naturalista sicil. 10: 30.

Type-species: Heteromorpha ragusae Failla-Tedaldi, I 890, by monotypy.

A junior homonym of Heteromorpha Hübner, I822, Syst.-alphab. Verz.: I 5, I 8, - Lepid., Noctuidae, suppressed for the purposes of the Law of Priority but not for those of the Law of Homonymy in Opin. Decl. int. Commn zool. Nom. (I957) 17 Opinion 494: 267.

The objective replacement name is Orrhodiella Spuler, 1907.

ORSA Walker, 1865, List Specimens lepid. Insects Colln Br. Mus. 33: I I 2.

OPHI

Type-species: Orsa erythrospila Walker, I865, ibidem 33: II 2, by monotypy. Holotype ơ, [BRazIL]: [R. Amazon], [Tefé] Ega (BIINH).

ORTHEAGA Walker, I865, List Specimens lepid. Insects Colln Br. Mus. 33: 927.

OPHI

Type-species: Ortheaga combusta Walker, I865, ibidem 33: 928, by monotypy. Holotype , JAVA (BMNH).

ORTHIA Herrich-Schäffer, 1853, Samml. neuer oder wenig bekannter aussereur. Schmett. 1 (I): wrapper, pl. 5, fig. I 8; I 856 \& I 858 , ibidem 1: I 3, 56, 78 .

AGAR

Type-species: Orthia augias Herrich-Schäffer, I853, ibidem 1 (I): wrapper, pl. 5, fig. I 8; I 856 \& I 858, ibidem 1: 13, 56, 78, by subsequent designation by Hampson, 190 I, Cat. Lepid. Phalaenae Br. Mus. 3: 655. Type(s), Brazil (BMNH).

Both Orthia and augias were attributed to Boisduval by Herrich-Schäffer. They are both dated from the wrappers to the plates and not the title page of the volume $1850-1858$. OTHRIA Westwood, r877, Trans. Linn. Soc. Lond. (Zool.) (2) 1: 201.

Type-species: Orthia augias Herrich-Schäffer, 1853 , by subsequent designation by Kirby, I892, Synonymic Cat. Lepid. Heterocera 1: 32 (but cited as łangias, an incorrect sibsequent spelling).

Othria is a junior objective synonym of Orthia Herrich-Schäffer, $18_{53}$.

ORTHOA Billberg, I820. See Orthosia Ochsenheimer, I8I6.

HAIE

ORTHOCLOSTERA Butler, I878, Trans. ent. Soc. Lond. 1878: 70.

EUTE

Type-species: Orthoclostera peculiaris Butler, 1878 , ilidem 1878: 70 , by original designation. Type(s), Brazil: Amazons, Rio Negro (BIINH).

O. peculiaris is a junior subjective synonym of Abrostola derincta Walker, 1858 , List Specimens lepid. Insects Colln Br. Mus. 15: I $78 \mathrm{r}$. 
ORTHOCRASPIS Hampson, I9I2, Cat. Lepid. Phalaenae Br. Mus. 11: xv, 586.

CHLO

Type-species: Carea rectimarginata Hampson, I896, Fauna Br. India (Moths) 4: 530, by original designation. Type(s) ô, BHutan (BMNH).

$\ddagger$ ORTHODEA, misspelling. See Orthodes Guenée, I 852 .

HADE

ORTHODES Guenée, I852, in Boisduval \& Guenée, Hist. nat. Insectes (Lépid.) 5: 371. HADE

Type-species: Orthodes infirma Guenée, I 852 , ibidem $5: 375$, by subsequent designation by Grote, I 874, Bull. Buffalo Soc. nat. Sci. 2: 23. Syntypes 2 ô. I 9, BrazIL: NouvelleFribourg (BMNH).

†ORTHODE $A$; Smith, 1906, Can. Ent. 38: 230.

An incorrect subsequent spelling.

ORTHOGONIA Felder \& Felder, I862, Wien. ent. Monatschr. 6: 38.

ACRO

Type-species: Orthogonia sera Felder \& Felder, i862, ibidem 6: 38, by monotypy. Syntypes 2 o, China: [Che-Kiang] Ning-po (BMNH).

ORTHOGONICA Hampson, I908, Cat. Lepid. Phalaenae Br. Mus. 7: vii, 45.

Proposed, unnecessarily, as an objective replacement name for Orthogonia Felder \&

Felder, I862, which Hampson considered to be a homonym of Orthogonius Dejean, I825,

Species gén. Coléopt. 1:279, - Coleoptera.

ORTHOGONICA Hampson, I908. See Orthogonia Felder \& Felder, I862.

ACRO

ORTHOGRAMMA R.L., I8I 7, Jenaische Allg. Lit.-Ztg 1: 285.

CUCU

Type-species: Noctua tersa [Denis \& Schiffermüller], I775, Ankündung syst. Werkes

Schmett. Wienergegend: 312, by PRESENT DESIGNATION. Type(s), [AusTria]: Vienna district (Collection destroyed, Horn \& Kahle, I936, Ent. Beih. Berl.-Dahlem 3: 243).

$N$. tersa was the second of the species originally included in Orthogramma and is designated in order to prevent unnecessary changes of valid generic names. $N$. tersa is congeneric with P. glaucina Esper, see Boursin, I95 I, Z. wien. ent. Ges. 36: 45-46; thus Orthogramma R.L., I8I7, is a junior subjective synonym of Episema Ochsenheimer, I8I6.

ORTHOGRAMMA Guenée, I852. See Archana Walker, I 865.

OPHI

ORTHOGRAMMICA Hampson, 1926, Descr. new Genera Species lepid. Phalaenae Subfamily Noctuinae Br. Mus.: 273.

OPHI

Type-species: Acantholipes bilineatus Hampson, I894, Fauna Br. India (Moths) 2: 525, by original designation. Syntypes $\widehat{0},+$,, [SIKkim] Sikhim. IndiA: [Assam], Nagas.

ORTHOLEUCA Hampson, I9I0, Cat. Lepid. Phalaenae Br. Mus. 10: 6, 486.

$\operatorname{ACON}$

Type-species: Ortholeuca albiluna Hampson, I9I0, ibidem 10: 487 , fig. I42, by original designation. Syntypes I $\hat{\sigma}, \mathrm{I}$ ㅇ, Panama: La Chorrera (BMNH).

ORTHOPHA Köhler, (I952) I95I, Acta zool. lilloana 12: I42.

CUCU

Type-species: Orthopha polypuncta Köhler, (I952) I95I, ibidem 12: I42, by original designation. Holotype $q$, Argentina: Rio Negro [Prov.], Cipolletti (ZSBS, Munich).

ORTHORUZA Warren, I913, in Seitz, Gross-Schmett. Erde 11: 248.

$\operatorname{ACON}$

Type-species: Orthoruza niveipuncta Warren, I9I3, ibidem 11: 248, by original designation. Syntypes 2 0 , New Guinea: [Papua], R. Angabunga (BMNH).

The date of publication of the part of Seitz containing page 248 , was stated on page $24 \mathrm{I}$ as 1913 September 15 th in both the German and the English editions.

ORTHOSIA Ochsenheimer, I 816, Schmett. Eur. 4: 79.

HADE

Type-species: Noctua instabilis [Denis \& Schiffermüller], I775, Ankïndung syst. Werkes Schmett. Wienergegend: 76 , by subsequent designation by Curtis, I828, Br. Ent. 5: 237, but cited as 'instabilis Fab.', an incorrect authorship. Type(s), [Austria]: Vienna district (Collection destroyed, Horn \& Kahle, I936, Ent. Beih. Berl.-Dahlem 3: 243). 
N. instabilis is a junior subjective synonym of Phalaena incerta Hufnagel, r 766 , Berlin . Mag. 3 (3): 298.

ORTHOA Billberg, I820, Enumeratio Insect. Mus. G. J. Billberg: 85.

Type-species: Noctua instabilis [Denis \& Schiffermüller], I 775, by subsequent designation by Berio, 1966, Annali Mus. civ. Stor. nat. Giacomo Doria 76: 63.

Orthoa is a junior objective synonym of Orthosia Ochsenheimer, I8I6.

Incorrect type-species designation: Phalaena incerta Hufnagel, I766, a name not originally included in Orthoa, and not linked with one of the originally included names when cited by Tams, 1939, Entomologist 72: I 40.

ORTHOZANCLA Turner, 1933, Trans. R. Soc. S. Aust. 57: i67.

OPHI

Type-species: Orthozancla rhythmotypa Turner, I 933, ibidem 57: 168, by monotypy. Holotype ô, Australia: Qd, near Nambour, Montrille (ANIC, Canberra).

ORTHOZONA Hampson, i895, Funna Br. India (Moths) 3: xvi, 94.

OPH I

Type-species: Madopa quadrilineata Moore, is82, in Hewitson \& Moore, Descr. new Inclian lepid. Insects Colln late MV WV.S. Atkinson: 193, by original designation. Syntypes o., f, India: [IV. Bengal], Darjiling (BMNH).

ORTOPLA Walker, [1859] I 858, List Specimens lepid. Insects Colln Br. Mus. 16: I56. OPH Type-species: Ortopla iarbasalis Walker, [I $\left.5_{50}\right]$ I 858, ibidem 16: I 57, by monotypy. Lectotype o., Borneo: Sarawak (UMI, Oxford), designated (as type) by Swinhoe, I90o, Cat. east. and Aust. Lepicl. Heterocera 2: 122.

ORTOSPANA Walker, I865, List Specimens lepid. Insects Colln Br. Mus. 33: 950. CATo Type-species: Ortospana connectens Vallker, i 865, ibidem 33: 950, by monotypy. Type(s) ö. Cerlon.

ORUZA Walker, 186I, List Specimens lepid. Insects Colln Br. Mus. 23: 947. Available, but without included species until Walker, r 862, ibidem 24: ro89. ACON

Type-species: Oruza costata Walker, I 862, ibidem 24: I089, by subsequent monotypy. Lectotype ㅇ. [INDIA]: Hindostan (UM, Oxford), designated (as type) by Swinhoe, I90o, Cat. east. and Aust. Lepid. Heterocera 2: 70.

Oruza when originally proposed contained a second species, but this was doubtfully included and, under the Colle, Article 68 (c), is not eligible for selection as type-species.

ORUZODES Warren, 1913, in Seitz, Gross-Schmett. Erde 11: 255.

ACON

Type-species: Oruza unipunctata Bethune-Baker, I 906, Norit. zool. 13:2 I 5, by original designation. Holotype $\hat{\delta}$, New Guinea: [Papua], Aroa River (BMNH).

The date of publication of the part of Seitz containing page 255 was stated on page 249 as I9I 3 September I5th in both the English and German editions.

ORYGMOPHORA Hampson, 1926, Descr. nexi Genera Species Lepid. Phalaenae Subfamily Nochinat Br. 11 us.: 587 .

OPH I

Type-species: Orygmophora mediofoveata Hampson, 1926, ibidem: 587 , by original designation. Syntypes 4 ô. Sierra Leone (BMNH).

OSDARA Walker, I865. See Radosa nom. n.

OPHI

OSERICANA Walker, [1866] 1865, List Specimens lepid. Insects Colln Br. Mus. 34: I2 I 4. I1re

Type-species: Osericana albistella Walker, [I 866] I865, ibidem 34: 12 I 4, by monotypy. Syntypes 20 . Sumatra (BMNH).

OSLARIA Dyar, 1904, Jl N.Y. ent. Soc. 12: 4 I.

ACRO

Type-species: Zotheca viridifera Grote, $1882, C a n$. Ent. 14:217, by original designation. Type(s), [U.S.A.]: Arizona.

OSSONOBA Walker, i866, List Specimens lepid. Insects Colln Br. Mus. 35: 1966.

OPHI

Type-species: Ossonoba torpiala Walker, I866, ibidem 35: 1966, by monotypy. Holotype $\delta^{\star},[$ INDIA $]$ : [W. Bengal], Darjeeling (BMNH). 
OSTACRONYCTA Bethune-Baker, I9I I, Ann. Mag. nat. Hist. (8) 8: 535.

OPHI

Type-species: Ostacronycta glaucopasta Bethune-Baker, I9I I, ibidem (8) 8: 535, by original designation. Type(s) ㅇ, [KeNYA]: Nairobi (BMNH).

OSTHA Walker, I86I, List Specimens lepid. Insects Colln Br. Mus. 23: 947. Available but without included species until Walker, I862, ibidem 24: Iog8.

OPHI

Type-species: Ostha sileniata Walker, I 862, ibidem 24: I099, by subsequent monotypy. Syntypes 2 \&, [BRAzIL]: [R. Amazon], [Tefé] Ega (BMNH).

Ostha seleniata Walker, [1863] I862, ibidem 26: I687, is an unjustified emendation of O. sileniata Walker, I862.

PSEPHIS Guenée sensu Felder \& Rogenhofer, I875, Reise öst. Fregatte Novara (Zool.) 2 (Abt. 2): pl. I20, fig. 4.

Cited by Neave, 1940, Nomencl.zool. 3: 958 as though it were a nomenclaturally available generic name distinct from Psephis Guenée, 1854, Hist. nat. Insectes (Lépid.) 8: 257,Lepid., Pyralidae. Felder \& Rogenhofer did not, however, propose a new genus but merely described a new species as 'Psephis? aega F. \& R.' which should have been placed in Ostha WValker.

OSTHELDERA nom. n. for Pfeifferella Osthelder, I933.

CUCU

Type-species: Pfeifferella gracilis Osthelder, 1933, Mitt. münch. ent. Ges. 23: 54, by monotypy (of Pfeifferella Osthelder). Syntypes I ô, 3 ㅇ․ [TURKey]: Nordsyrien, Marasch (BMNH).

PFEIFFERELLA Osthelder, 1933, Mitt. münch. ent. Ges. 23: 54 .

Type-species: Pfeifferella gracilis Osthelder, 1933, by monotypy.

A junior homonym of Pfeifferella Labbé, I899, Tierreich 5: 60,-Protozoa. The objective replacement name is Ostheldera nom. n.

OSTHOPIS Hampson, I926, Descr. new Genera Species Lepid. Phalaenae Subfamily Noctuinae Br. Mus.: 60I.

OPHI

Type-species: Osthopis exocausta Hampson, I926, ibidem: 60 I, by original designation. Holotype ô., BrazIL: Espiritu Sto. (BMNH).

OTACES Druce, I891, Biologia cent.-am. (Zool.) Lepid. Heterocera 1: 464 .

HYPE

Type-species: Otaces lineata Druce, I891, ibidem 1: 464, pl. 38, fig. I6, by original designation. Syntypes $\widehat{\partial}$, ㅇ, Panama: Chiriqui.

OTHORA Walker, I865, List Specimens lepid. Insects Colln Br. Mus. 33: 902.

SARR

Type-species: Othora velata Walker, 1865 , ibidem 33: 902 , by original designation Holotype ô. CEylon (BMNH).

$O$. velata is a junior subjective synonym of Gadivtha decrescens Walker, [I858] I857, ibidem 13: I Io4.

See also Plotheia Walker, [I858]; Egelesta Walker, I858; and Galleriomorpha Nietner, I861.

OTHREIS Hübner, [I823] I8I6, Verz. bekannter Schmett.: 264.

OPH1

Type-species: Phalaena pomona Cramer, I775, Uitlandsche Kapellen 1: 122, pl. 77 , fig. C, by subsequent designation by Moore, [I885] I887, Lepid. Ceylon 3: I3I, but cited as $\$$ fullonica Linnaeus (an incorrect subsequent spelling) which Moore placed as the senior synonym of pomona Cramer. Type(s), [IND1A]: Coromandel coast.

$P$. pomona is a junior subjective synonym of Phalaena fullonia Clerck, I764, Icones Insect. rariorum 2: pl. 48 .

OTHRYIS Agassiz, I846, Nomencl. zool. Index univl.: 266.

An unjustified emendation of Othreis Hübner, [1823].

OTHRES YPNA Berio, 1958, in Berio \& Fletcher, Annali Mus. civ. Stor. nat. Giacomo Doria 70 : 362.

CATO

Type-species: Cerbia subolivacea Walker, [1863] 1864, J. Proc. Linn. Soc. (Zool.) 7 : 
I79, by original designation. Type(s) $\overrightarrow{0}$, Borneo: Sarawak (UM, Oxford), designated (as type) by Swinhoe, I900, Cat. east. and Aust. Lepid. Heterocera 2: Io6.

Othresupna was proposed as a subgenus of Hypersypnoides Berio, I954.

OTHRIA Westwood, I877. See Orthia Herrich-Schäffer, I853.

AGAR

OTHRYIS Agassiz, I 4 6. See Othreis Hübner, [1823].

OPHI

OTOSEMA Hübner, [1823]. See Ascalapha Hübner, [1809].

OPHI

OUTAYA Chrétien, I9I I, Anmls Soc. ent. Fr. 79: 505.

$A C O N$

Type-species: Outaya grisescens Chrétien, I9I I, ibidem 79: 505, fig., by monotypy. Syntypes of, + , [Tunisia]: Gafsa (MNHN, Paris).

OVIOS Walker, I855, List Specimens lepid. Insects Colln Br. Mus. 3: $587,753$.

AGAR

Type-species: Eudryas capensis Herrich-Schäffer, I854, Samml. neuer oder wenig bekannter aussereur. Schmett. 1 (I): wrapper, pl. 25, figs I I5, I I 6, by subsequent designation by Kirby, I892, Synonymic Cat. Lepid. Heterocera 1: 40. Type(s), [Soutu Africa]: Cap[e of Good Hope] (from the wrapper of the plates).

OXAENANUS Swinhoe, I90o, Cat. east. and Aust. Lepid. Heterocera 2: 201.

HYPE

Type-species: Mastygophora brontesalis Walker, [1859] I858, List Specimens lepid. Insects Colln Br. MIus. 16: I5I, by original designation. Lectotype, Borneo: Sarawak (UM, Oxford), designated (as type) by Swinhoe, I900, ibidem 2: 202.

OXICESTA Huibner, [I819] I816, Verz. bekannter Schmett.: I44.

ACRO

Type-species: Noctua geographica Fabricius, 1787, Mantissa Insect. 2: 167, by subsequent designation by Hampson, 1906, Cat. Lepid. Phalaenae Br. Mus. 6: IIz. Type(s), Austria (Type(s) lost, Zimsen, I964, Type Material of I. C. Fabricius: 550).

CLIDIA Boisduval, [1837] I834, Icones hist. Lépid. nouv. ou peu connus 2: I5I.

Type-species: Nuctua geographica Fabricius, I787, by monotypy.

Clidia is a junior objective synonym of Oxicesta Hübner, [I8I9].

OXYCESTE Agassiz, I846, Nomencl. zool. Index univl.: 267.

An unjustified emendation of Oxicesta Hübner, [18I9].

†OXYCESTA; Neave, I940, Nomencl. zool. 3: 494.

An incorrect subsequent spelling of Oxyceste Agassiz, 1846 .

OXIDERCIA Hübner, [I825] I816, V'erz. bekannter Schmett.: $34^{\circ}$.

OPHI

Type-species: Phalaena toxea Stoll, I781, in Cramer, Uitlandsche Kapellen 4: 133, pl. 358 , figs $G, H$, by monotypy, but cited by Hübner, as $O$. toxealis, an unjustified emendation by him. Type(s), Surinam.

$P$. toxea is dated from the wrapper of the part and not from the title-page of the volume, I7 82 .

OXYDERCIA Agassiz, I846, Nomencl. zool. Index univl.: 267.

An unjustified emendation of Oxidercia Hübner, [1 825].

Neave, 1940, Nomencl. zool. 3: 495 was in error in citing 'Oxydercia Felder, 1873, Reise Novara, Zool. 2, Abt. 2: Lep. Het., Explan. Pls. cviii-cxl, 15. - Lep. (Noctuid.)' as the original reference of this name. In the caption to the figure itself (pl. I20, fig. 24) Oxydercia was attributed to 'Hüb. Verz. 340'.

PARANI'MPHA Butler, I879, Trans. ent. Soc. Lond. 1879: 73.

Type-species: Phalaena toxea Stoll, I78I, by original designation.

Paranympha is a junior objective synonym of Oxidercia Hübner, [1825].

OXIRA Walker, I865, List Specimens lepid. Insects Colln Br. Mus. 32: 656.

NOCT Type-species: Oxira ochracea Walker, 1865 , ibidem 32: 657, by monotypy. Holotype o. CEYLON (BMNH).

$\ddagger$ OXYCESTA, misspelling. See Oxicesta Hübner, [18I9].

ACRO 
OXYCESTE Agassiz, I846. See Oxicesta Hübner, [I819].

ACRO

OXYCILLA Grote, (1896) I895, Proc. Am. Phil. Soc. 34: 418.

OPHI

Type-species: Oxycilla tripla Grote, (I896) I895, ibidem 34: 4I8, by monotypy. Syntypes 0 , ㅇ, [U.S.A.]: Arizona.

OXYCNEMIS Grote, I882, Can. Ent. 14: I82.

ACRO

Type-species: Oxycnemis advena Grote, I882, ibidem 14: I82, by monotypy. Type(s) ๆ. [U.S.A.]: Arizona.

OXYDERCIA Agassiz, I846. See Oxidercia Hübner, [I825].

OPHI

$\ddagger$ OXYDONA Hampson, no published reference found.

HYPE

$\ddagger$ OXYGIA, misspelling. See Yigoga nom. n.

NOCT

OXYGONITIS Hampson, I893, Illust. typical Specimens Lepid. Heterocera Colln Br. Mus. 9: $22,103$.

OPHI

Type-species: Oxygonitis sericeata Hampson, I893, ibidem 9: 22, I03, pl. I63, fig. I, by monotypy. Type(s) , CEYlon: Wattegama (BMNH).

OXYLOS Grote, 1875, Check List Noctuidae Am. N. of Mexico: I9.

NOCT

Type-species: Heliothis citrinellus Grote \& Robinson, I87o, Trans. Am. ent. Soc. 3: I8o, pl. 2, fig. 79, by monotypy. Syntypes ô, ㅇ. [U.S.A.]: Texas (BMNH).

OXYODES Guenée, 1852, in Boisduval \& Guenée, Hist. nat. Insectes (Lépid.) 7: I28. OPHI

Type-species: Noctua scrobiculata Fabricius, I775, Syst. Ent.: 592, by subsequent designation by Hampson, 1894, Fauna Br. India (Moths) 2: 546. Type(s), [India] India orientali (Type(s) lost, Zimsen, 1964, Type Material of I. C. Fabricius: 522).

OXYTHAPHORA Dyar, I9I7, Insecutor Inscit. menstr. 5: 50.

ACRO

Type-species: Oxythaphora delta Dyar, I917, ibidem 5: $5 \mathrm{I}$, by monotypy. Holotype o., BrazIL: Natal (USNM, IVashington).

OXYTHRES Druce, I900, Ann. Mag. nat. Hist. (7) 5: 5 I 7.

ACRO

Type-species: Oxythres splendens Druce, 1900, ibidem (7) 5:5 I7, by original designation. Syntypes $\widehat{\jmath}, q$, Colombia: Minca (BMNH).

‡OXYTRIPIA Staudinger, I87I. See Oxytrypia Staudinger, I871.

NOCT

OXYTRITA Warren, I913, in Seitz, Gross-Schmett. Erde 11: 246.

ACON

Type-species: Zagira bipars Hampson, 1907, J. Bombay nat. Hist. Soc. 17: 648, by original designation. Syntypes $\delta$, , , IndiA: Assam, Khasis (BMNH); N. Kanara, Karwar (BMNH).

The date of publication of the part of Seitz containing page 246 was stated on page $24 \mathrm{I}$ as 1913 September I 5 th in both the English and German editions.

OXYTRYPIA Staudinger, I871, in Staudinger \& Wocke, Cat. Lepid. enr. Faunengeb.: 98, 423.

NOCT

Type-species: Phalaena orbiculosa Esper, [1799], Die Schmett. 3 Abschnitt: 93, pl. 93, fig. 8, by monotypy. Type(s) ठే, Hungary: Szeged.

Oxytrypia was a correction, allowable under the Code, Article 32 (b), of †Oxytripia Staudinger, I $87 \mathrm{I}$. The original spelling on page 98 was changed in the errata on page 423 published at the same time and within the same covers.

$\ddagger$ OXYTRIPIA Staudinger, I871, in Staudinger \& Wocke, Cat. Lepid. eur. Famnengeb.: 98. An incorrect original spelling.

OZANA Berio, 1950, Annali Mus. civ. Stor. nat. Giacomo Doria 64: 156.

$\mathrm{ACON}$

Type-species: Metachrostis chinensis Leech, 1900, Trans. ent. Soc. Lond. 1900: 156,

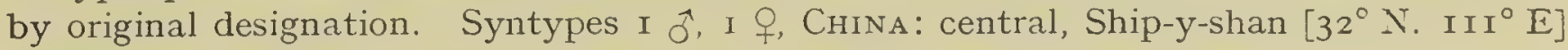
(BMNH). 
OZARBA Walker, r865, List Specimens lepid. Insects Colln Br. Mus. 32: 684.

ACON

Type-species: Ozarba punctigera Walker, 1865 , ibidem 32: 685, by monotypy. Syntypes $4(\hat{o}$, $)$, China (BMNH). [Australia]: [Qd], Moreton Bay.

OZOPTERYX Saalmüller, I891, Lepid. Madagascar: 423.

$\operatorname{ACON}$

Type-species: Ozopteryx basalis Saalmüller, r89r, ibidem: 424, by monotypy. Holotype $\widehat{\sigma}$, Madagascar: Nossi Bé I. (SNG, Frankfurt).

PACHETRA Guenée, r84I, Annls Soc. ent. Fr. 10:241.

HADE

Type-species: Noctua leucophaea [Denis \& Schiffermüller], 1775, Ankündung syst. Werkes Schmett. Wienergegend: 82, by monotypy, but included as leucophaea Bork[hausen] an incorrect authorship. Type(s), [AusrRIA]: Vienna district (Collection destroyed, Horn \& Kahle, 1936, Ent. Beih. Berl.-Dahlem 3: 243).

$N$. leucophaea is a junior subjective synonym of Phalaena sagittigera Hufnagel, 1766. Berlin. Mag. 3 (4): 4ro.

PACHNOBIA Guenée, 1852, in Boisduval \& Guenée, Hist. nat. Insectes (Lépid.) 5: 34I. Noct

Type-species: Pachnobia carnea Thunberg sensu Guenée, I852, ibidem 5: 342 [a misidentification already described as Noctua tecta Hübner, [I808], Samml. eur. Schmett. 4: pl. 8I, fig. 377], by subsequent designation by Grote, 1874, Bull. Buffalo Soc. nat. Sci. 2: I24, but cited as Noctua carnea Thunberg, the name included in Pachnobia, by Guenée. Type(s) of tecta Hübner, Europe.

$P$. carnea Thunberg sensu Guence has been long accepted as a misidentification of Noctua carnea Thunberg, x788. In fact, Guenée included tecta Hübner as a junior synonym of carnea Thunberg. Under the Code, Article 70 (a), this case should be referred to the Commission.

Incorrect type-species designation: Noctua rubricosa [Denis \& Schiffermüller], I775, a name not originally included in Pachnobia, and not linked with one of the originally included names when cited by Grote, 1874, ibidem 2: 23 .

PACHYAGROTIS Boursin, 1953, Z. wien. ent. Ges. 38: 212.

NOCT

Type-species: Episema ankarensis Rebel, (I93I) 1933, Annln naturh. Mus. Wien 46: 7, fig., pl. I, figs $\mathrm{r}, 2$, by original designation. Syntypes $\hat{\sigma}$, TuRkeY: Ankara (NM, Vienna).

PACHYCOA Hampson, igr6, in Poulton, Proc. zool. Soc. Lond. 1916: 109.

ACRO

Type-species: Pachycoa olivacea Hampson, I9I6, ibidem 1916: r Io, pl. I, fig. r2, by original designation. Holotype , , [Somalia] Somaliland: Mandera (BMNH).

PACHYDASYS Butler, I892, Proc. zool. Soc. Lond. 1892: I 25.

ACRO

Type-species: Eutelia consentanea Walker, [1863] I864, J. Proc. Linn. Soc. (Zool.) 7 : 68, by monotypy. Lectotype + , Borneo: Sarawak (UM, Oxford), designated (as type) by Swinhoe, I900, Cat. east. and Aust. Lepid. Heterocera 2: 38.

E. consentanea is a junior subjective synonym of Diethusa emiliusalis Walker, [1859].

See also Diethusa Walker, [I859].

PACHYGNATHESIS Hampson, r918, Novit. zool. 25: 156.

ACRO

Type-species: Pachygnathesis squamata Hampson, I9I8, ibidem 25: I56, by original designation. Holotype $\hat{\sigma}$, New Guinea: Papua, Louisiade Arch., Rossel I. (BMNH).

$\ddagger$ PACHYGNATHMA Hampson, no published reference found. HYPE

PACHYLEPIS Felder, I874. See Yepcalphis nom. n.

PACHYPLASTIS Felder, I874, in Felder \& Rogenhofer, Reise öst. Fregatte Novara (Zool.)

2 (Abt. 2): pl. 99; Erklärung: 7 .

OPHI

Type-species: Pachyplastis apicalis Felder, I874, ibidem 2 (Abt. 2): pl. 99, fig. 21 ; Erklärung: 7, by monotypy. Type(s) q, [BRAzIL]: R. Amazon (BMNH). 
PACHYPOLIA Grote, I874, Annual Rep. Trustees Peabody Acad Sci. 6: 27.

Type-species: Pachypolia atricornis Grote, 1874, ibidem 6:27, by monotypy. Type(s) o. [U.S.A.]: Illinois, Chicago (BMNH).

PACHYTHRIX Turner, I 942, Proc. R. Soc. Qd 53: 73.

ACRO

Type-species: Trachea smaragdistis Hampson sensu Hampson, I908, Cat. Lepid. Phalaenae Br. Mus. 7: I37, pl. II I, fig. 2 [a misidentification here named Pachythrix hampsoni sp. $\mathbf{n}$.$] , by original designation, as 'smaragdistis Hmps.'$

Holotype $\delta$. Australia: Queensland, Kuranda, I9 I I (Dodd) (BMNH).

Paratypes. Australia: 3 o, 3 우, Queensland, Kuranda (Dodd) (BMNH); 2 우, Queensland, Mackay (BMNH); I $\delta$, Queensland, Geraldton (Colln A. J. Turner, the specimen described and figured by Hampson).

Description of Pachythrix hampsoni sp.n., as for Trachea smaragdistis Hampson sensu Hampson, I908, ibidem 7: I37, pl. III, fig. 2. Very similar to Euplexia smaragdina Bethune-Baker, I906, Novit. zool. 13: 195, from New Guinea, but differs in distribution and in the postmedial fascia of the fore wing, almost straight anteriad in smaragdina but excurved in hampsoni.

See Frontispiece, fig. 7, Pachythrix hampsoni sp. n., holotype §;; fig. 8, Euplexia smaragdina Bethune-Baker, 0 .

Hampson, 1908, ibidem 7: I37, I 59, placed both E. smaragdina Bethune-Baker, I906, and Hadena smaragdina Neumoegen, I883, in the same genus Trachea Ochsenheimer, I8I6. E. smaragdina Bethune-Baker thus became a junior secondary homonym and Hampson proposed for it the objective replacement name Trachea smaragdistis Hampson, I908, ibidem 7: I37, pl. I I I, fig. 2, but actually described and figured an Australian species not conspecific with smaragdina Bethune-Baker. Warren, 1912, Novit. zool. 19: 21, noted this misidentification and proposed for smaragdina Bethune-Baker another objective replacement name Euplexia smaragdifera Warren, I9I2, so as to leave 'smaragdina [lapsus for smaragdistis] Hampson standing for the Australian [species]'. In doing this Warren was in error and should instead have named the misidentified Australian species described and figured by Hampson.

Trachea smaragdistis Hampson, objective replacement name for Euplexia smaragdina Bethune-Baker is here transferred to Pachythrix Turner (comb. n). Although E. smaragdina Bethune-Baker is thus no longer a junior secondary homonym, it was rejected as such prior to I96I and under the Code, Article 59(b)(i), is not to be restored as the valid name.

Pachythrix Turner has a misidentified type-species and under the Code, Article 70 (a), should be referred to the Commission.

PACIDARA Walker, I865, List Specimens lepid. Insects Colln Br. Mus. 33: 830.

EUTE

Type-species: Pacidara venustissima Walker, I865, ibidem 33: 831, by monotypy. Holotype ô. [South Africa]: Natal (BMNH).

$P$. venustissima is a junior subjective synonym of Caligatus angasii Wing, [1850].

See also Caligatus Wing, [1850].

PAECTES Hübner, 1818, Zuträge Samml. exot. Schmett. 1:21.

EUTE

Type-species: Paectes pygmaea Hübner, I \& 8, ibidem 1: 2I, figs I09, I Io, by monotypy.

Type(s) \%, [U.S.A.]: Georgia [formerly] in Florida.

$\ddagger$ TRIBONOPHORA Hübner, I808, Erste Zuträge Samml. exot. Schmett.: 5 .

Included in a work rejected for nomenclatural purposes by Int. Commn zool. nom., I966, Bull. zool. Nom. 23 Opinion 789:216. Placed on the Official Index of Rejected and Invalid Generic Names in Zoology: Name No. 1887.

Only included species: ${ }_{+}^{\dagger}$ Tribonophora py'gmaea Hübner, I808, later made nomenclaturally available as Paectes pygmaea Hübner, I8I8.

†PAESULA, misspelling. See Poesula Walker, 1858 .

OPHI 
PAETICA Walker, [1858] I857, List Specimens lepid. Insects Colln Br. Mus. 13: I I4I. ACRO Type-species: Phalaena eutropia Stoll, [1790] 1791, in Cramer, Uitlandsche Kapellen

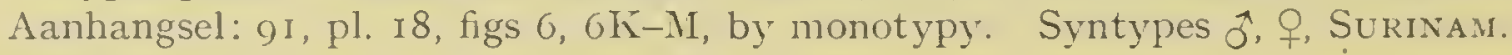

PAGETIA Prout, 1922. See Tegiapa nom. n. ACON

PAGYRA Scliaus, I90 I, Ann. Mag. nat. Hist. (7) 8:91.

OPHI

Type-species: Pagyra calgia Schaus, 1901, ibidem (7) 8: 91, by monotypy. Type(s), [Brazil]: Parana, Castro.

PAIDA Jordan, 1896, in Rothschild \& Jordan, Novit. zool. 3: 47.

AGAR

Type-species: Pais pulchra Trimen, i 863, Trans. ent. Soc. Lond. (3) 1:524, by original designation. Holotype , South WEST AFrICA: Damaraland.

Paida Jordan is not preoccupied by $\ddagger$ Paida; Mabille, 1878, Petites Nouv. Ent. 2: 274, an incorrect subsequent spelling of Paidia Huibner, [1819], - Lepid., Arctiidae.

$\ddagger$ PEIDA; Pagenstecher, 1909, Geogr. Verbreitung Schmett.: 423 .

An incorrect subsequent spelling.

PAIS Hübner, I821, Index exot. Lepid.: [4]. AGAR

Type-species: Phalaena decora Linnaeus, 1764, Mus. Ludovicae Ulricae: 382, by monotypy. Type(s), [Soutri AFrica]: [Cape of Good Hope] Cap. b. Spei (IZUU, Uppsala). ${ }_{t}^{+P A R I S}$; Pagenstecher, I go9, Geogr. I'erbreitung Schmett.: 423.

An incorrect subsequent spelling.

PALADA Smith, 1900, Proc. U.S. natn. Mus. 22: 480.

NOCT

Type-species: Palada scarletina Smith, 1900, ibidem 22:487, by monotypy. Syntypes 2 ô, I q. [U.S.A.]: California, San Diego County; Utah. (USNM, Washington).

PALAEAGROTIS Hampson, 1907, Ann. Mag. nat. Hist. (7) 19:252. Noct

Type-species: Hadena inops Lederer, i 853, Verh. zool.-bot. Ver. Wien 3: 371, pl. 3 , fig. 2, by original designation. Type(s), [L.S.S.R.]: Siberia.

PALAEAMATHES Boursin, 1954, Bonn. zool. Beitr. 5: 271.

NOCT

Type-species: Palaeamathes hoenei Boursin, I954, ibidem 5: 273, pl. 6, fig. 2, by original designation, but cited as $\ddagger P$. hönei, an incorrect original spelling. Holotype $\hat{o}$, CHina: N. Yünnan, Li-kiang (MAK, Bonn).

Under the Code, Article 32 (c) (i), the name hönei, being formed from a German personal name, must be corrected to hoenei.

PALAECHTHONA Hampson, 1926, Descr. new Genera Species Lepid. Phalaenae Subfamily Noctuinae Br. Muss.: 4.

OPHI

Type-species: Barcita hypoleuca Dognin, I914, Hétérocères nour. Am. Sud 8: 35, by original designation. Holotype ô. PERU: Angasmarca (USNM, Washington).

PALAEOPLUSIA Hampson, I913, Cat. Lepid. Phalaenae Br. MIus. 13: xiii, 581 . Plus

Type-species: Plusia venusta Walker, 1865, List Specimens lepid. Insects Colln Br. Mus. 33: 836, by original designation. Holotype q, Canada: [Ontario, Orillia] Orilla (BMINH).

PALAEOSAFIA Draudt, 1950, Mitt. münch. ent. Ges. 40: 154.

CATO

Type-species: Palcieosafia hoenei Draudt, 1950, ibidem 40: I5t, by monotypy, but cited as $¥ P$. hönei, an incorrect original spelling. Holotype q. CHiNa: [Yunnan Prov.], Li-kiang ( $M \wedge \mathrm{K}$, Bonn, note in collection 'Type verloren').

Under the Code, Article 32 (c) (i), the name hönei, being formed from a German personal name, must be corrected to hoenei.

PALAFRONTIA Hampson, igo8. See Amefrontia Hampson, i $899 . \quad$ ACro

$\ddagger$ PALARA, misspelling. See Palura Walker, 186 г.

$\operatorname{ACON}$ 
PALIMPSESTIS Hübner, [182I] I 816, Verz. behannter Schmett.: [237], but printed as 273.

THYATIRIDAE

Type-species: Phalaena octogesimea Hübner, I 786, Beitr. Gesch. Schmett. 1 (I): I3, pl. I, fig. G (but included by Hübner as ł'octogesima Hübn.', an incorrect subsequent spelling), by subsequent designation by Warren, I9r2, in Seitz, Gross-Schmett. Evde 2: 327 , but cited as 'ocularis L.' which Warren placed (on the same page) as the senior synonym of †octogesima.

Palimpsestis was originally proposed in the 'Noctuae'.

PALINDIA Guenée, I852, in Boisduval \& Guenée, Hist. nat. Insectes (Lépid.) 6: 274. OPHI

Type-species: Palindia hemileuca Guenée, I852, ibidem 6: 275, by subsequent designation by Dyar, I9I4, Proc. U.S. natn. Mus. 47: 96. Syntypes, Brazil (BMNH).

Incorrect type-species designation: Phalaena julianata Stoll, I787, cited by Butler, I892, Entomologist 25: 189, as '. . . : these are followed by the typical Palindiae, $P$. julianata and allies, .... This, under the Code, Article 67 (c), does not constitute a type-designation for Palindia Guenée, I 852 .

PALINDIONA Hampson, 1926, Descr. new Genera Species Lepid. Phalaenae Subfamily Noctuinae Br. Mus.: I 4 I.

OPHI

Type-species: Palindia micra Bar, 1876, Annls Soc. ent. Fr. 6: 433, pl. 7, fig. 25, by original designation. Type(s), French Guiana.

†PALINTHIS Hampson. See Lophophova Möschler, I89o.

HYPE

PALLACHIRA Grote, 1877, Can. Ent. 9: 197.

HYPE

Type-species: Pallachira bivittata Grote, I877, ibidem 9: 198, by monotypy. Holotype o. [U.S.A.]: [N.Y.], Buffalo (BMNH).

PALLUPERINA Hampson, I920. See Luperina Boisduval, I828.

ACRO

PALPANGULA Staudinger, I877, Stettin. ent. Ztg 38: 196.

OPHI

Type-species: Leucanitis henkei Staudinger, I877, ibidem 38: I96, by monotypy. Syntypes $3 \hat{0}, 2$ q, [U.S.S.R.]: steppe between Sarepta and Astrakhan (MNHU, Berlin).

Palpangula was proposed as a subgenus of Leucanitis Guenée, 1852 .

PALPONIMA Hampson, 1905, Cat. Lepid. Phalaenae Br. Mus. 5: xiii, 432.

HADE

Type-species: Semiophora orthosioides Butler, 1889, Illust. typical Specimens Lepid. Heteroceva Colln Br. Mus. 7: 10, 56, pl. 128, fig. I, by original designation. Syntypes, [InDIA]: [Himachal Pradesh], Mundi (BMNH).

PALTHIS Hübner, [1825] 1816, Verz. bekannter Schmett.: 342.

HYPE

Type-species: Pyralis angulalis Hübner, I796, Samml. eur. Schmett. 6: pl. I6, fig. I07, by monotypy. Type(s), TYPE-LOCALITY stated to be Europe, but this was probably an error as the species illustrated is not known to occur in the Old World but is widespread in U.S.A. and CANADA.

See also Mardava Walker, [1859].

CLANYMA Guenée, 1854, in Boisduval \& Guenée, Hist. nat. Insectes (Lépid.) 8: 94.

Type-species: Pyralis angulalis Hübner, I796, by subsequent designation by Desmarest, (1857), in Chenu, Encycl. Hist. nat. Papillons nocturnes: 219, but cited as \$angularis Hübner, an incorrect subsequent spelling.

Clanyma is a junior objective synonym of Palthis Hübner, [1825].

PALTHISOMIS Schaus, I916, Proc. U.S. natn. Mus. 50: 263, 344.

HYPE

Type-species: Herminia baresalis Walker, 1859, List Specimens lepid. Insects Colln $B r$. Mus. 19: 859, by original designation. Type(s) Jै, [BRAzIL]: Rio Janeiro (UM, Oxford).

PALURA Walker, 186r, List Specimens lepid. Insects Colln Br. Mus. 23: 950. Available, but without included species until Walker, I862, ibidem 24: Iogo (as ł'Palara'). ACON

Type-species: Palura implexata Walker, J862, ibidem 24: I090 (but proposed as AA 
¥Palara', an incorrect subsequent spelling), by monotypy. Lectotype $q$, Borneo: Sarawak (UM, Oxford), designated (as type) by Swinhoe, I900, Cat. east. and Aust. Lepid. Heterocera 2: 69 .

${ }_{\ddagger} P A L A R A$; Walker, I862, List Specimens lepid. Insects Colln Br. Mus. 24: Iogo.

An incorrect subsequent spelling.

PALYNA Guenée, I852, in Boisduval \& Guenée, Hist. nat. Insectes (Lépid.) 7: 387. HYPE

Type-species: Palyna semilunaris Guenée, 1852 , ibidem 7: 388, by subsequent designation by Berio, 1966, Annali Mus. civ. Stor. nat. Giacomo Doria 76: 63. Holotype [French Guiana]: Cayenne.

†AMILLA, misspelling. See Panilla Moore, [1885].

OPHI

PANARENIA Schaus, I9I6, Proc. U.S. natn. Mus. 50: 261, 303.

HYPE

Type-species: Panarenia subhirsuta Schaus, I9I6, ibidem 50:303, by original designation. Type(s) ô, Peru: Santo Domingo (USNM, Washington).

PANCHRYSIA Hübner, [I82I] I8I6, Verz. bekannter Schmett.: 252.

PLUS

Type-species: Noctua aurea Hübner, [1803], Samml. eur. Schmett. 4: pl. 59, fig. 288, by monotypy. Type(s), Europe.

$N$. aurea is a junior subjective synonym of Phalaena deaurata Esper, 1787, Die Schmett. 4 (I): pl. IIo, fig. 6; I790, ibidem: 203.

PANCRA Swinhoe, 1902, Ann. Mag. nat. Hist. (7) 9: 177.

HYPE

Type-species: Pancra sordida Swinhoe, I902, ibidem (7) 9: 178 , by monotypy. Type(s) §. [SUNDA Is]: Lombok [I.] (BMNH).

†PANCROIDES Prout, no published reference found.

HYPE

PANDESMA Guenée, I852, in Boisduval \& Guenée, Hist. nat. Insectes (Lépid.) 6: 438 . OPHI

Type-species: Pandesma quenavadi Guenée, I 852, ibidem 6: 438, pl. I5, fig. 3, by subsequent designation by Moore, [1885] I887, Lepid. Ceylon 3: 9I. Syntypes 2 . [Bangladesh]: [E. Bengal], Silhet (BMNH).

PANEMERIA Hübner, [I823] I8I6, Verz. bekannter Schmett.: 262.

ACRO

Type-species: Noctua heliaca [Denis \& Schiffermüller], I 775, Ankündung syst. Werkes Schmett. Wienergegend: 94, by monotypy. Type(s), [Austria]: Vienna district (Collection destroyed, Horn \& Kahle, I936, Ent. Beih. Berl.-Dahlem 3: 243).

$N$. heliaca is a junior subjective synonym of Phalaena tenebrata Scopoli, 1763 , Ent. Carniolica: 230.

See also Heliodes Guenée, I84I.

PANHEMERIA Spuler, 1907, in Hofmann, Schmett. Eur. 1: 279.

An unjustified emendation of Panemeria Hübner, [1823].

GYMNOPA Stephens, I829, Illust. Br. Ent. (Haustellata) 3: I Io.

Type-species: Noctua heliaca [Denis \& Schiffermüller], 1775, by monotypy.

A junior homonym of Gymnopa Fallén, I820, Oscinides Sveciae: Io,-Diptera. The objective replacement name is Panemeria Hübner, [I 823].

PANGLIMA Moore, I 858. See Eligma Hübner, [1819].

CHLO

PANGRAPTA Hübner, 1818, Zuträge Samml. exot. Schmett. 1: 18.

OPHI

Type-species: Pangrapta decoralis Hübner, I 8I 8, ibidem 1 : I 8, figs 91 , 92, by subsequent designation by Grote, I874, Bull. Buffalo Soc. nat. Sci. 2: 47. 'Type(s) ô. [U.S.A.]: Georgia [formerly] in Florida.

See also Marmorinia Guenée, I 852.

PANGRAPTELLA Hampson, 1926, Descr. new Genera Species Lepid. Phalaenae Subfamily Noctuinae Br. Muss.: 599.

OPHI 
Type-species: Pangrapta herbitecta Dyar, 1913, Proc. U.S. natn. Mus. 44: 302, by original designation. Syntypes 2 , Mexico: Misantla and Orizaba. (USNM, Washington).

PANGRAPTICA Wileman \& South, I92 I, Entomologist 54:273. EUTE

Type-species: Pangraptica albistigma Wileman \& South, I92I, ibidem 54: 273, by monotypy. Syntypes I $\hat{\text { Ò. I }}$ q, Philippines: Luzon I., Rizal, Manila (BMNH).

Pangraptica was used and unintentionally made nomenclaturally available by Wileman \& South prior to its proposal and generic description by Hampson for a concept having a type-species in a different subfamily.

PANGRAPTICA Hampson, I926. See Craptignapa nom. n.

OPHI

PANHEMERIA Spuler, I907. See Panemeria Hübner, [1823].

ACRO

PANILLA Moore, [1885] I887, Lepid. Ceylon 3: 93.

OPHI

Type-species: Homoptera dispila Walker, I865, List Specimens lepid. Insects Colln Br. Mus. 33: 89o, by original designation. Holotype ơ. TyPE-LOCality not known (BMNH) [INDIA. CEYLON].

$\ddagger P A M I L L A$; Neave, 1940, Nomencl. zool. 3: 543 .

An incorrect subsequent spelling. Neave attributed a misspelling to Bethune-Baker, 1906, Novit. zool. 13: 25I, when no error had been made.

$\ddagger P$ ANIOLA Boisduval. See Plotheia Walker, [I 858$]$ SARR

PANOLIS Hübner, [1821] I8I6, Verz. bekannter Schmett.: 214. HADE

Type-species: Noctua flammea [Denis \& Schiffermüller], I775, Ankündung syst. Werkes Schmett. Wienergegend: 87, by subsequent designation by Hampson, 1905, Cat. Lepid. Phalaenae Br. Mus. 5: 46r. Type(s), [Austria]: Vienna district (Collection destroyed, Horn \& Kahle, 1936, Ent. Beih. Berl.-Dahlem 3: 243).

See also Ilanus Boisduval, I 828.

PANOPODA Guenée, 1852, in Boisduval \& Guenée, Hist. nat. Insectes (Lépid.) 7: 324 . OPHI Type-species: Panopoda rubricosta Guenée, I852, ibidem 7: 324, by subsequent designation by Grote, I874, Bull. Buffalo Soc. nat. Sci. 2: 44, but cited as rufimargo Hübner, which Grote placed as the senior synonym of rubricosta Guenée. Holotype $\hat{\delta}$. NORTH AMERICA (BMNH).

P. rubricosta is a junior subjective synonym of Phobevia rufimargo Hübner, 181 8 , Zuträge Samml. exot. Schmett. 1: I 3, figs $45,46$.

PANSEMNA Turner, I920, Trans. R. Soc. S. Aust. 44: I40, I 42. ACRO

Type-species: Diphthera beryllodes Turner, 1903, ibidem 27: 5, by monotypy. Holotype $\hat{0}$. Australia: Qd, Dulong, Blackall Range.

PANTHAUMA Staudinger, r 892, in Romanoff, Mém. Lépid. 6: 404.

OPHI

Type-species: Panthauma egregia Staudinger, I 892, ibidem 6: 404, pl. 7, fig. 2, by monotypy. Holotype +, [U.S.S.R.]: Amur district, Baranowka (MNHU, Berlin).

PANTHEA Hübner, [1820] I 816, Vevz. bekannter Schmett.: 203.

PANT

Type-species: Phalaena coenobita Esper, I785, Die Schmett. 3: 196, pl. 37, fig. 7, by monotypy. Holotype + , [GERMANY].

ELATINA Duponchel, [1845] i844, Cat. méth. Lépid. Eur.: 98.

Type-species: Phalaena coenobita Esper, $\mathrm{x} 785$, by monotypy.

Elatina is a junior objective synonym of Panthea Hübner, [1820].

DIPHTHERA Hübner sensu Hampson, I913, Cat. Lepid. Phalaenae Br. Mus. 13: 365.

Hampson attributed Diphthera to Treitschke and based it on an incorrect type-species, $P$. coenobita Esper.

PANTURA Moore, [1885] I887, Lepid. Ceylon 3: 247.

OPHI

Type-species: Bocana ophiusalis Walker, [1859] I858, List Specimens lepid. Insects Colln Br. Mus. 16: I73, by original designation. Syntypes 3 q, CEYLON (BMNH). 
PANTYDIA Guenće, 1852, in Boisduval \& Guenée, Hist. nat. Insectes (Lépid.) 6: $436 . \quad$ OPHI Type-species: Pantydia sparsa Guenée, 1852, ibidem 6:437, by subsequent designation by Joannis, 1929, Annls Soc. ent. Fr. 98: 367. Lectotype ô, TYPE-LOCALITY not known (MNHN, Paris), designated by Viette, 1951, Bull. mens. Soc. linn. Lyon 20: I61. [Australia].

PANULA Guenée, 1852, in Boisduval \& Guenée, Hist. nat. Insectes (Lépid.) 7: 59.

OPHI

Type-species: Panula inconstans Guenée, 1852 , ibidem 7:59, pl. I3, fig. 9, by subsequent designation by Grote, 1874, Bull. Buffalo Soc. nat. Sci. 2: 39. Syntypes, North America (BMNH).

PAONIDIA Meyrick, 1902, Trans. ent. Soc. Lond. 35: 38.

HYPE

Type-species: Paonidia pentaptila Meyrick, 1902, ibidem 35: 39, by monotypy. Holotype ơ, Australia: N.S.W., Richmond River (BMNH).

PAPAIPEMA Smith, I899, Trans. Am. ent. Soc. 26: 12.

ACRO

Type-species: Gortyna cerina Grote, 1874, Proc. Acad. nat. Sci. Philad. 1874: 200, by subsequent designation by Hampson, r9 10, Cat. Lepid. Phalaenae Br. Mus. 9: 6o. Lectotype q. [U.S.A.]: Kansas (BMNH), designated (as type) by Hampson, r9Io, ibidem 9: 64 .

PAPESTRA Sukhareva, 1973, Ent. Obozr. 52: $409 . \quad$ HADE

Type-species: Phalaena biren Goeze, r 78r, Ent. Beyträge 3 (3): 206, by original designation. Type(s), TyPE-Locality not stated [Europe].

$P$. biven was originally proposed as 'bi-ren'. Under the Code, Article 26(a), the component words must be united without a hyphen.

Papestra was proposed as a subgenus of Mamestra Ochsenheimer, I8I6.

PAPUACOLA Hampson, I926, Descr. new Genera Species Lepid. Phalaenae Subfamily Noctuinae Br. Mus.: 406.

OPHI

Type-species: Papuacola lignicolor Hampson, 1926, ibidem: 407, by original designation. Holotype , New Guinea: [West Irian], Fak-fak (BMNH).

PARABARROVIA Gibson, 1920, Rep. Can. arct. Exped. I9I3-18, 3 (I): $33 . \quad$ Noct Type-species: Parabarrovia keelei Gibson, I920, ibidem 3 (1): 33, pl. 5, figs 9, 10, by original designation. Holotype $\delta^{2}$ CANADA: N.W. Territories, near Gravel river, Mt. below Twitya river.

PARABASIS Bethune-Baker, r904, Novit. zool. 11:428. Notodontidae

Type-species: Parabasis pratti Bethune-Baker, 1904, ibidem 11:429, pl. 4, fig. 26, by original designation.

Parabasis was originally placed in the Noctuidae.

PARABR YOPIILA Dyar, 1921, Insecutor Inscit. menstr. 9: 99.

ACRO

Type-species: Bryophilopsis edobasis Dyar, 1920, ibidem 8: 190, by monotypy (of Bryophilopsis Dyar, 1920). Holotype 9, Mexico: Cuernavaca (USNM, Washington).

Parabryophila was proposed as the objective replacement name for Bryophilopsis Dyar, I 920.

BRYOPHILOPSIS Dyar, 1920, Insecutor Inscit. menstr. 8: I9o.

Type-species: Bryophilopsis edobasis Dyar, r920, by monotypy.

A junior homonym of Bry'ophilopsis Hampson, i 894, Fauna Br. India (Moths) 2: 36 I, Lepid., Noctuidae. The objective replacement name is Parabryophila Dyar, r92r.

PARACAREA Bethune-Baker, r9o6, Novit. zool. 13: $245 . \quad$ CHLo

Type-species: Paracarea rubiginea Bethune-Baker, I906, ibidem 13: 246, by original designation. Type(s) + N New Guinea: [Papua], Dinawa (BMNH).

PARACAROIDES Kenrick, ig17, Trans. ent. Soc. Lond. 1917: 89.

ACRO

Type-species: Paracaroides pratti Kenrick, 1917, ibidem 1917: 89, pl. r, by monotypy. 
Lectotype + , MadaGascar (BMNH), designated (as holotype) by Viette, 1967, Faune Madagascar 20:675.

PARACARSIA Hampson, 1926, Descr. nev Genera Species Lepid. Phalaenae Subfamily Noctuinae Br. Mus.: 450 .

OPHI

Type-species: Paracarsia antitermina Hampson, 1926, ibidem: 45I, by original designation. Holotype 9 , BrazIL: Rio de Janeiro (BMNH).

PARACELIPTERA Draudt, 1940, in Seitz, Gross-Schmett. Erde 7: 445.

Type-species: Celiptera codo Dyar, 1912, Proc. U.S. natn. Mus. 42: 79, by original designation. Holotype $\hat{\delta}$, Mexico: Tehuacan (USNM, Washington).

The date of publication of the part of Seitz containing page 445, was stated on page 445 as I940 May 23rd in the German edition. Owing to the second World War the English edition of this part was not published.

PARACENTROPUS Boursin, I958, Bull. mens. Soc. linn. Lyon 27: 6.

ACRO

Type-species: Scythocentropus cyrus Brandt, 1938, Ent. Rdsch. 55: 523, figs 92-95. by original designation. Syntypes $\sigma^{t}$, Iran: Fort Sine-Sefid (NR, Stockholm).

PARACHABORA Warren, I 889, Trans. ent. Soc. Lond. 1889: 245.

Type-species: Noctua abydas Herrich-Schäffer, I869, Samml. neuer oder wenig bekannter aussereut. Schmett. 2: 4, fig. 565, by monotypy. Type(s), Venezuela.

PARACHAEA Hampson, 1926, Descr. new Genera Species Lepid. Phalaenae Subfamily Noctuinae Br. Mus.: 104.

OPHI

Type-species: Phalaena macaria Cramer, I 777, Uitlandsche Kapellen 2: 48, pl. I29, fig. D, by original designation. Type(s), Surinam.

$P$. macaria is dated from the wrapper of the part and not from the title-page of the volume, I 779 .

PARACHALCIOPE Hampson, I9I3 [January], Cat. Lepid. Phalaenae Br. Mus. 12: 9. Available but without included species until Hampson, I9I3 [November], ibidem 13: vii, 35.

CATO

Type-species: Fodina euclidicola Walker, 1858, List Specimens lepid. Insects Colln Br. Mus. 14: 1 438, by subsequent designation by Hampson, I9 13, ibidem 13: 35. Holotype + . West Africa (BMNH).

PARACODIA Druce, I909, Ann. Mag. nat. Hist. (8) 3: 465.

$\operatorname{ACON}$

Type-species: Paracodia albiceps Druce, I909, ibidem (8) 3: 465, by monotypy. Type(s) $\hat{0}$. Colombia: Valparaiso (BMNH).

Druce used this generic name without any indication that it was new, even though his new species described on the same page were marked sp. n. The generic name was probably obtained from Hampson as an identification and was unintentionally made nomenclaturally available by Druce prior to Hampson's generic description for a concept having a different type-species.

PARACODIA Hampson, igio. See Cadiorapa nom. n.

$\triangle C O N$

PARACOERIA Hampson, 1926, Descr. new Genera Species Lepid. Phalaenae Subfamily Noctuinae Br. Mus.: 236. OPHI

Type-species: Rivula orobena Druce, I891, Biologia cent.-am. (Zool.) Lepid. Heterocera 1: 444 , pl. 36 , fig. 8 , by original designation. Syntypes 3 ex, Panama: Chiriqui; Volcan de Chiriqui (BMNH).

PARACOLAX Hübner, [1825] I816, Verz. bekannter Schmett.: 344.

HYPE

Type-species: Pyralis derivalis Hübner, I 796, Samml. eur. Schmett. 6: pl. 3, fig. I9, by subsequent designation by Westwood, I840, Synopsis Genera Br. Insects: I05. Type(s), EUROPE. 
PARAHERMINIA Richards, 1932, Ent. News 43: I88.

Type-species: Pyralis derivalis Hübner, I 796, by original designation.

Paraherminia is a junior objective synonym of Paracolax Hübner, [1 825].

PARACRAMA Moore, I884, Lepid. Ceylon 3: 54.

Type-species: Nolasena dulcissima Walker, [1863] 1864, J. Proc. Linn. Soc. (Zool.)

7: 76, by monotypy. Lectotype ?, Borneo: Sarawak (UMI, Oxford), designated (as type) by Swinhoe, 1900, Cat. east. and Aust. Lepid. Heterocera 2: 590.

PARACRETONIA Dyar, I9I2, Proc. ent. Soc. Wash. 14: I67.

ACON

Type-species: Paracretonia xithon Dyar, I912, ibidem 14: I67, by monotypy. Syntypes 3 ex., [U.S.A.]: Calif., San Diego, La Puerta Valley (USNM, Washington).

PARACROMA Dognin, I9I4, Hétérocères nouv. Am. Sud 8: 73.

HYPE

Type-species: Paracroma zamora Dognin, I914, ibidem 8: 74, by original designation. Holotype §., EcUador: Vallée de la Zamora.

PARACRORIA Hampson, 1908, Cat. Lepid. Phalaenae Br. Mus. 7: 8. Available, but without included species until Hampson, I909, ibidem 8: x, 28I. ACRO

Type-species: Xanthoptera griseocincta Hampson, 1902, Ann. S. Afr. Mus. 2: 396, by

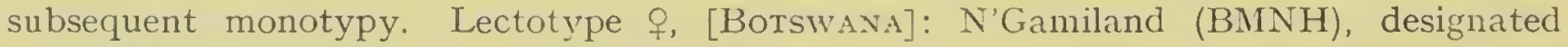
(as type) by Hampson, I909, ibidem 8: 282.

PARACULLIA Köhler, (1952) I951, Acta zool. lilloana 12: I 59.

CUCU

Type-species: Paracullia burmeisteri Köhler, (1952) I951, ibidem 12: I60, by original designation. Syntypes $\hat{0}$, 우 Argentina: Santa Cruz [Prov.], Rio del Fondo (ZSBS, Munich); Chubut [Prov.], Comodoro Rivadavia (ZSBS, Munich).

$\ddagger$ PARADELTA Hampson, no published reference found.

HY'PE

PARADIARSIA McDunnough, [1929] 1928, Bull. Dep. Mines, Can. 55: 48.

NOCT

Type-species: Agrotis littoralis Packard, 1867, Proc. Boston Soc. nat. Hist. 11: 36, by original designation. Syntypes $\hat{\sigma},[\mathrm{CANADA}]$ : Labrador, Str. of Belle I., Caribou I.

PARADIOPA Prout, I928, Sarawak Mus. J. 3: 464.

ACRO

Type-species: Paradiopa parthenia Prout, I 928, ibidem 3: 465, pl. I 5, fig. 5, by original designation. Holotype o, Borneo: Sarawak, Mt Dulit (BMNH).

PARADRINA Boursin, 1937, Ent. Rdsch. 54: 421 .

ACRO

Type-species: Caradrina selini Boisduval, I840, Genera Index meth. Eur. Lepid.: 137, by original designation. Type(s), [SwitzerLand]: [Valais] Valesia.

Paradrina was proposed as a subgenus of Elaphria Hübner, 1818, but has since been placed as a subgenus of Caradrina Ochsenheimer, i 816.

PARAEGLE Hampson, I908, Cat. Lepid. Phalaenae Br. Mus. 7: 4. Available, but without included species until Hampson, I910, ibidem 9: xiii, 379.

ACro

Type-species: Metoponia ochracea Ershov, 1874, in Fedchenko, Reise in Turkestan 2 (5) 3 : 53, pl. 3, fig. 52, by subsequent monotypy. Syntypes ${ }_{0}$, 오. [U.S.S.R.]: Turkestan, Kisil-kum desert (ZI, Leningrad).

PARAEGOCERA Hampson, I9o I, Cat. Lepid. Phalaenae Br. Mus. 3: xviii, 606.

AGAR

Type-species: Aegocera confluens Weymer, i 892, Stettin. ent. Ztg 53: roo, by original designation. Holotype $q$, [TANZANIA]: Zanzibar.

PARAFODINA Hampson, 1926, Descr. new Genera Species Lepid. Phalaenae Subfamily Noctuinae Br. Mus.: 204. OPHI

Type-species: Grammodes conjungens Walker, 1858, List Specimens lepid. Insects Colln Br. Mus. 14: 1446, by original designation. Holotype d. TYPE-Locality not known (BMNH). [Related species from the Indo-Australian Region]. 
PARAGABARA Hampson, 1926, Descr. new Genera Species Lepid. Phalaenae Subfamily Noctuinae Br. Mus.: 260.

OPHI

Type-species: Madopa flavomacula Oberthür, I880, Étud. Ent. 5: 87, pl. 4, fig. 5, by original designation. Syntypes $3 \hat{0}$, [U.S.S.R.]: near Vladivostok, Askold I.

PARAGARISTA Bethune-Baker, I906, Novit.zool. 13: 255.

CATO

Type-species: Paragarista albostriata Bethune-Baler, 1906, ibidem 13: 255, by original designation. Type(s) ô, New Guinea: [Papua], Aroa River (BMNH).

PARAGONA Staudinger, I892, in Romanoff, Mém. Lépid. 6: 557.

OPHI

Type-species: Acidalia multisignata Christoph, I88I, Bull. Soc. Nat. Moscou 55 (3): 50, by monotypy. Syntypes 5 đ̃. [U.S.S.R.]: Amur, Pompejefka (ZI, Leningrad).

PARAGONITIS Bethune-Baker, 1906, Novit. zool. 13: 238.

OPHI

Type-species: Paragonitis strigocrenulata Bethune-Baker, I906, ibiden 13: 239, by original designation. Type(s) ô, New Guinea: [Papua], Kebea Range (BMNH).

PARAGRIA Hampson, 1926, Descr. new Genera Species Lepid. Phalaenae Subfamily Noctuinae Br. Mus.: I 47 .

OPHI

Type-species: Paragria sesamiodes Hampson, I926, ibidem: I47, by original designation. Holotype $\hat{\sigma}$, [South Africa]: Natal, Durban (BMNH).

PARAGROTIS Dyar, I 902, Bull. U.S. natn. Mus. 52: I 40.

NOCT

Type-species: Carneades moerens Grote, 1883, Can. Ent. 15: 4, by monotypy (of Carneades Grote, I883). Type(s) ô, [U.S.A.]: Arizona.

Paragrotis was proposed as the objective replacement name for Carneades Grote, i 883; but Dyar attributed Paragrotis to F. C. Pratt, and this has been followed by Neave, 1940, Nomencl. zool. 3: 570 .

CARNEADES Grote, I883, Can. Ent. 15: 4.

Type-species: Carneades moerens Grote, I883, by monotypy.

A junior homonym of Carneades Bates, 1869, Trans. ent. Soc. Lond. 1869: 387,Coleoptera. The objective replacement name is Paragrotis Dyar, I902.

PARAHERMINIA Richards, 1932. See Paracolax Hübner, [1825).

HYPE

PARAHYPENODES Barnes \& McDunnough, i918, Contr. nat. Hist. Lepid. N. Am. 4: I23.

HYPE

Type-species: Parahypenodes quadralis Barnes \& McDunnough, rgr8, ibidem 4: I24, pl. 18 , fig. 6 , by original designation. Syntypes 3 s. [CANADA]: Que., St. John's Co., St. Thérèse Isl.

PARALECTRA Hampson, 1926, Descr. New Genera Species Lepid. Plialaenae Subfamily Noctuinae Br. Mus.: I6.

OPHI

Type-species: Ypsia exaggerata Schaus, 1914, Proc. U.S. natn. Mus. 46: 505, by original designation. Holotype ô. [GuYana]: Essequebo River, Rockstone (USNM, Washington).

Dognin in I9I4, used and made nomenclaturally available the generic name Parelectra prior to the proposal and generic description by Hampson of Paralectra, differing by one letter. Both generic names are used for a similar concept but have different type-species. Hampson's unpublished manuscript shows that he regarded these two species as congeneric.

PARALEPHANA Hampson, 1926, Descr. new Genera Species Lepid. Phalaenae Subfamily Noctuinae Br. Mus.: I50.

OPHI

Type-species: Paralephana poliotis Hampson, I926, ibidem: I $5 \mathrm{I}$, by original designation. Holotype ô, MadaGascar: Betsileo (BMNH).

PARALLELIA Hübner, i 8 18, Zuträge Samml. exot. Schmett. 1 : I 5.

CATO

Type-species: Parallelia bistriaris Hübner, I8 18, ibidem 1 : I 5 , figs 63,64 , by monotypy. Type(s) 오 [U.S.A.]: Georgia [formerly] in Florida. 
†ASCALAPHA Hübner, i 808, Erste Zuträge Samml. exot. Schmett. : 4-6.

Included in a work rejected for nomenclatural purposes by Int. Commn zool. Nom., 1966, Bull. zool. Nom. 23 Opinion 789: 213. Placed on the Official Index of Rejected and Invalid Generic Names in Zoology: Name No. I 828.

No type-species has been designated. The first of the five originally included species was $\ddagger A$. bistra Hübner, 1808 , which was later made nomenclaturally available as Parallelia bistriaris Hübner, 1818 .

PARALLELURA Berio, 1954, Boll. Soc. ent. ital. 84: 24.

CATO

Type-species: Ophiusa palumbioides Hampson, 1902, Ann. S. Afr. Mus. 2: 34I, by original designation. Syntypes, [South Africa]: Natal, Durban (BMNH).

PARALOPHA Bethune-Baker, I908, Novit. zool. 15: 204.

OPHI

Type-species: Paralopha rubiginea Bethune-Baker, 1908, ibidem 15: 204, by original designation. Holotype , NEw Guinea: [Papua], Owgarra (BMNH).

Hampson, I912, Cat. Lepid. Phalaenae Br. Mus. 11: I42, placed Paralopha in the Stictopterinae. It is here transferred to the Ophiderinae and placed next to Dinumma Walker, I $85^{8}$.

PARALOPHATA Bethune-Baker, I9I I, Ann. Mag. nat. Hist. (8) 8: 5 I 5.

Type-species: Paralophata ansorgei Bethune-Baker, I9I I, ibidem (8) 8: 5I6, by original designation. Syntypes $\hat{\alpha}$,, , [ANGoLA $]$ : N'Dalla Tando (BMNH).

PARAMATHES Boursin, 1954, Bonn. zool. Beitr. 5: 276.

NOCT

Type-species: Agrotis perigrapha Püngeler, 1900, Dt. ent. Z. Iris 12: 290, pl. 8, fig. Io, by original designation. Syntypes 20, I $q$, [CHINA]: [Tsinghai], Kuku-Noor (MNHU, Berlin).

PARAMIANA Barnes \& Benjamin, 1924, Contr. nat. Hist. Lepid. N. Am. 5: i 54 acro Type-species: Hadena laetabilis Smith, 1899, Can. Ent. 31: 263 (a primary homonym), by original designation. Syntypes $3 \hat{0}$, [U.S.A.]: New Mexico, Santa Fé.

$H$. laetabilis Sinith is a junior primary homonym of Hadena laetabilis Zetterstedt, I839, Insecta Lapponica: 940. There is no synonym to use as a replacement name so I here propose Paramiana perissa nom. n. for $H$. laetabilis Smith.

PARAMIMETICA Warren, I889, Trans, ent. Soc. Lond. 1889: 235.

HYPE

Type-species: Paramimetica subrufa Warren, I889, ibidem 1889: 235, by subsequent designation by Schaus, igi6, Proc. U.S. natn. Mus. 50:368. Syntypes I o. 4 f, [BraziL]: Amazon basin, R. Purus, Juruena and R. Jura (BMNH).

$P$. subrufa is a junior subjective synonym of Physula phtisialis Guenée, I 854.

See also Mursa Walker, I 859 , and Sisputa Möschler, I 890.

PARAMOCIS Roepke, I948, Tijdschr. Ent. 89: 225.

CATO

Type-species: Paramocis maculata Roepke, 1948, ibidem 89: 225, pl. 13, fig. 23, by original designation. Holotype 9 , Sumatra.

PARANAGIA Hampson, 1926, Descr. new Genera Species Lepid. Phalaenae Subfamily Noctuinae Br. MTus.: 64 .

OPHI

Type-species: Catephia rufostrigata Bethune-Baker, 1906, Novit. zool. 13: 251, by original designation. Holotype ô, NEw Guinea: [Papua], Ekeikei (BMNH).

PARANATAELIA Draudt, I935, Ent. Rdsch. 52: 192.

ACro

Type-species: Anataelia orotavae Draudt, 1933, ibidem 50: 169, pl. 2, fig. 9, by original designation (for Anataelia Draudt, 1933). Holotype ô. [CANARY Is]: Teneriffe, Orotava.

A. orotavae is a junior subjective synonym of Eumichtis tenerifica Hampson, 1906, Cat. Lepid. Phalaenae Br. Mus. 6: 326, pl. 108, fig. 5 .

Paranataelia was proposed as the objective replacement name for Anataelia Draudt, 1933. 
ANATAELIA Draudt, 1933, Ent. Rdsch. 50: 168.

Type-species: Anataelia orotavae Draudt, 1933, by original designation.

This genus was originally proposed as $\ddagger$ Anataëlia, an incorrect original spelling which must be corrected under the Code, Article 32 (c).

A junior homonym of Anataelia Bolivar, i 899, Actas Soc. esp. Hist. nat. 1899: 97, Dermaptera. The objective replacement name is Paranataelia Draudt, I935.

$\ddagger$ PARANGILIA Druce, I909. See Parangitia Druce, I909.

$\operatorname{ACON}$

PARANGITIA Druce, I909, Ann. Mag. nat. Hist. (8) 3: 463.

$A C O N$

Type-species: Parangitia veluta Druce, Igog, ibidem (8) 3: 463 , by subsequent designation by Collins, I962, ibidem (13) 5: I23. Type(s) $\widehat{\jmath}$, Peru: La Oroya, Carabaya (BMNH).

Druce used this generic name without any indication that it was new, even though his new species described on the same page were marked sp. n. The generic name was probably obtained from Hampson as an identification and was used and unintentionally made nomenclaturally available by Druce before Hampson's generic description was published.

Parangitia was the spelling adopted by Hampson, I9ro, as first reviser under the Code, Article 32 (b), from a multiple original spelling.

Parangitia was used by Druce as the generic name for the first of the included species and $\ddagger$ Parangilia was used for the other three.

$\ddagger$ PARANGILIA; Druce, I909, Ann. Mag. nat. Hist. (8) 3: 463 .

An incorrect original spelling.

PARANGITIA Hampson, i9io, Cat. Lepid. Phalaenae Br. Mus. 10: 335.

Type-species: Parangitia cervina Hampson, I910, ibidem 10: 337, fig. roo, by original designation. Syntypes I $\hat{\sigma}, \mathrm{I} q$, [Guyana] Br. Guiana: Rockstone (BMNH).

A junior homonym of Parangitia Druce, I909,-Lepid., Noctuidae. There is no objective replacement name but $P$. cervina is congeneric with Pavangitia veluta Druce, the type-species of Parangitia Druce, 1909. The latter is therefore available for use as a subjective replacement name.

PARANGITIA Hampson, I9Io. See Parangitia Druce, I909.

$\operatorname{ACON}$

PARANICLA Köhler, 1959, Revta Soc. ent. Argent. 21: Iоз.

NoCT

Type-species: Agrotis pesronii Guenée, 1852, in Boisduval \& Guenée, Hist. nat. Insectes (Lépid.) 5: 282, by original designation. Holotype ô. [CHrLe]: Magellan's Strait (MNHN, Paris).

PARANOLA Son, 1933, Ann. Transv. Mus. 15: 222.

NOLI

Type-species: Paranola bipartita Son, I933, ibidem 15: 222, pl. 3, fig. 2, pl. 7, fig. 2, pl. 8, fig. I 8, by original designation. Holotype ô, [Mozanibigue] Portuguese E. Africa: Magude (TM, Pretoria).

PARANORATHA Bethune-Baker, 1908, Novit. zool. 15: 240.

HYPE

Type-species: Paranoratha fusca Bethune-Baker, I908, ibidem 15: 240, by original designation. Type(s) $\hat{\sigma}$, NEw Guinea: [Papua], Aroa River (BMNH).

PARANYMPHA Butler, I879. See Oxidercia Hübner, [I825].

OPHI

PARAPADNA Hampson, 1926, Descr. new Genera Species Lepid. Phalaenae Subfamily Noctuinae Br. Mus. : 192.

OPHI

Type-species: Anachrostis zonophora Turner, 1908, Trans. R. Soc. S. Aust. 32: 7I, by original designation. Holotype $\widehat{\delta}$. Australia: N. Qd, Kuranda (ANIC, Canberra).

PARAPAMEA Bird, 1927, Jl N.Y. ent. Soc. 35: i 81.

ACRO

Type-species: Ochria buffaloensis Grote, 1877, Can. Ent. 9: 88, by original designation. Lectotype , , [U.S.A.]: [N.Y.], Buffalo (BMNH), designated (as type) by Hampson, rgro, Cat. Lepid. Phalaenae Br. Mus. 9: 33. 
${ }_{\ddagger}$ PARAPLUSIA Mukerji \& Krishnamorthy, 1955. See Macdunnoughia Kostrowicki, I96r.

PLUS

PARARCTE Hampson, 1926, Descr. new Genera Species Lepid. Phalaenae Subfamily Noctuinae Br. Mius.: 49.

Type-species: Phalaena schneideriana Stoll, 1780 , in Cramer, Uitlandsche Kapellen 4: 37. pl. 308, fig. A, by original designation. Type(s) Surinam.

$P$. schneideviana is dated from the wrapper of the part and not from the title-page of the volume, 1780 .

PARARGIDIA Hampson, 1926, Descr. new Genera Species Lepid. Phalaenae Subfamily Noctuinae Br. Mus.: $46 \mathrm{I}$.

OPHI

Type-species: Orthogramma vacillans Walker, 1858 , List Specimens lepid. Insects Colln Br. Mus. 15: I555, by original designation. Holotype ․, [BRAzIL]: [R. Amazon], [Tefé] Ega (BMNH).

PARASADA Hampson, 1910, Cat. Lepid. Phalaenae Br. Mus. 10: xi, 281.

ACON

Type-species: Lycauges carnosa Hampson, I893, Illust. typical Specimens Lepid. Heterocera Colln Br. Muss. 9: 40, I 50, pl. I69, fig. 9, by original designation. Lectotype q, CEYLON: Kelani Valley (BMNH), designated (as type) by Hampson, I91o, ibidem 10:282.

PARASCOTIA Hübner, [1825] I816, V'erz. bekannter Schmett.: 314.

OPHI

Type-species: Geometra carbonaria [Denis \& Schiffermüller], 1775, Ankündung syst. Werkes Schmett. Wienergegend: I08, by subsequent designation by Warren, I913, in Seitz, Gross-Schmett. Erde 3: 399, but cited as P. fuliginaria Linnaeus which Warren placed as the senior synonym of carbonaria Esper, an incorrect authorship. Type(s), [AusTRIA]: Vienna district (Collection destroyed, Horn \& Kahle, 1936, Ent. Beih. Berl.-Dahlem 3: 243).

G. carbonaria is a junior subjective synonym of Phalaena fuliginaria Linnaeus, 1761, Fauna Suecica (Edn 2): 327.

BOLETOBIA Boisduval, I 8,o, Genera Index meth. Eur. Lepid.: 201.

Type-species: Geometra carbonaria [Denis \& Schiffermüller], I775, by monotypy.

Boletobia is a junior objective synonym of Pavascotia Hübner, [1825].

BOLITOBIA Agassiz, I 846 , Nomencl. zool. Index univl.: 48.

An unjustified emendation of Boletobia Boisduval, i 840 .

PARASIOPSIS Bethune-Baker, 191 I, Ann. Mag. nat. Hist. (8) 8: 536.

HYPE

Type-species: Parasiopsis arcuata Bethune-Baker, I9I I, ibidem (8) 8: 537, by original designation. Type(s) Ô, Avgola: N’Dalla Tando.

PARASTENOPTERYGIA Berio, I955, Mém. Inst. scient. Madagascar (E) 6: I I 4. ACRo

Type-species: Parastenopterygia viettei Berio, I955, ibidem (E) 6: I I 4, fig. 4, by original designation. Holotype ô, Madagascar: Ankarafantsika, Ampijoroa (MNHN, Paris).

PARASTICHTIS Hübner, [1821] 1816, Verz. bekannter Schmett.: 213.

CUCU

Type-species: Noctua suspecta Hübner, [1817], Samml. eur. Schmett. 4: pl. I 38, fig. 633, by subsequent designation by Grote, I9oo, Can. Ent. 32: 212. Type(s), Europe.

PARASTICTUS Agassiz, I846, Nomencl. zool. Index univl.: 274.

An unjustified emendation of Parastichtis Hübner, [1821].

DYSCHORISTA Lederer, I 857 , Noctuinen Eur.: 39, 143.

Type-species: Noctua suspecta Hübner, [1817], hy subsequent designation by Grote, 1895, Abh. naturw. Ver. Bremen 14: 94.

Dyschorista is a junior objective synonym of Parastichtis Hübner, [1821].

PARASTICTUS Agassiz, 1846. See Parastichtis Hübner, [1821].

CUCU

${ }_{+}^{+} \boldsymbol{P A R A T A D A}$ Hampson, no published reference found.

HYPE 
PARATHERMES Hampson, I902, Ann. S. Afr. Mus. 2: 368.

OPHI

Type-species: Renodes nigriceps Walker, 1858, List Specimens lepid. Insects Colln Br. Mus. 15: I595, by original designation. Holotype ô. Sierra Leone (BMNH).

$R$. nigriceps is a junior subjective synonym of Ophiusa marchalii Boisduval, I833, Nouv. Annls Mus. Paris 2: 253, pl. I3, fig. 4.

PARA TOLNA Aurivillius, 1925, Ergebn. zweit. Dt. zentr.-Afr. Exped. I9I0-I9I 1 1: I320. CAto Type-species: Paratolna brunneovittata Aurivillius, 1925, ibidem 1: I321, fig. 78 , by monotypy. Holotype + , W. Africa: Fernando Poo I.

PARA TRACHEA Hampson, I908, Cat. Lepid. Phalaenae Br. Mus. 7: ix, 2 I 6.

ACRO Type-species: Valeria laches Druce, I889, Biologia cent.-am. (Zool.) Lepid. Heterocera 1: 292, pl. 27, fig. 20, by original designation. Holotype 9 , Panama: Volcan de Chiriqui (BMNH).

PARATUERTA Hampson, I902, Ann. S. Afr. Mus. 2: 282. AGAR Type-species: Paratuerta marshalli Hampson, 1902, ibidem 2: 282, by monotypy. Holotype , , [RHodesia]: Mashonaland, Salisbury (BMNH).

PARAUCHMIS Holland, I897, in Smith, Through unknown African Countries: 4I 4. NOTODONTIDAE

Type-species: Parauchmis smithii Holland, I897, ibidem: 4I 4, fig. 2, by monotypy.

Parauchmis was originally placed in the Noctuidae.

PARAXESTIA Hampson, 1903, Cat. Lepid. Phalaenae Br. Mus. 4: xix, 601.

NOCT

Type-species: Amphipyra flavicaudata Warren, 1888, Proc. zool. Soc. Lond. 1888: 3I2, by original designation. Syntypes 8 ㅇ, India: [Punjab], Abbottabad, Thundiani $(\mathrm{BMNH})$.

PARAXESTIS Hampson, I902, Ann. S. Afr. Mus. 2: 3 I 7.

CHLO

Type-species: Paraxestis rufescens Hampson, 1902, ibidem 2: 3I7, by monotypy. Holotype ô., South Africa: Natal, Durban (BMNH).

$\ddagger P A R E X I S T I S$; Grünberg, I9I2, Avch. Naturgesch. 77 (4) 2: I42.

An incorrect subsequent spelling.

PARAXIA Möschler, r89o, Abh. senckenb. naturforsch. Ges. 16: I 20.

SARR

Type-species: Paraxia chamaeleon Möschler, I89o, ibidem 16: I2I, by monotypy. Syntypes, Puerto Rico.

$P$. chamaeleon is a junior subjective synonym of Sarrothripus nilotica Rogenhofer, I882 (but proposed in $\ddagger$ Sarothripa, an incorrect subsequent spelling), Verh.zool.-bot. Ges. Wien 31 Sber.: 26.

PARCA Saalmüller, I89i, Lepid. Madagascar: 486.

ACRO Type-species: Parca inusitata Saalmüller, I89I, ibidem: 487, fig. I99, by monotypy. Holotype, Madagascar: N.W., Nossi-Bé I (SNG, Frankfurt).

Parca was originally placed in the 'Herminiidae'; it is here transferred to the Acronictinae.

PARDASENA Walker, I866, List Specimens lepid. Insects Colln Br. Mus. 35: I730. SARR Type-species: Pardasena acronyctella Walker, I866, ibidem 35: I730, by subsequent designation by Hampson, I912, Cat. Lepid. Phalaenae Br. Mus. 11: 245, but cited as roeselioides which Hampson placed (on page 247 ) as the senior synonym of acronyctella. Holotype , Sierra LeONE (BMNH).

$P$. acronyctella is a junior subjective synonym of Acontia roeselioides Wallier, I858, ibidem 15: 1760 .

$\ddagger P A R S A D E N A$; Legrand (I966) 1965, Mém. Mus. natn. Hist. nat. Paris (Zool.) 37: I4I.

An incorrect subsequent spelling. 
PARELECTRA Dognin, 1914, Hétérocères nonv. Am. Sud 8: 32.

OPH I

Type-species: Parelectra homochroa Dognin, I9I t, ibidem 8: 32, by monotypy. Holotype ô. Colombia: San Antonio (USNM, Washington).

The generic name Parelectra probably originated from Hampson but was used and unintentionally made nomenclaturally available by Dognin prior to the proposal and generic description of Paralectra Hampson, 1926, (differing by one letter) for a similar concept but having a different type-species. An unpublished manuscript by Hampson showed that he regarded these two species as congeneric.

PARELIA Berio, 1957, Annali Miıs. civ. Stor. nat. Giacomo Doria 69: 79.

EUTE

Type-species: Parelia albivirgula Berio, 1957, ibidem 69: 79, fig. 6, pl. I, figs 5-7, by original designation. Holotype $\widehat{\jmath}$, Madagascar: E., Dist. Ifanadiana, dist. de Ranomafana (MNHN, Paris).

PARELYDNA Bethune-Baker, 1906, Novit. zool. 13: 24 I.

CHLO

Type-species: Parelydna mirabilis Bethune-Balier, I906, ibidem 13: 242 , by original designation. Syntypes $\hat{0}$,, , New Guinea: [Papua], Ekeikei (BMINH).

PAREPISPARIS Bethune-Baker, I906, Novit. zool. 13: 269.

GEOMETRIDAE

Type-species: Parepisparis crenulata Bethune-Baker, I906, ibidem 13: 269, by original designation.

Parepisparis was originally proposed in the Noctuidae.

PARERASTRIA Warren, 1914, Novit. zool. 21: 407.

$\operatorname{ACON}$

Type-species: Parerastria castaneata Warren, I9I4, ibidem 21: 407, by original designation. Holotype $\hat{\sigma}$, [AUstralia]: Queensland, Mackay (BMNH).

PAREUPLEXIA Warren, I91 I, in Seitz, Gross-Schmett. Erde 3: i87.

ACRO

Type-species: Naenia chalybeata Moore, 1867, Proc. zool. Soc. Lond. 1867: 64, by original designation. Type(s) +, India: Bengal (BMNH).

The date of publication of the part of Seitz containing page I87, was stated on page r $8 \mathrm{I}$ as I9I I February Sth in both the German and the English editions.

PAREUXOA Forbes, 1933, Entomologica am. 14:27.

NOCT

Type-species: Noctua lineifera Blanchard, 1852, in Gay, Historia de Chile 7: 76, by original designation. Syntypes, Chile: Coquimbo (MNHN, Paris).

PAREUXOINA Köhler, 195t, Revia Soc. ent. argent. 17: 35.

NOCT

Type-species: Pareuxoina microstigmoides Köhler, 1954, ibidem 17: 36, figs I, 2, by monotypy. Holotype ô. Argentina: [Buenos Aires Prov.], Balcarce (ZSBS, Munich).

PAREXARNIS Boursin, 1946, Revue fr. Lépidopt. 10: I87.

NOCT

Type-species: Agrotis sollers Christoph, (1877) i 876, Horae Soc. ent. ross. 12: 200, 245 , pl. 6, fig. 19, by original designation. Syntypes $\hat{0}$. + , [Iran] Persia: [Elburz Mts], Shahrud (ZI, Leningrad).

${ }_{+}$PAREXISTIS, misspelling. See Paraxestis Hampson, I902.

CHLO

PARHYLOPHILA Hampson, 1912, Cat. Lepid. Phalaenae Br. Mus. 11 : xiv, 514.

CHLO

Type-species: Chloephora celsiana Staudinger, I887, in Romanoff, Mém. Lépid. 3: I77, pl. ro, fig. I, by original designation. Syntypes 2 ô. [U.S.S.R.]: Amur district, Raddefka and Chingan (MNHU, Berlin).

C. celsiana was proposed in $¥$ 'Chloeophora', an incorrect subsequent spelling.

PARHYPENA Bethine-Baker, I908, Novit. zool. 15: 273.

IIY PE

Type-species: Parhypena albopunctata Bethune-Baker, 1908, ibidem 15: 237, by original designation. Type(s) $\vec{\sigma}$, New Guinea: [Papua], Dinawa (BMNH). 
PARIAMBIA Hampson, igo8, Cat. Lepid. Phalaenae Br. Mus. 7 : 9, 66o.

ACRO Type-species: Pariambia aprepes Hampson, I908, ibidem 7:660, fig. I69, by original designation. Holotype $\sigma \overrightarrow{0}, \mathrm{CEYLON}$ : Willawaya (BMNH).

P. aprepes is a junior subjective synonym of Acontia pulla Swinhoe, 1885, Proc. zool. Soc. Lond. 1885: 456, pl. 27, fig. I 5.

PARILYRGIS Bethune-Baker, 1908, Novit. zool. 15: 204.

OPHI

Type-species: Parilyrgis concolor Bethune-Baker, I908, ibidem 15: 205, by original designation. Type(s) $\overrightarrow{0}$, NEw Guinea: [Papua], Dinawa and Aroa River (BMNH).

$\ddagger$ PARIS, misspelling. See Pais Hübner, I821.

AGAR

PAROLIGIA Warren, I9I3, in Seitz, Gross-Schmett. Erde 11: I28.

ACRO

Type-species: Erastria pallidisca Moore, I88 I, Proc. zool. Soc. Lond. 1881: 372, pl. 37, fig. $1_{4}$, by original designation. Syntypes $\sigma^{*}$, 우, [India]: [WV. Bengal], Darjiling.

The date of publication of the part of Seitz containing page 128 was stated on page I 2 I as 1913 August 5 th in both the German and the English editions.

PAROLULIS Hampson, 1926, Descr. new Genera Species Lepid. Phalaenae Subfamily Noctuinae Bv. Mus.: 477 .

OPHI

Type-species: Zethes renalis Moore, [1885] I887, Lepid. Ceylon 3: I94, pl. I72, fig. I2, by original designation. Syntypes, $\hat{\sigma}, \hat{q}$, CEYLon (BMNH).

PAROMIA Schaus, I9I3. See Ramopia nom. n.

HYPE

PAROMPHALE Hampson, 1908, Cat. Lepid. Phalaenae Br. Mus. 7: 8. Available, but without included species until Hampson, I909, ibidem 8: xii, $42 \mathrm{I}$ ACRO

Type-species: Caradrina caeca Swinhoe, 1902, Ann. Mag. nat. Hist. (7) 9: 42I, by subsequent monotypy. Syntypes $4^{8} \overrightarrow{0}, 69$ ․, Australia: W. Aust., Roebourne (BMNH).

PARORA Smith, I9oo, Proc. U.S. natn. Mus. 22: 49I.

OPH I

Type-species: Parora texana Smith, I900, ibidem 22: 49I, by monotypy. Syntypes 3 ㅇ, [U.S.A.]: Texas, Brownsville and San Diego and Corpus Christi. (USNM, Washington).

$\ddagger$ PARORAGROTIS, misspelling. See Porosagrotis Smith, I89o.

NOCT

PARORENA Hampson, I926, Descr. new Genera Species Lepid. Phalaenae Subfamily . Voctuinae Br. Mus.: 330.

OPHI

Type-species: Parorena sminthochroa Hampson, 1926, ibidem: 330, by original designation. Holotype $\widehat{\delta}$, Mexico: Guadalajara (BNNH).

PARORUZA Hampson, I902, Ann. S. Afr. Mus. 2: 406.

$\mathrm{ACON}$

Type-species: Acidalia subductata Walker, I86I, List Specimens lepid. Insects Colln Br. Mus. 23: 790, by monotypy. Lectotype ô., [South AfriCA]: Cape (BMNH), designated (as type) by Hampson, I910, Cat. Lepid. Phalaenae Br. Mus. 10: 273.

PAROSMIA Köhler, (1952) I95 I, Acta zool. lilloana 12: I62.

CUCU

Type-species: Parosmia simulans Köhler, (1952) I951, ibidem 12: 162, by original designation. Syntypes $\hat{\sigma}, \hat{+}$, Argentina: Chubut [Prov.], Comodoro Rivadavia (ZSBS, Munich).

PAROTHRIA Hampson, igoi, Cat. Lepid. Phalaenae Br. Muts. 3: xix, 654.

AGAR

Type-species: Othria ecuadorina Westwood, 1877, Trans. Linn. Soc. Lond. (2) 1: 202, pl. 29, fig. 23, by monotypy. Type(s), Ecuador (UM, Oxford).

$\ddagger$ PARSADENA, misspelling. See Pardasena Walker, I 866 .

SARR

PARTHENOS Hübner, [1823]. See Euparthenos Grote, I876. 
PARVAPENNA Ĺaye, I90I, Trans. ent. Soc. Lond. 1901: I29.

Type-species: Parvapenna sentalis Kaye, I90I, ibidem 1901: I 29, pl. 6, fig. 7, by original designation. Lectotype $\hat{j}$. TRINIDAD: Tabaquite (BMINH), designated (as type) by Hampson, I9 1о, Cat. Lepid. Phalaenae Br. Mus. 9: 3 го.

PARYRIAS Hampson, 1926, Descr. new Genera Species Lepid. Phalaenae Subfamily Noctuinae Br. Mus.: I 9.

OPHI

Type-species: Paryrias stygia Hampson, 1926, ibidem: 19, by original designation. Holotype ô, Guatemala: S. Geronimo (BMNH).

PASIPEDA Walker, i 858, List Specimens lepid. Insects Colln Br. Mus. 15: I794.

OPHI

Type-species: Pasipeda rufipalpis Walker, I858, ibidem 15: I795, by monotypy. Holotype $q$, Ceylon (BMNH).

PASIPEDA Moore, I882. See Macaldenia Moore, [1885].

CATO

PASIRA Moore, I882. See Rivula Guenée, [1845].

OPHI

PASTONA Walker, i 858, List Specimens lepid. Insects Colln Br. Mus. 15: 1754. HAdE

Type-species: Pastona rudis Walker, I 858 , ibidem 15: I754, by monotypy. Type(s)

․, BraziL (UM, Oxford).

See also Maguza Walker, [1866].

PATAETA Walker, I858, List Specimens lepid. Insects Colln Br. Mus. 15: I 748.

EUTE

Type-species: Pataeta conspicienda Walker, I858, ibidem 15: I748, by monotypy. Holotype ? (not ô as stated), [AUstralia]: [Qd], Moreton Bay (BMNH).

$P$. conspicienda is a junior subjective synonym of Phlegetonia carbo Guenée, 1852 , in Boisduval \& Guenée, Hist. nat. Insectes (Lépid.) 6: 302.

PATULA Guenée, I852. See Eupatula Ragonot, i 895.

CATO

PAUROPHYLLA Turner, I902, Proc. Linn. Soc. N.S.W. 27: I 24.

HYPE

Type-species: Paurophylla aleuropasta Turner, I902, ibidem 27: I24, by monotypy. Holotype +, Australia: Qd, Brisbane (ANIC, Canberra).

PAUROSCELES Turner, I945, Mem. Qd Mus. 12: I 56.

ACON

Type-species: Paurosceles geminipuncta Turner, 1945, ibidem 12: I56, by monotypy. Holotype ô., Australia: Qd, Cape York.

PAVENTIA Rossi, 1958, Physis B. Aires 21: 42.

ACON

Type-species: Asaphes asemantica Turner, 1945, Mem. Qd Mus. 12: 155, by monotypy

(of Asaphes Turner, 1945). Holotype, Australia: Qd, Cape York (QMI, Brisbane).

Paventia was proposed as the objective replacement name for Asaphes Turner, 1945. ASAPHES Turner, 1945, Mem. Qd Mus. 12: 155.

Type-species: Asaphes asemantica Turner, I945, ibidem 12: I 55, by monotypy.

A junior homonym of Asaphes Waller, i 834, Ent. Mag. 2: I5I,-Hymenoptera. The objective replacement name is Paventia Rossi, 1958.

PECHIPOGO Hübner, [1825] i 816, Verz. bekannter Schmett.: 345. HYPE

Type-species: Pyralis pectitalis Hübner, I796, Samml. eur. Schmett. 6 : pl. 19, fig. I22, by subsequent designation by Warren, I9I3, in Seitz, Gross-Schmett. Erde 3 : 42 I, but cited as ' $P$. barbalis Cl.' which on page 422 was placed as the senior synonym of $P$. pectitalis. Type(s), Europe.

$P$. pectitalis is a junior subjective synonym of Phalaena (Geometra) strigilata Linnacus, I 758, Sy'st. Nat. (Edn ro) 1:528. LECTOTYPE + , Europe, pin-label data 'strigilata' (LS, London), here designated [examined].

Invalid type-species designation: P. pectitalis was cited by Grote, r 896, Proc. Am. phil. Soc. $34: 424$ as 'unknown to me, may be the type'. A designation made in a qualified manner is invalid uncler the Code, Article 67(c). 
Incorrect type-species designation: Phalaena barbalis Clerck, I 759 (but cited as $P$. barbalis L., an incorrect authorship), designated by Westwood, I840, Synopsis Genera Br. Insects: 105, for ¥'Pechipogon Hb.' an incorrect subsequent spelling. One of the species originally included by Hübner in Pechipogon was cited as 'Pechipogo Plumigeralis. Barbalis Hübn. Pyr. I 8'. 'This species is distinct from P. barbalis Clerck, so the designation by Westwood is of a non-included species; neither was it synonymized with one of the originally included species.

$\ddagger$ PECHIPOGON; Stephens, I834, Illust. Br. Ent. (Haustellata) 4: 14.

An incorrect subsequent spelling, also used by Westwood, I840, Synopsis Genera Br. Insects: 105.

PECH YPOGON Agassiz, i 846, Nomencl. zool. Index univl.: 275 .

An unjustified emendation of Pechipogo Hübner, [1 825].

$\ddagger E R P Y Z O N$ Hübner, [1806], Tentamen determinationis digestionis . . . [2].

Included in a work rejected for nomenclatural purposes by Int. Commn zool. Nom., 1926, Smithson. misc. Collns 73 (4) Opinion 97: I9. Also idem, r954, Opin. Decl. int. Commn zool. Nom. 6 Opinion 278: I 40.

Only included species Pyralis barbalis Clerck sensu Hübner, r796, later made nomenclaturally available as Pechipogo plumigeralis Hübner, [1825].

There is no objective replacement name available for $\ddagger$ Erpyzon. P. plumigevalis is close to but probably not congeneric with $P$. pectitalis Hübner. Both species belong to a complex of Holarctic genera and species which require further systematic study.

†PECHIPOGON, misspelling. See Pechipogo Hübner, [1825).

HYPE

PECHYPOGON Agassiz, I 846. See Pechipogo Hübner, [1825].

HYPE

PECTINIFERA Berio, r964, Boll. Soc. ent. ital. 94: 89.

OPHI

Type-species: Pectinifera sypnaesimilis Berio, r964, ibidem 94: 89, by original designation. Holotype $\hat{O}$. [ZAIRE] Congo: Lusambo (MRAC, Tervuren).

PEDIARCHA Turner, I936, Proc. R. Soc. Od 47: 37.

ACON

Type-species: Pediarcha singularis Turner, 1936, ibidem 47: 37, by monotypy. Holotype, Australia: Qd, Cunnamulla (QM, Brisbane).

$\ddagger$ PEIDA, misspelling. See Paida Jordan, I 896 .

AGAR

PELAMIA Guenée, I 852, in Boisduval \& Guenée, Hist. nat. Insectes (Lépid.) 7: $286 . \quad$ Cato Type-species: Pelamia phasianoides Guenée, I852, ibidem 7: 286, pl. 22, fig. 8, by monotypy. Syntypes $\hat{0}$,,+ , [Uruguay]: Montevideo. Paraguay.

†PELOMIA; Warren, I913, in Seitz, Gross-Schmett. Erde 3: 332, 498.

An incorrect subsequent spelling in the German edition, but correctly spelled in the English edition.

PELECIA Hübner, I82 I, Index exot. Lepid.: [6].

OPHI

Type-species: Idia retusalis Hübner, [I 8I 8], Samml. exot. Schmett. 1: pl. [2I I], figs I-4. Syntypes $\hat{\sigma}, q$, TYPE-LOCALITY not stated [BRaZIL].

PELIALA Walker, r 865, List Specimens lepid. Insects Colln Br. Mus. 33: 1005.

HYPE

Type-species: Peliala tenebrosa Walker, I865, ibidem 33: 1005, by monotypy. Holotype ô. VenEzUela (BMNH).

PELODESIS Hampson, I926, Descr. new Genera Species Lepid. Phalaenae Subfamily Noctuinae Br. Mus.: 379.

OPHI

Type-species: Pelodesis viridifera Hampson, r926, ibidem: 380 , by original designation. Holotype ㅇ, Panama: La Chorrera (BMNH).

PELODIA Schaus, r9r3, Ann. Mag. nat. Hist. (8) 11: 5 .

Type-species: Pelodia rava Schaus, I9I3, ibidem (8) 11: 6, by original designation. Type(s) ơ, Costa Rica: Guapiles. 
${ }_{\ddagger}^{\ddagger}$ PELOMIA, misspelling. See Pelamia Guenée, 1852.

PENDINA Treitschke, I 829, Schmett. Eur. 7: 227.

Type-species: Tortrix salicana [Denis \& Schiffermüller], 1775, Ankündung syst. Werkes Schmett. Wienergegend: I3I, by subsequent designation by Duponchel, I834, in Godart \& Duponchel, Hist. nat. Lépid. Papillons Fr. 9: 2 I , but cited for Penthina Treitschke, I 830, an unjustified emendation.

T. salicana is a junior subjective synonym of Phalaena salicella Linnaeus, 1758, Syst. Nat. (Edn Io) 1: 536 .

The citation by Duponchel is acceptable as a type-species designation as it is contained in the continuation of a layout in which Duponchel stated, in the same work 7 (2): 102, that the species so cited were the types of genera.

PENTHINA Treitschke, 1830, Schmett. Eur. 8: 21.

An unjustified emendation of Pendina Treitschke.

Penthina has been used in the Noctuidae based on the incorrect type-species Phalaena revayana Scopoli, 1772, designated by Boisduval, 1836, Hist. nat. Insectes (Lépid.) 1: 137.

PENICILLARIA Guenćc, 1852, in Boisduval \& Guenéc, Hist. nat. Insectes (Lépid.) 6: 302.

EUTE

Type-species: Penicillaria nugatrix Guenée, 1852, ibidem 6: 303, pl. 14, fig. 7, by subsequent designation by Desmarest, (1857), in Chenu, Encycl. Hist. nat. Papillons nocturnes: 122. Syntypes f, Indis: central.

PENISA Warren, I91 I, in Seitz, Macrolepid. World 3: 260.

ACON

Type-species: Hyria oblataria Walker, I86r, List Specimens lepid. Insects Colln Br. Mus. 22: 665, by original designation. Syntypes 2 ㅇ, CEylon: Colombo (BMNH).

The date of publication of the part of Seitz containing page 260, was stated on page 253 as I9I October $15^{\text {th }}$ in the English edition and as 1912 January Ioth in the German edition.

PENNALTICOLA Berio, 1973, Annali Mus. civ. Stor. nat. Giacomo Doria 79 : i66. ACon

Type-species: Pennalticola rectangulum Berio, I973, ibidem $\mathbf{7 9}$ : 166 , fig. 56, by original designation. Holotype ô. BUrma: N.E., Kambaiti, $2000 \mathrm{~m}$ (NR, Stockholm).

PENTACLONIA Felder, I86I, Sber. Akad. Wiss., Wien 43 (1): 35.

CHLO

Type-species: Pentaclonia uniformis Felder, I 86I, ibidem 43 (I): 36, by monotypy. Type(s) ô. [Moluccas]: Amboina [I.].

Systematic position uncertain. Placed by Kirby, 1892, Synonymic Cat. Lepid. Heterocera 1: 285, in Cymbidae [Chlocphorinac]. Placed by Hampson in Tincidae, according to a manuscript note in Hampson's copy of Kirby's catalogue.

PENTHETRIA Edwards, i881. See Tantura Kirby, i 892.

$\triangle \mathrm{CON}$

PENTHINA Treitschke, i8zo. See Pendina Treitschke, I829.

OLETHREITIDAE

PENZA Walker, [1863] i 864, J. Proc. Limn. Soc. (Zool.) 7: 56.

HADE

Type-species: Penza puncticeps Walker, [1863] 1864, ibidem 7: 56, by monotypy. Lectotype ô. Borneo: Sarawak (UM, Oxford), designated (as type) by Swinhoe, igoo, Cat. east. and Aust. Lepid. Heterocera 2: 38.

PEOSINA Gucnće, I 852, in Boisduval \& Guenée, Hist. nat. Insectes (Lépid.) 7: i $31 . \quad$ opru

Type-species: Phalaena leontia Stoll, 1790 , in Cramer, Uitlandsche Kapellen Aanhangsel: I 55, pl. 34, fig. 6, by subsequent designation by 1)esmarest, (I 857), in Chenu, Encycl. Hist. nat. Papillons nocturnes: I zo. Type(s), Brazil: Rio Janeiro.

PEPERITA Hampson, 1910, Cat. Lepid. Phalaenae Br. Mus. 10: x, 202.

$\triangle \operatorname{CON}$

'Type-species: Erastroides molybdopasta Turner, 1908, Trans. R. Soc. S. Aust. 32: 6n, by original designation. Holotype q, Austral1a: N. Qd, Kuranda (ANIC, Canberra). 
PERANIA Barnes \& McDunnough, i910. See Pevaniana Strand, 1942.

ACRO

PERANIANA Strand, I942, Folia zool. hydrobiol. 11: 390.

ACRO

Type-species: Perania dissociata Barnes \& McDunnough, I91о, Can. Ent. 42: 249, by original designation (for Pevania Barnes \& McDunnough, I9Io). Syntypes 2 q, [U.S.A.]: Utah, Provo.

Peraniana was proposed as the objective replacement name for Perania Barnes \& McDunnough, I910.

PERANIA Barnes \& McDunnough, i9io, Can. Ent. 42: 249.

Type-species: Perania dissociata Barnes \& McDunnough, I91o, by original designation.

A junior homonym of Perania Thorell, i89o, Annali Mus. civ. Stor. nat. Genova 28: 3I 5, - Arachnida. The objective replacement name is Peraniana Strand, I942.

PERANUA Berio, 1959, Annali Mus. civ. Stor. nat. Giacomo Doria 71: 3 i6. Cato

Type-species: Achaea conspicienda Walker, 1858 , List Specimens lepid. Insects Colln Br. Mus. 14: I 392, by original designation. Holotype 9 , West Africa (BMNH).

PERASIA Hübner, [1823] I816, Verz. bekannter Schmett.: 268.

CATO

Type-species: Phalaena ora Cramer, I776, Uitlandsche Kapellen 1: 139, pl. 88, fig. B, by monotypy. Type(s), Type-Locality not stated. [Guyana. Venezuela. Trinidad. $P$. ora is dated from the wrapper of the part and not from the title-page of the volume, I 779 .

Pevasia Hübner had three originally included species of which two, $\ddagger P$. ule Hübner, [1823], and $\ddagger P$. strigillaris Hübner, [1823], were nomina nuda.

PERATA Swinhoe, I9I9, Ann. Mag. nat. Hist. (9) 4: I27.

HYPE

Type-species: Adrapsa curiosalis Swinhoe, 1905, ibidem (7) 15: 162, by original designation, but cited as ' $P$. curiosalis Swinhoe, I895, ibidem (6) 15: I6', which is an incorrect reference. Syntypes $\delta$, 우, [IND1A]: [Assam], Khasia Hills (BMNH).

$\ddagger$ PERATOPHAEA Hampson, no published reference found.

HYPE

${ }_{+}^{+}$PERATOSEMA Hampson, no published reference found.

HYPE

PERCALPE Berio, 1956, Memorie Soc. ent. ital. 35: i 10.

OPHI

Type-species: Calpe canadensis Bethune, 1865, Proc. ent. Soc. Philad. 4: 213, by original designation. Type(s) $\hat{o}$, CANADA: C.W., London.

PERCIANA Walker, I865, List Specimens lepid. Insects Colln Br. Mus. 33: 8г 2.

HYPE

Type-species: Perciana marmorea Walker, i865, ibidem 33: 8г 3, by monotypy. Lectotype $\hat{\jmath}$, [INDIA]: Hindostan (UM, Oxford), designated (as type) by Swinhoe, I9oo, Cat. east. and Aust. Lepid. Heterocera 2: 50.

PERICONTA Dyar, I912, Proc. U.S. natn. Mus. 42: 66.

ACRO

Type-species: Periconta obliqua Dyar, 1912, ibidem 42: 66, by original designation. Holotype $\delta$, Mexico: Tehuacan (USNM, Washington).

PERICYMA Herrich-Schäffer, [I85I] I 845, Syst. Bearb. Schmett. Eur. 2: $429 . \quad$ CATo

Type-species: Acidalia albidentaria Freyer, I842, Neueve Beitr. Schmett. 4: II5, pl. 354, fig. I, by monotypy. Type(s), [U.S.S.R.] : steppe.

PERIDROMA Hübner, [I82I] 18I6, Verz. bekannter Schmett.: 227.

NOCT

Type-species: Noctua saucia Hübner, [I8o8], Samml. eur. Schmett. 4: pl. 8I, fig. 378, by subsequent designation by Butler, I889, Trans. ent. Soc. Lond. 1889: 380. Type(s), EUROPE.

PERIGEA Guenée, 1852, in Boisduval \& Guenée, Hist. nat. Insectes. (Lépid.) 5: 225. ACRo Type-species: Perigea xanthioides Guenée, I 852 , ibidem 5: 227 , by subsequent designa$\mathrm{BB}$ 
tion by Grote, I874, Bull. Buffalo Soc, nat. Sci. 2: 16. Syntypes ơ, ㅇ, [U.S.A.]: Florida (BMNH).

${ }_{+}$PERIGIA; Smith, 1894, Trans. Am. ent. Soc. 21:60.

An incorrect subsequent spelling.

PERIGEODES Hampson, I908, Cat. Lepid. Phalaenae Br. Mus. 7: x, 287.

ACRO

Type-species: Orthosia rectivitta Moore, I88I, Proc. zool. Soc. Lond. 1881: 353, by original designation. Lectotype \%. [INDIA]: [W. Bengal], Darjiling (BMNH), designated as (type) by Hampson, 1908, ibidem 7: 291 .

$\$$ PERIGIA, misspelling. See Perigea Guenée, 1852 .

ACRO

PERIGONICA Smith, I 890, Entomologica am. 6: 123.

HADE

Type-species: Perigonica angulata Smith, I890, ibidem 6: 124 , by subsequent designation by Hampson, 1905, Cat. Lepid. Phalaenae Br. Mus. 5: 434. Syntypes ơ. + . [U.S.A.]: Cal., Sierra Nevada.

PERIGRAPHA Lederer, I857, Noctuinen Eur.: 38, I 36.

HADE

Type-species: Noctua i-cinctum [Denis \& Schiffermüller], I775, Ankündung syst. Werkes Schmett. Wienergegend: 78 , by subsequent designation by Grote, 1874, Bull. Buffalo Soc. nat. Sci. 2: i23. Type(s), [Austria]: Vienna district (Collection destroyed, Horn \& Kahle, 1936, Ent. Beih. Berl.-Dahlem 3: 243).

PERIMAGIA Strand, 1909. See Leucovis Hampson, 1908.

AGAR

PERINAENIA Butler, 1878, Ann. Mag. nat. Hist. (5) 1: 289.

NOCT

Type-species: Perinaenia lignosa Butler, i 878 , ibidem (5) $1: 289$, by original designation. Type(s), Japan: Yokohama (BMNH).

$P$. lignosa is a junior subjective synonym of Spintherops accipiter Felder \& Rogenhofer, I 874 .

See also Anepilecta Warren, I912.

PERIOPTA Turner, 1920, Trans. R. Soc. S. Aust. 44: 123, I27.

AGAR

Type-species: Agarista ardescens Butler, i 884, Ann. Mag. nat. Hist. (5) 14: 405, by original designation. Lectotype ㅇ, [Australia]: [N. Territ.], Port Darwin (BMNH), designated (as type) by Hampson, I9or, Cat. Lepid. Phalaenae Br. Mus. 3: 559.

†PERIPHANA, misspelling. See Periphanes Hübner, [1821].

NOCT

$\ddagger$ PERIPHANEA, misspelling. See Periphanes Hübner, [1821].

NOCT

PERIPHANES Hübner, [1821] I 816, I'erz. bekannter Schmett.: 247.

NOCT

Type-species: Phalaena delphinii Linnaeus, 1758, Syst. Nat. (Edn 10) 1: 518, by subsequent designation by Hampson, 1903, Cat. Lepid. Phalaenae Br. Mus. 4: 52, but cited as delphini, an unjustified emendation by Hampson, for †Periphana an incorrect subsequent spelling. Type(s), TYPE-LOCALITy not stated [EurOPE].

$\ddagger$ PERIPHANEA; Walker, 1857, List Specimens lepid. Insects Colln Br. Mus. 11: 672.

An incorrect subsequent spelling.

${ }_{+}$PERIPHANA; Hampson, 1903, Cat. Lepid. Phalaenae Br. Mus. 4: 52.

An incorrect subsequent spelling.

CHARICLEA Curtis, 1825, Br. Ent. 2: 76 .

Type-species: Phalaena delphinii Linnaeus, I $75^{8}$, by original designation.

Curtis attributed Chariclea to Stephens, but Stephens did not publish this name until 1829 .

Chariclea is a junior objective synonym of Periphanes Hübner, [182I].

CHARICLEA Stephens, i 829 [June], Nom. Br. Insects: 42.

Type-species: Phalaena delphinii Linnaeus, I 758 , by monotypy.

Chariclea Stephens was also proposed in 1829 September Ist., Illust. Br. Ent. (Haustellata) 3: 92 . 
A junior homonym of Chariclea Curtis, I825,-Lepid., Noctuidae. The objective replacement name is Periphanes Hübner, [I82 I].

†CHARIELLA; Bertkau, I889, Arch. Naturgesch. 55(2) (2): I 5 I.

An incorrect subsequent spelling of Chariclea Curtis, 1825 .

PERIPHRAGE Herrich-Schäffer, I856, Samml. neuer oder wenig bekannter aussereur. Schmett.

1 (I): wrapper, pl. 69, fig. 396; I 858, ibidem 1: 69, 82.

HYPE

Type-species: Periphrage barbatula Herrich-Schäffer, I856, ibidem 1 (I): wrapper, pl. 69, fig. 396; 1858, ibidem 1:69, 82, by monotypy. Type(s), Brazil.

PERIPLUSIA Holland, I894, Psyche, Camb. 7: 30.

CHLO

Type-species: Periplusia nubilicosta Holland, I894, ibidem 7: 30, pl. I, fig. 4, by original designation. Type(s) $\lesssim$, West Africa (CM, Pittsburgh).

PERIPYRA Hampson, 1908, Cat. Lepid. Phalaenae Br. Mus. 7: vii, 25.

ACRO

Type-species: Amphipyra sanguinipuncta Guenée, 1852, in Boisduval \& Guenée, Hist. nat. Insectes (Lépid.) 6: 4I2, pl. 18, fig. 2, by original designation. Holotype $\delta^{\star}$, Australia (MNHN, Paris).

PERISCEPTA Turner, I920, Trans. R. Soc. S. Aust. 44: I 24, I 26.

AGAR

Type-species: Agarista polysticta Butler, 1875, Ann. Mag. nat. Hist. (4) 15: 138, by monotypy. Type(s) ㅇ, [Australia]: between Sydney and Moreton Bay (BMNH).

PERISSANDRIA Warren, 1909, in Seitz, Macrolepid. World 3: 58.

NOCT

Type-species: Eicomorpha argillacea Alphéraky, I892, Horae Soc. ent. ross. 26: 445, by original designation. Type(s) $\hat{\sigma},[\mathrm{CHINA}$ : [Tibet], Tschan-husa to Amdo, Sinin (ZI, Leningrad).

The date of publication of the part of Seitz containing page 58 was stated on page 53 as 1909 October 28 th in the English edition and as 1909 November 19th in the German edition.

PEROPALPUS Blanchard, I852, in Gay, Historia de Chile (Zool.) 7: 85.

$\mathrm{OPHI}$

Type-species: Peropalpus albidus Blanchard, I852, ibidem 7:86, pl. 7, fig. 2, by monotypy. Type(s), CHILE: Coquimbo.

PEROPHIUSA Berio, 1959, Annali Mus. civ. Stor. nat. Giacomo Doria 71: 316.

CATO

Type-species: Anua pseudotirhaca Berio, 1956, Memorie Soc. ent. ital. 35: 24, by original designation. Holotype $\sigma^{\tau},\left[Z_{A I R E}\right]$ Belgian Congo: Lusambo (MRAC, Tervuren).

PERSECTANIA Hampson, 1905, Cat. Lepid. Phalaenae Br. Mus. 5: xii, 386. HADE

Type-species: Noctua ewingii Westwood, I839, in Ewing, Trans. ent. Soc. Lond. 2 Proc.: lv, pl. 20, fig. I, by original designation, but cited by Hampson as P. evingi, an unjustified emendation. Type(s), [Australia]: [Tasmania] Van Diemen's Land (UM, Oxford).

PERSIDIA Wiltshire, I946, Proc. R. ent. Soc. Lond. (B) 15: i 22.

ACRO

Type-species: Margelana achaemenica Wiltshire, I94I, J. Bombay nat. Hist. Soc. 42 : 477, fig. 5, by original designation. Holotype ơ, Iran: S.IV., Fars, Pir-i-Zan woods (Colln E. P. Wiltshire, London).

PERTA Walker, [1866] i 865, List Specimens lepid. Insects Colln Br. Mus. 34: I 195. OpHI Type-species: Perta arenalis Walker, [1866] I865, ibidem 34: II96, by monotypy. Holotype , [Dominican Republic] St. Domingo (BMNH).

PERYNEA Hampson, i9io, Cat. Lepid. Phalaenae Br. Mus. 10: x, 201.

ACON

Type-species: Rivula subrosea Butler, I88I, Trans. ent. Soc. Lond. 1881: 580, by original designation. Lectotype $\delta$, JAPAN: Tokei (BMNH), designated (as type) by Hampson, I9IO, ibidem 10: 202. 
PESSIDA Walker, I 858, List. Specimens lepid. Insects Colln Br. Mus. 15: I 547, I64I. opH Type-species: Pessida interlineata Walker, I858, ibidem 15: I642, by monotypy. Type(s) ô, [BraziL]: Parà.

$P$. interlineata is a junior subjective synonym of Pterhemia mutilatalis Guenée, 1854. See also Pterhemia Guenée, I 854.

PETALUMA Buckett \& Bauer, 1964. See Petalumaria Buckett \& Bauer, I967.

$\mathrm{ACRO}$

PETALUMARIA Buckett \& Bauer, 1967, J. Res. Lepid. 6: 52.

$\mathrm{ACRO}$

Type-species: Petaluma californica Buckett \& Bauer, I964, ibidem 3: I94, figs I-4, by original designation (for Petaluma Buckett \& Bauer). Holotype ô. [U.S.A.]: Calif., Sonoma County, Petaluma (Univ. Calif., Davis).

Petalumaria was proposed as the objective replacement name for Petaluma Buckett \& Bauer, 1964 .

PETALUMA Buckett \& Bauer, 1964, J. Res. Lepid. 3: 193.

Type-species: Petaluma californica Buckett \& Bauer, 1964, by original designation.

A junior homonym of Petaluma Hulst, i 888, Entomologica am. 4: I 16, - Lepid., Pyralidae.

The objective replacement name is Petalumaria Buckett \& Bauer, I967.

PETASIA Stephens, 1829. See Asteroscopus Boisduval, r 828.

CUCU

PETEROMA Schaus, 1901, Ann. Mag. nat. Hist. (7) 8: 78.

OPHI

Type-species: Peteroma jarinta Schaus, 1901, ibidem (7) 8: 78, by subsequent designation by Hampson, 1926, Descr. new Genera Species Lepid. Phalaenae Subfamily Noctuinae Br. Mus.: 431. Type(s) ô. [Braz1L]: Rio Janeiro, Petropolis.

PETEROMA Schaus, I906, Proc. U.S. natn. Mus. 30: I 13.

Type-species: Peteroma lignea Schaus, 1906, ibidem 30: II3, by monotypy. Type(s), Venezuela: Aroa (USNM, Washington).

A junior homonym of Peteroma Schaus, igor. There is no objective replacement name, but $P$. lignea is congeneric with $P$. jarinta Schaus, the type-species of Peteroma Schaus, Igor. The latter is therefore available for use as the subjective replacement name.

PETEROMA Schaus, igo6. See Peteroma Schaus, Igoi.

$\mathrm{OPHI}$

PETILAMPA Aurivillius, I 89I, Nordens Fjärilar: 156.

ACRO

Type-species: Noctua minima Haworth, 1809, Lepid. Br.: 216, by monotypy. Holotype, [Great Britain): [England], [Kent county] Cantio.

$N$. minima was first proposed as $¥ N$. minimina [Haworth], 1802, Prodromus Lepid. $B r$. I9, a nomen nudum.

$\ddagger$ PETOSIA; Swinhoe, 1893, Ann. Mag. nat. Hist. (6) 12: 216.

ARCTIIDAE

An incorrect subsequent spelling of Pelosia Hübner, [1819] I8I6, Verz. bekannter Schmett.: 165,-Arctiidae, Lithosinae. Cited by Neave, 1940, Nomencl. zool. 3: 679 as 'pro Petasia Stephens, I829', - Noctuidae, Cuculliinae.

PETRINIA Walker, r 869, in Chapman, Proc. nat. Hist. Soc. Glasgow 1: 347.

SARR

Type-species: Petrinia lignosa Walker, I869, ibidem 1: 348 , by monotypy. Lectotype ․ Congo (BMNH), designated (as type f) by Hampson, igi2, Cat. Lepid. Phalaenae Br. Mus. 11: 324 .

${ }_{+}^{+}$PETROPHORA Hühner, i808. See Ptichodis Hübner, I818.

CATO

PETROWSKYA köhler, 1958, Revta Soc. ent. argent. 20 : I 4.

Noct

Type-species: Petrowskya hiberna Köhler, 1958, ibidem 20: I4, fig., by original designation. Holotype ô. Argentina: [Rio Negro Prov.], Gral. Roca (ZSBS, Munich).

PEUCEPHILA Hampson, 1909, Trans. ent. Soc. Lond. 1909: $46 \mathrm{I}$.

HADE

Type-species: Peucephila essoni Hampson, I909, ibidem 1909: 461, pl. 16, by original designation. Holotype + , [Great Britain]: Scotland, Aberdeen (BMNH). 
P. essoni is a junior subjective synonym of Phalaena oleracea Linnaeus, I758, Syst. Nat. (Edn Io) 1: 5 I 7 .

$\ddagger$ PEXIPOGO Hampson, no published reference found.

HYPE

PFEIFFERELLA Osthelder, 1933. See Ostheldera nom. n.

CUCU

PHACHTHIA Walker, 1864, List Specimens lepid. Insects Colln Br. Mus. 30: 979. OPH I

Type-species: Phachthia lignigeralis Walker, I864, ibidem 30: 979, by monotypy. Type(s) ô. BORNEo: Sarawak (BMNH).

$P$. lignigeralis is a junior subjective synonym of Olulis puncticinctalis Walker, I 863.

See also Olulis Walker, 1863.

PHAENAGROTIS Köhler, I953, Revta Soc. ent. argent. 16: 20.

NOCT

Type-species: Phaenagrotis hecateia Köhler, 1953, ibidem 16:20, figs 3, 4, by original designation. Syntypes ô, Argentina: Neuquén, San Martî́n de los Andes (ZSBS, Munich).

PHAEOBLEMMA Hampson, 1926, Descr. new Genera Species Lepid. Phalaenae Subfamily Noctuinae Br. Mus.: 8.

OPHI

Type-species: Phalaena dares Stoll, I780, in Cramer, Uitlandsche Kapellen 4: 42, pl. 3 I o, fig. G, by original designation. Type(s), Surinam.

$P$. dares is dated from the wrapper of the part and not from the title-page of the volume, I 782 .

PHAEOCHLAENA Hübner, i 818, Zuträge Samml. exot. Schmett. 1: 18, 32. Dioptidae

Type-species: Phaeochlaena tendinosa Hübner, I8I8, ibidem 1: 18, figs 89, 90, by subsequent designation by Kirby, 1892, Synonymic Cat. Lepid. Heterocera 1 : 398.

Phaeochlaena has been used as the original generic name for species which are now in the Noctuidae.

PHAEOCYMA Hübner, i 818, Zuträge Samml. exot. Schmett. 1: i9.

CATO

Type-species: Phaeocyma lunifera Hübner, 1818, ibidem 1: 19, figs 97, 98, by subsequent designation by Grote, 1874, Bull. Buffalo Soc. nat. Sci. 2: 45, but cited for †Peocyma Hübner, an incorrect subsequent spelling. Type(s) ㅇ․ [U.S.A.]: Georgia [formerly] in Florida.

$\ddagger$ PHEOC YMA; Grote, I 874, Bull. Buffalo Soc. nat. Sci. 2: 45 .

An incorrect subsequent spelling.

${ }_{+}$PHAEOLITA Hampson, no published reference found.

HYPE

PHAEOMORPHA Turner, 1920, Trans. R. Soc. S. Aust. 44: I 4I, I 49.

ACRO

Type-species: Phaeomorpha acineta Turner, I920, ibidem 44: I49, by monotypy. Syntypes $2 q$ (not I $\hat{0}, \mathbf{I} q$ as stated), Australia: Qd, Montville, Blackall Range (ANIC, Canberra).

$P$. acineta is a junior subjective synonym of Syntheta xylitis Turner, I 902.

See also Syntheta Turner, 1902.

PHAEOPYRA Hampson, I908, Cat. Lepid. Phalaenae Br. Mus. 7: vii, I9. ACRO

Type-species: Protagrotis novaguinensis Bethune-Baker, 1906, Novit. zool. 13: 192, by original designation. Holotype ㅇ, New GunneA: [Papua], Ekeikei (BMNH).

PHAEOSCIA Hampson, 1926, Descr. new Genera Species Lepid. Phalaenae Subfamily Noctuinae Br. Mus.: 505.

OPHI

Type-species: Phaeoscia canipars Hampson, 1926, ibidem: 505, by original designation. Holotype ð, Nigeria: Sapele (BMNH).

PHAEOTHRIPA Hampson, 19i 2, Cat. Lepid. Phalaenae Br. Mus. 11: xi, 3 I8.

SARR

Type-species: Sarrothripa morena Swinhoe, Igo I, Ann. Mag. nat. Hist. (7) 7: 490, by 
original designation. Lectotype + , BoRneo: Sarawak (BMNH), designated (as type) by Hampson, 1912, ibidem 11: 319.

S. morena was originally proposed in †Sarothripa, an incorrect subsequent spelling.

PHAEOZONA Hampson, igro, Cat. Lepid. Phalaenae Br. Mus. 10: x, $200 . \quad$ ACon

Type-species: Acantholipes purpurascens Bethune-Baker, 1906, Novit. zool. 13: 265. by original designation. Holotype ô, New Guinea: [Papua], Dinawa (BMNH).

PHAGYTRA Walker, [1866] i 865, List Specimens lepid. Insects Colln Br. Mus. 34: I 508. opH Type-species: Phagytra leucogastralis Walker, [I866] I865, ibidem 34: I508, by monotypy. Holotype q, Java (BMNH).

$P$. leucogastralis is a junior subjective synonym of Masca abactalis Walker, [I859].

See also Masca Walker, [1859].

PHAIOECIA Dyar, I9I I, Proc. ent. Soc. Wash. 13: 69.

ACRO

Type-species: Cirrhophanus duplicatus Smith, I891, Trans. Am. ent. Soc. 18: I I2, by original designation. Holotype ㅇ. [U.S.A.]: Col., Platte Canon (USNM, Washington).

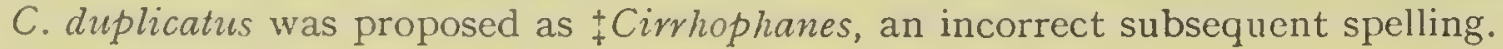

PHALACRA Staudinger, 1892. See Dysmilichia Speiser, I902. ACRO

PHALAENA Linnaeus, I 758. See Naenia Stephens, I829. NocT

PHALAENOIDES Lewin, I805, Nat. Hist. lepid. Insects N.S. W.: 2. AGAR

Type-species: Phalaenoides glycinae Lewin, I805, ibidem: 2, pl. I, by monotypy. Syntypes d, q. [Australis]: New South Wales.

†PH ALENOIDES; Pagenstecher, 1909, Geogr. Verbreitung Schmett.: 423.

An incorrect subsequent spelling.

PHALAENOPHANA Grote, I 873, Trans. Am. ent. Soc. 4: 304.

HYPE

Type-species: Phalaenophana rurigena Grote, i 873, ibidem 4: 305, by monotypy. Syntypes $\hat{\jmath}$, ㅇ, [U.S.A.]: New York and Pennsylvania (BMNH, but without locality).

$P$. vurigena is a junior subjective synonym of Herminia pyramusalis Walker, [1859].

See also Neoherminia Druce, I89I.

PHALAENOSTOLA Grote, I873, Trans. Am. ent. Soc. 4: 302.

HYPE

Type-species: Phalaenostola larentioides Grote, I873, ibidem 4: 302, by subsequent designation by Grote, 1874, Bull. Buffalo Soc. nat. Sci. 2: 47. Syntypes 18 (ô, ㅇ), [U.S.A.]: New York and Pennsylvania (BMNH, but without locality).

$\ddagger$ PHALAKRA, misspelling. See Dysmilichia Speiser, 1902.

ACRO

$\ddagger$ PHALENA Radermacher, I779. See Naenia Stephens, I829.

NOCT

$\ddagger_{\ddagger} P H A L E N A$, misspelling. See Naenia Stephens, 1829 .

NOCT

$\ddagger$ PHALENOIDES, misspelling. See Phalaenoides Lewin, I 805 .

AGAR

PHALERODES Hampson, 1908, Cat. Lepid. Phalaenae Br. Mus. 7: I I. Available, but without included species until Hampson, 1910, ibidem 9: xi, 263.

ACRO

Type-species: Charidea cauta Hampson, 1902, Ann. S. Afr. Mus. 2: 293, by subsequent monotypy. Syntypes $\overrightarrow{0}$, $q$, Nigeria. [Rhodesia]: Mashonaland, Salisbury (BMNH). [South AFrica]: Natal, Malvern (BMNH); Durban (BMNH); Cape Colony, Annshaw.

PHALGA Moore, i 88I, Proc. zool. Soc. Lond. 1881: 375.

EUTE

Type-species: Phalga sinuosa Moore, 1881, ibidem 1881:375, pl. 37, fig. 7, by monotypy. Type(s), [INDiA]: Darjiling (MNHU, Berlin).

PHANACA Wallier, I865, List Specimens lepid. Insects Colln Br. Mus. 33: 856.

CHLO

Type-species: Phanaca damnipennis Walker, I 865, ibidem 33: 857 , by monotypy. Holntype o, CEYLON (BMNH). 
PHANASPA Walker, [r866] 1865, List Specimens lepid. Insects Colln Br. Mus. 34: I I92. HYPE Type-species: Phanaspa dilatatalis Walker, [1866] 1865, ibidem 34: I I93, by monotypy. Type(s) ô. [South AFrica]: Cape (UM, Oxford).

$P$. dilatatalis is a junior subjective synonym of Hypena namaqualis Guenée, 1854 , in Boisduval \& Guenée, Hist. nat. Insectes (Lépid.) 8: 28.

PHANASPA Walker, [1866] I865, List Specimens lepid. Insects Colln Br. Mus. 34: I2 II. See Busmadis Walker, I 866.

PHARETRA Hübner, [1820]. See Viminia Chapman, r89o.

ACRO

PHARGA Walker, 1863, List Specimens lepid. Insects Colln Br. Mus. 27: $200 . \quad$ орні

Type-species: Pharga fasciculella Walker, I863, ibidem 27: 201, by monotypy. Holotype $\widehat{\jmath}$, Venezuela (BMNH).

$P$. fasciculella is a junior subjective synonym of Hormisa pholausalis Walker, [I859] I 858 , ibidem 16: 237 .

$\ddagger P H A R N A C I S A$, misspelling. See Phornacisa Walker, I862.

CUCU

PHASIDIA Hampson, rgo I, Cat. Lepid. Phalaenae Br. Mus. 3: xix, 659.

AGAR

Type-species: Phasis contraria Walker, [1865] I864, List Specimens lepid. Insects Colln $B r$. Mus. 31: 136, by original designation. Lectotype ㅇ, TyPE-Locality not known (BMNH), designated (as type) by Hampson, I90I, ibidem 3: 660. [BRAzIL].

PHASIS Walker, I854. See Shapis nom. n.

AGAR

†PHEOYMA, misspelling. See Phaeocyma Hübner, I818. Caro

PHERECHOA Turner, I 932, Trans. R. Soc. S. Aust. 56: I79. OpH

Type-species: Pherechoa crypsichlora Turner, 1932, ibidem 56: 179, by monotypy. Holotype $q$, Australia: N. Qd, Kuranda (ANIC, Canberra).

PHIALTA Schaus, I90I, Ann. Mag. nat. Hist. (7) 8: 44.

OPHI

Type-species: Phialta duomita Schaus, I9or, ibidem (7) 8:44, by monotypy. Type(s), VenEZUela: Aroa (USNM, Washington).

PHIBALAPTER YX Stephens, I 829, Nom. Br. Insects: 44.

GEOMETRIDAE

Type-species: Geometra lineolata [Denis \& Schiffermüller], I775, Ankündung syst. Werkes Schmett. Wienergegend: I02, by subsequent designation by Curtis, 1836, Br. Ent. $13: 623$.

Phibalapteryx has been used as the original generic name for species which are now in the Noctuidae.

PHIBALOCERA Stephens, I829 [June], Nom. Br. Insects: 48.

OECOPHORIDAE

Type-species: Pyralis quercana Fabricius, 1775, Syst. Ent.: 652, by monotypy.

Phibalocera has been incorrectly associated with Noctuidae owing to the similarity in name and date of its type-species with that of a species in the Chloephorinae, Tortrix quercana [Denis \& Schiffermüller], 1775, Anküindung syst. Werkes Schmett. Wienergegend: 125 .

PHIBROMIA Schaus, 1906, Proc. U.S. natn. Mus. 30: 106.

OPHI

Type-species: Phibromia narecta Schaus, I906, ibidem 30 : 106, by monotypy. Type(s), Brazil: Parana, Castro (USNM, Washington).

PHILECIA Walker, 1861, List Specimens lepid. Insects Colln Br. Muts. 23: 947. Available but without included species until Walker, I862, ibidem 24: Io88.

OPHI

Type-species: Philecia bracteigutta Walker, I862, ibidem 24: I088, by subsequent monotypy. Lectotype $\hat{\sigma}$, BorNEO: Sarawak (UM, Oxford), designated (as type) by Swinhoe, I9oo, Cat. east. and Aust. Lepid. Heterocera 2: 75. 
PHILIPPODAMIAS Clench, 1958, Ann. Cameg. Mus. 35: 73.

AGAR

Type-species: Philippodamias jocelyna Clench, 1958 , ibidem 35: 74 , by original designation. Holotype $\hat{\sigma}$, Philippre Is: Samar I., Matuguinao (CM,., Pittsburgh).

PHILOCHR YSA Grote, I863, Proc. ent. Soc. Philad. 2: 338.

HADE

Type-species: Philochrysa regnatrix Grote, 1863 , ibidem 2: $339, \mathrm{pl}$. 8, fig. 4, by monotypy. Syntypes $2 \hat{o}$, U.S.A.: Pennsylvania; D.C., Washington.

$P$. regnatrix is a junior subjective synonym of Phalaena timais Cramer, 1780 .

See also Xanthopastis Hübner, [1821].

PHILOGETHES Turner, 1939, Proc. R. Soc. Qd 50: I 49.

HYPE

Type-species: Philogethes metableta Turner, 1939, ibidem 50: I49, by monotypy. Syntypes, Australia: I $\hat{O}, \mathrm{~N}$. Qd, Ravenshoe (ANIC, Canberra); I $\hat{O}$, Lake Barrine (ANIC, Canberra); I $\hat{\delta}$, Kuranda (ANIC, Canberra).

PHILOMETRA Grote, I 872. See Epidelta nom 11.

HYPE

PHILOMMA Grote, I 86, Proc. ent. Soc. Philad. 3: 2.

NocT

Type-species: Philomma henrietta Grote, I864, ibidem 3: 3, by monotypy. Syntypes

ㅇ. [U.S.A.]: Eastern States.

$P$. henrietta is a junior subjective synonym of Derrima stellata Walker, [1858].

See also Derrima Walker, [1858].

PHILOPYRA Guenée, 1837. See Amphipyra Ochsenheimer, I8I6.

ACRO

PHILORGYIA Grote, I896, Mitt. Roemermus. Hildesh. 3: 6, I 7 .

ACRO

Type-species: Acronycta luteicoma Grote \& Robinson, 187o, Trans. Am. ent. Soc. 3: I79, pl. 2, fig. 83 , by original designation. Syntypes 3 ․ [U.S.A.]: Atlantic District, New York and Peunsylvania.

A. luteicoma is a junior subjective synonym of Acronycta impleta Walker, I856, List Specimens lepid. Insects Colln Br. Mus. 9:57.

PHILOSTOLA Billberg, I820. See Mythimna Ochsenheimer, I8I6.

HADE

PHIMODIUM Herrich-Schäffer, I 870, Corresp.-Bl.zool.-min. Ver. Regensbury 24: Io I, I04. OPHI Type-species: Phalaena sergilia Stoll, 1780, in Cramer, Uitlandsche Kapellen 4: 66, pl. 321 , fig. F, by monotypy. Type(s), Surinam.

$P$. sergilia is dated from the wrapper of the part and not from the title-page of the volume, 1782 .

PHINECA Walker, I856, List Specimens lepid. Insects Colln Br. Mus. 7: 1740.

PANT

Type-species: Phineca basistriga Walker, 1856, ibidem 7: 1747, by monotypy. Holotype 9 , TYPE-LOCALITY not known (BMNH) [EUROPE].

$P$. basistriga is a junior subjective synonym of Phalaena coryli Linnaeus, $\mathbf{I} 758$.

See also Colocasia Ochsenheimer, I816.

PHIPROSOPUS Dyar, I893. See Phyprosopus Grote, 1872.

OPHI

PHLEBOEIS Christoph, I 887, Stettin. ent. Ztg 48: I64.

NOCT

Type-species: Phleboeis petersi Christoph, I88\%, ibidem 48: 164, by monotypy.

Syntypes, [U.S.S.R.]: [Turkmenistan], Tekke, Askhabad (ZI, Leningrad).

This genus was originally proposed as ¥Phleboëis which, under the Code, Article 32(c),

is an incorrect original spelling.

PHLEBOIS Spuler, 1905, in Hofmann, Schmett. Eur. 1 : 166.

An unjustified emendation of Phleboeis Christoph, 1887.

PHLEBOIS Spuler, I905. See Phleboeis Christoph, I887.

NOCT 
PHLEGETONIA Guenée, r852, in Boisduval \& Guenée, Hist. nat. Insectes (Lépid.) 6: 3or.

Type-species: Phlegetonia catephioides Guenée, 1852, ibidem 6: 301, pl. I4, fig. 6, by subsequent designation by Hampson, I9r2, Cat. Lepid. Phalaenae Br. Mus. 11: 70. Lectotype $\hat{0}$. [South Africa]: Port Natal (MNHN, Paris), designated by Viette, I95I, Bull. mens. Soc.linn. Lyon 20: 161.

PHLOGOCHROA Hampson, 1926, Descr. new Genera Species Lepid. Phalaenae Subfamily Noctuinae Br. Mus.: $4 \mathrm{I} 6$. OPHI Type-species: Capnodes haematoessa Holland, i 894, Psyche, Camb. 7: I Io, pl. 5, fig. I9, by original designation. Syntypes $\hat{\sigma}, q$, WEST AFrica.

The type-species was proposed as $\ddagger C$. haematoëssa, an incorrect original spelling.

PHLOGOPHORA Treitschke, 1825, Schmett. Eur. 5 (1) : 369.

ACRO

Type-species: Phalaena meticulosa Linnaeus, 1758, Syst. Nat. (Edn Io) 1: 513, by subsequent designation by Duponchel, 1829, in Godart \& Duponchel, Hist. nat. Lépid. Papillons Fr. 7 (2): 71. Type(s), TYPe-Locality not stated (LS, London) [Europi].

Duponchel's type-species designation was ignored by Hampson, 19r8, Novit. zool. 25 : 190, 383, who cited as type-species Noctua adulatrix Hübner, [I8I3], and based a subfamily Phlogophorinae (now known as Euteliinae) on this concept.

SOLENOPTERA Duponchel [1845] I844, Cat. méth. Lépid. Eur. (2) : I34.

Type-species: Phalaena meticulosa Linnaeus, I758, by subsequent designation (for Brotolomia Lederer) by Grote, 1874, Bull. Buffalo Soc. nat. Sci. 2: 18.

A junior homonym of Solenoptera Audinet-Serville, I832, Annls Soc. ent. Fr. 1 (2): I83, Coleoptera. The objective replacement name is Phlogophora Treitschke, I825.

BROTOLOMIA Lederer, I857, Noctuinen Eur.: 35, I I 5 .

Type-species: Phalaena meticulosa Linnaeus, $175^{8}$, by subsequent designation by Grote, I 874, Bull. Buffalo Soc. nat. Sci. 2: 18.

Brotolomia was proposed as the objective replacement name for Solenoptera Duponchel,

[1845]; it is therefore, also, a junior objective synonym of Phlogophora Treitschle, I825.

RACOPTERA Scott, I858, Zoologist 16: 5962.

Type-species: Phalaena meticulosa Linnaeus, $175^{8}$, by monotypy.

Racoptera is a junior objective synonym of Phlogophora Treitschke, 1825.

PHLOSSA Walker, I 858, List Specimens lepid. Insects Colln Br. Mus. 15: I673. Limacodidae Type-species: Phlossa fimbriares Walker, i 858 , ibidem 15: 1673, by monotypy.

$P$. fimbriares is a junior subjective synonym of Limacodes conjuncta Walker, I855, ibidem

5: II 50.

Phlossa was originally placed in the Noctuidae.

PHLYCTAINA Möschler, I890, Abh. senckenb. naturforsch. Ges. 16: 228.

HYPE

Type-species: Phlyctaina irrigualis Möschler, I890, ibidem 16: 229, by monotypy. Holotype $\hat{\sigma}$, Puerto Rico.

APHL YCTAENA Kaye \& Lamont, 1927, Mem. Dep. Agric. Trin. 3: 77.

Type-species: Phlyctaina irrigualis Möschler, I890, by monotypy.

Aphlyctaena originated from Hampson, who intended to publish it as a replacement name for Phlyctaina Möschler, 1890 , which Hampson considered to be a homonym of Phlyctaenia Hübner, [1825], Verz. bekannter Schmett.: 359, - Lepid., Pyralidae.

Aphlyctaena is a junior objective synonym of Phlyctaina Möschler, r 890.

PHOBERIA Hübner, r818, Zuträge Samml. exot. Schmett. 1: 13, r6.

OPHI

Type-species: Phoberia atomaris Hübner, 1818 , ibidem 1: 16 , figs 75,76 , by subsequent designation by Grote, 1874, Bull. Buffalo Soc. nat. Sci. 2: 39. Type(s) ô, [U.S.A.]: Georgia [formerly] in Florida.

See also Lyssia Guenée, 1852. 
PHOBOLOSIA Dyar, 1908, Proc. ent. Soc. Wash. 10: 52.

Type-species: Phobolosia reincarnata Dyar, r9o8, ibidem 10: 52, by monotypy. Syntypes, [U.S.A.]: Cal., San Diego; S. Arizona; Tex., Kerrville. (USNM, Washington).

PHOEBOPHILUS Staudinger, I 888, Stettin. ent. Ztg 49: 21.

ACRO

Type-species: Phoebophilus amoenus Staudinger, 1888, ibidem 49:21, by monotypy. Syntypes ô. 9, [U.S.S.R.]: [Uzbekistan], Margelan (MNHU, Berlin); [Kirghizia], Issyk-Kul (MNHU, Berlin)

PHOENICOPHANTA Hampson, I9io, Cat. Lepid. Phalaenae Br. Mus. 10: 2, 653. ACoN Type-species: Phoenicophanta flavifera Hampson, 1910, ibidem 10: 653, fig. I 84 , by original designation. Syntypes $7 \delta^{\pi}, I$ P, Argentina: Tucuman, Los Vasquez (BMNH).

PHOPERIGEA Yiette, 1965, Bull. Soc. ent. Fr. 70: 86.

ACRO

Type-species: Hypoperigea variegata Kenrick, 1917, Trans. ent. Soc. Lond. 1917: 87 , pl. I, by original designation. Lectotype $\hat{0}$. NADAGASCAR (BMNH), designated (as holotype) by Viette, 1967, Fanne Madagascar 20(2): 493.

PHORICA Walker, I858, List Specimens lepid. Insects Colln Br. Mus. 15: I 732.

$\mathrm{OPHI}$

Type-species: Phorica phasipennis Walker, I858, ibidem 15: I732, by monotypy. Lectotype ô. [INDiA] Hindostan (UM, Oxford), designated (as type) by Swinhoe, I90o, Cat. east. and Aust. Lepid. Heterocera 2: iof.

PHORNACISA Walker, I862, Trans. ent. Soc. Lond. (3) 1: 3 I I.

CUCU

Type-species: Phornacisa piffardi Walker. I862, ibidem (3) 1: 312, by monotypy.

Type(s) $9,[$ CAnAda]: Nova Scotia, Halifax (BMNH).

See also Metahadena Morrison, i 876.

${ }_{\ddagger}$ PHARNACISA; Forbes, I954, Lepid. New York and neighboring States 3: I 25.

An incorrect subsequent spelling.

PHOROCERA Guenée, I 852. See Recoropha nom. n.

CUCU

PHOSPHILA Hübner, I8 8 8, Zuträge Samml. exot. Schmett. 1: I 5.

ACRO

Type-species: Phosphila turbulenta Hübner, I818, ibidem 1: I 5, figs 67, 68, by subsequent designation by Hampson, i 908, Cat. Lepid. Phalaenae Br. Mus. 7: I1 3. Type(s) ऽ. [U.S.A.]: Georgia [formerly] in Florida.

†PROPH YLA Hübner, I 808, Erste Zuträge Samml. exot. Schmett.: 4 .

Included in a work rejected for nomenclatural purposes by Int. Commn zool. Nom., 1966, Bull. zool. Nom. 23 Opinion 789: 2 I 6.

Only included species: $¥$ Prophyla turbulenta Hübner, 1808 , later made available as Phosphila turbulenta Hübner, I 8 I 8.

Prophyla was placed on the Official Index of Rejected and Invalid Generic Names in Zoology: Name No. I 882 .

PHOTEDES Lederer, I857, Noctuinen Eur.: 44, I89, 236.

ACRO

Type-species: Apamea captiuncula Treitschke, 1825, Schmett. Eur. 5 (2): 96, by monotypy. Type(s), Europe (TM, Budapest).

Photedes was the spelling adopted by Heinemann, I859, Schmett. Dtl. Schweiz 1: $5^{89}$, as first reviser from a multiple original spelling. Photedes was used by Lederer on page 44 and + Phothedes was used on page 189 and in the index on page 236 .

¡PHOTHEDES Lederer, I 857, Noctuinen Eur.: I 89, 236.

An incorrect original spelling.

$\ddagger$ PHOTHEDES, misspelling. See Photedes Lederer, 1857.

ACRO

PHRAGMATIPHILA Hampson, 1908, Cat. Lepid. Phalacnae Br. Mus. 7: 9. Available, but without included species until Hampson, ig I0, ibidem 9: 266.

ACRO

Type-species: Noctua nexa Hübner, [1808], Samml. eur. Schmett. 4: pl. 84, fig. 39.5, by subsecuent designation by Hampson, i910, ibidem 9: 266. Type(s), Europe. 
PHRICTIA Hübner, [I82I] I8I6, Vevz. bekannter Schmett.: 255.

OPHI

Type-species: Phalaena lingea Stoll, I782, in Cramer, Uitlandsche Kapellen 4: 239, pl. 399, fig. K, by subsequent designation by Berio, 1957 Memorie Soc. ent. ital. 36: I7. Type(s), [GuYana]: Berbices.

PHRODITA Schaus, I898, Jl N.Y. ent. Soc. 6: 120.

OPHI

Type-species: Phrodita bilinea Schaus, 1898 , ibidem 6: I20, by monotypy. Type(s), [BRAzIL]: Parana, Castro.

PHRODITA Hampson, 1926, Descr. new Genera Species Lepid. Phalaenae Subfamily Noctuinae Br. Mus.: 243.

Type-species: Phrodita bilinea Schaus, I898, by original designation.

A junior homonym of Phrodita Schaus, I 898 . This generic name was used and made nomenclaturally available by Schaus prior to its proposal and generic description by Hampson. The objective replacement name is Phrodita Schaus, I 898.

PHRODITA Hampson, I926. See Phrodita Schaus, I 898.

OPHI

PHUMANA Walker, [1863] i864, J. Proc. Linn. Soc. (Zool.) 7: i64.

EUTE

Type-species: Phumana canescens Walker, [1863] 1864, ibidem 7: 164, by monotypy. Lectotype , Borneo: Sarawak (UM, Oxford), designated (as type) by Swinhoe, I9oo, Cat. east. and Aust. Lepid. Heteroceva 2: 84.

PHUPHENA Walker, I 858, List Specimens lepid. Insects Colln Br. Mus. 15: I 845 . ACRO Type-species: Phuphena fusipennis Walker, I 858 , ibidem 15: I 846 , by monotypy. Type(s) \%, [BRAzIL]: Rio Janeiro (UM, Oxford).

PHURYS Guenée, I 852, in Boisduval \& Guenée, Hist. nat. Insectes (Lépid.) 7: 303. Cato Type-species: Noctua lineolaris Hübner, [1809], Samml. eur. Schmett. 4: pl. 96, fig. 454, by subsequent designation by Desmarest, (1857), in Chenu, Encycl. Hist. nat. Papillons nocturnes: I33. Type(s), Europe.

PHYCIDIMORPHA Hampson, 1893, Illust. typical Specimens Lepid. Heterocera Colln Br. Mus. 9: 22, I03. OPHI

Type-species: Phycidimorpha rosea Hampson, 1893, ibidem 9: 22, I04, pl. I63, fig. 22, by monotypy. Syntypes ô, Ceylon: Matale (BMNH); Nawala-pittia (BMINH).

$P$. rosea is a junior subjective synonym of Rhangena roseipennis Moore, [1886].

See also Rhangena Moore, [1886].

PHYCIDOPSIS Hampson, I893, Illust. typical Specimens Lepid. Heterocera Colln Br. Mus. 9: I6, 91 .

NOTODONTIDAE

Type-species: Phycidopsis albovittata Hampson, I893, ibidem 9: I6, 9I, pl. I6I, fig. I3, by monotypy.

Phycidopsis was originally placed in the Noctuidae.

PHYCODES Guenée, I852, in Boisduval \& Guenée, Hist. nat. Insectes (Lépid.) 6: 389.

GLYPHIPTERIGIDAE

Type-species: Phycodes hirudinicornis Guenée, I852, ibidem 6:389, pl. 13, fig. 5, by monotypy.

Phycodes was originally placed in the Hyblaeidae.

PHYCOMA Hübner, [1823] I816, Verz. bekannter Schmett.: [258].

OPHI

Type-species: Phalaena marcellina Stoll, I78o, in Cramer, Uitlandsche Kapellen 4: 48, pl. 3r2, fig. F, by subsequent designation by Berio, 1957, Memorie Soc. ent. ital. 36: I 7 . Type(s), Surinam.

PHYCOPTERUS Blanchard, 1852, in Gay, Historia de Chile (Zool.) 7: 103.

OPHI

Type-species: Phycopterus flavellus Blanchard, 1852, ibidem 7: 103, by subsequent designation by Hampson, 1926, Descr. new Genera Species Lepid. Phalaenae Subfamily Noctuinae Br. Mus.: 6r3. Type(s), CHILE: Coquimbo. 
PHYLLEDESTES Cockerell, r907, Can. Ent. 39: i88.

Type-species: Phylledestes vorax Cockerell, I907, ibidem 39: 188, fig. 9, by monotypy. Holotype larva, [U.S.A.]: Colorado, Florissant, in the Miocene shales.

PHYLLODES Boisduval, 1832, Voyage de Déconvertes de l'Astrolabe, sous d'Urville 1:246. Cato Type-species: Phalaena conspicillator Cramer, [1777], Uillandsche Kapellen 2: I, pl. 97, figs A, B, by monotypy. Type(s), [Moluccas]: Amboina [I.].

XENODRYAS Gistl, I848, Naturg. Thierreichs: $\mathrm{x}$.

Proposed, unnecessarily, as an objective replacement name for Phyllodes Boisduval, I832.

PHYLLOPHILA Guenée, I 852, in Boisduval \& Guenée, Hist. nat. Insectes (Lépid.) 6: 254. ACoN

Type-species: Anthophila wimmerii Treitschke, I835, Schmett. Eur. 10 (2): I48, by monotypy. Holotype, [Yugoslavia]: Syrmien [Syrmia was a former county $4.5^{\circ} \mathrm{N}$ I $9^{\circ}$ E.] (TM, Budapest).

A. vimmerii is a junior subjective synonym of Anthophila obliterata Rambur, I833, Annls Soc. ent. Fr. 2: 27, pl. 2, fig. I 7 .

$\ddagger$ PHYLLOPHYLA; Warren, 191 2, in Seitz, Gross-Scimelt. Erde 3: 273.

An incorrect subsequent spelling in the German edition; but correctly spelled in the English edition.

${ }_{+}^{+}$PHYLLOPHYLA, misspelling. See Phyllophila Guenée, I 852.

$\operatorname{ACON}$

PHYPROSOPUS Grote, I872 September, Trans. Am. ent. Soc. 4: 9o.

OPH I

Type-species: Phyprosopus callitrichoides Grote, I872, ibidem 4: 90, by monotypy. Syntypes ô. [U.S.A.]: New York (BMNH); Texas (BMNH).

$P$. callitrichoides was described as a new species by Grote for the misidentification Doryodes acutalis WValker, [1859] i858, List Specimens lepid. Insects Colln Br. Mus. 16: 73. Walker proposed acutalis as an emendation of Ligia acutaria Herrich-Schäffer, [1852], but because of the misidentification acutalis Walker has at times been incorrectly used for callitrichoides Grote.

See also Sudariophora Zeller, i 872 [December].

PHIPROSOPUS Dyar, i893, Bull. U.S. naln. Mus. 44: 192.

An unjustified emendation of Phyprosopus Grote, I 872.

PHYSETICA Meyrick, I887, Trans. N. Z. Inst. 19: 5.

HADE

Type-species: Agrotis coerulea Guenée, 1868, Entomologist's mon. Mag. 5: 38, by monotypy, but cited by Meyrick as $\ddagger A$. caerulea, an incorrect subsequent spelling. Syntypes $\hat{\jmath}, q$, New Zealand: Canterbury.

PHYSULA Guenée, I 854, in Boisduval \& Guenée, Hist. nat. Insectes (Lépid.) 8: 86. HY PE

Type-species: Physula migralis Guenée, I 854, ibidem 8: 87, by subsequent designation by Schaus, igi 6, Proc. U.S. natn. Mus. 50: 295. Holotype ô. [French Guiana]: Cayenne (BMNH).

PHYSULODES Warren, I 889, Trans. ent. Soc. Lond. 1889: 242.

HYPE

Type-species: Physula eupithecialis Guenéc, I854, in Boiscluval \& Guenée, Hist. nat. Insectes (Lépid.) 8: 87, by monotypy. Holotype ô. [French Guiana] : Cayenne.

PHYTOMETRA Haworth, I809, Lepid. Br.: 254.

OPHI

Type-species: Noctua aenea [Denis \& Schiffermüller], I775, Ankündung syst. Werkes Schmett. Wienergegend: 85 (but included by Haworth as 'aenea Hüb.', an incorrect authorship), by subsequent designation by Westwood, I840, Synopsis Genera Br. Insects: 98. Type(s), [Austria]: Vienna district (Collection destroyed, Horn \& Kahle, 1936, Ent. Beih. Berl.-Dahlem 3: 243).

Westwood's type-species designation was ignored by Hampson, I913, Cat. Lepid. Phalaenae. Br. Mus. 13: 452, who cited as type-species Phalaena festucae Limnaeus, i 758, and based a subfamily Phytometrinae (now known as the Plusiinac) on this concept. 
N. aenea is a junior subjective synonym of Phalaena viridaria Clerck, I759, Icones Insect. rariorum 1: pl. 9, fig. I2.

PROTHYMIA Hübner, [1823] x816, Verz. bekannter Schmett.: 282.

Type-species: Noctua aenea [Denis \& Schiffermüller], I775, by monotypy.

Prothymia is a junior objective synonym of Phytometra Haworth, I809.

PIADA Walker, 1858 , List Specimens lepid. Insects Colln Br. Mus. 15: 1746.

EUTE

Type-species: Piada multiplicans Walker, I858, ibidem 15: 1747 , by monotypy. Type(s) ô. [InDia]: [Mysore], Canara (BMNH).

PIALA Walker, I858, List Specimens lepid. Insects Colln Br. Mus. 15: 1766.

OPHI

Type-species: Piala basipunctum Walker, I858, ibidem 15: 1766 , by monotypy. Holotype , Sierra Leone (BMNH).

PIANA Walker, I869, Characters undescr. Lepid. Heterocera: 48.

OPHI

Type-species: Piana lignificta Walker, I869, ibidem: 48, by monotypy. Type(s) $q$,

[Australia]: [Qd], Moreton Bay (BMNH).

$P$. lignificta is a junior subjective synonym of Crioa acronyctoides Walker, [1858].

See also Crioa Walker, [1858].

PILIPECTUS Bethune-Baker, I910, Ann. Mag. nat. Hist. (8) 6: 443.

OPHI

Type-species: Pilipectus ocellatus Bethune-Baker, I9Io, ibidem (8) 6:443, by original designation. Holotype ơ, New Guinea: [West Irian], Arfak Mts (BMNH).

PILOSOCRURES Hampson, I89I, Illust. typical Specimens Lepid. Heterocera Colln Br. Mus. 8: $\mathrm{x} 8,85$.

OPHI

Type-species: Pilosocrures variegata Hampson, I891, ibidem 8: 18, 85, pl. 146, fig. 20, by monotypy. Syntypes ô. India: Nilgiri, W. Slopes (BMNH).

PIMPRANA Moore, 1879, in Hewitson \& Moore, Descr. new Indian lepid. Insects Colln late Mr W.S. Atkinson: $4^{\mathrm{I}}$.

AGAR

Type-species: Pimprana atkinsoni Moore, I879, ibidem: 42, pl. 2, fig. II, by monotypy. Syntypes $\widehat{\sigma}$. India: [VV. Bengal], Darjiling.

PINACIA Hübner, [I83I] I 825, Zuträge Samml. exot. Schmett. 3: І 3.

HYPE

Type-species: Pinacia molybdaenalis Hübner, [I83I] I825, ibidem 3: I3, figs 435, 436,

by monotypy. Holotype ô, [JAva]: [Djakarta swamp] Bataviens Sümpfe.

${ }_{\ddagger}$ PINACIA Walker, I859, List Specimens lepid. Insects Colln Br. Mus. 19: 9 19.

An incorrect subsequent authorship used by Swinhoe, I900, Cat. east. and Aust. Lepid.

Heterocera 2: 193. Also listed as an available name by Neave, I940, Nomencl. zool. 3: 763 .

Walker redescribed Pinacia Hübner and retained in it one species, P. molybdaenalis.

MIXTILA Swinhoe, r9oo, Cat. east. and Aust. Lepid. Heterocera 2: 193.

Proposed, unnecessarily, as the objective replacement name for $¥$ Pinacia Walker, 1859 . which Swinhoe considered to be preoccupied by Pinacia Hübner, [I83I]. These names are not homonyms as Walker was merely using, correctly, Hübner's name.

Mixtila is a junior objective synonym of Pinacia Hübner, [183I].

†PINACIA Walker, 1859 . See Pinacia Hübner, [r831].

HYPE

PINACOPLUS Hampson, I908, Cat. Lepid. Phalaenae Br. Mus. 7: 4. Available, but without included species until Hampson, I9ro, ibidem 9: $x, 229$.

ACRO

Type-species: Mycteroplus didymogramma Ershov, 1874 , in Fedchenlio, Reise in Turkestan 2 (5) 3: 44, pl. 3, fig. 43, by subsequent monotypy. Syntypes đ. ㅇ. [U.S.S.R.]: Turkestan, Kisil-kum desert (ZI, Leningrad).

PINCIA Walker, I869, Characters undescr. Lepid. Heterocera: 43.

OPHI

Type-species: Pincia continua Walker, 1869, ibidem: 44, by monotypy. Type(s) $q$, [HoNDURAS]: Limas (BMNH). 
$P$. contimua is a junior subjective synonym of Isogona natatrix Guenée, I 852 .

See also Isogona Guenée, 1852.

PINDARA Moore, [1885] I887, Lepid. Ceylon 3: I69.

CATO

Type-species: Noctua illibata Fabricius, 1775, Syst. Ent.: 592, by original designation. Type(s), India (BMNH).

PIPPONA Harvey, I875, Bull. Buffalo Soc. nat. Sci. 3: 9.

NOCT

Type-species: Pippona bimatris Harvey, I875, ibidem 3: 10, by monotypy. Type(s) ô. [U.S.A.]: Texas (BMNH).

PIRA TISCA Meyrick, I902, Trans. ent. Soc. Lond. 35: 42.

HYPE

Type-species: Piratisca minax Meyrick, 1902, ibidem 35: 42, by monotypy. Holotype ㅇ, New Guinea (BMNH).

See also Systaticospora Bethune-Baker, I908; and Megaloptera Bethune-Baker, 1908.

PISARA Walker, i 862, J. Proc. Linn. Soc. (Zool.) 6: 117.

NOLI

Type-species: Pisara opalina Valker, I862, ibidem 6: II 8, by monotypy. Lectotype o. Borneo: Sarawak (UM, Oxford), designated (as type) by Swinhoe, 1892, Cat. east. and Aust. Lepid. Heterocera 1 : I3 I.

Pisara when originally proposed contained a second species, but this was only doubtfully included and, under the Code, Article 68 (c), is not eligible for selection as type-species.

PITACOTA Moore, 1884, Lepid. Ceylon 3: 46. SARR

Type-species: Felinia terminigera Walker, r 858, List Specimens lepid. Insects Colln Br. Mus. 15: I85o, by monotypy. Type(s) \&. [INDIA]: Canara (BMNH).

See also Beana Walker, I862.

PITARA Walker, I858, List Specimens lepid. Insects Colln Br. Mus. 15: I663.

OPHI

Type-species: Pitara subcosta Walker, I858, ibidem 15: 1664, by monotypy. Holotype o., [Brazir] : [R. Amazon], [Tefé] Ega (BMNH).

PITRASA Moore, I882, in Hewitson \& Moore, Descr. new Indian lepid. Insects Colln late Mr W. S. Atkinson: 94 .

SARR

Type-species: Pitrasa variegata Moore, I882, ibidem: 94, pl. 4, fig. 2, by subsequent designation by Hampson, r9r2, Cat. Lepid. Phalaenae Br. Mus. 11: 424. Type(s) ô. IndIA: [W. Bengal], Darjiling (MNHU, Berlin).

PITYOLITA Grote, I873 January, Trans. Am. ent. Soc. 4: 309.

HYPE

Type-species: Herminia pedipilalis Guenée, in Boisduval \& Guenée, Hist. nat. Insectes

(Lépid.) 8: 57, by monotypy. Holotype $\hat{o}$. North America.

Pityolita was again proposed by Grote, 1873 May, Bull. Buffalo Soc. nat. Sci. 1: 39.

PLACEROBELA Turner, 1903, Trans. R. Soc. S. Aust. 27: I3.

OPHI

Type-species: Placerobela brachyphylla Turner, 1903, ibidem 27: 14, by monotypy. Holotype 9 , Australia: Qd, Townsville (ANIC, Canberra).

PLACODES Boisduval, I840. See Eucarta Lederer, 1857 .

ACRO

PLACONIA Möschler, I88o, Verh. zool.-bot. Ges. Wien 30 (Ablı.) : 4 ro.

CATO

Type-species: Placonia selene Möschler, I880, ibidem 30 (Abh.): 4ro, pl. 8, fig. I, by monotypy. Syntypes 2 ô. Surinam: interior (MNHU, Berlin).

PLAGIDEICTA Warren, 19I4, in Seitz, Macrolepid. World 11: 339.

ACRO

Type-species: Euplexia leprosticta Hampson, 1906, J. Bombay nat. Hist. Soc. 17: 469, by original designation. Holotype of. CEYLon: Matele (BMNH).

The date of publication of the part of Seitz containing page 339 was stated on page 337 as I9I4 June 29th in the English edition and as 1937 July 20 th in the German edition.

$+P L A G I D I E C T$ A ; Neave, r966, Nomencl. zool. 6: 228.

An incorrect subsequent spelling. 
+PLAGIDIECTA, misspelling. See Plagideicta Warren, I9I4.

PLAGIOGRAPTA Hampson, I907, J. Bombay nat. Hist. Soc. 17: 657 ,

CHLO

Type-species: Plagiograpta macrodonta Hampson, 1907, ibidem 17: 657, fig., by monotypy. Type(s) $\hat{\sigma}$. India: Canara, Malmani (BMNH).

PLAGIOMIMICUS Grote, x873, Bull. Buffalo Soc. nat. Sci. 1: I82.

ACRO

Type-species: Plagiomimicus pityochromus Grote, I873, ibidem 1: 182, by monotypy. Lectotype ô. [U.S.A.]: Alabama (BMNH), designated (as type) by Hampson, r9io, Cat. Lepid. Phalaenae Br. Mus. 9: 25x.

PLASMATICUS Bethune-Baker, 1906, Novit. zool. 13: 268.

CATO

Type-species: Plasmaticus angulata Bethune-Baker, 1906, ibidem 13: 268, by original designation. Type(s) q, New Guinea: [Papua], Dinawa (BMNH).

PLASTENIS Boisduval, 1840. See Ipimorpha Hübner, [I82 I].

ACRO

PLATAGARISTA Jordan, 1912, in Seitz, Gross-Schmett. Erde 11 : 2 x.

AGAR

Type-species: Agarista tetrapleura Meyrick, I89I, Trans. R. Soc. S. Aust. 14: 195, by monotypy. Holotype $q$, Australia: New South Wales (BMNH).

The date of publication of the part of Seitz containing page 2I was stated on page $\mathrm{I}_{7}$ as I912 February 22nd in both the German and the English editions.

PLATAGROTIS Smith, I89o, Bull. U.S. natn. Mus. 38: 9, 43.

NOCT

Type-species: Noctua speciosa Hübner, [18r3], Samml. eur. Schmett. 4: pl. Io4, fig. 49r, by original designation (on page 9). Type(s), Europe.

PLATAPLECTA Butler, I878, Ann. Mag. nat. Hist. (5) 1: 195.

ACRO

Type-species: Polia soluta Walker, I865, List Specimens lepid. Insects Colln Br. Mus.

33: 723, by original designation. Holotype , [INDIA]: N. Hindostan (BMNH).

$P$. soluta is a junior subjective synonym of Acronycta pruinosa Guenée, I852, in Boisduval

\& Guenée, Hist. nat. Insectes (Lépid.) 5: 53.

PLATHYPENA Grote, I873 January, Trans. Am. ent. Soc. 4: 310.

HYPE

Type-species: Hyblaea scabra Fabricius, I798, Suppl. Ent. Syst.: 448, by monotypy. Type(s), AmericA: northern (Type(s) not found by Zimsen, I964, Type Material of I. C. Fabricius: 583).

Plathypena was again proposed by Grote, I873 May, Bull. Buffalo Soc. nat. Sci. 1: 38.

PLAT YCERURA Packard, 1864, Proc. ent. Soc. Philad. 3: 373.

PANT

Type-species: Platycerura furcilla Packard, 1864, ibidem 3: 374, by monotypy. Type(s) J̄, U.S.A.: Mass.

PLATYCHASMA Butler, I881, Trans. ent. Soc. Lond. 1881: 596.

Type-species: Platychasma virgo Butler, r881, ibidem 1881: 596, by monotypy.

Type(s), Japan: Tokei (BMNH).

Platychasma was originally placed in the Notodontidae; it was transferred to the

Noctuidae by Kiriakoff, I963, J. Lepid. Soc. 17: 33 .

PLATYDASYS Butler, I892, Proc. zool. Soc. Lond. 1892: I 26.

ACRO

Type-species: Platydasys pryeri Butler, I892, ibidem 1892,: $\mathrm{x} 26$, pl. 6, fig. 6, by monotypy. Lectotype ô. BORNEO: Sarawak (BMNH), designated (as type) by Hampson, I908, Cat. Lepid. Phalaenae Br. Mus. 7: 535.

PLATYDIA Guenée, r854. See Yidalpta nom.n.

OPHI

$\boldsymbol{P L A T Y J A}$ Hübner, [1823] I8I6, Verz. bekannter Schmett.: 268.

OPHI

Type-species: Phalaena umminia Cramer, I780, Uitlandsche Kapellen 3: 137, pl. 267, fig. F, by subsequent designation by Moore, [1885] 1887, Lepid. Ceylon 3: 201, but cited as $\ddagger$ umminea, an incorrect subsequent spelling. Type(s), JAVA: N.W. coast, Samarang.

See also Ginaea Walker, $185^{8}$. 
PLATYJIONIA Hampson, 1926, Descr. new Genera Species Lepid. Phalaenae Subfamily Noctuinae Br. Mus.: 94.

Type-species: Platyja sada Swinhoe, I903, Fasc. malayenses (Zool.) 1: 79, by original designation. Type(s) , , [West Malaysia] : Malaya, Selangor-Pahang boundary, Semangko Pass (BMNH).

${ }_{\ddagger}^{+}$PLATYPERIGA, misspelling. See Platyperigea Smith, I 894.

ACRO

PLATYPERIGEA Smith, I894, Trans. Am. ent. Soc. $21: 60$.

ACRO

Type-species: Platyperigea camina Smith, I894, ibidem 21: 60, pl. 6, fig. 9, by original designation. Syntypes $3(\hat{o}, 9)$, [U.S.A.]: Colorado.

Platyperigea was the spelling used in the binomen of three new species and their figures; the spelling †Platyperiga used as the heading was an inadvertent error, and has not been subsequently used by other authors.

$\ddagger$ PLAT YPERIGA Smith, I894, Trans. Am. ent. Soc. 21:60.

An incorrect original spelling.

$\ddagger$ PLATYPERIGEA; Neave, I940, Nomencl. zool. 3: 798 .

Neave cited 'Platyperigea (pro -ga Smith, I894) Hampson, I909, Cat. Lep. Phalaenae Brit. Mus. 8, 4I. - Lep.' but Hampson had correctly cited Platyperigea Smith, I894, without any error or emendation.

${ }_{+}$PLATYPERIGEA; Neave, I940. See Platyperigea Smith, I 894.

ACRO

PLAT YPOLIA Grote, I 895, Abh. naturw. Ver. Bremen 14:82.

CUCU

Type-species: Pachypolia acutissima Grote, 1875, Check List Noctuidae Am. North of Mex.: 23, fig. 9, by original designation. Lectotype ô. [CANADA]: Montreal (BMNH), (lesignated (as type) by Hampson, I906, Cat. Lepid. Phalaenae Br. Mus. 6: 290.

$P$. acutissima is a junior subjective synonym of Miselia anceps Stephens, 1850 , List Specimens Br. Anim. Colln Br. Mus. 5: 285.

EUROTYPE Hampson, 1906, Cat. Lepid. Phalaenae Br. Mus. 6: xi, 289.

Type-species: Pachypolia acutissima Grote, I 875, by original designation.

Eurotype is a junior objective synonym of Platypolia Grote, I895.

PLATYPROSOPA Warren, 1913, in Seitz, Gross-Schmett. Erde 11: 133.

ACRO

Type-species: Bagada nigrostrigata Bethune-Baker, I906, Novit. zool. 13: 209, by original designation. Holotype $\hat{\jmath}$, New Guinea: [Papua], Dinawa (BMNH).

The date of publication of the part of Seitz containing page I33, was stated on page I 29 as I9r3 January 2oth in both the English and German editions.

PLATY SCIA Hampson, 1926, Descr. new Genera Species Lepid. Phalaenae Subfamily Noctuinae Br. Mus.: 90 .

OPHI

Type-species: Platyscia mesoscia Hampson, 1926, ibidem: 91, by original designation. Holotype ô. [GHana] Gold Coast: Kumasi (BMNH).

PLATYSENTA Grote, I 874, Annual Rep. Trustees Peabody Acad. Sci. 6: 28.

ACRO

Type-species: Platysenta atriciliata Grote, 1874 , itidem 6:28, by monotypy. Lectotype ô. U.S.A. (BNNH), designated (as type) by Hampson, 1909, Cat. Lepid. Phalaenae Br. Mus. 8: 449 .

$P$. atriciliata is a junior subjective synonym of Leucania videns Guenée, 1852 , in Boisduval \& Guenée, Hist. nat. Insectes (Lépid.) 5: 78.

PLAXIA Guenée, I 852, in Boisduval \& Guenée, Hist. nat. Insectes (Lépid.) 7: 386.

OPHI

Type-species: Phalaena macarea Cramer, 1777. Titlandsche Kapellen 2: 17, pl. 107. fig. F, by subsequent designation by Hampson, 1926, Descr. new Genera Species Lepid. Phalaenae Subfamily Noctuinae Br. Mus.: 444. Type(s), Suninam. 
PLECOPTERA Guenée, I852, in Boisduval \& Guenée, Hist. nat. Insectes (Lépid.) 6: 429.

OPHI

Type-species: Plecoptera reflexa Guenée, I 852, ibidem 6: 430, by monotypy. Holotype o. India: central (UM, Oxford).

PLECOPTERODES Hampson, i913, Cat. Lepid. Phalaenae Br. Mus. 12: 6. Available, but without included species until Hampson, I9I3, ibidem 13: viii, I4I. CATo

Type-species: Grammodes moderata Wallengren, I860, Wien. ent. Monatschr. 4: I 74, by subsequent designation by Hampson, I9I3, ibidem 13: I4I. Type(s) ㅇ․, [SouTh AFrICA]: Caffraria (NR, Stockholm).

PLECOPTEROIDES Strand, I9I 8, Z. öst. EntVer. 3: i Io.

OPHI

Type-species: Plecoptera chalciope Strand, I9I 8, ibidem 3: I Io, by original designation.

Syntypes $4 \hat{o}$, [ZAIRE] Congo: $345 \mathrm{~km}$ from Tïindu (MRAC, Tervuren).

Plecopteroides was proposed as a subgenus of Plecoptera Guenée, I852.

PLECTOTHRIPA Hampson, I9I8, Novit. zool. 25: 197.

SARR

Type-species: Plectothripa excisa Hampson, I918, ibidem 25: I98, by original designation. Syntypes $2 \pi$. Singapore (BMNH).

PLEONECTOPODA Grote, i873, Bull. Buffalo Soc. nat. Sci. 1: i 36.

NOCT

Type-species: Pleonectopoda lewisi Grote, I 873, ibidem 1 : I 37, pl. 4, fig. Io, by monotypy.

Lectotype o. [U.S.A.]: Colorado Territory (BMNH), designated (as type) by Hampson, 1903, Cat. Lepid. Phalaenae Br. Mus. 4: 279.

PLEONECTYPTERA Grote, r872. See Hemeroplanis Hübner, i818.

OPHI

PLEONOTROCTA Hampson, 1926, Descr. new Genera Species Lepid. Phalaenae Subfamily Noctuinae Br. Mus.: 537.

OPHI

Type-species: Pleonotrocta xerota Hampson, I926, ibidem: 538, by original designation. Holotype $\delta$, PERU: Huancabamba (BMNH).

PLEROMA Smith, I89i. See Pleromelloida nom. n.

CUCU

PLEROMELLA Dyar, I92 I, Insecutor Inscit. menstr. 9: 40.

cucu

Type-species: Pleromella opter Dyar, I921, ibidem 9: 40, by monotypy. Syntypes

I $\mathcal{O}^{\star}, \mathrm{I}$ ㅇ, U.S.A.: Calif., Eldridge; Placer Cty., Colfax. (USNM, Washington).

PLEROMELLOIDA nom. n. for Plevoma Sinith, I89i.

CUCU

Type-species: Pleroma obliquata Smith, I89r, Trans. Am. ent. Soc. 18: II 4, by monotypy (of Plevoma Smith). Syntypes ô, ㅇ․ [U.S.A.]: Colorado (USNM, Washington). PLEROMA Smith, I89i, Trans. Am. ent. Soc. 18: iा 3.

Type-species: Pleroma obliquata Smith, r89r, by monotypy.

A junior homonym of Pleroma Sollas, I888, Rep. scient. Results Voy. Challenger (Zool.) 25 (63): 3 I2, - Spongida. The objective replacement name is Pleromelloida nom. 11.

${ }_{\ddagger} \boldsymbol{P L E S I O P H Y S A}$ Hampson, no published reference found.

HYPE

PLEURASYMPIEZA Möschler, I890, Abh. senckenb. naturforsch. Ges. 16: I 46.

SARR

Type-species: Pleurasympieza smithii Möschler, I89o, ibidem 16: I47, fig. I8, by monotypy. Syntypes 4 ó, I 9 , Puerto Rico.

$P$. smithii is a junior subjective synonym of Motya abseuzalis Walker, 1859 .

See also Motya Walker, I859; and Lussa Grote, I883.

PLEURONA Walker, I866, List Specimens lepid. Insects Colln Br. Mus. 35: I 564.

OPHI

Type-species: Pleurona falcata Walker, I 866, ibidem 35: I564, by monotypy. Holotype + , [INDIA]: S. Hindostan (BMNH).

CC, 
PLEURONODES Hampson, 1926, Descr. new Genera Species Lepid. Phalaenae Subfamily Noctuinae Br. Mus.: 508.

OPHI

Type-species: Pleurona lepticyma Hampson, i909, Trans. zool. Soc. Lond. 19: I I4,

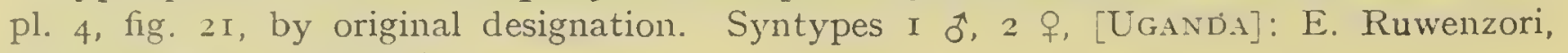
Mubuku Valley (BMNH).

PLEXIPHLEPS Warren, 1913, in Seitz, Gross-Schmett. Erde 11: I34. ACRO

Type-species: Dianthoecia stellifera Moore, I882, in Hewitson \& Moore, Descr. new Indian lepid. Insects Colln late Mr W.S. Atkinson: 123, by original designation. Lectotype o. India: [W. Bengal], Darjiling (BMNH), designated (as type) by Hampson, I908, Cat. Lepid. Phalaenae Br. Mus. 7: 126, but locality cited in error as Sikhim.

$D$. stellifera was originally proposed in $\ddagger$ Dianthecia, an incorrect subsequent spelling.

The date of publication of the part of Seitz containing page 134 was stated on page I 29 as I9I 3 January 2oth in both the German and the English editions.

PLINTHOPA Bethune-Baker, I908, Novit. zool. 15: 225.

PYRALIDAE

Type-species: Plinthopa rubra Bethune-Baker, 1908, ibidem 15: 225, by original designation.

Plinthopa was originally proposed in the Noctuidae.

PLOTHEIA Walker, [1858] i 857, List Specimens lepid. Insects Colln Br. Mus. 13: 1079, i 108. SARR

Type-species: Plotheia frontalis Walker, [1858] I 857, ibidem 13: I Io9, by monotypy. Holotype + , CEYLON (BMNH).

$P$. frontalis is a junior subjective synonym of Gadirtha decrescens Walker, [1858] i 857 , ibidem 13: $\mathrm{IO}_{4}$.

See also Egelesta Walker, I858; Galleriomorpha Nietner, I86I ; and Othora Walker, I865. $\ddagger$ PROTHENIA; Pagenstecher, 1909, Geogr. Verbreitung Schmett.: 424 .

An incorrect subsequent spelling of Plotheia Walker, [1858]. Neave, 1940, Nomencl. zool. 3: 936 cited 'pro Prothymia Huebner, I823' but from the context of Pagenstecher's work Plotheia was intended.

${ }_{\ddagger} P A N T O L A$ Boisduval, no published reference found. This name occurs on a specimen of Plotheia decrescens (Walker) in BMNH bearing a label $\$$ Paniola cristulina Boisduval, also probably unpublished.

PLOTHEIA Walker, I863. See Thopelia nom. n.

OPHI

PLUMIPALPIA Hampson, I898, J. Bombay nat. Hist. Soc. 11: 705.

HYPE

Type-species: Plumipalpia lignicolor Hampson, I898, ibidem 11: 705, fig., by original designation. Type(s) ô, India: N.W. Himalayas, Kasauli (BMNH).

${ }_{+} \boldsymbol{P L U S I A}$ Hübner, [1806]. See Diachrysia Hübner, [182 I]

PLUS

PLUSIA Ochsenheimer, I816, Schmett. Eur. 4: 89.

PLUS

Type-species: Phalaena festucae Linnaeus, I758, Syst. Nat. (Edn Io) 1: 5I3, by subsequent designation by Duponchel, 1826, in Godart \& Duponchel, Hist. nat. Lépid. Papillons Fr. 6: [(3)]. Type(s), Type-Locality not stated (LS, London) [Europe].

CHRYSASPIDIA Hübner, [1821] i 8I6, Verz. bekannter Schmett.: 252.

Type-species: Phalaena festucae Linnaeus, I758, by subsequent designation by Grote, I 896, Entomologist's Rec. J. Var. 8: 303 .

Chrysaspidia is a junior objective synonym of Plusia Ochsenheimer, I8I6.

PLUSIDIA Butler, I879, Illust. typical Specimens Lepid. Heterocera Colln Br. Mus. 3: 27.

PLUS

Type-species: Plusidia abrostoloides Butler, I 879, ibidem 3:28, pl. 47, fig. 5, by original designation. Type(s), [JAPAN]: Hakodaté (BMNH).

$P$. abrostoloides is a junior subjective synonym of Noctua cheiranthi Tauscher, I8o9, Mém. Soc. imp. Nat. Moscou 2: 322, pl. 20, fig. 6. 
PLUSILLA Staudinger, I892, in Romanoff, Mém. Lépid. 6: 533.

ACRO

Type-species: Plusilla rosalia Staudinger, I892, ibidem 6:533, pl. 9, fig. I I, by monotypy. Holotype ô. [U.S.S.R.]: Amur district, Wladiwostok (MNHU, Berlin).

PLUSIOCALPE Holland, I 894, Psyche, Camb. 7: 29.

CHLO

Type-species: Plusiocalpe pallida Holland, I894, ibidem 7:29, pl. I, fig. I, by original designation. Syntypes $\delta$, o, West Africa (CM, Pittsburgh).

PI.USIODES Guenée, I852. See Westermannia Hübner, [1 821 ] .

CHLO

PLUSIODONTA Guenée, I852, in Boisduval \& Guenée, Hist. nat. Insectes (Lépid.) 6: 359.

OPHI

Type-species: Plusiodonta chalsytoides Guenée, I852, ibidem $6: 360$, by subsequent designation by Desmarest, (I 857), in Chenu, Encycl. Hist. nat. Papillons nocturnes: I23 (but cited for ${ }_{+}^{+}$Pluciodonta, an incorrect subsequent spelling). Holotype $q$, JAVA (BMNH).

PLUSIOPALPA Holland, i894, Psyche, Camb. 7: 9.

PLUS

Type-species; Plusiopalpa dichora Holland, 1894, ibidem 7: 9, by original designation. Type(s) ô. West Africa (CM, Pittsburgh).

PLUSIOPHAES Prout, I921, Bull. Hill Mus. Witley 1: 123.

ACRO

Type-species: Plusiophaes metallica Prout, I92I, ibidem 1: I24, pl. I7, fig. 6, by monotypy. Holotype ô. [Tanzania]: E. Tanganyika, Urindi District, Upper Ruvubu R. $(\mathrm{BMNH})$.

In the original description this genus was written as 'Plusiophaës'. Under the Code, Article 32 (c) (i), the diaeresis has been deleted.

PLUSIOTRICHA Holland, i894, Psyche, Camb. 7: ro.

PLUS

Type-species: Plusiotricha livida Holland, I894, ibidem 7: 10, by original designation. Type(s) ô. West Africa (CM, Pittsburgh).

PLUXILLOIDES Berio, I944, Memorie Soc. ent. ital. 23: 75.

ACON

Type-species: Pluxilloides hartigi Berio, I 944, ibidem 23: 76 , by original designation. Holotype o, [Iran]: Sagan-Omo, Elolo (Colln E. Berio, Genoa).

PL YNTERIA Druce, I89I, Biologia cent.-am. (Zool.) Lepid. Heterocera 1: 489.

OPHI

Type-species: Plynteria marginata Druce, I89I, ibidem 1: 489, pl. 4I, figs 5, 6, by original designation. Syntypes $\hat{0}$,, , Panama: Chiriqui and Bugaba.

POAPHILA Guenée, 1852, in Boisduval \& Guenée, Hist. nat. Insectes (Lépid.) 7: 299. Caro

Type-species: Agnomonia quadrifilaris Hübner, [I83I] I825, Zuträge Samml. exot. Schmett. 3: 37, figs 569, 570, by subsequent designation by Grote, 1874, Bull. Buffalo Soc. nat. Sci. 2: 38. Type(s), U.S.A.

PODAGRA Smith, I902, Jl N.Y. ent. Soc. 10: 48.

NOCT

Type-species: Podagra crassipes Smith, I902, ibidem 10:48, by monotypy. Syntypes $3 \hat{0}, 4$ ㅇ, [U.S.A.]: Arizona, Yuma County, Quartzsite; California, Colorado Desert, Walters Station.

PODIOPLUSIA Ichinose, 1962. See Anadevidia Kostrowicki, I961.

PLUS

POECILIA Schrank, I802. See Bryophila Treitschke, I825.

ACRO

POECILOGRAMMA Butler, i892. See Aeologramma Strand, I9Io. ACRo

POECILONOLA Hampson, I9oo, Cat. Lepid. Phalaenae Br. Mus. 2: $47 . \quad$ Noli

Type-species: Selca plagiola Hampson, I898, J. Bombay nat. Hist. Soc. 11: 44I, pl. A, fig. 2, by original designation. Lectotype ${ }^{\star}$, CEYLON: Puttalam (BMNH), designated (as type) by Hampson, 1900, ibidem 2: $4^{8}$. 
POECILOPTERA Pagenstecher, I885. See Hexamitoptera Pagenstecher, I 885.

POECOPA Bowden, 1956, Bull. ent. Res. 47: 4r8.

Type-species: Poecopa mediopuncta Bowden, 1956, ibidem 47: 4 I9, figs 6-9, by origina] designation. Holotype $\widehat{\jmath},[$ GHANA $]$ Gold Coast: Kumasi, Kwadaso (BMINH).

POENA Druce, r89r, Biologia cent.-am. (Zool.) Lepid. Heterocera 1: ${ }_{4}{ }_{3}$.

HYPE

Type-species: Poena porrectalis Fabricius sensu Druce, I S9I, ibidem 1: 484 , pl. 4 o, fig. I 4 [a misidentification here named Poena drucella sp. n.], by original designation (but cited as Hypena porrectalis Guenée). Holotype o", Guatemala: Pantaleon I $700 \mathrm{ft}$ (Champion) (BMNH)

Paratypes. Guatemala: I ${ }^{\star}$ Pantaleon I $700 \mathrm{ft}$ (Champion) (BMNH); I 0 , San Isidro I6oo ft (Champion) (BMNH). Mexico: I 웅 Tabasco Teapa, ii. (H.H.S.) (BMNH).

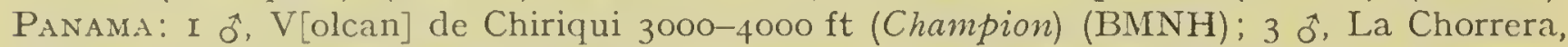
I.iv.-I 5.v.I 898 (Dolby-Tylor) (BMNH). TR1NIDAD: 2 (Jackson) (BMNH); I q, Cuparo, xi.I904 (BMNH).

Description of Poena drucella sp. n., as for Poena porrectalis Fabricius sensu Druce, I891, ibidem 1: $484, \mathrm{pl} .40$, fig. I 4 . Differs from $P$. tessellata Druce, $189 \mathrm{r}$, and $P$. albomarginata Druce, 1891 , in wing markings as shown in figures 15 and 16 . Differs in wing marlings from Phalaena porrectalis Fabricius, 1794, Ent. Sy'st. 3 (2): 223, which has not hitherto been figured but is similar to Hypena androma Druce, I89I: 434, pl. 35, fig. I 2.

Druce, r 89 r, listed the first of the three species he placed in Poena as follows:

'I. Poena porrectalis?' (Tab. XL. fig. I4.) Phalaena porrectalis, Fabr. Ent. Syst. iii.2, p. 223.

Hypena porrectalis, Guen. Sp. gén. des Lép. viii. p. 37 (o゙).'

Druce then gave the pin-label data of the specimens he was examining and continued, 'This insect varies considerably in size and colour. Our specimens are almost identical with those named Hypena porrectalis in the National Museum [BMNH]; but I do not feel quite certain that they belong to the species described by Guenéc, not having seen the type.'

Guenée, 1854, in Boisduval \& Guenée, Hist. nat. Insectes (Lépid.) 8: 37, correctly attributed Phalaena porrectalis to Fabricius, I 794. The name 'Hypena porrectalis Guenée' was not intended as the description of a new species and is not nomenclaturally available.

The type of Phalaena porrectalis Fabricius has not been found (Zimsen, 1964, Type Material of I.C. Fabricius: 574 ), but the specimens cited by Guenée under that name are in BMNH, and as Guenée himself stated are a good match for Fabricius' description. Until the type of porrectalis Fabricius is found I am accepting the Colln Guenée specimens as correctly determined.

See Frontispiece, fig. 9, Poena drucella sp. n., holotype ơ; fig. 10, Phalaena porrcctalis Fabricius, ô.

Poena Druce has a misidentified type-species and under the Code, Article 7o (a), this case should be referred to the Commission.

POENOMIA Schaus, rgr3, Ann. Mag. nat. Hist. (8) $11: 35$.

HYPE

Type-species: Poenomia turpis Schaus, r9r3, ibidem (8) 11:35, by original designation. Type(s) ô. Costa Rica: San José.

POENOPSIS Dyar, I922, Insecutor Inscit. menstr. 10: 12.

HYPE

Type-species: Poenopsis abstrusa Dyar, 1922, ibidem 10: 12, by monotypy. Holotype ô, Mexico: Guerrero.

POEONOMA Tams \& Bowden, i953, Bull. ent. Res. 43: 652.

ACRO

Type-species: Phragmatiphila serrata Hampson, I9Io, Cat. Lepid. Phalaenae Br. Mus. 9: 268, pl. 142, fig. 31, by original designation. Holotype ơ. UGANDA: Ruwenzori (BMNH).

POESULA Walker, 1858, List Specimens lepid. Insects Colln Br. Mus. 15: $18_{4} \mathrm{I}$.

OPHI

Type-species: Poesula delinquens Walker, 1858 , ibidem 15: $184 \mathrm{I}$, by monotypy. Holotype ơ, [Brazil): [R. Amazon], [Tefé] Ega (BMNH). 
$\ddagger$ PAESULA; Butler, x 879, Trans. ent. Soc. Lond. 1879: 39.

An incorrect subsequent spelling.

POETA Walker, 1865, List Specimens lepid. Insects Colln Br. Mus. 33: I Ior.

OPHI

Type-species: Poeta denotalis Walker, x865, ibidem 33: г гог, by monotypy. Lectotype 6. Borneo: Sarawak (UM, Oxford), designated (as type) by Swinhoe, 1900, Cat. east. and Aust. Lepid. Heterocera 2: 84.

POGONITIS Sodoffsky, 1837. See Herminia Latreille, 1802.

HYPE

POGOPUS Dyar, 1914, Proc. U.S. natn. Mus. 47: 217.

HYPE

Type-species: Pogopus mictochroma Dyar, I91 4, ibidem 47: 2 I 7, by original designation. Syntypes 3 0 , I 5 ㅇ, PANAMA : Cabima; La Chorrera; R. Trinidad (USNM, Washington).

POLACANTHOPODA Hampson, igoi, Cat. Lepid. Phalaenae Br. Muss. 3: xvii, 573. Agar Type-species: Hespagarista tigrina Druce, I882, Proc. zool. Soc. Lond. 1882: 788, pl. 6o, fig. 4, by original designation. Syntypes, [Nigeria]: Calabar (BMNH). Cameroun.

POLENTA Morrison, I875, Proc. Boston Soc. nat. Hist. 18: I 24.

ACRO

Type-species: Schinia tepperi Morrison, 1875, Proc. Acad. nat. Sci. Philad. 27: 68, by original designation. Type(s), [U.S.A.]: Texas.

${ }_{+}^{+}$POLIA Hübner, [1806]. See Polymixis Hübner, [1820].

CUCU

POLIA Ochsenheimer, I8I6, Schmett. Eur. 4: 73.

HADE

Type-species: Phalaena nebulosa Hufnagel, r 766, Berlin. Mag. 3 (4) : 4I 8, by subsequent designation by Curtis, I 829 February Ist, Br. Ent. 6: 248, but cited as 'nebulosa Och.', an incorrect authorship. Type(s), [Germany]: Berlin.

Incorrect type-species designation: Noctua compta [Denis \& Schiffermüller], 1775, a name not originally included in Polia, and not linked with one of the originally included names when cited by Duponchel, 1829 [March 2Ist], in Godart \& Duponchel, Hist. nat. Lépid. Papillons Fr. 7 (2): 7 I.

POLIA Boisduval, i828, Eur. Lepid. Index meth.: 73 .

Type-species: Phalaena nebulosa Hufnagel, 1766 , by subsequent designation by Curtis, I 829, Br. Ent. 6: 248, but cited as 'nebulosa Och.', an incorrect authorship.

A junior homonym of Polia Ochsenheimer, 1816 , which is also the objective replacement name.

APLECT A Guenée, I838, Amnls Soc. ent. Fr. 7: 2 I 7.

Type-species: Phalaena nebulosa Hufnagel, I 766 , by subsequent designation by Guenée, I 852, in Boisduval \& Guenée, Hist. nat. Insectes (Lépid.) 6: 77.

Aplecta is a junior objective synonym of Polia Ochsenheimer, I8I6.

POLIA Boisduval, 1828. See Polia Ochsenheimer, I8I6.

HADE

POLICOCNEMIS Benjamin, 1932, Bull. S. Calif. Acad. Sci. 31: 30.

ACRO

Type-species: Policocnemis ungulatus Benjamin, I932, ibidem 31: 3I, by original designation. Holotype ô. U.S.A.: Texas, Alpine (USNM, Washington).

POLIOBRYA Hampson, I908, Cat. Lepid. Phalaenae Br. Mus. 7: xiv, 6I 7.

ACRO

Type-species: Bryophila patula Püngeler, I907, Dt. ent. Z. Iris 19: 21 7, pl. 8, fig. I4, by original designation. Syntypes $2 \hat{0},\left[\mathrm{CHINA}_{1}\right.$ : [Sinkiang-Uigur], N. of Korla, Bergen (MNHU, Berlin).

POLIODES Hampson, 1905. See Poliodestra Hampson, I905.

HADE

POLIODESTRA Hampson, 1905, Cat. Lepid. Phalaenae Br. Mus. 5: xii, 6 Io.

HADE

Type-species: Mamestra flavidentula Schaus, 1903, Jl N.Y. ent. Soc. 11: 230, by original designation (for Poliodes Hampson). Type(s), Chile (USNM, Washington).

Poliodestra was proposed as the objective replacement name for Poliodes Hampson, 1905. 
POLIODES Hampson, 1905, Cat. Lepid. Phalaenae Br. Mus. 5: 359.

Type-species: Mamestra flavidentula Schaus, 1903, by original designation.

A junior homonym of Poliodes Rothschild \& Jordan, 1903, Novit. zool. 9 Suppl: 285, Lepid., Sphingidae. The objective replacement name is Poliodestra Hampson, I905.

POLIOFOCA Hampson, 1926, Descr. new Genera Species Lepid. Phalaenae Subfamily Noctuinae Br. Mus.: 5 I2.

OPHI

Type-species: Talapa gebenna Swinhoe, 1903, Ann. Mag. nat. Hist. (7) 11: 509, by original designation. Syntypes $\hat{o}$, [Thanland] Siam: Muok-lek (BMNH). Singapore.

POLIONYCTA Hampson, 1908, Cat. Lepid. Phalaenae Br. Mus. 7: I6. Available, but without included species until Hampson, I909, ibidem 8: 4 I.

ACRO

Type-species: Polionycta apicata Hampson, I909, ibidem 8: 4I, fig. ro, by subsequent monotypy. Holotype + , Panama: Obispo (BMNH).

POLIOTHRIPA Hampson, 1902, Ann. S. Afr. Mus. 2: 3 II.

NOLI

Type-species: Poliothripa niphostena Hampson, x902, ibidem 2: 312, by monotypy. Type(s) q, South Africa: Natal (B.MNH).

P. niphostena Hampson is a junior secondary homonym of Sor[o]costia niphostena Lower, 1896, Trans. R. Soc. S. Aust. 20: I 53, both having been placed in the genus Nola Leach by Son, I933, Amn. Transv. Mus. 15: 213. The objective replacement name for $P$. niphostena Hampson is Nola steniphona Son, I933, ibidem 15: 2 I3.

POLIOTHRIPA Hampson, I9I2. See Megastopolia, Hampson, I9I8.

OPHI

$\ddagger$ POL YCHRISIA, misspelling. Sec Polychrysia Hübner, [182 I].

PLUS

POL YCHRYSIA Hübner, [I821] I8I6, Verz. bekannter Schmett.: 251.

PLUS

Type-species: Noctua moneta Fabricius, 1787, Mantissa Insect. 2: I62, by monotypy.

Type(s), Austria (Type(s) lost, Zimsen, I964, Type Material of I. C. Fabricius: 550).

$\ddagger$ POL YCHRISIA ; Bethune-Baker, 1906, Novit. zool. 13: $27 \mathrm{I}$.

An incorrect subsequent spelling.

POLYDESMA Boisduval, 1833, Nouv. Amnls Mus. Hist. nat. Paris 2: 256.

OPHI

Type-species: Polydesma umbricola Boisduval, I 833, ibidem 2: 256, by subsequent designation by Desmarest, (I857), in Chenu, Encycl. Hist. nat. Papillons nocturnes: io8. Neotype ․ Mauritius (BMNH), designated by Viette, I967, Bull. Soc. ent. Fr. 72 : 202. This neotype designation fulfilled the requirements of the Code, Article 75 (c). A second neotype (also in BMNH), has been designated by Berio, 197I, Annali Mus. civ. Stor. nat. Giacomo Doria 78: 28I, for an externally similar but different species. Under the Code, Article 75 (d) the second neotype has no validity.

Polydesma and its type-species were also published in I833 in Faune ent. Madagascar, Bourbon et . Taurice Lépid. : I08, pl. 13, fig. 5.

See also Trichopolydesma Berio, 1954.

ANTHEMOISIA Blanchard, 1840, in Brullé, Hist. nat. Anim. articulés 3: $52 \mathrm{I}$.

Proposed, unnecessarily, as an objective replacement name for Polydesma Boisduval, I 833, which Blanchard considered to be a junior homonym of Polydesmus Latreille, i 802, in Sonnini’s Buffon, Hist. nat. gén. et particulière des Crustacés et Insectes 3 : 44, - Myriapoda. ANTHEMOESSA Agassiz, 1846, Nomencl. zool. Index univl.: 25.

An unjustified emendation of Anthemoisia Blanchard, i 8 qo. Agassiz proposed this emendation as $\ddagger A$ nthemoëssu, an incorrect original spelling which under the Code, Article 32 (c), must be corrected by the deletion of the diaeresis.

ANODAPHA Moore, [1885] i 887, Lepid. Ceylon 3: 91.

Proposed, unnecessarily, as an objective replacement name for Polydesma Boisduval, s 833, which MIoore considered to be a junior homonym of Polydesmus Latreille, i 802, in Sonnini's Buffon, Hist. nat. gén. et particulière des Crustacés et Insectes 3: 44, - Myriapoda. 
POLYDESMIOLA Strand, I916, Lepid. Niepeltiana 2: I.

OPHI

Type-species: Polydesmiola niepelti Strand, I9 16, ibidem 2: 2, pl. I5, fig. 4, by original designation. Holotype $\hat{\sigma}$, Solomon Is: Bougainville (BMNH).

POLYGNAMPTIA Schaus, I9I4, Proc. U.S. natn. Mus. 46: 54I.

OPHI

Type-species: Polygnamptia chloristicta Schaus, I9I 4, ibidem 46: 54I, by original designation. Type(s) ô, [French Guians]: R. Maroni, St. Jean (USNM, Washington).

POLYGONIODES Hampson, 1926, Descr. new Genera Species Lepid. Phalaenae Subfamily Noctuinae Br. Mus.: 523 .

OPH I

Type-species: Amphigonia laciniata Felder \& Rogenhofer, I874, Reise öst. Fregatte Novara (Zool.) 2 (Abt. 2) : pl. i 8 8, fig. 26, by original designation. Syntypes $q$, Venezuela (BMNH). Brazil: São Paulo.

POLYGRAMMATA Hampson, I908. See Polygrammate Hübner, i8r8.

$\Lambda \mathrm{CRO}$

POL YGRAMMATE Hübner, isr8, Zuträge Samml. exot. Schmett. 1: Io.

ACRO

Type-species: Polygrammate hebraeicum Hübner, I8I 8, ibidem 1: I0, figs 25, 26 by monotypy. Type(s) + , [U.S.A.]: Georgia [formerly] in Florida.

POL YGRA MMATA Hampson, Igo8, Cat. Lepid. Phalaenae Br. Mus. 7: 689.

An unjustified emendation of Polygrammate Hübner, I8 I8.

†IPHTHERA Hübner, I 808, Erste Zuträge Samml. exot. Schmett.: 4.

Included in a work rejected for nomenclatural purposes by Int. Commn zool. Nom., I966, Bull. zool. Nom. 23 Opinion 789:2 I 4. Placed on the Official Index of Rejected and Invalid Generic Names in Zoology: Name No. 1834.

Only included species: ${ }_{\ddagger}$ Diphthera hebraeicum Hübner, I 808, later made nomenclaturally available as Polygrammate hebraeicum Hübner, I8 8 .

GRAMMOPHORA Guenée, 1852, in Boisduval \& Guenée, Hist. nat. Insectes (Lépid.) 5: 30.

Type-species: Polygrammate hebraeicum Hübner, I 818, by subsequent designation by

Hampson, 1908, Cat. Lepid. Phalaenae Br. Mus. 7: 689, but cited as hebraica, an unjustified emendation by Hampson, I 908.

Grammophora is junior objective synonym of Polygrammate Hübner, I8 8 .

POLYGRAPTA Hampson, 1926, Descr. new Genera Species Lepid. Phalaenae Subfamily Noctuinae Br. Mus.: i 84 .

OPH I

Type-species: Polygrapta argyropasta Hampson, I926, ibidem: 185, by original designation. Syntypes $2 \sigma^{\widehat{T}},[\mathrm{GH} \Lambda \mathrm{NA}]$ Gold Coast: Kumasi (BMNH).

POL YMIXIS Hübner, [I820] I8I6, Vevz. bekannter Schmett.: 205.

CUCU

Type-species: Phalaena polymita Linnaeus, I76I, Fauna Suecica (Edn 2): 32I, by subsequent designation by Berio, 1957, Memorie Soc. ent. ital. 36: I7. Type(s), SwedEN (LS, London).

Incorrect type-species designation: Noctua filigrama Esper, I788, a name not originally included in Polymixis, cited by Hampson, 1905, Cat. Lepid. Phalaenae Br. Mus. 5: 6o. Hampson (on page I98) listed 'Noctua polymita, Hübn., [I803], Eur. Schmett., Noct. f. $4^{8}$ (1827) nec Linn.' as a junior synonym of $N$. filigrama Esper, I788. However, Hübner [1820] when he proposed Polymixis was including the true polymita Linnaeus, for he had by then realised that his polymita of $[\mathrm{I} 803]$ : fig. $4^{8}$ was a misidentification. Hübner renamed this fig. 48 as Xanthopastis flavivibica on page 2 II of his T'erzeichniss.

$\ddagger$ POLIA Hübner, [1806], Tentamen determinationis digestionis . ... [I].

Included in a work rejected for nomenclatural purposes by Int. Commn zool. Nom., 1926, Smithson. misc. Collns 73 (4) Opinion 97: 19. Also idem, 1954, Opin. Decl. int. Commn zool. Nom. 6 Opinion 278: 1 40.

Only included species: Noctua flavicincta [Denis \& Schiffermüller], 1775, which is congeneric with Phalaena polymita Linnaeus. 
POLYORYCTA Warren, IgI I, in Seitz, Macrolepid. World 3: 260.

ACON

Type-species: Phalaena dimidialis Fabricius, I 794, Eut. Sy'st. 3 (2): 224, by original designation. Type(s), India: eastern (UZM, Copenhagen).

The date of publication of the part of Seitz containing page 260 , was stated on page 253 as I9I I October I 5 th in the English edition, and 1912 January Ioth in the German edition.

POLYPHAENIS Boisduval, I840, Genera Index meth. Eur. Lepid.: I28.

ACRO

Type-species: Noctua sericata Esper, I787, Die Schmett. 4 (1): pl. 108, fig. +; I790, ibidem: 183 (as sericina, an unjustified emendation), by subsequent designation by Guenée, I852, in Boisduval \& Guenée, Hist. nat. Insectes (Lépid.) 6: 7 I, but cited as sericina Esper. Syntypes ơ, Italy: Florence (SM, Wiesbaden).

$\ddagger$ POL YPHLAENIS; Walker, 1857, List Specimens lepid. Insects Colln Br. Mus. 11: 547 .

An incorrect subsequent spelling.

$\ddagger$ †OL Y PHLENIS; Walker, I 857, List Specimens lepid. Insects Colln Br. Mus. 11 : 547.

An incorrect subsequent spelling.

$\ddagger$ POLYPHLAENIS, misspelling. See Polyphaenis Boisduval, i 8 †o.

ACRO

${ }_{+}$POLYPHLENIS, misspelling. See Polyphaenis Boisduval, I 840 .

$\triangle C R O$

POL YPLOCA Hübner, [1821] i 816, Verz. bekannter Schmett.: 273.

THYATIRIDAE

Type-species: Phalaena xanthoceros Borkhausen, I792, Naturg. Eur. Schmett. 4: 276, by monotypy.

$P$. xanthoceros is a junior subjective synonym of Noctua ridens Fabricius, I 787 , Mantissa Insect. 2: i 80 .

Polyploca was originally placed among the 'Noctuae'.

POLYPOGON Schrank, i802, Fauna Boica 2 (2): i61.

HYPE

Type-species: Phalaena tentacularia Linnaeus, i 758, Syst. Nat. (Edn Io) 1: 522 (but included by Schrank as Pyralis tentaculatis, see below), by subsequent designation by Grote, i 895, Jl N.Y. ent. Soc. 3: I 74. Type(s), Europe (LS, London).

Pyralis tentaculalis [Denis \& Schiffermüller], I775, Ankündung syst. Werkes Schmett. Wienergegend: I19, is an unjustified emendation under the present Code although at the time of its use a change of termination from the Geometrid ending-aria to the Pyralid-alis was customary. Schrank attributed tentaculalis to Hübner, an incorrect subsequent authorship. Grote designated the type-species as tentaculalis.

Incorrect type-species designation: Pyralis derivalis Hübner, I796, a name not originally included in Polypogon, and not linked with one of the originally included names when cited by Boisduval, I 836, Hist. nat. Insectes (Lépid.) 1: I 47.

Incorrect type-species designation: Pyvalis barbalis (Clerck) sensu Hübner, I796 [a misidentification later named as Pechipogo plumigeralis Hübner, [1825]] cited by Grote, I 896, Proc. Am. phil. Soc. 34:422. Grote designated two different type-species, each in a different work, each dated at the top of the pages December I895. One appeared in part 4 of the $J l N . Y$. ent. Soc. 3 which was received in BMNH on $25^{\text {th }}$ January I 896 according to a date-stamp on the wrapper of the part. The other appeared in Proc. Am. phil. Soc. 34, and printed at the bottom of page 4I 5 of the fascicle containing the citation was "Printed Feb. 5, I896". I have accepted the designation which had chronological priority.

$\ddagger$ †OPYPOGON ; Doubleday, I850, Synonymic List Br. Lepid.: I $4,27$.

An incorrect subsequent spelling.

POLYSCIERA Hampson, 1926, Descr. new Genera Species Lepid. Phalaenae Subfamily Noctuinae Br. Mus.: 588 .

OPIII

Type-species: Egnasia manleyi Leech, 1900, Trans. ent. Soc. Lond. 1900: 609, by original designation. Syntypes 5 오. JAPAN: Yokohama (BMNH). 
POLYTELA Guenée, I 852, in Boisduval \& Guenée, Hist. nat. Insectes (Lépid.) 5: I I3. HADE Type-species: Bombyx gloriosae Fabricius, I775, Syst. Ent.: 587, by subsequent designation by Moore, I884, Lepid. Ceylon 3: I3. Type(s), [IndiA]: Indiae orientalis (Type(s) lost, Zimsen, I964, Type Material of I. C. Fabricius: 522).

POL Y TELODES Hampson, 1905, Cat. Lepid. Phalaenae Br. Mus. 5: xiii, 454. HADE Type-species: Polytela florifera Walker, 1858 , List Specimens lepid. Insects Colln Br. Mus. 15: 1666, by original designation. Holotype $\delta$. TYPE-LOCALITY not stated (BMNH). [W. AFrica].

PONOMETIA Herrich-Schäffer, I868, CorrespBl. zool.-min. Ver. Regensburg 22: I 54. ACON Type-species: Ponometia ochricosta Herrich-Schäffer, I868, ibidem 22: I 54, by monotypy. Syntypes, Cuba.

POPPAEA Fawcett, 1916, Proc. zool. Soc. Lond. 1916: 722.

ACRO

Type-species: Poppaea sabina Fawcett, 1916, ibidem 1916: 722, pl. I, fig. 5, by original designation. Holotype $\widehat{o},[\mathrm{KENYA}]$ : Kedai (BMNH).

${ }_{+}$POP YPOGON, misspelling. See Polypogon Schrank, i 802.

HYPE

POROSAGROTIS Smith, I89o, Bull. U.S. natn. Mus. 38: I I, I23.

NOCT

Type-species: Agrotis muraenula Grote \& Robinson, I868, Trans. Am. ent. Soc. 1: 352, pl. 7, fig. 48, by original designation, on page i I. Syntypes + , [U.S.A.]: Atlantic District, New York and Rhode Island.

A. muraenula is a junior subjective synonym of Mythimna vetusta Walker, 1856 , List Specimens lepid. Insects Colln Br. Mus. 9: 78 .

†PARORAGROTIS; Dod, I 91 o, Ent. News 21: 396 .

An incorrect subsequent spelling.

POROSANA Schaus, i9i3, Ann. Mag. nat. Hist (8) 11: 32.

OPHI

Type-species: Porosana uruca Schaus, I9I3, ibidem (8) 11: 32, by original designation.

Type(s) ô, Costa Rica: La Uruca (USNM, Washington).

PORPHYRINIA Hübner, [I821] I8I6, Verz. bekannter Schmett.: 256.

ACON

Type-species: Noctua purpurina [Denis \& Schiffermïller], I775, Ankïndung syst.

Werkes Schmett. Wienergegend: 88, by subsequent designation by Hampson, I9Io, Cat.

Lepid. Phalaenae Br. Mus. 10: 63. Type(s), [Austria]: Vienna district (Collection destroyed, Horn \& Kahle, 1936, Ent. Beih. Berl.-Dahlem 3: 243).

$\ddagger$ ANTHOPHIL AE Hübner, [1806], Tentamen determinationis digestionis . . . : [2].

Included in a work rejected for nomenclatural purposes by Int. Commn zool. Nom., 1926, Smithson. misc. Collns 73 (4) Opinion 97: 19. Also idem, 1954, Opin. Decl. int. Commn zool. Nom. 6 Opinion 278: I 40.

Only included species: Noctua purpurina [Denis \& Schiffermüller], r 775.

$\ddagger$ ANTOPHILA Hübner, . [1806], Tentamen determinationis digestionis . . . : [2].

A multiple spelling of $\ddagger$ Anthophilae Hübner, [I 806].

ANTHOPHILA Ochsenheimer, 1816, Schmett. Eur. 4: 93.

Type-species: Noctua purpurina [Denis \& Schiffermüller], I775, by subsequent designation by Duponchel, I 829, in Godart \& Duponchel, Hist. nat. Lépid. Papillons Fr. 7 (2): 72, but cited for $\ddagger$ Antophyla, an incorrect subsequent spelling.

A junior homonym of Anthophila Haworth, I8 I I, Lepid. Br.: 47 I, - Lepid., Glyphipterigidae. The objective replacement name is Porphyrinia Hübner, [182 I].

$\ddagger$ ANTOPHYLA: Duponchel, 1829, in Godart \& Duponchel, Hist. nat. Lépid. Papillons Fr. $7(2): 72$.

An incorrect subsequent spelling of Anthophila Ochsenheimer, I816.

HELIOMANES Sodoffsky, I837, Bull. Soc. imp. Nat. Moscou 1837 (6): 89.

Proposed, unnecessarily, as an objective replacement name for Anthophila Ochsenheimer, I8I6. 
THALPOCHARES Lederer, i 853, l'erh. zool.-bot. V'er. Wien 3: 377.

Proposed, unnecessarily, as an objective replacement name for Anthophila Ochsenheimer, I 8 I 6 .

Thalpochares was also proposed (as though for the first time) in Lederer, 1857 , Noctuinen Eur.: 43,184 .

$\ddagger$ TALPOCHARES; Pagenstecher, 1909, Geogr. Verbreitung Schmett.: 423.

An incorrect subsequent spelling.

PORRIMA Grote, I877, in Uhler, Bull. U.S. geol. geogr. Surv. Territ. 3: 769, 798. Noct

Type-species: Oria sanguinea Geyer, I832, in Hübner, Zuträge Samml. exot. Schmett.

4: 9, figs 613, 6I 4, by monotypy. Type(s) ơ. [U.S.A.]: Georgia.

Porrima was used by Lhler on page 769 in his list of Colorado insects, but was proposed

by Grote on page 798 in an Appendix to the same paper.

ORIA Guenée, 1852, in Boisduval \& Guenée, Hist. nat. Insectes (Lépid.) 6: 166.

Ty'pe-species: Oria sanguinea Geyer, I832, by monotypy.

A junior homonym of Oria Hübner, [I82I],-Lepid., Noctuidae. The objective replacement name is Porrima Grote, 1877 .

PORROTHA Gistl, I848. Sec Acontia Ochsenheimer, I8r6.

$A C O N$

POTAMOPHORA Guenće, I 852. See Ischija Hübner, [1823].

OPHI

POTERIOPHORA Boursir1, I928, Encycl. ent. B. (3) 3: 54.

CUCU

Type-species: Poteriophora radoti Boursin, I y28, ibidem B. (3) 3: 55, Pl. 5, fig. 9, by original designation. Holotype ô. SPAin: Prov. Lerida, Juneda (I.N, Karlsruhe).

$\ddagger$ POTOPSIS, misspelling. See Fotopsis Dyar, I918.

ACRO

POWELLINIA Oberthiir, I912, Études Lépid. comparée 6: 330.

NOCT

Type-species: Luperina lasserrei Oberthür, r88 r, Etudes Ent. 6: 86, pl. I I, figs I 3, I 4 , by monotypy. Syntypes, Algeria: I ô, Magenta (BMNH); I 옹 Sebdou (BMNH).

PRADATTA Moore, i 88 I, Proc. znol. Soc. Lond. 1881: 364.

NOCT

Type-species: Pradatta beatrix Moore, I88 I, ibidem 1881: 365, by original designation. Lectotype ô, INvis: [Punjab], Dharmsala (BM[NH), designated by Seymour, 1972, Bull. Br. Mus. nat. Hist. (Fnt.) 27: 59 .

PRADIOTA Walker, i866, List Specimens lepid. Insects Colln Br. Mus. 35: 1572.

OPHI

Type-species: Pradiota sejunctata Walker, 1866, ibidem 35: 1572, by monotypy. Holotype ô, JAVA (BNINH).

Pradiota when originally proposed contained a second species, but this was doubtfully included and under the Code, Article 68 (c), is nut eligible for designation as type-species.

PRAESTILBIA Staudinger, I892, Dt. ent. Z. Iris 4: 288.

ACRO

Ty'je-species: Praestibia armeniaca Staudinger, i 892, ibidem 4:288, pl. 3, fig. Io, by monotypy. Syntypes ô, [Turkey]: Amasia (MNHU, Berlin).

PRAINA Schaus, $1898, J l$ X.Y. ent. Soc. 6: 11 .

NOCT

Tyle-species: Praina radiata Schans, i 898, ibidem 6: I I4, by monotypy. Type(s), [Brazil]: Parana, Castro (TSNMI, Washington).

PRASINOPYRA Hampsol1, I914, Amn. Mag. nat. Hist. (8) 13: 202.

$\operatorname{ACON}$

Type-species: Chlorhoda metacausta Hampson, Iy1o, Cat. Lepid. Phalaenue Br. Mus.

10: 479, fig. I 36 , by original designation (for Chlorhoda Hampson, 1910). Syntypes 3 万,

3 ‥ CUBA: Baracoa (BMNH).

Prasionopyra was proposed as the objective replacement name for Chlorhoda Hampson, I $) 10$.

CHI.()RH(J)A Hampson, igio, Cat. Lepid. Phalaenae Br. Mus. 10: 8, 479.

Type-species: Chlorhoda metacaust, Hampson, mo, by original designation. 
A junior homonym of Chlorhoda Hampson, I90I, ibidem 3: 42I, - Lepid., Arctiidae. The objective replacement name is Prasinopyra Hampson, I9I 4.

PRAXIS Guenée, I 852, in Boisduval \& Guenće, Hist. nat. Insectes (Lépid.) 7: $28 . \quad$ o PHI

Type-species: Praxis edwardsii Guenée, I 852, ibidem 7: 29, by subsequent designation by Viette, 1951, Bull. mens. Soc. limn. Lyon 20: i6I. Holotype ô. Australia (MNHN, Paris).

PREMUSIA Walker, I858, List Specimens lepid. Insects Colln Br. Mus. 15: I 78o.

OPHI

Type-species: Premusia intrahens Walker, I858, ibidem 15: I 780 , by monotypy.

Holotype ơ, Borneo: Sarawak (BMNH).

See also Dysedia Rogenhofer, 1874 .

$\ddagger$ REMUSIA; Walker, [1863] I864, J. Proc. Limn. Soc. (Zool.) 7: I 73.

An incorrect subsequent spelling.

PRICOMIA nom. n. for Micropia Hampson, I909.

ACRO

Type-species: Prometopus rhodocentra Lower, 1902, Trans. R. Soc. S. Aust. 26: 222, by subsequent monotypy (of Micropia Hampson). Syntypes 2 ․, Australia: N.S.W., Broken Hill.

MICROPIA Hampson, I9o8, Cat. Lepid. Phalaenae Br. Mus.. 7: 5. Available, but without included species until Hampson, I909, ibidem 8: xi, 373.

Type-species: Prometopus rhodocentra Lower, 1902, by subsequent monotypy.

A junior homonym of Micropia Gray, I868, Synopsis Species Whales Dolphins Colln Br. Mus.: 6, - Mammalia. The objective replacement name is Pricomia nom. n.

PRIONOFRONTIA Hampson, ig02, Ann. S. Afr. Mus. 2: 350.

OPHI

Type-species: Prionofrontia erygidia Hampson, I902, ibidem 2: 35I, by monotypy.

Holotype $\widehat{0}$. South Africa: Natal, Victoria District (BMNH).

PRIONOPHORA Meyrick, I879. See Meyrickella Berg, I898.

OPHI

PRIONOPTERA Herrich-Schäffer, I858, Samml. neuer oder wenig bekannter aussereur.

Schmett. 1: 69, 84 .

CATO

Type-species: Ophiusa serra Herrich-Schäffer, I856, ibidem 1 (I): wrapper, pl. 90, fig. 5I6, by monotypy. Type(s) $q$, Venezuela, cited on wrapper containing the plates.

$O$. serra is dated from the wrapper of the plates and not from the title-page of the work, I $850-1858$.

PRIONOPTERINA Hampson, I926, Descr. new Genera Species Lepid. Phalaenae Subfamily Noctuinae Br. Mus.: 6I5.

OPHI

Type-species: Prionophora grammatistis Meyrick, i 897, Trans. ent. Soc. Lond. 1897: 374 , by original designation. Syntypes $6(\hat{\sigma}, 9)$, Australia: Queensland, Duaringa (BMNH).

PRIONOXANTHIA Draudt, 1950, Mitt. münch. ent. Ges. 40: I 22.

ACRO

Type-species: Prionoxanthia cinnamomina Draudt, I950, ibidem 40 : I22, pl. 8, fig. 7 , by monotypy. Syntypes $\hat{0}$,, , China: [Shensi Prov.], Tai-pei-shan (MAK, Bonn).

$P$. cimnamomina is a junior subjective synonym of Pygopteryx suava Staudinger, I887. See also Pygopteryx Staudinger, i 887.

PRISTANEPA Hampson, I926, Descr. new Genera Species Lepid. Phalaenae Subfamily Noctuinae Br. Mus.: 206.

OPH I

Type-species: Pristanepa platti Hampson, 1926, ibidem: 206, by original designation. Syntypes I 0,3 , [Sourh AFrica] : Natal, Durban (BMNH).

PRIS TOCERAEA Karsch, I895, Ent. Nachr. 21: 349.

AGAR

Type-species: Agarista eriopis Herrich-Schäffer, 1853, Samml. nener oder wenig bekannter aussereur. Schmett. 1(I): wrapper, pl. 7, fig. 3I ; I856 \& I858 : I3, 7I, 78, by original designation. Type(s), MAdagascar. 
A. eriopis is dated from the wrapper of the plates and not from the title-page of the work, I $850-1858$.

†PROTOCERAEA; Kirby; I 896, Ann. Mag. nat. Hist. (6) 18: 377 .

An incorrect subsequent spelling.

PROANNAPHILA Rindge \& Smith, 1952, Bull. Am. Mus. nat. Hist. 98: 200.

ACRO

Type-species: Annaphila danistica Grote, I873, Bull. Buffalo Soc. nat. Sci. 1: I5I, pl. 4, fig. 7, by original designation. Syntypes ô, ㅇ, [U.S.A.]: Nevada Territory (BMNH).

Proannaphila was originally described as a subgenus of Annaphila Grote, I873.

PROCHLORIDEA Barnes \& MicDunnough, I9I I, Jl N.Y. ent. Soc. 19: I 53. ACRo

Type-species: Prochloridea modesta Barnes \& McDunnough, I9I I, ibidem 19: I53,

by original designation. Syntypes 4 우, [U.S.A.]: New Mexico, Ft Wingate.

PROCOERIA Hampson, 1926, Descr. new Genera Species Lepid. Phalaenae Subfamily Noctuinae Br. Mus.: 237. OPHI

Type-species: Procoeria mesoneura Hampson, 1926, ibidem: 238, by original designation. Holotype $\hat{o}$, BRAzIL: Rio Janeiro, Tijuca (BMNH).

PROCONIS Hampson, I902, Ann. S. Afr. Muts. 2: 362.

OPHI

Type-species: Proconis abrostoloides Hampson, 1902, ibidem 2: 362 , by monotypy. Holotype $\widehat{0}$, Sourh Africa: Cape Colony, Annshaw (BMNH).

PROCRATERIA Hampson, I905, Ann. S. Afr. Mus. 3: 426.

$\mathrm{AC} \mathrm{RO}$

Type-species: Procrateria noloides Hampson, I905, ibidem 3: 426, by original designation. Holotype , [RHodesia]: Mashonaland (BNINH).

PROCRIOSIS Hampson, I9Io, Cat. Lepid. Phalaenae Br. Mus. 10: 4, 7I I.

$\operatorname{ACON}$

Type-species: Procriosis dileuca Hampson, I910, ibidem 10:71 I, fig. 202, by original designation. Holotype $q$, [KENYA] Brit. E. Afr.: Voi (BMNH).

$\ddagger$ PROCUS Oken, I8 15 . See Procus Agassiz, i 846 .

ACRO

PROCUS Agassiz, I 846, Nomencl. zool. Nomina syst. Generum Animalium: $57 . \quad$ ACRo

Type-species: Noctua latruncula [Denis \& Schiffermüller], I775, Ankündung syst. Werkes Schmett. Wienergegend: 89, by monotypy. Type(s), Austria: Vienna district (Collection destroyed, Horn \& Kahle, 1936, Ent. Beih. Berl.-Dahlem 3: 2.43).

As Agassiz did not include any species but referred Procus to Oken, I8I5 (see below), then the type-species must be the one originally included by Oken.

$\ddagger$ PROCUS Oken, 1815, Okens Lehrbuch Naturg. 3: 682.

Included in a work rejected for nomenclatural purposes by Int. Commn zool. Nom., 1956, Opin. Decl. int. Commn zool. Nom. 14 Opinion 41 7:3.

Only included species: Noctua latruncula [Denis \& Schiffermüller], I 775 .

PRODENIA Guenée, I852, in Boisduval \& Guenée, Hist. nat. Insectes (Lépid.) 5: I $59 . \quad$ acro

Type-species: Hadena retina Freyer, I845, Nenere Beitr. Schmett. 5: i6I, pl. 478. figs 2, 3, by subsequent designation by Grote, 1874, Bull. Buffalo Soc. nat. Sci. 2: 17, but cited as 'retina Friv.', an incorrect authorship. Syntypes $\delta$., , CRETE I.

$H$. retina is a junior subjective synonym of Hadena littoralis Boisduval, I883, Nouv. Annls Mus. Paris. 2: 239, pl. 13, fig. 8.

PRODICELLA Hampson, I908, Cat. Lepid. Phalaenae Br. Mits. 7: 6. Available, but without included species until Hampson, 1909, ibidem 8: ix, 227.

ACRO

Type-species: Thalpochares darena Druce, I 898, Biologia cent.am. (Zool.) Lepid. Heterocera 2: 496, pl. 95, fig. 2I, by subsequent monotypy. Syntypes ô. Mexico: Mazatlan, Presidio (BMNH).

PRODOSIA Dyar, I9I \&, Proc. U.S. natn. Mus. 47: 194. $\operatorname{ACON}$

Type-species: Prodosia mycha Dyar, I9I4, ibidem 47: 194, by original designation. Syntypes 6 ex., Panama: Taboga Island and Porto Bello. (USNM, Washington). 
PRODOTIS John, i9io, Horae Soc. ent. ross. 39: 622.

Type-species: Noctua stolida Fabricius, 1775, Syst. Ent.: 599, by monotypy. Type(s), India: eastern (Type(s) lost, Zimsen, I964, Type Material of I. C. Fabricius: 523).

PROGONIA Hampson, I896, Fauna Br. India (Moths) 4: 538.

Type-species: Progonia reniferalis Hampson, I 896, ibidem 4: 538, fig. 278, by original designation. Syntypes ô, 우, CEYLON (BMNH).

$P$. veniferalis is a junior subjective synonym of Herminia oileusalis Walker, [I859] I 858, List Specimens lepid. Insects Colln Br. Mus. 16: I I6.

PROLOPHOTA Hampson, I 896, Fauna Br. India (Moths) 4: 546.

HYPE

Type-species: Prolophota trigonifera Hampson, I896, ibidem 4: 547, fig. 284, by original designation. Type(s) ô, CEYLON: Hambantota (BMNH).

PROLUTA Saalmüller, I89i, Lepid. Madagascar: 326.

OPHI

Type-species: Proluta deflexa Saalmüller, I 89 I, ibidem: 327 , pl. Io, fig. I78, by monotypy. Syntypes 2 ex., Madagascar: Nossi-Bé I. (SNG, Frankfurt).

Proluta Saalmüller is not preoccupied by $\ddagger$ Proluta Schmarda, I86I, Nene wirbellose Thieve... 1(2): pl. 22 (see also page 32), an incorrect subsequent spelling of Protula Risso, I826, - Vermes.

PROLYMNIA Hampson, I9I I, Ann. Mag. nat. Hist. (8) 8: 440.

ACRO

Type-species: Prolymnia viola Hampson, I9I I, ibidem (8) 8: 44I, by original designation. Holotype $\hat{\sigma},[$ Ghana] Gold Coast: Kumasi (BMNH).

PROLYNCESTIS Viette, I97I, Bull. Madagascar 298: 274.

OPHI

Type-species: Prolyncestis biplagiata Viette, I97I, ibidem 298: 276, figs 3, 4, by original designation. Holotype ơ, Madagascar: Tuléar, Sept-Lacs (MNHN, Paris).

PROMETOPUS Guenée, I 852, in Boisduval \& Guenée, Hist. nat. Insectes (Lépid.) 5: $38 . \quad$ ACro

Type-species: Prometopus inassueta Guenée, I852, ibidem 5: 38, pl. 3, fig. 9, by monotypy. Lectotype $\hat{\sigma}$, [Australia] Nouvelle-Hollande (MNHN, Paris), designated by Viette, I95I, Bull. mens. Soc. linn. Lyon 20: I 59.

PROMINEA Saalmüller, I89I, Lepid. Madagascar: 482.

ОРН I

Type-species: Capnodes porrecta Saalmüller, I880, Ber. senckenb. naturf. Ges. 1879-80: 286, by monotypy. Type(s), Madagascar (SNG, Frankfurt).

PROMIONIDES Berio, I966, Amali Mus. civ. Stor. nat. Giacomo Doria 76: i 16.

ACRO

Type-species: Promionides obliqua Berio, I966, ibidem 76: i I6, by original designation. Holotype + , MadaGascar: Massif de l'Ankaratra, Manjakatompo, Forêt d'Ambahona (MNHN, Paris).

PRONECA Sivinhoe, I 89o, Trans. ent. Soc. Lond. 1890: 193.

NOLI

Type-species: Proneca fola Swinhoe, I890, ibidem 1890: I94, pl. 6, fig. 8, by monotypy. Lectotype + , Burma: Thyetmyo (BMNH), designated (as type) by Hampson, Igoo, Cat. Lepid. Phalaenae Br. Mus. 2: 52.

PRONOCTUA Smith, I894, Trans. Am. ent. Soc. 21: 44.

NOCT

Type-species: Pronoctua typica Smith, I 894, ibidem 21: 45, pl. 4, fig. I, by original designation, under the Code, Article 68 (b). Type(s), [U.S.A.]: Colorado.

$\ddagger$ PRONOPHORA, misspelling. See Meyrickella Berg, I 898 .

OPHI

PRONOTESTRA Hampson, 1905, Cat. Lepid. Phalaenae Br. Mus. 5: x, 224.

HADE

Type-species: Mamestra silenides Staudinger, 1895, Dt. ent. Z. Iris 7: 273, pl. 9, fig. I 4 , by original designation. Syntypes 20,3 , [Spain]: Andalusia, Chiclana (MNHU, Berlin). 
¥PROTONESTRA; Warren, 1909, in Seitz, Macrolepid. World 3: 82.

An incorrect subsequent spelling in the English edition; and also in the following year in the German edition.

PROPATRIA Hampson, 1903, Cat. Lepid. Phalaenae Br. Mus. 4: xx, 651.

NOCT

Type-species: Heliothis neuroides Swinhoe, I901, Ann. Mag. nat. Hist. (7) 8: 128, by original designation. Syntypes $\delta$, ㅇ, Australia: W. Aust., Towranna Plains (UM, Oxford).

PROPERIGEA Barnes \& Benjamin, i 927, Pan-Pacif. Ent. 3: I1 2.

CUCU

Type-species: Perigea loculosa Grote, I88I, Papilio 1: I54, by original designation. Syntypes 0 , ㅇ, [U.S.A.]: Arizona, Tucson.

$\ddagger$ PROPHYLA Hübner, 1808. See Phosphila Hübner, I818.

ACRO

Prophyla has not been made nomenclaturally available in Zoology.

†PROPSALTA, misspelling. See Prospalta Walker, [1858].

ACRO

PRORACHIA Hampson, I908, Cat. Lepid. Phalaenae Br. Mus. 7: 9. Available, but without included species until Hampson, I909, ibidem 8: ix, 230.

ACRO

Type-species: Thalpochares daria Druce, I898, Biologia cent.-am. (Zool.) Lepid. Heterocera 2: 497, pl. 95, fig. 29, by subsequent monotypy. Syntypes ô, Mexico: Jalapa (BMNH).

PRORAGROTIS McDunnough, [1929] 1928, Bull. Dep. Mines, Can. 55: 33.

NOCT

Type-species: Feltia longidens Smith, I890, Bull. U.S. natn. Mus. 38: 217, by original designation. Syntypes I $\sigma^{2}$ I ㅇ. [U.S.A.]: New Mexico, Las Vegas.

PRORIVULA Schaus, I913, Ann. Mag. nat. Hist. (8) 11: 38.

OPHI

Type-species: Prorivula leucosticta Schaus, I913, ibidem (8) 11: 38, by original designation. Syntypes of, ㅇ, Costa Rica: Juan Viñas.

PROROBLEMMA Hampson, I910, Cat. Lepid. Phalaenae Br. Mus. 10: vii, 34.

ACON

Type-species: Acidalia stictopteris Butler, I88I, Trans. ent. Soc. Lond. 1881: 339, by original designation. Type(s), [BrazIL]: Amazons, Rio Jutahi, Barreira branca (BMNH).

PROROCOPIS Meyrick, I897, Trans. ent. Soc. Lond. 1897: 373.

OPHI

Type-species: Prorocopis melanochorda Meyrick, 1897, ibidem 1897: 373, by monotypy. Holotype 9 , Australia: W. Aust., Carnarvon.

PRORUACA Hampson, 1902, Ann. S. Afr. Mus. 2: 360.

OPH I

Type-species: Proruaca recurrens Hampson, 1902, ibidem 2: 360, by original designation. Holotype , [Botswana]: N'Gamiland (BMNH).

$\ddagger$ PROSAGROTIS, misspelling. See Orosagrotis Hampson, I903.

NOCT

PROSCHORA Turner, I945, Mem. Od Mus. 12: i6o.

ACON

Type-species: Proschora amaura Turner, 1945, ibidem 12: 160, by monotypy. Holotype $\delta$, Australia: Qd, Toowoomba.

PROSCRANA Turner, 1902, Proc. Linn. Soc. N. S. W. 27: 104.

Ol'H I

Type-species: Proscrana tephropis Turner, 1902, ibidem 27: 104, by subsequent designation by Berio, 1966, Annali Mus. civ. Stor. nat. Giacomo Doria 76: 64. Syntypes, Australia: $2 \hat{0}, 2$ q, Qd, Townsville (ANIC, Canberra).

PROSENIELLA Köhler, I953, Revta Soc. ent. argent. 16: 92.

CUCU

Type-species: Proseniella operosa Köhler, 1953, ibidem 16: 92, fig. 5, by original designation. Holotype $\delta$, Argentina: Santiago del Estero [Prov.], Campo Gallo (ZSBS, Munich). 
PROSOPARIA Grote, I883, Can. Ent. 15: r 30.

Type-species: Prosoparia perfuscaria Grote, I883, ibidem 15: I30, by monotypy. Type(s) ô. [U.S.A.]: Arizona.

See also Friesia Barnes \& McDunnough, rgr2.

PROSPALTA Walker, [1858] I 857, List Specimens lepid. Insects Colln Br. Mus. 13: I079, I I I4.

ACRO

Type-species: Prospalta leucospila Walker, [1858] i 857, ibidem 13: I I 4 4 , by monotypy. Lectotype ㅇ. [INDIA]: Hindostan (UM, Oxford), designated (as type) by Swinhoe, I9oo, Cat. east. and Aust. Lepid. Heterocera 2: $2 \mathrm{r}$.

¥PROPSALTA; Warren, I9I I, in Seitz, Gross-Schmett. Evde 3: 218, 500, 510.

An incorrect subsequent spelling (corrected on page 5 ro) in the German edition; but correctly spelled in the English edition. The incorrect spelling was also used by Draeseke, I928, Dt. ent. Z. Iris 42: 306.

PROSTHETA Turner, 1922, Proc. R. Soc. Vict. 35: 33.

AGAR

Type-species: Prostheta acrypta Turner, 1922, ibidem 35: 33, by monotypy. Holotype ${ }^{\text {. }}$ Australia: S. Aust., Tumby.

PROTAGROTIS Hampson, I903, Cat. Lepid. Phalaenae Br. Mus. 4: xx, 655.

NocT

Type-species: Agrotis viralis Grote, 1881, Bull. U.S. geol. geogr. Suvv. Tervit. 6: 260, by original designation. Lectotype $\widehat{\beta}$, [U.S.A.]: Nebraska (BMNH), designated (as type), by Hampson, I903, ibidem 4: 656 .

A. viralis is a junior subjective synonym of Agrotis niveivenosa Grote, 1879, ibidem 5 : 206.

PROTARACHE Hampson, i9 Io, Cat. Lepid. Phalaenae Br. Mus. 10: xiii, 373.

ACON

Type-species: Tarache eulepidea Hampson, I896, Fauna Br. India (Moths) 4: 5I 4, by original designation. Type(s) ㅇ, Ceylon: Puttalam (BMNH).

PROTEINANIA Hampson, I905, Cat. Lepid. Phalaenae Br. Mus. 5: xi, 290.

HADE

Type-species: Chloridea vigasia Schaus, 1894, Trans. Am. ent. Soc. 21: 239, by original designation. Lectotype 0 , Mexico: Las Vigas (USNM, Washington), designated (as type) by Hampson, 1905, Cat. Lepid. Phalaenae Br. Mus. 5: $29 \mathrm{I}$.

C. vigasia is a junior subjective synonym of Bombyx agavis Blasquez, 1870, Naturaleza 1: 285 , figs $5^{-1} 2$.

PROTEUXOA Hampson, I903, Cat. Lepid. Phalaenae Br. Mus. 4: xx, 649.

NOCT

Type-species: Agrotis amaurodes Lower, 1902, Proc. Linn. Soc. N.S.W. 26: 642, by original designation. Syntypes 2 ㅇ, Australia: Vic., Toorak (SAM, Adelaide).

PROTEXARNIS McDunnough, [1929] I928, Bull. Dep. Mines, Can. 55: 26.

NOCT

Type-species: Agrotis balanitis Grote, 1873, Bull. Buffalo Soc. nat. Sci. 1: 97, by original designation. Lectotype ㅇ․, [U.S.A.]: Colorado Territory (BMNH), designated (as type) by Hampson, 1903, Cat. Lepid. Phalaenae Br. Mus. 4: 313.

$\ddagger$ †ROTHENIA, misspelling. See Plotheia Walker, [1858].

SARR

PROTHEODES Swinhoe, I885, Proc. zool. Soc. Lond. 1885: 456.

ACRO

Type-species: Protheodes veprecola Swinhoe, I885, ibidem 1885: 456, by monotypy. Type(s), [INDiA]: Bombay, Poona (BMNH).

EUMILICHIA Warren, 1937, in Seitz, Gross-Schmett. Erde 11: 347.

Type-species: Protheodes veprecola Swinhoe, I885, by original designation.

The date of publication of the part of Seitz containing page 347 was stated on page 345 as 1937 June 3 oth in both the English and the German editions.

Eumilichia is a junior objective synonym of Protheodes Swinhoe, I 885. 
PROTHRINAX Hampson, igos, Cat. Lepid. Phalaenae Br. Mus. 7: 6. Available, but without included species until Hampson, 1909, ibidem 8: ix, 225. ACRO

Type-species: Aleptina luteomedia Smith, 1907, Trans. Am. ent. Soc. 33: 136, by subsequent monotypy. Syntypes $+\hat{0}, 2$, $[$ [U.S.A.]: southern Arizona, Poling.

PROTHYMIA Hübner, [1823]. See Phytometra Haworth, I809.

OPHI

$\ddagger$ PROTOCERAEA, misspelling. See Pristoceraea Karsch, I 895 .

AGARISTIDAE

PROTOCRYPHIA Barnes \& MIcl)unnough, 1918, Contr. nat. Hist. Lepid. N. Am. 4: II 2. ACON Type-species: Eustrotia secta Grote, IS79, Can. Ent. 11: 199, by original designation. Holotype $\tilde{\sigma}$, [U.S.A.]: Massachusetts (BMNH).

PROTOGYGIA McDunnough, [1929] 1928, Bull. Dep. Mines, Can. 55: 37.

NOCT

Type-species: Agrotis lagena Grote, IS75, Can. Ent. 7: 26, by original designation. Type(s), [U.S.A.]: California (B.INH).

PROTOLAMPRA McDunnough, [1929] 1928, Bull. Dep. Mines Can. 55: 66. NOCT

Type-species: Agrotis rufipectus Morrison, i 874, Proc. Boston Soc. nat. Hist. 17: 165, by original designation. Typc(s), [U.S.A.]: New York.

PROTOLEUCANIA McDunnough, 1937, Can. Ent. 69: I4I.

HADE

Type-specics: Leucania rubripennis Grote \& Robinson, i870, Trans. Am. ent. Soc.

3: 179, pl. 2, fig. 77, by original designation (for Psendolencania McDunnough). Lectotype O., [U.S.A.]: Texas (B.INH), designated (as type) by Hampson, 1905, Cat. Lepid. Phalaenae Br. Mus. 5: 577 .

Protolencania was proposed as the objective replacement name for Pseudoleucania McDunnough, 1937.

PSEUDOLEUCANIA McDunnough, 1937, Can. Ent. 69: 45.

Type-species: Leucania mbripennis Grote \& Robinson, 1870 , by original designation.

A junior homonym of Pseudolencania Staurlinger, 1899, Ergebn. Hamb. Magalhaensischen Sammelreise 4 (7): 73,- Iepicl., Noctuidac. The objective replacement name is Protoleucania McDunnough, 1937.

PROTOMECERAS Rebel, IgoI, in Staudinger \& Rebel, Cat. Lepid. palaearct. Fannengeb. 1: 240. ACRO

Type-species: Cimelia mimicaria Oberthür. I887, Bull. Soc. ent. Fr. 1887: lviii, by monotypy. Holotype ô. ALgeria: Sebdou.

C. mimicaria has been placed in the Gcometriclae and in the Thyatiridae.

PROTOMEROLEUCA Berio, 1966, Annali Mus. civ. Stor. nat. Giacomo Doria 76: I I3. ACro

Type-species: Protomeroleuca perlides Berio, i 966, ibidem 76 : I I 4, by original designation. Holotype , Madagascar: S., Bekily Reg. (MNHN, Paris).

PROTOMISELIA Sugi, I958, Tinea, Tokyo 4: i8I.

HADE

Type-species: Polia bilinea Hampson, i905, Cat. Lepid. Phalaenae Br. Mus. 5: 603, pl. 96, fig. I I, by original designation. Holotype f. JAPAN: Tokio (BMNH).

†PROTONESTRA, misspelling. Sce Pronotestra Hampson, I905.

HADE

PROTOPERIGEA McDunnough, I937, Can. Ent. 69:62.

ACRO

Type-species: Platyperigea anotha Dyar, 190., ibidem 36: 29, by original designation. Holotype $\hat{o}$. [CANADA]: British Columbia, Rerelstolic (USNM, Washington).

PROTOPHANA Hampson, i906, Cat. Lepid. Phalaenae Rr. Mass. 6: ix, izi.

CUCUT

Type-species: Herrichia cervina Edwards, 1890, Entomologica am. 6: II 4 , by original designation. Syntypes 4 ex., [U.S.A.]: N. California, Mt. Shasta (BMNH). 
PROTORTHODES McDunnough, 1943, Can. Ent. 75: 51.

HADE

Type-species: Taeniocampa curtica Smith, I890, Entomologica am. 6: 122, by original designation. Syntypes, [U.S.A.]: Cal., Sierra Nevada.

PROTOSCHINIA Hardwick, i970, Mem. ent. Soc. Can. 73: 32.

NOCT

Type-species: Noctua scutosa [Denis \& Schiffermüller], I775, Ankündung syst. Werkes Schmett. Wienergegend: 89, by original designation. Type(s), AusTria: Vienna district (Collection destroyed, Horn \& Kahle, 1936, Ent. Beih. Berl.-Dahlem 3: 243).

PROTOSEUDYRA Hampson, 1908, Cat. Lepid. Phalaenae Br. Mus. 7: 9. Available, but without included species until Hampson, I910, ibidem 9: xiv, 443. ACRO

Type-species: Caradrina picta Hampson, I 894, Fauna Br. India (Moths) 2: 263, by subsequent designation by Hampson, i9io, ibidem 9: 443. Syntypes, India: [Assam], Khasis (BMNH). [Srkkrm] Sikhim. China: Chekiang.

PROTOTRACHEA Viette, I965, Faune Madagascar 20:380.

ACRO

Type-species: Trachea leucopicta Kenrick, I9I7, Trans. ent. Soc. Lond. 1917: 90, pl. 2, by original designation. Lectotype $q$, MADAGASCAR: central (BMNH), designated (as holotype) by Viette, 1955, ibidem 20:382.

PROVIA Barnes \& McDunnough, I9ro, Can. Ent. 42: 248.

CUCU

Type-species: Provia argentata Barnes \& McDunnough, I9Io, ibidem 42: 248, by original designation. Syntypes $2 \hat{\jmath}, \mathrm{I}$ q, [U.S.A.]: Utah, Eureka.

PROXENUS Herrich-Schäffer, I845 [1850], Syst. Bearb. Schmett. Euv. 2: I90, $240 . \quad$ ACro

Type-species: Caradrina hospes Freyer, [1831] I 833, Neueve Beitr. Schmett. 1: 40, pl. 2 I, fig. 4, by monotypy. Type(s), [ITALY]: Sicily.

PSAMMATHODOXA Dyar, I92 I, Insecutor Inscit. menst. 9: 43.

OPHI

Type-species: Psammathodoxa cochlidioides Dyar, I92I, ibidem 9: 43, by monotypy. Holotype ${ }_{0}^{\lambda}$. U.S.A.: Texas, Brownsville (USNM, Washington).

PSAMMOPHILA Stephens, i85o. See Agrotis Ochsenheimer, i8i6. Nocr

PSAPHARA Walker, I 857, List Specimens lepid. Insects Colln Br. Mus. 11: 495, 607. NocT Type-species: Psaphara interclusa Walker, I857, ibidem 11: 607, by monotypy. Holotype ô, America: W. coast (BMNH). [Galapagos Is].

PSAPHIDA Walker, I 865, List Specimens lepid. Insects Colln Br. Mus. 32: 447. Cucu Type-species: Psaphida resumens Walker, I865, ibidem 32: 448, by monotypy.

Holotype $\sigma^{7}$, [U.S.A.]: Georgia (BMNH).

See also Dicopis Grote, 1874 .

$\ddagger P S A P H I D I A ;$ Dyar, I90 I, Jl N.Y. ent. Soc. 9: 84

An incorrect subsequent spelling.

$\ddagger P S A P H I D I A$, misspelling. 'See Psaphida Walker, I 865. CUCU

PSECTRAGLAEA Hampson, 1906, Cat. Lepid. Phalaenae Br. Mus. 6: xiii, 439. cucu

Type-species: Glaea carnosa Grote, I 877, Can. Ent. 9: 2 I, by original designation. Lectotype q, [U.S.A.]: Maine, Oldtown (BMNH), designated (as type) by Hampson, I906, ibidem 6: 439 .

PSECTRAXYLIA Fletcher, 1961, Ruwenzori Exped. I952 1: I83.

NOCT

Type-species: Psectraxylia boursini Fletcher, 196I, ibidem 1: I84, figs 7 \& I5I, by original designation. Holotype $\tilde{\sigma}$, Kenya: Aberdare Range, Mt Kinangop (BMNH).

PSECTROTARSIA Dognin, I907, Ammls Soc. ent. Belg. 51: 240.

Type-species: Psectrotarsia flava Dognin, 1907, ibidem 51: 240, by monotypy. Holotype $q$, PERU: Trujillo (USNM, Washington).

$\mathrm{DD}$ 
PSECTROTARSIA Hampson, I908, Cat. Lepid. Phalaenae Br. Mus. 7: 5. Available, but without included species until Hampson, I910, ibidem 9: 232.

Typc-species: Psectrotarsia fuscirena Hampson, 1910, ibidem 9: 232, fig. 93, by subsequent designation by Hampson, rgio, ibidem 9: 232. Holotype $\sigma^{x}$. Argentina: Mendoza, Trofero Suza (BMNH).

A junior homonym of Psectrotarsia Dognin, 1907. This generic name probably originated from Hampson but was used and made available by Lognin prior to its proposal and generic description by Hampson. There is no objective replacement name but $P$. fuscirena is congeneric with Psectrotarsia flac'a Dognin, the type-species of Psectrotarsia Dognin, I907. The latter is therefore available for use as a subjective replacement name.

PSECTROTARSIA Hampson, 1908. See Psectrotursia Dognin, 1907.

ACRO

PSEPHIS Guenée sensu Felder \& Rogenhofer, IS75. See Ostha Walker, I861.

OPHI

PSEUDACIDALIA Hampson, r894, Fauna Br. India (Moths) 2: xvi, 35I.

$\operatorname{ACON}$

Type-species: Aethia albicosta Moore, [1885], r887, Lepid. Ceylon 3: 232, by original designation. Type(s) 우, CExLON (BMNH).

PSEUDACONTIA Smith, IS83, Trans. Am. ent. Soc. 10: 246.

ACRO

Type-species: Tarache crustaria Norrison, i 875, Proc. Acad. nat. Sci. Philatl. 27: 70, by monotypy. Type(s), [U.S.A.]: Nebraska.

PSEUDAGLOSSA Grote, 1874, Bull. Buffalo Soc. nat. Sci. 2: 47. HYPE

Type-species: Epizeuxis lubricalis Geyer, 1832, in Hübner, Zuträge Samml. cxot. Schmett. 4: 19, figs 665, 666, by original designation. Type(s) ô, JAvA.

PSEUDALEA Turner, 1936, Proc. R. Soc. Qd 47: 39.

OPHI

Type-species: Pseudalea macrogastris Turner, 1936, ibidem 47: 40, by monotypy. Type(s) ô, Australia: N. Qd, Cape York (QM, Brisbane).

PSEUDALELIMMA Inoue, 1965, Konty't 33 (2): 221. HYPE

Type-species: Pseudalelinma miwai Inoue, 1965, ibidem 33 (2): 221, pl. Io, figs I-8, by original designation. Holotype ô. JAPAN: Miye Pref., Inabe Co., Sakamoto Valley (Colln H. Inoue, Tokyo).

PSEUDALETIA Franclemont, 1951, Proc. ent. Soc. W'ash. 53:64.

HADE

Type-species: Leucania unipuncta Haworth, I809, Lepid. Br.: 174, by original designation. LECTOTYPE ô, Great Britain: [England] Anglia (BMNH), here designated.

PSEUDALYPIA Edwards, i874, Proc. Calif. Acad. Sci. 5: i I I.

$\mathrm{AGAR}$

Type-species: Pseudalypia crotchii Edwards, I874, ibidem 5: I I, by monotypy. Syntypes 2 q, [U.S.A.]: Calif., San Diego, Warner's Ranch.

Pseudalypia was originally described in the Zygaenidae.

PSEUDAMATHES Hampson, ig Io, Cat. Lepid. Phalaenue Br. Mus. 9: xv, 508.

ACRO

Type-species: Amathes volloni Lucas, 1907, Bull. Soc. ent. Fr. 1907: 342, by original designation. Syntypes, Tunisia: Kćbili.

${ }_{\ddagger}$ PSEUDAMATHES Rothschild, 1920, Novit. zool. $27: 7$.

An incorrect subsequent citation by Neave, 1940, Nomencl. zool. 3: 961, as Rothschild was not proposing a new genus.

†PSEUDAMATHES Rothschild, I920. See P'seudamathes Hampson, 1910.

$\Lambda \mathrm{CRO}$

PSEUDANARTA Grote, 1878, Bull. IT.S. geol. geogr. Surv. Territ. 4: 178.

CUCU

Type-species: Anarta crocea Edwards, 1875, Proc. Calif. Acad. Sci. 6: 1 3.3, by monotypy. Type(s), [U.S.A.]: Oregon, Dalles. 
PSEUDANARTA Kozhanchikov, 1947. See Anartomima Boursin, 1952.

HADE

PSEUDANTHOECIA Smith, I882, Trans. Am. ent. Soc. 10: 2 I I, 2 I 3.

CUCU

Type-species: Lygranthoecia tumida Grote, I880, Bull. Brooklyn ent. Soc. 3: 30, by monotypy. Lectotype ô. [U.S.A.]: Colorado (BMNH), designated (as type) by Hampson, 1906, Cat. Lepid. Phalaenae Br. Mus. 6: II 5 .

PSEUDANTHRACIA Grote, I 874, Bull. Buffalo Soc. nat. Sci. 2: 46.

CATO

Type-species: Anthracia coracias Guenée, I852, in Boisduval \& Guenée, Hist. nat. Insectes (Lépid.) 7: 19, by original designation. Syntypes ô,

PSEUDARISTA Schaus, r9r6, Proc. U.S. natn. Mus. 50: 260, 286.

HYPE

Type-species: Pseudarista geldersi Schaus, I 916 , ibidem $50: 287$, by original designation. Type(s) ô, Surinam: Geldersland (USNM, Washington).

PSEUDATHETIS Boursin, 1937, Ent. Rdsch. 54: 366.

ACRO

Type-species: Pseudophia fixseni Christoph, i882, Horae Soc. ent. ross. 17: II3, by monotypy. Syntypes ơ, + , [U.S.S.R.] : Armenia, Ordubat (ZI, Leningrad).

PSEUDATHYRMA Butler, i892, Ann. Mag. nat. Hist. (6) 10: 299.

CATO

Type-species: Hypaetra complens Walker, I858, List Specimens lepid. Insects Colln Br. Mus. 14: i 4 I5, by original designation. Holotype ơ, Sumatra (BMNH).

PSEUDBARYDIA Hampson, I924, Ann. Mag. nat. Hist. (9) 13: 425.

OPHI

Type-species: Phalaena japeta Stoll, I78I, in Cramer, Uitlandsche Kapellen 4: Io9, pl. 346, fig. G, by original designation. Type(s), Surinam.

P. japeta is dated from the wrapper of the part and not from the title-page of the volume, I 782.

PSEUDCRASPEDIA Hampson, i898, J. Bombay nat. Hist. Soc. 11: 704.

ACON

Type-species: Pseudcraspedia punctata Hampson, I898, ibidem 11: 704, fig., by original clesignation. Lectotype $f$, [SIKkIM] Sikhim (BMNH), designated (as type) by Hampson, i 9 Iо, Cat. Lepid. Phalaenae Br. Mus. 10: 262.

${ }_{\ddagger}$ PSEUDOCRASPEDIA; Swinhoe, I901, Ann, Mag. nat. Hist. (7) 8: 17.

An incorrect subsequent spelling also used by Schaus, 1915, Trans. Am. ent. Soc. 41 : 3. Recently Todd, [1972] I97I, J.Wash. Acad. Sci. 61:267, commented that the spelling used by Schaus, I9I5, was an unjustified emendation. This is not so, as under the Code, Article 33 (a), there was no 'demonstrably intentional change in the original spelling' of the name.

†PSEUDEALODES, misspelling. See Pseudelaeodes Viette, I965. ACRo

PSEUDELAEODES Viette, 1965, Bull. Soc. ent. Fr. $70: 90$.

ACRO

Type-species: Elıeodes proteoides Kenrick, r9I7, Trans. ent. Soc. Lond. 1917: 90, pl. I, by original designation. Lectotype $\hat{\sigma}$, MADAGASCAR (BMNH), designated (as holotype) by Viette, 1967, Faune Madagascar 20 (2): 686.

†PSEUDEA LODES; Laporte, 1970, Bull. Inst. fond. Afr. noire (A) 32: 1048 .

An incorrect subsequent spelling.

PSEUDELYDNA Hampson, I894, Fauna Br. India (Moths) 2: xviii, 420.

CHLO

Type-species: Xanthia rufoflava Walker, [1857] I 856, List Specimens lepid. Insects Colln Br. MIus. 10: 467, by original designation. Syntypes I $\hat{o}, I$ f, INDIA: northern (BMNH).

PSEUDELYPTRON Berio, 1962, Annali Mus. civ. Stor. nat. Giacomo Doria 73: 201. Noct

Type-species: Pseudelyptron bilacteata Berio, r962, ibidem 73: 202, fig. 5, by original designation. Holotype $\hat{\sigma}$. [ZAIRE]: Lac Kiwu, Rwankwi (MRAC, Tervuren).

PSEUDENARGIA Boursin, 1956, Bull. mens. Soc. linn. Lyon 25: i 24.

ACRO

Type-species: Cosmia regina Staudinger, r892, Dt. ent. Z. Iris 4: 297, pl. 4, fig. 2, by original designation. Syntypes, [TURKEY]: Amasia (MNHU, Berlin). 
PSEUDEPHYRA Butler, r886, Trans. ent. Soc. Lond. 1886: 422.

$\operatorname{ACON}$

Type-species: Pseudephyra straminea Butler, I886, ibidem 1886: 422, by monotypy. Type(s), [Australia]: [Qd], Peak Downs (BMNH).

PSEUDEPUNDA Butler, i89o, Trans. ent. Soc. Lond. 1890:672.

ACRO

Type-species: Acronycta bicolor Moore, i88I, 'Proc. zool. Soc. Lond. 1881: 332, by original designation. Type(s), India: Punjab, Solun (BMNH).

PSEUDERASTRIA Hampson, I908, Cat. Lepid. Phalaenae Br. Mus. 7: xiv, 6I4. ACro

Type-species: Amyna larentica Hampson, 1894, Fanna Br. India (Moths) 2: 252, by original designation. Type(s), IndiA: [Assam], Nagas (BMNH).

PSEUDERIOPUS Warren, I9I3, in Seitz, Gross-Schmett. Erde 11: i69.

ACRO

Type-species: Zurobata albiscripta Hampson, I898, J. Bombay nat. Hist. Soc. 11: 449, by original designation. Holotype , Indi: [Assam], Khasis (BMNH).

The date of publication of the part of Seitz containing page r 69 was stated at the top of the page as 1913 April roth in both the German and the English editions.

PSEUDEUSTROTIA Warren, I9I3, in Seitz, Gross-Schmett. Erde 11: 288.

ACON

Type-species: Noctua candidula [Denis \& Schiffermüller], I775, Ankündung syst. Werkes Schmett. Wienergegend: 89, by original designation. Type(s), [AusTrIA]: Vienna district (Collection destroyed, Horn \& Kahle, 1936, Ent. Beih. Berl.-Dahlem 3: 243).

The date of publication of the part of Seitz containing page 288 was stated on page $28 \mathrm{I}$ as I9I 3 October 5 th in both the German and the English editions.

PSEUDEVA Hampson, I9I3, Cat. Lepid. Phalaenae Br. Mus, 13: xii, $447 . \quad$ Plus

Type-species: Deva purpurigera Walker, I858, List Specimens lepid. Insects Colln Br. Mus. 15: I79I, by original designation. Holotype $\delta$, CANADA: western, Orilla $(\mathrm{BMNH})$.

PSEUDINA Guenée, I 852, in Boisduval \& Guenée, Hist. nat. Insectes (Lépid.) 6: 226. ACro Type-species: Pseudina vellerea Guenée, I 852, ibidem 6: 226, pl. Io, fig. 6, by monotypy. Syntypes $2 \hat{0}, 2$ ㅇ, Br.AzIL: [Rio de Janeiro], Nouvelle-Fribourg (BMNH).

PSEUDINODES Hampson, I908, Cat. Lepid. Phalaenae Br. Mus. 7: 6. Available but without included species until Hampson, I9 Io, ibidem 9: 396.

ACRO

Type-species: Pseudinodes producta Hampson, I9I0, ibidem 9: 396, by subsequent monotypy. Holotype ô. Argentina: Entre Rios (BMNH).

PSEUDOARCTE Viette, I949, Bull. mens. Soc. linn. Lyon 18: 28.

CATO

Type-species: Arcte maurus Holland, I894, Psyche, Camb. 7: 50, pl. 2, fig. 2, by original designation. Lectotype, sex not stated, [?GABON]: Ogové (CM, Pittsburgh), designated by Clench, 1955, Revue Zool. Bot. afr. 51: 2 I.

A. maurus is a junior subjective synonym of Catephia melanis Mabille, I890, A m $\mathrm{mls}_{\mathrm{s}}$ Soc. ent. Fr. (6) $10: 43$.

PSEUDOBENDIS Butler, I 896, Entomologist 29: 254.

OPHI

Type-species: Itonia opistographa Guenće, 1852, in Boisduval \& Guence, Hist. nat. Insectes (Lépid.) 7: 2I 2, pl. 23, fig. 4, by monotypy. Holotype 0 , [Honduras]: Baie de Honduras.

PSEUDOBRYOMIMA Barnes \& Benjamin, I927, Pan-Pacif. Ent. 3: i I .

CUCU

Type-species: Bryomima distans Barnes \& McDunnough, I912, Contr. nat. Hist. Lepid. N.Am. $1(5):$ I 4, pl. 2, fig. Io, by original designation. Syntypes 1 ô, 2 ㅇ. [U.S.A.]: Arizona, Tucson and Redington.

PSEUDOBRYOPHILA Thierry-Mieg, 1905, Naturaliste (2) 19: 192. $\triangle \mathrm{CRO}$

Type-species: Pseudobryophila preciosa Thierry-Mieg, 1905, ibidem (2) 19: 193, by monotypy. Holotype q, PkRU: Rio-Colorado. 
PSEUDOCALPE Möschler, I890, Abh. senckenb. naturforsch. Ges. 16: I79.

OPHI

Type-species: Pseudocalpe tristriga Möschler, I89o, ibidem 16: I80, by PRESENT DESIGNATION. Syntypes $\sigma^{*},+$, CUBA.

$P$. tristriga was first described by Herrich-Schäffer, I868, Corresp.-Bl. zool.-min. Ver. Regensburg 22: r8 I but was without a generic name and so not nomenclaturally available. Gundlach, I88I, Contribucion Ent. Cubana 1: 329, repeated the description but still did not place tristriga in a genus.

Pseudocalpe was proposed primarily for tristriga but two other species were also mentioned.

P. tristriga was placed in Sudariophora Zeller, 1872, by Schaus, 1940, Scient. surv. P. Rico 12: 260; thus Pseudocalpe is a junior subjective synonym.

PSEUDOCALPE Hampson, I894. See Euxestis Lederer, I869.

CHLO

PSEUDOCERURA Butler, 1882, Trans. ent. Soc. Lond. 1882: I05.

CUCU

Type-species: Pseudocerura thoracica Butler, i 882, ibidem 1882: Io6, by monotypy. Type(s), Chile (BMNH).

PSEUDOCOPICUCULLIA Dumont, 1928, Encycl. ent. (B. 3) 3: 20.

CUCU

Type-species: Cucullia syrtana Mabille, I 888, Bull. Soc. ent. Fr. 1888: li, by original designation. Lectotype + , Tunisia: Gabès (MNHN, Paris), designated (as holotype) by Viette \& Fletcher, I968, Bull. Br. Mus, nat. Hist. (Ent.) 21: 4 I I.

PSEUDOCOPIVALERIA Buckett \& Bauer, I966, J. Lepid. Soc. $20: 84$.

Type-species: Psaphida sonoma McDunnough designation. Holotype $\sigma^{\star}$, [U.S.A.]: Calif., Sonoma Co., The Geysers (CNC, Ottawa).

†PSEUDOCRASPEDIA, misspelling. See Pseudcraspedia Hampson, 1898. ACON

PSEUDODELTOIDA Bethune-Baker, I906, Novit. zool. 13: 287 . OPH

Type-species: Pseudodeltoida aroa Bethune-Baker, 1906, ibidem 13:287, by original designation. Type(s) ô, New Guinea: [Papua], Aroa River (BMNH).

PSEUDOGERESPA Hampson, 1926, Descr. new Genera Species Lepid. Phalaenae Subfamily Noctuinae Br. Mus.: 535 .

OPH I

Type-species: Melipotis usipetes Druce, I898, Biologia cent.-am. (Zool.) Lepid. Heterocera 2: 5Io, pl. 96, fig. 23, by original designation. Syntypes, MExico: Jalapa (BMNH). Costa Rica: Volcan de Irazu (BMNH); Candelaria Mts (BMNH).

PSEUDOGIRIA Berio, I965, Boll. Soc. ent. ital. 95: I 47.

OPHI

Type-species: Pseudogiria polita Berio, I965, ibidem 95: I47, by original designation. Holotype ô. [ZAIRE] Congo: Lusambo (MRAC, Tervuren).

PSEUDOGLAEA Grote, i876, Can. Ent. 8: i 8.

NOCT

Type-species: Choephora blanda Grote, i876, Bull. Buffalo Soc. nat. Sci. 3: 86, by subsequent designation by Grote, 1895, Abh. naturw. Ver. Bremen 14: 92. Lectotype q, North America: Pacific Coast (BMNH), designated (as type) by Hampson, igo3, Cat. Lepid. Phalaenae Br. MIus. 4: 609.

C. blanda is a junior subjective synonym of Glaea olivata Harvey, I874, Bull. Buffalo Soc. nat. Sci. 2: r 20.

PSEUDOGONITIS Hampson, I894, Famna Br. India (Moths) 2: xviii, 407, 412. OPHI Type-species: Gonitis brunnea Moore, 1882, in Hewitson \& Moore, Descr. new Indian lepid. Insects Colln late $M r W$. S Atkinson: I53, by original designation. Syntypes ot, INDIA: Calcutta (BMNH).

$G$. brunnea was orginally proposed as $\ddagger$ Gonotis, an incorrect subsequent spelling.

PSEUDOGYRTONA Bethune-Baker, 1908, Novit. zool. 15: 2 18.

OPHI

Type-species: Pseudogyrtona fulvana Bethune-Baker, I908, ibidem 15: 218, by original designation. Type(s) $\delta^{\star}$, New Guinea: [Papua], Dinawa and Aroa River (BMNH). 
PSEUDOHADENA Alphéraky, I889, in Romanoff, Mém. Lépid. 5: i63.

ACRO

Type-species: Hadena armata Alphéraky, i 887, Stettin. ent. Ztg 48: 170, by monotypy. Type(s) ô. [U.S.S.R.]: Turkestan (ZI, Leningrad).

PSEUDOHEMICERAS Möschler, I890. Abh. senckenb. naturforsch. Ges. 16: I 76.

OPHI

Type-species: Pseudohemiceras krugii Möschler, I890, ibidem 16: 1 76, by monotypy. Syntypes I d . I ㅇ․ Puerto Rico (MNHU, Berlin).

${ }_{+}$PSEUDOIPS Hübner, [1806]. See Bena Billberg, 1820 .

CHLO

PSEUDOIPS Hübner, 1822, Syst.-alphab. Verz.: 59, 63.

CHLO

Type-species: Pseudoips prasinana Linnaeus sensu Hübner, I822 [a misidentification of Pyralis fagana Fabricius, I781, Species Insect. 2: 276], by subsequent designation by Fletcher, 1966, Entomologist's Gaz. 17: i6 (but cited as prasinana Linnaeus). LECTOTYPE 9 , EUROPE (UZMI, Copenhagen), here designated [photograph examined].

The type-species was originally inclucled by Hübner as 'Prasinana L. 158. Pseudoips ampla.' The 158 referred to Hiibner, [1 799], Samml. eur. Schmett. 7: pl. 25, fig. I58. This figure is of fagana Fabricius, not of prasinana Linnaeus as fixed by the lectotype designation of Lempke, 1947, Entomologist 80: I29. The type-species having been misidentified, the case should, under the Code, Article $70(\mathrm{a})$, be referred to the Commission.

Great confusion has existed between the interpretations of Pseudopis Hübner, I822 and the clistinct genus Bena Billberg, 1820, the type-species of which is the true Phalaena prasinana Linnaeus (see Bena Billberg, 1820). The main synonymy is as follows:

Pseudoips Hübner, I 822

fagana Fabricius, I $78 \mathrm{I}$

(The Green Silver Lines)

sylvana Fabricius, I 794

prasinana Linnaeus sensu Hübner
Bena Billberg, 1820.

prasinana Linnaeus, I 758

(The Scarce Silver Lines)

bicolorana Fuessly, I 775

quercana [Denis \& Schiffermüller), I 775 .

See Frontispiece, fig. I, Phalaena prasinana Linnaeus, lectotype 9 ; fig. 2, Bena prasinana (Linnaeus), ô; fig. 3, Pyralis fagana Fabricius, lectotype ơ; fig. 4, Pseudoips fagana (Fabricius), ㅇ. .

Incorrect type-species designation: Phaluena bicolorana Fuessly, 1775, a name not originally included in Pseudoips, and not linked with one of the originally included names when cited by Bode, 1907, Mitt. Roemermus., Hildesh. 22: 36.

See also Chloephila Costantini, 1920.

HYLOPHILA Hiibner, [1825] i816, Verz. bekannter Schmett.: 396.

Type-species: Hylophila prasinana Linnaeus sensu Hübner, [1825] [a misidentification of Pyralis fagana Fabricius, 178I], by subsequent designation by Westwood, $18_{4} 0$, Synopsis Genera Br. Insects: 107 (but cited as prasinana Linnaeus).

The type-species was originally included by Hübner, as ' 3842 . Hylophila Prasinana Linn. Syst. Phal. 285. Fagana et Sylvana Fabr. Ent. Pyr. 5.6. Hübn. Tor. 158.' As with Pseudoips the 158 referred to Hülner, [1799], and the same comments apply here.

The type-species having been misidentified, the case should, under the Code, Article 70 (a), be referred to the Commission.

Hylophila is a junior objective synonym of Pseudoips Hülmer, 1822 .

CHLOEPHORA Stephens, 1827, in Anonymous, Retrospective Rev. (2) 1: 242, 244.

Type-species: Chloephora prasinaria Fabricius sensu Stephens, 1827 [a misidentification of Pyralis fagana Fabricius, i 781 ], by subsequent designation by Guenée, 1 854 , in Boisduval \& Cruencé, Hist. nat. Insectes (Lépid.) 8: 3 (in a footnote referring to Herrich-Schäffer, 1845, Syst. Bearb. Schnett. Eur. 2: 446) but cited by Guence as prasinana. Stephens' use of $\ddagger$ prasinaria was the same as $\ddagger$ prasinaria Fabricius, 1794, Ent. Sy'st. 3 (2): 243, an incorrect subsequent spelling of Phalaena prasinana Linnaeus. Herrich-Schäffer on page $44^{6}$ placed 'prasinana L. - H ïbner, fig.] 158 ' as the senior subjective synonym of syllana F. and fagana F. Herrich-Schäffer had, however, followed Huilner who had misidentified the species and figured $P$. fagana over the caption prasinuma. 
The type-species having been misidentified, the case should, under the Code, Article 70 (a), be referred to the Commission.

Invalid designation of type-species: $P$. fagana was designated by Boisduval, I836, Hist. nat. Insectes (Lépid.) 1: r48. Boisduval in his lengthy Introduction, up to page 154 . reviewed earlier classifications and designated up to three different type-species for each generic name. In his 'Exposé de notre méthode' from pages I55-69o, no type-species designations were made for the genera he himself used. Under the Code, Article 69 (a) (iii), the type-designation of an author is eligible for consideration if he states that it is the type '. . . and if it is clear that he himself accepts it as the type-species.' Boisduval's type-designations although clearly stated do not fulfil the last requirement and so are invalid. Even though Boisduval's r 836 work was well known to lepidopterists the typedesignations contained in it have not in the past been accepted by Hemning or other authors. Acceptance of Boisduval's designation in this case would of course make no difference.

Chloëphora Agassiz, i 846, Nomencl. zool. Index univl.: 82, was proposed as an emendation but under the Code, Article 32 (c) (i), the diaeresis must be deleted.

Chloephora Stephens is a junior objective synonym of Pseudoips Hübner, r 822.

HALIAS Treitschke, r829, Schmett. Eur. 7: 227.

Type-species: Halias prasinana Linnaeus sensu Treitschke, I829 [a misidentification of Pyralis fagana Fabricius, I78I], by subsequent designation by Duponchel, 1834 , in Godart \& Duponchel, Hist. nat. Lépid. Papillons Fr. 9: r9 (but cited as 'prasinana Linn.').

The type-species was orginally included by Treitschke as 'Prasinana, Linn. W.V. Hübn. I58. Charp. (Pyr. Fagana et Sylvana, Fabr.).' As with Pseudoips the 158 referred to Hübner, [r 799], and the same comments apply here.

The citation by Duponchel is acceptable as a type-species designation as it is contained in the continuation of a layout in which Duponchel stated, in the same work 7 (2): 102, that the species so cited were the types of genera.

The type-species having been misidentified, the case should, under the Code, Article 7o (a), be referred to the Commission.

Halias is a junior objective synonym of Psendoips Hübner, I 822.

${ }_{\ddagger}$ CHLOEOPHORA; Staudinger, I887, in Romanoff, Mém. Lépid. 3: I 77.

An incorrect subsequent spelling.

PSEUDOLEUCANIA Staudinger, 1899, Evgebn. Hamb. Magalhaensischen Sammelreise 4 (7). 73.

Type-species: Pseudoleucania ignicola Staudinger, r899, ibidem: 73, fig. 25, by

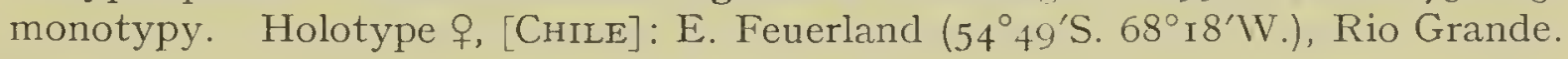

PSEUDOLEUCANIA McDunnough, 1937. See Protolencania McDunnough, 1937. Hade

PSEUDOLIGIA Staudinger, I901, in Staudinger \& Rebel, Cat. Lepid. palaearct. Faunengeb. 1: 242 .

ACRO

Type-species: Ligia similiaria Ménétriés, i 849, Mém. Acad. imp. Sci. St. Pétersb. (6) Sci. nat. 6: 296, pl. 6, fig. I2, by monotypy. Holotype q, [U.S.S.R.]: [Uzbekistan], Bolkhara.

PSEUDOLIMACODES Grote, I874, Proc. Acad. nat. Sci. Philad. 1874: 2 I 2. $\mathrm{ACRO}$

Type-species: Pseudolimacodes niveicostatus Grote, I8 $7_{4}$, ibidem 1874: 212, by monotypy. Holotype $\hat{0}$. [U.S.A.]: Massachusetts.

$P$. niveicostatus is a junior subjective synonym of Lencania littera Guenée, I 852.

See also Fagitana Walker, I865.

PSEUDOMECIA Hampson, I918, Novit. zool. 25: I 42.

CUCU

Type-species: Hypomecia lithoxylea Bang-Haas, 1912, Dt. ent. Z. Iris 26: 157, pl.

6, fig. 19, by original designation. Syntypes $3 \hat{0}$. Algeria: Batna (MNHU, Berlin).

ALLOMECIA Dumont, I928, Encycl. ent. (B. 3) 3: 91.

Type-species: Hypomecia lithoxylea Bang-Haas, I91 2, by original designation.

Allomecia is a junior objective synonym of Pseudomecia. Hampson, I918. 
PSEUDOMICRA Butler, 1892, Entomologist 25: 91.

OPHI

Type-species: Anthophila marginalis Vialker, 1865, List Specimens lepid. Insects Colln Br. Mus. 33: 802, by subsequent designation by Hampson, 1926, Descr. new Genera Species Lepid. Phalaenae Subfamily Nochinae Br. MIus.:6r6. Syntypes 2 ex., [AUstralia]: [Qd], Moreton Bay (BMNH).

PSEUDOMICRA Rebel, I907. See Pseudomicrodes Hampson, I9Io.

ACON

PSEUDOMICRODES Hampson, I9ro, Cat. Lepid. Phalaenae Br. MIus. 10: xit, 6I3. ACON

Type-species: Pseudomicra decolor Rebel, rgo7, Lepid. Sïdarabien und Insel Sokótra: 52. Republished in 1931, Denkschr. Akad. Wiss. Wien. 71 (2): 82, by monotypy (of Pseudomicra Rebel, I907). Syntypes $2 \hat{\jmath}$, I 오. Indian OcEan: Off Socotra I., Abd-al-Kuri I.

Pseudomicrodes was proposed as the objective replacement name for Pseudomicra Rebel, I907.

PSEUDOMICRA Rebel, i907, Lepid. Sïdarabien und Insel Sokotra: 52. Republished in 1931, Denkschr. Akad. Wiss. Wien 71 (2): 82.

Type-species: Pseudomicra decolor Rebel, I907, by monotypy.

Separates of this work by Rebel were dated 1907 on the original wrapper and were issued in that year, prior to its repaginated publication in I93I.

A junior homonym of Psendomicra Butler, I 892, Entomologist 25 : 9 I, - Lepid., Noctuidae. The objective replacement name is Pseudomicrodes Hampson, r9io.

PSEUDONYCTEROPHAETA Berio, 1934, Boll. Soc. ent. ital. 66: 124.

CUCU

Type-species: Pseudonycterophaeta melanoglossa Berio, I934, ibidem 66: I25, fig. I I, by original designation. Holotype ô, [LıßA]: Fezzan, Ubari.

PSEUDOPAIS Bartel, Ig03, Verh. zool.-bol. Ges. Wien 53: 127.

AGAR

Type-species: Pseudopais nigrobasalis Bartel, Igo3, ibidem 53: 127, by monotypy. Syntypes I ô, 1 f, [MALAwi]: N. Lake Nyasa, Unyika.

PSEUDOPANOLIS Inaba, 1927, Proc. imp. Acad. Japan 3: 449.

HADE

Type-species: Pseudopanolis takao Inaba, I927, ibidem 3: 449, figs I, 2, by monotypy. Syntypes $3 \hat{\jmath}, 2$, [JAPAN]: Musashi pref., Mt Takao; Nikko region.

PSEUDOPANTHEA McDunnough, I942, Can. Ent. 74: 94.

PANT

Type-species: Charadra palata Grote, I880, ibidem 12: 258 , by original designation. Lectotype ô. [U.S.A.]: Colorado (BNNH), designated (as type) by Hampson, ror3, Cat. Lepid. Phalaenae Br. Mus. 13: 37 I.

PSEUDOPHIA Guenée, 1852. See Clylie Hübner, [1823].

CATO

PSEUDOPHISMA Hampson, 1926, Descr. new Genera Species Lcpid. Phalaenae Subfamily Nochrinae Br. Mus.: ro3.

OPHI

Type-species: Phalaena pritanis Cramer, I777, Uitlandsche Kiapellen 2: 28 , pl. I I5. fig. D, by original designation. Type(s), Surinam.

$P$. pritanis is dated from the wrapper of the part and not from the title-page of the volume, 1779 .

PSEUDOPHYX Bethune-Baker, 1906, Novil. zool. 13: 260.

OPH I

Type-species: Pseudopliyx pratti Bethune-Baker, I906, ibidem 13: 260, by original designation. Syntypes $\hat{\jmath},+$, New Gunea: [Papua], Mafalu (BMNH); Babooni (BMNH); Ekeikei (BMNH); Aroa River.

PSEUDOPISARA Shiraki, 19i3, Spec. Rep. Fomosa agric. Exp. Stn 8: [429].

NOLI

Type-species: Pseudopisara quadripunctata Shiraki, I9 13, ibidem 8: [429], by original designation. Syntypes $\tilde{0}, \mathcal{q}$, [Taiwan] Formosa: Taihoku.

I'. quadripunctata was proposed as $\$ 4$-punctata, an incorrect original spelling. 
PSEUDOPLUSIA McDunnough, 1944, Mem. sth. Calif. Acad. Sci. 2: 206.

PLUS

Type-species: Phalaena oo Stoll, I780, in Cramer, Uitlandsche Kapellen 4: 45, fig. E but not fig. F ( a junior primary homonym), by original designation. Type(s) , , Surinam.

$P .00$ is dated from the wrapper of the part and not from the title-page of the volume, I782. Two coloured figures were published, the female in fig. E was the true oo, but the male in fig. F, was Noctua verruca Fabricius, I 794.

Phalaena oo Stoll is a junior primary homonym of Phalaena (Bombyx) oo Linnaeus, I 758, Syst. Nat. (Edn Io) 1: 507, - Noctuidae, Acronictinae. There is no objective replacement name but Plusia includens Walker, [1858] 1857, List Specimens lepid. Insects Colln Br. Mus. 12: 9I4, is the oldest of the junior subjective synonyms and so is available for use as a subjective replacement name.

PSEUDOPOLIA Turati, I924, Atti Soc. ital. Sci. nat. 63:84. cucu

Type-species: Pseudopolia aurora Turati, 1924, ibidem 63: 84, pl. 3, figs 21, 22, by monotypy. Syntypes I $\hat{o}$, I 9 , [LIBYA $]$ : [Benghazi] Bengasi.

P. aurora is a junior subjective synonym of Antitype rosea Rothschild, I920, Novit. zool. 27: 53 .

PSEUDOPSEUSTIS Hampson, i9io, Cat. Lepid. Phalaenae Br. Mus. 9: xv, 509. Acro

Type-species: Taeniocampa tellieri Lucas, 1907, Bull. Soc. ent. Fr. 1907: 196, by original designation. Syntypes $\hat{0}, q$, Tunisia: S. of Chotts, Kébili oasis.

TETRACHELA Hampson, I9I 8, Novit. zool. 25: I 26.

Type-species: Harpagophana diacrisioides Rothschild, 1914, ibidem 21: 326, by original designation. Syntypes I $\widehat{\delta}$, I $q$, Algeria: Alger Prov., Guelt-es-Stel (BMNH).

A junior homonym of Tetrachela Reuss, I858, Beitr. Paläont. Oest. 1(I): 5, - Crustacea. There is no objective replacement name but $H$. diacrisioides is a junior subjective synonym of Taeniocampa tellieri Lucas. Igo7. the type-species of Psendopsenstis Hampson, I9Io. The latter is thus available for use as a subjective replacement name.

PSEUDORGYIA Harvey, I 875, Bull. Buffalo Soc. nat. Sci. 2: 283.

$\mathrm{OPH}$

Type-species: Pseudorgyia versuta Harvey, 1875, ibidem 2: 284, by monotypy. Type(s) ô. [U.S.A.]: Texas (BMNH).

PSEUDORTHODES Morrison, I874, Can. Ent. 6: 253.

HADE

Type-species: Orthodes vecors Guenée, I 852, in Boisduval \& Guenée, Hist. nat. Insectes (Lépid.) 5: 376, by monotypy. Holotype ô, [U.S.A.]: New York State (BMNH).

PSEUDORTHOSIA Grote, I874, Bull. Buffalo Soc. nat. Sci. 2: i6r.

NOCT

Type-species: Pseudorthosia variabilis Grote, 1874, ibidem 2: I6I, by monotypy. Syntypes $5(\hat{0},+)$, [U.S.A.]: Sanzalito (BMNH).

PSEUDOSCHRANKIA Zimmerman, 1958, Insects Hawaii 7: 421.

HYPE

Type-species: Hypenodes epichalca Meyrick, I 899, in Sharp, Fauna Hawaiiensis 1 : I 54 , pl. 4 , fig. I 2 , by original designation. Syntypes $4(\hat{\sigma}, q)$, Hawaitan Is: Olaa (BMNH); Kilauea.

PSEUDOSEPTIS McDunnough [1929] I928, Bull. Dep. Mines, Can. 55: 29. NOCT

Type-species: Agrotis grandipennis Grote, I883, Ann. Mag. nat. Hist. (5) 11: 54, by original designation. Syntypes I $\hat{\sigma}$, I $q$, U.S.A.: New Mexico.

PSEUDOSICCIA Roeple, 1956, Tijdschr. Ent. 99: 25.

ACON

Type-species: Pseudosiccia lichenaria Roepke, I956, ibidem 99: 26, pl. 2, fig. 5, by original designation. Holotype $q$, Java: W., Tjibodas.

PSEUDOSMIA Köhler, (I952). See Despumosia nom. n.

CUCU

PSEUDOSPAELOTIS McDunnough, [1929] i928, Bull. Dep. Mines, Can. 55: 49. Noct Type-species: Agrotis haruspica Grote, I875, Bull. Buffalo Soc. nat. Sci. 2: 212, by 
original designation. Type(s) (of Agrotis unimacula Morrison), [U.S.A.]: Atlantic states (BMNH).

A. haruspica was proposed as the objective replacement name for. Agrotis unimacula Morrison, 1874, Proc. Boston Soc. nat. Hist. 17: 166, a junior primary homonym of Agrotis unimacula Staudinger, I859, Stettin. ent. Ztg 20: 213,-Noctuidae, Noctuinae.

PSEUDOSPHETTA Hampson, 1926, Descr. new Genera Species Lepid. Phalaenae Subfamily Noctuinae Br. Mus.: 22.

Type-species: Sphetta moorei Cotes d Swinhoe, I88-, Cat. Moths India (2): I72, by original designation. Type(s), [INDIA]: [W. Bengal], Darjiling (BMNH).

S. moovei was proposed as the objective replacement name for Sphetta apicalis Moore, 1879, Proc. zool. Soc. Lond. 1879: 405, a junior primary homonym of Sphetta apicalis Walker, 1865, List Specimens lepid. Insects Colln Br. Mus. 32: 457, - Noctuidae, Acronictinae.

PSEUDOSPIRIS Butler, i 895, Proc. zool. Soc. Lond. 1895: 267.

AGAR

Type-species: Pseudospiris paidiformis Butler, I895, ibidem 1895: 267, pl. I 5, figs 8, 9, by monotypy. Syntypes $1 \hat{0}, 2$ O [TaNzania]: L. Tanganyika, Fwambo (BMNH).

PSEUDOSTELLA Hampson, 1926, Descr. new Genera Species Lepid. Phalaenae Subfamily Noctuinae Br. Mus.: 60I. OPHI

Type-species: Megatomis cyanolepia Kaye, Igor, Trans. ent. Soc. Lond. 1901: I32, pl. 5, fig. I9, by original designation. Type(s), Trinidan: Verdant Vale (BMNH).

PSEUDOSTERRHA Rebel, IgoI. See Nereisana Strand, I9II.

$\operatorname{ACON}$

PSEUDOTAMILA Smith, I883, Trans. Am. ent. Soc. 10:212, 238.

NOCT

Type-species: Tamila vanella Grote, i 879, Can. Ent. 11: 197, by subsequent designation by Grote, I895, Abh. natume. Ver. Bremen 14: III. Holotype ô, [U.S.A.]: Nevada (BMNII).

T. ranella is a junior subjective synonym of Melicleptria vacciniae Edwards, i876, Proc. Calif. Acad. Sci. 6: 134.

PSEUDOTOLNA Hampson, 1926, Descr. new Genera Species Lepid. Phalaenae Subfamily Noctuinae Br. Mus.: 96.

OPHI

Type-species: Methorasa eximia Holland, 189.4, Psyche, Camb. 7: 7, by original designation. Type(s) ô, WEST AFrICA (CMI, Pittsburgh).

PSEUDOXESTIA Boursin, I953, Z. wien. ent. Ges. 38: 68. Noct

Type-species: Hiptelia apfelbecki Rebel, I901, Verh. zool.-bot. Ges. Wien 51: 798, by original designation. Holotype $\hat{o}$, [Yugoslavia]: Ivan planina.

PSEUDOXYLOMOEA Barnes \& Benjamin, 1927, Insecutor Inscit. menstr. 14: I83. ACro

Tyle-species: Apamea laetrina Druce, I 898, Biologia cent.-am. (Zool.) Lepid. Heterocera 2: 477, pl. 93, fig. 2 I, by original designation. Syntypes $0, q$, Mexico: Guerrero, Omilteme (BMNH).

PSEUDOZALISSA Bethune-Baker, Inof, Nonit. zool. 13: 250.

OPIII

Type-species: Pseudozalissa bella I3ethune-Baker, igo6, ibidem 13: 250, by original

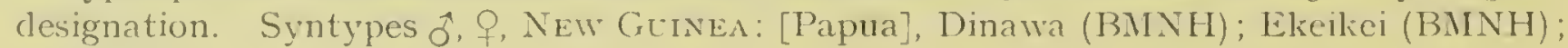
Aroa River.

PSEUDOZARBA Warren, 19г 3, in Seitz, Gross-Schmett. Erde 11: 289.

ACON

Type-species: Acontia opella Swinlioe, 1885, Proc. zool. Soc. Lond. 1885: 456, pl. 27 , fig. I6, by original designation. Lectotype $\hat{0}$. InDia: Bombay, Loma (13MNH), designated (as type) by Hampson, 1910, Cat. Lepid. Phalaenae Br. Mats. 10:597.

The date of publication of the part of Seitz containing page 289 was stated, on the same page, as 1913 October $5^{\text {th }}$ in hoth the German and the English editions. 
EUTHYTOMA Turner, I920, Trans. R. Soc. S. Aust. 44: I57, I65.

Type-species: Acontia opella Swinhoe, I 885 , by monotypy.

Euthytoma is a junior objective synonym of Pseudozarba Warren, I9I3.

PSEUDUGIA Fletcher \& Viette, I955, Bull. Inst. fr. Afr. noire 17 (A): r 79.

OPHI

Type-species: Pseudugia bistriata Fletcher \& Viette, I955, ibidem 17 (A): I79, pl. I, fig. 3, by original designation. Holotype $\hat{\sigma}$, [Guinea] French Guinea: Mont Nimba (MNHN, Paris.)

PSEUDYPSIA Dognin, I90o, Annls Soc. ent. Belg. 44: 437.

OPHI

Type-species: Pseudypsia dilectata Dognin, I900, ibidem 44: 437, by monotypy. Holotype ô, [EcUADor]: Loja (USNM, Washington).

PSEUDYRIAS Hampson, I926, Descr. new Genera Species Lepid. Phalaenae Subfamily Noctuinae Br. Mus.: I6. OPHI

Type-species: Ypsia lineata Druce, I890, Biologia cent.-am. (Zool.) Lepid. Heterocera 1: 346 , by original designation. Syntypes, Guatemala: Vera Paz; Senahu (BMNH); San Gerónimo. Panama: Volcan de Chiriqui.

PSILOMONODES Warren, I9I I, in Seitz, Macrolepid. World 3: 2 I 7.

ACRO

Type-species: Celaena agrotina Guenée, 1852, in Boisduval \& Guenée, Hist. nat. Insectes (Lépid.) 5: 22I, by original designation. Syntypes, Cuba. Brazil.

The date of publication of the part of Seitz containing page 2I 7 was stated on page 2 I3 as I9II March Ist in the English edition, and I9II December Ioth in the German edition.

PSIMADA Walker, I858, List Specimens lepid. Insects Colln Br. Mus. 15: i827.

OPHI Type-species: Psimada quadripennis Walker, I858, ibidem 15: I828, by monotypy. Type(s) \%, [IndiA]: [Mysore], Canara (BMNH).

PSORYA Schaus, I923, Zoologica, N.Y. 5: 43.

OPHI

Type-species: Psorya hadesia Schaus, I923, ibidem 5: 44, pl. 2, fig. I 8, by original designation. Lectotype + , Galapagos Is: South Seymour (USNM, Washington), designated by Todd, 1973, Proc. ent. Soc. Wash. 75: 39.

PS YCHOMORPHA Harris, I839, Am. J. Sci. 36: 3 I9.

AGAR

Type-species: Phalaena epimenis Drury, I782, Illust. nat. Hist. 3: 39, Index, pl. 29, fig. 2, by monotypy. Type(s), [U.S.A.]: Virginia.

Psychomorpha was originally described as a subgenus of Glaucopis Fabricius, 1807, Lepid., Zygaenidae.

PSYLLA Germar, I8I I. See Staurophova R.L., I8I 7.

CUCU

PTERAETHOLIX Grote, I873, Trans. Am. ent. Soc. 4: 298.

ACON

Type-species: Pteraetholix bullula Grote, I873, ibidem 4: 299, by monotypy. Syntypes $\widehat{\jmath}$, ㅇ, [U.S.A.]: central Alabama.

PTERHEMIA Guenée, I 854, in Boisduval \& Guenée, Hist. nat. Insectes (Lépid.) 8: 23.

$\mathrm{OPHI}$

Type-species: Pterhemia mutilatalis Guenée, 1854 , ibidem 8:24, pl. 7, fig. I, by monotypy. Syntypes I $\hat{0}$, I $q$, [French Guiana]: Cayenne (BMNH).

See also Pessida Walker, 1858.

PTEROCHAETA Holland, I900, Novit. zool. $7: 568$.

CATO

Type-species: Pterochaeta dohertyi Holland, I900, ibidem 7: 568, by original designation. Syntypes $5 \hat{\sigma}$, [Moluccas]: Buru I. (CM, Pittsburgh).

P. dohertyi is a junior subjective synonym of Hypaetra stigmata Moore, I877, Proc. zool. Soc. Lond. 1877: 6 I0. 
PTEROCYCLOPHORA Hampson, 1893, Illust. typical Specimens Lepid. Heterocera Colln Br. Mus. 9: 25, IO9.

Type-species: Pterocyclophora pictimargo Hampson, I893, ibidem 9: I Io, pl. I65, fig. I6 (see below), by monotypy. Lectotype q. CEYLON: Udagama (BMNH), designated (as type) by Hampson, i913, Cat. Lepid. Phalaenae Br. Mus. 12: 47 I.

$P$. pictimargo was the spelling adopted by Hampson, I 894 , Fauna Br. India (Moths) 2: 454 , as first reviser, from a multiple original spelling in which $P$. pictimargo was used on page I Io and $P$. marginalis was used on page 25 and on pl. I65, fig. I6.

PTERODONTA R.L., I8I7. See Scoliopteryx Germar, i8 I I.

OPHI

PTEROGONAGA Berio, I964, Doriana 3 (147): 4.

CHLO

Type-species: Pterogonaga chinensis Berio, I964, ibidem 3 (I 47): 5, fig. 5, by original designation. Holotype $\widehat{\sigma}$, Chrna: Chekiang, West Tien-Mu-Shan (Colln E. Berio, Genoa).

PTEROGONIA Swinhoe, i 891, Trans. ent. Soc. Lond. 1891: i 52.

CHLO

Type-species: Pterogonia episcopalis Swinhoe, I S9I, ibidem 1891: I 52, by subsequent designation by Hampson, I894, Fauna Br. India (Moths) 2: 362. Holotype ô. INDiA: S., Nilgiri Hills, Coonoor (BINH).

PTERONYCTA Fawcett, 1918, Proc, zool. Soc. Lond. 1917: 240.

OPHI

Type-species: Pteronycta fasciata Fawcett, I9I8, ibidem 1917: 24I, by subsequent designation by Gaede, 1939, in Seitz, Gross-Schmett. Evde 15: 287, but cited as fasciata Hampson, for Pteronycta Hampson (see below). LECTOTYPE $\widehat{\sigma}$, [MALAwi] Nyasaland: Zomba (E. Ballard) (BMNH), here designated.

The name Pteronycia fasciata originated from Hampson but was used, together with a bricf description, and unintentionally made nomenclaturally available by Fawcett prior to its proposal and description by Hampson.

PTERONYCTA Hampson, 1926, Descr. new Genera Species Lepid. Phalaenae Subfamily Noctuinae Br. Mus.: 122 .

Type-species: Pteronycia fasciata Hampson, 1926, ibidem: 123 (a junior primary homonym whose objective replacement name is Pteronycta fasciata Fawcett, I918), by original designation. LECTOTYPE $\delta$, [MALAwI] Nyasaland: Zomba (E. Ballard) (BMNH), here designated.

A junior homonym of Pteronycta Fawcett, I918,-Lepid., Noctuidae. This generic name originated from Hampson but was used and unintentionally made nomenclaturally available by Fawcett prior to its proposal and generic description by Hampson. The lectotype of $P$. fasciala Hampson is the same specimen as the lectotype of $P$. fasciala Fawcett, 1918. Therefore Pteronycta Fawcett, I918, is the objective replacement name.

PTERONYCTA Hampson, 1926. See Pteronycta Fawcett, 1918.

OPH I

PTEROPRISTA Warren, I889, Trans. ent. Soc. Lond. 1889: 256.

HYPE

Type-species: Pteroprista metallica Warren, I889, ibidem 1889: 257, by monotypy. Holotype $\hat{\jmath}$, [BraziL]: Amazon basin, R. Madeira, R. Mamellos (BMNH).

PTEROSCIA Morrison, I874, Proc. Boston Soc. nat. Hist. 17: I 55.

NOCT

Type-species: Pteroscia atrata Morrison, I 874, ibidem 17: I 56, by monotypy. Syntypes 2 ex., [U.S.A.]: N[ew] H[ampshire], Mt Washington (BMNH).

PTICHODIS Hübner, I8ı, Zuträge Samml. exot. Schmett. 1: 21.

CATO

Type-species: Ptichodis bistrigata Hübner, 1818, ibidem 1: 2I, figs III, II 2, by PRESENT DESIGNATION. Type(s) ö, [U.S.A.]: Neugeorgia.

Ptichodis was proposed primarily for P. bistrigata, but Geometra palumbaria [Denis \& Schiffermüller], [775, was also included for comparison.

$\ddagger$ PETROPHORA Hïbner, 1808, Erste Zuträge Samml. exot. Schmett.: 5 .

Included in a work rejected for nomenclatural purposes by Int. Commn zool. Nom., 
1966, Bull. zool. Nom. 23 Opinion 789:216. Placed on the Official Index of Rejected and Invalid Generic Names in Zoology: Name No. I880.

The only included species, $\ddagger$ Petrophora bistrigata Hübner, I808, ibidem: 5 , was later made nomenclaturally available as Ptichodis bistrigata Hübner, I8I8.

$\ddagger$ PTILODON Hübner, [1806]. See Ptilodon Hübner, I 822.

NOTODONTIDAE

$\ddagger$ PTILODON Hübner, I808. See Gonodonta Hübner, I818.

OPHI

PTILODON Hübner, I822, Syst.-alphab. Verz.: I4, I 5.

NOTODONTIDAE

Type-species: Phalaena camelina Linnaeus, 1758, Syst. Nat. (Edn 10) 1: 507, by subsequent designation by Grote, I 895, Abh. naturw. Ver. Bremen 14: 48.

This Notodontid generic name is included here because Ptilodon has in the past been used in the Noctuidae, based on an incorrect type-species designation.

$\ddagger$ PTILODON Hübner, [1806], Tentamen determinationis digestionis . . . : [1]

Included in a work rejected for nomenclatural purposes by Int. Commn zool. Nom., 1926, Smithson. misc. Collns 73 (4) Opinion 97: 19. Also idem, 1954, Opin. Decl. int. Commn zool. Nom. 6 Opinion 278: I40.

Only included species Phalaena camelina Linnaeus, 1758.

PTISCIANA Walker, I 865, List Specimens lepid. Insects Colln Br. Mus. 33: 912.

SARR

Type-species: Ptisciana seminivea Walker, I865, ibidem 33: 912, by monotypy. Lectotype $\hat{0}$. Borneo (BMNH), designated (as type) by Hampson, igi2, Cat. Lepid. Phalaenae Br. Mus. 11: 287.

See also Argyrothripa Hampson, I 894.

$\ddagger$ †TYNOTA, misspelling. See Ptyonota Hampson, I 894 .

CHLO

PTYONOTA Hampson, I 894, Fanna Br. India (Moths) 2: 407, 424.

CHLO

Type-species: Ptyonota formosa Hampson, I 894, ibidem 2: 425, fig. 239, by original designation. Type(s) ㅇ, [Burma]: Tenasserim Valley (BMNH).

$\ddagger$ PTYNOTA; Neave, I940, Nomencl. zool. 3: 1042.

An incorrect subsequent spelling.

PTYOPHORA Hampson, I893, Illust. typical Specimens Lepid. Heterocera Colln Br. Mus. 9 :

3I, I 23. HYPE

Type-species: Ptyophora ochreistigma Hampson, I893, ibidem 9: 31, I24, pl. I67, fig. 25, by monotypy. Type(s) ô, CEYLON: Nawala-pittia (BMNH).

PTYOPTEROTA Hampson, I894, Fauna Br. India (Moths) 2: 40, 75.

$\operatorname{ACON}$

Type-species: Ptyopterota obscura Hampson, I894, ibidem 2: 75, fig. 43, by original designation. Type(s) ô, [BURMA]: Tenasserim (BMNH).

PTYORHYNCHA Hampson, I 895, Trans. ent. Soc. Lond. 1895: 308.

OPHI

Type-species: Ptyorhyncha. argyresthis Hampson, I 895, ibidem 1895: 308, fig. [I5], by original designation. Type(s) + , BHUtan (BMNH).

PUCIALIA Walker, I 869, Characters undescr. Lepid. Heterocera: 25.

SARR

Type-species: Pucialia furcifera Walker, I869, ibidem: 25 , by monotypy. Lectotype ․, [Honduras]: Limas (BMNH), designated (as type) by Hampson, I912, Cat. Lepid. Phalaenae Br. Mus, 11: 336.

$P$. furcifera is a junior subjective synonym of Sebagena furcifera Walker, 1865, List Specimens lepid. Insects Colln Br. Mus. 33: 929.

See also Sebagena Walker, I 865 .

PUDORINA Gistl, I 848. See Lencania Ochsenheimer, I 8I6.

HADE

PULCHERIA Alphéraky, i 887, Stettin. ent. Ztg 48: I 70.

ACRO

Type-species: Pulcheria catomelas Alphéraky, I887, ibidem 48: I70, by monotypy. Syntypes $\hat{0}$. ㅇ. [U.S.S.R]: Turkestan (ZI, Leningrad). 
PUMORA Dyar, 1918, Proc. U.S. natn. Nus. 54: 348.

Type-species: Pumora hyperion Dyar, I918, ibidem 54:348, by original designation. Holotype \%, Mexico: Cuernaraca (USNM, Washington).

PURBIA Moore, I881, Trans. zool. Soc. Lond. 11: 70.

OPHI

Type-species: Ophideres discrepans Walker, [1858] I857, List Specimens lepid. Insects Colln Br. Mus. 13: 1227, by monotypy. Lectotype (sex not stated), Singapore (UM, Oxford), designated (as type) by Swinhoe, i goo, Cat. east. and Aust. Lepid. Heterocera 2: 175 .

PYCNODONTIS Felder, I874, in Felder \& Rogenhofer, Reise öst. Fregatte Novara (Zool.) 2 (Abt. 2): pl. 107.

AGAR

Type-species: Pycnodontis spadicea Felder, 1874, ibidem 2 (Abt. 2): pl. 107, fig. I2; Erklärung pls 75-107: 8, by subsequent designation by Hampson, 1910, Cat. Lepid. Phalaenae Br. Mus. 9: 4 II. Type(s), [Colombia]: Bogota.

PYGOPTERYX Staudinger, I887, in Romanoff, Mém. Lépid. 3: 230.

ACRO

Type-species: Pygopteryx suava Staudinger, I887, ibidem 3: 230, pl. I3 [recte I7], fig. 4, by monotypy. Syntypes I $\widehat{o}, 5$ ․, [U.S.S.R.]: Amur region, Sidemi (MNHU, Berlin); Vladivostok.

Pygoptery'x was originally placed in the Notodontidae.

See also Prionoxanthia Draudt, 1950.

PYRALIDESTHES Warren, I9I 3, in Seitz, Gross-Schmett. Erde 3: 401.

OPHI

Type-species: Rhodaria amata Butler, I879, Illust.typical Specimens Lepid. Heterocera Colln Br. Mus. 3: xri, $7^{2}$, pl. 58, fig. I I, by original designation. Type(s), Japan: Yokohama (BMNH).

The date of publication of the part of Seitz containing page 40r, was stated on page 397 as rgr 3 December ist in both the German and the English editions.

PYRALIS Linnaeus, I 758, Syst. Nat. (Edn io) 1: 533 .

PYRALIDAE

Type-species: Phalaena farinalis Linnaeus, I 758 , ibidem 1: 533, by subsequent designation by Int. Commn zool. Nom., I 957, Opin. Decl. int. Commn zool. Nom. 15 Opinion 450: 254 .

Pyralis was originally proposed as a subgenus of Phalaena Linnaeus, I758. Several species now in the Noctuidae were originally placed in Pyralis.

PYRALOIDES Rebel, I948, Z. wien. ent. Ges. 32: 55.

$\operatorname{ACON}$

Type-species: Pyraloides spodia Rebel, 1948, ibidem 32: 55, by monotypy. Holotype $\hat{o}$, EGYPT: Wadi Aideb, Gebel Elba.

PYRALOMORPHA Rebel, i917, in Rebel \& Zerny, Denkschr. Akad. W'iss. Wien 93: 432.

OPIII

Type-species: Pyralomorpha inscripta Rebel, I917, ibidem 93: 432, figs I, 2, pl. fig. ıI, by monotypy. Syntypes I ô, I $q$, Sudan: Kosti (NM, Vienna).

PYRAMARISTA Kirby, 1896, Ann. Mag. nat. Hist. (6) 18: 39I.

CATO

Type-species: Pyramarista rufescens Kirby, 1896, ibidem (6) 18: 391, by monotypy. Holotype ô. [Malawi]: Lake Nyassa, Parumbira (BMNH).

PYREFERRA Franclemont, 1937, Can. Ent. 69: 129.

cucu

Type-species: Scopelosoma pettiti (rote, I 875, Can. Ent. 7: 188, by original clesignation. Syntypes 2 ex., CANADA: Ont., Grimslyy (BMNH).

PYRGEIA köhler, 1959, Rev. Soc. ent. Argent. 21 : 101.

NOCT

Type-species: Pyrgeia rungsi Köhler, 1959, ibidem 21: 101, fig. 3, by original rlesignation. Holotype ô, [ARgentina/CunLE]: Tierre del Fuego (ZSBS, Munich). 
PYRGION Druce, I89 I, Biologia cent.-am. (Zool.) Lepid. Heterocera 1: 453.

HYPE

Type-species: Bleptina menippusalis Walker sensu Druce, I891, ibidem 1: 454, pl. 37, figs Io, II [a misidentification later described as Scopifera repanda Schaus, IgI2, Ann. Mag. nat. Hist. (8) 9: 208], by original designation. Syntypes of (of repanda), Costa Rica: San José and Juan Viñas.

Schaus, 1916, Proc. U.S. natn. Mus. 50:346 cited the type-species of Pyrgion as 'Pyrgion menippusalis of the Biologia, not Walker, = Scopifera repanda Schaus, I912.' This is a case of a misidentified type-species which under the Code, Article 70 (a), should be submitted to the Commission.

PYRIPNOA Turner, I920, Trans. R. Soc. S. Aust. 44: I57, I65.

ACON

Type-species: Thalpochares pyraspis Meyrick, I891, ibidem 14: I96, by original designation. Syntypes ô, ㅇ, Australia: Qd, Duaringa (BMNH).

PYROBLEMMA Hampson, 1926, Descr. new Genera Species Lepid. Phalaenae Subfamily Noctuinae Br. Mus.: 4 I8.

OPHI

Type-species: Pyroblemma villicosta Hampson, I926, ibidem: 4I8, by original designation. Holotype $\hat{\delta}$, [GHANA] Gold Coast: Bibianaha (BMNH).

$P$. villicosta is a junior subjective synonym of Lophiophora fulminans Bryk, igi5.

See also Lophiophora Bryk, I9I 5.

PYROCLEPTRIA Hampson, igo3, Cat. Lepid. Phalaenae Br. Muss. 4: ix, 25.

NOCT

Type-species: Heliothis cora Eversmann, I837, Bull. Soc. N'at. Moscou 1837 (6) : 47, by original designation. Syntypes, [U.S.S.R.]: [Kazakhstan], Orenburg (ZI, Leningrad); Kazan (ZI, Leningrad).

PYROIS Hübner, [I820] i 8I6, Vevz. bekannter Schmett.: 208.

ACRO

Type-species: Phalaena cinnamomea Goeze, I7SI, Ent. Beyträge 3 (3): 205 (but included by Hübner as 'cinnamomea Borkh.', an incorrect authorship), by subsequent designation by Hampson, igo8, Cat. Lepid. Phalaenae Br. Mus. 7: 26. Syntypes, TrPELOCALITY not stated [EUROPE].

SYNTOMOPUS Guenée, I 837, Amuls Soc. ent. Fr. 6: 223.

Type-species: Phalaena cimamomea Goeze, I $78 \mathrm{I}$, by monotypy.

Syntomopus is a junior objective synonym of Pyrois Hübner, [1820].

PYROPHILA Stephens, I829. See Amphipyra Ochsenheimer, I8I6. ACRO

†PYROPHILAE Ochsenheimer, I8I6. See Amphipyra Ochsenheimer, I8I6. ACRo

†PYROPHYLA Hübner, [1806]. See Amphipyra Ochsenheimer, I8I6. ACRO

PYRRHIA Hübner, [182I] I816, Verz. bekannter Schmett.: $233 . \quad$ NOCT

Type-species: Noctua rutilago [Denis \& Schiffermüller], I775, Ankündung syst. Werkes Schmett. Wienergegend: 86, by subsequent designation by Grote, I874, Bull. Buffalo Soc. nat. Sci. 2: 35. Type(s), [AustriA]: Vienna district (Collection destroyed, Horn \& Kahle, 1936, Ent. Beih. Berl.-Dahlem 3: 243).

$N$. rutilago is a junior subjective synonym of Phalaena umbra Hufnagel, I766, Berlin. Mag. 3 (3): 294 .

PYSNOIDES Berio, 1959. See Sypnoides Hampson, I9I3.

CATO

QUANDARA nom. n. for Lycangesia Hampson, rgio.

$\triangle \mathrm{CON}$

Type-species: Lycaugesia hypozonata Hampson, I9Iо, Cat. Lepid. Phalaenae Br. Mus. 10: 268, fig. 76, by original designation (for Lycaugesia Hampson). Syntypes 2 \%, 2 우 PANama: La Chorrera (BMNH).

LYCAUGESIA Hampson, igio [December gth], Cat. Lepid. Phalaenae Br. Mus. 10: 263.

Type-species: Lycaugesia hypozonata Hampson, r910, by original designation.

A junior homonym of Lycaugesia Dognin, I9 Io April, - Lepid., Noctuidae. This generic 
name probably originated from Hampson but was used and unintentionally made nomenclaturally available by Dognin prior to its proposal and generic description by Hampson for a different concept. The objective replacement name is Quandara nom. n.

RABILA Walker, I865, List Specimens lepid. Insects Colln Br. Mus. 32: $507 . \quad$ Acro Type-species: Rabila frontalis Walker, 1865 , ibidem 32: 508, by monotypy. Holotype ô. Cevlon (BMNH).

RACHIPLUSIA Hampson, i913, Cat. Lepid. Phalaenae Br. Mus. 13: xii, 4 Io.

PLUS

Type-species: Plusia nu Guenée, 1852 , in Boisduval \& Guenée, Hist. nat. Insectes (Lépid.) 6:347, by original designation. Lectotype (sex not stated), [URUGUAr]: MonteVideo (MNHN, Paris), designated by Viette, i95 I, Bull. mens. Soc. linn. Lyon 20: i6 .

RACLIA Walker, I 869, in Chapman, Proc. nat. Hist. Soc. Glasgow 1: $366 . \quad$ opH

Type-species: Raclia cervina Walker, I869, ibidem 1: 367 , by monotypy. Type(s) q. CONGo (BMNH).

RACOPTERA Scott, I 858 . See Phlogophora Treitschle, I 825.

ACRO

RADARA Walker, I 862, Trans. ent. Soc. Lond. (3) 1:94.

$\mathrm{OPHI}$

Type-species: Radara vacillans Walker, I 862, ibidem (3) 1:95, by monotypy. Type(s) ô. [South Africi] : Cape (UMI, Oxford).

RADDEA Alphéraky, 1892, Horae Soc. ent. ross. 26: 450.

VOCT

Type-species: Raddea digna Alphéraliy, i 892, ibidem 26: 450, by monotypy. Holotype 3 , [CHINA]: Amdo region, Myn-dyn-scha (ZI, Leningrad).

RADINACRA Butler, I878, Amn. Mag. nat. Hist. (5) 1: I6 I.

ACRO

Type-species: Radinacra palpalis Butler, i 878 , ibidem (5) 1 : I 6 I, by original designation. Type(s) ơ. JAPAN: Yoliohama (BMNH).

$R$. palpalis is a junior subjective synonym of Cosmia cinerascens Motschulsky, [I86I] i 860, Études ent. 9: 34 .

RADINOCERA Hampson, 1908, Cat. Lepid. Phalaenae Br. Mus. 7: 7. Available, but without included species until Hampson, 19I0, ibidem 9: xiv, 457.

$A G+1 R$

Type-species: Phalaenoides maculosus Rothschild, 1896, Novit.zool. 3: 94, pl. I.5, fig. 29, by subsequent designation by Hampson, I910, ibidem 9: 457. Holotype $\hat{\sigma}$, Australia: Qd, S. of Cooktown, Cedar Bay (BMNH).

RADINOGOES Butler, 1886, Trans. ent. Soc. Lond. 1886: 393.

ACRO

Type-species: Radinogoes tenuis Butler, I886, ibidem 1886: 394, by monotypy. Lectotype , [AUstrali ] : [Qd], Peak Downs (BMNH), designated (as type) by Hampson, 1909, Cat. Lepid. Phalaenae Br. Mus. 8: 434.

RADOSA nom. n. for Osdava Walker, i 865.

OPIII

Type-species: Osdara ordinata Walker, 1865, List Specimens lepid. Insects Colln Br. Mus. 33: i I04, by monotypy (of Osdara Walker). Holotype ô, [Br.ıziL]: [R. Amazon], [Tefé] Ega (BMNH).

OSDARA Walker, i 865, List Specimens lepid. Insects Colln Br. Mus. 33: I Io4.

Type-species: Osdara ordinata Valker, i 865 , by monotypy.

A junior homonym of Osdara Walker, I 858 , Ann. Magr. nat. Hist. (3) 2: 284, - Coleoptera. The oljective replacement name is Radosa nom. n.

RAGANA Swinloe, igoo, Cat. east. and Aust. Lepid. Heterncera 2: 204. IIYPE

Type-species: Bocana gravatalis Waller, [ 8 503] I 858, L.ist Specimens lepid. Insects Colln Br. Mus. 16: i75, by monotypy. Holotype f, CEYLON (BMLNH).

RAGHUVA Moore, 1881, Proc, zool. Soc. Lond. 1881: 362.

NOCT

Type-species: Lencania confertissima Walker, 1865, List Specimens lepid. Insects Colln Br. Mus. 32: 625, by monotypy. Holotype ô, [INDIA]: South Hindostan (BMNH). 
RAGONOTIA Staudinger, Igoo. See Doerriesa Staudinger, I 900.

ACRO

RAMADASA Moore, I 877, Proc. zool. Soc. Lond. 1877: 603.

OPHI Type-species: Chasmina pavo Walker, I856, List Specimens lepid. Insects Colln Br. Mus. 9: I 47, by monotypy. Holotype $q$, Ceylon (BMNH).

RAMESODES Hampson, I908, Cat. Lepid. Phalaenae Br. Mus. 7: I 7. Available, but without included species until Hampson, I9I0, ibidem 9: xi, 264.

ACRO

Type-species: Charidea divisa Hampson, I902, Ann. S. Afv. Mus. 2: 292, by subsequent designation by Hampson, I9Io, ibidem 9: 264. Syntypes, South Africa: Natal, Victoria District (BMNH); Cape Colony, Transkei (BMNH).

Ramesodes was the spelling adopted by Hampson, I9I0, as first reviser, from a multiple original spelling. Ramesodes was used in the phylogenetic table facing page 2 , and $\ddagger$ Rampsodes was used in the key to genera on page I 7 .

$\ddagger$ RAMPSODES; Hampson, 1908, Cat. Lepid. Phalaenae Br. Mus. 7: I 7.

An incorrect original spelling.

RAMOPIA nom. n. for Paromia Schaus, I913.

HYPE

Type-species: Paromia nigripunctata Schaus, 1913, Ann. Mag. nat. Hist. (8) 11: 23, by original designation (for Paromia Schaus). Type(s) ô, Costa Rica: Juan Vinas. PAROMIA Schaus, I913, Ann. Mag. nat. Hist. (8) 11: 23.

Type-species: Paromia nigripunctata Schaus, I9I3, by original designation.

A junior homonym of Paromia Westwood, I851, Trans. ent. Soc. Lond. (2) 1: I67,Coleoptera. The objective replacement name is Ramopia nom. $\mathrm{n}$.

RAMPHIA Guenée, I 852, in Boisduval \& Guenée, Hist. nat. Insectes (Lépid.). 7: I42. Cato Type-species: Noctua albizona Latreille, 1817, in Humboldt \& Bonpland, Voyage Régions equinoxiales Nouveau Continent, fait en I799-I804.... (Zool.) 2 (2): 136, pl. 43, figs 5, 6, by subsequent designation by Hampson, 1913, Cat. Lepid. Phalaenae Br. Mus. 13: i6i. Type(s), Equatorial South America.

$\ddagger R U M P H I A$; Pagenstecher, I909, Geogr. Verbreitung Schmett.: 424 .

An incorrect subsequent spelling.

$\ddagger$ †AMPSODES, misspelling. See Ramesodes Hampson, I 908.

ACRO

RANAJA Moore, I882, in Hewitson \& Moore, Descr. new Indian lepid. Insects Colln late Mr W. S. Atkinson: I2I.

CHLO

Type-species: Ranaja fasciata Moore, I882, ibidem: I2I, pl. 4, fig. I8, by monotypy. Type(s), IndiA: Darjiling (MNHU, Berlin).

RANCORA Smith, I 892, Ent. Neres 3: 253.

CUCU

Type-species: Rancora strigata Smith, I 892 , ibidem 3: 253, pl. Io, by monotypy. Holotype q, [CANADA]: B.C., Victoria.

$\ddagger$ RAPARA, misspelling. See Raparna Moore, I 882 .

OPHI

RAPARNA Moore, I882, in Hewitson \& Moore, Descr. new Indian lepid. Insects Colln late Mr W. S. Atkinson: I 77 . OPHI

Type-species: Raparna ochreipennis Moore, 1882 , ibidem: I 78 , pl. 6, fig. 8, by original designation. Syntypes, [INDiA]: Bengal, Parisnath (BMNH).

$\ddagger$ RAPARA; Pagenstecher, I909, Geogr. Verbreitung Schmett.: 424 .

An incorrect subsequent spelling.

RAPHIA Hübner, [I821] 1816, Verz. bekannter Schmett.: 212.

OPHI

Type-species: Noctua hybris Hübner, [1813], Samml. eur. Schmett. 4: pl. II I, fig. 518, by subsequent designation by Grote, 1874, Bull. Buffalo Soc. nat. Sci. 2: 6. Type(s), EUROPE. 
RHAPHIA Agassiz, I846, Nomencl. zool. Index univl.: 321, 323.

An unjustified emendation of Raphia Hübner, [I82I].

ANODONTA Rambur, I858, Cat. syst. Lépid. Andalousie: pl. 4, fig. 7 .

Type-species: Noctua hybris Hübner, [18I3], by monotypy.

A junior homonym of Anodonta Lamarck, I 799, Mém. Soc. Hist.nat. Paris: 87, - Mollusca.

The objective replacement name is Raphia Hübner, [1821].

RAPHISCOPA Hampson, 1925, Ann. Mag. nat. Hist. (9) 15: 408.

H $>$ PE

Type-species: Bertula invenusta Swinhoe, 1902, Ann. Mag. nat. Hist. (7) 9: I77, by original designation. Type(s) ô, Sumatra (BMNH).

$B$. invenusta is a junior subjective synonym of Spintherops undulata Felder \& Rogenhofer, I 875, Reise öst. Fregatte Novara (Zool.) 2 (Abt. 2): pl. I I 7, fig. 22.

REABOTIS Smith, [1903] 1902, Bull. U.S. natn. Mus. 52: 206.

HYPE

Type-species: Botis immaculalis Hulst, I 886, Trans. Am. ent.Soc. 13: I 54, by monotypy. Syntypes 2 ㅇ, [U.S.A.]: Montana.

$B$. immaculalis was originally described in the Pyralidae.

RECOROPHA nom. n. for Phorocera Guence, I 852.

CUCU

Type-species: Polia canteneri Duponchel, i 833, Rev. ent. (Silbermann) 1 (I): 37, pl. 3, by subsequent designation (for Phorocera Guenée) by Hampson, 1906, Cat. Lepid. Phalaenae Br. Mus. 6: 166. Type(s), France: Hyères.

PHOROCERA Guenée, I852, in Boisduval \& Guenée, Hist. nat. Insectes (Lépid.) 6: 3 I.

Type-species: Polia canteneri Duponchel, I 833, by subsequent designation by Hampson, I906, Cat. Lepid. Phalaenae Br. Mus. 6: i r6.

A junior homonym of Phorocera Robineau-Desvoidy, 1830, Mém. prés. div. Sav. Acad.

R. Sci. Inst. Fr. 2: I3I, - Diptera. The objective replacement name is Recoropha nom. 11 .

RECTANGULIPALPUS Bethune-Baker, I906, Novit. zool. 13: 286.

IIYPE

Type-species: Rectangulipalpus meeki Bethune-Baker, 1906, ibidem 13: 287, by original designation. Type(s) ô, New Guinea: [Papua], Aroa River.

RECTIPALPULA Joannis, I90o, Bull. Soc. ent. Fr. 1900: 280.

OPH I

Type-species: Oxyodes billeti Joannis, I900, ibidem 1900: 280, by monotypy. Syntypes, India: I $\hat{0}$, [W. Bengal], Darjeeling (BMNH). [North Vietnam]: i $q$, N.E. Tonkin, Cao-Bang.

Rectipalpula was proposed as a sub-genus of Oxyodes Guenée, I852.

REDECTIS nom. n. for Dercetis Grote, I 878.

OPHI

Type-species: Dercetis vitrea Grote, I878, Bull. U.S. geol. geogr. Surv. Territ. 4: I87, by subsequent designation by Grote, I896, Proc. Am. phil. Soc. 34: 434, (for Dercetis Grote). Syntypes ô, [U.S.A.]: N.Y., Buffalo (BMNH).

DERCETIS Grote, 1878, Bull. U.S. geol. geogr. Surv. Territ. 4: i 86.

Type-species: Dercetis vitrea Grote, I878, by subsequent designation by Grote, I 896 , Proc. Am. phil. Soc. 34: 434 .

A junior homonym of Dercetis Münster \& Agassiz, 1834, Nenes Jb. Miner. Geogn. Geol.

Petrefakt. 1834: 389, - Pisces. The objective replacement name is Redectis nom. 11.

†IRCETIS; Dyar, [1903] 1902, Bull. U.S. natn. Mus. 52: 245.

An incorrect subsequent spelling.

REDINGTONIA Barnes \& McDunnough, I9I2, Can. Ent. 44:9I.

Type-species: Redingtonia alba Barnes \& Mclunnough, I9I2, ibidem 44: 9I, by original designation. Syntypes 2 ㅇ, [U.S.A.]: Ariz., Redington.

REJECTARIA Guenée, I854, in Boisduval \& Guenée, Hist. nat. Insectes (Lépid.) 8: 85. Hy PE

Type-species: Rejectaria cocytalis Guenće, 1854, ibillem 8: 86, by sulsequent designation by Schaus, I9I6, Proc. U.S. natn. Mus. 50: 346. Holotype ô. [French Guiana]: Cayenne (BMNH). 
REMA Swinhoe, I90o, Cat. east. and Aust. Lepid. Heterocera 2: I2 I.

OPHI

Type-species: Toxocampa costimacula Guenée, I852, in Boisduval \& Guenée, Hist. nat. Insectes (Lépid.) 6: 429, by PRESENT DESIGNATION. Syntypes I $\overrightarrow{0}, 2$ q, [BANGLAdesh]: [E. Bengal], Silhet (BMNH).

REMIGIA Guenée, I852, in Boisduval \& Guenée, Hist. nat. Insectes (Lépid.) 7: 3 I2. C Cato Type-species: Remigia latipes Guenée, I852, ibidem 7:314, by subsequent designation by Grote, I874, Bull. Buffalo Soc. nat. Sci. 2: 44. LECTOTYPE ô. [West INdies]: Guadeloupe [I.] (M. Coppry) (BMNH), here designated, so as to fix the type-locality. In the original description the distribution included Bengal, Madagascar, North and South America.

REMIGIODES Hampson, I9I3, Cat. Lepid. Phalaenae Br. Mus. 12: 5. Available but without included species until Hampson, I9I3 ibidem 13: viii, 74 .

CATO

Type-species: Euclidia remigina Mabille, J884, C. r. Séanc. ent. Soc. Belg. 1884: cxci, by subsequent monotypy. Lectotype $\hat{\sigma}$, Madagascar (BMNH), designated by Viette $\&$ Fletcher, 1968, Bull. Br. Mus. nat. Hist. (Ent.) 21 : 4 I 2.

$\ddagger$ REMUSIA, misspelling. See Premusia Walker, 1858 .

OPHI

RENIA Guenée, I854, in Boisduval \& Guenée, Hist. nat. Insectes (Lépid.) 8: 80.

HYPE

Type-species: Renia orthosialis Guenée, I 854 , ibidem 8: 82, pl. 4, fig. 3, by subsequent designation by Desmarest, (I857), in Chenu, Encycl. Hist. nat. Papillons nocturnes: 2 I9. Syntypes 2 ㅇ. BrazIL (BMNH).

RENODES Guenée, I852, in Boisduval \& Guenée, Hist. nat. Insectes (Lépid.) 7: 367. OPHI

Type-species: Renodes curvicosta Guenée, 1852, ibidem 7: 367, pl. 24, fig. 8, by subsequent designation by Desmarest, (1857), in Chenu, Encycl. Hist. nat. Papillons nocturnes: I35. Syntypes 2 $\curvearrowright$, Brazil (BMNH).

${ }_{\ddagger}$ RESCIPHA, misspelling. See Rhescipha Walker, [1866].

OPHI

RETHMA Walker, i866, List Specimens lepid. Insects Colln Br. Mus. 35: I 570.

OPHI

Type-species: Rethma transcissaria Walker, I866, ibidem 35: I 570, by monotypy.

Holotype $\widehat{\jmath}$. [BrazIL]: [R. Amazon], [Tefé] Ega (BMNH).

$R$. transcissaria is a junior subjective synonym of $A$ sthana evecta Walker, I862.

See also Asthana Walker, I862.

RETICULANA Bethune-Baker, I906, Novit. zool. 13: 205.

OPHI

Type-species: Reticulana costilinea Bethune-Baker, I906, ibidem 13: 205, by original designation. Syntypes ơ. 오. New Guinea: [Papua], Ekeilkei (BMNH); Babooni (BMNH); Dinawa; Aroa River.

RHABDOPHANA Sodoffsky, I837. See Cleophana Boisduval, Rambur \& Graslin, I832.

CUCU

RHABDOPHERA Staudinger, I 898, Dt. ent. Z. Iris 10: 296.

CATO

Type-species: Rhabdophera messrae Staudinger, I898, ibidem 10: 296, pl. 9, fig. I8, by monotypy. Syntypes I2 $(\hat{\sigma}$,, ). [Jordan] Palestine: Dead Sea, Messra peninsula.

$R$. messrae is a junior subjective synonym of Remigia arefacta Swinhoe, I884, Proc. zool. Soc. Lond. 1884: 52 I, pl. 48, fig. 2.

RHABDOTINA Hampson, 1926, Descr. new Genera Species Lepid. Phalaenae Subfamily Noctuinae Br. Mus.: 329.

SARR

Type-species: Rhabdotina vittifera Hampson, 1926, ibidem: 329, by original designation. Holotype $\hat{\jmath}$, Venezuela: Esteban Valley, Las Quiguas (BMNH).

$R$. vittifera is a junior subjective synonym of Casandria phoenicias Hampson, I9I8, Novit. zool. 25: 199, but proposed as $\ddagger$ Cassandria, an incorrect subsequent spelling. 
RHABINOGANA Draudt, 1950, Mitt. mïnch. ent. Ges. 40: I 17.

Type-species: Rhabinogana albistriga Draudt, I950, ibidem 40: II 7, pl. 7, fig. 27 , by monotypy. Syntypes 2 $q, \mathrm{CH}_{\mathrm{N} A}$ : [Szechwan Prov.], Batang, Yangtse valley; [Yunnan Prov.], A-tun-tse (MAK, Bonn).

RHABINOPTERYX Christoph, I889, in Romanoff, Mém. Lépid. 5: 33.

ACRO

Type-species: Ligia turanica Ershov, 1874, in Fedchenko, Reise in Turkestan (2) 5 (3): 67 , pl. 4, fig. 69, by monotypy. Holotype + , [U.S.S.R.]: Turkestan town (ZI, Leningrad).

Rhabinopteryx was proposed as a new genus and not (as stated by Neave, I940, Nomencl. zool. 4: 29) as an objective replacement name for Ligia Duponchel, 1829,-Lepid., Geometridae.

RHAESENA Walker, 1866, List Specimens lepid. Insects Colln Br. Mus. 35: I 973.

OPHI

Type-species: Rhaesena transcissa Walker, 1866, ibidem 35: I974, by monotypy. Holotype ô. [Australia]: [W. Aust.] Swan River (BMNH).

$R$. transcissa is a junior subjective synonym of Bertula subcupralis Walker, [1866] I865, ibidem 34: I 67 .

See also Symplusia Holland, I 894 .

RHAMNOCAMPA Franclemont, 1949, Proc. ent. Soc. Wash. 51: 283.

$\mathrm{OPHI}$

Type-species: Herminodes albistriga Schaus, 1914, Proc. U.S. natn. Mus. 46: 507, by original designation. Holotype + , FReNCH GUiana: Cayenne (USNM, Washington).

$\ddagger$ RHANGANA, misspelling. See Rhangena Moore, I886.

OPH I

RHANGENA Moore, [1886] 1887, Lepid. Ceylon 3: 375.

OPHI

Type-species: Rhangena roseipennis Moore, [1886] I887, ibidem 3: 376, pl. I84, fig. I2, by monotypy. Type(s) 우, CEYLoN (BMNH).

See also Phycidimorpha Hampson, 1893.

$\ddagger R H A N G A N A$; Draudt, 1914, in Seitz, Gross-Schmett. Erde 10: 218.

An incorrect subsequent spelling in both the German and in the English editions.

RHANIDOPHORA Wallengren, 1858, Öfvers. K. Vetensk.-Akad. Förh. Stockh. 15: 21 3. орH

Type-species: Phalaena phedonia Stoll, 1781, in Cramer, Uitlandsche Kapellen 4: I Io, pl. 347, fig. C, by original designation. Type(s), [South Africa]. Cap de Bonne Espérance.

RHAPHIA Agassiz, I846. See Raphia Hübner, [I82I].

OPHI

RHAPSA Walker, [1866] I865, List Specimens lepid. Insects Colln Br. Mus. 34: I 149. opH Type-species: Rhapsa scotosialis Walker, [1866] I865, ibidem 34: I I 50, by monotypy. Syntypes $3 \hat{\sigma}$. New Zealand: Auckland (BMNH).

RHATTA Walker, 1857, List Specimens lepid. Insects Colln Br. Mus. 11: 763

cucu

Type-species: Rhatta cramboidina Walker, 1857, ibidem 11: 764, by monotypy. Holotype ô, [BrazIL]: [R. Amazon], Santarem.

RHAZUNDA Walker, 1866, List Specimens lepid. Insects Colln Br. Mus. 35: 1961.

OPHI

Type-species: Rhazunda distributa Walker, 1866, ibidem 35: 1962, by monotypy. Holotype $\precsim$, Venezuela (BMNH).

RHESALA Walker, I858, List Specimens lepid. Insects Colln Br. Mus. 15: 1776.

OPHI Type-species: Rhesala imparata Walker, I858, ibidem 15: I777, by monotypy. Holotype , CEYLoN (BMNH).

See also Hingula Moore, I882.

RHESALIDES Prout, I921, Ann. Mag.nat. Hist. (9) 8: 3 I.

OPHI

Type-species: Rhesalides keiensis Prout, I92I, ibidem (9) 8: 3I, pl. 5, fig. 4, by monotypy. Holotype q, [Moluccas]: Kei Is (BMNH). 
RHESALIDES Hampson, 1926, Descr. new Genera Species Lepid. Phalaenae Subfanily Noctuinae Br. Mus.: 274 .

Type-species: Rhesalides admiraltensis Hampson, I926, ibidem: 274, by original designation. Syntypes $3 \hat{o}$, [New Guinea]: Admiralty Is (BMNH).

A junior homonym of Rhesalides Prout, I921, - Lepid., Noctuidae. This generic name probably originated from Hampson but was used and unintentionally made nomenclaturally available by Prout prior to its proposal and generic description by Hampson. There is no objective replacement name but $R$. admivaltensis is congeneric with Rhesalides keiensis Prout, I92I, the type-species of Rhesalides Prout, I92I. The latter is therefore available for use as a subjective replacement name.

RHESALIDES Hampson, I926. See Rhesalides Prout, I92I.

OPHI

RHESALISTIS Hampson, 1926, Descr. new Genera Species Lepid. Phalaenae Subfamily Noctuinae Br. Mus.: 276.

OPHI

Type-species: Magulaba rotundata Rothschild, 1915, Lepid. Br. Orn. Union and Wollaston Exped.: 65, by original designation. Syntypes 3 J, I ㅇ, New Guinea: [West Irian], Snow Mts, Utakwa River (BMNH).

RHESCIPHA Walker, [1866] r865, List Specimens lepid. Insects Colln Br. Mus. 34: I I94. OPHI Type-species: Rhescipha obtusa Walker, [1866] I 865, ibidem 34: I 195, by monotypy. Holotype + , Brazil (BMNH).

$\ddagger$ RESCIPHA; Weymer, I 895, Stettin. ent. Ztg 55: 332.

An incorrect subsequent spelling.

${ }_{+}^{+}$RHINOGNATHA Hampson, no published reference found. HYPE

$\ddagger$ RHIPIPRORA Prout, no published reference found. HYPE

RHISCIPHA Walker, I 865, List Specimens lepid. Insects Colln Br. Mus. 33: 850. орH

Type-species: Rhiscipha scissa Walker, r865, ibidem 33: 85I, by monotypy. Holotype o. Congo (BMNH).

RHIZA Staudinger, r889, Stettin. ent. Ztg 50: 44.

ACRO

Type-species: Rhiza commoda Staudinger, I 889, ibidem 50: 44, by monotypy. Syntypes 4 ô, 3 ㅇ, [U.S.S.R.]: [Kirghizia], S. of Issyk Kul (MNHU, Berlin).

Rhiza when originally proposed contained a second species, but this was doubtfully included and, under the Code, Article 68 (c), is not eligible for selection as type-species.

RHIZAGROTIS Smith, I890, Bull. U.S. natn. Mus. 38: Iо, I03.

NOCT

Type-species: Agrotis cloanthoides Grote, I880, Bull. U.S. geol. geogr. Surv. Territ.

6: I 53, by original designation (on page ro). Type(s) ㅇ, [U.S.A.]: Colorado.

RHIZANA Walker, 1865, List Specimens lepid. Insects Colln Br. Mus. 33: 719.

CHLO

Type-species: Rhizana metarhoda Walker, I865, ibidem 33: 720, by monotypy. Holotype q, Java (BMNH).

R. metarhoda is a junior subjective synonym of Gadivtha semifervens IValker, [I863] I 864, J. Proc. Linn. Soc. (Zool.) 7: I63.

See also Erizada Walker, I865, List. . . 32: 506; and Tinosoma Hampson, I 894.

RHIZEDRA Warren, I9I I, in Seitz, Gross-Schmett. Erde 3: 234.

ACRO

Type-species: Noctua lutosa Hübner, [I803], Samml. eur. Schmett. 4: pl. 48, fig. 232, by original designation. Type(s), Europe.

The date of publication of the part of Seitz containing page 234, was stated on page 229 as rgrr March roth in both the English and the German editions.

RHIZOGRAMMA Lederer, 1857. See Auchmis Hübner, I821.

Cucu 
RHIZOLITHA Curtis, [1830] 1829, Guide Br. Insects: I 49.

CUCU

Type-species: Noctua lamda Fabricius, 1787, Mantissa Insect. 2: I74 (but included by Curtis as ‡lambda Haw.', an incorrect spelling by Haworth, [1809], Lepid. Br.: I8I), by subsequent designation by Curtis, 1833, Ent. Mag. 1: I 89, but cited as +'lambda Haw.' Type(s), Germany (Type(s) not found by Zimsen, 1964, Type Material of I. C. Fabricius: $550)$.

RHIZOTYPE Hampson, 1906, Cat. Lepid. Phalaenae Br. Mus. 6: xii, 373.

CUCU

Type-species: Bombyx flammea Esper, I785, Die Schmett. 3: 269, pl. 53, fig. 3. by original designation. Syntypes 9 , ITALY: southern.

See also Trigonophora Hübner, [182I].

RHODARIA Duponchel, I845, Cat. méth. Lépid. Eur.: I99.

PYRALIDAE

Type-species: Phalaena sanguinalis Linnaeus, i 767, Syst. Nat. (Edn I2) 1(2): 882, by subsequent designation by Guenée, 1854, in Boisduval \& Guenée, Hist. nat. Insectes (Lépid.) 8: I 70.

Rhodaria has been used as the original generic name for species which are now in the Noctuidae.

RHODINA Guenéc, I 854, in Boisduval \& Guenée, Hist. nat. Insectes (Lépid.) 8: I9. OPHI Type-species: Rhodina falculalis Guenée, I 854 , ibidem $8: 20$, pl. 4, fig. I, by monotypy. Syntypes I $\hat{\delta}, 2$ ㅇ, [Australia] Nouvelle-Hollande.

RHODOCHLAENA Hampson, 1906, Cat. Lepid. Phalaenae Br. Mus. 6: ix, I 31.

CUCU

Type-species: Dianthoecia botonga Felder \& Rogenhofer, 1875, Reise öst. Fregatte Novara (Zool.) 2 (Abt. 2): pl. Io9, fig. 28, by original designation. Type(s) ô, South AFrica: [Cape prov.], Knysna.

RHODOCLEPTRIA Hampson, 1903, Cat. Lepid. Phalaenae Br. Mus. 4: ix, 33.

NOCT

Type-species: Heliothis incarnata Freyer, [1838] 1839, Nenere Beitr. Schmett. 3: 91, pl. 256, fig. 4, by original designation. Syntypes, [TURKEY]: [Istanbul] Constantinople.

RHODODACTYLA Warren, I889, Trans. cnt. Soc. Lond. 1889: 247.

OPHI

Type-species: Colobochila elicrina Felder \& Rogenhofer, 1875, Reise öst. Fregatte Novara (Zool.) 2 (Abt. 2): pl. I 20, fig. I7, by monotypy. Type(s) 우, [Brazil]: R. Amazon (BMNH).

RHODODIPSA Grote, IS77, in Uhler, Bull. U.S. geol. geogr. Surv. Territ. 3: 769, 797. Noct Type-species: Alaria volupia Fitch, 1868, Trans. N.Y. State agric. Soc. 27: 907-908, by monotypy. Type(s), [U.S.A.]: Indian Territory W. of Arkansas.

Rhododipsa was used by Uhler on page 769 in his list of Colorado insects, but was proposed by Grote on page 797 in an Appendix to the same paper.

RHODOECIA Hampson, I908, Cat. Lepid. Phalaenae Br. Mus. 7: 5. Available, but without included species until Hampson, I910, ibidem 9: vii, 5 I.

NOCT

Type-species: Xanthia aurantiago Guencee, 1852, in Boisduval \& Guenéc, Hist. nat. Insectes (Lépid.) 5: 394, pl. 7 , fig. I, by subsequent monotypy. Syntypes 3 ô. America: northern $(\mathrm{BMNH})$.

RHODOPHORA Guenée, I $S_{52}$, in Boisduval \& Gucnée, Hist. nat. Insectes (Lépid.) 6: I jo.

NOCT

'lype-species: Rhodophora florida Guence, 1852, ibidem 6: 171, pl. 9, fig. 7 , by subsequent designation by Grote, 1874, Bull. Buffalo Soc. nat. Sci. 2: 33. Holotype , [U.S.A.]: New York state.

ALARIA J)uncan [\& Westwood], I8. I, in Jardine, Naturalist's Library (Edn I) 33 (Ent. 7): 200.

Type-species: Phalaena gaurae Smith, 1797, in Smith \& Abbot, Nat. Hist, raver lepid. Insects Georgia 2: 197, pl. 99, by monotypy. Syntypes, [U.S.A.]: Georgia. 
A junior homonym of Alaria Schrank, I788, Verz. bisher hinlänglich bekannten Eingeweidewïrmer: Gattungen \& 52,-Vermes. There is no objective replacement name but $P$. gauvae is congeneric with Rhodophora florida Guenée, I 852, the type-species of Rhodophora Guenée, $185^{2}$. The latter is therefore available for use as the subjective replacement name.

Alaria, although unacknowledged by Duncan, was attributed to Westwood by some authors. Justification can be found in Stainton, 1885, Entomologist's mon. Mag. 21: I 8I-I86, who quoted from a letter by Westwood.

RHODOSEA Grote, 1883, Can. Ent. 15: 4.

NOCT

Type-species: Rhodosea julia Grote, I883, ibidem 15: 5, by monotypy. Type(s), [U.S.A.]: New Mexico.

RHODOTARACHE Warren, I9I4, Ann. S. Afr. Mus. 10: 478.

$\mathrm{ACON}$

Type-species: Rhodotarache roseofusca Warren, I9I4, ibidem 10: 478, pl. 40, fig. I7, by original designation. Holotype , [South Africa]: Cape Albany.

RHOPALOGNATHA Hampson, 1926, Descr. new Genera Species Lepid. Phalaenae Subfamily Noctuinae Br. Mus.: 458.

OPH I

Type-species: Thermesia anterosticta Dognin, I9I4, Hétérocères nouv. Am. Sud 8: 44 , by original designation. Holotype $\hat{\sigma}$, Colombia: Monte Socorro.

RHOPTROPHALAENA Hampson, I9I8. See Cocytia Boisduval, I828.

COCYTIIDAE

RHOPTROTRICHIA Butler, I891, Ann. Mag. nat. Hist. (6) 8: 7 I, 76.

ACRO

Type-species: Callopistria recurvata Moore, I882, in Hewitson \& Moore, Descr. new Indian lepid. Insects Colln late MrW.S. Atkinson: r44, by original designation. Syntypes, India: [W. Bengal], Calcutta (BMNH); Darjiling. CEylon (BMNH).

C. recurvata is a junior subjective synonym of Eviopus maillardi Guenée, I862, in Maillard, Notes Île Réunion 2: G 39, pl. 22, fig. 8.

OBANA Walker, I862 November, J. Proc. Limn. Soc. (Zool.) 6: I9o.

ACRO

Type-species: Obana pulchrilinea Valker, 1862, ibidem 6: 190, by monotypy. Lectotype q, Borneo: Sarawak (UM, Oxford), designated (as type) by Swinhoe, igoo, Cat. east. and Aust. Lepid. Heterocera 2: 36 .

A junior homonym of Obana Walker, I862 [March], List Specimens lepid. Insects Colln Br. Mus. 24: I Io3,-Lepid., Noctuidae. There is no objective replacement name but O. pulchrilinea is congeneric with Callopistria recurvata Moore, I882, the type-species of Rhoptrotrichia Butler, I89I. The latter is therefore available for use as a subjective replacement name.

RHOSOLOGIA Walker, r865, List Specimens lepid. Insects Colln Br. Mus. 33: roo3. opH Type-species: Rhosologia porrecta Walker, I865, ibidem 33: roo3, by monotypy. Holotype $\hat{\sigma}$, Mexico (BMNH).

RHOSUS Walker, 1854, List Specimens lepid. Insects Colln Br. Mus. 2: 359. AGAR

Type-species: Rhosus posticus Wallier, I 854, ibidem 2:360, by monotypy. Syntypes I $\widehat{o}, \mathrm{I}$ Q , VENEZUELA (BMNH).

RHUBUNA Walker, I858, List Specimens lepid. Insects Colln Br. Mus. 15: I820. CATO Type-species: Rhubuna irresoluta Walker, I858, ibidem 15: I821, by monotypy. Syntypes $3(\widehat{\jmath}$, $)$, [Dominican Republic] St. Domingo (BMNH).

RHUSINA Spuler, I906. See Rusina Stephens, I829.

ACRO

RHYACIA Hübner, [I82I] 1816, Verz. bekannter Schmett.: 2ro.

NOCT

Type-species: Noctua lucipeta [Denis \& Schiffermüller], r775, Ankündung syst. Werkes Schmett. Wienergegend: 7r, by subsequent designation by Hampson, 1903, Cat. Lepid. Phalaenae Br. Mus. 4: 153. Type(s), [Austria]: Vienna district (Collection destroyed, Horn \& Kahle, 1936, Ent. Beih. Berl.-Dahlem 3: 243). 
RHYNCHAGLAEA Hampson, I906, Cat. Lepid. Phalaenae Br. Mus. 6: xiii, 435.

Type-species: Xylophasia scitula Butler, i879, Ann. Mag. nat. Hist. (5) 4: 359, by original designation. Type(s) $\widehat{\jmath}$, JAPAN (BMNH).

RHYNCHAGROTIS Smith, I89o, Bull. U.S. natn. Mus. 38: 9, I 3.

NOCT

Type-species: Noctua cupida Grote, 1864, Proc. ent. Soc. Philad. 3: 525, pl. 5, fig. 7 , by original designation (on page 9). Type(s) ô, [U.S.A.]: middle states.

RHYNCHINA Guenée, 1854, in Boisduval \& Guenée, Hist. nat. Insectes (Lépid.) 8: 20. HY PE

Type-species: Rhynchina pionealis Guenée, 1854 , ibidem 8: $2 \mathrm{I}$, by monotypy. Holotype , INDIA: central (BMINH).

RHYNCHODES Guenée, I852. See Hondryches nom. 11.

OPHI

RHYNCHODIA Hampson, igio, Cat. Lepid. Phalaenae Br. Mus. 10: 2, 489.

ACON

Type-species: Rhynchodia mesophaea Hampson, I9I0, ibidem 10: 489, fig. I45, by original designation. Syntypes I $\hat{\jmath}$, I ㅇ. [INDiA]: Travancore, Pirmad (BMNH).

$\ddagger R H Y N C O D I A$; Warren, 1913, in Seitz, Gross-Schmett Erde 11: 276.

An incorrect subsequent spelling, in both the German and the English editions.

RHYNCHODINA Hampson, 1926, Descr. new Genera Species Lepid. Phalaenae Subfamily Noctuinae Br. Mus.: 262.

OPHI

Type-species: Rhynchodina molybdota Hampson, I926, ibidem: 263, by original designation. Holotype , Burma: Tenasserim, Donat Hills (BMNH).

RHYNCHODONTODES Warren, 1913, in Seitz, Gross-Schmett. Erde 3: 430.

HYPE

Type-species: Pyralis antiqualis Huibner, [1809], Samml. eur. Schmett. 6: pl. 23 , fig. 152 , by original designation. Type(s), Eurore.

The date of publication of the part of Seitz, containing page 430 was stated on page 429 as 1913 November $25^{\text {th }}$ in both the English and the German editions.

RHYNCHOLITA Hampson, igro, Cat. Lepid. Phalaenae Br. Mus. 9: 2, 307.

$\triangle C R O$

Type-species: Rhyncholita atripuncta Hampson, I910, ibidem 9: 308, fig. I 30, by original designation. Syntypes I $\hat{0}, 2$ 우, Brazil: Castro Parana (BMNH).

RHYNCHOPALPUS Hampson, I893. Illust. typical Specimens Lepid. Heterocera Colln Br. Mus. 9: 89 .

NOLI

Type-species: Aglossa argentalis Moore, i867, Proc. zool. Soc. Lond. 1867: 89, by subsequent designation by Hampson, 1900, Cat. Lepid. Phalaenae Br. Mus. 2: 31. Syntypes ${ }^{\circ},[$ INDIA]: [WV.] Bengal, Darjeeling (BMNH).

RHYNCHOPLEXIA Hampson, 1908, Cat. Lepid. Phalaenae Br. Mus. 7: xii, 433. Acro

Type-species: Ancara rubra Hampson, i 894, Fauna Br. India (Noths) 2: 225, by original designation. Type(s) ô, [Sıkкim] Sikhim (BMNH).

RHYNCHOPSOTA Lower, 1903, Trans. R. Soc. S. Aust. 27: 196.

GEOMETRIDAE

Type-species: Rhynchopsota delogramma Lower, 1903, ibidem 27: i 96, by monotypy.

$R$. delogramma is congeneric and probably conspecific with Liodes neurogramma Turner. I906, which was later placed by Turner in the Ophiclerinae. In 1969 I. F. 13. Common (ANIC, Canberra), in a letter to D. S. Fletcher (BMNH), considered that neurogramma should be placed in the Geometridae.

$\ddagger$ RHYNCODES, misspelling. See Hondryches nom. n.

OPH I

$\ddagger$ RHYNCODIA, misspelling. See Rhynchodia Hampson, I9 Iо.

$\triangle \mathrm{CON}$

RHYPAGLA nom. n. for Glaphyra Guenée, $184 \mathrm{I}$.

ACON

Type-species: Anthophila glarea Treitschke, 1826, Schmett. Eur. 5 (3): 282, by subsequent designation (for Glaphyra Guenée) by Desmarest, (1857), in Clienu, Encycl. Hist. nat. Papillons nocturnes: 94. Type(s), [Yugoslavia]: Dalmatia (1M, Budapest). 
A. glarea is a junior subjective synonym of Geometra lacernaria Hübner, [1813], Samml. eur. Schmett. 5: pl. 82, fig. 422.

GLAPHYRA Guenée, 184I, Annls Soc. ent. Fr. 10: 232.

Type-species: Anthophila glavea Treitschke, 1826, by subsequent designation by Desmarest, (1857), in Chenu, Encycl. Hist. nat. Papillons nocturnes: 94.

A junior homonym of Glaphyra Newman, I840, Entomologist 1 (2): I9, - Coleoptera. The objective replacement name is Rhypagla nom. $\mathrm{n}$.

RHYTIA Hübner, [1823] I816, Verz. bekannter Schmett.: 264.

OPHI

Type-species: Phalaena cocalus Cramer, 1777, Uitlandsche Kapellen 2: 59, pl. 134, fig. B, by subsequent designation by Joannis, I929, Ammls Soc. ent. Fr. 98: 380. Type(s), EAST INDIES.

RIADHIA Wiltshire, I96I, J. Bombay nat. Hist. Soc. 58: 627.

OPHI

Type-species: Riadhia diehli Wiltshire, I961, ibidem 58: 627, pl. 2, figs. 23, 27, by original designation. Holotype $\hat{\sigma}$, Saudi Arabia: Riadh (ZSBS, Munich).

$\boldsymbol{R I A G A}$ Wileman \& West, I928, Entomologist's Rec. J. Var. 40: I74.

OPHI

Type-species: Riaga radiata Wileman \& West, 1928, ibidem 40: I 74, by original designation. Holotype + , Philippines: Luzon I., Benguet subprov., Palali (BMNH).

RIAGRIA Köhler, (1952) I95I, Acta zool. lilloana 12: I 5 I.

CUCU

Type-species: Riagria xylinoides Köhler, (1952) I95 I, ibidem 12: I5I, by original designation. Syntypes $\hat{0}$, , Argentina: Chubut [Prov.], Comodoro Rivadavia (ZSBS, Munich); Nequén [Prov.], Rio Agrio (ZSBS, Munich).

$\ddagger$ RICENSIA Hampson, no published reference found.

HYPE

RICHIA Grote, I887, Can. Ent. 19: 44.

NOCT

Type-species: Agrotis chortalis Harvey, 1875, Bull. Buffalo Soc. nat. Sci. 2: 272, by original designation. Syntypes ô, + , [U.S.A.]: Texas.

RICLA Walker, 1869, in Chapman, Proc. nat. Hist. Soc. Glasgow 1: 365.

HYPE

Type-species: Ricla expandens Walker, I869, ibidem 1: 365, by monotypy. Type(s)

ㅇ. CONGO (BMNH).

RICTONIS nom. n. for Nitocris Guenée, I 868.

ACRO

Type-species: Mamestra comma Walker, I856, List Specimens lepid. Insects Colln Br. Mus. 9: 239, by subsequent designation (for Nitocris Guenée) by Hampson, I9o9, Cat. Lepid. Phalaenae Br. Mus. 8: 383. Holotype q, New ZEaland (BMNH).

NITOCRIS Guenée, I868, Entomologist's mon. Mag. 5: 4.

Type-species: Mamestra comma Walker, 1856 , by subsequent designation by Hampson, I909, Cat. Lepid. Phalaenae Br. Mus. 8: 383.

A junior homonym of Nitocris Rafinesque, I8 15, Analyse Nature: I23,- Hymenoptera. The objective replacement name is Rictonis nom. $n$.

RILEYIANA Moucha \& Chvála, 1963, J. Lepid. Soc. 17: I69.

CUCU

Type-species: Phlogophora fovea Treitschle, I825, Schmett. Eur. 5 (I): 380, by monotypy (of Thecophora Lederer, I857). Syntypes ô, 오, Europe (TM, Budapest).

Rileyiana was proposed as the objective replacement name for Thecophora Lederer, I857.

THECOPHORA Lederer, 1857, Noctuinen Eur.: 33, 99.

Type-species: Phlogophora fovea Treitschke, 1825 , by monotypy.

A junior homonym of Thecophora Rondani, I845, Nuovi Ann. Sci. nat. Bologna (2) 3:

I 5, - Diptera. The objective replacement name is Rileyiana Moucha \& Chrála, I963.

RIMULIA Saalmüller, I89I, Lepid. Madagascar: 483.

OPHI

Type-species: Rimulia malgassica Heyden, I89I, in Saalmüller, ibidem: 483, fig.

I73, by monotypy. Type(s), MadaGascar (SNG, Frankfurt). 
R. malgassica is a junior subjective synonym of Magulaba moestalis Walker, [I866].

See also Magulaba Walker, [1866].

RIPOGENUS Grote, I 865, Proc. ent. Soc. Philad. 4: 325.

EUTE

Type-species: Ripogenus pulcherrimus Grote, I865, ibidem 4: 326, by monotypy. Syntypes 2 ex., [U.S.A.]: New Jersey, Hoboken (BMNH).

RISOBA Moore, i88 i, Proc. zool. Soc. Lond. 1881: 328.

SARR

Type-species: Thyatira repugnans Walker, 1856, List Specimens lepid. Insects Colln Br. Mus. 9: 9, by original designation. Lectotype , INDrA : northern (BMNH), designated (as type) by Hampson, I912, Cat. Lepid. Phalaence Br. Mus. 11: 426.

†RISOLA: Warren, I9I3, in Seitz, Gross-Schmett. Erde 3: 293.

An incorrect subsequent spelling in the German edition, but correctly spelled in the English edition.

$\ddagger$ RISOLA, misspelling. See Risoba Moore, I $88 \mathrm{I}$. SARR

RISTRA Walker, I858, List Specimens lepid. Insects Colln Br. Mus. 15: I 842.

OPHI

Type-species: Ristra tortuosa Walker, i 858 , ibidem 15: i $8 \downarrow_{2}$, by monotypy. Holotype ㅇ. HONDURAS (BINH).

$R$. tortuosa is a junior subjective synonym of Anomis illita Guenée, I $S_{5} 2$, in Boisduval \& Guenée, Hist. nat. Insectes (Lépid.) 6: „oo.

RIVULA Guenée, [18+5] I 8+4, in Duponchel, Cat. méth. Lépid. Eur. (2): 206.

OPHI

Type-species: Phalaena sericealis Scopoli, I763, Ent. Carniolica: 242, by monotypy, but cited as 'sericealis, W.V.', an incorrect authority. 'Type(s), [YugosLAvia]: [Slovenia] Carniola.

PASIRA Moore, i882, in Hewitson \& Moore, Descr. new Indian lepid. Insects Colln laie Mr W. S. Atkinson: 197 .

Type-species: Pasira ochracea Moore, I882, ibidem: 197, by monotypy. Syntypes A., , INDIA: Calcutta.

A junior homonym of Pasira Stål, I859, ufvers. K. Vetensh-Acad. Förh. Stockh. 16: I 9o, - Hemiptera. There is no objective replacement name but $P$. ochvacea is congeneric with Phalaena sericealis Scopoli, the type-species of Rivula Guenée, [18+5]. The latter is therefore available for use as a subjective replacement name.

RIVULANA Bethune-Baker, I9I 1, Ann. Mag. nat. Hist. (8) 8: 532.

Type-species: Rivulana ochrea Bethune-Baker, I9I I, ibidem (8) 8: 533, by original designation. Type(s) ô, AngolA: N'Dalla Tando (BMNH).

RODRIGUESIA Dyar, I912, Proc.ent. Soc. Wash. 14:6I.

ACRO

Type-species: Deva ornata Ottolengui, i 898, Can. Ent. 30: I06, pl. 5, fig. I, by monotypy. Holotype ô, [U.S.A.]: N. Mexico, Hot Springs (USNM, Washington).

D. ornata is a junior subjective synonym of Plusia howardi Edwards, 1877, Pacif. Coast Lepid. No. 25: 1.

ROESELIA Hübner, [1825] I816, Terz. bekannter Schmett.: 397.

NOLI

Type-species: Phalaena cucullatella Linnaeus, I 758, Syst. Nat. (Edn 10) 1: 537 (but included by Hübner as ¥'cuculatella Linn.', an incorrect subsequent spelling), by subsequent designation by Grote, I874, Bull. Buffalo Soc. nat. Sci. 2: i 52. Type(s), Type-Locality not stated (LS, London) [EUROPE].

See also Nola Leach, [1815].

ROLUA Dyar, 1915, Insecutor Inscit. menstr. 3:8 r.

ACRO

Type-species: Rolua monetifera Dyar, I9I5, ibidem 3: 8I, by original designation. Holotype ô. Mixico: Zacualpan (USNM, Washington).

ROPERUA nom. n. for Liuprova Hampson, 1926.

$\mathrm{ACRO}$

Type-species: Caradrina lichenophora Lower, 1902, Trans. R. Soc. S. Aust. 26: 224, 
by original designation (for Euprora Hampson). Holotype $q$, Australia: Victoria, Melbourne (SAM, Adelaide).

EUPRORA Hampson, 1926, Descr. new Genera Species Lepid. Phalaenae Subfamily Noctuinae Br. Mus.: 88.

Type-species: Caradrina lichenophora Lower, I902, by original designation.

A junior homonym of Euprora Busck, 1906, Proc. U.S. natn. Mus. 30: 732, - Lepid.,

Tineidae. The objective replacement name is Roperua nom. n.

${ }_{\ddagger}$ ROPTROPHALAENA, misspelling. See Cocytia Boisduval, I 828 .

COCYIIIDAE

ROSENIA Schawerda, 1922. See Rotoa Strand, I942.

ACRO

ROTHIA Westwood, I877, Trans. Linn. Soc. Lond. (2) Zool. 1: 204.

AGAR

Type-species: Agarista pales Gray, 1832, in Griffith, Animal Kingdom arranged in conformity with its Organization, by the Baron Cuvier, with supplementary Additions to each Order 15: 782, pl. 53, fig. I, by subsequent designation by Hampson, 1901, Cat. Lepid. Phalaenae Br. Mus. 3: 574. Type(s) 9 , Type-Locality not stated [Madagascar].

ROTOA Strand, 1942, Folia zool. hydrobiol. 11: 390.

ACRO

Type-species: Tapinostola distincta Bang-Haas, I912, Dt. ent. Z. Iris 26: I 54, pl. 6, fig. I 7b, by monotypy (of Rosenia Schawerda). Holotype o, [U.S.S.R.]: Amur, Ussuri, Nikolsk (MNHU, Berlin).

Rotoa was proposed as the objective replacement name for Rosenia Schawerda, I922. ROSENIA Schawerda, 1922, Z. öst. EntVer. 7 : 10.

Type-species: Tapinostola distincta Bang-Haas, I91 2, by monotypy.

A junior homonym of Rosenia Waagen \& Wentzel, r886, Pal. Ind. (13) 1 (6): 943 ,Protozoa. The objective replacement name is Rotoa Strand, 1942.

ROWDENIA nom. n. for Tomyris Druce, I 890 . HYPE

Type-species: Tonıyris nigropuncta Druce, I890, Biologia cent.-am. (Zool.) Lepid. Heterocera 1: 440, pl. 35, fig. 25, by original designation (for Tomyris Druce). Syntypes, Guatemala: I $\widehat{\jmath}$, Volcan de Atitlan (BMNH). Panama: I $\widehat{o}$, Chiriqui, Caldera (BMNH).

This name is proposed in honour of Mr Robert MI. Rowden in appreciation of his ever willing assistance during the later stages of the compilation of this catalogue.

TOMYRIS Druce, I890, Biologia cent.-am. (Zool.) Lepid. Heterocera 1: 440.

Type-species: Tomyris nigropuncta Druce, 1890 , by original designation.

A junior homonym of Tomyris Chapuis, I 874, in Lacordaire \& Chapuis, Hist. nat. Insectes (Coléoptères) 10: 265,-Coleoptera. The objective replacement name is Rowdenia nom. n.

RUACODES Hampson, 1908, Cat. Lepid. Phalaenae Br. Mus. 7: 7. Arailable, but without included species until Hampson, I909, ibidem 8: xiv, 537.

ACRO

Type-species: Hadena tela Smith, I900, Proc. U.S. natn. Mus. 22: 470, by subsequent monotypy. Holotype, [U.S.A.]: Arizona, Phoenix, Rio Verde Mts (USNM, Washington).

RUGOFRONTIA Köhler, 1947, Acta zool. lilloana 4: I03.

NOCT

Type-species: Rugofrontia unifera Köhler, 1947, ibidem 4: 103, by original designation. Holotype ㅇ, Argentina: [Chubut Prov.], Comodoro Rivadavia.

$\ddagger \boldsymbol{R U M P H I A}$, misspelling. See Ramphia Guenée, I852.

CATO

RUNGSIANEA Viette, [1963] 1962, Amnls Soc. ent. Fr. 131: I 49.

HADE

Type-species: Xylomania hecate Viette, (1960), Bull. Soc. ent. Fr. 64: 224, by original designation. Holotype 9 , Madagascar: N.E., Marojejy massif. Andasy (MNHU, Paris).

RUSICADA Walker, [1858] 1857, List Specimens lepid. Insects Colln Br. Mus. 13:984, I oo6. opH Type-species: Rusicada nigritarsis Walker, [1858] I857, ibidem 13: 1006, by monotypy. Holotype ô, CEYLon (BMNH). 
RUSIDRINA Staudinger, I 892, in Romanoff, Mém. Lépid. 6: $49 \mathrm{I}$.

ACRO

Type-species: Rusidrina rasdolnia Staudinger, I892, ibidem 6: 49I, pl. 7, fig. 9, by monotypy. Holotype ô, [U.S.S.R.]: Amur district, Suifun, Rasdolnaja.

R. rasdolnia is a junior subjective synonym of Agrotis depravata Butler, 1879, Ann. Mag. nat. Hist. (5) 4: 360 .

RUSINA Stephens, I 829, Illust. Br. Ent. (Haustellata) 2: I I I.

ACRO

Type-species: Bombyx ferruginea Esper, I 785, Die Schmett. 3: 246, pl. 47, figs 5, 6, by monotypy. Lectotype $\delta^{\hat{0}}$ [Germaxy]: Fränkischen district, designated by Franclemont, 1950, Bull. Brooklyn ent. Soc. 45: I 49: I 49 as 'the figure of the male.' Under the Code, Article 74 , this is to be treated as a designation of the specimen represented by the figure.

Hampson, I908, Cat. Lepid. Phalaenae Br. Mus. 7 : 14 implied that ferruginea as used by Stephens, I829, was not the same as fernuginea Esper. This is not the case, the following are all conspecific:

Phalaena (Bombyx) umbratica Goeze, i $78 \mathrm{I}$, [ a junior primary homonym]

$=$ Bombyx ferruginea Esper, [1 785$]$

= Noctua tenebrosa Hübner, [1803]

$=$ Rusina ferruginea (Esper); Stephens, I 829 .

See also Stygiostola Hampson, I 908.

RHUSINA Spuler, I906, in Hofmann, Schmett. Eur. 1: 235.

An unjustified emendation of Rusina Stephens, I 829.

SAALMUELLERANA Fletcher \& Viette, I962, Bull. mens. Soc. linn. Lyon 31: $6 . \quad$ hade

Type-species: Dianthoecia glebosa Saalmüller, I89I, Lepid. Madagascar: 306, pl. I3, fig. 253, by original designation. Holotype ${ }^{\text {, MAdagascar. }}$

Saalmuellerana was proposed as †Saalmüllerana, an incorrect original spelling. This name is formed from a modern German personal name and under the Code, Article $3^{2}(\mathrm{c})(\mathrm{i})$, must be changed.

SAALUNCIFERA Berio, I966, Annali Mus. civ. Stor. nat. Giacomo Doria 76: I I4. ACro

Type-species: Saaluncifera uncinata Berio, I966, ibidem 76: I I5, fig. 4, by original designation. Holotype ô, MADagascar: S., Bekil Reg. (MNHN, Paris).

Berio himself stated in the original description that the type-species (described intentionally as 'n. sp.') was probably the same as Hy'pogramma uncinata Saalmüller, I89 I, Lepid. Madagascar: 43I, fig. I62.

SABLIA Sukhareva, 1973, Ent. Obozr. 52: 405, 4I3.

HADE

Type-species: Leucania anderreggii Boisduval, 1840 , Genera Index meth. Eur. Lepid.: I32, by original designation. Type(s), [Switzerland]: Alps.

Sablia was proposed as a subgenus of Mythimna Ochsenheimer, I816.

SACADODES Dyar, I9I 2, Proc. ent. Soc. Wash. 14: I67.

Type-species: Sacadodes pyralis Dyar, I912, ibidem 14: I68, by monotypy. Syntypes 2 t, I \&, TRINIDAD (USNM, Washington).

SACCHAROPHAGOS Schaus, I923, Proc. ent. Soc. Wash. 25: i6 6.

OPHI

Type-species: Saccharophagos mochisa Schaus, I929, ibidem 25: I65, by original designation. Type(s) ô., Mexico: Sinaloa, Los Mochis (USNM, Waslington).

SADARSA Moore, I882, in Hewitson \& Moore, Descr. new Indian lepid. Insects Colln late Mr W. S. Atkinson: 164 .

$\operatorname{STIC}$

Type-species: Sadarsa longipennis Moore, I882, ibidem: 165, pl. 5, fig. 14, by original designation. Type(s) ô, InnIa: Darjiling (MNHU, Berlin).

SAFIA Guenée, I 852, in Boisduval \& Guenće, Hist. nat. Insectes (Lépid.) 7: 20. CATO

Type-species: Phalaena celia Stoll, 1781, in Cramer, Uitlandsche liapellen 4: Io9, pl. 346 , figs E, F, by monotypy. Type(s), Surinam.

$P$. celia is dated from the wrapper of the part and not from the title-page of the volume, I 782 
SAFIDIA Hampson, I913, Cat. Lepid. Phalaenae Br. Mus. 12: 4. Available, but without included species until Hampson, I9r3, ibidem 13: ix, I63.

Type-species: Safidia azteca Schaus sensu Hampson, I913, ibidem 13: I63 [a misidentification here named Safidia druceria sp.n.], by subsequent designation by Hampson, I9I3, ibidem 13: I63 (but cited as $S$. azteca Schaus). Holotype ô., Mexico: Jalapa (M. Trujillo) (BMNH). Allotype 9 , same data (BMNH).

Paratypes. Mexico: I $\hat{\sigma}$, Jalapa ( $M$. Trujillo) (type of S. azteca ab. I Hampson, I9I3 = type of $S$. azteca ab. aztecoides Strand, I9I7) (BMNH); I , same data (type of S. azteca ab. 2 Hampson, I9I3 = type of $S$. azteca ab. aztecella Strand, I9I7) $(\mathrm{BMNH})$; I ㅇ․, Orizaba, iv.1896 (W. Schaus) (BMNH); I 우, Orizaba (Boucard) (BMNH); I 9 , Vera Cruz, Huatuxco (BMNH). Costa RICA: I ㅇ, Candelaria Mts (Underwood) (type of S. azteca ab. 3 Hampson, I9I3 = type of S. azteca ab. aztecana Strand, I9I 7) (BMNH);

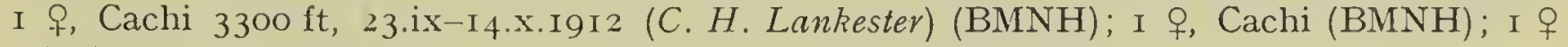
(Underwood) (BMNH); Colombia: i ㅇ, Bogota (Child) (BMNH).

Description of Safidia druceria sp. n., as for Sypna azteca Schaus sensu Druce, I898, Biologia cent.-am. (Zool.) Lepid. Heterocera 2: 5I4, pl. 97, figs 2, 3: also as for Safidia azteca Schaus sensu Hampson, 1913, Cat. Lepid. Phalaenae Br. Mus. 13: 163, fig. 50.

Safidia when proposed by Hampson contained only one nominal species Hydraecia azteca Schaus, 1894, Trans. Am. ent. Soc. $21: 231$. I have compared the original description by Schaus; a colour photograph of its type in USNM, Washington; and a specimen in BMNH determined by Schaus, and found that they are of a species in the Hadeninae which is very different from the species in the Catocalinae described and illustrated by Druce, I898, and by Hampson, I9I3. In his personal interleaved copy, Hampson has subsequently crossed out the Schaus reference. H. azteca Schaus, 1894, was proposed as +'Hadroecia azteca sp. nov.' without any indication that a new genus was being proposed, although in the same work four new genera were clearly indicated and described. $\ddagger_{\dagger}$ Hadroecia should therefore be treated as an incorrect subsequent spelling.

See Frontispiece, fig. II, Safidia druceria sp. n., holotype ô; fig. I2, Hydraecia azteca Schaus, ㅇ․

Safidia has a misidentified type-species and under the Code, Article 70 (a), this case should be referred to the Commission.

SAIGONITA Kiriakoff, I97I, Lambillionea 71:5.

AGAR

Type-species: Saigonita paradoxa Kiriakoff, I971, ibidem $71: 7$, by original designation. Holotype ${ }^{\star}$. [South Vietnam]: Saigon (MNHN, Paris).

SAJANIA Kozhanchikov, 1947, Ent. Obozr. 29: I3, I7.

HADE

Type-species: Anarta devagor Kozhanchikov, 1923, Jb. Martjanov'schen Staatsmus. $1: 45$, by original designation. Syntypes I I $\hat{~}^{*} 4$ 우 [U.S.S.R.]: Sajan Mts, R. Kasyr Suk (ZI, Leningrad).

SALACIA Boie, I839. See Discestra Hampson, 1905.

HADE

${ }_{+} \boldsymbol{S A L I A}$ Hübner, [1806]. See Colobochyla Hübner, [I 825].

OPHI

SALIA Hübner, [18I8], Samml. exot. Schmett. 1: pl. [208].

OPHI

Type-species: Salia mimalis Hübner, [1818], ibidem 1 : pl. [208], figs I-4, by monotypy.

Syntypes 0 , 9 , Type-locality not stated [EQUatorial South America].

MEGATOMIS Hübner, I82 I, Index exot. Lepid.: [5].

Type-species: Salia mimalis Hübner, [18I8], by monotypy.

Megatomis is a junior objective synonym of Salia Hübner, [I8I8].

HOMOGRAMMA Guenée, I 854, in Boisduval \& Guenée, Hist. nat. Insectes (Lépid.) 8: 88.

Type-species: Salia mimalis Hübner, [I8I8], by subsequent designation by Desmarest,

(1857), in Chenu, Encycl. Hist. nat. Papillons nocturnes: 219, but cited as †miacalis

Hübner, an incorrect subsequent spelling.

Homogramma is a junior objective synonym of Salia Hübner, [1818]. 
SALIGENA Walker, I865, List Specimens lepid. Insects Colln Br. Mus. 32: 605.

Type-species: Saligena personata Walker, I865, ibidem 32: 606, by monotypy. Holotype j, U.S.A. (BMNH).

S. personata is a junior subjective synonym of Raphia frater Grote, 1864, Proc. ent. Soc. Philad. 2: 435 , pl. 9, fig. 7 .

SALTIA Tams, 1952, Ann. Mag. nat. Hist. (1 2) 5: 870.

HADE

Type-species: Saltia acrophylax Tams, I952, ibidem (I2) 5: 870, figs 3-I I, by original designation. Holotype $\hat{\sigma}$, [TANZANiA]: Tanganyika, Kilimanjaro, I4 $500 \mathrm{ft}$ (BMNH).

SANACEA Walker, [1866] I865, List Specimens lepid. Insects Colln Br. Mus. 34: I203. opHI Type-species: Sanacea truncatalis Walker, [1866] 1865, ibidem 34: I203, by monotypy. Holotype $\hat{\jmath}, V$ TENEZUELa (BMINH).

${ }_{\ddagger} S A N D A S A$, misspelling. See Sandava Walker, [1863].

OPHI

SANDAVA Walker, [I863] I862, List Specimens lepid. Insects Colln Br. Mus. 26: I623. OpHI Type-species: Sandava melaleucata Walker, [1863] I 862, ibidem 26: I623, by monotypy. Syntypes $\hat{0}$, ㅇ, [Australia]: [Qd], Moreton Bay (BMNH).

S. melalencata is a junior subjective synonym of Cidaria scitisignata Walker, I862, ibidem 25: I 423 .

See also Istarba Walker, [1866].

†SANDASA; Kaye, I90 I, Trans. ent. Soc. Lond. 1901: I35.

An incorrect subsequent spelling.

SANTIAXIS Schaus, I9I6, Proc. U.S. natn. Mus. 50: 333.

HYPE

Type-species: Santiaxis copima Schaus, I9I6, ibidem 50:334, by original designation. Type(s) $\overrightarrow{0}$, Cuba: Santiago (USNM, Washington).

SANYS Guenée, I 852, in Boisduval \& Guenée, Hist. nat. Insectes (Lépid.) 7: 349.

OPHI

Type-species: Sanys carnina Guenéc, I852, ibidem 7: 350, by subsequent designation by Desmarest, (1857), in Chenu, Encycl. Hist. nat. Papillons nocturnes: 134. Holotype ot, BRazIL: Nouvelle-Fribourg (BMNH).

SARACA Walker, [1866] i 865, List Specimens lepid. Insects Colln Br. Mus. 34: I I9o. OpH I Type-species: Saraca disruptalis Walker, [1866] I 865, ibidem 34: I I90, by monotypy. Holotype q. [CHINA]: Shanghai (BMNII).

$S$. dismptalis is a junior subjective synonym of Egnasia curtalis Walker, [1866] I865, ibidem 34: II 77 .

SARAGOSSA Staudinger, I900, Dt. ent. Z. Iris 13: Io9.

HADE

Type-species: Saragossa seeboldi Staudinger, Igoo, ibidem 13: I09, by monotypy. Holotype ô, Spain: Saragossa, Upper Duro (MNHU, Berlin).

SARBANISSA Walker, I865, List Specimens lepid. Insects Colln Br. Mus. 33: $746 . \quad$ AGAR Type-species: Sarbanissa insocia WValker, I 865, ibidem 33: 746, by monotypy. Type(s) $\hat{o}$, [NDIA] : Darjeeling.

SARBENA Walker, I862, J. Proc. Limn. Soc. (Zool.) 6: 137.

NOLI Type-species: Sarbena lignifera Walker, I862, ibidem 6: 137, by monotypy. Lectotype ô., Borneo: Sarawak (UNI, Oxford), designated (as type) by Swinhoe, Igoo, Cat. east and Aust. Lepid. Heterocera 2: 589.

See also Cyphotopsyche Hampson, I895. 
SARCOPTERON Hampson, I893, Illust. typical Specimens Lepid. Heterocera Colln Br. Mus. 9: 28, II 5 . OPHI

Type-species: Sarcopteron punctimargo Hampson, I893, ibidem 9: II5, pl. I66, fig. 22, by PRESENT DESIGNATION. Type(s) ㅇ, Ceylon: Pundaloya (BMNH).

SARMATIA Guenée, I854, in Boisduval \& Guenée, Hist. nat. Insectes (Lépid.) 8: 25 . HyPE Type-species: Sarmatia interitalis Guenée, I854, ibidem $8: 25$, pl. 5, fig. I, by monotypy. Lectotype ô, [South Africa]: Cap de Bonne-Espérance (MNHN, Paris), designated by Viette, I951, Bull. mens. Soc. linn. Lyon 20: I62.

See also Sıma Walker, [1866].

SAROBA Walker, I865, List Specimens lepid. Insects Colln Br. Mus. 33: 1096.

OPHI

Type-species: Saroba pustulifera Walker, I865, ibidem 33: 1097, by monotypy. Holotype , , [INDiA]: N. Hindostan (BMNH).

SAROBELA Turner, 1936, Proc. R. Soc. Qd 47: 44.

HYPE

Type-species: Sarobela spectabilis Turner, I936, ibidem 47: 44, by monotypy. Syntypes 07, , Australia: N. Qd, Cape York (QM, Brisbane).

S. spectabilis is a junior subjective synonym of Plusia litterata Pagenstecher, I888, Jb. nassau. Ver. Naturk. 41: 137 .

SAROBIDES Hampson, 1926, Descr. new Genera Species Lepid. Phalaenae Subfamily Noctuinae Br. Mus.: 4ro.

OPHI

Type-species: Cosmophila inconclusa Walker, [1863] r864, J. Proc. Linn. Soc. (Zool.) 7: 77, by original designation. Lectotype, Borneo: Sarawak (UM, Oxford), designated (as type) by Swinhoe, I900, Cat. east and Aust. Lepid. Heterocera 2: 193.

SAROPTILA Turner, 1909, Proc. Linn. Soc. N.S.W. 34: 353.

HYPE

Type-species: Saroptila milichias Turner, 1909, ibidem 34:354, by original designation.

Holotype $\hat{\sigma}$, Australia: N. Qd, Kuranda (ANIC, Canberra).

$\ddagger$ SAROTHRIPA, misspelling. See Sarrothripus Curtis, I824.

SARR

SAROTHRIPUS Agassiz, 1846. See Sarrothripus Curtis, I824.

SARR

SAROTHROCERAS Mabille, I889, Bull. Soc. ent. Fr. (6) 9: xcix.

HYPE

Type-species: Sarothroceras alluaudi Mabille, I889, ibidem (6) $9:$ xcix, by monotypy. Lectotype $\hat{\sigma}$, [Ivory COAST]: Assinie (BMNH), designated (as holotype) by Viette \& Fletcher, 1968, Bull. Br. Mus, nat. Hist. (Ent.) 21: 4I5.

SAROTRICHA Meyrick, I888. See Sarrothripus Curtis, I824.

SARR

$\ddagger$ SARROTHRIPA, misspelling. See Sarrothripus Curtis, I824.

SARR

SARROTHRIPA Duponchel, [1845]. See Sarrothripus Curtis, I824.

SARR

SARROTHRIPUS Curtis, 1824, Br. Ent. 1: 29.

SARR

Type-species: Tortrix degenerana Hübner, [1799], Samml. eur. Schmett. 7: pl. 2, fig. 8, by original designation. Type(s), EuRope.

$¥ N$ YCTEOLA Hübner, [1806], Tentamen determinationis digestionis . . .: [2].

Included in a work rejected for nomenclatural purposes by Int. Commn zool. Nom., 1926, Smithson. misc. Collns 73 (4) Opinion 97: 19. Also idem, 1954, Opin. Decl. int. Commn zool. Nom. 6 Opinion 278: 140.

Only included species: Tortrix degenerana Hübner, [I799].

$\ddagger$ SARROTHRIPA; Duponchel, 1834, in Godart \& Duponchel, Hist. nat. Lépid. Papillons Fr. 9: 19, 556 .

An incorrect subsequent spelling.

SARROTHRIPA Duponchel, [1845] 1844, Cat. méth. Lépid. Eur.: 284 .

An unjustified emendation of Sarrothripus Curtis, r 824 . 
SAROTHRIPUS Agassiz, I846, Nomencl. zool. Index univl.: 331.

An unjustified emendation of Sarrothripus Curtis, I 824.

${ }_{\ddagger}^{+}$SAROTHRIPA; Rogenhofer, 1882, Verh. zool.-bot. Ges. Wien 31 Sber.: 26.

An incorrect subsequent spelling; also used by Swinhoe, igor, Ann. Mag. nat. Hist.

(7) $7: 490$.

SAROTRICHA Meyrick, i888, Proc. Linn. Soc. N.S.W. (2) 2: 924.

An unjustified emendation of Sarrothripa Duponchel, [1845].

SARTHA Staudinger, I891, Stettin. ent. Ztg 52: 229.

OPHI

Type-species: Namangana mirabilis Staudinger, 1888, ibidem 49: 52, by monotypy (of Namangana Staudinger, I888, ibidem 49: 52). Syntypes 2 ô, 2 ㅇ․ [U.S.S.R.]: [Uzbekistan], Namangan.

Sartha was proposed as the objective replacement name for Namangana Staudinger, I 888, ibidem 49: 52 .

NAMANGANA Staudinger, 1888, Stettin. ent. Ztg 49: 52.

Type-species: Namangana mirabilis Staudinger, I 888, by monotypy.

A junior homonym of Namangana Staudinger, i 888, ibidem 49: 28, - Lepid., Noctuidae. The objective replacement name is Sartha Staudinger, I89r.

SARTHIDA Walker, [1863] i 864, J. Proc. Linn. Soc. (Zool.) 7: 8o.

OPH I

Type-species: Sarthida signifera Walker, [1863] I 864, ibidem 7: 81, by monotypy. Lectotype $\hat{0}$, Borneo: Sarawak (UM, Oxford), designated (as type) by Swinhoe, I90o, Cat. east. and Aust. Lepid. Heterocera 2: i94.

S. signifera is a junior subjective synonym of Ugia disjungens Walker, 1858 .

See also Ugia Walker, $18,8$.

SARUNGA Walker, I 869, Characters undescr. Lepid. Heterocera: I Io.

OPHI

Type-species: Sarunga calida Walker, I 869, ibidem: I Io, by monotypy. Type(s) J. [Bangladesh]: [E. Bengal], Silhet.

SASERNA Druce, 1891, Biologia cent.-am. (Zool.) Lepid. Heterocera 1: 444.

OPHI

Type-species: Hypena scissilinea Walker, 1862, Trans. ent. Soc. Lond. (3) 1: 261, by original designation. Type(s) $\hat{\sigma},[$ BrazIL $]$ : Rio Janeiro.

SASUNAGA Moore, I881, Proc. zool. Soc. Lond. 1881: 342.

ACRO

Type-species: Hadena tenebrosa Moore, I867, ibidem 1867: 59, by monotypy. Type(s) o., [INDIA]: Bengal.

SATRAPODES Hampson, 1908, Cat. Lepid. Phalaenae Br. Muts. 7: 8. Available, but without included species until Hampson, I9I0, ibidem 9: x, 217.

ACRO

Type-species: Basilodes mina Schaus, 1894, Trans. Am. ent. Soc. 21 : 239, by subsequent monotypy. Type(s), Mexico: Jalapa (USNM, Washington).

SAVARA Walker, I862, J. Proc. Linn. Soc. (Zool.) 6: I84.

OPH I

Type-species: Savara contraria Walker, I862, ibidem 6: i 84 , by monotypy. Lectotype o. Borneo: Sarawak (UM, Oxford), designated (as type) by Swinhoe, 190o, Cat. east. and Aust. Lepid. Heterocera 2: 97.

SAVOCA Walker, I864, List Specimens lepid. Insects Colln Br. Mus. 30: $996 . \quad$ STIC

Type-species: Savoca sarawakana Walker, I864, ibidem 30: 996, by monotypy. Holotype 9 , Borneo: Sarawak (BMNH).

S. sarawakana is a junior subjective synonym of Gyrtona divitalis Walker, I863, ibidem 27: 91 .

SCAMBINA Walker, i865, List Specimens lepid. Insects Colln Br. Mus. 33: i i 8.

OPHI

Type-species: Scambina aliena Walker, I 865, ibidem 33: I I 19, by original designation. Holotype $\hat{o}$. Sierra LeONe (BMNH). 
SCEDOPLA Butler, I878, Ann. Mag. nat. Hist. (5) 1: 201.

OPHI

Type-species: Scedopla regalis Butler, I878, ibidem (5) 1:201, by original designation. Type(s) ô, Japan: Yokohama (BMNH).

SCELESCEPON Möschler, I89o, Abh. senckenb. naturforsch. Ges. 16: 230.

HYPE

Type-species: Scelescepon mutatalis Möschler, I890, ibidem 16: 230, by monotypy. Syntypes 0 . q. Puerto Rico (MNHU, Berlin).

SCHACHOWSKOYA Köhler, I953, Revta Soc. ent. argent. 16: 90.

NOCT

Type-species: Schachowskoya indecora Köhler, I953, ibidem 16: 9o, fig. 3, by original designation. Holotype $\tilde{\sigma}$, Argentina: Neuquén [Prov.], Pucará (ZSBS, Munich).

SCHALIDOMITRA Strand, I9 I I, Dt. ent. Z., 1911: 584.

OPHI

Type-species: Schalidomitra ambages Strand, I9I I, ibidem 1911: 584, by original designation. Holotype + , [Tanzania] Dt.-Ost-Afr.: [Lake] Nyassa, Kilondo-Mündung.

SCHALIFRONTIA Hampson, igoi, Cat. Lepid. Phalaenae Br. Muss. 3: 518, 654. Agar Type-species: Schalifrontia furcifer Hampson, I9oI, ibidem 3: 654, by monotypy. Holotype q, BRAzIL: Sta Catherina (BMNH).

SCHAUSIA Karsch, I895, Ent. Nachr. 21:346.

AGAR

Type-species: Aegocera leona Schaus, 1893, in Schaus \& Clements, Colln Sierra Leone Lepid.: 20, pl. I, fig. I, by original designation. Type(s), Sierra Leone.

SCHAZAMA Schaus, 1906, Proc. U.S. natn. Mus. 30: 1o8.

EUTE

Type-species: Schazama angustipennis Schaus, I906, ibidem 30: I09, by monotypy. Type(s), Brazil: São Paulo (USNM, Washington).

SCHINIA Hübner, I8I8, Zuträge Samml. exot. Schmett. 1: 8, I I.

N OCT

Type-species: Schinia trifascia Hübner, I8 18, ibidem 1: I I, figs 33, 34, by subsequent designation by Grote, I874, Bull. Buffalo Soc.nat.Sci. 2: 32. Type(s) §̋, [U.S.A.]: Georgia [formerly] in Florida.

$\ddagger$ EUCLIDIA Hübner, I808, Erste Zuträge Samml. exot. Schmett.: 3.

Included in a work rejected for nomenclatural purposes by Int. Commn zool. Nom., I966, Bull. zool. Nom. 23 Opinion 789:214. Placed on the Official Index of Rejected and Invalid Generic Names in Zoology: Name No. I842.

One of the five originally included names (all nomenclaturally unavailable), $\ddagger$ Euclidia gracilis, was cited as type-species by Tams, 1939, Entomologist 72: 138. This species, later made available as Schinia gracilenta Hübner, I8I8, is congeneric with Schinia trifascia Hübner, I8I8, the type-species of Schinia Hübner, I8'18.

TRILEUCA Grote, I883, Trans. Am. ent. Soc. 10: 265.

Type-species: Schinia trifascia Hübner, I818, by subsequent designation by Hampson, 1903, Cat. Lepid. Phalaenae Br. Mus. 4: 72.

Incorrect type-species designation: Schinia buxea Grote, I882, a name not originally included in Trilenca, and not linked with one of the originally included names when cited by Grote, I895, Abh. naturw. Ver. Bremen 14: 93.

Trileuca is a junior objective synonym of Schinia Hübner, I8I8.

CANIDIA Grote, I890, Revised Check List N. Am. Noctuidae 1: 36.

Type-species: Lygranthoecia scissa Grote, I877, Proc. Boston Soc. nat. Hist. 18: 4I5, by monotypy. Type(s), [U.S.A.]: Florida, Appalachicola.

A junior homonym of Canidia Thomson, 1857, Arch. ent. Paris 1: 193,-Coleoptera. There is no objective replacement name but L. scissa is congeneric with Schinia trifascia Hübner, 1818, the type-species of Schinia Hübner, I8I8, which is therefore available for use as the subjective replacement name.

$\ddagger$ TRILENCA; Neave, I9ło, Nomencl. zool. 4: 558 .

An incorrect subsequent spelling.

FF 
SCHIRACES Schaus, I916, Proc. U.S. natn. Mus. 50: 265, 395.

HYPE Type-species: Schiraces mopsus Schaus, I9I6, ibidem 50: 396, by original designation. Type(s) 9 , French Guiana: Cayenne (USNM, Washington).

SCHISTORHYNX Hampson, I898, J. Bombay nat. Hist. Soc. 11: 706. OPHI

Type-species: Schistorhynx argentistriga Hampson, I898, ibidem 11: 706, fig., by original designation. Type(s) $\delta$. INDiA: Assam, Khasis (BMNH).

SCHOYENIA Aurivillius, I883, Ent. Tidskr. 4: I9I.

NOCT

Type-species: Schoyenia arctica Aurivillius, I883, ibidem 4: I93, by monotypy. Syntypes, [U.S.S.R.]: [Novaya Zemlya I.] Novaja Semlia I. (NR, Stockholm).

S. arctica is a junior subjective synonym of Amphidasis unifasciata Ménétriés, I 85I, in Middendorff, Reise aussersten Norden und Osten Sibiriens 2 (I): 58, pl. 3, fig. I2.

Schoyenia was proposed as †Schöyenia, an incorrect original spelling. This name is formed from the modern personal name Schöyen which is Scandinavian and not German The ' $\ddot{\circ}$ ' must therefore be changed under the Code, Article 32 (c) (i) to ' 0 ' and not to 'oe.'

SCHRANCKIA Walker, [I859]. See Tholomiges Lederer, I 857 HYPE

SCHRANKIA Hübner, [I825] I8I6, Verz. bekannter Schmett.: 345. HYPE

Type-species: Pyralis taenialis Hübner, [I809], Samml. eur. Schmett. 6: pl. 23, fig. I 5 I, by monotypy. Type(s), Europe.

The type-species was originally proposed as $+P$. tänialis. Under the Code, Article 32 (c), this is regarded as an incorrect original spelling. Hübner himself emended it to taenialis when Schrankia was proposed.

HYPENODES Guenée, I 854, in Boisduval \& Guenée, Hist. nat. Insectes (Lépid.) 8: 4 I.

Type-species: Crambus albistrigatis Haworth, [1809], Lepid. Br.: 368, by subsequent designation by Hampson, I895, Fauna Br. India (Moths) 3: 98, but cited as +'albistrigalis Haw.', an incorrect subsequent spelling. Type(s), [GREAT] Britain.

A junior homonym of Hypenodes Doubleday, I 850,- Lepid., Noctuidae. This generic name originated from Guenée, but was used and unintentionally made nomenclaturally available by Doubleday prior to its proposal and generic description by Guenée for a similar concept but having a different type-species. There is no objective replacement name but $C$. albistrigatis Haworth, [1809], is a junior subjective synonym of Pyralis taenialis Hübner, [ISog], the type-species of Schrankia Hubner. The latter is therefore available for use as a subjective replacement name.

SCHRANKIA Herrich-Schäffer, [I85I]. See Tholomiges Lederer, I857.

HYPE

SCIOMESA Tams \& Bowden, I953, Bull. ent. Res. 43: 648.

ACRO

Type-species: Conicofrontia mesoscia Hampson, I9I 8, Novit.zool. 25: I56, by original designation. Holotype 9 , [South Africa]: Natal, Durban (BMNH).

SCIOPTILA Warren, I9I I, in Seitz, Macrolepid. World 3: 220.

ACRO

Type-species: Ophiusa eriopoda Herrich-Schäffer, I85I, Syst. Bearb. Schmett. Eur. 2: 4I3, pl. I I6, fig. 596, by original designation. Holotype ô, [TuRkEY]: [Anatolia], [Bursa] Brussa.

O. eviopoda is dated from the original wrappers.

The date of publication of the part of Seitz containing page 220 , was stated on page 2 I3 as I9I I March Ist in the English edition and as I9I I December Ioth in the German edition.

SCLEROPLUSIA Ichinose, I962. See Macdunnoughia Kostrowicki, I96i.

PLUS

SCODIONYX Staudinger, I899, Dt. ent. Z. Iris 12: 397.

CATO

Type-species: Scodionyx mysticus Staudinger, I899, ibidem 12: 397, pl. 5, fig. 2, by monotypy. Holotype oే. [ISRAEL]: J)ead Sea, W. bank (MNHU, Berlin). 
SCOEDISA Walker, [1858] I857, List Specimens lepid. Insects Colln Br. Mus. 13: 984, 1007.

OPHI

Type-species: Scoedisa designans Walker, [1858] I857, ibidem 13: 1007, by monotypy. Type(s) ô. BrazIL.

S. designans is a junior subjective synonym of Phalaena picta Sepp, I848, Surinaamsche Vlinders: 99 , pl. 46.

SCOLECOCAMPA Guenée, 1852, in Boisduval \& Guenée, Hist. nat. Insectes (Lépid.) 5: I3I.

OPHI

Type-species: Scolecocampa ligni Guenée, I 852, ibidem 5: I 3 I, pl.6, fig. 3, by monotypy. Lectotype ô. [U.S.A.] Amérique Septentrionale: Géorgie (MNHN, Paris), designated by Viette, 195x, Bull. mens. Soc. linn. Lyon 20: I60, locality cited as Santa Cruz.

S. ligni is a junior subjective synonym of Clytie liburna Geyer, I837, in Hübner, Zuträge Samml. exot. Schmett. 5: 40, figs 963, 964.

†SCOLICOCAMPA; Walker, I856, List Specimens lepid. Insects Colln Br. Mus. 9: I65.

An incorrect subsequent spelling.

$¥ S C O L I C O C A M P A$, misspelling. See Scolecocampa Guenée, I 852 .

OPHI

SCOLIOPTER YX Germar, I81o, Diss. sistens Bombycum Species: I4.

OPHI

Type-species: Phalaena libatrix Linnaeus, 1758, Syst. Nat. (Edn 10) 1: 507, by monotypy. Type(s), TYPE-LOCALITY not stated (LS, London) [EUROPE].

PTERODONTA R.L., I8I 7, Jenaische Allg. Lit.-Ztg 1: 286.

Type-species: Phalaena libatrix Linnaeus, $175^{8}$, by PRESENT DESIGNATION.

Pterodonta R.L., is a junior objective synonym of Scoliopteryx Germar, I8I I.

EPHEMIAS Hübner, [I82I] I8I6, Verz. bekannter Schmett.: 248.

Type-species: Phalaena libatrix Linnaeus, I758, by monotypy.

Ephemias is a junior objective synonym of Scoliopteryx Germar, I8I I.

EUPHAIS Hübner, I822, Syst.-alphab. Verz.: 21, 29.

Type-species: Phalaena libatrix Linnaeus, 1758 , by subsequent designation by Berio, 1957, Memorie Soc. ent. ital. 36: I4.

Euphais is a junior objective synonym of Scoliopteryx Germar, I8I I.

GONOPTERA Berthold, I827, in Latreille, Natürliche Familien Thierreichs: 483.

Type-species: Phalaena libatrix Linnaeus, 1758 , by monotypy, but cited as 'Noct. libatrix

Fabr.,' an incorrect authorship.

Billberg, I820, Enumeratio Insect. Mus. G. J. Billberg: 76, published the following 'Gonrptera Eg[0]. - Gonepteryx L[ea]ch . . ' in the butterfly family Pieridae. Scudder, I 875, Proc. Am. Acad. Arts. Sci. 10: I8I, interpreted Billberg's emendation as a misspelling which should have read Gonoptera Billberg, I820. Sherborn, I926, Index Anim. (I80I1850): 2758; Neave, I939, Nomencl. zool. 2: 504; and Hemming, I967, Bull. Br. Mus. nat. Hist. (Ent.) Suppl. 9: 201, have all listed Gonoptera Billberg, I820. If this is accepted as a nomenclaturally available name, then Gonoptera Berthold, r927, as well as being a junior objective synonym, is a junior homonym and has Scoliopteryx Germar, I8I I, as its objective replacement name.

$\ddagger$ LIBATRIX Berthold, I827; Sherborn, I927, Index Anim. (I80I-I850): 3547.

Sherborn cited Libatrix as a generic name with the comment '? usu. gen.' Berthold, 1827, in Latreille, Nat. Familien Thierreichs: 483, cited 'Noct. Libatrix, Fabr.' as a specific and not a generic name.

SCOLOPOCNEME Felder \& Felder, I862, Wien. ent. Monatschr. 6: 37.

SARR

Type-species: Scolopocneme bufonia Felder \& Felder, I862, ibidem 6:38, by monotypy. Type(s) o, China: [Che-Kiang] Ning-po.

SCOPARIOPSIS Strand, I909, Ent. Rdsch. 26: I4I.

ACRO

Type-species: Scopariopsis viridigrisea Strand, I909 ibidem 26: I45, by original designation. Holotype o, CAmeroun: Victoria (MNHU, Berlin). 
†SCOPELOPUS Stephens, I 829 [June]. See Scopelopus Stephens, I 829 [August].

SCOPELOPUS Stephens, I829 [August], Sy'st. Cat. Br. Insects 2: i I o.

OPH I

Type-species: Phytometra scopulepes Haworth, 1809, Lepid. Bx.: 260 (but included as $¥ P$. scopulaepes, an incorrect subsequent spelling), by monotypy. Type(s), TrPELOCALITY stated to be Great Britain: England, but this was probably an error as this species is not known to occur in the Old World, but does occur in soutliern U.S.A. and Mexico.

Stephens when he proposed Scopelopus, also proposed Scopelopus inops as an unnecessary objective replacement name for $\ddagger P$. scopulaepes.

See also Hemeroplanis Hübner, I8I8; and Coptocnemia Zeller, I872.

${ }_{\ddagger}$ SCOPELOPUS Stephens, I 829 [June], Nom. Br. Insects: 43.

OPHI

Type-species: ${ }_{\uparrow}$ Scopelopus inops Stephens, I 829, ibidem: 43 , nomen nudum, by monotypy.

Both the generic and the specific names were nomina nuda.

SCOPELOSOMA Curtis, I836. See Eupsilia Hübner, [1821].

CUCU

$\ddagger$ SCOPIBLEPTA Hampson, no published reference found.

HYPE

SCOPIFERA Herrich-Schäffer, 1870, CorrespBl. zool.-min. Ver. Regensburg 24: 103, 108.

Available, but without included species until Felder $\&$ Rogenhofer, i 874 . HYPE

Type-species: Scopifera longipalpis Felder \& Rogenhofer, 1874, Reise öst. Fregatte Novara (Zool.) 2 (Abt. 2): pl. I20, fig. 35, by subsequent monotypy. Syntypes ô, , Venezuela. Brazil: Marabitanas (BMNH) ; Nov. Friburg (BMNH).

S. longipalpis is a junior subjective synonym of Bleptina menippusalis Walker, [1 859] I 858, List Specimens lepid. Insects Colln Br. Mus. 16: I 26.

SCOPULA Schrank, I802, Fauna Boica 2(2): I62.

GEONETRIDAE

Type-species: Phalaena paludata Linnaeus, I 767 , Syst. Nat. (Edn I2) 1(2): 873, by subsequent designation by Prout, I9I I, Trans. Cy Lond. ent. nat. Hist. Soc. 20 : 25, where ornata Scopoli, 1763 , is cited and at the same time reference is made to Prout, 1906, Entomologist 39: 266, where ornata is placed as a synonym of paludata.

Scopula has been used as the original generic name for species which are now in the Noctuidae.

$\ddagger$ SCOPULOMIA Hampson, no published reference found.

HYPE

SCOTIA Hübner, [I821] I816, l'erz. bekannter Schmett.: 226.

NOCT

Type-species: Noctua cinerea [Denis \& Schiffermüller], r 775, Ankündung syst. Werkes Schmett. Wienergegend: 80, by subsequent designation by Hampson, 1903, Cat. Lepid. Phalaenae Br. Mus. 4: I53. Type(s), [AustriA]: Vienna district (Collection destroyed, Horn \& Kahle, 1936, Ent. Beih. Berl.-Dahlem 3: 243).

$N$. cinerea is congeneric with Noctua segetum [Denis \& Schiffermuiller], I 775, the typespecies of Agrotis Ochsenheimer, I8I6. Scotia is therefore a junior subjective synonym.

SCOTOCAMPA Staudinger, r 888, Stettin. cnt. Ztg 49: 36.

$\mathrm{ACRO}$

Type-species: Scotocampa indigesta Staudinger, I 888, ibidem 49: 36, by monotypy.

Syntypes 20. [CHINA]: [Sin Kiang], Kuldja District (MNHU, Berlin).

SCOTOCHROSTA Lederer, I857, Noctuinen Eur.: 40, I55.

CUCU

Type-species: Noctua pulla Denis \& Schiffermuiller, I775, Ankiindung sy'st. Werkes Schmett. Wienergegend: 76, by monotypy. Type(s), [Austrin]: Vienna district (Collection destroyed, Horn \& Kahle, 1936, Ent. Beih. Berl.-Dahlem 3: 243).

SCOTOGRAMMA Smith, I 897, Proc. U.S. natn. Mus. 10: 469.

HADE

Type-species: Anarta submarina Grote, I883, Can. Ent. 15: 4, by original designation. Syntypes 4 ex., [U.S.A.]: Montana.

SCOTOPHILA Hübner, [1821]. See Amphipyra Ochsenheimer, i 816.

$\triangle C R O$ 
SCOTOPHILA Stephens, I829. See Lycophotia Hübner, [1821]. NocT

SCOTOSTENA Hampson, I910, Cat. Lepid. Phalaenae Br. Mus. 10: xiii, 372. ACoN

Type-species: Scotostena lugens Hampson, I910, ibidem 10: 372, fig. I I5, by original designation. Syntypes 4 ㅇ, New Guinea: [Papua], Port Moresby (BMNH).

SCRIPTANIA Hampson, I905, Cat. Lepid. Phalaenae Br. Mus. 5: xiv, 464.

HADE

Type-species: Agrotis michaelseni Staudinger, I899, Ergebn. Hamb. Magalhaensischen Sammelveise $4(7)$ : 6o, pl. I, fig. I3, by original designation. Syntypes 2 o, [ARGENTINA]: [Tierra del Fuego], Uschuaia.

SCROBIGERA Jordan, 1896, in Rothschild \& Jordan, Novit. zool. 3: 37, 40.

AG.AR

Type-species: Eusemia amatrix Westwood, I 848 , Cabinet oriental Ent.: 68, pl. 33, fig. 4, by original designation. Type(s), INDiA: Assam (UM, Oxford).

SCULPTIFRONTIA Berio, I966, Annali Mus. civ. Stor. nat. Giacomo Doria 76: I34. oPHI

Type-species: Sculptifrontia arcuata Berio, I966, ibidem 76: I35, fig. 25, by original designation. Holotype 0 , MAdagascar: E., Route d’Anosibe, lim 57 (MNHN, Paris).

SCUTIRODES Schaus, I916, Proc. U.S. natn. Mus. 50 : 260, 265.

HYPE

Type-species: Hypenodes apis Druce, I89I, Biologia cent.-ann. (Zool.) Lepid. Heterocera 1: 442, pl. 36, fig. 3, by original designation. Syntypes 2 ex., Guatemala: Vera Paz, Balheu (BMNH). PANAMA: Chiriqui.

SCYTHOBR YA Boursin, I960, Bull. mens. Soc.linn. Lyon 29: I 70.

$\triangle C R O$

Type-species: Bryophila subliterata Filipjev, I931, Abh. der Pamir-Exped. r928 (Akad. Wiss. U.S.S.R.) 8: I56, fig. 4, pl. 4, fig. 4, by original designation. Syntypes I $0^{\star}$, I ㅇ, U.S.S.R.: Tadzhikistan, Pamirs, Chorog (ZI, Leningrad).

Scythobrya was proposed as a subgenus of Cryphia Hübner, I8I8.

†SCYTHOCENTROPIA, misspelling. See Scythocentropus Speiser, I9o2.

ACRO

SCYTHOCENTROPUS Speiser, I902, Berl. ent. Z. 47: I 40.

ACRO

Type-species: Xylina scripturosa Eversmann, I854, Bull. Soc. Nat. Moscou 27 (2): I90, by original designation. Syntypes ô. , [U.S.S.R.]: Kazak, S. Kirghiz steppe (ZI, Leningrad).

Scythocentropus was proposed as the objective replacement name for Centropus Christoph, I889.

CENTROPUS Christoph, I889, in Romanoff, Mém. Lépid. 5: 30.

Type-species: Xylina scripturosa Eversmann, I854, by subsequent designation by Speiser, I902, Berl. ent. Z. 47: I40.

A junior homonym of Centropus Illiger, I8I I, Prod. Syst. Mamm. Avium: 205, - Aves. The objective replacement name is Scythocentropus Speiser, I902.

HUGONIA Alphéraky, r9o8, Revue russe Ent. 7: 267.

Proposed, unnecessarily, as an objective replacement name for Centropus Christoph, I889. The objective replacement name Scythocentropus Speiser, I902, had already been proposed.

CENTROPODIA Hampson, I9o8, Cat. Lepid. Phalaenae Br. Mus. 7: xii, 452.

Proposed, unnecessarily, as an objective replacement name for Centropus Christoph, I889. The objective replacement name Scythocentropus Speiser, I902, had already been proposed.

†SCYTHOCENTROPIA; Oberthür, I912, Etudes Lépid. compavée 6: 332.

An incorrect subsequent spelling.

+SCYTOGNATHA Hampson, no published reference found.

HYPE

SEBAGENA Walker, I865, List Specimens lepid. Insects Colln Br. Mus. 33: 929.

SARR

Type-species: Sebagena furcifera Walker, I865, ibidem 33: 929, by monotypy. Type(s) 오, [Colombia]: Bogota (BMNH).

See also Pucialia Walker, I869. 
SEDINA Urbahn, 1933, Stettin. ent. Ztg 94: I50.

Type-species: Arsilonche buettneri Hering, I858, ibidem 19:442, pl. 3, by original designation. Syntypes 20,8 ㅇ, [?Germany]: Grabow.

$S$. buettneri was originally proposed as $\ddagger A$. bütmeri which under the Code, Article 32 (c)(i), is an incorrect original spelling, and being formed from a German name the 'ü' must be changed to ' $u e$. '

SEGETIA Stephens, I829, Illust. Br. Ent. (Haustellata) 2: I 53.

NOCT

Type-species: Noctua xanthographa [Denis \& Schiffermüller], 1775, Ankündung syst. Werkes Schmett. Wienergegend: 83 , by subsequent designation by Westwood, I 840 , Synopsis Genera Br. Insects: 94. Type(s), [AustrIA]: Vienna district (Collection destroyed, Horn \& Kahle, 1936, Ent. Beih. Berl.-Dahlem 3: 243).

Invalid type-species designation by Boisduval, I836, Hist. nat. Insectes (Lépid.) 1: I 44 , who also cited xanthographa. Boisduval in his lengthy I54 page Introduction reviewed earlier classifications and designated up to three different type-species for each generic name. In his 'Exposé de notre méthode' from pages I 55-69o, no type-species designations were made for the genera he himself used. Under the Code, Article 69(a)(iii), the typedesignation of an author is eligible for consideration if he states that it is the type '... and if it is clear that he himself accepts it as the type-species.' Boisduval's type-designations, although clearly stated, do not fulfil the last requirement and so are invalid. Even though Boisduval's i 836 work was well known to lepidopterists, the type-designations contained in it have not in the past been accepted by Hemming for Rhopalocera, or by other authors.

SEIROCASTNIA Grote, I 866, Proc. ent. Soc. Philad. 6: I 75.

AGAR

Type-species: Ephialtias tribuna Hübner, [183I] I 825, Zuträge Samml. exot. Schmett. 3: 24, figs 49I, 492, by monotypy. Type(s), CuBA.

SELAMBINA Valker, I 858, List Specimens lepid. Insects Colln Br. Mus. 15: I $796 . \quad$ ACRO

Type-species: Selambina trajiciens Walker, I 858, ibidem 15: I796, by monotypy. Type(s) ô, [Brazil]: Rio Janeiro (UM, Oxford).

SELCA Walker, [1866] I865, List Specimens lepid. Insects Colln Br. Mus. 34: I 218.

NOLI

Type-species: Selca latifascialis Walker, [1866] I865, ibidem 34: I219, by subsequent designation by Hampson, I9oo, Cat. Lepid. Phalaenae Br. Mus. 2: 31. Lectotype ô, Borneo: Sarawak (UM, Oxford), designated (as type) by Swinhoe, i 892, Cat. east. and Aust. Lepid. Heterocera 1: 132.

SELENIS Guenée, I852. See Selenisa Hayward, I967.

OPHI

SELENISA Hayward, 1967, Acta zool. lilloana 22: 133.

OPHI

Type-species: Phalaena suero Cramer, I777, Uitlandsche Kapellen 2: 3, pl. 97, fig. F, by subsequent designation (for Selenis Guenée) by Desmarest, (1857), in Chenu, Encycl. Hist. nat. Papillons nocturnes: I 35, but cited as † sueva Cramer, an incorrect subsequent spelling. Type(s), Surinam.

Selenisa was proposed as the objective replacement name for Selenis Guenée, I 852.

SELENIS Guenée, I 852, in Boisduval \& Guenée, Hist. nat. Insectes (Lépid). 7: 36 I.

Type-species: Phalaena suero Cramer, I777, by subsequent designation by Desmarest, (1857), in Chenu, Encycl. Hist. nat. Papillons nocturnes: I 35, but cited as $\ddagger$ sueva Cramer, an incorrect subsequent spelling.

A junior homonym of Selenis Hope, 1839, Ann. nat. Hist. 3: 96,-Coleoptera. The objective replacement name is Selenisa Hayward, 1967.

SELENISTIS Hampson, 1908, Cat. Lepid. Phalaenae Br. Mus. 7: 8. Available, but without included species until Hampson, I910, ibidem 9: xiii, 392. ACRO

Type-species: Tarache annulella Hampson, 1902, Ann. S. Afr. Mus, 2: 383, by subsequent monotypy. Lectotype ô. [RHoDESIA]: Mashonaland, Salisbury (BMNH), designated (as type) by Hampson, 1910, ibidem 9: 393. 
SELENOPERAS Hampson, 1926, Descr. new Genera Species Lepid. Phalaenae Subfamily Noctuinae Br. Mus.: $42 \mathrm{I}$.

OPHI

Type-species: Capnodes caustiplaga Hampson, I896, Fauna Br. India (Moths) 4: 537, by original designation. Type(s) o+, BHutan (BMNH).

SELENOSCOPUS Heinemann, I859. See Brachionycha Hübner, [1819].

CUCU

SELEPA Moore, I858, in Horsfield \& Moore, Cat. lepid. Insects 2: 353.

SARR

Type-species: Selepa celtis Moore, I 858, ibidem 2: 353, pl. 9a, fig. 9, by monotypy. Syntypes, JAVA (BMNH). INDIA: northern (BMNH).

SELICANIS Smith, I90o, Proc. U.S. natn. Mus. 22: $48 \mathrm{I}$.

ACRO

Type-species: Selicanis cinereola Smith, I900, ibidem 22: $48 \mathrm{I}$, by original designation.

Syntypes 3 ot. 3 ㅇ, [U.S.A.]: Colorado, Denver and Glenwood Springs. (USNM, Washington).

SEMAPHORA Guenée, I841. See Triaena Hübner, I818.

ACRO

SEMATOPHORA Agassiz, I846. See Triaena Hübner, I8I8.

ACRO

SEMIOPHORA Stephens, I829, Illust. Br. Ent. (Haustellata) 2: I 38.

HADE

Type-species: Phalaena gothica Linnaeus, I758, Syst. Nat. (Edn Io) 1: 5i6, by monotypy. Type(s), Europe (LS, London).

$\ddagger$ GRAPHIPHOR A Hübner, [r806], Tentamen determinationis digestionis . . . : [1] .

Included in a work rejected for nomenclatural purposes by Int. Commn zool. Nom., 1926, Smithson misc. Collns 73 (4) Opinion 97: 19. Also idem, 1954, Opin. Decl. int. Commn zool. Nom. 6 Opinion 278: I 40.

Only included species: Phalaena gothica Linnaeus, I 758.

ANCATA Căpușe, 1958, Polskie Pismo ent. 28: 167.

Type-species: Phalaena gothica Linnaeus, I 758 , by monotypy.

Ancata is a junior objective synonym of Semiophora Stephens, I829.

$\ddagger A N A C T A$; Kristensen, 1966, Ent. Meddr 34: 211 .

An incorrect subsequent spelling.

SEMIOTHISOPS Hampson, I926, Descr. new Genera Species Lepid. Phalaenae Subfamily Noctuinae Br. Mus.: 593. OPHI

Type-species: Zethes macariata Hampson, I902, J. Bombay nat. Hist. Soc, 14: 216, by original designation. Holotype ${ }^{\text {, }}$ [SIKKIM] Sikhim (BMNH).

$\ddagger$ SEMNIODES Hampson, no published reference found.

HYPE

+ SENDYRA, misspelling. See Seudyra Stretch, I 875 .

AGAR

SENERATIA Moore, [1885] I 887, Lepid. Ceylon 3: 202.

OPHI

Type-species: Thermesia praecipua Walker, 1865, List Specimens lepid. Insects Colln Br. Mus. 33: I 056, by original designation. Holotype q, CEYLON (BMNH).

SENTA Stephens, I 834, Illust. Br. Ent. (Haustellata) 4: 297.

HADE

Type-species: Melia flammea Curtis, I 828, Br. Ent. 5: 201, by subsequent designation by Westwood, I840, Synopsis Geneva Br. Insects: II3. Holotype, Great Britain: London, Lewisham.

MELIANA Curtis, I836, Br. Ent. 13: 587 .

Type-species: Melia flammea Curtis, I828, by subsequent designation by Hampson, 1905, Cat. Lepid. Phalaenae Br. Mus. 5: 576.

The genus Melia Curtis, I 828 , was proposed to contain the type-species, by original designation, Phalaena (Tinea) sociella Linnaeus, I 758, - Pyralidae, Galleriinae, together with Melia flammea Curtis, - Noctuidae, Hadeninae; Melia sericea Curtis; and one other species. Curtis, 1836, Br. Ent. 13: $5^{8} 7$ listed $T$. sociella as a synonym of $T$. colonella which he placed in Ilithyia Berthold, 1827 , in Latreille. Curtis then used Meliana for 
the two species Melia flammea Curtis and Melia sericea Curtis. Confusion has arisen because some authors have taken Meliana Curtis, I836, to be a new genus proposed for a concept different from that of Melia Curtis, 1828 , while other authors have taken Meliana Curtis to be an objective replacement name for Melia Curtis, which is a junior homonym. There need be no further confusion for if Meliana Curtis was intended to be an objective replacement name for Melia Curtis then it sinks as a junior objective synonym of Aphomia Hübner, [1825], - Pyralidae, Gallerinae; but if (as I accept for this catalogue) Meliana Curtis was intended to be a new genus then it sinks as a junior objective synonym of Senta Stephens, I 834 .

Sherborn, 1928, Index Anim. (I801-1850): 3989, and Neave, 1940, Nomencl. zool. 3: 92 both cited the original reference as Meliana Curtis, I839, Br. Ent. 16 Syst. Index: 1 3.

SEPTIS Hübner, [1821] 1816, Vevz. bekannter Schmett.: $2+3$.

ACRO

Type-species: Noctua lithoxylaea [Denis \& Schiffermüller], I775, Ankiindung syst. Werkes Schmett. W'ienergegend: 75 (but included by Hübner as +lithoxylea Schiff.', an incorrect subsequent spelling), by subsequent designation by Hampson, 19o8, Cat. Lepid.

Phalaenae Br. Mus. 7: 76, but cited as †'lithoxylea Schiff.' Type(s), [Austria]: Vienna district (Collection destroyed, Horn \& Kahle, I936, Ent. Beih. Berl.-Dahlem 3: 243).

$+X Y L E N A$ Hübner, [1806], Tentamen determinationis digestionis . . . [ [1].

Included in a work rejected for nomenclatural purposes by Int. Commn zool. Nom., 1926, Smithson. misc. Collns 73 (4) Opinion 97: 19. Also idem, 1954, Opin. Decl. int. Comm. zool. Nom. 6 Opinion 278: I 4 o.

Only included species: Noctua lithoxylaea [Denis \& Schiffermüller], I775, but cited as as $+X$. lythoxylea, an incorrect subsequent spelling.

SERIA Walker, i862, J. Proc. Linn. Soc. (Zool.) 6: I93.

HADE

Type-species: Seria cyathicornis Walker, I862, ibidem 6: 194, 'by monotypy. Lectotype ô. Borneo: Sarawak (UM, Oxford), designated (as type) by Swinhoe, I9oo, Cat. east. and Aust. Lepid. Heterocera 2: 39.

SERICAGLAEA Franclemont, I9+1, Ent. N'ews 52: 201.

CUCU

Type-species: Orthosia signata French, I879, Can. Ent. 11: 76, by original designation. Holotype $q$, TYPE-LOCALITY not stated, but from context probably U.S.A.: Illinois.

SERICIA Guenée, I852, in Boisduval \& Guenée, Hist. nat. Insectes (Lépid.) 7: I72. OpH Type-species: Sericia spectans Guenée, 1852, ibidem 7: I 73, by subsequent designation by Desmarest, (1857), in Chenu, Encycl. Hist. nat. Papillons nocturnes: I30. Lectotype ô. [Australia]: Tasmania (MNHN, Paris), designated by Viette, 195I, Bull. mens. Soc. linn. Lyon 20: 162.

SERRODES Guenée, I 852, in Boiscluval \& Guenée, Hist. nat. Insectes (Lépid.) 7: 251. OPHI Type-species: Phalaena inara Cramer, I779, Uitlandsche Kapellen 3: 78, pl. 239, fig. E, by subsequent designation by Desmarest (1857), in Chenu, Encycl. Hist. nat. Papillons nocturnes: 132 . Type(s), [ IND1A]: Coromandel coast.

P. inara is a junior subjective synonym of Noctua partita Fabricius, I775, Syst. Ent.: 604.

SESAMIA Guenée, I 852, in Boisduval \& Guenée, Hist. nat. Insectes (I.épid.) 5: 95.

ACRO

Type-species: Cossus nonagrioides Lefebrre, 1827, Mém. Soc. linn. Paris 6: 98, pl. 5, fig. 3, by moliotypy. Type(s) ô, [Italy]: Sicily, Palermo.

SETAGROTIS Smith, i89o, Bull. U.S. natu. MIus. 38: 9, 59.

NOCT

Type-species: Agrotis planifrons Smith, I890, Trans. Am. ent. Soc. 17:42, by original designation (on page 9). Holotype, [CANaIs] : N.W. British Columbia. 
SETIDA Walker, 1858, List Specimens lepid. Insects Colln Br. Mus. 15: I853.

OPHI

Type-species: Setida quadrisignata Walker, I858, ibidem 15: 1853, by monotypy. Syntypes $5(\hat{0}$, o), Australia: northern (BMNH).

S. quadrisignata is a junior subjective synonym of Diatenes aglossoides Guenée, 1852 , in Boisduval \& Guenée, Hist. nat. Insectes (Lépid.) 6: 443.

SETOCTENA Wallengren, I863, Wien. ent. Monatschr. 7: I44.

CHLO

Type-species: Setoctena ledereri Wallengren, 1863, ibidem 7: I44, by subsequent designation by Kirby, I892, Synonymic Cat. Lepid. Heterocera 1:280. Type(s) ơ. [SourH WEST AFrica]: R. Swakop (NR, Stockholm).

SEUDYRA Stretch, I875, Cistula ent. 2: 19.

AGAR

Type-species: Eusemia transiens Walker, 1856, List Specimens lepid. Insects Colln Br. Mus. 7: 1588, by original designation. Syntypes 2 ơ. JAVA (BMNH).

${ }_{+}$SENDYRA; Butler, 1878, Entomologist's mon. Mag. 14: 206.

An incorrect subsequent spelling.

SEXSERRATA Barnes \& Benjamin, 1922, Contr. nat. Hist. Lepid. N. Am. 5: $42 . \quad$ ACON

Type-species: Sexserrata hampsoni Barnes \& Benjamin, I922, ibidem 5:42, by original designation. Holotype ơ. [U.S.A.]: Calif., Imperial Co., Dixieland.

SHAPIS nom n. for Phasis Walker, I 854.

AG.AR

Type-species: Josia noctilux Walker, 1854 , List Specimens lepid. Insects Colln Br. Mus. 2: 312, by monotypy (of Phasis Walker). Holotype ô, Brazil: Tapajos (BMNH). PHASIS Walker, 1854, List Specimens lepid. Insects Colln Br. Mus. 2: 312.

Type-species: Josia noctilux Walker, I854, by monotypy.

A junior homonym of Phasis Hübner, [1819] i 8I6, Verz. bekannter Schmett.: 73, - Lepid., Lycaenidae. The objective replacement name is Shapis nom. n.

Phasis Walker was proposed as a subgenus of Josia Hübner, [I8I9] I8I6, ibidem: I 76, Lepid., Dioptidae.

SIAVANA Walker, [1858] I 857, List Specimens lepid. Insects Colln Br. Mus. 13:984, Ioo9. орнI Type-species: Siavana repanda Walker, [1858] 1857, ibidem 13: roog, by monotypy. Syntypes $5\left(\sigma^{*}\right.$, o $)$, [U.S.A.]: Florida (BMNH).

See also Harveya Grote, 1873 .

SICCYNA nom. n. for Cynisca Fawcett, I918.

OPHI

Type-species: Cynisca thisbe Fawcett, 1918, Proc. zool. Soc. Lond. 1917: 240, pl. I, fig. I3, by original designation (of Cynisca Fawcett). Holotype ${ }_{+}$, [KeNYA] Br. E. Africa: [Teita Hills], Kedai (BMNH).

CYNISCA Fawcett, 1918, Proc. zool. Soc. Lond. 1917: 240.

Type-species: Cynisca thisbe Fawcett, 1918, by original designation.

A junior homonym of Cynisca Gray, 1844, Cat. Tortoises, Crocodiles, Amphisbaenians Colln Br. Mus.: 7I, - Reptilia. The objective replacement name is Siccyna nom. 1 .

SIDEMIA Staudinger, I892, in Romanoff, Mém. Lépid. 6: 459.

ACRO

Type-species : Sidemia snelleni Staudinger, I 892, ibidem 6: 459, pl. 7, fig. 8, by monotypy. Syntypes 5 ot, I 우, [U.S.S.R.]: [S. of Vladivostok], Sidemi (MNHU, Berlin); S. E. Amur district.

S. snelleni is a junior subjective synonym of Noctua speciosa Bremer, 1861, Bull. Acad. imp. Sci. St. Pétersb. 3: 486; but as the latter is a junior primary homonym of Noctua speciosa Hübner, [I8I3], Samml. eur. Schmett. 4: pl. I04, fig. 491, and as S. snelleni is the subjective replacement name, then snelleni remains the valid name for the species.

SIDERIDIS Hübner, [1821] 1816, Vevz. bekannter Schmett.: 232.

HADE

Type-species: Noctua evidens Hübner, [1808], Samml. eur. Schmett. 4: pl. 79, fig. 369 (a primary homonym), by subsequent designation by Hampson, 1905, Cat. Lepid. Phalaenae Br. Mus. 5: 436 . Type(s), Europe. 
N. evidens Hübner, is a junior primary homonym of Noctua evidens Thunberg, 1784 , Diss. Ent. sistens Insecta Suecica (I):2, pl. [I], fig. [2], which is a junior subjective synonym of Phalaena trigrammica Hufnagel, I 766 , - Noctuidae, Acronictinae. There is no synonym to use as a replacement name so I here propose Sideridis anapheles nom. n. for $N$. evidens Hübner.

SIGELA Hulst, r896, Trans. Am. ent. Soc. 23: 296, 307.

$\operatorname{ACON}$

Type-species: Sigela penumbrata Hulst, I 896 , ibidem 23 : 308, by original designation, but cited as $\ddagger$ perumbrata, an incorrect (multiple) original spelling. Syntypes, [U.S.A.]: Fla, Archer.

Sigela was originally placed in the Geometridae.

SIGLOPHORA Butler, I892, Proc. zool. Soc. Lond. 1892: r 23.

CHLO

Type-species: Siglophora bella Butler, r 892, ibidem 1892: I24, pl. 6, fig. 2, by monotypy. Syntypes ô. 우, Borneo: North Borneo, Sandakan (BMNH).

SILACIDA Swinhoe, I900, Cat. east. and Aust. Lepid. Heterocera 2: 86.

EUTE

Type-species: Eutelia inextricata Moore, 1882, in Hewitson \& Moore, Descr. new Indian lepid. Insects Colln late Mr W. S. Atkinson: 147, by subsequent designation by Hampson, I912, Cat. Lepid. Phalaenae Br. Mus. 11: I5. Syntypes ${ }^{7}$, ㅇ, India: [Assam], Cherra Punji (BNINH); [W. Bengal], Darjiling.

E. inextricata is a junior subjective synonym of Eurhipia geyeri Felder \& Rogenhofer, I874, Reise öst. Fregatte Novara (Zool.) 2 (Abt. 2): pl. Iro, fig. 23.

SILDA Walker, I863, List Specimens lepid. Insects Colln Br. Mus. 27: I 31.

ACON

Type-species: Silda truncatalis Walker, i 863, ibidem 27: 131, by monotypy. Lectotype o. Borneo: Sarawak (BMNH), designated (as type) by Hampson, rgio, Cat. Lepid. Phalaenae Br. Muss. 10: 69 .

SILIGNEA Hampson, 1926, Descr. new Genera Species Lepid. Phalaenae Subfamily Noctuinae Br. Mus.: 4 IO.

OPHI

Type-species: Zethes albopunctata Bethune-Baker, 1906, Novit. zool. 13: 276, by original designation. Holotype $\delta$, New Guinea: [Papua], Aroa River.

SILLOPHORA Warren, I9I2. See Trichoptya Warren, I9I2.

OPHI

SIMPLICIA Guenée, I854, in Boisduval \& Guenée, Hist. nat. Insectes (Lépid.) 8: I $5 . \quad$ HyPE

Type-species: Herminia rectalis Eversmann, I842, Bull. Soc. imp. Nat. Moscou 15 (3): 558, by subsequent designation by Desmarest, (1857), in Chenu, Encycl. Hist. nat. Papillons nocturnes: 217. Syntypes, [U.S.S.R.] E. Russia: Casan and Orenburg provinces (ZI, Leningrad).

$\ddagger$ SIMPLICULA Hampson, no published reference found.

HYPE

SIMYRA Ochsenheimer, I8I6, Schmett. Eur. 4: 8I.

ACRO

Type-species: Noctua nervosa [Denis \& Schiffermüller], 1775, Ankündung syst. Werkes Schmett. Wienergegend: 85 , by subsequent designation by Westwood, r84o, Synopsis Genera Br. Insects: 97. Type(s), [AtsTr1A]: Vienna district (Collection destroyed, Horn \& Kahle, s936, Ent. Beih. Berl.-Dahlem 3: 243).

Invalid type-species designation by Boisduval, r 836, Hist. nat. Insectes (Lépid.) 1: r 35 , who also cited nervosa. Boisduval in his lengthy 154 page Introduction, reviewed carlier classifications and designated up to three different type-species for each generic name. In his 'Exposé de notre méthode' from pages I 55-69o, no type-species designations were made for the genera he himself used. Under the Code, Article on (a)(iii), the typedesignation of an author is eligible for consideration if he states that it is the type '... and if it is clear that he himself accepts it as the type-species.' Boisduval's type-designations although clearly stated, do not fulfil the last requirement and so are invalid. Even though 
Boisduval's 1836 work was well known to lepidopterists, the type-designations contained in it have not in the past been accepted by Hemming for Rhopalocera, or by other authors.

CNEPHATA Billberg, r820, Enumeratio Insect. Mus. G.J. Billberg: 85.

Proposed, unnecessarily, as an objective replacement name for Simyra Ochsenheimer, I 8 I 6.

$\ddagger S Y M I R A$; Hübner, [r82r] r8r6, Verz. bekannter Schmett.: 240.

An incorrect subsequent spelling.

ASEM A Sodoffsky, r 837, Bull. Soc. imp. Nat. Moscou 1837 (6): 87.

Proposed, unnecessarily, as an objective replacement name for Simyra Ochsenheimer, I 8 I 6.

$\ddagger N I M Y R A$; Guenée, I84 I, Annls Soc. ent. Fr. 10: 237.

An incorrect subsequent spelling.

†SIYMRA; Warren, rgr 2, in Seitz, Macrolepid. World $11: 39$.

An incorrect subsequent spelling in the English but not in the German edition.

SINA Walker, r865, List Specimens lepid. Insects Colln Br. Mus. 32: 6 ro.

SARR

Type-species: Sina tenera Walker, I865, ibidem 32: 6ro, by monotypy. Holotype ô. Australia (BMNH).

S. teneva is a junior subjective synonym of Calathusa basicunea Walker, $185^{8}$, ibidem 15: I645.

See also Calathusa Walker, I858; and Hisbanda Walker, [1866].

SINARELLA Bryk, I949, Ark. Zool. 41 (A) I: I44.

HYPE

Type-species: Sinarella stigmatophora Bryk, 1949, ibidem 41 (A) I: 144, pl. 6, fig. 2 ,

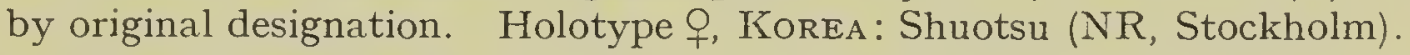

SINARIOLA Bethune-Baker, I906, Novit. zool. 13: 238.

OPHI

Type-species: Sinariola owgarra Bethune-Baker, r9o6, ibidem 13: 238, by original designation. Type(s) ㅇ, NEw Gurnea: [Papua], Owgarra (BMNH).

SINEUGRAPHE Boursin, 1954, Bonn. zool. Beitr. 5: 266.

NOCT

Type-species: Eugraphe disgnosta Boursin, 1948, Z. wien. ent. Ges. 33: 109, pl. 2, fig. 2, by original designation, but cited as $S$. dysgnosta Boursin. Holotype $\hat{0}$. JAPAN: Prov. Hohki, Mt Daisen (NM, Vienna).

In citing the type-species, Boursin emended its spelling. Under the Code, Article 32(a)(ii), S. dysgnosta Boursin, I954, is an unjustified emendation.

SINGARA Walker, I865, List Specimens lepid. Insects Colln Br. Mus. 33: I I 3.

OPH I

Type-species: Singara diversalis Walker, r865, ibidem 33: III3, by monotypy. Holotype ô., [Bangladesh]: [E. Bengal], Silhet (BMNH).

SINNA Walker, I 865, List Specimens lepid. Insects Colln Br. Mus. 32: 64I.

CHLO

Type-species: Sinna calospila Walker, r 865 , ibidem 32:642, by monotypy. Syntypes 2 우, JAva (BMNH).

SINOCHARIS Püngeler, I9I2, in Korb, Mitt. münch. ent. Ges. 3: 7 .

$\mathrm{ACON}$

Type-species: Sinocharis korbae Püngeler, I9r 2, ibidem. 3: 7, pl., by monotypy.

Holotype $\widehat{\jmath}$, [U.S.S.R.]: Ussuri district, Kasakewitsch (MNHU, Berlin).

See also Noshimea Matsumura, r93r.

SINOSIA Schaus, 1914, Proc. U.S. natn. Mus. 46:5I4.

OPH I

Type-species: Sinosia inornata Schaus, $\mathrm{I}_{9 \mathrm{I}} 4$, ibidem $46: 5^{\mathrm{I}} 4$, by original designation. Type(s) $\tilde{\sigma},[$ French Guiana]: R. Maroni, St. Jean (USNM, Washington).

SIPATOSIA Hampson, 1926, Descr. new Genera Species Lepid. Phalaenae Subfamily Noctuinae Br. Mus.: 433 .

OPHI

Type-species: Apistis onerosa Schaus, I914, Proc. U.S. natn. Mus. 46: 520, by original designation. Holotype $\hat{\sigma}$, PERU (USNM, Washington). 
SIRIOBA nom. n. for Borisia Filipjev, I949.

cucu

Type-species: Borisia hiemalis Filipjev, 1949, Ent. Obozr. 30: 247, by monotypy (of Borisia Filipjev). Syntypes 7 $\hat{0}$, U.S.S.R.: Turcmania, Repeteka (ZI, Leningrad).

BORISI.t Filipjev, I979, Ent. Obozr. 30: 246.

Type-species: Borisia hiemalis Filipjev, 1949, by monotypy.

A junior homonym of Borisia Rohdendorf, 1924, Ent. Mitt. 13: 125,-Diptera. The objective replacement name is Sirioba nom. n.

SISPUTA Möschler, I890, Abh. senckenb. naturforsch. Ges. 16: 222.

HYPE

Type-species: Sisputa gracilis Möschler, 1890, ibidem 16: 222, fig. 10, by monotypy.

Syntypes $\hat{0}, 0$, Puerto Rico. [Colombia]: Bogota (MNHU, Berlin).

S. gracilis is a junior subjective synonym of Physula phtisialis Guenée, 1854 .

See also Mursa Walker, I859, and Paramimetica Warren, I889.

SISYRHYPENA Grote, 1873, Can. Ent. 5: 227.

HYPE

Type-species: Sisyrhypena pupillaris Grote, 1873, ibidem 5: 227, by monotypy. Syntypes, [U.S.A.]: I ô. Philadelphia; I ô, 2 q, Texas (BMNH).

SITOPHORA Guenée, I854, in Boisduval \& Guenée, Hist. nat. Insectes (Lépid.) 8: 74. HYPE Type-species: Sitophora vesiculalis Guenée, 1854, ibidem 8: 75, by monotypy. Holotype jo, BrazIL (BIINH).

$\ddagger S I Y M R A$, misspelling. See Simy'ra Ochsenheimer, 1816 .

ACRO

SMICROLOBA Warren, 1913, in Seitz, Gross-Schmett. Evde 11: 215.

$\operatorname{ACON}$

Type-species: Mestleta quadrapex Hampson, I891, Illust. typical Specimens Lepid. Heterocera Colln Br. Muts. 8: 22, 91, pl. 147, fig. 15, by original designation. Lectotype 9. INDIA: [Madras prov.], Nilgiri $N$. slopes (BMNH), designated (as type) by Hampson, I9Io, Cat. Lepid. Phalaenae Br. Mus. 10:75.

The date of publication of the part of Seitz containing page 215, was stated on page 209 as I9I3 August 2oth in both the German and English editions.

SMICROPUS Warren, 1895, Novit. zool. 2: 85.

GEOMET1R1DA

Type-species: Micropus longalis Hübner, 1818, Zuträge Samml. exot. Schmett. 1: 24 , figs 131, I32, by monotypy.

Smicropus was proposed as the objective replacement name for Micropus Hübner, 18 I8, a junior homonym.

†IDIA Hübner, I808, Erste Zuträge Samml. exot. Schmett.: 5.

Included in a work rejected for nomenclatural purposes by Int. Commn zool. Nom., 1966, Bull. zool. Nom. 23 Opinion 789:215. Placed on the Official Inder of Rejected and Invalid Generic Names in Zoology: Name No. 1863.

Only included species: $\ddagger$ Idia longalis Hübner, 1808 , later made available as Micropus longalis Hüibner, 1818.

SMILEPHOLCIA Prout, 192.4, in Prout \& Talbot, Bull. Hill Mus., Witley 1: 406.

PANT

Type-species: Trisuloides luteifascia Hampson, I894, Fauna Br. India (Motlis) 2: 437, by original designation. I.ectotype +, [INDIA]: [Assam], Khásis (BMNH), designated (as type) by Hampson, 1913, Cat. Lepid. Phalaenae Br. Muss. 13: 342.

In the original description of the type-species Hampson attributed the specific name to Swinlıe .M.S.

SMINTHOPSES Hampson, 1926, Descr. new Genera Species Lepid. Phalaenae Subfamily Noctuinae Br. Mus.: 528 . LYMANTRIIDAE

Type-species: Sminthopses aeschra Hampson, 1926, ibidem: 528, by original designation.

Sminthopses was originally placed in the Noctuidae. 
SMYRA Möschler, I880, Verh. zool.-bot. Ges. Wien 30 (Abh.): 408.

OPHI

Type-species: Smyra recurvicornis Möschler, I 880, ibidem 30 (Abh.): 409, pl. 9, fig. 50, by PRESENT DESIGNATION. Holotype ㅇ, Surinam: interior (MNHU, Berlin).

S. recurvicornis is a junior subjective synonym of Homoptera stipatura Walker, I858, List Specimens lepid. Insects Colln Br. Mus. 15: I8oo.

SOLENOPTERA Duponchel, [1845]. See Phlogophora Treitschke, I825.

ACRO

SOLEPIA Herrich-Schäffer, I 870, Corresp.-Bl. zool.-min. Ver. Regensburg 24: Iо , I05. opH I

Nomenclaturally available, but without any included species. Proposed in a work on the Lepidoptera of Cuba.

SONAGARA Moore, I882, in Hewitson \& Moore, Descr. new Indian lepid. Insects Colln late Mr W. S. Atkinson: I 79.

THYRIDIDAE

Type-species: Sonagara strigipennis Moore, I882, ibidem: I8o, by original designation.

Sonagara was originally placed in the Noctuidae.

SOPHAGA Moore, i 88r, Proc. zool. Soc. Lond. 1881: 362.

NOCT

Type-species: Sophaga sinuata Moore, I881, ibidem 1881: 363 , by original designation. Syntypes ô. 우, [INDIA]: Bombay (BMNH).

SOPHRONIA Duponchel, [1845]. See Trisateles Tams, I939.

HYPE

SOPHTA Walker, [1863] I 862, List Specimens lepid. Insects Colln Br. Mus. 26: I758. ACON Type-species: Sophta concavata Walker, [1863] I862, ibidem 26: I759, by monotypy. Holotype 우, [Australia]: [Qd], Moreton Bay (BMNH).

See also Nacerasa Walker, I 866.

$\ddagger$ SOPHTHA; Draudt, I935, in Seitz, Gross-Schmett. Erde 3 (Suppl.) : 205.

An incorrect subsequent spelling in both the German and English editions.

$\ddagger$ SОРНTHA, misspelling. See Sophta Walker, [I863].

$\operatorname{ACON}$

SORA Heinemann, I859. See Gypsitea Tams, I939.

NOCT

†SORCOSTIA, misspelling. See Sorocostia Rosenstock, r885. NOLI

SOROCOSTIA Rosenstock, I 885, Ann. Mag. nat. Hist. (5) 16: 435. NOLI

Type-species: Eromene vetustella Walker, I866, List Specimens lepid. Insects Colln Br. Mus. 35: 1763, by monotypy. Holotype ô., [Australia]: [Qd], Moreton Bay.

E. vetustella is a junior subjective synonym of Hypena albalis Walker, [I 866] I 865, ibidem 34: II 43.

†SORCOSTIA; Lower, I896, Trans. R. Soc. S. Aust. 20: I53.

An incorrect subsequent spelling.

SORYGAZA Walker, [1866] I865, List Specimens lepid. Insects Colln Br. Mus. 34: I I I. Hy PE

Type-species: Sorygaza didymata Walker, [1 866] I 865, ibidem 34: II 81, by monotypy. Type(s) ō, Venezuela (UM, Oxford).

SOSXETRA Walker, I 862, Trans. ent. Soc. Lond. (3) 1:84.

OPHI

Type-species: Sosxetra grata Walker, r862, ibidem (3) 1: 84 , by monotypy. Type(s) ô, Brazil.

See also Chaetoloma Felder, i 874 .

SOTIGENA Druce, I 89o, Biologia cent.-am. (Zool.) Lepid. Heterocera 1: 437. HyPE

Type-species: Sotigena notodontoides Druce, I89o, ibidem 1:438, pl. 35, fig. 22, by original designation. Syntypes, Mexico: Coatepec (BMNH); Las Vigas.

SPAELOTIS Boisduval, i 840, Genera Index meth. Eur. Lepid.: io6.

NOCT

Type-species: Noctua ravida [Denis \& Schiffermüller], I775, Ankündung syst. Werkes Schmett. Wienergegend: 8o (but included by Boisduval as 'ravida $\mathrm{H}_{\text {.,' }}$ an incorrect 
authorship), by subsequent designation by Desmarest, 1848 , in d'Orbigny, Dict. univl Hist. nat. 11: 706, but cited as 'ravida H.' Type(s), [Austria]: Vienna district (Collection destroyed, Horn \& Kahle, 1936, Ent. Beih. Berl.-Dahlem 3: 243).

SPARGALOMA Grote, I873, Trans. Am. ent. Soc. 4: 299.

OPH I

Type-species: Spargaloma sexpunctata Grote, I873, ibidem 4:300, pl. I, fig. 90, by original designation. Syntypes $\hat{0}$, 우, [U.S.A.]: Pennsylvania (BMNH).

SPARKIA nom. n. for Cea Grote, I883.

ACRO

Type-species: Cea immacula Grote, 1883, Papilio 3: 78, by monotypy (of Cea Grote). Type(s), [U.S.A.]: Arizona.

This name is proposed in honour of Mrs Brenda A. Carter, née Spark, in appreciation of her great assistance in the early stages of the compilation of this catalogue.

CEA Grote, I883, Papilio 3: 78 .

Type-species: Cea immacula Grote, I 883 , by monotypy.

A junior homonym of Cea Haliday, 1837, in Walker, Entomological Mag. 4 (4): 355, Hymenoptera. The objective replacement name is Sparkia nom. n.

SPARTINIPHAGA McDunnough, 1937, Can. Ent. 69: 46.

ACRO

Type-species: Orthosia inops Grote, I881, Bull. U.S. geol. geogr. Surv. Territ. 6: 270, by original designation. Lectotype q. [U.S.A.]: [New Hampshire], Kittery Point (BMNH), designated (as type) by Hampson, 1906, Cat. Lepid. Phalaenae Br. Mus. 6: 49I.

SPATHOPTILA Turner, I944, Proc. R. Soc. Qd 55: 47.

NOLI

Type-species: Spathoptila cyclophora Turner, I944, ibidem 55: 48, by monotypy. Holotype , Australia: W. Aust., Nornalup (ANIC, Canberra).

SPECTROPHYSA Swinhoe, I900, Cat. east. and Aust. Lepid. Heterocera 2: 190.

OPHI

Type-species: Spectrophysa distorta Swinhoe, I90o, ilidem 2: I9I, by monotypy. Holotype of, BORnEO (UM, Oxford).

SPEIA Tams \& Bowden, 1953, Bull. ent. Res. 43: 647.

ACRO

Type-species: Phalaena vuteria Stoll, I790, in Cramer, Uitlandsche Kapellen Aanhangsel: I6r, pl. 36, fig. 5, by original designation. Type(s), [South Africa]: Cape of Good Hope.

$P$. vuteria is dated from the wrapper of the part and not from the title-page of the volume, I 79 I.

SPEIREDONIA Hübner, [1823] I816, Verz. bekannter Schmett.: 272.

OPH I

Type-species: Phalaena feducia Stoll, I790, in Cramer, Uitlandsche Kapellen Aanhangsel: I60, pl. 36, fig. 3, by subsequent designation by Desmarest, (1857), in Chenu, Encycl. Hist. nat. Papillons nocturnes: 130 (but cited for Spiredonia, an unjustified emendation). Type(s), TYPE-LOCALITy stated to be Surinam, but this was probably an error as the species illustrated is not known to occur in the New World but is present in southern India and Ceylon.

P. feducia is a junior subjective synonym of Noctua itynx Fabricius, 1787, Mantissa Insect. 2: 136.

SPIREDONIA Agassiz, I846, Nomencl. zool. Index univl.: $345,348$.

An unjustified emendation of Speiredonia Hübner, [1823].

†SPIREDONIA Felder, I875, in Felder \& Rogenhofer, Reise öst. Fregatte Novara (Zool.) 2 (Abt. 2): pl. I I3, fig. 7 .

†Spiredonia Felder was cited by Neave, 1940, Nomencl.zool. 4:259, as a nomenclaturally available name. This is not so as Felder was merely using Spiredonia and not proposing it as a new name.

SPEOCROPIA Hampson, I908, Cat. Lepid. Phalaenae Br. Mus. 7: x, $255 . \quad$ acro

Type-species: Hadena scriptura Walker, 1858, List Specimens lepid. Insects Colln 
Br. Mus. 15: I723, by original designation. Syntypes $3 \hat{\partial}$, [Dominican Republic] St. Domingo (BMNH).

SPERSARA Walker, [1863] i864, J. Proc. Linn. Soc. (Zool.) 7: I74.

EUTE

Type-species: Spersara glaucopoides Walker, [1863] I864, ibidem 7: I75, by monotypy.

Lectotype 01 . BorNeo: Sarawak (UM, Oxford), designated (as type) by Swinhoe, Igoo, Cat. east. and Aust. Lepid. Heterocera 2: 84.

S. glaucopoides is a junior subjective synonym of Anuga constricta Guenée, I852.

See also Anuga Guenée, i852; and Caecila Walker, I858.

SPHETTA Walker, 1865, List Specimens lepid. Insects Colln Br. Mus. 32: 457.

ACRO

Type-species: Sphetta apicalis Walker, I865, ibidem 32:457, by monotypy. Holotype

o. CEYLON (BMNH).

SPHIDA Grote, I878, Bull. U.S. geol. geogr. Surv. Territ. 4: I 79.

ACRO

Type-species: Arzama obliquata Grote \& Robinson, I868, Trans. Am. ent. Soc. 1: 339, pl. 6, fig. 47, by monotypy. Syntypes $\sigma^{*}$, ㅇ, [U.S.A.]: N.Y., Atlantic District.

A. obliquata is a junior subjective synonym of Edema obliqua Walker, 1865, List Specimens lepid. Insects Colln Br. Mus. 32: 428.

SPHINGIFORMA Bethune-Baker, 1906, Novit. zool. 13: 24 I.

CHLO

Type-species: Sphingiforma pratti Bethune-Baker, I906, ibidem 13: 24I, by original designation. Syntypes $\hat{\sigma}$, ㅇ․, New Guinea: [Papua], Dinawa (BMNH).

SPHINGOMORPHA Guenée, I852, in Boisduval \& Guenée, Hist. nat. Insectes (Lépid.) 7: 220.

OPHI

Type-species: Phalaena chlorea Cramer, I777, Uitlandsche Kapellen 2: 12, pl. IO4, fig. C, by subsequent designation by Moore, [1885] 1887, Lepid. Ceylon 3: 157. Type(s), SURINAM.

$\ddagger$ SPHINTEROPS, misspelling. See Apopestes Hübner, [1823].

OPHI

SPHRAGIFERA Staudinger, I892, in Romanoff, Mém. Lépid. 6: 554.

ACRO

Type-species: Anthoecia sigillata Ménétriés, 1859, Bull. Classe phys.-math. Acad. imp. Sci. St. Pétersb. 17: 219, by monotypy. Holotype ô, [U.S.S.R.]: rives méridionales de l'Amour (ZI, Leningrad).

SPILOLOMA Grote, I873, Bull. Buffalo Soc. nat. Sci. 1: I26.

OPHI

Type-species: Spiloloma lunilinea Grote, I873, ibidem 1: 127, by monotypy. Type(s) §. [U.S.A.]: West Virginia (BMNH).

STRENOLOMA Grote, I880, N. Am. Ent. 1: 99.

Proposed, unnecessarily, as an objective replacement name for Spiloloma Grote, I873, which Grote considered to be a name too similar to Spilosoma Curtis, I825, - Lepid., Arctiidae.

SPINIPALPA Alphéraky, I 892, Horae Soc. ent. ross. 26: 444.

NOCT

Type-species: Spinipalpa maculata Alphéraky, I892, ibidem 26: 445, by monotypy. Holotype $\sigma^{\circ},[\mathrm{CHinA}]$ : Amdo region $\left[36^{\circ} \mathrm{N}\right.$. $\left.100^{\circ} \mathrm{W}.\right]$ (ZI, Leningrad).

SPINTHEROPS Boisduval, I840. See Apopestes Hübner, [1823].

OPHI

SPIRAMA Guenée, I852, in Boisduval \& Guenée, Hist. nat. Insectes (Lépid.) 7: i94. CATo

Type-species: Phalaena retorta Clerck, I764, Icones Insect. rariorum 2: pl. [54], by original designation, but cited as 'retorta Lin. Mus. Lud. Ulr. I I - Clerck t. 54, f. 2, 3'. Type(s), TyPE-LOCALITy not stated. [CHINA. JaPAN. India.].

Linnaeus, I764, Mus. Ludovicae Ulvicae: 376 , No. I I, cited retorta without any reference to Clerck but on page 379 referred to Clerck t.54, f.I. In the absence of more exact dating I am following Sherborn, I902, Index Anim. (I758-I800): 829, and giving Clerck priority. SPIRAMIA Walker, I858, List Specimens lepid. Insects Colln Br. Mus. 14: I3I8.

An unjustified emendation of Spirama Guenée, I $85^{2}$. 
SPIRAMIA Walker, I858. See Spivama Guenée, I852. CATo

SPIREDONIA Agassiz, I846. See Speiredonia Hübner, [I823]. OPHI

${ }_{+}^{+}$SPIREDONIA Felder, I875. See Speivedonia Hübner, [I 823]. OPHI

SPODOPTERA Guenée, I 852, in Boisduval \& Guenée, Hist. nat. Insectes (Lépid.) 5 : I $53 . \quad$ ACRO Type-species: Hadena mauritia Boisduval, I 833, Nouv. Anmls Mus. Hist. nat. Paris 2: 240 , by subsequent designation by Hampson, I894, Fauna Br. India (Moths) 2: 248. Type(s), Mauritius. [RÉunion] Bourbon.

H. mauritia was also published in I833 in Faume ent. Madagascar, Bourbon et Maurice (Lépid.): 92, pl. I3, fig. 9 .

†SPODOSO.MA; Anonymous, I966, Eur. Pl. Prot. Orgn Rep. Serv. 66/12-285: I.

An incorrect subsequent spelling; confirmed as such in Rev. appl. Ent. (A) 55: Ioo.

†SPODOSOMA, misspelling. See Spodoptera Guenée, I 852.

ACRO

SPRAGUEIA Grote, I875, Check List Noctuidae Am., N. of Mex.: 20, 25.

ACON

Type-species: Agrophila leo Guenée, I 852, in Boisduval \& Guenée, Hist. nat. Insectes (Lépid.) 6:205, by original designation. Type(s), AmERICA: northern.

SPUDAEA Snellen, I867, De Vlinders van N'ederland 1867: 289.

CUCU

Type-species: Phalaena ruticilla Esper, I79I, Die Schmett. 4 (2) Abschnitt I: pl. I57. fig. I; [I 798-I804], ibidem: 525, by monotypy. Type(s) ơ, [IrALy]: Florence district.

SQUAMIPALPIS Bethune-Baker, I908, Novit. zool. 15: 206.

HY PE

'Type-species: Squamipalpis unilineata Bethune-Baker, I908, ibidem 15: 206, by original designation. Syntypes $\hat{\sigma}, q$, NEw Guines: [Papua], Dinawa (BMNH); Aroa River.

STADNA Swinhoe, I 900, Cat. east. and Aust. Lepid. Heterocera 2: 2 I6.

HYPE

Type-species: Stadna nigriplaga Swinhoe, Igoo, ibidem 2: 217 , pl. 4, fig. 24, by monotypy. Holotype ơ, Borneo: Sarawak (UM, Oxford).

STAGA Mabille, Igoo, Annls Soc. ent. Fr. 68: 735.

HYPE

Type-species: Staga producta Mabille, I900, ibidem 68: 735, by monotypy. Lectotype o. MAdigascar: Baie d'Antongil (MNHN, Paris), designated (as holotype) by Viette, I954, Mém. Inst. scient. Madagascar (E) 5: 386 .

STANDFUSSIA Spuler, I907. See Trisateles Tams, I939.

HYPE

STANDFUSSIANA Boursin, I946, Revue fr. Lépidopt. 10 : I 90.

NOCT

Type-species: Phalaena lucernea Linnacus, I 758, Syst. $\Lambda^{\top}$ at. (Edn Io) 1: 5IO, by original designation. Type(s), Europe (LS, London).

STAUROPHORA R.L., I 8I 7, Jenaische Allg. Lit.-Ztg 1: 286.

$\Lambda C R O$

Type-species: Phalaena celsia Linnaeus, I 758, Syst. Nat. (Edn Io) 1: 507, by monotypy.

Type(s), Type-Locality not stated (LS, London) [Europe].

PSYLLA Germar, i 8i I, Syst. Glossatorum Prodromus, sistens Bombycum Spec.: I4.

Type-species: Phalaena celsia Linnaeus, $x 758$, by PRESENT DESIGNATION

A junior homonym of Psylla Geoffroy, I 762, Hist. Insect. Paris. 1: $482,-$ Hemiptera. This work has been rejected for nomenclatural purposes by Int. Commn zool. Nom., I 954, Opin. Decl. int. Commn zool. Nom. 4 Opinion 228: 2 I I. The generic name Psylla Geoffroy has, however, been subsequently validated under the plenary powers of the Commission, 1965, Bull. zool. Nom. 22 Opinion 731: 86. Psylla Geoffroy has been placed on the Official List of Generic Names in Zoology: Name No. I67I. The objective replacement name for P'sylla Germar is Staurophora R.L., I 8 I 7.

DIACOPE Hiibner, [1820] I816, Verz. belinnter Schmett.: 204.

Type-species: Phalaena celsia Linnaeus, $175^{8}$, by monotypy. 
A junior homonym of Diacope Cuvier, 1817, Règne anim. 2:275, - Pisces. The objective replacement name is Staurophora R.L., I8I 7.

$\ddagger$ CELSI A Stephens, I829 [June], Nom. Br. Insects: 42 .

Only included species $\ddagger$ Celsia viridis Stephens, 1829 [June], ibidem: 42, later made available as an unnecessary objective replacement name for $P$. celsia. Both Celsia and viridis were nomina nuda.

CELSIA Stephens, 1829 [August], Syst. Cat. Br. Insects 2: 105.

Type-species: Phalaena celsia Linnaeus, I758, by monotypy, but cited by Stephens as $C$. viridis Stephens, 1829 [August], with $P$. celsia in synonymy. $C$. viridis was an unnecessary objective replacement name.

Although $P$. celsia was included in his lists and catalogues of British insects Stephens, I 829, Illust. Bv. Ent. (Haustellata) 3: 99 stated ' . . as I have never seen an indigenous example in any collection, I presume that it does not inhabit Britain: it is a native of Hungary, Austria, etc.; but it has been found in Sweden.'

Celsia Stephens is a junior objective synonym of Staurophora R.L., I9I 7.

CALOT AENIA Stephens, I830, Illust. Br. Ent. (Haustellata) 3: 99.

Proposed, unnecessarily, as an objective replacement name for Celsia Stephens, I829 [August], which Stephens considered to be a junior homonym of Celsia Linnaeus, applied to a genus of plants.

CALLIT AENIA Agassiz, 1846, Nomencl. zool. Index univl.: 60, 61.

An unjustified emendation of Calotaenia Stephens, I830.

JASPIDIA Boisduval, 1840, Genera Index meth. Eur. Lepid.: I28.

Type-species: Phalaena celsia Linnaeus, I758, by monotypy.

A junior homonym of Jaspidia Hübner, 1822,-Lepid., Noctuidae. The objective replacement name is Staurophora R.L., I8I 7 .

$\ddagger J A S P I D E A$; Staudinger, I861, in Staudinger \& Wocke, Cat. Lepid. Eur. angrenzenden Länder (I): 45 .

An incorrect subsequent spelling.

STAUROPIDES Hampson, 1908, Cat. Lepid. Phalaenae Br. Mus. 7: 6. Available, but without included species until Hampson, I909, ibidem 8: x, 269. ACRO

Type-species: Antachara superba Druce, 1894, Ann. Mag. nat. Hist. (6) 13: 361, by subsequent designation by Hampson, 1909, ibidem 8: 269. Syntypes 20 , I $q$, EcuAdor: Sarayacu (BMNH). Costa RicA: Santa Clara Valley.

STEGANIODES Hampson, I910, Cat. Lepid. Phalaenae Br. Mus. 10: 3, 268.

$\operatorname{ACON}$

Type-species: Steganiodes mesophaea Hampson, 1910, ibidem 10: 269, fig. 77, by original designation. Holotype ${ }^{\star}$, NIGERIA: Sapele (BMNH).

STEIRIA Walker, [1858] I857, List Specimens lepid. Insects Colln Br. Mus. 13: I I22, I I35. STIC Type-species: Steiria subobliqua Walker, [1858] 1857, ibidem 13: I I36, by subsequent designation by Hampson, I912, Cat. Lepid. Phalaenae Br. Mus. 11: 146. Syntypes 2 đ. CEYLON (BMNH).

S. subobliqua is a junior subjective synonym of Stictoptera cucullioides Guenée, 1852.

See also Stictoptera Guenée, 1852.

STELLIDIA Guenée, 1857, in Boisduval \& Guenée, Hist. nat. Insectes (Lépid.) 9: 328 . opH Type-species: Stellidia planetaria Guenée, I857, ibidem 9:329, pl. 20, fig. 4, by monotypy. Holotype 우 Brazil.

STEMMAPHORA Staudinger, I888, Stettin. ent. Ztg 49: 43.

ACRO

Type-species: Stemmaphora viola Staudinger, I 888, ibidem 49: 43, by monotypy. Syntypes ô, ㅇ․ [U.S.S.R.]: [Uzbekistan], Samarkand province (MNHU, Berlin).

STENAGROTIS Hampson, I903, Cat. Lepid. Phalaenae Br. Mus. 4: xi, I39.

NOCT

Type-species: Trachodopalpus edmondsii Butler, I882, Trans. ent. Soc. Lond. 1882:

I 6 , by original designation. Type(s), CHILE: Mts of the hacienda of Cauquenes (BMNH). GG 
STENBERGMANIA Bryli, 1949, Ark. Zool. 41 (A) I : I 2.

HYPE

Type-species: Herminia albomaculalis Bremer, I 864, Mém. Acad. imp. Sci. St. Pétersb.

(7) 8 (I): 65, pl. 5, fig. 24, by original designation. Type(s), [U.S.S.R.]: Ussuri, above the Ema estuary.

In the original description this genus was written as Sten-Bergmania. Under the Code, Article 32(c)(i), the hyphen has been deleted.

STENHYPENA Hampson, I895, Fauna Br. India (Moths) 3: xvi, 93.

HYPE

Type-species: Hypena adustalis Hampson, I893, Illust. typical Specimens Lepid. Heterocera Colln Br. Mus. 9: 30, 120, pl. 166, fig. 7, by original designation. Type(s) $q$, CEYLON: Pundaloya (BMNH).

STENISCADIA Hampson, I912, Cat. Lepid. Phalaenae Br. Mus. 11: 221, 376.

SARR

Type-species: Steniscadia poliophaea Hampson, I9I2, ibidem 11: 376, fig. I42, by original designation. Holotype , Ecuador: Paramba (BMNH).

${ }_{\ddagger} S T E N O B A D I Z A$ Prout, no published reference found.

HYPE

STENOCARSIA Hampson, 1926, Descr. new Genera Species Lepid. Phalaenae Subfamily Noctuinae Br. Mus.: 445 . OPHI

Type-species: Thermesia sthenoptera Swinhoe, I895, Ann. Mag. nat. Hist. (6) 15: I2, by original designation. Holotype ô, [INDIA]: [Assam], Cherra Punji (BMNH).

STENOCODIA Hampson, I9I0, Cat. Lepid. Phalaenae Br. Mus. 10: 3, 555 ACoN

Type-species: Stenocodia purpurascens Hampson, I9lo, ibidem 10: 555, fig. I5I, by original designation. Holotype ô. [French Guinna]: Maroni, St. Jean (BMNH). The type-locality in the original description was incorrectly cited as 'Br. Guinea.'

STENOCRYPTIS Warren, I913, in Seitz, Gross-Schmett. Erde 11:230. ACON

Type-species: Stenocryptis punctata Warren, 19I3, ibidem 11:23I, pl. 24, line e, by original designation. Holotype + , [India]: Assam, Khasia Hills (BMNH).

The date of publication of the part of Seitz containing page 230 was stated on page 225 as I9I 3 August 2oth in both the German and the English editions.

STENODRINA Boursin, 1937, Ent. Rdsch. 54: 368.

ACRO

Type-species: Caradrina paupera Romanoff, I885, Mém. Lépid. 2: 56, pl. 3, fig. 4, by original designation, but cited as 'paupera Chr.', an incorrect authorship. Holotype o., [U.S.S.R.]: [Armenia], [Yerevan] Erivan.

C. paupera was attributed to Christoph by Romanoff.

STENOECIA Warren, I9I I, in Seitz, Macrolepid. World 3: 258.

ACRO

Type-species: Heliothis dos Freyer, [1838] 1839, Nenere Beitr. Schmett. 3: 98, pl. 263, fig. 4, by original designation. Syntypes 2 ex., [TuRkEY]: [Istanbul] Constantinople.

The date of publication of the part of Seitz containing page 258 was stated on page 253 as I9I I October I 5 th in the English edition and as I9I 2 January Ioth in the German edition.

†STENOGNATHMA Hampson, no published reference found.

HYPE

STENOGRAPTA Sugi, I959, Tinea, Tokyo 5: 280.

OPHI

Type-species: Stenograpta stenoptera Sugi, 1959, ibidem 5: 281, pl. 38, fig. 6, I5, by original designation. Holotype $\delta$, JAPAN: Gumma Pref., Kawarayu (Colln S. Sugi, Tokyo).

STENOLA Möschler, I890, Abh. senckenb. naturforsch. Ges. 16: I I9.

NOLI

Type-species: Stenola bistriga Möschler, 1890, ibidem 16: 119, by monotypy. Syntypes 2 \%, [Puerto Rico]: Portorico I.

STENOLOBA Staudinger, 1892, in Romanoff, Mém. Lépid. 6: 38I.

$\Lambda \mathrm{CON}$

Type-species: Dichagyris jankowskii Oberthür, I 884, Etudes Int. 10: 28, pl. 3, fig. 5 , by monotypy. Syntypes I ô, 3 ․, [UT.S.S.R.]: [S. of Vladivostok], Sirlemi. 
STENOPALTIS Swinhoe, I9oI, Ann. Mag. nat. Hist. (7) 8: 23.

HYPE

Type-species: Stenopaltis lithina Swinhoe, Igor, ibidem (7) 8: 23, by monotypy. Syntypes $\hat{0}$, 우, Australia: Qd, Coomoo (BMNH).

STENOPIS Mabille, I880, Annls Soc. ent. Belg. 1880: cvii.

CATO

Type-species: Stenopis reducta Mabille, I880, ibidem 1880: cviii, by monotypy. Lectotype 0 . MADAGascar (BMNH), designated by Viette \& Fletcher 1968, Bull. Br. Mus. nat. Hist. (Ent.) 21: $4 \mathrm{I} 6$.

†STENOPSIS; Hampson, I9I3, Cat. Lepid. Phalaenae Br. Mus. 12: 45I (in key).

An incorrect subsequent spelling.

STENOPRORA Hampson, I926, Descr. new Genera Species Lepid. Phalaenae Subfamily Noctuinae Br. Mus.: 83 .

OPHI

Type-species: Prorocopis adelopis Lower, 1903, Trans. R. Soc. S. Aust. 27: 45, by original designation. Holotype đ̃, Australia: N.S.W., Broken Hill (SAM, Adelaide).

† STENOPSIS, misspelling. See Stenopis Mabille, I88o.

CATO

STENOPTERYGIA Hampson, I908, Cat. Lepid. Phalaenae Br. Mus. 7: vii, 61.

ACRO

Type-species: Hadena subcurva Walker, 1857, List Specimens lepid. Insects Colln Br. Mus. 11 : 592, by original designation. Holotype đ̊, CEYLON (BMNH).

STENOPYDNA Roepke, 1943, Natuırh. Maandbl. 32: 94.

PYRALIDAE

Type-species: Stenopydna lanceolata Roepke, I943, ibidem $32: 94$, fig. I2, by monotypy.

Stenopydna was originally placed in the Notodontidae, but was transferred to the Noctuidae by Kiriakoff, I968, in Wytsman, Genera Insect. 217(C):2. I am now transferring Stenspydna to the Pyralidae, Crambinae as a junior subjective synonym of Schoenobiodes Hampson, 1917, Novit. zool. 24: 57.

STENORACHE Hampson, I9 10, Cat. Lepid. Phalaenae Br. Mus. 10: xvi, 612.

$\mathrm{ACON}$

Type-species: Tarache nubilosa Hampson, I894, Fauna Br. India (Moths) 2: 315, by original designation. Type(s) ô. India: Punjab, Simla (BMNH).

STENOSOMA Turati, I924. See Stenosomides Strand, I942.

NOCT

STENOSOMIDES Strand, 1942, Folia zool. hydrobiol. 11: 390.

NOCT

Type-species: Stenosoma synesia Turati, 1924, Atti Soc. ital. Sci. nat. 63: 74, pl. 4, fig. I, by monotypy (of Stenosoma Turati). Holotype ô, [LrByA]: Bengasi.

S. synesia is a junior subjective synonym of Agrotis mansoura Chrétien, I9II, Annls Soc. ent. Fr. 79: 498.

Stenosomides was proposed as the objective replacement name for Stenosoma Turati, I924.

STENOSOMA Turati, I924, Atti Soc. ital. Sci. nat. 63: 74.

Type-species: Stenosoma synesia Turati, 1924, by monotypy.

A junior homonym of Stenosoma Leach, [18I4] 1830, in Brewster Edinburgh Encycl.

7 (2): 404, 433,-Crustacea. The objective replacement name is Stenosomides Strand, 1942 .

STENOSTICTA Hampson, I9I2, Cat. Lepid. Phalaenae Br. Mus. 11: I42, 206.

STIC

Type-species: Stenosticta grisea Hampson, I912, ibidem 11: 206, fig. 63, by original designation. Syntypes 2 đ̃, [Kenya] Brit. E. Afr.: R. Athi, Kitwi (BMNH).

STENOSTIGMA Warren, I910, in Seitz, Macrolepid. World 3: 138.

CUCU

Type-species: Rhiza curva Staudinger, I889, Stettin. ent. Ztg 50: 46, by original designation. Syntypes 2 ơ, 3 ㅇ, [U.S.S.R.]: [Kirghizia], S. of Issyk-Kul (MNHU, Berlin).

The date of publication of the part of Seitz containing page 138 was stated on page I33 as I9Io May $5^{\text {th }}$ in the English edition and as I9II January $3^{\text {th }}$ in the German edition. 
STENOSTYGIA Hampson, 1910, Cat. Lepid. Phalaenae Br. Mus. 10: xiii, 371.

ACON

Type-species: Tarache nigritula Hampson, I896, Fauna Br. India (Moths) 4: 5I4, by original designation. Syntypes 으, CEYLon: Puttalam (BMNH). .[PAkistan]: Sind.

STENOXIA Hampson, 1926, Descr. new Genera Species Lepid. Phalaenae Subfamily Noctuinae Br. Mus.: 602.

$\mathrm{OPHI}$

Type-species: Stenoxia erythropis Hampson, 1926, ibidem: 602, by original designation. Holotype ô, British GuiAna: R. Potaro (BMNH).

STENOZETHES Hampson, 1926, Descr. new Genera Species Lepid. Phalaenae Subfamily Noctuinae Br. Mus.: 556 .

OPHI

Type-species: Marmorinia obscurata Butler, 1879, Illust. typical Specimens Lepid. Heterocera Colln Br. Mus. 3: xri, 68, pl. 57, fig. II, by original designation. Type(s), [JAPAN]: Hakodate (BMNH).

STEPHANIA Guenée, I852. See Mycteroplus Herrich-Schäffer, [1850].

ACRO

STIBADIUM Grote, I874, Bull. Buffalo Soc. nat. Sci. 2: 32, 74.

ACRO

Type-species: Stibadium spumosum Grote, I 874, ibidem 2: 74, by original designation. Type(s) o. [U.S.A.]: Kansas (BMNH).

STIBAENA Walker, I858, List Specimens lepid. Insects Colln Br. Mus. 15: I814. OPH

Type-species: Stibaena hostilis Walker, I858, ibidem 15: I 8 I4, by monotypy. Type(s)

ㅇ. [BRAZIL]: Parà.

STIBAERA Walker, I 857, List Specimens lepid. Insects Colln Br. Mus. 11: 494, 608. OPH

Type-species: Stibaera costiplaga Walker, 1857, ibidem 11: 608, by monotypy. Holotype ô, Brazil (BMNH).

STICTIGRAMMA Hampson, 1926, Descr. new Genera Species Lepid. Phalaenae Subfamily Noctuinae Br. Mus.: r 86.

$\mathrm{OPHI}$

Type-species: Stictigramma steniptera Hampson, 1926, ibidem: I86, by original designation. Syntypes $2 \hat{0}$, New Guinea: [Papua], Louisiades, St. Aignan (BMNH).

STICTOPTERA Guenée, 1852, in Boisduval \& Guenée, Hist. nat. Insectes (Lépid.) 7: 51.

STIC

Type-species: Stictoptera cucullioides Guenée, I852, ibidem 7: 52, by subsequent designation by Grote, i 874, Bull. Buffalo Soc. nat. Sci. 2: 39. Holotype ó, Java (BMNH).

See also Steiria Walker, [1858].

STICTOTHRIPA Schaus, I9 I August, Ann. Mag. nat. Hist. (8) 8: 213.

SARR

Type-species: Stictothripa delaia Schaus, I9II, ibidem (8) 8: 213, by monotypy. Type(s) + , Costa RicA: Sixola (USNM, Washington).

STICTOTHRIPA Hampson, 1912. See Hayesia nom. n.

SARR

STIGMOPLUSIA Dufay, 1970, Fanne Madagascar 31: i65.

PLUS

Type-species: Plusia chalcoides Dufay, 1968, Bull. mens. Soc. linn. Lyon 37: 210, by original designation. Holotype ô, MADAGASCAR: Sakaraha, Lambomakandro (MNHN, Paris)

STILBIA Stephens, 1829 [June], Nom. Br. Insects: 43.

ACRO

Type-species: Phytometra anomala Haworth, 1812, Trans. ent. Soc. Lond. 1: 336, by monotypy, but cited by Stephens as +'S. anomalata Haw.', an incorrect subsequent spelling. Type(s), Great Britain.

STILBINA Staudinger, I892, Dt. ent. Z. Iris 4: 290.

$\mathrm{ACRO}$

Type-species: Stilbina hypaenides Staudlinger, I 892, ibidem 4: 290, pl. 3, fig. I 2, by monotypy. Syntypes ô, f, [LEBANon]: Beirut. [ISRAEL]: Jerusalem (MNHU, Berlin). 
STILBOTIS Berio, I962, Annali Mus. civ. Stor. nat. Giacomo Doria 73: 206.

NOCT

Type-species: Stilbotis nigra Berio, I962, ibidem 73: 206, fig. I I, by original designation. Holotype ô, [ZAIRE]: Lac Kiwu, Rwankwi (MRAC, Tervuren).

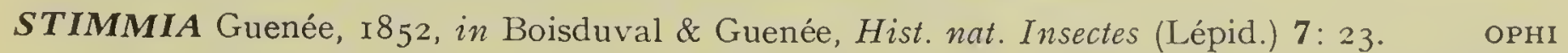
Type-species: Stimmia scoria Guenée, I852, ibidem 7: 24, by subsequent designation by Schaus, rgr6, Proc. U.S. natn. Mus. 50: 374. Holotype ơ, [French Guiana]: Cayenne.

S. scoria is a junior subjective synonym of Metalectra praecisalis Hübner, 1823 .

See also Metalectra Hübner, I 823.

STIRIA Grote, 1874, Bull. Buffalo Soc. nat. Sci. 2: 32, 73.

ACRO

Type-species: Stiria rugifrons Grote, 1874 , ibidem 2: 73 , by original designation. Syntypes, [U.S.A.]: Kansas (BMNH); Colorado.

STIRIODES Hampson, igo8, Cat. Lepid. Phalaenae Br. Mus. 7: 6. Available, but without included species until Hampson, I910, ibidem 9: x, 245.

ACRO

Type-species: Noct[ua] obtusa Herrich-Schäffer, I854, Samml. newer oder wenig bekannter aussereur. Schmett. 1 (1): wrapper, pl. 42, fig. 210 ; r858, ibidem 1: 68, 80, by subsequent designation by Hampson, r9ro, ibidem 9: 245. Type(s), [U.S.A.]: Tennessee.

$N$. obtusa is dated from the wrapper of the plates and not from the title-page of the volume, $1850-1858$

STOMAFRONTIA Hampson, 1905, Ann. S. Afr. Mus. 3: 436.

ACRO

Type-species: Stomafrontia albifasciata Hampson, 1905, ibidem 3: 437 , by original designation. Holotype , South Africa: Natal, Mooi River (BMNH).

STONYCHOTA Turner, 194I, Mem. Qd Mus. 12: 49.

ACRO

Type-species: Stonychota angustula Turner, I941, ibidem 12: 49, by monotypy. Holotype $q$, Australia: Qd, Injune (QM, Brisbane).

STORTHOPTERA Herrich-Schäffer, I856, Samml. neuer oder wenig bekannter aussereur. Schmett. 1 (1): wrapper, pl. 69, fig. 394; 1858, ibidem 1: 69, 82.

cATO

Type-species: Storthoptera tripuncta Herrich-Schäffer, I 856 , ibidem 1 (I): wrapper, pl. 69, fig. 394; 1858 , ibidem 1: 69, 82, by monotypy. Type(s), Africa.

STRABEA Schaus, i894, Trans. Am. ent. Soc. $21: 235$.

OPHI

Type-species: Strabea punctulata Schaus, 1894, ibidem 21: 235, by monotypy. Syntypes $\hat{\sigma}$, 우, Brazil : Parana, Castro.

STRATHOCLES Druce, I89I, Biologia cent.-am. (Zool.) Lepid..Heterocera 1: 45I. HYPE Type-species: Strathocles ribbei Druce, I 89 I, ibidem 1:452, pl. 37, figs 7, 8, by original designation. Syntypes $\hat{\jmath}, q$, Panama: Chiriqui (BMNH); Volcan de Chiriqui. Mexico: Tabasco, Teapa.

STRENOLOMA Grote, r880. See Spiloloma Grote, $1873 . \quad$ OPHI

STREPSELYDNA Warren, I9I I, in Seitz, Gross-Schmett. Erde 3: 229.

ACRO

Type-species: Elydna truncipennis Hampson, rgro, Cat. Lepid. Phalaenae Br. Mus. 9: 168 , fig. 59, by original designation. Holotype $\widehat{\sigma},[$ IndiA] : Assam, Khasis (BMNH).

E. truncipennis is a junior subjective synonym of Graphiphora stellata Moore, I882, in Hewitson \& Moore, Descr. new Indian lepid. Insects Colln late Mr W. S. Atkinson: I I9.

The date of publication of the part of Seitz containing page 229, was stated at the top of the page as rgr I March roth in both the English and German editions.

STRETCHIA Edwards, I874, Proc. Calif. Acad. Sci. 5: 266.

HADE

Type-species: Stretchia plusiaeformis Edwards, I874, ibidem 5: 267, by monotypy.

Type(s), [U.S.A.]: Nevada, White Pine.

The genus was originally described in the Notodontidae. 
STRIAPTERA Hampson, I926, Descr. new Genera Species Lepid. Phalaenae Subfamily Noctuinae Br. Mus.: $35^{8}$. OPHI Type-species: Camptochilus cinnamomeus Bethune-Baker, Igo8, Novit. zool. 15: 224, by original designation. Holotype ㅇ, NEw Guinea: [Papua], R. Aroa, Ekeikei (BMNH).

STRIDOVA Walker, I869, in Chapman, Proc. nat. Hist. Soc. Glasgow 1: 353.

ACON

Type-species: Stridova albigutta Walker, I869, ibidem 1: 354, by monotypy. Lectotype + , Congo (BMINH), designated (as type) by Hampson, I9Io, Cat. Lepid. Phalaenae Br. Mus. 10: 469.

S. albigutta is a junior subjective synonym of Perigea octo Guenée, 1852.

See also Ilattia Walker, [1859], and Chytoryza Grote, I876.

STRIGANIA Hampson, 1905, Cat. Lepid. Phalaenae Br. Mus. 5: xiv, 466.

HADE

Type-species: Heliophobus lithophilus Butler, I882, Trans. ent. Soc. Lond. 1882: I I9, by original designation. Syntypes, CHILE: Valparaiso (BMNH).

STRIGINA Savigny, I8I6, Mém. Anim. sans Vertèbres 1: 33.

HYPE

Type-species: Strigina poae Savigny, I 8 I6, ibidem 1:33, pl. 3, fig. I, by monotypy Type(s), TYPE-LOCALiTy not stated.

STRIGIPHLEBIA Hampson, 1908, Cat. Lepid. Phalaenae Br. Mus. 7: I7. Available, but without included species until Hampson, 1909, ibidem 8:283. ACRO

Type-species: Strigiphlebia flavirena Hampson, I909, ibidem 8: 283, fig. 79, by subsequent monotypy. Syntypes I $\hat{\delta}$, I $q$, PERU: Oroya (BMNH).

STRONGYLOSIA Hampson, 1926, Descr. new Genera Species Lepid. Phalaenae Subfamily Noctuinae Br. Mus.: 357 . OPHI

Type-species: Strongylosia semiflava Hampson, 1926, ibidem: 358 , by original designation. Syntypes 20 , [Ghana] Gold Coast: Kumasi (BMNH).

STYGIATHETIS Hampson, I908, Cat. Lepid. Phalaenae Br. Mus. 7: 8. Available, but without included species until Hampson, r9o9, ibidem 8: xiii, 444. ACRO

Type-species: Radinacra mus Hampson, I89I, Illust. typical Specimens Lepid. Heterocera Colln Br. Muss. 8: I4, 77, pl. I 45, fig. 9, by subsequent monotypy. Syntypes o. 오. India: [Madras prov.], Nilgiri plateau $6700 \mathrm{ft}$ (BMNH).

STYGIODRINA Boursin, I937, Ent. Rdsch. 54: 366.

ACRO

Type-species: Caradrina maurella Staudinger, I888, Stettin. ent. Ztg 49: 25.5, by monotypy. Syntypes ô, 우, [U.S.S.R.]: Amur district, Blagoweschtschensk (MNHU, Berlin); Ussuri (MNHU, Berlin); Suifun (MNHU, Berlin); Sidimi (MNHU, Berlin).

STYGIONYX Hampson, 1926, Descr. new Genera Species Lepid. Phalaenae Subfamily Noctuinae Br. Mus. : 360 .

Type-species: Hypena montana Joicey \& Talbot, I9I7, Ann. Mag. nat. Hist. (8) 20 : 54, pl. I, fig. 7, by original designation. Syntypes 40 , New Guinea: [West Irian], Wandammen Mts (BMNH).

STYGIOSTOLA Hampson, I908, Cat. Lepid. Phalaenae Br. Mus. 7: vii, 44.

Type-species: Phalaena unbratica Goeze, I 78I, Ent. Beyträge 3 (3): 67, by original designation. Type(s), TYPE-LOCALITY not stated. [EUROPE].

Phalaena (Bombyx) umbratica Groeze is a junior homonym of Phalaena (Noctua) umbratica Linnaeus, 1758, Syst. Nat. (Edn I0) 1:515,-Cuculliinae. There is no objective replacement name but Bombyx ferruginea Esper, 1785, Die Schmett. 3: 246, pl. 47, fig. 5, is a junior subjective synonym of $P$. umbratica Croeze. The former is therefore available for use as the subjective replacement name.

See also Rusina Stephens, i 829. 
STYLOPODA Smith, I89I, Trans. Am. ent. Soc. 18: I3r.

CUCU

Type-species: Stylopoda cephalica Smith, I89I, ibiden 18: I3I, by monotypy. Syntypes 2 ㅇ, [U.S.A.]: California (USNM, Washington).

STYLORACHE Hampson, I9Io, Cat. Lepid. Phalaenae Br. Mus. 10: 7, 701.

$\operatorname{ACON}$

Type-species: Stylorache albida Hampson, r910, ibidem 10: 70I, fig. I98, by original designation. Holotype $\hat{\sigma}$. Argentina: Gran Chaco, Florenzia (BMNH).

SUBACRONICTA Kozhanchikov, I950, Fauna SSSR (Insecta) Lepid. 12: 456.

ACRO

Type-species: Noctua megacephala [Denis \& Schiffermüller], 1775, Ankündung Syst. Werkes Schmett. Wienergegend: 67, by original designation. Type(s), [AUSTRIA]: Vienna district (Collection destroyed, Horn \& Kahle, 1936, Ent. Beih. Berl.-Dahlem 3: 243).

SUBANUA Berio, 1959, Annali Mus. civ. Stor. nat. Giacomo Doria 71: 316.

CATO

Type-species: Anua flavociliata Aurivillius, 1925, Ergebn. zweiten Dt. cent.-Afr. Exped. I9IO-I9II (Zool.) 1 (I8): I3 I8, fig. 77, by original designation, but cited as 'A. flavociliata Strd.', an incorrect authorship. Holotype ${ }^{\circ}$, [CAmeroun]: Süd-Kamerun, Nemayong.

SUBLEUCONYCTA Kozhanchikov, I950, Fauna SSSR (Insecta) Lepid. 12: $447 . \quad$ ACro Type-species: Leuconycta palshkovi Filipjev, 1937, Lambillionea 37: 64, figs I, 2 , by original designation, but cited as $\ddagger L$. palschkovi, an incorrect subsequent spelling. Holotype ô, U.S.S.R.: Ussuri, Sutshan (ZI, Leningrad).

SUBNOCTUA Berio, 1962, Annali Mus. civ. Stor. nat. Giacomo Doria 73: 207.

NOCT

Type-species: Subnoctua tanganykae Berio, I962, ibidem 73: 207, fig. I2, by original designation. Holotype $\hat{\sigma}$, [Tanzania]: Tangany[i]ka, Marangu (Colln E. Berio, Genoa).

SUBPANDESMA Berio, 1966. See Thria Walker, [1 858].

OPHI

SUBRITA Walker, I866, List Specimens lepid. Insects Colln Br. Mus. 35: I 743.

SARR

Type-species: Subrita bilineatella Walker, I866, ibidem 35: I744, by subsequent designation by Hampson, 1912, Cat. Lepid. Phalaenae Br. Mus. 11: 261, but cited as revayana Scopoli, I772, which Hampson placed (on page 265) as the senior synonym of bilineatella Walker. Type(s) $\hat{o}$, [INDIA]: Hindostan.

S. bilineatella is a junior subjective synonym of Phalaena revayana Scopoli, 1772.

See also Nycteola Hübner, I 822.

SUBSIMPLICIA Prout, I928, Sarawak Mus. J. 3: 480.

HYPE

Type-species: Subsimplicia punctilinea Prout, 1928, ibidem 3: 48 I, pl. I5, fig. I2, by monotypy. Holotype $\delta$, Borneo: Sarawak, Mt Poi $4300 \mathrm{ft}$ (BMNH).

SUDARIOPHORA Zeller, I872 [December], Verh.zool.-bot. Ges. Wien 22 (Abh.): 489. OPHI

Type-species: Sudariophora nasutaria Zeller, I872, ibidem 22 (Abh.): 490, pl. 2, fig. II, by monotypy. Syntypes I ${ }^{\lambda}$, I 9 , [U.S.A.]: Texas.

S. nasutaria was described as a new species by Zeller for the misidentification Doryodes acutalis Walker, [1859] I858, List Specimens lepid. Insects Colln Br. Mus. 16: 73. Walker proposed acutalis as an emendation of Ligia acutaria Herrich-Schäffer, I 852 , but because of the misidentification acutalis Walker has at times been incorrectly used for nasutaria Zeller.

S. nasutaria Zeller, 1872 [December], is a junior subjective synonym of Phyprosopus callitrichoides Grote, 1872 September.

See also Phyprosopus Grote, 1872 September.

SUGITANIA Matsumura, 1926, Insecta matsum. 1: 53.

CUCU

Type-species: Sugitania maculifera Matsumura, 1926, ibidem 1: 53, pl. 1, fig. I3, by original designation. Syntypes 2 ô. JAPAN: Honshu, Kobe; Kyoto (EIHU, Sapporo).

S. maculifera is a junior subjective synonym of Graphiphora lepida Butler, 1879, Ann. Mag. nat. Hist. (5) 4:362. 
SUMA Walker, [1 866] I 865, List Specimens lepid. Insects Colln Br. Mus. 34: I I96. HYPE Type-species: Suma incongrualis Walker, [1866] 1865, ibidem 34: 1197, by monotypy. Holotype , South AFrica (BMNH).

S. incongrualis is a junior subjective synonym of Sarmatia interitalis Guencee, I 854 .

See also Sarmatia Guenée, 1854 .

SUNDWARDA Swinhoe, I9o I, Ann. Mag. nat. Hist. (7) 8: г 3 I.

OPHI

Type-species: Sundwarda efulgida Swinhoe, I90I, ibidem (7) 8: I3I, by monotypy.

Type(s) o. Borneo: Sarawak (BMNH).

S. efulgida is a junior subjective synonym of Cerura dohertyi Druce, 190I, Ann. Mag. nat. Hist. (7) $7:$ : 7 .

SUNIRA Franclemont, I950, Bull. Brooklyn ent. Soc. 45: I 48.

cucu

Type-species: Xanthia bicolorago Guenée, I852, in Boiscluval \& Guenée, Hist. nat. Insectes (Lépid.) 5: 397, by original designation. Holotype ô. [U.S.A.]: New York State (BMNH)

SUPERNOLA Hampson, I9I 8, Novit. zool. 25: 96.

NOLI

Type-species: Supernola subrufa Hampson, I918, ibidem $25: 96$, by original designation. Holotype ․ Borneo: [Sabah], Kina Balu (BMNH).

SUPERSYPNOIDES Berio, 1958, in Berio \& Fletcher, Annali Mus. civ. Stor. nat. Giacomo Doria $70: 344$.

CATO

Type-species: Sypna erebina Hampson, 1926, Descr. new Genera Species Lepid. Phalaenae Subfamily Noctuinae: 5, by original designation. Syntypes 200,1 q, CHINA: [Szechwan Prov.], Ta-chien-lu (BMNH).

Supersypnoides was proposed as a subgenus of Sypuoides Hampson, I9I3.

SUPRALATHOSEA Barnes \& Benjamin, 1924, Contr. nat. Hist. Lepid. N. Am. 5: r 33. cucu

Type-species: Supralathosea baboquivariensis Barnes \& Benjamin, I924, ibidem 5: I33, by monotypy. Holotype ô, [U.S.A.]: Arizona, Pima Co., Baboquivari Mts.

SURINA Walker, I869, in Chapman, Proc. nat. Hist. Soc. Glasgow 1:333. chlo

Type-species: Surina hypsoides Walker, I 869, ibidem 1: 333, by monotypy. Type(s)

P. CONGO (UM, Oxford).

SUT YNA Todd, 1958, J. Kansas ent. Soc. 31 : 154:

NOCT

Type-species: Xylina sculpta Grote, I873, Bull. Buffalo Soc. nat. Sci. 1: I I4, pl. 3. fig. I, by subsequent designation (for Anytus Grote) by Grote, I874, ibidem 2: 27. Syntypes ơ. ․ [U.S.A.]: Philadelphia and New York (BMNH).

$X$. sculpta is a junior subjective synonym of Polia privata Walker, 1857 , List Specimens lepid. Insects Colln Br. Mus. 11: 52 I.

Sutyna was proposed as the objective replacement name for Anytus Grote, 1873 .

ANYTUS Grote, i 873, Bull. Buffalo Soc. nat. Sci. 1: I 44

Type-species: Xylina sculpta Grote, I873, by subsequent designation by Grote, I874, ibidem 2: 27 .

A junior homonym of Anytus Stảl, 1865, Hemiptera Africana 3: 74,- Hemiptera. The objective replacement name is Sutyna Todd, r958.

† AMYTUS; Grünberg, 1910, Arch. Naturgesch. (1908) 74 (2) 2: 230.

An incorrect subsequent spelling.

SVINHOEA Hampson, 1902. See Swinhoea Hampson, I 894.

$\operatorname{ACON}$

SWINHOEA Hampson, I894, Fauna Br. India (Moths) 2: xv, 324.

$\operatorname{ACON}$

Type-species: Spadix vegetus Swinhoe, I885, Proc. zool. Soc. Lond. 1885: 475. pl. 28, fig. I 4, by original designation, but cited as S. vegeta, a justified emendation. Type(s), [INDIA]: Bombay (BMNH).

SVINHOEA Hampson, igo2, Ann. S. Afr. Mus. 2: 295.

An unjustified emendation. 
SYDIVA Moore, I882, in Hewitson \& Moore, Descr. new Indian lepid. Insects Colln late Mr W.S. Atkinson: 95. CuCU

Type-species: Sydiva nigrogrisea Moore, I882, ibidem: 96, by monotypy. Syntypes Ђ. India: Darjiling (BMNH).

SYFANIA Oberthür, I893, Études Ent. 18: I9.

AGAR

Type-species: Agarista bieti Oberthür, I886, Bull. Soc. ent. Fr. (6) 5: ccxxviii, by subsequent designation by Hampson, I90 I, Cat. Lepid. Phalaenae Br. Mus. 3: 632. Type(s), ChINA: Tibet, Tâ-tsien-loû (BMNH).

$\ddagger$ SYPHANIA; Strand, I9I 2, in Aurivillius \& Wagner, Lepid. Cat. 5: 74 .

An incorrect subsequent spelling.

SYFANOIDEA Bartel, I903, Verh. zool.-bot. Ges. Wien 53: 125.

AGAR

Type-species: Syfanoidea schencki Bartel, I903, ibidem 53: I26, by monotypy. Holotype , , [South AFRICA]: Natal, Durban.

$\ddagger$ \YPHANOIDEA; Strand, I9I 2, in Aurivillius \& Wagner, Lepid. Cat. 5: 74.

An incorrect subsequent spelling.

† S YLECTRA, misspelling. See Syllectra Hübner, [1819].

OPHI

SYLLECTRA Hübner, [18I9], Samml. exot. Schmett. 2: pl. [223].

OPHI

Type-species: Syllectra mirandalis Hübner, [1819], ibidem 2: pl. [223], figs I-4, by monotypy. Syntypes $\hat{\sigma}$, + , Type-locality not stated. [West Indies. Equatorial South America].

S. mirandalis is a junior subjective synonym of Phalaena erycata Cramer, 1780 , Uitlandsche Kapellen 3: I 7o, pl. 287, fig. D.

$\ddagger$ SYLECTRA; Hübner, [1825] I816, Verz. bekannter Schmett.: 34I.

An incorrect subsequent spelling.

SYMA Stephens, I850. See A pamea Ochsenheimer, I8I6.

ACRO

$\ddagger$ S YMIRA, misspelling. See Simyra Ochsenheimer, I8I6.

ACRO

SYMITHA Walker, I 866, List Specimens lepid. Insects Colln Br. Mrıs. 35: 173I.

SARR

Type-species: Symitha nolalella Walker, I866, ibidem 35: I73I, by monotypy. Lectotype $\hat{o}$ (not $Q$ as stated by Walker), JAVA (BMNH), designated (as type) by Hampson. I9I 2, Cat. Lepid. Phalaenae Br. Mus. 11: 244.

SYMMERISTA Hübner, [I82I] I8I6, Verz. bekannter Schmett.: 248.

NOTODONTIDAE

Type-species: Noctua albicosta Hübner, [1809], Samml. eur. Schmett. 4: pl. 93, fig. $44^{0}$, by subsequent designation by Kirby, I892, Synonymic Cat. Lepid. Heterocera 1: 572 .

Symmerista was originally proposed in the Noctuidae.

S YMMOLPIS Turner, I902, Proc. Linn. Soc. N.S.W. 27: I 27.

OPHI

Type-species: Symmolpis hyporrhoda Turner, I902, ibidem 27: 128, by monotypy. Holotype , Australia: Qd, Stradbroke I. (ANIC, Canberra).

SYMPIS Guenée, I852, in Boisduval \& Guenée, Hist. nat. Insectes (Lépid.) 7: $343 . \quad$ OPHI Type-species: Sympis rufibasis Guenée, 1852, ibidem 7:344, pl. 24, fig. I, by subsequent designation by Desmarest, (I 857), in Chenu, Encycl. Hist. nat. Papillons nocturnes: I34. Syntypes, Java (BMNH).

S YMPISTIS Hübner, [1823] I8I6, Verz. bekannter Schmett.: 261.

cucu

Type-species: Noctua melaleuca Thunberg, I79I, Diss. Ent. sistens Insecta Suecica (2): 42, pl. 2, fig. I2 (a junior primary homonym), by subsequent designation by Hampson, 1906, Cat. Lepid. Phalaenae Br. Mus. 6: 4I2. Syntypes, [Scandinavia]: Lappland.

N. melaleuca Thunberg, is a junior homonym of Noctua melaleuca Vieweg, I790, 
Tabellarisches Verz. Churmark Brandenburg einheimischen Schmett. 2: 68, - Noctuidae, Hadeninae. There is no objective replacement name but Noctua heliophila Paykull, I 793, Skr. Naturh.-Selskabet 2 (2): 102, pl. 2, fig. 5, is a junior surbjective synonym of $N$. melalenca Thunberg. The former is therefore available for use as the subjective replacement name.

Sympistis was also used by Hübner, [1823], Samml. exot. Schmett. 2: pl. [202]. If accepted from this work it has the same type-species, by monotypy.

SYMPIS TOIDES Kozhanchikov, I947, Ent. Obozr. 29: I 4, 19, 33.

CUCU

Type-species: Heliophobus grumi Alphéraky, i892, Horae Soc. ent. ross. 26: 447, by original designation. Syntypes $\hat{0}, q$, , [CHINA]: [Tsinghai], L. Kuku-noor (ZI, Leningrad).

SYMPLUSIA Holland, I894, Psyche, Camb. 7: 28.

OPHI

Type-species: Symplusia frequens Holland, I 894, ibidem 7:28, pl. I, fig. 19, by original designation. Syntypes $25(\hat{0}$, o $)$, WEST AFrica.

S. frequens is a junior subjective synonym of Bertula subcupralis Walker, [I866] I 865, List Specimens lepid. Insects Colln Br. Mus. 34: I 67.

See also Rhaesena Walker, r 866.

SYNALISSA Möschler, I88o, Verh. zool.-bot. Ges. Wien 30 (Abh.) : 475.

OPHI

Type-species: Synalissa tempaca Möschler, I880, ibidem 30 (Abh.): 475, pl. 9, fig. 3I, by monotypy. Holotype ․ Surivam: Paramaribo.

SYNAPHE Hübner, [1825] I 816, Verz. bekannter Schmett.: 347.

PYRALIDAE

Type-species: Pyralis angustalis [Denis \& Schiffermüller], I775, Ankündung syst.

Werkes Schmett. Wienergegend: I 20, by subsequent designation by Westwood, I840, Synopsis Genera Br. Insects: 105.

Symaphe is included in this catalogue as it is the senior objective synonym of Cledeobia Stephens, I829, which has been used in the Noctuidac.

$\ddagger I D I A$ Hübner, [1806], Tentamen determinationis digestionis . . . [2].

Included in a work rejected for nomenclatural purposes by Int. Commn zool. Nom., I926, Smithson. misc. Collns 73 (4) Opinion 97: I9. Also idem, 1954, Opin. Decl. int. Comm zool. Nom. 6 Opinion 278: I 40.

Only included species: P'yralis bombycalis [Denis \& Schiffermüller], 1775, which is congeneric with Pyralis angustalis [Denis \& Schiffermüller], I775.

CLEDEOBIA Stephens, I829, Nom. Br. Insects: 45.

Type-species: Pyralis angustalis [Denis \& Schiffermüller], I 775, by subsequent designation by Duponchel, I831, in Godart \& Duponchel, Hist. nat. Lépid. Papillons Fr. 8 (2): 9.

The citation by Duponchel is acceptable as a type-species designation as it is contained in the continuation of a layout in which Duponchel stated, in the same work 7 (2): I02, that the species so cited were the types of genera. Cledeobia has been used incorrectly in the Noctuidae.

SYNCALAMA Hampson, 1908, Cat. Lepid. Phalaenae Br. Mus. 7: 7. Available, but without included species until Hampson, 1910, ibidem 9: 228.

ACRO

Type-species: Syncalama mimica Hampson, igro, ibidem 9:228, fig. 89, by subsequent inonotypy. Holotype ô. [Soutr Afric.1]: Cape Colony, Deelfontein (BMNH).

SYNCLEROSTOLA Berg, I 877, An. Soc. cient. argent. 4: 202.

NOCT

Type-species: Synclerostola pampeana Berg, 1877, ibidem 4: 204, by monotypy. Syntypes io $(\hat{0}$, ) $)$, Argentina: Santa Cruz.

See also Noctulizeria Köhler, 1945.

SYNEDA Guenćc, I 852. Sce Drasteria Hübner, 1818.

OPIII

SYNEDOIDA Erlwards, I878, Pacific Coast Lepid. No. 29: ๆ.

OPHI

Type-species: Synedoida scrupulosa Edwards, 1 878, ibidem No. 29: 9, by subsequent 
designation by Richards, 1936, Revta Ent. Rio de J. 6: 358. Syntypes 3 ơ, 2 q, [U.S.A.]: [Calif.], Kern Co., Havilah.

SYNGATHA Bethune-Baker, 1913, Ann. Mag. nat. Hist. (8) 11:563.

ACON

Type-species: Syngatha elegans Bethune-Baker, I913, ibidem (8) 11: 563 , by origina 1 designation. Holotype $\widehat{\jmath}$, ANGola: N'Dalla Tando (BMNH).

SYNGRAPHA Hübner, [I821] I816, Verz. bekannter Schmett.: 250.

PLUS

Type-species: Noctua devergens Hübner, [I8I3], Samml. eur. Schmett. 4: pl. Io7, figs 500, 50I, by subsequent designation by Grote, I895, Abh. naturw. Ver. Bremen 14: IO3. Syntypes, Europe.

SYNIA Duponchel, [1845]. See Oria Hübner, [1821].

ACRO

SYNOLULIS Hampson, I926, Descr. new Genera Species Lepid. Phalaenae Subfamily Noctuinae Br. Mus.: 485 .

Type-species: Prionophora rhodinastis Meyrick, I902, Trans. ent. Soc. Lond. 1902: 44, by original designation. Syntypes 3 \%, New GuineA: [Papua], Port Moresby. Australia: Queensland.

SYNOMERA Schaus, I916, Proc. U.S. natn. Mus. 50: 264, 368.

HYPE

Type-species: Synomera cyllarus Schaus, I9I6, ibidem 50:368, by original designation. Type(s) of, French Guiana : Cayenne (USNM, Washington).

SYNTHACA Turner, 1908, Trans. R. Soc. S. Aust. 32: 69.

ACON

Type-species: Synthaca gilviceps Turner, I908, ibidem 32: 70, by monotypy.

Holotype đ̆, Australia: Qd, Kuranda (ANIC, Canberra).

SYNTHETA Turner, 1902, Proc. Linn. Soc. N.S.W. 27: 84.

ACRO

Type-species: Syntheta xylitis Turner, 1902, ibidem 27: 85, by original designation.

Holotype ơ, Australia: Qd, Townsville (ANIC, Canberra).

See also Phaeomorpha Turner, 1920.

SYNTHIMIA Walker, [1858]. See Synthymia Hübner, [I823]. ACRO

SYNTHYMIA Hübner, [I823] I816, Verz. bekannter Schmett.: 282. ACRO

Type-species: Noctua monogramma Hübner, [1808], Samml. eur. Schmett. 4: pl. 76,

fig. 353, by monotypy. Type(s), [Europe].

N. monogramma is a junior subjective synonym of Noctua fixa Fabricius, I 787 , Mantissa Insect. 2: I 47 .

METOPTRIA Guenée, [1845], in Duponchel, Cat. méth. Lépid. Eur. (2): I9I.

Type-species: Noctua monogramma Hübner, [1808], by monotypy.

Metoptria is a junior objective synonym of Synthymia Hübner, [I823].

SYNTHIMIA Walker, [1858] I857, List Specimens lepid. Insects Colln Br. Mus. 12: 838.

An unjustified emendation of Synthymia Hübner, [1823].

SYNTOMOPUS Guenée, I837. See Pyrois Hübner, [1820]. ACro

SYNVALERIA Butler, I89o, Trans. ent. Soc. Lond. 1890:672. CUCU

Type-species: Phalaena jaspidea Villers, I 789 , Caroli Linnaei Entomologia 2: 284, pl. 5, fig. 28, by original designation. Type(s), Europe.

SYNYRIAS Hampson, 1926, Descr. new Genera Species Lepid. Phalaenae Subfamily Noctuinae Br. Mus.: I6. $\mathrm{OPHT}$

Type-species: Celaena diffundens Walker, 1858 , List Specimens lepid. Insects Colln Br. Mus. 15: i688, by original designation. Holotype q, Venezuela (BMNH).

$\ddagger$ SYPHANIA, misspelling. See Syfania Oberthür, I893.

AGAR

$\ddagger_{\ddagger}$ SYPHANOIDEA, misspelling. See Syfanoidea Bartel, I903.

AGAR 
SYPNA Guenée, I 852, in Buisduval \& Guenée, Hist. nat. Insectes (Lépid.) 7: 144.

OPHI

Type-species: Sypna omicronigera Guenée, 1852, ibidem 7: 145, by monotypy. Holotype ô, Indis: central (BMNH).

SYPNOIDES Hampson, I9I3 [January 3oth], Cat. Lepid. Phalaenae Br. Mus. 12: 5. Comenclaturally available, but without included species until Warren, I9I3 February 2oth, in Seitz, Gross-Schmett. Erde 3: 335 .

Type-species: Sypna mandarina Leech, I 9oo, Trans. ent. Soc. Lond. 1900: 545, by subsequent designation by Warren, I9I3 February 2oth, ibidem 3: 335. Syntypes 6 o , Chrss: W., Chow-pin-sa (BMNH).

Sipnoides was made available by Hampson, I9I3 [January 3oth], in a Key to Genera, but it was not until the next volume (Hampson, I9I3 [November I9th], ibidem 13: 248) that Hampson gave the full generic description, included three species (Sypna equatorialis Holland, Sypna mandarina Leech, Sypna pannosa Moore), and designated S. pannosa as type-species. In between the publication of Hampson's volumes I 2 and I 3 , Warren used Sypnoides for a single species (S. mandarina) and designated it as type-species.

The exact dates of publication of Hampson's catalogue are taken from Collins, 1962, -tnn. Mag. nat. Hist. (13) 5: I26. The date of publication of the part of Seitz containing page 335 was stated on page 333 as being I913 February 2oth in both the German and the English editions.

PYS.NOIDES Berio, 1959, Annali Mus. civ. Stor. nat. Giacomo Doria 71: $30+$.

Type-species: Sypua mandarina Leech, I900, by original designation.

Py'snoides is a junior objective synonym of Sypnoides Hampson, I9I3.

SYRNIA Hübner, [182I], Samml. exot. Schmett. 2: pls [207], [208], [210].

OPHI

Type-species: Syrnia hypnois Hübner, [182I], ibidem 2: pl. [210], by subsequent designation by Desmarest, (I 857), in Chenu, Encycl. Hist. nat. Papillons nocturnes: I 30.

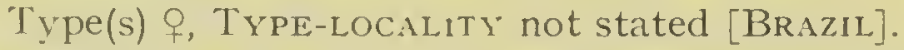

SYRRUSIS Hampson, I 908, Cut. Lepid. Phalaenae Br. Mus. 7: xiii, 508.

ACRO

Type-species: Panolis notabilis Butler, 1879, Ann. Mag. nat. Hist. (5) 4: 243, by original designation. Lectotype $\mathrm{O}^{4}$ MADAG.ASCAR: Antananarivo (BMNH), designated (as holotype) by Viette, In65, Faune Madagascar 20(I): I 52.

$\ddagger$ SYSSTREMA, misspelling. See Sy'stremma Herrich-Schäffer, I 855 .

OPHI

SYSTATICOSPORA Bethune-Baker, 1908, Novit. zool. 15: 2 ro.

HYPE

Type-species: Systaticospora orphnina Bethune-Baker, 1908, ibidem 15: 210, by original designation. Type(s) ․, New Guines: [Papua], R. Aroa.

S. orphnina is a junior subjective synonym of Piratisca minax Meyrick, I902.

See also Piratisca Meyrick, I902; and Megaloptera Bethune-Baker, I 908.

SYSTREMMA Herrich-Schäffer, 1856, Samml. neuer oder wenig bekannter aussereur. Schmett. 1 (1): wrapper, pl. 69, fig. 393; I 858, ibidem 1: 69, 82.

OPIII

Type-species: Systremma crassicornis Herrich-Schäffer, I 856 , ibidem 1 (I): wrapper, pl. 69, fig. 393; 1 8.58, ibidem 1: 69, 82, by monotypy. Type(s), [Brazil]: Rio.

†SISSTREMA; Hampson, 1926, Descr. new Genera Species lepid. Phalaenae Subfamily

Nuctuinae Br. Mus.: 423 .

An incorrect subsequent spelling.

TABOMEERES nom. n. for Evemobates Turner, 1939.

$\operatorname{ACON}$

Trpe-species: Eremobates dolera Turner, 1939, Proc. R. Soc. Qd 50: 1 45, by monotypy (of Eremobates Turner). Type(s) ơ. Australia: [N. Territory], Tennant's Creek (NMV, Melbourne).

EREMOBATES Turner, I939, Proc. R. Soc. Qd 50: 145.

Type-species: Eremobates doleva Turner, 1939, ibidem, by monotypy.

A junior homonym of Eremobates Banks, I900, Am. Nat. 34: 426, - Arachnida. The objective replacement name is Tabomeeres nom. $\mathrm{n}$. 
TACHOSA Walker, I869, in Chapman, Proc. nat. Hist. Soc. Glasgow 1: 345.

CATO

Type-species: Tachosa acronyctoides Walker, I869, ibidem 1: 345 , by subsequent designation by Hampson, I913, Cat. Lepid. Phalaenae Br. Mus. 12: 241. Lectotype $\hat{\sigma}$, Congo (BMNH), designated (as type) by Hampson, I9I3, ibidem 12: 242.

See also Anabathra Möschler, i 887 .

TADAXA nom. n. for Daxata Walker, I 865.

OPH I

Type-species: Daxata bijungens Walker, 1865, List Specimens lepid. Insects Colln Br. Mus. 33: i io9, by monotypy (of Daxata Walker, i 865). Holotype ðૈ, CEYlon (BMNH). DAXATA Walker, i 865, List Specimens lepid. Insects Colln Br. Mins. 33: I 108.

Type-species: Daxata bijungens Walker, I 865 , by monotypy.

A junior homonym of Daxata Pascoe, i 864, Trans. ent. Soc. Lond. (3) 3: 88, - Coleoptera. The objective replacement name is Tadaxa nom. n.

TAENEREMA Draudt, I950, Mitt. mïnch. ent Ges. 40: 100.

ACRO

Type-species: Taenerema hoenei Draudt, 1950, ibidem 40 : Ior, pl. 7, fig. 6, by monotypy, but cited as $\ddagger T$. hönei. Holotype ô. China: [Szechwan Prov.], Mien-shan (MAK, Bonn). Under the Code, Article 32 (c)(i), the name thönei is an incorrect original spelling. Being formed from a German personal name, it must be replaced by hoenei.

TAENIOCAMPA Guenée, I839. See Cuphanoa Hübner, [1821]. HADE

TAENIOPYGA Wallengren, I 858, Öfvers. K. VetenskAkad. Förh. Stockh. 15: 212. HAdE

Type-species: Phalaena eumela Stoll, I78I, in Cramer, Uitlandsche Kapellen 4: III, pl. 347, fig. G, by original designation. Type(s), [South Africa]: Cape of Good Hope.

See also Diaphone Hübner, [1 820].

TAENIOSEA Grote, I 874, Bull. Buffalo Soc. nat. Sci. 2: i 43.

ACRO

Type-species: Taeniosea gentilis Grote, 1874 , ibidem 2: I 43, by original designation (at bottom of page r 44). Syntypes ô, $q$, [CANADA]: Ontario, St. Catharines (BMNH).

$T$. gentilis is a junior subjective synonym of Bryophila discivaria Walker, 1856 , List Specimens lepid. Insects Colln Br. Mus. 9: 27.

TAF ALLA Walker, I 869, Characters undescr. Lepid. Heterocera: 42.

OPH I

Type-species: Tafalla clavifera Walker, I 869, ibidem: 43, by monotypy. Type(s), [HONDURAS]: Limas (BMNH).

TALAPA Moore, i 867, Proc. zool. Soc. Lond. 1867: 82.

$\mathrm{OPHI}$

Type-species: Remigia caliginosa Walker, I865, List Specimens lepid. Insects Colln $B r . M u s$. 33: ror 7 , by monotypy, but cited by Moore as $T$. caliginosalis, an unjustified emendation by Moore, I 867 . Holotype, India: N. Hindostan.

TALAPOPTERA Hampson, 1926, Descr. new Genera Species Lepid. Phalaenae Subfamily Noctuinae Br. Mus.: 263. OPHI

Type-species: Talapa confluens Hampson, I898, J. Bombay nat. Hist. Soc. 11: 705, by original designation. Holotype ô. InDIA: [Assam], Khasis (BMNH).

$T$. confluens is a junior subjective synonym of Iluza duplexa Moore, I 882, in Hewitson \& Moore, Descr. new Indian lepid. Insects Colln late Mr WW. S. Atkinson: 174, pl. 6, fig. 7.

TALARIGA Walker, I 858, List Specimens lepid. Insects Colln Br. Mus. 15: 1546, I634. opH Type-species: Talariga capacior Walker, 1858 , ibidem 15: 1634, by monotypy. Holotype ठ․ Sumatra (BMNH).

TALMELA nom. n. for Matella Moore, [1885].

OPHI

Type-species: Egnasia accingalis Walker, [1 859] I 858, List Specimens lepid. Insects Colln $B r$. Mus. 16: 21 8, by original designation (for Matella Moore). Holotype ô, CEYLoN (BMNH). 
MATELLA Moore, [1885] i 887, Lepid. Ceylon 3: 196.

Type-species: Egnasia accingalis Walker, [1 859 ], by original designation.

A junior homonym of Matella Gistl, i 848, Naturg. Thierreichs: ix, - Lepid., Geometridae. The objective replacement name is Talmela nom. $\mathrm{n}$.

$\ddagger$ TALPOCHARES, misspelling. See Porphyrinia Hübner, [I $82 \mathrm{I}]$. ACON

$\ddagger$ TALPOPHILA, misspelling. See Thalpophila Hübner, [1820]. ACRO

TAMBA Waller, i 869, Characters undescr. Lepid. Heterocera: $94 . \quad$ орH

Type-species: Tamba submicacea Walker, 1869, ibidem: 94, by monotypy. Type(s) ․, [INDIA]: [Varanasi] Benares (BMNH).

T. submicacea is a junior subjective synonym of Focilla decolor Walker, i 865, List Specimens lepid. Insects Colln Br. Mus. 33: 1029.

TAMBANA Moore, 1882, in Hewitson \& Moore, Descr. new Indian lepid. Insects Colln late Mr W. S. Atkinson: I 55 .

PANT

Type-species: Tambana variegata Moore, I 882, ibidem: I55, by original designation. Syntypes, INDiA: Darjiling (BMNH).

TAMILA Guenée, I852, in Boisduval \& Guenée, Hist. nat. Insectes (Lépid.) 6: i $76 . \quad$ Noct Type-species: Phalaena nundina Drury, I 773, Illust. nat. Hist. 1 ( 1770 ): 36, pl. I8, fig. 5, but without names until the Index, issued with 2 (I773), by monotypy. Type(s), [U.S.A.]: New York.

TAMSEALE nom. n. for Eleale Walker, I 862.

EUTE

Type-species: Eleale plusioides Walker, I 862, J. Proc. Linn. Soc. (Zool.) 6: 135, by monotypy (of Eleale Walker, I862). Lectotype + (not $\delta^{*}$ as stated by Walker), BorNEo: Sarawak (UM, Oxford), designated (as type) by Swinhoe, 1900, Cat. east. and Aust. Lepid. Heterocera 2: 85 .

This new name is proposed in honour of my mentor, $\mathrm{Mr} \mathrm{W}$. H. T. Tams, in appreciation of his willing help, advice and textual criticism.

ELEALE Walker, i 862, J. Proc. Linn. Soc. (Zool.) 6: I 35.

Type-species: Eleale plusioides Walker, I 862, by monotypy.

A junior homonym of Eleale Newman, i 84I, Entomologist 1: 36,-Coleoptera. The objective replacement name is Tamseale nom. 11.

† TAMSEUXOA Köhler, I954. See Tamsenxoa Köhler, I967.

NOCT

TAMSEUXOA Köhler, 1967, Acta zool. lilloana $21: 266,290$.

NOCT

Type-species: Agrotis ingoufii Mabille, I885, Bull. Soc. philomath. Paris (7) 9: 59, by original designation but cited as $\ddagger A$. ingonffii, an incorrect subsequent spelling. Lectotype + , [Argentina]: Patagonia, Santa Cruz (MNHN, Paris), designated (as holotype) by Viette \& Fletcher, 1968, Bull. Br. Mus. nat. Hist. (Ent.) 21: 410.

+ TA.MSEUXOA Köhler, I954, Revta Soc. ent. argent. 17: 37.

Not nomenclaturally available under the Code, Article I $3(\mathrm{~b})$, as the name was published after 1930 and was not accompanied by the fixation of a type-species.

TAMSIA Roepke, I938, Bull. Mus. r. Hist. nat. Belg. 14 (13): 52, 72.

OPHI

Type-species: Tamsia elegantula Roepke, I938, ibidem 14 (13): 52, by monotypy. Holotype ô. [Sulawesi] N. Celebes (IRSNB, Brussels).

$T$. elegantula is a junior subjective synonym of Oxaenanus hieroglyphica Swinhoe, igoz, Ann. Mag. nat. Hist. (7) 9: I 78.

Tamsia was proposed for $T$. elegantula, but in the last paragraph of the description Roepke also proposed the name $T$. javanica for a o specimen from Java, if it proved to be different. Although this latter name is available, it was at that time doubtfully identified and, under the Code, Article 68(c), is not eligible for selection as type-species. 
$\ddagger$ TAMSOLA Wiltshire, I946. See Tamsola Wiltshire, I949.

HYPE

TAMSOLA Wiltshire, 1949, Bull. Soc. Fouad I. Ent. 33: 362.

HYPE

Type-species: Tamsola tarda Wiltshire, 1946, Entomologist's Rec. J. Var. 58: 3I, pl. 7, fig. $\mathrm{H}$, by original designation. Holotype, IRAQ: Kurdistan, Rowanduz Gorge (Colln E. P. Wiltshire, London).

Tamsola was first used in the binomen of the type-species, but without any generic description. Under the Code, Article $\mathrm{r}_{3}$ (a), Tamsola is not nomenclaturally available from 1946. Under Article I7 (3) this does not, however, prevent the availability of the specific name.

$+T$ A MSOL $A$ Wiltshire, I946, Entomologist's Rec.J. Var. 58: 3 r.

Not nomenclaturally available under the Code, Article I 3 (a), as the name was published after 1930 and was not accompanied by any statement differentiating the taxon.

TAMUSIDA Walker, I866, List Specimens lepid. Insects Colln Br. Mus. 35: r 732.

SARR

Type-species: Tamusida vittalis Walker, r866, ibidem 35: r733, by monotypy. Lectotype + , Borneo: Sarawak (BMNH), designated (as type) by Hampson, rgra, Cat. Lepid. Phalaenae Br. Mus. 11: 255.

TANDILIA Köhler, I954, Revta Soc. ent. argent. 17: 33. NOCT

Type-species: Lycophotia microstigma Hampson, 1903, Cat. Lepid. Phalaenae Br. Mus. 4: 55I, pl. 78, fig. 4, by monotypy. Type(s) ô, BrAzil: Castro, Parana (USNM, Washington).

L. microstigma was described and made nomenclaturally available by Hampson, r903 [October], prior to its proposal and description by Schaus, rgo3 December, $J l ~ N . Y$. ent. Soc. 11: 23o. The bibliographic reference of the type-species as cited by Köhler, 1954, is entirely incorrect, but has been corrected in Köhler, 1967, Acta zool. lilloana 21: 3 I2.

TANOCRYX Viette, I965, Bull. Soc. ent. Fr. 70: 86.

ACRO

Type-species: Acronycta pseudobamra Rothschild, 1924, Ann. Mag. nat. Hist. (9) 14: 3 I2, by original designation. Holotype ô, MADAGASCAR: Diego Suarez (BMNH).

TANTURA Kirby, r 892, Synonymic Cat. Lepid. Heterocera 1: 86.

$\operatorname{ACON}$

Type-species: Penthetria majuscula Edwards, I88I, Papilio 1: 80, by subsequent designation by Kirby, r892, ibidem 1:86. Syntypes $\delta$, ㅇ, [U.S.A.]: Greorgia.

Tantura was proposed as the objective replacement name for Penthetria Edwards, I88I. PENTHETRIA Edwards, I88r, Papilio 1: 8o.

Type-species: Penthetria majuscula Edwards, I881, by subsequent designation by Kirby, I892, Synonymic Cat. Lepid. Heterocera 1: 86.

A junior homonym of Penthetria Meigen, I803, Magazin Insektenk. (Illiger) 2: 264,Diptera. The objective replacement name is Tantura Kirby, I892.

TAPHONIA Schaus, I9I6, Proc. U.S. natn. Mus. 50: 262, 329.

HYPE

Type-species: Taphonia peonis Schaus, I9I6, ibidem 50: 330, by original designation. Type(s) ơ, Mexico: Jalapa, Orizaba (USNM, Washington).

TAPINOSTOLA Lederer, I857. See Oria Hübner, [I82 r].

ACRO

TARACHE Hübner, [r823] r8r6, Vevz. bekannter Schmett.: 26r.

$\operatorname{ACON}$

Type-species: Noctua aprica Hübner, [r8o8], Samml. eur. Schmett. 4: pl. 8o, fig. 37 r, by subsequent designation by Grote, I874, Bull. Buffalo Soc. nat. Sci. 2: 36 . Type(s), EUROPE.

TARACHEPHIA Hampson, 1926, Descr. new Genera Species Lepid. Phalaenae Subfamily Noctuinae Br. Mus.: 87 . OPHI

Type-species: Acontia hueberi Ershov, 1874, in Fedchenko, Reise in Turkestan 2 (5) 3: 49, pl. 3, fig. 47, by original designation. Syntypes đo, ․, [U.S.S.R.]: Turkestan, Kisil-kum desert (ZI, Leningrad). 
TARACHIDIA Hampson, 1898, Trans. ent. Soc. Lond. 1898: 248.

ACON

Type-species: Tarachidia flavibasis Hampson, I 898, ibidem 1898: 248, pl. I 7, fig. 3 , by original designation. Holotype ô. Lesser ANTilles: Grenadines, Union I. (BMNH).

TARACONICA Berio, 1959, Annali Mus. civ. Stor. nat. Giacomo Doria 71: 97.

$\operatorname{ACON}$

Type-species: Taraconica transversa Berio, 1959, ibidem 71:97, by original designation. Holotype , MAdagascar: Perinet, forêt d'Analamazoatra (MNHN, Paris).

TARAMINA Noore, [1885] i 887, Lepid. Ceylon 3: 153.

CATO

Type-species: Entomogramma torsa Guenée, 1852, in Boisduval \& Guenée, Hist. nat.

Insectes (Lépid.) 7: 204, by monotypy. Syntypes o․ ㅇ. JAVA (BMNH).

TARGALLA Walker, [1858] i 857, List Specimens lepid. Insects Colln Br. Mus. 13: 98 4, 1007.

EUTE

Type-species: Targalla infida Walker, [1858] I857, ibidem 13: I008, by monotypy. Holotype $\widehat{\delta}$, [INDIA]: Hindostan (BMNH).

T. infida is a junior subjective synonym of Penicillaria delatrix Guenée, 1852, in Boisduval \& Guenée, Hist. nat. Insectes (Lépid.) 6 : 304.

TARGALLODES Holland, I894, Psyche, Camb. 7: 31.

EUTE

Type-species: Targallodes rufula Holland, I894, ibidem 7: 31, fig., by original designation. Type(s) + , West Africa (CM, Pittsburgh).

TARISTA Schaus, 1913, Ann. Mag. nat. Hist. (8) 11: 23.

HัPE

Type-species: Tarista morosa Schaus, I9I3, ibidem (8) 11:24, by original designation. Type(s) ô, Costa Rica: Juan Viñas.

TARSICOPIA Köhler, (1952) 195 I, Acta zool. lilloana 12: I 58.

CUCU:

Type-species: Tarsicopia robusta Köhler, (I952) I951, ibidem 12: I58, by original designation. Syntypes $\hat{0}$, ․ ARgentina: Chubut [Prov.], Comodoro Rivadavia (ZSBS, Munich); Mendoza.

TARSOLEPIS Butler, 1872, Ann. Mag. nat. Hist. (4) 10: 125.

NOTODONTIDAE

Type-species: Tarsolepis remicauda Butler, i 872, ibidem (4) 10: I25, pl. 8, by original designation.

Tarsolepis resembles Antiophlebia Felder, 1874, and has been incorrectly, used in the Noctuidae.

CRINO Hübner, [1821], Samml. exot. Schmett. 2: pl. [197].

Type-species: Crino sommeri Hübner, [182I], ibidem 2: pl. [197], by monotypy, as a second species Crino besckei Hübner, pl. [196], was not published until [1823] according to Hemming, 1937, Hïbner 1: 4 Io.

A junior homonym of Crino Lamarck, (1798), - Vermes. There is no objective replacement name, but $C$. sommeri Hübner is congeneric with Tarsolepis remicauda Butler, I872, the type-species of Tarsolepis Butler, 1872. The latter is therefore available for use as a subjective replacement name.

Hübner's Notodontid genus has been included in this catalogue to show its correct usage because Crino Hübner sensu Hampson, I906, was used with an incorrect type-species in the Noctuidae, Cuculliinae.

$\ddagger$ TARVIA, misspelling. See Tavia Walker, I 858 .

OPHI

TASEOPTERYX Butler, I883, Trans. ent. Soc. Lond. 1883: 63.

ACON

Type-species: Taseopteryx sericea Butler, I883, ibidem 1883: 64, by monotypy. Syntypes, Chile: Las Zorras (BMNH).

TATHODELTA Hampson, I893, Illust. typical Specimens Lepid. Heterocera Colln Br. Mus.

9: 32,135

OPHI

Type-species: Tathodelta purpurascens Hampson, 1893, ibidem 9: 32, I35, pl. I68,

fig. I 5, by monotypy. Syntypes, Cerron: i $\hat{O}$, Pundaloya (BMNH); i $q$, Dickoya (BMNH). 
TATHORHYNCHUS Hampson, I 894, Fanna Br. India (Moths) 2: xiii, 268.

ACRO

Type-species: Scopula vinctalis Walker, [1 866] I 865, List Specimens lepid. Insects Colln Br. Mus. 34: 1476 , by original designation, but cited as ' $T$. vinctale Wlk'. Syntypes 5 (Ј., P), [India]: S. Hindostan (BMNH). Australia: Sydney.

S. vinctalis is a junior subjective synonym of Spintherops exsiccata Lederer, I 855 , Verh. zool.-bot. Vev. Wien 5 (Sber.): 204, pl. 2, fig. I 2.

$\ddagger_{\ddagger}$ TATHORHYNCUS; Bethune-Baker, I9II, Ann. Mag. nat. Hist. (8) 7: 538 .

An incorrect subsequent spelling.

$\ddagger$ TATHORHYNCUS, misspelling. See Tathorhynchus Hampson, I 894.

ACRO

TATHOTHRIPA Hampson, i9I2, Cat. Lepid. Phalaenae Br. Mus. 11: xi, 307. SARR Type-species: Ariola continua Walker, 1862, J. Proc. Linn. Soc. (Zool.) 6: 196, by original designation. Lectotype , BoRneo: Sarawak (UM, Oxford), designated (as type) by Swinhoe, 1900, Cat. east. and Aust. Lepid. Heterocera 2: 76.

$\ddagger$ TATORHINIA, misspelling. See Tatorinia Butler, I 875 .

OPHI

TATORINIA Butler, I875, Ann. Mag. nat. Hist. (4) 16: 408.

Type-species: Tatorinia burrowsii Butler, 1875, ibidem (4) 16: 408, by original designation. Type(s), South Africa: Natal (BMNH).

T. burrowsii is a junior subjective synonym of Gracilodes fumipennis Felder \& Rogenhofer, I 874, Reise öst. Fregatte Novara (Zool.) 2 (Abt. 2): pl. I I9, fig. 29.

$\ddagger$ TATORHINIA; Holland, I894, Psyche, Camb. 7: I I 4 .

An incorrect subsequent spelling.

TAUTOBRIGA Walker, I869, Characters undescr. Lepid. Heterocera: 56.

OPHI

Type-species: Tautobriga euspila Walker, I869, ibidem: 56, by monotypy. Type(s) ㅇ, [HONDURAS]: Limas (BMNH).

TAVETA Fawcett, I9I6, Proc. zool. Soc. Lond. 1916: 7 I 8.

OPHI

Type-species: Taveta syrinx Fawcett, I9I6, ibidem 1916: 7I8, pl. I, fig. I3, by original designation. Syntypes I ô, I ㅇ, [Kenya]: Kedai (BMNH).

TAVIA Walker, I858, List Specimens lepid. Insects Colln Br. Mus. 14: I238, I275. OPHI

Type-species: Tavia instruens Walker, I858, ibidem 14: I275, by subsequent designation by Hampson, I902, Ann. S. Afr. Mus. 2: 353, but cited as nycterina Boisduval, I833, which Hampson placed as the senior synonym of instruens Walker. Holotype +, CONGO (BMNH).

$\ddagger$ TARVIA; Felder \& Rogenhofer, I 875, Reise öst. Fregatte Novara (Zool.) 2 (Abt 2): pl. I I4, fig. 6.

An incorrect subsequent spelling.

TA VILA Walker, I869, in Chapman, Proc. nat. Hist. Soc. Glasgow 1 (2): 349.

ACON

Type-species: Tavila indeterminata Walker, 1869, ibidem 1 (2): 350, by monotypy. Type(s) 0 , CONGo.

TAVIODES Hampson, 1926, Descr. new Genera Species Lepid. Phalaenae Subfamily Noctuinae Br. Mus.: 28.

OPHI

Type-species: Taviodes discomma Hampson, I926, ibidem: 31 , by original designation. Holotype ô, [GHana] Gold Coast: Bibianaha (BMNH).

TECHNEMON Turner, I945, Mem. Qd Mus. 12: I 59.

$\operatorname{ACON}$

Type-species: Technemon epichares Turner, I945, ibidem 12: I59, by monotypy.

Holotype, Australia: Qd, Injune (QM, Brisbane).

TECTOREA Berio, I955, Mem. Inst. sci. Madagascar (E) 6: i I8.

ACRO

Type-species: Caradrina nitens Saalmüller, I891, Lepid. Madagascar: 276, p1. I3,

fig. 232, by original designation. Syntypes, Madagascar: S. Betsileo-Land.

Tectorea was proposed as a subgenus of Athetis Hübner, [182I]. 
TEGARPAGON Berio, 1955, Mém. Inst. scient. Madagascar (E) 6: Ir8. ACRO Type-species: Caradrina tenebrata Hampson sensu Berio, I955, ibidem (E) 6: I i 8, fig. 8 [a misidentification later described as Elyptron berioi Viette, (1958) 1957, Bull. Soc. ent. Fr. 62: 278], by original designation, but cited as Caradrina tenebrata Hampson, a misidentification by Berio (see below). Holotype ô (of E. berioi), MADAGascar: Massif de l'Ankaratra, forêt d'Ambahona (MNHN, Paris).

Viette (1958), ibidem: 278 stated that the species figured by Berio as C. tenebrata Hampson, 1902, Amn. S. Afr. Mus. 2: 30r, was a misidentification and represented a new species E. berioi. Under the Code, Article 70, this case should be referred to the Commission.

Tegarpagon was proposed as a subgenus of Athetis Hübner, [I82I].

TEGENA Fletcher, I96I, Ruwenzori Exped. 19521 (7): 227.

CHLO

Type-species: Tegena steeleae Fletcher, 1961, ibidem 1 (7): 227, figs 53, 100, 104 \& 296, by original designation. Holotype $\hat{\sigma}$, [CAMEroun]: Mt Cameroon, Onyanga (BMNH).

TEGIAPA nom. n. for Pagetia Prout, r922.

ACON

Type-species: Pagetia larentiodes Prout, 1922, in Prout \& Prout, Ann. Transv. Mins. 8: I83, pl. I, fig. 26, by monotypy (of Pagetia Prout). Holotype ठิ. South Africa: Pretoria (TM, Pretoria).

PAGETIA Prout, 1922, in Prout \& Prout, Ann. Transv. Mus. 8: i83.

Type-species: Pagetia larentiodes Prout, 1922, by monotypy.

A junior homonym of Pagetia Walcott, 1916, Smithson. misc. Collns 64: 407, - Trilobita.

The objective replacement name is Tegiapa nom. $\mathrm{n}$.

TEG TEZA Walker, I869, Characters undescr. Lepid. Heterocera: 104.

OPHI

Type-species: Tegteza palpalis Walker, I869, ibidem: 104, by monotypy. Type(s) o. [Colombia]: Bogota.

TEINANOMIS Hampson, I926. See Ipnista Schaus, I9I6.

OPH I

TEINOLETIS Hampson, 1926, Descr. new Genera Species Lepid. Phalaenae Subfamily

Noctuinae Br. Mus.: II9.

OPH I

Type-species: Cyclopis simoenta Guenée, I852, in Boisduval \& Guenée, Hist. nat. Insectes Lépid. 7: I66, pl. I9, fig. 4, by original designation. Holotype q, Brazil (cited in Explication des Planches: 3) (BMNH).

TEINOPTERA Calberla, I89I, Dt. ent. Z. Iris 4: 46.

OPHI

Type-species: Teinoptera culminifera Calberla, I891, ibidem 4: 46, by monotypy. Syntypes $2 \hat{0}, \mathrm{I}$ ㅇ. [EGYPT/ISRAEL]: Palestine, El-Arisch desert.

TEINOPYGA Felder, 1874, in Felder \& Rogenhofer, Reise öst. Fregatte Novara (Zool.) 2 (Abt. 2): pl. Io6; Erklärung: 9. CHLO

Type-species: Teinopyga reticularis Felder, I874, ibidem 2 (Abt. 2): pl. ro6, fig. r8; Erklärung: 9, by monotypy. Type(s) $\sigma^{2}$. [CHina]: Shanghai (BMNH).

$T$. reticularis is a junior subjective synonym of Deiopeia extrema Walker, I854, List Specimens lepid. Insects Colln Br. Mus. 2: 573.

TELESILLA Herrich-Schäffer, I 856. See Eucarta Lederer, I857.

ACRO

TELMIA Hübner, [1821] 1816, Verz. bekannter Schmett.: 228.

NOCT

Type-species: Noctua sagitta Hübner, [1813], Samml. eur. Schmett. 4: pl. I 3o, fig. 596 (a junior primary homonym), by subsequent designation by Hampson, I903, Cat. Lepid. Phalacnae Br. Mus. 4: I 53, but cited as 'cursoria Hufn.', which Hampson placed (on page $248)$ as the senior synonym of sagitta. Type(s), Europe.

N. sagitta Hübner, [1813] (a junior primary homonym of Noctua sagitta Fabricius, I775. Syst. Ent.: 597), is a junior subjective synonym of Phalaenae cursoria Hufnagel, I766. Berlin. Mag. 3: $4 \mathrm{I} 6$. The latter is therefore available for 11 se as subjective replacement name. 
$\ddagger$ TELOLOPHA Hampson, no published reference found.

HYPE

TELORTA Warren, I9 10, in Seitz, Macrolepid. World 3: I 56.

CUCU

Type-species: Mesogona divergens Butler, 1879, Ann. Mag. nat. Hist. (5) 4: 364 , by original designation. Type(s), JAPAN (BMNH).

The date of publication of the part of Seitz containing page ${ }_{5} 6$ was stated on page $r_{49}$ as I9Io May 2oth in the English edition and I9I I January 23rd in the German edition.

TEMNOPTERA Bethune-Balier, 1908, Novit. zool. 15: 223.

OPHI

Type-species: Temnoptera meeki Bethune-Balser, 1908, ibidem 15: 223, by original designation. Type(s) J̃, New Guinea: [Papua], Aroa River (BMNH).

TENDARBA Walker, [1866] 1865, List Specimens lepid. Insects Colln Br. Mus. 34: I I85. ACRo Type-species: Tendarba lineosa Walker, [1866] I865, ibidem 34: I I86, by monotypy. Holotype ô., [BRAzIL]: Rio Janeiro (BMNH).

$T$. lineosa is a junior subjective synonym of Licha undilinealis Walker, $\mathrm{r} 859$.

See also Licha Walker, 1859; and Bononia Walker, I862.

TEPHRIALIA Hampson, I926, Descr. new Genera Species Lepid. Phalaenae Subfamily Noctuinae Br. Mus.: I 80 . OPHI

Type-species: Baniana trigonospila Hampson, r9ro, Proc. zool. Soc. Lond. 1910: 435, pl. 37, fig. 19, by original designation. Holotype $\hat{\sigma}$. [ZAmBIA]: Rhodesia: N.E., upper Luangwa R. (BMNH).

TEPHRIAS Wallengren, r860, Wien. ent. Munatschr. 4: 170.

OPHI

Type-species: Tephrias plumipes Wallengren, I860, ibidem 4: I70, by monotypy. Type(s), [South Africa]: Caffraria (NR, Stockholm).

See also Baxagha Walker, I 865.

GALAPHA Walker, 1858, List Specimens lepid. Insects Colln Br. Mus. 15: 1850.

Type-species: Galapha anguliplaga Walker, 1858, ibidem 15: I85I, by monotypy. Type(s) ô. [South Africa]: Natal.

$G$. anguliplaga is a junior subjective synonym of Toxocampa atriplaga Walker, I858, ibidem 15: I795.

A junior homonym of Galapha Walker, r858, ibidem 15: r 537 , I 544, - Lepid., Noctuidae. There is no objective replacement name but G. anguliplaga is congeneric with Tephrias plumipes Wallengren, r860, the type-species of Tephrias Wallengren, I860. The latter is therefore available for use as the subjective replacement name.

TEPHRINOPS Hampson, 1926, Descr. new Genera Species Lepid. Phalaenae Subfamily Noctuinae Br. Mus.: I60.

OPHI

Type-species: Tephrinops velutisigna Hampson, 1926, ibidem: 160 , by original designation. Holotype $\sigma^{7}$, BrazIL: Espiritu Santo (BMNH).

TEPHRIOPIS Hampson, 1926, Descr. new Genera Species Lepid. Phalaenae Subfamily Noctuinae Br. Mus.: I 7 I.

OPHI

Type-species: Athyrma divulsa Walker, I865, List Specimens lepid. Insects Colln Br. M Ius. 33: 966, by original designation. Syntypes $3(\hat{\sigma},+$ ) , [BANGLAdESH]: [E. Bengal], Silhet $(\mathrm{BMNH})$.

TEPHROCHARES Zerny, 1933, Dt. ent. Z. Iris 47: 80.

$\operatorname{ACON}$

Type-species: Madopa inquinata Lederer, I857, Wien. ent. MTonatschr. 1: 98, by original designation. Syntypes $\mathrm{I} \hat{\sigma}, \mathrm{r}$ ㅇ, [LEBANON]: Beirut.

TERA TOCERA Guenée, I 852, in Boisduval \& Guenée, Hist. nat. Insectes (Lépid.) 7: 339 . OPHI

Type-species: Syllectra congemmalis Hübner, r823, Zuträge Samml. exot. Schmett. 2: 2r, figs 309, 3 ro, by subsequent designation by Desmarest, (1857), in Chenu, Encycl. Hist. nat. Papillons nocturnes: 134. Type(s) + , SuRinam.

${ }_{+}$TETRATOCERA; Butler, r 878, Proc. zool. Soc. Lond. 1878: 498.

An incorrect subsequent spelling. 
TERATOGLAEA Sugi, I958, Tinea, Tokyo 4: 208.

Type-species: Teratoglaea pacifica Sugi, 1958, ibidem 4: 209, pl. 3I, fig. 20, pl. 28, figs 9, 9a, by original designation. Holotype ô, JApax: Tokyo, Takao-san (Colln S. Sugi, Tokyo).

TESOMONODA nom. n. for Odontosema Warren, I9I3. ACON

Type-species: Ligidia endolopha Hampson, I910, Cat. Lepid. Phalaenae Br. Mus. 10: 799, pl. I74, fig. 7, by original designation (for Odontosema Warren). Syntypes I $\hat{\delta}$, 2 , New Gurnea: [Papua], Milne Bay (BMNH).

ODONTOSEMA Warren, I9I 3, in Seitz, Gross-Schmett. Erde 11: 233.

Type-species: Ligidia endolopha Hampson, I910, by original designation.

The date of publication of the part of Seitz containing page 233 was stated on the same page as I9I 3 August 20th in both the English and German editions.

A junior homonym of Odontosema Kieffer, 1909, Bull. Soc. Hist. nat. Metz 26: 58, Hymenoptera. The objective replacement name is Tesomonoda nom. n.

TETANOLITA Grote, I 873, Trans. Am. ent. Soc. 4: 305.

HYPE

Type-species: Tetanolita lixalis Grote, I 873, ibidem 4: 306 , by monotypy. Syntypes I $\tilde{\sigma}, \mathrm{I}$ \%, [U.S.A.]: Alabama and Texas.

$T$. lixalis is a junior subjective synonym of Bleptina mynesalis Walker, I859, List Specimens lepid. Insects Colln Br. Mus, 19:860.

TETHEA Ochsenheimer, i 816, Schmett. Eur. 4:64. THYATIRIDAE

Type-species: Noctua or [Denis \& Schiffermüller], 1775, Ankiindung syst. Werkes Schmett. Wienergegend: 87, by subsequent designation by Curtis, 1829, Br. Ent. 6: 272, but cited as ' $N$. or Hüb.', an incorrect authorship.

Tethea was originally placed in the Noctuidae.

$\ddagger B O M B Y C I A$ Hübner, [1806], Tentamen determinationis digestionis . . . : [1].

Included in a work rejected for nomenclatural purposes by Int. Commn zool. Nom., I926, Smithson. misc. Collns 73 (4) Opinion 97: 19. Also idem, 1954, Opin. Decl. int. Commn zool. Nom. 6 Opinion 278: 140.

Only included species: Noctua or [Denis \& Schiffermüller], I775.

${ }_{\ddagger} B O M B Y C I A E$ Ochsenheimer, 1816, Schmett. Eur. 4: 64 .

Published as a junior synonym of Tethea Ochsenheimer, I8I6, and not subsequently treated as an available name under the Code, Article II(d).

BOMBYCIA Hübner, 1822, Syst.-alphab. Verz.: 22, 31.

Type-species: Noctua or [Denis \& Schiffermüller], I775, by subsequent designation by Harvey, I874, Bull. Buffalo Soc. nat. Sci. 1: 278.

The type-species was originally included in this genus as $\ddagger$ oo $\mathrm{S}_{\llcorner}^{-}$chiff.], 2 10, but reference to figure 2 I o of Hübner's Samml. eur. Schmett. 4 shows that too was an incorrect subsequent spelling of Noctua or. Confusion has arisen as Bombycia has been used by authors, based on the incorrect type-species Phalaena oo Linnaeus, I758, - Noctuidae, Acronictinae.

Bombycia is a junior objective synonym of Tethea Ochsenheimer, I8I6.

CEROPACHA Stephens, I829 [June], Nom. Br. Insects: 42.

Type-species: Noctua or [Denis \& Schiffermüller], I775, by subsequent designation by Westwood, I840, Synopsis Genera Br. Insects: 96.

Ceropacha was originally placed among Noctuidae. It is a junior objective synonym of Tethea Ochsenheimer, I8 6 .

$\ddagger_{\dagger}$ ERATOPACHA ; Stephens, 183I, Illust. Br. Ent. (Haustellata) 3: 33 I, 334 .

An incorrect subsequent spelling.

CERATOPACHA Stephens, i 85o, List Specimens Br. Anim. Colln Br. Mus. 5: I 19.

An unjustified emendation of Ceropacha Stephens, I 829. 
TET RACME Hampson, I902, Ann. S. Afr. Mus. 2: 439.

HYPE

Type-species: Erosia truncataria Walker, I86I, List Specimens lepid. Insects Colln Br. Mus. 23: 847, by monotypy. Holotype q, South Africa (BMNH).

TETRAPYRGIA Walker, I865, List Specimens lepid. Insects Colln Br. Mus. 33: 7II. Nocr Type-species: Tetrapyrgia graphiphorides Walker, I 865, ibidem 33: 7 I 2, by monotypy. Holotype $\hat{\sigma}$. [Australia]: Tasmania (BMNH).

T. graphiphorides is a junior subjective synonym of Agrotis porphyricollis Guenée, 1852, in Boisduval \& Guenée, Hist. nat. Insectes (Lépid.) 5: 259.

$\ddagger$ TETRATOCERA, misspelling. See Teratocera Guenée, 1852 .

OPHI

TETRISIA Walker, [1867] I 868, J. Linn. Soc. (Zool.) 9: I86.

OPHI

Type-species: Tetrisia florigera Walker, [1867] I868, ibidem 9: I 87, by monotypy. Type(s) ㅇ. [Colombia]: Bogota.

TEUCOCRANON Berio, 1937, Annali Mus. civ. Stor. nat. Giacomo Doria 59: 392. ACON

Type-species: Teucocranon microcallia Berio, I937, ibidem 59: 392, by original designation. Holotype, [Somali Republic] Somalia Ital.: Ogaden, Uarder (Colln E. Berio, Genoa).

${ }_{+}^{+}$THALALTA, misspelling. See Thalatta Walker, [18 88 ].

OPHI

$\ddagger$ THALATA, misspelling. See Thalatha Walker, I862.

ACRO

THALATHA Walker, I862, J. Proc. Linn. Soc. (Zool.) 6: i 87.

ACRO

Type-species: Orthosia sinens Walker, I857, List Specimens lepid. Insects Colln Br. Mus. 11: 746, by monotypy. Holotype 오, [InDIA] Hindostan (BMNH).

$\ddagger$ THALATA; Grünberg, I9I I, Arch. Naturgesch. 76 (4) 2: I 20.

An incorrect subsequent spelling of Thalatha Walker, I862, not of Thalatta Valker, [1858], as stated by Neave, I940, Nomencl. zool. 4: 449.

THALATTA Walker, [I858] I857, List Specimens lepid. Insects Colln Br. Mus. 13: 984, 996.

OPHI

Type-species: Thalatta precedens Walker, [1858] I 857, ibidem 13: 996, by subsequent designation by Moore, i 884, Lepid. Ceylon 3: 87. Holotype q, [INDia]: Hindostan (BMNH). THALALTA; Candèze, 1927, Encycl. ent. (B. 3) 2: 98.

An incorrect subsequent spelling of Thalatta Walker, [1858], not of Thalatha Walker, I862, as stated by Neave, I940, Nomencl. zool. 4: 447.

THALERASTRIA Staudinger, I 897, Dt. ent. Z. Iris 10: 294.

$\operatorname{ACON}$

Type-species: Erastria diaphora Staudinger, 1879, Horae Soc. ent. ross. 14: 4I 5, by subsequent designation by Hampson, igro, Cat. Lepid. Phalaenae Br. Mus. 10: 618. Holotype \%, [Turkey]: Armenia, Kerasdere (MNHU, Berlin).

THALOMICRA Spuler, I907, in Hofmann, Schmett. Eur. 1: 290, footnote.

ACON

Type-species: Thalpochares debilis Christoph, I884, in Romanoff, Mém. Lépid. 1 : 129, by monotypy. Syntypes ô, [U.S.S.R.]: [Turkmenistan], Achal-Telke, Nuchur (ZI, Leningrad); [Dagestan], Derbent.

THALPOCHARES Lederer, I 853. See Porphyrinia Hübner, [1821].

$\operatorname{ACON}$

THALPOPHILA Hübner, [1820] I816, Verz. bekannter Schmett.: 208.

ACRO

Type-species: Phalaena texta Esper, I787, Die Schmett. 4 (I): pl. I08, figs 5, 6; I790, ibidem: 185 , by subsequent designation by Moore, I884, Lepid. Ceylon 3: 24. Syntypes o. 오. ITALy.

P. texta is a junior subjective synonym of Phalaena matura Hufnagel, I766, Berlin. Mag. $3(4): 4$ I 4 . 
CERIGO Stephens, I829, Illust. Br. Ent. (Haustellata) 2: Io6.

Type-species: Phalaena texta Esper, 1787, by monotypy.

Cerigo is a junior objective synonym of Thalpophila Hübner, [1820].

$\ddagger$ TALPOPHILA; Warren, I9I I, in Seitz, Gross-Schmett. Erde 3: I99.

An incorrect subsequent spelling in the German edition, but correctly spelled in the English edition.

THARGELIA Püngeler, 1899, Dt. ent. Z. Iris 12: I0 †.

HADE

Type-species: Scotochrosta distincta Christoph, I88ł, in Romanoff, Mém. Lépid. 1: I24, pl. 8, fig. 3, by subsequent designation by Hampson, 1905, Cat. Lepid. Phalaenae $\mathrm{Br}$. Mus. 5: 220. Syntypes $\tilde{o}$, q, [U.S.S.R.]: [Turkmenistan], Achal-Tekke district (ZI, Leningrad).

THAUMASIODES Turner, 1939, Proc. R. Soc. Qd 50: I 44.

ACON

Type-species: Thaumasiodes eurymitra Turner, I939, ibidem 50: I +5, by monotypy. Holotype 9 , Australia: N. Qd, Lake Barrine (ANIC, Canberra).

THAUMASTA Staudinger, I87I. See Athaumasta Hampson, I906.

CUCU

THAUSGEA Viette, 1966, Bull. Soc. ent. Fr. 71 : 150.

OPHI

Type-species: Thausgea lolo Viette, I966, ibidem 71: 150, by original designation. Holotype ô. MAdagascar: enviruns d'Ambohimahasao, forêt d'Ankafina (MNHN, Paris).

THECAMICHTIS Draudt, 1937, in Seitz, Macrolepid. World 3 Suppl.: 270.

CUCU

Type-species: Thecamichtis meissneri Draudt, 1937, ibidem 3 Suppl. : 270 , by monotypy. Syntypes [CHina]: I $\hat{o}, \mathbf{N}$. Yunnan, Likiang (MAK, Bonn); I $q$, Shantung, Tai-shan (MAK, Bonn).

The date of publication of the part of Seitz containing page 270 was stated on page 265 as 1937 June $15^{\text {th }}$ in the English edition and 1937 June $24^{\text {th }}$ in the German edition.

THECOPHORA Lederer, I857. See Rileyiana Moucha \& Chvála, I963.

CUCU

THEGALEA Turner, I920, Trans. R. Soc. S. Aust. 44: I 40, I47. ACro

Type-species: Hypoperigea haemorrhanta Turner, I909 September I5th, Proc. Linn. Soc. N.S.W. 34: 342 (a junior homonym), by monotypy. Holotype ô. Australia: Qd, Adavale (far west) (ANIC, Canberra).

$H$. haemorrhanta Turner is a junior primary homonym and a junior objective synonym of Hypoperigea haemorrhanta Hampson, 1909 [Nay 25th], Cat. Lepid. Phalaenae Br. Mus. 8: 425. This species was described and made nomenclaturally available by Hampson, based on the same specimen as Turner's description published four months later.

THELIDORA Möschler, I88o, V'erh. zool.-bot. Ges. Wien 30 (Abh.): 391.

OPHI

Type-species: Thelidora splendens Möschler, I88o, ibidem 30 (Abh.) : 392, pl. 9, fig. 46, by monotypy. Syntypes, Jamaica: i o (MNHU, Berlin). Surinam: I $q$, Paramaribo (MNHU, Berlin). Möschler stated that the description was based on the male from Jamaica.

T. splendens is a junior subjective synonym of Concana mundissima Walker, [1858].

See also Concana Walker, [1858].

THELXINOA Turner, 1902, Proc. Limn. Soc. N.S.W. 27: 13 I.

$\operatorname{ACON}$

Type-species: Thelxinoa epiphracta Turner, 1902, ibidem 27: 132, by original designation. Syntypes, Australia: I $\hat{0}, 2$ ㅇ, Qd, Brisbane (ANIC, Canberra).

THEMMA Walker, I863, List Specimens lepid. Insects Colln Br. Mus. 27: 186.

Type-species: Themma divisa Walker, I863, ibidem 27: 186, by monotypy. Syntypes I I $\left(\sigma^{\prime}\right.$, $)$, [U.S.A.]: East Florida, St. John's Bluff (BMNll).

T. divisa is a junior subjective synonym of Dorvodes spaclaria Gucnce, 1857 , in Boisduval

\& Guenée, Hist. nat. Insectes (Lépid.) 10: 234.

See also Tunza Walker, 1863. 
THEOTINUS Druce, I 89I, Biologia cent.-am. (Zool.) Lepid. Heterocera 1: $460 . \quad$ Hy PE Type-species: Bleptina virbiusalis Walker, [1859] I858, List Specimens lepid. Insects Colln Br. Mus. 16: I26, by original designation. Holotype + , Venezuela (BMNH).

THERASEA Grote, I 895, Abh. naturw. Ver. Bremen 14: I I 5.

Type-species: Tarache augustipennis Grote, 1875, Proc. Acad. nat.Sci.Philad. 1875: 426 , by monotypy, but cited as $\ddagger T$. angustipennis, an incorrect subsequent spelling. Holotype , [U.S.A.]: Texas, Bosque Co. (BMNH).

$T$. augustipennis has subsequently been written as $\ddagger$ angustipennis by Grote and other authors. In the original description the name was only used once, and the printed ' $u$ ' was not an inverted ' $n$ '.

† THERMECIA, misspelling. See Thermesia Hübner, I823.

$\mathrm{OPHI}$

THERMESIA Hübner, I 823, Zutväge Samml. exot. Schmett. 2: I 2.

OPHI

Type-species: Thermesia abadirina Hübner, 1823, ibidem 2: 12, figs 237, 238, by subsequent designation by Berio, 1966, Annali Mits. civ. Stor. nat. Giacomo Doria 76: 65 . Syntype(s) $q$, Surinam.

Thermesia was also used by Hübner, [1823] I8I6, Verz. bekannter Schmett.: 270, but according to Hemming, I 937, Hübner 2: 263, the Zuträge usage has priority.

Incorrect type-species designation: Anticarsia gemmatalis Hübner, a name not originally included in either the Verzeichniss or the Zutragge genus and not linked with any of the originally included names when cited by Hampson, I 894, Fauna Br. India (Moths) 2: 533 .

Incorrect type-species designation: Phalaena acron Cramer (a name not originally included in the $Z$ utväge genus although it was included in the Verzeichniss genus) cited by Hampson, 1926, Descr. new Genera Species Lepid. Phalaenae Subfamily Noctuinae Br. Mus.: i 20.

$\ddagger$ THERMECI A; Swainson, I goo, Jl N.Y. ent. Soc. 8: 34 .

An incorrect subsequent spelling.

THERMOSARA Hampson, 1926, Descr. new Genera Species Lepid. Phalaenae Subfamily Noctuinae Br. Mus.: 408. OPHI

Type-species: Thermosara diapyra Hampson, I926, ibidem: 408, by original designation. Holotype $\hat{\sigma}$, New Guinea: [West Irian], Arfak Mts, Ninay Valley (BMNH).

THIACHROIA Schaus, I921, Proc. U.S. natn. Mus. 59:372.

Type-species: Thiachroia deilinias Schaus, I92 I, ibidem 59: 372, by monotypy. Type(s) $\hat{\sigma}$, Guatemala: Cayuga (USNM, Washington).

THIACIDAS Walker, I 855, List Specimens lepid. Insects Colln Br. Mus. 5: 979, I 027.

Type-species: Thiacidas postica Walker, I 855 , ibidem 5: I028, by monotypy. Holotype $\vec{\sigma}$, NePal (BMNH).

$T$. postica was originally placed in the Notodontidae.

THIGANUSA Walker, I 865, List Specimens lepid. Insects Colln Br. Mus. 33 : 979.

cHLO Type-species: Thiganusa euproctisoides Walker, i 865, ibidem 33: 979, by monotypy. Holotype , [South AFrica]: Natal (BMNH).

T. euproctisoides is a junior subjective synonym of Leocyma appollinis Guenée, I 852 .

See also Leocyma Guenée, I 852.

THIOCHROA Hampson, 1926, Descr. new Genera Species Lepid. Phalaenae Subfamily Noctuinae Br. Mus.: 3 I 4 .

OPHI

Type-species: Thiochroa flavala Hampson, 1926, ibidem: 314, by original designation. Syntypes I ô, I $q$, [Guyana] Br. Guiana: Demerara (BMNH).

THIONA Guenée, I852, in Boisduval \& Guenée, Hist. nat. Insectes (Lépid.) 7: 352. OPHI Type-species: Thiona phalaena Guenée, I 852 , ibidem 7:353, pl. 23, fig. 9, by monotypy. Type(s), Brazil (BMNH). 
THIOPTERA Franclemont, I950, Proc. ent. Soc. Wash. 52:271.

Type-species: Xanthoptera nigrofimbria Guenée, I852, in Boisduval \& Guenée, Hist. nat. Insectes (Lépid.) 6: 24I, pl. Io, fig. I2, by subsequent designation (for Xanthoptera Guenée) by Grote, i 874, Bull. Buffalo Soc. nat. Sci. 2: 36. Holotype +, AmErica: northern.

Thioptera was proposed as the objective replacement name for Xanthoptera Guenée, I 852.

XANTHOPTERA Guenée, I852, in Boisduval \& Guenée, Hist. nat. Insectes (Lépid.) 6: 240.

Type-species: Xanthoptera nigrofimbria Guenée, 1852, by subsequent designation by Grote, 1874, Bull. Buffalo Soc. nat. Sci. 2: 36.

A junior homonym of Xanthoptera Sodoffsky, i837, Bull. Soc. imp. Nat. Moscou 1837 (6): 86, - Lepid., Noctuidae. The objective replacement name is Thioptera Franclemont, I950.

FLAVALA Berio, 1966, Annali Mus. civ. Stor. nat. Giacomo Doria 76: 66.

Proposed, unnecessarily, as an objective replacement name for Xanthoptera Guenée, I852.

THOLERA Hübner, [182I] I816, Verz. bekannter Schmett.: 215.

HADE

Type-species: Noctua cespitis [Denis \& Schiffermiuller], I775, Ankïndung syst. Werkes Schmett. Wienergegend: 82, by subsequent designation by Hampson, I905, Cat. Lepid. Phalaenae Br. Mus. 5: 216. Type(s), [Austria]: Vienna district (Collection destroyed, Horn \& Kahle, 1936, Ent. Beih. Berl.-Dahlem 3: 243).

CHARAEAS Stephens, I 829, Illust. Br. Ent. (Haustellata) 2: 108.

Type-species: Noctua cespitis [Denis \& Schiffermüller], I 775, by subsequent designation by Westwood, I8+o, Synopsis Genera Br. Insects: 93.

Incorrect type-species designation: Agrotis aethiops Ochsenheimer, i816 [ = Noctua nigra Haworth, 1809], cited by Boisduval, 1836, IIist. nat. Insectes (Lépid.) 1: I 44. Boisduval in his lengthy 154 page Introduction, reviewed earlier classifications and designated up to the three different type-species for each generic name. In his 'Exposé de notre méthode' from pages 155-690, no type-species designations were made for the genera he himself used. Under the Code, Article 69(a)(iii), the type-designation of an author is eligible for consideration if he states that it is the type '... and if it is clear that he himself accepts it as the type-species.' Boisduval's type-designations although clearly stated, do not fulfil the last requirement and so are invalid. Even though Boisduval's I 836 work was well known to lepidopterists, the type-designations contained in it have not in the past been accepted by Hemming or otler authors.

Charaeas is a junior objective synonym of Tholeva Hübner, [I82I].

THOLOMIGES Lederer, I857, Noctuinen Enr.: f HYPE

Type-species: Hypena turfosalis Wocke, 1850, Z. Ent. Breslau 4 (16) Lepid.: pl. 5, fig I 7, by monotypy (of Schrankia Herrich-Schäffer, [1851]. Syntypes ${ }_{0}$, + , TYPE-Locality llot stated but from context probably [Czecuoslovakia]: Altvater Mts.

Tholomiges was proposed as the objective replacement name for Schrankia HerrichSchäffer, [I 851 I]. Lederer considered that Schrankia Herrich-Schäffer was proccupied by a botanical genus. This is not now the case as under the Code, Article 2, zoological nomenclature is independent of other systems. However, Schrankia Herrich-Schäffer is also preoccupied in zoological nomenclature (see below).

See also Hypenodes Doubleday, 1850.

SCHRANKIA Herrich-Schäffer, [I85I] i 845, Syst. Bearl. Schmett. Eur. 2: 148.

Type-species: Hypena turfosalis Wocke, I 850 , by monotypy.

A junior homonym of Schrankia Hübner, [1825], - Lepid., Noctuidac. The objective replacement name is Tholomiges Lederer, 1857.

SCHRANCIIA Walker, [1859] 1858, List Specimens lepid. Insects Colln Rr. Mus. 16: 70.

An unjustified emendation of Schrankia Herrich-Schäffer, [1 851 ]

THOPELIA nom. n. for Plotheia Walker, I863. 
Br. Muss. 28: 46I, by monotypy (of Plotheia Walker, I863). Type(s) $q$, Borneo: Sarawak (BMNH).

PLOTHEIA Walker, I863, List Specimens lepid. Insects Colln Br. Mus. 28: 460.

Type-species: Plotheia innotabilis Walker, 1863 , by monotypy.

A junior homonym of Plotheia Walker, [1858],-Lepid., Noctuidae. The objective replacement name is Thopelia nom. $\mathrm{n}$.

THORACOLOPHA Turner, 1939, Proc. R. Soc. Qd 50: 143.

ACRO

Type-species: Thoracolopha pissonephra Turner, 1939, ibidem 50: 143, by original designation. Syntypes, Australia: $20 \hat{0}, \mathrm{I}$ ․, W. Aust., R. Margaret (ANIC, Canberra) ; Perth; Narrogin; Albany.

THORACOLOPHOTOS Bethune-Baker, I906, Novit. zool. 13: 261.

$\mathrm{OPHI}$

Type-species: Thoracolophotos ekeikei Bethune-Baker, 1906, ibidem 13: 261, by original designation. Type(s) $\hat{0}$, NEw Guinea: [Papua], Ekeikei (BMNH).

THRIA Walker, [1858] I 857, List Specimens lepid. Insects Colln Br. Mus. 13: Io79, I I I. OPHI Type-species: Thria robusta Walker, [1858] I857, ibidem 13: II I2, by monotypy. Holotype ơ. South Africa (BMNH).

See also Cerbia Walker, I 858.

SUBPANDESMA Berio, I966, Boll. Soc. ent. ital. 96: I40.

Type-species: Pandesma anysa Guenée sensu Berio, I966, ibidem 96: I40 [a misidentification of Thria robusta Walker, [1858]], by original designation.

The type-species was misidentified; this was noted by Berio, 1968, Annali Mus. civ. Stor. nat. Giacomo Doria 77: 2 Io, who placed Subpandesma as a synonym of Thria. Under Article $70(\mathrm{a})$ of the Code this case should be referred to the Commission.

THRIPONEA Hulstaert, 1924, Ann. Mag. nat. Hist. (9) 13: I07.

SARR

Type-species: Thriponea muricata Hulstaert, I924, ibidem, (9) 13: 107, by original designation. Holotype , , [PHilippines]: E. Mindanao, Higakit.

THROANA Walker, [1859] I858, List Specimens lepid. Insects Colln Br. Mus. 16: 224. OPHI Type-species: Throana amyntoralis Walker, [1 859] I 858, ibidem 16:225, by monotypy. Lectotype $\hat{\sigma}$. Borneo: Sarawak (UM, Oxford), designated (as type) by Swinhoe, rgoo, Cat. east. and Aust. Lepid. Heterocera 2: I92.

THRYPTICODES Lucas, 189o, Proc. Limn. Soc. N.S.W. (2) 4: 1073.

SARR

Type-species: Thrypticodes xyloglypta Lucas, I890, ibidem (2) 4: 1073, by monotypy. Syntypes ô, 9 , Australia: Brisbane.

T. xyloglypta is a junior subjective synonym of Nanaguna breviuscula Walker, 1863.

See also Nanaguna Walker, 1863; Clettharra Walker, I863; and Bagistana Walker, I864. $\ddagger$ THR Y PTICODES Turner, I 899, Trans. R. Soc. S. Aust. 23: I 7.

Neave, 1940, Nomencl. zool. 4: 482 was in error in citing the above reference as the first establishment of the genus.

†THR YPTICODES Turner, 1899. See Thrypticodes Lucas, I 890.

SARR

THURBERIPHAGA Dyar (1920) 1919, Insecutor Inscit. menstr. 7: i88.

ACRO

Type-species: Thurberiphaga catalina Dyar, (1920) I919, ibidem 7: 188, by monotypy. Syntypes 3 o, 2 q. [U.S.A.]: Arizona, Santa Catalina Mts, Sabino Canyon (USNM, Washington).

THURSANIA Schaus, I913, Ann. Mag. nat. Hist. (8) 11: I4.

HYPE

Type-species: Thursania decocta Schaus, I913, ibidem (8) 11: I4, by original designation. Type(s) ơ, Costa Rrca: Sixola. 
THYAS Hïbner, [1824j, Samml. exot. Schmett. 2: pl. [203].

Type-species: Thyas honesta Hübner, [1824], ibidem 2: pl. [203], figs I, 2, by monotypy. Type(s) ô. Type-Locality not stated [INdia. East Indies].

THYATIRA Ochsenheimer, i816, Schmett. Eur. 4: 77.

Type-species: Phalaena batis Linnaeus, 1758 , Syst. Nat. (Edn 10) 1:509, by subsequent designation by Curtis, I 825, Br. Ent. 2: 72 .

Thyatira was originally placed amongst Noctuidae.

THYATIRIDES Kozhanchikov, I950. See Trispila Houlbert, 1921.

OPHI

THYATIRINA Hampson, igio August, Proc. zool. Soc. Lond. 1910: 4I I.

ACON

Type-species: Thyatira achatina Weymer, i 896, Berl. ent. Z. 41: 90, pl. 8, fig. 3, by monotypy. Holotype + , [TANZANIA]: [Tanganyika], [L.] Victoria-Nyanza, Muanza.

Thyatirina was also described as new in Hampson, I91o [December], Cat. Lepid. Phalaenae Br. Mus. 10: xvii, 650, having the same type-species.

THYATIRODES Hampson, I908, Cat. Lepid. Phalaenae Br. Mus. 7: 8. Nomenclaturally available, but without included species until Hampson, I909, ibidem 8: x, 282 . ACRO

Type-species: Thyatira godalma Schaus, i904, Trans. Am. ent. Soc. 30: 152, by subsequent monotypy. Type(s), Mexico: Guadalajara (USNM, Washington).

THYREION Smith, 1891, Trans. Am. ent. Soc. 18: I 21.

NOCT

Type-species: Thyreion rosea Smith, i 891 , ibidem 18: I I, by monotypy. Holotype J. [U.S.A.]: Colorado.

THYRESTRA Hampson, I905, Cat. Lepid. Phalaenae Br. Mus. 5: vii, 6.

HADE

Type-species: Euplexia hyalophora Hampson, 1898, J. Bombay nat. Ilist. Soc. 11: 442, by original designation. Holotype $\hat{o}$, Indis: [Assam], Khasis (BMNH).

THYRIA Cruenće, i 852, in Boisduval \& Guenće, Hist. nat. Insectes (Lépid). 6: $357 . \quad$ ACRO

Type-species: Thyria bellinita Guenée, 1852 , ibidem 6: 357 , by subsequent designation by Hampson, 1908, Cat. Lepid. Phalaenae Br. Mus. 7: 517. Holotype ô, Brazil: Pernambuco district (BMNH).

THYRIDOSPILA Guenéc, I 852, in Boiscluval \& Guenée, Hist. nat. Insectes (Lépid.) 7: 331.

OPII

Type-species: Thyridospila ennomoides Guenée, 1852, ibidem 7: 331, by monotypy. Holotype ô, BrazIL: Bahia.

THYRIODES Guenée, I 852, in Boiscluval \& Gnenée, Hist. nat. Insectes (Lépid.) 7: 364. EuTE

Type-species: Thyriodes flabellum Guenée, I 852 , ibidem 7: 365, pl. 23, fig. I I, by monotypy. Lectotype 3̂, Mexico (MNHN, Paris), designated by Viette, I95 r, Bull. mens. Sor. linn. Lyon 20: 162.

THYROSTIPA Hampson, 1926, Descr. new Genera Species Lepid. Phalaenae Subfamily Nortuinae Br. Mus.: 574 .

Type-species: Thyridospila sphaeriphora Noore, I867, Proc. zool. Soc. Lond. 1867: 79, by original designation. Syntypes +. Inda: Bengal (BMNH).

‡THYRSOLOMIA Hampson, no published reference found.

HYPE

THYRSOSCELIS Meyrick, I889, Trans. ent. Soc. Lond. 1889: 473.

CHLO

Type-species: Thyrsoscelis iridias Meyrick, 1889, ibidem 1889: 473, by monotypy. Syntypes i os, I $q$, New Guined: [Papua], Nt Obree or adjoining ranges (B.INH).

THYSANIA Dalman, I 824, K. suenska Vetensk.1cad. Handl. 1824: $407 . \quad$ OrH I

Type-species: Phalaena agrippina Cramer, i776, L'itlandsche Kapellen 1: I36, p1. 87, fig. A, by subsequent designation by Berio, 1957, Memorie Soc. ent. ital. 36: I9. Syntypes, SURINAM. 
$\ddagger$ THYSANOGNATHA Hampson, no published reference found.

HY PE

TIAUSPA Walker, [1858]. See Monogona Guenée, 1852.

OPHI

TIBIOCILLARIA Bethune-Baker, I906, Novit. zool. 13: 23 I.

EUTE

Type-species: Tibiocillaria pratti Bethune-Baker, 1906, ibidem 13: 231, by original designation. Syntypes $0^{7}$,, , NEw Guinea: [Papua], Kebea Range (BMNH).

TIBRACANA Walker, [1866] I865, List Specimens lepid. Insects Colln Br. Mus. 34: I I98. HypE Type-species: Tibracana xanthialis Walker, [1866] I865, ibidem 34: I I99, by monotypy. Type(s) ․ BrazIL (UM, Oxford).

TIGRANA Walker, [1 866] I 865, List Specimens lepid. Insects Colln Br. Mus. 34: I209. Hy PE Type-species: Tigrana detritalis Walker, [1866] I865, ibidem 34: 1210, by monotypy. Holotype ơ, [Australia]: [Qd], Moreton Bay.

TILIACEA Tutt, I896, Entomologist's Rec. J. Var. 8: I7.

CUCU

Type-species: Phalaena citrago Linnaeus, I758, Syst. Nat. (Edn Io) 1:518, by monotypy. Type(s), TyPE-LOCALITY not stated (LS, London) [Europe].

Tiliacea when originally proposed contained a second species, but this was doubtfully included and, under the Code, Article 68 (c), is not eligible for selection as type-species.

TIMA Walker, [1858]. See Desmophova Stephens, 1829.

$\operatorname{ACON}$

TIMAEA Saalmüller, I89I. See Compsotata Prout, 1907.

CUCU

TIMANDRA Duponchel, I829, in Godart \& Duponchel, Hist. nat. Lépid. Papillons Fr. 7 (2): IO5, 224 .

GEOMETRIDAE

Type-species: Phalaena amataria Linnaeus sensu Hübner [a misidentification now known as Timandra griseata Petersen, I902], by original designation.

$\ddagger$ ERASTRI A Hübner, [I806], Tentamen determinationis digestionis . . . : [2].

Included in a work rejected for nomenclatural purposes by Int. Commn zool. Nom., 1926, Smithson. misc. Collns 73 (4) Opinion 97: 19. Also idem, 1954, Opin. Decl. int. Commn zool. Nom. 6 Opinion 278: I 40.

Only included species: Phalaena amataria Linnaeus sensu Hübner.

TIMORA Walker, I856, List Specimens lepid. Insects Colln Br. Mus. 9: 69, I32.

NOCT

Type-species: Nonagria senegalensis Guenée, 1852, in Boisduval \& Guenée, Hist. nat. Insectes (Lépid.) 5: r ro, by monotypy. Lectotype + , SENEGal (MNHN, Paris), designated by Viette, I951, Bull. mens. Soc.linn. Lyon 20: 160.

TIMORODES Meyrick, 1902, Trans. ent. Soc. Lond. 35: 46.

SARR

Type-species: Timorodes blepharias Meyrick, I902, ibidem 35: 46, by monotypy. Holotype ơ, New Guinea (BMNH).

TINEOCEPHALA Dyar, I914, Proc. U.S. natn. Mus. 47: 220.

HYPE

Type-species: Tineocephala judis Dyar, I9I 4, ibidem 47: 22I, by original designation. Syntypes 4 ex., Panama: La Chorrera (USNM, Washington).

TINNODOA nom. n. for Odontina Guenée, I 862.

OPH I

Type-species: Odontina excavata Guenée, I862, in Maillard, Notes Ille Réunion 2:

G43, pl. 22, fig. ro, by monotypy (of Odontina Guenée). Syntypes ô, ㅇ, Réunion I. (BMNH).

ODONTINA Guenée, I862, in Maillard, Notes Ile Réunion 2: $\mathrm{G}_{4} 2$.

Type-species: Odontina excavata Guenée, r862, by monotypy.

A : unior homonym of Odontina Zborzewski, i 834, Nouv. Mém. Soc. imp. Nat. Mosc. 3 3ro, - Mollusca. The objective replacement name is Tinnodoa nom. n.

TINOLIUS Waller, I855, List Specimens lepid. Insects Colln Br. Mus. 3: 587, 62 I. OPHI

Type-species: Tinolius eburneigutta Walker, 1855, ibidem 3: 621, by monotypy. Syntypes I $\vec{\jmath}$, I + , Ceylon (BMNH). [India]: Malabar Coast. [BAngladesh]: [E. Bengal], Silhet. EAST Indies. 
TINOSOMA Hampson, 1894, Fanna Br. India (Moths) 2: xviii, 426.

Type-species: Tinosoma hyperythrum Hampson, 1894, ibidem 2: 426, fig. 24I, by original designation. Syntypes $\hat{\sigma}$, Ixdi.: Naga Hills. Andamas., Borneo (BMNH). T. hyperythrum is a junior subjective synonym of Gadirtha semifervens Walker [I863] I86 4, J. Proc. Linn. Soc. (Zool.) 7: 163.

See also Erizada Walker, 1865, List . . 32: 506; and Rhizana Walker, I865, List . . 33: 7 I 9.

TIPASA Walker, I863, List Specimens lepid. Insects Colln Br. Mus. 27: I 29.

OPH I

Type-species: Tipasa nebulosella Walker, I863, ibidem 27: I 29, by monotypy. Type(s) , BorNeo: Sarawak (BMNH).

TIPASODES Hampson, I 926, Descr. nei" Genera Species Lepid. Phalaenae Subfamily' Noctuinae Br. Mus.: 474 .

OPHI

Type-species: Tipasodes melalepidia Hampson, 1926, ibidem: 474, by original designation. Holotype ?, New Gune.1: [Papua], R. Nambare, Biagi (BMNH).

TIPRA Walker, i869, Characters undescr. Lepid. Heterocera: Io8.

$\mathrm{OPHI}$

Type-species: Tipra latipes Walker, I869, ibidem: I09, by monotypy. Type(s) ô. GUIANA.

TIRACOLA Mloore, i 88I, Proc. zool. Soc. Lond. 1881: 35 I.

HADE

Type-species: Agrotis plagiata Walker, 1857, List Specimens lepid. Insects Colln Br.

Mus. 11: $7 \nmid 0$, by original designation. Holotype ô, CEYLON (BMNH).

TIRIDATA Willker, i 865, List Specimens lepid. Insects Colln Br. Mus. 33: 87o.

OPHI

Type-species: Tirialata colligata Walker, I 865 , ibidem 33: 870 , by monotypy. Holotype ô, CEYLLON (BMNH).

T. colligata is a junior subjective synonym of Gonitis sabulifera Guenée, I852, in Boisdual \& Guenée, Hist. nat. Insectes (Lépid.) 6: 404, pl. I I, fig. 6.

TIRUVACA Swinhoe, Igor, Ann. Wag. nat. Hist. (7) $7: 497$.

OPHI

Type-species: Thermesia subcostalis Walker, I865, List Specimens lepid. Insects Colln Br. Mus. 33: I 059, by original designation. Holotype q, [INDIA]: Hindostan [probably an error for East Inclies] (BMNH).

TISAGRONIA Kiöhler, I967, Acta zool. lilloana 21: 278.

NOCT

Type-species: Tisagronia fleissiana liöhler, 1967, ibidem 21: 279, fig. I25, by original designation. Holotype, Argentrna: Rio Negro, Paso Flores.

TITULCIA Walker, r864, List Specimens lepid. Insects Colln Br. Mus. 29: 8 Io.

CHLO

Type-species: Titulcia eximia Walker, I 864, ibidem 29: 8 I I, by subsequent designation

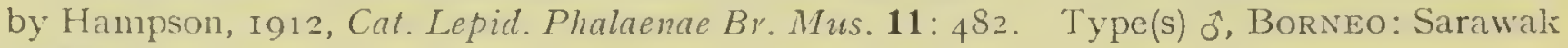
(BMNH).

TMETOLOPHOTA Hampson, 1905, Cat. Lepid. Phalaenue Br. Mus. 5: xiv, 47o. HaDe

Type-species: Leucania propria Walker, I856, List Specimens lepid. Insects Colln $\mathrm{Br}$. Mus. 9: I 1, by original designation. Holotype ô, NEW ZEALAND (BMNH).

TOANA Walker, 1865, List Specimens lepid. Insects Colln Br. Mus. 32: 500.

$\Lambda$ CON

Type-species: Toana semiochrealis Walker, 1865, ibidem 32: 50I, by monotypy. Lectotype ô, Borneo: Sarawak (UM, Oxford), designated (as type) by Sivinhoe, ig0o, Cat. east. and Aust. Lepid. Heterocera 2: 62.

† GOAN.1; Eecke, 1925, in Strand, Lepid. Cat. 32:67.

An incorrect subsequent spelling

TOANODES Warren, I913, in Seitz, Gross-Schmett. Frde 11: 234.

$\triangle \mathrm{CON}$

Type-species: Toana rotundipennis Hampsun, i9ı, Cat. Lepid. Phalaenae Br. Mus. 10: 207 , fig. 48, by original designation. Holotype ${ }^{-}$. New Guinea: [Papua], Woodlark I. (BMNH) 
The date of publication of the part of Seitz containing page 234 was stated on page 233 as r9r3 August 2oth in both the English and the German editions.

TOANOPSIS Prout, 1926, Sarawak Mus. J. 3: 219.

ACON

Type-species: Toanopsis engenes Prout, 1926, ibidem 3: 220, pl. 8, fig. If, by original designation. Holotype 0 , [Borneo]: Sarawak, Mt Penrissen $3500 \mathrm{ft}$ (BMINH).

TOCHARA Moore, 1882, in Hewitson \& Moore, Descr. new Indian Lepid. Insects Colln late MrW. S. Atkinson: 175 .

OPHI

Type-species: Tochara obliqua Moore, I882, ibidem: 175, pl. 6, fig. 27, by monotypy. Syntypes $\overrightarrow{0}, q$, [INDrA]: [Assam], Khasia Hills and Cherra.

T. obliqua is a junior subjective synonym of Thermesia creberrima Walker, I858, List Specimens lepid. Insects Colln Br. Mus. 15: I 574 .

TOLNA Walker, 1869, in Chapman, Proc. nat. Hist. Soc. Glasgow 1: 355.

CATO

Type-species: Tolna versicolor Walker, I869, ibidem 1:355, pl. 7, fig. Io, by monotypy. Type(s) ð, Congo.

TOLNAODES Hampson, 19г3, Cat. Lepid. Phalaenae Br. Mus. 12: 8. Nomenclaturally available, but without included species until Hampson, 1913, ibidem 13: x, 252. CATO

Type-species: Achaea dasynota Felder \& Rogenhofer, 1875, Reise öst. Fregatte Novara (Zool.) 2 (Abt. 2): pl. II2, fig. Io, by subsequent monotypy. Type(s), French GUIANA (BMNH).

TOLNAOPSIS Hampson, I926, Descr. new Genera Species Lepid. Phalaenae Subfamily Noctuinae Br. Mus.: 95. OPHI

Type-species: Tolnaopsis eusciasta Hampson, 1926, ibidem: 96, by original designation. Holotype , Congo: upper (BMNH).

TOLNOSPHINGIA Berio, 1964, Boll. Soc. ent. ital. $94: 88$.

CATO

Type-species: Tolna bolengensis Holland, I920, Bull. Am. Mus. nat. Hist. 43: 280, pl. $x_{3}$, fig. 9 , by original designation. Holotype ơ, [ZAIRE $]$ Congo: Bolengi o $5^{\prime}$ S. I $8^{\circ} 10^{\prime} \mathrm{E}$. (AMNH, New York).

TOLPIA Walker, 1863, List Specimens lepid. Insects Colln Br. Mus. 28: 449.

OPHI Type-species: Tolpia conscitulana Valker, r863, ibidem 28: 45o, by monotypy. Type(s) \%, BORnEo: Sarawak (BMNH).

TOLPIODES Hampson, 1926, Descr. new Genera Species Lepid. Phalaenae Subfamily' Noctuinae Br. Mus.: I 92 .

OPHI

Type-species: Narosodes fasciata Rothschild, 1913, Novit. zool. 20: 224, by original designation. Type(s) ô. New Gurnea: [Papua], Milne Bay (BMNH).

$N$. fasciata was originally proposed as $\$$ Narasodes, an incorrect subsequent spelling.

TOMYRIS Druce, I 890 . See Rowdenia nom. n.

HYPE

TOPADESA Moore, I888, in Hewitson \& Moore, Descr. new Indian lepid. Insects Colln late Mr W.S. Atkinson: 280. CHLO

Type- species: Topadesa sanguinea Moore, r 888 , ibidem: 280 , by monotypy. Syntypes o, India: [W. Bengal], Darjiling (BMNH).

†TOPIDOSA; Pagenstecher, 1909, Geogr. Verbreitung Schmett.: 422.

An incorrect subsequent spelling.

† TOPIDOSA, misspelling. See Topadesa Moore, r 888 .

CHLO

TORNACONTIA Smith, I9oo, Trans. Am. ent. Soc. 27:49, 55.

$\mathrm{ACON}$

Type-species: Tarache sutrix Grote, I 880, Can. Ent. 12: I 54, by subsequent designation by Hampson, rgro, Cat. Lepid. Phalaenae Br. IIus. 10: 675. Syntypes 2 ex., [U.S.A.]: Colorado (BMNH). 
TORNOCONIA Berio, I966, Annali Mus. civ. Stor. nat. Giacomo Doria 76: 124.

CHLO

Type-species: Tornoconia royi Berio, 1966, ibidem 76: 125, fig. I4, by original designation. Holotype ô. Senegal: Parc National du Niokolo Kioba, Badi (MINHN, Paris).

TORNOSINUS Bethune-Baker, 1906, Novit. zool. 13: 286.

HYPE

Type-species: Tornosinus niger Bethune-Baker, 1906, ibidem 13: 286 , by original designation. Type(s) $\hat{o}$, NEw Guinea: [Papua], Aroa River (BMNH).

TORONE Walker, I863, List Specimens lepid. Insects Colln Br. Mus. 27: 5. HyblaEIdaE Type-species: Torone hyblaeoides Walker, 1863, ibidem 27:6, by monotypy. Syntypes 3 우, [BRAzIL]: [R. Amazon], [Tefé] Ega (BMNH).

T. hyblaeoides is a junior subjective synonym of Erythrochrus bicolor Herrich-Schäffer, 1855 .

See also Erythrochrus Herrich-Schäffer, 1855.

TORTRICIFORMA Hampson, I 894, Fauna Br. India (Moths) 2: 408, 425.

CHLO

Type-species: Tortriciforma viridipuncta Hampson, I 894, ibidem 2: 425, fig. 240, by original designation. Type(s), [SIKkim] Sikhim (BMNH).

TORTRICODES Guenée, 1854. See Alberticodes Biezanko \& Ruffinelli, I963.

HYPE

TORTRIX Linpaeus, 1758, Syst. Nat. (Edn 1o) 1: 530.

TORTRICIDAE

Type-species: Phalaena viridana Linnaeus, I758, ibidem 1: 530, by subsequent designation by Int. Commn zool. Nom., 1957, Opin. Decl. int. Commn zool. Nom. 15 Opinion $450: 254$.

Tortrix was originally proposed as a subgenus of Phalaena Linnaeus, 1758 , and has been used as the original generic name for species which are now in the Noctuidae.

TOSACANTHA Fletcher, 1961, Ruwenzori Exped. 19521 1: 250.

HYPE

Type-species: Tosacantha atmocyma Fletcher, 196r, ibidem 1:250, figs 81, i16, 304, 307 \& 308 , by original designation. Holotype ô. UGanda: Ruwenzori, Kilembe (BMNH).

TOXOCAMPA Guenée, I841, Amnls Soc. ent. Fr. 10: 75.

OPHI

Type-species: Ophiusa limosa Treitschke, i 826, Schmett. Eur. 5 (3):298, by subsequent designation by Desmarest, I 848, in d'Orbigny, Dict. univl Hist. nat. 12: 623. Syntypes, [Hungary]: Ofen Mts (TM, Budapest).

O. limosa is a junior subjective synonym of Noctua procax Hübner, [1813].

See also Asticta Hïbner, [1823].

TOXOLOMA Felder, 187ł, in Felder \& Rogenhofer, Reise öst. Fregatte Novara (Zool.) 2 (Abt. 2) : pl. Ioo, fig. I6; Erklärung: 9. NOLI

Type-species: Toxoloma australe Felder, 1874, ibidem 2 (Abt. 2): pl. 1oo, fig. I6; Erklärung: 9, by monotypy. Type(s), Australia.

$T$. australe is a junior subjective synonym of Uraba lugens Walker, 1863.

See also Lraba Walker, I863; and Cioesa Wallser, I866.

TOXONPRUCHA Möschler, r 890, Abh. senckenb. naturforsch. Ges. 16: 198.

Type-species: Toxonprucha amoena Möschler, I890, ibidem 16: I98, fig. I, by monotypy. Syntypes ô. o, Puerto Rico (MNHU, Berlin).

TOXOPHLEPS Hampson, I893, Illust. typical Specimens Lepid. Heterocera Colln Br. Mus. 9: $3 \mathrm{I}, \mathrm{I} 24$. ACON

Type-species: Tarache optiva Swinhoe, I890, Trans. ent. Soc. Lond. 1890: 224, by original clesignation. Syntypes, Burma: Rangoon (BMNH).

T. optiva is a junior subjective synonym of Cophanta funestalis Walker, 1864.

See also Cophanta Walker, 1864.

†TRACHAEA, misspelling. See Trachea Ochsenheimer, I8r6.

ACRO 
TRACHEA Ochsenheimer, i 816, Schmett. Eur. 4: 75.

ACRO

Type-species: Phalaena atriplicis Linnaeus, I758, Syst. Nat. (Edn I0) 1: 517, by subsequent designation by Curtis, I 832, Br. Ent. 9: 431. Type(s), Type-Locality not stated (LS, London) [EUROPE].

$\ddagger$ ACHATIA Hübner, [I806], Tentamen determinationis digestionis... . [I].

Included in a work rejected for nomenclatural purposes by Int. Commn zool. Nom., I926, Smithson. misc. Collns 73 (4) Opinion 97: 19. Also idem, 1954, Opin. Decl. int. Commn zool. Nom. 6 Opinion 278: 140.

Only included species: Phalaena atriplicis Linnaeus, 1758.

ACHATIS Billberg, I820, Enumeratio Insect. Mus. G. J. Billberg: 87.

Type-species: Phalaena atriplicis Linnaeus, 1758 , by subsequent designation by Tams, 1939, Entomologist 72: г36.

Achatis is a junior objective synonym of Trachea Ochsenheimer, I8I6.

$\ddagger T R A C H A E A$; Schaus, I923, Zoologica, N.Y. 5 (2): 33.

An incorrect subsequent spelling.

TRACHEOIDES Prout, I926, Entomologist 59: 64.

ACRO

Type-species: Tracheoides tamsi Prout, I926, ibidem 59: 65, by original designation. Syntypes 26 ô, 49 ㅇ. [Moluccas]: Buru, Gamoe 'Mrapat (BMNH).

TRACHEPLEXIA Janse, I937, Moths S. Afr. 3: 7 I.

ACRO

Type-species: Euplexia amaranta Felder \& Rogenhofer, 1875, Reise öst. Fregatte Novara (Zool.) 2 (Abt. 2): pl. I Io, fig. 8, by original designation. Type(s) ㅇ, [South Africa]: Natalia, Durban.

TRACHODOPALPUS Blanchard, 1852, in Gay, Historia de Chile (Zool.) 7 : 72.

HYPE

Type-species: Trachodopalpus cinereus Blanchard, I852, ibidem 7: 72, pl. 7, fig. I by monotypy. Syntypes, Chile: Coquimbo (MNHN, Paris).

TRACHYSMATIS Schaus, I9I6, Proc. U.S. natn. Mus. 50: 260, 274.

HYPE

Type-species: Trachysmatis ignobilis Schaus, I9I6, ibidem 50: 274, by original designation. Type(s) ô. Panama: R. Trinidad (USNM, Washington).

TRACTA Saalmüller, I89I, Lepid. Madagascar: 479.

OPH I

Type-species: Capnodes albooculata Saalmüller, I88o, Ber. senckenb. naturf. Ges. 1879-1880: 286, by monotypy. Type(s), MADAGascar (SNG, Frankfurt).

TRAMA Harvey, I875. See Lepidotrama Cockerell, 1903.

OPHI

TRANOSES Schaus, I913, Ann. Mag. nat. Hist. (8) 11:4.

ACRO

Type-species: Tranoses hirtipuncta Schaus, I9I3, ibidem (8) 11: 4, by original designation. Type(s) ô, Costa RrcA: Juan Viñas.

TRAPEZOPTERA Hulstaert, 1924, Annls Soc. ent. Belg. 64: 95.

OPHI

Type-species: Trapezoptera lobata Hulstaert, I924, ibidem 64: 95, by original designation. Holotype ơ, [Moluccas]: [Kai 1s], Grande Kei, Har.

TRAUAXA Walker, [1866] i 865, List Specimens lepid. Insects Colln Br. Mus. 34: I2 I6. HyPE Type-species: Trauaxa obliqualis Walker, [1866] I865, ibidem 34: I 216, by monotypy. Holotype đ., [BraziL]: [R. Amazon], [T’efé] Ega (BMNH).

TRIAENA Hübner, I818, Zuträge Samml. exot. Schmett. 1: 21.

ACRO

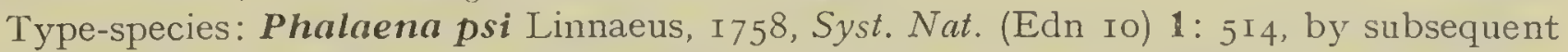
designation by Grote, I873, Bull. Buffalo Soc. nat. Sci. 1: 78. Type(s), Type-Locality not stated (LS, London) [EUROPE].

$\ddagger$ APATELE Hübner, I808, Erste Zuträge Samml. exot. Schmett.: 5 .

Included in a work rejected for nomenclatural purposes by Int. Commn zool. Nom., 1966, Bull. zool. Nom. 23 Opinion 789:213. Placed on the Official Index of Rejected and Invalid Generic Names in Zoology: Name No. I 827. 
Only included species: † Apatele tritona Hübner, I808, later made nomenclaturally available as Triaena tritona Hübner, i8 8 8, Zutvïge Samml. exot. Schmett. 1: figs Io7, Io8. T. tritona is congeneric with Phalaena psi Linnaeus, 1758 , the type-species of Triaena Hübner, I818.

SEMAPHORA Guenée, i 84I, Annls Soc. ent. Fr. $10: 235$.

Type-species: Phalaena psi Linnaeus, I758, by PRESENT DESIGNATION.

Semaphora is a junior objective synonym of Triaena Hübner, I818.

SEMATOPHORA Agassiz, i846, Nomencl. zool. Index univl.: 338.

An unjustified emendation of Semaphora Guenée, I84I.

CUSPIDIA Chapman, I890, Entomologist's Rec. J. Var. 1:27.

Type-species: Phalaena psi Linnaeus, I 758, by subsequent designation by Butler, i 89 I, Entomologist 24: II I.

Cuspidia is a junior objective synonym of Triaena Hübner, I8I8.

${ }_{+}^{+}$TRIBONOPHORA Hübner, [1806]. See Cucullia Schrank, I802.

CUCU

$\ddagger$ TRIBONOPHORA Hübner, I808. See Paectes Hübner, I8I8.

EUTE

†TRIBONOPHORAE Ochsenheimer, i8I6. See Cucullia Schrank, i80z.

CUCU

TRIBUNOPHORA Hübner, I822. See Cucullia Schrank, 1802.

CUCU

TRIBUNTA Walker, [1866] i 865, List Specimens lepid. Insects Colln Br. Mus. 34: I506. NolI

Type-species: Tribunta biguttalis Walker, [1866] i 865, ilidem 34: 1507, by subsequent designation by Kirby, 1892, Synonymic Cat. Lepid. Heterocera 1: 376 . Holotype $\delta$. Australia (BMNH).

TRICHAGROTIS McDunnough [1929] 1928, Bull. Dep. Mines, Can. 55: 60.

HADE

Type-species: Trichorthosia spinosa Barnes \& McDunnough, I912, Contr. wat. Hist. Lepid. N.Am.1(5): 9, pl. I, fig. I8, by original designation. Syntypes 2 f, [CT.S.A.]: Arizona, Palmerlee.

TRICHANARTA Hampson, I 896, Fauna Br. India (Noths) 4: xxv, 507.

Type-species: Anarta ladakensis Felder \& Rogenhofer, 1875, Reise öst. Fregatte Novara (Zool.) 2 (Abt. 2): pl. 108, fig. 38, by original designation. Type(s) 9 , [INDIA]: Himalaya.

ALA Staudinger, r 882, Stettin. ent. Ztg 43: 49.

Type-species: Ala picteti Staudinger, i 882, ibidem 43: 49 , by monotypy. Syntypes $\hat{j}, q$, [U.S.S.R.]: [Tadzhikistan], [Alai Tagh] Ala Tau (MINHU, Berlin).

A junior homonym of Ala Lockington, i877, Proc. Calif. Acad. Sci. 7: 65,-Crustacea. There is no objective replacement name but $A$. picteti is congeneric with Anarta ladakensis Felder \& Rogenhofer, 1874, the type-species of Trichanarta Hampson, I896. The latter should therefore be used as the subjective replacement name.

TRICHANUA Berio, 1954, Amnali Mus. civ. Stor. nat. Giacomo Doria 66: 337.

CATO

Type-species: Anua mimetica Berio, 1954, ibidem 66: 337, by monotypy. Holotype o. [Zarre]: Sankuru, Dimbelenge (MRAC, Tervuren).

Trichamua was proposed as a subgenus of Anua IValker, I 858.

$\ddagger$ TRICHCLEA, misspelling. See Trichoclea Grote, 1883.

HADE

TRICHESTRA Hampson, I9n5, Cat. Lepid. Phalaenae Br. Mus. 5: vii, 8.

HADE

Type-species: Celaena arbuticolens Butler, 1882, Trans. ent. Soc. Loncl. 1882: I 2 I by original designation. Syntypes, CHILE: Las Zorras (BIINH).

TRICHEUROIS Hampson, I905, Ann. Mag. nat. Hist. (7) 15: 45I.

NOC'T

Type-species: Mamestra nigrocuprea Monre, 1867, Proc. zool. Soc. I.nnd. 1867: 52, by original designation. Syntypes $\hat{\sigma}$, INDIA: Bengal (BMNH). 
TRICHOBATHRA Hampson, 1926, Descr. new Genera Species Lepid. Phalaenae Subfamily Noctuinae Br. Mus.: 434 .

Type-species: Trichobathra triplogramma Hampson, 1926, ibidem: 435, by original designation. Holotype $\widehat{\sigma}$, [GHANA] Gold Coast: Kumasi (BMNH).

TRICHOBLEMMA Hampson, I926, Descr. new Genera Species Lepid. Phalaenae Subfamily Noctuinae Br. Mus.: 473.

$\mathrm{OPHI}$

Type-species: Capnodes lophophora Hampson, I895, Fauna Br. India (Moths) 3: 22, by original designation. Type(s) ふ઼, Burma: Toungngoo (BMNH).

TRICHOCALA Dyar, I902. See Kallitrichia Ottolengui, I898.

ACRO

TRICHOCERAPODA Benjamin, 1932, Bull. S. Calif. Acad.Sci. 31: 27.

CUCU

Type-species: Trichocerapoda comstocki Benjamin, 1932, ibidem 31: 28, by original designation. Holotype ô. [U.S.A.]: Calif., Coahuilla Valley, Snow Creek (USNM, Washington).

TRICHOCLEA Grote, I883, Papilio 3: 30.

HADE

Type-species: Trichoclea decepta Grote, I883, ibidem 3: 30, by monotypy. Syntypes $3(\hat{0}$, O), [U.S.A.]: Arizona.

$\ddagger$ TRICHCLEA; Smith, 1903, Trans. Am. ent. Soc. 29: 198.

An incorrect subsequent spelling.

TRICHOCOSMIA Grote, I883, Can. Ent. 15: 6.

ACRO

Type-species: Trichocosmia inornata Grote, I883, ibidem 15: 6, by monotypy. Syntypes $6(\widehat{0}$, $)$, [U.S.A.]: Arizona.

TRICHOFELTIA McDunnough [1929] I928, Bull. Dep. Mines, Can. 55: 61.

HADE

Type-species: Agrotis circumdata Grote, 1883, Ann. Mag. nat. Hist. (5) 11: 53, by original designation. Holotype, U.S.A.: New Mexico.

TRICHOGATHA Warren, I9I3, in Seitz, Gross-Schmett. Erde 11: 258.

ACON

Type-species: Oruza variegata Bethune-Baker, 1906, Novit. zool. 13: 215, by original designation. Holotype $\widehat{\sigma}$, [PAPUA] Br. New Guinea: Dinawa (BMNH).

The date of publication of the part of Seitz containing page 258 was stated on page 257 as 1913 October 5 th in both the German and English editions.

TRICHOLITA Grote, I875 January, Bull. Buffalo Soc. nat. Sci. 2: 2 I 5.

HADE

Type-species: Hydroecia semiaperta Morrison, 1874, Can. Ent. 6: 105, by original designation. Syntypes, [U.S.A.]: Mass., and New York.

H. semiaperta is a junior subjective synonym of Nephelodes signata Walker, I860, Can. Nat. \& Geol. 5 : 253.

Neave, 1940, Nomencl. zool. 4: 543, cited Tricholita Grote, 1875, Stettin. ent. Ztg 36: 341, but the part containing this page was dated July-September 1875 and is not the original description.

TRICHOLONCHE Grote, I896, Mitt. Roemermus. Hildesh. 3: 6, I6.

ACRO

Type-species: Acronycta afflicta Grote, i864, Proc. ent. Soc. Philad. 2: 438, pl. 9, fig. 4, by original designation. Type(s) ठ̃. [U.S.A.]: Texas.

TRICHOPALPINA Hampson, I926, Descr. new Genera Species Lepid. Phalaenae Subfamily Noctuinae Br. Mrts.: 232. OPHI

Type-species: Elyra gabunalis Holland sensu Hampson, 1926, ibidem: 232 [a misidentification later described as Trichopalpina simplex Berio, 1956, Memorie Soc. ent. ital. 35: 28], by original designation. Holotype $\vec{\sigma}$, of $T$. simplex, [ZAIRE] Congo Belge: Sankuru, Katako-Kombe (MRAC, Tervuren).

From the generic description of Trichopalpina and from the specimens in BMNH named by Hampson, which I have compared with colour transparencies of the type of Elyra gabunalis Holland, 1894, Psyche, Camb. 7: 126, pl. 4, fig. 17 , it is certain that gabunalis 
Holland sensu Hampson, 1926, was a misidentification of what was at that time a new species, later described as $T$. simplex Berio, 1956. This is a case of a misidentified typespecies which under the Code, Article 70 (a), should be referred to the Commission.

TRICHOPHOTIA Hampson, I9I3, Ann. Mag. nat. Hist. (8) 12: 586.

NOCT

Type-species: Trichophotia homogenea Hampson, I9I3, ibidem (8) 12:586, by original designation. Holotype ô, P'ERU: Agualani (BMNH).

TRICHOPLEXIA Hampson, 19o8, Cat. Lepid. Phalaenae Br. Mus. 7: xiii, 482.

ACRO

Type-species: Hadena exornata Möschler, i860, Wien. ent. Monatschr. 4: 364, pl. 9, fig. 5, by original designation. Syntypes ơ, $q,[\mathrm{CANADA}]$ : Labrador.

TRICHOPLUSIA IICDunnough, I944, Mem. sth. Calif. Acad. Sci. 2: 204.

PLUS

Type-species: Plusia brassicae Riley, 1870, Rep. noxious beneficial \& other Insects,

Missouri 2: IIo, fig. 81, by original designation. Syntypes ô. ㅇ, U.S.A.: Missouri state.

$P$. brassicae is a junior subjective synonym of Noctua ni Hübner, [1803], Samml. eur. Schmett. 4: pl. 58, fig. 284 .

TRICHOPOLIA Grote, I 883, Papilio 3: 76.

HADE

Type-species: Trichopolia dentatella Grote, I883, ibidem 3:77, by monotypy. Type(s) o, [U.S.A.]: Arizona.

EUPOLIA Smith, I894, Trans. Am. ent. Soc. $21: 69$.

Type-species: Eupolia licentiosa Smith, 1894, ibidem 21:70, pl. 3, fig. 8, by monotypy. Syntypes I $\hat{0}$, I $q$, [U.S.A.]: Utah.

A junior homonym of Eupolia Hubrecht, I887, Rep. Challenger Exped. (Zool.) 19 (54): Io, - Vermes. There is no objective replacement name but E. licentiosa is a junior subjective synonym of Trichopolia dentatella Grote, I 883 , the type-species of Trichopolia Grote, I 883. The latter is thus available for use as a subjective replacement name.

TRICHOPOLYDESMA Berio, 1954, Doriana 1 (50): 6.

OPHI

Type-species: Coenipeta collutrix Geyer, 1837, in Hübner, Zuträge Samml. exot.

Schmett. 5: 22, tigs 885, 886, by original designation. Neotype + , South AFrica: Natal, Durban (BMNH), designated by Berio, 1971, Annali Mus. civ. Stor. nat. Giacomo Doria 78: 291 .

C. collutrix is a junior subjective synonym of Polydesma umbricola Boiscluval, I 833 .

See also Polydesma Boisduval, i 833 .

TRICHOPTYA Warren, I912, Novit. zool. 19: 53.

$\mathrm{OPHI}$

Type-species: Leucania sejuncta Walker, I856, List Specimens lepid. Insects Colln Br. Mus. 9: I09, by original designation. Syntypes 20 , [INDIA]: Hindostan (BMNH).

SILLOPHORA Warren, I912, Novit. zool. 19: 54 .

Type-species: Sillophora bimaculata Warren, I912, ibidem 19: 54, by original designation. Holotype of. [Sulawesi] Celebes: N. of Palos Bay, Tawaya (BMNH).

S. bimaculata Warren is a junior subjective synonym of Herminodes bimaculata Snellen, I880, Tijdschr. Ent. 23: 78, pl. 6, fig. 7 .

A junior homonym of Sillophora Warren, 1907, Novit. zool. 14: 159, - Lepid., Geometridae. There is no objective replacement name but $S$. bimaculata Warren is congeneric with Leucania sejuncta Walker, 1856 , the type-species of Trichoptya Warren, I912. The latter is therefore available for use as the subjective replacement name.

TRICHORHIZA Hampson, 1905, Cat. Lepid. Phalaenae Br. Mus. 5: vii, I 3. ACRO Type-species: Rhizogramma peterseni Christoph, I887, in Romanoff, Mém. Lépid. 3: 76 , pl. 4, fig. 3, by original designation. Syntypes ô, q, [U.S.S.R.]: [Turkmenistan] Askhabad (7, Leningrad); Daghestan, Kurusch. [Iran] Persia: Schahrud.

TRICHORIDIA Hampson, igo6, Cat. Lepid. Phalaenae Br. Mus. 6: xii, foo. CUCU

Type-species: Crymodes herchatra Swinhoe, I893, Ann. Mag. nat. Hist. (6) 12: 260, by original designation. Syntypes 2 ot, Sikkim (BMNH). 
TRICHORTHOSIA Grote, I883, Papilio 3: 3I.

Type-species: Trichorthosia parallela Grote, 1883 , ibidem 3: 3 I, by monotypy. Type(s) [U.S.A.]: New Mexico.

Trichorthosia was also proposed as new in Grote, i883, Trans. Kans. Acad. Sci. 8: 38, 50, having the same type-species.

TRICHOSEA Grote, I875, Bull. Buffalo Soc. nat. Sci. 2 : 213.

PANT

Type-species: Phalaena ludifica Linnaeus, I758, Syst. Nat. (Edn Io) 1: 5I4, by monotypy. Type(s), Europe.

TRICHOSELLUS Grote, I89o, Revised Check List N. Am. Noctuidae 1: 36.

NOCT

Type-species: Heliothis cupes Grote, I875, Trans. Am. ent. Soc. 5: I I3, by monotypy. Type(s) ô, [U.S.A.]: Texas (BMNH).

TRICHOSILIA Hampson, I918, Novit zool. 25 : I 2.

NOCT

Type-species: Noctua acarnea Smith, 1905, Jl N.Y. ent. Soc. 13: 194, by original designation. Holotype $\hat{\delta}$, [CANADA $]$ : Alberta, Banff.

TRICHOSPOLAS Draudt, I936, Ent. Rdsch. 53: 470.

HADE

Type-species: Trichospolas arterialis Draudt, I936, ibidem 53: 470, pl. 4 [recte 5], fig. 2e, by monotypy. Syntypes I $\hat{0}$, I (Collection destroyed in World War II).

TRICHOSTERNUM Draudt, I950. See Zutragum nom. n.

CUCU

TRICHOTARACHE Grote, I875, Can. Ent. 7: 48.

$\operatorname{ACON}$

Type-species: Trichotarache assimilis Grote, 1875, ibidem 7: 48, by monotypy. Type(s) ô. [U.S.A.]: California (BMNH).

TRICHYPENA Joannis, I9I 5, Annls Soc. ent. Fr. 84: 6.

HYPE

Type-species: Trichypena quadra Joannis, I9I 5 , ibidem $84: 6$, pl. I, fig. 4, by monotypy. Syntypes $4 \hat{o}$, [Mauritrus]: Curepipe (MNHN, Paris).

TRICOPIS Grote, I874, Bull. Buffalo Soc. nat. Sci. 2: 34, 75.

NOCT

Type-species: Tricopis chrysellus Grote, I 874 , ibidem 2: 76, by original designation. Syntypes ô. ㅇ [U.S.A.]: Texas (BMNH); Kansas (BMNH).

TRICRATERIFRONTIA Berio, I940, Boll. Soc. ent. ital. 72: i64.

ACRO

Type-species: Tricraterifrontia xanthiata Berio, I940, ibidem 72: I65, fig., by original designation. Holotype ô, [Eтhiopia]: Eritrea, Adi-Abuna, Adua (Colln E. Berio, Genoa).

TRIDENTIFRONS Warren, I9I2, Novit. zool. 19: 45.

ACRO

Type-species: Tridentifrons insularis Warren, I9I 2 , ibidem 19:46, by original designation. Syntypes 2 ㅇ, JAVA: Palabuan (BMNH).

TRIDEPIA McDunnough, I937, Can. Ent. 69:4I.

$\mathrm{HADE}$

Type-species: Trichoclea nova Smith, I903, Trans. Am. ent. Soc. 29: 198, by original designation. Syntypes 6 ex., [U.S.A.]: Arizona, Tucson and Phoenix; New Mexico, Mesilla Park.

$T$. nova was described by Smith in $\ddagger$ Trichclea, an incorrect subsequent spelling.

TRIGONIA Guenée, I854. See Claterna Walker, I 858.

OPHI

TRIGONIS TIS Meyrick, I902, Trans. ent. Soc. Lond. 35: 39.

HYPE

Type-species: Trigonistis demonias Meyrick, I902, ibidem 35: 39, by original designation. Holotype $\delta$, Australia: Qd, Brisbane (BMNH).

TRIGONOCHROSTIA Hampson, I926, Descr. new Genera Species Lepid. Phalaenae Subfamily Noctuinae Br. Mus.: 198.

Type-species: Trigonochrostia excisa Hampson, 1926, ibidem: I98, by original designation. Holotype +, BORNEO: Pulo Laut (BMNH). 
TRIGONODES Guenée, I 852, in Boisduval \& Guenée, Hist. nat. Insectes (Lépid.) 7: 28I. CATo

Type-species: Phalaena hyppasia Cramer, I779, Uitlandsche Liapellen 3: 99, pl. 250, fig. E, by subsequent designation by Moore, [1885] i887, Lepid. Ceylon 3: I86. Type(s), [INDIA]: Coromandel Coast.

$\ddagger_{\ddagger}$ TRIGONODESMA Hampson, no published reference found.

OPH I

TRIGONODESMA Wileman \& South, I92 I, Entomologist 54: 203. OPHI

Type-species: Trigonodesma bimacula Wileman \& South, I921, ibidem 54: 203, by monotypy. Holotype , Philippines: Luzon I., Benguet, Baguio.

Wileman \& South were not intending to propose a new genus but were using one of Hampson's unpublished generic names in the BMNH collection.

TRIGONOPHORA Hübner, [I82I] I816, Verz. bekannter Schmett.: 2 I 7.

CUCU

Type-species: Noctua empyrea Hübner, [1803], Samml. eur. Schmett. 4: pl. I3, fig. 63, by subsequent designation by Grote, I874, Bull. Buffalo Soc. nat. Sci. 2: I7. Type(s), EUROPE.

N. empyrea is a junior subjective synonym of Bombyx flammea Esper, [1 785].

See also Rhizotype Hampson, 1906.

${ }_{+}^{+}$TRILENCA, misspelling. See Schinia Hübner, I818.

TRILEUCA Grote, I883. See Schinia Hübner, I818.

TRILOPHIA Turner, 1943, Mem. Qd Mus. 12: i 2.

ACRO

Type-species: Trilophia niphadospila Turner, I943, ibidem 12: I I2, by monotypy. Holotype $q$, Australia: Qd, Rivertree near Stanthorpe.

TRILOPHONOTA Hampson, I 898, J. Bombay nat. Hist. Soc. 11: 700.

HY'PE

Type-species: Trilophonota caerulilineata Hampson, I898, ibidem 11:700, fig., by original designation. Type(s) ô, India: Assam, Khasis (BMNH).

TRINGILBURRA Lucas, I90i, Proc. R. Soc. Od 16: 8 I.

Type-species: Tringilburra lugens Lucas, I90I, ibidem 16:8 1 , by monotypy. Type(s) O. [Australia]: Qd, Bellenden Ker (SAM, Adelaide).

TRIOCNEMIS Grote, I88I, Papilio 1: 77.

ACRO

Type-species: Triocnemis saporis Grote, $188 \mathrm{I}$, ibidem 1: 77, by monotypy. Type(s) o., [U.S.A.]: Washington Ter. (BMNH).

TRIOMMATODES Warren, I889, Trans, ent. Soc. Lond. 1889: 25 I.

OPHI

Type-species: Triommatodes plumosa Warren, I889, ibidem 1889: 252, by monotypy. Holotype ô. [Brazil]: Amazon basin, R. Purus, Gepatiny (BMNH).

TRIORBIS Hampson, I 894, Fauna Br. India (Moths) 2: xvi, 366.

Type-species: Hyperaeschra annulata Swinhoe, I890, Trans. ent. Soc. Lond. 1890: I 89, pl. 6, fig. I 5, by original designation. Type(s) 오․ Burma: Thyetmyo (BMNH).

TRIPHAENA Ochsenheimer, I8I6. See Noctua Linnaeus, I758.

TRIPHAENOPSIS Butler, I878, Ann. Mag. nat. Hist. (5) 1: 163.

Type-species: Triphaenopsis lucilla Butler, I878, ibidem (5) 1: I63, by original designation. Type(s), JAPAN: Yokohama (BMNH).

TRIPSEUXOA Hampson, I903, Cat. Lepid. Phalaenae Br. Mus. 4: 8, I 39.

Type-species: Tripseuxoa strigata Hampson, 1903, ibidem 4: 139, fig. 51, by original designation. Syntypes I ô, 4 f, Argentina: Goya (BMNH).

TRIPUDIA Grote, 1877, Can. Ent. 9: 69.

$\operatorname{ACON}$

'Type-species: Erastria quadrifera Zeller, I874, Verh.zool.-bot. Ges. Wien 24 (Abh.): 
425, pl. I2, fig. 2, by subsequent designation by Grote, I882, Can. Ent. 14: I96. Holotype $\hat{\sigma}$, Mexico: Mazatlan.

TRISATELES Tams, I939, Entomologist 72: I4I.

HYPE

Type-species: Pyralis emortualis [Denis \& Schiffermüller], I775, Ankündung syst. Werkes Schmett. Wienergegend: I20, by subsequent designation (for Standfussia Spuler) by Tams, I939, ibidem 72: I4I. Type(s), [Austria]: Vienna district (Collection destroyed, Horn \& Kahle, 1936, Ent. Beih. Berl.-Dahlem 3: 243).

Trisateles was proposed as the objective replacement name for Standfussia Spuler, i 907. AETHIA Hübner, [I825] i 8I6, Verz. bekannter Schmett.: 340.

Type-species: Pyralis emortualis [Denis \& Schiffermüller], I 775, by subsequent designation by Westwood, I 840, Synopsis Genera Br. Insects: 105, but cited as 'emortralis Hb.', an incorrect authorship.

A junior homonym of Aethia Merrem, I788, Vers. Grundrisses allg. Gesch. . . Voegel 1 (Tentamen nat. Syst. Avium) : 7, 20,-Aves. The objective replacement name is Trisateles Tams, I939.

SOPHRONIA Duponchel, [1845], Cat. méth. Lépid. Eur. (2): 2 I I.

Type-species: Pyralis emortualis [Denis \& Schiffermüller], I 775, by subsequent designation (for Standfussia Spuler, I907) by Tams, I939, Entomologist 72: I4I.

Invalid designation of type-species: $P$. emortwalis had earlier been cited amongst 'types' by Guenée, 1854, in Boisduval \& Guenée, Hist. nat. Insectes (Lépid.) 8: 3, in a footnote reviewing Herrich-Schäffer, I 845 , Syst. Bearb. Schmett. Eur. 2. Within the same sentence Guenée cited up to three nominal species for each genus. The French word 'types' was being used to mean the same as the English 'examples'.

A junior homonym of Sophronia Hübner, [I825] I8I6, Verz. bekannter. Schmett.: 407, Lepid., Tineidae. The objective replacement name is Trisateles Tams, I939.

ST ANDFUSSIA Spuler, I907, in Hofmann, Schmett. Eur. 1: 327.

Type-species: Pyralis emortualis [Denis \& Schiffermüller], I775, by subsequent designation by Tams, I939, Entomologist 72: I 4 I.

Standfussia was proposed as the objective replacement name for Sophronia Duponchel, [1845].

A junior homonym of Standfussia Tutt, I9oo, Nat. Hist. Br. Lepid. 2: 4I 5, - Lepid., Psychidae. The objective replacement name is Trisateles Tams, I939.

TRISPILA Houlbert, I92I, in Oberthür, Étud. Lépid. compavée 18 (2): 235.

OPHI

Type-species: Thyatira trimaculata Bremer, I86I, Bull. Acad. imp. Sci. St. Petersb. 3 : 483, by original designation. Syntypes, [U.S.S.R.]: Amur, between the Ussuri estuary and the Noor.

THYATIRIDES Kozhanchiliov, I950, Fauna SSSR (Insecta) Lepid. 12: 45I.

Type-species: Thyativa trimaculata Bremer, I86I, by original designation.

Thyatirides is a junior objective synonym of Trispila Houlbert, I92I.

TRISSERNIS Meyrick, I902, Trans. ent. Soc. Lond. 35: 37.

$\mathrm{ACON}$

Type-species: Trissernis prasinoscia Meyrick, I902, ibidem 35: 37, by monotypy.

Holotype $\widehat{o}$ (not o), Australia: Qd, Brisbane (BMNH).

BRYOMIMA Turner, I902, Proc. Lim. Soc. N.S.W. 27: I34.

Type-species: Bryonima elaeodes Turner, I902, ibidem 27: I34, by subsequent designation by Hampson, I9Io, Cat. Lepid. Phalaenae Br. Mus. 10: 3I, but cited as T. prasinoscia Meyrick which Hampson placed (on page 32) as the senior synonym of B. elaeodes. Syntypes, Australia: $2 \hat{0}, 2$ 으, Qd, Brisbane (ANIC, Canberra).

A junior homonym of Bryomima Staudinger, I90o,-Lepid., Noctuidae. There is no objective replacement name but $B$. elaeodes Turner, 1902 August 22nd, is a junior subjective synonym of Trissernis prasinoscia Meyrick, I902 April I4th, the type-species of Trissernis Meyrick, I 902 . The latter is therefore available for use as a subjective replacement name. 
TRISSOPHAES Hübner, [1823] I8I6, Verz. bekannter Schmett.: 264.

Type-species: Phalciena collusoria Cramer, I777, Uitlandsche Kapellen 2: 116, pl. 172, fig. F (but included by Hübner as †'collusaria Cram.', an incorrect subsequent spelling), by subsequent designation by Berio, I966, Annali Mus. civ. Stor. nat. Giacomo Doria 76: 66. Type(s), Surinam.

TRISTYLA Smith, I893, Insect Life, Wash. 5: 332.

ACRO

Type-species: Tristyla alboplagiata Smith, I893, ibidem 5: 332, by monotypy. Syntypes $3(\hat{0}$, of $)$ [U.S.A.]: Death Valley, Argus MIts.

TRISULA Moore, 1858, in Horsfield \& Moore, Cat. lepid. Insects 2: 420.

OPHI

Type-species: Trisula variegata Moore, 1858 , ibidem 2: 420 , pl. I 2a, fig. I, by monotypy. Syntypes $\delta$, ㅇ, INDIA: northern (BMNH); Madras.

TRISULANA Bethune-Baker, I9I I, Ann. Mag. nat. Hist. (8) 8: 534.

Type-species: Trisulana senex Bethune-Baker, I9I I, ibidem (8) 8: 534 , by original designation. Syntypes ô, 오, Angola: N'Dalla Tando (BMNH).

TRISULIPSA Berio, I973, Annali Mus. civ. Stor, nat. Giacomo Doria 79: 160.

PANT

Type-species: Trisulipsa quadrata Berio, 1973, ibidem 79: 160, fig. 52, by monotypy. Holotype ô, Burma: N.E., Kambaiti, $2000 \mathrm{~m}$ (NR, Stockholm).

Trisulipsa when originally proposed contained a second species, but this was doubtfully included and, under the Code, Article 68(c), is not eligible for consideration as type-species.

TRISULOIDES Butler, I88I, Ann. Mag. nat. Hist. (5) 7: 36.

PANT

Type-species: Trisuloides sericea Butler, 188I, ibidem (5) 7: 36, by monotypy. Syntypes, [INDIA]: Darjiling (BMNH); Assam, Shillong (BMNH).

TRISULOPSIS Strand, 1909, Ent. Rdsch. 26: 141.

OPHI

Type-species: Trisula clathrata Grünberg, 1907, Berl. cnt. Z. 52: 72, fig. \&, by monotypy. Type(s) + , [CAMEroun] Süd-Kamerun: J.obomündung (MNHU, Berlin).

TROCTOPTERA Hampson, I893, Illust. typical Specimens Lepid. Heterocera Colln Br. Mus. 9: 20,99 .

$A C O N$

Type-species: Troctoptera erubescens Hampson, I893, ibidem 9: 20, 99, pl. 157, fig. I6, by monotypy. Type(s) ơ, Ceylon: Pundaloya (BMNH).

TROGACONTIA Hampson, I892, Fanna Br. India (Moths) 4: xxvi, 520.

$\triangle \mathrm{CON}$

Type-species: Timandra albifrontata Moore, 1888, in Hewitson \& Moore, Descr. new Indian lepid. Insects Colln late Mr. IT. S. Atkinson: 255, by original designation. Type(s) +, Indis: [W. Bengal], Darjiling (MNHU, Berlin).

TROGATHA Hampson, igio, Cat. Lepid. Phalaenae Br. Mus. 10: xi, 283.

$\mathrm{ACON}$

Type-species: Corgatha poecilota Turner, Igo8, Trans. R. Soc. S. Aust. 32: 66, by original designation. Holotype ô, Australia: N.Q., Kuranda (NMV, Melbourne).

TROGOBLEMMA Hampson, ig1o, Cat. Lepid. Phalaenae Br. Mus. 10: 796.

$\triangle C O N$

Type-species: Sorygaza acutalis Schaus, 1906, Proc. U.S. natn. Mus. 30: I2 I, by original designation. Holotype, BraziL: São Paulo (USNM, Washington).

TROGOBRIGA Hampson, I9I2, Cat. Lepid. Phalaenae Br. Mus. 11: 456, 6 I6.

CIILO

Type-species: Trogobriga albifera Hampson, 1912, ibidem 11: 6I6, fig. 24\%, by original designation. Holotype ${ }^{\circ}$, UGanda: Entebbe (BMNH).

$\ddagger$ TROGOCRASPIA Hampson, no published reference found.

HYPE

TROGOCRASPIS Hampson, I9 I 8, Nonit. zool. 25: 162.

$\operatorname{ACON}$

'Type-species: Trogocraspis durbanica Hampson, 1918, ibidem 25: I62, hy original designation. Holotype ô. [Sourn ArRiCA]: Natal, I)urban (BMNH). 
TROGOGONIA Hampson, 1926, Descr. new Genera Species Lepid. Phalaenae Subfamily Noctuinae Br. Mus.: 523 .

OPHI

Type-species: Focilla abrupta Walker, 1862, Trans. ent. Soc. Lond. (3) 1: 105, by original designation. Type(s) ô, BrazIL: Amazon region (UM, Oxford).

TROGOTORNA Hampson, igio, Cat. Lepid. Phalaenae Br. Mus. 10: 3, 33 I.

ACON

Type-species: Trogotorna persecta Hampson, I9Io, ibidem 10: 33I, fig. 98, by original designation. Syntypes 3 , PARAguay: Sapucay (BMNH).

TROGOXESTIS Hampson, I9I4, Ann. Mag. nat. Hist. (8) 13: 222.

CHLO

Type-species: Eublemma crenularia Bethune-Baker, I9I I, ibidem (8) 8: 520, by original designation. Type(s) $\widehat{\delta},[$ Angol 1$]$ Portuguese W. Africa: Malange (BMNH).

TROPIDTAMBA Hampson, 1926, Descr. new Genera Species Lepid. Phalaenae Subfamily Noctuinae Br. Mus.: 544 .

OPHI

Type-species: Zethes lepraota Hampson, I898, J. Bombay nat. Hist. Soc. 11: 458, by original designation. Syntypes 9 , India : [Assam], Khasis (BMNH). Singapore (BMNH).

TROTHISA Hübner, [I82I] i 816, Verz. bekannter Schmett.: 256.

$\mathrm{ACON}$

Type-species: Noctua paula Hübner, [1809], Samml. eur. Schmett. 4: pl. 96, fig. 452, by subsequent designation by Hampson, I910, Cat. Lepid. Phalaenae Br. Mus. 10: 63, but cited as Pyralis noctualis Hübner, which Hampson placed on page I 39 as the senior synonym of $N$. paula. Type(s) Europe.

$N$. paula is a junior subjective synonym of Pyralis noctualis Hübner, I 796, ibidem 6 : pl. 6 , fig. 38 .

MICRA Guenée, I84I, Annls Soc. ent. Fr. 10: 224.

Type-species: Noctua minuta Hübner, [I8og], Samml. eur. Schmett. 4: pl. 96, fig. 45I, by subsequent designation by Guenée, 1852 , in Boisduval \& Guenée, Hist. nat. Insectes (Lépid.) 6: 242 , but cited as ' $M$. candidana Fab.' which Guenée placed as the senior synonym of N. minuta.

N. minuta is a junior subjective synonym of Pyralis candidana Fabricius, I794, Ent. Syst. $3(2): 245$.

A junior homonym of Micra Zetterstedt, I8 38 , Insecta Lapponica descr.: 631, - Diptera. There is no objective replacement name but $N$. minuta is congeneric with Noctua paula Hübner, [I809], the type-species of Trothisa Hübner, [1821]. The latter is therefore available for use as a subjective replacement name.

TROTOSEMA Butler, I879, Ann. Mag. nat. Hist. (5) 4: 448.

HYPE

Type-species: Trotosema sordidum Butler, I879, ibidem (5) 4: 449, by original designation. Type(s), JaPAN (BMNH).

TRUDESTRA McDunnough, 1937, Can. Ent. 69: 4I.

HADE

Type-species: Mamestra hadeniformis Smith, 1894, Trans. Am. ent. Soc. 21: 55, pl. 5, fig. 4 , by original designation. Syntypes I $\hat{\sigma}, \mathrm{I}$ q. [U.S.A.]: Colorado.

TRYPANA Guenée, I84I. See Melicleptria Hübner, [I823].

NOCT

TRYPHAENA Meigen, I83I. See Noctua Linnaeus, I758.

NOCT

TSCHET WERIKOVIA Bundel, 1966, Ent. Obozr. 45: 2 I 3.

CUCU

Type-species: Tschetwerikovia rjabovi Bundel, I966, ibidem 45: 2 I3, figs I, 2, by original designation. Holotype ô, U.S.S.R.: [Tadzhikistan], Pamir, Khorog (ZI, Leningrad).

TUERTA Walker, I869, in Chapman, Proc. nat. Hist. Soc. Glasgow 1: 346.

AGAR

Type-species: Tuerta chrysochlora Walker, 1869, ibidem 1: $347, \mathrm{pl}$. 6, fig. 7 , by monotypy. Type(s) ơ, Congo (BMNH). 
TUMIDIFRONTIA Hampson, I902, Ann. S. Afr. Mus. 2: 282.

Type-species: Tumidifrontia castaneotincta Hampson, 1902, ibidem 2: 283, by monotypy. Holotype ô., [RHodesia]: Mashonaland, Salisbury (BMNH).

TUNOCARIA Viette, I96ז, Bull. Soc. ent. Fr. 66: 48.

CUCU

Type-species: Tunocaria rubiginosa Viette, $196 \mathrm{I}$, ibidem $66: 48$, by original designation. Holotype ô, Madagascar: N., Montagne d'Ambre, Les Roussettes (MNHN, Paris).

TUNZA Walker, 1863, List Specimens lepid. Insects Colln Br. Muss. 27: 196.

CATO

Type-species: Tunza promptella Walker, I863, ibidem 27: 196, by monotypy. Syntypes 2 o, TYPE-LOCALITY not known (BMINH) [U.S.A.].

T. promptella is a junior subjective synonym of Doryodes spadaria Guenée, I 857.

See also Themma Walker, 1863.

TURACINA Warren, $191 \mathrm{I}$, in Seitz, Gross-Schmett. Evde 3: 248.

Type-species: Bryophila ceratopyga Püngeler, I902, Dt. ent. Z. Iris 15: I49, pl. 5. fig. If, by original designation. Syntypes 2 , [U.S.S.R.]: [Turkmenistan], Merw district (MNHU, Berlin).

The date of publication of the part of Seitz containing page 248 was stated on page 245 as I9I I November 1 oth in both the German and the English editions.

Turacina was attributed to Püngeler by Warren, but no record of its earlier publication has been found.

TURANICA Boursin, 1963, Bull. mens. Soc. linn. Ly'on 32: 299.

CUCU

'Type-species: Polia haeretica Püngeler, I902, Dt. ent. Z. Iris 15: I 53, pl. 5, fig. I2, by original designation. Syntypes $2 \hat{j}$, [U.S.S.R.]: [Turkmenistan] Aschabad (MNHU, Ber!in).

TURBULA Walker, 1869, Characters undescr. Lepid. Heterocera: 26.

ACRO

Type-species: Turbula petruea Walker, 1869 , ibidem: 26, by monotypy. Type(s) $\sigma^{*}$.

TYPE-LOCALITI not stated (BMNH) [U.S.A.].

$T$. petraea is a junior subjective synonym of Catabena iineolata Walker, 1865.

See also Catabena Walker, 1865; and Adipsophanes Grote, I873.

TYANA Walker, i 866, List Specimens lepid. Insects Colln Br. Mus. 35: 1776

CHLO

Type-species: Tyana callichlora Walker, 1866, ibidem 35: I776, by subsequent designation by Kirby, 1892, Synonymic Cat. Lepid. Heterocera 1:283. Syntypes I ô. I + , [lNDA] : [W. Bengal], Darjeeling (BMNH).

TYCOMARPTES Fletcher, 1961, Ruwenzori Exped. I952 1: 195.

HADE

Type-species: Apamea inferior Guenée, 1852, in Boisduval \& Guenée, Hist. nat. Insectes (Lépid.) 5: 2II, by original designation. Holotype ô, [EThiopia] Abyssinia (MNHN, Paris).

TYCRACONA Moore, I882, in Hewitson \& Moore, Descr. new Indian lepid. Insects Colln late Mr W. S. Atkinson: 95. ACRO

Type-species: Tycracona obliqua Moore, I882, ibidem: 95, by subsequent desigation by Hampson, 1909, Cat. Lepid. Phalaenae Br. Mus. 8: 50. Syntypes ô, + . India: Cherra; Darjiling (MNHU, Berlin).

TYMPANISTES Moore, I867, Proc. zool. Soc. Lond. 1867: 48.

CHLO

Type-species: Tympanistes pallida Moore, I867, ibidem 1867: 49, pl. 6, fig. I, by subsequent designation by Hampson, I894, Fanna Br. India (Moths) 2: 420. Syntypes ふ.. P. [INDIA]: [W. Bengal], Darjeeling (BMNH).

†TYMPANISTIS; Prout, 1925, Ann. Mag. nat. Hist. (9) 16: foo.

An incorrect subsequent spelling.

$\ddagger$ TYMPANISTIS, misspelling. See Tympanistes Moore, I 867 .

CHLO 
TYMPANOBASIS Hampson, 1926, Descr. new Genera Species Lepid. Phalaenae Subfamily Noctuinae Br. Mus.: 322.

Type-species: Tympanobasis thyrsipalpis Hampson, 1926, ibidem: 322, by original designation. Holotype of, Paraguay: Sapucay (BMNH).

TYRISSA Walker, I866, List Specimens lepid. Insects Colln Br. Mus. 35: 1975.

OPHI

Type-species: Tyrissa recurva Walker, 1866 , ibidem 35 : 1976, by monotypy. Holotype o. [Dominican Republic] St. Domingo (BMnH).

TY TA Billberg, I820, Enumeratio Insect. Mus. G. J. Billberg: 88.

OPH I

Type-species: Noctua italica Fabricius, I78I, Species Insect. 2: 218, by monotypy. Type(s), Italy (Type(s) not found by Zimsen, I964, Type Material of I. C. Fabricius: 538).

$N$. italica is a junior subjective synonym of Noctua luctuosa [Denis \& Schiffermüller], I 775 .

See also Dysthymia Newman, I868.

TYTROCA Wiltshire, I970, Veröff. zool. St Samml. Münch. 14: го I.

CATO

Type-species: Hypaetra leucoptera Hampson, I895, in Walsingham \& Hampson, Proc. zool. Soc. Lond. 1896:264, pl. Io, fig. I, by original designation. Type(s), [Southern YEMEN]: Aden (BMNH).

UFEUS Grote, 1873, Bull. Buffalo Soc. nat. Sci. 1: Iо I.

NOCT

Type-species: Ufeus satyricus Grote, 1873, ibidem 1: Ior, pl. 3, fig. 4, by original designation. Syntypes 9, CANAda. [U.S.A.]: N.Y., Albany.

UGANA Swinhoe, 1909, Ann. Mag. nat. Hist. (8) 3: 97.

OPHI

Type-species: Ugana piana Swinhoe, 1909, ibidem (8) 3: 98, by monotypy. Syntypes 5 o. UGanda: Entebbe (BMNH).

$U$. piana is a junior subjective synonym of Aburina sobrina Möschler, I887.

See also Aburina Möschler, I887.

UGIA Walker, I858, List Specimens lepid. Insects Colln Br. Mus. 15: 1860.

OPH I

Type-species: Ugia disjungens Walker, I 858, ibidem 15: I86 I, by monotypy. Lectotype 0 . Singapore (UM, Oxford), designated (as type) by Swinhoe, I90o, Cat. east. and Aust. Lepid. Heterocera 2: 194.

See also Sarthida Walker, [1863].

UGIODES Hampson, I926, Descr. new Genera Species Lepid. Phalaenae Subfamily Noctuinae Br. Mus.: 422.

OPHI

Type-species: Ugiodes cinerea Hampson, I926, ibidem: 422, by original designation. Holotype $\hat{o}$. [GHana] Gold Coast: Kumasi (BMNH).

ULOCHLAENA Lederer, I857, Noctuinen Eur.: 33, 96.

CUCU

Type-species: Noctua hirta Hübner, [1813], Samml. eur. Schmett. 4: pl. I28, fig. 59I, by monotypy. Type(s), Europe.

ULOLONCHE Smith, I887, Proc. U.S. natn. Muss. $10: 47$ I.

HADE

Type-species: Mamestra niveiguttata Grote, I873. Bull. Buffalo Soc. nat. Sci. 1: I 40, pl. 4, fig. I6, by subsequent designation by Grote, 1895, Abh. naturw. Ver. Bremen 14: 74 . Syntypes $5(0$, o) $)$ [U.S.A.]: California (BMNH).

ULOSYNEDA Smith, [1903] I902, in Dyar, Bull. U.S. natn. Mus. 52: 220.

OPH I

Type-species: Synedoida valens Edwards, I88ז, Papilio 1: II9, by monotypy. Holotype $\sigma^{\prime},[$ U.S.A.]: S. Utah, Kanab.

ULOTHRICHOPUS Wallengren, I865. See Ulotrichopus Wallengren, I 860. CATo

$\ddagger$ ULOTHRICHOPUS Prout, 1928. See Ulotrichopus Wallengren, I860.

CATO 
ULOTRICHOPUS Wallengren, 1860, Wien. ent. Monatschr. 4: I74.

CATO

Type-species: Ulotrichopus tortuosus Wallengren, I860, ibidem 4: I 74, by monotypy. Type(s) ô. [South AFRICA] Caffraria (NR, Stockholm).

ULOTHRICHOPUS Wallengren, I865, K. suenska Y'etenskAkad. Handl. (N.F.) 5(4): 76.

Type-species: Ulotrichopus [sic] tortuosus Wallengren, i 860, by monotypy.

Ulothrichopus was described as a new genus having as its only included species ' $U$. tortuosus Wallengr. Wiener Ent. Monatschr. I860 No. 6.' It is therefore a junior objective synonym of Ulotrichopus Wallengren, is6o.

$\ddagger$ ULOTHRICHOPUS Prout, 1928; Neave, 1940, Nomencl. zool. 4: 608.

A normal usage by Prout but cited by Neave as an emendation.

$\ddagger$ UNCA Oken, I815. See Abrostola Ochsenheimer, I816.

PLUS

UNCA Lhomme, [1929,. See Abrostola Ochsenheimer, i816.

PLUS

UNCULA Swinhoe, i 900, Cat. east. and Aust. Lepid. Heterocera 2: 52.

$A C O N$

Type-species: Hiccoda herbaria Swinhoe, I 886, Proc. zool. Soc. Lond. 1886: 446, by monotypy. Type(s), India: central, Mhow (BMNH).

UNIPTENA nom. n. for Neptunia Barnes \& McDunnough, I9I I.

$\mathrm{ACON}$

Type-species: Azenia pulchra Barnes \& McDunnough, I9Iо, Jl N.Y. ent. Soc. 18: I 58, by original designation (for Neptunia Barnes \& McDunnough). Holotype ô. [U.S.A.]: Texas, Kerrville.

NEPT UNIA Barnes \& McDunnough, 191 I, Jl N.Y. ent. Soc. 19: 159.

Type-species: Azenia pulchra Barnes \& McDunnough, I9ro, by original designation.

A junior homonym of Neptunia Locard, r 886, Cat. gén. Mollusques vivants Fr.: 174, Mollusca. The objective replacement name is Uniptena nom. n.

UOLLEGA Berio, 1945, Memorie Soc. ent. ital. 24:81.

NOCT

Type-species: Uollega ungemachi Berio, I 945, ibidem 24: 8I, by original designation. Holotype , Eтuiopia: Galla Sidamo, Uollega (Colln E. Berio, Genoa).

UPOTHENIA Schaus, I913, Ann. Mag.nat. Hist. (8) $11: 44$.

HYPE

Type-species: Megachyta acutipennis Schaus, I9I2, ibidem (8) 9: 2II, by original designation. Syntypes $\hat{O}, \mathcal{O}$, Costa RicA: Carillo and Laguna.

URABA Walker, I863, List Specimens lepid. Insects Colln Br. Mus. 28: $4+8 . \quad$ NOLI Type-species: Uraba lugens Walker, 1863 , ibidem 28: 479, by monotypy. Holotype ㅇ, [AUstralia]: Tasmania (BMLNH).

See also Coesa Walker, I866; and Toxoloma Feleler, I874.

$\ddagger$ †RUBA; Kirby, i 892, Svnonvmic Cat. Lepid. Heteroceva 1: $37 \mathrm{I}$.

An incorrect subsequent spelling.

URBONA Walker, i 862, J. Proc. Linn. Soc. (Zool.) 6: ir 83.

CHLO

Type-species: Urbona sublineata Walker, i 862 , ibidem 6: i 84 , by monotypy. Lectotype ô. Borneo: Sarawak (U.M, Oxford), clesignated (as type) by Swinhoe, 1900, Cat. east. and Aust. Lepid. Heterocera 2: 73.

See also Nagasena Moore, I882.

URIPAO Hampson, 1926, Descr. new Genera Species Lepid. Phalaenae Subfamily Noctuinae Br. Mus.: 89 .

OPHI

Type-species: Uripao albizonata Hampson, 1926, ibidem: 90, by original designation. Syntypes i ô, i O Siriza LEONe (BMNH).

URSOGASTRA Smith, igor, Jl N.Y. ent. Soc. 14: I 2.

HADE

Type-species: Ursogastra lunata Smith, igo6, ibidem 14: 13, hy monotypy. Syntypes

2 ô, [U.S.A.]: Ariz., Huachuca Mts; Ariz, Cochise Co., Palmerly.

$\ddagger$ URUBA, misspelling. Sce Lraba Walker, I 863 .

NOLI 
USBECA Püngeler, I9I 4, Dt. ent. Z. Iris 28: 46.

ACRO

Type-species: Usbeca cornuta Püngeler, 1914, ibidem 28: 46, pl. 2, fig. I 5, by monotypy.

Syntypes 3 ot, I + , [U.S.S.R.]: Syr-Daria, Baigacum (MNHU, Berlin).

See also Acrosphalia Rebel, igr8.

UZINIA Schaus, 1913, Ann. Mag. nat. Hist. (8) $11: 31$.

OPHI

Type-species: Uzinia hyas Schaus, I913, ibidem (8) 11: 3 I, by original designation.

Syntypes đ, Costa Rica: Esperanza; Sixola (USNM, Washington).

UZOMATHIS Schaus, I9I6, Proc. U.S. natn. Mus. 50: 261, 300.

HYPE

Type-species: Uzomathis dissensa Schaus, 1916, ibidem $50: 300$, by original designation.

Type(s) ô, [French Guiana]: R. Maroni, St. Jean (USNM, Washington).

$\boldsymbol{V} \boldsymbol{A L E R I A}$ Stephens, i 829 [June 3rd], Nom. Br. Insects : 42.

CUCU

Type-species: Bombyx oleagina [Denis \& Schiffermüller], I775, Ankündung syst.

Werkes Schmett. Wienergegend: 59, by monotypy. Type(s), [AustriA]: Vienna district (Collection destroyed, Horn \& Kahle, 1936, Ent. Beih. Berl.-Dahlem 3: 243).

The date of publication of Valeria Stephens was ascertained from the Athenaeum of I 829 June 3 rd, page 352 on which the Nom. Br. Insects was cited under a 'List of books published during the week.' Stephens attributed Valeria to Germar, but no earlier published reference has been found. Valevia was also proposed in Stephens, 1829 August, Illust. Br. Ent. (Haustellata) 3: 22 .

Valeria Stephens is not preoccupied by Valeria Horsfield, I829 [June 24th], Descr. Cat. lepid. Insects Mus. Hon. East India Company: 139,-Lepid., Danaidae. The date of publication is accepted from Corbet, 1939, J. Soc. Biblphy nat. Hist. 1 : 196.

VALERIETTA Draudt, I938, Mitt. münch. ent. Ges. 28: 30.

CUCU

Type-species: Valerietta forsteri Draudt, 1938, ibidem 28: 30, by monotypy. Syntypes I J., I +, [IRAN] Persia: Elburz Mts, Vandarban (ZSBS, Munich).

VALERIODES Warren, 1913, in Seitz, Gross-Schmett. Erde 11: 132.

CUCU

Type-species: Euplexia icamba Swinhoe, I893. Ann. Mag. nat. Hist. (6) 12: 260, by original designation. Syntypes $3 \hat{0}$. Sikkim (BMNH).

The date of publication of the part of Seitz containing page 132 was stated on page I 29 as I9I 3 January 20 th in both the English and German editions.

VANDAMIA Son, 1933, Ann. Transv. Mus. 15: 205.

NOLI

Type-species: Vandamia typica Son, 1933, ibidem 15: 206, pl. 2, fig. 1, pl. 5, fig. 7 , pl. 8, fig. 9, by original designation. Holotype $\widetilde{\jmath}$. South Africa: Transvaal, Pietersburg dist., Malta (TM, Pretoria).

VANDANA Moore, I881, Trans. zool. Soc. Lond. 11: 72.

OPHI

Type-species: Ophideres dividens Walker, [1858] I857. List Specimens lepid. Insects Colln Br. Mus. 13: 1228, by monotypy. Holotype, Java (BMNH).

VAPARA Moore, 1882, in Hewitson \& Moore, Descr. new Indian lepid. Insects Colln late MrW. S. Atkinson: i63.

CHLO

Type-species: Vapara fasciata Moore, I882, ibidem: I63, by original designation. Syntypes, India: [W. Bengal], Darjiling (BMNH).

VARIA Walker, [1867] i 868, J. Linn. Soc. (Zool.) 9: I90.

OPHI

Type-species: Varia rubiginea Walker, [I867] I868, ibidem 9: I90, by monotypy. Type(s) ô, [Colombia]: Bogota.

$V$. rubiginea is a junior subjective synonym of Chamyna homichlodes Hübner, [I 82 I].

See also Chamyna Hübner, [I82I].

VARICOSIA Hampson, 1924, Entomologist 57: 183.

OPHI

Type-species: Varicosia venata Hampson, I924, ibidem 57 : I83, by original designation. Syntypes, Ceylon: 2 o, 3 우, Kandy (BMNH). JAva: I 우 (BMNH). 
VARINA Neumoegen, I884, Papilio 4:94.

Type-species: Varina ornata Neumoegen, I 884, ibidem 4:94, by monotypy. Holotype ô. [U.S.A.]: central Florida.

I. ornata is a junior subjective synonym of Acherdoa fervaria Walker, I 865.

See also Acherdoa Walker, i 865.

VEIA Walker, [1863] i 864, J. Proc. Linn. Soc. (Zool.) 7: i6o.

OPHI

Type-species: Veia homopteroides Walker, [1863] I 864, ibidem 7: 16I, by monotypy. Lectotype ô, BorNeo: Sarawak (UM, Oxford), designated (as type) by Swinhoe, I90o, Cat. east. and Aust. Lepid. Heterocera 2: 128.

VELAZCONIA Köhler, (1952) i95I, Acta zool. lilloana 12: I 46.

CUCU

Type-species: Velazconia difficilis Köhler, (1952) 1951, ibidem 12: I46, by originaI designation. Syntypes $\hat{\sigma}, \underline{C}$, Argentina: La Rioja [Prov.], La Rioja (ZSBS, Munich); Mendoza, Tupungato.

VELUTINIA Herrich-Schäffer, i 8jo, Corresp.-Bl.zool-min. Ver. Regensburg 24: I02. HYPE

Nomenclaturally available, but without any included species. Proposed in a work on the Lepidoptera of Cuba.

VESCISA Walker, 1864, J. Proc. Linn. Soc. (Zool.) 7: i 9 1.

ACON

Type-species: Vescisa commoda Walker, I864, ibidem 7: 191, by monotypy. Lectotype, Borneo: Sarawak (UM, Oxford), designated (as type) by Swinhoe, I970, Cat. east. and Aust. Lepid. Heterocera 2: 196.

VESPOLA Walker, [1867] 1868, J. Linn. Soc. (Zool.) 9: i 85.

Type-species: Vespola caeruleifera Walker, [1867] i 868, ibidem 9: I 86, by monotypy. Type(s) ô, [Colombia]: Bogota (BMNH).

VESTERMANNIA Hampson, 1912. See Hestermannia Hübner, [I82I]. Chlo

VESTURA Swinhoe, 1904, Trans. ent. Soc. Lond. 1904: 142.

OPH I

Type-species: Vestura adeba Swinhoe, 1904, ibidem 1904: 142, by monotypy. Syntypes $2 \hat{0}$. SiNGAPORE (BMNH)

$\Gamma^{\circ}$. adeba is a junior subjective synonym of Egnasia minereusalis Walker, [1859] 1858, List Specimens lepid. Insects Colln Br. Mus. 16: 223.

VEYMERIA Hampson, I901. See IIeymeria Karsch, I 895.

AGAR

VIA Dyar, I9I4, Proc. U.S. natu. Mus. 47: 193.

$\operatorname{ACON}$

Type-species: Via vindicia Dyar, I9I4, ibidem 47: I94, by original designation. Syntypes 8 ex., PANAma: la Chorrera; Taboga I. (USNM, Washington).

VICTRIX Staudinger, I 879, in Romanoff, Horae Soc. ent. ross. 14: 489.

ACRO

Type-species: Victrix karsiana Staudinger, I 879, ibidem 14: 490, pl. 3, fig. 2, by monotypy. Holotype, [Turiey]: Armenia, Kars (MNHU, Berlin).

See also Amelina Draudt, I934.

VIETTEANIA Rungs, 1955, Mem. Inst. scient. Madagascur (E)6: Io I.

HADE

Type-species: Leucania torrentium Guenée, 1852, in Boisduval \& Guenée, Hist. nat. Insectes (Lépid.) 5: 88, by original designation. Lectotype ô. MADAGASCAR (MNHN, Parisi, designated by Viette, 195x, Bull. mens. Soc. limn. Lyon 20: 59.

VILLOSA Koch, I865, Indo-Australische Lepid.-Fauna: 108.

OPHI

Type-species: Villosa leichardtii Koch, i 865, ibidem: 108, pl. I, by monotypy. Type(s), At'stralia: northern.

VIMINIA Chapnan, I 890 May; Entomologist's Rec. J. Vur. 1: 26. 
subsequent designation by Chapman, I890 December, ibidem 1: 221. Type(s), TypeLOCALITY not stated (LS, London) [EUROPE].

PHARETRA Hübner, [1820] i816, Verz. bekannter Schmett.: 202.

Type-species: Noctua auricoma [Denis \& Schiffermüller], I775, Ankündung syst. W' erkes Schmett. Wienergegend: 67, by subsequent designation by Grote, I 895, Entomologist's Rec. J. Var. 7: 57. Type(s), [AusTria]: Vienna district (Collection destroyed, Horn \& Kahle, 1936, Ent. Beih. Berl.-Dahlem 3: 243).

A junior homonym of Pharetra Bolten, I 798, Mus. Boltenianum 2: I59, - Brachiopoda. There is no objective replacement name but $N$. auricoma is congeneric with Phalaena rumicis Linnaeus, I 758, the type-species of Viminia Chapman, I89o. The latter is therefore available for use as a subjective replacement name.

CHAMAEPORA Warren, I909, in Seitz, Macrolepid. World 3: I6.

Type-species: Phalaena rumicis Linnaeus, $175^{8}$, by original designation in the English edition (but not in the German).

The date of publication of the part of Seitz containing page 16 was stated on page 13 as I 909 February $24^{\text {th }}$ in the English edition and as I909 May 2 Ist in the German edition.

Chamaepora is a junior objective synonym of Viminia Chapman.

VIRGO Staudinger, I892, in Romanoff, Mém. Lépid. 6: 467.

ACRO

Type-species: Nonagria amoena Staudinger, I 888, Stettin. ent. Ztg 49: 254, by monotypy. Syntypes 20 , [U.S.S.R.]: Amur district, Sidimi (MNHU, Berlin).

$N$. amoena is a junior subjective synonym of Nephelodes datanidia Butler, I885, Cistula ent. 3: 132 .

VIRIDEMAS Smith, 1908, Amn. N.Y. Acad. Sci. 18: 91.

ACRO

Type-species: Viridemas galena Smith, I 908, ibidem 18: 92, by monotypy. Syntypes 2 o, 1 q, [U.S.A.]: Arizona, Huachuca Mts and Cochise County, Palmerly.

VITTAPPRESSA Bethune-Baker, I906, Novit. zool. 13: 206.

$\operatorname{ACON}$

Type-species: Vittappressa rufiplaga Bethune-Balier, I906, ibidem 13: 206, by original designation. Syntypes $\hat{\jmath}$,, , New Guinea: [Papua], Dinawa (BMNH).

$\ddagger$ VITT APRESSA; Neave, I940, Nomencl. zool. 4: 644 .

An incorrect subsequent spelling.

$\ddagger$ VITTAPRESSA, misspelling. See Vittappressa Bethune-Baker, I906.

$\operatorname{ACON}$

VIZAGA Swinhoe, I 901, Amm. Mag. nat. Hist. (7) 7: 491.

CHLO

Type-species: Ophiusa cyanea Snellen, I88I, Tijdschr. Ent. 24: 129, pl. I4, fig. 2, by original designation. Holotype $q$, Philippines: Luzon.

VOGIA Walker, I858, List Specimens lepid. Insects Colln. Br. Mus. 15: I 818.

OPHI

Type-species: Vogia amplivitta Walker, I858, ibidem 15: I 818, by monotypy. Holotype đ̆, [Brazil]: [R. Amazon], [Tefé] Ega (BMNH).

VOLAZAHA Viette, I971, Bull. Madagascar 298: 277.

OPH I

Type-species: Volazaha iridoplitis Viette, I97 I, ibidem 298:279, by original designation. Holotype ô, MAdagascar: Sakaraha, forêt de Lambomakandro (MNHN, Paris).

VULCANICA Berio, 1970, Boll. Soc. ent. ital. 102: 21.

NOCT

Type-species: Vulcanica gentilis Berio, I970, ibidem 102: 21, by original designation.

Holotype $q$, [ZAIRE] Congo: Katanga, Tshifundgi (MRAC, Tervuren).

$\ddagger$ WALKARA, misspelling. See Wilkara Swinhoe, I9I 8.

HYPE

WALTERELLA Dyar, I 92I, Insecutor Inscit. menstr. 9: 62.

ACRO

Type-species:Walterella eudesmia Dyar, I92I, ibidem 9:62, by monotypy. Holotype o, U.S.A.: Arizona, Tempe (USNM, Washington).

W. eudesmia is a junior subjective synonym of Prothrinax ocellata Barnes \& McDunnough, I910, Can. Ent. 42: 246. 
WESTERMANNIA Hübner, [1821] 1816, Vevz. bekannter Schmett.: 250. Nomenclaturally' available, but with the only included species a nomen nudum until Hübner, 1823, Zuträge Samml. exot. Schmett. 2: 23 .

Type-species: Westermannia superba Hübner, 1823, ibidem 2: 23, figs 323, 324, by subsequent monotypy. Type(s) ô, JAvA.

PLUSIODES Guenée, I 852, in Boisduval \& Guenée, Hist. nat. Insectes (Lépid.) 6: 385.

Type-species: Westermannia superba Hübner, by monotypy, but cited as Plusiodes westermannii Guenée, 1852 , an unnecessary objective replacement name.

Plusiodes is a junior objective synonym of Westermannia Hübner, [I82 I].

VESTERMANNIA Hampson, rgr2, Cat. Lepid. Phalaenae Br. Mus. 11: xvi, 603.

An unjustified emendation of Westermannia Hübner, [182 r ] .

WEYMERIA Karsch, I895, Ent. Nachr. 21 : 347.

AGAR

Type-species: Xanthospilopteryx athene Weymer, r892, Stettin. ent. Ztg 53: ror, by original designation. Holotype ô, EAST AFricA.

VEYMERIA Hampson, I901, Cat. Lepid. Phalaenae Br. Mus. 3: xviii, 584.

An unjustified emendation of Weymeria Karsch, 1895.

WILKARA Swinhoe, I918, Ann. Mag. nat. Hist. (9) 2: 92.

HYPE

Type-species: Wilkara nigerrima Swinhoe, I9r8, ibidem (9) 2: 93, by original designation. Type(s) ô, [Sumatra]: central Nias [I.], Kalim Bungo (BMNH).

$W$. nigerrima was originally described as $\ddagger W$ alkara, an incorrect original spelling.

${ }_{\ddagger}$ WALKARA; Swinhoe, I918, Ann. Mag. nat. Hist. (9) 2: 92.

An incorrect original spelling. The genus was described as 'Wilkara, nov.', but the only included species was clescribed on the next page as 'Walkara nigerrima, nov.' In the absence of a reviser I am following Neave, r940, Nomencl. zool. 4: 657 .

XANTHA Billberg, i 820. See Xanthia Ochsenheimer, r816.

CUCU

XANTHALIA Berg, I894, in Fairmaire, Annls Soc. ent. Belg. 38: 395.

COLEOPTERA

Proposed as the objective replacement name for Xanthia Fairmaire, I 893, - Coleoptera; preoccupied by Xanthia Ochsenheimer, r816, - Lepid., Noctuidae.

Xanthalia Berg is therefore a coleopterous name and Hampson, 1905, Cat. Lepid. Phalaenae Br. Mus. 5: 6o, was incorrect in citing it together with Xanthia Ochsenheimer, as a synonym of Polia Ochsenheimer, 1816, - Lepid., Noctuidae.

XANTHANOMIS Hampson, 1926, Descr. new Genera Species Lepid. Phalaenae Subfamily Noctuinae Br. Mus. : 355.

Type-species: Cosmophila fuscifrons Walker, [1863] i 864, J. Proc. Linn. Soc. (Zool.)

7: 77, by original designation. Lectotype \&, BornEo: Sarawak (UM, Oxford), designation (as type) by Swinhoe, 1900, Cat. east. and Aust. Lepid. Heterocera 2: 96.

${ }_{+} \boldsymbol{X} \boldsymbol{A N T H I A}$ Hübner, [1806]. See Cirrhia Hübner, [1821].

CUCU

$\ddagger \boldsymbol{X A N T H I A}$ Hübner, I808. See Xystopeplus Franclemont, 1937.

CUCU

XANTHIA Ochsenheimer, r8r6, Schmett. Eur. 4: 82.

CUCU

Type-species: Noctua flavago Fabricius, I787, Mantissa Insect. 2: I60 (a junior primary homonym), by subsequent designation by Curtis, 1825, Br. Ent. 2: 84. Type(s), [Gernany']: [Kiel] Kilia (Type(s) not found by Zimsen, 1964, Type Material of I. C. Fabricius: 549).

The type-species is preoccupied by Noctua flavago [Denis \& Schiffermüller], 1775, Ankïndung syst. Werkes Schmett. Wienergegend: 86, - Noctuidac, Acronictinae. There is no objective replacement name but Phaluena togata Esper, 1788, Die Schmett. 4 (1): 1)1. 124 , fig. I; I79r, ibidem: $33^{6}$, is a junior subjective synonym and is therefore available for use as a subjective replacement nane.

N. flavago Fabricius, $\mathrm{I} 787$, is also a junior subjective synonym of Phalaena (Noctua) 
lutea Ström, I783, Nye Samling Kongelige Danske Videnskabers Selskabs Skrifter 2: 78, fig. 26, but this is a junior homonym of Phalaena (Geometra) lutea Stoll, I $78 \mathrm{I}$, and so cannot be used as a replacement name.

XANTHA Billberg, I8zo, Enumeratio Insect. Mus. G.J. Billberg: 88.

An unjustified emendation of Xanthia Ochsenheimer.

CITRIA Hübner, [182I] I816, Vevz. bekannter Schmett.: 234.

Type-species: Noctua flavago Fabricius, I 787 , by subsequent designation by Grote, I 896 , Entomologist's Rec.J. Var. 8: I7.

Citria is a junior objective synonym of Xanthia Ochsenheimer, I8r6.

EUTHEMONIA Gistl, I848, Naturg. Thierreichs: xi.

Proposed, unnecessarily, as an objective replacement name for Xanthia Ochsenheimer, I8I6.

A junior homonym of Euthemonia Stephens, I828, Illust. Br. Ent. (Haustellata) 2: 55 , 68, - Lepid., Arctiidae.

XANTHIA Boisduval, I828. See Jodia Hübner, I8r8.

CUCU

XANTHIRIA Hampson, I908, Cat. Lepid. Phalaenae Br. Mus. 7: 5. Nomenclaturally available, but without included species until Hampson, I9Io, ibidem 9: x, 244. ACRO

Type-species: Metoponia primulina Druce, 1889, Biologia cent.-am. (Zool.) Lepid. Heterocera 1: 303, pl. 28, fig. Io, by subsequent monotypy. Syntypes, Mexico: Guerrero, Omilteme (BMNH).

XANTHIRINOPSIS Dyar, 1926, Insecutor Inscit. menstr. 14: 146.

NOCT

Type-species: Xanthirinopsis cataplaga Dyar, I926, ibidem 14: I46, by original designation. Holotype $\tilde{\sigma}$, Mexico: Zacualpan (USNM, Washington).

XANTHODES Guenée, I 852, in Boisduval \& Guenée, Hist. nat. Insectes (Lépid.) 6: 209. CHLO Type-species: Phalaena malvae Esper, [I796], Die Schmett. 4 (2) Abschnitt 2: 63, pl. I95, fig. 4, by original designation. Syntypes, HungarY.

P. malvae has also been cited as Noctua malvae Hübner, [1808], Samml. eur. Schmett. 4: pl. 77 , fig. $35^{8}$.

N. malvae is a junior subjective synonym of Noctua albago Fabricius, 1794, Ent. Syst. $3(2): 74$.

$\ddagger A C O N T I A$ Hübner, [1823] I816, Verz. bekannter Schmett.: 257.

Only included species: Noctua malváe Hübner, [1808]. Hemming, 1937, Hübner 2: I46 considered that Hübner's genus was distinct from Acontia Ochsenheimer, i8í. Neave, 1939, Nomencl. zool. 1: 38 cited both. As N. malvae was the first of the species originally included in Acontia Ochsenheimer, I8r6, - Lepid., Noctuidae, it is evident that Hübner is not to be regarded, under the present Code, as proposing a new monotypic genus.

XANTHODESMA Aurivillius, I910, in Sjöstedt, Kilimandjaro Meru Exped. 9: $34 . \quad$ OPHI Type-species: Xanthodesma aurata Aurivillius, I910, ibidem 9: 34, pl. 2, fig. 3, by subsequent designation by Gaede, 1939, in Seitz, Gross-Schmett. Erde 15: 3 I8. Holotype ô, [Tanzania]: Kilimandjaro, Kibonoto (NR, Stockholm).

XANTHOECIA Hampson, I908. See Gortyna Ochsenheimer, I8r6.

ACRO

XANTHOGRAPTA Hampson, ig Io, Cat. Lepid. Phalaenae Br. Mus. 10: xvi, 563. ACoN Type-species: Pyralis trilatalis Walker, [1866] 1865, List Specimens lepid. Insects Colln Br. Mus. 34: 1237, by original designation. Holotype o, [INDIA]: N. Hindostan (BMNH).

XANTHOLEPIS Hampson, Igo9, Cat. Lepid. Phalaenae Br. Muss. 8: 2. Available, but without included species until Hampson, r9ro, ibidem 9: xv, 5I4. ACRO

Type-species: Acontia dicycla Walker, 1866, List Specimens lepid. Insects Colln Br. Mus. 35: 1964, by subsequent monotypy. Holotype ô., [INDia]: N. Hindostan (BMNH).

XANTHOLEUCA Stephens, I831. See Jodia Hübner, I8I8.

CUCU 
XANTHOLEUCA Hampson, r9io. See Chionoxantha Hampson, I9I4.

XANTHOMANTIS Warren, I gog, in Seitz, Macrolepid. World 3: r 8.

Type-species: Acronycta cornelia Staudinger, r888, Stettin. ent. Ztg 49: 246, by monotypy. Syntypes 2 우 [U.S.S.R.]: E. Amur district, Askold and Suifun.

The date of publication of the part of Seitz containing page r 8 was stated on page 13 as 1909 February $24^{\text {th }}$ in the English edition and as 1909 May 2 Ist in the German edition.

XANTHOMERA Hampson, I914, Ann. Mag. nat. Hist. (8) 13: 202.

$\mathrm{ACON}$

Type-species: Erastria leucoglene Mabille, I880, C. r. Séanc. Soc. ent. Belg. 1880: xviii, by original designation (for Xanthozona Hampson, I9I0). Lectotype , MADAGASCAR: Nossy-Bé (MNHN, Paris), designated by Viette, 1954, Mém. Inst. Sci. Madag. (E) 5: 384.

Xanthomera was proposed as the objective replacement name for Xanthozona Hampson, I9IO.

XANTHOZONA Hampson, rgro, Cat. Lepid. Phalaenae Br. Mus. 10: xv, 487.

Type-species: Erastria leucoglene Mabille, I880, by original designation.

A junior homonym of Xanthozona Townsend, rgo8, Smithson. misc. Collns 51 (1803): I I6, - Diptera. The objective replacement name is Xanthomera Hampson, I9I 4.

XANTHOPASTIS Hübner, [1821] I816, Verz. bekannter Schmett. : 2 I I.

HADE

Type-species: Phalaena timais Cramer, I780, Uitlandsche Kapellen 3: 148, pl. 275. fig. B, by subsequent designation by Grote, r895, Abh. naturw. Ver. Bremen 14: 85 . Type(s), TYPE-LOCALITY stated to be [India]: Coromandel Coast, but this was probably an error as the species illustrated is not known to occur in the Oriental Region but is widespread in Central America (BMNH).

See also Philochrysa Grote, 1863.

EUTHISANOTIA Hübner, [I83I] r 825, Zuträge Samml. exot. Schmett. 3: 12, 39.

Type-species: Phalaena timais Cramer, I 780 , by subsequent designation by Grote, 1874 , Bull. Buffalo Soc. nat. Sci. 2: 20.

Euthisanotia is a junior objective synonym of Xanthopastis Hübner, [1821].

XANTHOPTERA Sodoffsky, I837. See Noctua Linnaeus, I 758.

NOCT

XANTHOPTERA Guenée, 1852. See Thiopteva Franclemont, 1950.

$\operatorname{ACON}$

XANTHOSPILOPTERYX Wallengren, 1858, Öfvers. K. VetenskAkad. Förh. Stockh. 15: 83.

AGAK

Type-species: Noctua geryon Fabricius, I781, Species Insect. 2: 2 I6, by original designation. Type(s), AfricA: equinoctial (Type(s) not found by Zimsen, r964, Type Material of I. C. Fabricius: 538 ).

See also Hevaclia Hübner, [1820].

XANTHOSTHA Hampson, I926, Descr. new Genera Species Lepid. Phalaenae Subfamily Noctuinae Br. Mus.: 600 .

OPHI

Type-species: Pyrinia xantharia Walker, I866, List Specimens lepid. Insects Colln Br. Mus. 35: r 543, by original designation. Type(s) ô, [BRAzIL]: Amazon region (LM, Oxford).

XANTHOTHRIX Edwards, r878, Pacific Coast Lepid. No. 29: 7.

ACRO

Type-species: Xanthothrix ranunculi Edwards, I 878, ibidem No. 29: 7 , by monotypy. Syntypes i I 0 , 8 ㅇ, [U.S.A.]: [Calif.], Kern Co., Havilah.

XANTHOZONA Hampson, Igro. See Xanthomera Hampson, I9I4.

$\mathrm{ACON}$

XENAPAMEA Sugi, r970, Tinea, Tokyo 8: 220.

ACRO

Type-species: Xenapamea pacifica Sugi, I970, ibidem 8: 220, figs 13-15, 28-30, by original designation. Holotype ō, Ryukyu Is: Okinawa I., Yona (Colln S. Sugi, Tokyo). 
XENOCHROA Felder, I874, in Felder \& Rogenhofer, Reise öst. Fregatte Novara (Zool.) 2 (Abt. 2): pl. 97, fig. 23.

Type-species: Xenochroa notodontina Felder, 1874, ibidem 2 (Abt. 2): pl. 97, fig. 23; Erklärung: Io, by monotypy. Type(s) ô. [JAVA: Buitenzorg] not R. Amazonas. (BMNH).

XENODRYAS Gistl, I 848 . See Phyllodes Boisduval, 1832.

CATO

XENONOLA Wileman \& West, I928, Entomologist 61: 277.

NOLI

Type-species: Erastroides limbata Wileman, I9I 5, Entomologist 48: I60, by original designation. Holotype + , [TAIWAN] Formosa: Kanshirei (BMNH).

XENOPHYSA Boursin, 1969, Entomops, Nice 2: 221.

NOC'T

Type-species: Agrotis junctimacula Christoph, I887, in Romanoff, Mém. Lépid. 3: 67, pl. 3, fig. II, by original designation. Syntypes, [U.S.S.R.]: I đ̇, [Turkmenistan], Askhabad, Kopet-dagh. [IRAN] Persia: I $\hat{0}, 2$, northern, Schahkuh.

XENOPSEUSTIS Meyrick, I 897, Trans. ent. Soc. Lond. 1897: 369.

ACRO

Type-species: Xenopseustis poecilastis Meyrick, I897, ibidem 1897 : 370, by monotypy. Syntypes $3(\widehat{0}$, $)$, Australia: Qd, Duaringa (BMNH).

XENOTRACHEA Sugi, 1958, Tinea, Tokyo 4: 184.

ACRO

Type-species: Hadena albidisca Moore, I867, Proc. zool. Soc. Lond. 1867: 59, pl. 6, fig. I 7 , by original designation. Type(s) $\partial$, [INDIA/Bangladesh]: Bengal (BMNH).

XEROCIRIS Cockerell, I904, Ent. News 15: 76.

AGAR

Type-species: Ciris wilsonii Grote, I863, Proc. ent. Soc. Philad. 2: 65, pl. 3, fig. I, by monotypy (of Ciris Grote, I 863). Holotype 'which I regard (somewhat doubtfully) as a female', [U.S.A.]: Western Texas.

Xerocivis was proposed as the objective replacement name for Ciris Grote, 1863.

CIRIS Grote, I 863, Proc. ent. Soc. Philad. 2: 65.

Type-species: Ciris wilsonii Grote, 1863 , by monotypy.

A junior homonym of Ciris Koch, i 846, Die Arachniden 14 (2): 85, - Arachnida. The objective replacement name is Xerociris Cockerell.

$\ddagger$ XEROPHYLLIDIA Hampson, no published reference found.

HYPE

XESTIA Hübner, I818, Zuträge Samml. exot. Schmett. 1 : I6.

NOCT

Type-species: Noctua ochreago Hübner, [1809], Samml. euv. Schmett. 4: pl..92, fig. 43 I, by subsequent designation by Hampson, 1903, Cat. Lepid. Phalaenae Br. Mus. 4: 593. Type(s), EUROPE.

HIPTELIA Guenée, I852, in Boisduval \& Guenée, Hist. nat. Insectes (Lépid.) 5: 399.

Type-species: Noctua ochreago Hübner, [1809], by subsequent designation by Hampson, 1903, Cat. Lepid. Phalaenae Br. Mus. 4: 593.

Hiptelia is a junior objective synonym of Xestia Hübner, I818.

XIANA nom. n. for Naxia Guenée, 1852.

CATO

Type-species: Naxia absentimacula Guenée, I 852, in Boisduval \& Guenée, Hist. nat. Insectes (Lépid.) 7: 255, by subsequent designation (for Naxia Guenée) by Desmarest, (1857), in Chenu, Encycl. Hist. nat. Papillons nocturnes: I32. Syntypes, Java (BMNH). INDIA: central (BMNH).

NAXIA Guenée, I 852, in Boisduval \& Guenée, Hist. nat. Insectes (Lépid.) 7: 254.

Type-species: Naxia absentimacula Guenée, 1852 , by subsequent designation by Desmarest, (1857), in Chenu, Encycl. Hist. nat. Papillons nocturnes: I32.

A junior homonym of Naxia Leach, 1823, Dictionnaire Sci. nat. 28: 266, - Crustacea. The objective replacement name is Xiana nom. $\mathrm{n}$.

XIPHOLEUCANIA Sugi, 1970, Tinea, Tokyo 8: 219.

HADE

Type-species: Leucania roseilinea Walker, I862, J. Proc. Linn. Soc. (Zool.) 6: I79, by KK 
original designation. Lectotype $\widehat{\jmath}$, BORNEO: Sarawak (UM, Oxford) designated (as type) by Swinhoe, 1900, Cat. east. and Aust. Lepid. Heterocera 2: 45.

Xipholeucania was proposed as a subgenus of Mythimna Ochsenheimer, 1816.

XORIA nom. n. for Orixa Walker, I869.

HYPE

Type-species: Orixa filifera Walker, 1869, Proc. nat. Hist. Soc. Glasgow 1: 367 , by monotypy (of Orixa Walker). Type(s), Congo (BMNH).

ORIXA Walker, 1869, in Chapman, Proc. nat. Hist. Soc. Glasgow $1: 367$.

Type-species: Orixa filifera Walker, 1869, ibidem 1: 367 , by monotypy. Type(s) o. CONGO (BMNH).

A junior homonym of Orixa Gray, 1852, Synopsis Mollusca Gt Br.:277, - Mollusca. The objective replacement name is Xoria nom. $\mathrm{n}$.

$\ddagger X S Y L I N A$ Frivaldsky, 1835. See Xylena Ochsenheimer, 1816.

CUCU

$\$ X Y \boldsymbol{L A} \boldsymbol{E N A}$, misspelling. See Xylena Ochsenheimer, 1816 .

CUCU

$+X Y L E N A$ Hübner, [1806]. See Septis Hübner, [1821].

ACRO

XYLENA Ochsenheimer, 1816, Schmett. Eur. 4:85.

CUCU

Type-species: Phalaena exsoleta Linnacus, 1758, Syst. Nat. (Edn I0) 1: 5 I5 (but included by Ochsenheimer as †'exoleta Linn.', an incorrect subsequent spelling), by subsequent designation (for Xylina Treitschke) by Duponchel, I829 [March 21st], in Godart \& Duponchel, Hist. nat. Lépid. Papillons Fr. 7 (2): 72. Type(s), Type-Locality not stated (LS, London) [EUROPE].

$P$. exsoleta was also designated (as ‘'exoleta Linn.') as the type-species of Xylina Treitschke, by Curtis, 1829 April Ist, Br. Ent. 6: 256.

XYLITES R. L., I817, Jenaische Allg. Lit.-Ztg 1: 287.

Type-species: Phalaena exsoleta Linnaeus, 1758 , by PRESENT DESIGNATION.

Xylites R. L., is a junior objective synonym of Xylena Ochsenheimer, I816.

$\ddagger X Y L A E N A$; Hübner, 1822, Syst. - alphab. Verz.: 21 .

An incorrect subsequent spelling.

XYLINA Treitschke, 1826, Schmett. Eur. 5 (3): 3.

An unjustified emendation of Xylena Ochsenheimer, 1816.

CALOCAMPA Stephens, I829, Illust. Br. Ent. (Haustellata) 2: 172.

Type-species: Phalaena exsoleta Linnaeus, $175^{8}$ (but included by Stephens as ‡'exoleta

L.', misspelling), by subsequent designation by Westwood, I840, Synopsis Genera Br.

Insects: 94 , but cited as ¥'exoleta $\mathrm{L}$.'

$\ddagger$ XSYLINA Frivaldszky, 1835, Magy. Tudos Társ. evk.-Mad. Koetet. 2: 273.

An incorrect subsequent spelling.

$+H Y L I N A ;$ Freyer, I840, Nenere Beitr. Schmett. $4: 37$.

An incorrect subsequent spelling.

CALLICAMPA Agassiz, 1846, Nomencl. zool. Index univl.: 58, 60.

An unjustified emendation of Calocampa Stephens, I829.

${ }_{+}^{+}$COLOCAMPA; Stichel, 1908, Berl. ent. Z. 53: 103.

An incorrect subsequent spelling.

XYLINA Treitschke, 1826. See Xylena Ochsenheimer, 1816.

CUCU

XYLINISSA Hampson, 1906 [December]. See Xylinissa Schaus, I906 April.

CUCU

XYLINISSA Schaus, 1906 April, Proc. U.S. natn. Mus. 30: 97.

Cucu

Type-species: Xylinissa lignitis Schaus, 1906, ibidem 30:97, by monotypy. Type(s),

Colombia: Bogota (USNM, Washington).

XYLINISSA Hampson, Igo6 [1)ccember], Cat. Lepid. Phalaenae Br. Mus. 6: 355.

Type-species: Xylina cossoides Butler, 1882, Trans. ent. Soc. Lond. 1882: 136, by original designation. Syntypes $\hat{f}, q$, CHule: Las Zorras (BMNH). 
A junior homonym of Xylinissa Schaus, I906 April. This generic name probably originated from Hampson but was used and unintentionally made nomenclaturally available by Schaus prior to its proposal and generic description by Hampson for a similar concept but having a different type-species. I have compared the syntypes of $X$. cossoides with a colour photograph of the type of $X$. lignitis Schaus and consider them to be congeneric. Until a more detailed comparison can be made no objective replacement name for Xylinissa Hampson should be proposed.

XYLIODES Guenée, I857, in Boisduval \& Guenée, Hist. nat. Insectes (Lépid.) 9: 329. OPHI Type-species: Xyliodes fortunaria Guenée, I857, ibidem 9: 329, by monotypy. Holotype 0 , CHINA: northern (BMNH).

XYLIS Guenée, I852, in Boisduval \& Guenée, Hist. nat. Insectes (Lépid.) 7: 7.

CATO

Type-species: Xylis setipes Guenée, 1852, ibidem 7: 7, pl. I 5, fig. 6, by monotypy. Holotype ô, BraziL: [Rio de Janeiro], Nouvelle-Fribourg (BMNH).

XYLITES R. L., I817. See Xylena Ochsenheimer, i 816.

CUCU

XYLOCAMPA Guenée, I 837, Annls Soc. ent. Fr. 6: 227.

CUCU

Type-species: Phalaena lithoriza Borkhausen, I792, Naturgeschichte Eur. Schmett.

4: 339, by subsequent designation by Desmarest, (1857), in Chenu, Encycl. Hist. nat. Papillons nocturnes: 83 (but cited as $\ddagger$ lithorhiza, an incorrect subsequent spelling). Syntypes, Germany: Mainz. Sweden.

P. lithoriza is a junior subjective synonym of Phalaena areola Esper, I789, Die Schmett.

4 (2) Abschnitt I : pl. I4I, fig. 4; [I 798-I804], ibidem: 448.

XYLOMANIA Hampson, 1905, Cat. Lepid. Phalaenae Br. Mus. 5: xii, 389.

HADE

Type-species: Xylomiges hiemalis Grote, I874, Bull. Buffalo Soc. nat. Sci. 2: 71, by original designation. Syntypes $4(\hat{o}$,, ) , [U.S.A.]: Oakland $(\mathrm{BMNH})$; California (BMNH).

$\ddagger$ XYLOMIGES, misspelling. See Egiva Duponchel, [1845].

HADE

XYLOMOEA Hampson, I909. See Xylomoia Staudinger, I892.

ACRO

XYLOMOIA Staudinger, I892, in Romanoff, Mém. Lépid. 6: 451.

ACRO

Type-species: Xylomoia separata Staudinger, I892, ibidem 6: 45 I, pl. r2, fig. 8, by monotypy. Syntypes 207,2 ․ [U.S.S.R.]: Amur district, Sutschan area (MNHU, Berlin). XYLOMOEA Hampson, I909, Cat. Lepid. Phalaenae Br. Mus. 8: 534.

An unjustified emendation of Xylomoia Staudinger, 1892.

XYLOMYGES Guenée, I852. See Egira Duponchel, [1 845].

HADE

XYLOPHASIA Stephens, I 829. See Abromias Billberg, I820.

ACRO

XYLOPHYLLA Hampson, I913, Cat. Lepid. Phalaenae Br. Mus. 12: xi, 387.

CATO

Type-species: Phyllodes punctifascia Leech, I900, Trans, ent. Soc. Lond. 1900: 576, by original designation. Syntypes I Ĵ, 2 우 CHINA : western, Omei-shan (BMNH); Chia-kou-ho (BMNH).

XYLORMISA Forbes, I922, Ent. News 33: iо I.

HYPE

Type-species: Xylormisa louisiana Forbes, I922, ibidem 33: ror, by original designation. Holotype ${ }^{\star}$, [U.S.A.]: Sabine River Ferry (Cornell Univ., Ithaca).

XYLOSTOLA Hampson, 1908, Cat. Lepid. Phalaenae Br. Mus. 7: Io. Available, but without included species until Hampson, I910, ibidem 9: xii, 335 . ACRO

Type-species: Vapara indistincta Moore, I882, in Hewitson \& Moore, Descr. new Indian lepid. Insects Colln late $M r W$. S. Atkinson: I63, by subsequent designation by Hampson, I 9 I o, ibidem 9: 335. Syntypes $\lesssim$, + , India: [W. Bengal], Darjiling (BMNH). 
XYLOTYPE Hampson, I906, Cat. Lepid. Phalaenae Br. Mus. 6: xi, 288.

Type-species: Xylina capax Grote \& Robinson, 1868, Trans. Am. ent. Soc. 1: 355, pl. 7, fig. 57 , by original designation. Syntypes $\hat{0}$,,+ , [U.S.A.]: Atlantic District, Mass. to Penn[sylvani]a (B.MNH).

XYMEHOPS Viette, 1954, Annls Mus. r. Congo Belge (N.S. 4o Sci. zool.) 1: 557.

OPHI

Type-species: Xymehops nigra Viette, 1954, ibidem 1: 558, fig. I 5, by original designation. Holotype 0 . Madagascar: centr., Tananarive, Parc de Tsimbazaza (MHNN, Paris).

XYSTOPEPLUS Franclemont, 1937, Can. Ent. 69: 128.

CUCU

Type-species: Jodia rufago Hübner, 1818, Zuträge Samml. exot. Schmett. 1: 15, figs 6r, 62, by original designation. Type(s) $\sigma^{2}$ [U.S.A.]: Georgia [formerly] in Florida.

$\ddagger_{+}$XNTHIA Hübner, I808, Erste Zuträge Samml. exot. Schmett.: 4.

Included in a work rejected for nomenclatural purposes by Int. Commn zool. Nom., 1966, Bull. zool. Nom. 23 Opinion 789: 216. Placed on the Official Index of Rejected and invalid Generic Names in Zoology: Name No. I 888.

Only included species: $¥$ Xanthia rufago Hübner, 1808 , later made nomenclaturally available as Jodia rufago Hübner, I8r8.

YEPCALPHIS nom. n. for Pachylepis Felder, I874.

ACRO

Type-species: Pachylepis limacodina Felder, 1874, in Felder \& Rogenhofer, Reise öst.

Fregatte Novara (Zool.) 2 (Abt. 2): pl. 83, fig. 12; Erklärung: 7, by monotypy (of Pachylepis Felder). Type(s), BorNeo: Sarawak (BMNH).

$P$. limacodina is a junior subjective synonym of Ariola dilectissima Walker, 1858, List Specimens lepid. Insects Colln Br. Mus. 15: I 75 I.

PACHYLEPIS Felder, I874, in Felder \& Rogenhofer, Reise öst. Fregatte Novara (Zool.) 2

(Abt. 2): pl. 83; Erklärung: 7 .

Type-species: Pachylepis limacodina Felder, 1874, by monotypy.

A junior homonym of Pachylepis Pander, 1856, Monogr. fossilen Fische . . : 67, - Pisces. The objective replacement name is Yepcalphis nom. $\mathrm{n}$.

YERONGPONGA Lucas, igor, Proc. R. Soc. Qd 16: 82.

PLUS

Type-species: Yerongponga exequialis Lucas, I90I, ibidem 16: 83, by monotypy. Holotype ô. [Australia]: Qd, Bellenden Ker.

YIDALPTA nom. n. for Platydia Guenée, I854.

OPHI

Type-species: Platydia flavagalis Guenée, 1854, in Boisduval \& Guenée, Hist. nat.

Insectes (Lépid.) 8: 1 5, pl. 6, fig. I, by PRESENT DESIGNATION (for Platydia Guenée). Syntypes i ô, 2 ㅇ, Brazil (BMNH). Colombia.

PLAT YDIA Guenée, 1854, in Boisduval \& Guenée, Hist. nat. Insectes (Lépid.) 8: I 5.

Type-species: Platydia flavagalis Guenée, 1854 , by PIRESENT DESIGNATION.

A junior homonym of Platydia Costa, 1852, Fauna Regno Napoli 10 Anim. molli (5).

Brachiopodi: 47, - Brachiopoda. The objective replacement name is Yidalpta nom. 11.

YIGOGA nom. n. for Ogygia Hübner, [1821].

NOCT

Type-species: Noctua signifera [Denis \& Schiffermüller], 1775, Ankündung syst.

Werkes Schmett. Wienergegend: 8o, by subsequent designation (for Ogygia Hübner) by

Grote, 1895, Abh. naturw. Ver. Bremen 14:63, 64. Type(s), [Austria]: Vienna district

(Collection destroyed, Horn \& Kahle, 1936, Ent. Beih.'Berl.-Dahlem 3: 243).

OGYGIA Hübner, [I821] I 816, Verz. bekannter Schmett.: 225.

Type-species: Noctua signifera [Denis \& Schiffermiiller], I 775, by subsequent designation by Grote, 1895, Abh. naturw. Ver. Bremen 14: 63, 64.

A junior homonym of Ogygia Brongniart, I8I7, in Desmarest, Nouv. Dict. Hist. nat.

(Nouv. Edn) 8: 5I6, - Trilobita. The objective replacement name is Yigoga nom. $\mathrm{n}$.

${ }_{\ddagger} O X Y G I A$; Walker, I856, List Specimens lepid. Insects Colln Br. Mus. 9: 243.

An incorrect subsequent spelling. 
YPSIA Guenée, 1852, in Boisduval \& Guenée, Hist. nat. Insectes (Lépid.) 7: 16.

Type-species: Ypsia aeruginosa Guenée, I $85_{2}$, ibidem $7: 17$, pl. I 8, fig. 7, by subsequent designation by Grote, I874, Bull. Buffalo Soc. nat. Sci. 2: 46. Type(s), North America.

YPSORA Schaus, I901, Ann. Mag. nat. Hist. (7) 8: 77.

OPHI

Type-species: Ypsora santaris Schaus, I90I, ibidem (7) 8:77, by monotypy. Type(s), BrazIL (USNM, Washington).

YRIAS Guenée, 1852, in Boisduval \& Guenée, Hist. nat. Insectes (Lépid.) 7: 21.

Type-species: Phalaena acharia Stoll, I78I, in Cramer, Uitlandsche Kapellen 4: 108, pl. 346, fig. C, by subsequent designation by Desmarest, (1857), in Chenu, Encycl. Hist. nat. Papillons nocturnes: 127 . Type(s), Surinam.

YULA Bethune-Baker, 1906, Novit. zool. 13: 193.

ACRO

Type-species: Yula novaeguineae Bethune-Baker, I906, ibidem 13: I93, by original designation. Syntypes $\lesssim$, 우, New Guinea: [Papua], Ekeikei (BMNH).

ZACTHYS Viette, 1973, Nouv. Rev. Ent. 3: 189.

ACRO

Type-species: Zacthys biplaga Viette, I973, ibidem 3: I89, figs 5, 8, by original designation. Holotype $\hat{\sigma}$, Madagascar: $N$. de Morondava, Forêt de Marofandilia (MNHN, Paris).

ZAGIRA Walker, I866, List Specimens lepid. Insects Colln Br. Mus. 35: I637.

$A C O N$ Type-species: Zagira acidaliaria Walker, I 866, ibidem 35: 1638, by monotypy. Lectotype , BorNEo (UM, Oxford), designated (as type) by Swinhoe, I9oo, Cat. east. and Aust. Lepid. Heterocera 2: 68.

$Z$. acidaliaria is a junior subjective synonym of Carmara subcervina Walker, [1863].

See also Carmara Walker, [1863]; and Gyrognatha Hampson, I893.

ZAGORISTA Schaus, I916, Proc. U.S. natn. Mus. 50: 262, 318.

OPHI

Type-species: Drobeta debora Druce, I 898, Biologia cent.am. (Zool.) Lepid. Heterocera 2: 494, pl. 95, fig. 9, by original designation. Syntypes 2 ơ. Costa Rica: Candelaria Mts (BMNH).

ZALACA Walker, [1866] 1865, List Specimens lepid. Insects Colln Br. Mus. 34: I202. ACoN

Type-species: Zalaca anticalis Walker, [1866] I 865, ibidem 34: I202, by monotypy. Holotype ô. [South Africa]: Natal (BMNH).

$\boldsymbol{Z} \boldsymbol{A} \boldsymbol{L} \boldsymbol{E}$ Hübner, 1818, Zuträge Samml. exot. Schmett. 1: I I.

CATO

Type-species: Zale horrida Hübner, I8I8, ibidem 1: II, figs 3I, 32, by monotypy. Type(s) 中. [U.S.A.]: Georgia [formerly] in Florida.

$\ddagger$ LEMUR Hübner, I808, Erste Zuträge Samml. exot. Schmett.: 4, 5 .

Included in a work rejected for nomenclatural purposes by Int. Commn zool. Nom., I 966, Bull. zool. Nom. 23 Opinion 789: 213. Placed on the Official Index of Rejected and Invalid Generic Names in Zoology: Name No. 358.

Of the two originally included names, $\ddagger$ Lemur horrida (later made nomenclaturally available as Zale horrida Hübner, I8I8) was designated as type-species by Berio, I957, Memorie Soc. ent. ital. 36: 19.

$\ddagger$ LEMUR Hübner, 1818, Zuträge Samml. exot. Schmett. 1: II, I9, 34.

Not nomenclaturally available as a genus-group name. Hübner used Lemur, on all three pages, in a suprageneric sense for one of the names of his tribes (Namen der Stämme) listed on pages 33-34. Hübner listed his genus-group names on page 35.

ZALEODES Hampson, 1926, Descr. new Genera Species Lepid. Phalaenae Subfamily Noctuinae Br. Mus.: 429.

OPHI

Type-species: Zaleodes xylochroa Hampson, I926, ibidem : 430, by original designation. Holotype +, PERU: Yahuarmayo (BMNH). 
ZALEOPS Hampson, 1926, Descr. new Genera Species Lepid. Phalaenae Subfamily Noctuinae Br. Mus.: i 4 .

Type-species: Pheocyma umbrina Grote, 1883, Can. Ent.15:3, by original designation. Syntypes ô, ㅇ. [U.S.A.]: Arizona.

ZALISSA Walker, I 865, List Specimens lepid. Insects Colln Br. Mus. 33: 936.

$A G A K$

Type-species: Zalissa catocalina Walker, I865, ibidem 33: 936, by monotypy. Holotype , , [Australia]: [W. Aust.], R. Swan (B.INH).

$\ddagger$ EULISSA; Pagenstecher, 1909, Geogr. Verbreitung Schmett.: 423.

An incorrect subsequent spelling.

$\ddagger J A L I S S A$; Pagenstecher, 1909, Geogr. Verbreitung Schmett.: 423 .

An incorrect subsequent spelling.

ZANCLOGNATHA Lederer, I857, Noctuinen Eur.: 45, 2 II.

HYPE

Type-species: Pyralis tarsiplumalis Hübner, 1796, Samml. eur. Schmett. 6: pl. I9, fig. I25, by subsequent designation by Grote, I874, Bull. Buffalo Soc. nat. Sci. 2: 48. Type(s), Europe.

$P$. tarsiplumalis is a junior subjective synonym of Phalaena hunalis Scopoli, I763, Ent. Carniolica: $24 \mathrm{I}$.

See Frontispiece, fig. 5, Zanclognatha lunalis (Scopoli), ơ.

ZANCLOPALPUS Hampson, i 893, Illust. typical Specimens Lepid. Heterocera Colln Br. Mus.

9: 31,127 .

HYPE

Type-species: Micreremites rasalis Warren, 1891, Ann. Mag. nat. Hist. (6) 8: 6ך, by

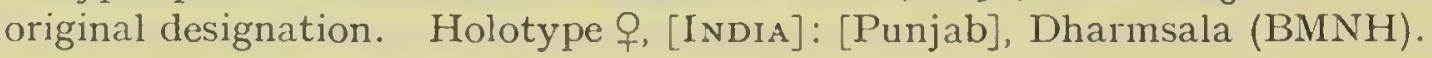

ZANCLOSTA THME Draudt, I937, in Seitz, Gross-Schmett. Erde 3 Suppl. : 279.

OPH I

Type-species: Zanclostathme elbursalis Draudt, 1937, ibidem 3 Suppl: 280, by monotypy. Syntypes I $\hat{o}$, I 9, [IRAN] Persia: Elburz Mts, Demavend, [Lar] Tar Valley (Collection destroyed in World War II).

The date of publication of the part of Seitz containing page 279 was stated on page 273 as 1937 September 27 th in both the German and the English editions.

ZARIMA Moore, I882, in Hewitson \& Moore, Descr. new Indian lepid. Insects Colln late Mr W. S. Atkinson: 162.

OPHI

Type-species: Zarima dentifera Moore, I882, ibidem: 162, p1. 5, fig. 19, by monotypy. Syntypes $\hat{\sigma}$, 오. India: Darjiling.

ZATILPA Dyar, 1920, Insecutor Inscit. menstr. 8: 50.

ACRO

Type-species: Grypotes dentifer Dyar, 1917, ibidem 5:66, by monotypy (of Grypotes Dyar, 1917). Holotype ô. U.S.A.: New Mexico, Las Vegas, Hot Springs (USNM, Washington).

G. dentifer is a junior subjective synonym of Acopa perpallida Grote, i 878, Can. Ent. 10: 68.

Zatilpa was proposed as the objective replacement name for Grypotes Dyar, I9I 7.

GRYPOTES Dyar, I9I7, Insecutor Inscit. menstr. 5: 66.

Type-species: Grypotes dentifer Dyar, I917, by monotypy.

A junior homonym of Grypotes Fieber, 1866, Verh. zool.-bot. Ges. Wien 16 (Abh.): 503, - Hemiptera. The objective replacement name is Zatilpa Dyar, I920.

ZAZUNGA Dyar, I9Io, Proc. U. S. natn. Mus. 38: 25I.

Type-species: Zazunga opinor Dyar, 1910, ibidem 38: 25I, by original designation. Syntypes $2 \hat{0}$, Mexico: Zacualpan and Orizaba. (USNM, Washington).

ZEBEEBA Kirby, 1892, Synonymic Cat. Lepid. Heterocera 1: 279.

$\operatorname{ACON}$

Type-species: Hypena falsalis Herrich-Schäffer, I839, in Panzer, Deutschlands Insecten Heft 166 : front cover, fig. I, by monotypy (of Nycteola Herrich-Schäffer). Type(s), ITALY: Sicily. 
Zebeeba was proposed as the objective replacement name for Nycteola Herrich-Schäffer, [1851].

NYCTEOLA Herrich-Schäffer, [I85I] I845, Syst. Bearb. Schmett. Eur. 2: 444.

Type-species: Hypena falsalis Herrich-Schäffer, I839, by monotypy.

A junior homonym of Nycteola Hübner, I 822,-Lepid., Noctuidae. The objective replacement name is Zebeeba Kirby, I 892 .

ZEKELITA Walker, I863, List Specimens lepid. Insects Colln Br. Mus. 27: I99.

HYPE

Type-species: Zekelita equalisella Walker, I863, ibidem 27: 199, by monotypy. Holotype $\widehat{\jmath}$, South AFRICA (BMNH).

ZELICODES Grote, (I896) I 895, Proc. Am. phil. Soc. 34: 4 I 8.

OPHI

Type-species: Litognatha linearis Grote, I883, Can. Ent. 15: I 2 I, by monotypy. Type(s) ㅇ․ [U.S.A.]: Arizona.

+ ZENOBIA Oken, I 815. See Ipimorpha Hübner, [1821].

ACRO

ZENOBIA Agassiz, I846. See Ipimorpha Hübner, [1821].

ACRO

ZENOMIA Dognin, I9I 4, Hétérocères nouv. Am. Sud 8: 52.

OPHI

Type-species: Zenomia alcisa Dognin, I9I4, ibidem 8: 52, by original designation. Holotype $\sigma$, Colombia: San Antonio.

ZETEOLYGA Billberg, I 820, Enumeratio Insect. Mus. G. J. Billberg: 87.

HADE

Type-species: Bombyx annulata Fabricius, [I777], Genera Insect.: 28I, by subsequent designation by Berio, I966, Annali Mus. civ. Stor. nat. Giacomo Doria 76: 67. Type(s), [Germany]: Hamburg (Type(s) not found by Zimsen, i964, Type Material of I. C. Fabricius: 532).

B. annulata is a junior subjective synonym of Phalaena confusa Hufnagel, I766, Berlin. Mag. 3 (4): 4I 4.

Incorrect type-species designation: Noctua conspersa [Denis \& Schiffermüller], I775, a name not originally included in Zeteolyg $a$ and not linked with one of the originally included names, when cited by Tams, 1939, Entomologist 72: 14I.

ZETHES Rambur, I 833, Annls Soc. ent. Fr. 2: 29.

OPHI

Type-species: Zethes insularis Rambur, I833, ibidem 2: 29, pl. 2, figs I, 2, by monotypy. Type(s), [France]: Corsica.

ZETHESIDES Hampson, 1926, Descr. new Genera Species Lepid. Phalaenae Subfamily Noctuinae Br. Mus.: 594. OPHI

Type-species: Zethes hesperioides Guenée, 1852, in Boisduval \& Guenée, Hist. nat. Insectes (Lépid.) 7: 330, pl. 24, fig. I I, by original designation. Lectotype ㅇ. [Australia]: [N.S.W.], Port Jackson (MNHN, Paris), designated by Viette, I95I, Bull. mens. Soc. linn. Lyon 20: 162.

ZEUXINIA Legrand, (1966) I965, Mém. Mus. natn. Hist. nat. Paris (A) 37: I 53. Hy Type-species: Zeuxinia aeschrina Legrand, (1966) 1965, ibidem 37: I54, figs 8, 9, by original designation. Holotype $\overrightarrow{0}$. ALDABRA I. (Indian Ocean) (MNHN, Paris).

Legrand attributed Zeuxinia to Hampson (M.S), and aeschrina to Gaede (M.S.).

ZIA Walker, I863. See Aquita Walker, i 863

NOLI

ZIELA Fletcher \& Viette, 1955, Bull. Inst. fr. Afr. noive 17(A): I 80.

HYPE

Type-species: Ziela biplaga Fletcher \& Viette, I955, ibidem 17(A): I80, pl. I, fig. 4, by original designation. Holotype $\widetilde{\sigma}$. [Guinea] French Guinea: Mont Nimba, Ziela (MNHN, Paris).

ZIGERA Walker, i862, J. Proc. Limn. Soc. (Zool.) 6: i82.

OPHI

Type-species: Zigera orbifera Walker, I862, ibidem 6: 182, by monotypy. Lectotype 
o., [BORNEO]: Sarawak (UM, Oxford), designated (as type) by Swinhoe, 1900, Cat. east, and Aust. Lepid. Heterocera 2: 193.

ZINNA Walker, I869, Characters undescr. Lepid. Heterocera: 95.

OPHI

Type-species: Zinna nigripalpis Walker, 1869, ibidem: 96, by monotypy. Type(s) o, [INDIA]: Benares (BMNH).

$O B B A$ Walker, I869, in Chapman, Proc. nat. Hist. Soc. Glasgow 1: 362.

Type-species: Obba prompta Walker, I 869, ibidem 1: 362, by monotypy. Type(s) J., CONGo (BMNH).

A junior homonym of Obba Beck, 1837, Index Mollusc. praesentis aevi Mus. Principis Augustissimi C. Frederici 1: 3o, - Mollusca. There is no objective replacement name but O. prompta is congeneric with Zinna nigripalpis Walker, 1869 , the type-species of Zinna Walker, I 869. The latter is therefore available for use as a subjective replacement name.

ZIRONA Walker, I 869, in Chapman, Proc. nat. Hist. Soc. Glasgow 1: 363.

OPHI

Type-species: Zirona marginata Walker, I 869, ibidem 1:363, by monotypy. Type(s)

Q. CONGO (BINH).

ZITNA Walker, [1866] I865, List Specimens lepid. Insects Colln Br. Mus. 34: I 276. ACON Type-species: Zitna albicinctalis Walker, [1866] I865, ibidem 34: I277, by monotypy. Lectotype, Borneo: Sarawak (UM, Oxford), designated (as type) by Swinhoe, I90o, Cat. east. and Aust. Lepid. Heterocera 2: 70.

ZIZA Walker, I863, List Specimens lepid. Insects Colln Br. Mus. 27: i 8.

HYPE

Type-species: Ziza ostentalis Walker, I863, ibidem 27: I 19, by monotypy. Type(s) $\hat{o}$ [BORNEO]: Sarawak (BMNH).

ZOBIA Saalmüller, 1891, Lepid. Madagascar: 384.

EUTE

Type-species: Ingura snelleni Saalmüller, I 88 I, Stettin. ent. Ztg 42: 433, by monotypy. Type(s) ô, Madagascar: Nossi-Bé (SNG, Frankfurt).

ZONESTHIOUSA Thierry-Mieg, I907. See Coccidiphaga Spuler, I907.

$\operatorname{ACON}$

ZOPHOCHROA Turner, 1902, Proc. Linn. Soc. N.S.W. 27 : i 8.

HYPE

Type-species: Zophochroa leucochorda Turner, I902, ibidem 27: I 18 , by subsequent designation by Berio, I966, Annali Mus. civ. Stor. nat. Giacomo Doria 76: 67, but cited as $\ddagger Z$. leucorhoda Turner, an incorrect subsequent spelling. Syntypes 20 . Australia: Qd, Townstille (ANIC, Canberra).

ZOROTHIS Schaus, i9i6, Proc. U.S. natn. Mus. 50: 265, 39I.

HYPE

Type-species: Zorothis zacualpana Schaus, I916, ibidem 50:391, by original designation. Type(s) ô, Mexico: Zacualpan (USNM, Washington).

ZORZINES Druce, I89I, Biologia cent.-am. (Zool.) Lepid. Heterocera 1: 446.

HҮִPE

Type-species: Zorzines plumula Druce, I891, ibidem 1: 446, pl. 36, figs I6, i 6a, by original designation. Holotype ô, Panama: Chiriqui.

ZOSTEROPODA Grote, 1874, Bull. Buffalo Soc. nat. Sci. 2: 22, 67.

HADE:

Type-species: Zosteropoda hirtipes Grote, r874, ibillem 2: 68, by original designation. Type(s) ô. [U.S.A.]: California (BMNH).

ZOTHECA Grote, 1874, Bull. Buffalo Soc. nat. Sci. 2: 23, 68.

ACRO

Type-species: Zotheca tranquilla Grote, 1874, ibidem 2: 69, by original designation. Syntypes ô. [U.S.A.]: California (BMNH).

ZUROBATA Walker, [1866] i 865, List Specimens lepid. Insects Colln Br. Mus. 34: 1276. ACON

Type-species: Zurobata rorata Walker, [1866] I 86.5, ibidem 34: 1276, by monotypy. Lectotype \%. Bornfon: Sarawak (UM, Oxford), designated (as type) by Swinhoe, r9oo, Cat. east. and Aust. Lepid. Heterocera 2: 68. 
ZUTRAGUM nom. n. for Trichosternum Draudt, I950.

CUCU

Type-species: Trichosternum likianga Draudt, I950, Mitt. mïnch. ent. Ges. 40: 80, pl. 5, fig. 20, by monotypy (of Trichostermum Draudt). Type(s), China: [Yunnan Prov.], Li-kiang (MAK, Bonn).

TRICHOSTERNUM Draudt, 1950, Mitt. münch. ent. Ges. 40: 79.

Type-species: Trichosternum likianga Draudt, I950, by monotypy.

A junior homonym of Trichostermum Wollaston, I8I6, Ann. Mag. nat. Hist. (3) 7: 205, Coleoptera. The objective replacement name is Zutragum nom. n.

\section{Summary of New Genus-group Names Proposed}

The following list summarises the junior homonyms for which new objective replacement names have been proposed in this catalogue.

Preoccupied name

Boana Walker, I 862

Borisia Filipjev, I949

Brachyblemma Hampson, 1926

Callogonia Hampson, 1908

Calymniodes Hampson, 1908

Cea Grote, 1883

Cremnodes Felder, 1874

Cynisca Fawcett, 1918

Dapha Walker, [I863 May I3th]

Daxata Walker, 1865

Dercetis Grote, 1878

Eleale Walker, I 862

Epiphanis Schaus, I9I3

Evemobates Turner, 1939

Englyphis Hübner, [I 825]

Euprora Hampson, 1926

Glaphyra Guenée, I 84I

Hansa Berio, 1959

Harmatelia Moore, I 882

Langona Barnes \& Lindsey, I92 I

Lasionota Warren, I9I2

Lena Herz, I903

Lithopoma Schaus, I9I6

Lycangesia Hampson, I910

Manobia Staudinger, I 896

Matella Moore, [I 885]

Microblemma Hampson, I9Io

Micropia Hampson, I908

Naxia Guenée, I 852

Neptunia Barnes \& McDunnough, I9I I

Niphosticta Turner, 1936

Nitocris Guenée, I 868

Odontina Guenée, I 862

Odontosema Warren, I9I3

Ogygia Hübner, [I82I]

Orixa Walker, I 869

Osdara Walker, 1865
Replacement name

Naboa nom. n.

Sirioba nom. n.

Habershonia nom. n.

Goonallica nom. n.

Hampsonodes nom. n.

Sparkia nom. n.

Mocrendes nom. $\mathbf{n}$.

Siccyna nom. $\mathbf{n}$.

Hapda nom. n.

Tadaxa nom. n.

Redectis nom. $\mathbf{n}$.

Tamseale nom. $\mathbf{n}$.

Ipanephis nom. $\mathbf{n}$.

Tabomeeres nom. $\mathbf{n}$.

Hulypegis nom. n.

Roperua nom. $\mathbf{n}$.

Rhypagla nom. n.

Beriohansa nom. n.

Imaharela nom. $\mathbf{n}$.

Gloanna nom. n.

Isatoolna nom. $\mathbf{n}$.

Knappia nom. n.

Mopothila nom. n.

Quandara nom. n.

Niaboma nom. n.

Talmela nom. $\mathbf{n}$.

Abablemma nom. $\mathbf{n}$.

Pricomia nom. $\mathbf{n}$.

Xiana nom. $\mathrm{n}$.

Uniptena nom. $\mathbf{n}$.

Hurworthia nom. n.

Rictonis nom. n.

Tinnodoa nom. n.

Tesomonoda nom. $\mathbf{n}$.

Yigoga nom. n.

Xoria nom. n.

Radosa nom. n. 
Preoccupied name

Pachylepis Felder, I 874

Pagetia Prout, 1922

Pangraptica Hampson, 1926

Paracodia Hampson, I 9 ro

Paromia Schaus, I9I 3

Pfeifferella Osthelder, I 933

Phasis Walker, I 854

Philometra Grote, 1872

Phorocera Guenée, I 852

Platydia Guenée, 1854

Pleroma Smith, I89 I

Plotheia Walker, 1863

Pseudosmia Köhler, (1952)

Rhynchodes Guenée, I 852

Setagrotis Warren, I9I 2

Stictothripa Hampson, I9I 2

Tomyris Druce, I 890

Trichosternum Draudt, $195^{\circ}$
Replacement name

Yepcalphis nom. n.

Tegiapa nom. n.

Craptignapa nom. $\mathbf{n}$.

Cadiorapa nom. n.

Ramopia nom. $\mathbf{n}$.

Ostheldera nom. n.

Shapis nom. n.

Epidelta nom. n.

Recoropha nom. n.

Yidalpta nom. n.

Pleromelloida nom. n.

Thopelia nom. n.

Despumosia nom. n.

Hondryches nom. n.

Estagrotis nom. n.

Hayesia nom. n.

Rowdenia nom. $\mathbf{n}$.

Zutragum nom. $\mathbf{n}$.

\section{Summary of New Species-group Names Proposed}

In three genera the nominal species which have been designated as type-species have been shown to be misidentifications of hitherto unnamed species. These have now been named in this catalogue and cases should be submitted to the Commission under the Code, Article 7o(a). The genera and new species-group names are as follows.

\section{Pachythrix Turner, 1942 Pachythrix hampsoni sp. n. \\ Poena Druce, I89I Poena drucella sp.n. \\ Safidia Hampson, r913 Safidia druceria sp. n.}

In two genera the type-species were each found to be junior primary homonyms and neither of them had a synonym which could be used as a replacement name. New species-group names have therefore been proposed.

Preoccupied name

Hadena laetabilis Smith, I 899

Noctua evidens Hübner, [1808]
Replacement name

Paramiana perissa nom. n.

Sideridia anaphelea nom. n.

\section{Index to Species-group Names}

Species-group names are listed with author and original genus. The names following a semicolon are the genus-group names, listed in the catalogue in bold type, under which the species-group names will be found.

abactalis Walker, Masca; Masca

abadirina Hübner, Thermesia; Thermesia

abavalis WValker, Echana; Echana

abditalis Walker, Platydia; Gustiana

abjecta Hübner, Noctua; Elecmosia abjudicalis Walker, Bertula; Bertula ablualis Walker, Adrapsa; Adrapsa ablunaris Guence, Ophisma; Geria abnormalis Schaus, Goednes; Goednes abrostolina 13utler, Inguridia; Inguridia 
abrostoloides Butler, Plusidia; Plusidia abrostoloides Hampson, Proconis; Proconis abrupta Grote, Raphia; Certila abrupta Walker, Focilla; Trogogonia abrupta Walker, Mestleta; Mestleta abscissa Saalmüller, Daula; Daula abscondens Walker, Coenipeta;

Hypogrammodes absentimacula Guenée, Naxia; Xiana abseuzalis Walker, Motya; Motya absidum Harvey, Ablepharon; Copablepharon absinthiata Clerck, Phalaena; Eupithecia absita Felder \& Rogenhofer, Metoptria;

Cytothymia absorptalis Walker, Hormisa; Hormisa abstrusa Dyar, Poenopsis; Poenopsis absumens Walker, Thermesia; Metallata abunda Felder \& Rogenhofer, Epimecia; Epimeciodes abydas Herrich-Schäffer, Noctua;

\section{Parachabora}

ahyssinia Guenée, Spodoptera; Eulaphygma acanthusalis Walker, Dierna; Dierna acarnea Smith, Noctua; Trichosilia accingalis Walker, Egnasia; Talmela accipiens Walker, Blenina; Blenina accipiter Felder \& Rogenhofer, Spintherops;

Anepilecta acclamalis Walker, Gisira; Gisira acclinalis Hübner, Antiblemma; Antiblemma aceris Linnaeus, Phalaena; Arctomyscis acetosellae [Denis \& Schiffermüller], Noctua; Mesogona achaemenica Wiltshire, Margelana; Persidia achaeoides Walker, Elpia; Elpia acharia Stoll, Phalaena; Yrias achatina Weymer, Thyatira; Thyatirina acholi Bethune-Baker, Catephia;

Amblyprora acidaliaria Walker, Zagira; Zagira acineta Turner, Phaeomorpha; Phaeomorpha acosmopis Turner, Epitripta; Epitripta acroleuca Dyar, Melanchroiopsis;

Melanchroiopsis acronyctella Walker, Pardasena; Pardasena acronyctoides Walker, Audela; Audela acronyctoides Walker, Barasa; Barasa acronyctoides Walker, Crioa; Crioa acronyctoides Walker, Hisbanda; Hisbanda acronyctoides Walker, Tachosa; Tachosa;

Anabathra acrophylax Tams, Saltia; Saltia acrypta Turner, Prostheta; Prostheta actinobola Felder, Chaetoloma;

Chaetoloma

acuminata Hampson, Acutipenna;

Acutipenna

acuta Walker, Baratha; Baratha

acuta Walker, Plusia; Neoplusia

acutalis Schaus, Sorygaza; Trogoblemma acutalis Walker, Doryodes; Doryodes acutangula Hampson, Gonelydna;

Gonelydna

acutangula Hampson, Neaxestis; Neaxestis acutaria Herrich-Schäffer, Ligia; Doryodes acutipennis Schaus, Megachyta; Upothenia acutissima Bethune-Baker, Parathermes;

Hypopleurona

acutissima Grote, Pachypolia; Platypolia acutus Smith, Conochares; Conochares acynodonta Dognin, Anthodes; Anthodes acypera Hampson, Talapa; Malagonia adeba Swinhoe, Vestura; Vestura adelopis Lower, Prorocopis; Stenoprora adin Schaus, Neolita; Ablita adjutrix Cramer, Phalaena; Athyrma admiraltensis Hampson, Rhesalides;

Rhesalides adspersa Felder \& Rogenhofer, Felinia;

Attonda adulatrix Hübner, Noctua; Eutelia adustalis Hampson, Hypena; Stenhypena advena Grote, Oxycnemis; Oxycnemis adversa Grote, Litosea; Litosea aemula Hübner, Idia; Idia aenea Butler, Honorana; Distagma aenea [Denis \& Schiffermüller], Noctua;

Phytometra aenochromoides Walker, Ombrea; Ombrea aeolia Druce, Isogona; Bendisodes aequa Walker, Ausinza; Ausinza aequalis Walker, Motina; Motina aequatoria Mabille, Diphthera; Halochroa aerea Hübner, Noctua; Allagrapha aeruginea Hübner, Noctua; Dichonia aeruginosa Guenée, Ypsia; Ypsia aeschra Hampson, Sminthopses;

Sminthopses aeschrina Legrand, Zeuxinia; Zeuxinia aetheria Grote, Thalpochares; Hyperstrotia affabilis Möschler, Anateinoma;

Anateinoma affinis Boisduval, Agarista; Idalima afflicta Grote, Acronycta; Tricholonche africana Holland, Deinopalpus; Deinopalpus agavis Blasquez, Bombyx; Proteinania agenjoi Fernández, Esteparia; Esteparia 
aglossoides Guenće, Diatenes; Setida agonax Druce, Anarta; Gonostygia agrippina Cramer, Phalaena; Thysania agropoides Walker, Caedesa; Caedesa agrotina Guenée, Celaena; Psilomonodes agrotina Staudinger, Amphidrina;

Amphidrina

ahenea Hübner, Agrapha; Agrapha

alabamae Grote, Anicla; Anicla

alabastraria Hübner, Eulepidotis;

Eulepidotis

alala Druce, Xanthia; Hrdroeciodes alampeta Bethune-Baker, Aneliopis;

Aneliopis

alaskae Grote, Agrotis; Epipsiliamorpha alba Barnes \& McDunnough, Redingtonia;

Redingtonia

alba Rothschild, Pristoceraea; Leucovis albago Fabricius, Noctua; Xanthodes albalis Walker, Hypena; Sorocostia albapex Hampson, Lobotorna; Lobotorna albatra Hulstaert, Mesenıbreosa;

Mesembreosa

albavitta Ottolengui, Autographa; Adeva albescens Moore, Nagasena; Nagasena albibasis Draudt, Melaleucantha;

Melaleucantha albicans Walker, Elousa; Elousa albiceps Druce, Paracodia; Paracodia albicilia Hampson, Clapronia; Clapronia albicinctalis Walker, Zitna; Zitna albicinctus Kollar, Erebus; Cariona albicola Walker, Felinia; Bamra albicosta Hübner, Noctua; Synmerista albicosta Moore, Aethia; Pseudacidalia albicosta Walker, Carthara; Carthara albida Hampson, Stylorache; Stylorache albida Hampson, Timora; Lecerfia albida Walker, Bantana; Bantana albidens Walker, Moepa; Moepa albidentaria Freyer, Acidalia; Pericyma albidentata Hampson, Avittonia; Avittonia albidisca Moore, Hadena; Xenotrachea albidus Blanchard, Peropalpus; Peropalpus albifascia Hampson, Gyrtonides; Gyrtonides albifasciata Hampson, Stomafrontia;

Stomafrontia albifera Hampson, Trogobriga; Trogobriga albifissa Druce, Gonodes; Gonodes albifrontata Moore, Timandra; Trogacontia albigutta Karsch, Pristoceraea;

Charitosemia albigutta Swinhoe, Talapa; Athyrmina albigutta WValker, Stridova; Stridova albiguttalis Walker, Maguza; Maguza albilinea Hampson, Nanamonodes; Nanamonodes albiluna Hampson, Atrephes; Atrephes albiluna Hampson, Ortholeuca; Ortholeuca albinotata Butler, Amblygoes; Capnistis albiocula Barnes \& McDunnough,

Hoplotarache; Airamia

albipoda Berio, Acremma; Acremma albipuncta [Denis \& Schiffermüller], Noctua; Hyphilare albipuncta Druce, Ogoas; Ogoas albirena Christoph, Photedes; Metoponrhis albirena Druce, Mictochroa; Mictochroa albirena Moore, Mamestra; Lophotyna albiscripta Hampson, Zurobata;

Pseuderiopus

albisigna Moore, Mathura; Mathura albisignata Janse, Neocucullia; Neocucullia albistella Walker, Osericana; Osericana albistigma IVileman \& South, Pangraptica; Pangraptica

albistriata Hampson, Aphorisma;

Aphorisma

albistriga Draudt, Rhabinogana;

Rhabinogana

albistriga Schaus, Herminodes;

Rhamnocampa

albistrigata Bethune-Baker, Argillana;

Argillana

albistrigatis Haworth, Crambus; Schrankia albivenata Warren, Euonychodes;

Euonychodes

albivirgula Berio, Parelia; Parelia albizona Latreille, Noctua; Ramphia albizonata Hampson, Uripao; Uripao alboarcuata Bethune-Baker, Engorna;

Heoeugorna

albocincta Warren, Erebostrota;

Erebostrota

albolineata Blachier, Calophasia;

Criophasia

albolunata Moore, Hingula; Hingula

albomacula Hulstaert, Eopaectes:

Eopaectes

albomaculalis Bremer, Herminia;

Stenberginania

albomaculata Stoll, Phalaena; Leucosemia albonigra Bytinski-Salz \& Brandt,

Brandtina; Brancltina

albonitens Bremer, Acontia; Chasminodes albooculata Saalmuiller, Capmocles; Tracta alboplagiata Smith, Tristyla; Tristyla 
albopunctata Bethune-Baker, Gonopteronia; Gonopteronia

albopunctata Bethune-Baker, Parhypena; Parhypena

albopunctata Bethune-Baker, Zethes;

Silignea

albopunctata Walker, Homoptera; Caduca

albopunctella Walker, Cutina; Cutina

albosignata Staudinger, Chara; Chara

albostriata Bethune-Baker, Paragarista;

Paragarista

albotentata Walker, Nechesia; Nechesia

albovenosa Goeze, Phalaena; Arsilonche

albovittata Hampson, Phycidopsis;

Phycidopsis

albula Bowden, Carelis; Carelis

albulalis Walker, Characoma; Characoma

alceste Druce, Capnodes; Micranthops

alchymista [Denis \& Schiffermüller],

Noctua; Catephia

alcisa Dognin, Zenomia; Zenomia

aleada Smith, Faronta; Faronta

aleuca Guenée, Odontodes; Odontodes

aleuropasta Turner, Paurophylla;

Paurophylla

algae Fabricius, Noctua; Euthales

algira Linnaeus, Phalaena; Dysgonia

algoa Felder \& Rogenhofer, Hadena;

Hypoplexia

aliena Walker, Arugisa; Arugisa

aliena Walker, Girpa; Girpa

aliena Walker, Scambina; Scambina

alluaudi Mabille, Sarothroceras;

Sarothroceras

alni Linnaeus, Phalaena; Jocheaera

alope Cramer, Phalaena; Lacera

alpigena Boisduval, Hadena; Heterommiola

alpium Osbeck, Phalaena; Moma

alpnista Fletcher, Homonacna;

Homonacna

alsines Brahn, Phalaena; Hoplodrina

alstoni Hampson, Eublemma;

Mesophractias

alsusalis Walker, Onevatha; Onevatha amabilis Drury, Phalaena; Crameria amaranta Felder \& Rogenhofer, Euplexia;

Tracheplexia

amasia Esper, Phalaena; Eunetis

amata Bremer \& Grey, Catocala;

Chrysorithrum

amata Butler, Rhodaria; Pyralidesthes

amataria Linnaeus sensu Hübner, Phalaena;

Timandra

amatrix Hübner, Noctua; Lamprosia amatrix Westwood, Eusemia; Scrobigera amaura Turner, Proschora; Proschora amaurodes Lower, Agrotis; Proteuxoa ambages Strand, Schalidomitra;

Schalidomitra ambigualis Walker, Gaberasa; Gaberasa ambusta [Denis \& Schiffermüller], Noctua;

Brachycosmia

ambusta Mabille, Hypsa; Arctiopais

anrella Guenée, Campometra; Campometra

ameria Druce, Polia; Megastopolia

amethystina Hübner, Hemeroblemma;

Hemeroblemma

amethystina Hübner, Noctua; Eucarta amica Hübner, Ephesia; Corisce

amica Treitschke, Hadena; Blepharita

amicina Staudinger, Hadena; Euxenistis

amoena Hübner, Noctua; Eublemma

amoena Möschler, Toxonprucha;

Toxonprucha

amoena Staudinger, Nonagria; Virgo

amoenita Stoll, Phalaena; Argyrosticta

amoenus Staudinger, Phoebophilus;

Phoebophilus

amphidecta Butler, Marmorinia;

Amphitrogia

amphipyroides Guenée, Latebraria;

Latebraria

amphix Cramer, Phalaena; Lyncestis

ampla Schaus, Gorosina; Gorosina

ampla Walker, Chabuata; Chabuata

amplificans Walker, Empelathra;

Empelathra

amplior Walker, Anua; Anua

amplipennis Turner, Epigrypodes;

Epigrypodes

amplivitta IValker, Vogia; Vogia

ampolleta Draudt, Chamyrisilla;

Chamyrisilla

amynta Cramer, Phalaena; Ceroctena amyntoralis Walker, Throana; Throana anaemica Hampson, Microzada; Microzada anaphelea nom. n., Sideridis; Sideridis anarrhini Duponchel, Cleophana; Amephana anartoides Walker, Plusiodonta; Chirconia anartoides Warnecke, Metoponrhis;

Beihania

anceps Mabille, Thermesia; Mecodinops anceps Staudinger, Hepatica; Hepatica anceps Stephens, Miselia; Platy polia anderreggii Boisduval, Leucania; Sablia angasii Angas, Agarista; Cremnophora angasii Walker, Apina; Cremnophora angasii Wing, Caligatus; Caligatus 
angelica Smith, Copibryophila;

Copibryophila

angelica Smith, Heliodes; Microhelia

angelicus Smith, Cobalos; Cobalos

angola Bethune-Baker, Enispades;

Enispades

angola Bethune-Baker, Neuranethes;

Neuranethes

angulalis Hübner, Pyralis; Palthis

angulata Barnes \& Lindsey, Nothophila;

Bandelia

angulata Bethune-Baker, Plasmaticus;

Plasmaticus

angulata Fabricius, Bombyx; Dabarita

angulata Schaus, Coscaga; Coscaga

angulata Smith, Perigonica; Perigonica

angulatilinea Bethune-Baker, Episparis;

Episparonia

angulifera Moore, Bankia; Euxestis

angulipennis Butler, Mitothemma;

Mitothemma

angulipennis Moore, Amrella; Amrella

angulipennis Moore, Corcobara; Corcobara

anguliplaga Walker, Galapha; Tephrias

angusta Butler, Gerbatha; Crerbathodes

angustalis [Denis \& Schiffermüller], Pyralis;

Synaphe

angustipennis Hampson, Aethodes;

Aethodes

angustipennis Schaus, Schazama; Schazama angustula Turner, Stonychota; Stonychota anilis Drury, Phalaena; Argyrostrotis ankarensis Rebel, Episema; Pachyagrotis ankasoka Viette, Decarynodes; Decarynodes annulata Fabricius, Bombyx; Zeteolyga annulata Swinhoe, Hyperaeschra; Triorbis annulella Hampson, Tarache; Selenistis annulifera Walker, Mouralia; Mouralia anodonta Guenée, Phlogophora;

Conservula anomala Haworth, Phytometra; Stilbia anormalis Barnes \& McDunnough, Friesia;

Friesia

anormalis Hampson, Tarache; Iambiodes anotha Dyar, Platyperigea; Protoperigea ansorgei Bethune-Baker, Paralophata;

Paralophata

antecedens Walker, Galapha; Galapha antennalis Berio, Audea; Davea antennalis Strand, Euheterospila;

Euheterospila

antennata Butler, Roeselia; Callinola

antennata Moore, Lugana; Lugana

antennata Smith, Meleneta; Meleneta anterosticta Dognin, Thermesia;

Rhopalognatha

anthracia Meyrick, Elaphristis; Elaphristis anticalis Walker, Zalaca; Zalaca antinympha Hübner, Ephesia; Catabapta antiqua Staudinger, Eicomorpha;

Eicomorpha

antiqualis Hübner, Pyralis;

Rhynchodontodes

antirrhinii Hübner, Noctua; Omphalophana antitermina Hampson, Paracarsia;

Paracarsia

antonito Barnes, Mamestra; Mimobarathra

aperta Walker, Evonima; Evonima

aperta Walker, Iscadia; Iscadia

apfelbecki Rebel, Hiptelia; Pseudoxestia

aphelioides Möschler, Haplostola;

Haplostola

apiata Grote, Orthosia; Epiglaea

apicalis Felder, Pachyplastis;

Pachyplastis

apicalis Guenée, Erygia; Erygia; Calicula apicalis Moore, Doranaga; Doranaga

apicalis Moore, Dordura; Dordura

apicalis WValker, Lochia; Lochia

apicalis Walker, Mosara; Mosara

apicalis Walker, Sphetta; Sphetta

apicata Dognin, Ephyrodes; Herpoperasa

apicata Druce, Aristaria; Drucuma

apicata Hampson, Polionycta; Polionycta

apicella Grote, Emmelia; Heliocontia

apicina Herrich-Schäffer, Orodesma;

Orodesma

apicinota Turner, Eremnophanes;

Eremnophanes

apicipuncta Turner, Niphosticta;

Hurworthia

apicosa Haworth, Phytometra; Neoerastria apis Druce, Hypenodes; Scutirodes apotheina Brandt, Sidemia; Eremophysa appollina Guenée, Calliodes; Calliodes appollinis Guenée, Leocyma; Leocyma apposita Barnes \& McDunnough, Stilbia;

Hemistilbia

approximata Walker, Eutelia; Atacira aprepes Hampson, Pariambia; Pariambia aprica Hübner, Noctua; Tarache apriformis Walker, Capotena; Capotena aprilina Linnaeus, Phalaena; Griposia aracinthusalis Walker, Mardara; Mardara arbosi Dognin, Gaujonia; Gaujonia arbuti Fabricins, Noctua; Heliodes arbuticolens Butler, Celaena; Trichestra archesia Cramer, Phalaena; Cauninda 
arcifera Mabille, Orthosia; Argyphia arcta Lederer, Hadena; Dexiadena arctica Aurivillius, Schoyenia; Schoyenia arctomys Alphéraky, Isochlora; Chamyla arcuata Berio, Mythymima; Mythymima arcuata Berio, Sculptifrontia; Sculptifrontia arcuata Bethune-Baker, Parasiopsis;

Parasiopsis arcuata Walker, Xanthodes; Maurilia arcuinna Hübner, Phalaena; Odice ardalus Druce, Ipnea; Mopothila ardescens Butler, Agarista; Periopta arduine Druce, Ledaea; Dialoxa arefacta Swinhoe, Remigia; Rhabdophera arenacea Hampson, Ozarba; Arenarba arenalis Walker, Perta; Perta areola Esper, Phalaena; Xylocampa areos Cramer, Phalaena; Habershonia argentalis Moore, Aglossa; Rhynchopalpus argentata Barnes \& McDunnough, Provia;

Provia argentata Butler, Micardia; Micardia argentata Druce, Euthisanotia; Laquea argentea Hufnagel, Phalaena; Argyrogalea;

Argyritis

argentea Walker, Libunca; Libunca argenteostriata Barnes, Hemigrotella;

Hemigrotella argentescens Schaus, Makapta; Makapta argentifascia Hampson, Leiorhynx;

Leiorhynx

argentifera Berio, Eublarginea;

Eublarginea argentifera Hampson, Homodina;

Homodina argentilinea Druce, Didugua; Didugua argentilinea Walker, Adra; Adra argentilinea Walker, Gariga; Gariga argentina Köhler, Austrandesia;

Austrandesia argentipes Walker, Gomora; Gomora argentistriga Hampson, Schistorhynx;

Schistorhynx argentula Hübner, Phalaena; Deltote argillacea Alphéraky, Eicomorpha;

Perissandria argillacea Butler, Egnasia; Aventina argillacea Hübner, Aletia; Alabama argillosa Boursin, Datungia; Datungia argyra Druce, Plusia; Metaplusia argyresthis Hampson, Ptyorhyncha;

Ptyorhyncha argyritis Hampson, Elaeognatha; Elaeognatha argyrofulva Köhler, Desertullia;

Desertullia

argyrogramma Hampson, A paegocera;

Apaegocera

argyrolepia Hampson, Lophodelta;

Lophodelta

argyropasta Hampson, Polygrapta;

Polygrapta

argyrophora Hampson, Lophocryptis;

Lophocryptis

argyrozona Joannis, Leucophanera;

Leucophanera

aridoxa Turner, Cycloprora; Hedymiges

armata Alphéraky, Hadena; Pseudohadena

armata Grote, Fota; Fota

armata Walker, Bolica; Bolica

armeniaca Staudinger, Praestilbia;

Praestilbia

armigera Hübner, Noctua; Helicoverpa

aroa Bethune-Baker, Pseudodeltoida;

Pseudodeltoida

aroa Bethune-Baker, Zethes; Carpheria

aroensis Bethune-Baker, Foveades;

Foveades

arrosa Harvey, Trama; Lepidotrama

artega Barnes, Tricholita; Lophoceramica

artemisiae [Denis \& Schiffermüller],

Noctua; Argyritis

arterialis Draudt, Trichospolas;

Trichospolas

artia Turner, Clytophylla; Clytophylla arvalis Grote, Axenus; Axenus asaphes Turner, Aethalina; Aethalina asemantica Turner, Asaphes; Paventia asiatica Alphéraky, Monostola; Monostola asna Schaus, Neocodia; Neocodia assimilis Grote, Trichotarache;

Trichotarache

astarte Doubleday, Heterocampa;

Heterocampa

asteris [Denis \& Schiffermüller], Noctua;

Euderaea

asthenoides Möschler, Clapra; Clapra

atava Felder \& Rogenhofer, Achantodes;

Disticta

athene Weymer, Xanthospilopteryx;

Weymeria

atkinsoni Moore, Adisura; Adisura

atkinsoni Moore, Pimprana; Pimprana

atlantica Herrich-Schäffer, Amblygonia;

Amblygonia

atmocyma Fletcher, Tosacantha;

Tosacantha

atomaris Hübner, Phoberia; Phoberia 
atomifera W'alker, Devena; Devena atrapex Hampson, Raparna; Acygnatha atrata Butler, Pseudaglossa; Falcimala atrata Morrison, Pteroscia; Pteroscia atriceps Hampson, Gyrognatha;

Gyrognatha

atriciliata Grote, Platysenta; Platysenti atricollaris Harvey, Homohadena;

Copihadena

atricornis Grote, Pachypolia; Pachypolia atrifasciata Morrison, Metahadena;

Metahadena

atrigutta Walker, Lyclene; Metaemene atrilineella Grote, Cleptomita; Cleptomita atripalpis Hampson, Haemaphlebia;

Haemaphlebia atriplaga Walker, Toxocampa; Tephrias atriplicis Linnaeus, Phalaena; Trachea atripuncta Hampson, Rhyncholita;

Rhyncholita atripustula Walker, Gangra; Gangra atrirena Hampson, Caradrina; Anathetis atrisigna Hampson, Galleridia; Galleridia atrisignata Hampson, Nolasodes; Nolasodes atrisignata Hampson, Oglasodes; Oglasodes atrosignata Walker, Anumeta; Anumeta atrosignata Walker, Ariathisa; Ariathisa atrosignata Walker, Chadaca; Chadaca attenuata Hampson, Araea; Araea augias Herrich-Schäffer, Orthia; Orthia augur Fabricius, Noctua; Graphiphora augusta Stoll, Phalaena; Gorgone augustipennis Grote, Tarache; Therasea aurantia Moore, Ophideres; Khadira aurantiaca Alphéraky, Omorphina;

Omorphina

aurantiaca Edwards, Annaphila; Incita aurantiago Guenée, Xanthia; Rhodoecia aurariae Oberthür, Taeniocampa;

Clavipalpula

aurata Aurivillius, Xanthodesma;

Xanthodesma

aurea Grote, Hadena; Copanarta

aurea Hübner, Noctua; Panchrysia

aureopicta Kenrick, Eutelia; Eutelephia

aureus Bang-Haas, Chrysoptera;

Chrysonicara

auricinctaria Grote, Melanomma;

Melanomma

auricoma [Ienis \& Schiffermüller], Noctua;

Viminia

auricularis Hübner, Noctua; Meropis

aurigera Walker, Berrhaea; Berrhaea

aurilinea Walker, Mareura; Mareura auripennis Grote, Harveya; Harveya auripennis Schaus, Hypogramma;

Cryptochrysa

auritincta Hampson, Carea; Lophocrama aurora Joannis, Miniophyllodes;

Miniophyllodes aurora Turati, Pseudopolia; Pseudopolia auroralis [Denis \& Schiffermüller], Pyralis;

Anteois australe Felder, Toxoloma; Toxoloma australis Boisduval, Xylina; A porophyla autoplusia Dyar, Chalcamistis; Chalcamistis auxiliaris Grote, Agrotis; Chorizagrotis axrlides Hampson, Micragrotis; Micragrotis azelinoides Schaus, Ampelasia; Ampelasia azteca Schaus sensu Hampson, Safidia; Safidia

baboquivariensis Barnes \& Benjamin, Supralathosea; Supralathosea hadistriga Grote, Hadena; Homohadena baeota Turner, Dendrothripa; Microthripa baetica Rambur, Cleophana; Cleonymia baja [Denis \& Schiffermüller], Noctua; Anathes balanitis Grote, Agrotis; Protexarnis balma Guenée, Hypogramma;

Hypogrammodes bambusalis Leech, Hypoepa; Hypoepa bankiana Fabricius, Pyralis; Deltote barbalis Clerck, Phalaena; Pechipogo barbata 1 Holland, Hypodeva; Hypodeva barbitula Herrich-Schäffer, Periphrage;

Periphrage baresalis Walker, Herminia; Palthisomis basalis Moore, Harmatelia; Itmaharela basalis Saalmüller, Ozopteryx; Ozoptery basalis Walker, Andrapha; Andrapha basalis Walker, Etanna; Etanna basalis Walker, Marathyssa; Marathyssa basicarnea Walker, Hadena; Hampsonodes basicunea Walker, Calathusa; Calathusa basifusca Bethune-Baker, Acatapanstus; Acatapaustus

basilinea Bowden, Manga; Manga basilinea [Denis \& Schiffermüller], Noctua; Apamea basilinea Hampson, Polydesma; Archephia basilinea Köhler, Galeana; Galeana basipuncta Schultze, Crusiseta; Crusiseta basipunctum Walker, Piala: Piala 
basistriga Walker, Phineca; Phineca batis Linnaeus, Phalaena; Thyatira batisella Hampson, Micracontia;

Micracontia

bayni, Hampson, Callargyra; Callargyra beatrix Moore, Pradatta; Pradatta behri Angas, Agarista; Comocrus belangeri Guérin-Méneville, Agarista; Fleta belfragiana Harvey, Catocala; Andrewsia bella Bethune-Baker, Cirrodiana; Cirrodiana bella Bethune-Baker, Pseudozalissa;

Pseudozalissa

bella Butler, Siglophora; Siglophora belladonna Edwards, Melicleptria;

Dysocnemis bellicula Hübner, Lithacodia; Lithacodia bellinita Guenée, Thyria; Thyria benedicta Dyar, Cirrodistis; Cirrodistis benenotata Warren, Pandesma; Hypoglaucitis

berenice Fawcett, Galactomoia; Galactomoia bergi Köhler, Metacullia; Metacullia berioi Viette, Elyptron; Tegarpagon bertha Schaus, Eustrotia; Neomonodes beryllodes Turner, Diphthera; Pansemna betsileo Viette, Xylomania; Maghadena biasalis Walker, Bocana; Hipoepa bicolor Barnes \& McDunnough,

Phoenicophanta; Callostolis bicolor Herrich-Schäffer, Erythrochrus; Erythrochrus bicolor Mabille, Arctia; Lepidopalpia bicolor Moore, Acronycta; Pseudepunda bicolor Rothschild, Arrothia; Arrothia bicolorago Guenée, Xanthia; Sunira bicolorana Fuessly, Phalaena; Hylophilina bi[c]olorata Hufnagel, Phalaena; Mamestra bicostata Berio, Episcotia; Episcotia bicruris Hufnagel, Phalaena; Hadena bieti Oberthür, Agarista; Syfania bifacies Walker, Cryassa; Cryassa bifacies Walker, Menecina; Menecina bifaria Walker, Lacibisa; Lacibisa bifasciata Hampson, Anachrostis; Mimeugoa bifidalis Grote, Tortricodes; Eulintneria biformis Walker, Dandaca; Belciana bigutta Walker, Thermesia; Epicarsia biguttalis Walker, Tribunta; Tribunta biguttata Walker, Britha; Britha bijungens Walker, Daxata; Tadaxa bilacteata Berio, Pseudelyptron;

Pseudelyptron bilinea Hampson, Polia; Protomiselia bilinea Hübner, Noctua; Grammesia bilinea Schaus, Phrodita; Phrodita bilinea Swinhoe, Cletthora; Homophlebia bilineata Berio, Engusanacantha;

Engusanacantha bilineatella Walker, Subrita; Subrita bilineatus Hampson, Acantholipes;

Orthogrammica billeti Joannis, Oxyodes; Rectipalpula bimacula Walker, Nebrissa; Nebrissa bimacula Wileman \& South, Trigonodesma;

Trigonodesma

bimaculata Herrich-Schäffer, Agarista;

Alypiodes

bimaculata Snellen, Herminodes;

Trichoptya

bimaculata Walker, Arabriga; Arabriga

bimaculata Warren, Sillophora; Sillophora

bimaculosa Linnaeus, Phalaena; Meganephria

bimarginata Walker, Culicula; Culicula bimatris Harvey, Pippona; Pippona binotata Walker, Maresia; Maresia bipars Hampson, Zagira; Oxytrita biparticolor Hampson, Haritalopha; Haritalopha

bipartita Smith, Anepischetos; Anepischetos bipartita Son, Paranola; Paranola bipartita Walker, Buciara; Buciara biplaga Fletcher \& Viette, Ziela; Ziela biplaga Viette, Zacthys; Zacthys biplagiata Viette, Prolyncestis; Prolyncestis bipuncta Joannis, Celazia; Celazia bipuncta Morrison, Eucalyptra;

Eucalyptra

bipunctalis Walker, Egone; Egone bipunctalis Warren, Hyphypena;

Hyphypena

bipunctata Hulstaert, Meizoglossa;

Meizoglossa

bipunctata Walker, Audea; Audea biramata Warren, Isolasia; Isolasia biren Goeze, Phalaena; Papestra birenosa Felder \& Rogenhofer, Renodes;

Discosema

bisacutum Hampson, Latirostrum;

Latirostrum

bisignata Walker, Boethantha; Boethantha bisignata Walker, Naarda; Naarda bistrialis Geyer, Agriphila; Doryodes bistriaris Hübner, Parallelia; Parallelia bistriata Fletcher \& Viette, Pseudugia; Pseudugia

bistriga Möschler, Stenola; Stenola 
bistrigata Barnes \& McDunnough, Acopa; Nacopa

bistrigata Bethune-Baker, Perciana; Goniotermasia

bistrigata Hübner, Ptichodis; Ptichodis bivittata Grote, Pallachira; Pallachira blanda Grote, Choephora; Pseudoglaea blandiatrix Guenée, Eurhipia; Alotsa blandula Staudinger, Bryophilina; Bryophilina blepharias Meyrick, Timorodes; Timorodes boarmioides Vialker, Steiria; Hypotrisula bochus Morrison, Agrotis; Crassivesica bohemani Staudinger, Anarta; Anartomima bolengensis Holland, Tolna; Tolnosphingia bolinalis Walker, Arsisaca; Arsisaca bolinoides Guenée, Hypospila; Hypospila bombycalis [Denis \& Schiffermuiller],

Pyralis; Idia

bombycoicles Walker, Bagisara; Caroia bonema Schaus, Clargia; Clargia bormia Schaus, Ilsea; Ilsea borneensis Roepke, Cyrtandra; Cyrtandra bostrycodes Bethune-Baker, Lophotagonia; Lophotagonia

botonga Felder $\&$ Rogenhofer, Dianthoecia;

Rhodochlaen:

botyoides Guenée, Xanthoptera; Marimatha bourgaulti Bar, Calydia; Calydia boursini Draudt, Ipiristis; Ipiristis boursini Fletcher, Psectraxylia; Psectraxylia brachyphylla Turner, Placerobela;

Placerobela

bracteata Felder, Antiophlebia;

Antiophlebia

bracteigutta WValker, Philecia; Philccia brassicae Linnaeus, Phalaena; Mamestra brassicae Riley, Plusia; Trichoplusia braziliensis Hampson, Neogalea; Neogalea braziliensis Schaus, Dectocraspedon;

Dcctocraspedon

braziliensis Schaus, Hypena; Burdettia brevicornis Walker, Xanthia; Nyodes breviuscula Walker, Nanagnna; Nanaguna brillians Neuntoegen, Edwardsia;

Eupseudomorpha

brocha Smith, Carneades; Crassivesica brontesalis Walker, Mastygophora;

Oxaenanus

brunnea Berio, Elaemima; Elaemima

brunnea Moorc, Gonitis; Pscudogonitis

brunnea Turner, Bathystolma; Bathystolma

brunneaplaga Bethune-Balicr,

Xanthograpta; Ethionodes brunnearis Guenée, Bolina; Bulia brunneiplaga Swinhoe, Metachrostis; Neachrostia

brunneovittata Aurivillius, Paratolna; Paratolna brunneum Bethune-Baker,

Ceilodiastrophon; Ceilodiastrophon bryophilina Hampson, Apsaranycta;

Apsaranycta

bryophiloides Butler, Eurythmus;

Eurythmus

bryophiloides Draudt, Chytobrya;

Chytobrya

bubastis Fawcett, Giria; Giria

buettneri Hering, Arsilonche; Sedina buffaloensis Grote, Ochria; Parapamea bufo Guenée, Barydia; Barydia

bufonia Felder \& Felder, Scolopocneme;

Scolopocneme

bugaba Druce, Margites; Margitesia bullula Grote, Pteraetholix; Pteraetholix burmeisteri Köhler, Paracullia; Paracullia burrowsii Butler, Tatorinia; Tatorinia busalis Walker, Dantona; Dantona butesalis Walker, Libisosa; Libisosa buxtoni Tams, Cymodegma; Cymodcgma

cacata Guenée, Ephyrodes; Ephyrodes cacuminalis Walker, Hypena; Hypenula cadburyi Franclemont, Comachara;

Comachara

cadmia Guencee, Hemiceras; Hemiceras cadoreli Viette, Madecathymia;

Madecathymia caducus Dyar, Mcnopsimus; Menopsimus caeca Swinhoe, Caradrina; Paromphale caecimacula [Denis \& Schiffermüller],

Noctua; Ammoconia caecuticns Hübner, Cyclopis; Cyclopis caerulea Butler, Trisuloides; Disepholcia caerulca Grote, Drasteria; Caenurgina caerıleifera Walker, Vespola; Vcspola caeruleocephala Linnaens, Phalaena;

Diloba caerulilineata Hampson, Trilophonota;

Trilophonota caffra Girence, Gracilodes; Gracilodes caicus Schaus, Neoptodes; Neoptodes cailino Lcfelovre, Heliothis; Aleucanitis caja Linnaeus, Phalaena; Arctia cajeta Cramcr, Plialaena; Corycia calamitosa Walker, Olyssa; Olyssa 
calgia Schaus, Pagyra; Pagyra

calida Walker, Sarunga; Sarunga

californica Bucliett \& Bauer, Petaluma;

Petalumaria

caliginea Butler, Aplectoides; Anacronicta caliginosa Hübner, Noctua; Acosmetia caliginosa Schaus, Oidemastis; Oidemastis caliginosa Walker, Remigia; Talapa

calisalis Walker, Mursa; Mursa

calisto Boisduval, Amazela; Amazela

callichlora Walker, Tyana; Tyana

calligramma Hübner, Helia; Helia

callima Fletcher, Euxootera; Euxootera

callista Holland, Geometrimima;

Geometrimima

callisto Walker, Apina; Apina

callitrichoides Grote, Phyprosopus;

Phyprosopus

calodesna Rothschild \& Jordan, Buzara;

Haemabasis

calopasa Walker, Brana; Brana

calora Barnes, Grotella; Grotellaforma

calorifica Walker, Naxia; Delgamma

calospila WValker, Sinna; Sinna

calvaria [Denis \& Schiffermüller], Noctua;

Epizeuxis

camelina Linnaeus, Phalaena; Ptilodon camina Smith, Platyperigea; Platyperigea

camptogramma Hampson, Gonoglasa;

Gonoglasa

campyla Hampson, Delta; Campydelta

canadensis Bethune, Calpe; Percalpe

candida Walker, Arbasera; Arbasera

candidula [Denis \& Schiffermüller], Noctua;

Pseudeustrotia

canescens Walker, Cortyta; Cortyta

canescens Walker, Phumana; Phumana

canina Felder, Acronycta; Ectochela

canioralis Walker, Necla; Necla

canipars Hampson, Phaeoscia; Phaeoscia

canosparsa Walker, Epicoria; Epicoria

canteneri Duponchel, Polia; Recoropha

capacior Walker, Talariga; Talariga

capax Grote \& Robinson, Xylina; Xylotype

capensis Cramer, Phalaena; Anthocitta

capensis Herrich-Schäffer, Eudryas; Ovios

capitalis Strand, Camadeniana;

Camadeniana

capsincola [Denis \& Schiffermüller], Noctua;

Hadena

captata Butler, Euclidia; Brevipecten

captiuncula Treitschke, Apamea; Photedes

cara Strand, Erna; Erna

caradrinalis Guenée, Bleptina; Bleptina caradrinoides Guenée, Bocula; Bocula

caranea Cramer, Phalaena; Hulodes

carbo Guenée, Phlegetonia; Pataeta

carbonaria [Denis \& Schiffermüller],

Geometra; Parascotia

carbonaria Harvey, Lithophane;

Euharveya

carda Swinhoe, Hopetounia; Hopetounia

carducha Staudinger, Bryomima;

Bryomima

cardui Hübner, Phalaena; Melicleptria

carina Harvey, Acopa; Acopa

caripina Felder \& Rogenhofer, Catamelas;

Catamelas

carmelitoides Guenée, Canodia; Canodia

carnea Thunberg sensu Guenée, Pachnobia;

Pachnobia

carnescens Schaus, Makapta; Macapta

carnicolor Hampson, Chalciope; Ctenusa

carnina Guenée, Sanys; Sanys

carnosa Grote, Glaea; Psectraglaea

carnosa Hampson, Lycauges; Parasada

carunalis Schaus, Cloniatarphes;

Cloniatarphes

cassinia [Denis \& Schiffermüller], Bombyx;

Asteroscopus

casta Borkhausen, Phalaena; Cleophana

castalia Fabricius, Bombyx; Eucyclomma

castanea Hampson, Diparopsis; Diparopsis

castanea Moore, Egnasia; Eutrogia

castanea Moore, Nikara; Nikara

castanea Turati, Draudtiana; Draudtiana

castaneata Warren, Parerastria; Parerastria

castaneotincta Hampson, Tumidifrontia;

Tumidifrontia

castanitis Hampson, Zethes; Malatrogia

catagrapha Schaus, Compsenia; Compsenia

catagrapha Turner, Cymatophoropsis;

Gryposoba

cataleuca Herrich-Schäffer, Hypena;

Naboa

catalina Dyar, Thurberiphaga;

Thurberiphaga

cataplaga Dyar, Xanthirinopsis;

Xanthirinopsis

catena Sowerby, Phalaena; Desmophora

catephioides Guenée, Phlegetonia;

Phlegetonia

catharina Schaus, Herminodes; Elegocampa catherina Grote, Matuta; Cerastis

catocala Felder \& Rogenhofer, Phoberia;

Alura

catocalina Walker, Zalissa; Zalissa

catomelas Alphéraky, Pulcheria; Pulcheria 
caudaequina Karsch, Andrhippuris; Andrhippuris

caupona Möschler, Alura; Alura c-aureum Knoch, Phalaena; Lamprotes caustiplaga Hampson, Capnodes;

Selenoperas cauta Hampson, Charidea; Phalerodes cavatalis Walker, Agamana; Agamana cavernosa Eversmann, Orthosia; Hyssia cedermarki Bryk, Erythrotis; Erythrotis celadon Schaus, Maronia; Maronia celia Stoll, Phalaena; Safia cellulalis Guenée, Aristaria; Aristaria celsia Linnaeus, Phalaena; Staurophora celsiana Staudinger, Chloeophora;

Parhylophila celtis Moore, Selepa; Selepa ceneusalis Walker, Elusa; Elusa centrago Haworth, Noctua; Cirroedia centurialis Hübner, Euclystis; Euclystis cephalica Smith, Stylopoda; Stylopoda cephusalis Walker, Ilattia; Ilattia cerasina Swinhoe, Chrysocraspeda;

Chrysocraspeda cerata Franclemont, Chaetaglaea;

Chaetaglaea ceratopyga Püngeler, Bryophila; Turacina ceraunias Meyrick, Ichneutica; Ichneutica ceres Butler, Leucocosmia; Leucocosmia cerina Grote, Gortyna; Papaipema cerintha Treitschke, Acontia; Chamyris certa Walker, Archana; Archana certissima Walker, Egabra; Egabra cerusicosta Guenée, Achantodes;

Achantodes cervicornis Fawcett, Pteronycta; Crionica cervina Edwards, Herrichia; Protophana cervina Hampson, Parangitia; Parangitia cervina Walker, Raclia; Raclia

cespitis [Denis \& Schiffermüller], Noctua;

Tholera

chalciope Strand, Plecoptera; Plecopteroides chalcites Esper, Phalaena; Chrysodeixis chalcoela Hampson, Callyna; Opsyra chalcoides Dufay, Plusia; Stigmoplusia chalcospilans Dyar, Neophaeus; Neophaeus chalsytoides Guenée, Plusiodonta;

Plusiodonta chalybeata Moore, Naenia; Pareuplexia chamaeleon Möschler, Paraxia; Paraxia characteria Stoll, Phalaena; Henicephalis chartaria Grote, Mamestra; Discestra cheiranthi Tauscher, Noctua; Plusidia chephise Stoll, Phalaena; Melanchroia chi Linnaeus, Phalaena; Antitype chilensis Hampson, Mesembreuxoa;

Mesembreuxoa chinensis Berio, Pterogonaga; Pterogonaga chinensis Leech, Metachrostis; Ozana chionanthi Smith, Phalaena; Adita chionobola Turner, Macroprora;

Macroprora; Conocrana chionostigma Turner, Barybela; Barybela chitinipyga Dumont, Lecerfia; Lecerfia chloe Schaus, Mastixis; Mastixis chlorana Hampson, Sarrothripa;

Chloethripa

chlorea Cramer, Phalaena; Sphingomorpha chloristicta Schaus, Polygnamptia;

Polygnamptia chloristis Schaus, Chytonidia; Chytonidia chloriza Guenée, Eriopus; Dissolophus chlorochrysa Hampson, Centrogone;

Centrogone chlorogastra Felder, Jochroa; Jochroa chlorota Hampson, Eustrotiopis;

Eustrotiopis chlorozonea Hampson, Eublemmistis;

Eublemmistis chordophoides Lucas, Zethes; Amphiongia chorinea Stoll, Phalaena; Athysania. chortalis Harvey, Agrotis; Richia christophi Ershov, Calophasia;

Marsipiophora chromatophila Walker, Boecula; Dyops chryselectra Grote, Hadena; Hemibryomima chrysellus Grote, Tricopis; Tricopis chrysochlora Walker, Eliocroea; Eliocroea chrysochlora Walker, Tuerta; Tuerta chrysomela Walker, Buzara; Buzara chryson Esper, Phalaena; Diachrysia chrysopasa Walker, Deobriga; Deobriga chrysorrhaea Hampson, Dilophothripa;

Dilophothripa chrysosticta Hampson, Basilodes; Basilica cidaria Druce, Dida; Dida cidarioides Boursin, Hoeneidia; Hoeneidia cidarioides Moore, Motama; Motama ciliaria Ménétriés, Ligia; Imitator cilicoides Grote, Argyrophyes;

Argyrophyes cilipes Walker, Dugaria; Dugaria cimolia Guenće, Dyomyx; Dyomyx cincta Smith, Kakopoda; Kakopoda cinctigutta Walker, Enydra; Enydra cinctum Saalmüller, Elyptron; Elyptron cinerascens Motscliulsky, Cosmia; Radinacra cinerea Butler, Capnodes; Hypostrotia 
cinerea Butler, Carpholithia; Carpholithia cinerea [Denis \& Schiffermüller], Noctua; Scotia

cinerea Hampson, Ugiodes; Ugiodes cinerea Smith, Epidemas; Epidemas cinereola Guenée, Placodes; Ogdoconta cinereola Smith, Selicanis; Selicanis cinereovariegata Le Cerf, Melanephia; Melanephia cinereus Blanchard, Trachodopalpus; Trachodopalpus cinerosa Felder, Darcetina; Darcetina cinnamomea Goeze, Phalaena; Pyrois cinnamomeus Bethune-Baker, Camptochilus; Striaptera cinnamomina Draudt, Prionoxanthia; Prionoxanthia cinnamomina Rothschild, Catamecia; Oedibrya circulifera Walker, Acronycta; Charadra circumdata Grote, Agrotis; Trichofeltia circumdata Walker, Hydrelia; Docela circumflexa Linnaeus, Phalaena;

Cornutiplusia

circumflexa Linnaeus sensu Hübner, Noctua; Macdunnoughia circumflexalis Guenée, Heterogramma; Heterogramma circumscripta Walker, Aginna; Aginna citrago Linnaeus, Phalaena; Tiliacea citrinea Berio, Melionica; Melionica citrinellus Grote, Heliothis; Oxylos clandestina Walker, Coeriana; Coeriana clanymoides Möschler, Lophophora;

Lophophora clara Stoll, Phalaena; Nagara clathrata Grünberg, Trisula; Trisulopsis clathrum Guenée, Homaea; Homaea clauda Grote, Escaria; Escaria claudens Walker, Hadena; Eremobina clavata Köhler, Cotarsina; Cotarsina clavifera Walker, Nahara; Nahara clavifera Walker, Tafalla; Tafalla cleoriformis Barnes \& McDunnough,

Homocerynea; Homocerynea clevia Miöschler, Daedalina; Daedalina cliopis Dyar, Eumuelleria; Eumuelleria cloanthoides Grote, Agrotis; Rhizagrotis clorana Linnaeus, Phalaena; Earias closteroides Walker, Hiaspis; Hiaspis clotho Schaus, Eromidia; Eromidia cocalus Cramer, Phalaena; Rhytia cochlidioides Dyar, Psammathodoxa;

Psammathodoxa cocophaga Franclemont, Echinocampa; Echinocampa cocytalis Guenée, Rejectaria; Rejectaria codeti Oberthür sensu Hampson,

Metopoceras; Ammetopa codo Dyar, Celiptera; Paraceliptera coelisigna Walker, Ariola; Ariola coelonota Kollar, Plusia; Deva coenobita Esper, Phalaena; Panthea coenotype Hampson, Zethes; Eucampima coerula Guenée, Cocytodes; Cocytodes coerulea Guenée, Agrotis; Physetica coerulina Möschler, Gabyna; Gabyna colligata Walker, Tiridata; Tiridata colliquens Hübner, Coenipeta; Coenipeta collusoria Cramer, Phalaena; Trissophaes collutrix Geyer, Coenipeta; Trichopolydesma colon Dognin, Hypermilichia; Hypermilichia colorada Smith, Arsilonche; Benjaminiola colorado Smith, Agrotiphila; Agrotiphila coloraria Fabricius, Phalaena; Erastria columba Walker, Dandaca; Dandaca columbina Walker, Armactica; Armactica combinans WValker, Achaea; Chrysopera combusta Walker, Dacira; Dacira combusta Walker, Ortheaga; Ortheaga comma Linnaeus, Phalaena; Leucania comma Walker, Mamestra; Rictonis commiscens Walker, Boryza; Boryza commoda Staudinger, Rhiza; Rhiza commoda Walker, Vescisa; Vescisa communimacula [Denis \& Schiffermüller],

Noctua; Calymma comosa Guenée, Calesia; Calesia compactilis Swinhoe, Zethes;

Hyperlophoides complens Walker, Hypaetra;

Pseudathyrma complexa Walker, Caecila; Caecila composita Edwards, Eulithosia; Eulithosia comprimens IValker, Amphigonia;

Lopharthrum compripalpis Strand, Chusaris; Luceriola compsias Meyrick, Leucania; Haliophyle compta [Denis \& Schiffermüller], Noctua;

Miselia comstocki Benjamin, Trichocerapoda;

Trichocerapoda comstocki Grote, Feralia; Momophana concavata Walker, Sophta; Sophta concha Druce, Oroscopa; Oroscopa concha Fabricius, Noctua; Lamprotes conchiformis Grote, Behrensia; Behrensia conchyfera Guenée, Nystalea; Nystalea 
concinnula Mabille, Hypsa; Mila

concisa Walker, Moepa; Orrea

concolor Bethune-Baker, Crenularia;

Crenularia

concolor Bethune-Baker, Parilyrgis;

Parilyrgis

condecoralis Walker, Larassa; Larassa condita Guenée, Aplecta; Aplectoides conducens Walker, Deva; Deva confertissima Walker, Leucania; Raghuva conflua Hampson, Euminucia; Euminucia confluens Hampson, Talapa; Talapoptera confluens Valker, Minica; Minica confluens Weymer, Aegocera; Paraegocera conformis [Denis \& Schiffermüller], Noctua;

Graptolitha

confusa Freyer, Amphipyra; Oncocnemis confusa Hufnagel, Phalaena; Zeteolyga confusa Leech, Moma; Lophonycta confusa Stephens, Plusia; Macdunnoughia confusalis Guenée, Bleptina; Opotura confutalis Walker, Canatha; Canatha congemmalis Hübner, Syllectra; Teratocera congenitalis Hampson, Nephelistis;

Nephelistis

congoensis Berio, Hypersypnoides;

Hypersypnoides

congressa Walker, Abrostola; Habrostolodes conisema Hampson, Mecodopsis;

Mecodopsis

conjuncta Walker, Limacodes; Phlossa conjungens Walker, Grammodes; Parafodina connectens Walker, Ortospana; Ortospana conscitulana Walker, Tolpia; Tolpia consentanea Walker, Eutelia; Pachydasys consignata Walker, Hermonassa;

Hermonassa

conspicienda Walker, Acliaea; Peranua conspicienda Walker, Pataeta; Pataeta conspicillaris Linnaeus, Phalaena; Egira conspicillator Cramer, Phalaena; Phyllodes conspicua Dyar, Meganola; Meganola conspicua Leech, Polia; Gaurenopsis conspicua Leech, Remigia; Hypocarea constricta Guenée, Anuga; Anuga contaninei Eversmann, Noctua; Eogena contigua Walker, Charadra; Charadra continens Edwards, Perigea; Neperigea continua Walker, Ariola; Tathothripa continua Walker, Pincia; Pincia contorta Walker, Agarista; Comocrus contracta Walker, Naharra; Naharra contrahens Walker, Celaena; Anhimella contraria Walker, Phasis; Plıasidia contraria Walker, Savara; Savara contrariata Wallier, Negeta; Negeta contrasta Barnes \& MçDunnough, Perigea;

Euamiana

contrita Christoph, Agrotis; Mesoplus convalescens Guenée, Drasteria; Caenurgia convergens Hampson, Leoniloma;

Leoniloma copima Schaus, Santiaxis; Santiaxis coppryi Guenée, Orthogramma; Archana cora Eversmann, Heliothis; Pyrocleptria cora Hübner, Cerma; Cerma coracias Guenée, Anthracia; Psendanthracia coraxa Püngeler, Rusina; Netrocerocora cornelia Staudinger, Acronycta;

Xanthomantis

cornifrons Moore, Mudaria; Mudaria cornifrons Snellen, Metecia; Metecia cornigera Butler, I pana; I panica cornuta Grote, Pachnobia; Metalepsis cornuta Püngeler, Usbeca; Usbeca coronides Druce, Plusia; Kallitrichia corticea Strand, Bertulania; Bertulania cortytoides Berio, Heteropalpia;

Heteropalpia coryli Linnaeus, Phalaena; Colocasia coryphata Dyar, Dymba; Dymba cosmion Dyar, Meropleon; Meropleon cosmopis Lower, Britha; Metaxanthiella cossoides Butler, Xylina; Xylinissa cossoides Walker, Carissa; Carissa costalis Moore, Acharya; Leptotroga costalis Moore, Anoratha; Anoratha costalis Moore, Chutapha; Chutapha costalis Schaus, Caroga; Caroga costalis Walker, Acontia; Heliodora costalis Walker, Cirphis; Cirphis costata Moore, Cephena; Cephena costata Walker, Oruza; Oruza costibarbata Hampson, Argyrolopha;

Argyrolopha costilinea Bethune-Baker, Reticulana;

Reticulana

costimacula Guenée, Toxocampa; Rema costimacula Joannis, Neoniga; Neoniga costimacula Staudinger, Aventiola;

Aventina

costinotata Hampson, Hyperbaniana;

Hyperbaniana

costiplaga Walker, Stibaera; Stibaera costistrigata Bethune-Baker, Cheillophota;

Cheillophota

crambisata IValker, Hileia; Hileia cramboidina Walker, Rhatta; Rhatta 
crassalis Fabricius, Phalaena; Bomolocha crassicornis Herrich-Schäffer, Systremma; Systremma

crassicornis Moore, Acharya; Acharya crassipes Smith, Podagra; Podagra crassisquama Strand, Bonaberiana;

Bonaberiana craushayi Hampson, Conservula;

Calpiformis creberrima Walker, Thermesia; Tochara crenata Esper, Bombyx: Gluphisia crenata Hufnagel, Phalaena; Apamea crenelata Hampson, Acantholipes;

Cymoblemma

crenularia Bethune-Baker, Eublemma;

Trogoxestis crenulata Bethune-Baker, Parepisparis;

Parepisparis

crepuscularis Linnaeus, Phalaena; Erebus crescentica Hampson, Atimaea; Metappana cretacea Staudinger, Namangana;

Namangana cribralis Hübner, Pyralis; Macrochilo cribrumalis Hübner, Phalaena; Macrochilo crinalis Treitschke, Herminia; Pechipogo crinipes Felder \& Rogenhofer, Remigia;

Hirsutipes crinipuncta Schaus, Ateneria; Ateneria cristagalli Köhler, Cuculluna; Cuculluna cristata Morrison, Homophoberia;

Homophoberia cristatrix Guenée, Ingura; Callingura cristatum Hampson, Lophocraspedon;

Lophocraspedon cristifera Walker, Ephyrodes; Hyperlopha crocea Edwards, Anarta; Pseudanarta crocea Guenée, Homodes; Homodes crocea Walker, Mandela; Mandela croceago [Denis \& Schiffermüller], Noctua; Jodia

croceicosta Schaus, Micramma; Micramma croceipennis Walker, Ophisma; Heliophisma crossodora Meyrick, Mormoscopa;

Mormoscopa crotchii Edwards, Pseudalypia; Pseudalypia crustaria Morrison, Tarache; Pseudacontia crypsichlora Turner, Pherechoa; Pherechoa crysipyrrha Turner, Brachycyttara;

Brachycyttara crystallodes Meyrick, Chaograptis;

Chaograptis cubilis Grote, Copipanolis; Copipanolis cucullatalis Walker, Lambana; Lambana;

Anablemma cucullatella Linnaeus, Phalaena; Nola cucullidea Benjamin, Emariannia;

Emariannia

cucullioides Guenée, Stictoptera;

Stictoptera

cucullioides Walker, Chaladra; Chaladra culminifera Calberla, Teinoptera; Teinoptera culta [Denis \& Schiffermüller], Noctua;

Lamprosticta cumatilis Grote, Euleucyptera;

Euleucyptera cupentia Cramer, Phalaena; Condica cupes Grote, Heliothis; Trichosellus cupida Grote, Noctua; Rhynchagrotis cuprea Moore, Gortyna; Estagrotis cuprea Schaus, Phaeochlaena; Bostrycharia cuprina Moore, Apamea; Lasiplexia curiosa Clench, Noctasota; Noctasota curiosa Swinhoe, Globosusa; Globosusa curiosa Swinhoe, Selepa; Hypothripa curiosalis Swinhoe, Adrapsa; Perata cursoria Hufnagel, Phalaena; Telmia curtalis Walker, Egnasia; Saraca curtica Smith, Taeniocampa; Protorthodes curva Staudinger, Rhiza; Stenostigma curvata Grote, Capis; Capis curvicosta Guenée, Renodes; Renodes cuspidea Hübner, Drasteria; Euclidina cyanastis Meyrick, Musothyma;

Musothyma cyanea Snellen, Ophiusa; Vizaga cyanolepia Kaye, Megatomis; Pseudostella cyathicornis Walker, Seria; Seria cyclophora Turner, Spathoptila; Spathoptila cycloptera Dognin, Diplolopha; Diplolopha cydonia Cramer, Phalaena; Claterna cygnus Walker, Chasmina; Chasmina cylindrica Grote, Hadena; Afotella cyllarus Schaus, Synomera; Synomera cyma Hübner, Dioptis; Dioptis cymaea Turner, Hypobleta; Hypobleta cymatistis Meyrick, Ceparcha; Ceparcha cymbalariae Hübner, Noctua; Omia cynara Cramer, Phalaena; Macrodes cynaralis Guenée, Macrodes; Macrodes cyrus Brandt, Scythocentropus; Paracentropus

dahlii Hübner, Noctua; Diarsia damnipennis Walker, Phanaca; Phanaca damoetesalis Walker, Ecregma; Glympis 
danistica Grote, Annaphila; Proannaphila daona Druce, Ophiodes; Herminiocala darena Druce, Thalpochares; Prodicella dares Stoll, Phalaena; Phaeoblemma daria Druce, Thalpochares; Prorachia darwini Staudinger, Orthosia; Boursinidia dasychira Hübner, Noctua; Athetis dasynota Felder \& Rogenlıofer, Achaea;

Tolnaodes dasypterus Kollar, Erebus; Calesia datanidia Butler, Nephelodes; Virgo deaurata Esper, Phalaena; Panchrysia debilis Christoph, Thalpochares; Thalomicra debora Druce, Drobeta; Zagorista decepta Grote, Trichoclea; Trichoclea deceptalis Walker, Hypena; Macrhypena deceptrix Staudinger, Pseudohadena;

Anamecia

decimalis Poda, Phalaena; Neuronia decipiens Hampson, Mixomelia; Mixomelia decisa Walker, Iluza; Iluza

decisissima Walker, Ligidia; Ligidia

declivis Turner, Amblyzancla;

Amblyzancla

decocta Schaus, Thursania; Thursania

decolor Rebel, Pseudomicra;

Pseudomicrocles

decolor Walker, Focilla; Tamba

decora [Denis \& Schiffermüller], Noctua;

Euxoa

decora Linnaeus, Phalaena; Pais

decora Saalmüller, Maxia; Maxia

decoralis Hübner, Pangrapta; Pangrapta

decorata Frivaldszky, Heliothis; Micriantha

decorata Moore, Karana; Karana

decorata Moore, Mamestra; Oroplexia

decoripennis Mabille, Tarache; Aulotarache decrescens Walker, Gadirtha; Plotheia deducta Morrison, Syneda; Cirrhobolina defigurata Walker, Xylina; Bityla defixa Waller, Barbesola; Barbesola deflexa Saalmüller, Proluta; Proluta deflorata Fabricius, Hyblaea; Hypocala degenerana Hübner, Tortrix; Sarrothripus deilinias Schaus, Thiachroia; Thiachroia clelaia Schaus, Stictothripa; Stictothripa delatrix Guenée, Penicillaria; Targalla deleta Moore, Niaccaba; Decticryptis delicata Bethune-Baker, Egnasia;

Craptignapa

delicata Möschler, Amabela; Amabela delicata Staudinger, Nudifrons; Nudifrons delicatula Berio, Micragrotis; Micraxylia delinquens Walker, Poesula; Poesula delogramma Lower, Rhynchopsota; Rhynchopsota

delospila Prout, Ableptina; Ableptina delphinii Linnaeus, Phalaena; Periphanes delta Dyar, Oxythaphora; Oxythaphora delunaris Guenée, Ophisma; Mimophisma demonias Meyrick, Trigonistis; Trigonistis denotalis Walker, Poeta; Poeta densa Walker, Arzama; Arzama dentata Grote, Apatela; Apharetra dentata Staudinger, Acontia; Armada dentata Walker, Cetola; Cetola dentatella Grote, Trichopolia; Trichopolia dentatrix Westwood, Eusemia; Exsula denticulata Moore, Apanda; Apanda denticulata Warren, Ochropleura;

Hypernaenia denticulosa Walker, Miana; Cytocanis dentifer Dyar, Grypotes; Zatilpa dentifera Moore, Zarima; Zarima dentina [Denis \& Schiffermüller], Noctua; Hada dentistrigatae Alphéraky, Imitator;

Imitator

dentula Lederer, Euxestis; Euxestis depravata Butler, Agrotis; Rusidrina derasa Linnaeus, Phalaena; Habrosyne derivalis Hübner, Pyralis; Paracolax derna Swinhoe, Abriesa; Abriesa describens Walker, Aegilia; Aegilia deserticola Barnes \& Benjamin, Neotarache;

Neotarache deserticola Hampson, Aglossestra;

Aglossestra deserticola Staudinger, Epipsammia;

Epipsammia

deserticola Turner, Ammophanes;

Ammophanes

designans Walker, Scoedisa; Scoedisa desolata Staudinger, Distagma; Distagma determinata Walker, Bithiasa; Bithiasa detersa Esper, Noctua; Auchmis detersalis Valker, Catada; Daona detrahens WValker, Poaphila; Lepidotrama detritalis Walker, Tigrana; Tigrana devagor Kozhanchikov, Anarta; Sajania devergens Hübner, Noctua; Syngrapha devia Walker, Manbuta; Manbuta devincta Walker, Abrostola; Orthoclostera diacrisioides Rothschild, Harpagophana;

Tetrachela

diagonalis Collenette, Fautaua; Fautaua diagrapha Turner, Lophotoma; Lophotoma diagrapta Hampson, Cataloxia; Cataloxia 
diana Schaus, Stellidia; Aglossostola dianthoecioides Boursin, Kuruschia;

Kuruschia

diaperas Hampson, Acaenica; Acaenica

diaphora Staudinger, Erastria;

Thalerastria

diapyra Hampson, Thermosara; Thermosara

dichora Holland, Plusiopalpa; Plusiopalpa

dichroa Hampson, Chelaprora; Chelaprora

dichromella Walker, Beara; Beara

dicycla Walker, Acontia; Xantholepis

dicyma Hampson, Hesperothripa;

Hesperothripa

didyma Esper, Noctua; Mesapamea

didymata Walker, Sorygaza; Sorygaza

didymogramma Ershov, Mycteroplus;

Pinacoplus

diehli Wiltshire, Riadhia; Riadhia

difficilis Köhler, Velazconia; Velazconia

difficilis Walker, Corrha; Corrha

diffinis Linnaeus, Phalaena; Cosmia

difformis Roepke, Acygonia; Acygonia

diffumata Möschler, Arctinia; Arctinia

diffundens Walker, Celaena; Synyrias

diffusa Grote, Arzama; Arzamopsis

diffusa Walker, Xanthodes; Naranga

digestalis Walker, Nazuda; Nazuda

digitalis Grote, Anchoscelis; Lemmeria

digna Alphéraky, Raddea; Raddea

digonia Turner, Diplothecta; Diplothecta

digramma Walker, Bocana; Byturna

dilatata Butler, Mesogona; Hypoxestia

dilatatalis Walker, Phanaspa; Phanaspa

dilecta Hübner, Noctua; Astiotes

dilectata Dognin, Pseudypsia; Pseudypsia

dilectissima Walker, Ariola; Yepcalphis

dileuca Hampson, Procriosis; Procriosis

dilucida Hübner, Noctua; Autophila

dimidialis Fabricius, Phalaena; Polyorycta

dimidiata Grote, Antaplaga; Antaplaga

dimidiata Walker, Baputa; Baputa

dimidiata Walker, Massala; Massala

diminuens Walker, Medava; Medava

diminuta Guenée, Xylophasia; Antachara.

diminutivus Grote, Heliothis; Heliothodes

dinawa Bethune-Baker, Catephia;

Eucatephia

dinawa Bethune-Baker, Eublemmoides;

Eublemmoides

dinawa Bethune-Baker, Focillodes;

Focillodes

dinotis Meyrick, Miaromima; Miaromima dinumeratalis Walker, Marimatha;

Marimatha diopis Hampson, Tarache; Meneptera

diphteroides Guenée, Microcoelia;

Leuconycta

diplogramma Hampson, Catephia;

Chitasida

diplogramma Hampson, Neochrostis;

Neochrostis

diplolopha Druce, Acroriodes; Acroriodes

diplostigma Hampson, Elwesia; Elwesia

dipsacea Linnaeus, Phalaena; Heliothis

direae Berio, Hyperfrontia; Hyperfrontia

directa Walker, Angitia; Blancharditia

discalis Moore, Agriopis; Bamra

discalis Walker, Abacena; Abacena

discalis Walker, Chobata; Chobata

discerpta Walker, Gerisa; Gerisa

discessalis Walker, Alinza; Alinza

discipuncta Hampson, Microblemma;

Abablemma

discistriga Smith, Lythrodes; Hoplolythra

discitriga Walker, Eutelia; Alotsa

discivaria Walker, Bryophila; Taeniosea

discolor Fabricius, Noctua; Avatha

discolor Guenée, Miniodes; Miniodes

discolor Smith, Cleoceris; Lomilysis

discomma Hampson, Taviodes; Taviodes

discrepans Walker, Ophideres; Purbia

discreta Walker, Dioptis; Erocha

discursa Walker, Amarna; Amarna

disgnosta Boursin, Eugraphe; Sineugraphe

disgrega Möschler, Hadena; Adiopa

disjuncta Walker, Leucanimorpha;

Leucanimorpha

disjungens Wallier, Heliophobus; Graphania

disjungens Walker, Ugia; Ugia

dispar Hampson, Gonodontodes;

Gonodontodes

dispar Herrich-Schäffer, Asymbletia;

Asymbletia

disparalis Walker, Motina; Hypercodia

disparatalis Walker, Aganzagara;

Aganzagara

disparilis Herrich-Schäffer, Heterandra;

Heterandra

dispila Walker, Homoptera; Panilla

displosa Schaus, Elecussa; Elecussa

dispunctalis Walker, Gaala; Gaala

disruptalis Walker, Saraca; Saraca

dissensa Schaus, Uzomathis; Uzomathis

disseverans Walker, Remigia; Baratha

dissimilaria Hübner, Erastria; Erastria

dissimilis Distant, Polydesma; Cnodifrontia

dissimilis Warren, Dysglyptogona;

Dysglyptogona 
dissimulans Hampson, Hypena; Aphypena dissociata Barnes \& McDunnough, Perania;

Peraniana

distans Barnes \& McDunnough, Bryonima;

Pseudobryomima

distema Grote, Cilla; Cilla

distigmata Hampson, Cosmia; Apostema

distigmata Hampson, Erastria; Amynodes distincta Bang-Haas, Tapinostola; Rotoa

distincta Christoph, Scotochrosta;

Thargelia

distincta Hübner, Achatia; Achatia

distincta Leech, Gonitis; Clethrophora

distorta Hampson, Eutelia; Noctasota

distorta Swinhoe, Spectrophysa;

Spectrophysa

distorta Warren, Diastreptoneura;

Diastreptoneura

distorta Warren, Zethes; Contortivena

distracta Eversmann, Caradrina;

Hypostilbia

distributa Walker, Rhazunda; Rhazunda distriga Hampson, Acanthodelta;

Acanthodelta

ditata I.ucas, I.uperina; Diplonephra

diva Grote, Annaphila; Annaphila

divergens Butler, Mesogona; Telorta

divergens Walker, Agrotis; I.ongivesica

diversalis Walker, Singara; Singara

diversicolor Warren, Autanthema;

Autanthema

diversipennis Walker, Erchcia; Ercheia dives Butler, Metaculasta; Metaculasta dividens WValker, Ophideres; Vanclana dividua Grote, Eustrotia; Nerastria divisa Hampson, Charidea; Ramesodes divisa Walker, Themma; Themma divisalis Walker, Betousa; Bucinna divitalis Walker, Gyrtona; Savoca divitiosa Walker, Ophideres; Halastus divulsa Walker, Athyrma; Tephriopis docla Swinhoe, Hypena; Anaepa dohertvi Druce, Cerurá; Sundwarda dohertyi Holland, Pterochaeta; Pterochaeta dolera Turner, Eremobates; Tabomecres dolon Cramer, Phalaena; Hemeroblemma domestica Hufnagel, Phalaena; Bryophila dominula I.innaeıs, Phalaena; Callimorpha donovani Boisdnval, Agarista; Cruria dormitans Guence, Leptina; Baileya dorsicinis Walker, Agrotis; Elegarda dorsilinea Dognin, Capnodes;

Dolichosomastis

dorsivitta Walker, Juncaria; Juncaria dos Freyer, Heliothis; Stenoecia dosaroides Moore, Hiccoda; Hiccoda dotata Walker, Arvaduca; Arvaduca drepanoides Walker, Cotuza; Cotuza dromedarius Linnaeus, Phalaena;

Notodonta

drosera Meyrick, Callipyris; Callipyris

drucella sp.n., Poena; Poena

clruceria sp.n., Safidia; Safidia

dryopa Meyrick, Acrarmostis; Acrarmostis dubia Butler, Mataeomera; Mataeomera dubia Warren, Leptoctenista; Leptoctenista dubiosa Strand, Alelimminola; Alelimminola ducens Walker, Feltia; Feltia duchesnei Viette, Perigea; Andobana ducta Grote, Hadena; Mniotype dudgeoni Hampson, Trisula; Eumichtochroa dulcissima Walker, Nolasena; Paracrama dulcistriga Walker, Anophia; Ecpatia dumetorum, Geyer, Noctua; Heterophysa duomita Schaus, Phialta; Phialta duplexa Moore, Iluza; Talapoptera duplicatus Smith, Cirrhophanus; Phaioecia durbanica Hampson, Trogocraspis;

Trogocraspis durvillii Boisduval, Cocytia; Cocytia dux Saalmüller, Phyllodes; Huebnerius dyari Hampson, Acanthermia;

Acanthermia

dysodea [Denis \& Schiffermüller], Noctua;

Hecatera

cburneifera Walker, Choluata; Choluata eburneigutta Walker, Tinolius; Tinolius echana Swinhoe, Lignicida; Lignicida echephurealis Walker, Ilyrgis; Ilyrgis echii Borkhausen, Phalaena; Anepia echione Boisduval, Agarista; Hespagarista eclipsia Hampson, Focillopis; Focillopis ectorhoda Hampson, Oederastria;

Oederastria ecuadorina Westwood, Othria; Parothria edemoides Walker, Burdria; Burdria edentifascia Hampson, Mimeugoa;

Mimeugoa

edictalis Walker, Hypena; Meghypena editrix Guenée, Gonitis; Gonitis edmondsii Butler, Trachodopalpus;

Stenagrotis

edobasis Dyar, Bryophilopsis;

Parabryophila

eductalis Walker, llypena; Lomanaltes 
edusa Drury, Phalaena; Nephelina edwardsii Guenée, Praxis; Praxis efulgida Swinhoe, Sundwarda; Sundwarda egena Guenée, Plusia; Autoplusia egregia Schaus, Leioselia; Leioselia egregia Staudinger, Panthauma; Panthauma ekeikei Bethune-Baker, Thoracolophotos;

Thoracolophotos ekeikei Bethune-Baker, Zalissa; Leucogonia elaeodes Turner, Bryonima; Trissernis elataria Walker, Hyria; Micraeschus elbursalis Draudt, Zanclostathme;

Zanclostathme

electrica Schaus, Thermesia; Goniocarsia elegans Bethune-Baker, Syngatha; Syngatha elegans Donovan, Phalaena; Cosmodes elegans Fabricius, Bombyx; Diaphone elegans Hoeven, Ophideres; Lagoptera elegans Hübner, Diphthera; Diphthera elegans Saalmüller, Erastria; Microplexia elegantissima Berio, Acropserotarache;

Acropserotarache elegantissima Guenée, Charidea; Compsotata elegantula Köhler, Noctulizeria;

Noctulizeria

elegantula Roepke, Tamsia; Tamsia elicrina Felder \& Rogenhofer, Colobochila;

Rhododactyla

elingua Smith, Manruta; Manruta elinguis Püngeler, Jaxartia; Jaxartia elongata Möschler, Cephalospargeta;

Cephalospargeta elongatus Grote, Litomitus; Litomitus elonympha Hübner, Ephesia; Allotria elota Möschler, Collomena; Collomena emarginata Fabricius, Noctua; Oraesia emarginata Hampson, Ozarba;

Androlymnia emessa Druce, Anthoecia; Chalcoecia emiliusalis Walker, Diethusa; Diethusa emittens Walker, Casandria; Casandria emortualis [Denis \& Schiffermüller], Pyralis;

Trisateles empyra Turner, Neocleta; Neocleta empyrea Hübner, Noctua; Trigonophora endesma Lower, Prometopus; Dinoprora endoleucus Guérin-Méneville, Erebus;

Lygniodes endolopha Hampson, Ligidia; Tesomonoda endosticta Hampson, Eutornoptera;

Eutornoptera engenes Prout, Toanopsis; Toanopsis ennomoide Hampson, Goniocraspidum;

Goniocraspidum ennomoides Guenée, Thyridospila;

Thyridospila

ensipalpis Guenée, Coxina; Coxina

enthea Grote, Fishia; Fishia

eosarialis Walker, Enispa; Enispa

ephialtes Hübner, Noctua; Anthracia

ephyrodalis Walker, Egnasia; Egnasia

epiastra Meyrick, Leucania; Dipaustica

epichalca Meyrick, Hypenodes;

Pseudoschrankia

epichares Turner, Technemon; Technemon epimenis Drury, Phalaena; Psychomorpha epione Drury, Phalaena; Mormonia epionoides Guenée, Marmorinia;

Marmorinia

epipaschia Grote, Homohadena; Achytonix epiphracta Turner, Thelxinoa; Thelxinoa epiplemoides Hampson, Nicevillea;

Nicevillea

episcopalis Swinhoe, Pterogonia; Pterogonia epitusalis Walker, Mastygophora; Mastigia equalisella Walker, Zekelita; Zekelita equatorialis Holland, Sypna; Equatosypna eraconalis Walker, Glympis; Glympis erateinalis Walker, Cossedia; Cossedia erebina Hampson, Sypna; Supersypnoides erebipennis Walker, Casperia; Casperia ereboides Walker, Badiza; Badiza erebusalis Walker, Arrade; Arrade erebusalis Walker, Ipnea; Ipnea erecta Walker, Asthana; Asthana eremistis Püngeler, Mamestra; Cardiestra eremita Fabricius, Noctua; Dryobotodes eremna Franclemont, Apsaphida;

Apsaphida eremnopa Turner, Nestiodes; Nestiodes erigida Swinhoe, Aletia; Floccifera erinusalis Walker, Opotura; Opotura eriogona Hampson, Epigrypera; Epigrypera eriopioides Guenée, Heterochroma;

Heterochroma eriopis Herrich-Schäffer, Agarista;

Pristoceraea eriopoda Herrich-Schäffer, Ophiusa;

Scioptila

eriopoides Roepke, Euteliella; Euteliella ernesti Draudt, Metopodicha; Metopodicha ernestinana Blanchard, Nanthilda;

Nanthilda erratica Smith, Agrotis; Abagrotis erschoffi Christoph, Phothedes; Metopistis erubescens Hampson, Troctoptera;

Troctoptera erycata Cramer, Phalaena; Syllectra 
erygidia Hampson, Prionofrontia;

Prionofrontia

erythrias Meyrick, Haplopseustis;

Haplopseustis

erythropis Hampson, Stenoxia; Stenoxia

erythrospila Waller, Orsa; Orsa

eschata Franclemont, Euxoamorpha;

Euxoamorpha

esmeralda Brandt, Fredina; Fredina

esmeralda Dyar, Centrochlora; Centrochlora esperanza Schaus, Ophisma; Achaeops esperanzalis Schaus, Epiphanis; Epiphanis

esquina Dognin, Noctua; Eugrammodes

essoni Hampson, Peucephila; Peucephila

esula Druce, Xylina; Neogalea

etialis Dyar, Alesua; Alesua

eubolialis WValker, Aluaca; Aluaca

eucesta Turner, Acanthocoles; Acanthocoles

euclidica Walker, Colbusa; Colbusa

euclidicola Walker, Fodina; Parachalciope

eucraspedica Dyar, Calocea; Calocél

eudesmia Dyar, Walterella; Walterella

eudoxia Staudinger, Erythrophaia;

Erythrophaia

euenemus Dyar, Hortonius; Hortonius

eulepidea Hampson, Tarache; Protarache

eulepis Grote, Cleophana; Copicucullia

eumela Stoll, Phalaena; Diaphone;

Taeniopyga

euphancra Dyar, Metasarca; Metasarca

euphanera Hampson, Metasarca; Metasarca

euphemia Stoll, Phalaena; Heraclia

eupithecialis Guencee, Physula; Physulodes

eupithecioides Walker, Dagassa; Dagassa

euplexiodes Sugi, Hemictenophora;

Hemictenophora

euprepes Hampson, Euippodes; Euippodes

euprepia Hampson, Westermannia;

Hemichloridia

euproctisoides Walker, Thiganusa;

Thiganusa

eurapta Turner, Aedemon; Aedemon

eureka Turati \& Krüger, Aneureta;

Aneureta

euristea Stoll, Phalaena; Metria

euritiusalis Walker, Macella; Macella

eurychlora Wallker, Dandaca; Donda

eurymitra Turner, Thaumasiodes;

Thaumasiodes

eusciasta Hampson, Tolnaopsis; Tolnaopsis

euspila Walker, Tautobriga; Tautobriga

eustratii Alphéraliy, Heptapotamia;

Heptapotamia

entropia Stoll, Phalaena; Patica eversmanni Lederer, Hadena; Nyssocnemis evicta Grote, Cloantha; Morrisonia evidens Hübner, Noctua; Sideridis evingi Hampson, Persectania; Persectania evulsalis Walker, Apphadana; Amblygoes ewingii Westwood, Noctua; Persectania exacta Hübner, Anomis; Anomis exagens Walker, Claterna; Claterna exaggerata Schaus, Ipsia; Paralectra excarata Bethune-Baker, Ercheia;

Metatacha excavata Guenée, Odontina; Tinnodoa excavata Swinhoe, Cosmophila; Goniophila excellens Köhler, Argyrana; Argyrana excelsa Christoph, Clidia; Metopoplus excisa Hampson, Plectothripa; Plectothripa excisa Hampson, Trigonochrostia;

Trigonochrostia excisa Herrich-Schäffer, Noctua;

Ariathisa; Eulaphygma

excludens Walker, Crambopsis; Crambopsis exempta Walker, Calicula; Calicula exequialis Lucas, Yerongponga;

Yerongponga

exesa Warren, Lasionotella; Lasionotella exhibens Walker, Dapha; Hapda exigua Hübner, Noctua; Laphygma eximia Freyer, Cosmia; Megalodes eximia Holland, Methorasa; Pseudotolna eximia Pagenstecher, Hypena; Brontypena eximia Walker, Titulcia; Titulcia exocausta Hampson, Osthopis; Osthopis exornata Möschler, Hadena; Trichoplexia exotica Guenée, Eriopus; Hyperdasys expandens Walker, Obucola; Obucola expandens IValker, Ricla; Ricla expetita Walker, Bryophila; Melagramma expressa Lederer, Polia; Athaumasta exprimata Staudinger, Dasypolia;

Anhausta

exscendens Walker, Drobeta; Drobeta exscripta Walker, Asinduma; Asinduma exsiccata Lederer, Spintherops;

Tathorhynchus exsoleta Linnaeus, Phalaena; Xylena extensa Butler, Belosticta; Belosticta extensa Walker, Bematha; Bematha extollens Walker, Escua; Escua extorris Warren, Microedma; Microedma extranea Walker, Homopterá; Mulelocha extrema Walker, Deiopeia; Teinopyga exulis Lefebvre, Hadena; Crymodes exusta Butler, Gelastocera; Gelastocera exusta Gruenée, Ceramica; Ceramica 
fagana Fabricius, Pyralis; Pseudoips falcata Berio, Hypersophtha; Hypersophtha falcata Graeser, Calymnia; Eccopteroma falcata Swinhoe, Chilkasa; Chilkasa falcata Walker, Pleurona; Pleurona falcifera Kirby, Plusia; Anagrapha falcigera Berio, Falcapyris; Falcapyris falcigera Walker, Plaxia; Drepanoperas falculalis Guenće, Rhodina; Rhodina falernaria Guenée, Monoctenia; Monoctenia fallax Herrich-Schäffer, Moma; Agripodes falsalis Herrich-Schäffer, Hypena; Zebeeba famelica Walker, Masebia; Masebia fannia Schaus, Dusponera; Dusponera farinalis Linnaeus, Phalaena; Pyralis fasciata Bethune-Baker, Hirsutopalpis; Hirsutopalpis

fasciata Butler, Daphoenura; Daphoenura fasciata Butler, Eulocastra; Eulocastra fasciata Fawcett, Pteronycta; Pteronycta fasciata Moore, Borolia; Borolia fasciata Moore, Calpe; Hypocalpe fasciata Moore, Coarica; Coarica fasciata Moore, Ranaja; Ranaja fasciata Moore, Vapara; Vapara fasciata Rothschild, Narosodes; Tolpiodes fasciata Skinner, Psychophora; Barrovia fasciata Walker, Lazanda; Lazanda fasciata Walker, Minnagara; Minnagara fasciatella Grote, Spragueia; Fruva fasciatus Tams, Machaeropalpus;

Machaeropalpus

fasciculella Walker, Pharga; Pharga fasciculosa Walker, Acontia; Carandana fasciolata Butler, Erastria; Epopsima fastuosa Guenée, Noropsis; Noropsis fatua Püngeler, Lithocampa; Lophoterges fatua Warren, Micreremites; Micreremites fautrix Guenée, Entomogramma; Entomogramma featheri Hampson, Odontoretha; Odontoretha februalis Grote, Feralia; Arthrochlora feducia Stoll, Phalaena; Speiredonia feisthamelii Herrich-Schäffer, Agarista; Agaristodes felicina Donzel, Polia; Metopoceras felicita Smith, Baptarma; Baptarma fellearis Hübner, Apistis; Apistis fenestrata Boisduval, Hecatesia; Hecatesia ferraria Walker, Acherdoa; Acherdoa ferrifervens Walker, Nolasena; Nolasena ferrinalis Walker, Evia; Evia ferrogrisea Hampson, Heliophobus; Caffristis

ferruginea Esper, Bombyx; Rusina ferruginea Hampson, Eutrinita; Eutrinita ferruginea Walker, Nedroma; Nedroma fervens Butler, Macrochthonia;

Macrochthonia fessa Grote, Amolita; Amolita festiva Donovan, Phalaena; Calogramma festiva Fabricius, Bombyx; Diphthera festucae Linnaeus, Phalaena; Plusia fidelis Grote, Himella; Himella filamentosa Felder \& Rogenhofer, Thiona; Dochmiogramma filaria Smith, Egryrlon; Egryrlon filifera Walker, Orixa; Xoria filipalpis Walker, Ansa; Ansa fimbria Linnaeus, Phalaena; Lampra fimbrialis Dyar, Escandia; Escandia fimbriares Walker, Phlossa; Phlossa fimbriaris Guenée, Heliophobus;

Eucoptocnemis

fimbriata Schreber, Phalaena; Lampra fimbripes Walker, Agraga; Agraga finifascia Walker, Nephelodes; Anua fiorii Berio, Donuctenusa; Donuctenusa fiorii Berio, Microraphe; Microraphe fiorii Costantini, Hylophila; Chloephila fixa, Fabricius, Noctua; Synthymia fixseni Christoph, Pseudophia; Pseudathetis flabellum Guenée, Thyriodes; Thyriodes flaminia Möschler, Gonuris; Gonuris flammea Curtis, Melia; Senta flammea [Denis \& Schiffermüller], Noctua; Panolis flammea Esper, Bombyx; Rhizotype flandriana Berio, Hypersypnoides; Hyposypnoides flava Dognin, Psectrotarsia; Psectrotarsia flava Fabricius, Noctua; Cosmophila flava Janse, Oncotibialis; Oncotibialis flavagalis Guenée, Platydia; Yidalpta flavago [Denis \& Schiffermüller], Noctua;

Gortyna

flavago Fabricius, Noctua; Xanthia

flavala Hampson, Thiochroa; Thiochroa

flavalis Gaede, Leptamma; Leptamma

flavellus Blanchard, Phycopterus; Phycopterus

flavibasis Hampson, Tarachidia; Tarachidia flavicaudata Warren, Amphipyra;

Paraxestia

flavicornis Hampson, Callophisma; Callophisma 
flavicornis Linnaeus, Phalaena; Achlya flavicosta Smith, Conacontia; Conacontia flavida Felder, Earias; Digba

flavida Ochsenheimer, Anthophila; Metoponia

flavidentula Schaus, Mamestra; Poliodestra

flavifera Hampson, Phoenicophanta;

Phoenicophanta

flavilinguis Grote, Alypiodes; Alypiodes

flavirena Hampson, Strigiphlebia;

Strigiphlebia

flavirosea Hampson, Carcharoda;

Carcharoda

flavistriaria Hübner, Crochiphora;

Crochiphora

flavizonata Hampson, Callostrotia;

Callostrotia

flavociliata Aurivillius, Anua; Subanua

flavocostata Möschler, Bavilia; Bavilia

flavofimbria Saalmüller, Anchiroe;

Anchiroe

flavomacula Oberthïr, Madopa;

Paragabara

fleissiana Köhler, Tisagronia; Tisagronia

fletcheri Orfila \& Schajovskoi, Laninia;

Laninia

flexilineata Hampson, Nola;

Melanographia

flexula [Denis \& Schiffermüller], Bombyx;

Laspeyria

flexuosa Walker, Certila; Certila

floccalis Zeller, Coptocnemia; Coptocnemia

floccosa Walker, Declana; Declana

flora Alphéraky, Grumia; Grumia

florens Walker, Gaurena; Gaurena

florescens Walker, Celaena; Omphaletis

florida Guenée, Rhodophora; Rhodophora

florifera Walker, Polytela; Polytelodes

florigera Walker, Tetrisia; Tetrisia

fluviatilis Lucas, Imleanga; Imleanga

fola Swinhoe, Proneca; Proneca

formosa Blanchard, Caloptera; Egybolis

formosa Grote, Leptina; Chrysanympha

formosa Hampson, Ptyonota; Ptyonota

formosalis Walker, Nigetia; Nigetia

formosana Matsumura, Conicophoria;

Conicophoria

formosanus Matsumura, Akonus; Akonus

forsteri Draudt, Valerietta; Valerietta

fortis Grote, Homohadena; Homoncocnemis

fortissina Moore, Checupa; Checupa

fortunaria Guenée, Xyliodes; Xyliodes

fotelloides Barnes \& McDunnough, Stilbia;

Anycteola fovea Treitschke, Phlogophora; Rileyiana fragariae Vieweg, Noctua; Orbona fragilis Guenée, Micrococlia; Microcoelia fragilis Turner, Goniophylla; Goniophylla frater Grote, Raphia; Saligena

fraternella Strand, Calesidesma;

Calesidesma

fraxini Linnaeus, Phalaena; Hemigeometra frequens Holland, Symplusia; Symplusia frigida Mabille, Agrotis; Noctubourgognea friwaldszkii Duponchel, Heliothis;

Janthinea

frontalis Strand, Amyna; Formosamyna frontalis Walker, Arrhapa; Arrhapa frontalis Walker, Maxilua; Maxilua frontalis Walker, Mulelocha; Mulelocha frontalis Walker, Plotheia; Plotheia frontalis Walker, Rabila; Rabila frustulum Guenée, Celiptera; Celiptera fuegensis Hampson, Metalepsis; Beriotisia fugitiva Walker, Cerbia; Cerbia fulgularis Matsumura, Noshimea; Noshimea fulgurea Guenée, Heterospila; Heterospila fulgurita Lederer, Hypeuthina; Hypeuthina fuliginaria Linnaeus, Phalaena; Parascotia fuliginosa Druce, Hypocchana; Hypoechana fullonia Clerck, Phalaena; Othreis fulminans Bryk, Lophiophora; Lophiophora fulminea Scopoli, Phalaena; Ephesia fulvago Clerck sensu Hübner, Noctua; Enargia

fulvana Bethune-Baker, Pseudogyrtona;

Pseudogyrtona

fulvescens Hampson, Ozarba; Fulvarba fulvirufa Hampson, Lophorache;

Lophorache

fumida Schaus, Aplocampa; Aplocampa fumipennis Felder \& Rogenhofer,

Gracilodes; Tatorinia

fumosa [Denis \& Schiffermüller], Noctua;

Brotis

funebris Moore, Eusemia; Cruriopsis

funesta Esper, Noctua; Aedia

funestalis Walker, Cophanta; Cophanta

fungorum Grote \& Robinson, Choephora;

Choephora

furcifer Hampson, Schalifrontia;

Schalifrontia

furcifera Hufnagel, Phalaena; Graptolitha

furcifera Walker, Pucialia; Pucialia furcifera Walker, Sebagena; Sebagena furcilla Grote, Argillophora; Argillophora furcilla Packard, Platycerura; Platycerura furcivitta Hampson, Centrartha; Centrartha 
furcula Walker, Diopa; Diopa

furfurata Grote, Himella; Homorthodes

furihatai Okano, Neoplusia; Neoplusia

furtiva Dognin, Compsenia; Compsenia

furtiva Turner, Anomophlebia;

Anomophlebia

furuncula [Denis \& Schiffermüller], Noctua;

Mesoligia

furvula Hübner, Noctua; Athetis

fusca Bethune-Baker, Paranoratha;

Paranoratha

fusca Hampson, Sesamia; Calamistis;

Busseola

fuscescens Walker, Edema; Adrana

fuscicollis Butler, Triphaena; Isoura

fuscifascia Dognin, Lycaugesia; Lycaugesia

fuscifrons Walker, Cosmophila;

Xanthanomis

fuscirena Hampson, Psectrotarsia;

Psectrotarsia

fuscomarginata Bethune-Baker, Zethes;

Cryptastria

fusifera Walker, Mazacyla; Mazacyla

fusipennis Walker, Phuphena; Phuphena

gabunalis Holland, Elyra; Trichopalpina galatheae Wallengren, Leocyma; Curubasa galena Smith, Viridemas; Viridemas

gallinago Felder \& Rogenhofer, Selenis;

Goniohelia

gallinalis Felder \& Rogenhofer, Blemmatia;

Blemmatia

gallopavo Walker, Baecula; Baecula

gamma Linnaeus, Phalaena; Autographa

gammophora Guenée, Emarginea;

Emarginea

gastropachoides Guenée, Calesia; Mecyra

gaurae Smith, Phalaena; Rhodophora

gebenna Swinhoe, Talpa; Poliofoca

geldersi Schaus, Pseudarista; Pseudarista

gemella Leech, Perigea; Dysmilichia

geminipuncta Turner, Paurosceles;

Paurosceles

geminipunctalis Schaus, Macristis;

Macristis

gemmata Hampson, Pisara; Dialithoptera

gemmatalis Hübner, Anticarsia; Anticarsia

gemmea Treitschlse, Miselia; Crypsedra

gemmifera Hübner, Dialithis; Dialithis

genisusalis Waller, Lista; Lista

genitrix Grote, Hadena; Aseptis

gentilis Berio, Vulcanica; Vulcanica gentilis Grote, Taeniosea; Taeniosea gentiusalis Walker, Edessena; Edessena geographica Fabricius, Noctua; Oxicesta geometrica Fabricius, Noctua; Grammodes geometriformis Strand, Biareolifera;

Biareolifera

geometroides Walker, Corula; Corula geometroides Walker, Lusia; Amilaga geraldi Kirby, Pristoceraea; Charitosemia gerula Guenée, Diatenés; Diatenes geryon Fabricius, Noctua;

Xanthospilopteryx geyeri Felder \& Rogenhofer, Eurhipia;

Silacida

giacomellii Berio, Argyrargenta;

Argyrargenta

gilvago [Denis \& Schiffermüller], Noctua;

Mellinia

gilviceps Turner, Synthaca; Synthaca gilvipennis Grote, Agrotis; Cryptocala gladiata Butler, Cidariplura; Cidariplura glarea Treitschke, Anthophila; Rhypagla glaucescens Hampson, Aspidifrontia;

Aspidhampsonia glaucina Esper, Bombyx; Episema glaucineta Walker, Bulna; Bulna glaucochlora Hampson, Ancara;

Chlorognesia

glaucopasta Bethune-Baker, Ostacronycta;

Ostacronycta

glaucopoides Walker, Spersara; Spersara

glebosa Saalmüller, Dianthoecia;

Saalmuellerana

globulipes Walker, Homoptera; Lophotavia glomeralis Walker, Catada; Catada gloriosae Fabricius, Bombyx; Polytela gloveri Grote \& Robinson, Euscirrhopterus; Copidryas

glycinae Lewin, Phalaenoides;

Phalaenoides

glyphica Linnaeus, Phalaena; Euclidia gnaphalii Hübner, Noctua; Eucalimia godalma Schaus, Thyatira; Thyatirodes goniosema Hampson, Epicerynea;

Epicerynea

gortynoides Walker, Bellura; Bellura gortynoides Walker, Elocussa; Elocussa gothica Linnaeus, Phalaena; Semiophora gouzzakouli Dumont, Mesaegle; Mesaegle gracilis Möschler, Sisputa; Sisputa gracilis Osthelder, Pfeifferella; Ostheldera gracilis Wagner, Amelia; Amelina graminicolens Butler, Dianthoecia;

Leumicamia 
graminis Linnaeus, l'halaena; Cerapteryx graminosa Walker, Erana; Feredayia grammatistis Meyrick, Prionophora;

Prionopterina grammivora Walker, Dargida; Dargida grandipennis Grote, Agrotis; Pseudoseptis grandis Schaus, Acanthodica; Acanthodica graphica Hübner, Drasteria; Drasteria graphiphorides Walker, Tetrapyrgia;

Tetrapyrgia

grata Butler, Leptina; Antha

grata Hübner, Elaphria; Elaphria grata Walker, Sosxetra; Sosxetra gratissima Walker, Nolasena; Chloriola gravata Guenée, Ophisma; Ophisma gravatalis Walker, Bocana; Ragana gravipes Walker, Nagia; Nagia grisea Bethune-Baker, Cellacrinata;

Cellacrinata grisea Hampson, Stenosticta; Stenosticta grisea Moore, Hyada; Hyada griseata Hampson, Bryophilopsis;

Bryophilopsis griseata Petersen, Timandra; Timandra griseifusa Hampson, I obocraspis;

Lobocraspis

griseifusa Hampson, Mecistoptera;

Mecistoptera

griseisigna Wileman \& South, Eucampima;

Eucampima

grisella Schaus, Thalpochares; Hayesia griseocincta Hampson, Xanthoptera;

Paracroria

grisescens Barnes \& Lindsey, Langona;

Gloanna

grisescens Chrétien, Outaya; Outaya grisescens Osthelder, Maraschia; Maraschia groenlandica Duponchel, Hadena;

Crymodes

grotei Morrison, Valeria; Copivaleria grotelliformis Barnes \& McDunnough,

Homolagoa; Homolagoa

grumi Alphéraky, Heliophobus;

Sympistoides

guarama Schaus, Thalpochares;

Micromonodes

guarana Schaus, Ocalaria; Ocalaria

guianensis Hampson, Gracilopsis;

Gracilopsis

gulo Herrich-Schäffer, Lichnoptera;

l.ichnoptera

guttalis Walker, Molvena; Molvena

guttiventris Walker, Chlumetia; Chlumetia

gyona Schaus, Boalda; Boalda hachem Dupont, Crosia; Crosia hadeniformis Smith, Mamestra; Trudestra hadenoides Walker, Cropia; Cropia hadesia Schaus, Psorya; Psorya haemaplaga Hampson, Hoplarista; Hoplarista

haematina Boursin, Erebophasma; Erebophasma

haematoessa Holland, Capnodes; Phlogochroa

haemorranta Hampson, Narangodes; Narangodes

haemorrhanta Hampson, Hypoperigea; Thegalea

haemorrhanta Turner, Hypoperigea;

Thegalea

haeretica Püngeler, Polia; Turanica haidingeri Heer, Noctuites; Noctuites hampsoni Barnes \& Benjamin, Sexserrata; Sexserrata

hampsoni Brèthes, Allorhodoecia; Allorhodoecia

hampsoni sp. n., Pachythrix; Pachythrix hampsoni Wileman, Asinduma;

Negritothripa

hansali Felder \& Rogenhofer, Remigia;

Beriohansa

harmina Schaus, Acontia; Monocymia harminella Dyar, Cymonia; Cymonia harmonia Schaus, Carillade; Carillade harmonica Hampson, Chalciope; Euphiusa hartigi Berio, Pluxilloides; Pluxilloides haruspica Grote, Agrotis; Pseudospaelotis hastatalis Walker, Hypena; Crymona hastulifera Smith, Phalaena; Megacronycta hatuey Poey, Noctua; Litoprosopus hebraeicum Hübner, Polygrammate;

Polygrammate

hebrusalis Walker, Galanda; Galanda hecate Viette, Xylomania; Rungsianea hecateia Köhler, Phaenagrotis; Phaenagrotis heliaca [Denis \& Schiffermüller], Noctua;

Panemeria

heliophila Hübner, Noctua; Apaustis heliophila Paykull, Noctua; Sympistis hemileuca Guence, Palindia; Palindia hemiochra Hampson, Omphalagria;

Omphalagria henkei Staudinger, I.eucanitis; Palpangula henrici Grote, Leucania; Ablepharon henrietta Grote, Philomma; Philomma hepara Guence, Cralgula; Galgula hepatica Moore, Nasaya; Nasaya 
hepatizans Guenée, Amphigonia;

Amphigonia

hepialoides Guenée, Amphia; Amphia

herbaria Swinhoe, Hiccoda; Uncula

herbealis Walker, Labanda; Labanda

herbida Walker, Dumatha; Dumatha

herbitecta Dyar, Pangrapta; Pangraptella

herchatra Swinhoe, Crymodes; Trichoridia

herrichschaefferi Alphéraky, Agrotis;

Estimata

hesparia Cramer, Phalaena; Massaga

hesperina Herrich-Schäffer, Darceta;

Darceta

hesperioides Guenée, Zethes; Zethesides

hesperioides Pagenstecher, Dahlia; Dahlia

hesperoides Köhler, Stenagrotis;

Atlantagrotis

hesychima Turner, Ecnomia; Ecnomia

hiberna Köhler, Petrowskya; Petrowskya

hiemalis Filipjev, Borisia; Sirioba

hiemalis Grote, Xylomiges; Xylomania

hieroglyphica Cramer, Phalaena; Noropsis

hieroglyphica Drury, Phalaena; Argiva

hieroglyphica Holland, Episparis;

Episparina

hieroglyphica Moore, Baorisa; Baorisa

hieroglyphica Saalmüller, Hemiceras;

Hemiceratoides

hieroglyphica Swinhoe, Oxaenanus; Tamsia hieroglyphica Walker, Erymella; Erymella hilaris Staudinger, Metopoceras;

Harpagophana

himalayensis Hampson, Pachypolia;

Isopolia

himerata Walker, Erosia; Ectrogatha

hircina Morrison, Homoglaea; Homoglaea

hirta Hübner, Noctua; Ulochlaena

hirticornis Haworth, Noctua; Apamea

hirtipalpis Walker, Bleptina; Metina

hirtipes Grote, Zosteropoda; Zosteropoda

hirtipuncta Schaus, Tranoses; Tranoses

hirtissima Walker, Premusia; Herchunda

hirudinicornis Guenée, Phycodes; Phycodes

hispanalis Guenée, Nodaria; Nodaria

hispida Hübner, Noctua; Leucochlaena

histigma Dyar, Hopothia; Hopothia

hochenwarthi Hochenwarth, Phalaena;

Caloplusia

hoedularia Guenée, Azelina; Egabra

hoenei Boursin, Palaeamathes;

Palaeamathes

hoenei Draudt, Palaeosafia; Palaeosafia

hoenei Draudt, Taenerema; Taenerema

hollandiae Guenée, Dasygaster; Dasygaster holophaea Bethune-Baker, Gortynodes; Gortynodes

holzi Pagenstecher, Alamis; Macrocarsia

homichlodes Hübner, Chamyna; Chamyna

homochroa Dognin, Parelectra; Parelectra

homogenea Hampson, Trichophotia;

Trichophotia

homopteroides Walker, Veia; Veia

honesta Hübner, Thyas; Thyas

honestalis Treitschke, Pyralis; Actenia

hopkinsi Tams, Anomocala; Anomocala

hormos Hübner, Hypsoropha; Monogona

horrida Hübner, Zale; Zale

horridella Walker, Aquita; Aquita

horridipes Walker, Crithote; Crithote

horsfieldi Guenée, Lineopalpa; Lineopalpa

hospes Freyer, Caradrina; Proxenus

hostilis Walker, Stibaena; Stibaena

howardi Edwards, Plusia; Rodriguesia

huallatani Staudinger, Andicola; Andicola

hueberi Ershov, Acontia; Tarachephia

humeralis Walker, Molynda; Molynda

humidalis Doubleday, Hypenodes;

Hypenodes

huntei Warren, Thyrsoscelis; Careades

hyalophora Hampson, Euplexia; Thyrestra

hyas Schaus, Uzinia; Uzinia

hyblaeoides Walker, Torone; Torone

hybris Hübner, Noctua; Raphia

hydrocampata Guenée, Phibalapteryx;

Hyposada

hylea Stoll, Phalaena; Neomilichia

hypaenides Staudinger, Stilbina; Stilbina

hypenalis Walker, Bocana; Hadennia

hypenoides Moore, Acantholipes; Isatoolna

hypenoides Schaus, Phiprosopus;

Leucogramma

hypenoides Walker, Badausa; Badausa

hypenoides Walker, Condate; Condate

hyperacantha Berio, Cassania; Cassania

hyperici [Denis \& Schiffermïller], Noctua;

Chloantha

hyperion Dyar, Pumora; Pumora

hyperythrum Hampson, Tinosoma;

Tinosoma

hyphinoe Cramer, Phalaena;

Cryptochrostis

hypnois Hübner, Syrnia; Syrnia

hypoleuca Dognin, Barcita; Palaechthona

hypopyroides Walker, Enmonodia;

Enmonodia

hyporrhoda Turner, Symmolpis; Symmolpis

hypozonata Hampson, Lycaugesia;

Quandara 
hyppasia Cramer, Phalaena; Trigonodes

hypsoides Walker, Surina; Surina

hyrtacides Schaus, Anorena; Anorena

iarbasalis Walker, Ortopla; Ortopla iaspis Guenće, Apamea; Chytonix icamba Swinhoe, Euplexia; Valeriodes i-cinctum [Denis \& Schiffermüller], Noctua;

Perigrapha

icterias Eversmann, Xylina; Hypobarathra icteritia Hufnagel, Phalaena; Cirrhia idella Barnes, Cerathosia; Hemispragueia idia Staudinger, Chamyla; Chamyla idonea Walker, Maxula; Maxula igneola Swinhoe, Egnasia; Chrysograpta ignicola Staudinger, Pseudoleucania;

Pseudoleucania

igniflua IVileman \& South, Eugrapta;

Eugrapta

ignifusa Hampson, Acroriesis; Acroriesis

ignobilis Schaus, Trachysmatis;

Trachysmatis

ikondae Berio, Ikondiana; Ikondiana

illattioides Hampson, Lobocheilos;

Lobocheilos

illaudabilis Grote, Mamestra; Lacinipolia

illegitima Wallengren, Anophia;

Lipatephia

illibata Fabricius, Noctua; Pindara

illita Guenée, Anomis; Ristra

illocata Warren, Dyspyralis; Dyspyralis

illunaris Hübner, Noctua; Clytie

illustris Fabricius, Noctua; Euchalcia

imbecilla Fabricius, Noctua; Eriopygodes

imitata Druce, Myrtale; Myrtale

immacula Grote, Cea; Sparkia

immaculalis Hulst, Botis; Reabotis

immortua Grote, Melaporphyria;

Nelaporphyria

immundalis Walker, Maguda; Maguda

imparata Wallker, Rhesala; Rhesala

impartialis Harvey, Eubolina; Eubolina

imperialis Jordan, Aletopus; Aletopus

impingens Walker, Gadirtha; Gadirtha

impleta Walker, Acronycta; Philorgyia

implexata Walker, Palura; Palura

implora Grote, Azenia; Azenia

improba Schans, Bradunia; Bradunia

inamoena Hübner, Noctua; Odice

inara Cramer, Phalaena; Serrodes

inassueta Guenée, Prometopus; Prometopus inca Dyar, Aleptina; Aleptina incana Dognin, Carteris; Carteris incarnata Freyer, Heliothis; Rhodocleptria incendiata Guenée, Erosia; Erosia incerta Hufnagel, Phalaena; Orthosia incerta Roepke, Feliniopsis; Feliniopsis incertalis Walker, Herminia; Hyposemeia incidens Walker, Bagisara; Bagisara incidens Walker, Bareia; Bareia incitans Walker, Gadera; Gadera includens Walker, Avatha; Avatha includens Walker, Plusia; Pseudoplusia inconclusa Walker, Cosmophila; Sarobides incondita Dognin, Leucatomis; Leucatomis incondita Schaus, Nyctipolia; Nyctipolia incongrualis Walker, Suma; Suma inconspicua Walker, Corna; Corna inconstans Guenée, Panula; Panula inconstans Walker, Ctypansa; Ctypansa incrassata IValker, Dyrzela; Maxilua incurvata Schaus, Capnodes;

Drepanoblemma indecisa Moore, Culasta; Culasta indecora Köhler, Schachowskoya;

Schachowslioya indecoralis Walker, Nagadeba; Nagadeba indeterminata Walker, Tavila; Tavila indica Moore, Phlogophora; Appana indigesta Staudinger, Scotocampa; Scotocampa indigna Walker, Decelea; Decelea indistans Guenée, Hadena; Eutamsia indistincta Moore, Vapara; Xylostola ineffectalis Walker, Hypena; Naarda inermis Dyar, Cacofota; Cacofota inexacta Walker, Gadirtha; Gadirtha inexacta Walker, Thermesia; Euthermesia inextricata Moore, Eutelia; Silacida inextricata Moore, Rhizogramma; Euscotia infecta Ochsenheimer, Agrotis; Anicla inferalis Walker, Iambia; Iambia inferior Guenće, Apamea; Tycomarptes infida Walker, Targalla; Targalla infirma Guenée, Orthodes; Orthodes infixaria Walker, Narulla; Narulla inflexa Möschler, Asylaea; Asylaea infusa Walker, Decelea; Listonia ingoufii Mabille, Agrotis; Tamseuxoa iniqua Guenée, Nymbis; Nymbis innocua Butler, Nola; Epizeuctis innotabilis Waller, Plotheia; Thopelia inops Grote, Orthosia; Spartiniphaga inops Lederer, Hadena; Palaeagrotis inops Stephens, Scopelopus; Scopelopus 
inornata Grote, Trichocosmia; Trichocosmia inornata Schaus, Sinosia; Sinosia inornata Walker, Deva; Arytrurides inquieta Walker, Celaena; Gaphara inquinata Lederer, Madopa; Tephrochares inquirendus Strand, Cremopalpus; Cremopalpus

inscripta Rebel, Pyralomorpha;

Pyralomorpha inscripta Walker, Marcipa; Marcipa insecuta Walker, Leucania; Neoborolia insignata Lederer, Graphiphora;

Eugnorisma insignis Aurivillius, Ichthyopselapha;

Ichthyopselapha insocia Walker, Acontia; Metapioplasta insocia Walker, Sarbanissa; Sarbanissa insolita Staudinger, Hadula; Hadula insolitata Walker, Cacyparis; Cacyparis instabilis [Denis \& Schiffermüller], Noctua; Orthosia instans Smith, Minofala; Minofala instruens Walker, Tavia; Tavia insularis Rambur, Zethes; Zethes insularis VVarren, Tridentifrons;

Tridentifrons insulata Walker, Birtha; Birtha insulicola Guenée, Leucania; Boursinania intacta Hampson, Ilyrgodes; Ilyrgodes interclusa Walker, Psaphara; Psaphara interitalis Guenée, Sarmatia; Sarmatia interlecta Walker, Hespagarista;

Hespagarista interlineata Walker, Pessida; Pessida internalis Robinson, Hypena; Euhypena interruptalis Walker, Aegara; Aegara intracta Walker, Azirista; Azirista intractata Morrison, Taeniocampa; Himella intrahens Walker, Premusia; Premusia intricatus Butler, Halastus; Halastus intrusa Krüger, Africalpe; Africalpe inusitata Saalmuiller, Parca; Parca invaria Walker, Leucania; Canthylidia invaria Walker, Mecyra; Mecyra invenusta Swinhoe, Bertula; Raphiscopa involuta Walker, Chiripha; Chiripha iochlora Turner, Dumigania; Dumigania ioglauca Zerny, Agrotis; Apoxestia iomelas Draudt, Lasiridia; Lasiridia iorrhoda Turner, Clytoscopa; Clytoscopa iphida Swinhoe, Clettharra; Apothripa irene Guenée, Capnodes; Capnodes iridescens Dognin, Baniana; Neathyrma iridias Meyrick, Thyrsoscelis; Thyrsoscelis iridocosma Turner, Alypophanes; Alypophanes iridoplitis Viette, Volazaha; Volazaha iris Guenée, Phlogophora; Mesolomia iris Zetterstedt, Hadena; Crasia irregularis Hübner, Noctua; Bendis irregularis Hufnagel, Phalaena; Anepia irrenosa Guenée, Lepidomys; Lepidomys irresoluta Walker, Rhubuna; Rhubuna irretita Hübner, Eusceptis; Eusceptis irrigualis Möschler, Phlyctaina;

Phlyctaina

irrisoria Ershov, Mamestra; Cardepia italica Fabricius, Noctua; Tyta iter Guenée, Calyptis; Calyptis itynx Fabricius, Noctua; Speiredonia

jacksoni Bethune-Baker, Agrotana; Agrotana jactatalis Walker, Hypena; Dogninades jamaicalis Schaus, Diplodira; Diplodira jamaicensis Möschler, Listonia; Listonia janata Linnaeus, Phalaena; Achaea janeira Schaus, Arpia; Arpia jankowslkii Oberthür, Apatela; Hampsonidia jankowskii Oberthür, Dichagyris; Stenoloba jansei Berio, Afrenella; Afrenella jansei Romieux, Ophiosema; Ophiosema janthina [Denis \& Schiffermüller], Noctua; Euschesis japana Motschulsky, Chelonomorpha; Chelonomorpha

japeta Stoll, Phalaena; Pseudbarydia japonica Leech, Dipterygia; Dipterygina japonica Leech, Piada; Mimanuga japonica Ogata,Pelamia; Melapia jarinta Schaus, Peteroma; Peteroma jaspidea Villers, Phalaena; Synvaleria jezoensis Matsumura, Colocasiodes; Colocasiodes jezoensis Matsumura, Maikona; Maikona joa Boisduval, Cyligramma; Cyligramma jocelyna Clench, Philippodamias;

Philippodamias jocosa Guenée, Diphtera; Feralia jocosatrix Guenée, Penicillaria; Bombotelia jordana Staudinger, Catamecia; Catamecia josioides Walker, Aucula; Aucula jourdanaria Serres, Geometra; Compsoptera jucunda Hübner, Melipotis; Melipotis jucunda Hübner, Noctua; Microphisa jucunda Jordan, Pseudospiris; Choeropais 
judis Dyar, Tineocephala; Tineocephala jugorum Ershov, Heliothis; Hypsophila julia Cramer, Phalaena; Brephos julia Grote, Rhodosea; Rhodosea julitae Benjamin, Ommatostolidea;

Ommatostolidea

juncta Hampson, Fodina; Drepanofoda junctimacula Christoph, Agrotis; Xenophysa juno Dalman, Noctua; Lagoptera jutalis Walker, Bocana; Hadennia juturna Cramer, Phalaena; Antaea juvenilis Bremer, Agnomonia; Leucomelas juventina Stoll, Phalaena; Callopistria

kaekeritziana Hübner, Tortrix; Aegle karsiana Staudinger, Victrix; Victrix kebea Bethune-Baker, Ancaroides;

Ancaroides

kebea Bethune-Baker, Erygansa; Erygansa keelei Gibson, Parabarrovia; Parabarrovia keiensis Prout, Rhesalides; Rhesalides kenricki Bethune-Baker, Diptheroides;

Diptheroides

kinabaluensis Rothschild, Crinocula;

Crinocula

klugii Boisduval, Ophiusa; Heliophisma kneuckeri Rebel, Acrobyla; Acrobyla kohleriana Petrowsky, Epioecia; Epioecia korbae Püngeler, Sinocharis; Sinocharis krugii Möschler, Pseudohemiceras;

Pseudohemiceras

kuehni Warren, Mesoruza; Mesoruza kulmburgi Rebel, Acrosphalia; Acrosphalia kuyanianus Matsumura, Akoniodes;

Akoniodes

kuznetzovi Daricheva, Mervia; Mervia

laba Druce, Focilla; Cymosafia labandina Hampson, Lophodaxa;

Lophodaxa

labecula Esper, Phalaena; Dryobota lacernaria Hübner, Geometra; Rhypagla laches Druce, Valeria; Paratrachea lachnogyia Tams, Mormecia; Mormecia laciniata Felder \& Rogenhofer, Amphigonia;

Polygoniodes

lacista Holland, Deinypena; Deinypena

lactaria Graeser, Nola; Nolathripa

lactea Fabricius, Noctua; Empusada

lactea Stretch, Cisthene; Grotellaforma ladakensis Felder \& Rogenhofer, Anarta; Trichanarta

laeta Jordan, Musurgina; Musurgina

laeta Walker, Ballatha; Ballatha

laetabilis Smith, Hadena; Paramiana

laetabilis Walker, Epitausa; Epitausa

laetabilis Zetterstedt, Hadena; Anomogyna

laetrina Druce, Apamea; Pseudoxylomoea

laetulus Grote, Lomanaltes; Lomanaltes

lagena Grote, Agrotis; Protogygia

lagenifera Moore, Luperina; Daseuplexia

lagusalis Walker, Oglasa; Oglasa

lama Piingeler, Heliophobus; Blepharosis

lanıbertoni Oberthür, Hypsiforma;

Hypsiforma

lamda Fabricius, Noctua; Rhizolitha

laminata Butler, Tyrissa; Diagrapta

laminifera Saalmüller, Ovios; Ancarista

lamusalis Walker, Ensipia; Ensipia

lainuta Hertz, Anarta; Anartodes

lanceola Guenée, Mecodina; Mecodina

lanceolata Roepke, Stenopydna;

Stenopydna

lanceolata Walker, Alaria; Curubasa

lanifera Walker, Eragisa; Eragisa

lanigera Butler, Epicausis; Epicausis

lapidaria WValker, Clina; Clina

lapsalis Walker, Hipoepa; Hipoepa

larentica Hampson, Amyna; Pseuderastria

larentiodes Prout, Pagetia; Tegiapa

larentioides Grote, Phalaenostola;

Phalaenostola

laronia Druce, Thalpochares; Argyrhoda

larusalis Walker, Isana; Isana

lasserrei Oberthür, Luperina; Powellinia

latalis Walker, Meranda; Meranda

latens Hübner, Noctua; Epipsilia

lateritia Hufnagel, Phalaena; Agroperina

lathridia Turner, Epicyrtica; Epicyrtica

laticincta Walker, Gerbatha; Gerbatha

latifascialis Walker, Selca; Selca

latiflavaria Swinhoe, Chrysocraspeda;

Chrysozonata

latinus Donovan, Phalaena; Eutrichopidia latiorella Walker, Acrobasis; Diallagma latipalpis Walker, Hydrelia; Hormoschista latipennis Herrich-Schäffer, Mastigophora;

Drepanopalpia

latipennis Walker, Coruncala; Coruncala

latipes Guenée, Remigia; Remigia

latipes Walker, Tipra; Tipra

lativitta Moore, Eudorea; Erastriopis

lativitta Moore, Jarasana; Jarasana

latreillei Duponclicl, Noctua; Methorasa 
latruncula [Denis \& Schiffermüller], Noctua; Procus

laudeti Boisduval, Cleophana; Enterpia

laurentia Schaus, Bleptiphora; Bleptiphora

lauta Püngeler, Mamestra; Lasianobia

lawinda Pagenstecher, Poeciloptera;

Hexamitoptera

lawsoni Felder \& Rogenhofer, Diatenes;

Eudesmeola

lebana Schaus, Anablemma; Anablemma

lectrix Linnaeus, Phalaena; Episteme

ledereri Wallengren, Setoctena; Setoctena

legrandi Berio, Apothriguna; Apothriguna

leichardtii Koch, Villosa; Villosa

leineri Freyer, Apamea; Conisania

lemonia Berio, Ecthymia; Ecthymia

lemur Felder, Cremnodes; Mocrendes

lenis Staudinger, Phoebophilus; Eremopola

lentalis Guenée, Hydrillodes; Hydrillodes

leo Guenée, Agrophila; Spragueia

leona Schaus, Aegocera; Schausia

leona Schaus, Gonitis; Deinopalpus

leontia Stoll, Phalaena; Peosina

lepida Butler, Graphiphora; Sugitania

lepidula Grote, Jaspidia; Bryocodia

leporina Linnaeus, Phalaena; Acronicta

lepraota Hampson, Zethes; Tropidtamba

leprosticta Hampson, Euplexia; Plagideicta

lepticyma Hampson, Pleurona; Pleuronodes leptochroma Turner, Crioa; Ochrothripa

leptomera Walker, Ipana; Ipana

leucania Barnes \& Lindsey, Mammifrontia;

Mammifrontia

leucanioides Schaus, Hyponeuma;

Hyponeuma

leucanioides Walker, Cholimma; Cholimma leucobasis Hampson, Euplexia; Monoptya

leucochorda Turner, Zophochroa;

Zophochroa

leucocosmalis Walker, Madoce; Madoce leucocraspis Hampson, Hyriodes; Hyriodes leucogastralis Valker, Phagytra; Phagytra leucoglene Mabille, Erastria; Xanthomera

leucogrammica Hampson, Dolichosomastis;

Dolichosomastis

leucographa [Denis \& Schiffermuiller],

Noctua; Gypsitea

leucomelas Linnaeus, Phalaena; Anophia

leucomelas Linnaeus sensu Hübner, Noctua;

Aedia

leucomera Dyar, Concana; Epiconcana leucomera Hampson, Dischalis; Dischalis leuconephra Hampson, Crlaucicodia;

Glaucicodia leuconeura Hampson, Grammoscelis;

Grammoscelis

leucophaea [Denis \& Schiffermüller],

Noctua; Pachetra

leucopicta Kenrick, Trachea; Prototrachea

leucoplaga Hampson, Neolaphygma;

Neolaphygma

leucopolia Turner, Hypolispa; Hypolispa

leucoptera Hampson, Hypaetra; Tytroca

leucorhabda Hampson, Brachygalea;

Brachygalea

leucorhabda Jones, Doryodes; Microlita leucospila Walker, Capnodes; Antapistis leucospila Walker, Prospalta; Prospalta leucosticta Schaus, Prorivula; Prorivula leucostigma Hübner, Noctua; Helotropha leucostrepta Hampson, Crambiforma;

Crambiforma

leucotelus Walker, Euthisanotia; Leonides

leucozona Turner, Axiorata; Axiorata

lewinii Butler, Leucania; Eurypsyche

lewisi Grote, Pleonectopoda; Pleonectopoda

libatrix Linnaeus, Phalaena; Scoliopteryx

libitina Druce, Gustiana; Aggustiana

liburna Geyer, Clytie; Scolecocampa

licentiosa Smith, Eupolia; Trichopolia

lichenaria Roepke, Pseudosiccia;

Pseudosiccia

lichenaria Walker, Erizada; Erizada

lichenea Hampson, Catephia; Catephiona

lichenea Hampson, Mionides; Mionides

lichenea Hübner, Noctua; Eumichtis

lichenigera Hampson, Giaura; Mniothripa

lichenodes Graeser, Graphiphora;

Acronictoides

lichenoides Nietner, Galleriomorpha;

Galleriomorpha

lichenophora Lower, Caradrina; Roperua

lichenosa Moore, Mithila; Mithila

lidia Stoll, Phalaena; Mesoeuxoa

ligaminosa Eversmann, Amphipyra;

Cheirophanes

lignaris Hübner, Itomia; Itomia

lignea Butler, Eurabila; Eurabila

lignea Swinhoe, Hepsidera; Hepsidera

ligni Guenée, Scolecocampa; Scolecocampa

lignicolor Hampson, Papuacola; Papuacola

lignicolor Hampson, Plumipalpia;

Plumipalpia

lignicolora Walker, Corsa; Corsa

lignifera Walker, Sarbena; Sarbena

lignificta Walker, Piana; Piana

lignigeralis Walker, Phachthia; Phachthia

ligniperda Fabricius, Cossus; Cossus 
lignitis Schaus, Xylinissa; Xylinissa lignosa Butler, Perinaenia; Perinaenia lignosa Walker, Petrinia; Petrinia lignulina Walker, Hamaxia; Olulis ligustri [Denis \& Schiffermüller], Noctua;

Craniophora

likianga Draudt, Trichosternum; Zutragum lilacina Butler, Mesoplectra; Mesoplectra lilacina Moore, Symitha; Argyrothripa limacodella Walker, Nabara; Nabara linuacodina Felder, Pachylepis; Yepcalphis limacodoides Walker, Alimala; Alimala limata Christoph, Photedes; Drasteriodes limbata Butler, Apamea; Euchoristea limbata Butler, Eudragana; Eudragana limbata Holland, Selenis; Euaethiops limbata Wileman, Erastroides; Xenonola limbatis Strand, Anugana; Anugana limbirena Guenée, Plusia; Ctenoplusia limbulata Berio, Trachea; Berioana limbulata Guenée, Lepidodes; Lepidodes limonalis Schaus, Metacinia; Metacinia limosa Treitschke, Ophiusa; Toxocampa limula Möschler, Epistona; Epistona linariae [Denis \& Schiffermüller], Noctua; Calophasia

lincea Cramer, Phalaena; Ophthalmis lindsayi Hampson, Koptoplax; Koptoplax linearis Grote, Litognatha; Zelicodes lineata Druce, Otaces; Otaces lineata Druce, Ypsia; Pseudyrias lineata Walker, Nipista; Nipista lineifera Blanchard, Noctua; Pareuxoa lineola Berio, Conochuza; Conochuza lineolaris Hübner, Noctua; Phurys lineolata [Denis \& Schiffermüller], Geometra; Phibalapteryx lineolata Walker, Ariolica; Ariolica lineolata Walker, Catabena; Catabena lineosa Moore, Dadica; Dadica lineosa Walker, Tendarba; Tendarba lingea Stoll, Phalaena; Phrictia linogrisea [Denis \& Schiffermüller], Noctua; Epilecta

lintneri Crote, Ommatostola; Ommatostola liparisalis Walker, Celama; Celama liquida Möschler, Ipinıorpha; Gonodes litarga Turner, Astonycha; Astonycha lithina Swinhoe, Stenopaltis; Stenopaltis lithophilus Butler, Heliophobus; Strigania lithoplasta Hampson, Oederemia; Oederemia lithoriza Borkhausen, Phalaena; Xylocampa lithosiana Hampson, Acanthofrontia;

Acanthofrontia lithosiana Hampson, Asiccia; Asiccia lithoxylaea [Denis \& Schiffermüller], Noctua; Septis lithoxylea Bang-Haas, Hypomecia; Pseudomecia

littera Guenée, Leucania; Fagitana litterata Pagenstecher, Plusia; Sarobela littoralis Boisduval, Hadena; Prodenia littoralis Packard, Agrotis; Paradiarsia lituralis Hübner, Epizeuxis; Megachyta liturata Christoph, Megalodes; Metalopha liturata Fabricius, Phalaena; Neviasca liturata Walker, Apphadana; Apphadana livida Holland, Plusiotricha; Plusiotricha lividalis Hübner, Phalaena; Ophiuche lixalis Grote, Tetanolita; Tetanolita lobata Fletcher \& Viette, Eunimbatana; Eunimbatana

lobata Hulstaert, Trapezoptera; Trapezoptera

loculosa Grote, Perigea; Properigea lolo Viette, Thausgea; Thausgea longa Guenée, Acronycta; Mastiphanes longalis Hübner, Micropus; Smicropus longidens Smith, Feltia; Proragrotis longilabris Grote, Herminia; Epidelta longimacula Saalmüller, Diadocis; Diadocis longinasus Joannis, Bifurca; Bifurca longipalpis Felder \& Rogenhofer, Scopifera; Scopifera

longipalpis Walker, Selenis; Eugnathia longipennis Moore, Norraca; Norraca longipennis Moore, Sadarsa; Sadarsa longipes Druce, Diamuna; Gerrodes longivalvis Guenée, Arcyophora;

Arcyophora

lophophora Hampson, Capnodes;

Trichoblemma

lophota Bethune-Baker, Megaloptera;

Megaloptera

loreyi Duponchel, Noctua; Acantholeucania lorina Druce, Polia; Boryzola

lorquinii Grote \& Robinson, Alypia; Androloma

lota Clerck, Phalaena; Leptologia lou isiana Forbes, Xylormisa; Xylormisa loxographa Bethune-Baker, Cyttaralopha; Cyttaralopha

loxoscia Prout, Elaeodopsis; Elaeodopsis lubina Möschler, Maurilia; Maurilia lubricalis Geyer, Epizeuxis; Pseudaglossa lubricans Guencee, Noctua; Euagrotis lucala Swinhoe, Megalodes; Calophasidia 
lucens Morrison, Heliothis; Dasyspoudaea lucernea Linnaeus, Phalaena; Standfussiana lucida Hufnagel, Phalaena; Acontia lucidata Walker, Fagitana; Fagitana lucilla Butler, Triphaenopsis;

Triphaenopsis

lucina Druce, Stibaera; Craterestra lucipara Linnaeus, Phalaena; Euplexia lucipeta [Denis \& Schiffermüller], Noctua; Rhyacia

luctuosa [Denis \& Schiffermüller], Noctua; Dysthymia

ludicra Hübner, Phalaena; Eccrita ludifica Linnaeus, Phalaena; Trichosea lugens Hampson, Scotostena; Scotostena lugens Lucas, Tringilburra; Tringilburra lugens Walker, Uraba; Uraba luminosa Cramer, Phalaena; Ommatophora luna Hampson, Avitta; Eclipsea luna Morrison, Cucullia; Epinyctis; Nycterophaeta

lunalis Scopoli, Phalaena; Zanclognatha lunaris [Denis \& Schiffermüller], Noctua; Minucia

lunaris Walker, Bocana; Coria lunata Drury, Phalaena; Omoptera lunata Möschler, Lycoselene; Lycoselene lunata Smith, Ursogastra; Ursogastra lunifera Druce, Toxocampa; Imleanga lunifera Hübner, Phaeocyma; Phaeocyma lunilinea Grote, Spiloloma; Spiloloma lunodes Guenée, Ingura; Ingura lunosa Haworth, Noctua; Omphaloscelis lunula Hufnagel, Phalaena; Calophasia luperinoides Guenée, Cyrebia; Cyrebia lusoria Linnaeus, Phalaena; Lygephila lutea Smith, Diallagma; Diallagma lutea Ström, Phalaena; Xanthia lutea Walker, Ophisma; Kalmina luteicoma Grote \& Robinson, Acronycta; Philorgyia

luteifascia Hampson, Trisuloides; Smilephoicia Iuteomedia Smith, Aleptina; Prothrinax lutosa Hübner, Noctua; Rhizedra lutosa Karsch, Entomogramma;

Holoxanthina

lutosa Staudinger, Hadjina; Hadjina luxa Grote, Bessula; Bessula lychnidis [Denis \& Schiffermüller], Noctua; Agrochola

lyncestidis Strand, Anereuthinula; Anereuthinula mabillei Saalmüller, Cryptomeria; Cryptomeria macarea Cramer, Phalaena; Plaxia macaria Cramer, Phalaena; Parachaea macariata Hampson, Zethes; Semiothisops maccullochii Kirby, Alypia; Androloma macrocula Berio, Gigaglossa; Gigaglossa macrodonta Hampson, Plagiograpta;

Plagiograpta macrogastris Turner, Pseudalea; Pseudalea macrops Linnaeus, Phalaena; Eupatula macula Druce, Hypenodes; Hypenopsis maculapex Hampson, Bracharthron;

Bracharthron maculata Alphéraky, Spinipalpa; Spinipalpa maculata Eversmann, Leucania;

Argyrospila maculata Roepke, Paramocis; Paramocis maculifera Matsumura, Sugitania;

Sugitania maculifera Staudinger, Aventiola; Aventiola maculifera Staudinger, Gynaephila;

Gynaephila maculosa Walker, Chorsia; Chorsia maculosus Rothschild, Phalaenoides;

Radinocera madagascariensis Rothschild, Eudryas;

Madathisanotia madates Druce, Neopalthis; Neopalthis magdalena Hulst, Nycterophaeta;

Nycterophaeta magna Aurivillius, Hoplotarsia; Hoplotarsia magna Leech, Zethes; Diapolia magnifica Neumoegen, Heliodora;

Heliodora magnifica Schaus, Eupalindia; Eupalindia magniplaga Walker, Gammace; Gammace maillardi Guenée, Eriopus; Rhoptrotrichia majorcula Dyar, Crambophilia;

Crambophilia

majuscula Edwards, Penthetria; Tantura malagassica Hampson, Thalatha;

Madegalatha malana Fitch, Brachytaenia; Nolaphana malgassica Heyden, Rimulia; Rimulia malgassica Kenrick, Daseochaeta;

Mageochaeta malitiosa Guenée, Brujas; Blosyris malonia Schaus, Eustrotia; Neostrotia malvae Esper, Phalaena; Xanthodes mamestrina Butler, Agrotis; Blepharoa mamestrina Butler, Epunda; Ebertidia mammida Druce, Apamea; Aeschradia 
mandarina Leech, Nodaria; Megaloctena mandarina Leech, Sypina; Sypnoides mandschuriana Oberthür, Erastria;

\section{Mimerastria}

mandschurica Graeser, Pachnobia; Heraema manifestalis Walker, Bocana; Bocana manleyi Leeclı, Egnasia; Polyssciera manlia Cramer, Phalaena; Ischyja mansoura Chrétien, Agrotis: Stenosomides mansueta Walker, Daona; Daona mansueta Walker, Maceda; Maceda manto Cramer, Phalaena; Acigona marcellina Stoll, Phalaena; Phycoma marchalii Boisduval, Ophiusa; Parathermes marchandi Guencee, Agyra; Agyra margarita Hübner, Noctua; Axia margaritata Drury, Phalaena; Desmophora margiana Püngeler, Thargelia; Odontelia marginalis Walker, Agrotis; Ctenusa marginalis Walker, Anthophila;

Pseudomicra marginalis Walker, Batina; Batina marginata Druce, Plynteria; Plynteria marginata Noore, Aramuna; Aramuna marginata Walker, Zirona; Zirona marginatus Haworth, Crambus;

Lygranthoecia marginea [Denis \& Schiffermüller], Noctua;

Catastia

marginifera Walker, Agrotis; Ctenusa marina Druce, Ipnea; Ipnista maritima Tauscher, Noctua; Chilodes marmarides Turati, Libyana; Libyana marmorata Hampson, Hypertrocta;

Hypertrocta marmorea Swinhoe, Delocoma; Delocoma marmorea Walker, Perciana; Perciana marshalli Hampson, Paratuerta;

Paratuerta materna Linnaeus, Phalaena; Elygea matura Hufnagel, Phalaena; Thalpophila maura Linnaeus, Phalaena; Mormo maura Saalmüller, Polia; Brithysana maurella Staudinger, Caradrina; Stygiodrina mauritia Boisduval, Hadena; Spodoptera maurus Holland, Arcte; Pseudoarcte mediafricana Berio, Interdelta; Interdelta mediofoveata Hampson, Orygmophora;

Orygmophora

mediopunctat Bowden, Poecopa; Poecopa mediovitta Rothschild, Acronycta;

Negalonycta

meeki Bethune-Baker, Catephiodes;

Anophiodes meeki Bethune-Baker, Rectangulipalpus; Rectangulipalpus meeki Bethune-Baker, 'Temnoptera; Temnoptera meeki Rothschild \& Jordan, Eucocytia; Eucocytia megacephala [Denis \& Schiffermüller], Noctua; Subacronicta megalops Hampson, Gigides; Gigides megaspila Walker, Mosopia; Mosopia megastigma Püngeler, Senta; Hypostilbia meissneri Draudt, Thecamichtis;

Thecamichtis melachlora Staudinger, Bryophila; Bryomoia melalepidia Hampson, Tipasodes; Tipasodes melaleuca Thunberg, Noctua; Sympistis melaleucata Walker, Sandava; Sandava melanchlaena Swinhoe, Tarache;

Ceratostrotia melaneplira Hampson, Lamprolopha;

La mprolopha melanis Mabille, Catephia; Pseudoarcte melanochorda Meyrick, Prorocopis;

Prorocopis melanodonta Hampson, Melipotis; Asplenia melanoclonta Hampson, Trachea;

Leucotrachea melanoglossa Berio, Pseudonycterophaeta;

Pseudonycterophaeta

melanoleuca Hampson, Acanthoprora;

Acanthoprora

melanoleuca Hampson, Bryophila;

Leucotrachea

melanophaes Turner, Corethrobela;

Corethrobela

melanospila Guenée, Euperia; Janseodes melanura Carrara, Agrotis; Dichagyris meleagris Felder \& Rogenhofer, Alamis;

Caduca

melicerta Drury, Phalaena; Achaea memnonia Karsch, Misa; Misa mendax Walker, Alamis; Dugaria mendozina Hampson, Syneda; Asyneda mendozina Köhler, Borsania; Borsania menete Cramer, Phalaena; Mitrophrys menippusalis Walker, Bleptina;

Scopifera menippusalis Walker sensu Druce, Pyrgion; Pyrgion merolenca Hampson, Gonioscia; Gonioscia mesoleuca Lower, Corula; Eurynola mesomelana Hampson, Euplexia; Bryotype mesoneura Hampson, Procoeria;

Procoeria 
mesophaea Hampson, Oediplexia; Oediplexia

mesophaea Hampson, Rhynchodia;

Rhynchodia

mesophaea Hampson, Steganiodes;

Steganiodes

mesoscia Hampson, Conicofrontia; Sciomesa

mesoscia Hampson, Platyscia; Platyscia

mesosticta Hampson, Neonola; Neonola

mesostipa Hampson, Microselene;

Microselene

messala Schaus, Girtesma; Girtesma

messrae Staudinger, Rhabdophera;

Rhabdophera

metableta Turner, Philogethes; Philogethes

metabrachys Hampson, Gonagyra;

Gonagyra

metacausta Hampson, Chlorhoda;

Prasinopyra

metachrostina Rungs, Duhemia; Duhemia

metachryseis Hampson, Micriscadia;

Micriscadia

metaleuca Hampson, Authadistis;

Authadistis

metallica Prout, Plusiophaes; Plusiophaes metallica Warren, Pteroprista; Pteroprista metallicella Dyar, Metaxyllia; Metaxyllia metalophota Hampson, Barasa;

Barastrotia

metaphaearia Walker, Tachosa; Abseudrapa metarhoda Walker, Rhizana; Rhizana metaspilaris Walker, Cobubatha; Cobubatha metaxantha Hampson, Hypercalymnia;

Hypercalymnia

meterythra Hampson, Arachnognatha;

Arachnognatha

meterythra Hampson, Bombycia;

Hypocalamia

meticulosa Linnaeus, Phalaena; Phlogophora metonalis Walker, Herminia; Epidelta

mi Clerck, Phalaena; Callistege

micacea Esper, Phalaena; Hydraecia

micacea Hampson, Leucania; Analetia

micaceella Walker, Chalenata; Chalenata

michaelseni Staudinger, Agrotis;

Scriptania

micra Bar, Palindia; Palindiona

micraeola Meyrick, Essonistis; Essonistis

microcallia Berio, Teucocranon;

Teucocranon

microglossa Rambur, Poecilia; Moureia microscopica Berio, Hypendalia; Hypendalia microstigma Hampson, Lycophotia;

Tandilia microstigmoides Köhler, Pareuxoina;

Pareuxoina

mictochroma Dyar, Pogopus; Pogopus

midasalis Walker, Gizama; Gizama

migralis Guenée, Physula; Physula

milichias Turner, Saroptila; Saroptila

mima Harvey, Homoptera; Heteranassa

mimalis Hübner, Salia; Salia

mimetica Berio, Anua; Trichanua

mimetica Rothschild, Crinala; Crinala

mimica Hampson, Syncalama; Syncalama

mimica Walker, Eggyna; Eggyna

mimicaria Oberthür, Cimelia; Protomeceras

mina Schaus, Basilodes; Satrapodes

minax Meyrick, Piratisca; Piratisca

minereusalis Walker, Egnasia; Vestura

minians Guenée, Nephelodes; Nephelodes

miniatus Grünberg, Huebnerius;

Lobophyllodes

minima Guenée, Micra; Eumicremma

minima Haworth, Noctua; Petilampa

minima Swinhoe, Apamea; Catamecia

miniosa [Denis \& Schiffermüller], Noctua;

Monima

minor Butler, Crambodes; Eremaula

minuscula Butler, Daphoenura;

Adaphaenura

minuta Barnes \& McDunnough, Viridemas;

Eviridemas

minuta Butler, Callopistria; Gnamptocera

minuta Hübner, Noctua; Micra

minuta Püngeler, Pseudohadena;

Gryphadena

minutum Turner, Icasma; Icasma

mionides Hampson, Tarache; Hoplotarache miophaea Hampson, Bryothripa;

Bryothripa

mira Butler, Selenis; Curvatula

mira Staudinger, Luperina; Heterographa

mirabilis Bethune-Baker, Parelydna;

Parelydna

mirabilis Butler, Lophocoleus; Lophocoleus

mirabilis Neumoegen, Basilodes;

Heminocloa

mirabilis Rothschild, Depalpata; Depalpata

mirabilis Schaus, Erastria; Micrantha

mirabilis Staudinger, Namangana; Sartha

mirabilis Sivinhoe, Clettharra; Camptozada

mirandalis Hübner, Syllectra; Syllectra

miricornis Walker, Donacesa; Donacesa

mirifica Ershov, Euclidia; Anydrophila

mirificum Strecker, Aenigma; Hyblaea

miscellus Grote, Adipsophanes;

Adipsophanes 
miscidisce Dyar, Araeopterella; Araeopterella miselioides Guenée, Hadena; Helioscota misella Püngeler, Margelana; Ecbolemia mistura Swinhoe, Nagadeba;

Goniocraspedon

mitis Grote, Heliothis; Heliophana mitrula Guenée, Eriocera; Eriocera miwai Inoue, Pseudalelimma;

Pseudalelimma

mixta Staudinger, Euromoia; Euromoia mixtura Walker, Milyas; Milyas mocha Möschler, Cladenia; Cladenia mochisa Schaus, Saccharophagos;

Saccharophagos

moderata Eversmann, Apamea; Mesapamea moderata Wallengren, Grainmodes;

Plecopterodes

modesta Barnes \& McDunnough,

Prochloridea; Prochloridea

modesta Butler, Renodes; Metaprosphera modesta Moore, Bryophila; Hymenocryphia modesta Saalmüller, Argyphia; Argyphia modesta Swinhoe, Heterormista;

Heterormista

modicella Grote, Heliolonche; Heliolonche moechilla Püngeler, Euxoa; Hemiexarnis moeonalis Walker, Herminia; Acmana moerens Grote, Carneades; Paragrotis moesta Walker, Naesia; Naesia moestalis Walker, Magulaba; Magulaba mollis Butler, Poaphila; Leiostola mollis Möschler, Algonia; Algonia mollis Walker, Eudrapa; Eudrapa mollis Walker, Eusemia; Longicella mollissima Guenée, Eriopıs; Haploolophus molybdaenalis Hübner, Pinacia; Pinacia molybdopasta Turner, Erastroides; Peperita molybdota Hampson, Rhynchodina;

Rhynchodina

moneta Fabricius, Noctua; Polychrysia monetifera Dyar, Rolua; Rolua monetifera Guenée, Eriopus; Euherrichia monilis Fabricius, Noctua; Hypsoropha monochronatea Morrison, Agrotis;

Hemipachnobia

monoflex Dyar, Lois; Lois monoglypha Hufnagel, Phalaena; Abromias monogramma Hübner, Noctua; Synthymia monogramma Joicey, Clavipalpa; Clavipalpa monophyes Turner, Coenotoca; Coenotoca monovittata Berin, I permarca; I permarca monstrosa Dognin, Orotermes; Orotermes montana Joicey \& Talbot, Hypena;

Stygionyx montana Morrison, Agrotis; Orosagrotis monticola Hulst, Mycterophora;

Mycterophora

moorei Cotes \& Swinhoe, Sphetta;

Pseudosphetta

mopsus Schaus, Schiraces; Schiraces morbidalis Guenée, Herminia; Chytolita morena Swinhoe, Sarrothripa; Phaeothripa morga Dyar, Gorgora; Gorgora mori Linnaeus, Phalaena; Bombyx morosa Schaus, Tarista; Tarista morpheus Hufnagel, Phalaena; Caradrina morrisii Dale, Acosmetia; Chortodes mosca Dyar, Melipotis; Ianius moses Staudinger, Hypoglaucitis;

Hypoglaucitis

muirii Edwards, Oribates; Gyros multilineata Hampson, Gnamptogyia;

Gnamptogyia multiplicans Walker, Piada; Piada multiscripta Holland, Eudrapa;

Hesperochroa multisignata Christoph, Acidalia; Paragona mummia Cramer, Phalaena; Erocha mundalis Walker, Gauzania; Gauzania mundiferalis Walker, Anitha; Anitha mundissima Walker, Concana; Concana mundula Zeller, Thalpochares; Ommatocliila munita Hübner, Noctua; Gonospileia muraenula Grote \& Robinson, Agrotis;

Porosagrotis

muralis Forster, Phalaena; Bryopsis muralis Grote, Dicopis; Dicopis muricata Hufnagel, Phalaena; Anteois muricata Hulstaert, Thriponea; Thriponea mus Hampson, Radinacra; Stygiathetis mus Oberthür, Diloba; Colocasiodes muscosa Geyer, Mentaxya; Mentaxya muscosa Möschler, Barcita; Barcita muscosa Schaus, Cyrima; Cyrima musculana Ershov, Sarrothripa;

Erschoviella musculina Walker, Aiteta; Aiteta musculosa Hübner, Noctua; Oria musculus Ménétriés, Zethes; Arytrura mutatalis Möschler, Scelescepon; Scelescepon mutilatalis Guenée, Pterhemia; Pterhemia mycha Dyar, Prodosia; Prodosia mygdon Cramer, Phalaena; Chalciope mynesalis Walker, Bleptina; Tetanolita myriostigma Son, Metanola; Metanoli myrtaea Drury, Phalaena; Durdara myrtilli linnaeus, Phalaena; Anarta mysticus Staudinger, Scodionyx; Scodionyx 
nabis Dyar, Cola; Cola

namaqualis Guenée, Hypena; Phanaspa

nana Hübner, Cryphia; Hyperstrotia

nana Hufnagel, Phalaena; Hada

nana Staudinger, Leucanitis;

Epharmottomena

nana Staudinger, Tapinostola; Coenagria

nana Walker, Nacerasa; Nacerasa

napaea Morrison, Scopelosoma; Litholomia

narcissus Cramer, Phalaena; Eligma

narecta Schaus, Phibromia; Phibromia

nasuta Mabille, Ametropalpis; Ametropalpis

nasutaria Zeller, Sudariophora;

Sudariophora

natalis Walker, Berresa; Berresa

natatrix Guenée, Isogona; Isogona

nea Druce, Heterocampa; Heterodelta

nealcesalis Walker, Megatomis; Duriga

neavi Hampson, Ethioterpia; Ethioterpia

nebula Schaus, Kohlera; Kohlera

nebulilinea Walker, Carteia; Carteia

nebulosa Hübner, Noctua; Euxoa

nebulosa Hufnagel, Phalaena; Polia

nebulosella Walker, Tipasa; Tipasa

nectarea Möschler, Cecharismena;

Cecharismena

negata Walker, Obroatis; Obroatis

neleusalis IValker, Lysimelia; Lysimelia

neonympha Esper, Phalaena; Eucora

nervosa [Denis \& Schiffermüller], Noctua;

Simyra

neumoegeni Edwards, Xanthothrix;

Euedwardsia

neuritis Hampson, Lophocalama;

Lophocalama

neuroides Swinhoe, Heliothis; Propatria

nexa Hübner, Noctua; Phragmatiphila

nexilis Morrison, Eutricopis; Eutricopis

ni Hübner, Noctua; Trichoplusia

niama Dyar, Amiana; Amiana

nictitans Linnaeus, Phalaena; Amphipoea

niepelti Strand, Polydesmiola; Polydesmiola

niger Bethune-Baker, Tornosinus;

Tornosinus

nigerrima Swinhoe, Wilkara; Wilkara

nigra Berio, Stilbotis; Stilbotis

nigra Viette, Xymehops; Xymehops

nigraericta Swinhoe, Armana; Armana

nigralba Draudt, Charierges; Charierges

nigrescens Wallengren, Anophia;

Malanephia

nigribasis Holland, Metaleptina;

Metaleptina

nigricans Linnaeus, Phalaena; Brotis nigriceps Hampson, Mimoruza; Mimoruza

nigriceps Walker, Renodes; Parathermes

nigricostata Hampson, Parastichtis;

Neostichtis

nigrilinea Druce, Mamerthes; Mamerthes

nigrilinea Walker, Obdora; Obdora

nigripalpis Guenée, Herminodes;

Herminodes

nigripalpis Walker, Marimatha; Leucotela nigripalpis Walker, Zinna; Zinna

nigripes Köhler, Chubutiana; Chubutiana

nigriplaga Swinhoe, Stadna; Stadna

nigripuncta Hampson, Anachrostis;

Anachrostis

nigripunctata Schaus, Paromia; Ramopia

nigrisigna Hampson, Catada; Anatatha

nigrisigna Moore, Churia; Churia

nigritarsis Walker, Rusicada; Rusicada

nigritula Hampson, Tarache; Stenostygia

nigrobasalis Bartel, Pseudopais; Pseudopais

nigrocuprea Moore, Mamestra; Tricheurois

nigrofasciatum Pinhey, Capillamentum;

Capillamentum

nigrofimbria Guenée, Xanthoptera;

Thioptera

nigrofusca Walker, Facidia; Facidia

nigrogrisea Moore, Sydiva; Sydiva

nigroguttata Grote, Lussa; Lussa

nigropuncta Druce, Neostictoptera;

Neostictoptera

nigropuncta Druce, Tomyris; Rowdenia

nigropunctata Bethune-Baker, Corgatha;

Dysgnathia

nigrosignata Walker, Artigisa; Artigisa

nigrostrigata Bethune-Baker, Bagada;

Platyprosopa

nigroviridis Walker, Diphtera; Neurois

nilotica Rogenhofer, Sarrothripus; Paraxia

niphadospila Turner, Trilophia; Trilophia

niphosema Lower, Monoctenia;

Baryphanes

niphostena Hampson, Poliothripa;

Poliothripa

nitens Butler, Celeopsyche; Celeopsyche

nitens Butler, Spaelotis; Atrachea

nitens Hampson, Clettharina; Clettharina

nitens Saalmüller, Caradrina; Tectorea

nitida [Denis \& Schiffermüller], Noctua;

Anchoscelis

nitida Köhler, Neuquenia; Neuquenioa

nitidula Fabricius, Bombyx; Desmophora

niveicosta Smith, Neleucania; Neleucania

niveicostatus Grote, Pseudolimacodes;

Pseudolimacodes 
niveigutta Walker, Eulepa; Eulepa niveiguttata Grote, Mamestra; Ulolonche niveiguttata Hampson, Frastria; Goenycta niveilinea Walker, Bononia; Bononia niveipuncta Warren, Orthoruza; Orthoruza niveivenosa Grote, Agrotis; Protagrotis nivens Hübner, Euxoa; Euxoa niveola Motschoulsky, Habrostola; Belciades nivifascia Walker, Borbotana; Borbotana;

Choluata nivosa Turner, Chorizomena; Chorizomena noa Dyar, Letaba; Letaba nobilitella Cramer, Phalaena; Cydosia noctilux Walker, Josia; Shapis noctualis Hübner, Pyralis; Trothisa noctuidalis Dyar, Pleonectyptera;

Nychioptera noctuiformis Hampson, Euplexidia;

Euplexidia

noctuiformis Neumoegen, Aon; Aon noctuina Butler, Seudyra; Asteropetes nocturna Hampson, Lophonotidia:

Lophonotidia

nocturnus Hampson, Corythurus;

Corythurus nodicornis Guenée, Hapigia; Hapigia nodosalis Herrich-Schäffer, Herminia;

Nodaria

nodyna Turner, Prometopus; Cycloprora noela Druce, Mychonia; Bistica noela Dyar, Bistica; Bistica nohirae Matsumura, Neoborolia;

Neoborolia nolalella Walker, Symitha; Symitha noliformis Strand, Dilophothripoides;

Dilophothripoides

noloides Hampson, Procrateria; Procrateria nolophaea Hampson, Nanarhyncha;

Nanarhyncha

nomas Erschov, Agrotis; Menada nomimus Dyarr, Egchiretes; Egchiretes nonagrica Wallier, Curgia; Leucocosmia nonagrioides Lefebvre, Cossus; Sesamia nonagrioides Walker, Juncaria; Hyamia nonogriella Walker, Archanara; Archanara norma Karsch, Aegoceropsis; Aegoceropsis normalis Crote, Acerra; Acerra normani Grote, Perigrapha; Crocigrapha notabilis Butler, Panolis; Syrrusis notabilis Walker, Deinopa; Deinopa notabilis Walker, Dorsippa; Dorsippal notalis Grote, Fotella; Fotella notatella Grote, Epinyctis; Epinyctis notodontina Felder, Xenochroa; Xenochroa notodontoides Druce, Sotigena; Sotigena nova Smith, Trichoclea; Tridepia novaeguineae Bethune-Baker, Yula; Yula novaguinensis Bethune-Baker, Protagrotis; Phaeopyra novatusalis Walker, I uceria; Luceria nu Guenée, Plusia; Rachiplusia nubeculosa Esper, Bombyx; Brachionycha nubes Hampson, Craspedogonia;

Craspedogonia nubifera Hampson, Chelichares;

Chelichares nubifera Walker, Dimirica; Dimirica nubilicosta Holland, Periplusia; Periplusia nubilifascia Grote, Litognatha; Litognatha nubilis Hübner, Parthenos; Euparthenos nubilosa Hampson, Tarache; Stenorache nucicolora Guenée, Monodes; Monodes nugatrix Guenée, Penicillaria; Penicillaria nullifera Walker, Agrotis; Alysina numisma Hübner, Noctua; Antarchaea nundina Drury, Phalaena; Tamila nupta Linnaeus, Phalaena; Catocala nyctichroa Hampson, Microphaca;

Microphaea nygmia Swinhoe, Acontia; Chloroplaga nymbisalis Schaus, Iuberta; Luberta

obconica Druce, Neocalynınia; Neocalymnia obeditalis Walker, Gesonia; Gesonia oberratalis Walker, Lamura; Lamura oberthueri Culot, Simyra; Metlaouia obesa Eversmann, Noctua; Nyssocnemis obesa Walker, Dosa; Dosa oblataria Walker, Hyria; Penisa oblinita Smith, Phalaena; Eulonche obliqua Berio, Promionides; Promionides obliqua Dyar, Marzigetta; Marzigetta obliqua Dyar, Periconta; Periconta obliqua Moore, Phurys; Carsina obliqua Moore, Tochara; Tochara obliqua Moore, Tycracona; Tycracona obliqua Walker, Batracharta; Batracharta obliciıa Walker, Edema; Sphida obliqua Walker, Gigia; Gigia obliqualis Fabricius, Plalacna; Eumestleta obliqualis IValker, Trauaxa; Trauaxa obliquata Grote \& Robinson, Arzama; Sphicla obliquata Smith, Pleroma; Pleromelloida obliquata Walker, Erysthia; Erysthia 
obliquifasciata Hampson, Nola; Melaleucia obliquifera Walker, Balsa; Balsa

obliquilineata Leech, Talapa; Eurogramma obliquisigna Hampson, Polia; Dichoniopsis obliterata Rambur, Anthophila; Phyllophila oblonga Haworth, Noctua; Eleemosia obscura Berio, Mauriliadia; Mauriliadia obscura Hampson, Ptyopterota;

Ptyopterota

obscura Schaus, Carbona; Carbona obscurata Butler, Marmorinia; Stenozethes obscurissima Holland, Panilla; Lophopanilla observalis Guenée, Cýclopteryx; Cyclopteryx ¥'obsita, Felder', Metoptria; Cytothymia obstans Walker, Trigonodes; Cuneisigna obtenta Walker, Calduba; Calduba obtusa Herrich-Schäffer, Noctua; Stiriodes obtusa Walker, Rhescipha; Rhescipha occlusa Hübner, Noctua; Dryobota occulta Linnaeus, Phalaena; Eurois occulta Swinhoe, Selepa; Cryptothripa ocellata Barnes \& McDunnough,

Prothrinax; Walterella ocellata Berio, Cryphioides; Cryphioides ocellata Cramer, Phalaena; Dypos ocellata Saalmüller, Bryophila; Cryphioides ocellata Walker, Allia; Allia ocellata Walker, Nystalea; Opsigalea ocellatus Bethune-Baker, Pilipectus;

Pilipectus ochracea Ershov, Metoponia; Paraegle ochracea Leech, Adrapsa; Apladrapsa ochracea Moore, Pasira; Rivula ochracea Swinhoe, Kalmina; Kalmina ochracea Walker, Oxira; Oxira ochrea Barnes \& McDunnough,

Aleptinoides; Aleptinoides ochrea Bethune-Baker, Rivulana; Rivulana ochreago Hübner, Noctua; Xestia ochreipennis Moore, Raparna; Raparna ochreistigma Hampson, Ptyophora;

Ptyophora ochreoplagata Kenrick, Eutelia; Leucotelia ochreopunctata Bethune-Baker,

Insolentipalpus; Insolentipalpus ochrias Turner, Gyroprora; Gyroprora ochricosta Herrich-Schäffer, Ponometia;

Ponometia ochrodiscata Hampson, Enmonodiops;

Enmonodiops

ochroleuca [Denis \& Schiffermüller],

Noctua; Eremobia ochroprocta Hampson, Lophotarsia;

Lophotarsia ochroptera Guenée, Ophyx; Ophyx ochrospila Turner, Auchmophanes;

Auchmophanes

ochthera Turner, Conocrana; Conocrana ochthias Meyrick, Heteronota; Heteronota octias Meyrick, Hyperaucha; Hyperaucha octo Guenée, Perigea; Ilattia octogesimea Hübner, Phalaena; Palimpsestis octomaculalis Hübner, Alypia; Alypia octomaculata Fabricius, Sesia; Alypia oculea Linnaeus, Phalaena; Amphipoea oculifera Hübner, Glenopteris; Glenopteris oda Lattin, Cranionycta; Cranionycta odiosa Staudinger, Mamestra; Hadulipolia odorata Linnaeus, Phalaena; Ascalapha oeneus Fawcett, Cladocerotis; Eucladodes oenistis Hampson, Andesia; Andesia oenocrossa Turner, Acachmena; Acachmena oenoplex Turner, Axiocteta; Axiocteta oileusalis Walker, Herminia; Progonia oleagina [Denis \& Schiffermüller], Bombyx; Valeria oleracea Linnaeus, Phalaena; Peucephila olivacea Bethune-Baker, Aroana; Aroana olivacea Hampson, Archephia; Archephia olivacea Hampson, Pachycoa; Pachycoa olivacea Köhler, Oortiana; Oortiana olivacea Walker, Idicara; Idicara olivacea Wileman, Chytonix; Conicochyta olivata Hampson, Metopoplacis;

Metopoplacis

olivata Harvey, Glaea; Pseudoglaea olivens Bethune-Baker, Cristatopalpus;

Cristatopalpus

olivescens Hampson, Chlorocodia;

Chlorocodia olivescens Warren, Bleptina; Monochroides oliviaria Hampson, Erastroides; Erastroides olivina Herrich-Schäffer, Cleophana;

Copiphana

omega Hübner, Argyrogramma;

Argyrogramma

omicronigera Guenée, Sypna; Sypna omma Hoeven, Erebus; Cyclodes omphisalis Walker, Cerynea; Cerynea onelia Guenée, Naxia; Caranilla onerosa Schaus, Apistis; Sipatosia onesalis Schaus, Batyma; Batyma onusta Grote, Macronoctua; Macronoctua oo Linnaeus, Phalaena; Dicycla oo Stoll, Phalaena; Pseudoplusia opacalis Grote, Hypenula; Hypenula opalina Walker, Pisara; Pisara opella Swinhoe, Acontia; Pseudozarba 
operatrix Wallengren, Eurhipia;

Colpocheilopteryx

operosa Köhler, Proseniella; Proseniella

uperta Möschler, Krugia; Krugia

ophiodesma Neyrick, Crypsiprora;

Crypsiprora

ophiusalis Walker, Bocana; Pantura

ophiusioides Möschler, Anagoa; Anagoa

opigena Drury sensu Hübner, Phalaena;

Blosyris

opina Grote, Dryobota; Dryotype

opinor Dyar, Zazunga; Zazunga

opistographa Guenée, Itonia; Pseudobendis

oppilata Guenće, Heteropygas; Heteropygas

optabilis Boisduval, Heliophobus;

Cladocerotis

opter Dyar, Pleromella; Pleromella

optiva Swinhoe, Tarache; Toxophleps

opulenta MIöschler, Ophisma; Calligraphidia

or [Denis \& Schiffermüller], Noctua; Tethea

ura Crainer, Phalaena; Perasia

ora Schaus, Epistrema; Epistrema

orana Lucas, Episema; Eremochlaena

oranaria Lucas, Chesias; Nereisana

orbiculosa Esper, Phalaena; Oxytrypia

orbifera Walker, Xylina; Magusa

orbifera Walker, Zigera; Zigera

ordinata Walker, Osdara; Radosa

oreophila Staudinger, Fergana; Fergana

orgyoides Walker, Murgisa; Murgisa

orichalcea Fabricius sensu Hübner, Noctua;

Diachrysia

orientalis Berio, Giubicolanta; Giubicolanta

orientalis Hampson, Halias; Hylophilodes

oriolus Guenée, Fodina; Fodina

orionalis Walker, Bocana; Coremagnatha

ornata Moore, Phyllodes; Gloriana

ornata Neumoegen, Varina; Varina

ornata Ottolengui, Deva; Rodriguesia

orobena Druce, Rivula; Paracoeria

orosia Cramer, Phalaena; Dichromia

orotavae Draudt, Anataelia; Paranataelia

orphna Hübner, Ascalapha; Gorgone

orphnina Bethune-Baker, Systaticospora;

Systaticospora

orthosialis Guence, Renia; Renia

orthosioicles Butler, Semiophora; Palponima

orthosioides Guenée, Lyssia; Lyssia

orthosioides Warren, Hyalobole; Hyalobole

orthozona Meyrick, Micrapatetis;

Nicrapatetis

ortilia Stoll, Phalaena; Hypenaria

ossicolor Rosenstock, Lithilaria; Lithilaria

ostentalis Walker, Ziza; Ziza ostrina Hübner, Noctua; Eromene othello Fawcett, Lecasia; Lecasia ovata Grote, Acronycta; Lepitoreuma ovipennis Hampson, Oedebasis; Oedebasis owgarra Bethune-Baker, Sinariola;

Sinariola

oxyacanthae Linnaeus, Phalaena; Allophyes oxydata Hampson, Hypena; Folka

oxylopha Turner, Capelica; Capelica oxymorus Grote, Admetovis; Admetovis

pacifica Sugi, Teratoglaea; Teratoglaea pagenstecheri MIöschler, Hormoschista;

Hormoschista

pagodae Alphéraky, Dasypolia; Copitype paidiformis Butler, Pseudospiris;

Pseudospiris palata Grote, Charadra; Pseudopanthea paleacea Esper, Phalaena; Enargia pales Gray, Agarista; Rothia palleago Hübner, Noctua; Mellinia pallescens Schaus, Adrocampa; Adrocampa pallescens WValker, Liviana; Liviana pallicostata Staudinger, Curvatula;

Curvatula

pallida Butler, Cantlyylidia; Canthylidia pallida Butler, Hyperaeschra; Hyperaeschra pallida Butler, Microsemyra; Microsemyra pallida Hampson, Calliodes; Ctenusa pallida Holland, Plusiocalpe; Plusiocalpe pallida Moore, Diphthera; Diphtherocome pallida Moore, Tympanistes; Tympanistes pallida Staudinger, Metoponia; Metaegle pallida Walker, Leida; Leida pallidifusca Hampson, Alelimma; Alelimma pallidisca Moore, Erastria; Paroligia pallimedia Smith, Crimona; Crimona palliola [Denis \& Schiffermüller], Noctua;

Nola

pallipars Dyar, Bouda; Bouda

pallirena Felder \& Rogenhofer, Hadena;

Eucropia

pallustris Hübner, Noctua; Hydrillula palpalis Butler, Radinacra; Radinacra palpalis Walker, Condica; Condica palpalis Walker, Emea; Erebostrota palpalis Valker, Tegteza; Tegteza palpigera Walker, Modunga; Modunga palpitatalis Walker, Hyamia; Hyamia palshkovi Filipjev, Leuconycta;

Subleuconycta

paludata Linnaeus, Phalaena; Scopula 
palumba Guenée, Hulodes; Macaldenia

palumbioides Hampson, Ophiusa;

Parallelura

pampeana Berg, Synclerostola;

Synclerostola; Noctulizeria

pamphilia Stoll, Phalaena; Argyrolepidia

pampina Guenée, Cirroedia; Eucirroedia

panagralis Walker, Masoga; Masoga

panamensis Druce, Nicetas; Nicetas

pancratii Cyrillo, Noctua; Brithys

pangraptalis Strand, Ectogoniella;

Ectogoniella

panimera Turner, Lampadephora;

Lampadephora

pannosa Guenée, Epidromia; Epidromia pansalis Walker, Dragana; Dragana paolii Berio, Aegleoides; Aegleoides paradoxa Hampson, Acidon; Acidon paradoxa Kiriakoff, Saigonita; Saigonita paradoxa Saalmüller, Hesperimorpha;

Hesperimorpha paradoxus Grote, Heliocheilus; Heliocheilus parallela Grote, Trichorthosia; Trichorthosia parallelipipeda Guenée, Ophiodes;

Dermaleipa paranympha Linnaeus, Phalaena; Ephesia parmelia Toulgoët, Nola; Bryonola parra Poey, Mastigophorus; Mastigophorus parthenia Prout, Paradiopa; Paradiopa parthenias Linnaeus, Phalaena; Archiearis parthenomorpha Dyar, Nyctodryas;

Nyctodryas partita Fabricius, Noctua; Serrodes partita Guenée, Cultripalpa; CuItripalpa partita Guenée, Galgula; Galgula partita Moore, Chandata; Chandata partita Walker, Gorua; Gorua partitalis Walker, Aradrapha; Aradrapha pascuella Linnaeus, Phalaena; Crambus pastillicans Morrison, Glaea; Harpaglaea patanei Turati, Coelites; Coeloturatia patibulum Fabricius, Noctua; Dierna;

Nalıara

patricei Viette, Brachypteragrotis;

Brachypteragrotis patula Morrison, Tarache; Eumestleta patula Püngeler, Bryophila; Poliobrya paucula Walker, Poaphila; Coenobela paula Hübner, Noctua; Trothisa pauliani Berio, Callopistria; Miropalpa pauliani Viette, Fletcherea; Fletcherea paupera Romanoff, Caradrina; Stenodrina pauropis Turner, Alapadna; Alapadna pavo Walker, Chasmina; Ramadasa pectinicornis Bethune-Baker, Ophiusa; Giria pectinicornis Smith, Feltia; Aretypa pectitalis Hübner, Pyralis; Pechipogo pectoralis Walker, Ginaea; Gespanna peculiaris Butler, Brachyxanthia;

Brachyxanthia

peculiaris Butler, Orthoclostera;

Orthoclostera pedipilalis Guenée, Herminia; Pityolita peguense Hampson, Deltapterum;

Deltapterum peleusalis Walker, Eordaea; Eordaea pelidnalis Hübner, Lesmone; Lesmone pellex Hübner, Noctua; Blepharonia pellicosta Felder \& Rogenhofer, Abolla;

Abolla pelopsalis Walker, Meliaba; Meliaba pendula Ottolengui, Kallitrichia;

Kallitrichia penetrata Walker, Episparis; Episparis penicillalis Geyer, Corynitis; Corynitis peninsulata Walker, Plusia; Desana pentaptila Meyrick, Paonidia; Paonidia penthima Ershov, Erastria; Hyptioxesta penumbrata Hulst, Sigela; Sigela peonis Schaus, Taphonia; Taphonia pepita Guenée, Basilodes; Basilodes peponis Fabricius, Noctua; Anadevidia peralta Barnes, Papaipema; Homoanarta peratopis Hampson, Hypangitia;

Hypangitia peratosema Hampson, Nyctennomos;

Nyctennomos .

percara Morrison, Bryophila; Cyathissa perditalis Walker, Hyamia; Ledaea perfundis Smith, Oxycnemis; Leucocnemis perfuscaria Grote, Prosoparia; Prosoparia pergentilis Grote, Hadenella; Hadenella perigrapha Püngeler, Agrotis; Paramathes perillalis Walker, Laugasa; Laugasa perissa nom. n., Paramiana; Paramiana perissalis Schaus, Alogonia; Alogonia perla [Denis \& Schiffermüller], Noctua;

Bryophila perlaeta Edwards, Melipotis; Forsebia perlides Berio, Protomeroleuca;

Protomeroleuca pernana Grote, Chariclea; Chamaeclea perpallida Grote, Acopa; Zatilpa perscripta Guenée, Lepipolys; Lepipolys persecta Hampson, Trogotorna; Trogotorna persicariae Linnaeus, Phalaena; Melanchra persimilis Butler, Mimeusemia; Mimeusemia 
persimilis Hampson, Bombycia; Nyctycia personata IValker, Saligena; Saligena perspicillaris Linnaeus, Phalaena;

Actinotia

perspicillaris Möschler, Lophoditta;

Lophoditta

perstriata Hampson, Mimleucania;

Mimleucania

perstriata Hampson, Neolita; Neolita

pcrstriata Hampson, Prolophota;

Acidaliodes

pertorrida Berio, Gondysia; Gondysia

perumbrosa Hampson, Nodaria; Bleptinodes

perusia Möschler, Gonippa; Gonippa

peruviana Druce, Copidryas; Cisaucula

pesronii Guenée, Agrotis; Paranicla

peterseni Christoph, Rhizogramma;

Trichorhiza

petersi Christoph, Phleboeis; Phleboeis

petraea Walker, Turbula; Turbula

petrificata [Denis \& Schiffermüller], Noctua;

Lithophane

petroriza Borkhausen, Phalaena; Auchmis

petrowskyi Köhler, Boursinidia;

Boursinidia

pettiti Grote, Scopelosoma; Pyreferra

phalaena Guenée, Thiona; Thiona

phalaeniformis Guence, Rhynchodes;

Hondryches

phasianoides Guenée, Pelamia; Pelamia

phasianura Lucas, Holocryptis; Holocryptis

phasianus Walker, Betusa; Betusa

phasipennis Walker, Phorica; Phorica

phasis Cramer, Phalaena; Eudipna

phedonia Stoll, Phalaena; Rhanidophora

phenax Boursin, Lucasidia; Lucasidia

phenax Meyrick, Eporectis; Eporectis

phlegeusalis Walker, Elyra; Elyra;

Eordaea

phoca Möschler, Dianthoecia; Lasiestra

phocea Jones, Atrephes; Atrephes

phoenicea Hampson, Cautatha; Cautatha

phoenicea Hampson, Cirrodes; Cirrodes

phoenicias Hampson, Casandria;

Rhabdotina

phoenicolepia Hampson, Nolatypa;

Nolatypa

phoenicoxantha Hampson, Lophocyttarra;

Lophocyttarra

pholausalis Walker, Hormisa; Pharga

phormisalis Walker, Lascoria; Lascoria

phragmitidis Hübner, Noctua; Arenostola

phryganealis Walker, Nagara; Nagara

phtisialis Guenće, Physula; Mursa phyllophora Grote, Agrotis; Heptagrotis

pi Barnes \& Lindsey, Hyperepia; Hyperepia piana Swinhoe, Ugana; Ugana

picatum Butler, Poecilogramma;

Aeologramma

picta Guérin-Méneville, Polia; Calogramma picta Hampson, Caradrina; Protoseudyra picta Leach, Agarista; Agarista picta Moore, Calymera; Calymera picta Sepp, Phalaena; Scoedisa

pictale Hampson, Araeopteron; Araeopteron

pictalis Hampson, Mimoruza;

Eugraptoblemma

picteti Staudinger, Ala; Trichanarta

pictimargo Hampson, Pterocyclophora;

Pterocyclophora

pictipennis Grote, Heliosea; Heliosea

pictula Taylor, Dianthoecia; Meterana

pictura Saalmüller, Timaea; Atimaea

pienaari Distant, Mcgalodes; Euterpiodes

piffardi Walker, Phornacisa; Phornacisa

pilcheri Hampson, Leocyma; Clethrorasa

pilifera Walker, Agrotis; Dimorphinoctua

pilosa Druce, Argania; Argania

pima Barnes \& Benjamin, Hemioslaria;

Hemioslaria

pinastri Linnaeus, Phalaena; Dypterygia pineti Staudinger, Bryophila; Bryonycta piniperda Panzer, Phalaena; Ilarus pionealis Guenćc, Rhynchina; Rhynchina pirata Herz, Koraia; Koraia

pissonephra Turner, Thoracolopha;

Thoracolopha

pistacina [Denis \& Schiffermüller], Noctua;

Agrochola

pityochromus Grote, Plagiomimicus;

Plagiomimicus

placens Walker, Dinumma; Dinumma placens Walker, Diphthera; Hypnotype placida Butler, Amphigonia; Gabyna placodoides Guenée, Eriopus; Cotanda plagiata Smith, Nocloa; Nocloa plagiata Walker, Agrotis; Tiracola plagiata Walker, Dyrzela; Dyrzela plagifera Walker, Thermesia; Metaphoenia plagiola Hampson, Selca; Poecilonola plagiola Wileman \& West, Neogabara;

Neogabara plagiota Meyrick, Nesamiptis; Ncsamiptis plana Swinhoe, Apphadana; Lobocheilos planalis Swinhoe, Molopa; Molopa planctaria Guenée, Stcllidia; Stellidia planifrons Smith, Agrotis; Sctagrotis platti Hampson, Pristanepa; Pristanepa 
platyphaeella Walker, Cretonia; Cretonia plebeia Smith, Noctua; Hemigraphiphora plecopteridia Hampson, Bocula;

Gnathogonia

plecopteroides Strand, Acantholipes;

Nolaseniola

plecta Linnaeus, Phalaena; Ochropleura

plena Walker, Erana; Graphania

plumifera Swinhoe, Eucosmocara;

Eucosmocara

plumigeralis Hübner, Pechipogo; Pechipogo

plumipars Hampson, Hemipsectra;

Hemipsectra

plumipes Wallengren, Tephrias; Tephrias

plumosa Warren, Triommatodes;

Triommatodes

plumula Druce, Zorzines; Zorzines

plusiaeformis Edwards, Stretchia; Stretchia

plusina Staudinger, Metalopha; Metalopha

plusioides Walker, Eleale; Tamseale

plutonis Lucas, Thalpochares; Heterorta

poae Savigny, Strigina; Strigina

poaphiloides Guenée, Bendis; Epidromia

podaresalis Walker, Chalestra; Chalestra

poecilastis Meyrick, Xenopseustis;

Xenopseustis

poecilota Turner, Corgatha; Trogatha

poetica Grote, Neumoegenia; Neumoegenia

poeyi Grote, Euscirrhopterus;

Euscirrhopterus

poinimerina Viette, Andrianam; Andrianam

polianus Staudinger, Dasythorax;

Dasythorax

poliastis Draudt, Dasyerges; Dasyerges

poliophaea Hampson, Steniscadia;

Steniscadia

poliophasma Turner, Cryphimaea;

Cryphimaea

poliotis Hampson, Gerarctia; Gerarctia poliotis Hampson, Paralephana;

Paralephana

polita Berio, Pseudogiria; Pseudogiria

polita Walker, Nonagria; Archanara

polybapta Butler, Egnasia; Lophomilia

polycesta Turner, Zagira; Metasada

polychroma Walker, Beana; Beana

polycletusalis Walker, Megatomis; Aegara

polycyma Hampson, Drepanopalpia;

Drepanopalpia

polydamia Stoll, Phalaena; Cubena

polygona [Denis \& Schiffermüller], Noctua;

Opigena

polygrapha Kollar, Arcte; Arcte

polymesata Walker, Lycimna; Lycimna

$\mathrm{NN}$ polymita Linnaeus, Phalaena; Polymixis polyodon Clerck, Phalaena; Actinotia

polyodon Clerck sensu Linnaeus, Phalaena;

Abromias

polypuncta Köhler, Orthopha; Orthopha

polyrrhoda Walker, Callostolis; Callostolis

polyspila Walker, Athyrma; Bessacta

polyspilalis Walker, Gabala; Gabala

polysticta Butler, Agarista; Periscepta

polystigma Lower, Ischyja; Facidina

pomona Cramer, Phalaena; Othreis

poppiusi Herz, Lena; Knappia

popularis Fabricius, Bombyx; Neuronia

populi Strecker, Cleoceris; Brachylomia

porcia Stoll, Phalaena; Lesmone

porphyrea [Denis \& Schiffermüller], Noctua;

Lycophotia

porphyricollis Guenée, Agrotis; Tetrapyrgia

porrecta Saalmüller, Capnodes; Prominea

porrecta Walker, Rhosologia; Rhosologia

porrectalis Fabricius sensu Druce,

Phalaena; Poena

porrigens Walker, Dimona; Dimona

porrima Schaus, Encruphion; Encruphion

postica Walker, Thiacidas; Thiacidas

posticalis Walker, Moscha; Moscha

posticata Walker, Eupithecia; Mesocopsis

posticus Walker, Rhosus; Rhosus

postvittata Moore, Lycauges; Hyposada

potanini Alphéraky, Anartomorpha;

Anartomorpha

praecipua Walker, Thermesia; Seneratia

praecisalis Hübner, Metalectra; Metalectra

praecox Linnaeus, Phalaena; Actebia

praetiosa Köhler, Grisana; Grisana

prasinana Linnaeus, Phalaena; Bena

prasinana Linnaeus sensu Hübner,

Phalaena; Pseudoips

prasinoscia Meyrick, Trissernis; Trissernis

pratti Bethune-Baker, Avirostrum;

Avirostrum

pratti Bethune-Baker, Epa; Epa

pratti Bethune-Baker, Lasiopoderes;

Lasiopoderes

pratti Bethune-Baker, Lasiosceles;

Lasiosceles

pratti Bethune-Baker, Parabasis; Parabasis

pratti Bethune-Baker, Pseudophyx;

Pseudophyx

pratti Bethune-Baker; Sphingiforma;

Sphingiforma

pratti Bethune-Baker, Tibiocillaria;

Tibiocillaria

pratti Bethune-Baker, Zethes; Conosema 
pratti Kenrick, Paracaroides; Paracaroides pratti Kenrick, Plytometra; Eutheiaplusia precedens Walker, Briarda; Briarda precedens Malker, Thalatta; Thalatta preciosa Thierry-Mieg, Pseudobryophila;

Pseudobryophila

pressus Grote, Eurois; Anaplectoides pretiosa Staudinger, Antha; Antha pretiosa Viette, Madeuplexia; Madeuplexia priangani Roepke, Athyrmella;

Athyrmella

prima Smith, Anorthodes; Anorthodes primulina Druce, Metoponia; Xanthiria princeps Boisduval, Ophideres; Ophideres prisca Walker, Leucania; Acrapex pritanis Cramer, Phalaena; Pseudophisma privata Walker, Polia; Sutyna problematica Strand, Agrotis;

Hermonassoides proboscidalis Linnaeus, Phalaena; Hypena proboscidata Herrich-Schäffer, Helia; Orectis procax Hübner, Noctua; Asticta procinctus Grote, Eupsephopaectes;

Eupsephopaectes proclinata Saalmüller, Marca; Marca proclivis Smith, Agrotis; Loxagrotis procopialis Hübner, Euglyphis; Hulypegis procus Cramer, Phalaena; Acacallis prodigiosa Draudt, Miracopa; Miracopa producta Hampson, Pseudinodes;

Pseudinodes producta Mabille, Staga; Staga progenies Guenée, Yrias; Meridyrias prolata Möschler, Amphodia; Amphodia prolata Walker, Gerespa; Gerespa proleuca Hampson, Bostrodes; Bostrodes proleuca Hampson, Miselia; Dicerogastra prolixa Turner, Lophozancla; Lophozancla prompta Walker, Obba; Zinna promptella Walker, Tunza; Tunza pronuba Linnaeus, Phalaena; Noctua propitia Boisduval, Opliiusa; Hamodes propria TValker, Biula; Bulia propria Walker, Leucania; Tmetolophota proprius Edwards, Euros; Euros proserpina Stoll, Phalaena; Clitis prosthenia Hampson, Gracillina; Gracillina prosticta Holland, Plusiocalpe; Odontestis protea [Denis \& Schiffermüller], Noctua;

Dryobotodes

proteoides Kenrick, Elaeodes; Pseudelaeodes proximalis Walker, Gyrtona; Gyrtona

pruinosa Berio, Euncophlelsia;

Euneophlebia pruinosa Guenée, Acronycta; Plataplecta pruinosa Snellen, Notocyma; Notocyma pryeri Butler, Platydasys; Platydasys psammias Meyrick, Eremochroa;

Eremochroa pseudastigma Eecke, Celamoides;

Celamoides pseudobamra Rothschild, Acronycta; Tanocryx pseudopsis Walker, Adrana; Adrana pseudotirhaca Berio, Anua; Perophiusa psi Linnaeus, Phalaena; Triaena psilozona Turner, Clytomorpha;

Clytomorpha

psychodidarum Dyar, Gelenipsa; Gelenipsa pterophoralis Guenée, Tortricodes;

Alberticodes pterylota Meyrick, Plusia; Lophoplusia ptilopleura Turner, Eremaula; Eremaula ptycophora Grote, Fala; Fala pudens Mabille, Agrotis; Mabilleana puella Schaus, Tarache; Cadiorapa puera Cramer, Phalaena; Hyblaea puerpera Giorna, Phalaena; Blepharonia;

Eunetis

pulchella Smith, Xanthia; Lycanades pulcherrima Butler, Egnasia; Lophograpta pulcherrima Hampson, Conservula;

Eulymnia

pulcherrimus Grote, Ripogenus; Ripogenus

pulchra Barnes \& McDunnough, Azenia;

Uniptena

pulchra Bethune-Baker, Corgatha;

Calloruza

pulchra Bethume-Baker, Farara; Farara pulchra Bethune-Baker, Olulodes; Olulodes pulchra Möschler, Alibama; Alibama pulchra Trimen, Pais; Paida pulchrilinea Waller, Obana; Rhoptrotrichia pulchripennis Grote, Heliothis; Adonisea pulchripicta Walker, Canna; Nacna pulchristrigata Bethune-Baker, Catephia; Leistera

pulla [Denis \& Schiffermüller], Noctua;

Scotochrosta

pulla Grote, Lathosea; I athosea pulla Swinhoe, Acontia; Pariambia pulmonaris Esper, Phalacua; Atypha pulverata Guence, Sanys; Marapana pulverca Hampson, Baniopis; Baniopis pulverea Smith, Narthecophora;

Narthecophora

pulverilinea Grote, Matigramma; Matigramma. 
punctalis Fabricius, Phalaena; Botys punctana Walker, Tospitis; Narosodes punctata Bethune-Baker, Catadoides;

Catadoides

punctata Bethune-Baker, Phanaspa;

Lophocerynea

punctata Hampson, Pseudcraspedia;

Pseudcraspedia

punctata Warren, Stenocryptis;

Stenocryptis

puncticeps Walker, Penza; Penza

puncticinctalis Walker, Olulis; Olulis punctifascia Leech, Phyllodes; Xylophylla punctifera Walker, Heliothis; Neocleptria punctigera Roepke, Calesiodes; Calesiodes punctigera Walker, Ozarba; Ozarba punctilinea Dognin, Enedena; Enedena punctilinea Jones, Parvapenna; Amphilita punctilinea Prout, Subsimplicia;

Subsimplicia

punctilineata Moore, Kerala; Kerala

punctimargo Hampson, Sarcopteron;

Sarcopteron

punctistigma Hampson, Neothripa;

Neothripa

punctulata Schaus, Strabea; Strabea

punctulum Guenée, Eriopyga; Eriopyga

punctum Fabricius, Noctua; Amyna

puniceago Boisduval, Xanthia; Mycteroplus

punkikonis Matsumura, Mesocrapex;

Mesocrapex

pupillaris Grote, Sisyrhypena; Sisyrhypena

pura Hübner, Noctua; Ecthetis

purissima Dyar, Iscadia; Boryzops

purpuraria Dognin, Margiza; Lophuda

purpurascens Bethune-Baker, Acantholipes;

Phaeozona

purpurascens Hampson, Stenocodia;

Stenocodia

purpurascens Hampson, Tathodelta;

Tathodelta

purpurascens Kaye, Epitomiptera;

Epitomiptera

purpurascens Schaus, Lophosema;

Lophosema

purpurea Bethune-Baker, Echanella;

Echanella

purpurea Butler, Graphiphora;

Austramathes

purpurea Hampson, Amefrontia;

Amefrontia

purpurea Hampson, Enispa; Enispodes

purpurea Hampson, Oenoptera; Oenoptera

purpurea Warren, Hypaenistis; Hypaenistis purpureoscripta Walker, Didigua; Didigua purpurigera Guenée, Hypotrix; Hypotrix purpurigera IValker, Deva; Pseudeva purpurina [Denis \& Schiffermüller], Noctua;

Porphyrinia

pusilla Butler, Egnasia; Aventiola pusilla Moore, Gyrtona; Gyrtothripa pustularia Hübner, Hypagyrtis; Hypagyrtis pustulifera Walker, Ciasa; Ciasa pustulifera Walker, Saroba; Saroba puta Grote \& Robinson, Xanthia; Anathix putrefacta Guenée, Axylia; Amazonides putrescens Guérin-Méneville, Erebus;

Omoptera

putris Linnaeus, Phalaena; Axylia

pygmaea Hübner, Paectes; Paectes

pyralella [Denis \& Schiffermüller], Tinea;

Eudorea

pyralidia Hampson, Archinola; Archinola

pyraliformis Moore, Arasada; Arasada

pyralina Hampson, Constantiodes;

Constantiodes

pyralis Dyar, Sacadodes; Sacadodes

pyralis Hübner, Hemeroplanis;

Hemeroplanis

pyraloides Walker, Chuduca; Chuduca

pyraloides Walker, Obrima; Obrima

pyramusalis Walker, Herminia;

Neoherminia

pyraspis Meyrick, Thalpochares; Pyripnoa

pyrochroma Walker, Bagada; Bagada

pyrostrota Dognin, Calymniodes;

Calymniodes

pyrrhias Turner, Acnissa; Acnissa

pyrula Hopffer, Spirama; Cometaster

quadra Joannis, Trichypena; Trichypena

quadralis Barnes \& McDunnough,

Parahypenodes; Parahypenodes

quadrapex Hampson, Mestleta; Smicroloba quadrata Berio: Trisulipsa; Trisulipsa quadratifera Walker, Nigramma; Nigramma quadratipennis Walker, Bessara; Bessara quadricolor Walker, Giscala; Giscala quadrifasciata Walker, Flammona;

Flammona quadrifera Zeller, Erastria; Tripudia quadrifilaris Hübner, Agnomonia; Poaphila quadrilinea Walker, Asta; Asta quadrilineata Bethune-Baker, Brithodes;

Brithodes

quadrilineata Moore, Madopa; Orthozona 
quadrilineata Moore, Thalpochares;

Gyophora

quadrilineata Walker, Borsippa; Borsippa quadrimacula Herrich-Schäffer,

Mystrocephala; Mystrocephala

quadripennis Moore, Chandica; Chandica quadripennis Walker, Psimada; Psimada quadriplaga Bartel, Netrocerocora;

Netrocerocora

quadripuncta Hampson, Mimasura;

Mimasura

quadripunctata Shiraki, Pseudopisara;

Pseudopisara

quadrisignata Walker, Daddala; Daddala quadrisignata Walker, Homoptera;

Homopyralis quadrisignata Walker, Setida; Setida quadrivirgula Mabille, Epimecia;

Hypomecia quassa Walker, Azeta; Massala quenavadi Guenée, Pandesma; Pandesma quercana Fabricius, Pyralis; Phibalocera quercus Fabricius, Noctua; Charanyca quieta Hübner, Noctua; Archanarta

rada Boisduval, Microphisa; Leucanitis radians Westwood, Noctua; Apsarasa radiata Moore, Masalia; Masalia radiata Schaus, Praina; Praina radiata Wileman \& West, Riaga; Riaga radiatus Snith, Lythrodes; Lythrodes radicalis Walker, Gerra; Gerra radoti Boursin, Poteriophora; Poteriophora ragusae Failla-Tedaldi, Heteromorpha;

Orrhodiella

ramosa Esper, Bombyx; Calliergis ramosula Guenée, Cloantha; Nedra rangnovi Püngeler, Mamestra; Anartodes ranunculi Edwards, Xanthothrix;

Xanthothrix raralis Walker, Hypena; Marapana rasalis Warren, Micreremites;

Zanclopalpus rasdolnia Staudinger, Rusidrina; Rusidrina rava Schaus, Pelodia; Pelodia ravida [Denis \& Schiffermüller], Noctua;

Spaelotis recens Saalmüller, Biregula; Biregula receptalis Walker, Crymona; Crymona receptricula Hübner, Noctua; Cryphia recta Guenée, Micra; Eumestleta rectalis Eversmann, Herminia; Simplicia rectalis Walker, Midea; Midea

rectangula [Denis \& Schiffermüller], Noctua;

Chersotis

rectangularis Hübner, Noctua; Exophyla rectangulum Berio, Pennalticola;

Pennalticola

rectifasciata Ménétriés, Spirama; Metopta rectigramma Hampson, Codonodes;

Codonodes

rectilinea Esper, Phalaena; Hyppa

rectilinea Moore, Harita; Harita

rectimarginata Hampson, Carea;

Orthocraspis

rectivitta Moore, Orthosia; Perigeodes recurrens Hampson, Proruaca; Proruaca recurva Walker, Tyrissa; Tyrissa recurvata Moore, Callopistria;

Rhoptrotrichia recurvata Möschler, Ballonicha; Ballonicha recurvicornis Möschler, Smyra; Smyra reducta Mabille, Stenopis; Stenopis redunca Swinhoe, Ophiusa; Bastilla reflexa Guenée, Plecoptera; Plecoptera regalis Butler, Scedopla; Scedopla regalis Moore, Hypercompa; Attatha regina Guenée, Ophideres; Graphigona regina Staudinger, Cosmia; Pseudenargia regnatrix Grote, Philochrysa; Philochrysa regularis Hübner, Noctua; Acantholipes reincarnata Dyar, Phobolosia; Phobolosia relapsa Walker, Poaphila; Baniana relinquenda Walker, Briada; Oromena remicauda Butler, Tarsolepis; Tarsolepis remigina Mabille, Euclidia; Remigiodes remissa Walker, Ephyrodes; Bendisopis removens Walker, Ginaea; Ginaea renalis Hübner, Pyralis; Haemerosia renalis Moore, Zethes; Parolulis rendalli Rothschild, Metagarista;

Chaetostephana renifera Boisduval, Haemerosia; Haemerosia reniferalis Guenće, Ceraptila; Ceraptila reniferalis Hampson, Progonia; Progonia reniplaga Felder, Closteromorpha;

Closteromorpha renosa Hübner, Anereuthina; Anereuthina repanda Fabricius, Bombyx; Bagisara repanda Schaus, Scopifera; Pyrgion repanda IValker, Siavana; Siavana repandens Walker, Chora; Chora replenens WValker, Beregra; Beregra repletana Walker, Giaura; Giaura replicans Walker, Ancara; Ancara reposita Möschler, Adyroma; Adyroma 
repugnans Walker, Thyatira; Risoba respersa Hübner, Phalaena; Eublemma respondens Walker, Polyphaenis;

Neophaenis restituta Walker, Geria; Geria resumens Walker, Psaphida; Dicopsis; Psaphida retardens Walker, Azatha; Azatha retatalis Walker, Chusaris; Chusaris reticularis Felder, Teinopyga; Teinopyga reticulata Goeze, Phalaena; Heliophobus reticulatis Leech, Adrapsa; Adrapsoides retina Freyer, Hadena; Prodenia retorta Clerck, Phalaena; Spirama retracta Hampson, Audea; Hypotacha retracta Hampson, Cosmophila;

Isadelphina retusa Linnaeus, Phalaena; Ipimorpha retusalis Hübner, Idia; Pelecia revayana Scopoli, Phalaena; Nycteola revellata Barnes, Namangana; Draudtia reversa Moore, Aletia; Hypopteridia reversa Walker, Nertobriga; Nertobriga rhexiae Smith, Phalaena; Chloridea rhizoleuca Brabant, Pseudacontia; Brabantia rhodinastis Meyrick, Prionophora; Synolulis rhodites Eversmann, Heliothis; Aedophron rhodocentra Lower, Prometopus; Pricomia rhombalis Guenée, Hypena; Badausa rhopalophora Turner, Euzancla; Euzancla rhythmotypa Turner, Orthozancla;

Orthozancla ribbei Druce, Strathocles; Strathocles ribbei Pagenstecher, Nyctipao;

Megistoclisma ridens Fabricius, Noctua; Polyploca rigida Smith, Agrotiphila; Orosagrotis rileyana Morrison, Agrotis; Onychagrotis ripae Hübner, Noctua; Agrotis rivosa Saalmüller, Maronis; Maronis rivularis Fabricius, Noctua; Aneda rivularis Walker, Callopistria;

Hemipachycera rivulosa Guenée, Anthoecia; Lygranthoecia rivulosa Walker, Thermesia; Blasticorhinus rivulosum Saalmüller, Megacephalon;

Megacephalomana rjabovi Bundel, Tschetwerikovia;

Tschetwerikovia roastis Hampson, Melapera; Melapera robertsi Berio, Eupsoropsis; Eupsoropsis robinsoni Rothschild, Gravodos; Gravodos robusta Hampson, Nonagria; Hygrostola robusta Köhler, Tarsicopia; Tarsicopia robusta Walker, Thria; Thria

robustior Strand, Hygrostolides;

Hygrostolides

†'roda Herrich-Schäffer', Euclidia;

Leucanitis

roeselioides Walker, Acontia; Pardasena rogenhoferi Möschler, Metaponpneumata;

Metaponpneumata

rolandi Grote, Eutolype; Eutolype roma Druce, Dacira; Bryolymnia rorata Walker, Zurobata; Zurobata rosacea Bethune-Baker, Hypenarana;

Hypenarana

rosalia Staudinger, Plusilla; Plusilla rosea Hampson, Phycidimorpha;

Phycidimorpha rosea Rothschild, Antitype; Pseudopolia rosea Smith, Thyreion; Thyreion rosealutea Berio, Gaedonea; Gaedonea roseilinea Walker, Leucania; Xipholeucania roseipennis Moore, Rhangena; Rhangena roseiventris Gerstaecker, Asymbata;

Asymbata

roseoalba Walker, Heorta; Heorta roseofusca Warren, Rhodotarache;

Rhodotarache rothschildi Bethune-Baker, Amphoraceras;

Amphoraceras rotunda Berio, Brachytegma; Brachytegma rotunda Hampson, Anodontodes;

Anodontodes rotundata Rothschild, Magulaba;

Rhesalistis rotundata Walker, Antachara; Antachara rotundata Walker, Diomea; Diomea rotundipennis Hampson, Toana; Toanodes rotundipennis Walker, Garella; Garella royi Berio, Tornoconia; Tornoconia ruber Hampson, Arboricornus:

Arboricornus

rubescens Köhler, Pseudosmia; Despumosia rubida Walker, Anigraea; Anigraea rubidata Kenrick, Perigea; Kenrickodes rubiginea Bethune-Baker, Paracarea;

Paracarea rubiginea Bethune-Baker, Paralopha;

Paralopha rubiginea [Denis \& Schiffermüller], Noctua;

Dasycampa rubiginea IValker, Varia; Varia rubiginosa Scopoli, Phalaena; Orrhodia rubiginosa Viette, Tunocaria; Tunocaria rubra Bethune-Baker, Plinthopa; Plinthopa 
rubra Hampson, Ancara; Rhynchoplexia rubrescens Hampson, Asplenia; Asplenia rubricans Boisduval, Ophiusa; Azazia rubricosa [Denis \& Schiffermüller], Noctua;

Cerastis

rubricosa Walker, Marcillada; Marcillada rubricosta Guenée, Panopoda; Panopoda rubripennis Grote \& Robinson, Leucania;

Protoleucania rubrisparsa Walker, Bithiga; Bithiga rubromarginata Schaus, Rhaesena;

Matiloxis ruckesi Barnes \& Benjamin,

Mesembragrotis; Mesembragrotis rudens Harvey, Agrotis; Hemieuxoa rudis Walker, Amphigonia; Herpeperas rudis Walker, Bagistana; Bagistana rudis Walker, Pastona; Pastona rudivitta Walker, Egelesta; Egelesta rudmuna Swinhoe, Egnasia; Egnasides rufa Haworth, Phytometra; Coenobia rufa Schaus, Arsaciodes; Arsaciodes rufago Hübner, Jodia; Xystopeplus rufescens Hampson, Paraxestis; Paraxestis rufescens Kirby, Pyramarista;

Pyramarista

rufibasis Guenée, Sympis; Sympis ruficollis [Denis \& Schiffermüller], Noctua;

Asphalia

rufimargo Hübner, Phoberia; Panopoda rufimedia Hampson, Sarrothripa;

Megathripa

rufinans Guenée, Capnodes; Gerisa rufipalpis Walker, Pasipeda; Pasipeda rufipectus Morrison, Agrotis; Protolampra rufipicta Hampson, Drepanopses;

Drepanopses

rufiplaga Bethune-Baker, Vittappressa;

Vittappressa

rufipuncta Hampson, Dendrothripa;

Dendrothripa

rufoflava Walker, Xanthia; Pseudelydna

rufostrigata Bethune-Baker, Catephia;

Paranagia

rufostrigata Packard, Leucania; Hypocoena rufula Holland, Targallodes; Targallodes

rufulus Shchetkin, Aridagricola;

Aridagricola

rugifrons Grote, Stiria; Stiria

rumicis I innaeus, Phalaena; Viminia

rungsi Köhler, Pyrgeia; Pyrgeia

rupicola [Denis \& Schiffermüller], Noctua;

Apaustis

ruptellus Walker, Crambus; Meyrickella rureoides Möschler, Erioscele; Erioscele rurigena Grote, Phalaenophana;

Phalaenophana

ruris Hübner, Noctua; Exarnis russula Grote, Pseudorgyia; Aretypa ruticilla Esper, Phalaena; Spudaea rutilago [Denis \& Schiffermüller], Noctua;

Pyrrhia

rutilans Walker, Banassa; Banassa rutilifrons Walker, Plusia; Erythroplusia

sabina Fawcett, Poppaea; Poppaea sabulifera Guenée, Gonitis; Tiridata sabulosa Fletcher, Apospasta; Apospasta sabulosa Schaus, Apustis; Apustis sabulosa Schaus, Capnodes; Neocerynea saca Püngeler, Diadochia; Diadochia sacelli Staudinger, Cteipolia; Cteipolia sachalinensis Matsumura, Kitanola;

Kitanola sada Swinhoe, Platyja; Platyjionia saga Fabricius, Noctua; Hyblaea sagata Walker, Diphtera; Bathyra sagitta Fabricius, Noctua; Dichromia sagitta Hübner, Noctua; Telmia sagittalis Strand, Metoposcopa;

Metoposcopa sagittata Köhler, Graptocullia;

Graptocullia sagittigera Hufnagel, Phalaena; Pachetra salebrosa Guenée, Anisoneura; Anisoneura salicalis [Denis \& Schiffermüller], Pyralis;

Colobochyla

salicana [Denis \& Schiffermüller], Tortrix;

Pendina

salicella Linnaeus, Phalaena; Pendina salsoma Swinhoe, Zethes; Focillistis sancta Staudinger, Acontiola; Acontiola sandix Guenée, Achatodes; Achatodes sanguifusa Jones, Doryodes; Acylita sanguiguttata Hampson, Haematosticta;

Haematosticta sanguilinea Hampson, Mesasteria;

Mesasteria

sanguinalis Linnaeus, Phalaena; Rhodaria sanguinata I ucas, Chariclea; Metopiora sanguinea Bethune-Baker, Careades;

Careades sanguinea Cieyer, Oria; Porrima sanguinea Moore, Topadesa; Topadesa sanguinipuncta Guenée, Amphipyra:

Peripyra 
sanguinolenta Moore, Dorika; Dorika sanmartini Köhler, Mendozania;

Mendozania

santaris Schaus, Ypsora; Ypsora santista Jones, Iheringia; Lophomyra saphobasis Hampson, Brachyherca;

Brachyherca

saponariae Borkhausen, Phalaena;

Heliophobus

saporis Grote, Triocnemis; Triocnemis sapota Felder \& Rogenhofer, Megatomis;

Eugoniella

sarawakana Walker, Savoca; Savoca satellitia Linnaeus, Phalaena; Eupsilia satinea Rougemont, Amphipyra; Neocomia saturata Walker, Ariola; Lasiolopha saturata Walker, Eusemia; Immetalia saturatalis Walker, Arsacia; Arsacia saturnina Mabille, Ancistris; Ancistris satyricus Grote, Ufeus; Ufeus saucia Hübner, Noctua; Peridroma sauzalitae Grote, Ochria; Emboloecia scabra Fabricius, Hyblaea; Plathypena scabriuscula Linnaeus, Phalaena;

Dypterygia scapulosa Hübner, Noctua; Cerocala scarletina Smith, Palada; Palada schaldusalis Walker, Bocana; Nabartha;

Culicula schausi Hampson, Auchenisa; Auchenisa schawerdae Reisser, Evisa; Evisa schedocala Dyar, Erastrifacies;

Erastrifacies

schencki Bartel, Syfanoidea; Syfanoidea schildei Möschler, Encalypta; Encalypta schiniodes Dognin, Dipinacia; Dipinacia schneideriana Stoll, Phalaena; Pararcte schoutedeni Strand, Makaia; Makaia schwingenschussi Wagner, Hyrcanypena;

Hyrcanypena

scira Druce, Anthoecia; Chrysoecia scissa Walker, Massava; Massava scissa Walker, Rhiscipha; Rhiscipha scissilinea Walker, Hypena; Saserna scissuralis Moore, Arthisma; Arthisma scita Hübner, Phalaena; Habryntis scitisignata Walker, Cidaria; Istarba scitula Butler, Xylophasia; Rhynchagaea scitula Rambur, Erastria; Coccidiphaga scitula Walker, Thermesia; Dunira scopariae Dorfmeister, Cucullia; Cheligalea scoparioides Walker, Corticata; Corticata scopulepes Haworth, Phytometra;

Scopelopus scoria Guenée, Stimmia; Stimmia scoriacea Esper, Phalaena; Cleoceris scotaea Turner, Dnopheropis; Dnopheropis scotia Hampson, Ptisciana; Lamprothripa scotomista Hampson, Polia; Hypotype scotosialis Walker, Rhapsa; Rhapsa scriptura Walker, Hadena; Speocropia scripturosa Eversmann, Xylina:

Scythocentropus scrobiculata Fabricius, Noctua; Oxyodes scrupulosa Edwards, Synedoida; Synedoida sculpta Felder \& Rogenhofer, Ophisma;

Metaprionota sculpta Grote, Xylina; Sutyna scutosa [Denis \& Schiffermüller], Noctua;

Protoschinia secalis Linnaeus, Phalaena; Mesapamea seclusalis Walker, Margana; Margana secta Grote, Eustrotia; Protocryphia seeboldi Staudinger, Saragossa; Saragossa segetum [Denis \& Schiffermüller], Noctua; Agrotis sejuncta Walker, Leucania; Trichoptya sejunctata Walker, Pradiota; Pradiota selecta Schaus, Colodes; Colodes selenampha Guenée, Amyna; Amyna selene Möschler, Placonia; Placonia seleniata Walker, Ostha; Ostha selenis Fletcher, Numeniastes;

Numeniastes selenisoides Walker, Blanona; Blanona selenophora Guenée, Dasypodia; Dasypodia selenotypa Turner, Camptocrossa;

Camptocrossa

selini Boisduval, Caradrina; Paradrina semaphora Hampson, Nephelemorpha;

Nephelemorpha semialba Walker, Boana; Naboa semiaperta Morrison, Hydroecia; Tricholita semicrocea Guenée, Xanthoptera; Exyra semidolosa Walker, Automala; Automala semifasciana Haworth, Tortrix;

Brachytaenia semifervens Walker, Gadirtha; Erizada semiflava Hampson, Strongylosia;

Strongylosia semilineata Walker, Legna; Ledaea semilunaris Guenée, Palyna; Palyna seminivea Walker, Ptisciana; Ptisciana;

Argyrothripa semiochrealis IValker, Toana; Toana semipallida Hampson, Aspidifrontia;

Aspidifrontia semipardata Walker, Guriauna; Guriauna 
semipars Waller, Bryophila; Gerbatha semirufa Barnes \& McDunnough, Euaontia;

Euaontia

semiusta Warren, Erebothrix; Erebothrix semograpta Meyrick, Sorocostia; Nestiodes senegalensis Guenée, Nonagria; Timora senescens Grote, Hadena; Hillia senex Bethune-Baker, Trisulana; Trisulana sentalis Kaye, Parvapenna; Parvapenna separata Staudinger, Xylomoia; Xylomoia separata Walker, Maliattha; Maliattha septempunctata Harvey, Grotella; Grotella sequistriaris Hübner, Agnomonia;

Agnomonia

sera Felder \& Felder, Orthogonia;

Orthogonia

serena [Denis \& Schiffermüller], Noctua;

Mamestra

sergilia Stoll, Phalaena; Phidmodium

sericata Esper, Noctua; Polyphaenis sericea Butler, Taseopteryx; Taseopteryx sericea Butler, Trisuloides; Trisuloides sericea Hampson, Clinophlebia;

Clinophlebia

sericea Hampson, Metaptya; Metaptya sericea Zerny, Caradjia; Caradjia sericealis Hampson, Erizada; Goniocalpe sericealis Scopoli, Phalaena; Rivula sericeata Hampson, Oxygonitis; Oxygonitis sericina Turner, Ochthophora; Ochthophora serotina Ochsenheimer, Cerastis; Orbona serpentina Walker, Baxagha; Baxagha serpentinum Felder, Leiosoma; Leonides serra Herrich-Schäffer, Ophiusa;

Prionoptera

serrata Hainpson, Phragmatiphila;

Poeonoma

serrata Smith, Trichopolia; Hexorthodes serratilinea Ochsenheimer, Polia; Chera sesamiodes Hampson, Paragria; Paragria sesamioides Rebel, Neosema; Neosema sesamoides Hampson, Conicofrontia;

Conicofrontia

seticornis Walker, Nonagria; Didigua setipes Guence, Xylis; Xylis

setula Druce, Ariphrades; Ariphrades severa Stoll, Phalaena; Diamuna sevorsa Grote, Fonaria; Fenaria sexguttata Harris, Notodonta;

Harrisimemna

sexmaculata Walker, Capnorles; Eucapnodes sexpunctata Grote, Spargaloma;

Spargaloma

sexsignata Harvey, Lita; Litocala sexstrigata Haworth, Noctua; Lytaea shelfordi Swinhoe, Pseudaglossa;

Drepanorhina

siccanorum Staudinger, Mamestra;

Onychestra

sicheas Cramer, Phalaena; Gronodonta siculipalpis Strand, Niaccabana; Niaccabana siderea Guenée, Callyna; Callyna siderosticta Holland, Rhescipha;

Drepanophiletis sidus Guenée, Scopelosoma; Dichagramma sigillata Butler, Hollandia; Hollandia sigillata Ménétriés, Anthoecia; Sphragifera sigina [Denis \& Schiffermüller], Noctua;

Eugraphe sigmoides Guenée, Noctua; Eueretagrotis signalis Treitschke, Scopula; Mesotrosta signata Butler, Actenia; Marojala signata French, Orthosia; Sericaglaea signata Walker, Ariolica; Doranaga signata Walker, Nephelodes; Tricholita signicosta Strand, Alikangiana;

Alikangiana signicosta Walker, Enea; Enea signifera [Denis \& Schiffermüller], Noctua; Yigoga signifera Walker, Sarthida; Sarthida significans Walker, Baniana; Baniana significans Walker, Edyma; Edyma signipennis Strand, Biagicola; Biagicola silenalis Guenće, Arsina; Arsina sileniata Walker, Ostha; Ostha silenides Staudinger, Mamestra;

Pronotestra silenusalis Walker, Bocana; Asthala silvandra Stoll, Phalaena; Hypsa simia Saalmüller, Zethes; Mepantadrea similiaria Ménétriés, Ligia; Pseudoligia similis Aurivillius, Haplocestra;

Haplocestra

similis Butler, Eurypsyche; Eurypsyche similis Hampson, Megalodes; Cyclopera similis Moore, Phurys; Loxioda simo Wallengren, Acontia; Metapioplasta simoenta Guencee, Cyclopis; Teinoletis simonyi Rogenhofer, Bryophila; Nyctobrya simplex Holland, Episparis; Libystica simplex Möschler, Berocynta; Berocynta simplex Schaus, Lutogonia; Lntogonia simplex Smith, Schinia; Chlorocleptria simulans Köhler, Parosmia; Parosmia simulatrix Wilker, Deremma; Deremma simyrides Staudinger, Lciometopon;

Lciometopon 
sinens Walker, Orthosia; Thalatha singha Guenée, Marmorinia; Hyposemansis singularis Staudinger, Orbifrons; Orbifrons singularis Turner, Pediarcha; Pediarcha singularis Warren, Atopomorpha;

Atopomorpha sinuata Moore, Dimya; Apameá sinuata Moore, Gluphisia; Cymatophoropsis sinuata Moore, Sophaga; Sophaga sinuosa Moore, Phalga; Phalga sisera Schaus, Bryogramma; Bryogramma skraelingia Herrich-Schäffer, Phlogophora;

Lasionycta

smaragdina Bethune-Baker, Euplexia;

Pachythrix

smaragdistis Hampson sensu Hampson,

Trachea; Pachythrix

smerinthoides Walker, Corymbia; Corymbia smilacis Bourquin, Speocropia; Cromobergia sminthochroa Hampson, Parorena; Parorena smithii Holland, Parauchmis; Parauchmis smithii Mabille, Daphoenura; Epicausis smithii Möschler, Pleurasympieza;

Pleurasympieza snelleni Möschler, Aglaonice; Aglaonice snelleni Saalmüller, Ingura; Zobia snelleni Staudinger, Sidemia; Sidemia snowi Smith, Parora; Diapera sobria Walker, Arcilasisa; Arcilasisa sobria Walker, Ericeia; Ericeia sobria Walker, Gaphara; Gaphara sobrina Möschler, Aburina; Aburina socia Hufnagel, Phalaena; Lithophane sociabilis Graslin, Hadena; Cardepia sociella Linnaeus, Phalaena; Aphomia socors Walker, Caenurgia; Caenurgia sodae Boisduval, Mamestra; Discestra solaris [Denis \& Schiffermüller], Noctua; Acontia solidaginis Hübner, Noctua; Lithomoia sollers Christoph, Agrotis; Parexarnis solstitialis Holland, Gigantoceras;

Gigantoceras

soluta Walker, Polia; Plataplecta sommeri Hübner, Crino; Tarsolepis sonoma McDunnough, Psaphida;

Pseudocopivaleria sopora Swinhoe, Myana; Myana sordens Hufnagel, Phalaena; A pamea sordescens Rosenstock, Bleptina;

Mormoscopa

sordida Moore, Falana; Falana sordida Swinhoe, Pancra; Pancra sordidula Walker, Chodda; Chodda sordidum Butler, Trotosema; Trotosema sorghicida Thurau, Busseola; Busseola spadaria Guenée, Doryodes; Themma spadicea Felder, Pycnodontis; Pycnodontis spadix Cramer, Phalaena; Cissusa spaldingi Barnes \& McDunnough, Grotella;

Neogrotella sparganiotis Dyar, Fotopsis; Fotopsis sparsa Guenée, Pantydia; Pantydia spartiata Herbst, Phalaena; Chesias specifica Guenée, Alysia; Alysina speciosa Bremer, Noctua; Sidemia speciosa Hübner, Noctua; Platagrotis spectabilis Turner, Sarobela; Sarobela spectabilis Walker, Astha; Astha spectabilis Walker, Donuca; Donuca spectans Guenée, Sericia; Sericia spectrum Esper, Noctua; Apopestes specularis Hübner, Letis; Letis speiplena Walker, Aphusia; Aphusia sphaeriphora Moore, Thyridospila;

Thyrostipa sphendonistis Hampson, Tarache;

Cardiosace

sphinx Hufnagel, Phalaena; Asteroscopus spicea Guenée, Perigea; Bagada spinea Grote, Apatela; Merolonche spinosa Barnes \& McDunnough,

Trichorthosia; Trichagrotis spinosae Guenée, Heliothis; Eupanychis spiramioides Walker, Niguza; Niguza spissa Guenée, Felinia; Felinia splendens Butler, Mydrodoxa; Mydrodoxa splendens Druce, Oxythres; Oxythres splendens Hübner, Noctua; Diataraxia splendens Möschler, Thelidora; Thelidora splendens Viette, Epicausis; Eudaphaenura splendida Köhler, Comodoria; Comodoria splendida Stoll, Phalaena; Argyromata spodia Rebel, Pyraloides; Pyraloides spodochroa Turner, Lathraeolis;

Lathraeolis spoliatricula [Denis \& Schiffermüller],

Noctua; Jaspidia spreta Dognin, Athurmodes; Athurmodes spumosum Grote, Stibadium; Stibadium spurcata Walker, Detounda; Detounda squamata Hampson, Pachygnathesis;

Pachygnathesis squamivaria Walker, Marthama; Marthama squammigera Guenée, Lophoptera;

Lophoptera

stabilis [Denis \& Schiffermüiller], Noctua; Cuphanoa 
staccata Guenée, Peosina; Obucola staudingeri Möschler, Agrotis; Agrotimorpha staudingeri Standfuss, Erastria;

Chionoxantha

stecleae Fletcher, Tegena; Tegena

stellaris Grote, Agrotis; Adelphagrotis

stellata Moore, Graphiphora;

Strepselydna

stellata WValker, Derrima; Derrima stellifera Moore, Dianthoecia; Plexiphleps stenelea Stoll, Phalaena; Erebostrota steniphona Son, Nola; Poliothripa steniptera Hampson, Stictigramma;

Stictigramma

stenoptera Sugi, Stenograpta; Stenograpta stenothyris Hampson, Neopangrapta;

Neopangrapta

sterrha Staudinger, Gyrohypsoma;

Gyrohypsoma

sthenoptera Swinhoe, Thermesia;

Stenocarsia

stictopteris Butler, Acidalia; Proroblemma stigmagrapta Hampson, Niphosticta;

Niphosticta

stigmata Moore, Hypaetra; Pterochaeta stigmata Smith, Miodera; Miodera stigmatophora Bryk, Sinarella; Sinarella. stillata Guenée, Nonagria; Dantona stipatura Walker, Homoptera; Smyra stolida Fabricius, Noctua; Prodotis stolifera Saalmiiller, Delta; Lepidodelta stolliana Stoll, Plialaeıra; Ferenta straminea Bang-Haas, Palpangula;

Eremonoina

straminea Butler, Pseudephyra;

Pseudephyra

straminea Dyar, Dasyblemma; Dasyblemma straminealis Walker, Aniana; Aniana striata Staudinger, Ragonotia; Doerriesa strigaria Hübner, Geometra; Cymatida strigata Hampson, Athetis; Exathetis strigata Hampson, Tripseuxoa; Tripseuxoa strigata Smith, Rancora; Rancora strigicincta WValker, Mazuca; Mazuca strigicosta Hampson, Microphysa;

Cingalesa

strigidisca Moore, Apanea; Isopolia strigifera Pagenstecher, Alamis;

Cycloprosopus

strigifera WValker, Bolina; Coronta

strigifera Walker, Magusa; Magusa

strigilata Linndeus, Phalacna; Pechipogo

strigilis I-innaeus, Thalaena; Oligia

strigipennis Moore, Sonagara; Sonagara strigocrenulata Bethune-Baker, Paragonitis; Paragonitis

strigosa [Denis \& Schiffermüller], Noctua; Hyboma

stygia Bryk, Heteroscotia; Heteroscotia stygia Hampson, Paryrias; Paryrias stylata Smith, Cerapoda; Cerapoda suava Staudinger, Pygopteryx; Pygopteryx suavis Edwards, Heliothis; Erythroecia suavis Staudinger, Ammogrotis;

Ammogrotis

subalbiplaga Warren, Anigraeopsis;

Anigraeopsis

subarschanica Staudinger, Hadena;

Haderonia

subaspersa IValker, Melanchroia;

Coenotoca

subcervina Walker, Carmara; Carmara subcosta IValker, Pitara; Pitara subcostalis Walker, Thermesia; Tiruvaca subcupralis Walker, Bertula; Rhaesena subcurva WValker, Hadena; Stenopterygia subductata Walker, Acidalia; Paroruza subfervida Hampson, Oedicraspis;

Oedicraspis subfervida Walker, Eusimara; Eusimara subflexata IValker, Gustiana; Gustiana subglauca Walker, Elesma; Elesma;

Amaloptila subhirsuta Schaus, Panarenia; Panarenia subhyalina Hampson, Agrotisia; Agrotisia sublata Walker, Bepara; Darcetina sublineata IValker, Capitaria; Capitaria sublineata IValker, Urbona; Urbona subliterata Filipjev, Bryophila; Scythobrya submarina Grotc, Anarta; Scotogramina submedianata Hampson, Scotogramma;

Omphalestra submicacea Malker, Tamba; Tamba submira IValker, Artena; Artena submurina Walker, Michera; Michera submuscosa Walker, Bariana; Bariana subnivosella IValker, Gabara; Gabara subnotata Walker, Monosca; Monosca subobliqua IValker, Steiria; Steiria subocellata WValker, Elixoia; Elixoia subocellata Walker, Maxera; Maxera subolivacea Walker, Acripia; Acripia subolivacea Walker, Cerbia; Othresypna subrosea Butler, Rivula; Perynca subrosea Stephens, Graphiphora; Coenophila subrufa Hampson, Supernola; Supernola subrufa WVarren, Paramimetica;

Paranimetica 
subrufescens Walker, Ectopatria; Ectopatria subsatura Guenée, Hypocala; Eudragana subsignans Walker, Avitta; Avitta subtilis Walker, Dabarita; Dabarita subtusa [Denis \& Schiffermüller], Noctua; Ipimorpha subviridescens Walker, Hadena; Barcita succinea Esper, Phalaena; Argyrospila suero Cramer, Phalaena; Selenisa sulima Stoll, Phalaena; Hypogramma sulphuralis Linnaeus, Phalaena; Emmelia sulphurea [Denis \& Schiffermüller], Noctua; Emmelia

sumbavensis Hampson, Catoblemma; Catoblemma sumptualis Walker, Niaccaba; Niaccaba superba Druce, Antachara; Stauropides superba Hübner, IVestermannia;

Westermannia

superba Moore, Tyana; Chionomera suppulchraria Hübner, Cryptochrostis;

Cryptochrostis

surrepens Walker, Coronta; Coronta surrigens Walker, Oroba; Oroba suspecta Hübner, Noctua; Parastichtis sutrix Grote, Tarache; Tornacontia swinhoei Butler, Anthracia; Calloecia sylvia Dyar, Momaphana; Miracavira symmicta Brandt, Boursinia; Boursinia synesia Turati, Stenosoma; Stenosomides syngenes Turner, Alophosoma; Alophosoma sypnaesimilis Berio, Pectinifera; Pectinifera sypnoides Butler, Edmondsia; Edmondsia syrinx Fawcett, Taveta; Taveta syrtana Mabille, Cucullia;

Pseudocopicucullia syrticola Staudinger, Hebdomochondra; Hebdomochondra

tacita Schaus, Lophomyra; Lophomyra tactalis Walker, Zia; Aquita tactus Grote, Homopyralis; Homopyralis taenialis Hübner, Pyralis; Schrankia takao Inaba, Pseudopanolis;

Pseudopanolis talboti Prout, Anepholcia; Anepholcia talidiformis Guenée, Crambodes; Crambodes talpa Draudt, Bombyciella; Bombyciella taltula Schaus, Herminodes; Hyponeuma tamsi Prout, Tracheoides; Tracheoides tanais Cramer, Phalaena; Acolasis tandoana Bethune-Baker, Eublemmara; Eublemmara

tanganykae Berio, Subnoctua; Subnoctua tanyphylla Turner, Euprora; Litoscelis tanzaniae Berio, Euxoullia; Euxoullia tarassota Hampson, Phytometra;

Acanthoplusia tarchon Cramer, Phalaena; Azirista tarda Guenée, Caradrina; Anorthodes tarda Wiltshire, Tamsola; Tamsola tarsicrinalis Knoch, Phalaena; Herminia tarsiplumalis Hübner, Pyralis;

Zanclognatha

tauralis Walker, Chabora; Duriga tavetensis Holland, Ogovia; Ogovia tecta Grote, Chytoryza; Chytoryza tecta Hübner, Noctua; Pachnobia teichii Berg, Cucullia; Cromobergia tela Smith, Hadena; Ruacodes tellieri Lucas, Taeniocampa;

Pseudopseustis

temera Hübner, Noctua; Exarnis tempaca Möschler, Synalissa; Synalissa templada Schaus, Eudipna; Cropia templi Thunberg, Noctua; Dasypolia tendinosa Hübner, Phaeochlaena;

Phaeochlaena

tenebrata Scopoli, Phalaena; Panemeria tenebrifera Walker, Hadena; Matuta tenebrosa Hampson, Caradrina;

Apocalymnia tenebrosa Holland, Bareia; Eutermina tenebrosa Moore, Hadena; Sasunaga tenebrosa Walker, Peliala; Peliala tenera Walker, Sina; Sina tenerifica Hampson, Eumichtis; Paranataelia tentacularia Linnaeus, Phalaena; Polypogon tenuis Butler, Radinogoes; Radinogoes tenuis Grote, Eutoreuma; Eutoreuma tenuis Prout, Ctenypena; Ctenypena tephropis Turner, Proscrana; Proscrana tepperi Morrison, Schinia; Polenta terens Walker, Hadena; Acroria terminigera Walker, Felinia; Pitacota terranea Schaus, Margiza; Margiza terrea Freyer, Caradrina; Hymenodrina territans Edwards, Basilodes; Chalcopasta tersa [Denis \& Schiffermüller], Noctua;

Orthogramma

tespisalis Walker, Neviasca; Cardalena testacea [Denis \& Schiffermüller], Noctua; Luperina 
testacea Fletcher \& Viette, Gaedeodes;

Gaedeodes

testacea Schaus, Corubia; Corubia

tetraphorella Walker, Lephana; Lephana

tetrapleura Meyrick, Agarista; Platagarista

texana Hampson, Focillidia; Focillidia

texana Smith, Parora; Parora

texta Esper, Phalaena; Thalpophila

thalictri Borkhausen, Phalaena; Calyptra

thalpophiloides Walker, Data; Data

thelephusalis Walker, Olybama; Olybama

theodori Grote, Apatela; Andropolia

thermesialis Walker, Phanaspa; Busmadis

thermisioides Walker, Maltana; Maltana

thermochroa Hampson, Eugatha; Eugatha

thermodesa Viette, Hadenella; Anedhella

thermopera Hampson, Argyropasta;

Argyropasta

thiochroa Turner, Himerois; Himerois

thisbe Fawcett, Cynisca; Siccyna

thomae Prout, Elesmoides; Elesmoides

thomensis Jordan, Tuerta; Acantuerta

thomensis Prout, Camphypena; Camphypena

thoracica Butler, Pseudocerura; Pseudocerura

thoracica Walker, Bityla; Bityla

thyatyroides Guenée, Plusia;

Eosphoropteryx

thyodes Dyar, Neomanobia; Neomanobia

thyrophora Hampson, Heteromala;

Heteromala

thyrsipalpis Hampson, Tympanobasis;

Tympanobasis

tibetanum Staudinger, Dasysternum;

Dasysternum

tibialis Fabricius, Bombyx; Chasmina

tibiata Guenée, Agrotis; Androdes

tigrina Druce, Hespagarista; Polacanthopoda

tigris Guenée, Diastema; Diastema

timais Cramer, Phalaena; Xanthopastis

tirhaca Cramer, Phalaena; Ophiusa

todilla Möschler, Arbinia; Arbinia

togata Esper, Phalaena; Xanthia

togataria Walker, Anaitis; Litosea

tomyris Cramer, Phalaena; Argidia

tonsa Guenće, Perigea; Hypoperige‘

toreuta Grote, Hypena; Euhypena

tornotis Meyrick, Sorocostia; Idiocyttara

torpida Walker, Ossonoba; Ossonoba

torrentium Guenée, Leucania; Vietteania

torsa Guencee, Entomogramma; Taramina

torsivena Hampson, Lithosiopsis;

I.ithosiopsis

tortriciformis Möschler, Mindora; Mindora tortriciform is Walker, Azamora; Azamora tortricoides IValker, Orosa; Orosa tortuosa Walker, Ristra; Ristra

tortuosus Wallengren, Ulotrichopus;

Ulotrichopus

toxea Stoll, Phalaena; Oxidercia trabealis Scopoli, Phalaena; Emmelia trachylepis Prout, Epigiaura; Epigiaura tragopoginis Clerck, Phalaena; Amphipyra trajiciens Walker, Selambina; Selambina tranquilla Grote, Zotheca; Zotheca transcissa Walker, Rhaesena; Rhaesena transcissaria Walker, Rethma; Rethma transducta Walker, Burgena; Burgena transfossa Möschler, Leianophera;

Leianophera

transiens WValker, Eusemia; Seudyra transversa Berio, Taraconica; Taraconica transversa Hufnagel, Phalaena; Eupsilia transversa Walker, Complutia; Complutia transversa Walker, Elydna; Elydna transversa Walker, Nachaba; Chlumetia transvitta Moore, Hingula; Diascia trapezata Moore, Elydna; Calymniops trapezina Linnaeus, Phalaena; Calymnia trapezoides Draudt, Calliocloa; Calliocloa trefoliata Butler, Gnamptonyx; Chelecala triangula Berio, Dallolmoia; Dallolmoia triangularis Bethune-Baker, Deinypena; Caryonopera

triangularis Mabille, Eusemia; Omphaloceps triangulifer Grote, Cirrhophanus;

Cirrhophanus

triangulifera Hampson, Bleptina;

Ochrotrigona

triangulum Hufnagel, Phalaena; Megasema tribuna Hübner, Ephialtias; Seirocastnia trichoptycha Hampson, Geniascota;

Geniascota

trichostrota Meyrick, Mnesipyrga;

Mnesipyrga

tricolor Smith, Cerathosia; Cerathosia tricycla Turner, Euryschema; Euryschema tridens Hufnagel, Phalaena; Calamia trifascia Hübner, Schinia; Schinia trifasciata Moore, Cabralia; Cabralia trifasciata Moore, Capnodes; Attonda trifasciata Swinhoe, Hypaetra; Hirsutipes trigonica Hampson, IVestermannia;

Neonegeta

trigonifera Hampson, Prolophota;

Prolophota

trigonoides Walker, Anoba; Anoba

trigonospila Hampson, Baniana;

Tephrialia 
trigrammica Hufnagel, Phalaena; Charanyca trijuncta Walker, Hadena; Neumichtis trilatalis Walker, Pyralis; Xanthograpta trilinea Bethune-Baker, Miana; Bryoleuca trilinea [Denis \& Schiffermüller], Noctua;

Meristis

trilinea Schaus, Diodines; Diodines

trilinealis Bremer, Herminia; Crinisinus

trilineata Hampson, Ditrogoptera;

Ditrogoptera

trilineata Mabille, Coelophoris; Coelophoris

trilineata Walker, Agabra; Agabra

trimacula Strand, Acripioides; Acripioides trimaculata Bremer, Thyatira; Trispila trinotata Walker, Lebena; Lebena triorbis Turner, Amaloptila; Amaloptila tripartita Semper, Ingura; Aplotelia triphaenoides Walker, Metagarista;

Metagarista

tripla Grote, Oxycilla; Oxycilla triplaga Walker, Ausava; Ausava triplasia Linnaeus, Phalaena; Abrostola triplex Walker, Laphygma; Micrathetis triplogramma Hampson, Trichobathra;

Trichobathra

tripuncta Hampson, Xanthoptera; Mimasura

tripuncta Herrich-Schäffer, Storthoptera; Storthoptera

triquetrana Fitch, Brachytaenia; Lebena trisema Hampson, Lophotidia; Lophotidia trisema Mabille, Anarta; Magellana trisignata Walker, Grammophora;

Harrisimemna tristifica Hübner, Eutactis; Futactis tristriga Möschler, Pseudocalpe;

Pseudocalpe tristrigella Walker, Gargaza; Gargaza trogoptera Hampson, Oediblemma;

Oediblemma truncata Moore, Bibacta; Bibacta truncata Walker, Brada; Aiteta truncata Walker, Leucania; Bathytricha truncatalis Walker, Sanacea; Sanacea truncatalis Walker, Silda; Silda truncataria Walker, Erosia; Tetracme truncipennis Hampson, Elydna;

Strepselydna tsara Viette, Butleronea; Butleronea tuberculata Herrich-Schäffer, Physula;

Lophoditta tullia Perty, Goniapteryx; Goniapteryx tumida Grote, Lygranthoecia;

Pseudanthoecia turanica Ershov, Ligia; Rhabinopteryx turbata Herrich-Schäffer, Polia; Copitarsia turbo Bryk, Crinisinus; Crinisinus turbulenta Hübner, Phosphila; Phosphila turca Linnaeus, Phalaena; Mythimna turcomanica Christoph, Micromania;

Micromania turfosalis Wocke, Hypena; Tholomiges turpis Schaus, Poenomia; Poenomia tusa Swinhoe, Megalodes; Austrazenia tymber Cramer, Phalaena; Ceromacra typhae Thunberg, Noctua; Nonagria typica Distant, Matopo; Matopo typica Hampson, Mesosciera; Mesosciera typica Linnaeus, Phalaena; Naenia typica Smith, Pronoctua; Pronoctua typica Son, Vandamia; Vandamia typica Strand, Alika; Alika typica Strand, Myalila; Myalila tyra Swinhoe, Prorocopis; Hypoprora tyrannus Guenée, Ophideres; Adris

u-album Guenée, Ceramica; Iodopepla uliginosalis Guenée, Hydrillodes; Olybama ulmi [Denis \& Schiffermüller], Noctua;

Exaereta ulvae Hübner, Noctua; Chilodes umbra Hufnagel, Phalaena; Pyrrhia umbratica Goeze, Phalaena; Stygiostola umbratica Linnaeus, Phalaena; Cucullia umbricola Boisduval, Polydesma;

Polydesma umbrina Doubleday, Iontha; Iontha umbrina Grote, Pheocyma; Zaleops umbrina Guenée, Alamis; Alamis umbripennis Strand, Meristis; Meristides umbrosa Bethune-Baker, Contortivena;

Contortivena umbrosa Hampson, Araeognatha;

Araeognatha

umbrosa Hübner, Noctua; Lytaea umminia Cramer, Phalaena; Platyja una Möschler, Anabathra; Anabathra unca [Denis \& Schiffermüller], Noctua;

Eustrotia uncana Linnaeus, Phalaena; Eustrotia uncas Guenée, Azeta; Azeta uncifera Druce, Leucosigma; Leucosigma uncina Hübner, Gonodonta; Gonodonta uncinata Berio, Saaluncifera; Saaluncifera uncinata Saalmüller, Hypogramma;

Saaluncifera 
uncula Clerck, Phalaena; Eustrotia undata Fabricius, Noctua; Mocis undilinealis Walker, Licha; Licha undulalis Stephens, Camptylochila;

Camptylochila undulana Hübner, Tortrix; Nycteola undulans Walker, Caularis; Caularis undulata Felder \& Rogenhofer, Spintherops;

Raphiscopa undulifera Walker, Chabora; Chabora ungemachi Berio, Uollega; Uollega ungulatus Benjamin, Policocnemis;

Policocnemis unicolor Walker, Mamestra; Amphitrota unifasciata Ménétriés, Amphidasis;

Schoyenia unifera Köhler, Rugofrontia; Rugofrontia uniformis Berio, Marcipopsis; Marcipopsis uniformis Felder, Pentaclonia; Pentaclonia unilinea Dyar, Charoblemma; Charoblemma unilineata Bethune-Baker, Squamipalpis;

Squamipalpis unimacula Morrison, Agrotis;

Pscudospaelotis uninotata Walker, Digba; Digba unio Hübner, Euthisanotia; Eudryas unipuncta Haworth, Leucania;

Pseudaletia unipuncta Son, Nolidia; Nolidia unipunctata Bethune-Baker, Oruza;

Oruzodes unipunctata Haworth, Phalaena; Hypagyrtis unistrigata Guenée, Hypopyra; Maxula urbicola Behr, Agassizia; Agassizia ursina Smith, Lathosea; Engelhardtia uruca Schaus, Porosana; Porosana usipetes Druce, Melipotis; Pseudogerespa ustata Hampson, Macellopis; Macellopis ustata Mampson, Tathodelta; Metacausta ustipennis Hampson, Cyphotopsyche;

Cyphotopsyche

ustipennis Hampson, Micraeschus;

Cautaeschra ustula Freyer, Cymatophora; Epimecia ustulata Boisduval, Cleophana; Epimecia ustulata Westwood, Phyllodes; Oporophylla utuadae Schaus, Kyneria; Kyneria vacillans Walker, Orthogramma; Parargidia vacillans Walker, Radara; Radara vacillans Walker, Tavia; Facidia vacillatrix Hampson, Lophoruza;

Lophoruza

vagalis Walker, Bocana; Catada vagipennata Walker, Obana; Obana vaillantina Stoll, Phalaena; Egybolis valens Edwards, Synedoida; Ulosyneda valeria Cramer, Papilio; Pareronia valeusalis Walker, Dapha; Dapha valida Walker, Clettharra; Clettharra valligera [Denis \& Schiffermüller], Noctua;

Agronoma

vanderweelei Roepke, Loboplusia;

Loboplusia vaneeckei Roepke, Eubryopterella;

Eubryopterella vanella Grote, Tamila; Pseudotamila varia Walker, Damias; Burgena variabilis Druce, Freilla; Freilla variabilis Grote, Pseudorthosia;

Pseudorthosia variabilis Möschler, Metallata; Metallata variabilis Piller \& Mitterpacher, Noctua;

Euchalcia varialis Walker, Istarba; Istarba varialis Walker, Neviasca; Neviasca varians Hampson, Mesogenea; Mesogenea varians Wallengren, Agrotis; Ctenusa variegata Bethune-Baker, Oruza;

Trichogatha

variegata Hampson, Pilosocrures;

Pilosocrures variegata Kenrick, Hypoperigea;

Phoperigea variegata Leech, Carea; Elydnodes variegata Lucas, Jugurthia; Duhemia variegata Moore, Pitrasa; Pitrasa variegata Moore, Tambana; Tambana variegata Moore, Trisula; Trisula variegata Walker, Pradiota; Chlorograpta varipes Walker, Carea; Carea vaupunctatum Esper, Bombyx; Orrhodia vecors Guenée, Arthodes; Pseudorthodes vectis Möschler, Charmodia; Charmodia vegetus Swinhoe, Spadix; Swinhoea velans WValker, Aucha; Aucha velata Walker, Othora; Othora velifera Grote, Meghypcna; Meghypena vellerea Cruenée, Pseudina; Pseudina velox Hiibuer, Noctua; Metachrost is veluta Druce, Parangitia; Parangitia velutina Hampson, Mclamera; Melamera vacciniae Edwards, Melicleptria;

Pseuclotamila

vaccinii Linnacus, Phalaena; Gloia 
velutina Houlbert, Gaurenopsis; Gaurenopsis velutisigna Hampson, Tephrinops;

Tephrinops

venata Hampson, Varicosia; Varicosia

venosula Staudinger, Lithophasia;

Lithophasia

ventilabris Fabricius, Phalaena; Herminia

ventilator Grote, Marasmalus; Marasmalus

venulia Cramer, Phalaena; Aegocera

venusta Hampson, Egnasia; Eugrapta

venusta Walker, Plusia; Palaeoplusia venustissima Walker, Pacidara; Pacidara venustula Hübner, Phalaena; Hapalotis veprecola Swinhoe, Protheodes;

Protheodes

verna Hampson, Erizada; Chlorozada veronicae Hübner, Noctua; Conistra verruca Fabricius, Noctua; Argyrogramma versicolor Fabricius, Noctua; Chabora versicolor Staudinger, Margelana;

Margelana

versicolor Turner, Esthlodora; Esthlodora versicolor Walker, Autoba; Autoba versicolor Walker, Tolna; Tolna versicolora Saalmüller, Callixena; Callixena versuta Harvey, Pseudorgyia; Pseudorgyia vesiculalis Guenée, Sitophora; Sitophora vespertilio Fabricius, Noctua; Hypopyra vestigialis Hufnagel, Phalaena; Agronoma vetusta Walker, Mythimna; Porosagrotis vetustalis Walker, Docela; Docela vetustella Walker, Eromene; Sorocostia vexataria Walker, Acidalia; Hypenagonia vexatariola Strand, Hypenagoniodes;

Hypenagoniodes

vialis Moore, Acontia; Hyelopsis viatica Grote, Orthosia; Metaxaglaea vicina Staudinger, Caradrina; Eremodrina videns Guenée, Leucania; Platysenta vidua Holland, Eugorna; Eugorna viduella Walker, Coesa; Coesa viettei Berio, Corgathalia; Corgathalia viettei Berio, Parastenopterygia;

Parastenopterygia vieui Viette, Griveaudia; Griveaudia vigasia Schaus, Chloridea; Proteinania vilis Walker, Homoptera; Gnamptonyx villalis Schaus, Neoptista; Neoptista villica Schaus, Tarache; Gonophaea villicosta Hampson, Pyroblemma;

Pyroblemma villicosta Walker, Alpesa; Alpesa villipes Walker, Acroria; Acroria villosa Druce, Narcaea; Narcaea villosus Alphéraky, Comophorus;

Lycophorus

viminalis Fabricius, Noctua; Iteophaga vinctalis Walker, Scopula; Tathorhynchus vindicia Dyar, Via; Via vinosa Hampson, Charidea; Ethiopica viola Hampson, Camptochilus; Ectogonia viola Hampson, Prolymnia; Prolymnia viola Staudinger, Stemmaphora;

Stemmaphora

violacea Prout, Aulocheta; Aulocheta violascens Hampson, Oedicodia; Oedicodia violetta Schaus, Tarache; Euchromalia viralis Grote, Agrotis; Protagrotis virbia Cramer, Phalaena; Mocis virbiusalis Walker, Bleptina; Theotinus virens Linnaeus, Phalaena; Calamia virescens Fabricius, Noctua; Chloridea virescens Hampson, Libyphaenis;

Libyphaenis virescens Hampson, Polia; Bryopolia virgo Butler, Platychasma; Platychasma virgo Treitschke, Abrostola; Goonallica viridana Linnaeus, Phalaena; Tortrix viridaria Clerck, Phalaena; Phytometra viridata Stoll, Phalaena; Fracara viridescens Hampson, Ectolopha;

Ectolopha viridicosta Schaus, Rhyncholita; Lepteria viridifera Grote, Zotheca; Oslaria viridifera Hampson, Pelodesis; Pelodesis viridigrisea Strand, Scopariopsis;

Scopariopsis

viridimicta Hampson, Stenoloba;

Lepidopyrga

viridinotata Hampson, Perciana;

Neopistria

viridipuncta Hampson, Tortriciforma;

Tortriciforma

viridis Druce, Arbostola; Arbostola viridis Leech, Agriopis; Daseochaeta viridis Staudinger, Isochlora; Isochlora viridisquama Walker, Aquis; Aquis viriditrina Berio, Callhyccoda; Callhyccoda viriplaca Hufnagel, Phalaena; Heliothis vitea Swinhoe, Selepa; Lophothripa vitellina Hübner, Noctua; Aletia vitiosa Schaus, Artiloxis; Artiloxis vitrea Grote, Dercetis; Redectis vitta Esper, Phalaena; Metaxyja vittalis IValker, Tamusida; Tamusida vittella Fabricius, Tinea; Aphusia vittifera Hampson, Rhabdotina;

Rhabdotina 
vittigera Hampson, Mamestra; Odontestra vohilava Viette, Catalana; Catalana volloni Lucas, Amathes; Pseudamathes volupia Fitch, Alaria; Rhododipsa vorax Cockerell, Phylledestes; Phylledestes vulgaris Butler, Polydesma; Bambusiphila vulpina Staudinger, Episema; Catasema vuteria Stoll, Phalaena; Speia

wadicola Amsel, Micronola; Micronola walkerii Grote, Dichagramma;

Dichagramma williamsi Schaus, Harrisonia; Agrotisia wilsonii Grote, Ciris; Xerociris wiltshirei Boursin, Evisa; Evanina wimmerii Treitschke, Anthophila;

Phyllophila witzenmanni Standfuss, Orthosia;

Ammopolia w-latinum Hufnagel, Phalaena; Lacanobia wollastoni Rothschild, Crypsotidia;

Crypsotidia wutzdorff Püngeler; Euxestis; Microxestis

xantharia Walker, Pyrinia; Xanthostha xantherythra Hampson, Copifrontia;

Copifrontia

xanthia Hampson, Megalodes; Ochrocalama xanthialis Walker, Tibracana; Tibracana xanthiata Berio, Tricraterifrontia;

Tricraterifrontia xanthindyma Boisduval, Cosmophila;

Cosmophila xanthioides Guenée, Perigea; Perigea xanthoceros Borkhausen, Phalaena;

Polyploca xanthochlora Walker, Cyphanta; Cyphanta xanthographa [Denis \& Schiffermiiller],

Noctua; Segetia xantholepis Dyar, Dyops; Eudyops xantholopha Hampson, Blenina;

Nacrobarasa xanthosticha Turner, Dysapura; Dysapura xanthostola Hampson, Bocula; Aramuna xanthostola Mabille, Agrotis; Caphornia xena Staudinger, Manobia; Niaboma xeneusalis Walker, Geroda; Geroda xerampelina Esper, Phalaena; Atethınia xerampelina Esper sensu Hübner, Noctua;

Cirroedia

xerota Hampson, Pleonotrocta;

Pleonotrocta

xithon Dyar, Paracretonia; Paracretonia xuthophanes Turner, Eccleta; Eccleta xuthosoma Turner, Tarache; Habrophyes xyliniformis Guenée, Acronycta;

Mastiphanes

xylinoides Guenée, Acronycta; Mastiphanes xylinoides Köhler, Riagria; Riagria xylochroa Hampson, Zaleodes; Zaleodes xylodesina Hampson, Brachyona; Brachyona xyloglypta Lucas, Thrypticodes;

Thrypticodes

zacualpana Schaus, Zorothis; Zorothis zanbesita Walker, Calesia; Asymbata zamora Dognin, Paracroma; Paracroma zanderi Felder \& Rogenhofer, Arcyophora;

Gonioxestis zeae Harris, Gortyna; Achatodes zebrina Viette, Perigea; Chopardiana zelleri Christoph, Mamestra; Heterographa zelleri Grote, Asisyra; Asisyra zelotypa Lederer, Xanthia; Brachyxanthia zernyi Berio, Obarza; Obarza zibellina Felder \& Rogenhofer, Dysedia;

Dysedia zonalis Walker, Corgatha; Corgatha zonata Walker, Casamba; Casamba zonella Druce, Miana; Mictochroa zonophora Turner, Anachrostis; Parapadna zopissa Möschler, Buphana; Buphana zothecaea Dyar, Chlorothrix; Chlorothrix zuelana Schaus, Hadena; Catephiodes
1. IV. 13. Nye, Pli.D., B.Sc., D.I.C.

Department of Entomology

British Museum (Natural History)

CrOMWELL ROAD

LONDON $\mathrm{SIV}_{7}{ }_{5} \mathrm{BL}$ 



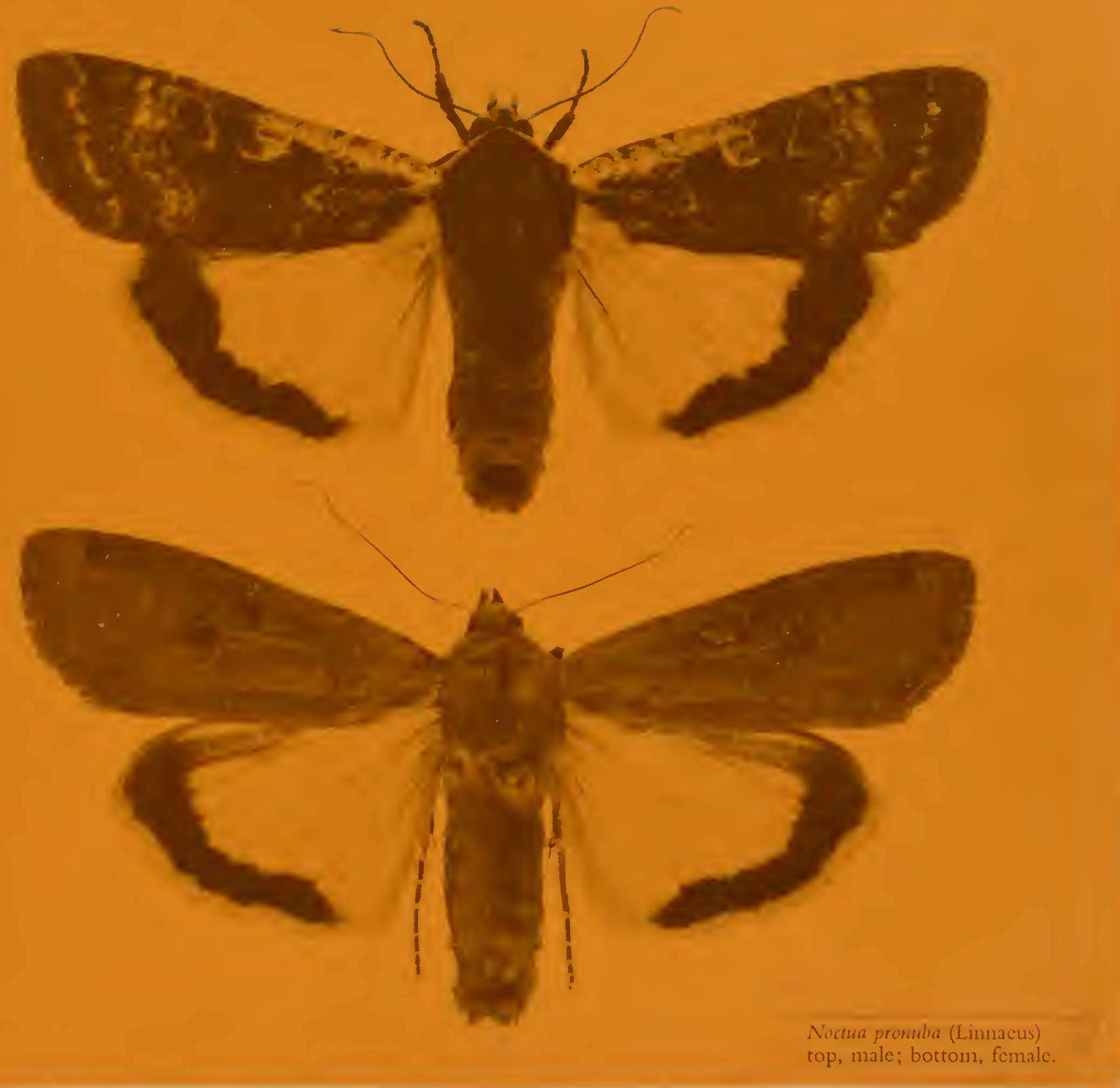


